\title{
The Continuum Limit of Causal Fermion Systems
}

From Planck Scale Structures to Macroscopic Physics

Felix Finster 
Felix Finster

Fakultät für Mathematik

Universität Regensburg

Regensburg

Germany

finster@ur.de 


\section{Preface to the First Online Edition}

In the two years since this book appeared, I got a lot of feedback which led to a number of small corrections and improvements. Although all changes are minor, I decided to replace the book on the arXiv, simply in order to improve readability. I also took the opportunity to correct a few typos. Apart from these small changes, the present online version coincides precisely with the Springer book. In particular, all equation numbers are the same.

I am grateful to all the readers who gave me feedback. More specifically, I would like to thank José Isidro, Maximilian Jokel and Andreas Platzer for helpful comments.

Felix Finster, Berlin, October 2018 



\section{Preface}

This book is devoted to explaining how the causal action principle gives rise to the interactions of the standard model plus gravity on the level of second-quantized fermionic fields coupled to classical bosonic fields. It is the result of an endeavor which I was occupied with for many years. Publishing the methods and results as a book gives me the opportunity to present the material in a coherent and comprehensible way.

The four chapters of this book evolved differently. Chapters 1 and 2 are based on the notes of my lecture "The fermionic projector and causal variational principles" given at the University of Regensburg in the summer semester 2014. The intention of this lecture was to introduce the basic concepts. Most of the material in these two chapters has been published previously, as is made clear in the text by references to the corresponding research articles. We also included exercises in order to facilitate the selfstudy. Chapters 35, however, are extended versions of three consecutive research papers written in the years 2007-2014 (arXiv:0908.1542 [math-ph], arXiv:1211.3351 [math-ph], arXiv:1409.2568 [math-ph]). Thus the results of these chapters are new and have not been published elsewhere. Similarly, the appendix is formed of the appendices of the above-mentioned papers and also contains results of original research.

The fact that Chapters 35 originated from separate research papers is still visible in their style. In particular, each chapter has its own short introduction, where the notation is fixed and some important formulas are stated. Although this leads to some redundancy and a few repetitions, I decided to leave these introductions unchanged, because they might help the reader to revisit the prerequisites of each chapter.

We remark that, having the explicit analysis of the continuum limit in mind, the focus of this book is on the computational side. This entails that more theoretical questions like the existence and uniqueness of solutions of Cauchy problems or the non-perturbative methods for constructing the fermionic projector are omitted. To the reader interested in mathematical concepts from functional analysis and partial differential equations, we can recommend the book "An Introduction to the Fermionic Projector and Causal Fermion Systems" [FKT]. The intention is that the book [FKT] explains the physical ideas in a non-technical way and introduces the mathematical background from a conceptual point of view. It also includes the non-perturbative construction of the fermionic projector in the presence of an external potential and introduces spinors in curved space-time. The present book, on the other hand, focuses on getting a rigorous connection between causal fermion systems and physical systems in Minkowski space. Here we also introduce the mathematical tools and give all the technical and computational details needed for the analysis of the continuum limit. With this different perspective, the two books should complement each other and when combined should give a mathematically and physically convincing introduction to causal fermion systems and to the analysis of the causal action principle in the continuum limit. 
We point out that the connection to quantum field theory (in particular to secondquantized bosonic fields) is not covered in this book. The reader interested in this direction is referred to [F17] and [F20.

I would like to thank the participants of the spring school "Causal fermion systems" hold in Regensburg in March 2016 for their interest and feedback. Moreover, I am grateful to David Cherney, Andreas Grotz, Christian Hainzl, Johannes Kleiner, Simone Murro, Joel Smoller and Alexander Strohmaier for helpful discussions and valuable comments on the manuscript. Special thanks goes to Johannes Kleiner for suggesting many of the exercises. I would also like to thank the Max Planck Institute for Mathematics in the Sciences in Leipzig and the Center of Mathematical Sciences and Applications at Harvard University for hospitality while I was working on the manuscript. I am grateful to the Deutsche Forschungsgemeinschaft (DFG) for financial support.

Felix Finster, Regensburg, May 2016 


\section{Contents}

Preface to the First Online Edition

Preface vii

Chapter 1. Causal Fermion Systems - An Overview

1.1. The Abstract Framework 1

1.1.1. Basic Definitions 1

1.1.2. Space-Time and Causal Structure 3

1.1.3. The Kernel of the Fermionic Projector

1.1.4. Wave Functions and Spinors 6

1.1.5. The Fermionic Projector on the Krein Space 8

1.1.6. Geometric Structures 9

1.1.7. Topological Structures 12

1.2. Correspondence to Minkowski Space 13

1.2.1. Concepts Behind the Construction of Causal Fermion Systems 13

1.2.2. Introducing an Ultraviolet Regularization 18

1.2.3. Correspondence of Space-Time 20

1.2.4. Correspondence of Spinors and Physical Wave Functions 22

1.2.5. Correspondence of the Causal Structure 25

1.3. Underlying Physical Principles 34

1.4. The Dynamics of Causal Fermion Systems 35

1.4.1. The Euler-Lagrange Equations 35

1.4.2. Symmetries and Conserved Surface Layer Integrals 42

1.4.3. The Initial Value Problem and Time Evolution 45

1.5. Limiting Cases 47

1.5.1. The Quasi-Free Dirac Field and Hadamard States 47

1.5.2. Effective Interaction via Classical Gauge Fields 49

1.5.3. Effective Interaction via Bosonic Quantum Fields 53

Exercises 55

Chapter 2. Computational Tools 65

2.1. The Fermionic Projector in an External Potential 66

2.1.1. The Fermionic Projector of the Vacuum 66

2.1.2. The External Field Problem 67

2.1.3. Main Ingredients to the Construction 67

2.1.4. The Perturbation Expansion of the Causal Green's Functions 71

2.1.5. Computation of Operator Products 74

2.1.6. The Causal Perturbation Expansion 78

2.1.7. Introducing Particles and Anti-Particles 83

2.2. The Light-Cone Expansion 84 
2.2.1. Basic Definition 84

2.2.2. Inductive Light-Cone Expansion of the Green's Functions 85

2.2.3. Structural Results for Chiral Potentials 91

2.2.4. Reduction to the Phase-Free Contribution 98

2.2.5. The Residual Argument 108

2.2.6. The Non-Causal Low Energy Contribution 113

2.2.7. The Non-Causal High Energy Contribution 115

2.2.8. The Unregularized Fermionic Projector in Position Space 119

2.3. Description of Linearized Gravity 119

2.4. The Formalism of the Continuum Limit 121

2.4.1. Example: The $i \varepsilon$-Regularization 122

2.4.2. Example: Linear Combinations of $i \varepsilon$-Regularizations 128

2.4.3. Further Regularization Effects 130

2.4.4. The Formalism of the Continuum Limit 131

2.4.5. Outline of the Derivation 134

2.5. Computation of the Local Trace 141

2.6. Spectral Analysis of the Closed Chain 144

2.6.1. Spectral Decomposition of the Regularized Vacuum 144

2.6.2. The Double Null Spinor Frame 147

2.6.3. Perturbing the Spectral Decomposition 150

2.6.4. General Properties of the Spectral Decomposition 151

2.6.5. Spectral Analysis of the Euler-Lagrange Equations 153

Exercises 154

Chapter 3. An Action Principle for an Interacting Fermion System and its Analysis in the Continuum Limit

3.1. Introduction

3.2. An Action Principle for Fermion Systems in Minkowski Space 169

3.3. Assuming a Vacuum Minimizer 172

3.4. Introducing an Interaction 175

3.4.1. A Dirac Equation for the Fermionic Projector 175

3.4.2. The Interacting Dirac Sea 177

3.4.3. Introducing Particles and Anti-Particles 177

3.4.4. The Light-Cone Expansion and Resummation 178

3.4.5. Clarifying Remarks 179

3.4.6. Relation to Other Approaches 181

3.5. The Continuum Limit 183

3.5.1. Weak Evaluation on the Light Cone 183

3.5.2. The Euler-Lagrange Equations in the Continuum Limit 187

3.6. The Euler-Lagrange Equations to Degree Five 191

3.6.1. The Vacuum 191

3.6.2. Chiral Gauge Potentials 195

3.7. The Euler-Lagrange Equations to Degree Four 197

3.7.1. The Axial Current Terms and the Mass Terms 198

3.7.2. The Dirac Current Terms 199

3.7.3. The Logarithmic Poles on the Light Cone 199

3.7.4. A Pseudoscalar Differential Potential 201

3.7.5. A Vector Differential Potential 203 
3.7.6. Recovering the Differential Potentials by a Local Axial Transformation 204

3.7.7. General Local Transformations 207

3.7.8. The Shear Contributions by the Local Axial Transformation 208

3.7.9. Homogeneous Transformations in the High-Frequency Limit 210

3.7.10. The Microlocal Chiral Transformation 217

3.7.11. The Shear Contributions by the Microlocal Chiral Transformation 220

3.8. The Field Equations 222

3.8.1. The Smooth Contributions to the Fermionic Projector at the Origin 222

3.8.2. Violation of Causality and the Vacuum Polarization 226

3.8.3. Higher Order Non-Causal Corrections to the Field Equations 232

3.8.4. The Standard Quantum Corrections to the Field Equations 233

3.8.5. The Absence of the Higgs Boson 238

3.8.6. The Coupling Constant and the Bosonic Mass in Examples 240

3.9. The Euler-Lagrange Equations to Degree Three and Lower 242

3.9.1. Scalar and Pseudoscalar Currents 242

3.9.2. Bilinear Currents and Potentials 243

3.9.3. Further Potentials and Fields 243

3.9.4. The Non-Dynamical Character of the EL Equations to Lower Degree 245]

3.10. Nonlocal Potentials 247

3.10.1. Homogeneous Transformations in the Low-Frequency Regime 247

3.10.2. Homogeneous Perturbations by Varying the Momenta 248

3.10.3. The Analysis of Homogeneous Perturbations on the Light Cone 252

3.10.4. Nonlocal Potentials, the Quasi-Homogeneous Ansatz 257

$\begin{array}{lll}\text { 3.10.5. Discussion and Concluding Remarks } 260 & \end{array}$

Chapter 4. The Continuum Limit of a Fermion System Involving Neutrinos: Weak and Gravitational Interactions $\quad 263$

4.1. Introduction 263

4.2. Regularizing the Neutrino Sector 266

4.2.1. A Naive Regularization of the Neutrino Sector 266

4.2.2. Instability of the Naively Regularized Neutrino Sector 267

4.2.3. Regularizing the Vacuum Neutrino Sector - Introductory Discussion 270

4.2.4. Ruling out the Chiral Neutrino Ansatz 275

4.2.5. A Formalism for the Regularized Vacuum Fermionic Projector 276

4.2.6. Interacting Systems, Regularization of the Light-Cone Expansion 279

4.2.7. The $\iota$-Formalism 282

4.3. The Euler-Lagrange Equations to Degree Five 285

4.3.1. The Vacuum 285

4.3.2. The Gauge Phases 287

4.4. The Euler-Lagrange Equations to Degree Four 293

4.4.1. General Structural Results 293

\begin{tabular}{ll} 
4.4.2. The Vacuum 296 \\
\hline
\end{tabular}

4.4.3. The Current and Mass Terms 297

4.4.4. The Microlocal Chiral Transformation 300

4.4.5. The Shear Contributions 308

4.5. The Energy-Momentum Tensor and the Curvature Terms 311

4.5.1. The Energy-Momentum Tensor of the Dirac Field 311

4.5.2. The Curvature Terms 312 
4.5.3. The Energy-Momentum Tensor of the Gauge Field 315

4.6. Structural Contributions to the Euler-Lagrange Equations 319

4.6.1. The Bilinear Logarithmic Terms 319

4.6.2. The Field Tensor Terms 325

4.7. The Effective Action in the Continuum Limit 329

4.7.1. Treating the Algebraic Constraints 329

4.7.2. The Effective Dirac Action 333

4.7.3. Varying the Effective Dirac Action 335

4.8. The Field Equations for Chiral Gauge Fields 339

4.9. The Einstein Equations 342

Chapter 5. The Continuum Limit of a Fermion System Involving Leptons and

Quarks: Strong, Electroweak and Gravitational Interactions 347

5.1. Introduction 347

5.2. Preliminaries 348

5.2.1. The Fermionic Projector and its Perturbation Expansion 348

5.2.2. Chiral Gauge Potentials and Gauge Phases 351

5.2.3. The Microlocal Chiral Transformation 352

5.2.4. The Causal Action Principle 353

5.3. Spontaneous Block Formation 354

5.3.1. The Statement of Spontaneous Block Formation 354

5.3.2. The Sectorial Projection of the Chiral Gauge Phases 356

5.3.3. The Bilinear Logarithmic Terms 361

5.3.4. The Field Tensor Terms 362

5.3.5. Proof of Spontaneous Block Formation 363

Left-handed $\mathfrak{s u}(2)$-potentials 363

Arranging the free gauge group of maximal dimension 364

Proof that $\mathfrak{g}$ is maximal $\quad 366$

Non-triviality of the mixing matrices $\quad 369$

Proof that $\mathfrak{g}$ is admissible 369

5.4. The Effective Action 370

5.4.1. The General Strategy 370

5.4.2. The Effective Lagrangian for Chiral Gauge Fields 371

General structure of the effective Lagrangian 371

Correspondence to electroweak theory 373

Additional relations between the regularization parameters 375

5.4.3. The Effective Lagrangian for Gravity 381

5.5. The Higgs Field 383

Appendix A. Testing on Null Lines 385

Appendix B. Spectral Analysis of the Closed Chain 393

B.1. The General Procedure 393

B.2. Vector and Axial Contributions 394

B.3. Scalar and Pseudoscalar Contributions 399

B.4. Shear Contributions 399

B.5. The Energy-Momentum Tensor of Chiral Gauge Fields 400

Appendix C. Ruling out the Local Axial Transformation 403 
Appendix D. Resummation of the Current and Mass Terms at the Origin 409

Appendix E. The Weight Factors $\rho_{\beta}$

Appendix F. The Regularized Causal Perturbation Theory with Neutrinos 419

F.1. The General Setting 419

F.2. Formal Introduction of the Interaction 421

F.3. Compatibility Conditions for the Interaction 422

F.4. The Causal Perturbation Expansion with Regularization 426

F.5. The Behavior under Gauge Transformations 427

F.6. The Regularized Light-Cone Expansion 428

Bibliography 431

Notation Index

Subject Index

Back Cover 



\section{CHAPTER 1}

\section{Causal Fermion Systems - An Overview}

Causal fermion systems were introduced in [FGS] as a reformulation and generalization of the setting used in the fermionic projector approach [F7]. In the meantime, the theory of causal fermion systems has evolved to an approach to fundamental physics. It gives quantum mechanics, general relativity and quantum field theory as limiting cases and is therefore a candidate for a unified physical theory. In this chapter, we introduce the mathematical framework and give an overview of the different limiting cases. The presentation is self-contained and includes references to the corresponding research papers. The aim is not only to convey the underlying physical picture, but also to lay the mathematical foundations in a conceptually convincing way. This includes technical issues like specifying the topologies on the different spaces of functions and operators, giving a mathematical definition of an ultraviolet regularization, or specifying the maps which identify the objects of the causal fermion system with corresponding objects in Minkowski space. Also, we use a basis-independent notation whenever possible. The reader interested in a non-technical introduction is referred to [FK1].

\subsection{The Abstract Framework}

1.1.1. Basic Definitions. For conceptual clarity, we begin with the general definitions.

DEFinition 1.1.1. (causal fermion system) Given a separable complex Hilbert space $\mathcal{H}$ with scalar product $\langle. \mid .\rangle_{\mathcal{H}}$ and a parameter $n \in \mathbb{N}$ (the "spin dimension"), we let $\mathcal{F} \subset \mathrm{L}(\mathcal{H})$ be the set of all self-adjoint operators on $\mathcal{H}$ of finite rank, which (counting multiplicities) have at most $n$ positive and at most $n$ negative eigenvalues. On $\mathcal{F}$ we are given a positive measure $\rho$ (defined on a $\sigma$-algebra of subsets of $\mathcal{F}$ ), the so-called universal measure. We refer to $(\mathcal{H}, \mathcal{F}, \rho)$ as a causal fermion system.

We remark that the separability of the Hilbert space (i.e. the assumption that $\mathcal{H}$ admits an at most countable Hilbert space basis) is not essential and could be left out. We included the separability assumption because it seems to cover all cases of physical interest and is useful if one wants to work with basis representations. A simple example of a causal fermion system is given in Exercise 1.1 .

As will be explained in detail in this book, a causal fermion system describes a spacetime together with all structures and objects therein (like the causal and metric structures, spinors and interacting quantum fields). In order to single out the physically admissible causal fermion systems, one must formulate physical equations. To this end, we impose that the universal measure should be a minimizer of the causal action principle, which we now introduce. For any $x, y \in \mathcal{F}$, the product $x y$ is an operator of rank at most $2 n$. We denote its non-trivial eigenvalues counting algebraic multiplicities by $\lambda_{1}^{x y}, \ldots, \lambda_{2 n}^{x y} \in \mathbb{C}$ (more specifically, denoting the rank of $x y$ by $k \leq 2 n$, we choose $\lambda_{1}^{x y}, \ldots, \lambda_{k}^{x y}$ as all the non-zero eigenvalues and set $\left.\lambda_{k+1}^{x y}, \ldots, \lambda_{2 n}^{x y}=0\right)$. We introduce the spectral weight $|$. 
of an operator as the sum of the absolute values of its eigenvalues. In particular, the spectral weights of the operator products $x y$ and $(x y)^{2}$ are defined by

$$
|x y|=\sum_{i=1}^{2 n}\left|\lambda_{i}^{x y}\right| \quad \text { and } \quad\left|(x y)^{2}\right|=\sum_{i=1}^{2 n}\left|\lambda_{i}^{x y}\right|^{2} .
$$

We introduce the Lagrangian and the causal action by

$$
\begin{aligned}
& \text { Lagrangian: } & \mathcal{L}(x, y) & =\left|(x y)^{2}\right|-\frac{1}{2 n}|x y|^{2} \\
& \text { causal action: } & \mathcal{S}(\rho) & =\iint_{\mathcal{F} \times \mathcal{F}} \mathcal{L}(x, y) d \rho(x) d \rho(y) .
\end{aligned}
$$

The causal action principle is to minimize $\mathcal{S}$ by varying the universal measure under the following constraints:

$$
\begin{array}{rc}
\text { volume constraint: } & \rho(\mathcal{F})=\text { const } \\
\text { trace constraint: } & \int_{\mathcal{F}} \operatorname{tr}(x) d \rho(x)=\text { const } \\
\text { boundedness constraint: } & \mathcal{T}(\rho):=\iint_{\mathcal{F} \times \mathcal{F}}|x y|^{2} d \rho(x) d \rho(y) \leq C,
\end{array}
$$

where $C$ is a given parameter (and tr denotes the trace of a linear operator on $\mathcal{H}$ ).

In order to make the causal action principle mathematically well-defined, one needs to specify the class of measures in which to vary $\rho$. To this end, on $\mathcal{F}$ we consider the topology induced by the operator norm

$$
\|A\|:=\sup \left\{\|A u\|_{\mathcal{H}} \text { with }\|u\|_{\mathcal{H}}=1\right\} .
$$

In this topology, the Lagrangian as well as the integrands in (1.1.4) and (1.1.5) are continuous. The $\sigma$-algebra generated by the open sets of $\mathcal{F}$ consists of the so-called Borel sets. A regular Borel measure is a measure on the Borel sets with the property that it is continuous under approximations by compact sets from inside and by open sets from outside (for basics see for example $[\mathbf{H a}, \S 52]$ ). The right prescription is to vary $\rho$ within the class of regular Borel measures on $\mathcal{F}$. In the so-called finite-dimensional setting when $\mathcal{H}$ is finite-dimensional and the total volume $\rho(\mathcal{F})$ is finite, the existence of minimizers is proven in $[\mathbf{F 1 0}, \mathbf{F 1 3}$, and the properties of minimizing measures are analyzed in $\mathbf{F S}, \mathbf{B F}$.

The causal action principle is ill-posed if the total volume $\rho(\mathcal{F})$ is finite and the Hilbert space $\mathcal{H}$ is infinite-dimensional (see Exercises 1.2 and 1.3). But the causal action principle does make mathematical sense in the so-called infinite-dimensional setting when $\mathcal{H}$ is infinite-dimensional and the total volume $\rho(\mathcal{F})$ is infinite. In this case, the volume constraint 1.1.3 is implemented by demanding that all variations $(\rho(\tau))_{\tau \in(-\varepsilon, \varepsilon)}$ should for all $\tau, \tau^{\prime} \in(-\varepsilon, \varepsilon)$ satisfy the conditions

$$
\left|\rho(\tau)-\rho\left(\tau^{\prime}\right)\right|(\mathcal{F})<\infty \quad \text { and } \quad\left(\rho(\tau)-\rho\left(\tau^{\prime}\right)\right)(\mathcal{F})=0
$$

(where |.| denotes the total variation of a measure; see [Ha, §28]). The existence theory in the infinite-dimensional setting has not yet been developed. But it is known that the Euler-Lagrange equations corresponding to the causal action principle still have a mathematical meaning (as will be explained in 1.4 .1 below). This will make it possible to analyze the causal action principle without restrictions on the dimension of $\mathcal{H}$ nor on 
the total volume. One way of getting along without an existence theory in the infinitedimensional setting is to take the point of view that on a fundamental physical level, the total volume is finite and the Hilbert space $\mathcal{H}$ is finite-dimensional, whereas the infinitedimensional setting merely is a mathematical idealization needed in order to describe systems in infinite volume involving an infinite number of quantum particles.

We finally explain the significance of the constraints. Generally speaking, the constraints $1.1 .3-1.1 .5$ are needed to avoid trivial minimizers and in order for the variational principle to be well-posed. More specifically, if we dropped the constraint of fixed total volume 1.1.3, the measure $\rho=0$ would be a trivial minimizer. Without the boundedness constraint (1.1.5), the loss of compactness discussed in [F13, Section 2.2] implies that no minimizers exist (see Exercises 1.2 and 1.4). If, on the other hand, we dropped the trace constraint 1.1.4, a trivial minimizer could be constructed as follows: We let $x$ be the operator with the matrix representation

$$
x=\operatorname{diag}(\underbrace{1, \ldots, 1}_{n \text { times }}, \underbrace{-1, \ldots,-1}_{n \text { times }}, 0,0, \ldots)
$$

and choose $\rho$ as a multiple of the Dirac measure supported at $x$. Then $\mathcal{T}>0$ but $\mathcal{S}=0$.

1.1.2. Space-Time and Causal Structure. A causal fermion system $(\mathcal{H}, \mathcal{F}, \rho)$ encodes a large amount of information. In order to recover this information, one can for example form products of linear operators in $\mathcal{F}$, compute the eigenvalues of such operator products and integrate expressions involving these eigenvalues with respect to the universal measure. However, it is not obvious what all this information means. In order to clarify the situation, we now introduce additional mathematical objects. These objects are inherent in the sense that we only use information already encoded in the causal fermion system.

We define space-time, denoted by $M$, as the support of the universal measure ${ }^{1}$,

$$
M:=\operatorname{supp} \rho \subset \mathcal{F} .
$$

Thus the space-time points are symmetric linear operators on $\mathcal{H}$. On $M$ we consider the topology induced by $\mathcal{F}$ (generated by the sup-norm 1.1 .6 on $\mathrm{L}(\mathcal{H})$ ). Moreover, the universal measure $\left.\rho\right|_{M}$ restricted to $M$ can be regarded as a volume measure on spacetime. This makes space-time to a topological measure space. Furthermore, one has the following notion of causality:

Definition 1.1.2. (causal structure) For any $x, y \in \mathcal{F}$, the product $x y$ is an operator of rank at most $2 n$. We denote its non-trivial eigenvalues (counting algebraic multiplicities) by $\lambda_{1}^{x y}, \ldots, \lambda_{2 n}^{x y}$. The points $x$ and $y$ are called spacelike separated if all the $\lambda_{j}^{x y}$ have the same absolute value. They are said to be timelike separated if the $\lambda_{j}^{x y}$ are all real and do not all have the same absolute value. In all other cases (i.e. if the $\lambda_{j}^{x y}$ are not all real and do not all have the same absolute value), the points $x$ and $y$ are said to be lightlike separated.

Restricting the causal structure of $\mathcal{F}$ to $M$, we get causal relations in space-time. To avoid confusion, we remark that in earlier papers (see [FG2, [FGS]) a slightly different

${ }^{1}$ The support of a measure is defined as the complement of the largest open set of measure zero, i.e.

$$
\operatorname{supp} \rho:=\mathcal{F} \backslash \bigcup\{\Omega \subset \mathcal{F} \mid \Omega \text { is open and } \rho(\Omega)=0\} .
$$

It is by definition a closed set. This definition is illustrated in Exercise 1.5 
definition of the causal structure was used. But the modified definition used here seems preferable.

The Lagrangian (1.1.1) is compatible with the above notion of causality in the following sense (the correspondence to the causal structure of Minkowski space and the notion of lightlike separation will be explained in Section 1.2 .5 below). Suppose that two points $x, y \in \mathcal{F}$ are spacelike separated. Then the eigenvalues $\lambda_{i}^{x y}$ all have the same absolute value. Rewriting (1.1.1) as

$$
\mathcal{L}=\sum_{i=1}^{2 n}\left|\lambda_{i}^{x y}\right|^{2}-\frac{1}{2 n} \sum_{i, j=1}^{2 n}\left|\lambda_{i}^{x y}\right|\left|\lambda_{j}^{x y}\right|=\frac{1}{4 n} \sum_{i, j=1}^{2 n}\left(\left|\lambda_{i}^{x y}\right|-\left|\lambda_{j}^{x y}\right|\right)^{2},
$$

one concludes that the Lagrangian vanishes. Thus pairs of points with spacelike separation do not enter the action. This can be seen in analogy to the usual notion of causality where points with spacelike separation cannot influence each other ${ }^{2}$, This analogy is the reason for the notion "causal" in "causal fermion system" and "causal action principle."

The above notion of causality is symmetric in $x$ and $y$, as we now explain. Since the trace is invariant under cyclic permutations, we know that

$$
\operatorname{tr}\left((x y)^{p}\right)=\operatorname{tr}\left(x(y x)^{p-1} y\right)=\operatorname{tr}\left((y x)^{p-1} y x\right)=\operatorname{tr}\left((y x)^{p}\right)
$$

(where tr again denotes the trace of a linear operator on $\mathcal{H}$ ). Since all our operators have finite rank, there is a finite-dimensional subspace $I$ of $\mathcal{H}$ such that $x y$ maps $I$ to itself and vanishes on the orthogonal complement of $I$. Then the non-trivial eigenvalues of the operator product $x y$ are given as the zeros of the characteristic polynomial of the restriction $\left.x y\right|_{I}: I \rightarrow I$. The coefficients of this characteristic polynomial (like the trace, the determinant, etc.) are symmetric polynomials in the eigenvalues and can therefore be expressed in terms of traces of powers of $x y$. As a consequence, the identity (1.1.10) implies that the operators $x y$ and $y x$ have the same characteristic polynomial and are thus isospectral. This shows that our notions of causality are indeed symmetric in the sense that $x$ and $y$ are spacelike separated if and only if $y$ and $x$ are (and similarly for timelike and lightlike separation). One also sees that the Lagrangian $\mathcal{L}(x, y)$ is symmetric in its two arguments.

A causal fermion system also distinguishes a direction of time. To this end, we let $\pi_{x}$ be the orthogonal projection in $\mathcal{H}$ on the subspace $x(\mathcal{H}) \subset \mathcal{H}$ and introduce the functional

$$
\mathcal{C}: M \times M \rightarrow \mathbb{R}, \quad \mathcal{C}(x, y):=i \operatorname{tr}\left(y x \pi_{y} \pi_{x}-x y \pi_{x} \pi_{y}\right)
$$

(this functional was first stated in [FK, Section 8.5], motivated by constructions in [FG2, Section 3.5]). Obviously, this functional is anti-symmetric in its two arguments. This makes it possible to introduce the notions

$$
\left\{\begin{array}{cl}
y \text { lies in the future of } x & \text { if } \mathcal{C}(x, y)>0 \\
y \text { lies in the past of } x & \text { if } \mathcal{C}(x, y)<0 .
\end{array}\right.
$$

By distinguishing a direction of time, we get a structure similar to a causal set (see for example [BLMS]). But in contrast to a causal set, our notion of "lies in the future of" is not necessarily transitive. This corresponds to our physical conception that the transitivity of the causal relations could be violated both on the cosmological scale (there might be closed timelike curves) and on the microscopic scale (there seems no compelling reason why the causal relations should be transitive down to the Planck scale). This

\footnotetext{
${ }^{2}$ For clarity, we point out that our notion of causality does allow for nonlocal correlations and entanglement between regions with space-like separation. This will become clear in $\$ 1.1 .4$ and $\$ 1.5 .3$
} 
is the reason why we consider other structures (namely the universal measure and the causal action principle) as being more fundamental. In our setting, causality merely is a derived structure encoded in the causal fermion system.

In Exercise 1.6, the causal structure is studied in the example of Exercise 1.1.

1.1.3. The Kernel of the Fermionic Projector. The causal action principle depends crucially on the eigenvalues of the operator product $x y$ with $x, y \in \mathcal{F}$. For computing these eigenvalues, it is convenient not to consider this operator product on the (possibly infinite-dimensional) Hilbert space $\mathcal{H}$, but instead to restrict attention to a finite-dimensional subspace of $\mathcal{H}$, chosen such that the operator product vanishes on the orthogonal complement of this subspace. This construction leads us to the spin spaces and to the kernel of the fermionic projector, which we now introduce. For every $x \in \mathcal{F}$ we define the spin space $S_{x}$ by $S_{x}=x(\mathcal{H})$; it is a subspace of $\mathcal{H}$ of dimension at most $2 n$. For any $x, y \in M$ we define the kernel of the fermionic operator $P(x, y)$ by

$$
P(x, y)=\left.\pi_{x} y\right|_{S_{y}}: S_{y} \rightarrow S_{x}
$$

(where $\pi_{x}$ is again the orthogonal projection on the subspace $x(\mathcal{H}) \subset \mathcal{H}$ ). Taking the trace of 1.1.13) in the case $x=y$, one finds that $\operatorname{tr}(x)=\operatorname{Tr}_{S_{x}}(P(x, x))$, making it possible to express the integrand of the trace constraint (1.1.4) in terms of the kernel of the fermionic operator. In order to also express the eigenvalues of the operator $x y$, we introduce the closed chain $A_{x y}$ as the product

$$
A_{x y}=P(x, y) P(y, x): S_{x} \rightarrow S_{x} .
$$

Computing powers of the closed chain, one obtains

$$
A_{x y}=\left.\left(\pi_{x} y\right)\left(\pi_{y} x\right)\right|_{S_{x}}=\left.\pi_{x} y x\right|_{S_{x}}, \quad\left(A_{x y}\right)^{p}=\left.\pi_{x}(y x)^{p}\right|_{S_{x}} .
$$

Taking the trace, one sees in particular that $\operatorname{Tr}_{S_{x}}\left(A_{x y}^{p}\right)=\operatorname{tr}\left((y x)^{p}\right)$. Repeating the arguments after (1.1.10), one concludes that the eigenvalues of the closed chain coincide with the non-trivial eigenvalues $\lambda_{1}^{x y}, \ldots, \lambda_{2 n}^{x y}$ of the operator $x y$ in Definition 1.1.2. Therefore, the kernel of the fermionic operator encodes the causal structure of $M$. The main advantage of working with the kernel of the fermionic operator is that the closed chain (1.1.14 is a linear operator on a vector space of dimension at most $2 n$, making it possible to compute the $\lambda_{1}^{x y}, \ldots, \lambda_{2 n}^{x y}$ as the eigenvalues of a finite matrix.

Next, it is very convenient to arrange that the kernel of the fermionic operator is symmetric in the sense that

$$
P(x, y)^{*}=P(y, x) .
$$

To this end, one chooses on the spin space $S_{x}$ the spin scalar product $\prec$.|. $\succ_{x}$ by

$$
\left.\prec u \mid v \succ_{x}=-\langle u \mid x v\rangle_{\mathcal{H}} \quad \text { (for all } u, v \in S_{x}\right) .
$$

Due to the factor $x$ on the right, this definition really makes the kernel of the fermionic operator symmetric, as is verified by the computation

$$
\begin{aligned}
\prec u \mid P(x, y) v \succ_{x} & =-\langle u \mid x P(x, y) v\rangle_{\mathcal{H}}=-\langle u \mid x y v\rangle_{\mathcal{H}} \\
& =-\left\langle\pi_{y} x u \mid y v\right\rangle_{\mathcal{H}}=\prec P(y, x) u \mid v \succ_{y}
\end{aligned}
$$

(where $u \in S_{x}$ and $\left.v \in S_{y}\right)$. The spin space $\left(S_{x}, \prec . \mid \cdot \succ_{x}\right)$ is an indefinite inner product of signature $(p, q)$ with $p, q \leq n$ (for textbooks on indefinite inner product spaces see [B2, GLR ). In this way, indefinite inner product spaces arise naturally when analyzing the mathematical structure of the causal action principle. 
The kernel of the fermionic operator as defined by $(1.1 .13)$ is also referred to as the kernel of the fermionic projector, provided that suitable normalization conditions are satisfied. Different normalization conditions have been proposed and analyzed (see the discussion in [FT2, Section 2.2]). More recently, it was observed in [FK2 that one of these normalization conditions is automatically satisfied if the universal measure is a minimizer of the causal action principle (see $\$ 1.4 .2$ below). With this in mind, we no longer need to be so careful about the normalization. For notational simplicity, we always refer to $P(x, y)$ as the kernel of the fermionic projector.

1.1.4. Wave Functions and Spinors. For clarity, we sometimes denote the spin space $S_{x}$ at a space-time point $x \in M$ by $S_{x} M$. A wave function $\psi$ is defined as a function which to every $x \in M$ associates a vector of the corresponding spin space,

$$
\psi: M \rightarrow \mathcal{H} \quad \text { with } \quad \psi(x) \in S_{x} M \quad \text { for all } x \in M .
$$

We now want to define what we mean by continuity of a wave function. For the notion of continuity, we need to compare the wave function at different space-time points, being vectors $\psi(x) \in S_{x} M$ and $\psi(y) \in S_{y} M$ in different spin spaces. Using that both spin spaces $S_{x} M$ and $S_{y} M$ are subspaces of the same Hilbert space $\mathcal{H}$, an obvious idea is to simply work with the Hilbert space norm $\|\psi(x)-\psi(y)\|_{\mathcal{H}}$. However, in view of the factor $x$ in the spin scalar product (1.1.16), it is preferable to insert a corresponding power of the operator $x$. Namely, the natural norm on the spin space $\left(S_{x}, \prec . \mid . \succ_{x}\right)$ is given by

$$
|\psi(x)|_{x}^{2}:=\langle\psi(x)|| x \mid \psi(x)\rangle_{\mathcal{H}}=\|\sqrt{|x|} \psi(x)\|_{\mathcal{H}}^{2}
$$

(where $|x|$ is the absolute value of the symmetric operator $x$ on $\mathcal{H}$, and $\sqrt{|x|}$ is the square root thereof). This leads us to defining that the wave function $\psi$ is continuous at $x$ if for every $\varepsilon>0$ there is $\delta>0$ such that

$$
\|\sqrt{|y|} \psi(y)-\sqrt{|x|} \psi(x)\|_{\mathcal{H}}<\varepsilon \quad \text { for all } y \in M \text { with }\|y-x\| \leq \delta .
$$

Likewise, $\psi$ is said to be continuous on $M$ if it is continuous at every $x \in M$. We denote the set of continuous wave functions by $C^{0}(M, S M)$. Clearly, the space of continuous wave functions is a complex vector space with pointwise operations, i.e. $(\alpha \psi+\beta \phi)(x):=$ $\alpha \psi(x)+\beta \phi(x)$ with $\alpha, \beta \in \mathbb{C}$.

It is an important observation that every vector $u \in \mathcal{H}$ of the Hilbert space gives rise to a unique wave function. To obtain this wave function, denoted by $\psi^{u}$, we simply project the vector $u$ to the corresponding spin spaces,

$$
\psi^{u}: M \rightarrow \mathcal{H}, \quad \psi^{u}(x)=\pi_{x} u \in S_{x} M .
$$

We refer to $\psi^{u}$ as the physical wave function of $u \in \mathcal{H}$. The estimate

$$
\begin{gathered}
\left\|\sqrt{|y|} \psi^{u}(y)-\sqrt{|x|} \psi^{u}(x)\right\|_{\mathcal{H}}=\|\sqrt{|y|} u-\sqrt{|x|} u\|_{\mathcal{H}} \\
\leq\|\sqrt{|y|}-\sqrt{|x|}\|\|u\|_{\mathcal{H}} \stackrel{(\star)}{\leq}\|y-x\|^{\frac{1}{4}}\|y+x\|^{\frac{1}{4}}\|u\|_{\mathcal{H}}
\end{gathered}
$$

shows that $\psi^{u}$ is indeed continuous (for the inequality $(\star)$ see Exercise 1.7). The physical picture is that the physical wave functions $\psi^{u}$ are those wave functions which are realized in the physical system. Using a common physical notion, one could say that the vectors in $\mathcal{H}$ correspond to the "occupied states" of the system, and that an occupied state $u \in$ $\mathcal{H}$ is represented in space-time by the corresponding physical wave function $\psi^{u}$. The shortcoming of this notion is that an "occupied state" is defined only for free quantum 
fields, whereas the physical wave functions are defined also in the interacting theory. For this reason, we prefer not use the notion of "occupied states."

For a convenient notation, we also introduce the wave evaluation operator $\Psi$ as an operator which to every Hilbert space vector associates the corresponding physical wave function,

$$
\Psi: \mathcal{H} \rightarrow C^{0}(M, S M), \quad u \mapsto \psi^{u} .
$$

Evaluating at a fixed space-time point gives the mapping

$$
\Psi(x): \mathcal{H} \rightarrow S_{x} M, \quad u \mapsto \psi^{u}(x) .
$$

The kernel of the fermionic projector can be expressed in terms of the wave evaluation operator:

Lemma 1.1.3. For any $x, y \in M$,

$$
\begin{aligned}
x & =-\Psi(x)^{*} \Psi(x) \\
P(x, y) & =-\Psi(x) \Psi(y)^{*} .
\end{aligned}
$$

Proof. For any $v \in S_{x} M$ and $u \in \mathcal{H}$,

$$
\prec v\left|\Psi(x) u \succ_{x}=\prec v\right| \pi_{x} u \succ_{x} \stackrel{1.1 .16}{-}-\langle v \mid x u\rangle_{\mathcal{H}}=\langle(-x) v \mid u\rangle_{\mathcal{H}}
$$

and thus

$$
\Psi(x)^{*}=-\left.x\right|_{S_{x} M}: S_{x} M \rightarrow \mathcal{H}
$$

Hence

$$
\Psi(x)^{*} \Psi(x) u=\Psi(x)^{*} \psi_{x}^{u}=-x \psi_{x}^{u \stackrel{1.1 .18}{-}}-x \pi_{x} u=-x u,
$$

proving 1.1.21). Similarly, the relation 1.1.22) follows from the computation

$$
\Psi(x) \Psi(y)^{*}=-\left.\pi_{x} y\right|_{S_{y}}=-P(x, y) .
$$

This completes the proof.

The structure of the wave functions (1.1.17) taking values in the spin spaces is reminiscent of sections of a vector bundle. The only difference is that our setting is more general in that the base space $M$ does not need to be a manifold, and the fibres $S_{x} M$ do not need to depend smoothly on the base point $x$. However, comparing to the setting of spinors in Minkowski space or on a Lorentzian manifold, one important structure is missing: we have no Dirac matrices and no notion of Clifford multiplication. The following definition is a step towards introducing these additional structures.

Definition 1.1.4. (Clifford subspace) We denote the space of symmetric linear operators on $\left(S_{x}, \prec \cdot \mid \cdot \succ_{x}\right)$ by $\operatorname{Symm}\left(S_{x}\right) \subset \mathrm{L}\left(S_{x}\right)$. A subspace $K \subset \operatorname{Symm}\left(S_{x}\right)$ is called a Clifford subspace of signature $(r, s)$ at the point $x$ (with $r, s \in \mathbb{N}_{0}$ ) if the following conditions hold:

(i) For any $u, v \in K$, the anti-commutator $\{u, v\} \equiv u v+v u$ is a multiple of the identity on $S_{x}$.

(ii) The bilinear form $\langle.,$.$\rangle on K$ defined by

$$
\frac{1}{2}\{u, v\}=\langle u, v\rangle \mathbb{1} \quad \text { for all } u, v \in K
$$

is non-degenerate and has signature $(r, s)$. 
In view of the anti-commutation relations (1.1.23), a Clifford subspace can be regarded as a generalization of the space spanned by the usual Dirac matrices. However, the above definition has two shortcomings: First, there are many different Clifford subspaces, so that there is no unique notion of Clifford multiplication. Second, we are missing the structure of tangent vectors as well as a mapping which would associate a tangent vector to an element of the Clifford subspace.

These shortcomings can be overcome by using either geometric or measure-theoretic methods. In the geometric approach, one gets along with the non-uniqueness of the Clifford subspaces by working with suitable equivalence classes. Using geometric information encoded in the causal fermion system, one can then construct mappings between the equivalence classes at different space-time points. This method will be outlined in $\$ 1.1 .6$. In the measure-theoretic approach, on the other hand, one uses the local form of the universal measure with the aim of constructing a unique Clifford subspace at every space-time point. This will be outlined in \$1.1.7. Before entering these geometric and measure-theoretic constructions, we introduce additional structures on the space of wave functions.

1.1.5. The Fermionic Projector on the Krein Space. The space of wave functions can be endowed with an inner product and a topology. The inner product is defined by

$$
<\psi\left|\phi>=\int_{M} \prec \psi(x)\right| \phi(x) \succ_{x} d \rho(x) .
$$

In order to ensure that the last integral converges, we also introduce the scalar product $\langle\langle. \mid\rangle$.$\rangle by$

$$
\langle\langle\psi \mid \phi\rangle\rangle=\int_{M}\langle\psi(x)|| x \mid \phi(x)\rangle_{\mathcal{H}} d \rho(x)
$$

(where $|x|$ is again the absolute value of the symmetric operator $x$ on $\mathcal{H}$ ). The one-particle space $(\mathcal{K},<. \mid .>)$ is defined as the space of wave functions for which the corresponding norm $\||\cdot| \mid$ is finite, with the topology induced by this norm, and endowed with the inner product $<$.|. $>$. Such an indefinite inner product space with a topology induced by an additional scalar product is referred to as a Krein space (see for example [B2, $\mathbf{L}$ ).

When working with the one-particle Krein space, one must keep in mind that the physical wave function $\psi^{u}$ of a vector $u \in \mathcal{H}$ does not need to be a vector in $\mathcal{K}$ because the corresponding integral in 1.1.24 may diverge. Similarly, the scalar product $\left\langle\left\langle\psi^{u} \mid \psi^{u}\right\rangle\right\rangle$ may be infinite. One could impose conditions on the causal fermion system which ensure that the integrals in (1.1.24) and (1.1.25) are finite for all physical wave functions. Then the mapping $u \mapsto \psi^{u}$ would give rise to an embedding $\mathcal{H} \hookrightarrow \mathcal{K}$ of the Hilbert space $\mathcal{H}$ into the one-particle Krein space. However, such conditions seem too restrictive and are not really needed. Therefore, here we shall not impose any conditions on the causal fermion systems but simply keep in mind that the physical wave functions are in general no Krein vectors.

Despite this shortcoming, the Krein space is useful because the kernel of the fermionic projector gives rise to an operator on $\mathcal{K}$. Namely, choosing a suitable dense domain of definition ${ }^{3} \mathcal{D}(P)$, we can regard $P(x, y)$ as the integral kernel of a corresponding

\footnotetext{
${ }^{3}$ For example, one may choose $\mathcal{D}(P)$ as the set of all vectors $\psi \in \mathcal{K}$ satisfying the conditions$$
\phi:=\int_{M} x \psi(x) d \rho(x) \in \mathcal{H} \quad \text { and } \quad\|\phi\|<\infty .
$$ 
operator $P$,

$$
P: \mathcal{D}(P) \subset \mathcal{K} \rightarrow \mathcal{K}, \quad(P \psi)(x)=\int_{M} P(x, y) \psi(y) d \rho(y),
$$

referred to as the fermionic projector. The fermionic projector has the following two useful properties:

- $P$ is symmetric in the sense that $\langle P \psi \mid \phi\rangle=\langle\psi \mid P \phi\rangle$ for all $\psi, \phi \in \mathcal{D}(P)$ :

The symmetry of the kernel of the fermionic projector 1.1.15) implies that

$$
\prec P(x, y) \psi(y)\left|\psi(x) \succ_{x}=\prec \psi(y)\right| P(y, x) \psi(x) \succ_{y} .
$$

Integrating over $x$ and $y$ and applying (1.1.26) and (1.1.24) gives the result.

- $(-P)$ is positive in the sense that $\langle\psi|(-P) \psi>\geq 0$ for all $\psi \in \mathcal{D}(P)$ :

This follows immediately from the calculation

$$
\begin{aligned}
<\psi \mid(-P) \psi> & =-\iint_{M \times M} \prec \psi(x) \mid P(x, y) \psi(y) \succ_{x} d \rho(x) d \rho(y) \\
& =\iint_{M \times M}\left\langle\psi(x) \mid x \pi_{x} y \psi(y)\right\rangle_{\mathcal{H}} d \rho(x) d \rho(y)=\langle\phi \mid \phi\rangle_{\mathcal{H}} \geq 0,
\end{aligned}
$$

where we again used 1.1.24 and 1.1.13 and set

$$
\phi=\int_{M} x \psi(x) d \rho(x) .
$$

In Exercise 1.8 the wave functions and the Krein structure are studied in the example of Exercise 1.1.

1.1.6. Geometric Structures. A causal fermion system also encodes geometric information on space-time. More specifically, in the paper [FG2 notions of connection and curvature are introduced and analyzed. We now outline a few constructions from this paper. Recall that the kernel of the fermionic projector 1.1 .13 is a mapping from one spin space to another, thereby inducing relations between different space-time points. The idea is to use these relations for the construction of a spin connection $D_{x, y}$, being a unitary mapping between the corresponding spin spaces,

$$
D_{x, y}: S_{y} \rightarrow S_{x}
$$

(we consistently use the notation that the subscript $x y$ denotes an object at the point $x$, whereas the additional comma $x, y$ denotes an operator which maps an object at $y$ to an object at $x$ ). The simplest method for constructing the spin connection would be to form a polar decomposition, $P(x, y)=A_{x y}^{-\frac{1}{2}} U$, and to introduce the spin connection as the unitary part, $D_{x, y}=U$. However, this method is too naive, because we want the spin connection to be compatible with a corresponding metric connection $\nabla_{x, y}$ which should map Clifford subspaces at $x$ and $y$ (see Definition 1.1.4 above) isometrically to each other. A complication is that, as discussed at the end of \$1.1.4, the Clifford subspaces at $x$ and $y$ are not unique. The method to bypass these problems is to work with several Clifford subspaces and to use so-called splice maps, as we now briefly explain.

First, it is useful to restrict the freedom in choosing the Clifford subspaces with the following construction. Recall that for any $x \in M$, the operator $(-x)$ on $\mathcal{H}$ has at most $n$ positive and at most $n$ negative eigenvalues. We denote its positive and negative 
spectral subspaces by $S_{x}^{+}$and $S_{x}^{-}$, respectively. In view of 1.1 .16$)$, these subspaces are also orthogonal with respect to the spin scalar product,

$$
S_{x}=S_{x}^{+} \oplus S_{x}^{-} \text {. }
$$

We introduce the Euclidean sign operator $s_{x}$ as a symmetric operator on $S_{x}$ whose eigenspaces corresponding to the eigenvalues \pm 1 are the spaces $S_{x}^{+}$and $S_{x}^{-}$, respectively. Since $s_{x}^{2}=\mathbb{1}$, the span of the Euclidean sign operator is a one-dimensional Clifford subspace of signature $(1,0)$. The idea is to extend $s_{x}$ to obtain higher-dimensional Clifford subspaces. We thus define a Clifford extension as a Clifford subspace which contains $s_{x}$. By restricting attention to Clifford extensions, we have reduced the freedom in choosing Clifford subspaces. However, still there is not a unique Clifford extension, even for fixed dimension and signature. But one can define the tangent space $T_{x}$ as an equivalence class of Clifford extensions; for details see [FG2, Section 3.1]. The bilinear form $\langle.,$. in 1.1.23) induces a Lorentzian metric on the tangent space.

Next, for our constructions to work, we need to assume that the points $x$ and $y$ are both regular and are properly timelike separated, defined as follows:

Definition 1.1.5. A space-time point $x \in M$ is said to be regular if $x$ has the maximal possible rank, i.e. $\operatorname{dim} x(\mathcal{H})=2 n$. Otherwise, the space-time point is called singular.

In most situations of physical interest (like Dirac sea configurations to be discussed in Sections 1.2 and 1.5 below), all space-time points are regular. Singular points, on the other hand, should be regarded as exceptional points or "singularities" of space-time.

Definition 1.1.6. The space-time points $x, y \in M$ are properly timelike separated if the closed chain $A_{x y},(1.1 .14)$, has a strictly positive spectrum and if all eigenspaces are definite subspaces of $\left(S_{x}, \prec . \mid \cdot \succ_{x}\right)$.

By a definite subspace of $S_{x}$ we mean a subspace on which the inner product $\prec . \mid . \succ_{x}$ is either positive or negative definite.

The two following observations explain why the last definition makes sense:

- Properly timelike separation implies timelike separation (see Definition 1.1.2):

Before entering the proof, we give a simple counter example which shows why the assumption of definite eigenspaces in Definition 1.1.6 is necessary for the implication to hold. Namely, if the point $x$ is regular and $A_{x y}$ is the identity, then the eigenvalues $\lambda_{1}, \ldots, \lambda_{2 n}$ are all strictly positive, but they are all equal.

If $I \subset S_{x}$ is a definite invariant subspace of $A_{x y}$, then the restriction $\left.A_{x y}\right|_{I}$ is a symmetric operator on the Hilbert space $\left(I, \pm \prec \cdot \mid \cdot \succ_{I \times I}\right)$, which is diagonalizable with real eigenvalues. Moreover, the orthogonal complement $I^{\perp}$ of $I \subset S_{x}$ is again invariant. If $I^{\perp}$ is non-trivial, the restriction $\left.A_{x y}\right|_{I^{\perp}}$ has at least one eigenspace. Therefore, the assumption in Definition 1.1.6 that all eigenspaces are definite makes it possible to proceed inductively to conclude that the operator $A_{x y}$ is diagonalizable and has real eigenvalues.

If $x$ and $y$ are properly timelike separated, then its eigenvalues are by definition all real and positive. Thus it remains to show that they are not all the same. If conversely they were all the same, i.e. $\lambda_{1}=\cdots=\lambda_{2 n}=\lambda>0$, then $S_{x}$ would necessarily have the maximal dimension $2 n$. Moreover, the fact that $A_{x y}$ is diagonalizable implies that $A_{x y}$ would be a multiple of the identity on $S_{x}$. Therefore, the spin space $\left(S_{x}, \prec . \mid . \succ_{x}\right)$ would have to be definite, in contradiction to the fact that it has signature $(n, n)$. 
- The notion is symmetric in $x$ and $y$ :

Suppose that $A_{x y} u=\lambda u$ with $u \in S_{x}$ and $\lambda>0$. Then the vector $w:=P(y, x) u \in S_{y}$ is an eigenvector of $A_{y x}$ again to the eigenvalue $\lambda$,

$$
\begin{aligned}
A_{y x} w & =P(y, x) P(x, y) P(y, x) u \\
& =P(y, x) A_{x y} u=\lambda P(y, x) u=\lambda w .
\end{aligned}
$$

Moreover, the calculation

$$
\begin{aligned}
\lambda \prec u \mid u \succ & =\prec u\left|A_{x y} u \succ=\prec u\right| P(x, y) P(y, x) u \succ \\
& =\prec P(y, x) u|P(y, x) u \succ=\prec w| w \succ
\end{aligned}
$$

shows that $w$ is a definite vector if and only if $u$ is. We conclude that $A_{y x}$ has positive eigenvalues and definite eigenspaces if and only if $A_{x y}$ has these properties.

So far, the construction of the spin connection has been worked out only in the case of spin dimension $n=2$. Then for two regular and properly timelike separated points $x, y \in$ $M$, the spin space $S_{x}$ can be decomposed uniquely into an orthogonal direct sum $S_{x}=$ $I^{+} \oplus I^{-}$of a two-dimensional positive definite subspace $I^{+}$and a two-dimensional negative definite subspace $I^{-}$of $A_{x y}$. We define the directional sign operator $v_{x y}$ of $A_{x y}$ as the unique operator on $S_{x} M$ such that the eigenspaces corresponding to the eigenvalues \pm 1 are the subspaces $I^{ \pm}$.

Having the Euclidean sign operator $s_{x}$ and the directional sign operator $v_{x y}$ to our disposal, under generic assumptions one can distinguish two Clifford subspaces at the point $x$ : a Clifford subspace $K_{x y}$ containing $v_{x y}$ and a Clifford extension $K_{x}^{(y)}$ (for details see [FG2, Lemma 3.12]). Similarly, at the point $y$ we have a distinguished Clifford subspace $K_{y x}$ (which contains $v_{y x}$ ) and a distinguished Clifford extension $K_{y}^{(x)}$. For the construction of the spin connection $D_{x, y}: S_{y} \rightarrow S_{x}$ one works with the Clifford subspaces $K_{x y}$ and $K_{y x}$ and demands that these are mapped to each other. More precisely, the spin connection is uniquely characterized by the following properties (see [FG2, Theorem 3.20]):

(i) $D_{x, y}$ is of the form

$$
D_{x, y}=e^{i \varphi_{x y} v_{x y}} A_{x y}^{-\frac{1}{2}} P(x, y) \quad \text { with } \quad \varphi_{x y} \in\left(-\frac{3 \pi}{4},-\frac{\pi}{2}\right) \cup\left(\frac{\pi}{2}, \frac{3 \pi}{4}\right) .
$$

(ii) The spin connection maps the Clifford subspaces $K_{x y}$ and $K_{y x}$ to each other, i.e.

$$
D_{y, x} K_{x y} D_{x, y}=K_{y x}
$$

The spin connection has the properties

$$
D_{y, x}=\left(D_{x, y}\right)^{-1}=\left(D_{x, y}\right)^{*} \quad \text { and } \quad A_{x y}=D_{x, y} A_{y x} D_{y, x} .
$$

All the assumptions needed for the construction of the spin connection are combined in the notion that $x$ and $y$ must be spin-connectable (see [FG2, Definition 3.17]). We remark that in the limiting case of a Lorentzian manifold, the points $x$ and $y$ are spin-connectable if they are timelike separated and sufficiently close to each other (see [FG2, Section 5]).

By composing the spin connection along a discrete "path" of space-time points, one obtains a "parallel transport" of spinors. When doing so, it is important to keep track of the different Clifford subspaces and to carefully transform them to each other. In order to illustrate in an example how this works, suppose that we want to compose the spin connection $D_{y, z}$ with $D_{z, x}$. As mentioned above, the spin connection $D_{z, x}$ at the point $z$ is constructed using the Clifford subspace $K_{z x}$. The spin connection $D_{y, z}$, however, takes 
at the same space-time point $z$ the Clifford subspace $K_{z y}$ as reference. This entails that before applying $D_{y, z}$ we must transform from the Clifford subspace $K_{z x}$ to the Clifford subspace $K_{z y}$. This is accomplished by the splice map $U_{z}^{(y \mid x)}$, being a uniquely defined unitary transformation of $S_{x}$ with the property that

$$
K_{z y}=U_{z}^{(y \mid x)} K_{z x}\left(U_{z}^{(y \mid x)}\right)^{*} .
$$

The splice map must be sandwiched between the spin connections in combinations like

$$
D_{y, z} U_{z}^{(y \mid x)} D_{z, x} \text {. }
$$

In order to construct a corresponding metric connection $\nabla_{x, y}$, one uses a similar procedure to relate the Clifford subspaces to corresponding Clifford extensions. More precisely, one first unitarily transforms the Clifford extension $K_{y}^{(x)}$ to the Clifford subspace $K_{y x}$. Unitarily transforming with the spin connection $D_{x y}$ gives the Clifford subspace $K_{x y}$. Finally, one unitarily transforms to the Clifford extension $K_{x}^{(y)}$. Since the Clifford extensions at the beginning and end are representatives of the corresponding tangent spaces, we thus obtain an isometry

$$
\nabla_{x, y}: T_{y} \rightarrow T_{x}
$$

between the tangent spaces (for details see [FG2, Section 3.4]).

In this setting, curvature is defined as usual as the holonomy of the connection. Thus the curvature of the spin connection is given by

$$
\mathfrak{R}(x, y, z)=U_{x}^{(z \mid y)} D_{x, y} U_{y}^{(x \mid z)} D_{y, z} U_{z}^{(y \mid x)} D_{z, x}: S_{x} \rightarrow S_{x},
$$

and similarly for the metric connection. In [FG2, Sections 4 and 5] it is proven that the above notions in fact reduce to the spinorial Levi-Civita connection and the Riemannian curvature on a globally hyperbolic Lorentzian manifold if the causal fermion system is constructed by regularizing solutions of the Dirac equation (similar as will explained in the next section for the Minkowski vacuum) and removing the regularization in a suitable way. These results show that the notions of connection and curvature defined above indeed generalize the corresponding notions in Lorentzian spin geometry.

1.1.7. Topological Structures. From a mathematical perspective, causal fermion systems provide a framework for non-smooth geometries or generalized "quantum geometries." In this context, it is of interest how the topological notions on a differentiable manifold or a spin manifold generalize to causal fermion systems. Such topological questions are analyzed in [FK], as we now briefly summarize.

By definition, space-time $M$ is a topological space (see $\$ 1.1 .2$ ). Attaching to every space-time point $x \in M$ the corresponding spin space $S_{x}$ gives the structure of a sheaf, making it possible to describe the topology by sheaf cohomology. If one assumes in addition that all space-time points are regular (see Definition 1.1.5), then all spin spaces are isomorphic, giving rise to a topological vector bundle.

In order to get the connection to spinor bundles, one needs the additional structure of Clifford multiplication. As explained in $\$ 1.1 .4$, the notion of a Clifford subspace (see Definition 1.1.4 makes it possible to define Clifford structures at every space-time point, but the definition is not unique and does not give the connection to tangent vectors of the base space. In $\$ 1.1 .6$ these shortcomings where bypassed by working with suitable equivalence classes of Clifford subspaces. From the topological point of view, the basic question is whether one can choose a representative of this equivalence class at each space-time point in such a way that the representative depends continuously on the base 
point. This leads to the notion of a Clifford section $\mathcal{C} \ell$, being a continuous mapping which to every space-time point $x \in M$ associates a corresponding Clifford subspace $\mathcal{C} \ell_{x}$ (for details see [FK, Section 4.1]). Choosing a Clifford section leads to the structure of a so-called topological spinor bundle. An advantage of working with topological spinor bundles is that no notion of differentiability is required.

If $M$ has a differentiable structure, one would like to associate a tangent vector $u \in$ $T_{x} M$ to a corresponding element of the Clifford subspace $\mathcal{C} \ell_{x}$. This leads to the notion of a spin structure $\gamma$ on a topological spinor bundle, being a continuous mapping which to every $x \in M$ associates a mapping $\gamma_{x}: T_{x} M \rightarrow \mathcal{C} \ell_{x}$. The topological obstructions for the existence of a spin structure on a topological spinor bundle generalize the spin condition on a spin manifold (for details see [FK, Sections 4.2 and 4.5]).

A useful analytic tool for the construction of Clifford sections are so-called tangent cone measures (see [FK, Section 6]). These measures make it possible to analyze the local structure of space-time in a neighborhood of a point $x \in M$ (again without any differentiability assumptions). The tangent cone measures can be used to distinguish a specific Clifford subspace $\mathcal{C} \ell_{x}$ and to relate $\mathcal{C} \ell_{x}$ to neighboring space-time points.

We close with two remarks. First, all the above constructions generalize to the Riemannian setting if the definition of causal fermion systems is extended to so-called topological fermion systems (see $[\mathbf{F K}$, Definition 2.1]). We thus obtain a mathematical framework to describe spinors on singular spaces (see [FK, Sections 8 and 9] for many examples). Second, one can introduce nontrivial topological notions even for discrete space-times by constructing neighborhoods of $M$ in $\mathcal{F}$ (using the metric structure of $\mathcal{F}$ induced by the norm on the Banach space $\mathrm{L}(\mathcal{H})$ ) and by studying the topology of these neighborhoods (see [FK, Section 9.4]).

\subsection{Correspondence to Minkowski Space}

In order to put the abstract framework into a simple and concrete context, we now explain how to describe Dirac spinors in Minkowski space by a causal fermion system.

1.2.1. Concepts Behind the Construction of Causal Fermion Systems. We let $(\mathscr{M},\langle.,\rangle$.$) be Minkowski space (with the signature convention (+---))$ and $d \mu$ the standard volume measure (thus $d \mu=d^{4} x$ in a reference frame $x=\left(x^{0}, \ldots, x^{3}\right)$ ). We denote the spinor space at a point $x \in \mathscr{M}$ by $S_{x} \mathcal{M}$, so that a Dirac wave function $\psi$ takes values in

$$
\psi(x) \in S_{x} \mathscr{M} \simeq \mathbb{C}^{4} .
$$

The spinor space at $x$ is endowed with an indefinite inner product of signature $(2,2)$, which as in physics textbooks we denote by $\bar{\psi} \phi$ (where $\bar{\psi}=\psi^{\dagger} \gamma^{0}$ is the usual adjoint spinor). Clearly, in Minkowski space one has a trivial parallel transport of spinors, making it possible to identify the spinor spaces at different space-time points. Thus the space-time index $S_{x} \mathscr{M}$ of the spinor space is added only for notational clarity.

We now consider solutions of the Dirac equation of mass $m$,

$$
\left(i \gamma^{j} \partial_{j}-m\right) \psi=0 \text {. }
$$

For a solution $\psi$, the function $\left(\bar{\psi} \gamma^{0} \psi\right)(t, \vec{x})$ has the interpretation as the probability density of the Dirac particle at time $t$ to be at the position $\vec{x}$. The spatial integral of this probability density is time independent (for more details in the presence of an 
external potential see Exercise 2.5). Considering the bilinear form corresponding to this probability integral gives the scalar product

$$
(\psi \mid \phi):=2 \pi \int_{\mathbb{R}^{3}}\left(\bar{\psi} \gamma^{0} \phi\right)(t, \vec{x}) d^{3} x
$$

This scalar product is indeed independent of time and does not depend on the choice of the reference frame. In order to ensure that the integral in 1.2 .2 is well-defined and finite, we first consider solutions which at time $t$ are smooth and have compact support. Taking the completion, the solution space becomes a separable Hilbert space. We note that the factor $2 \pi$ in 1.2 .2 is not quite standard, but our convention has the advantage that many formulas become simpler.

Next, we choose $\mathcal{H}$ as a closed subspace of this Hilbert space with the induced scalar product $\langle. \mid .\rangle_{\mathcal{H}}:=\left.(. \mid)\right|_{.\mathcal{H} \times \mathcal{H}}$. Clearly, $\mathcal{H}$ is again a separable Hilbert space. In order to describe the vacuum (i.e. the physical system where no particles and anti-particles are present), one chooses $\mathcal{H}$ as the subspace spanned by all the negative-energy solutions (the "Dirac sea vacuum"). To describe particles or anti-particles, one includes positiveenergy solutions or leaves out negative-energy solutions, respectively. But any other closed subspace of the solution space may be chosen as well. We remark for clarity that in this section, we only consider the vacuum Dirac equation (1.2.1), so that the Dirac particles do not interact (interacting systems will be discussed in Section 1.5 below).

In order to get into the framework of causal fermion systems, to every space-time point $x \in \mathcal{M}$ we want to associate a linear operator $F(x) \in \mathcal{F}$. Once this has been accomplished, the resulting mapping

$$
F: \mathscr{M} \rightarrow \mathcal{F}
$$

can be used to introduce a measure $\rho$ on $\mathcal{F}$. Namely, we say that a subset $\Omega \subset \mathcal{F}$ is measurable if and only if its pre-image $F^{-1}(\Omega)$ is a measurable subset of $\mathscr{M}$. Moreover, we define the measure of $\Omega$ as the space-time volume of the pre-image, $\rho(\Omega):=\mu\left(F^{-1}(\Omega)\right)$. This construction is commonly used in mathematical analysis and is referred to as the push-forward measure, denoted by

$$
\rho=F_{*} \mu
$$

(see for example [B1, Section 3.6] or Exercise 1.5 (b)). Then $(\mathcal{H}, \mathcal{F}, \rho)$ will be a causal fermion system.

The basic idea for constructing $F(x)$ is to represent the inner product on the spinors at the space-time point $x$ in terms of the Hilbert space scalar product, i.e.

$$
\langle\psi \mid F(x) \phi\rangle_{\mathcal{H}}=-\overline{\psi(x)} \phi(x) \quad \text { for all } \psi, \phi \in \mathcal{H} .
$$

The operator $F(x)$ gives information on the densities and correlations of the Dirac wave functions at the space-time point $x$. It is referred to as the local correlation operator at $x$. Since the spinor space at $x$ is four-dimensional, it follows that the operator $F(x)$ has rank at most four. Moreover, the fact that the spin scalar product has signature $(2,2)$ implies that $F(x)$ has at most two positive and at most two negative eigenvalues. Therefore, the local correlation operator $F(x)$ is indeed an element of $\mathcal{F}$ if we choose the spin dimension $n=2$. However, the equation (1.2.4) is problematic because Dirac solutions $\psi, \phi \in \mathcal{H}$ are in general not continuous, so that the pointwise evaluation on the right side of (1.2.4) makes no mathematical sense. This is the reason why we need to introduce an ultraviolet regularization (UV regularization). Before entering the analysis, we first outline our method and explain the physical picture in a few remarks. The mathematical construction will be given afterwards in $\$ 1.2 .2$. 
In order to put our constructions in the general physical context, we first note that UV regularizations are frequently used in relativistic quantum field theory as a technical tool to remove divergences. A common view is that the appearance of such divergences indicates that the physical theory is incomplete and should be replaced for very small distances by another, more fundamental theory. The renormalization program is a method to get along with standard quantum field theory by finding a way of dealing with the divergences. The first step is the UV regularization, which is usually a set of prescriptions which make divergent integrals finite. The next step of the renormalization program is to show that the UV regularization can be taken out if other parameters of the theory (like masses and coupling constants) are suitably rescaled. Conceptually, in the renormalization program the UV regularization merely is a technical tool. All predictions of theory should be independent of how the regularization is carried out.

In the context of causal fermion systems, however, the physical picture behind the UV regularization is quite different. Namely, in our setting the regularized objects are to be considered as the fundamental physical objects. Therefore, the regularization has a physical significance. It should describe the microscopic structure of physical space-time.

Before explaining this physical picture in more detail, we need to introduce a microscopic length scale $\varepsilon>0$ on which the UV regularization should come into play. Regularization lengths are often associated to the Planck length $\ell_{P} \approx 1.6 \cdot 10^{-35} \mathrm{~m}$. The analysis of the gravitational field in this book suggests that $\varepsilon$ should be chosen even much smaller than the Planck length (see Section 4.9 and \$5.4.3). Even without entering a detailed discussion of the length scales, it is clear that $\varepsilon$ will be by many orders of magnitude smaller than most other physical length scales of the system. Therefore, it is a sensible method to analyze the causal action principle in the asymptotics when $\varepsilon$ is very small. In order to make such an asymptotics mathematically precise, it is necessary to consider the regularization length $\varepsilon$ as a variable parameter taking values in an interval $\left(0, \varepsilon_{\max }\right)$. Only for such a variable parameter, it will be possible later in this book to analyze the asymptotics as $\varepsilon \searrow 0$.

For any $\varepsilon \in\left(0, \varepsilon_{\max }\right)$, similar to $(1.2 .3)$ we shall construct a mapping $F^{\varepsilon}: \mathscr{M} \rightarrow \mathcal{F}$ by suitably inserting an UV regularization into (1.2.4). Then we construct the corresponding universal measure as the push-forward by $F^{\varepsilon}$, i.e.

$$
\rho^{\varepsilon}:=F_{*}^{\varepsilon} \mu \text {. }
$$

This will give rise to a causal fermion system $\left(\mathcal{H}, \mathcal{F}, \rho^{\varepsilon}\right)$. We will also explain how to identify the objects in Minkowski space with corresponding objects of the causal fermion system:

\begin{tabular}{|c|c|}
\hline Minkowski space & causal fermion system \\
\hline space-time point $x \in \mathscr{M}$ & space-time point $x \in M^{\varepsilon}:=\operatorname{supp} \rho^{\varepsilon}$ \\
topology of $\mathscr{M}$ & topology of $M^{\varepsilon}$ \\
spinor space $S_{x} \mathscr{M}$ & spin space $S_{x} M^{\varepsilon}$ \\
causal structure of Minkowski space & causal structure of Definition 1.1 .2 \\
\hline
\end{tabular}

With these identifications made, the structures of Minkowski space will no longer be needed. They are encoded in the causal fermion system, and we may describe the physical space-time exclusively by the causal fermion system. We consider the objects with UV 
regularization as described by the causal fermion system as the fundamental physical objects.

In the following remarks we elaborate on the physical picture behind the UV regularization and explain why our setting is sufficiently general to describe the physical situation we have in mind.

REMARK 1.2.1. (method of variable regularization) As just explained, the only reason for considering a family of causal fermion systems is to give the asymptotics $\varepsilon \searrow 0$ a precise mathematical meaning. But from the physical point of view, a specific regularization for a specific value of $\varepsilon$ should be distinguished by the fact that the corresponding causal fermion system $\left(\mathcal{H}, \mathcal{F}, \rho^{\varepsilon}\right)$ describes our physical space-time. We again point out that this concept is different from standard quantum field theory, where the regularization merely is a technical tool used in order to remove divergences. In our setting, the regularization has a physical significance. The regularized objects are to be considered as the fundamental physical objects, and the regularization is a method to describe the microscopic structure of physical space-time.

This concept immediately raises the question how the "physical regularization" should look like. Generally speaking, the regularized space-time should look like Minkowski space down to distances of the scale $\varepsilon$. For distances smaller than $\varepsilon$, the structure of space-time may be completely different. The simplest method of regularizing is to "smear out" or "mollify" all wave functions on the scale $\varepsilon$ (this corresponds to Example 1.2.4 below). But it is also conceivable that space-time has a non-trivial microstructure on the scale $\varepsilon$, which cannot be guessed or extrapolated from the structures of Minkowski space. Since experiments on the length scale $\varepsilon$ seem out of reach, it is completely unknown what the microscopic structure of space-time is. Nevertheless, we can hope that we can get along without knowing this micro-structure, because the detailed form of this micro-structure might have no influence on the effective physical equations which are valid on the energy scales accessible to experiments. More precisely, the picture is that the general structure of the effective physical equations should be independent of the micro-structure of spacetime. Values of mass ratios or coupling constants, however, may well depend on the micro-structure (a typical example is the gravitational constant, which is closely tied to the Planck length, which in turn is related to $\varepsilon$ as explained in Section 4.9 below). In more general terms, the unknown micro-structure of space-time should enter the effective physical equations only by a finite (hopefully small) number of free parameters, which can then be taken as empirical free parameters of the effective macroscopic theory.

Clearly, the above picture must be questioned and supported by mathematical results. To this end, one needs to analyze in detail how the effective macroscopic theory depends on the regularization. For this reason, it is not sufficient to consider a specific family of regularizations. Instead, one must analyze a whole class of regularizations which is so large that it covers all relevant regularization effects. This strategy is referred to as the method of variable regularization (for a longer explanation see [F7, Section 4.1]). It is the reason why in Definition 1.2.3 below we shall only state properties of the regularization, but we do not specify how precisely it should look like.

REMARK 1.2.2. (sequences of finite-dimensional regularizations) The critical reader may wonder why we consider a family of regularizations $\left(\mathcal{H}, \mathcal{F}, \rho^{\varepsilon}\right)$ parametrized by a continuous parameter $\left(0, \varepsilon_{\max }\right)$. Would it not be more suitable to consider instead a sequence of causal fermion systems $\left(\mathcal{H}_{\ell}, \mathcal{F}_{\ell}, \rho_{\ell}\right)$ which asymptotically as $\ell \rightarrow \infty$ describes 
Minkowski space? A related question is why we constructed the measure $\rho$ as the pushforward of the Lebesgue measure (1.2.5). Would it not be better to work with more general measures such as to allow for the possibility of discrete micro-structures? The answer to these questions is that it is no loss of generality and a simply a matter of convenience to work with the family $\left(\mathcal{H}, \mathcal{F}, \rho^{\varepsilon}\right)$ with $\varepsilon \in\left(0, \varepsilon_{\max }\right)$, as we now explain.

We first point out that we do not demand our family $\left(\mathcal{H}, \mathcal{F}, \rho^{\varepsilon}\right)$ to be in any sense "continuous" in the parameter $\varepsilon$. Therefore, one can also describe a sequence $\left(\mathcal{H}, \mathcal{F}, \rho_{\ell}\right)$ simply by choosing the family $\rho^{\varepsilon}$ to be piecewise constant, for example

$$
\rho^{\varepsilon}=\rho_{\ell} \quad \text { if } \quad \frac{1}{\ell} \leq \varepsilon<\frac{1}{\ell+1} .
$$

Similarly, it is no loss of generality to take $\rho$ as the push-forward measure of the Lebesgue measure because $F^{\varepsilon}(x)$ need not depend continuously on $x \in M$. For example, one can arrange a discrete space-time like a space-time lattice by choosing $F^{\varepsilon}$ as a mapping which is piecewise constant on little cubes of Minkowski space. Clearly, this mapping is not continuous, but it is continuous almost everywhere. Moreover, its image is a discrete set, corresponding to a discrete micro-structure of space-time. For the method for representing a general measure $\rho$ as the push-forward of for example the Lebesgue measure we refer the interested reader to the proof of [F13, Lemma 1.4].

The remaining question is why we may keep the Hilbert space $\mathcal{H}$ fixed. In particular, we noted in $\$ 1.1 .1$ that the existence of minimizers of the causal action principle has been proven only if $\mathcal{H}$ is finite-dimensional. Therefore, should one not consider a filtration $\mathcal{H}_{1} \subset$ $\mathcal{H}_{2} \subset \cdots \subset \mathcal{H}$ of $\mathcal{H}$ by finite-dimensional subspaces? Indeed, from the conceptual point of view, this would be the correct way to proceed. Nevertheless, the following consideration explains why we can just as well replace all the Hilbert spaces $\mathcal{H}_{\ell}$ by the larger space $\mathcal{H}$ : For a given causal fermion system $\left(\mathcal{H}_{\ell}, \mathcal{F}_{\ell}, \rho_{\ell}\right)$ with $\mathcal{H}_{\ell} \subset \mathcal{H}$, by extending all operators by zero to the orthogonal complement of $\mathcal{H}_{\ell}$, one obtains the so-called extended causal fermion system $\left(\mathcal{H}, \mathcal{F}, \rho_{\ell}\right)$. The fact that the causal fermion system was extended can still be seen by forming the so-called effective Hilbert space as

$$
\mathcal{H}^{\mathrm{eff}}=\overline{\operatorname{span}\{x(\mathcal{H}) \mid x \in \operatorname{supp} \rho\}} .
$$

Namely, for an extended causal fermion system, the effective Hilbert space still is a subset of the original Hilbert space, $\mathcal{H}^{\text {eff }} \subset \mathcal{H}_{\ell}$. Moreover, the support of the extended causal fermion system is still contained in $\mathcal{F}_{\ell} \subset \mathrm{L}\left(\mathcal{H}_{\ell}\right)$. Therefore, we do not lose any information by extending a causal fermion system. Conversely, when analyzing a causal fermion system, it seems preferable to always make the Hilbert space as small as possible by taking $\mathcal{H}^{\text {eff }}$ as the underlying Hilbert space.

The delicate point about extending causal fermion systems is that the causal action principle does depend sensitively on the dimension of the underlying Hilbert space $\mathcal{H}$. More specifically, the infimum of the action is known to be strictly decreasing in the dimension of $\mathcal{H}$ (see the estimates in [F10, Lemma 5.1], which apply similarly in the more general setting of [F13]). Therefore, a minimizer $\rho$ of the causal action principle will no longer be a minimizer if the causal fermion system is extended. However, the first order Euler-Lagrange equations (for details see \$1.4.1 below) are still satisfied for the extended causal fermion system, and this is all we need for the analysis in this book. Therefore, for convenience we fix the Hilbert space $\mathcal{H}$ and consider a family of causal fermion systems $\left(\mathcal{H}, \mathcal{F}, \rho^{\varepsilon}\right)$ thereon. In order for the causal action principle to be well-defined and for $\rho^{\varepsilon}$ to be a minimizer, one should replace $\mathcal{H}$ by the corresponding 
effective Hilbert space $\mathcal{H}^{\text {eff }}$, which may depend on $\varepsilon$ and should be arranged to be finitedimensional. For the analysis of the Euler-Lagrange equations, however, the restriction to $\mathcal{H}^{\text {eff }}$ is unnecessary, and it is preferable to work with the extended Hilbert space $\mathcal{H}$.

We finally remark that the hurried reader who wants to skip the following constructions may read instead the introductory section [FGS, Section 1.1] where formal considerations without UV regularization are given. Moreover, a more explicit analysis of fourdimensional Minkowski space with a particularly convenient regularization is presented in [FG2, Section 4]. For a somewhat simpler analysis of two-dimensional Minkowski space we refer to [FK, Section 8.2].

1.2.2. Introducing an Ultraviolet Regularization. We now enter the construction of the UV regularization. We denote the continuous Dirac wave functions (i.e. the continuous sections of the spinor bundle, not necessarily solutions of the Dirac equation) by $C^{0}(\mathscr{M}, S \mathscr{M})$. Similarly, the smooth wave functions with compact support in a subset $K \subset \mathscr{M}$ are denoted by $C_{0}^{\infty}(K, S \mathscr{M})$. For the $C^{k}$-norms we use the notation

$$
|\eta|_{C^{k}(K)}=\sum_{|\alpha| \leq k} \sup _{x \in K}\left|\partial^{\alpha} \eta(x)\right| \quad \text { for } \eta \in C_{0}^{\infty}(K, S \mathscr{M}),
$$

where the $\alpha$ are multi-indices. Here $|$.$| is any pointwise norm on the spinor spaces$ (we again identify all spinor spaces via the trivial parallel transport). Since any two such norms can be estimated from above and below by a constant, the $C^{k}$-norms corresponding to different choices of the norms |.| are also equivalent. For example, one can choose $|\psi|^{2}:=\bar{\psi} \gamma^{0} \psi$ similar to the integrand in the scalar product 1.2 .2 . But clearly, other choices are possible just as well.

The UV regularization is performed most conveniently with so-called regularization operators, which we now define.

Definition 1.2.3. Consider a family of linear operators $\left(\mathfrak{R}_{\varepsilon}\right)$ with $0<\varepsilon<\varepsilon_{\max }$ which map $\mathcal{H}$ to the continuous wave functions,

$$
\mathfrak{R}_{\varepsilon}: \mathcal{H} \rightarrow C^{0}(\mathscr{M}, S \mathscr{M}) .
$$

The family is called a family of regularization operators if the following conditions hold:

(i) The image of every regularization operator is pointwise bounded, meaning that for every $\varepsilon \in\left(0, \varepsilon_{\max }\right)$ and all $x \in \mathcal{M}$ there is a constant $c>0$ such that for all $u \in \mathcal{H}$,

$$
\left|\left(\mathfrak{R}_{\varepsilon} u\right)(x)\right| \leq c\|u\|_{\mathcal{H}} .
$$

(ii) The image of every regularization operator is equicontinuous almost everywhere in the sense that for every $\varepsilon \in\left(0, \varepsilon_{\max }\right)$, almost all $x \in \mathcal{M}$ and every $\delta>0$, there is an open neighborhood $U \subset \mathcal{M}$ of $x$ such that for all $u \in \mathcal{H}$ and all $y \in U$,

$$
\left|\left(\mathfrak{R}_{\varepsilon} u\right)(x)-\left(\mathfrak{R}_{\varepsilon} u\right)(y)\right| \leq \delta\|u\|_{\mathcal{H}} .
$$

(iii) In the limit $\varepsilon \searrow 0$, the family converges weakly to the identity, meaning that for every compact subset $K \subset \mathcal{M}$ and every $\delta>0$ there is a constant $\varepsilon_{0}>0$, such that for all $\varepsilon \in\left(0, \varepsilon_{0}\right), u \in \mathcal{H}$ and $\eta \in C_{0}^{\infty}(K, S \mathcal{M})$,

$$
\left|\int_{\mathscr{M}} \overline{\eta(x)}\left(\mathfrak{R}_{\varepsilon}(u)-u\right)(x) d^{4} x\right| \leq \delta\|u\|_{\mathcal{H}}|\eta|_{C^{1}(K)} .
$$


We point out that we do not demand that the regularized wave function $\mathfrak{R}_{\varepsilon} \psi$ is again a solution of the Dirac equation. This could be imposed (as is done in [FR2, Section 4]), but doing so seems too restrictive for the physical applications. We also note that "almost all" in (ii) refers to the standard volume measure $d \mu$ on $\mathscr{M}$.

For the mathematically interested reader we remark that the above properties (i) and (ii) are very similar to the assumptions in the Arzelà-Ascoli theorem (see for example [D1, Section VII.5] or [Ru, Theorem 7.25]). In fact, if we replaced "almost all" in (ii) by "all", one could apply the Arzelà-Ascoli theorem and restate the properties (i) and (ii) equivalently by saying that taking the image $\mathfrak{R}_{\varepsilon}\left(B_{1}(0)\right)$ of the unit ball in $\mathcal{H}$ and restricting the resulting family of functions to any compact set $K \subset \mathcal{M}$, one obtains a relatively compact subset of $C^{0}(K, S \mathscr{M})$. It is remarkable that the properties (i) and (ii) come up naturally as conditions for a sensible UV regularization, although we shall never use compactness arguments in our proofs. Weakening "all" by "almost all" in (ii) makes it possible to describe discrete space-times like space-time lattices, as was mentioned in Remark 1.2 .2 above.

Simple examples of regularization operators are obtained by mollifying the wave functions on the scale $\varepsilon$ :

EXAMPLE 1.2.4. (regularization by mollification) Let $h \in C_{0}^{\infty}(\mathscr{M}, \mathbb{R})$ be a nonnegative test function with

$$
\int_{\mathscr{M}} h(x) d^{4} x=1
$$

We define the operators $\mathfrak{R}_{\varepsilon}$ for $\varepsilon>0$ as the convolution operators

$$
\left(\mathfrak{R}_{\varepsilon} u\right)(x):=\frac{1}{\varepsilon^{4}} \int_{\mathscr{M}} h\left(\frac{x-y}{\varepsilon}\right) u(y) d^{4} y .
$$

Let us prove that the family $\left(\mathfrak{R}_{\varepsilon}\right)_{0<\varepsilon<1}$ is a family of regularization operators. First,

$$
\left|\left(\Re_{\varepsilon} u\right)(x)\right| \leq \frac{|h|_{C^{0}}}{\varepsilon^{4}} \int_{K}|u(y)| d^{4} y \leq \frac{|h|_{C^{0}}}{\varepsilon^{4}} \sqrt{\mu(K)}\left(\int_{K}|u(y)|^{2} d^{4} y\right)^{\frac{1}{2}},
$$

where in the last step we used the Schwarz inequality. We now rewrite the obtained space-time integral of $|u|^{2}$ with the help of Fubini's theorem as a bounded time integral and a spatial integral. In view of $(1.2 .2)$, the spatial integral can be estimated by the Hilbert space norm. We thus obtain

$$
\int_{K}|u(y)|^{2} d^{4} y \leq C \int_{K}\left(\bar{u} \gamma^{0} u\right)(y) d^{4} y \leq C \int_{t_{0}}^{t_{1}}\|u\|_{\mathcal{H}}^{2}=C\left(t_{1}-t_{0}\right)\|u\|_{\mathcal{H}}^{2},
$$

where $t_{0}$ and $t_{1}$ are chosen such that $K$ is contained in the time strip $t_{0}<t<t_{1}$. We conclude that

proving 1.2 .6 .

$$
\left|\left(\mathfrak{R}_{\varepsilon} u\right)\right| \leq \frac{|h|_{C^{0}}}{\varepsilon^{4}} \sqrt{\mu(K) C\left(t_{1}-t_{0}\right)}\|u\|_{\mathcal{H}}^{2},
$$

In order to derive the inequality (1.2.7), we begin with the estimate

$$
\left|\left(\Re_{\varepsilon} u\right)(x)-\left(\mathfrak{R}_{\varepsilon} u\right)(y)\right| \leq \frac{1}{\varepsilon^{4}} \sup _{z \in \mathscr{M}}\left|h\left(\frac{x-z}{\varepsilon}\right)-h\left(\frac{y-z}{\varepsilon}\right)\right| \int_{K}|u(y)| d^{4} y .
$$

Again applying (1.2.9) and using that $h$ is uniformly continuous, one obtains (1.2.7).

It remains to prove (1.2.8). We first write the integral on the left as

$$
\int_{\mathscr{M}} \overline{\eta(x)}\left(\mathfrak{R}_{\varepsilon}(u)-u\right)(x) d^{4} x=\int_{\mathscr{M}} \overline{\left(\eta_{\varepsilon}(y)-\eta(y)\right)} u(y) d^{4} y,
$$


where we set

$$
\eta_{\varepsilon}(y)=\frac{1}{\varepsilon^{4}} \int_{\mathscr{M}} \eta(x) h\left(\frac{x-y}{\varepsilon}\right) d^{4} x .
$$

Now we use the standard estimate for convolutions

$$
\begin{aligned}
& \left|\eta_{\varepsilon}(y)-\eta(y)\right|=\frac{1}{\varepsilon^{4}}\left|\int_{\mathscr{M}}(\eta(x)-\eta(y)) h\left(\frac{x-y}{\varepsilon}\right) d^{4} x\right| \\
& =\left|\int_{\mathscr{M}}(\eta(y+\varepsilon z)-\eta(y)) h(z) d^{4} z\right| \leq|\eta|_{C^{1}(K)} \int_{\mathscr{M}}|\varepsilon z| h(z) d^{4} z
\end{aligned}
$$

(where in the last step we used the mean value theorem). This gives rise to the estimate

$$
\left|\eta_{\varepsilon}-\eta\right|_{C^{0}(K)} \leq c \varepsilon|\eta|_{C^{1}(K)},
$$

where $c$ may depend on $K$ and the choice of $h$, but is independent of $\eta$. This makes it possible to estimate $(1.2 .10)$ by

$$
\left|\int_{\mathscr{M}} \overline{\eta(x)}\left(\mathfrak{R}_{\varepsilon}(u)-u\right)(x) d^{4} x\right| \leq \varepsilon|\eta|_{C^{1}(K)} \int_{K}|u(y)|_{y} d^{4} y .
$$

Again applying (1.2.9), we conclude that

$$
\left|\int_{\mathscr{M}} \overline{\eta(x)}\left(\Re_{\varepsilon}(u)-u\right)(x) d^{4} x\right| \leq \delta|\eta|_{C^{1}(K)} \sqrt{\mu(K)} \sqrt{C\left(t_{1}-t_{0}\right)}\|u\|_{\mathcal{H}}
$$

proving 1.2.8.

Given a family of regularization operators, we can construct causal fermion systems as follows. We fix $\varepsilon \in\left(0, \varepsilon_{\max }\right)$. For any $x \in \mathscr{M}$, we consider the bilinear form

$$
b_{x}: \mathcal{H} \times \mathcal{H} \rightarrow \mathbb{C}, \quad b_{x}(u, v)=-\overline{\left(\Re_{\varepsilon} u\right)(x)}\left(\Re_{\varepsilon} v\right)(x) .
$$

This bilinear form is well-defined and bounded because $\mathfrak{R}_{\varepsilon}$ is defined pointwise and because evaluation at $x$ gives a linear operator of finite rank. Thus for any $v \in \mathcal{H}$, the anti-linear form $b_{x}(., v): \mathcal{H} \rightarrow \mathbb{C}$ is continuous. By the Fréchet-Riesz theorem (see for example [Lax, Section 6.3]), there is a unique vector $w \in \mathcal{H}$ such that $b_{x}(u, v)=\langle u \mid w\rangle_{\mathcal{H}}$ for all $u \in \mathcal{H}$. The mapping $v \mapsto w$ is linear and bounded. We thus obtain a bounded linear operator $F^{\varepsilon}(x)$ on $\mathcal{H}$ such that

$$
b_{x}(u, v)=\left\langle u \mid F^{\varepsilon}(x) v\right\rangle_{\mathcal{H}} \quad \text { for all } u, v \in \mathcal{H},
$$

referred to as the local correlation operator. Taking into account that the inner product on the Dirac spinors at $x$ has signature $(2,2)$, the local correlation operator $F^{\varepsilon}(x)$ is a symmetric operator on $\mathcal{H}$ of rank at most four, which has at most two positive and at most two negative eigenvalues. Finally, we introduce the universal measure $\rho^{\varepsilon}=F_{*}^{\varepsilon} \mu$ as the push-forward of the volume measure on $\mathscr{M}$ under the mapping $F^{\varepsilon}$. In this way, for every $\varepsilon \in\left(0, \varepsilon_{0}\right)$ we obtain a causal fermion system $\left(\mathcal{H}, \mathcal{F}, \rho^{\varepsilon}\right)$ of spin dimension $n=2$.

1.2.3. Correspondence of Space-Time. We now explain the connection between points of Minkowski space and points of space-time $M^{\varepsilon}:=\operatorname{supp} \rho^{\varepsilon}$ of the corresponding causal fermion system $\left(\mathcal{H}, \mathcal{F}, \rho^{\varepsilon}\right)$. We begin with a general characterization of $M^{\varepsilon}$.

Proposition 1.2.5. For any $\varepsilon \in\left(0, \varepsilon_{\max }\right)$, there is a subset $E \subset \mathcal{M}$ of $\mu$-measure zero such that the mapping $\left.F^{\varepsilon}\right|_{\mathscr{M} \backslash E}: \mathcal{M} \backslash E \rightarrow \mathcal{F}$ is continuous. Moreover, the support of the universal measure $M^{\varepsilon}:=\operatorname{supp} \rho^{\varepsilon}$ is given by

$$
M^{\varepsilon}={\overline{F^{\varepsilon}(\mathscr{M} \backslash E)^{\mathrm{L}(\mathcal{H})}}} .
$$


Proof. In order to show continuity, we need to estimate the sup-norm $\| F^{\varepsilon}(x)-$ $F^{\varepsilon}(y) \|$. We first write the expectation value of the corresponding operator by

$$
\begin{aligned}
& \left\langle u \mid\left(F^{\varepsilon}(x)-F^{\varepsilon}(y)\right) v\right\rangle_{\mathcal{H}}=-\overline{\left(\mathfrak{R}_{\varepsilon} u\right)(x)}\left(\mathfrak{R}_{\varepsilon} v\right)(x)+\overline{\left(\Re_{\varepsilon} u\right)(y)}\left(\Re_{\varepsilon} v\right)(y) \\
& \quad=-\overline{\left(\mathfrak{R}_{\varepsilon} u\right)(x)}\left(\left(\mathfrak{R}_{\varepsilon} v\right)(x)-\left(\mathfrak{R}_{\varepsilon} v\right)(y)\right)-\overline{\left(\left(\mathfrak{R}_{\varepsilon} u\right)(x)-\left(\mathfrak{R}_{\varepsilon} u\right)(y)\right)}\left(\mathfrak{R}_{\varepsilon} v\right)(y),
\end{aligned}
$$

giving rise to the estimate

$$
\begin{aligned}
& \left|\left\langle u \mid\left(F^{\varepsilon}(x)-F^{\varepsilon}(y)\right) v\right\rangle_{\mathcal{H}}\right| \\
& \left.\quad \leq\left|\left(\mathfrak{R}_{\varepsilon} u\right)(x)\right| \mid\left(\mathfrak{R}_{\varepsilon} v\right)(x)-\mathfrak{R}_{\varepsilon} v\right)(y)|+|\left(\mathfrak{R}_{\varepsilon} u\right)(x)-\left(\mathfrak{R}_{\varepsilon} u\right)(y)||\left(\mathfrak{R}_{\varepsilon} v\right)(y) \mid .
\end{aligned}
$$

We now estimate the resulting spinor norms with the help of properties (i) and (ii) of Definition 1.2.3. First, we denote the exceptional set of $\mu$-measure zero where (1.2.7) does not hold by $E \subset \mathscr{M}$. Combining (1.2.6) and (1.2.7), one immediately sees that every point $x \in \mathscr{M} \backslash E$ has a neighborhood $U$ such that the boundedness property $(1.2 .6)$ holds uniformly on $U$ (i.e. $\left|\left(\mathfrak{R}_{\varepsilon} u\right)(y)\right| \leq c\|u\|_{\mathcal{H}}$ for all $\left.y \in U\right)$. We thus obtain the estimate

$$
\left|\left\langle u \mid\left(F^{\varepsilon}(x)-F^{\varepsilon}(y)\right) v\right\rangle_{\mathcal{H}}\right| \leq 2 c \delta\|u\|_{\mathcal{H}}\|v\|_{\mathcal{H}},
$$

valid for all $y \in U$ and $u, v \in \mathcal{H}$. Hence the sup-norm is bounded by $\left\|F^{\varepsilon}(x)-F^{\varepsilon}(y)\right\| \leq$ $2 c \delta$, showing that $F^{\varepsilon}$ is continuous on $\mathcal{M} \backslash E$.

It remains to prove (1.2.12). Since $\mu(E)=0$, the set $E$ can be disregarded when forming the push-forward measure. Therefore, taking into account that the support of a measure is by definition a closed set, it suffices to show that for every $x \in \mathscr{M} \backslash E$, the operator $p:=F^{\varepsilon}(x)$ lies in the support of $\rho^{\varepsilon}$. Let $U \subset \mathcal{F}$ be an open neighborhood of $p$. Then the continuity of $F^{\varepsilon}$ at $x$ implies that the preimage $\left(F^{\varepsilon}\right)^{-1}(U)$ is an open subset of $\mathcal{M}$. Hence the Lebesgue measure of this subset is non-zero, $\mu\left(\left(F^{\varepsilon}\right)^{-1}(U)\right)>0$. By definition of the push-forward measure, it follows that $\rho^{\varepsilon}(U)>0$. Hence every neighborhood of $p$ has a non-zero measure, implying that $p \in \operatorname{supp} \rho^{\varepsilon}$. This concludes the proof.

In order to have a convenient notation, in what follows we always identify a point in Minkowski space with the corresponding operator of the causal fermion system,

$$
\text { identify } \quad x \in \mathscr{M} \quad \text { with } \quad F^{\varepsilon}(x) \in \mathcal{F} \text {. }
$$

In general, this identification is not one-to-one, because the mapping $F^{\varepsilon}$ need not be injective. In the latter case, there are two points $x, y \in \mathscr{M}$ such that the bilinear forms $b_{x}$ and $b_{y}$ coincide (see (1.2.11)). In other words, all correlations between regularized wave functions coincide at the points $x$ and $y$. Using a more physical language, this means that the points $x, y$ of Minkowski space are not distinguishable by any measurements performed on the fermionic wave functions. We take the point of view that in such situations, the points $x$ and $y$ should not be distinguished physically, and that it is reasonable and desirable that the two points are identified in the causal fermion system with the same space-time point $F^{\varepsilon}(x)=F^{\varepsilon}(y) \in M^{\varepsilon}:=\operatorname{supp} \rho^{\varepsilon}$. In philosophical terms, our construction realizes the principle of the identity of indiscernibles.

We also remark that, due to the closure in (1.2.12), it may happen that the spacetime $M^{\varepsilon}$ contains a point $z$ which does not lie in the image of $F^{\varepsilon}$, but is merely an accumulation point in $F^{\varepsilon}(\mathscr{M})$. In this case, the corresponding bilinear form $b(u, v):=$ $\langle u \mid z v\rangle_{\mathcal{H}}$ can be approximated with an arbitrarily small error by bilinear forms $b_{x}$ with $x \in$ $\mathcal{M}$. Since experiments always involve small imprecisions, we take the point of view that it is again reasonable and desirable mathematically to include $z$ to the space-time points. 
Generally speaking, the just-discussed cases that $F^{\varepsilon}$ is not injective or its image is not closed seem mostly of academic interest. In all applications in this book, the mapping $F^{\varepsilon}$ will be injective and closed. In all these situations, Proposition 1.2 .5 will give us a oneto-one correspondence between points $x \in \mathcal{M}$ and points $F^{\varepsilon}(x) \in M^{\varepsilon}$.

We finally note that, working with the push-forward measure 1.2 .5 , the volume measure on space-time $M^{\varepsilon}$ as defined by the universal measure $d \rho^{\varepsilon}$ always agrees under the identification $(1.2 .13)$ with the Lebesgue measure $d \mu$ on $\mathscr{M}$.

1.2.4. Correspondence of Spinors and Physical Wave Functions. We proceed by explaining the connection between the spinor space $S_{x} \mathscr{M}$ at a point $x \in \mathscr{M}$ of Minkowski space and the corresponding spin space $S_{x} M \subset \mathcal{H}$ of the causal fermion system (where we use the identification (1.2.13)). This will also make it possible to get a connection between Dirac wave functions in Minkowski space and wave functions as defined in 1.1.4. In preparation, we derive useful explicit formulas for the local correlation operators. To this end, for any $x \in \mathcal{M}$ we define the evaluation map $e_{x}^{\varepsilon}$ by

$$
e_{x}^{\varepsilon}: \mathcal{H} \rightarrow S_{x} \mathscr{M}, \quad e_{x}^{\varepsilon} \psi=\left(\mathfrak{R}_{\varepsilon} \psi\right)(x) .
$$

Its adjoint is defined as usual, taking into account the corresponding inner products on the domain and the target space, i.e.

$$
\left\langle\left(e_{x}^{\varepsilon}\right)^{*} \chi \mid \psi\right\rangle_{\mathcal{H}}=\bar{\chi}\left(e_{x}^{\varepsilon} \psi\right) \quad \text { for all } \chi \in S_{x} \mathcal{M} .
$$

We denote this adjoint by $\iota_{x}^{\varepsilon}$,

$$
\iota_{x}^{\varepsilon}:=\left(e_{x}^{\varepsilon}\right)^{*}: S_{x} \mathcal{M} \rightarrow \mathcal{H} .
$$

Multiplying $e_{x}^{\varepsilon}$ by $\iota_{x}^{\varepsilon}$ gives us back the local correlation operator $F^{\varepsilon}(x)$. Namely,

$$
\left\langle\psi \mid F^{\varepsilon}(x) \phi\right\rangle_{\mathcal{H}}=-\overline{\left(\mathfrak{R}_{\varepsilon} \psi\right)(x)}\left(\Re_{\varepsilon} \phi\right)(x)=-\overline{\left(e_{x}^{\varepsilon} \psi\right)}\left(e_{x}^{\varepsilon} \phi\right)=-\left\langle\psi \mid \iota_{x}^{\varepsilon} e_{x}^{\varepsilon} \phi\right\rangle_{\mathcal{H}}
$$

and thus

$$
F^{\varepsilon}(x)=-\iota_{x}^{\varepsilon} e_{x}^{\varepsilon}=-\iota_{x}^{\varepsilon}\left(\iota_{x}^{\varepsilon}\right)^{*}: \mathcal{H} \rightarrow \mathcal{H} .
$$

The next proposition gives the desired connection between the spinor space $S_{x} \mathcal{M}$ and the corresponding spin space $S_{x} M$. We first state and prove the proposition and explain it afterwards.

Proposition 1.2.6. The mapping

$$
\left.e_{x}^{\varepsilon}\right|_{S_{x}}: S_{x} M \rightarrow S_{x} \mathcal{M} \quad \text { is an isometric embedding. }
$$

If the point $x$ is regular (see Definition 1.1.5), then this mapping is an isomorphism. Moreover, the physical wave function of a vector $u$ at $x$ is mapped to the regularized Dirac wave function at $x$,

$$
\left.e_{x}^{\varepsilon}\right|_{S_{x}} \psi^{u}(x)=\left(\Re_{\varepsilon} u\right)(x) .
$$

Finally, the inverse of $\left.e_{x}^{\varepsilon}\right|_{S_{x}}$ is given by

$$
\left(\left.e_{x}^{\varepsilon}\right|_{S_{x}}\right)^{-1}=-\left(\left.x\right|_{S_{x}}\right)^{-1} \iota_{x}^{\varepsilon}: S_{x} \mathcal{M} \rightarrow S_{x} M .
$$

Proof. Let $\psi, \phi \in S_{x} M$. Then

$$
\overline{\left(e_{x}^{\varepsilon} \psi\right)}\left(e_{x}^{\varepsilon} \phi\right)=\left\langle\psi \mid\left(e_{x}^{\varepsilon}\right)^{*} e_{x}^{\varepsilon} \phi\right\rangle_{\mathcal{H}}=\left\langle\psi \mid \iota_{x}^{\varepsilon} e_{x}^{\varepsilon} \phi\right\rangle_{\mathcal{H}} \frac{1.2 .15}{-}-\langle\psi \mid x \phi\rangle_{\mathcal{H}}=\prec \psi \mid \phi \succ,
$$

proving that $\left.e_{x}^{\varepsilon}\right|_{S_{x}}$ is an isometry. Since the inner product $\prec . \mid \succ_{x}$ on $S_{x} M$ is nondegenerate, it follows that the mapping $\left.e_{x}^{\varepsilon}\right|_{S_{x}}$ is injective. Hence it is an isometric embedding. 
If $x$ is regular, a dimensional argument shows that $\left.e_{x}^{\varepsilon}\right|_{S_{x}}$ is an isomorphism. Again a dimensional argument yields that the image of the operator product $F^{\varepsilon}(x)=-\iota_{x}^{\varepsilon}\left(\iota_{x}^{\varepsilon}\right)^{*}$ coincides with the image of the operator $\iota_{x}^{\varepsilon}$, i.e.

$$
\iota_{x}^{\varepsilon}\left(S_{x} \mathcal{M}\right)=S_{x} M .
$$

As a consequence, for any vector $u \in S_{x}^{\perp}$ and any $\chi \in S_{x} \mathscr{M}$,

$$
0=\left\langle\iota_{x}^{\varepsilon} \chi \mid u\right\rangle=\prec \chi \mid e_{x}^{\varepsilon} u \succ_{x},
$$

showing that the operator $e_{x}^{\varepsilon}$ vanishes on the orthogonal complement of $S_{x}$. Therefore,

$$
\left.e_{x}^{\varepsilon}\right|_{S_{x}} \psi^{u}(x)=\left.e_{x}^{\varepsilon}\right|_{S_{x}} \pi_{x} u=e_{x}^{\varepsilon} u=\left(\Re_{\varepsilon} u\right)(x),
$$

proving (1.2.16). Finally,

$$
-\left.\left.\left(\left.x\right|_{S_{x}}\right)^{-1} \iota_{x}^{\varepsilon} e_{x}^{\varepsilon}\right|_{S_{x} M} \stackrel{1.2 .15}{=}\left(\left.x\right|_{S_{x}}\right)^{-1} x\right|_{S_{x}}=\mathbb{1}_{S_{x}},
$$

so that that the inverse of $\left.e_{x}^{\varepsilon}\right|_{S_{x}}$ is indeed given by the expression in (1.2.17).

This proposition makes it possible to identify the spin space $S_{x} M \subset \mathcal{H}$ endowed with the inner product $\prec . \mid . \succ_{x}$ with a subspace of the spinor space $S_{x} \mathscr{M}$ with the inner product $\bar{\psi} \phi$. If the space-time point $x$ is singular (see Definition 1.1.5), this is all we can expect, because in this case the spaces $S_{x} M$ and $S_{x} \mathscr{M}$ have different dimensions and are clearly not isomorphic. As already mentioned after Definition 1.1.5, in most situations of physical interest the point $x$ will be regular. In this case, we even obtain an isomorphism of $S_{x} M$ and $S_{x} \mathcal{M}$ which preserves the inner products on these spaces. The identity (1.2.16) shows that, under the above identifications, the physical wave function $\psi^{u}$ (as defined by (1.1.18) ) goes over to the regularized Dirac wave function $\left(\mathfrak{R}_{\varepsilon} u\right)(x)$. This shows again that the causal fermion system involves the regularized objects. Moreover, one sees that the abstract formalism introduced in Section 1.1 indeed gives agreement with the usual objects in Minkowski space. We remark that the above isomorphism of $S_{x} M$ and $S_{x} \mathcal{M}$ also makes it possible to use unambiguously the same notation for the corresponding inner products. Indeed, it is convenient to denote the inner product $\bar{\psi} \phi$ on the Dirac spinors at a time point $x \in \mathscr{M}$ by

$$
\prec .\left|. \succ_{x}: S_{x} \mathscr{M} \times S_{x} \mathscr{M} \rightarrow \mathbb{C}, \quad \prec \psi\right| \phi \succ_{x}=\bar{\psi} \phi .
$$

In order to avoid confusion, we avoided this notation so far. But from now on, we will sometimes use it.

In the next proposition we compute the kernel of the fermionic projector $P^{\varepsilon}(x, y)$ (as defined by (1.1.13), where the subscript $\varepsilon$ clarifies the dependence on the UV regularization) in Minkowski space. Moreover, we prove that the limit $\varepsilon \searrow 0$ exists in the distributional sense.

Proposition 1.2.7. Assume that the points $x$ and $y$ are regular. Then, under the above identification of $S_{x} M$ with $S_{x} \mathcal{M}$, the kernel of the fermionic projector has the representation

$$
P^{\varepsilon}(x, y)=-e_{x}^{\varepsilon} \iota_{y}^{\varepsilon}: S_{y} \mathscr{M} \rightarrow S_{x} \mathscr{M} .
$$

Moreover, choosing an orthonormal basis $\left(u_{\ell}\right)$ of $\mathcal{H}$, the kernel of the fermionic projector can be written as

$$
P^{\varepsilon}(x, y)=-\sum_{\ell}\left(\Re_{\varepsilon} u_{\ell}\right)(x) \overline{\left(\mathfrak{R}_{\varepsilon} u_{\ell}\right)(y)}
$$


In the limit $\varepsilon \searrow 0$, the kernel of the fermionic projector $P^{\varepsilon}(x, y)$ converges as a bi-distribution to the unregularized kernel defined by

$$
P(x, y):=-\sum_{\ell} u_{\ell}(x) \overline{u_{\ell}(y)} .
$$

More precisely, for every compact subset $K \subset \mathcal{M}$ and every $\delta>0$, there is a constant $\varepsilon_{0}>$ 0 such that for all $\varepsilon \in\left(0, \varepsilon_{0}\right)$ and for all test wave functions $\eta, \tilde{\eta} \in C_{0}^{\infty}(K, S \mathcal{M})$,

$$
\left|\iint_{\mathcal{M} \times \mathcal{M}} \overline{\eta(x)}\left(P^{\varepsilon}(x, y)-P(x, y)\right) \tilde{\eta}(y) d^{4} x d^{4} y\right| \leq \delta|\eta|_{C^{1}(K)}|\tilde{\eta}|_{C^{1}(K)} .
$$

We remark that, since $\mathcal{H}$ is separable, we can always choose an at most countable orthonormal basis $\left(u_{\ell}\right)$ of $\mathcal{H}$. The inequality (1.2.21) is discussed in Exercise 1.9.

Proof of Proposition 1.2.7. We first note that

$$
P^{\varepsilon}(x, y)=e_{x}^{\varepsilon} \pi_{x} y\left(\left.e_{y}^{\varepsilon}\right|_{S_{y}}\right)^{-1}=-e_{x}^{\varepsilon} \pi_{x} y\left(\left.y\right|_{S_{y}}\right)^{-1} \iota_{y}^{\varepsilon}=-e_{x}^{\varepsilon} \pi_{x} \iota_{y}^{\varepsilon}=-e_{x}^{\varepsilon} \iota_{y}^{\varepsilon} .
$$

In an orthonormal basis $(u)_{\ell}$, the completeness relation yields for any spinor $\chi \in S_{y} \mathscr{M}$

$$
P^{\varepsilon}(x, y) \chi=-e_{x}^{\varepsilon} \iota_{y}^{\varepsilon} \chi=-\sum_{\ell}\left(e_{x}^{\varepsilon} u_{\ell}\right)\left\langle u_{\ell} \mid \iota_{y}^{\varepsilon} \chi\right\rangle_{\mathcal{H}}=-\sum_{\ell}\left(e_{x}^{\varepsilon} u_{\ell}\right)\left(\overline{e_{x}^{\varepsilon} u_{\ell}} \chi\right),
$$

and using (1.2.14) gives $(1.2 .19)$.

In order to prove (1.2.21), we introduce the functionals

$$
\Phi_{\eta}^{\varepsilon}: \mathcal{H} \rightarrow \mathbb{C}, \quad \Phi_{\eta}^{\varepsilon} u=\int_{\mathscr{M}} \overline{\eta(x)}\left(\Re_{\varepsilon} u\right)(x) d^{4} x
$$

and similarly without UV regularization,

$$
\Phi_{\eta}: \mathcal{H} \rightarrow \mathbb{C}, \quad \Phi_{\eta} u=\int_{\mathscr{M}} \overline{\eta(x)} u(x) d^{4} x .
$$

Then the left side of 1.2.21 can be written in the compact form

$$
\left|\Phi_{\eta}^{\varepsilon}\left(\Phi_{\tilde{\eta}}^{\varepsilon}\right)^{*}-\Phi_{\eta}\left(\Phi_{\tilde{\eta}}\right)^{*}\right|
$$

which can be estimated with the triangle inequality by

$$
\left|\Phi_{\eta}^{\varepsilon}\left(\Phi_{\tilde{\eta}}^{\varepsilon}\right)^{*}-\Phi_{\eta}\left(\Phi_{\tilde{\eta}}\right)^{*}\right| \leq\left\|\Phi_{\eta}^{\varepsilon}\right\|\left\|\Phi_{\tilde{\eta}}^{\varepsilon}-\Phi_{\tilde{\eta}}\right\|+\left\|\Phi_{\eta}^{\varepsilon}-\Phi_{\eta}\right\|\left\|\Phi_{\tilde{\eta}}\right\|
$$

It remains to estimate the operator norms in 1.2 .22 . To this end, we use property (iii) of Definition 1.2 .3 in the following way: First, the norm of $\Phi_{\eta}$ can be estimated by

$$
\left|\Phi_{\eta} u\right|=\int_{\mathcal{M}} \overline{\eta(x)} u(x) d^{4} x \leq|\eta|_{C^{0}(K)} \sqrt{\mu(K)}\left(\int_{K}|u(x)| d^{4} x\right)^{\frac{1}{2}},
$$

and again by applying 1.2 .9 . This gives

$$
\left\|\Phi_{\eta}\right\| \leq c|\eta|_{C^{0}(K)} .
$$

Next, we use the triangle inequality together with (1.2.8) to obtain the inequality

$$
\left\|\Phi_{\eta}^{\varepsilon}\right\| \leq\left\|\Phi_{\eta}^{\varepsilon}-\Phi_{\eta}\right\|+\left\|\Phi_{\eta}\right\| \leq \delta|\eta|_{C^{1}(K)}+c|\eta|_{C^{0}(K)} \leq 2 c|\eta|_{C^{1}(K)}
$$

valid uniformly for all $\varepsilon \in\left(0, \varepsilon_{0}\right)$ (note that property (i) cannot be used to obtain such a uniform estimate because we have no control on how the constant $c$ in $(1.2 .6)$ depends on $\varepsilon$ ). Finally, again applying (1.2.8), we also know that

$$
\left\|\Phi_{\eta}^{\varepsilon}-\Phi_{\eta}\right\| \leq \delta|\eta|_{C^{1}(K)} .
$$


Using these inequalities in $(1.2 .22)$ gives the result.

1.2.5. Correspondence of the Causal Structure. We now explain how the causal structure of Minkowski space is related to the corresponding notions of a causal fermion system (see Definition 1.1.2 and the time direction (1.1.12). To this end, we need to specify $\mathcal{H}$ as a closed subspace of the solution space of the vacuum Dirac equation (1.2.1). Clearly, this Dirac equation can be solved by the plane-wave ansatz

$$
\psi(x)=e^{-i k x} \chi_{k}
$$

with a constant spinor $\chi_{k}$. Evaluating the resulting algebraic equation for $\chi$ shows that the momentum $k$ must lie on the mass shell $k^{2}=m^{2}$ (where $k^{2} \equiv k^{j} k_{j}$ is the Minkowski inner product). The solutions on the upper and lower mass shell are the solutions of positive and negative energy, respectively. In order to avoid potential confusion with other notions of energy (like energy densities or energy expectation values), we here prefer the notion of solutions of positive and negative frequency. Taking Dirac's original concept literally, we here describe the vacuum in Minkowski space by the completely filled Dirac sea. Thus we choose $\mathcal{H}$ as the subspace of the solution space spanned by all plane-wave solutions of negative frequency. We refer to this choice as a Dirac sea configuration.

Lemma 1.2.8. If $\mathcal{H}$ is the subspace of the solution space of the Dirac equation (1.2.1) spanned by all negative-frequency solutions, then the unregularized kernel of the fermionic projector as defined by 1.2 .20 is the tempered bi-distribution

$$
P(x, y)=\int \frac{d^{4} k}{(2 \pi)^{4}}(\not k+m) \delta\left(k^{2}-m^{2}\right) \Theta\left(-k_{0}\right) e^{-i k(x-y)},
$$

where $\delta$ is Dirac's delta distribution, $\Theta$ is the Heaviside function, $k(x-y)$ is a short notation for the Minkowski inner product $k_{j}(x-y)^{j}$, and the slash in $\not k=k^{j} \gamma_{j}$ denotes contraction with the Dirac matrices (the "Feynman dagger").

ProOF. The integrand in (1.2.23) clearly is a tempered distribution. Hence its Fourier transform $P(x, y)$ is also a tempered distribution (in the vector $y-x$ and also in both vectors $x$ and $y)$. In addition, one verifies by direct computation that $P(x, y)$ is a distributional solution of the Dirac equation,

$$
\begin{aligned}
\left(i \not \partial_{x}-m\right) P(x, y) & =\int \frac{d^{4} k}{(2 \pi)^{4}}(\not k-m)(\not k+m) \delta\left(k^{2}-m^{2}\right) \Theta\left(-k_{0}\right) e^{-i k(x-y)} \\
& =\int \frac{d^{4} k}{(2 \pi)^{4}}\left(k^{2}-m^{2}\right) \delta\left(k^{2}-m^{2}\right) \Theta\left(-k_{0}\right) e^{-i k(x-y)}=0 .
\end{aligned}
$$

Due to the factor $\Theta\left(-k_{0}\right)$, the distribution $P(x, y)$ is composed of solutions of negative frequency. Moreover, since the matrix $(\not k+m)$ has rank two, one sees that $P(x, y)$ is indeed composed of all negative-frequency solutions. It remains to show that the normalization of $P(x, y)$ is compatible with 1.2 .20 , meaning that

$$
-2 \pi \int_{\mathbb{R}^{3}} P(x,(t, \vec{y})) \gamma^{0} P((t, \vec{y}), z) d^{3} y=P(x, z) .
$$


This identity follows by a straightforward computation: First,

$$
\begin{array}{rl}
\int_{\mathbb{R}^{3}} & P(x,(t, \vec{y})) \gamma^{0} P((t, \vec{y}), z) d^{3} y \\
& =\int_{\mathbb{R}^{3}} d^{3} y \int \frac{d^{4} k}{(2 \pi)^{4}} e^{-i k(x-y)} \int \frac{d^{4} q}{(2 \pi)^{4}} e^{-i q(y-z)} P_{m}(k) \gamma^{0} P_{m}(q) \\
& =\left.\int \frac{d^{4} k}{(2 \pi)^{4}} \int_{\mathbb{R}} \frac{d \lambda}{2 \pi} e^{-i k x+i q z} P_{m}(k) \gamma^{0} P_{m}(q)\right|_{q=(\lambda, \vec{k})}
\end{array}
$$

Setting $k=(\omega, \vec{k})$, we evaluate the $\delta$-distributions inside the factors $P_{m}$,

$$
\begin{gathered}
\left.\delta\left(k^{2}-m^{2}\right) \delta\left(q^{2}-m^{2}\right)\right|_{q=(\lambda, \vec{k})}=\delta\left(\omega^{2}-|\vec{k}|^{2}-m^{2}\right) \delta\left(\lambda^{2}-|\vec{k}|^{2}-m^{2}\right) \\
=\delta\left(\lambda^{2}-\omega^{2}\right) \delta\left(\omega^{2}-|\vec{k}|^{2}-m^{2}\right) .
\end{gathered}
$$

This shows that we only get a contribution if $\lambda= \pm \omega$. Using this fact together with the mass shell property $\omega^{2}-|\vec{k}|^{2}=m^{2}$, we can simplify the Dirac matrices according to

$$
\begin{aligned}
(\not k+m) & \gamma^{0}(\not 1+m)=\left(\omega \gamma^{0}+\vec{k} \vec{\gamma}+m\right) \gamma^{0}\left( \pm \omega \gamma^{0}+\vec{k} \vec{\gamma}+m\right) \\
& =\left(\omega \gamma^{0}+\vec{k} \vec{\gamma}+m\right)\left( \pm \omega \gamma^{0}-\vec{k} \vec{\gamma}+m\right) \gamma^{0} \\
& =\left(\left( \pm \omega^{2}+|\vec{k}|^{2}+m^{2}\right) \gamma^{0}+(1 \pm 1) \omega(\vec{k} \vec{\gamma})+(1 \pm 1) m \omega\right) \\
& =\left\{\begin{array}{cl}
2 \omega(\not k+m) & \text { in case }+ \\
0 & \text { in case }-.
\end{array}\right.
\end{aligned}
$$

Hence we only get a contribution if $\lambda=\omega$, giving rise to the identity

$$
\delta\left(\lambda^{2}-\omega^{2}\right)=\frac{1}{2|\omega|} \delta(\lambda-\omega) .
$$

Combining these formulas, we obtain

$$
\begin{array}{rl}
\int_{\mathbb{R}^{3}} & P(x,(t, \vec{y})) \gamma^{0} P((t, \vec{y}), z) d^{3} y \\
& =\int \frac{d^{4} k}{(2 \pi)^{4}} \int_{\mathbb{R}} \frac{d \lambda}{2 \pi} e^{-i k(x-z)} \delta(\lambda-\omega) \delta\left(k^{2}-m^{2}\right) \frac{2 \omega}{2|\omega|}(\not k+m) \Theta\left(-k_{0}\right) \\
& =-\frac{1}{2 \pi} \int \frac{d^{4} k}{(2 \pi)^{4}} e^{-i k(x-z)} \delta\left(k^{2}-m^{2}\right)(\not k+m) \Theta\left(-k_{0}\right) .
\end{array}
$$

This gives the result.

The Fourier integral 1.2 .23 can be computed in closed form, giving an expression involving Bessel functions. Since the general structure of the resulting formula will be important later on, we give the computation in detail. In preparation, it is useful to pull the Dirac matrices out of the Fourier integral. To this end, one rewrites the factor $(\not k+m)$ in 1.2 .23 in terms of a differential operator in position space,

$$
P(x, y)=\left(i \not \partial_{x}+m\right) T_{m^{2}}(x, y),
$$

where $T_{m^{2}}$ is the scalar bi-distribution

$$
T_{m^{2}}(x, y):=\int \frac{d^{4} k}{(2 \pi)^{4}} \delta\left(k^{2}-m^{2}\right) \Theta\left(-k_{0}\right) e^{-i k(x-y)} .
$$

In the next lemma, we determine the singular structure of this distribution. The method is to subtract an explicit singular distribution and to show that the difference is a regular 
distribution (i.e. a locally integrable function, denoted by $L_{\text {loc }}^{1}$ ). The distribution $\mathrm{PP} / \xi^{2}$, denoted by principal value, is defined by evaluating weakly with a test function $\eta \in$ $C_{0}^{\infty}(\mathscr{M})$ and by removing the positive and negative parts of the pole in a symmetric way. There are different equivalent ways of writing the principal part, each of which could serve as a possible definition (for mathematical details see Exercises 1.11 and 1.12):

$$
\begin{aligned}
& \int \frac{\mathrm{PP}}{\xi^{2}} \eta(\xi) d^{4} \xi=\lim _{\nu \searrow 0} \int \Theta\left(\left|\xi^{2}\right|-\nu\right) \frac{1}{\xi^{2}} \eta(\xi) d^{4} \xi \\
& \quad=\lim _{\nu \searrow 0} \frac{1}{2} \sum_{ \pm} \int \frac{1}{\xi^{2} \pm i \nu} \eta(\xi) d^{4} \xi=\lim _{\nu \searrow 0} \frac{1}{2} \sum_{ \pm} \int \frac{1}{\xi^{2} \pm i \nu \xi^{0}} \eta(\xi) d^{4} \xi
\end{aligned}
$$

(here $\xi^{2} \equiv \xi^{j} \xi_{j}$ is again the Minkowski inner product).

LEMma 1.2.9. On the light cone, the distribution $T_{m^{2}}$ has the singularity structure

$$
T_{m^{2}}(x, y)=-\frac{1}{8 \pi^{3}}\left(\frac{\mathrm{PP}}{\xi^{2}}+i \pi \delta\left(\xi^{2}\right) \epsilon\left(\xi^{0}\right)\right)+r(x, y)
$$

where we set $\xi:=y-x$, and $r \in L_{\mathrm{loc}}^{1}(\mathscr{M} \times \mathcal{M})$ is a regular distribution. Here $\epsilon$ is the sign function $\epsilon(x)=1$ for $x \geq 0$ and $\epsilon(x)=-1$ otherwise. Away from the light cone (i.e. for $\left.\xi^{2} \neq 0\right), T_{m^{2}}(x, y)$ is a smooth function given by

$$
T_{m^{2}}(x, y)=\left\{\begin{array}{cl}
\frac{m}{16 \pi^{2}} \frac{Y_{1}\left(m \sqrt{\xi^{2}}\right)}{\sqrt{\xi^{2}}}+\frac{i m}{16 \pi^{2}} \frac{J_{1}\left(m \sqrt{\xi^{2}}\right)}{\sqrt{\xi^{2}}} \epsilon\left(\xi^{0}\right) & \text { if } \xi \text { is timelike } \\
\frac{m}{8 \pi^{3}} \frac{K_{1}\left(m \sqrt{-\xi^{2}}\right)}{\sqrt{-\xi^{2}}} & \text { if } \xi \text { is spacelike },
\end{array}\right.
$$

where $J_{1}, Y_{1}$ and $K_{1}$ are Bessel functions.

Proof. The Fourier integral is computed most conveniently by inserting a convergence-generating factor. Thus for any $\varepsilon>0$ we consider the Fourier integral

$$
T_{m^{2}}^{\varepsilon}(x, y):=\int \frac{d^{4} k}{(2 \pi)^{4}} \delta\left(k^{2}-m^{2}\right) \Theta\left(-k_{0}\right) e^{-i k(x-y)} e^{-\varepsilon\left|k_{0}\right|}
$$

This Fourier integral can be computed pointwise, showing that $T^{\varepsilon}(x, y)$ is a regular distribution. Taking the limit $\varepsilon \searrow 0$ in the distributional sense, we will then obtain $T_{m^{2}}(x, y)$.

Setting $\xi=y-x$ and $t=\xi^{0}$, we first carry out the integral over $k_{0}$ to obtain

$$
\begin{aligned}
T_{m^{2}}^{\varepsilon}(x, y) & =\int \frac{d^{4} k}{(2 \pi)^{4}} \delta\left(k^{2}-m^{2}\right) \Theta\left(-k_{0}\right) e^{i k \xi} e^{-\varepsilon\left|k_{0}\right|} \\
& =\int_{\mathbb{R}^{3}} \frac{d^{3} k}{(2 \pi)^{4}} \frac{1}{2 \sqrt{\vec{k}^{2}+m^{2}}} e^{-i \sqrt{\vec{k}^{2}+m^{2}} t-i \vec{k} \vec{\xi}} e^{-\varepsilon \sqrt{\vec{k}^{2}+m^{2}}}
\end{aligned}
$$


Next, for the spatial momentum $\vec{k}$ we introduce polar coordinates $(p=|\vec{k}|, \vartheta, \varphi)$, where $\vartheta$ is the angle between $\vec{k}$ and $\vec{\xi}$, and $\varphi$ is the azimuthal angle. Also setting $r=|\vec{\xi}|$, we get

$$
\begin{aligned}
T_{m^{2}}^{\varepsilon}(x, y) & =\int_{0}^{\infty} \frac{d p}{2(2 \pi)^{3}} \int_{-1}^{1} d \cos \theta \frac{p^{2}}{\sqrt{p^{2}+m^{2}}} e^{-(\varepsilon+i t) \sqrt{p^{2}+m^{2}}} e^{-i p r \cos \theta} \\
& =\frac{1}{r} \int_{0}^{\infty} \frac{d p}{(2 \pi)^{3}} \frac{p}{\sqrt{p^{2}+m^{2}}} e^{-(\varepsilon+i t) \sqrt{p^{2}+m^{2}}} \sin (p r) \\
& =\frac{m^{2}}{(2 \pi)^{3}} \frac{K_{1}\left(m \sqrt{r^{2}+(\varepsilon+i t)^{2}}\right)}{m \sqrt{r^{2}+(\varepsilon+i t)^{2}}}
\end{aligned}
$$

where the last integral was carried out using [GR, formula (3.961.1)]. Here the square root and the Bessel function $K_{1}$ are defined as usual using a branch cut along the negative real axis.

When taking the limit $\varepsilon \searrow 0$, one must be careful for two reasons. First, a pole forms on the light cone $t= \pm r$. Second, the Bessel function $K_{1}$ involves logarithms, which must be evaluated in the complex plane using the branch cut along the negative real axis. For clarity, we treat these two issues after each other. The asymptotic expansion of the Bessel function (see [OLBC, (10.31.1)])

$$
K_{1}(z)=\frac{1}{z}+\mathcal{O}(z \log z)
$$

yields that the pole on the light cone is of the form

$$
T_{m^{2}}^{\varepsilon}(x, y)=\frac{1}{(2 \pi)^{3}} \frac{1}{r^{2}+(\varepsilon+i t)^{2}}+\mathcal{O}\left(\log \left|\xi^{2}\right|\right),
$$

uniformly in $\varepsilon$. Therefore, after subtracting the pole, we can take the limit $\varepsilon \searrow 0$ as a locally integrable function, i.e.

$$
\lim _{\varepsilon \searrow 0}\left(T_{m^{2}}^{\varepsilon}(x, y)-\frac{1}{(2 \pi)^{3}} \frac{1}{r^{2}+(\varepsilon+i t)^{2}}\right) \in L_{\mathrm{loc}}^{1}(\mathscr{M} \times \mathscr{M}) .
$$

For the subtracted pole, the limit $\varepsilon \searrow 0$ can be computed in the distributional sense by

$$
\lim _{\varepsilon \searrow 0} \frac{1}{r^{2}+(\varepsilon+i t)^{2}}=\lim _{\varepsilon \searrow 0} \frac{1}{r^{2}-t^{2}+i \varepsilon t}=-\frac{P P}{\xi^{2}}-i \pi \delta\left(\xi^{2}\right) \epsilon\left(\xi^{0}\right),
$$

where we used the distributional equations

$$
\begin{aligned}
\lim _{\varepsilon \searrow 0}\left(\frac{1}{x-i \varepsilon}-\frac{1}{x+i \varepsilon}\right) & =2 \pi i \delta(x) \\
\frac{1}{2} \lim _{\varepsilon \searrow 0}\left(\frac{1}{x-i \varepsilon}+\frac{1}{x+i \varepsilon}\right) & =: \frac{P P}{x} .
\end{aligned}
$$

(for details see Exercises 1.101 .12 . Here $\epsilon$ is again the sign function $\epsilon(x)=1$ for $x \geq 0$ and $\epsilon(x)=-1$ otherwise. This gives 1.2.28.

In order to compute the regular part of the distribution $T_{m^{2}}$, we may disregard the singularity on the light cone and may consider the case that $\xi$ is either spacelike or timelike. In the first case, the argument $m \sqrt{r^{2}+(\varepsilon+i t)^{2}}$ of the Bessel function converges to the positive real axis, where the Bessel function is analytic. This gives the lower equation in $(1.2 .29)$. In the remaining case that $\xi$ is timelike, the argument $m \sqrt{r^{2}+(\varepsilon+i t)^{2}}$ converges to the imaginary axis (more precisely, to the upper imaginary axis if $t>0$ and to the lower imaginary axis if $t<0$; see Figure 1.1). Using the relations $\mathbf{O L B C},(10.27 .9)$ 


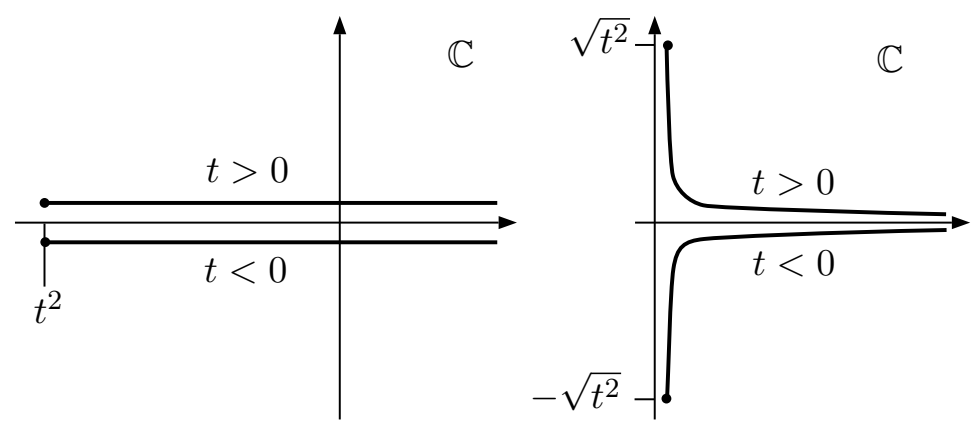

Figure 1.1. The set $\left\{r^{2}+(\varepsilon+i t)^{2} \mid r \in \mathbb{R}\right\}$ (left) and its square root (right)

and (10.27.10)]

$$
i \pi J_{1}(z)=-i K_{1}(-i z)-i K_{1}(i z) \quad \text { and } \quad-\pi Y_{1}(z)=-i K_{1}(-i z)+i K_{1}(i z)
$$

(valid if $|\arg z|<\frac{\pi}{2}$ ), one can express $K_{1}$ near the upper and lower imaginary axis by

$$
K_{1}( \pm i z)=-\frac{\pi}{2}\left(J_{1}(z) \mp i Y_{1}(z)\right) .
$$

Using these identities in 1.2.31 and using that the Bessel functions $J_{1}$ and $K_{1}$ are analytic in a neighborhood of the positive real axis, one can take the limit $\varepsilon \searrow 0$ to obtain the upper equation in (1.2.29).

We point out that the Bessel functions in 1.2 .29 are all real-valued. In particular, one sees that $T(x, y)$ is real-valued if the vector $\xi$ is spacelike. This fact can also be understood from a general symmetry argument (see Exercise 1.13.

Using the result of Lemma 1.2.9 in 1.2.25), one can derive corresponding formulas for $P(x, y)$. In particular, differentiating (1.2.28), one sees that $P(x, y)$ has an even stronger singularity on the light cone which involves terms of the form $1 / \xi^{4}$ and $\delta^{\prime}\left(\xi^{2}\right)$. Differentiating (1.2.29), carrying out the derivatives with the chain rule and using formulas for the derivatives of Bessel functions (see [OLBC, (10.6.6) and (10.29.4)]), one can also express the fermionic projector $P(x, y)$ in terms of Bessel functions (see Exercise 1.14). We do not give the resulting formulas, because we do not need the detailed form later on. Instead, we here prefer to argue with structural properties of the distribution $P(x, y)$. This makes it possible to infer qualitative properties of the eigenvalues of $A_{x y}$, even without referring to the detailed form of the formulas in Lemma 1.2.9. From Lorentz symmetry, we know that for all $x$ and $y$ with spacelike or timelike separation, $P(x, y)$ can be written as

$$
P(x, y)=\alpha \xi_{j} \gamma^{j}+\beta \mathbb{1}
$$

with two complex-valued functions $\alpha$ and $\beta$ (where again $\xi=y-x$ ). Taking the adjoint with respect to the spin scalar product, we see that

$$
P(y, x)=\bar{\alpha} \xi_{j} \gamma^{j}+\bar{\beta} \mathbb{1} .
$$

As a consequence,

$$
A_{x y}=P(x, y) P(y, x)=a \xi_{j} \gamma^{j}+b \mathbb{1}
$$

with two real parameters $a$ and $b$ given by

$$
a=\alpha \bar{\beta}+\beta \bar{\alpha}, \quad b=|\alpha|^{2} \xi^{2}+|\beta|^{2} .
$$


Applying the formula $\left(A_{x y}-b \mathbb{1}\right)^{2}=a^{2} \xi^{2} \mathbb{1}$, the roots of the characteristic polynomial of $A_{x y}$ are computed by

$$
b \pm \sqrt{a^{2} \xi^{2}} .
$$

Therefore, the eigenvalues of the closed chain are either real, or else they form a complex conjugate pain 4 . Which of the two cases occurs is determined by the sign of the factor $\xi^{2}$. This gives the agreement of the different notions of causality in the following sense:

Proposition 1.2.10. Assume that $P(x, y)$ is the unregularized kernel of the fermionic projector of the vacuum (1.2.23), and that the eigenvalues $\lambda_{1}^{x y}, \ldots, \lambda_{4}^{x y}$ are computed as the eigenvalues of the closed chain (1.1.14). Then the following statements hold:

If the points $x, y \in M$ have spacelike separation in Minkowski space, then they are also spacelike separated in the sense of Definition 1.1.2. If, on the other hand, the points $x, y \in$ $M$ have timelike separation in Minkowski space, then they are also timelike separated in the sense of Definition 1.1.2. Even more, they are properly timelike separated (see Definition 1.1.6) in the sense that the closed chain $A_{x y}$ has strictly positive eigenvalues and definite eigenspaces. Finally, if the points $x, y \in \mathscr{M}$ have lightlike separation in Minkowski space, then the causal structure of Definition 1.1.2 is ill-defined.

The fact that the causal structure is ill-defined for lightlike separation again explains why an UV regularization must be introduced.

Proof of Proposition 1.2.10. If the vector $\xi=y-x$ is spacelike, then the term $\xi^{2}$ is negative. Thus the eigenvalues in 1.2 .39 form a complex conjugate pair, implying that they all have the same absolute value. Thus the points are spacelike separated in the sense of Definition 1.1.2.

If the vector $\xi$ is timelike, the term $\xi^{2}$ in 1.2 .39 is positive, so that the $\lambda_{j}$ are all real. In order to show that they do not have the same absolute value, we need to verify that the parameters $a$ and $b$ are both non-zero. This makes it necessary to refer to the explicit formula involving Bessel functions (1.2.29): The Bessel functions $Y_{1}$ and $J_{1}$ do not have joint zeros on the positive real axis (this can be understood abstractly from the fact that these Bessel functions form a fundamental system of solutions of the Bessel equation; see Exercise 1.16). As a consequence, the parameter $\beta$ in 1.2 .35 is non-zero. Likewise, the derivatives $Y_{1}^{\prime}$ and $J_{1}^{\prime}$ do not have joint zeros (as can again be verified from the fact that the Bessel functions form a fundamental system). This implies that the parameter $\alpha$ in 1.2.35 is non-zero. We conclude that the parameter $b$ in 1.2 .38 is non-zero. The combination of $\alpha$ and $\beta$ in the formula for $a$ in 1.2 .38 can be rewritten in terms of a Wronskian of the Bessel function. This Wronskian can be computed explicitly using OLBC, (10.5.2)], implying that $a$ is non-zero (see Exercise 1.14). We conclude that the points $x$ and $y$ are timelike separated in the sense of Definition 1.1.2.

In order to get the connection to proper timelike separation, recall that if $\xi$ is a timelike vector of Minkowski space, then the closed chain has the form $(1.2 .38)$ with $a, b \neq 0$. A direct computation shows that this matrix is diagonalizable and that the eigenspaces are definite with respect to the spin scalar product (see Exercise 1.17). Moreover, applying the Schwarz inequality to the explicit formulas (1.2.38), one obtains

$$
|a| \sqrt{\xi^{2}}=2 \operatorname{Re}\left(\alpha \sqrt{\xi^{2}} \bar{\beta}\right) \stackrel{(\star)}{\leq}|\alpha|^{2} \xi^{2}+|\beta|^{2}=b
$$

\footnotetext{
${ }^{4}$ It is a general property of the closed chain that if $\lambda$ is an eigenvalue, then so is $\bar{\lambda}$; see Exercise 1.15
} 
proving that the eigenvalues in $(1.2 .39)$ are non-negative. It remains to show that none of these eigenvalues vanishes. To this end, it suffices to show that the inequality $(\star)$ in 1.2.40 is strict, which in turn is equivalent to proving that

$$
\operatorname{Im}(\alpha \bar{\beta}) \neq 0 .
$$

This inequality follows by a detailed analysis of the Bessel functions (see [FG2, proof of Lemma 4.3]). We conclude that $x$ and $y$ are indeed properly timelike separated.

If the vector $\xi$ is lightlike, then $P(x, y)$ is not defined pointwise. As a consequence, the closed chain is ill-defined.

This proposition cannot be applied directly to causal fermion systems because, as explained in 1.2 .1 and $\$ 1.2 .2$, constructing a causal fermion system makes it necessary to introduce an UV regularization. Nevertheless, the above proposition also gives the correspondence of the different notions of causality for causal fermion systems describing the Minkowski vacuum, as we now explain. Thus let us consider the causal fermion system corresponding to the regularized fermionic projector of the vacuum $P^{\varepsilon}(x, y)$. In the limit $\varepsilon \searrow 0$, the kernel of the fermionic projector $P^{\varepsilon}(x, y)$ converges to the unregularized kernel $P(x, y)$ (see (1.2.21) in Proposition 1.2.6). If this convergence is pointwise, i.e. if for given space-time points $x, y \in \mathscr{M}$,

$$
\lim _{\varepsilon \searrow 0} P^{\varepsilon}(x, y)=P(x, y),
$$

then the results of Proposition 1.2.10 also apply to the causal fermion system, up to error terms which tend to zero as $\varepsilon \searrow 0$ (for the stability of the notions of causality see Exercises 1.18 and 1.19). Thinking of $\varepsilon$ as the Planck scale, this means physically that the notion of causality of Definition 1.1 .2 agrees with the usual notion of causality in Minkowski space, up to corrections which are so small that they cannot be observed. The subtle point of this argument is that it requires pointwise convergence (1.2.41). Clearly, such a pointwise convergence cannot hold if $x$ and $y$ are lightlike separated, because the right side of (1.2.41) is ill-defined pointwise. Expressed for a causal fermion system for fixed $\varepsilon$ on the Planck scale, this means that the notion of causality of Definition 1.1.2 does not agree with the usual notion of causality if the vector $\xi$ is almost lightlike in the sense that ||$\xi^{0}|-| \vec{\xi}|| \lesssim \varepsilon$. This is not surprising because we cannot expect that the notion of causality in Minkowski space holds with a higher resolution than the regularization scale $\varepsilon$. The remaining question is whether we have pointwise convergence $(1.2 .41)$ if the points $x$ and $y$ have timelike or spacelike separation. The answer is yes for a large class of regularizations (like for example the regularization by mollification in Example 1.2.4. However, the general notion of Definition 1.2 .3 only gives weak convergence of the kernels (1.2.21). This shortcoming could be removed by adding a condition to Definition 1.2 .3 which ensures pointwise convergence away from the light cone. On the other hand, such an additional condition will not be needed for the constructions in this book, and therefore it seems preferable not to impose it. Nevertheless, the physical picture is that the regularized kernel should converge pointwise, at least for generic points $x$ and $y$ which lie sufficiently far away from the light cone. With this in mind, Proposition 1.2.10 indeed shows that the notion of causality of Definition 1.1.2 corresponds to the usual notion of causality in Minkowski space, up to corrections which are so small that they are irrelevant in most situations of interest.

We next make a remark on the notion of lightlike separation. At first sight, Definition 1.1.2 leaves many possibilities for lightlike separation: two eigenvalues could be real 
and two could form a complex conjugate pair, or there could be two complex conjugate pairs with different abolute values, etc. However, in the above example of Minkowski space, we saw that lightlike separation appears only as the boundary case between timelike and spacelike separation when all eigenvalues are degenerate. More generally, all known examples and numerical studies suggest that lightlike separation is not favorable when minimizing the causal action. This can be understood intuitively as follows: Since the Lagrangian vanishes for spacelike separation, the causal action principle aims at arranging spacelike separation for as many pairs or points as possible. But in view of the constraints, it is impossible to arrange that all pairs of points are spacelike separated. It seems preferable to satisfy the constraints by arranging timelike separation for certain pairs or points, whereas the other pairs of points are arranged to be spacelike separated. In this mechanism, lightlike separation appears typically only for few pairs of points as the boundary case between timelike and spacelike separation.

We conclude this section by explaining why the functional $\mathcal{C}$ introduced in (1.1.11) gives information on the time direction. Our first task is to rewrite this functional in terms of the regularized kernel of the fermionic projector $P^{\varepsilon}(x, y)$.

Lemma 1.2.11. Assume that the operator $P^{\varepsilon}(x, x): S_{x} \mathcal{M} \rightarrow S_{x} \mathcal{M}$ is invertible. Then, setting

$$
\nu(x)=P^{\varepsilon}(x, x)^{-1}: S_{x} \mathscr{M} \rightarrow S_{x} \mathscr{M},
$$

the functional $\mathrm{C}, 1.1 .11$, can be written as

$$
\mathcal{C}(x, y)=i \operatorname{Tr}_{S_{x}}\left(P^{\varepsilon}(x, y) \nu(y) P^{\varepsilon}(y, x)\left[\nu(x), A_{x y}\right]\right) .
$$

Proof. Since $P(x, x)=\left.\pi_{x} x\right|_{S_{x}}=\left.x\right|_{S_{x}}$, we know that $\nu(x)=\left(\left.x\right|_{S_{x}}\right)^{-1}$. Thus

$$
\begin{aligned}
\left.\pi_{x} y x \pi_{y} \pi_{x}\right|_{S_{x}} & =\left.\pi_{x} y \pi_{y} x \pi_{x} y \nu(y) \pi_{y} x \nu(x)\right|_{S_{x}} \\
& =\left.P^{\varepsilon}(x, y) P^{\varepsilon}(y, x) P^{\varepsilon}(x, y) \nu(y) P^{\varepsilon}(y, x) \nu(x)\right|_{S_{x}} .
\end{aligned}
$$

Using this formula in (1.1.11), we obtain

$$
\begin{aligned}
\mathcal{C}(x, y)= & i \operatorname{Tr}_{S_{x}}\left(\left.y x \pi_{y} \pi_{x}\right|_{S_{x}}-\left.y \pi_{x} \pi_{y} x\right|_{S_{x}}\right) \\
= & i \operatorname{Tr}_{S_{x}}\left(P^{\varepsilon}(x, y) P^{\varepsilon}(y, x) P^{\varepsilon}(x, y) \nu(y) P^{\varepsilon}(y, x) \nu(x)\right. \\
- & \left.P^{\varepsilon}(x, y) P^{\varepsilon}(y, x) \nu(x) P^{\varepsilon}(x, y) \nu(y) P^{\varepsilon}(y, x)\right) \\
= & i \operatorname{Tr}_{S_{x}}\left(P^{\varepsilon}(x, y) \nu(y) P^{\varepsilon}(y, x) \nu(x) P^{\varepsilon}(x, y) P^{\varepsilon}(y, x)\right. \\
- & \left.P^{\varepsilon}(x, y) \nu(y) P^{\varepsilon}(y, x) P^{\varepsilon}(x, y) P^{\varepsilon}(y, x) \nu(x)\right) .
\end{aligned}
$$

This gives the result.

We point out that the operator $\nu(x)$ in $(1.2 .42)$ is ill-defined without UV regularization because evaluating the distribution $P(x, y)$ on the diagonal $x=y$ has no mathematical meaning. As a consequence, the functional $\mathcal{C}$ is ill-defined without UV regularization, even if $x$ and $y$ have timelike separation. This makes the following computation somewhat delicate. In order to keep the analysis reasonably simple, we assume that the regularized kernel of the fermionic projector has vector-scalar structure, meaning that it is of the general form

$$
P^{\varepsilon}(x, y)=v_{j}^{\varepsilon}(x, y) \gamma^{j}+\beta^{\varepsilon}(x, y) \mathbb{1}
$$


with a vector and a scalar component. Here $v^{\varepsilon}(x, y)$ is a complex vector field (i.e. it can be written as $v^{\varepsilon}=u^{\varepsilon}+i w^{\varepsilon}$ with Minkowski vectors $u^{\varepsilon}$ and $w^{\varepsilon}$ which need not be collinear). Moreover, we only consider the case that $x$ and $y$ have timelike separation (for points with spacelike separation see Exercise 1.20). Then, evaluating (1.2.44 for $x=y$, one sees that $P^{\varepsilon}(x, x)$ can be written as

$$
P^{\varepsilon}(x, x)=v_{j}^{\varepsilon}(x) \gamma^{j}+\beta^{\varepsilon}(x) \mathbb{1}
$$

(where we set $v^{\varepsilon}(x)=v^{\varepsilon}(x, x)$ and $\beta^{\varepsilon}(x)=\beta^{\varepsilon}(x, x)$ ). Since $P^{\varepsilon}(x, x)$ is a symmetric operator on $S_{x} \mathscr{M}$, it follows that $v^{\varepsilon}$ is a real vector field, and $\beta$ a real-valued function. For a large class of regularizations, the matrix $P^{\varepsilon}(x, x)$ is invertible because the vectorial component dominates the scalar component (see Exercise 1.21). With this in mind, we here assume that $\nu(x)$ exists. Then it is given by

$$
\nu(x)=\frac{1}{\rho(x)}\left(v_{j}^{\varepsilon}(x) \gamma^{j}-\beta^{\varepsilon}(x) \mathbb{1}\right),
$$

where $\rho:=v_{j}^{\varepsilon}\left(v^{\varepsilon}\right)^{j}-\left(\beta^{\varepsilon}\right)^{2}$. Now we can compute the composite expression in 1.2.43), working for all other terms with the unregularized formulas (which is again justified if we have pointwise convergence (1.2.41) $)$. This gives the following result.

Proposition 1.2.12. Using (1.2.45) and replacing $P^{\varepsilon}(x, y), P^{\varepsilon}(y, x)$ and $A_{x y}$ by the unregularized expressions (1.2.35), (1.2.36) and (1.2.37), the functional $\mathrm{C}$ is given by

$$
\mathcal{C}(x, y)=\frac{16 a}{\rho(x) \rho(y)} \operatorname{Im}(\alpha \bar{\beta})\left(v^{\varepsilon}(x)^{j} \xi_{j} v^{\varepsilon}(y)^{k} \xi_{k}-\xi^{2} v^{\varepsilon}(x)^{j} v^{\varepsilon}(y)_{j}\right) .
$$

Proof. Using (1.2.45) and (1.2.37) in 1.2 .43$)$ gives

$$
\begin{aligned}
\mathcal{C}(x, y) & =i \operatorname{Tr}_{S_{x}}\left(P(x, y) \nu(y) P(y, x)\left[\nu(x), A_{x y}\right]\right) \\
& =\frac{i a}{\rho(x)} \operatorname{Tr}_{S_{x}}\left(P(x, y) \nu(y) P(y, x)\left[\psi^{\varepsilon}(x), \Varangle\right]\right),
\end{aligned}
$$

where in the last step we used that the scalar components of $A_{x y}$ and $\nu(x)$ drop out of the commutator. Taking the scalar component of $\nu(y)$, the two factors $P(x, y)$ and $P(y, x)$ combine to the closed chain, which according to (1.2.37) has no bilinear component, so that the trace vanishes. Therefore, we only need to take into account the vectorial component of $\nu(y)$. Using 1.2 .35 and 1.2 .36 , we obtain

$$
\begin{aligned}
\mathcal{C}(x, y) & =\frac{i a}{\rho(x) \rho(y)} \operatorname{Tr}_{S_{x}}\left((\alpha \not+\beta \mathbb{1}) \psi^{\varepsilon}(y)(\bar{\alpha} \not+\bar{\beta} \mathbb{1})\left[\psi^{\varepsilon}(x), \not\right]\right) \\
& =-\frac{a}{\rho(x) \rho(y)} \operatorname{Im}(\alpha \bar{\beta}) \operatorname{Tr}_{S_{x}}\left(\left[\not \xi^{\prime}, \psi^{\varepsilon}(y)\right]\left[\psi^{\varepsilon}(x), \not\right]\right) .
\end{aligned}
$$

Computing the trace of the product of Dirac matrices gives the result.

The critical reader may wonder why the functional which distinguishes the time direction has the specific form 1.1.11. This question is addressed in Exercise 1.22, where another similar functional is analyzed.

For the interpretation of the formula (1.2.46), we first consider the case that $y$ and $x$ have space-like separation. In this case, it turns out that the prefactor $\operatorname{Im}(\alpha \bar{\beta})$ vanishes, so that (1.2.46) gives no information on a time direction. This is consistent with the fact that for points in Minkowski space with space-like separation, the notions of future- and past-directed depend on the observer and cannot be defined in a covariant manner. However, if $y$ and $x$ have timelike separation, then the factors $a$ and $\operatorname{Im}(\alpha \bar{\beta})$ are indeed both 
non-zero (see the proof of Proposition 1.2.10). Therefore, the functional $\mathcal{C}$ is non-zero, provided that the vector $\xi$ is non-degenerate in the sense that it is linearly independent of both $v^{\varepsilon}(x)$ and $v^{\varepsilon}(y)$. Since the set of directions $\xi$ for which these vectors are linearly dependent has measure zero, we may always restrict attention to non-degenerate directions. Moreover, the formula (1.2.46) shows that the functional $\mathcal{C}$ does not change sign for $\xi$ inside the upper or lower light cone. On the other hand, $\mathcal{C}$ is antisymmetric under sign flips of $\xi$ because interchanging $x$ and $y$ in 1.1.11 obviously gives a minus sign.

We conclude that for the regularized Dirac sea vacuum, the sign of the functional $\mathcal{C}$ distinguishes a time direction. Asymptotically as $\varepsilon \searrow 0$, this time direction agrees with the distinction of the causal past and causal future in Minkowski space.

To summarize, in this section we saw how the intrinsic structures of a causal fermion system correspond to the usual structures in Minkowski space. To this end, we constructed causal fermion systems from a regularized Dirac sea configuration and analyzed the asymptotics as the UV regularization is removed. For brevity, we only considered the topological and causal structure of space-time as well as spinors and wave functions. The reader interested in geometric structures like connection and curvature is referred to the detailed exposition in [FG2]. Moreover, in Section 1.5 below we shall explain how the methods and results introduced in this section can be generalized to interacting systems.

\subsection{Underlying Physical Principles}

In order to clarify the physical concepts, we now briefly discuss the underlying physical principles. Causal fermion systems evolved from an attempt to combine several physical principles in a coherent mathematical framework. As a result, these principles appear in the framework in a specific way:

- The principle of causality is built into a causal fermion system in a specific way, as was explained in 1.1 .2 above.

- The Pauli exclusion principle is incorporated in a causal fermion system, as can be seen in various ways. One formulation of the Pauli exclusion principle states that every fermionic one-particle state can be occupied by at most one particle. In this formulation, the Pauli exclusion principle is respected because every wave function can either be represented in the form $\psi^{u}$ (the state is occupied) with $u \in \mathcal{H}$ or it cannot be represented as a physical wave function (the state is not occupied). Via these two conditions, the fermionic projector encodes for every state the occupation numbers 1 and 0 , respectively, but it is impossible to describe higher occupation numbers. More technically, one may obtain the connection to the fermionic Fock space formalism by choosing an orthonormal basis $u_{1}, \ldots, u_{f}$ of $\mathcal{H}$ and forming the $f$-particle Hartree-Fock state

$$
\Psi:=\psi^{u_{1}} \wedge \cdots \wedge \psi^{u_{f}}
$$

Clearly, the choice of the orthonormal basis is unique only up to the unitary transformations

$$
u_{i} \rightarrow \tilde{u}_{i}=\sum_{j=1}^{f} U_{i j} u_{j} \quad \text { with } \quad U \in \mathrm{U}(f) .
$$

Due to the anti-symmetrization, this transformation changes the corresponding Hartree-Fock state only by an irrelevant phase factor,

$$
\psi^{\tilde{u}_{1}} \wedge \cdots \wedge \psi^{\tilde{u}_{f}}=\operatorname{det} U \psi^{u_{1}} \wedge \cdots \wedge \psi^{u_{f}} .
$$


Thus the configuration of the physical wave functions can be described by a fermionic multi-particle wave function. The Pauli exclusion principle becomes apparent in the total anti-symmetrization of this wave function.

- A local gauge principle becomes apparent once we choose basis representations of the spin spaces and write the wave functions in components. Denoting the signature of $\left(S_{x}, \prec . \mid . \succ_{x}\right)$ by $(p(x), q(x))$, we choose a pseudo-orthonormal basis $\left(\mathfrak{e}_{\alpha}(x)\right)_{\alpha=1, \ldots, p+q}$ of $S_{x}$. Then a wave function $\psi$ can be represented as

$$
\psi(x)=\sum_{\alpha=1}^{p+q} \psi^{\alpha}(x) \mathfrak{e}_{\alpha}(x)
$$

with component functions $\psi^{1}, \ldots, \psi^{p+q}$. The freedom in choosing the basis $\left(\mathfrak{e}_{\alpha}\right)$ is described by the group $\mathrm{U}(p, q)$ of unitary transformations with respect to an inner product of signature $(p, q)$. This gives rise to the transformations

$$
\mathfrak{e}_{\alpha}(x) \rightarrow \sum_{\beta=1}^{p+q} U^{-1}(x)_{\alpha}^{\beta} \mathfrak{e}_{\beta}(x) \quad \text { and } \quad \psi^{\alpha}(x) \rightarrow \sum_{\beta=1}^{p+q} U(x)_{\beta}^{\alpha} \psi^{\beta}(x)
$$

with $U \in \mathrm{U}(p, q)$. As the basis $\left(\mathfrak{e}_{\alpha}\right)$ can be chosen independently at each space-time point, one obtains local gauge transformations of the wave functions, where the gauge group is determined to be the isometry group of the spin scalar product. The causal action is gauge invariant in the sense that it does not depend on the choice of spinor bases.

- The equivalence principle is incorporated in the following general way. Space-time $M:=\operatorname{supp} \rho$ together with the universal measure $\rho$ form a topological measure space, being a more general structure than a Lorentzian manifold. Therefore, when describing $M$ by local coordinates, the freedom in choosing such coordinates generalizes the freedom in choosing general reference frames in a space-time manifold. Therefore, the equivalence principle of general relativity is respected. The causal action is generally covariant in the sense that it does not depend on the choice of coordinates.

\subsection{The Dynamics of Causal Fermion Systems}

Similar to the Einstein-Hilbert action in general relativity, in the causal action principle one varies space-time as well as all structures therein globally. This global viewpoint implies that it is not obvious what the causal action principle tells us about the dynamics of the system. The first step for clarifying the situation is to derive the Euler-Lagrange (EL) equations corresponding to the causal action principle (1.4.1). Similar to the Einstein or Maxwell equations, these EL equations should describe how the system evolves in time. Additional insight is gained by studying Noether-like theorems which specify the quantities which are conserved in the dynamics (1.4.2). Finally, we review results on the initial value problem $(\$ 1.4 .3$. We remark that more explicit information on the dynamics is obtained by considering limiting cases in which the EL equations corresponding to the causal action reduce to equations of a structure familiar from classical field theory and quantum field theory (see Section 1.5).

1.4.1. The Euler-Lagrange Equations. We return to the abstract setting of Section 1.1. Our goal is to derive the EL equations corresponding to the causal action principle in the form most useful for our purposes. Let $(\mathcal{H}, \mathcal{F}, \rho)$ be a causal fermion system. We assume that $\rho$ is a minimizer of the causal action principle. However, we do not 
want to assume that the total volume $\rho(\mathcal{F})$ be finite. Instead, we merely assume that $\rho$ is locally finite in the sense that $\rho(K)<\infty$ for every compact subset $K \subset \mathcal{F}$. Moreover, we only consider variations of $\rho$ of finite total variation (see the inequality in (1.1.7)). We treat the constraints with Lagrange multipliers (this procedure is justified in $[\overline{\mathbf{B F}}]$ ). Thus for each constraint (1.1.3)-(1.1.5), we add a corresponding Lagrange Lagrange multiplier term to the action. We conclude that first variations of the functional

$$
\mathcal{S}_{\kappa, \lambda, \nu}:=\mathcal{S}+\kappa\left(\mathcal{T}-C_{1}\right)-\lambda\left(\int_{\mathcal{F}} \operatorname{tr}(x) d \rho-C_{2}\right)-\nu\left(\rho(\mathcal{F})-C_{3}\right)
$$

vanish for suitable values of the Lagrange parameters $\kappa, \lambda, \nu \in \mathbb{R}$, where the constants $C_{1}$, $C_{2}$ and $C_{3}$ are the prescribed values of the constraints. For clarity, we point out that the boundedness constraint merely is an inequality. The method for handling this inequality constraint is to choose $\kappa=0$ if $\mathcal{T}(\rho)<C$, whereas in the case $\mathcal{T}(\rho)=C$ the Lagrange multiplier $\kappa$ is in general non-zero (for details see again $[\mathbf{B F}]$ ). Introducing the short notation

$$
\mathcal{L}_{\kappa}(x, y):=\mathcal{L}(x, y)+\kappa|x y|^{2},
$$

we can write the effective action as

$$
\begin{aligned}
\mathcal{S}_{\kappa, \lambda, \nu}(\rho)=\iint_{\mathcal{F} \times \mathcal{F}} \mathcal{L}_{\kappa}(x, y) d \rho(x) d \rho(y)-\lambda \int_{\mathcal{F}} \operatorname{tr}(x) d \rho(x)-\nu \rho(\mathcal{F}) \\
-\kappa C_{1}+\lambda C_{2}+\nu C_{3} .
\end{aligned}
$$

When considering first variations of the measure $\rho$, it is useful to distinguish between two types of variations. One possible variation is to multiply $\rho$ by a positive function $f_{\tau}$ : $M \rightarrow \mathbb{R}^{+}$,

$$
\rho_{\tau}=f_{\tau} \rho
$$

Clearly, this does not change the support of the measure. In order to change the support, one can consider a function $F_{\tau}: M \rightarrow \mathcal{F}$ and take the push-forward measure,

$$
\rho_{\tau}=\left(F_{\tau}\right)_{*} \rho .
$$

Combining these two variations, we are led to considering the family of measures

$$
\rho_{\tau}=\left(F_{\tau}\right)_{*}\left(f_{\tau} \rho\right) .
$$

Clearly, $\rho_{0}$ should coincide with our minimizing measure $\rho$, leading to the condition

$$
f_{0} \equiv 1 \quad \text { and } \quad F_{0} \equiv \mathbb{1} .
$$

Moreover, in order to ensure that the variation has finite total variation, we assume that it is trivial outside a compact set $K \subset M$, i.e. for all $\tau \in(-\delta, \delta)$,

$$
\left.f_{\tau}\right|_{M \backslash K} \equiv 1 \quad \text { and }\left.\quad F_{\tau}\right|_{M \backslash K} \equiv \mathbb{1} .
$$

Finally, we assume that the functions $f_{\tau}$ and $F_{\tau}$ are defined and smooth in $\tau$ for all $\tau \in$ $(-\delta, \delta)$ for some $\delta>0$. Variations of the form (1.4.6) are sufficiently general for all the purposes of this book (more general variations will be discussed in Remark 1.4.4 below).

Choosing the function $F_{\tau}$ in a specific way, one gets the following result.

Proposition 1.4.1. If $\rho$ is a minimizing measure of the causal action principle, then there is a real constant $c$ such that

$$
\operatorname{tr}(x)=c \quad \text { for all } x \in M .
$$

We often refer to $\operatorname{tr}(x)$ as the local trace at the point $x$. Then the above proposition can be stated that for a minimizing measure, the local trace is constant in space-time. 
Proof of Proposition 1.4.1. Using the definition of the push-forward measure and the fact that the variation is trivial outside $K$, the integral over a function $\phi$ on $\mathcal{F}$ can be written conveniently as

$$
\begin{aligned}
\int_{\mathcal{F}} \phi(x) d \rho_{\tau}(x) & =\int_{\mathcal{F}} \phi\left(F_{\tau}(x)\right) f_{\tau}(x) d \rho(x) \\
& =\int_{K} \phi\left(F_{\tau}(x)\right) f_{\tau}(x) d \rho(x)+\int_{M \backslash K} \phi(x) d \rho(x) .
\end{aligned}
$$

We choose the mapping $F_{\tau}$ as

$$
F_{\tau}(x)=\frac{x}{\sqrt{f_{\tau}(x)}}
$$

Using that $\mathcal{L}_{\kappa}(x, y)$ is homogeneous in $y$ of degree two, it follows that

$$
\begin{gathered}
\int_{K} \mathcal{L}_{\kappa}\left(x, F_{\tau}(y)\right) f_{\tau}(y) d \rho(y)=\int_{K} \mathcal{L}_{\kappa}\left(x, \frac{y}{\sqrt{f_{\tau}(y)}}\right) f_{\tau}(y) d \rho(y) \\
=\int_{K} \mathcal{L}_{\kappa}(x, y) \frac{1}{f_{\tau}(y)} f_{\tau}(y) d \rho(y)=\int_{K} \mathcal{L}_{\kappa}(x, y) d \rho(y) .
\end{gathered}
$$

Arguing similarly in the variable $x$, one sees that the variation does not change the integrals over $\mathcal{L}_{\kappa}$ in 1.4 .3 . Hence it remains to consider the variation of the other terms in 1.4 .3 ,

$$
\begin{aligned}
\mathcal{S}_{\kappa, \lambda, \nu}\left(\rho_{\tau}\right)-\mathcal{S}_{\kappa, \lambda, \nu}(\rho) & =-\lambda \int_{K}\left(\operatorname{tr}\left(F_{\tau}(x)\right) f_{\tau}(x)-\operatorname{tr}(x)\right) d \rho(x)-\nu \int_{K}\left(f_{\tau}(x)-1\right) d \rho(x) \\
& =-\lambda \int_{K}\left(\sqrt{f_{\tau}(x)}-1\right) \operatorname{tr}(x) d \rho(x)-\nu \int_{K}\left(f_{\tau}(x)-1\right) d \rho(x),
\end{aligned}
$$

where in the last step we used the linearity of the trace. Choosing $f_{\tau}=1+\tau g$ (where $g$ is a bounded function supported in $K$ ), the first order variation, denoted by

is computed by

$$
\delta \mathcal{S}_{\kappa, \lambda, \nu}=\left.\frac{d}{d \tau} \mathcal{S}_{\kappa, \lambda, \nu}\right|_{\tau=0}
$$

$$
\delta \mathcal{S}_{\kappa, \lambda, \nu}=-\frac{\lambda}{2} \int_{K} g(x) \operatorname{tr}(x) d \rho(x)-\nu \int_{K} g(x) d \rho(x)=-\int_{K} g(x)\left[\frac{\lambda}{2} \operatorname{tr}(x)+\nu\right] d \rho(x) .
$$

Since $g$ is arbitrary, it follows that the square brackets vanish identically. This gives the result.

The result of this proposition is important because it tells us that seeking for minimizers of the causal action, we should always arrange that the local trace is constant on $M$. If this constant is zero, then the measure supported at one point $x$ given by 1.1 .8 is a trivial minimizer. Therefore, we shall always restrict attention to the case $c \neq 0$. Then we can arrange 1.4 .9 by the scaling

$$
\rho \rightarrow F_{*} \rho \quad \text { with } \quad F(x)=\frac{c}{\operatorname{tr}(x)} x .
$$

Clearly, this transformation can be used only if the local trace has no zeros in $M$. In order to avoid the analysis of the zeros of the local trace, we note that if the local trace has zeros, then the measure cannot be a minimizer because the condition $(1.4 .9)$ is violated, and we cannot arrange this condition by rescaling. Thus we may take the point of view that this measure is not useful for us and should be discarded. In other words, our strategy 
for constructing minimizers is to start from a measure $\rho$ for which the local trace has no zeros in $M$, and to perform the rescaling (1.4.11). The resulting measure satisfies (1.4.9). With this in mind, in what follows we shall always assume that $(1.4 .9)$ holds.

In Proposition 1.4.1 we considered variations of the form 1.4 .6$)$ with an arbitrary function $f_{\tau}$. Therefore, in what follows we may restrict attention to variations obtained by taking the push-forward 1.4.5) (more precisely, every linear perturbation can be decomposed uniquely into the sum of a variation of the form $(1.4 .6)$ with $F_{\tau}$ given by 1.4 .10 and a variation of the form (1.4.5)). Variations of the form (1.4.5) can be described conveniently by working with so-called variations of the physical wave functions, which we now introduce. Our starting point is the wave evaluation operator $\Psi$ introduced in $(1.1 .20)$,

$$
\Psi: \mathcal{H} \rightarrow C^{0}(M, S M), \quad u \mapsto \psi^{u} .
$$

We want to vary the wave evaluation operator. Thus for given $\delta>0$ and any $\tau \in(-\delta, \delta)$ we consider a mapping $\Psi_{\tau}: \mathcal{H} \rightarrow C^{0}(M, S M)$. For $\tau=0$, this mapping should coincide with the wave evaluation operator $\Psi$. The family $\left(\Psi_{\tau}\right)_{\tau \in(-\delta, \delta)}$ can be regarded as a simultaneous variation of all physical wave functions of the system. In fact, for any $u \in \mathcal{H}$, the variation of the corresponding physical wave function is given by

$$
\psi_{\tau}^{u}:=\Psi_{\tau}(u) \in C^{0}(M, S M) .
$$

Next, we introduce the corresponding local correlation operators $F_{\tau}$ by

$$
F_{\tau}(x):=-\Psi_{\tau}(x)^{*} \Psi_{\tau}(x) \quad \text { so that } \quad F_{\tau}: M \rightarrow \mathcal{F} .
$$

In view of 1.1.21, we know that $F_{0}(x)=x$. Therefore, the family $\left(F_{\tau}\right)_{\tau \in(-\delta, \delta)}$ is a variation of the local correlation operators. Taking the push-forward measure $(1.4 .5)$ gives rise to a variation $\left(\rho_{\tau}\right)_{\tau \in(-\delta, \delta)}$ of the universal measure. Indeed, if all points of $K$ are regular (see Definition 1.1.5), every variation of the universal measure of the form (1.4.5) can be realized by a variation of the wave functions (see Exercise 1.23).

We now work out the EL equations for the resulting class of variations of the universal measure. In order for the constructions to be mathematically well-defined, we need a few technical assumptions which are summarized in the following definition.

DEFINITION 1.4.2. The variation of the physical wave functions is smooth and compact if the family of operators $\left(\Psi_{\tau}\right)_{\tau \in(-\delta, \delta)}$ has the following properties:

(a) The variation is trivial on the orthogonal complement of a finite-dimensional subspace $I \subset \mathcal{H}$, i.e.

$$
\left.\Psi_{\tau}\right|_{I^{\perp}}=\Psi \quad \text { for all } \tau \in(-\delta, \delta) .
$$

(b) There is a compact subset $K \subset M$ outside which the variation is trivial, i.e.

$$
\left.\left(\Psi_{\tau}(u)\right)\right|_{M \backslash K}=\left.(\Psi(u))\right|_{M \backslash K} \quad \text { for all } \tau \in(-\delta, \delta) \text { and } u \in \mathcal{H} .
$$

(c) The Lagrangian is continuously differentiable in the sense that the derivative

$$
\left.\frac{d}{d \tau} \mathcal{L}\left(x, F_{\tau}(y)\right)\right|_{\tau=0}
$$

exists and is continuous on $M \times M$.

With the conditions (a) and (b) we restrict attention to variations which are sufficiently well-behaved (similar as in the classical calculus of variations, where one restricts attention to smooth and compactly supported variations). It is a delicate point to satisfy the condition (c), because (due to the absolute values of the eigenvalues in (1.1.1)) the Lagrangian is only Lipschitz continuous on $\mathcal{F} \times \mathcal{F}$. Therefore, the derivative in (1.4.13) 
does not need to exist, even if $F_{\tau}(y)$ is smooth. This means that in the applications, one must verify that the condition (c) holds (for details see Sections 3.5, 3.6 and many computations in subsequent sections). Right now, we simply assume that the variation of the wave functions is smooth and compact.

By definition of the push-forward measure 1.4.5), we know that for any integrable function $f$ on $\mathcal{F}$,

$$
\int_{\mathcal{F}} f(x) d \rho_{\tau}=\int_{\mathcal{F}} f\left(F_{\tau}(x)\right) d \rho
$$

In this way, the variation of the measure can be rewritten as a variation of the arguments of the integrand. In particular, the variation of the action can be written as

$$
\iint_{M \times M} \mathcal{L}\left(F_{\tau}(x), F_{\tau}(y)\right) d \rho(x) d \rho(y)
$$

(and similarly for the other integrals). Another benefit of working with the push-forward measure (1.4.5) is that the total volume is preserved. Namely, combining the identity (1.4.14) with the assumption in Definition 1.4.2 (b), one readily verifies that the volume constraint (1.1.3) is satisfied in the sense that $\rho_{\tau}$ satisfies the conditions (1.1.7).

Now we can compute the first variation by differentiating with respect to $\tau$. It is most convenient to express the causal action and the constraints in terms of the kernel of the fermionic projector (just as explained at the beginning of 1.1 .3 ). Moreover, it is preferable to consider the Lagrangian $\mathcal{L}_{\kappa}(x, y)$ as a function only of $P_{\tau}(x, y)$ by writing the closed chain as

$$
A_{x y}^{\tau}=P_{\tau}(x, y) P_{\tau}(x, y)^{*}
$$

(where the index $\tau$ clarifies the dependence on the parameter $\tau \in(-\delta, \delta)$, and $P_{\tau}(x, y)^{*}$ denotes similar to (1.1.15 the adjoint with respect to the spin scalar product). When computing the variation of the Lagrangian, one must keep in mind that $\mathcal{L}_{\kappa}(x, y)$ depends both on $P_{\tau}(x, y)$ and on its adjoint $P_{\tau}(x, y)^{*}$ (cf. (1.4.15)). Therefore, when applying the chain rule, we obtain contributions which are complex linear and complex anti-linear in $\delta P(x, y)$. We write the first variation in terms of traces as

$$
\delta \mathcal{L}_{\kappa}(x, y)=\operatorname{Tr}_{S_{y}}(B \delta P(x, y))+\operatorname{Tr}_{S_{x}}\left(C \delta P(x, y)^{*}\right)
$$

with linear operators $B: S_{x} \rightarrow S_{y}$ and $C: S_{y} \rightarrow S_{x}$. Since $\delta P(x, y)$ can be chosen arbitrarily, this equation uniquely defines both $B$ and $C$. Since the variation of the Lagrangian is always real-valued, it follows that $C=B^{*}$. Using furthermore the symmetry of the Lagrangian in the arguments $x$ and $y$, we conclude that the first variation of the Lagrangian can be written as (see also [F7, Section 5.2])

$$
\delta \mathcal{L}_{\kappa}(x, y)=\operatorname{Tr}_{S_{y}}(Q(y, x) \delta P(x, y))+\operatorname{Tr}_{S_{x}}\left(Q(x, y) \delta P(x, y)^{*}\right)
$$

with a kernel $Q(x, y): S_{y} \rightarrow S_{x}$ which is symmetric in the sense that

$$
Q(x, y)^{*}=Q(y, x) \text {. }
$$

The EL equations are expressed in terms of the kernel $Q(x, y)$ as follows.

Proposition 1.4.3. (Euler-Lagrange equations) Let $\rho$ be a minimizer of the causal action principle. Then for a suitable choice of the Lagrange parameters $\lambda$ and $\kappa$, the integral operator $Q$ with kernel defined by 1.4.16 satisfies the equations

$$
\int_{M} Q(x, y) \psi^{u}(y) d \rho(y)=\frac{\lambda}{2} \psi^{u}(x) \quad \text { for all } u \in \mathcal{H} \text { and } x \in M .
$$


We note for clarity that by writing the equation (1.4.18) we imply that the integral must exist and be finite.

Proof of Proposition 1.4.3. Using (1.4.16), the first variation of $\mathcal{S}_{\kappa, \lambda, \nu}$ is computed by

$$
\begin{aligned}
\delta \mathcal{S}_{\kappa, \lambda, \nu}= & \iint_{M \times M}\left(\operatorname{Tr}_{S_{y}}(Q(y, x) \delta P(x, y))+\operatorname{Tr}_{S_{x}}\left(Q(x, y) \delta P(x, y)^{*}\right)\right) d \rho(x) d \rho(y) \\
& -\lambda \int_{M} \operatorname{Tr}(\delta P(x, x)) d \rho(x) .
\end{aligned}
$$

Noting that $\delta P(x, y)=\delta P(y, x)^{*}$, after renaming the integration variables in the first summand of the double integral, we obtain

$$
\begin{aligned}
\delta \mathcal{S}_{\kappa, \lambda, \nu}=2 & \iint_{M \times M} \operatorname{Tr}_{S_{x}}(Q(x, y) \delta P(y, x)) d \rho(x) d \rho(y) \\
& -\lambda \int_{M} \operatorname{Tr}_{S_{x}}(\delta P(x, x)) d \rho(x) .
\end{aligned}
$$

Next, we express $\delta P$ in terms of the variation of the physical wave functions. By Lemma 1.1.3, we know that

$$
P_{\tau}(y, x)=-\Psi_{\tau}(y) \Psi_{\tau}(x)^{*} .
$$

Differentiating this relation gives

$$
\delta P(y, x)=-(\delta \Psi)(y) \Psi(x)^{*}-\Psi(y)(\delta \Psi)(x)^{*} .
$$

We now specialize to the case that the variation is trivial on the orthogonal complement of a one-dimensional subspace $I=\operatorname{span}(u) \subset \mathcal{H}$. Then for any $\phi \in S_{y}$,

$$
\delta P(y, x) \phi=-\delta \psi^{u}(y) \prec \psi^{u}(x)\left|\phi \succ_{x}-\psi^{u}(y) \prec \delta \psi^{u}(x)\right| \phi \succ_{x} .
$$

By inserting a phase factor according to

$$
\delta \psi^{u} \rightarrow e^{i \varphi} \delta \psi^{u},
$$

one sees that $\delta \psi^{u}$ can be varied independently inside and outside the spin scalar product (more precisely, denoting the variation of the action 1.4 .19 ) corresponding to $\delta \psi^{u}$ by $\delta \mathcal{S}_{\kappa, \lambda, \nu}\left[\delta \psi^{u}\right]$, the linear combination $\delta \mathcal{S}_{\kappa, \lambda, \nu}\left[\delta \psi^{u}\right]+i \delta \mathcal{S}_{\kappa, \lambda, \nu}\left[i \delta \psi^{u}\right]$ involves only the complex conjugate of $\delta \psi^{u}$, whereas $\delta \psi^{u}$ without complex conjugation drops out). We conclude that it suffices to consider variations inside the spin scalar product. Thus the vanishing of the first variation 1.4 .19 yields the condition

$$
0=2 \iint_{M \times M} \prec \delta \psi^{u}(x)\left|Q(x, y) \psi^{u}(y) \succ_{x}-\lambda \int_{M} \prec \delta \psi^{u}(x)\right| \psi^{u}(x) \succ_{x} .
$$

Since $\delta \psi^{u}$ is arbitrary (within the class of smooth and compactly supported variations), the result follows.

We remark that the kernel $Q(x, y)$ also gives rise to an operator on the one-particle Krein space $(\mathcal{K},<. \mid .>)$ as introduced in 1.1 .5 . Thus, in analogy to 1.1 .26 , one sets

$$
Q: \mathcal{D}(Q) \subset \mathcal{K} \rightarrow \mathcal{K}, \quad(Q \psi)(x)=\int_{M} Q(x, y) \psi(y) d \rho(y),
$$

where the domain $\mathcal{D}(Q)$ can be chosen for example as the continuous wave functions with compact support. The symmetry property of the kernel (1.4.17) implies that the 
operator $Q$ is symmetric on the $\operatorname{Krein}$ space $(\mathcal{K},<. \mid .>)$. The equation 1.4 .18 can be written in a compact form as the operator equation

$$
(2 Q-\lambda \mathbb{1}) \Psi=0
$$

(where $\Psi$ is again the wave evaluation operator 1.1 .20$)$ ). In words, this equation means that the operator $(2 Q-\lambda \mathbb{1})$ vanishes on the physical wave functions. However, the operator equation 1.4 .20 is not satisfying mathematically because the physical wave functions in the image of $\Psi$ are in general not vectors of the Krein space $(\mathcal{K},<. \mid .>$ ) (see \$1.1.5). Nevertheless, (1.4.20) is useful as a short notation for the EL equations (1.4.18).

REMARK 1.4.4. (more general variations) Clearly, the ansatz (1.4.6) only covers a certain class of variations of the universal measure. As a consequence, the resulting EL equations 1.4 .9$)$ and $(1.4 .18)$ are only necessary conditions for $\rho$ to be a critical point of the action (1.4.1). We now explain how these necessary conditions are related to the stronger EL equations as derived in $\mathbf{B F}$.

Variations of the form (1.4.6) have the property that the support of the universal measure changes continuously (in the sense that for every compact set $K \subset \mathcal{F}$ and every open neighborhood $U$ of $K \cap \operatorname{supp} \rho$ there is $\varepsilon>0$ such that $\operatorname{supp} \rho_{\tau} \cap K \subset U$ for all $\tau$ with $|\tau|<\varepsilon$ ). In fact, up to regularity and smoothness issues which we shall not enter here, every variation of $\rho$ which changes its support continuously can be written in the form (1.4.6) (this could be proved abstractly using arguments as in [F13, Lemma 1.4]). Such variations can be regarded as the analogs of variations of the potentials, the metric or the wave functions in classical field theory or quantum mechanics. However, in the setting of causal fermion systems there are also more general smooth variations for which the support of the measure $\rho_{\tau}$ changes discontinuously. A typical example is to let $\rho$ be a bounded measure and to set

$$
\rho_{\tau}=\left(1-\tau^{2}\right) \rho+\tau^{2} \rho(\mathcal{F}) \delta_{x},
$$

where $\delta_{x}$ is the Dirac measure supported at $x \notin \operatorname{supp} \rho$. The EL equations corresponding to such variations have a different mathematical structure, which we cannot explain in detail here. Generally speaking, for interacting systems in Minkowski space, the EL equations of Proposition 1.4 .3 give rise to an effective interaction via classical gauge fields (this so-called continuum limit will be discussed in \$1.5.2). The EL equations corresponding to more general variations like (1.4.21), however, give rise to an effective interaction via bosonic quantum fields (see Exercise 1.24). We will come back to this point in 1.5 .3 .

REMARK 1.4.5. (unitary variations in Krein space) Rather than generalizing (1.4.6), one can also proceed in the opposite way and restrict attention to a more special class of variations of the universal measure. If this is done, one obtains weaker equations, meaning that the resulting EL equations are only necessary conditions for the EL equations 1.4.18) of Proposition 1.4.3 to hold. Nevertheless, this procedure has its benefits in cases when the weaker EL equations are easier to handle and/or if the weaker EL equations capture the essence of (1.4.18) in certain limiting cases. A specific class of variations which is of interest in this context are so-called unitary variations in the Krein space. Such variations were first considered in [F7, Section 3.5]. It turns out that in the continuum limit, the resulting EL equations are equivalent to (1.4.18). The advantage of working with unitary variations in Krein spaces is that the volume and trace constraints 
are respected by the variation, making it unnecessary to treat these constraints with Lagrange multipliers. This method is also used in Chapter 3 (see Section 3.2 and \$3.5.2). We now briefly outline the method and put it into the context of the variations (1.4.6).

We let $U_{\tau}$ be a family of unitary operators on the Krein space $(\mathcal{K},<. \mid .>)$. Setting

$$
\Psi_{\tau}=U_{\tau} \circ \Psi
$$

we obtain a corresponding variation of the physical wave functions. Following 1.4.12 and (1.4.5) gives a corresponding variation of the universal measure, i.e.

$$
\rho_{\tau}=\left(F_{\tau}\right)_{*} \rho \quad \text { with } \quad F_{\tau}(x):=-\Psi_{\tau}(x)^{*} \Psi_{\tau}(x) .
$$

Since $\rho_{\tau}$ is the push-forward of the measure $\rho$, the volume constraint is clearly satisfied. In order to verify the trace constraint, we note that formally,

$$
\begin{aligned}
\int_{\mathcal{F}} \operatorname{tr}(x) d \rho_{\tau}(x) & =\int_{M} \operatorname{tr}\left(F_{\tau}(x)\right) d \rho_{\tau}(x) \\
& =-\int_{M} \operatorname{tr}\left(\Psi_{\tau}(x)^{*} \Psi_{\tau}(x)\right) d \rho=-\operatorname{tr}\left(\Psi_{\tau}^{*} \Psi_{\tau}\right),
\end{aligned}
$$

where the adjoint $\Psi_{\tau}^{*}: \mathcal{K} \rightarrow \mathcal{H}$ is defined using the respective inner products, i.e.

$$
<\Psi_{\tau} u|\phi\rangle=\left\langle u \mid \Psi_{\tau}^{*} \phi\right\rangle_{\mathcal{H}} \quad \text { for } u \in \mathcal{H}, \phi \in \mathcal{K} .
$$

Therefore, using (1.4.22) together with the fact that the operators $U_{\tau}$ are unitary, we conclude that

$$
\int_{\mathcal{F}} \operatorname{tr}(x) d \rho_{\tau}(x)=-\operatorname{tr}\left(\Psi_{\tau}^{*} \Psi_{\tau}\right)=-\operatorname{tr}\left(\Psi^{*} U_{\tau}^{*} U_{\tau} \Psi_{\tau}\right)=-\operatorname{tr}\left(\Psi^{*} \Psi_{\tau}\right)=\int_{M} \operatorname{tr}(x) d \rho(x),
$$

showing that the trace constraint is indeed respected. Clearly, this computation has the shortcoming that the integral in (1.4.23) may diverge (see before (1.1.7) ), and that $\Psi$ does not necessarily map to $\mathcal{K}$ (see $\$ 1.1 .5$. But the above consideration can be given a mathematical meaning when assuming that the operators $\mathbb{1}-U_{\tau}$ can be represented as integral operators with integral kernels which vanish outside a compact subset of $M \times M$. We refer to the details to Definition 3.2.1 and the constructions in Section 3.2.

1.4.2. Symmetries and Conserved Surface Layer Integrals. In FK2 it is shown that symmetries of the Lagrangian give rise to conservation laws. These results can be understood as adaptations of Noether's theorem to the causal action principle. Since the mathematical structure of the causal action principle is quite different from that of the Lagrangian formulation of classical field theory, these adaptations are not straightforward. We now explain a few concepts and results from FK2 which are important for understanding the general physical picture.

We first recall that the conservation laws obtained from the classical Noether theorem state that the integral of a certain density over a Cauchy surface $\mathcal{N}$ does not depend on the choice of $\mathcal{N}$. For example, charge conservation states that the spatial integral of the charge density gives a constant. As another example, energy conservation states that in a static space-time background, the integral of the energy density is a constant. In general terms, the conserved quantities are surface integrals over a Cauchy surface $\mathcal{N}$ (see the left of Figure 1.2). In the setting of causal fermion systems, it is unclear how such surface integrals should be defined, in particular because we do not have a measure on hypersurfaces and because it is not clear what the normal $\nu$ on the hypersurface should be. This is the reason why in the Noether-like theorems in [FK2] one works instead of surface integrals with so-called surface layer integrals where one integrates over a boundary layer 

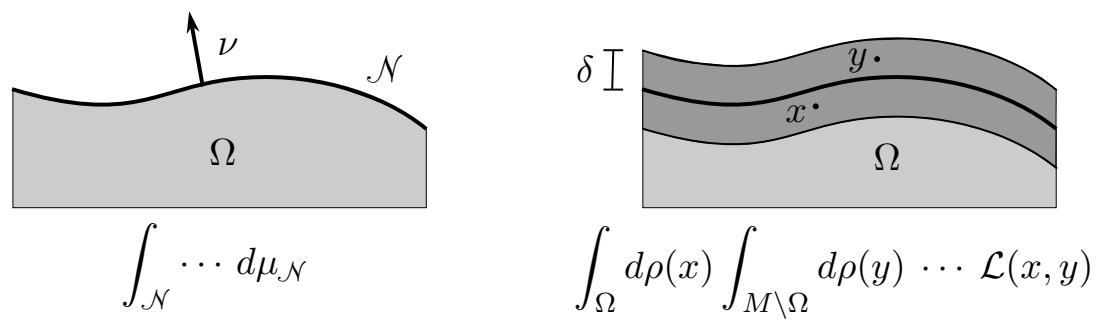

FiguRE 1.2. A surface integral and a corresponding surface layer integral

of a set $\Omega \subset M$ (see the right of Figure 1.2 and Exercise 1.25 for an illustration of how such double integrals arise). The width $\delta$ of this layer is the length scale on which $\mathcal{L}(x, y)$ decays. For a system composed of Dirac particles (similar as explained in Section 1.2 for the Minkowski vacuum and in $\$ 1.5 .2$ for interacting systems), this length scale can be identified with the Compton scale $\sim m^{-1}$ of the Dirac particles. Thus the width of the surface layer is a non-zero macroscopic length scale. In particular, the surface layer integrals cannot be identified with or considered as a generalization of the surface integrals of the classical Noether theorem. However, in many situations of interest the surface $N$ is almost flat on the Compton scale (the simplest example being a spatial hyperplane in Minkowski space). Then the surface layer integral can be well-approximated by a corresponding surface integral. It turns out that in this limiting case, the conservation laws obtained from the Noether-like theorems in FK2 go over to corresponding classical conservation laws.

From the conceptual point of view, the most interesting conservation law is charge conservation. In order to construct the underlying symmetry, we let $\mathcal{A}$ be a bounded symmetric operator on $\mathcal{H}$ and let

$$
\mathcal{U}_{\tau}:=\exp (i \tau \mathcal{A}) \quad \text { for } \tau \in \mathbb{R}
$$

be the corresponding one-parameter family of unitary transformations. We introduce the family of transformations

$$
\Phi_{\tau}: \mathcal{F} \rightarrow \mathcal{F}, \quad \Phi_{\tau}(x)=\mathcal{U}_{\tau} x \mathcal{U}_{\tau}^{-1} .
$$

Since the Lagrangian is defined via the spectrum of operators on $\mathcal{H}$, it clearly remains unchanged if all operators are unitarily transformed, i.e.

$$
\mathcal{L}\left(\Phi_{\tau}(x), \Phi_{\tau}(y)\right)=\mathcal{L}(x, y)
$$

In other words, the transformations $\Phi_{\tau}$ describe a symmetry of the Lagrangian. Next, one constructs a corresponding one-parameter family of universal measures by taking the push-forward,

$$
\rho_{\tau}:=\left(\Phi_{\tau}\right)_{*} \rho .
$$

As a consequence of the symmetry (1.4.24), this variation of the universal measure leaves the action invariant. Under suitable differentiability assumptions, this symmetry gives rise to the identity

$$
\left.\frac{d}{d \tau} \int_{\Omega} d \rho(x) \int_{M \backslash \Omega} d \rho(y)\left(\mathcal{L}\left(\Phi_{\tau}(x), y\right)-\mathcal{L}\left(\Phi_{-\tau}(x), y\right)\right)\right|_{\tau=0}=0,
$$

valid for any compact subset $\Omega \subset M$.

We now explain how the identity 1.4 .25 is related to a conservation law. To this end, for simplicity we consider a system in Minkowski space (similar as explained for 
the vacuum in Section 1.2 and choose a sequence of compact sets $\Omega_{n}$ which exhaust the region between two Cauchy surfaces at times $t=t_{0}$ and $t=t_{1}$. Then the surface layer integral 1.4 .25 reduces to the difference of integrals over surface layers at times $t \approx t_{0}$ and $t \approx t_{1}$. Next, we choose $\mathcal{A}=\pi_{\langle u\rangle}$ as the projection operator on the one-dimensional subspace generated by a vector $u \in \mathcal{H}$. Then in the limit $\varepsilon \searrow 0$ in which the UV regularization is removed, the resulting surface layer integral at time $t \approx t_{0}$ reduces to the integral

$$
\int_{\mathbb{R}^{3}} \prec u\left(t_{0}, \vec{x}\right) \mid \gamma^{0} u\left(t_{0}, \vec{x}\right) \succ_{\left(t_{0}, \vec{x}\right)} d^{3} x
$$

thereby reproducing the probability integral in Dirac theory. As a consequence, the representation of the scalar product $\langle. \mid\rangle_{\mathcal{H}}$ as an integral over a Cauchy surface $(1.2 .2)$ has a natural generalization to the setting of causal fermion systems, if the surface integral is replaced by a corresponding surface layer integral. This result also shows that the spatial normalization of the fermionic projector (where one works with spatial integrals of the form 1.2.24); for details see [FT2]) really is the correct normalization method which reflects the intrinsic conservation laws of the causal fermion system.

The conservation laws in [FK2 also give rise to the conservation of energy and momentum, as we now outline. In the classical Noether theorem, these conservation laws are a consequence of space-time symmetries as described most conveniently using the notion of Killing fields. Therefore, one must extend this notion to the setting of causal fermion systems. Before explaining how this can be accomplished, we recall the procedure in the classical Noether theorem: In the notion of a Killing field, one distinguishes the background geometry from the additional particles and fields. The background geometry must have a symmetry as described by the Killing equation. The additional particles and fields, however, do not need to have any symmetries. Nevertheless, one can construct a symmetry of the whole system by actively transporting the particles and fields along the flow lines of the Killing field. The conservation law corresponding to this symmetry transformation gives rise to the conservation of energy and momentum.

In a causal fermion system, there is no clear-cut distinction between the background geometry and the particles and fields of the system, because all of these structures are encoded in the underlying causal fermion system and mutually depend on each other. Therefore, instead of working with a symmetry of the background geometry, we work with the notion of an approximate symmetry. By actively transforming those physical wave functions which do not respect the symmetry, such an approximate symmetry again gives rise to an exact symmetry transformation, to which the Noether-like theorems in FK2 can be applied. More precisely, one begins with a $C^{1}$-family of transformations $\left(f_{\tau}\right)_{\tau \in(-\delta, \delta)}$ of space-time,

$$
f_{\tau}: M \rightarrow M \quad \text { with } \quad f_{0}=\mathbb{1}
$$

which preserve the universal measure in the sense that $\left(f_{\tau}\right)_{*} \rho=\rho$. The family $\left(f_{\tau}\right)$ can be regarded as the analog of a flow in space-time along a classical Killing field. Moreover, one considers a family of unitary transformations $\left(\mathcal{U}_{\tau}\right)_{\tau \in(-\delta, \delta)}$ on $\mathcal{H}$ with the property that

$$
\mathcal{U}_{-\tau} \mathcal{U}_{\tau}=\mathbb{1} \quad \text { for all } \tau \in(-\delta, \delta) .
$$

Combining these transformations should give rise to an approximate symmetry of the wave evaluation operator 1.1 .20 in the sense that if we compare the transformation of the space-time point with the unitary transformation by setting

$$
E_{\tau}(u, x):=(\Psi u)\left(f_{\tau}(x)\right)-\left(\Psi \mathcal{U}_{\tau}^{-1} u\right)(x) \quad(x \in M, u \in \mathcal{H}),
$$


then the operator $E_{\tau}: \mathcal{H} \rightarrow C^{0}(M, S M)$ should be sufficiently small. Here "small" means for example that $E$ vanishes on the orthogonal complement of a finite-dimensional subspace of $\mathcal{H}$; for details see [FK2, Section 6]. Introducing the variation $\Phi_{\tau}$ by

$$
\Phi_{\tau}: M \rightarrow \mathcal{F}, \quad \Phi_{\tau}(x)=\mathcal{U}_{\tau} x \mathcal{U}_{\tau}^{-1},
$$

we again obtain a symmetry of the Lagrangian (1.4.24). This gives rise to conserved surface layer integrals of the form (1.4.25). In order to bring these surface layer integrals into a computable form, one decomposes the first variation of $\Phi_{\tau}$ as

$$
\delta \Phi(x):=\left.\partial_{\tau} \Phi_{\tau}(x)\right|_{\tau=0}=\delta f(x)+v(x),
$$

where $\delta f$ is the first variation of $f_{\tau}, 1.4 .26$, and $v(x)$ is a vector field on $\mathcal{F}$ along $M$ which is transversal to $M \subset \mathcal{F}$. Expressing $v$ in terms of the operator $E$ in (1.4.27) shows that $v$ is again small, making it possible to compute the corresponding variation of the Lagrangian in 1.4.25). We remark that in the decomposition 1.4.28, the vector field $\delta f$ describes a transformation of the space-time points. The vector field $v$, however, can be understood as an active transformation of all the objects in space-time which do not have the space-time symmetry (similar as described above for the parallel transport of the particles and fields along the flow lines of the Killing field in the classical Noether theorem).

In order to get the connection to classical conservation laws, one again studies a system in Minkowski space and considers the limiting case where a sequence $\Omega_{n}$ exhausts the region between two Cauchy surfaces at times $t=t_{0}$ and $t=t_{1}$. In this limiting case, the conserved surface layer integral reduces to the surface integral

$$
\int_{\mathbb{R}^{3}} T_{i 0} K^{i} d^{3} x
$$

where $T_{i j}$ is the energy-momentum tensor of the Dirac particles and $K=\delta f$ is a Killing field. This shows that the conservation of energy and momentum is a special case of more general conservation laws which are intrinsic to causal fermion systems.

1.4.3. The Initial Value Problem and Time Evolution. In order to get a better understanding of the dynamics described by the causal action principle, it is an important task to analyze the initial value problem. The obvious questions are: What is the initial data? Is it clear that a solution exists? Is the solution unique? How do solutions look like? Giving general answers to these questions is a difficult mathematical problem. In order to evaluate the difficulties, one should recall that $\rho$ describes space-time as well as all structures therein. Therefore, similar as in the Cauchy problem for the Einstein equations, solving the initial value problem involves finding the geometry of space-time together with the dynamics of all particles and fields. In view of the complexity of this problem, at present there are only a few partial results. First, in the paper [FG3 an initial value problem is formulated and some existence and uniqueness theorems are proven. We now review a few methods and results of this paper. Moreover, at the end of this section we mention an approach proposed in [FK3 for obtaining more explicit information on the dynamics by analyzing perturbations of a given minimizing measure.

Since the analysis of the causal action principle is technically demanding, in [FG3] one considers instead so-called causal variational principles in the compact setting. In order to get into this simplified setting, one replaces $\mathcal{F}$ by a compact metric space (or a smooth manifold). The Lagrangian is replaced by a non-negative Lipschitz-continuous 
function $\mathcal{L} \in C^{0,1}\left(\mathcal{F} \times \mathcal{F}, \mathbb{R}_{0}^{+}\right)$which is symmetric in its two arguments. Similar to 1.1.2 one minimizes the action

$$
\mathcal{S}(\rho)=\iint_{\mathcal{F} \times \mathcal{F}} \mathcal{L}(x, y) d \rho(x) d \rho(y)
$$

in the class of all normalized regular Borel measures on $\mathcal{F}$, but now leaving out the constraints $(1.1 .4)$ and $(1.1 .5)$. Space-time is again defined by $M:=\operatorname{supp} \rho$. The resulting causal structure is defined by saying that two space-time points $x, y \in M$ are called timelike separated if $\mathcal{L}(x, y)>0$, and spacelike separated if $\mathcal{L}(x, y)=0$. The principle of causality is again incorporated in the sense that pairs of points with spacelike separation do no enter the action. But clearly, in this setting there are no wave functions. Nevertheless, causal variational principles in the compact setting have many features of the causal action principle and are therefore a good starting point for the analysis (for a more detailed introduction and structural results on the minimizing measures see [FS]).

When solving the classical Cauchy problem, instead of searching for a global solution for all times, it is often easier to look for a local solution around a given initial value surface. This concept of a local solution also reflects the common physical situation where the physical system under consideration is only a small subsystem of the whole universe. With this in mind, we would like to "localize" the variational principle to a subset $\mathfrak{I} \subset \mathcal{F}$, referred to as the inner region. There is the complication that the Lagrangian $\mathcal{L}(x, y)$ is nonlocal in the sense that it may be non-zero for points $x \in \mathfrak{I}$ and $y \in \mathcal{F} \backslash \mathfrak{I}$. In order to take this effect into account, one describes the influence of the "outer region" $\mathcal{F} \backslash \mathfrak{I}$ by a so-called external potential $\phi: \mathcal{F} \rightarrow \mathbb{R}_{0}^{+}$. In the limiting case when the outer region becomes large, this gives rise to the so-called inner variational principle, where the action defined by

$$
\mathcal{S}_{\mathfrak{I}}[\rho, \phi]=\iint_{\mathfrak{I} \times \mathfrak{I}} \mathcal{L}(x, y) d \rho(x) d \rho(y)+2 \int_{\mathfrak{I}}(\phi(x)-\mathfrak{s}) d \rho(x)
$$

is minimized under variations of $\rho$ in the class of regular Borel measures on $\mathfrak{I}$ (not necessarily normalized because the volume constraint is now taken care of by the corresponding Lagrange parameter $\mathfrak{s}>0$ ).

The initial values are described by a regular Borel measure $\rho_{0}$ (which is to be thought of as the universal measure restricted to a time slice around the initial value surface in space-time). The initial conditions are implemented by demanding that

$$
\rho \geq \rho_{0} \text {. }
$$

The naive method of minimizing $(1.4 .29)$ under the constraint 1.4 .30 is not a sensible concept because the constraint 1.4.30) would give rise to undesirable Lagrange multiplier terms in the EL equations. Instead, one minimizes 1.4 .29 without constraints, but chooses the external potential $\phi$ in such a way that the minimizing measure satisfies the initial values 1.4 .30 . It turns out that this procedure does not determine the external potential uniquely. Therefore, the method proposed in [FG3 is to optimize the external potential by making it in a suitable sense "small." As is made precise in [FG3 in various situations, the resulting interplay between minimizing the action and optimizing the external potential gives rise to unique solutions of the initial-value problem with an optimal external potential.

We point out that, due to the mathematical simplifications made, the results in [FG3] do not apply to physically interesting situations like the initial value problem for interacting Dirac sea configurations. Moreover, the methods in [FG3] do not seem to give explicit 
information on the dynamics of causal fermion systems. Therefore, it is a promising complementary approach to consider perturbations of a given minimizing measure (which should describe the "vacuum configuration") and to analyze the dynamics of the perturbations by studying the resulting EL equations. This approach is pursued in FK3 in the following way. In order to describe the perturbations of the minimizing measure $\rho$, one considers smooth variations for which the support of $\rho$ changes continuously. Combining (1.4.5) and (1.4.4), these variations can be written as

$$
\tilde{\rho}_{\tau}=\left(F_{\tau}\right)_{*}\left(f_{\tau} \rho\right)
$$

with a family of mappings $F_{\tau}: M \rightarrow \mathcal{F}$ and a family of non-negative functions $f_{\tau}$. Expanding in powers of $\tau$, these variations can be described conveniently in terms of sections of jet bundles over $M$. The EL equations yield conditions on the jets, which can be rewritten as dynamical equations in space-time.

\subsection{Limiting Cases}

We now discuss different limiting cases of causal fermion systems.

1.5.1. The Quasi-Free Dirac Field and Hadamard States. We now turn attention to interacting systems. The simplest interaction is obtained by inserting an external potential into the Dirac equation (1.2.1),

$$
\left(i \gamma^{j} \partial_{j}+\mathcal{B}-m\right) \psi(x)=0 .
$$

Another situation of physical interest is to consider the Dirac equation in an external classical gravitational field as described mathematically by a globally hyperbolic Lorentzian manifold $(\mathscr{M}, g)$. In this section, we explain how the methods and results of Section 1.2 generalize to the situation when an external field is present. This will also give a connection to quasi-free Dirac fields and Hadamard states. In order to keep the explanations as simple as possible, we here restrict attention to an external potential $\mathcal{B}$ in Minkowski space, but remark that many methods and results could or have been worked out also in the presence of a gravitational field.

The obvious conceptual difficulty when extending the constructions of Section 1.2 is that one no longer has the notion of "negative-frequency solutions" which were essential for introducing Dirac sea configurations (see Lemma 1.2.8). In order to overcome this difficulty, one needs to decompose the solution space of the Dirac equation (1.5.1) into two subspaces, in such a way that without external potential, the two subspaces reduce to the subspaces of positive and negative frequency. This external field problem was solved perturbatively in [F3, FG1 and non-perturbatively in [FR2, FR3, FMR] (for a more detailed exposition see $\$ 2.1 .2$ or [F7, Section 2.1]).

We now briefly outline the non-perturbative treatment, which relies on the construction on the so-called fermionic signature operator. Choosing again the scalar product (1.2.2), the solution space of the Dirac equation (1.5.1) forms a Hilbert space denoted by $\left(\mathcal{H}_{m},(. \mid \cdot)_{m}\right)$. Moreover, on the Dirac wave functions (not necessarily solutions of the Dirac equations) one may introduce a dual pairing by integrating the spin scalar product over all of space-time,

$$
<.\left|.>: C^{\infty}(\mathscr{M}, S \mathscr{M}) \times C_{0}^{\infty}(\mathscr{M}, S \mathscr{M}) \rightarrow \mathbb{C}, \quad<\psi\right| \phi>=\int_{\mathscr{M}} \prec \psi \mid \phi \succ_{x} d^{4} x .
$$


The basic idea is to extend this dual pairing to a bilinear form on the Hilbert space $\mathcal{H}_{m}$ and to represent this bilinear form in terms of the Hilbert space scalar product

$$
<\phi_{m} \mid \psi_{m}>=\left(\phi_{m} \mid \mathcal{S} \psi_{m}\right)_{m} .
$$

If $\mathcal{M}$ is a space-time of finite lifetime, this construction can indeed be carried out and defines the fermionic signature operator $\mathcal{S}$ being a bounded symmetric operator on $\mathcal{H}_{m}$ (see FR2 ). The positive and negative spectral subspaces of $\mathcal{S}$ give the desired decomposition of $\mathcal{H}_{m}$ into two subspaces. We remark that the fermionic signature operator makes it possible to study spectral geometry for Lorentzian signature (see [FM] and [F19] for the connection to index theory).

In space-times of infinite lifetime like Minkowski space, the above method does not work because 1 1.5.2 does not extend to a continuous bilinear form on $\mathcal{H}_{m} \times \mathcal{H}_{m}$. The underlying problem is that the time integral in 1.5 .2 in general diverges for solutions of the Dirac equation. In order to circumvent this problem, one considers families of Dirac solutions $\left(\psi_{m}\right)_{m \in I}$ (for an open interval $I=\left(m_{a}, m_{b}\right) \subset(0, \infty)$ ) and makes use of the fact that integrating over the mass parameter generates decay of the wave functions for large times (the mass oscillation property; for details see [FR3]). As a result, one can make sense of the equation

$$
<\int_{I} \psi_{m} d m \mid \int_{I} \psi_{m^{\prime}} d m^{\prime}>=\int_{I}\left(\psi_{m} \mid \mathcal{S}_{m} \phi_{m}\right)_{m} d m
$$

which uniquely defines a family of bounded symmetric operators $\left(\mathcal{S}_{m}\right)_{m \in I}$. Now the positive and negative spectral subspaces of the operator $\mathcal{S}_{m}$ again give the desired decomposition of $\mathcal{H}_{m}$ into two subspaces.

Having decomposed the solution space, one may choose the Hilbert space $\mathcal{H}$ of the causal fermion system as one of the two subspaces of the solution space. Choosing an orthonormal basis $\left(u_{\ell}\right)$ of $\mathcal{H}$ and introducing the unregularized kernel of the fermionic projector again by 1.2 .20 , one obtains a two-point distribution $P(x, y)$. Using that this two-point distribution comes from a projection operator in the Hilbert space $\mathcal{H}_{m}$, there is a canonical construction which gives a quasi-free Dirac field together with a Fock representation such that the two-point distribution coincides with $P(x, y)$. In the canonical formalism, this result can be stated as follows (for a formulation in the language of algebraic quantum field theory see [FMR, Theorem 1.4]):

THEOREM 1.5.1. There are fermionic field operators $\hat{\Psi}^{\alpha}(x)$ and $\hat{\Psi}^{\beta}(y)^{*}$ together with a ground state $\mid 0>$ with the following properties:

(a) The canonical anti-commutation relations hold: $:^{5}$

$$
\left\{\hat{\Psi}^{\alpha}(x), \hat{\Psi}^{\beta}(y)^{*}\right\}=\tilde{k}_{m}(x, y)_{\beta}^{\alpha}, \quad\left\{\hat{\Psi}^{\alpha}(x), \hat{\Psi}^{\beta}(y)\right\}=0=\left\{\hat{\Psi}^{\alpha}(x)^{*}, \hat{\Psi}^{\beta}(y)^{*}\right\} .
$$

(b) The two-point function is given by

$$
<0\left|\hat{\Psi}^{\alpha}(x) \hat{\Psi}^{\beta}(y)^{*}\right| 0>=-P(x, y)_{\beta}^{\alpha} .
$$

This theorem means that before introducing an UV regularization, the description of the Dirac system using the fermionic projector is equivalent to the usual description of a non-interacting Dirac field in quantum field theory.

\footnotetext{
${ }^{5}$ In order to avoid confusion, we note that the operators $\hat{\Psi}(x)^{\dagger}$ which appear in the usual equal-time canonical commutation relations $\left\{\hat{\Psi}^{\alpha}(t, \vec{x}), \hat{\Psi}^{\beta}(t, \vec{y})^{\dagger}\right\}=\delta_{\beta}^{\alpha} \delta^{3}(\vec{x}-\vec{y})$ are related to the above operators by $\hat{\Psi}^{\alpha}(x)^{\dagger}=2 \pi \sum_{\beta=1}^{4} \hat{\Psi}^{\beta}(x)^{*}\left(\gamma^{0}\right)_{\alpha}^{\beta}$.
} 
Moreover, it is shown in FMR that the two-point distribution $P(x, y)$ is of Hadamard form, provided that $\mathcal{B}$ is smooth, not too large and decays faster than quadratically for large times (for details see [FMR, Theorem 1.3] and the references in this paper). This result implies that the representation of the quasi-free Dirac field as obtained from the fermionic projector is a suitable starting point for a perturbative treatment of the resulting interacting theory (see for example $[\mathbf{B D F}]$ ).

In our context, the fact that $P(x, y)$ is of Hadamard form implies that the results in $\$ 1.1 .2$ also apply in the presence of an external potential, as we now explain. The Hadamard property means in words that the bi-distribution $P(x, y)$ in the presence of the external potential has the same singularity structure as in the Minkowski vacuum. As a consequence, the arguments in $\$ 1.1 .2$ remain true if the points $x$ and $y$ are sufficiently close to each other. More precisely, the relevant length scale is given by the inverse of the amplitude $|\mathcal{B}(x)|^{-1}$ of the external potential. On the other hand, the separation of the points $x$ and $y$ must be larger than the scale $\varepsilon$ on which regularization effects come into play. Therefore, the causal structure of a causal fermion system agrees with that of Minkowski space on the scale $\varepsilon \ll\left|x^{0}-y^{0}\right|+|\vec{x}-\vec{y}| \ll|\mathcal{B}|^{-1}$ (where $|\mathcal{B}|$ is any matrix norm). Thinking of $\varepsilon$ as being at least as small as the Planck length, in most situations of interest the lower bound is no restriction. The upper bound is also unproblematic because the causal structure on the macroscopic scale can still be recovered by considering paths in space-time and subdividing the path on a scale $\delta \ll|\mathcal{B}|^{-1}$ (similar as explained in [FG2, Section 4.4] for the spin connection). With this in mind, we conclude that the causal structure of a causal fermion system indeed agrees with that of Minkowski space, even in the presence of an external potential.

1.5.2. Effective Interaction via Classical Gauge Fields. We now outline how to describe interacting systems in Minkowski space by analyzing the EL equations corresponding to the causal action principle as worked out in Proposition 1.4.3. In this so-called continuum limit, the interaction is described by classical gauge fields. Working out the details of this procedure is the main objective of this book (see Sections 2.4 and 2.6 for the general formalism and Chapters 35 for the explicit analysis of different models). Therefore, we here merely explain a few basic concepts.

Let us begin with the Minkowski vacuum. As shown in $\$ 1.2 .2$, regularizing a vacuum Dirac sea configuration gives rise to a causal fermion system $\left(\mathcal{H}, \mathcal{F}, \rho^{\varepsilon}\right)$. Moreover, we saw in the following sections 1.2 .311 .2 .4 that the inherent structures of the causal fermion system can be identified with those of Minkowski space (in particular, see $(1.2 .13)$ as well as Propositions 1.2.6 and 1.2.7). This makes it possible to write the EL equations 1.4.18 as

$$
\int_{\mathcal{M}} Q^{\varepsilon}(x, y)\left(\Re_{\varepsilon} u_{\ell}\right)(y) d^{4} y=\frac{\lambda}{2}\left(\Re_{\varepsilon} u_{\ell}\right)(x) \quad \text { for all } u \in \mathcal{H}
$$

where the regularized kernel $Q^{\varepsilon}(x, y)$ is again defined via (1.4.16) as the derivative of the Lagrangian. Next, one chooses the Hilbert space $\mathcal{H}$ as in $\$ 1.2 .5$ as the Dirac sea configuration formed of all negative-energy solutions of the Dirac equation. Then $P^{\varepsilon}(x, y)$ can be computed explicitly by regularizing the distribution $P(x, y)$ as given in momentum space by (1.2.23) and in position space by (1.2.25) and Lemma 1.2.9. Computing $Q^{\varepsilon}(x, y)$, it turns out that the EL equations are mathematically well-defined if the convolution integral in 1.5.3 is rewritten with the help of Plancherel's theorem as a multiplication in momentum space. The analysis of the continuum limit gives a procedure for studying these equations in the asymptotics $\varepsilon \searrow 0$ when the regularization is removed. The 
effective equations obtained in this asymptotic limit are evaluated most conveniently in a formalism in which the unknown microscopic structure of space-time (as described by the regularization) enters only in terms of a finite (typically small) number of socalled regularization parameters. According to the method of variable regularization (see Remark 1.2.1), one needs to analyze the dependence of the regularization parameters in detail. It turns out that the causal fermion systems obtained from the vacuum Dirac sea configuration satisfy the EL equations in the continuum limit, for any choice of the regularization parameters.

The first step towards interacting systems is to consider systems involving particles and/or anti-particles. To this end, one simply modifies the constructions in $\$ 1.2 .5$ by choosing the Hilbert space $\mathcal{H}$ differently. Namely, instead of choosing all negative-energy solutions, one chooses $\mathcal{H}$ as a subspace of the solution space which differs from the space of all negative-energy solutions by a finite-dimensional subspace. In other words, $\mathcal{H}$ is obtained from the space of all negative-energy solutions by taking out a finite number $n_{\mathrm{a}}$ of states and by adding a finite number of states $n_{\mathrm{p}}$ of positive energy. Thus, denoting the regularized kernel of the fermionic projector of the Minkowski vacuum for clarity by $P_{\text {sea }}^{\varepsilon}(x, y)$, the kernel of the fermionic projector 1.2 .19 can be written as

$$
P^{\varepsilon}(x, y)=P_{\text {sea }}^{\varepsilon}(x, y)-\sum_{k=1}^{n_{\mathrm{p}}}\left(\mathfrak{R}_{\varepsilon} \psi_{k}\right)(x) \overline{\left(\mathfrak{R}_{\varepsilon} \psi_{k}\right)(y)}+\sum_{l=1}^{n_{\mathrm{a}}}\left(\mathfrak{R}_{\varepsilon} \phi_{l}\right)(x) \overline{\left(\mathfrak{R}_{\varepsilon} \phi_{l}\right)(y)},
$$

where $\psi_{k}$ and $\phi_{l}$ are suitably normalized bases of the particle and anti-particle states, respectively. In this procedure, we again take Dirac's concept of a "sea" of particles literally and describe particles and anti-particles by occupying positive-energy states and creating "holes" in the Dirac sea, respectively. We also remark that the construction (1.5.4) modifies the kernel of the fermionic projector only by smooth contributions and thus preserves the singularity structure of $P^{\varepsilon}(x, y)$ as $\varepsilon \searrow 0$. As a consequence, the correspondence of the inherent structures of the causal fermion systems to the structures in Minkowski space remains unchanged (just as explained at the end of \$1.5.1 for an external potential).

According to (1.5.4), the particle and anti-particle states modify the kernel of the fermionic projector. It turns out that this has the effect that the EL equations in the continuum limit no longer hold. In order to again satisfy these equations, we need to introduce an interaction. In mathematical terms, this means that the universal measure $\rho$ must be modified. The basic question is how to modify the universal measure in such a way that the EL equations in the continuum limit again hold. It turns out that it is a useful first step to insert an external potential $\mathcal{B}$ into the Dirac equation (1.2.1) by going over to the Dirac equation (1.5.1). Choosing $\mathcal{H}$ as a subspace of the solution space of this Dirac equation, the constructions of Section 1.2 again apply and give rise to causal fermion systems $\left(\mathcal{H}, \mathcal{F}, \rho^{\varepsilon}\right)$. The potential $\mathcal{B}$ modifies the dynamics of all physical wave functions in a collective way. Now one can ask the question whether the resulting causal fermion systems satisfy the EL equations in the continuum limit. It turns out that this is the case if and only if the potential $\mathcal{B}$ satisfies certain equations, which can be identified with classical field equations for the potential $\mathcal{B}$. In this way, the causal action principle gives rise to classical field equations. In order to make our concepts clear, we point out that the potential $\mathcal{B}$ merely is a convenient device in order to describe the collective behavior of all physical wave functions. It should not be considered as a fundamental object of the theory. We also note that, in order to describe variations of the physical wave functions, the potential in 1.5.1) can be chosen arbitrarily (in particular, the potential does not need to satisfy any field equations). Each choice of $\mathcal{B}$ describes a different variation of 
the physical wave functions. It is the EL equations in the continuum limit which single out the physically admissible potentials as being those which satisfy the field equations.

Before going on, we briefly explain how the subspace $\mathcal{H}$ is chosen. Clearly, the Dirac equation (1.5.1) cannot in general be solved in closed form. Therefore, for an explicit analysis one must use perturbative methods. When performing the perturbation expansion, one must be careful about the proper normalization of the fermionic states (in the sense that spatial integrals of the form (1.2.24) should be preserved). Moreover, one must make sure that the singular structure of $P(x, y)$ in position space is compatible with the causal action principle (meaning that the light-cone expansion of $P(x, y)$ only involves bounded integrals of $\mathcal{B}$ and its derivatives). Satisfying these two requirements leads to the causal perturbation expansion (see $\$ 2.1 .6$ or [FT2] and the references therein). We also mention that regularizing the perturbation expansion is a delicate issue. This can already be understood for the simple regularization by mollification in Example 1.2.4, in which case it is not clear whether one should first mollify and then introduce the interaction or vice versa. The correct method for regularizing the perturbation expansion is obtained by demanding that the behavior under gauge transformations should be preserved by the regularization. This leads to the regularized causal perturbation expansion as developed in [F7, Appendix D] and Appendix F.

We proceed with a brief overview of the results of the analysis of the continuum limit. In the following Chapters 35 the continuum limit is worked out in several steps beginning from simple systems and ending with a system realizing the fermion configuration of the standard model. For each of these systems, the continuum limit gives rise to effective equations for second-quantized fermion fields coupled to classical bosonic gauge fields (for the connection to second-quantized bosonic fields see $\$ 1.5 .3$ below). To explain the structure of the obtained results, it is preferable to first describe the system modelling the leptons as analyzed in Chapter 4. The input to this model is the configuration of the leptons in the standard model without interaction. Thus the fermionic projector of the vacuum is assumed to be composed of three generations of Dirac particles of masses $m_{1}, m_{2}, m_{3}>0$ (describing $e, \mu, \tau$ ) as well as three generations of Dirac particles of masses $\tilde{m}_{1}, \tilde{m}_{2}, \tilde{m}_{3} \geq 0$ (describing the corresponding neutrinos). Furthermore, we assume that the regularization of the neutrinos breaks the chiral symmetry (implying that we only see their left-handed components). We point out that the definition of the model does not involve any assumptions on the interaction.

The detailed analysis in Chapter 4 reveals that the effective interaction in the continuum limit has the following structure. The fermions satisfy the Dirac equation coupled to a left-handed $\mathrm{SU}(2)$-gauge potential $A_{L}=\left(A_{L}^{i j}\right)_{i, j=1,2}$,

$$
\left[i \not \partial+\left(\begin{array}{cc}
A_{L}^{11} & A_{L}^{12} U_{\mathrm{MNS}}^{*} \\
\mathcal{A}_{L}^{21} U_{\mathrm{MNS}} & -\mathcal{A}_{L}^{11}
\end{array}\right) \chi_{L}-m Y\right] \psi=0,
$$

where we used a block matrix notation (in which the matrix entries are $3 \times 3$-matrices). Here $m Y$ is a diagonal matrix composed of the fermion masses,

$$
m Y=\operatorname{diag}\left(\tilde{m}_{1}, \tilde{m}_{2}, \tilde{m}_{3}, m_{1}, m_{2}, m_{3}\right),
$$

and $U_{\mathrm{MNS}}$ is a unitary $3 \times 3$-matrix (taking the role of the Maki-Nakagawa-Sakata matrix in the standard model). The gauge potentials $A_{L}$ satisfy a classical Yang-Mills-type equation, coupled to the fermions. More precisely, writing the isospin dependence of the gauge potentials according to $A_{L}=\sum_{\alpha=1}^{3} A_{L}^{\alpha} \sigma^{\alpha}$ in terms of Pauli matrices, we obtain 
the field equations

$$
\partial^{k} \partial_{l}\left(A_{L}^{\alpha}\right)^{l}-\square\left(A_{L}^{\alpha}\right)^{k}-M_{\alpha}^{2}\left(A_{L}^{\alpha}\right)^{k}=c_{\alpha} \bar{\psi}\left(\chi_{L} \gamma^{k} \sigma^{\alpha}\right) \psi,
$$

valid for $\alpha=1,2,3$ (for notational simplicity, we wrote the Dirac current for one Dirac particle; for a second-quantized Dirac field, this current is to be replaced by the expectation value of the corresponding fermionic field operators). Here $M_{\alpha}$ are the bosonic masses and $c_{\alpha}$ the corresponding coupling constants. The masses and coupling constants of the two off-diagonal components are equal, i.e. $M_{1}=M_{2}$ and $c_{1}=c_{2}$, but they may be different from the mass and coupling constant of the diagonal component $\alpha=3$. Generally speaking, the mass ratios $M_{1} / m_{1}, M_{3} / m_{1}$ as well as the coupling constants $c_{1}, c_{3}$ depend on the regularization. For a given regularization, they are computable.

Finally, our model involves a gravitational field described by the Einstein equations

$$
R_{j k}-\frac{1}{2} R g_{j k}+\Lambda g_{j k}=\kappa T_{j k},
$$

where $R_{j k}$ denotes the Ricci tensor, $R$ is scalar curvature, and $T_{j k}$ is the energy-momentum tensor of the Dirac field. Moreover, $\kappa$ and $\Lambda$ denote the gravitational and the cosmological constants, respectively. We find that the gravitational constant scales like $\kappa \sim \delta^{2}$, where $\delta \geq \varepsilon$ is the length scale on which the chiral symmetry is broken. We remark that the regularization is not necessarily constant in space-time but may have a dynamical behavior, in which case also the gravitational constant would become dynamical. The resulting effect, referred to as dynamical gravitational coupling, will not be covered in this book, but we refer the interested reader to [FR.

In Chapter 5, a system is analyzed which realizes the configuration of the leptons and quarks in the standard model. The result is that the field equation 1.5 .6 is replaced by field equations for the electroweak and strong interactions after spontaneous symmetry breaking (the dynamics of the corresponding Higgs field has not yet been analyzed). Furthermore, the system again involves gravity (1.5.7).

A few clarifying remarks are in order. First, the above field equations come with corrections which for brevity we cannot discuss here (see Sections 3.8, 4.4 and 4.6). Next, it is worth noting that, although the states of the Dirac sea are explicitly taken into account in our analysis, they do not enter the field equations. More specifically, in a perturbative treatment, the divergences of the Feynman diagram describing the vacuum polarization drop out of the EL equations of the causal action. Similarly, the naive "infinite negative energy density" of the sea drops out of the Einstein equations, making it unnecessary to subtract any counter terms. We finally remark that the only free parameters of the theory are the masses in (1.5.5) as well as the parameter $\delta$ which determines the gravitational constant. The coupling constants, the bosonic masses and the mixing matrices are functions of the regularization parameters which are unknown due to our present lack of knowledge on the microscopic structure of space-time. The regularization parameters cannot be chosen arbitrarily because they must satisfy certain relations. But except for these constraints, the regularization parameters are currently treated as free empirical parameters.

To summarize, the dynamics in the continuum limit is described by Dirac spinors coupled to classical gauge fields and gravity. The effective continuum theory is manifestly covariant under general coordinate transformations. The only limitation of the continuum limit is that the bosonic fields are merely classical. We shall come back to secondquantized bosonic fields in $\$ 1.5 .3$ below. 
1.5.3. Effective Interaction via Bosonic Quantum Fields. In 1.5 .2 it was outlined that and in which sense the regularized Dirac sea vacuum satisfies the EL equations (1.4.18). In simple terms, these results mean that the regularized Dirac sea vacuum is a critical point of the causal action under variations of the physical wave functions (see Definition 1.4.2). We now explain why the regularized Dirac sea vacuum is not a minimizer of the causal action principle. This argument will lead us to a method for further decreasing the causal action. It also gives some insight on the structure of the minimizing measure. Working out this method systematically reveals that the resulting interaction is to be described effectively by bosonic quantum fields.

Our argument is based on the general observation that a convex combination of universal measures is again a universal measure. More precisely, let $\rho_{1}, \ldots, \rho_{L}$ be positive measures on $\mathcal{F}$. Choosing coefficients $c_{\ell}$ with

$$
c_{\ell} \geq 0 \quad \text { and } \quad \sum_{\ell=1}^{L} c_{\ell}=1,
$$

the convex combination

$$
\tilde{\rho}:=\sum_{\ell=1}^{L} c_{\ell} \rho_{\ell}
$$

is again a positive measure on $\mathcal{F}$. Moreover, if the $\rho_{\ell}$ satisfy the linear constraints (i.e. the volume constraint (1.1.3) and the trace constraint (1.1.4)), then these constraints are again respected by $\tilde{\rho}$. Taking convex combinations of universal measures resembles superpositions of quantum states in quantum field theory. However, as a major difference, the coefficients in the convex combination 1.5.8 must be real-valued and non-negative.

Taking convex combinations of measures is a useful method for decreasing the causal action. Thus we want to choose the measures $\rho_{\ell}$ and the coefficients $c_{\ell}$ in $(1.5 .8)$ in such a way that the boundedness constraint $(1.1 .5)$ is satisfied and the causal action $[1.1 .2)$ is smaller than that of $\rho$. A simple but useful method for constructing the measures $\rho_{\ell}$ is to work with unitary transformations. For a unitary operator $V \in \mathrm{U}(\mathcal{H})$, we define the measure $V(\rho)$ by

$$
(V \rho)(\Omega)=\rho\left(V \Omega V^{-1}\right) .
$$

Choosing unitary transformations $V_{1}, \ldots, V_{L}$ we set $\rho_{\ell}=V_{\ell} \rho$ and introduce $\tilde{\rho}$ as a convex combination (1.5.8) where for simplicity we choose $c_{\ell}=1 / L$,

$$
\tilde{\rho}=\frac{1}{L} \sum_{\mathfrak{a}=1}^{L} V_{\mathfrak{a}} \rho .
$$

The action becomes

$$
\begin{aligned}
\mathcal{S}(\tilde{\rho}) & =\frac{1}{L^{2}} \sum_{\mathfrak{a}, \mathfrak{b}=1}^{L} \iint_{\mathcal{F} \times \mathcal{F}} \mathcal{L}(x, y) d\left(V_{\mathfrak{a}} \rho\right)(x) d\left(V_{\mathfrak{b}} \rho\right)(y) \\
& =\frac{\mathcal{S}(\rho)}{L}+\frac{1}{L^{2}} \sum_{\mathfrak{a} \neq \mathfrak{b}} \iint_{\mathcal{F} \times \mathcal{F}} \mathcal{L}(x, y) d\left(V_{\mathfrak{a}} \rho\right)(x) d\left(V_{\mathfrak{b}} \rho\right)(y) .
\end{aligned}
$$

In order to analyze this equation more concretely, we consider the situation that $(\mathcal{H}, \mathcal{F}, \rho)$ is a causal fermion system describing a regularized Dirac sea configuration (see $\$ 1.2 .5$ ). Then, due to the factor $1 / L$, the first summand becomes small as $L$ increases. The second summand involves all the contributions for $\mathfrak{a} \neq \mathfrak{b}$. If we can arrange that these 
contributions become small, then the action of the new measure $\tilde{\rho}$ will indeed be smaller than the action of $\rho$.

Let us consider the contributions for $\mathfrak{a} \neq \mathfrak{b}$ in more detail. In order to simplify the explanations, it is convenient to assume that the measures $V_{\mathfrak{a}} \rho$ have mutually disjoint supports (this can typically be arranged by a suitable choice of the unitary transformations $\left.V_{\mathfrak{a}}\right)$. Then the space-time $\tilde{M}:=\operatorname{supp} \tilde{\rho}$ can be decomposed into $L$ "sub-space-times" $M_{\mathfrak{a}}:=\operatorname{supp} \rho_{\mathfrak{a}}$,

$$
\tilde{M}=M_{1} \cup \cdots \cup M_{L} \quad \text { and } \quad M_{\mathfrak{a}} \cap M_{\mathfrak{b}}=\varnothing \quad \text { if } \mathfrak{a} \neq \mathfrak{b} .
$$

Likewise, a physical wave function $\psi^{u}$ can be decomposed into the contributions in the individual sub-space-times,

$$
\psi^{u}=\sum_{\mathfrak{a}=1}^{L} \psi_{\mathfrak{a}}^{u} \quad \text { with } \quad \psi_{\mathfrak{a}}^{u}:=\chi_{M_{\mathfrak{a}}} \psi^{u}
$$

(and $\chi_{M_{\mathfrak{a}}}$ is the characteristic function). This also gives rise to a corresponding decomposition of the fermionic projector:

LEMMA 1.5.2. Every sub-space-time $M_{\mathfrak{a}}$ of $\tilde{M}$ is homeomorphic to $M$, with a homeomorphism given by

Moreover, the mapping

$$
\phi_{\mathfrak{a}}: M \rightarrow M_{\mathfrak{a}}, \quad \phi_{\mathfrak{a}}(x):=V_{\mathfrak{a}}^{*} x V_{\mathfrak{a}} .
$$

$$
\left.V_{\mathfrak{a}}^{*}\right|_{S_{x}}: S_{x} \rightarrow S_{\phi_{\mathfrak{a}}(x)}
$$

is an isomorphism of the corresponding spin spaces. Identifying the spin spaces in different sub-space-times via this isomorphism, the fermionic projector can be written as

$$
\begin{aligned}
P(x, y) & =-\sum_{\mathfrak{a}, \mathfrak{b}=1}^{L} \chi_{M_{\mathfrak{a}}}(x) P_{\mathfrak{a}, \mathfrak{b}}(x, y) \chi_{M_{\mathfrak{b}}}(y) \quad \text { with } \\
P_{\mathfrak{a}, \mathfrak{b}}(x, y) & :=\Psi(x) V_{\mathfrak{a}} V_{\mathfrak{b}}^{*} \Psi(y)^{*} .
\end{aligned}
$$

Proof. The definition of $V \rho, \sqrt{1.5 .9})$, immediately implies that the transformation 1.1.18 maps $M$ to $M_{\mathfrak{a}}$ and is a homeomorphism. By definition of the physical wave function (1.1.18,

$$
\psi^{u}\left(\phi_{\mathfrak{a}}(x)\right)=\pi_{\phi_{\mathfrak{a}}(x)}=\pi_{V_{\mathfrak{a}}^{*} x V_{\mathfrak{a}}} u=V_{\mathfrak{a}}^{*} \pi_{x} V_{\mathfrak{a}} u .
$$

The identification 1.5.11 makes it possible to leave out the factor $V_{\mathfrak{a}}^{*}$. Then we can write the wave evaluation operator (1.1.20) as

$$
\tilde{\Psi}(x)=\sum_{\mathfrak{a}=1}^{L} \chi_{M_{\mathfrak{a}}}(x) \Psi(x) V_{\mathfrak{a}} .
$$

Applying 1.1.22 gives the result.

This lemma makes it possible to rewrite the action 1.5.10 as

$$
\mathcal{S}(\tilde{\rho})=\frac{\mathcal{S}(\rho)}{L}+\frac{1}{L^{2}} \sum_{\mathfrak{a} \neq \mathfrak{b}} \iint_{M \times M} \mathcal{L}\left[P_{\mathfrak{a}, \mathfrak{b}}(x, y)\right] d \rho(x) d \rho(y),
$$

where the square bracket means that the Lagrangian is computed as a function of the kernel of the fermionic projector $P_{\mathfrak{a}, \mathfrak{b}}(x, y)$ (just as explained after $(1.1 .14)$ for the kernel $P(x, y))$. The identities (1.5.13) and (1.5.14) give a good intuitive understanding of 
how the action depends on the unitary operators $V_{\mathfrak{a}}$. We first note that in the case $\mathfrak{a}=\mathfrak{b}$, the unitary operators in 1.5 .13 drop out, so that $P_{\mathfrak{a}, \mathfrak{a}}(x, y)=P(x, y)$. This also explains why the first summand in (1.5.14) involves the original action $\mathcal{S}(\rho)$. In the case $\mathfrak{a} \neq \mathfrak{b}$, however, the unitary operators in 1.5 .13 do not drop out. In particular, this makes it possible to introduce phase factors into the fermionic projector. For example, one may change the phase of each physical wave function $\psi_{\mathfrak{a}}^{u}$ arbitrarily while keeping the physical wave functions $\psi_{\mathfrak{b}}^{u}$ for $\mathfrak{b} \neq \mathfrak{a}$ unchanged. Choosing the resulting phases randomly, one gets destructive interference, implying that the kernel $P_{\mathfrak{a}, \mathfrak{b}}(x, y)$ becomes small. Making use of this dephasing effect, one can make the summands in (1.5.14 for $\mathfrak{a} \neq \mathfrak{b}$ small. A detailed analysis of the involved scalings reveals that this indeed makes it possible to decrease the causal action while respecting all constraints (see [F17]).

We refer to the measures $\rho_{\mathfrak{a}}=V_{\mathfrak{a}} \rho$ as decoherent replicas of $\rho$. Thus in the above example, the universal measure $\tilde{\rho}$ consists of a convex combination of many decoherent replicas of $\rho$. Likewise, space-time $M$ is decomposed many sub-space-times $M_{1}, \ldots, M_{L}$. Each of these sub-space-times describes the same physical system because the geometric structures are identical for all sub-space-times. However, the physical wave functions in the different sub-space-times involve relative phases, with the effect that the correlations between the sub-space-times (as described by the kernels $P_{\mathfrak{a}, \mathfrak{b}}(x, y)$ ) become small. This means physically that the decoherent replicas do not interact with each other. The resulting picture is that space-time looks effectively like a "superposition" of the different sub-space-times. The dephasing can be understood similar to decoherence effects in standard quantum field theory (see for example [JZKGKS]).

Instead of taking decoherent replicas of the same measure $\rho$, one can consider the situation that each of the measures $\rho_{\ell}$ describes a sub-space-time which involves a different classical bosonic field. In this way, one obtains effectively a superposition of classical field configurations. This makes it possible to describe second-quantized bosonic fields (see [F14]). However, as the different sub-space-times do not interact with each other, each sub-space-time has it own independent dynamics. This dynamics is described by the classical bosonic field in the corresponding sub-space-time.

In order to obtain an interaction via second-quantized bosonic fields, one needs to consider another limiting case in which the dephasing involves only some of the physical wave functions. In this case, the contributions $P_{\mathfrak{a}, \mathfrak{b}}$ with $\mathfrak{a} \neq \mathfrak{b}$ to the fermionic projector are not necessarily small. This also implies that relations which arise as a consequence of the collective behavior of all physical wave functions (like the causal relations or classical bosonic fields) still exist between the sub-space-times $M_{\mathfrak{a}}$ and $M_{\mathfrak{b}}$. In more physical terms, the sub-space-times still interact with each other. This scenario is studied in [F17] and is referred to as the microscopic mixing of physical wave functions. In order to describe the effective interaction, one describes the unitary operators $V_{\mathfrak{a}}$ by random matrices. Taking averages over the random matrices, one finds that the effective interaction can be described perturbatively in the Fock space formalism working with fermionic and bosonic field operators. Working out the detailed combinatorics and the implications of the resulting quantum field theory is work in progress (for the first step in this program see [F20]).

\section{Exercises}

EXERCISE 1.1. (a causal fermion system on $\ell_{2}$ ) Let $\left(\mathcal{H}=\ell^{2},\langle. \mid\right.$.$\rangle ) be the Hilbert$ space of square-summable complex-valued sequences. Thus, writing the vectors of $\mathcal{H}$ 
as $u=\left(u_{i}\right)_{i \in \mathbb{N}}$, the scalar product is defined by

$$
\langle u \mid v\rangle=\sum_{i=1}^{\infty} \overline{u_{i}} v_{i} .
$$

For any $k \in \mathbb{N}$, we let $x_{k}$ be an operator on $\mathcal{H}$ defined by

$$
\left(x_{k} u\right)_{k}=u_{k+1}, \quad\left(x_{k} u\right)_{k+1}=u_{k}
$$

and $\left(x_{k} u\right)_{i}=0$ for all $i \notin\{k, k+1\}$. In other words,

$$
x_{k} u=(\underbrace{0, \ldots, 0}_{k-1 \text { entries }}, u_{k+1}, u_{k}, 0, \ldots) .
$$

Finally, we let $\mu$ be the counting measure on $\mathbb{N}$ (i.e. $\mu(X)=\# X$ equals the number of elements of $X \subset \mathbb{N}$ ).

(a) Show that every operator $x_{k}$ has rank two, is symmetric, and has one positive and one negative eigenvalue. Make yourself familiar with the concept that every operator is a point in $\mathcal{F}$ (as introduced in Definition 1.1.1) for spin dimension $n=1$.

(b) Let $F: \mathbb{N} \rightarrow \mathcal{F}$ be the mapping which to every $k$ associates the corresponding operator $x_{k}$. Show that the push-forward measure $\rho=F_{*} \mu$ defined by $\rho(\Omega)=$ $\mu\left(F^{-1}(\Omega)\right)$ defines a measure on $\mathcal{F}$. Show that this measure can also be characterized by

$$
\rho(\Omega)=\#\left\{k \in \mathbb{N} \mid x_{k} \in \Omega\right\} .
$$

(Clearly, we could also have taken this equation as the definition of $\rho$. But working with the push-forward measure is a good preparation for the constructions in Section 1.2.)

(c) Show that $(\mathcal{H}, \mathcal{F}, \rho)$ is a causal fermion system of spin dimension one.

EXERCISE 1.2. This exercise shows that the trace constraint ensures that the action is non-zero. Let $(\mathcal{H}, \mathcal{F}, \rho)$ be a causal fermion system of spin dimension $n$.

(a) Assume that $\operatorname{tr}(x) \neq 0$. Show that $\mathcal{L}(x, x)>0$. (For a quantitative statement of this fact in the setting of discrete space-times see [F10, Proposition 4.3].)

(b) Assume that $\int_{\mathcal{F}} \operatorname{tr}(x) d \rho \neq 0$. Show that $\mathcal{S}(\rho)>0$.

EXERCISE 1.3. This exercise explains why the causal action principle is ill-posed in the case $\operatorname{dim} \mathcal{H}=\infty$ and $\rho(\mathcal{F})<\infty$. The underlying estimates were first given in the setting of discrete space-times in [F10, Lemma 5.1].

(a) Let $\mathcal{H}_{0}$ be a finite-dimensional Hilbert space of dimension $n$ and $\left(\mathcal{H}_{0}, \rho_{0}, \mathcal{F}_{0}\right)$ be a causal fermion system of finite total volume $\rho_{0}\left(\mathcal{F}_{0}\right)$. Let $\iota: \mathcal{H}_{0} \rightarrow \mathcal{H}$ be an isometric embedding. Construct a causal fermion system $(\mathcal{H}, \rho, \mathcal{F})$ which has the same action, the same total volume and the same values for the trace and boundedness constraints as the causal fermion system $\left(\mathcal{H}_{0}, \rho_{0}, \mathcal{F}_{0}\right)$.

(b) Let $\mathcal{H}_{1}=\mathcal{H}_{0} \oplus \mathcal{H}_{0}$. Construct a causal fermion system $\left(\mathcal{H}_{1}, \rho_{1}, \mathcal{F}_{1}\right)$ which has the same total volume and the same value of the trace constraint as $\left(\mathcal{H}_{0}, \rho_{0}, \mathcal{F}_{0}\right)$ but a smaller action and a smaller value of the boundedness constraint. Hint: Let $F_{1 / 2}$ : $\mathrm{L}\left(\mathcal{H}_{0}\right) \rightarrow \mathrm{L}\left(\mathcal{H}_{1}\right)$ be the linear mappings

$$
\left(F_{1}(A)\right)(u \oplus v)=(A u) \oplus 0, \quad\left(F_{2}(A)\right)(u \oplus v)=0 \oplus(A v) .
$$

Show that $F_{1 / 2}$ map $\mathcal{F}_{0}$ to $\mathcal{F}_{1}$. Define $\rho_{1}$ by

$$
\rho_{1}=\frac{1}{2}\left(\left(F_{1}\right)_{*} \rho+\left(F_{2}\right)_{*} \rho\right) .
$$


(c) Iterate the construction in (b) and apply (a) to obtain a series of universal measures on $\mathcal{F}$ of fixed total volume and with fixed value of the trace constraint, for which the action and the values of the boundedness constraint tend to zero. Do these universal measures converge? If yes, what is the limit?

EXERCISE 1.4. The following example explains why the boundedness constraint (1.1.5) is needed to ensure the existence of minimizers. This example was first given in $[\mathbf{F 1 3}$, Example 2.9]. Let $\mathcal{H}=\mathbb{C}^{4}$. For a given parameter $\tau>1$ consider the following mapping from the sphere $S^{3} \subset \mathbb{R}^{4}$ to the linear operators on $\mathcal{H}$,

$$
F: S^{3} \rightarrow \mathrm{L}(\mathcal{H}), \quad F(x)=\sum_{i=1}^{4} \tau x^{i} \gamma^{i}+\mathbb{1} .
$$

Here $\gamma^{i}$ are the four matrices

$$
\gamma^{\alpha}=\left(\begin{array}{cc}
\sigma^{\alpha} & 0 \\
0 & -\sigma^{\alpha}
\end{array}\right), \quad \alpha=1,2,3 \quad \text { and } \quad \gamma^{4}=\left(\begin{array}{cc}
0 & \mathbb{1} \\
\mathbb{1} & 0
\end{array}\right)
$$

(and $\sigma^{\alpha}$ are the Pauli matrices).

(a) Verify by explicit computation that $F(x)$ has two positive and two negative eigenvalues. Hint: To simplify the computation one can make use of the fact that the matrices $\gamma^{i}$ satisfy the anti-commutation relations $\left\{\gamma^{i}, \gamma^{j}\right\}=2 \delta^{i j} \mathbb{1}$ (in other words, these matrices generate the Clifford algebra on Euclidean $\mathbb{R}^{4}$ ).

(b) Let $\mu$ be the normalized Lebesgue measure on $S^{3} \subset \mathbb{R}^{4}$. Show that setting $\rho=F_{*} \mu$ defines a causal fermion system of spin dimension two and total volume one. Show that $M:=\operatorname{supp} \rho$ is homeomorhic to $S^{3}$.

(c) Compute the eigenvalues of $F(x) F(y)$. What is the causal structure of the causal fermion system?

(d) We now analyze the dependence on the parameter $\tau$. Show that the value of the trace constraint is independent of $\tau$, whereas

$$
\lim _{\tau \rightarrow \infty} \mathcal{S}=0 \quad \text { and } \quad \lim _{\tau \rightarrow \infty} \mathcal{T}=\infty .
$$

Do the universal measures converge in the limit $\tau \rightarrow \infty$ ? If yes, what is the limit?

EXERCISE 1.5. (support of a measure)

(a) We return to the example of Exercise 1.1. Show that the support of $\rho$ consists precisely of all the operators $x_{k}$.

(b) In order to illustrate how to encode geometric information in the support of a measure, let $\mathscr{M} \subset \mathbb{R}^{3}$ be a smooth surface described in a parametrization $\Phi$. Thus given an open subset $\Omega \subset \mathbb{R}^{2}$, we consider a smooth injective map

$$
\Phi: \Omega \rightarrow \mathbb{R}^{3}
$$

with the property that $\left.D \Phi\right|_{p}: \mathbb{R}^{2} \rightarrow \mathbb{R}^{3}$ has rank two for all $p \in \Omega$. Then the surface $\mathcal{M}$ is defined as the image $\Phi(\Omega) \subset \mathbb{R}^{3}$. We now introduce the measure $\rho$ as the push-forward measure of the Lebesgue measure on $\mathbb{R}^{2}$ : Let $\mu$ be the Lebesgue measure on $\mathbb{R}^{2}$. We define a set $U \subset \mathbb{R}^{3}$ to be $\rho$-measurable if and only if its preimage $\Phi^{-1}(U) \subset \mathbb{R}^{2}$ is $\mu$-measurable. On the $\rho$-measurable sets we define the measure $\rho$ by

$$
\rho(U)=\mu\left(\Phi^{-1}(U)\right) .
$$

Verify that the $\rho$-measurable sets form a $\sigma$-algebra, and that $\rho$ is a measure. What are the sets of $\rho$-measure zero? What is the support of the measure $\rho$ ? 
Suppose that $\Phi$ is no longer assumed to be injective. Is $\rho$ still a well-defined measure? Is $\rho$ well-defined if $\Phi$ is only assumed to be continuous? What are the minimal regularity assumptions on $\Phi$ needed for the push-forward measure to be well-defined? What is the support of $\rho$ in this general setting?

EXERCISE 1.6. (space-time and causal structure of the causal fermion system on $\ell_{2}$ ) We return to the example of Exercise 1.1. What is space-time M? (Hint: See Exercise 1.5 (a).) What is the causal structure on $M$ ? What is the resulting causal action? Discuss the last result in the context of the trace constraint and Exercise 1.2.

EXERCISE 1.7. This exercise is devoted to the inequality

$$
\|\sqrt{|y|}-\sqrt{|x|}\| \leq\|y-x\|^{\frac{1}{4}}\|y+x\|^{\frac{1}{4}}
$$

used in 1.1.19) (the solution to this exercise can be found in [F18]).

(a) Let $A$ and $B$ be symmetric linear operators of finite rank. Construct an explicit counter example for $2 \times 2$-matrices to the inequality

$$
\||A|-|B|\| \leq\|A-B\|
$$

(a similar exercise can be found in [RS1, Exercise 7 on page 217]).

(b) Prove the inequality

$$
\||A|-|B|\|^{2} \leq\left\|A^{2}-B^{2}\right\| .
$$

Hint: First show that there is a vector $u \in \mathcal{H}$ such that

$$
(|A|-|B|) u= \pm|||A|-|B||| u
$$

and deduce the inequality

$$
\||A|-|B||| \leq\langle u \mid(|A|+|B|) u\rangle .
$$

Then use (1.5.18) once again.

(c) Iterate 1.5.17 to obtain 1.5.16.

EXERCISE 1.8. (Krein structure of the causal fermion system on $\ell_{2}$ ) We return to the example of Exercise 1.1 and Exercise 1.6.

(a) For any $k \in \mathbb{N}$, construct the spin space $S_{x_{k}}$ and its spin scalar product.

(b) Given a vector $u \in \mathcal{H}$, what is the corresponding wave function $\psi^{u}\left(x_{k}\right)$ ? What is the Krein inner product $<. \mid$. $>$ ?

(c) What is the topology on the Krein space $\mathcal{K}$ ? Does the wave evaluation operator 1.1.20 give rise to a well-defined and continuous mapping $\Psi: \mathcal{H} \rightarrow \mathcal{K}$ ? If yes, is it an embedding? Is it surjective?

(d) Repeat part (c) of this exercise for the causal fermion system obtained if the operators $x_{k}$ in (1.5.15) are multiplied by $k$, i.e.

$$
x_{k} u=(\underbrace{0, \ldots, 0}_{k-1 \text { entries }}, k u_{k+1}, k u_{k}, 0, \ldots) .
$$

ExERCISE 1.9. The goal of this exercise is to explore possible modifications of the definition of regularization operators. 
(a) Show that for the regularization operators in Example 1.2.4, the estimate 1.2.21) can be improved to

$$
\begin{aligned}
& \left|\iint_{\mathscr{M} \times \mathcal{M}} \overline{\eta(x)}\left(P^{\varepsilon}(x, y)-P(x, y)\right) \tilde{\eta}(y) d^{4} x d^{4} y\right| \\
& \quad \leq \delta\left(|\eta|_{C^{0}(K)}|\tilde{\eta}|_{C^{1}(K)}+|\eta|_{C^{1}(K)}|\tilde{\eta}|_{C^{0}(K)}\right) .
\end{aligned}
$$

Hint: Using the notation in the proof of Proposition 1.2.7, one should first prove that

$$
\left\|\Phi_{\eta}^{\varepsilon}\right\| \leq c|\eta|_{C^{0}(K)} .
$$

(b) Can Definition 1.2 .3 be modified so that the stronger estimate 1.5 .19 holds? Is there a natural way of doing so?

EXERCISE 1.10. This exercise is devoted to a clean proof of the distributional relation $(1.2 .33)$ in one dimension. More precisely, we want to prove the slightly more general statement that for any function $\eta \in C^{1}(\mathbb{R}) \cap L^{1}(\mathbb{R})$,

$$
\lim _{\varepsilon \searrow 0} \int_{\mathbb{R}} \eta(x)\left(\frac{1}{x-i \varepsilon}-\frac{1}{x+i \varepsilon}\right) d x=2 \pi i \eta(0) .
$$

(a) Let $\eta \in C^{1}(\mathbb{R}) \cap L^{1}(\mathbb{R})$ with $\eta(0)=0$. Show with the help of Lebesgue's dominated convergence theorem that $(1.5 .20)$ holds.

(b) Show with residues that 1.5.20) holds for the function $\eta(x)=1 /\left(x^{2}+1\right)$.

(c) Combine the results of (a) and (b) to prove (1.5.20) for general $\eta \in C^{1}(\mathbb{R}) \cap L^{1}(\mathbb{R})$.

EXERCISE 1.11. This exercise recalls basics on the principal value in one dimension 1.2 .34 .

(a) Repeat the method in Exercise 1.10 to show that the limit of the left side of (1.2.34) exist for any $\eta \in C^{1}(\mathbb{R}) \cap L^{1}(\mathbb{R})$. Derive a corresponding estimate which shows that $\mathrm{PP}$ is a well-defined tempered distribution.

(b) Show that for any $\eta \in C^{1}(\mathbb{R}) \cap L^{1}(\mathbb{R})$,

$$
\operatorname{PP}(\eta)=\lim _{\varepsilon \searrow 0}\left(\int_{-\infty}^{-\varepsilon}+\int_{\varepsilon}^{\infty}\right) \frac{\eta(x)}{x} d x .
$$

ExERCISE 1.12. The goal of this exercise is to justify that the one-dimensional relations 1.2.33 and 1.2.34 can be used in the four-dimensional setting 1.2.32).

(a) Let $T$ be a distribution on $\mathbb{R}, \Omega \subset \mathcal{M}$ be an open subset of Minkowski space and $f$ : $\Omega \rightarrow \mathbb{R}$ a smooth function with nowhere vanishing gradient. Show that the relation

$$
\left(f^{*} T\right)(\eta):=T\left(\phi_{f}(\eta)\right), \quad \eta \in C_{0}^{\infty}(\Omega)
$$

with

$$
\phi_{f}(\eta)(t):=\frac{\partial}{\partial t} \int_{\Omega} \Theta(t-f(x)) \eta(x) d^{4} x
$$

(where $\Theta$ is the Heaviside function) defines $f^{*} T$ as a distribution on $\Omega$ (this is the so-called pullback of $T$ under $f$; for details see $[\mathbf{F r}$, Section 7.2]).

(b) Choosing $\Omega$ as the half space in the future, $\Omega=\left\{x \in \mathcal{M}, x^{0}>0\right\}$, one can rewrite the expression on the left of 1.2 .32 as

$$
\lim _{\varepsilon \searrow 0} \frac{1}{r^{2}-t^{2}+i \varepsilon} \text {. }
$$


Use (a) to conclude that this expression is a well-defined distribution for any $\varepsilon>0$. Show that the limit $\varepsilon \searrow 0$ exist in the distributional sense.

(c) Repeating the procedure of (b) for the half space in the past, one obtains a distribution on $\mathscr{M} \backslash\{t=0\}$. Show that this distribution coincides with the limit in (1.2.32). Hint: Similar as in Exercise 1.10, one can estimate the behavior at the origin with Lebesgue's dominated convergence theorem (to this end, first consider a test function which vanishes to a certain order at the origin).

EXERCISE 1.13. Show with a symmetry argument (without explicit computation of Fourier integrals!) that the imaginary part of the distribution $T(x, y)$ vanishes if $x$ and $y$ have space-like separation.

EXERCISE 1.14. This exercise is concerned with the Bessel functions in Lemma 1.2.9.

(a) Express the vectorial component of $P(x, y)$ similar to 1.2 .29 in terms of Bessel functions. Hint: Use 1.2.25 together with the relations for the derivatives of Bessel functions (see OLBC, (10.6.6) and (10.29.4)]).

(b) Use $(1.2 .29)$ together with the results of (a) to compute the parameters $a$ and $b$ in 1.2 .38 in the case that $x$ and $y$ have timelike separation. Simplify the formula for $a$ using the relations for the Wronskians of Bessel functions (see [OLBC, $(10.5 .2)])$.

EXERCISE 1.15. This exercise is devoted to analyzing general properties of the spectrum of the closed chain.

(a) As in Definition 1.1 .2 , we let $x$ and $y$ be symmetric operators of finite rank on a Hilbert space $\left(\mathcal{H},\langle. \mid .\rangle_{\mathcal{H}}\right)$. Show that there is a finite-dimensional subspace $I \subset \mathcal{H}$ on which both $x$ and $y$ are invariant. By choosing an orthonormal basis of $I$ and restricting the operators to $I$, we may represent both $x$ and $y$ by Hermitian matrices. Therefore, the remainder of this exercise is formulated for simplicity in terms of Hermitian matrices.

(b) Show that for any matrix $Z$, the characteristic polynomials of $Z$ and of its adjoint $Z^{*}$ (being the transposed complex conjugate matrix) are related by complex conjugation, i.e. $\operatorname{det}\left(Z^{*}-\bar{\lambda} \mathbb{1}\right)=\overline{\operatorname{det}(Z-\lambda \mathbb{1})}$.

(c) Let $X$ and $Y$ be symmetric matrices. Show that the characteristic polynomials of the matrices $X Y$ and $Y X$ coincide.

(d) Combine (b) and (c) to conclude that the characteristic polynomial of $X Y$ has real coefficients, i.e. $\operatorname{det}(X Y-\bar{\lambda} \mathbb{1})=\overline{\operatorname{det}(X Y-\lambda \mathbb{1})}$. Infer that the spectrum of the matrix product $X Y$ is symmetric about the real axis, i.e.

$$
\operatorname{det}(X Y-\lambda \mathbb{1})=0 \Longrightarrow \operatorname{det}(X Y-\bar{\lambda} \mathbb{1})=0 .
$$

(e) For the closed chain (1.1.14), the mathematical setting is somewhat different, because $A_{x y}$ is a symmetric operator on the indefinite inner product space $\left(S_{x}, \prec\right.$.|. $\left.\succ_{x}\right)$. On the other hand, we saw after (1.1.14 that $A_{x y}$ is isospectral to $x y$. Indeed, the symmetry result 1.5.21 can be used to prove a corresponding statement for $A_{x y}$,

$$
\operatorname{det}\left(A_{x y}-\lambda \mathbb{1}\right)=0 \Longrightarrow \operatorname{det}\left(A_{x y}-\bar{\lambda} \mathbb{1}\right)=0 .
$$

This result is well-known in the theory of indefinite inner product spaces (see for example the textbooks [B2, GLR or [F10, Section 3]). In order to derive it from (1.5.21), one can proceed as follows: First, represent the indefinite inner product in the form $\prec . \mid . \succ=\langle. \mid S x\rangle$, where $\langle. \mid$.$\rangle is a scalar product and S$ is an invertible operator which is symmetric (with respect to this scalar product). Next, show that 
the operator $B:=A_{x y} S$ is symmetric (again with respect to this scalar product). Finally, write the closed chain as $A_{x y}=B S^{-1}$ and apply (1.5.21).

EXERCise 1.16. This exercise recalls a few basic facts from the theory of ordinary differential equations which are relevant in the context of the Bessel functions in Lemma 1.2.9 (for more material in this direction see for example the textbook [CL]). Let $\phi_{1}$ and $\phi_{2}$ be two linearly independent real-valued solutions of the linear ordinary differential equation of second order

$$
\phi^{\prime \prime}(x)+a(x) \phi^{\prime}(x)+b(x) \phi(x)=0,
$$

where $a$ and $b$ are two smooth, real-valued functions on an open interval $I$.

(a) Show that at every $x \in I$, either $\phi_{1}(x)$ or $\phi_{2}(x)$ is non-zero. Moreover, either $\phi_{1}^{\prime}(x)$ or $\phi_{2}^{\prime}(x)$ is non-zero. Hint: Combine the statement of the Picard-Lindelöf theorem with the fact that a general solution can be written as a linear combination of $\phi_{1}$ and $\phi_{2}$.

(b) Show that the Wronskian defined by $w\left(\phi_{1}, \phi_{2}\right)=\phi_{1}^{\prime}(x) \phi_{2}(x)-\phi_{1}(x) \phi_{2}^{\prime}(x)$ is independent of $x$ and non-zero.

EXERCISE 1.17. Let $\xi$ be a timelike vector, for simplicity normalized to $\xi^{2}=1$. Let $A$ be the $4 \times 4$-matrix $A=a \notin+b$. Show that the operators

$$
F_{ \pm}:=\frac{1}{2}(\mathbb{1} \pm \not ̈)
$$

have rank two and map to eigenspaces of $A$. What are the corresponding eigenvalues? Show that the operators $F_{ \pm}$are idempotent and symmetric with respect to the spin scalar product. Show that the image of the operators $F_{ \pm}$is positive or negative definite. Moreover, the image of $F_{+}$is orthogonal to that of $F_{-}$(again with respect to the spin scalar product). The results of this exercise can be summarized by saying that the $F_{ \pm}$ are the spectral projection operators of $A$.

EXERCISE 1.18. The goal of this exercise is to analyze in which sense the notion of causality is stable under perturbations.

(a) Show by a counter example with $3 \times 3$-matrices that the notion of timelike separation (see Definition 1.1.2) is not stable under perturbations.

(b) Show that the notion of properly timelike separation (see Definition 1.1.6) is stable under perturbations.

(c) We now analyze a setting in which the notion of spacelike separation (see Definition 1.1.2 is stable under perturbations: Assume that the regularized kernel $P^{\varepsilon}(x, y)$ converges to the unregularized kernel (1.2.41). Moreover, assume that the eigenvalues of the regularized closed chain are at least two-fold degenerate for every $\varepsilon>0$. Finally, assume that the eigenvalues of the unregularized closed chain form a complex conjugate pair. Show that under these assumptions, the eigenvalues of the regularized closed chain also form a complex conjugate pair for sufficiently small $\varepsilon$.

In Exercise 1.19 a setting is given in which the assumptions in (c) are satisfied.

ExERCISE 1.19. This exercise explains why the assumptions in Exercise 1.18 (c) are reasonable. It is a good preparation for the computation of the eigenvalues of the closed chain in the vacuum to be carried out in 3.6.1. Assume that the regularized kernel $P^{\varepsilon}(x, y)$ has vector-scalar structure (1.2.44). Compute the eigenvalues of the closed chain. Why are they always two-fold degenerate? Explain why the bilinear contribution to the closed chain tends to gives rise to complex conjugate pairs of eigenvalues. 
In order to put these results into context, we remark that the picture in 33.6 .1 is that in space-like directions, the bilinear contribution gives rise to complex conjugate pairs of eigenvalues. These are stable under perturbations according to Exercise 1.18 (c).

EXERCise 1.20. The goal of this exercise is to analyze the functional $\mathcal{C}$ for the regularized Dirac sea vacuum in spacelike directions.

(a) Let $\xi$ be a spacelike vector. Show that in the representation 1.2 .35 of the kernel of the fermionic projector, the parameter $\alpha$ is imaginary, whereas $\beta$ is real. Hint: Use 1.2 .25 with either the formula $(1.2 .29)$ or the result of Exercise 1.13.

(b) Deduce that the parameter $a$ in (1.2.37) vanishes if $\xi$ is spacelike.


commutator in 1.2.43).

EXERCISE 1.21. Consider the kernel of the fermionic projector regularized by a convergence-generating factor $e^{\varepsilon\left|k_{0}\right|}$, i.e. similar to 1.2 .30 ,

$$
P^{\varepsilon}(x, y)=\int \frac{d^{4} k}{(2 \pi)^{4}}(\not k+m) \delta\left(k^{2}-m^{2}\right) \Theta\left(-k_{0}\right) e^{-i k(x-y)} e^{-\varepsilon\left|k_{0}\right|} .
$$

Compute $P^{\varepsilon}(x, x)$. How do the scalar and vectorial components scale in $\varepsilon$ ?

EXERCISE 1.22. We now explore a functional which at first sight might seem a promising alternative to (1.1.11) for distinguishing a time direction. Clearly, for the sign of a functional to distinguish a time direction, the functional should be anti-symmetric in its arguments $x$ and $y$. The simplest functional with this property is given by

$$
\mathcal{B}: M \times M \rightarrow \mathbb{R}, \quad \mathcal{B}(x, y):=\operatorname{tr}\left(y \pi_{x}-x \pi_{y}\right) .
$$

(a) Write the functional $\mathcal{B}$ similar to 1.2 .43 in the form

$$
\mathcal{B}(x, y)=\operatorname{Tr}_{S_{x}}\left(\nu(x) A_{x y}^{\varepsilon}\right)-\operatorname{Tr}_{S_{y}}\left(\nu(y) A_{y x}^{\varepsilon}\right) .
$$

(b) Now assume that in a given spinor basis, the fermionic projector has vector-scalar structure (1.2.44). Show that only the scalar and vectorial components of $A_{x y}^{\varepsilon}$ contribute to the trace in 1.5 .23 (whereas the bilinear component drops out). Deduce that, in the chosen spinor basis, the relations $A_{x y}^{\varepsilon}=A_{y x}^{\varepsilon}$ holds and

$$
\mathcal{B}(x, y)=\operatorname{Tr}\left((\nu(x)-\nu(y)) A_{x y}^{\varepsilon}\right) .
$$

Show that the last equation vanishes if the fermionic projector is homogeneous in the sense that $P^{\varepsilon}(x, x)=P^{\varepsilon}(y, y)$ for all $x, y \in M$.

In non-technical terms, these results show that the functional $\mathcal{B}$ gives information on the "deviation from homogeneity." But this functional cannot be used to distinguish a time direction. In particular, it vanishes for regularized Dirac sea configurations in Minkowski space. We remark that seeking for an anti-symmetric functional which does not vanish for homogeneous fermionic projectors with vector-scalar structure leads directly to the functional (1.1.11).

EXERCISE 1.23. This exercise explains how a variation of the universal measure described by a push-forward $(1.4 .5)$ can be realized by a variation of the physical wave functions. Thus we let $F_{\tau}: M \rightarrow \mathcal{F}$ with $\tau \in(-\delta, \delta)$ be a family of functions which satisfy the conditions in (1.4.7) and (1.4.8). Moreover, we assume that $F_{\tau}$ is differentiable in $\tau$ and that all points in $K$ are regular (see Definition 1.1.5). 
(a) We first fix $x \in K$. Show that by decreasing $\delta$, one can arrange that the operators $F_{\tau}(x)$ have maximal rank $2 n$ for all $\tau \in(-\delta, \delta)$. Hint: Make use of the fact that the spectrum of the operators $F_{\tau}(x)$ depends continuously on $\tau$.

(b) We introduce the spin spaces $S_{x}^{\tau}$ endowed with corresponding inner products $\prec$.|. $\succ_{x}^{\tau}$ in analogy to 1.1 .16 by

$$
S_{x}^{\tau}=\left(F_{\tau}(x)\right)(\mathcal{H}), \quad \prec \cdot\left|\cdot \succ_{x}^{\tau}=-\left\langle\cdot \mid F_{\tau}(x) \cdot\right\rangle_{\mathcal{H}}\right|_{S_{x}^{\tau} \times S_{x}^{\tau}} .
$$

Construct a family of isometries

$$
V_{\tau}(x):\left(S_{x}, \prec \cdot \mid \cdot \succ_{x}\right) \rightarrow\left(S_{x}^{\tau}, \prec \cdot \mid \cdot \succ_{x}^{\tau}\right)
$$

which is differentiable in $\tau$ (where "isometric" refers to the corresponding spin scalar product). Hint: For example, one can work with the orthogonal projections in $\mathcal{H}$ and take the polar decomposition with respect to the spin scalar products.

(c) Consider the variation of the wave evaluation operator given by

$$
\Psi_{\tau}(x)=\left(V_{\tau}(x)\right)^{-1} \pi_{F_{\tau}(x)}: \mathcal{H} \rightarrow S_{x}
$$

(where $\pi_{F_{\tau}(x)}$ is the orthogonal projection in $\mathcal{H}$ on $S_{x}^{\tau}$ as defined above). Show that the relation (1.4.12 gives us back the family of functions $F_{\tau}(x)$ we started with.

(d) So far, the point $x \in K$ was fixed. We now extend the construction so that $x$ can be varied: Use a compactness argument to show that there is $\delta>0$ such that the operators $F_{\tau}(x)$ have maximal rank $2 n$ for all $\tau \in(-\delta, \delta)$ and all $x \in K$. Show that the mappings $V_{\tau}(x)$ can be introduced such that they depend continuously in $x$ and are differentiable in $\tau$.

ExERCISE 1.24. The goal of this exercise is to illustrate the more general EL equations as derived in $[\mathbf{B F}]$. In order to simplify the setting, we leave out the constraints and replace $\mathcal{F}$ by a compact manifold. Thus let $\mathcal{F}$ be a smooth compact manifold and $\mathcal{L} \in$ $C^{0,1}\left(\mathcal{F} \times \mathcal{F}, \mathbb{R}_{0}^{+}\right)$be a non-negative Lipschitz-continuous function which is symmetric, i.e.

$$
\mathcal{L}(x, y)=\mathcal{L}(y, x) \quad \text { for all } x, y \in \mathcal{F} .
$$

The causal variational principle is to minimize the action $\mathcal{S}$ defined by

$$
\mathcal{S}(\rho)=\iint_{\mathcal{F} \times \mathcal{F}} \mathcal{L}(x, y) d \rho(x) d \rho(y)
$$

under variations of $\rho$ in the class of (positive) normalized regular Borel measures. Let $\rho$ be a minimizer.

(a) Show by analyzing variations of the form 1.4 .21 that the function $\ell \in C^{0,1}(\mathcal{F})$ defined by

$$
\ell(x)=\int_{\mathcal{F}} \mathcal{L}(x, y) d \rho(y)
$$

is minimal on the support of $\rho$,

$$
\left.\ell\right|_{\operatorname{supp} \rho} \equiv \inf _{\mathcal{F}} \ell .
$$

(b) We now consider second variations. Let $\left(\mathcal{H}_{\rho},\langle., .\rangle_{\rho}\right)$ be the Hilbert space $L^{2}(\mathcal{F}, d \rho)$. Show that the operator $\mathcal{L}_{\rho}$ defined by

$$
\mathcal{L}_{\rho}: \mathcal{H}_{\rho} \rightarrow \mathcal{H}_{\rho}, \quad\left(\mathcal{L}_{\rho} \psi\right)(x)=\int_{\mathcal{F}} \mathcal{L}(x, y) \psi(y) d \rho(y)
$$

is Hilbert-Schmidt. Show that it is non-negative. Hint: Consider suitable variations of the form $d \tilde{\rho}_{\tau}=d \rho+\tau \psi d \rho$ with $\psi \in \mathcal{H}_{\rho}$. 
We refer the reader interested in the analysis of the causal variational principle in this compact setting to [F13, FS.

EXERCise 1.25. The goal of this exercise is to illustrate the Noether-like theorems mentioned in 1.4 .2 . In order to simplify the problem as far as possible, we again consider the compact setting of Exercise 1.24 and assume furthermore that the Lagrangian is smooth, i.e. $\mathcal{L} \in C^{\infty}\left(\mathcal{F} \times \mathcal{F}, \mathbb{R}_{0}^{+}\right)$. Let $\rho$ be a minimizer of the action (1.5.25) under variations of $\rho$ in the class of (positive) normalized regular Borel measures. Let $u$ be a vector field on $\mathcal{F}$. Assume that $u$ is a symmetry of the Lagrangian in the sense that

$$
\left(u(x)^{j} \frac{\partial}{\partial x^{j}}+u(y)^{j} \frac{\partial}{\partial y^{j}}\right) \mathcal{L}(x, y)=0 \quad \text { for all } x, y \in \mathcal{F} .
$$

Prove that for any measurable set $\Omega \subset \mathcal{F}$,

$$
\int_{\Omega} d \rho(x) \int_{\mathcal{F} \backslash \Omega} d \rho(y) u(x)^{j} \frac{\partial}{\partial x^{j}} \mathcal{L}(x, y)=0 .
$$

Hint: Integrate $(1.5 .28)$ over $\Omega \times \Omega$. Transform the integrals using the symmetry of the Lagrangian (1.5.24). Finally, make use of the EL equations (1.5.27) and the smoothness of the function $\ell$. 


\section{CHAPTER 2}

\section{Computational Tools}

In this chapter we introduce the computational methods needed for the analysis of the causal action principle in the continuum limit. These methods are the backbone of the analysis given in Chapters 35. Nevertheless, in order to facilitate the reading of the book, we made the subsequent chapters accessible even without a detailed knowledge of the computational tools. To this end, all the technical computations are given in the appendices, whereas in the main Chapters 35 these results are merely stated and explained. Therefore, a reader who is willing to accept the results of the detailed computations may skip the present chapter in a first reading.

Our main objective is to construct the fermionic projector in the presence of an external potential and to analyze it in position space. The first task is to define the unregularized fermionic projector $P(x, y)$ in the presence of the external potential. In this setting, the fermionic projector was constructed in a perturbation expansion in $\mathcal{B}$ in [F3, FG1, FT2]. More recently, a non-perturbative construction was given in [FR2, FR3, FMR (see also the brief review in 1.5.1). For the explicit analysis of the causal action principle to be carried out in this book, we need the detailed formulas of the perturbation expansion. In order to focus on what is really needed in this book, we here restrict attention to the perturbative treatment (Section 2.1). The reader interested in non-perturbative methods is referred to the introduction in [FKT] or to the research papers [FR2, FR3, FMR].

Our next task is to derive detailed formulas for the fermionic projector in position space. Such formulas are most conveniently obtained using the so-called light-cone expansion as first developed in [F5, F6]. In Section 2.2 we give a self-contained introduction to the light-cone expansion.

In Section 2.3 the causal perturbation expansion and the light-cone expansion are adapted to the description of linearized gravity.

In Section 2.4 we turn attention to the ultraviolet regularization of the fermionic projector. This leads us to the so-called formalism of the continuum limit, which makes it possible to analyze how the different contributions to the causal action depend on the regularization. In order to make the presentation easily accessible, we begin with the example of an $i \varepsilon$-regularization (2.4.1). Then we consider linear combinations of such regularizations ( 2.4 .2 ) and explain further regularization effects $(2.4 .3)$. Then the formalism of the continuum is introduced (\$2.4.4), and its derivation is outlined ( 2.4.5). Our presentation is not as general as the original derivation as given in [F7, Chapter 4], but instead it aims at clarifying the main points of the construction.

In Section 2.5 we explain how to compute the local trace. This is important in view of the rescaling procedure explained in $\$ 1.4 .1$ (see (1.4.11)).

Finally, in Section 2.6 it is explained how the EL equations as derived in \$1.4.1 can can be analyzed in the formalism of the continuum limit. 


\subsection{The Fermionic Projector in an External Potential}

2.1.1. The Fermionic Projector of the Vacuum. Our starting point is the unregularized kernel of the fermionic projector of the vacuum which we already encountered in 1.2 .5 (see Lemma 1.2.8, (1.2.25) and Lemma 1.2.9). For the later constructions, it is convenient to clarify that we are in the Minkowski vacuum by adding an index "vac." Moreover, we denote the mass by an additional index $m$. Thus we define the kernel of the fermionic projector of the vacuum as the bi-distribution

$$
P_{m}^{\mathrm{vac}}(x, y)=\int \frac{d^{4} k}{(2 \pi)^{4}} P_{m}^{\mathrm{vac}}(k) e^{-i k(x-y)},
$$

where $P_{m}^{\mathrm{vac}}(k)$ is the distribution in momentum space

$$
P_{m}^{\mathrm{vac}}(k)=(\not k+m) \delta\left(k^{2}-m^{2}\right) \Theta\left(-k^{0}\right)
$$

(and $\Theta$ denotes the Heaviside function). We also consider the distribution $P_{m}^{\mathrm{vac}}(x, y)$ as the integral kernel of an operator acting on wave functions in space-time, i.e.

$$
P_{m}^{\mathrm{vac}}: C_{0}^{\infty}(\mathscr{M}, S \mathscr{M}) \rightarrow C^{\infty}(\mathscr{M}, S \mathscr{M}), \quad\left(P_{m}^{\mathrm{vac}} \psi\right)(x)=\int_{M} P_{m}^{\mathrm{vac}}(x, y) \psi(y) d^{4} y .
$$

This operator is the so-called fermionic projector of the vacuum.

Before going on, we briefly recall the physical picture. In 2.1.1 we integrate over all the plane-wave solutions of the Dirac equation of negative frequency (the decomposition into plane-wave solutions was explained in detail in Chapter 1; see (1.2.20) and Lemma 1.2.8). Thus $P_{m}^{\mathrm{vac}}$ describes the ensemble of all negative-frequency solutions of the Dirac equation. As already mentioned in 1.2 .5 , we use this Dirac sea configuration to describe the vacuum in Minkowski space. In order to describe a system with an additional particle, we simply add the corresponding bra/ket-combination by setting

$$
P(x, y)=P_{m}^{\mathrm{vac}}(x, y)-\frac{1}{2 \pi} \psi(x) \overline{\psi(y)},
$$

where $\psi$ is a positive-frequency solution of the Dirac equation (for the prefactor $-1 /(2 \pi)$ and the normalization of the wave function see $\$ 2.1 .7$ below). Similarly, we occupy several states by adding the bra/ket-combinations of several particle states,

$$
P(x, y)=P_{m}^{\mathrm{vac}}(x, y)-\frac{1}{2 \pi} \sum_{k=1}^{n_{\mathrm{p}}} \psi_{k}(x) \overline{\psi_{k}(y)}
$$

(which need to be suitably ortho-normalized; see again $\$ 2.1 .7$ below). In order to introduce anti-particles, we similarly subtract bra/ket-combinations

$$
P(x, y)=P_{m}^{\mathrm{vac}}(x, y)-\frac{1}{2 \pi} \sum_{k=1}^{n_{\mathrm{p}}} \psi_{k}(x) \overline{\psi_{k}(y)}+\frac{1}{2 \pi} \sum_{l=1}^{n_{\mathrm{a}}} \phi_{l}(x) \overline{\phi_{l}(y)},
$$

where $\phi_{1}, \ldots, \phi_{n_{\mathrm{a}}}$ are the wave functions of negative-frequency solutions. Thus in simple terms, we take Dirac's concept of the Dirac sea literally and describe particles by additional occupied states and anti-particles by "holes" in the sea.

With the methods introduced so far, this description of particles and anti-particles by occupying states and creating "holes" can only be performed in the non-interacting situation in which we can work with plane-wave solutions of the Dirac equation. But it is not obvious how the construction should be carried out if an external potential is present. In order to tackle this problem, we first analyze how to describe the completely 
filled Dirac sea in the presence of an external potential (see $2.1 .2-82.1 .6$ ). Afterwards, we will come back to the description of systems involving particles and anti-particles (see $\S 2.1 .7$ ).

2.1.2. The External Field Problem. We now return to the Dirac equation in the presence of an external potential 1.5.1,

$$
(i \not \partial+\mathcal{B}-m) \psi(x)=0,
$$

where $\mathcal{B}$ is a smooth potential with suitable decay properties at spatial infinity and for large times (to be specified in Lemma 2.1.2 below). We now explain the basic problem in defining the fermionic projector in the presence of an external potential.

The definition of the fermionic projector of the vacuum (2.1.1) and (2.1.2) makes essential use of the fact that the solution space of the Dirac equation splits into two subspaces of negative and positive frequency, respectively. Indeed, this made it possible in (2.1.2) to integrate only over the solutions of negative frequency. In order to extend the definition of the fermionic projector to the case when an external potential is present 2.1.5), one needs to again decompose the solution space into two subspaces. In the special case that $\mathcal{B}$ is static, one can still separate the time dependence by the plane wave ansatz $\psi(t, \vec{x})=e^{-i \omega t} \psi_{\omega}(\vec{x})$, so that the sign of $\omega$ gives a canonical splitting of the solution space. This procedure is often referred to as the frequency splitting. In the general time-dependent setting, however, no plane wave ansatz can be used, so that the frequency splitting breaks down. Therefore, it is no longer obvious if there still is a canonical decomposition of the solution space into two subspaces.

This problem is sometimes referred to as the external field problem (for more details see Exercise 2.1 or the exposition in [F7, Section 2.1]). It is a common belief that in the presence of a general time-dependent external potential, there no longer exists a canonical decomposition of the solution space into two subspaces. Nevertheless, it is still possible to decompose the solution space into two subspaces, for example by using the sign of the spectrum of the Dirac Hamiltonian on a distinguished Cauchy surface. But the decomposition is no longer canonical in the sense that it involves an arbitrariness. This arbitrariness is often associated to an observer, so that the choice of the subspaces depends on the observer. As a consequence, the interpretation of the fermionic manyparticle state in terms of particles and anti-particles also depends on the observer. This observer dependence of the particle interpretation becomes most apparent in the Unruh effect in which the vacuum of the observer at rest is described by a uniformly accelerated observer in terms of a thermal state involving particles and anti-particles.

Nevertheless, this reduction to particles and anti-particles as being objects associated to observers only tells part of the truth. Namely, as shall be developed in what follows, even in the presence of a time-dependent external potential there is a canonical decomposition of the solution space into two subspaces. In the static situation, this decomposition reduces to the frequency splitting. In the time-dependent situation, however, this decomposition depends on the global behavior of $\mathcal{B}$ in space-time. In particular, this decomposition cannot be associated to a local observer. Starting from the canonical decomposition of the solution space, one can again generate particles and holes, giving rise to an interpretation of the many-particle state in terms of particles and anti-particles. This particle interpretation is again independent of the choice of an observer. All constructions are explicitly covariant. 
2.1.3. Main Ingredients to the Construction. Before entering the constructions, we explain a few ingredients and ideas. Generally speaking, we shall make use of additional properties of the fermionic projector of the vacuum, which are not immediately apparent in the Fourier decomposition (2.1.1) and (2.1.2). One ingredient is to use that causality is built into $P^{\mathrm{vac}}(x, y)$. To see how this comes about, we decompose $P_{m}^{\mathrm{vac}}$ as

$$
P_{m}^{\mathrm{vac}}(x, y)=\frac{1}{2}\left(p_{m}(x, y)-k_{m}(x, y)\right),
$$

where $p_{m}(x, y)$ and $k_{m}(x, y)$ are the Fourier transforms of the distributions in momentum space

$$
\begin{aligned}
& p_{m}(q)=(q+m) \delta\left(q^{2}-m^{2}\right) \\
& k_{m}(q)=(q+m) \delta\left(q^{2}-m^{2}\right) \epsilon\left(q^{0}\right)
\end{aligned}
$$

(and $\epsilon$ in (2.1.8) is again the sign function $\epsilon(x)=1$ for $x \geq 0$ and $\epsilon(x)=-1$ otherwise). All these Fourier integrals are well-defined tempered distributions, which are also distributional solutions of the vacuum Dirac equation. The point is that the distribution $k_{m}(x, y)$ is causal in the sense that it vanishes if $x$ and $y$ have spacelike separation. In order to see this, it is useful to introduce the advanced and the retarded Green's functions by

$$
s_{m}^{\vee}(q)=\lim _{\nu \searrow 0} \frac{\not q+m}{q^{2}-m^{2}-i \nu q^{0}} \quad \text { and } \quad s_{m}^{\wedge}(q)=\lim _{\nu \searrow 0} \frac{\not q+m}{q^{2}-m^{2}+i \nu q^{0}},
$$

respectively (with the limit $\nu \searrow 0$ taken in the distributional sense). Taking their Fourier transform

$$
s_{m}(x, y)=\int \frac{d^{4} q}{(2 \pi)^{4}} s_{m}(q) e^{-i q(x-y)},
$$

we obtain corresponding bi-distributions $s_{m}^{\vee}(x, y)$ and $s_{m}^{\wedge}(x, y)$. By direct computation one verifies that these Green's functions satisfy the distributional equation

$$
\left(i \not \partial_{x}-m\right) s_{m}(x, y)=\delta^{4}(x-y) \text {. }
$$

Moreover, computing the Fourier integral 2.1.10 with residues, one sees that the support of these Green's functions lies in the upper respectively lower light cone, i.e.

$$
\operatorname{supp} s_{m}^{\vee}(x, .) \subset J_{x}^{\vee}, \quad \operatorname{supp} s_{m}^{\wedge}(x, .) \subset J_{x}^{\wedge},
$$

where $J_{x}^{\vee}$ and $J_{x}^{\wedge}$ denote the points in the causal future respectively past of $x$,

$$
\begin{aligned}
& J_{x}^{\vee}=\left\{y \in M \mid(y-x)^{2} \geq 0,\left(y^{0}-x^{0}\right) \geq 0\right\} \\
& J_{x}^{\wedge}=\left\{y \in M \mid(y-x)^{2} \geq 0,\left(y^{0}-x^{0}\right) \leq 0\right\}
\end{aligned}
$$

(for details see Exercise 2.2 or [FKT, Chapter 4]). In view of (2.1.11), the difference of the advanced and retarded Greens' functions is a solution of the homogeneous Dirac equation. In order to compute it in detail, we again make use of 1.2 .33 to obtain

$$
\begin{aligned}
s_{m}^{\vee}(q)-s_{m}^{\wedge}(q) & =(\not q+m) \lim _{\nu \searrow 0}\left[\frac{1}{q^{2}-m^{2}-i \nu q^{0}}-\frac{1}{q^{2}-m^{2}+i \nu q^{0}}\right] \\
& =(\not q+m) \lim _{\nu \searrow 0}\left[\frac{1}{q^{2}-m^{2}-i \nu}-\frac{1}{q^{2}-m^{2}+i \nu}\right] \epsilon\left(q^{0}\right) \\
& =2 \pi i(\not q+m) \delta\left(q^{2}-m^{2}\right) \epsilon\left(q^{0}\right)
\end{aligned}
$$


(for details see Exercise 2.4). Comparing with (2.1.8), we conclude that the difference of the advanced and retarded Green's functions is a multiple of $k_{m}$

$$
k_{m}(x, y)=\frac{1}{2 \pi i}\left(s^{\vee}(x, y)-s^{\wedge}(x, y)\right) .
$$

In particular, this shows that $k_{m}$ is indeed causal, i.e.

$$
\operatorname{supp} k_{m}(x, .) \subset J_{x},
$$

where $J_{x}:=J_{x}^{\vee} \cup J_{x}^{\wedge}$. We refer to $k_{m}$ as the causal fundamental solution.

Now (2.1.6) can be understood as the decomposition of the vacuum fermionic projector into a causal part (the distribution $k_{m}$ ) and a part which is not causal (the distribution $p_{m}$; note that the explicit formulas in 1.2 .25 and Lemma 1.2 .9 show that $p_{m}(x, y)$ is indeed non-zero for spacelike distances). One idea behind our constructions is to perform the perturbation expansion in such a way that the decomposition of $P(x, y)$ in to a causal and a non-causal part is preserved.

Another ingredient to our constructions is that the distributions $p_{m}$ and $k_{m}$ are related to each other by a functional calculus, as we now explain. We first point out that for the space-time integral in $(2.1 .3)$ to exist, we had to assume that the wave function $\psi$ has suitable decay properties at infinity. More specifically, the time integral in $(2.1 .3)$ in general diverges if $\psi$ is a physical wave function, being a solution of the Dirac equation. In particular, the operator in (2.1.3) cannot be defined as an operator from a vector space to itself, but it necessarily maps one function space to another function space. As a consequence, it is impossible to multiply the operator $P_{m}$ by itself. This is obvious because the formal integral

$$
\int P_{m}^{\mathrm{vac}}(x, z) P_{m}^{\mathrm{vac}}(z, y) d^{4} z
$$

is ill-defined. This problem can be understood similarly in momentum space. Namely, using that convolution in position space corresponds to multiplication in momentum space, the integral in 2.1.16 corresponds to the formal product

$$
P_{m}^{\mathrm{vac}}(q) P_{m}^{\mathrm{vac}}(q),
$$

which is again ill-defined because the square of the $\delta$-distribution in 2.1.2 makes no mathematical sense. As we shall see, these obvious problems in the naive treatment of the fermionic projector are not only a mathematical subtlety. On the contrary, the methods for overcoming these problems will involve a careful analysis of the causal structure of the fermionic projector and of its proper normalization.

It is important to observe that the above operator product does make sense if we consider two different mass parameters. Namely,

$$
\begin{aligned}
P_{m}^{\mathrm{vac}}(q) P_{m^{\prime}}^{\mathrm{vac}}(q) & =(\not++m) \delta\left(q^{2}-m^{2}\right) \Theta\left(-q^{0}\right)\left(\not t+m^{\prime}\right) \delta\left(q^{2}-\left(m^{\prime}\right)^{2}\right) \Theta\left(-q^{0}\right) \\
& =\left(q^{2}+\left(m+m^{\prime}\right) \not q+m m^{\prime}\right) \delta\left(m^{2}-\left(m^{\prime}\right)^{2}\right) \delta\left(q^{2}-m^{2}\right) \Theta\left(-q^{0}\right) \\
& =\left(q^{2}+\left(m+m^{\prime}\right) \not q+m m^{\prime}\right) \frac{1}{2 m} \delta\left(m-m^{\prime}\right) \delta\left(q^{2}-m^{2}\right) \Theta\left(-q^{0}\right) \\
& =\delta\left(m-m^{\prime}\right)(\not q+m) \delta\left(q^{2}-m^{2}\right) \Theta\left(-q^{0}\right) .
\end{aligned}
$$

giving rise to the distributional identity

$$
P_{m}^{\mathrm{vac}} P_{m^{\prime}}^{\mathrm{vac}}=\delta\left(m-m^{\prime}\right) P_{m}^{\mathrm{vac}} .
$$


This resembles idempotence, but it involves a $\delta$-distribution in the mass parameter. We remark that this $\delta$-normalization in the mass parameter can be treated in a mathematically convincing way using the notion of the mass oscillation property as introduced in [FR3. For brevity, we shall not enter these constructions here. Instead, we are content with the fact that 2.1.17) is well-defined if we test in both $m$ and $q$.

This calculus can be used similarly for the operators $p_{m}$ and $k_{m}$ obtained by considering the distributions (2.1.7) and 2.1.8 as multiplication operators in momentum space. In particular, this gives rise to the relation

$$
k_{m} k_{m^{\prime}}=\delta\left(m-m^{\prime}\right) p_{m}
$$

(for details see Lemma 2.1.3 below). This identity is very useful because it allows us to deduce $p_{m}$ from $k_{m}$. Therefore, our strategy is to first construct $k_{m}$ in the presence of an external potential using the underlying causal structure (2.1.14). Then we take 2.1.18) to define $p_{m}$ in the presence of the external potential. Finally, we use $(2.1 .6)$ to define the fermionic projector.

There is one subtle point in the construction which we want to mention here: the proper normalization of the states of the fermionic projector. The most obvious method is to interpret and use the identity 2.1.17) as a normalization condition. This so-called mass normalization was used in [F3, FG1]; see also [F7, Chapter 2]. More recently, the non-perturbative construction in FR3 revealed that on a general globally hyperbolic manifold, the mass normalization cannot be used and should be replaced by the so-called spatial normalization. In [FT2 the causal perturbation expansion is worked out for both the mass and the spatial normalizations, and the methods and results are compared. In [FT2, Section 2.2] the advantages of the spatial normalization are discussed, but no decisive argument in favor of one of the normalization methods is given. Finally, the Noether-like theorems in FK2 showed that the spatial normalization is the proper normalization method, because it reflects the intrinsic conservation laws of the causal fermion system (see [FK2, Remark 5.13] or the brief outline in $\$ 1.4 .2$ ).

With these results in mind, we here restrict attention to the spatial normalization, which we now introduce. Recall that for a Dirac wave function $\psi$, the quantity $\left(\bar{\psi} \gamma^{0} \psi\right)\left(t_{0}, \vec{x}\right)$ has the interpretation as the probability density for the particle at time $t_{0}$ to be at position $\vec{x}$. Integrating over space and polarizing, we obtain the scalar product (1.2.2), which we also denote by

$$
(\psi \mid \phi)_{t_{0}}=2 \pi \int_{\mathbb{R}^{3}} \overline{\psi\left(t_{0}, \vec{y}\right)} \gamma^{0} \phi\left(t_{0}, \vec{y}\right) d^{3} y .
$$

It follows from current conservation that for any solutions $\psi, \phi$ of the Dirac equation, this scalar product is independent of the choice of $t_{0}$. This is the case even in the presence of an external potential (2.1.5), provided that the potential is symmetric with respect to the inner product on the spinors (1.2.18), i.e.

$$
\prec \psi|\mathcal{B} \phi \succ=\prec \mathcal{B} \psi| \phi \succ
$$

(see Exercise 2.5). Since the kernel of the fermionic projector is a solution of the Dirac equation, one is led to evaluating the integral in $\overline{2.1 .19}$ for $\phi(y)=P(y, z)$ and $\overline{\psi(y)}=$ $P(x, y)$. In the vacuum, the resulting integral can be computed, giving a simple result.

Lemma 2.1.1. For any $t \in \mathbb{R}$, there is the distributional relation

$$
2 \pi \int_{\mathbb{R}^{3}} P_{m}^{\mathrm{vac}}(x,(t, \vec{y})) \gamma^{0} P_{m}^{\mathrm{vac}}((t, \vec{y}), z) d^{3} y=-P_{m}^{\mathrm{vac}}(x, z) .
$$


Proof. The identity follows by a straightforward computation, which was already given in the proof of Lemma 1.2 .8 (see 1.2 .24 and the computation thereafter).

We refer to 2.1.21) as the spatial normalization of the fermionic projector. It has the advantage that it is well-defined even for fixed $m$. Moreover, the normalization method is closely related to the probabilistic interpretation of the Dirac equation.

In the following sections 2.1 .4 2.1.6, we shall carry out the construction of the fermionic projector describing the completely filled Dirac sea in the presence of the external potential $\mathcal{B}$. Our method will make essential use of generalizations of the underlying causal structure (as is apparent in 2.1.6) and 2.1.12), of the relation between $k_{m}$ and $p_{m}$ as expressed by (2.1.18), and of the spatial normalization (2.1.21). Finally, in 82.1 .7 we shall extend the construction to allow for particles and anti-particles.

2.1.4. The Perturbation Expansion of the Causal Green's Functions. Using the causal support property, the advanced and retarded Green's functions $\tilde{s}_{m}^{\vee}$ and $\tilde{s}_{m}^{\wedge}$ are uniquely defined even in the presence of an external potential (2.1.5). They can be constructed non-perturbatively using the theory of symmetric hyperbolic systems (see $[\mathbf{J}]$ or [FKT, Chapter 5]). For our purposes, it is sufficient to work out their perturbation expansions: The retarded Green's function is characterized by the conditions

$$
(i \not \partial+\mathcal{B}-m) \tilde{s}_{m}^{\wedge}(x, y)=\delta^{4}(x-y) \quad \text { and } \quad \operatorname{supp} \tilde{s}_{m}^{\wedge}(x, .) \subset J_{x}^{\wedge} .
$$

Employing the perturbation ansatz

$$
\tilde{s}_{m}^{\wedge}=\sum_{n=0}^{\infty} s_{(n)}^{\wedge} \quad \text { with } \quad s_{(0)}^{\wedge}=s_{m}^{\wedge}
$$

(where the subscript $(n)$ denotes the order of perturbation theory), we obtain for $n=$ $1,2, \ldots$ the inductive conditions

$$
(i \not \partial-m) s_{(n)}^{\wedge}=-\mathcal{B} s_{(n-1)}^{\wedge} \quad \text { and } \quad \operatorname{supp} \tilde{s}_{(n)}^{\wedge}(x, .) \subset J_{x}^{\wedge} .
$$

Using the defining property of the Green's function 2.1.11), one sees that the left equation in 2.1.22 can be solved in the case $n=1$ by

$$
s_{(1)}^{\wedge}=-s_{m} \mathcal{B} s_{m}^{\wedge},
$$

where the operator product is defined as follows,

$$
\left(s_{m} \mathcal{B} s_{m}^{\wedge}\right)(x, y):=\int d^{4} z s_{m}(x, z) \mathcal{B}(z) s_{m}^{\wedge}(z, y)
$$

(the analytic justification of this and all other operator products in this section will be given in Lemma 2.1.2 below). The operator $s_{m}$ in (2.1.23) is any Green's function (like the advanced, retarded or the symmetric Green's function). In order to determine which Green's function to choose, we evaluate the condition on the right side of (2.1.22). Namely, if we choose $s_{m}$ in 2.1.23 again as the retarded Green's function, then the integral in 2.1.24 vanishes if $x$ lies in the past of $y$ because in this case the supports of the distributions $s_{m}^{\wedge}(x,$.$) and s_{m}^{\wedge}(., y)$ do not intersect. This leads us to setting

$$
s_{(1)}^{\wedge}=-s_{m}^{\wedge} \mathcal{B} s_{m}^{\wedge} .
$$

Now we can evaluate 2.1 .22 inductively to obtain

$$
s_{(n)}^{\wedge}=-s_{m}^{\wedge} \mathcal{B} s_{(n-1)}^{\wedge}=\left(-s_{m}^{\wedge} \mathcal{B}\right)^{n} s_{m}^{\wedge} .
$$


Proceeding similarly for the advanced Green's function, we obtain the unique perturbation series

$$
\tilde{s}_{m}^{\vee}=\sum_{n=0}^{\infty}\left(-s_{m}^{\vee} \mathcal{B}\right)^{n} s_{m}^{\vee}, \quad \tilde{s}_{m}^{\wedge}=\sum_{n=0}^{\infty}\left(-s_{m}^{\wedge} \mathcal{B}\right)^{n} s_{m}^{\wedge} .
$$

Having derived a perturbation series for the causal Green's functions, we can also define the causal fundamental solution in generalization of 2.1.14 by

$$
\tilde{k}_{m}:=\frac{1}{2 \pi i}\left(\tilde{s}_{m}^{\vee}-\tilde{s}_{m}^{\wedge}\right)
$$

We now specify a class of potentials for which all the operator products appearing here and later in this book are all well-defined in the distributional sense:

Lemma 2.1.2. Let $\left(C_{j}\right), 0 \leq j \leq n$, be a choice of operators $C_{j} \in\left\{k_{m}, p_{m}, s_{m}^{\vee}, s_{m}^{\wedge}\right\}$ (and $p_{m}, k_{m}$ according to 2.1.7) and (2.1.8)). If the external potential $\mathcal{B}$ is smooth and decays so fast at infinity that the functions $\mathcal{B}(x), x^{i} \mathcal{B}(x)$, and $x^{i} x^{j} \mathcal{B}(x)$ are integrable, then the operator product

$$
\left(C_{n} \mathcal{B} C_{n-1} \mathcal{B} \cdots \mathcal{B} C_{0}\right)(x, y)
$$

is a well-defined tempered distribution on $\mathbb{R}^{4} \times \mathbb{R}^{4}$.

Proof. Calculating the Fourier transform of (2.1.27) gives the formal expression

$$
\begin{aligned}
& M\left(q_{2}, q_{1}\right):=\int \frac{d^{4} p_{1}}{(2 \pi)^{4}} \cdots \int \frac{d^{4} p_{n-1}}{(2 \pi)^{4}} C_{n}\left(q_{2}\right) \hat{\mathcal{B}}\left(q_{2}-p_{n-1}\right) \\
& \quad \times C_{n-1}\left(p_{n-1}\right) \hat{\mathcal{B}}\left(p_{n-1}-p_{n-2}\right) \cdots C_{1}\left(p_{1}\right) \hat{\mathcal{B}}\left(p_{1}-q_{1}\right) C_{0}\left(q_{1}\right),
\end{aligned}
$$

where we consider the $C_{j}$ as multiplication operators in momentum space and where $\hat{\mathcal{B}}$ denotes the Fourier transform of the function $\mathcal{B}$ (it is more convenient to work in momentum space because the operators $C_{j}$ are then diagonal). We will show that $M\left(q_{2}, q_{1}\right)$ is a well-defined tempered distribution; the Lemma then immediately follows by transforming back to position space.

The assumptions on $\mathcal{B}$ yield that $\hat{\mathcal{B}}$ is $C^{2}$ and has rapid decay at infinity, i.e.

$$
\sup _{q \in \mathbb{R}^{4},|\kappa| \leq 2}\left|q^{i_{1}} \cdots q^{i_{n}} \partial_{\kappa} \hat{\mathcal{B}}(q)\right|<\infty
$$

for all $n$, all tensor indices $i_{1}, \ldots, i_{n}$ and all multi-indices $\kappa$ (with $\kappa=\left(\kappa^{1}, \ldots, \kappa^{q}\right)$, $|\kappa|:=q)$. As is verified explicitly in momentum space, the distributions $k_{m}, p_{m}$ and $s_{m}$ are bounded in the Schwartz norms of the test functions involving derivatives of only first order. More precisely,

$$
|C(f)| \leq \text { const }\|f\|_{4,1} \quad \text { with } \quad C=k_{m}, p_{m} \text { or } s_{m} \text { and } f \in \mathcal{S}\left(\mathbb{R}^{4}, \mathbb{C}^{4}\right),
$$

where $\mathcal{S}\left(\mathbb{R}^{4}, \mathbb{C}^{4}\right)$ is the Schwartz space, and the Schwartz norms are defined as usual by

$$
\|f\|_{p, q}=\max _{|I| \leq p,|J| \leq q} \sup _{x \in \mathbb{R}^{4}}\left|x^{I} \partial_{J} f(x)\right|
$$

(for basics on the Schwartz space and distributions see for example $[\mathbf{F r}]$ ). As a consequence, we can apply the corresponding operators even to functions with rapid decay which are only $C^{1}$. Furthermore, we can form the convolution of such functions with $C$; this gives continuous functions (which will no longer have rapid decay, however). Since $C$ involves first derivatives, a convolution decreases the order of differentiability of the function by one. 
We consider the combination of multiplication and convolution

$$
F\left(p_{2}\right):=\int \frac{d^{4} p_{1}}{(2 \pi)^{4}} f\left(p_{2}-p_{1}\right) C\left(p_{1}\right) g\left(p_{1}\right),
$$

where we assume that $f \in C^{2}$ has rapid decay and $g \in C^{1}$ is bounded together with its first derivatives, $\|g\|_{0,1}<\infty$. For any fixed $p_{2}$, the integral in 2.1 .29 is well-defined and finite because $f\left(p_{2}-.\right) g($.$) is C^{1}$ and has rapid decay. The resulting function $F$ is $C^{1}$ and bounded together with its first derivatives, more precisely

$$
\|F\|_{0,1} \leq \text { const }\|f\|_{4,2}\|g\|_{0,1} \text {. }
$$

After these preparations, we can estimate the integrals in 2.1 .28 from the right to the left: We choose two test functions $f, g \in \mathcal{S}\left(\mathbb{R}^{4}, \mathbb{C}^{4}\right)$ and introduce the functions

$$
\begin{aligned}
& F_{1}\left(p_{1}\right)=\int \frac{d^{4} q_{2}}{(2 \pi)^{4}} \hat{\mathcal{B}}\left(p_{1}-q_{1}\right) C_{0}\left(q_{1}\right) g\left(q_{1}\right) \\
& F_{j}\left(p_{j}\right)=\int \frac{d^{4} p_{j-1}}{(2 \pi)^{4}} \hat{\mathcal{B}}\left(p_{j}-p_{j-1}\right) C_{j-1}\left(p_{j-1}\right) F_{j-1}\left(p_{j-1}\right), \quad 1<j \leq n .
\end{aligned}
$$

The integral (2.1.31) is of the form $(2.1 .29)$ and satisfies the above assumptions on the integrand. Using the bound 2.1.30), we can proceed inductively in 2.1 .32 . Finally, we perform the $q_{2}$-integration,

$$
M(f, g)=\int \frac{d^{4} q_{2}}{(2 \pi)^{4}} f\left(q_{2}\right) C_{n}\left(q_{2}\right) F_{n}\left(q_{2}\right) .
$$

We conclude that $M$ is a linear functional on $\mathcal{S}\left(\mathbb{R}^{4}, \mathbb{C}^{4}\right) \times \mathcal{S}\left(\mathbb{R}^{4}, \mathbb{C}^{4}\right)$, which is bounded in the Schwartz norm $\|\cdot\|_{4,1}$ of the test functions.

We remark that the assumptions in this lemma are stronger than what is needed for the operator products in 2.1.25) and 2.1.26 to be well-defined: First of all, the smoothness assumption for $\mathcal{B}$ is unnecessarily strong; for example, it would be sufficient to assume that $\mathcal{B}$ is twice differentiable. Moreover, using the causal structure, the contributions to the above perturbation expansions are well-defined even without the decay assumptions in Lemma 2.1.2. Namely, these perturbation expansions are all causal in the sense that for any given $x, y \in \mathcal{M}$, the distributions $\tilde{s}^{\vee}(x, y)$ and $\tilde{s}^{\wedge}(x, y)$ depend on the potential $\mathcal{B}$ only on in the so-called

$$
\text { causal diamond } \quad\left(J_{x}^{\vee} \cap J_{y}^{\wedge}\right) \cup\left(J_{x}^{\wedge} \cap J_{y}^{\vee}\right) .
$$

Since the causal diamond is a bounded region of space-time, we may modify $\mathcal{B}$ outside this bounded set to arrange the decay assumptions without changing the contributions to the above perturbation expansions.

The reason why we prefer to impose with the stronger assumptions in Lemma 2.1.2 is that they will be needed later on. Indeed, for the operator products appearing in the causal perturbation expansion of the Dirac sea, the decay assumptions in Lemma 2.1.2 will be required. Moreover, the smoothness of $\mathcal{B}$ will be needed for the light-cone expansion.

The summands of the above perturbation expansions 2.1 .25$)$ and $(2.1 .26)$ arise similarly in quantum field theory and are then depicted by Feynman diagrams (see Figure 2.1). Using the language of quantum field theory, we also refer to the summands of our perturbation expansions as Feynman diagrams. Then the result of the last lemma can be understood from the fact that in the presence of an external field one only encounters tree diagrams, which are all finite. 


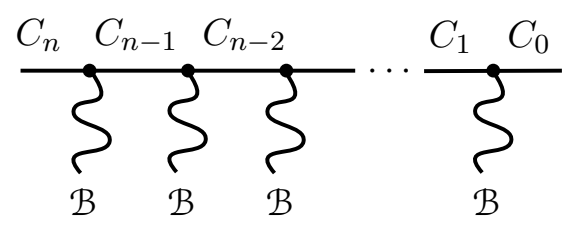

Figure 2.1. A Feynman tree diagram

2.1.5. Computation of Operator Products. We saw in 2.1.17) and 2.1.18 that operator products can be formed if the mass is considered as a variable parameter. We now develop this method more systematically. It is usually most convenient to work with the symmetric Green's function defined by

$$
s_{m}=\frac{1}{2}\left(s_{m}^{\vee}+s_{m}^{\wedge}\right) .
$$

Lemma 2.1.3. The following identities hold:

$$
\begin{aligned}
& p_{m} p_{m^{\prime}}=k_{m} k_{m^{\prime}}=\delta\left(m-m^{\prime}\right) p_{m} \\
& p_{m} k_{m^{\prime}}=k_{m} p_{m^{\prime}}=\delta\left(m-m^{\prime}\right) k_{m} \\
& p_{m} s_{m^{\prime}}=s_{m^{\prime}} p_{m}=\frac{\mathrm{PP}}{m-m^{\prime}} p_{m} \\
& k_{m} s_{m^{\prime}}=s_{m^{\prime}} k_{m}=\frac{\mathrm{PP}}{m-m^{\prime}} k_{m} \\
& s_{m} s_{m^{\prime}}=\frac{\mathrm{PP}}{m-m^{\prime}}\left(s_{m}-s_{m^{\prime}}\right)+\pi^{2} \delta\left(m-m^{\prime}\right) p_{m},
\end{aligned}
$$

where $\mathrm{PP}$ denotes the principal value defined in analogy to (1.2.27) alternatively by

$$
\begin{aligned}
\int_{-\infty}^{\infty} \frac{\mathrm{PP}}{m} \eta(m) d m & =\lim _{\nu \searrow 0}\left(\int_{-\infty}^{-\nu}+\int_{\nu}^{\infty}\right) \frac{\eta(m)}{m} d m \\
& =\lim _{\nu \searrow 0} \frac{1}{2} \sum_{ \pm} \int_{-\infty}^{\infty} \frac{\eta(m)}{m \pm i \nu} d m
\end{aligned}
$$

Proof. Calculating pointwise in momentum space, we obtain

$$
\begin{aligned}
p_{m}(q) p_{m^{\prime}}(q) & =(\not q+m) \delta\left(q^{2}-m^{2}\right)\left(\not q+m^{\prime}\right) \delta\left(q^{2}-m^{\prime 2}\right) \\
& =\delta\left(m^{2}-m^{\prime 2}\right) \delta\left(q^{2}-m^{2}\right)\left(q^{2}+\left(m+m^{\prime}\right) \not+m m^{\prime}\right) \\
& =\frac{1}{2 m} \delta\left(m-m^{\prime}\right) \delta\left(q^{2}-m^{2}\right)\left(m^{2}+\left(m+m^{\prime}\right) \not++m m^{\prime}\right) \\
& =\frac{1}{2 m} \delta\left(m-m^{\prime}\right) \delta\left(q^{2}-m^{2}\right) 2 m(m+\not q)=\delta\left(m-m^{\prime}\right) p_{m}(q) .
\end{aligned}
$$

This gives the first part of 2.1.35). The second part of this formula as well as formula (2.1.36) are obtained analogously. The formulas $(2.1 .37)$ and 2.1 .38 are obtained as 
follows:

$$
\begin{aligned}
2 & p_{m}(q) s_{m^{\prime}}(q)=\lim _{\nu \searrow 0} \delta\left(q^{2}-m^{2}\right)(q+m)\left(\frac{\not q+m^{\prime}}{q^{2}-m^{\prime 2}-i \nu q^{0}}+\frac{\not q+m^{\prime}}{q^{2}-m^{\prime 2}+i \nu q^{0}}\right) \\
= & \lim _{\nu \searrow 0} \delta\left(q^{2}-m^{2}\right)\left(q^{2}+\left(m+m^{\prime}\right) \not q+m m^{\prime}\right)\left(\frac{1}{q^{2}-m^{\prime 2}-i \nu q^{0}}+\frac{1}{q^{2}-m^{\prime 2}+i \nu q^{0}}\right) \\
= & \lim _{\nu \searrow 0} \delta\left(q^{2}-m^{2}\right)\left(m^{2}+\left(m+m^{\prime}\right) \not q+m m^{\prime}\right)\left(\frac{1}{m^{2}-m^{\prime 2}-i \nu q^{0}}+\frac{1}{m^{2}-m^{\prime 2}+i \nu q^{0}}\right) \\
= & \lim _{\nu \searrow 0} \delta\left(q^{2}-m^{2}\right)(q 1+m)\left(\frac{\left(m+m^{\prime}\right)}{\left(m+m^{\prime}\right)\left(m-m^{\prime}\right)-i \nu q^{0}}+\frac{\left(m+m^{\prime}\right)}{\left(m+m^{\prime}\right)\left(m-m^{\prime}\right)+i \nu q^{0}}\right) \\
= & 2 \frac{\mathrm{PP}}{m-m^{\prime}} p_{m}(q) .
\end{aligned}
$$

The derivation of 2.1.39 is a bit more involved. Combining 2.1.14 and 2.1.34, we obtain

$$
s_{m}=s_{m}^{\vee}-i \pi k_{m}=s_{m}^{\wedge}+i \pi k_{m} .
$$

Thus we can express the product $s_{m}(q) s_{m^{\prime}}(q)$ in two ways, namely as

$$
\begin{aligned}
s_{m}(q) s_{m^{\prime}}(q)= & \left(s_{m}^{\vee}(q)-i \pi k_{m}(q)\right)\left(s_{m^{\prime}}^{\vee}(q)-i \pi k_{m^{\prime}}(q)\right) \\
= & s_{m}^{\vee}(q) s_{m^{\prime}}^{\vee}(q)-\pi^{2} \delta\left(m-m^{\prime}\right) p_{m}(q) \\
& \quad-i \pi \lim _{\nu \searrow 0}\left(k_{m^{\prime}}(q) \frac{1}{m^{\prime}-m-i \nu q^{0}}+k_{m}(q) \frac{1}{m-m^{\prime}-i \nu q^{0}}\right),
\end{aligned}
$$

or alternatively as

$$
\begin{aligned}
& s_{m}(q) s_{m^{\prime}}(q)=\left(s_{m}^{\wedge}(q)+i \pi k_{m}(q)\right)\left(s_{m^{\prime}}^{\wedge}(q)+i \pi k_{m^{\prime}}(q)\right) \\
& =s_{m}^{\wedge}(q) s_{m^{\prime}}^{\wedge}(q)-\pi^{2} \delta\left(m-m^{\prime}\right) p_{m}(q) \\
& \quad+i \pi \lim _{\nu \searrow 0}\left(k_{m^{\prime}}(q) \frac{1}{m^{\prime}-m+i \nu q^{0}}+k_{m}(q) \frac{1}{m-m^{\prime}+i \nu q^{0}}\right) .
\end{aligned}
$$

Adding these two formulas yields

$$
\begin{aligned}
2 & s_{m}(q) s_{m^{\prime}}(q)-\left(s_{m}^{\vee}(q) s_{m^{\prime}}^{\vee}(q)+s_{m}^{\wedge}(q) s_{m^{\prime}}^{\wedge}(q)\right)+2 \pi^{2} \delta\left(m-m^{\prime}\right) p_{m}(q) \\
= & i \pi \lim _{\nu \searrow 0} k_{m^{\prime}}(q)\left(\frac{1}{m^{\prime}-m+i \nu q^{0}}-\frac{1}{m^{\prime}-m-i \nu q^{0}}\right) \\
& \quad+i \pi \lim _{\nu \searrow 0} k_{m}(q)\left(\frac{1}{m-m^{\prime}+i \nu q^{0}}-\frac{1}{m-m^{\prime}-i \nu q^{0}}\right) \\
& \stackrel{(*)}{=} i \pi k_{m^{\prime}}(q) \epsilon\left(-q^{0}\right) 2 \pi i \delta\left(m^{\prime}-m\right)+i \pi k_{m}(q) \epsilon\left(-q^{0}\right) 2 \pi i \delta\left(m-m^{\prime}\right) \\
= & -2 \pi^{2} \delta\left(m^{\prime}-m\right)\left(-p_{m^{\prime}}(q)\right)-2 \pi^{2} \delta\left(m-m^{\prime}\right)\left(-p_{m}(q)\right),
\end{aligned}
$$

where in $(*)$ we applied 1.2 .33$)$, and in the last line we used the definitions of $p_{m}$ and $k_{m}$. We thus obtain

$$
s_{m} s_{m^{\prime}}=\frac{1}{2}\left(s_{m}^{\vee} s_{m^{\prime}}^{\vee}+s_{m}^{\wedge} s_{m^{\prime}}^{\wedge}\right)+\pi^{2} \delta\left(m-m^{\prime}\right) p_{m} .
$$

It remains to derive the relations

$$
s_{m}^{\vee} s_{m^{\prime}}^{\vee}=\frac{\mathrm{PP}}{m-m^{\prime}}\left(s_{m}^{\vee}-s_{m^{\prime}}^{\vee}\right) \quad \text { and } \quad s_{m}^{\wedge} s_{m^{\prime}}^{\wedge}=\frac{\mathrm{PP}}{m-m^{\prime}}\left(s_{m}^{\wedge}-s_{m^{\prime}}^{\wedge}\right),
$$


which can be regarded as "resolvent identities" for the causal Green's functions. It suffices to consider the case of the advanced Green's function. Clearly, the operators on the right side of (2.1.43) satisfy the support condition $\operatorname{supp}\left(\left(s_{m}^{\vee}-s_{m^{\prime}}^{\vee}\right)(x,).\right) \subset J_{x}^{\vee}$, and from

$$
s_{m}^{\vee} s_{m^{\prime}}^{\vee}(x, y)=\int d^{4} z s_{m}^{\vee}(x, z) s_{m^{\prime}}^{\vee}(z, y)
$$

we see that the operators on the left side of (2.1.43) satisfy this support condition as well. Moreover, the calculations

$$
\left(i \not \partial_{x}-m\right) s_{m}^{\vee} s_{m^{\prime}}^{\vee}(x, y)=s_{m^{\prime}}^{\vee}(x, y)
$$

and

$$
\begin{aligned}
\left(i \not \partial_{x}\right. & -m) \frac{\mathrm{PP}}{m-m^{\prime}}\left(s_{m}^{\vee}-s_{m^{\prime}}^{\vee}\right)(x, y) \\
& =\frac{\mathrm{PP}}{m-m^{\prime}}\left(\delta(x-y)-\left(m^{\prime}-m\right) s_{m^{\prime}}^{\vee}(x, y)-\delta(x-y)\right)=s_{m^{\prime}}^{\vee}(x, y)
\end{aligned}
$$

show that both sides of (2.1.43) satisfy the same inhomogeneous Dirac equation. Hence their difference is a distributional solution of the homogeneous Dirac equation which vanishes outside $J_{x}^{\vee}$. The uniqueness of the solution of the Cauchy problem for hyperbolic PDEs yields that this difference vanishes identically. This proves (2.1.43) and thus concludes the proof of (2.1.39).

In the above operator products we get contributions of two different forms: those involving a factor $\delta\left(m-m^{\prime}\right)$ and those involving the principal value of $1 /\left(m-m^{\prime}\right)$. In order to simplify the structure of the multiplication rules, it is useful to get rid of the principal values by restricting attention to combinations in which all principal values drop out in telescopic sums. To this end, we introduce the series of operator products

$$
b_{m}^{<}=\sum_{n=0}^{\infty}\left(-s_{m} \mathcal{B}\right)^{n}, \quad b_{m}=\sum_{n=0}^{\infty}\left(-\mathcal{B} s_{m}\right)^{n} \mathcal{B}, \quad b_{m}^{>}=\sum_{n=0}^{\infty}\left(-\mathcal{B} s_{m}\right)^{n}
$$

Corollary 2.1.4. Let $C \in\left\{p_{m}, k_{m}\right\}$ and $C^{\prime} \in\left\{p_{m^{\prime}}, k_{m^{\prime}}\right\}$ as well as $b_{m}^{<}, b_{m}^{>}$as in 2.1.44. Then the following calculation rule holds:

$$
C b_{m}^{>} b_{m^{\prime}}^{<} C^{\prime}=C C^{\prime}+\delta\left(m-m^{\prime}\right) \pi^{2} C b_{m} p_{m} b_{m} C^{\prime} .
$$

Proof. Using the calculation rules of the previous lemma, we obtain

$$
\begin{gathered}
C\left(\sum_{l=0}^{1}\left(\mathcal{B} s_{m}\right)^{l}\left(s_{m^{\prime}} \mathcal{B}\right)^{n-l}\right) C^{\prime}=C s_{m^{\prime}} \mathcal{B} C^{\prime}+C \mathcal{B} s_{m} C^{\prime} \\
=\frac{\mathrm{PP}}{m-m^{\prime}}\left(C \mathcal{B} C^{\prime}-C \mathcal{B} C^{\prime}\right)=0 .
\end{gathered}
$$


The same method also applies to higher order. We again get a telescopic sum, but the last summand in (2.1.39) gives additional contributions. More precisely, for any $n \geq 2$,

$$
\begin{aligned}
C & \left(\sum_{l=0}^{n}\left(\mathcal{B} s_{m}\right)^{l}\left(s_{m^{\prime}} \mathcal{B}\right)^{n-l}\right) C^{\prime} \\
= & C\left(\mathcal{B} s_{m}\right)^{n} C^{\prime}+C\left(s_{m^{\prime}} \mathcal{B}\right)^{n} C^{\prime}+C\left[\sum_{l=1}^{n-1}\left(\mathcal{B} s_{m}\right)^{l}\left(s_{m^{\prime}} \mathcal{B}\right)^{n-l}\right] C^{\prime} \\
= & C \frac{\mathrm{PP}}{m-m^{\prime}}\left[-\left(\mathcal{B} s_{m}\right)^{n-1} \mathcal{B}+\mathcal{B}\left(s_{m^{\prime}} \mathcal{B}\right)^{n-1}\right] C^{\prime} \\
& +C \sum_{l=1}^{n-1}\left(\mathcal{B} s_{m}\right)^{l-1} \mathcal{B}\left(\frac{\mathrm{PP}}{m-m^{\prime}}\left(s_{m}-s_{m^{\prime}}\right)+\delta\left(m-m^{\prime}\right) \pi^{2} p_{m}\right) \mathcal{B}\left(s_{m^{\prime}} \mathcal{B}\right)^{n-l-1} C^{\prime} \\
= & \frac{\mathrm{PP}}{m-m^{\prime}} C\left[-\left(\mathcal{B} s_{m}\right)^{n-1} \mathcal{B}+\mathcal{B}\left(s_{m^{\prime}} \mathcal{B}\right)^{n-1}\right] C^{\prime} \\
& +\frac{\mathrm{PP}}{m-m^{\prime}} C\left(\sum_{l=1}^{n-1}\left(\mathcal{B} s_{m}\right)^{l}\left(\mathcal{B} s_{m^{\prime}}\right)^{n-l-1} \mathcal{B}-\sum_{l=0}^{n-2}\left(\mathcal{B} s_{m}\right)^{l}\left(\mathcal{B} s_{m^{\prime}}\right)^{n-l-1} \mathcal{B}\right) C^{\prime} \\
& +\delta\left(m-m^{\prime}\right) \pi^{2} C \sum_{l=1}^{n-1}\left(\mathcal{B} s_{m}\right)^{l-1} \mathcal{B} p_{m} \mathcal{B}\left(s_{m^{\prime}} \mathcal{B}\right)^{n-l-1} C^{\prime} \\
= & \delta\left(m-m^{\prime}\right) \pi^{2} C \sum_{l=1}^{n-1}\left(\mathcal{B} s_{m}\right)^{l-1} \mathcal{B} p_{m} \mathcal{B}\left(s_{m^{\prime}} \mathcal{B}\right)^{n-l-1} C^{\prime} \\
= & \delta\left(m-m^{\prime}\right) \pi^{2} C \sum_{l=0}^{n-2}\left(\mathcal{B} s_{m}\right)^{l} \mathcal{B} p_{m} \mathcal{B}\left(s_{m^{\prime}} \mathcal{B}\right)^{n-l-2} C^{\prime} .
\end{aligned}
$$

Thus, performing an index shift, we obtain

$$
\begin{aligned}
C b_{m}^{>} & b_{m^{\prime}}^{<} C^{\prime}=C \sum_{n=0}^{\infty}\left(-\mathcal{B} s_{m}\right)^{n} \sum_{n^{\prime}=0}^{\infty}\left(-s_{m^{\prime}} \mathcal{B}\right)^{n^{\prime}} C^{\prime} \\
= & C \sum_{n=0}^{\infty} \sum_{l=0}^{n}\left(-\mathcal{B} s_{m}\right)^{l}\left(-s_{m^{\prime}} \mathcal{B}\right)^{n-l} C^{\prime} \\
= & C C^{\prime}+\delta\left(m-m^{\prime}\right) \pi^{2} \sum_{n=2}^{\infty}(-1)^{n} C\left(\sum_{l=0}^{n-2}\left(\mathcal{B} s_{m}\right)^{l} \mathcal{B} p_{m} \mathcal{B}\left(s_{m^{\prime}} \mathcal{B}\right)^{n-l-2}\right) C^{\prime} \\
= & C C^{\prime}+\delta\left(m-m^{\prime}\right) \pi^{2} \sum_{n=0}^{\infty}(-1)^{n} C\left(\sum_{l=0}^{n}\left(\mathcal{B} s_{m}\right)^{l} \mathcal{B} p_{m} \mathcal{B}\left(s_{m^{\prime}} \mathcal{B}\right)^{n-l}\right) C^{\prime} \\
= & C C^{\prime}+\delta\left(m-m^{\prime}\right) \pi^{2} C b_{m} p_{m} b_{m} C^{\prime} .
\end{aligned}
$$

This concludes the proof.

In what follows, we rewrite all operator products in terms of $\mathcal{B}$ and $p_{m}, k_{m}$ as well as the above combinations $b_{m}^{<}, b_{m}$ and $b_{m}^{>}$. In order to explain how this can be done, we rewrite the perturbation expansion of $\tilde{k}_{m}$ in this form. 
Proposition 2.1.5. The perturbation expansion of $\tilde{k}_{m}$ as given by 2.1 .26 can be written as

$$
\tilde{k}_{m}=\sum_{\beta=0}^{\infty}(-i \pi)^{2 \beta} b_{m}^{<} k_{m}\left(b_{m} k_{m}\right)^{2 \beta} b_{m}^{>},
$$

where the factors $b_{m}^{<}, b_{m}$ and $b_{m}^{>}$are again the operator products in (2.1.44),

Proof. An explicit calculation shows that

$$
(i \not \partial+\mathcal{B}-m) b_{m}^{<}=0 \text {. }
$$

As all operator products in (2.1.46) have a factor $b_{m}^{<}$at the left, the series in 2.1 .46 is a solution of the Dirac equation.

From 2.1.14 and (2.1.34), we have

$$
s_{m}^{\vee}=s_{m}+i \pi k_{m}, \quad s_{m}^{\wedge}=s_{m}-i \pi k_{m} .
$$

We substitute the series 2.1.25) into (2.1.26), insert (2.1.47) and expand. A reordering of the resulting sum gives the claim. The details of the reordering process can be found in $[$ F3.

2.1.6. The Causal Perturbation Expansion. We follow the constructions in FT2. Recall that, in the presence of an external potential $\mathcal{B}$, the perturbation expansion of the advanced and retarded Green's functions is unique by causality 2.1.25). Moreover, Proposition 2.1.5 gave us a unique perturbation expansion of the causal fundamental solution (2.1.46).

In the following constructions, we need to multiply the operator products in 2.1.46). These products have a mathematical meaning as distributions in the involved mass parameters. Namely, according to Lemma 2.1.3 and Corollary 2.1.4.

$$
\begin{aligned}
p_{m} p_{m^{\prime}} & =k_{m} k_{m^{\prime}}=\delta\left(m-m^{\prime}\right) p_{m} \\
p_{m} k_{m^{\prime}} & =k_{m} p_{m^{\prime}}=\delta\left(m-m^{\prime}\right) k_{m} \\
k_{m} b_{m}^{>} b_{m^{\prime}}^{<} k_{m^{\prime}} & =\delta\left(m-m^{\prime}\right)\left(p_{m}+\pi^{2} k_{m} b_{m} p_{m} b_{m} k_{m}\right) .
\end{aligned}
$$

Since these formulas all involve a common prefactor $\delta\left(m-m^{\prime}\right)$, we can introduce a convenient notation by leaving out this factor and omitting the mass indices. For clarity, we denote this short notation with a dot, i.e. symbolically

$$
A \cdot B=C \quad \text { stands for } \quad A_{m} B_{m^{\prime}}=\delta\left(m-m^{\prime}\right) C_{m} .
$$

With this short notation, the above multiplication rules can be written in the compact form

$$
p \cdot p=k \cdot k=p, \quad p \cdot k=k \cdot p=k, \quad k b^{>} \cdot b^{<} k=p+\pi^{2} k b p b k .
$$

Writing (2.1.46) as

$$
\tilde{k}=\sum_{\beta=0}^{\infty}(-i \pi)^{2 \beta} b^{<} k(b k)^{2 \beta} b^{>},
$$

powers of the operator $\tilde{k}$ with the product (2.1.51) are well-defined using the multiplication rules (2.1.52). This makes it possible to develop a spectral calculus for $\tilde{k}$. In particular, in [FG1] the operator $P^{\text {sea }}$ is constructed as the projection operator on the negative spectral subspace of $\tilde{k}$. We now give an equivalent construction using contour 
integrals, which gives a more systematic procedure for computing all the contributions to the expansion (for basics on the resolvent and contour integrals see Exercise 2.6.

We introduce the resolvent by

$$
\tilde{R}_{\lambda}=(\tilde{k}-\lambda)^{-1}
$$

Writing $\tilde{k}$ as

$$
\tilde{k}=k+\Delta k,
$$

(where $k$ is the corresponding distribution in the vacuum), the resolvent $\tilde{R}_{\lambda}$ can be written as a Neumann series,

$$
\tilde{R}_{\lambda}=(k-\lambda+\Delta k)^{-1}=\left(1+R_{\lambda} \cdot \Delta k\right)^{-1} \cdot R_{\lambda}=\sum_{n=0}^{\infty}\left(-R_{\lambda} \cdot \Delta k\right)^{n} \cdot R_{\lambda} .
$$

The multiplication rules 2.1.52 imply that $p$ is idempotent and thus has the eigenvalues 1 and 0 . Since the operator $k$ commutes with $p$ and its square equals $p$, it has the eigenvalues \pm 1 and 0 . A short computation shows that the corresponding spectral projection operators are $(p \pm k) / 2$ and $\mathbb{1}-p$, respectively. Hence we can write the unperturbed resolvent $R_{\lambda}:=(k-\lambda)^{-1}$ as

$$
R_{\lambda}=\frac{p+k}{2}\left(\frac{1}{1-\lambda}\right)+\frac{p-k}{2}\left(\frac{1}{-1-\lambda}\right)-\frac{\mathbb{1}-p}{\lambda} .
$$

Using this formula in 2.1.56), to every order in perturbation theory we obtain a meromorphic function in $\lambda$ having poles only at $\lambda=0$ and $\lambda= \pm 1$.

We now use contour integral methods to develop a spectral calculus. To this end, we choose a contour $\Gamma_{-}$which encloses the point -1 in counter-clockwise direction and does not enclose the points 1 and 0 . Similarly, $\Gamma_{+}$is a contour which encloses the point +1 in counter-clockwise direction and does not enclose the points -1 and 0 . Moreover, we let $f$ be a holomorphic function defined on an open neighborhood of the points \pm 1 . We define $f(\tilde{k})$ as the contour integral

$$
f(\tilde{k}):=-\frac{1}{2 \pi i} \oint_{\Gamma_{+} \cup \Gamma_{-}} f(\lambda) \tilde{R}_{\lambda} d \lambda .
$$

Using 2.1.56 together with the fact that to every order in perturbation theory, the integrand is a meromorphic function in $\lambda$ having poles only at $\lambda=0$ and $\lambda= \pm 1$, one sees that the operator $f(\tilde{k})$ is well-defined to every order in perturbation theory and is independent of the choice of the contours $\Gamma_{+}$and $\Gamma_{-}$.

THEOREM 2.1.6. (functional calculus) For any functions $f, g$ which are holomorphic in discs around \pm 1 which contain the contours $\Gamma_{ \pm}$,

$$
\begin{aligned}
(i \not \partial+\mathcal{B}-m) f(\tilde{k}) & =0 \\
f(\tilde{k}) \cdot g(\tilde{k}) & =(f g)(\tilde{k}) .
\end{aligned}
$$

Proof. Since the operator $\tilde{k}$ maps to solutions of the Dirac equation, we know that

$$
(i \not \partial+\mathcal{B}-m) \tilde{R}_{\lambda}=(i \not \partial+\mathcal{B}-m)\left(-\lambda^{-1}\right) .
$$

Taking the contour integral 2.1.58) gives 2.1.59).

The starting point for proving (2.1.60) is the resolvent identity

$$
\tilde{R}_{\lambda} \cdot \tilde{R}_{\lambda^{\prime}}=\frac{1}{\lambda-\lambda^{\prime}}\left(\tilde{R}_{\lambda}-\tilde{R}_{\lambda^{\prime}}\right) .
$$


We set $\Gamma=\Gamma_{+} \cup \Gamma_{-}$and denote the corresponding contour for $\lambda^{\prime}$ by $\Gamma^{\prime}$. Since the integral 2.1.58) is independent of the precise choice of the contour, we may choose

$$
\Gamma=\partial B_{\delta}(1) \cup \partial B_{\delta}(-1) \quad \text { and } \quad \Gamma^{\prime}=\partial B_{2 \delta}(1) \cup \partial B_{2 \delta}(-1)
$$

for sufficiently small $\delta<1 / 2$. Then $\Gamma$ does not enclose any point of $\Gamma^{\prime}$, implying that

$$
\oint_{\Gamma} \frac{f(\lambda)}{\lambda-\lambda^{\prime}} d \lambda=0 \quad \text { for all } \lambda^{\prime} \in \Gamma^{\prime}
$$

On the other hand, $\Gamma^{\prime}$ encloses every point of $\Gamma$, so that

$$
\oint_{\Gamma^{\prime}} f(\lambda) g\left(\lambda^{\prime}\right) \frac{\tilde{R}_{\lambda}}{\lambda-\lambda^{\prime}} d \lambda^{\prime}=-2 \pi i f(\lambda) g(\lambda) \tilde{R}_{\lambda} \quad \text { for all } \lambda \in \Gamma .
$$

Combining (2.1.61) with (2.1.62) and (2.1.63), we obtain

$$
\begin{aligned}
f(\tilde{k}) \cdot g(\tilde{k}) & =-\frac{1}{4 \pi^{2}} \oint_{\Gamma} f(\lambda) d \lambda \oint_{\Gamma^{\prime}} g\left(\lambda^{\prime}\right) d \lambda^{\prime} \frac{1}{\lambda-\lambda^{\prime}}\left(\tilde{R}_{\lambda}-\tilde{R}_{\lambda^{\prime}}\right) \\
& =-\frac{1}{2 \pi i} \oint_{\Gamma} f(\lambda) g(\lambda) \tilde{R}_{\lambda} d \lambda=(f g)(\tilde{k}) .
\end{aligned}
$$

This concludes the proof.

The fermionic projector $P^{\text {sea }}$ is obtained by choosing a specific function $f$, as we now explain. First, the desired splitting of the solution space of the Dirac equation into two subspaces (see $\$ 2.1 .2$ can now be obtained using the sign of the spectrum of $\tilde{k}$. More precisely, we choose $P^{\text {sea }}$ such that its image coincides with the negative spectral subspace of $\tilde{k}$. To this end, we choose a function $f$ which vanishes identically in a neighborhood of +1 . In a neighborhood of -1 , on the other hand, the form of $f$ is determined by the spatial normalization condition (see (2.1.21)). Namely, the correct definition is

$$
P^{\text {sea }}=-\frac{1}{2 \pi i} \oint_{\Gamma_{-}}(-\lambda) \tilde{R}_{\lambda} d \lambda
$$

as becomes clear in the next proposition.

Proposition 2.1.7. The expansion $P^{\text {sea }}$ has the properties

$$
\begin{aligned}
(i \not \partial+\mathcal{B}-m) P^{\text {sea }} & =0 \\
2 \pi \int_{\mathbb{R}^{3}} P^{\text {sea }}(x,(t, \vec{y})) \gamma^{0} P^{\text {sea }}((t, \vec{y}), z) d^{3} y & =-P^{\text {sea }}(x, z) .
\end{aligned}
$$

Moreover, $P^{\text {sea }}$ is symmetric

$$
\left(P^{\text {sea }}\right)^{*}=P^{\text {sea }},
$$

where the star denotes the adjoint with respect to the space-time inner product 1.5 .2 .

We note for clarity that for the kernel of the fermionic projector, the symmetry property 2.1.67) means that

$$
\left(P^{\text {sea }}(x, y)\right)^{*}=P^{\text {sea }}(y, x),
$$

where the star denotes the adjoint with respect to the spin scalar product (1.2.18).

In order to simplify the notation in the proof, we abbreviate the spatial integral in 2.1.66) by $\left.\right|_{t}$, i.e.

$$
\left(\left.A\right|_{t} B\right)(x, z):=2 \pi \int_{\mathbb{R}^{3}} A(x,(t, \vec{y})) \gamma^{0} B((t, \vec{y}), z) d^{3} y
$$

We begin with a preparatory lemma. 
Lemma 2.1.8. For any $t_{0} \in \mathbb{R}$, the distribution 2.1.26) has the property

$$
\left.\tilde{k}_{m}\right|_{t_{0}} \tilde{k}_{m}=\tilde{k}_{m}
$$

ProOF. Clearly, it suffices to prove the relation when evaluated by a test function $f$. Then $\tilde{\phi}:=\tilde{k}_{m}(f)$ is a smooth solution of the Dirac equation with spatially compact support. Therefore, it suffices to show that for any such solution,

$$
\tilde{\phi}(t, \vec{x})=2 \pi \int_{\mathbb{R}^{3}} \tilde{k}_{m}\left(t, \vec{x} ; t_{0}, \vec{y}\right) \gamma^{0} \tilde{\phi}_{0}(\vec{y}) d^{3} y .
$$

Since $\tilde{\phi}$ and $\tilde{k}_{m}$ satisfy the Dirac equation, it suffices to prove this equation in the case $t>$ $t_{0}$. In this case, the equation simplifies in view of 2.1.26 to

$$
\tilde{\phi}(x)=\left.i \int_{\mathbb{R}^{3}} \tilde{s}_{m}^{\wedge}(x, y) \gamma^{0} \tilde{\phi}_{0}(y)\right|_{y=\left(t_{0}, \vec{y}\right)} d^{3} y,
$$

where we set $x=(t, \vec{x})$. This identity is derived as follows: We choose a non-negative function $\eta \in C^{\infty}(\mathbb{R})$ with $\left.\eta\right|_{\left[t_{0}, t\right]} \equiv 1$ and $\eta_{\left(-\infty, t_{0}-1\right)} \equiv 0$. We also consider $\eta=\eta\left(x^{0}\right)$ as a function of the time variable in space-time. Then

$$
\left.\tilde{\phi}(x)=(\eta \tilde{\phi})(x)=\tilde{s}_{m}^{\wedge}((i \not \partial+\mathcal{B}-m)(\eta \tilde{\phi}))=\tilde{s}_{m}^{\wedge}\left(i \gamma^{0} \dot{\eta} \tilde{\phi}\right)\right),
$$

where we used the defining equation of the Green's function $\tilde{s}_{m}^{\wedge}\left(i \not \partial_{x}+\mathcal{B}-m\right)=\mathbb{1}$ together with the fact that $\tilde{\phi}$ is a solution of the Dirac equation. To conclude the proof, we choose a sequence $\eta_{l}$ such that the sequence of derivatives $\dot{\eta}_{l}$ converges as $l \rightarrow \infty$ in the distributional sense to the $\delta$-distribution $\delta_{t_{0}}$ supported at $t_{0}$. Then

$$
\begin{aligned}
\left.\tilde{s}_{m}^{\wedge}\left(i \gamma^{0} \dot{\eta} \tilde{\phi}\right)\right)(x) & =\int\left(\tilde{s}_{m}^{\wedge}(x, y)\left(i \gamma^{0} \dot{\eta}\left(y^{0}\right) \tilde{\phi}(y)\right)\right) d^{4} y \\
& \left.\rightarrow \int_{\mathbb{R}^{3}}\left(\tilde{s}_{m}^{\wedge}(x, y)\left(i \gamma^{0} \tilde{\phi}\right)\right)\right|_{y=\left(t_{0}, \vec{y}\right)} d^{3} y,
\end{aligned}
$$

giving the result.

An alternative, more computational proof of this lemma is sketched in Exercise 2.7.

Proof of Proposition 2.1.7. The Dirac equation 2.1.65 follows immediately from the identity (2.1.59). In order to prove 2.1.66), we integrate the relations

$$
\tilde{R}_{\lambda} \cdot(\tilde{k}-\lambda)=\mathbb{1}=(\tilde{k}-\lambda) \cdot \tilde{R}_{\lambda},
$$

to obtain

$$
\oint_{\Gamma_{-}} \tilde{R}_{\lambda} \cdot \tilde{k} d \lambda=\oint_{\Gamma_{-}} \tilde{R}_{\lambda} \lambda d \lambda=\oint_{\Gamma_{-}} \tilde{k} \tilde{R}_{\lambda} d \lambda
$$

As a consequence,

$$
\left.P^{\text {sea }}\right|_{t} P^{\text {sea }}=-\left.\frac{1}{4 \pi^{2}} \oint_{\Gamma_{-}} d \lambda \oint_{\Gamma_{-}^{\prime}} d \lambda^{\prime} \tilde{R}_{\lambda} \cdot \tilde{k}\right|_{t} \tilde{k} \cdot \tilde{R}_{\lambda^{\prime}}
$$

and applying Lemma 2.1 .8 for $t_{0}=t$ gives

$$
P^{\text {sea }}{ }_{t} P^{\text {sea }}=-\frac{1}{4 \pi^{2}} \oint_{\Gamma_{-}} d \lambda \oint_{\Gamma_{-}^{\prime}} d \lambda^{\prime} \tilde{R}_{\lambda} \cdot \tilde{k} \cdot \tilde{R}_{\lambda^{\prime}}=-\frac{1}{4 \pi^{2}} \oint_{\Gamma_{-}} \lambda d \lambda \oint_{\Gamma_{-}^{\prime}} d \lambda^{\prime} \tilde{R}_{\lambda} \cdot \tilde{R}_{\lambda^{\prime}}
$$


Now we can again apply 2.1.61) and 2.1.62) (which remains valid if the integrand involves an additional factor $\lambda$ ) as well as (2.1.63). We thus obtain

$$
\left.P^{\text {sea }}\right|_{t} P^{\text {sea }}=-\frac{1}{2 \pi i} \oint_{\Gamma_{-}} \lambda \tilde{R}_{\lambda} d \lambda=-P^{\text {sea }} .
$$

It remains to prove the symmetry property (2.1.67). The operators $p_{m}, k_{m}$ and $s_{m}$ are obviously symmetric (with respect to the inner product (1.5.2)). According to 2.1.46), the operator $\tilde{k}_{m}$ is also symmetric. Hence the resolvent $\tilde{R}_{\lambda}$ defined by 2.1.54 has the property

$$
\tilde{R}_{\lambda}^{*}=\tilde{R}_{\bar{\lambda}}
$$

This property implies that if we consider the Laurent expansion of $-\lambda \tilde{R}_{\lambda}$ around $\lambda=-1$,

$$
-\lambda \tilde{R}_{\lambda}=\frac{A_{-1}}{\lambda+1}+A_{0}+A_{1}(1+\lambda)+\cdots,
$$

then the operators $A_{-1}, A_{0}, \ldots$ are all symmetric with respect to $(1.5 .2)$. Since the contour integral (2.1.64) simply gives the residue $-A_{-1}$, we obtain (2.1.67). This concludes the proof.

In order to illustrate the above constructions, we now compute the first orders of the perturbation expansion (2.1.64). We first recall that in the computation rules $(2.1 .48)$ (2.1.50 no principal values occur. Using these rules in (2.1.56) and 2.1.64), one sees that also $P^{\text {sea }}$ involves no principal values. With this in mind, we may omit all principal values in the computation, even if we consider other operator products. In particular, we may write the computation rules of Lemma 2.1 .3 as

$$
p \cdot s=s \cdot p=k \cdot s=s \cdot k=0 \quad \text { and } \quad s \cdot s=\pi^{2} p .
$$

Combining 2.1.52 and 2.1.69) with 2.1.57), we obtain

$$
\begin{aligned}
& R_{\lambda} \cdot s=s \cdot R_{\lambda}=-\frac{1}{\lambda} s \\
& R_{\lambda} \cdot k=k \cdot R_{\lambda}=\frac{p+k}{2}\left(\frac{1}{1-\lambda}\right)-\frac{p-k}{2}\left(\frac{1}{-1-\lambda}\right)
\end{aligned}
$$

According to 2.1.53) and 2.1.55),

$$
\Delta k=-s \mathcal{B} k-k \mathcal{B} s+k \mathcal{B} s \mathcal{B} s+s \mathcal{B} k \mathcal{B} s+s \mathcal{B} s \mathcal{B} k-\pi^{2} k \mathcal{B} k \mathcal{B} k+\mathcal{O}\left(\mathcal{B}^{3}\right) .
$$

Hence, using 2.1.56,

$$
\begin{aligned}
\tilde{R}_{\lambda}= & \sum_{n=0}^{\infty}\left(-R_{\lambda} \cdot \Delta k\right)^{n} \cdot R_{\lambda}=R_{\lambda}-R_{\lambda} \cdot \Delta k \cdot R_{\lambda}+R_{\lambda} \cdot \Delta k \cdot R_{\lambda} \cdot \Delta k \cdot R_{\lambda}+\mathcal{O}\left(\mathcal{B}^{3}\right) \\
= & R_{\lambda}-R_{\lambda} \cdot\left(-s \mathcal{B} k-k \mathcal{B} s+k \mathcal{B} s \mathcal{B} s+s \mathcal{B} k \mathcal{B} s+s \mathcal{B} s \mathcal{B}-\pi^{2} k \mathcal{B} k \mathcal{B} k\right) \cdot R_{\lambda} \\
& +R_{\lambda} \cdot(-s \mathcal{B} k-k \mathcal{B} s) \cdot R_{\lambda} \cdot(-s \mathcal{B} k-k \mathcal{B} s) \cdot R_{\lambda}+\mathcal{O}\left(\mathcal{B}^{3}\right) .
\end{aligned}
$$

Using (2.1.57) and computing the contour integrals, one obtains to first order

$$
\begin{aligned}
P^{\text {sea }} & =-\lambda \frac{p-k}{2}-s \mathcal{B} \frac{p-k}{2}-\left.\frac{p-k}{2} \mathcal{B} s\right|_{\lambda=-1}+\mathcal{O}\left(\mathcal{B}^{2}\right) \\
& =\frac{p-k}{2}-s \mathcal{B} \frac{p-k}{2}-\frac{p-k}{2} \mathcal{B} s+\mathcal{O}\left(\mathcal{B}^{2}\right) .
\end{aligned}
$$


To second and higher orders, the resolvent $\tilde{R}_{\lambda}$ involves higher poles at $\lambda=-1$. This gives rise to derivatives of the factor $(-\lambda)$ in $(2.1 .64)$, having an influence of the combinatorics of the perturbation expansion (see Exercise 2.8). The reader interested in more details is referred to [FT2, Appendix A]. A few structural results of the causal perturbation expansion are treated in Exercises 2.9 2.11.

2.1.7. Introducing Particles and Anti-Particles. We shall now make the method of occupying particle and anti-particle states 2.1.4 precise in the presence of an external potential. To this end, it is useful to construct out of the kernel of the fermionic projector a projection operator on a Hilbert space, as we now explain. On the smooth solutions of the Dirac equation (2.1.5) with spatially compact support one can introduce the scalar product 11.2 .2 . Due to current conservation, this scalar product is again independent of the choice of $t$. Taking the completion, the solution space of the Dirac equation becomes a Hilbert space, which we denote by $\left(\mathcal{H}_{m},(. \mid .)_{m}\right)$. We now introduce on the Dirac wave functions at time $t$ the operator

$$
\begin{aligned}
& \Pi^{\text {sea }}: C_{0}^{\infty}\left(\mathcal{N}_{t}, S \mathscr{M}\right) \rightarrow C^{\infty}(\mathscr{M}, S \mathcal{M}), \\
& \left(\Pi^{\text {sea }} \psi\right)(x)=-2 \pi \int_{\mathbb{R}^{3}} P^{\text {sea }}(x,(t, \vec{y})) \gamma^{0} \psi(\vec{y}) d^{3} y,
\end{aligned}
$$

where $\mathcal{N}_{t}:=\{t\} \times \mathbb{R}^{3} \subset \mathcal{M}$ denotes the spatial hyperplane at time $t$. According to 2.1.65), this operator maps to the solutions of the Dirac equation. Moreover, the spatial normalization property 2.1 .66 implies that $\Pi^{\text {sea }}$ can be extended by continuity to a projection operator on $\mathcal{H}_{m}$, i.e.

$$
\Pi^{\text {sea }}: \mathcal{H}_{m} \rightarrow \mathcal{H}_{m} \quad \text { with } \quad\left(\Pi^{\text {sea }}\right)^{*}=\Pi_{\text {sea }}=\Pi_{\text {sea }}^{2}
$$

(where the star now denotes the adjoint with respect to the scalar product 1.2 .2 ; ; note that the last equation follows from the symmetry of the kernel (2.1.68)).

Now we can form another operator by adding and subtracting projection operators. More precisely, the operator

$$
\Pi:=\Pi^{\text {sea }}+\Pi_{\text {span }\left(\psi_{1}, \ldots, \psi_{n_{\mathrm{p}}}\right)}-\Pi_{\mathrm{span}\left(\phi_{1}, \ldots, \phi_{n_{\mathrm{a}}}\right)}
$$

(where $\Pi_{U}: \mathcal{H}_{m} \rightarrow \mathcal{H}_{m}$ denotes the orthogonal projection to a subspace $U \subset \mathcal{H}_{m}$ ) is again a projection operator, provided that the functions $\phi_{l}$ are vectors in $\mathcal{H}_{m}$ which lie in the image of $\Pi^{\text {sea }}$, whereas the vectors $\psi_{k} \in \mathcal{H}_{m}$ are in the orthogonal complement of the image of $\Pi^{\text {sea }}$. In order to comply with the usual normalization of wave functions in quantum mechanics, we orthonormalize these vectors as follows,

$$
\left(\psi_{k} \mid \psi_{k^{\prime}}\right)_{m}=2 \pi \delta_{k, k^{\prime}} \quad \text { and } \quad\left(\phi_{l} \mid \phi_{l^{\prime}}\right)_{m}=2 \pi \delta_{l, l^{\prime}}
$$

(we included the factor $2 \pi$ in order to account for the factor $2 \pi$ in $(1.2 .2)$ ). Then we can write $\Pi$ more explicitly as

$$
\Pi \psi:=\Pi^{\mathrm{sea}} \psi+\frac{1}{2 \pi} \sum_{k=1}^{n_{\mathrm{p}}} \psi_{k}\left(\psi_{k} \mid \psi\right)_{m}-\frac{1}{2 \pi} \sum_{l=1}^{n_{\mathrm{a}}} \phi_{l}\left(\phi_{l} \mid \psi\right)_{m} .
$$

This new projection operator can again be written in the form 2.1.71) with the distribution

$$
P(x, y)=P_{m}^{\mathrm{vac}}(x, y)-\frac{1}{2 \pi} \sum_{k=1}^{n_{\mathrm{p}}} \psi_{k}(x) \overline{\psi_{k}(y)}+\frac{1}{2 \pi} \sum_{l=1}^{n_{\mathrm{a}}} \phi_{l}(x) \overline{\phi_{l}(y)} .
$$


This relation gives a mathematical justification for 2.1.4 in the presence of an external potential. Note that the wave functions $\psi_{k}$ and $\phi_{l}$ must be solutions of the Dirac equation (2.1.5). Moreover, the $\phi_{l}$ must be in the image of $\Pi^{\text {sea }}$, whereas the $\psi_{k}$ must be in the orthogonal complement of the image of $\Pi^{\text {sea }}$. Finally, the normalization conditions 2.1.72 can be written as

$$
\int_{\mathbb{R}^{3}}\left(\overline{\psi_{k}} \gamma^{0} \psi_{k^{\prime}}\right)(t, \vec{x}) d^{3} x=\delta_{k, k^{\prime}}, \quad \int_{\mathbb{R}^{3}}\left(\overline{\phi_{l}} \gamma^{0} \phi_{l^{\prime}}\right)(t, \vec{x}) d^{3} x=\delta_{l, l^{\prime}}
$$

\subsection{The Light-Cone Expansion}

The light-cone expansion is a powerful tool for analyzing the fermionic projector in position space. We now outline the constructions and results as first given in [F5] and [F6]. Before beginning, we point out that the light-cone expansion is closely tied to the causal perturbation expansion. Namely, we shall see that the "causality" of the perturbation expansion (as built in via (2.1.26) into the resolvent (2.1.54) will become apparent in the light-cone expansion of $P(x, y)$ in the fact that all appearing line integrals will be bounded integrals along the line segment $\overline{x y}$. This specific feature of the light-cone expansion is of central importance for the analysis of the continuum limit.

2.2.1. Basic Definition. We first give the basic definition of the light-cone expansion and explain it afterwards.

Definition 2.2.1. A distribution $A(x, y)$ on $M \times M$ is of the order $\mathcal{O}\left((y-x)^{2 p}\right)$ for $p \in \mathbb{Z}$ if the product

$$
(y-x)^{-2 p} A(x, y)
$$

is a regular distribution (i.e. a locally integrable function). An expansion of the form

$$
A(x, y)=\sum_{j=g}^{\infty} A^{[j]}(x, y)
$$

with $g \in \mathbb{Z}$ is called light-cone expansion if the $A^{[j]}(x, y)$ are distributions of the order $\mathcal{O}\left((y-x)^{2 j}\right)$ and if $A$ is approximated by the partial sums in the sense that for all $p \geq g$,

$$
A(x, y)-\sum_{j=g}^{p} A^{[j]}(x, y) \quad \text { is of the order } \mathcal{O}\left((y-x)^{2 p+2}\right) \text {. }
$$

The parameter $g$ gives the leading order of the singularity of $A(x, y)$ on the light cone. We point out that we do not demand that the infinite series in (2.2.1) converges. Thus, similar to a formal Taylor series, the series in (2.2.1) is defined only via the approximation by the partial sums (2.2.2). The notion of the light-cone expansion is illustrated in Exercise 2.12.

As a simple example for a light-cone expansion, we consider the distribution $T_{m^{2}}(x, y)$ as introduced in (1.2.26) and analyzed in Lemma 1.2.9. Expanding the Bessel functions in 1.2.29) in a power series, one obtains (see OLBC, (10.2.2), (10.8.1) and (10.25.2), 
$(10.31 .1)])$

$$
\begin{aligned}
T_{m^{2}}(x, y)= & -\frac{1}{8 \pi^{3}}\left(\frac{\mathrm{PP}}{(y-x)^{2}}+i \pi \delta\left((y-x)^{2}\right) \epsilon\left((y-x)^{0}\right)\right) \\
& +\frac{m^{2}}{32 \pi^{3}} \sum_{j=0}^{\infty} \frac{(-1)^{j}}{j !(j+1) !} \frac{\left(m^{2}(y-x)^{2}\right)^{j}}{4^{j}} \\
& \quad \times\left(\log \left|m^{2}(y-x)^{2}\right|+c_{j}+i \pi \Theta\left((y-x)^{2}\right) \epsilon\left((y-x)^{0}\right)\right)
\end{aligned}
$$

with real coefficients $c_{j}$ (here $\Theta$ and $\epsilon$ are again the Heaviside and the sign function, respectively). Due to the factors $(y-x)^{2 j}$, this series representation is a light-cone expansion. The term with the leading singularity becomes integrable after multiplying by $(y-x)^{2}$, showing that $g=-1$.

The light-cone expansion of the kernel of the fermionic projector of the vacuum $P^{\mathrm{vac}}(x, y)$ (see (2.1.1) and $(2.1 .2)$ ) is readily obtained using the relation (1.2.25). To this end, one simply applies the differential operator $i \not \partial+m$ to the above series expansion of $T_{m^{2}}$ and computes the derivatives term by term. Since differentiation increases the order of the singularity on the light cone by one, we thus obtain a light-cone expansion of the form (2.2.1) with $g=-2$.

2.2.2. Inductive Light-Cone Expansion of the Green's Functions. We now return to the perturbation series for the causal Green's functions 2.1.25) derived in $\$ 2.1 .4$. Our goal is to develop a method for performing the light-cone expansion of each summand of this perturbation series. In order to get a first idea for how to proceed, we begin by considering the free advanced Green's function $s_{m}^{\vee}$ of a the Dirac equation of mass $m$ in position space: Similar to (1.2.25), it is again convenient to pull the Dirac matrices out of $s_{m}^{\vee}$ by setting

$$
s_{m}^{\vee}(x, y)=\left(i \not \partial_{x}+m\right) S_{m^{2}}^{\vee}(x, y),
$$

where $S_{m^{2}}^{\vee}$ is the advanced Green's function of the Klein-Gordon operator,

$$
S_{m^{2}}^{\vee}(x, y)=\lim _{\nu \searrow 0} \int \frac{d^{4} p}{(2 \pi)^{4}} \frac{1}{p^{2}-m^{2}-i \nu p^{0}} e^{-i p(x-y)} .
$$

Computing this Fourier integral and expanding the resulting Bessel function in a power series gives (for details see Exercise 2.13)

$$
\begin{aligned}
S_{m^{2}}^{\vee}(x, y)= & -\frac{1}{2 \pi} \delta\left((y-x)^{2}\right) \Theta\left(y^{0}-x^{0}\right) \\
& +\frac{m^{2}}{4 \pi} \frac{J_{1}\left(\sqrt{m^{2}(y-x)^{2}}\right)}{\sqrt{m^{2}(y-x)^{2}}} \Theta\left((y-x)^{2}\right) \Theta\left(y^{0}-x^{0}\right) \\
= & -\frac{1}{2 \pi} \delta\left((y-x)^{2}\right) \Theta\left(y^{0}-x^{0}\right) \\
& +\frac{m^{2}}{8 \pi} \sum_{j=0}^{\infty} \frac{(-1)^{j}}{j !(j+1) !} \frac{\left(m^{2}(y-x)^{2}\right)^{j}}{4^{j}} \Theta\left((y-x)^{2}\right) \Theta\left(y^{0}-x^{0}\right) .
\end{aligned}
$$

This computation shows that $S_{m^{2}}^{\vee}(x, y)$ has a $\delta\left((y-x)^{2}\right)$-like singularity on the light cone. Furthermore, one sees that $S_{m^{2}}^{\vee}$ is a power series in $m^{2}$. The important point for what 
follows is that the higher order contributions in $m^{2}$ contain more factors $(y-x)^{2}$ and are thus of higher order on the light cone. More precisely,

$$
\left.\left(\frac{d}{d m^{2}}\right)^{n} S_{m^{2}}^{\vee}(x, y)\right|_{m=0} \quad \text { is of the order } \mathcal{O}\left((y-x)^{2 n-2}\right) .
$$

According to (2.2.4), the Dirac Green's function is obtained by computing the first partial derivatives of (2.2.7). Therefore, $s_{m}^{\vee}(x, y)$ has a singularity on the light cone which is even $\sim \delta^{\prime}\left((y-x)^{2}\right)$. The higher order contributions in $m$ are again of increasing order on the light cone. This means that we can view the Taylor expansion of $(2.2 .4)$ in $m$,

$$
s_{m}^{\vee}(x, y)=\left.\sum_{n=0}^{\infty}(i \not \partial+m) \frac{1}{n !}\left(\frac{d}{d m^{2}}\right)^{n} S_{m^{2}}^{\vee}(x, y)\right|_{m=0},
$$

as a light-cone expansion of the free Green's function. Our idea is to generalize this formula to the case with interaction. More precisely, we want to express the perturbed Green's function in the form

$$
\tilde{s}^{\vee}(x, y)=\left.\sum_{n=0}^{\infty} F_{n}(x, y)\left(\frac{d}{d m^{2}}\right)^{n} S_{m^{2}}^{\vee}(x, y)\right|_{m=0}
$$

with factors $F_{n}$ which depend on the external potential. We will see that this method is very convenient; especially, we can in this way avoid working with the rather complicated explicit formula (2.2.7). Apart from giving a motivation for the desired form 2.2 .9$)$ of the formulas of the light-cone expansion, the mass expansion (2.2.7) leads to the conjecture that even the higher order contributions in the mass to the perturbed Green's functions might be of higher order on the light cone. If this conjecture was true, it would be a good idea to expand the perturbation expansion of $\tilde{s}$ with respect to the parameter $m$. Therefore, our strategy is to first expand (2.1.25) with respect to the mass and to try to express the contributions to the resulting expansion in a form similar to 2.2.9.

The expansion of 2.1 .25 with respect to $m$ gives a double sum over the orders in the mass parameter and in the external potential. It is convenient to combine these two expansions in a single perturbation series. To this end, we rewrite the Dirac operator as

$$
i \not \partial+\mathcal{B}-m=i \not \partial+B \quad \text { with } \quad B:=\mathcal{B}-m .
$$

For the light-cone expansion of the Green's functions, we will always view $B$ as the perturbation of the Dirac operator. This has the advantage that the unperturbed objects are massless. Expanding in powers of $B$ gives the mass expansion and the perturbation expansion in one step. In order to simplify the notation, for the massless objects we usually omit the index $m$. Thus we write the Green's function of the massless Dirac equation in the Minkowski vacuum as

$$
s^{\vee}(x, y)=\left.i \not \partial_{x} S_{m^{2}}^{\vee}(x, y)\right|_{m=0}, \quad s^{\wedge}(x, y)=\left.i \not \partial_{x} S_{m^{2}}^{\wedge}(x, y)\right|_{m=0} .
$$

Then the interacting Green's functions are given by the perturbation series

$$
\tilde{s}^{\vee}=\sum_{k=0}^{\infty}\left(-s^{\vee} B\right)^{k} s^{\vee}, \quad \tilde{s}^{\wedge}=\sum_{k=0}^{\infty}\left(-s^{\wedge} B\right)^{k} s^{\wedge} .
$$

The constructions of the following subsections are exactly the same for the advanced and retarded Green's functions. In order to treat both cases at once, in the remainder of this section we will omit all superscripts ${ }^{\vee} \vee,{ }^{\prime} \wedge$ '. The formulas for the advanced and retarded Green's functions are obtained by either adding ' ${ }^{\vee}$, or ' $\wedge$ ' to all factors $s, S$. 
We now explain how the individual contributions to the perturbation expansion 2.2.12 can be written similar to the right side of 2.2 .9 as a sum of terms of increasing order on the light cone. For the mass expansion of $S_{m^{2}}$, we set $a=m^{2}$ and use the notation

$$
S^{(l)}=\left.\left(\frac{d}{d a}\right)^{l} S_{a}\right|_{a=0}
$$

In preparation, we derive some computation rules for the $S^{(l)}: S_{a}$ satisfies the defining equation of a Klein-Gordon Green's function

$$
\left(-\square_{x}-a\right) S_{a}(x, y)=\delta^{4}(x-y) .
$$

Differentiating with respect to $a$ and setting $a=0$ gives

$$
-\square_{x} S^{(l)}(x, y)=\delta_{l, 0} \delta^{4}(x-y)+l S^{(l-1)}(x, y), \quad l \geq 0 .
$$

(For $l=0$, this formula does not seem to make sense because $S^{(-1)}$ is undefined. The expression is meaningful, however, if one keeps in mind that in this case the factor $l$ is zero, and thus the whole second summand vanishes. We will also use this convention in the following calculations.) Next, we differentiate the formulas for $S_{a}$ in momentum space,

$$
S_{a}^{\vee}(p)=\frac{1}{p^{2}-a-i \nu p^{0}}, \quad S_{a}^{\wedge}(p)=\frac{1}{p^{2}-a+i \nu p^{0}}
$$

with respect to both $p$ and $a$. Comparing the results gives the relation

$$
\frac{\partial}{\partial p^{k}} S_{a}(p)=-2 p_{k} \frac{d}{d a} S_{a}(p)
$$

or, after expanding in the parameter $a$,

$$
\frac{\partial}{\partial p^{k}} S^{(l)}(p)=-2 p_{k} S^{(l+1)}(p), \quad l \geq 0
$$

This formula also determines the derivatives of $S^{(l)}$ in position space; namely

$$
\begin{gathered}
\frac{\partial}{\partial x^{k}} S^{(l)}(x, y)=\int \frac{d^{4} p}{(2 \pi)^{4}} S^{(l)}(p)\left(-i p_{k}\right) e^{-i p(x-y)} \\
\stackrel{2.2 .16}{=} \frac{i}{2} \int \frac{d^{4} p}{(2 \pi)^{4}} \frac{\partial}{\partial p^{k}} S^{(l-1)}(p) e^{-i p(x-y)} \\
=-\frac{i}{2} \int \frac{d^{4} p}{(2 \pi)^{4}} S^{(l-1)}(p) \frac{\partial}{\partial p^{k}} e^{-i p(x-y)} \\
=\frac{1}{2}(y-x)_{k} S^{(l-1)}(x, y), \quad l \geq 1
\end{gathered}
$$

We iterate this relation to calculate the Laplacian,

$$
\begin{aligned}
-\square_{x} S^{(l)}(x, y) & =-\frac{1}{2} \frac{\partial}{\partial x^{k}}\left((y-x)^{k} S^{(l-1)}(x, y)\right) \\
& =2 S^{(l-1)}(x, y)+\frac{1}{4}(y-x)^{2} S^{(l-2)}(x, y), \quad l \geq 2 .
\end{aligned}
$$

After comparing with (2.2.14), we conclude that

$$
(y-x)^{2} S^{(l)}(x, y)=-4 l S^{(l+1)}(x, y), \quad l \geq 0 .
$$


Finally, $S^{(l)}(x, y)$ is only a function of $(y-x)$, which implies that

$$
\frac{\partial}{\partial x^{k}} S^{(l)}(x, y)=-\frac{\partial}{\partial y^{k}} S^{(l)}(x, y), \quad l \geq 0
$$

The following lemma gives the light-cone expansion of an operator product which is linear in the external potential. We will later use it for the iterative light-cone expansion of more complicated operator products; in this case, the potential will be a composite expression in $B$ and its partial derivatives. In order to avoid confusion then, we denote the external potential by $V$.

Lemma 2.2.2. (light-cone expansion to first order) For any $l, r \geq 0$, the operator product $S^{(l)} V S^{(r)}$ has the light-cone expansion

$$
\begin{aligned}
& \left(S^{(l)} V S^{(r)}\right)(x, y) \\
& =\sum_{n=0}^{\infty} \frac{1}{n !} \int_{0}^{1} \alpha^{l}(1-\alpha)^{r}\left(\alpha-\alpha^{2}\right)^{n}\left(\square^{n} V\right)_{\mid \alpha y+(1-\alpha) x} d \alpha S^{(n+l+r+1)}(x, y) .
\end{aligned}
$$

Proof. The method of proof is to first compute the Laplacian of both sides of 2.2.20). The resulting formulas will have a similar structure, making it possible to proceed inductively.

On the left side of 2.2.20), we calculate the Laplacian with the help of 2.2.14 to

$$
-\square_{x}\left(S^{(l)} V S^{(r)}\right)(x, y)=\delta_{l, 0} V(x) S^{(r)}(x, y)+l\left(S^{(l-1)} V S^{(r)}\right)(x, y)
$$

The Laplacian of the integral on the right side of 2.2 .20 can be computed with 2.2.17) and (2.2.14,

$$
\begin{aligned}
&- \square_{x} \\
& \int_{0}^{1} \alpha^{l}(1-\alpha)^{r}\left(\alpha-\alpha^{2}\right)^{n}\left(\square^{n} V\right)_{\mid \alpha y+(1-\alpha) x} d \alpha S^{(n+l+r+1)}(x, y) \\
&=- \int_{0}^{1} \alpha^{l}(1-\alpha)^{r+2}\left(\alpha-\alpha^{2}\right)^{n}\left(\square^{n+1} V\right)_{\mid \alpha y+(1-\alpha) x} d \alpha S^{(n+l+r+1)}(x, y) \\
&-\int_{0}^{1} \alpha^{l}(1-\alpha)^{r+1}\left(\alpha-\alpha^{2}\right)^{n}\left(\partial_{k} \square^{n} V\right)_{\mid \alpha y+(1-\alpha) x} d \alpha(y-x)^{k} S^{(n+l+r)}(x, y) \\
&+(n+l+r+1) \int_{0}^{1} \alpha^{l}(1-\alpha)^{r}\left(\alpha-\alpha^{2}\right)^{n}\left(\square^{n} V\right)_{\mid \alpha y+(1-\alpha) x} d \alpha S^{(n+l+r)}(x, y) .
\end{aligned}
$$

In the second summand, we rewrite the partial derivative as a derivative with respect to $\alpha$,

$$
(y-x)^{k}\left(\partial_{k} \square^{n} V\right)_{\mid \alpha y+(1-\alpha) x}=\frac{d}{d \alpha}\left(\square^{n} V\right)_{\mid \alpha y+(1-\alpha) x}
$$


(as is verified immediately by computing the right side with the chain rule). This makes it possible to integrate in $\alpha$ by parts. We thus obtain

$$
\begin{aligned}
\int_{0}^{1} \alpha^{l}(1-\alpha)^{r+1}\left(\alpha-\alpha^{2}\right)^{n}\left(\partial_{k} \square^{n} V\right)_{\mid \alpha y+(1-\alpha) x} d \alpha(y-x)^{k} \\
=\int_{0}^{1} \alpha^{l}(1-\alpha)^{r+1}\left(\alpha-\alpha^{2}\right)^{n} \frac{d}{d \alpha}\left(\left.\left(\square^{n} V\right)\right|_{\alpha y+(1-\alpha) x}\right) d \alpha \\
=-\delta_{n, 0} \delta_{l, 0} V(x)-(n+l) \int_{0}^{1} \alpha^{l}(1-\alpha)^{r+2}\left(\alpha-\alpha^{2}\right)^{n-1}\left(\square^{n} V\right)_{\mid \alpha y+(1-\alpha) x} d \alpha \\
\quad+(n+r+1) \int_{0}^{1} \alpha^{l}(1-\alpha)^{r}\left(\alpha-\alpha^{2}\right)^{n}\left(\square^{n} V\right)_{\mid \alpha y+(1-\alpha) x} d \alpha \\
=-\delta_{n, 0} \delta_{l, 0} V(x) \\
\quad-n \int_{0}^{1} \alpha^{l}(1-\alpha)^{r+2}\left(\alpha-\alpha^{2}\right)^{n-1}\left(\square^{n} V\right)_{\mid \alpha y+(1-\alpha) x} d \alpha \\
\quad+(n+l+r+1) \int_{0}^{1} \alpha^{l}(1-\alpha)^{r}\left(\alpha-\alpha^{2}\right)^{n}\left(\square^{n} V\right)_{\mid \alpha y+(1-\alpha) x} d \alpha \\
\quad-l \int_{0}^{1} \alpha^{l-1}(1-\alpha)^{r}\left(\alpha-\alpha^{2}\right)^{n}\left(\square^{n} V\right)_{\mid \alpha y+(1-\alpha) x} d \alpha .
\end{aligned}
$$

We substitute back into the original equation to obtain

$$
\begin{aligned}
& 2.2 .22=\delta_{n, 0} \delta_{l, 0} V(x) S^{(r)}(x, y) \\
& +l \int_{0}^{1} \alpha^{l-1}(1-\alpha)^{r}\left(\alpha-\alpha^{2}\right)^{n}\left(\square^{n} V\right)_{\mid \alpha y+(1-\alpha) x} d \alpha S^{(n+l+r)}(x, y) \\
& -\int_{0}^{1} \alpha^{l}(1-\alpha)^{r+2}\left(\alpha-\alpha^{2}\right)^{n}\left(\square^{n+1} V\right)_{\mid \alpha y+(1-\alpha) x} d \alpha S^{(n+l+r+1)}(x, y) \\
& +n \int_{0}^{1} \alpha^{l}(1-\alpha)^{r+2}\left(\alpha-\alpha^{2}\right)^{n-1}\left(\square^{n} V\right)_{\mid \alpha y+(1-\alpha) x} d \alpha S^{(n+l+r)}(x, y) .
\end{aligned}
$$

After dividing by $n$ ! and summation over $n$, the last two summands are telescopic and cancel each other. Thus one gets

$$
\begin{aligned}
& -\square \sum_{n=0}^{\infty} \frac{1}{n !} \int_{0}^{1} \alpha^{l}(1-\alpha)^{r}\left(\alpha-\alpha^{2}\right)^{n}\left(\square^{n} V\right)_{\mid \alpha y+(1-\alpha) x} d \alpha S^{(n+l+r+1)}(x, y) \\
& =\delta_{l, 0} V(x) S^{(r)}(x, y) \\
& \quad+l \sum_{n=0}^{\infty} \frac{1}{n !} \int_{0}^{1} \alpha^{l-1}(1-\alpha)^{r}\left(\alpha-\alpha^{2}\right)^{n}\left(\square^{n} V\right)_{\mid \alpha y+(1-\alpha) x} d \alpha S^{(n+l+r)}(x, y) .
\end{aligned}
$$

We now compare the formulas 2.2 .21$)$ and 2.2 .23 for the Laplacian of both sides of 2.2 .20 . In the special case $l=0$, these formulas coincide, and we can use a uniqueness argument for the solutions of the wave equation to prove $(2.2 .20)$ : We assume that we consider the advanced Green's function (for the retarded Green's function, the argument is analogous). For given $y$, we denote the difference of both sides of 2.2 .20 by $F(x)$. Since the support of $F(x)$ is in the past light cone $x \in L_{y}^{\wedge}, F$ vanishes in a neighborhood of the hypersurface $\mathcal{H}=\left\{z \in \mathbb{R}^{4} \mid z^{0}=y^{0}+1\right\}$. Moreover, the Laplacian of $F$ is identically 
zero according to (2.2.21) and (2.2.23). We conclude that

$$
\square F=0 \quad \text { and } \quad F_{\mid \mathcal{H}}=\partial_{k} F_{\mid \mathcal{H}}=0 .
$$

Since the wave equation has a unique solution for given initial data on the Cauchy surface $\mathcal{H}, F$ vanishes identically.

The general case follows by induction in $l$ : Suppose that 2.2.20 holds for given $\hat{l}$ (and arbitrary $r$ ). Then, according to (2.2.21), 2.2.23), and the induction hypothesis, the Laplacian of both sides of $(2.2 .20)$ coincides for $l=\hat{l}+1$. The above uniqueness argument for the solutions of the wave equation again gives 2.2 .20 .

We recall for clarity that, according to $(2.2 .8)$, the higher $a$-derivatives of $S_{a}(x, y)$ are of higher order on the light cone. Thus the summands in (2.2.20) are of increasing order on the light cone, and the infinite sum is mathematically well-defined in the sense of Definition 2.2.1 via the approximation by the partial sums 2.2 .2 .

Lemma 2.2.2 can be used for the light-cone expansion of more complicated operator products. To explain the method, we look at the simplest example of three factors $S^{(0)}$ and two potentials $V$ and $W$,

$$
\left(S^{(0)} V S^{(0)} W S^{(0)}\right)(x, y)=\int d^{4} z S^{(0)}(x, z) V(z)\left(S^{(0)} W S^{(0)}\right)(z, y) .
$$

Having split up the operator product in this form, we can apply Lemma 2.2 .2 to the factor $S^{(0)} W S^{(0)}$,

$$
=\sum_{n=0}^{\infty} \frac{1}{n !} \int d^{4} z S^{(0)}(x, z)\left\{V(z) \int_{0}^{1}\left(\alpha-\alpha^{2}\right)^{n}\left(\square^{n} W\right)_{\mid \alpha y+(1-\alpha) z} d \alpha\right\} S^{(n+1)}(z, y) .
$$

Now we rewrite the $z$-integral as the operator product $\left(S^{(0)} g_{y} S^{(0)}\right)(x, y)$, where $g_{y}(z)$ is the function in the curly brackets. The $y$-dependence of $g_{y}$ causes no problems because we can view $y$ as a fixed parameter throughout the expansion. Thus we can simply apply Lemma 2.2 .2 once again to obtain

$$
\begin{aligned}
=\sum_{m, n=0}^{\infty} & \frac{1}{m ! n !} \int_{0}^{1} d \beta(1-\beta)^{n+1}\left(\beta-\beta^{2}\right)^{m} \int_{0}^{1} d \alpha\left(\alpha-\alpha^{2}\right)^{n} \\
& \times \square_{z}^{m}\left(V(z)\left(\square^{n} W\right)_{\mid \alpha y+(1-\alpha) z}\right)_{\mid z=\beta y+(1-\beta) x} S^{(m+n+2)}(x, y) .
\end{aligned}
$$

The Laplacian $\square_{z}^{m}$ could be computed further with the Leibniz rule. Notice that the manipulations of the infinite sums are unproblematic because to every order on the light cone, the number of terms is actually finite (the situation would be more difficult if we studied the convergence of the sum (2.2.1), but, as pointed out earlier, the light-cone expansion is defined merely via the partial sums).

We want to iteratively perform the light-cone expansion of the operator products in 2.2.12). This is not possible directly with the method just described, because 2.2.12 contains the Dirac Green's function $s$ (instead of $S$ ). We must think about how to deal with this complication. Relation (2.2.11) allows us to replace the factors $s$ by $S$, but this gives additional partial derivatives in the operator product. These derivatives can be carried out after each iteration step by applying the Leibniz rule and using the 
differentiation rule (2.2.17). In the simplest example, we have

$$
\begin{aligned}
& \left(s^{(0)} V S^{(0)}\right)(x, y)=\left(i \not \partial_{x}\right)\left(S^{(0)} V S^{(0)}\right)(x, y) \\
& =i \not \partial_{x} \sum_{n=0}^{\infty} \frac{1}{n !} \int_{0}^{1}\left(\alpha-\alpha^{2}\right)^{n}\left(\square^{n} V\right)_{\mid \alpha y+(1-\alpha) x} d \alpha S^{(n+1)}(x, y) \\
& =i \sum_{n=0}^{\infty} \frac{1}{n !} \int_{0}^{1}(1-\alpha)\left(\alpha-\alpha^{2}\right)^{n}\left(\not \partial \square^{n} V\right)_{\mid \alpha y+(1-\alpha) x} d \alpha S^{(n+1)}(x, y) \\
& \quad+\frac{i}{2} \sum_{n=0}^{\infty} \frac{1}{n !} \int_{0}^{1}\left(\alpha-\alpha^{2}\right)^{n}\left(\square^{n} V\right)_{\mid \alpha y+(1-\alpha) x} d \alpha(y-x)_{j} \gamma^{j} S^{(n)}(x, y) .
\end{aligned}
$$

The only problem with this method is that the partial derivatives might hit a factor $S^{(0)}$, in which case the rule (2.2.17) cannot be applied. In order to resolve this problem, we extend our constructions in a way which allows us to use all previous formulas also in this special case. To this end, we take (2.2.17) as the defining equation for $(y-x)_{k} S^{(-1)}(x, y)$,

$$
(y-x)_{k} S^{(-1)}(x, y):=2 \frac{\partial}{\partial x^{k}} S^{(0)}(x, y)
$$

(notice that $S^{(-1)}$ itself remains undefined, only the combination $(y-x)_{k} S^{(-1)}(x, y)$ makes mathematical sense as the partial derivative of the distribution $\left.S^{(0)}\right)$. It turns out that with this definition, all our computation rules as well as the light-cone expansion of Lemma 2.2 .2 remain valid for $S^{(-1)}$ :

Lemma 2.2.3. (light-cone expansion to first order for $r=-1$ ) The operator product $\left(S^{(l)} . S^{(-1)}\right), l \geq 0$, has the light-cone expansion

$$
\begin{aligned}
& \int d^{4} z S^{(l)}(x, z) V(z)(y-z)_{k} S^{(-1)}(z, y) \\
& =\left.\sum_{n=0}^{\infty} \frac{1}{n !} \int_{0}^{1} \alpha^{l}(1-\alpha)^{-1}\left(\alpha-\alpha^{2}\right)^{n} \square_{z}^{n}\left(V(z)(y-z)_{k}\right)\right|_{z=\alpha y+(1-\alpha) x} d \alpha S^{(n+l)}(x, y) .
\end{aligned}
$$

Since the proof is straightforward, we omit it here but refer to Exercise 2.14 or [F6, proof of Lemma 2.2]. We note for clarity that the pole of the factor $(1-\alpha)^{-1}$ at $\alpha=1$ in the formula of the above lemma does not cause any problems. Namely, in the case $n=0$ it disappears because $(1-\alpha)^{-1}(y-z)=y-x$, whereas in the case $n>0$ it is compensated by the zero of the factor $\left(\alpha-\alpha^{2}\right)^{n}$.

2.2.3. Structural Results for Chiral Potentials. In the previous section, we gave a constructive procedure for performing the light-cone expansion of each summand of the perturbation expansion for the causal Green's functions (2.2.12). In this and the next section, we shall explain how to use this method to uncover the structure of the Green's functions in position space. To this end, we need to specify the form of the external potential $\mathcal{B}$ in the Dirac equation 2.1.5). We are mostly interested in the situation that $\mathcal{B}$ is composed of left- or right-handed potentials, i.e.

$$
\mathcal{B}=\chi_{L} A_{R}+\chi_{R} A_{L}
$$

(here $\chi_{L / R}=\frac{1}{2}(\mathbb{1} \mp \Gamma)$ are the chiral projectors, and $\Gamma=i \gamma^{0} \gamma^{1} \gamma^{2} \gamma^{3}$ is the usual pseudoscalar matrix). Such so-called chiral potential are of central interest because they allow for the description of gauge fields. For example, an electromagnetic field is described by 
choosing $A_{L}=A_{R}=A$, where $A$ is the electromagnetic potential. A left-handed potential is needed for example for describing the weak interaction in the standard model. In this context, it is important to describe non-abelian gauge fields. In this case, the potentials $A_{L}$ and $A_{R}$ take values in a Lie algebra. For simplicity, we here always represent the potentials by matrices acting on $\mathbb{C}^{g}$ with $g \in \mathbb{N}$. In order to describe the coupling of the gauge gauge fields to the fermions, the Dirac wave functions must also carry an index $a=1, \ldots, g$. Moreover, we want to allow for the situation that the system involves Dirac matrices of different rest masses, which we label again by an index $a$. This leads to the following setup. We define the fermionic projector of the vacuum and the Green's functions as direct sums of the corresponding operators with rest masses $m_{1}, \ldots, m_{g}$, i.e.

$$
P^{\mathrm{vac}}=\bigoplus_{a=1}^{g} P_{m_{a}}^{\mathrm{vac}} \quad \text { and } \quad s=\bigoplus_{a=1}^{g} s_{m_{a}}
$$

with $P_{m_{a}}^{\mathrm{vac}}$ and $s_{m}$ according to 2.1 .2$)$ and 2.1 .9 . We write the Dirac equation as

$$
(i \not \partial+\mathcal{B}-m Y) \psi(x)=0
$$

with $\mathcal{B}$ as in 2.2.25. Here $Y$ is the mass matrix defined by

$$
Y=\frac{1}{m} \operatorname{diag}\left(m_{1}, \ldots, m_{a}\right)
$$

(here $m$ is introduced merely as an expansion parameter; the picture is that $Y$ is dimensionless, whereas $m$ carries the dimension of inverse length). For later use, it is also convenient to allow for scalar and pseudoscalar potentials. In order to built these potentials into the Dirac equation (2.2.27), it is most convenient to replace the mass matrix by a space-time dependent matrix. ${ }^{1}$

$$
Y=Y(x):=\chi_{L} Y_{L}(x)+\chi_{R} Y_{R}(x),
$$

referred to as the dynamical mass matrix.

In analogy to (2.2.10), we combine the mass term with the potential by setting

$$
B=\chi_{L} A_{R}+\chi_{R} A_{L}-m Y .
$$

Then the perturbation expansion of the causal Green's functions can again be written in the form (2.2.12). The light-cone expansion can be carried out exactly as explained in the previous section. The only point to keep in mind is that the chiral potentials at different space-time points do not necessarily commute. Moreover, the chiral potentials in general do not commute with the mass matrix. Therefore, in what follows we need to be careful in keeping track of the order of multiplication.

Before going on, we explain our convention for the chiral indices of potentials in 2.2 .28 and 2.2 .29 . We follow the usual rule that a left-handed potential couples to the lefthanded component of the Dirac wave function, whereas the right-handed potential couples to the right-handed component of the wave function. Indeed, decomposing the Dirac wave function as

$$
\psi=\chi_{L} \psi_{L}+\chi_{R} \psi_{R}
$$

\footnotetext{
${ }^{1}$ To avoid confusion, we point out that our convention differs from that used in [F6, F7, where the dynamical mass matrix is defined instead by $Y=\chi_{L} Y_{R}+\chi_{R} Y_{L}$. Our convention fits to our general rule that left- and right-handed potentials should couple to the left- and right-handed component of the Dirac spinors, respectively (see also 2.2.30 and the explanation thereafter).
} 
the Dirac equation (2.2.27) becomes

$$
\begin{aligned}
0 & =\left(i \not \partial+\chi_{L} A_{R}+\chi_{R} A_{L}-m \chi_{L} Y_{L}(x)-m \chi_{R} Y_{R}(x)\right)\left(\chi_{L} \psi_{L}+\chi_{R} \psi_{R}\right) \\
& =\chi_{L}\left(\left(i \not \partial+A_{R}\right) \psi_{R}-m Y_{L} \psi_{L}\right)+\chi_{R}\left(\left(i \not \partial+A_{L}\right) \psi_{L}-m Y_{R} \psi_{R}\right) .
\end{aligned}
$$

(here we use that the chirality is reversed at each Dirac matrix). This shows that our conventions 2.2.28) and 2.2.29) indeed imply that left-handed potentials couple to $\psi_{L}$ and right-handed potentials to $\psi_{R}$.

The next theorem gives a structural result on the contributions to the light-cone expansion of the Green's functions. For the line integrals, we introduce the short notation

$$
\int_{x}^{y}[l, r \mid n] d z f(z):=\int_{0}^{1} d \alpha \alpha^{l}(1-\alpha)^{r}\left(\alpha-\alpha^{2}\right)^{n} f(\alpha y+(1-\alpha) x) .
$$

Furthermore, we abbreviate the following products with multi-indices,

$$
\partial_{z}^{J}:=\frac{\partial}{\partial z^{j_{1}}} \cdots \frac{\partial}{\partial z^{j_{l}}}, \quad(y-x)^{J}:=(y-x)^{j_{1}} \cdots(y-x)^{j_{l}}, \quad \gamma^{J}:=\gamma^{j_{1}} \cdots \gamma^{j_{l}},
$$

where $J=\left(j_{1}, \ldots, j_{l}\right)$.

TheOREM 2.2.4. In the presence of chiral potentials (2.2.29), the light-cone expansion of the $k^{\text {th }}$ order contribution $\left((-s B)^{k} s\right)(x, y)$ to the perturbation series 2.2.12 can be written as an infinite sum of expressions, each of which has the form

$$
\begin{gathered}
\chi_{c_{0}} C(y-x)^{I} \int_{x}^{y}\left[l_{1}, r_{1} \mid n_{1}\right] d z_{1} \partial_{z_{1}}^{I_{1}} \square_{z_{1}}^{p_{1}} V_{J_{1}, c_{1}}^{(1)}\left(z_{1}\right) \int_{z_{1}}^{y}\left[l_{2}, r_{2} \mid n_{2}\right] d z_{2} \partial_{z_{2}}^{I_{2}} \square_{z_{2}}^{p_{2}} V_{J_{2}, c_{2}}^{(2)}\left(z_{2}\right) \\
\cdots \int_{z_{k-1}}^{y}\left[l_{k}, r_{k} \mid n_{k}\right] d z_{k} \partial_{z_{k}}^{I_{k}} \square_{z_{k}}^{p_{k}} V_{J_{k}, c_{k}}^{(k)}\left(z_{k}\right) \gamma^{J} S^{(h)}(x, y) .
\end{gathered}
$$

In this formula, $C$ is a complex number and the parameters $l_{a}, r_{a}, n_{a}$, and $p_{a}$ are nonnegative integers; the indices $c$ and $c_{a}$ can take the two values $L$ or $R$. The functions $V_{J_{a}, c_{a}}^{(a)}$ (where $J_{a}$ is a multi-index and $c_{a} \in\{L, R\}$ is a chiral index) coincide with any of the individual potentials in (2.2.29) and 2.2.28) with chirality $c_{a}$, i.e.

$$
\begin{array}{lll}
V_{c_{a}}^{(a)}=A_{c_{a}} & & \text { (in which case } \left.\left|J_{a}\right|=1\right) \\
V_{c_{a}}^{(a)}=m Y_{c_{a}} & & \text { (in which case } \left.\left|J_{a}\right|=0\right) .
\end{array}
$$

The chirality $c_{a}$ of the potentials is determined by the following rule:

(i) The chirality is reversed precisely at every mass matrix, i.e.

$$
c_{a-1} \text { and } c_{a}\left\{\begin{array}{cl}
\text { coincide } & \text { if } V_{c_{a}}^{(a)}=A_{c_{a}} \\
\text { are opposite } & \text { if } V_{c_{a}}^{(a)}=m Y_{c_{a}}
\end{array}\right.
$$

for all $a=1, \ldots, k$.

The tensor indices of the multi-indices in (2.2.32) are all contracted with each other, according to the following rules:

(a) No two tensor indices of the same multi-index are contracted with each other.

(b) The tensor indices of the factor $\gamma^{J}$ are all contracted with different multi-indices, in the order of their appearance in the product (2.2.32) (i.e., for $J=\left(j_{1}, \ldots, j_{l}\right)$ and $1 \leq a<b \leq l$, the multi-index with which $j_{a}$ is contracted must stand to the left of the multi-index corresponding to $j_{b}$ ). 
The parameter $h$ is given by

$$
2 h=k-1-|I|+\sum_{a=1}^{k}\left(\left|I_{a}\right|+2 p_{a}\right) .
$$

The number of factors $(y-x)$ is bounded by

$$
|I| \leq k+1-\sum_{a=1}^{k}\left|I_{a}\right| .
$$

Basically, this theorem states that the light-cone expansion of the $k^{\text {th }}$ order Feynman diagrams can be written with $k$ nested line integrals. Notice that the potentials $V^{(a)}\left(z_{a}\right)$ do in general not commute with each other, so that the order of multiplication is important in 2.2.32. In order to avoid misunderstandings, we point out that the derivatives $\partial_{z_{a}}^{I_{a}}$ and $\square_{z_{a}}^{p_{a}}$ do not only act on $V^{(a)}\left(z_{a}\right)$, but also on all the following factors $V^{(a+1)}\left(z_{a+1}\right)$, $V^{(a+2)}\left(z_{a+2}\right), \ldots$ (note that the variables $z_{a+1}, z_{a+2}, \ldots$ implicitly depend on $z_{a}$ via the inductive definition of the line integrals). Clearly, these derivatives could be carried out further with the Leibniz rule, but it is easier not to do this at the moment. The restrictions (a) and (b) on the possible contractions of the tensor indices were imposed in order to avoid an abuse of our multi-index notation. More precisely, (a) prevents factors $(y-x)^{2}$ in $(y-x)^{I}$, an unnecessary large number of $\gamma$-matrices in $\gamma^{J}$, and "hidden" Laplacians in the partial derivatives $\partial_{z_{a}}^{I_{a}}$. The rule (b), on the other hand, prevents factors $(y-x)^{2}$ and hidden Laplacians in combinations of the form $(y-x)_{i}(y-x)_{j} \gamma^{i} \gamma^{j}$ and $\partial_{i j} V_{J_{a}}^{(a)} \gamma^{i} \gamma^{j}$, respectively. Our ordering condition for the $\gamma$-matrices is just a matter of convenience. Relation 2.2.34) is very useful because it immediately tells for any configuration of the line integrals and potentials in $(2.2 .32)$ what the corresponding order on the light cone is. Notice that 2.2.34 and 2.2.35 imply the inequality

$$
h \geq-1+\sum_{a=1}^{k}\left(\left|I_{a}\right|+p_{a}\right) .
$$

In particular, one sees that $h \geq-1$. In the case $h=-1$, (2.2.34) yields that $|I|>0$, so that 2.2.32 must contain at least one factor $(y-x)$. Therefore, the factor $S^{(h)}$ in 2.2.32 is always well-defined by either 2.2.13) or 2.2.24).

We point out that, although the total number of summands 2.2 .32 is infinite, the number of summands for any given value of the parameter $h$ is finite. This is clear because, for fixed $h$, the relations (2.2.34) and 2.2.35) only allow for a finite number of possibilities to choose the parameters $|I|,\left|I_{a}\right|$, and $p_{a}$, giving rise to only a finite number of expressions of the form 2.2.32). Since, according to (2.2.8), the contributions for higher values of $h$ are of higher order on the light cone, we conclude that the number of summands 2.2 .32 is finite to every order on the light cone. Therefore, the light-cone expansion of Theorem 2.2.4 makes mathematical sense in terms of Definition 2.2.1.

Proof of Theorem 2.2.4. We proceed inductively in $k$. For $k=0$, the assumption is true because in view of 2.2.11 and 2.2.24 we can write the free Dirac Green's function as

$$
s(x, y)=\left(\chi_{L}+\chi_{R}\right) \frac{i}{2}(y-x)^{j} \gamma_{j} S^{(-1)}(x, y),
$$


which is of the desired form (2.2.32). The conditions $(i),(a),(b)$, and the relations (2.2.34, 2.2.35 are clearly satisfied.

Assume that the theorem holds for a given $k$. With the formula

$$
\left((-s B)^{k+1} s\right)(x, y)=-i \not \partial_{x} \int d^{4} z S^{(0)}(x, z) B(z)\left((-s B)^{k} s\right)(z, y),
$$

we can express the $(k+1)^{\text {st }}$ order contribution to the perturbation series 2.2 .12 in terms of the $k^{\text {th }}$ order contribution. We must show that (2.2.38) can again be written as a sum of expressions of the form (2.2.32) (with $k$ replaced by $k+1$ ), and that (i), (a), (b), and 2.2.34, 2.2.35 are satisfied. This is done in several construction steps:

(1) Chiral decomposition:

We substitute the induction hypothesis 2.2 .32 into 2.2 .38 . This gives a sum of expressions of the form

$$
\begin{gathered}
C i \not \partial_{x} \int d^{4} z S^{(0)}(x, z)\left\{(y-z)^{I} B(z) \chi_{c} \int_{z}^{y}\left[l_{1}, r_{1} \mid n_{1}\right] d z_{1} \partial_{z_{1}}^{I_{1}} \square^{p_{1}} V_{J_{1}, c_{1}}^{(1)}\left(z_{1}\right)\right. \\
\left.\ldots \int_{z_{k-1}}^{y}\left[l_{k}, r_{k} \mid n_{k}\right] d z_{k} \partial_{z_{k}}^{I_{k}} \square^{p_{k}} V_{J_{k}, c_{k}}^{(k)}\left(z_{k}\right) \gamma^{J}\right\} S^{(h)}(z, y) .
\end{gathered}
$$

We insert the specific form of the potential $B$, $(2.2 .29)$, and expand. Using the commutation rule $\gamma^{i} \chi_{L / R}=\chi_{R / L} \gamma^{i}$, we bring all chiral projectors to the very left, where they can be combined with the formula $\chi_{c} \chi_{d}=\delta_{c d} \chi_{c}$ to a single chiral projector. Next, we bring the $\gamma$-matrices of $B$ to the right and write them together with the factor $\gamma^{J}$ in 2.2.39 (notice that the Dirac matrices commute with the potentials $V_{c_{a}}^{(a)}$, which act non-trivially only on the Dirac sea index). Denoting the individual potentials of the factor $B$ in 2.2 .39 by $V_{J_{0}, c_{0}}^{(0)}$, we thus get for $2.2 .39 \mathrm{a}$ sum of expressions of the form

$$
\begin{gathered}
\chi_{c} C i \not \partial_{x} \int d^{4} z S^{(0)}(x, z)\left\{(y-z)^{I} V_{J_{0}, c_{0}}^{(0)}(z) \int_{z}^{y}\left[l_{1}, r_{1} \mid n_{1}\right] d z_{1} \partial_{z_{1}}^{I_{1}} \square^{p_{1}} V_{J_{1}, c_{1}}^{(1)}\left(z_{1}\right)\right. \\
\left.\ldots \int_{z_{k-1}}^{y}\left[l_{k}, r_{k} \mid n_{k}\right] d z_{k} \partial_{z_{k}}^{I_{k}} \square^{p_{k}} V_{J_{k}, c_{k}}^{(k)}\left(z_{k}\right) \gamma^{J}\right\} S^{(h)}(z, y) .
\end{gathered}
$$

The chiral decomposition in 2.2 .29$)$ and $(2.2 .28)$ imply that the chiralities in $(2.2 .40)$ satisfy the rule (i) (after relabeling the indices in an obvious way). The chirality of the potentials will not be affected in all the following construction steps; to simplify the notation, we will omit the indices $c_{a}$ from now on.

(2) Light-cone expansion:

Since $y$ can be considered as a fixed parameter, we can in 2.2 .40 apply Lemma 2.2 .2 with $V$ given by the expression in the curly brackets,

$$
\begin{aligned}
&(2.2 .40)= \chi_{c} C i \not \partial_{x} \sum_{n=0}^{\infty} \frac{1}{n !} \int_{x}^{y}[0, h \mid n] d z \\
& \times \square_{z}^{n}\left((y-z)^{I} V_{J_{0}}^{(0)}(z) \int_{z}^{y}\left[l_{1}, r_{1} \mid n_{1}\right] d z_{1} \partial_{z_{1}}^{I_{1}} \square^{p_{1}} V_{J_{1}}^{(1)}\left(z_{1}\right)\right. \\
&\left.\quad \ldots \int_{z_{k-1}}^{y}\left[l_{k}, r_{k} \mid n_{k}\right] d z_{k} \partial_{z_{k}}^{I_{k}} \square^{p_{k}} V_{J_{k}}^{(k)}\left(z_{k}\right)\right) \gamma^{J} S^{(n+h+1)}(x, y) .
\end{aligned}
$$


(3) Computation of the Laplacian $\square_{z}^{n}$ :

We carry out the $z$-derivatives in (2.2.41) inductively with the Leibniz rule. Each derivative can act either on the factors $(y-z)^{I}$ or on the functions $V^{(a)}$. In the first case, one of the factors $(y-z)$ disappears. Thus we get a sum of expressions of the form

$$
\begin{gathered}
\chi_{c} C i \not \partial_{x} \int_{x}^{y}[0, h \mid n] d z(y-z)^{\hat{I}} \partial_{z}^{I_{0}} \square_{z}^{p_{0}} V_{J_{0}}^{(0)}(z) \int_{z}^{y}\left[l_{1}, r_{1} \mid n_{1}\right] d z_{1} \partial_{z_{1}}^{I_{1}} \square^{p_{1}} V_{J_{1}}^{(1)}\left(z_{1}\right) \\
\cdots \int_{z_{k-1}}^{y}\left[l_{k}, r_{k} \mid n_{k}\right] d z_{k} \partial_{z_{k}}^{I_{k}} \square^{p_{k}} V_{J_{k}}^{(k)}\left(z_{k}\right) \gamma^{J} S^{(n+h+1)}(x, y)
\end{gathered}
$$

with $|\hat{I}| \leq|I|$ and

$$
2 n=|I|-|\hat{I}|+\left|I_{0}\right|+2 p_{0} .
$$

We can assume that no tensor indices of $\partial_{z}^{I_{0}}$ are contracted with each other (otherwise we rewrite the corresponding partial derivatives as additional Laplacians). Then all the partial derivatives $\partial_{z}$ in 2.2 .42 were generated in the case when one derivative of a Laplacian $\square_{z}$ in $(2.2 .41)$ hit a factor $(y-z)$ whereas the other derivative acted on the $V^{(a)}$. Thus the number of factors $(y-z)$ which disappeared by carrying out the Laplacians in 2.2.41) is larger or equal than the number of partial derivatives $\partial_{z}$,

$$
|I|-|\hat{I}| \geq\left|I_{0}\right|
$$

(4) Extraction of the factors $(y-x)$ :

In 2.2 .42 , we iteratively apply the identity

$$
\int_{x}^{y}[0, r \mid n] d z(y-z) \cdots=(y-x) \int_{x}^{y}[0, r+1 \mid n] d z \cdots .
$$

This gives $(k+1)$ nested line integrals of the form

$$
\begin{gathered}
2.2 .42=\chi_{c} C i \not \phi_{x}(y-x)^{\hat{I}} S^{(\hat{h})}(x, y) \int_{x}^{y}\left[l_{0}, r_{0} \mid n_{0}\right] d z_{0} \partial_{z_{0}}^{I_{0}} \square^{p_{0}} V_{J_{0}}^{(0)}\left(z_{0}\right) \\
\cdots \int_{z_{k-1}}^{y}\left[l_{k}, r_{k} \mid n_{k}\right] d z_{k} \partial_{z_{k}}^{I_{k}} \square^{p_{k}} V_{J_{k}}^{(k)}\left(z_{a}\right) \gamma^{J}
\end{gathered}
$$

with

$$
\begin{aligned}
& l_{0}=0, \quad r_{0}=h+|\hat{I}|, \quad n_{0}=n \\
& 0 \leq 2 \hat{h}=2(n+h+1) \stackrel{\frac{2.2 .43)}{-}}{2} 2 h+2+|I|-|\hat{I}|+\left|I_{0}\right|+2 p_{0} .
\end{aligned}
$$

We can arrange that the parameters $l_{0}, r_{0}$, and $n_{0}$ are all positive: The only parameter which might be negative is $r_{0}$; in this case, $h=-1,|\hat{I}|=0$, and thus $r_{0}=-1$. The induction hypothesis (2.2.34) yields that $|I|>0$. Thus $|I|>|\hat{I}|$, and relation 2.2.43 gives that $\left(n_{0}=\right) n>0$. Therefore, we can apply the identity

$$
\left[l_{0}, r_{0} \mid n_{0}\right]=\left[l_{0}+1, r_{0}+1 \mid n_{0}-1\right]
$$

to make all the parameters in this bracket positive.

(5) Computation of the partial derivative $\not_{x}$ :

The $x$-derivative in 2.2.45 can act on the factors $S^{(\hat{h})},(y-x)^{\hat{I}}$, or $V^{(a)}\left(z_{a}\right)$. The first case can be computed with the rules (2.2.17) or (2.2.24); it decreases $\hat{h}$ by one and 
gives one additional factor $(y-x)$. In the second case, one factor $(y-x)$ disappears, and thus $|\hat{I}|$ is decremented. The last case can be handled with the rule

$$
\frac{\partial}{\partial x^{k}} \int_{x}^{y}[l, r \mid n] d z f(z, y)=\int_{x}^{y}[l, r+1 \mid n] \frac{\partial}{\partial z^{k}} f(z, y),
$$

which increases $\left|I_{0}\right|$ by one. As is immediately verified in each of these cases, equation 2.2.47) transforms into

$$
2 \hat{h}=2 h+1+|I|-|\hat{I}|+\left|I_{0}\right|+2 p_{0},
$$

whereas inequality (2.2.44) must be weakened to

$$
|\hat{I}| \leq 1+|I|-\left|I_{0}\right|
$$

Finally, we combine the $\gamma$-matrix of the factor $\not_{x}$ with $\gamma^{J}$.

After these transformations, the $(k+1)^{\text {st }}$ order Feynman diagram consists of a sum of terms of the form

$$
\begin{aligned}
\chi_{c} C(y-x)^{\hat{I}} & \int_{x}^{y}\left[l_{0}, r_{0} \mid n_{0}\right] d z_{0} \partial_{z_{0}}^{I_{0}} \square_{z_{0}}^{p_{0}} V_{J_{0}}^{(0)}\left(z_{0}\right) \\
& \cdots \int_{z_{k-1}}^{y}\left[l_{k}, r_{k} \mid n_{k}\right] d z_{k} \partial_{z_{k}}^{I_{k}} \square_{z_{k}}^{p_{k}} V_{J_{k}}^{(k)}\left(z_{k}\right) \gamma^{J} S^{(\hat{h})}(x, y) .
\end{aligned}
$$

Notice that the parameters $I_{a}, p_{a}, a=1, \ldots, k$, were not changed by the above construction steps; they are still the same as in the induction hypothesis (2.2.32). After renaming the indices and the integration variables, 2.2.51) is of the required form 2.2.32). The conditions ( $a$ ) and (b) for the contractions of the tensor indices, however, will in general be violated. Therefore we need two further computation steps:

(6) Simplification of the Dirac matrices:

If any two of the tensor indices of the factor $\gamma^{J}$ are contracted with each other, we reorder the $\gamma$-matrices with the anti-commutation relations

$$
\left\{\gamma^{i}, \gamma^{j}\right\}=2 g^{i j} \mathbb{1}
$$

until the corresponding matrices are next to each other. Applying the identity $\gamma^{i} \gamma_{i}=$ $4 \mathbb{1}$, both Dirac matrices disappear. We iterate this procedure until no tensor indices of $\gamma^{J}$ are contracted with each other (notice that the iteration comes to an end because the number of $\gamma$-factors is decreased by two in each step). Again using the anti-commutation rule 2.2.52, we reorder the Dirac matrices until they are in the same order in which the factors to which their tensor indices are contracted appear in the product (2.2.51). If any two of the $\gamma$-matrices are contracted with the same multi-index, these $\gamma$-matrices are next to each other, and we can use the symmetry in the tensor indices to eliminate them both, more precisely

$$
\begin{aligned}
(y-x)_{i}(y-x)_{j} \cdots \gamma^{i} \gamma^{j} & =(y-x)^{2} \cdots \mathbb{1} \\
\partial_{i j} V^{(a)} \cdots \gamma^{i} \gamma^{j} & =\square V^{(a)} \cdots \mathbb{1} .
\end{aligned}
$$

After all these transformations, condition (b) is satisfied.

Notice that the parameters $\left|I_{a}\right|$ and $p_{a}$ are in general changed in this construction step. More precisely, each transformation 2.2.54 modifies the parameters according to

$$
\left|I_{a}\right| \rightarrow\left|I_{a}\right|-2 \quad \text { and } \quad p_{a} \rightarrow p_{a}+1
$$


(7) Handling of the new contractions:

If any two tensor indices of a factor $\partial_{z_{a}}^{I_{a}}$ are contracted with each other, we rewrite the corresponding partial derivatives as a Laplacian; this changes the parameters $\left|I_{a}\right|$ and $p_{a}$ according to (2.2.55). If two tensor indices of the factor $(y-x)^{\hat{I}}$ are contracted with each other, this gives a factor $(y-x)^{2}$. Using the identity (2.2.18), we inductively absorb the factors $(y-x)^{2}$ into $S^{(\hat{h})}(x, y)$, which transforms $\hat{h}$ and $|\hat{I}|$ as

$$
\hat{h} \rightarrow \hat{h}+1 \quad \text { and } \quad|\hat{I}| \rightarrow|\hat{I}|-2 .
$$

After these transformations, condition (a) is also satisfied.

After all these construction steps, the $(k+1)^{\text {st }}$ order Feynman diagram is a sum of terms of the form (2.2.51) satisfying the conditions $(a)$ and (b). It remains to show that the relations 2.2.34) and 2.2.35 remain valid in our inductive construction: As mentioned earlier, the parameters $I_{a}, p_{a}, a=1, \ldots, k$ are not changed in the construction steps (1) to (5). In the steps (6) and (7), the transformations (2.2.55) and (2.2.56) preserve both the induction hypothesis (2.2.34), 2.2.35) and the relations $(2.2 .49),(2.2 .50)$, as is immediately verified. By substituting (2.2.49) and 2.2.50 into (2.2.55, 2.2.56), we obtain

$$
2 \hat{h}=(k+1)-1-|\hat{I}|+\sum_{a=0}^{k}\left|I_{a}\right|+2 p_{a}, \quad|\hat{I}| \leq(k+1)+1-\sum_{a=0}^{k}\left|I_{a}\right| .
$$

This concludes the proof.

2.2.4. Reduction to the Phase-Free Contribution. The shortcoming of the constructions of the previous section is that the resulting formulas become more and more involved to higher order in perturbation theory. Moreover, to any order on the light cone, one gets an infinite number of contributions. In order to clarify the structure of the singularities on the light-cone, it is therefore essential to collect and rearrange the different contribution to the light-cone expansion. This procedure is called resummation of the light-cone expansion. After the resummation, the light-cone expansion of $\tilde{s}(x, y)$ will, to every order on the light cone, consist of only a finite number of terms. Before beginning, we remark that the resummation technique can also be understood from underlying gauge symmetries. In order no to mix mathematical constructions with physical considerations, we postpone the explanation of gauge phases and gauge transformations to 3.6 .2 (however, the idea of working with local transformations will be used in our constructions; see 2.2.82) and the computations thereafter).

In order to give a first idea of how the resummation works, we consider the leading singularity on the light cone by neglecting all terms of the order $\mathcal{O}\left((y-x)^{-2}\right)$. According to (2.2.8), we need to take into account only the contributions 2.2 .32 with $h=-1$. The inequality $(2.2 .36)$ implies that no derivatives of the potentials appear. Moreover, we obtain from (2.2.34) that $|I|=k+1$. Using the rules (a) and (b), we conclude that one tensor index of the multi-index $I$ is contracted with a Dirac matrix, whereas all the remaining $k$ indices of $I$ are contracted with chiral potentials. Therefore, all $k$ potentials are chiral, and no dynamical mass matrices appear. A detailed calculation yields for 
the $k^{\text {th }}$ order Feynman diagram a term of precisely this structure,

$$
\begin{aligned}
& \chi_{c}\left((-s B)^{k} s\right)(x, y)=\chi_{c}(-i)^{k} \int_{x}^{y} d z_{1}(y-x)_{j_{1}} A_{c}^{j_{1}}\left(z_{1}\right) \\
& \quad \times \int_{z_{1}}^{y} d z_{2}\left(y-z_{1}\right)_{j_{2}} A_{c}^{j_{2}}\left(z_{2}\right) \cdots \int_{z_{k-1}}^{y} d z_{k}\left(y-z_{k}\right)_{j_{k}} A_{c}^{j_{k}}\left(z_{k}\right) s(x, y)+\mathcal{O}\left((y-x)^{-2}\right) .
\end{aligned}
$$

The obtained nested line integrals can be identified with the summands of the familiar Dyson series. This allows us to carry out the sum over all Feynman diagrams,

$$
\chi_{c} \tilde{s}(x, y)=\chi_{c} \operatorname{Pexp}\left(-i \int_{x}^{y}(y-x)_{j} A_{c}^{j}(z) d z\right) s(x, y)+\mathcal{O}\left((y-x)^{-2}\right),
$$

where Pexp is defined as follows.

DeFinition 2.2.5. For a smooth one-parameter family of matrices $F(\alpha), \alpha \in \mathbb{R}$, the ordered exponential $\operatorname{Pexp}\left(\int F(\alpha) d \alpha\right)$ is given by the Dyson series

$$
\begin{aligned}
\operatorname{Pexp}\left(\int_{a}^{b} F(\alpha) d \alpha\right)=\mathbb{1} & +\int_{a}^{b} F\left(t_{0}\right) d t_{0}+\int_{a}^{b} d t_{0} F\left(t_{0}\right) \int_{t_{0}}^{b} d t_{1} F\left(t_{1}\right) \\
& +\int_{a}^{b} d t_{0} F\left(t_{0}\right) \int_{t_{0}}^{b} d t_{1} F\left(t_{1}\right) \int_{t_{1}}^{b} d t_{2} F\left(t_{2}\right)+\cdots .
\end{aligned}
$$

For ordered exponentials over the chiral potentials, we use the short notations

$$
\begin{aligned}
& \operatorname{Pexp}\left(-i \int_{x}^{y}(y-x)_{j} A_{c}^{j}(z) d z\right)=\operatorname{Pexp}\left(-i \int_{x}^{y} A_{c}^{j}(y-x)_{j}\right)=\mathrm{Pe}^{-i \int_{x}^{y} A_{c}^{j}(y-x)_{j}} \\
& \quad:=\operatorname{Pexp}\left(-\left.i \int_{0}^{1} A_{c}^{j}\right|_{\alpha y+(1-\alpha) x}(y-x)_{j} d \alpha\right) .
\end{aligned}
$$

Sometimes, we shall find it more convenient to write $\operatorname{Pexp}(\cdots)$ as $\mathrm{Pe}^{(\cdots)}$. For elementary properties of the ordered exponentials we refer to Exercise 2.15. For the general background on the ordered exponential we refer to [RS2, X.12] or to the closely related time-ordered or path-ordered exponential in the physics literature (see for example $\mathbf{P S}$, Section 4.2]). The connection to local gauge transformations is explained in Exercise 2.16.

To lower order on the light cone, the situation clearly is more complicated. The idea is to rearrange the contributions of the light-cone expansion in a such a way that certain subseries can be summed up to again obtain ordered exponentials of the chiral potentials. This idea is made precise in the following proposition and theorem, which we state and explain before giving their proofs.

Note that the partial derivatives in 2.2 .32 may be contracted with the factors $y-x$. If this is the case, the corresponding combination

$$
(y-x)^{j} \frac{\partial}{\partial z_{k}^{j}}
$$

is a derivative in the direction of the vector $y-x$. Since the direction $y-x$ is tangential to the corresponding line integral, such so-called tangential derivatives can be rewritten as derivatives with respect to the corresponding integration variable (for details see Exercise 2.17 or the proof of Proposition 2.2.6 below). Integrating by parts, the tangential derivatives disappear. Proceeding in this way, one can in fact eliminate all tangential derivatives, as is made precise in the following Proposition. 
Proposition 2.2.6. (elimination of tangential derivatives)

Every contribution 2.2.32 to the light cone expansion of Theorem 2.2.4 can be written as a finite sum of expressions of the form

$$
\begin{aligned}
\chi_{c} C(y-x)^{K} W^{(0)}(x) & \int_{x}^{y}\left[l_{1}, r_{1} \mid n_{1}\right] d z_{1} W^{(1)}\left(z_{1}\right) \int_{z_{1}}^{y}\left[l_{2}, r_{2} \mid n_{2}\right] d z_{2} W^{(2)}\left(z_{2}\right) \\
& \cdots \int_{z_{\alpha-1}}^{y}\left[l_{\alpha}, r_{\alpha} \mid n_{\alpha}\right] d z_{\alpha} W^{(\alpha)}\left(z_{\alpha}\right) \gamma^{J} S^{(h)}(x, y)
\end{aligned}
$$

with $\alpha \leq k$, where the factors $W^{(\beta)}$ are composed of the potentials and their partial derivatives,

$$
W^{(\beta)}=\left(\partial^{K_{a_{\beta}}} \square^{p_{a_{\beta}}} V_{J_{a_{\beta}, c_{a_{\beta}}}^{\left(a_{\beta}\right)}}^{(\beta)} \cdots\left(\partial^{K_{b_{\beta}}} \square^{p_{b_{\beta}}} V_{J_{b_{\beta}}, c_{b_{\beta}}}^{\left(b_{\beta}\right)}\right)\right.
$$

with $a_{1}=1, a_{\beta+1}=b_{\beta}+1, b_{\beta} \geq a_{\beta}-1$ (in the case $b_{\beta}=a_{\beta}-1, W^{(\beta)}$ is identically one), and $b_{\alpha}=k$. The parameters $l_{a}, r_{a}$, and $n_{a}$ are non-negative integers, $C$ is a complex number, and $c=L / R, c_{a}=L / R$ are chiral indices. The potentials $V^{(a)}$ are again given by (2.2.33); their chirality is determined by the rule (i) in Theorem 2.2.4. The tensor indices of the multi-indices $J, K, J_{a}$, and $K_{a}$ are all contracted with each other, according to the rules (a),(b) of Theorem 2.2.4 and

(c) The tensor indices of $(y-x)^{K}$ are all contracted with the tensor indices of the factors $V_{J_{a}}^{(a)}$ or $\gamma^{J}$ (but not with the factors $\partial^{K_{a}}$ ).

We have the relation

$$
2 h=k-1-|K|+\sum_{a=1}^{k}\left(\left|K_{a}\right|+2 p_{a}\right) .
$$

Before coming to the proof, we make precise how this proposition can be used to simplify the light-cone expansion.

Definition 2.2.7. A contribution of the form (2.2.32) to the light-cone expansion of Theorem 2.2.6 is called phase-free if all the tangential potentials $V_{J_{a}}^{(a)}$ are differentiated, i.e.

$$
\left|K_{a}\right|+2 p_{a}>0 \quad \text { whenever } \quad J_{a} \text { is contracted with }(y-x)^{K} .
$$

From every phase-free contribution the corresponding phase-inserted contribution is obtained as follows: We insert ordered exponentials according to the replacement rule

$$
W^{(\beta)}\left(z_{\beta}\right) \longrightarrow W^{(\beta)}\left(z_{\beta}\right) \operatorname{Pexp}\left(-i \int_{z_{\beta}}^{z_{\beta+1}} A_{c_{\beta}}^{j_{\beta}}\left(z_{\beta+1}-z_{\beta}\right)_{j_{\beta}}\right), \quad \beta=0, \ldots, \alpha,
$$

where we set $z_{0}=x$ and $z_{\alpha+1}=y$. The chiralities $c_{\beta}$ are determined by the relations $c_{0}=$ $c$ and

$$
\begin{aligned}
c_{\beta-1} \text { and } c_{\beta}\left\{\begin{array}{c}
\text { coincide } \\
\text { are opposite }
\end{array}\right\} \\
\text { if } W^{(\beta-1)} \text { contains an }\left\{\begin{array}{c}
\text { even } \\
\text { odd }
\end{array}\right\} \text { number of factors } Y .
\end{aligned}
$$

THEOREM 2.2.8. To every order on the light cone, the number of phase-free contributions is finite. The light-cone expansion of the Green's function $\tilde{s}(x, y)$ is given by the sum of the corresponding phase-inserted contributions. 
This theorem gives a convenient procedure for performing the light-cone expansion of the Green's function. The only task is to compute to any order on the light cone the finite number of phase-free contributions. Then one inserts ordered exponentials according to Definition 2.2.7. Note that this method is constructive in the sense that it gives a procedure with which the light-cone expansion of every Feynman diagram can be carried out explicitly. Indeed, this procedure is implemented in the $\mathrm{C}++$-program class_commute $\mathrm{2}^{2}$. These computations are illustrated in Exercise 2.18.

The remainder of this section is devoted to the proof of Proposition 2.2.6 and Theorem 2.2.8. We begin with a preparatory lemma which controls the number of tangential derivatives in the contributions 2.2.32 in Theorem 2.2.4.

Lemma 2.2.9. For any $a \in\{1, \ldots, k\}$, we let $t_{a}$ be the number of tensor indices of the multi-index $I_{a}$ in 2.2 .32 which are contracted with the factor $(y-x)^{I}$. Then the following inequalities hold for all $a=1, \ldots, k$ :

$$
l_{a}+n_{a} \geq t_{a}-1 \quad \text { and } \quad r_{a}+n_{a} \geq \sum_{b=a}^{k} t_{b} .
$$

Proof. As in the proof of Theorem 2.2.4, we proceed inductively in the order $k$ of the perturbation theory. For $k=0$, the inequalities 2.2.64) are trivially satisfied according to (2.2.37). Assume that (2.2.64) is true for a given $k$. We go through the construction steps (1) to (7) of Theorem 2.2.4 and check that the inequalities (2.2.64) then also hold in 2.2.51 for $a=0, \ldots, k$.

We first consider the case $a>0$. The parameters $l_{a}, r_{a}$, and $n_{a}$ remain unchanged in all the construction steps of Theorem 2.2.4. Furthermore, it is obvious that the parameters $t_{a}$ are not affected in the steps (1), (2), (4) and (7). In the steps (3) and (5), the computation of the derivatives $\square_{z}^{n}$ and $\not_{x}$ might annihilate some of the factors $(y-x)$ which were contracted with the factors $\partial_{z_{a}}^{I_{a}}$; this may decrease the parameters $t_{a}$. For the analysis of step (6), note that all $\gamma$-matrices which are contracted with factors $(y-x)$ stand to the left of those $\gamma$-matrices which are contracted with the $\partial_{z_{a}}^{I_{a}}, a=1, \ldots, k$ (this follows from the ordering condition (b) in the induction hypothesis and the fact that additional factors $(y-x)^{j} \cdots \gamma_{j}$ are only generated during the construction if the partial derivative $\not_{x}$ hits $S^{(\hat{h})}$ in step (5); in this case, the corresponding $\gamma$-matrix stands at the very left in $\gamma^{J}$ ). Therefore the commutations of the Dirac matrices do not lead to additional contractions between factors $(y-x)$ and $\partial_{z_{a}}^{I_{a}}$, which implies that the parameters $t_{a}$ remain unchanged in step (6). We conclude that the $l_{a}, r_{a}$, and $n_{a}$ remain unchanged whereas the $t_{a}$ may only decrease, and thus (2.2.64) holds for $a=1, \ldots, k$ throughout all the construction steps.

It remains to show that the inequalities (2.2.64) hold in $(2.2 .51)$ for $a=0$. We first look at the situation after step (4) in 2.2.45): The values 2.2 .46$)$ for $l_{0}, r_{0}$, and $n_{0}$ give in combination with 2.2 .43 the equations

$$
\begin{aligned}
l_{0}+n_{0} & =\frac{1}{2}\left(|I|-|\hat{I}|+\left|I_{0}\right|+2 p_{0}\right) \\
r_{0}+n_{0} & =h+\frac{1}{2}\left(|I|+|\hat{I}|+\left|I_{0}\right|+2 p_{0}\right) .
\end{aligned}
$$

\footnotetext{
${ }^{2}$ The $\mathrm{C}++$ program class_commute and its computational output as well as the resulting Mathematica worksheets were included as ancillary files to the arXiv submission arXiv:1211.3351 [math-ph].
} 
Moreover, the number of tangential derivatives $t_{0}$ at the first potential is clearly bounded by the total number of derivatives there,

$$
\left|I_{0}\right| \geq t_{0}
$$

Furthermore, the total number of tangential derivatives is smaller than the number of factors $(y-x)$,

$$
|\hat{I}| \geq \sum_{a=0}^{k} t_{a}
$$

Substituting (2.2.44) and (2.2.67) into (2.2.65) yields the inequalities

$$
l_{0}+n_{0} \geq\left|I_{0}\right|+p_{0} \geq t_{0} .
$$

In order to get a bound for $r_{0}+n_{0}$, we must distinguish two cases. If $h \geq 0$, we substitute 2.2.44 into 2.2.66) and get with 2.2.68 the inequality

$$
r_{0}+n_{0} \geq|\hat{I}|+\left|I_{0}\right|+p_{0} \geq|\hat{I}| \geq \sum_{a=0}^{k} t_{a} .
$$

In the case $h=-1,(2.2 .36)$ shows that $\left|I_{a}\right|$, and consequently also $t_{a}$, vanish for $1 \leq a \leq$ $k$. Furthermore, (2.2.34) yields that $|I| \neq 0$. Thus (2.2.66) and (2.2.67), 2.2.68) give the bound

$$
r_{0}+n_{0} \geq h+\frac{|I|}{2}+\frac{1}{2} \sum_{a=0}^{k} t_{a}+\frac{1}{2} t_{0} \geq \frac{1}{2} \sum_{a=0}^{k} t_{a}+\frac{1}{2} t_{0},
$$

where we used in the last inequality that $h+|I| / 2 \geq-1 / 2$ and that all the other terms are integers. Since $t_{0}=\sum_{a=0}^{k} t_{a}$, we conclude that inequality 2.2 .70 also holds in the case $h=-1$.

We finally consider how the bounds $(2.2 .69)$ and $(2.2 .70)$ for $l_{0}+n_{0}$ and $r_{0}+n_{0}$ must be modified in the subsequent construction steps. In step (5), the partial derivative $\not_{x}$ may annihilate a factor $(y-x)$, in which case the parameters $t_{a}$ might decrease. On the other hand, the partial derivatives $\partial_{x}$ may produce an additional factor $\partial_{z_{0}}$; in this case, $r_{0}$ is incremented according to 2.2.48). In step (6), only this additional factor $\partial_{z_{0}}$ may be contracted with $(y-x)^{\hat{I}}$. Step $(7)$ does not change $l_{0}, r_{0}, n_{0}$, and $t_{0}$. Putting these transformations together, we conclude that the inequality 2.2 .69 for $l_{0}+n_{0}$ must be weakened by one, whereas the bound $(2.2 .70)$ for $r_{0}+n_{0}$ remains valid as it is. This gives precisely the inequalities (2.2.64) for $a=0$.

Proof of Proposition 2.2.6. The basic method for the proof is to iteratively eliminate those partial derivatives $\partial_{z_{a}}^{I_{a}}$ in 2.2 .32 which are contracted with a factor $(y-x)$. This is accomplished with the integration-by-parts formula

$$
\begin{aligned}
& (y-x)^{j} \int_{x}^{y}[l, r \mid n] d z \partial_{j} f(z) \stackrel{2.2 .31}{-} \int_{0}^{1} d \alpha \alpha^{l}(1-\alpha)^{r}\left(\alpha-\alpha^{2}\right)^{n} \frac{d}{d \alpha} f(\alpha y+(1-\alpha) x) \\
& =\delta_{r+n, 0} f(y)-\delta_{l+n, 0} f(x) \\
& \quad-(l+n) \int_{x}^{y}[l-1, r \mid n] d z f(z)+(r+n) \int_{x}^{y}[l, r-1 \mid n] d z f(z) .
\end{aligned}
$$


In order to see the main difficulty, we consider the example of two nested line integrals with two tangential derivatives

$$
\begin{aligned}
(y-x)^{j}(y-x)^{k} \int_{x}^{y}[0,1 \mid 0] d z_{1} V^{(1)}\left(z_{1}\right) \int_{z_{1}}^{y}[0,1 \mid 0] d z_{2} \partial_{j k} V^{(2)}\left(z_{2}\right) \\
=(y-x)^{j} \int_{x}^{y}[0,0 \mid 0] d z_{1} V^{(1)}\left(z_{1}\right)\left(y-z_{1}\right)^{k} \int_{z_{1}}^{y}[0,1 \mid 0] d z_{2} \partial_{j k} V^{(2)}\left(z_{2}\right) \\
=-(y-x)^{j} \int_{x}^{y} d z_{1} V^{(1)}\left(z_{1}\right) \partial_{j} V^{(2)}\left(z_{1}\right) \\
\quad+(y-x)^{j} \int_{x}^{y} d z_{1} V^{(1)}\left(z_{1}\right) \int_{z_{1}}^{y} d z_{2} \partial_{j} V^{(2)}\left(z_{2}\right) .
\end{aligned}
$$

Although the line integrals in (2.2.71) satisfy the conditions of Theorem 2.2.4, the expression cannot be transformed into the required form $(2.2 .59)$. Namely, in $(2.2 .72)$ we cannot eliminate the remaining tangential derivative (because partial integration would yield a term $\left.(y-x)^{j} \partial_{j} V^{(1)}\left(z_{1}\right)\right)$. In $(2.2 .73)$, on the other hand, we can successfully perform a second partial integration

$$
2.2 .73=\int_{x}^{y}[0,-1 \mid 0] d z_{1} V^{(1)}\left(z_{1}\right)\left(V^{(2)}(y)-V^{(2)}\left(z_{1}\right)\right),
$$

but then the second parameter in the bracket [.,.|.] becomes negative. More generally, we must ensure that the boundary terms contain no tangential derivatives, and that the parameters $l_{a}, r_{a}$, and $n_{a}$ stay positive in the construction.

Since the chirality of the potentials is not affected by the partial integrations, it is obvious that the rule (i) in Theorem 2.2.4 will remain valid. For ease in notation, in the remainder of the proof we usually omit the indices $c_{a}$.

First of all, we split up the factor $(y-x)^{I}$ in $(2.2 .32)$ in the form $(y-x)^{I}=(y-$ $x)^{K}(y-x)^{L}$, where $L$ are those tensor indices which are contracted with the partial derivatives $\partial_{z_{a}}^{I_{a}}, a=1, \ldots, k$. Setting $b=1$ and $z_{0}=x$, the first line integral in 2.2 .32 can be written as

$$
\cdots\left(y-z_{b-1}\right)^{L} \int_{z_{b-1}}^{y}\left[l_{b}, r_{b} \mid n_{b}\right] d z_{b} \partial_{z_{b}}^{I_{b}} \square_{z_{b}}^{p_{b}} V_{J_{b}}^{(b)}\left(z_{b}\right) \cdots .
$$

We rewrite the tangential derivatives in this line integral as derivatives in the integration variable,

$$
=\cdots\left(y-z_{b-1}\right)^{N} \int_{0}^{1} d \alpha \alpha^{l}(1-\alpha)^{r}\left(\frac{d}{d \alpha}\right)^{q} \partial_{z_{b}}^{K_{b}} \square_{z_{b}}^{p_{b}} V_{J_{b}}^{(b)}\left(z_{b}\right) \cdots
$$

with $|L|=|N|+q$ and $l=l_{b}+n_{b}, r=r_{b}+n_{b}$. Lemma 2.2.9 gives the bounds

$$
l \geq q-1 \quad \text { and } \quad r \geq q+|N| .
$$

More generally, we use (2.2.75) and 2.2.76) as our induction hypothesis, where the left factor '...' stands for all previous line integrals (which contain no tangential derivatives), and the right factor '.. ' stands for subsequent line integrals. The tensor indices of the factor $\left(y-z_{a-1}\right)^{N}$ must all be contracted with the partial derivatives $\partial_{z_{a}}^{I_{a}}$ for $a>b$ and thus give tangential derivatives in the subsequent line integrals. The induction step is to show that all the $\alpha$-derivatives in 2.2 .75 can be eliminated, and that we can write the resulting expressions again in the form (2.2.75) and (2.2.76) with $b$ replaced by $b+1$. Under the assumption that this induction step holds, we can eliminate all tangential derivatives in $k$ steps. The resulting expressions are very similar to 2.2.59) and 2.2.60). 
The only difference is that the derivatives $\partial^{K_{a}}$ and $\square^{p_{a}}$ in the resulting expressions are differential operators acting on all the following factors $V^{(a)}, V^{(a+1)}, \ldots$; in $(2.2 .60)$, on the other hand, the partial derivatives act only on the adjacent potential $V^{(a)}$. In order to bring the resulting expressions into the required form, we finally carry out all the derivatives with the Leibniz rule and the chain rule (2.2.48).

For the proof of the induction step, we integrate in (2.2.75) $q$ times by parts (if $q$ is zero, we can skip the partial integrations; our expression is then of the form (2.2.78). Since the powers of the factors $\alpha$ and $(1-\alpha)$ are decreased at most by one in each partial integration step, 2.2.76) implies that the boundary values vanish unless in the last step for $\alpha=0$. We thus obtain a sum of terms of the form

$$
\cdots\left(y-z_{b-1}\right)^{N} \partial_{z_{b}}^{K_{b}} \square_{z_{b}}^{p_{b}} V_{J_{b}}^{(b)}\left(z_{b}\right) \cdots{ }_{\mid z_{b} \equiv z_{b-1}}
$$

and

$$
\cdots\left(y-z_{b-1}\right)^{N} \int_{z_{b-1}}^{y}[l, r \mid n=0] d z_{b} \partial_{z_{b}}^{K_{b}} \square_{z_{b}}^{p_{b}} V_{J_{b}}^{(b)}\left(z_{b}\right)
$$

$$
\text { with } l \geq 0, r \geq|N| \text {. }
$$

In 2.2.78), we iteratively use the relation

$$
(y-x)^{j} \int_{x}^{y}[l, r \mid n] d z \cdots=\int_{x}^{y}[l, r-1 \mid n] d z(y-z)^{j} \cdots
$$

to bring all factors $\left(y-z_{b-1}\right)$ to the right. We thus obtain expressions of the form

$$
2.2 .78=\cdots \int_{z_{b-1}}^{y}[l, r \mid n=0] d z_{b}\left(y-z_{b}\right)^{N} \partial_{z_{b}}^{K_{b}} \square_{z_{b}}^{p_{b}} V_{J_{b}}^{(b)}\left(z_{b}\right) \cdots \quad \text { with } l, r \geq 0 .
$$

In both cases (2.2.77) and (2.2.79), we have an expression of the form

$$
\cdots\left(y-z_{b}\right)^{N} \partial_{z_{b}}^{K_{b}} \square_{z_{b}}^{p_{b}} V_{J_{b}}^{(b)}\left(z_{b}\right) \cdots,
$$

where the first factor '...' stands for line integrals without tangential derivatives, and where none of the factors $\left(y-z_{b}\right)$ are contracted with $\partial_{z_{b}}^{K_{b}}$. Applying the "inverse Leibniz rules"

$$
\begin{gathered}
(y-x)^{j} \frac{\partial}{\partial x^{k}}=\frac{\partial}{\partial x^{k}}(y-x)^{j}+\delta_{k}^{j} \\
(y-x)_{j} \square_{x}=\square_{x}(y-x)_{j}+2 \frac{\partial}{\partial x^{j}},
\end{gathered}
$$

we iteratively commute all factors $\left(y-z_{b}\right)$ in $(2.2 .80)$ to the right. This gives a sum of expressions of the form

$$
\cdots \partial_{z_{b}}^{K_{b}} \square_{z_{b}}^{p_{b}} V_{J_{b}}^{(b)}\left(z_{b}\right)\left(y-z_{b}\right)^{L} \cdots,
$$

where the factors $\left(y-z_{b}\right)$ are all contracted with the partial derivatives $\partial_{z_{a}}^{I_{a}}, a=b+$ $1, \ldots, k$. The Leibniz rules may have annihilated some factors $\left(y-z_{b}\right)$ (i.e., $|L|$ might be smaller than $|N|)$; in this case, the parameters $t_{a}, a=b+1, \ldots, k$ have decreased. As a consequence, the inequalities of Lemma 2.2.9 are still valid for all expressions (2.2.81). If we write (2.2.81) in the form 2.2.74) with $b$ replaced by $b+1$, we can thus split up the tangential derivatives in the form (2.2.75) and 2.2.76). This concludes the proof of the induction step.

It remains to derive equation 2.2.61): Note that each integration by parts decreases both the number of factors $\left(y-z_{a-1}\right)$ and the total number of partial derivatives by one. If we carry out the remaining derivatives with the Leibniz rule (in the last step of the 
proof), this does not change the total order $\sum_{a=1}^{k}\left|K_{a}\right|+2 p_{a}$ of the derivatives. Therefore, relation (2.2.34) in Theorem 2.2.4 transforms into 2.2.61).

We come to the proof of Theorem 2.2.8. A possible method would be to rearrange all the contributions to the light-cone expansion of Theorem 2.2.4 until recovering the Dyson series of the ordered exponentials in (2.2.62). However, this method has the disadvantage of being rather involved. It is more elegant to use a particular form of local gauge invariance of the Green's function for the proof (for basics see Exercise 2.16). To this end, for given $x$ and $y$ we will transform the spinors locally. The transformation will be such that the light-cone expansion of the transformed Green's function $\hat{s}(x, y)$ consists precisely of all phase-free contributions. Using the transformation law of the Green's function, we then show that the light-cone expansion of $\tilde{s}(x, y)$ is obtained from that of $\hat{s}(x, y)$ by inserting unitary matrices into the line integrals. Finally, we prove that these unitary matrices coincide with the ordered exponentials in Definition 2.2.7.

In preparation, we consider the transformation law of the Dirac operator and the Green's function under generalized local phase transformations of the spinors. We let $U_{L}(x)$ and $U_{R}(x)$ be two unitary matrices acting on the Lie algebra index of the gauge potential. We transform the wave functions according to

$$
\psi(x) \rightarrow \hat{\psi}(x)=U(x) \psi(x) \quad \text { with } \quad U(x)=\chi_{L} U_{L}(x)+\chi_{R} U_{R}(x) .
$$

Thus $U_{L}$ and $U_{R}$ transform the left and right handed component of the wave functions, respectively. We point out that transformation $U$ is not unitary with respect to the spin scalar product because $\chi_{L}^{*}=\chi_{R}$ and therefore

$$
\begin{aligned}
V & :=U^{-1}=\chi_{L} U_{L}^{-1}+\chi_{R} U_{R}^{-1} \quad \text { but } \\
U^{*} & =\gamma^{0} U^{\dagger} \gamma^{0}=\chi_{R} U_{L}^{-1}+\chi_{L} U_{R}^{-1} .
\end{aligned}
$$

Therefore, in what follows we carefully distinguish between $U, U^{*}$ and their inverses $V$ and $V^{*}$. As an immediate consequence of the Dirac equation $(i \not \partial+\mathcal{B}-m) \psi=0$, the transformed wave functions $\hat{\psi}$ satisfies the equation

$$
V^{*}(i \not \partial+B) V \hat{\psi}=0 .
$$

A short computation yields for the transformed Dirac operator

$$
V^{*}(i \not \partial+B) V=i \not \partial+\hat{B}
$$

with

$$
\hat{B}=\chi_{L}\left(\hat{A}_{R}-m \hat{Y}_{L}\right)+\chi_{R}\left(\hat{A}_{L}-m \hat{Y}_{R}\right),
$$

where $\hat{A}_{L / R}$ and $\hat{Y}_{L / R}$ are the potentials

$$
\begin{aligned}
& \hat{A}_{L / R}^{j}=U_{L / R} A_{L / R}^{j} U_{L / R}^{-1}+i U_{L / R}\left(\partial^{j} U_{L / R}^{-1}\right) \\
& \hat{Y}_{L / R}=U_{R / L} Y U_{L / R}^{-1} .
\end{aligned}
$$

We denote the advanced and retarded Green's functions of the transformed Dirac operator $i \not \partial+\hat{B}$ by $\hat{s}$. They satisfy the equation

$$
\left(i \not \partial_{x}+\hat{B}(x)\right) \hat{s}(x, y)=\delta^{4}(x-y) .
$$


Since we can view $\hat{B}$ as the perturbation of the Dirac operator, the Green's function $\hat{s}$ has, in analogy to 2.2.12, the perturbation expansion

$$
\hat{s}=\sum_{n=0}^{\infty}(-s \hat{B})^{n} s .
$$

The important point for what follows is that the Green's functions $\tilde{s}$ and $\hat{s}$ are related to each other by the local transformation

$$
\hat{s}(x, y)=U(x) \tilde{s}(x, y) U(y)^{*} .
$$

This is verified as follows: The right side of (2.2.87) also satisfies the defining equation 2.2.85) of the Green's functions; namely

$$
\begin{aligned}
& \left(i \not \partial_{x}+\hat{B}(x)\right) U(x) \tilde{s}(x, y) U(y)^{*}=V(x)^{*}\left(i \not \partial_{x}+B(x)\right) V(x) U(x) \tilde{s}(x, y) U(y)^{*} \\
& \quad=V(x)^{*}\left(i \not \partial_{x}+B(x)\right) \tilde{s}(x, y) U(y)^{*}=V(x)^{*} \delta^{4}(x-y) U(y)^{*} \\
& \quad=V(x)^{*} U(x)^{*} \delta^{4}(x-y)=\delta^{4}(x-y) .
\end{aligned}
$$

Furthermore, the supports of both sides of (2.2.87) lie (depending on whether we consider the advanced or retarded Green's functions) either in the upper or in the lower light cone. A uniqueness argument for the solutions of hyperbolic differential equations yields that both sides of (2.2.87) coincide.

We next specify the unitary transformations $U_{L}$ and $U_{R}$ : We fix the points $x$ and $y$. For any point $z$ on the line segment $\overline{x y}$, we chose $U_{L / R}(z)$ as

$$
U_{L / R}(z)=\operatorname{Pexp}\left(-i \int_{x}^{z} A_{L / R}^{j}(z-x)_{j}\right) .
$$

Using the differential equation for the ordered exponential (see Exercise 2.15)

$$
(y-x)^{k} \frac{\partial}{\partial x^{k}} \mathrm{Pe}^{-i \int_{x}^{y} A_{c}^{j}(y-x)_{j}}=i(y-x)_{k} A_{c}^{k}(x) \mathrm{Pe}^{-i \int_{x}^{y} A_{c}^{j}(y-x)_{j}},
$$

we obtain

$$
\begin{aligned}
(y-x)^{j} U_{c}(z)\left(\partial_{j} U_{c}(z)^{-1}\right) & =\mathrm{Pe}^{-i \int_{x}^{z} A_{c}^{k}(z-x)_{k}}(y-x)^{j} \frac{\partial}{\partial z^{j}} \mathrm{Pe}^{-i \int_{z}^{x} A_{c}^{k}(x-z)_{k}} \\
& =\mathrm{Pe}^{-i \int_{x}^{z} A_{c}^{k}(z-x)_{k}} i(y-x)_{j} A_{c}^{j}(z) \mathrm{Pe}^{-i \int_{z}^{x} A_{c}^{k}(x-z)_{k}} \\
& =i(y-x)_{j} U_{c}(z) A_{c}^{j}(z) U_{c}(z)^{-1}
\end{aligned}
$$

Using this formula in 2.2 .83 gives

$$
\hat{A}_{L / R}^{j}(z)(y-x)_{j}=0 \quad \text { for } z \in \overline{x y} .
$$

Thus our choice of $U_{L}$ and $U_{R}$ makes the potentials $\hat{A}_{L}(z)$ and $\hat{A}_{R}(z)$ for $z \in \overline{x y}$ orthogonal to the vector $(y-x)$. Before going on, we point out that we did not specify $U_{L / R}(z)$ away from the line segment $z \in \overline{x y}$; the unitary transformation $U_{L / R}$ may be arbitrary there. This also implies that also $\hat{A}_{L / R}$ is undetermined outside the line segment $\overline{x y}$. In particular, all the non-tangential derivatives of $\hat{A}_{L / R}(z)$ for $z \in \overline{x y}$ are undetermined. However, 2.2.88) does give constraints for the tangential derivatives. For example, differentiating (2.2.90) in the direction $(y-x)$ yields

$$
(y-x)^{j}(y-x)_{k} \partial_{j} \hat{A}_{L / R}^{k}(z)=0 \quad \text { for } \quad z \in \overline{x y} .
$$


We now consider the perturbation expansion (2.2.86). The light-cone expansion of all Feynman diagrams according to Theorem 2.2.4 gives a sum of terms of the form

$$
\begin{gathered}
\chi_{c} C(y-x)^{K} \hat{W}^{(0)}(x) \int_{x}^{y}\left[l_{1}, r_{1} \mid n_{1}\right] d z_{1} \hat{W}^{(1)}\left(z_{1}\right) \int_{z_{1}}^{y}\left[l_{2}, r_{2} \mid n_{2}\right] d z_{2} \hat{W}^{(2)}\left(z_{2}\right) \\
\ldots \int_{z_{\alpha-1}}^{y}\left[l_{\alpha}, r_{\alpha} \mid n_{\alpha}\right] d z_{\alpha} \hat{W}^{(\alpha)}\left(z_{\alpha}\right) \gamma^{J} S^{(h)}(x, y),
\end{gathered}
$$

where the factors $\hat{W}^{(\beta)}$ are of the form

$$
\hat{W}^{(\beta)}=\left(\partial^{K_{a_{\beta}}} \square^{p_{a_{\beta}}} \hat{V}_{J_{a_{\beta}}, c_{a_{\beta}}}^{\left(a_{\beta}\right)}\right) \cdots\left(\partial^{K_{b_{\beta}}} \square^{p_{b_{\beta}}} \hat{V}_{J_{b_{\beta}}, c_{b_{\beta}}}^{\left(b_{\beta}\right)}\right) .
$$

Because of 2.2.90), all the contributions which are not phase-free vanish. Furthermore, according to Theorem 2.2.4, the contributions (2.2.91) and (2.2.92) contain no tangential derivatives. Clearly, the derivatives in these formulas may have a component in direction of $(y-x)$. But the contribution of the derivatives transversal to $(y-x)$ uniquely determines the form of each derivative term. Therefore, all the phase-free contributions of the form (2.2.91) and 2.2.92) are independent in the sense that we have no algebraic relations between them. We conclude that, as long as the potentials $\hat{A}_{L / R}$ and $\hat{Y}_{L / R}$ are only specified by (2.2.83), 2.2.84) and (2.2.88), the light-cone expansion (2.2.91) and (2.2.92) consists precisely of all phase-free contributions.

Next, we exploit the local transformation law (2.2.87) of the Green's functions: We solve this equation for $\tilde{s}$,

$$
\tilde{s}(x, y)=V(x) \hat{s}(x, y) V(y)^{*} .
$$

The transformation $U_{L / R}$ does not enter on the left side of this equation. Thus the right side of 2.2.93 is also independent of $U_{L / R}$. In particular, we conclude that the lightcone expansion of $\hat{s}(x, y)$ must be independent of the derivatives of $U_{L / R}$ along the line segment $\overline{x y}$. At first sight, this might seem inconsistent because the individual contributions 2.2.91 and 2.2.92 do depend on the derivatives of $U_{L / R}$ (this is obvious if one substitutes (2.2.83) and (2.2.84) into 2.2.92) and carries out the derivatives with the Leibniz rule). The right way to understand the independence of $\hat{s}(x, y)$ on the derivatives of $U_{L / R}$ is that all derivative terms of $U_{L / R}$ cancel each other to every order on the light cone if the (finite) sum over all contributions (2.2.91) to the light-cone expansion of $\hat{s}(x, y)$ is carried out. Since we will form the sum over all contributions to the light-cone expansion in the end, it suffices to consider only those contributions to the light-cone expansion which contain no derivatives of $U_{L / R}$. This means that we can substitute (2.2.83) and 2.2.84 into 2.2.92, forget about the derivative term $i U_{L / R}\left(\partial^{j} U_{L / R}^{-1}\right)$ in 2.2.83), and pull the unitary transformations $U_{L / R}, U_{L / R}^{-1}$ out of the derivatives. In other words, we can replace $\hat{W}^{(\beta)}, 2.2 .92$, by

$$
\hat{W}^{(\beta)}=U_{d_{a_{\beta}}}\left(\partial^{K_{a_{\beta}}} \square^{p_{a_{\beta}}} V_{J_{a_{\beta}}, c_{a_{\beta}}}^{\left(a_{3}\right)}\right) U_{c_{a_{\beta}}}^{-1} \cdots U_{d_{b_{\beta}}}\left(\partial^{K_{b_{\beta}}} \square^{p_{b_{\beta}}} V_{J_{b_{\beta}}, c_{b_{\beta}}}^{\left(b_{\beta}\right)}\right) U_{c_{b_{\beta}}}^{-1}
$$

with chiral indices $c_{a}, d_{a}=L / R$. The light-cone expansion of $\hat{s}(x, y)$ consists precisely of the sum of all phase-free contributions of the form (2.2.91) and (2.2.94).

The chiralities $c_{a}, d_{a}$ of the unitary transformations $U_{L / R}, U_{L / R}^{-1}$ in (2.2.94) are determined by the rule (i) in Theorem 2.2.4 and by 2.2 .83 ) and (2.2.84). According to this rule, the indices $c_{a-1}$ and $c_{a}$ coincide iff $V^{(a)}$ is a chiral potential. According to 2.2 .83 ) and 2.2.84, on the other hand, the indices $d_{a}$ and $c_{a}$ coincide iff $V^{(a)}=A_{L / R}$. We 
conclude that the indices $c_{a-1}$ and $d_{a}$ always coincide. Thus all the intermediate factors $U_{c_{a-1}}^{-1} U_{d_{a}}$ give the identity, and 2.2.94 simplifies to

$$
\hat{W}^{(\beta)}=U_{d_{\beta}} W^{(\beta)} U_{c_{\beta}}^{-1} .
$$

Furthermore, the chiralities $d_{\beta}$ and $c_{\beta}$ coincide if and only if $W^{(\beta)}$ contains an even number of dynamic mass matrices.

Finally, we substitute the light-cone expansion 2.2.91) for $\hat{s}(x, y)$ as well as 2.2.95) into 2.2.93). This gives for the light-cone expansion of $\tilde{s}(x, y)$ a sum of expressions of the form

$$
\begin{gathered}
\chi_{c} C(y-x)^{K} U_{c}^{-1}(x)\left(U_{d_{0}} W^{(0)} U_{c_{0}}^{-1}\right)(x) \int_{x}^{y}\left[l_{1}, r_{1} \mid n_{1}\right] d z_{1}\left(U_{d_{1}} W^{(1)} U_{c_{1}}^{-1}\right)\left(z_{1}\right) \\
\ldots \int_{z_{\alpha-1}}^{y}\left[l_{\alpha}, r_{\alpha} \mid n_{\alpha}\right] d z_{\alpha}\left(U_{d_{\alpha}} W^{(0)} U_{c_{\alpha}}^{-1}\right)\left(z_{\alpha}\right) U_{c_{\alpha+1}}(y) \gamma^{J} S^{(h)}(x, y),
\end{gathered}
$$

where the sum runs over all phase-free contributions of this type. Similar to the considerations before (2.2.95), one sees that adjacent unitary transformations always have the same chirality. Therefore, renaming the chiral indices, the expressions 2.2 .96$)$ can be written in the simpler form

$$
\begin{aligned}
\chi_{c} C & (y-x)^{K} W^{(0)}(x) \int_{x}^{y}\left[l_{1}, r_{1} \mid n_{1}\right] d z_{1} U_{c_{1}}(x)^{-1} U_{c_{1}}\left(z_{1}\right) W^{(1)} \\
& \cdots \int_{z_{\alpha-1}}^{y}\left[l_{\alpha}, r_{\alpha} \mid n_{\alpha}\right] d z_{\alpha} U_{c_{\alpha}}\left(z_{\alpha-1}\right)^{-1} U_{c_{\alpha}}\left(z_{\alpha}\right) W^{(0)}\left(z_{\alpha}\right) U_{c_{\alpha+1}}\left(z_{\alpha}\right)^{-1} \\
& \times U_{c_{\alpha+1}}(y) \gamma^{J} S^{(h)}(x, y),
\end{aligned}
$$

where the chiral indices $c_{a}$ satisfy the rule 2.2.63). According to (2.2.88), the factors $U_{c}^{-1}(.) U_{c}($.$) coincide with the ordered exponentials in 2.2.62). This concludes the proof$ of Theorem 2.2.8.

2.2.5. The Residual Argument. In the previous sections, the light-cone expansion was performed for the causal Green's functions. We now want to extend our methods and results to the fermionic projector. We begin by describing how the light-cone expansion of the Green's functions can be understood in momentum space. Apart from giving a different point of view, this will make it possible to get a connection to the light-cone expansion of the fermionic projector. For notational simplicity, we restrict attention to the case $g=1$ where in 2.2 .26 there is only one direct summand (the generalization to several direct summands is obtained in a straightforward way by replacing all vacuum operators as in (2.2.26) by corresponding direct sums). As in (2.2.10), we again combine the rest mass and the external potential in a potential $B$. Furthermore, we only consider the advanced Green's function; for the retarded Green's function, the calculation is analogous.

Suppose that we want to perform the light-cone expansion of the $k^{\text {th }}$ order contribution to the perturbation series (2.2.12). Using that the Green's function is diagonal in momentum space and that multiplying by $B$ in position space corresponds to a convolution in momentum space, we can write the contribution as a multiple Fourier integral,

$$
\begin{aligned}
(( & \left.\left.-s^{\vee} B\right)^{k} s^{\vee}\right)(x, y) \\
& =\int \frac{d^{4} p}{(2 \pi)^{4}} \int \frac{d^{4} q_{1}}{(2 \pi)^{4}} \cdots \int \frac{d^{4} q_{k}}{(2 \pi)^{4}} \Delta s^{\vee}\left(p ; q_{1}, \ldots, q_{k}\right) e^{-i\left(p+q_{1}+\cdots+q_{k}\right) x+i p y},
\end{aligned}
$$


where the distribution $\Delta s^{\vee}\left(p ; q_{1}, \ldots, q_{k}\right)$ is the Feynman diagram in momentum space,

$$
\begin{gathered}
\Delta s^{\vee}\left(p ; q_{1}, \ldots, q_{k}\right)=(-1)^{k} s^{\vee}\left(p+q_{1}+\cdots+q_{k}\right) \hat{B}\left(q_{k}\right) s^{\vee}\left(p+q_{1}+\cdots+q_{k-1}\right) \hat{B}\left(q_{k-1}\right) \\
\cdots \hat{B}\left(q_{2}\right) s^{\vee}\left(p+q_{1}\right) \hat{B}\left(q_{1}\right) s^{\vee}(p)
\end{gathered}
$$

(here $\hat{B}$ denotes the Fourier transform of the potential $B$, and $s^{\vee}(p)$ is the multiplication operator in momentum space). For the arguments of the Green's functions, we introduce the abbreviation

$$
p_{0}:=p \quad \text { and } \quad p_{l}:=p+q_{1}+\cdots+q_{l}, \quad 1 \leq l \leq k .
$$

Substituting the explicit formulas 2.2.4 and 2.2.15) into 2.2.98, we obtain

$$
\begin{aligned}
& \Delta s^{\vee}(p\left.; q_{1}, \ldots, q_{k}\right)=(-1)^{k} \not p_{k} \hat{B}\left(q_{k}\right) \not p_{k-1} \cdots \not p_{1} \hat{B}\left(q_{1}\right) \not p_{0} \\
& \quad \times \lim _{\nu_{0}, \ldots, \nu_{k} \searrow 0} \frac{1}{\left(p_{k}\right)^{2}-i \nu_{k} p_{k}^{0}} \frac{1}{\left(p_{k-1}\right)^{2}-i \nu_{k-1} p_{k-1}^{0}} \cdots \frac{1}{\left(p_{0}\right)^{2}-i \nu_{0} p_{0}^{0}} .
\end{aligned}
$$

We already know that the limits $\nu_{0}, \ldots, \nu_{k} \searrow 0$ exist in the distributional sense. This can be understood directly from the fact that, fixing the momenta $q_{1}, \ldots q_{k}$ as well as $\vec{p}$, the above expression for $\Delta s^{\vee}$ is a meromorphic function in $p^{0}$ having poles only in the lower half plane. Computing the Fourier transform with residues, we obtain a well-defined expression which remains finite as $\nu_{0}, \ldots, \nu_{k} \searrow 0$. This consideration also shows that we may choose the $\nu_{0}, \ldots, \nu_{k}$ to be equal, i.e.

$$
\begin{aligned}
\Delta s^{\vee}(p & \left.; q_{1}, \ldots, q_{k}\right)=(-1)^{k} \not p_{k} \hat{B}\left(q_{k}\right) \not p_{k-1} \cdots \not p_{1} \hat{B}\left(q_{1}\right) \not p_{0} \\
& \times \lim _{\nu \searrow 0} \frac{1}{\left(p_{k}\right)^{2}-i \nu p_{k}^{0}} \frac{1}{\left(p_{k-1}\right)^{2}-i \nu p_{k-1}^{0}} \cdots \frac{1}{\left(p_{0}\right)^{2}-i \nu p_{0}^{0}} .
\end{aligned}
$$

We now expand the Klein-Gordon Green's functions in 2.2 .100 with respect to the momenta $p_{l}-p$. If we expand the terms $i \nu p_{l}^{0}$ with a geometric series,

$$
\frac{1}{\left(p_{l}\right)^{2}-i \nu p_{l}^{0}}=\sum_{n=0}^{\infty} \frac{\left(i \nu\left(p_{l}^{0}-p^{0}\right)\right)^{n}}{\left(\left(p_{l}\right)^{2}-i \nu p^{0}\right)^{1+n}}
$$

all contributions with $n \geq 1$ contain factors $\nu$ and vanish in the limit $\nu \searrow 0$. Therefore, we must only expand with respect to the parameters $\left(\left(p_{l}\right)^{2}-p^{2}\right)$. This gives, again with geometric series,

$$
\begin{aligned}
& \Delta s^{\vee}(p ;\left.q_{1}, \ldots, q_{k}\right)=(-1)^{k} \not p_{k} \hat{B}\left(q_{k}\right) \not p_{k-1} \cdots \\
&\left.\quad \times \sum_{n_{1}, \ldots, n_{k}=0}^{\infty}\left(p^{2}-p_{k}^{2}\right)^{n_{k}} \cdots\left(p_{1}\right) \not p_{0}-p_{1}^{2}\right)^{n_{1}} \lim _{\nu \searrow 0} \frac{1}{\left(p^{2}-i \nu p^{0}\right)^{1+k+n_{1}+\cdots+n_{k}}} .
\end{aligned}
$$

Rewriting the negative power of $\left(p^{2}-i \nu p^{0}\right)$ as a mass-derivative,

$$
\begin{aligned}
& \frac{1}{\left(p^{2}-i \nu p^{0}\right)^{1+k+n_{1}+\cdots+n_{k}}} \\
& \quad=\left.\frac{1}{\left(k+n_{1}+\cdots+n_{k}\right) !}\left(\frac{d}{d a}\right)^{k+n_{1}+\cdots+n_{k}} \frac{1}{p^{2}-a-i \nu p^{0}}\right|_{a=0},
\end{aligned}
$$


we obtain a formula containing only one Green's function. Namely, using the notation 2.2.13, we get

$$
\begin{aligned}
& \Delta s^{\vee}\left(p ; q_{1}, \ldots, q_{k}\right)=(-1)^{k} \not p_{k} \hat{B}\left(q_{k}\right) \not p_{k-1} \cdots \not p_{1} \hat{B}\left(q_{1}\right) \not p_{0} \\
& \times \sum_{n_{1}, \ldots, n_{k}=0}^{\infty} \frac{1}{\left(k+n_{1}+\cdots+n_{k}\right) !}\left(p^{2}-p_{k}^{2}\right)^{n_{k}} \cdots\left(p^{2}-p_{1}^{2}\right)^{n_{1}} S^{\vee\left(k+n_{1}+\cdots+n_{k}\right)}(p) .
\end{aligned}
$$

This is the basic equation for the light-cone expansion of the Green's functions in momentum space. Similar to the light-cone expansion of the previous section, 2.2.102 involves

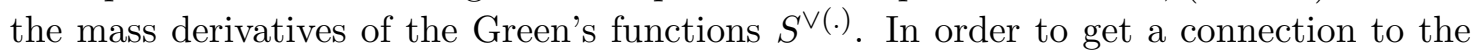
nested line integrals of, say, Theorem 2.2.4. it remains to transform the polynomials in the momenta $p_{0}, \ldots, p_{k}$ as follows: Using (2.2.99), we rewrite (2.2.102) in terms of the momenta $p, q_{1}, \ldots, q_{k}$ and multiply out. Furthermore, we simplify the Dirac matrices with the anti-commutation rules 2.2.52). This gives for 2.2.102 a sum of terms of the form

$$
\chi_{c} C \gamma^{I} q_{k}^{I_{k}} \cdots q_{1}^{I_{1}} \tilde{V}_{J_{k}, c_{k}}^{(k)}\left(q_{k}\right) \cdots \tilde{V}_{J_{1}, c_{1}}^{(1)}\left(q_{1}\right) p^{L} S^{\vee(h)}(p) \quad(h \geq[|L| / 2]),
$$

where the tensor indices of the multi-indices $I, I_{l}, J_{l}$, and $L$ are contracted with each other (similar to the notation of Theorem 2.2.4, the factors $\tilde{V}_{J_{l}, c_{l}}^{(l)}$ stand for the individual potentials of $\hat{B}$ ). If tensor indices of the power $p^{L}$ are contracted with each other, we can eliminate the corresponding factors $p^{2}$ iteratively with the rule 2.2.14), more precisely

$$
p^{2} S^{\vee(h)}(p)=h S^{\vee(h-1)}(p) \quad(h \geq 1) .
$$

In this way, we can arrange that the tensor indices of $p^{L}$ in (2.2.103) are all contracted with tensor indices of the factors $\gamma^{I}, q_{l}^{I_{l}}$, or $\tilde{V}_{J_{l}, c_{l}}^{(l)}$. By iteratively applying the differentiation rule (2.2.16), we can now rewrite the power $p^{L}$ in 2.2.103) with $p$-derivatives, e.g.

$$
\begin{aligned}
p_{j} p_{k} S^{\vee(2)}(p) & =-\frac{1}{2} p_{j} \frac{\partial}{\partial p^{k}} S^{\vee(1)}(p)=-\frac{1}{2} \frac{\partial}{\partial p^{k}}\left(p_{j} S^{\vee(1)}(p)\right)+\frac{1}{2} g_{j k} S^{\vee(1)}(p) \\
& =\frac{1}{4} \frac{\partial^{2}}{\partial p^{j} \partial p^{k}} S^{(0)}(p)+\frac{1}{2} g_{j k} S^{(1)}(p) .
\end{aligned}
$$

In this way, we obtain for $\Delta s^{\vee}\left(p ; q_{1}, \ldots, q_{k}\right)$ a sum of terms of the form

$$
\chi_{c} C \gamma^{I} q_{k}^{I_{k}} \cdots q_{1}^{I_{1}} \tilde{V}_{J_{k}, c_{k}}^{(k)}\left(q_{k}\right) \cdots \tilde{V}_{J_{1}, c_{1}}^{(1)}\left(q_{1}\right) \partial_{p}^{K} S^{\vee(h)}(p),
$$

where no tensor indices of the derivatives $\partial_{p}^{K}$ are contracted with each other. We substitute these terms into (2.2.97) and transform them to position space. Integrating the derivatives $\partial_{p}^{K}$ by parts gives factors $(y-x)^{K}$. The factors $q_{l}^{I_{l}}$, on the other hand, can be written as partial derivatives $\partial^{I_{l}}$ acting on the potentials $V^{(l)}$. More precisely, substituting into (2.2.97), the term (2.2.105) gives the contribution

$$
\chi_{c} C i^{\left|I_{1}\right|+\cdots+\left|I_{k}\right|}(-i)^{|K|} \gamma^{I}\left(\partial^{I_{k}} V_{J_{k}, c_{k}}^{(k)}(x)\right) \cdots\left(\partial^{I_{1}} V_{J_{1}, c_{1}}^{(1)}(x)\right)(y-x)^{K} S^{\vee(h)}(x, y),
$$

where the tensor indices of the factor $(y-x)^{K}$ are all contracted with tensor indices of the multi-indices $I, I_{l}$, or $J_{l}$. The Feynman diagram $\left((-s B)^{k} s\right)(x, y)$ coincides with the sum of all these contributions.

This expansion has much similarity with the light-cone expansion of Theorem 2.2.4. Namely, if one expands the nested line integrals in 2.2.32) in a Taylor series around 
$x$, one gets precisely the expansion into terms of the form 2.2 .106$)$. Clearly, the lightcone expansion of Theorem 2.2.4 goes far beyond the expansion (2.2.106), because the dependence on the external potential is described by non-local line integrals. Nevertheless, the expansion in momentum space $(2.2 .102)$ and subsequent Fourier transformation give an easy way of understanding in principle how the formulas of the light-cone expansion come about. We remark that, after going through the details of the combinatorics and rearranging the contributions (2.2.106), one can indeed recover the Taylor series of the line integrals in 2.2 .32 . This gives an alternative method for proving Theorem 2.2.4. However, it is obvious that this becomes complicated and does not yield the most elegant approach (the reader interested in the details of this method is referred to [F5], where a very similar technique is used for the light-cone expansion to first order in the external potential).

Next, we want to generalize the previous construction to other types of Green's functions. Since, similar to (2.2.101), we must rewrite a product of Green's functions as the mass derivative of a single Green's function, we can only expect the construction to work if all Green's functions in the product (2.2.98) are of the same type (e.g. the construction breaks down for a "mixed" operator product containing both advanced and retarded Green's functions). But we need not necessarily work with the advanced or retarded Green's functions. Instead, we can use Green's functions with a different location of the poles in the complex $p^{0}$-plane: We consider the Green's functions

$$
s^{ \pm}(p)=\not p S_{a \mid a=0}^{ \pm}(p) \quad \text { with } \quad S_{a}^{ \pm}(p)=\lim _{\nu \searrow 0} \frac{1}{p^{2}-a \mp i \nu}
$$

and again use the notation (2.2.13),

$$
S^{ \pm(l)}=\left(\frac{d}{d a}\right)^{l} S_{a \mid a=0}^{ \pm} .
$$

The distribution $s^{-}$is referred to as the Feynman propagator (see Exercise 2.3). The perturbation expansion of these Dirac Green's functions is, similar to 2.1.25) or (2.2.12), given by the formal series

$$
\tilde{s}^{+}:=\sum_{n=0}^{\infty}\left(-s^{+} B\right)^{n} s^{+} \quad \text { and } \quad \tilde{s}^{-}:=\sum_{n=0}^{\infty}\left(-s^{-} B\right)^{n} s^{-} .
$$

The light-cone expansion in momentum space is performed exactly as for the advanced and retarded Green's functions. In analogy to (2.2.97) and 2.2.102), we thus obtain the formula

$$
\begin{aligned}
& \left(\left(-s^{ \pm} B\right)^{k} s^{ \pm}\right)(x, y) \\
& \quad=\int \frac{d^{4} p}{(2 \pi)^{4}} \int \frac{d^{4} q_{1}}{(2 \pi)^{4}} \cdots \int \frac{d^{4} q_{k}}{(2 \pi)^{4}} \Delta s^{ \pm}\left(p ; q_{1}, \ldots, q_{k}\right) e^{-i\left(p+q_{1}+\cdots+q_{k}\right) x+i p y}
\end{aligned}
$$

with

$$
\begin{aligned}
& \Delta s^{ \pm}\left(p ; q_{1}, \ldots, q_{k}\right)=(-1)^{k} \not p_{k} \hat{B}\left(q_{k}\right) \not p_{k-1} \cdots \not p_{1} \hat{B}\left(q_{1}\right) \not p_{0} \\
& \quad \times \sum_{n_{1}, \ldots, n_{k}=0}^{\infty} \frac{1}{\left(k+n_{1}+\cdots+n_{k}\right) !}\left(p^{2}-p_{k}^{2}\right)^{n_{k}} \cdots\left(p^{2}-p_{1}^{2}\right)^{n_{1}} S^{ \pm\left(k+n_{1}+\cdots+n_{k}\right)} .
\end{aligned}
$$

Since $S^{ \pm}$are Green's functions of the Klein-Gordon equation, they clearly also satisfy the identity (2.2.104). Furthermore, the differentiation rule (2.2.16) is also valid for $S^{ \pm}$; 
namely

$$
\begin{aligned}
\frac{\partial}{\partial p^{j}} S^{ \pm(l)}(p) & =\left.\left(\frac{d}{d a}\right)^{l} \lim _{\nu \searrow 0} \frac{\partial}{\partial p^{j}}\left(\frac{1}{p^{2}-a \mp i \nu}\right)\right|_{a=0} \\
& =\left.\left(\frac{d}{d a}\right)^{l} \lim _{\nu \searrow 0} \frac{-2 p_{j}}{\left(p^{2}-a \mp i \nu\right)^{2}}\right|_{a=0}=-2 p_{j} S^{ \pm(l+1)}(p) .
\end{aligned}
$$

Therefore we can, exactly as in $(2.2 .105)$, rewrite the power $p^{L}$ with $p$-derivatives. Thus the expansion (2.2.106) is valid in the same way for the Green's functions $s^{ \pm}$if one only replaces the index "V" in 2.2.106) by " \pm ". As explained before, the expansion 2.2.106 can be obtained from the light-cone expansion of Theorem 2.2.4 by expanding the potentials around the space-time point $x$. Since the formulas of the light-cone expansion are uniquely determined by this Taylor expansion, we immediately conclude that the statement of Theorem 2.2.4 is also valid for the $k^{\text {th }}$ order contribution to the perturbation expansion (2.2.108) if the factor $S^{(h)}$ in 2.2 .32 stands more generally for $S^{+(h)}$ or $S^{-(h)}$, respectively. This simple analogy between the formulas of the light-cone expansions of the Feynman diagrams $\left(\left(-s^{\vee / \wedge} B\right)^{k} s^{\vee / \wedge}\right)$ and $\left(\left(-s^{ \pm} B\right)^{k} s^{ \pm}\right)$, which are obtained by changing the location of the poles of the vacuum Green's functions in momentum space, is called the residual argument (the name is motivated by the fact that the effect of changing the location of the poles becomes apparent when taking the Fourier integral with residues).

Having other Green's functions to our disposal, one can also form more general solutions of the homogeneous equation. Namely, taking the difference of $s^{+}$and $s^{-}$, we obtain similar to (2.1.13),

$$
s^{+}(q)-s^{-}(q)=\not \lim _{\nu \searrow 0}\left[\frac{1}{q^{2}-i \nu}-\frac{1}{q^{2}+i \nu}\right]=2 \pi i \not \phi \delta\left(q^{2}\right)=2 \pi i p(q)
$$

with $p$ according to (2.1.7). Replacing the Green's functions by those in the external potential, one gets a canonical perturbation series for $p$. As we shall see below (see $\$ 2.2 .7$ ), this perturbation series does not agree with the causal perturbation expansion (2.1.64). Therefore, we denote the obtained operator with an additional index res. Similar to (2.1.26), we thus introduce the residual fundamental solution $\tilde{p}^{\text {res }}$ by

$$
\tilde{p}^{\text {res }}:=\frac{1}{2 \pi i}\left(\tilde{s}^{+}-\tilde{s}^{-}\right) .
$$

We now introduce the residual fermionic projector by replacing the operators $p_{m}$ and $k_{m}$ in 2.1.6) by the corresponding perturbation series.

Definition 2.2.10. The residual fermionic projector $\tilde{P}^{\mathrm{res}}(x, y)$ is defined by

$$
\tilde{P}^{\mathrm{res}}(x, y)=\frac{1}{2}\left(\tilde{p}^{\mathrm{res}}-\tilde{k}\right)(x, y),
$$

where the operator $\tilde{p}^{\text {res }}$ is defined in 2.2.110, and $\tilde{k}$ is again given by 2.1.53).

Similar to (2.1.64), the residual fermionic projector also has a contour integral representation (see Exercise 2.19).

Applying the residual argument, the light-cone expansion of the Green's functions immediately carries over to $\tilde{P}^{\text {res: }}$ As in $(1.2 .26)$ we denote the lower mass shell by $T_{a}$, i.e. in momentum space

$$
T_{a}(q)=\Theta\left(-q^{0}\right) \delta\left(q^{2}-a\right)
$$


In analogy to the mass expansion of the Green's functions 2.2.13), we set

$$
T_{\text {formal }}^{(l)}=\left.\left(\frac{d}{d a}\right)^{l} T_{a}\right|_{a=0} .
$$

In order not to distract from the main idea, we postpone the analysis of whether these derivatives exist to 2.2 .6 . This is why we added the index "formal."

Proposition 2.2.11. The light-cone expansion of the residual fermionic projector $\tilde{P}^{\mathrm{res}}(x, y)$ is obtained from that of the causal Green's functions by the replacement

$$
S^{(l)} \rightarrow T_{\text {formal }}^{(l)} \text {. }
$$

Proof. The starting point is the light-cone expansion of the causal Green's functions (see Theorem 2.2.4. Theorem 2.2.6 and Theorem 2.2.8). By linearity, this light-cone expansion also hold for $\tilde{k}$ defined by (2.1.26), after the replacements

$$
S^{(l)} \rightarrow \frac{1}{2 \pi i}\left(S^{\vee(l)}-S^{\wedge(l)}\right) .
$$

Using the residual argument, the light-cone expansion of the Green's functions $\tilde{s}^{ \pm}$is obtained by the replacements $S^{(l)} \rightarrow S^{ \pm(l)}$. It follows by linearity that $\tilde{p}^{\text {res }}$ as defined by 2.2.110 also has a light-cone expansion obtained by the replacements

$$
S^{(l)} \rightarrow \frac{1}{2 \pi i}\left(S^{+(l)}-S^{-(l)}\right) .
$$

Finally, again by linearity, we obtain the light-cone expansion of residual fermionic projector (2.2.111) by the replacements

$$
S^{(l)} \rightarrow \frac{1}{4 \pi i}\left(S^{+(l)}-S^{-(l)}-S^{\vee(l)}+S^{\wedge(l)}\right) .
$$

A direct computation in analogy to 2.1 .13$)$ and $(2.2 .109)$ shows that

$$
\frac{1}{4 \pi i}\left(S^{+}-S^{-}-S^{\vee}+S^{\wedge}\right)=T_{a} .
$$

This concludes the proof.

We point out that the result of Proposition 2.2.11 is only formal because we have not yet analyzed whether the factors $T_{\text {formal }}^{(l)}$ are mathematically well-defined. This will be done in the next section.

2.2.6. The Non-Causal Low Energy Contribution. We now want to put the residual argument and the formal light-cone expansion of Proposition 2.2.11 on a satisfying mathematical basis. In order to explain what precisely we need to do, we first recall how the light-cone expansion of the Green's functions makes mathematical sense: Theorem 2.2.4 gives a representation of every Feynman diagram of the perturbation series 2.2.12 as an infinite sum of contributions of the form 2.2.32). According to the bound (2.2.36), there are, for any given $h$, only a finite number of possibilities to choose $I_{a}$ and $p_{a}$; as a consequence, we get, for fixed $h$, only a finite number of contributions 2.2.32. Thus we can write the light-cone expansion in the symbolic form

$$
\left((-s B)^{k} s\right)(x, y)=\sum_{h=-1}^{\infty} \sum_{\text {finite }} \cdots S^{(h)}(x, y),
$$


where '...' stands for a configuration of the $\gamma$-matrices and nested line integrals in 2.2.59). According to the explicit formula (2.2.7), the higher $a$-derivatives of $S_{a}(x, y)$ contain more factors $(y-x)^{2}$ and are thus of higher order on the light cone. This makes it possible to make mathematical sense of the infinite series in (2.2.114) as a light-cone expansion.

According to Proposition 2.2.11, all the results for the Green's function are, on a formal level, also valid for the residual fermionic projector. We begin by considering the light-cone expansion of the individual Feynman diagrams in more detail. Similar to 2.2.114, the $k^{\text {th }}$ order contribution $\Delta P^{\text {res }}$ to the residual fermionic projector has an expansion of the form

$$
\Delta P^{\mathrm{res}}(x, y)=\sum_{h=-1}^{\infty} \sum_{\text {finite }} \cdots T_{\text {formal }}^{(h)}(x, y),
$$

where $T_{\text {formal }}^{(h)}$ is the $a$-derivative 2.2 .113$)$ of the lower mass shell $T_{a}, 2.2 .112$. In position space, $T_{a}$ is given explicitly in $(2.2 .3)$. The basic difference between the light-cone expansions (2.2.114) and (2.2.115) is related to the logarithmic pole $\log |a|$ in (2.2.3). Namely, as a consequence of this logarithm, the higher $a$-derivatives of $T_{a}$ are not of higher order on the light cone. To the order $\mathcal{O}\left((y-x)^{2}\right)$, for example, one has

$$
\left(\frac{d}{d a}\right)^{n} T_{a}(x, y)=\frac{1}{32 \pi^{3}}\left(\frac{d}{d a}\right)^{n}(a \log |a|)+\mathcal{O}\left((y-x)^{2}\right) \quad(n \geq 2) .
$$

In our context of an expansion around $a=0$, the situation is even worse, because the $a$-derivatives of $T_{a}$ are singular for $a \rightarrow 0$ (as one sees e.g. in (2.2.116). Thus not even the individual contributions to the light-cone expansion make mathematical sense. These difficulties arising from the logarithm in (2.2.3 are called the logarithmic mass problem (see [F5] for a more detailed discussion in a slightly different setting). Since we know from Lemma 2.1.2 that the Feynman diagrams are all well-defined, the logarithmic mass problem is not a problem of the perturbation expansion, but shows that something is wrong with the light-cone expansion of Proposition 2.2.11.

In order to resolve the logarithmic mass problem, we first "regularize" the formal light-cone expansion by taking out the problematic $\log |a|$ term. By resumming the formal light-cone expansion, we then show that the difference between the residual Dirac sea and the "regularized" Dirac sea is a smooth function in position space. We introduce the notation

$$
\begin{aligned}
T_{a}^{\mathrm{reg}}(x, y) & =T_{a}(x, y)-\frac{a}{32 \pi^{3}} \log |a| \sum_{j=0}^{\infty} \frac{(-1)^{j}}{j !(j+1) !} \frac{\left(a \xi^{2}\right)^{j}}{4^{j}} \\
T^{(l)} & =\left(\frac{d}{d a}\right)^{l} T_{a \mid a=0}^{\mathrm{reg}}
\end{aligned}
$$

(where $\xi^{2} \equiv \xi^{j} \xi_{j}$ denotes again the Minkowski inner product).

DEFINITION 2.2.12. The causal contribution $\tilde{P}^{\text {causal }}$ to the fermionic projector is obtained from the residual Dirac sea $\tilde{P}^{\text {res }}$ by replacing all factors $T_{\text {formal }}^{(h)}$ in the formal light-cone expansion by $T^{(h)}$. The non-causal low energy contribution $\tilde{P}^{\text {le }}$ to the fermionic projector is given by

$$
\tilde{P}^{\mathrm{le}}(x, y)=\tilde{P}^{\mathrm{res}}(x, y)-\tilde{P}^{\text {causal }}(x, y) .
$$


By the replacement $T_{\text {formal }}^{(h)} \rightarrow T^{(h)}$, the formal light-cone expansion of Proposition 2.2.11 becomes mathematically meaningful in the sense of Definition 2.2.1. Thus we can restate this result as a theorem, leaving out the word "formal."

TheOREM 2.2.13. The light-cone expansion of the causal Green's functions also holds for the causal contribution $\tilde{P}^{\text {causal }}$ to the fermionic projector if one simply replaces $S^{(l)} \rightarrow$ $T^{(l)}$ with $T^{(l)}$ according to 2.2 .118$)$.

Since $T_{a}-T_{a}^{\mathrm{reg}}$ is a smooth function in $x$ and $y$, it is natural to expect that the non-causal low energy contribution should also be smooth. This is indeed the case, in the following sense.

THEOREM 2.2.14. To every order in the external potential $\mathcal{B}$, the non-causal low energy contribution $\tilde{P}^{\mathrm{le}}(x, y)$ is a smooth function in $x$ and $y$.

The subtle point in the proof is that, to every order in perturbation theory, the non-causal low energy contribution involves an infinite number of summands. Although each summand is smooth, it is not clear whether the infinite sum converges and gives rise to a smooth function. This makes it necessary to use a resummation technique for the smooth contributions to the light-cone expansion. For brevity, we do not enter these constructions here but instead refer the interested reader to [F6, Proof of Theorem 3.8]. The resummation technique will also be introduced and applied in Appendix D.

2.2.7. The Non-Causal High Energy Contribution. In the previous sections ( $\$ 2.2 .5$ and $\$ 2.2 .6)$ we performed the light-cone expansion of the residual fermionic projector $\vec{P}^{\text {res }}$ (see Definition 2.2.10). The remaining task is to deduce the light-cone expansion of the fermionic projector $P^{\text {sea }}$ with spatial normalization (as defined by (2.1.64)). We now prove that $P^{\text {sea }}$ and $\tilde{P}^{\text {res }}$ have the same light-cone expansion.

We begin by giving the difference between the fermionic projector and the residual fermionic projector a name.

Definition 2.2.15. The non-causal high energy contribution $\tilde{P}^{\text {he }}(x, y)$ to the fermionic projector is given by

$$
\tilde{P}^{\text {he }}(x, y)=P^{\text {sea }}(x, y)-\tilde{P}^{\text {res }}(x, y) .
$$

TheOREM 2.2.16. To every order in the external potential $\mathcal{B}$, the non-causal high energy contribution $\tilde{P}^{\text {he }}(x, y)$ is a smooth function in $x$ and $y$.

PROOF. Our first task is to rewrite the perturbation expansion of $\tilde{P}^{\text {res }}$ in terms of the potential $\mathcal{B}$. To this end, one combines the rest masses of the Dirac particles with the unperturbed Green's functions. Thus for the advanced and retarded Green's functions, we return to the perturbation expansions (2.1.25). Similarly, for the Green's functions $\tilde{s}^{ \pm}$, we rewrite 2.2.108) as

$$
\tilde{s}_{m}^{+}=\sum_{n=0}^{\infty}\left(-s_{m}^{+} \mathcal{B}\right)^{n} s_{m}^{+} \quad \text { and } \quad \tilde{s}_{m}^{-}=\sum_{n=0}^{\infty}\left(-s_{m}^{-} \mathcal{B}\right)^{n} s_{m}^{-} .
$$

Then $\tilde{k}$ and $\tilde{p}^{\text {res }}$ are defined again by 2.1 .26$)$ and 2.2 .110$)$, respectively. As a result, the operators $\tilde{k}$ and $\tilde{p}^{\text {res }}$ are defined as sums of operator products of the form

$$
C_{n} \mathcal{B} C_{n-1} \mathcal{B} \cdots \mathcal{B} C_{0},
$$


where the factors $C_{l}$ coincide with either $k, p$ or $s$.

Next, we need a few structural properties of the causal perturbation expansion. These results are derived in Exercises 2.9 2.11. Alternatively, these results are obvious from the detailed formulas in the research papers [FG1, FT2. First, the operator $\tilde{k}$ has the contour integral representation (see Exercise 2.9 (a))

$$
\tilde{k}=-\frac{1}{2 \pi i} \oint_{\Gamma_{+} \cup \Gamma_{-}} \lambda \tilde{R}_{\lambda} d \lambda
$$

As a consequence, the fermionic projector $P^{\text {sea }}$, 2.1.64), can be represented as

$$
P^{\text {sea }}=\frac{1}{2}(\tilde{p}-\tilde{k}),
$$

where $\tilde{p}$ is defined by

$$
\tilde{p}:=-\frac{1}{2 \pi i}\left(\oint_{\Gamma_{+}}-\oint_{\Gamma_{-}}\right) \lambda \tilde{R}_{\lambda} d \lambda
$$

(see Exercise 2.9 (b)). Comparing with 2.2.111) and Definition 2.2.15, we conclude that

$$
\tilde{P}^{\text {he }}=\frac{1}{2}\left(\tilde{p}-\tilde{p}^{\text {res }}\right) \text {. }
$$

Next, the operator $\tilde{p}$ has the following properties:

(i) Every contribution to the perturbation expansion of $\tilde{p}$ contains an even number of factors $k$.

(ii) If in the perturbation series for $\tilde{p}$ one replaces all factors $k$ by factors $p$, one gets precisely the perturbation series for $\tilde{p}^{\text {res }}$.

These properties can be read off from the explicit formulas for $\tilde{p}$ and $\tilde{p}^{\text {res }}$ given in [FG1, FT2 . For abstract proofs, one can proceed as follows. Property (i) is shown in Exercise 2.10. In order to prove (ii), we first bring the perturbation expansion of the residual fundamental solution into a more explicit form. Comparing 2.2 .110$)$ with $(2.1 .26)$ and noting that in view of 2.2 .109$)$, the Green's functions $s_{m}^{ \pm}$satisfy in analogy to (2.1.41) the relations

$$
s=s^{+}-i \pi p=s^{-}+i \pi p,
$$

we find that the perturbation expansion of $\tilde{p}^{\text {res }}$ is obtained from that for $\tilde{k}$, 2.1.46), simply by replacing all factors $k$ by factors $p$,

$$
\tilde{p}^{\text {res }}=\sum_{\beta=0}^{\infty}(-i \pi)^{2 \beta} b^{<} p(b p)^{2 \beta} b^{>} .
$$

In Exercise 2.11 it is shown that exactly the same perturbation series is obtained if in the perturbation series for $\tilde{p}$ one replaces all factors $k$ by factors $p$. This proves (ii).

Using the above properties (i) and (ii), we can convert the perturbation series for $\tilde{p}$ into that for $\tilde{p}^{\text {res }}$ by iteratively replacing pairs of factors $k$ in the operator products by pairs of factors $p$. Thus the difference $\tilde{p}-\tilde{p}^{\text {res }}$ can, to every order in perturbation theory, be written as a finite sum of expressions of the form

$$
\begin{array}{rl}
C_{n} \mathcal{B} \cdots C_{b+1} & \mathcal{B}\left(p \mathcal{B} C_{b-1} \cdots C_{a+1} \mathcal{B} p\right. \\
& \left.-k \mathcal{B} C_{b-1} \cdots C_{a+1} \mathcal{B} k\right) \mathcal{B} C_{a-1} \cdots \mathcal{B} C_{0},
\end{array}
$$

where the factors $C_{l}$ again stand for $k, p$ or $s$. Therefore, it remains to show that 2.2.120 is a smooth function in position space. 
We first simplify our problem: Once we have shown that the bracket in 2.2 .120 is smooth and bounded in position space, the additional multiplications to the very left and right can be carried out by iteratively multiplying with $\mathcal{B}$ and forming the convolution with $C_{l}$, which again gives a smooth and bounded function in each step (notice that, according to the assumptions of Lemma 2.1.2. $\mathcal{B}$ decays sufficiently fast at infinity). Thus we must only consider the bracket in (2.2.120). We rewrite this bracket with the projectors $\frac{1}{2}(p-k)$ and $\frac{1}{2}(p+k)$ on the lower and upper mass shells,

$$
\begin{aligned}
p \mathcal{B} & C_{n-1} \cdots C_{1} \mathcal{B} p-k \mathcal{B} C_{n-1} \cdots C_{1} \mathcal{B} k \\
& =\frac{1}{2}(p+k) \mathcal{B} C_{n-1} \cdots C_{1} \mathcal{B}(p-k)+\frac{1}{2}(p-k) \mathcal{B} C_{n-1} \cdots C_{1} \mathcal{B}(p+k) .
\end{aligned}
$$

For symmetry reasons, it suffices to consider the first summand of this decomposition,

$$
\left((p+k) \mathcal{B} C_{n-1} \cdots C_{1} \mathcal{B}(p-k)\right)(x, y),
$$

where the factors $C_{l}$ again stand for $k, p$, or $s$. Our task is to show that $(2.2 .121)$ is a smooth function in position space.

We proceed in momentum space. We say that a function $f(q)$ has rapid decay for positive frequency if it is $C^{1}$, bounded together with its first derivatives (i.e. sup $|f|, \sup \left|\partial_{l} f\right|<$ $\infty)$, and satisfies for every $\alpha>0$ the bounds

$$
\sup _{\omega>0, \vec{k} \in \mathbb{R}^{3}}\left|\omega^{\alpha} f(\omega, \vec{k})\right|, \sup _{\omega>0, \vec{k} \in \mathbb{R}^{3}}\left|\omega^{\alpha} \partial_{l} f(\omega, \vec{k})\right|<\infty .
$$

After setting $C_{0}=p-k$ and $C_{n}=p+k$, the operator product (2.2.121) is of the form (2.1.27). We choose a function $g$ with rapid decay for positive frequency and decompose the operator product in the form 2.1.31,, 2.1 .32 . It follows by induction that the functions $F_{j}$ all have rapid decay for positive frequency: The induction hypothesis is obvious by setting $F_{0}=g$. The induction step is to show that for a function $F_{j-1}$ with rapid decay for positive frequency, the convolution

$$
F_{j}(\omega, \vec{k})=\int \frac{d \omega^{\prime}}{2 \pi} \int \frac{d \vec{k}^{\prime}}{(2 \pi)^{3}} \hat{B}\left(\omega-\omega^{\prime}, \vec{k}-\vec{k}^{\prime}\right) C_{j-1}\left(\omega^{\prime}, \vec{k}^{\prime}\right) F_{j-1}\left(\omega^{\prime}, \vec{k}^{\prime}\right)
$$

also has rapid decay for positive frequency. In Lemma 2.1.2 it was shown that $F_{j}$ is $C^{1}$ and bounded together with its first derivatives. As a consequence, we must only establish the bounds 2.2 .122 for $\omega>1$. Moreover, because of the monotonicity $\omega^{\alpha}<\omega^{\beta}$ for $\alpha<\beta$ (and $\omega>1$ ), it suffices to show that there are arbitrarily large numbers $\alpha$ satisfying the bounds 2.2.122); we only consider $\alpha=2 n$ with $n \in \mathbb{N}$. For $\omega>1$ and $\omega^{\prime} \in \mathbb{R}$, we have the inequality

$$
\omega^{2 n} \leq\left(2 \omega^{\prime}\right)^{2 n} \Theta\left(\omega^{\prime}\right)+\left(2\left(\omega-\omega^{\prime}\right)\right)^{2 n},
$$

as is immediately verified by checking the three regions $\omega^{\prime} \leq 0,0<\omega^{\prime} \leq \omega / 2$, and $\omega^{\prime}>\omega / 2$. We combine this inequality with (2.2.123) and obtain for $\omega>1$ the estimate

$$
\left|\omega^{2 n} F_{j}(\omega, \vec{k})\right| \leq\left|\int \frac{d \omega^{\prime}}{(2 \pi)} \int \frac{d \vec{k}^{\prime}}{(2 \pi)^{3}}\left(E_{1}+E_{2}\right)\right|,
$$

where $E_{1}$ and $E_{2}$ are given by

$$
\begin{aligned}
& E_{1}=\hat{B}\left(\omega-\omega^{\prime}, \vec{k}-\vec{k}^{\prime}\right) C_{j-1}\left(\omega^{\prime}, \vec{k}^{\prime}\right)\left[\left(2 \omega^{\prime}\right)^{2 n} \Theta\left(\omega^{\prime}\right) F_{j-1}\left(\omega^{\prime}, \vec{k}\right)\right] \\
& E_{2}=\left[\left(2\left(\omega-\omega^{\prime}\right)\right)^{2 n} \hat{B}\left(\omega-\omega^{\prime}, \vec{k}-\vec{k}^{\prime}\right)\right] C_{j-1}\left(\omega^{\prime}, \vec{k}^{\prime}\right) F_{j-1}\left(\omega^{\prime}, \vec{k}\right) .
\end{aligned}
$$


According to the induction hypothesis, the square bracket in 2.2 .125 is bounded together with its first derivatives. Since $\hat{B}$ has rapid decay at infinity, the square bracket in (2.2.126) also has rapid decay at infinity. As a consequence, the integral in (2.2.124) satisfies the hypothesis considered in Lemma 2.1.2 for $(2.1 .29)$ and is therefore bounded. In order to estimate the expression $\left|\omega^{2 n} \partial_{l} F_{j}\right|$, we differentiate (2.2.123) and obtain similar to 2.2.125 and 2.2.126 the inequality

$$
\begin{aligned}
& \left|\omega^{2 n} \partial_{l} F_{j}(\omega, \vec{k})\right| \\
& \leq\left|\int \frac{d \omega^{\prime}}{2 \pi} \int \frac{d \vec{k}^{\prime}}{(2 \pi)^{3}} \partial_{l} \hat{B}\left(\omega-\omega^{\prime}, \vec{k}-\vec{k}^{\prime}\right) C_{j-1}\left(\omega^{\prime}, \vec{k}^{\prime}\right)\left[\left(2 \omega^{\prime}\right)^{2 n} \Theta\left(\omega^{\prime}\right) F_{j-1}\left(\omega^{\prime}, \vec{k}\right)\right]\right| \\
& \quad+\left|\int \frac{d \omega^{\prime}}{d \omega} \int \frac{d \vec{k}^{\prime}}{(2 \pi)^{3}}\left[\left(2\left(\omega-\omega^{\prime}\right)\right)^{2 n} \partial_{l} \hat{B}\left(\omega-\omega^{\prime}, \vec{k}-\vec{k}^{\prime}\right)\right] C_{j-1}\left(\omega^{\prime}, \vec{k}^{\prime}\right) F_{j-1}\left(\omega^{\prime}, \vec{k}\right)\right| .
\end{aligned}
$$

This concludes the proof of the induction step.

We just showed that for a function $g$ with rapid decay for positive frequency, the function

$$
F_{n}(q)=\int \frac{d^{4} q_{1}}{(2 \pi)^{4}}\left(\mathcal{B} C_{n-1} \mathcal{B} \cdots \mathcal{B} C_{1} \mathcal{B} C_{0}\right)\left(q, q_{1}\right) g\left(q_{1}\right)
$$

has rapid decay for positive frequency. We now consider what this means for our operator product 2.2.121 in position space. For a given four-vector $y=\left(y^{0}, \vec{y}\right)$, we choose

$$
g(\omega, \vec{k})=\eta(\omega) e^{-i\left(\omega y^{0}-\vec{k} \vec{y}\right)},
$$

where $\eta$ is a smooth function with $\eta(\omega)=1$ for $\omega \leq 0$ and $\eta(\omega)=0$ for $\omega>1$ (this choice of $g$ clearly has rapid decay for positive frequency). Since the support of the factor $C_{0}=(p-k)$ is the lower mass cone $\left\{q^{2} \geq 0, q^{0} \leq 0\right\}, g(\omega, \vec{k})$ enters into the integral 2.2.127) only for negative $\omega$. But for $\omega \leq 0$, the cutoff function $\eta$ is identically one. Thus the integral (2.2.127) is simply a Fourier integral; i.e., with a mixed notation in momentum and position space,

$$
F_{n}(q)=\left(\mathcal{B} C_{n-1} \mathcal{B} \cdots \mathcal{B} C_{1} \mathcal{B}(p-k)\right)(q, y) .
$$

Next, we multiply from the left with the operator $(p+k)$,

$$
\left((p+k) \mathcal{B} C_{n-1} \mathcal{B} \cdots \mathcal{B} C_{1} \mathcal{B}(p-k)\right)(q, y)=(p+k)(q) F_{n}(q) .
$$

Since $F_{n}$ has rapid decay for positive frequency and $(p+k)$ has its support in the upper mass cone $\left\{q^{2} \geq 0, q^{0}>0\right\}$, their product decays fast at infinity. More precisely,

$$
\left|q^{I}(p+k)(q) F_{n}(q)\right| \leq \operatorname{const}(I)(p+k)(q)
$$

for any multi-index $I$. As a consequence, the Fourier transform of (2.2.128) is even finite after multiplying with an arbitrary number of factors $q$, i.e.

$$
\left|\int \frac{d^{4} q}{(2 \pi)^{4}} q^{I}(p+k)(q) F_{n}(q) e^{-i q x}\right| \leq \operatorname{const}(I)<\infty
$$

for all $x$ and $I$. This shows that our operator product in position space (2.2.121) is bounded and, for fixed $y$, a smooth function in $x$ (with derivative bounds which are uniform in $y$ ). Similarly, one obtains that $(2.2 .121)$ is, for fixed $x$, a smooth function in $y$. We conclude that the distribution 2.2.121) is a smooth and bounded function. 
2.2.8. The Unregularized Fermionic Projector in Position Space. The previous constructions give a representation of the fermionic projector in the presence of chiral and scalar/pseudoscalar potentials (see 2.2.27), 2.2.25) and (2.2.28) ) of the form

$$
\begin{aligned}
P^{\text {sea }}(x, y)= & \left.\sum_{n=-1}^{\infty} \text { (phase-inserted line integrals }\right) \times T^{(n)}(x, y) \\
& +\tilde{P}^{\mathrm{le}}(x, y)+\tilde{P}^{\mathrm{he}}(x, y)
\end{aligned}
$$

Here the series is a light-cone expansion which describes the singular behavior of the fermionic projector on the light cone non-perturbatively. It is obtained from the lightcone expansion of the Green's functions by the simple replacement rule

$$
S^{(n)} \longrightarrow T^{(n)}
$$

(with $T^{(n)}$ as defined in $(2.2 .118)$ ). In particular, the phase-inserted line integrals are exactly the same as those for the Green's functions (see Definition 2.2.7). The contributions $\tilde{P}^{\text {le }}$ and $\tilde{P}^{\text {he }}$, on the other hand, are both given perturbatively by a series of terms which are all smooth on the light cone. The "causality" of the causal perturbation expansion can be understood from the fact that the phase-inserted line integrals in 2.2.129 are all bounded integrals along the line segment joining the points $x$ and $y$ (whereas the light-cone expansion of general operator products involves unbounded line integrals). In particular, when $y$ lies in the causal future or past of $x$, the light-cone expansion in 2.2.129) depends on the external potential only inside the causal diamond $\left(J_{x}^{\vee} \cap J_{y}^{\wedge}\right) \cup\left(\overline{J_{x}^{\wedge} \cap J_{y}^{\vee}}\right)$. Nevertheless, the light-cone expansion is not causal in this strict sense because there are contributions for $y \notin J_{x}$. Furthermore, the low and high energy contributions cannot be described with line integrals and violate locality as well as causality. This non-locality can be understood from the fact that the fermionic projector is a global object in space-time. We conclude that the singular behavior of the fermionic projector on the light-cone can be described explicitly by causal line integrals, whereas the smooth contributions to the fermionic projector are governed by non-local effects.

We finally remark that the decomposition $(2.2 .129$ ) is also a suitable starting point for analyzing the smooth contributions to the fermionic projector. Indeed, the low energy contribution $\tilde{P}^{\text {le }}$ can be computed effectively by resumming the perturbation expansion, as is explained in Appendix D. The high energy contribution $\tilde{P}^{\text {he }}$, on the other hand, is given in terms of operator products, which can be analyzed with Fourier methods.

\subsection{Description of Linearized Gravity}

We now outline how our computational tools apply in the presence of a gravitational field. Note that so far, the external potential $\mathcal{B}$ in the Dirac equation $(2.1 .5)$ was assumed to be a multiplication operator. When describing a gravitational field, however, the derivative terms in the Dirac equation are modified. The gravitational field can still be described by the Dirac equation (2.1.5) if we allow for $\mathcal{B}$ to be a first order differential operator. This means that the causal perturbation expansion of 2.1 .6 still applies. An analysis similar to that in Lemma 2.1.2 shows that the contributions to the perturbation series are again all well-defined and finite, provided that the gravitational field is smooth and decays sufficiently fast at infinity. In order to perform the light-cone expansion of the Green's functions, it is convenient to commute the differential operators contained 
in $\mathcal{B}$ to the very left to obtain operator products of the form

$$
\frac{\partial}{\partial x^{I}}\left[s Z_{1} \cdots Z_{n} s\right](x, y)
$$

where the $Z_{1}, \ldots, Z_{n}$ are again multiplication operators (which contain tensor indices contracted with the multi-index $I$ ). This makes it possible to perform the light-cone expansion of the square brackets with the inductive procedure described in $\$ 2.2 .2$. Carrying out the derivatives $\partial_{x}^{I}$ gives the desired light-cone expansion of the Green's function.

The basic difficulty with this construction is that, due to the additional derivatives, the contributions to higher order in perturbation theory become more and more singular on the light cone. In particular, the structural results of $\$ 2.2 .3$ no longer hold, and the resummation method of 2.2 .4 no longer applies. These difficulties are closely related to the fact that in the presence of a gravitational field, the light cone is no longer the light cone of Minkowski space, but it is generated by the null geodesics of the Lorentzian metric. This "deformation of the light cone" by the gravitational field is an effect which cannot be properly described by a light-cone expansion in Minkowski space. A possible way out is to use to use the non-perturbative construction in [FR3, FMR. The structure of the singularities on the light-cone can then be analyzed with the so-called Hadamard expansion (for explicit computations for the fermionic projector we refer to FG2, Appendix A]). Since we do not want to enter these techniques here, we simply describe how linearized gravity can be described with our methods. For more details we refer to [F5. Appendix B].

For the metric, we consider a first order perturbation $h_{j k}$ of the Minkowski metric $\eta_{j k}=\operatorname{diag}(1,-1,-1,-1)$,

$$
g_{j k}(x)=\eta_{j k}+h_{j k}(x) .
$$

As in the usual formalism (see for example [LL, $\S 105$ and $\S 107]$ ), we raise and lower tensor indices with the Minkowski metric. Using the transformation of $h_{j k}$ under infinitesimal coordinate transformations, we can assume [LL, §105] that

$$
\partial^{k} h_{j k}=\frac{1}{2} \partial_{j} h \quad \text { with } \quad h:=h_{k}^{k} .
$$

A straightforward computation (using for example the formalism introduced in [F4]) shows that in the so-called symmetric gauge, the Dirac operator takes the form

$$
i \not \partial_{x}-\frac{i}{2} \gamma^{j} h_{j k} \eta^{k l} \frac{\partial}{\partial x^{l}}+\frac{i}{8}(\not \partial h) \text {. }
$$

In contrast to 2.1.5), now the perturbation itself is a differential operator.

One complication arises from the fact that the integration measure in curved space is $\sqrt{|g|} d^{4} x=\left(1+\frac{h}{2}\right) d^{4} x$, whereas the formula 2.1.70 for the perturbation of the fermionic projector is valid only if one has the integration measure $d^{4} x$ of Minkowski space. Therefore we first transform the system such that the integration measure becomes $d^{4} x$, then apply (2.1.70), and finally transform back to the original integration measure $\sqrt{|g|} d^{4} x$. Rewriting the space-time inner product 1.5.2 as

$$
\int_{\mathscr{M}} \prec \psi\left|\phi \succ d \mu(x)=\int_{\mathbb{R}^{4}} \prec \psi\right| \phi \succ \sqrt{|g|} d^{4} x=\int_{\mathbb{R}^{4}} \prec\left(|g|^{\frac{1}{4}} \psi\right) \mid\left(|g|^{\frac{1}{4}} \phi \succ d^{4} x,\right.
$$


the transformation to the measure $d^{4} x$ is accomplished by

$$
\begin{aligned}
\psi(x) & \rightarrow \hat{\psi}(x)=|g|^{\frac{1}{4}}(x) \psi(x) \\
i \not \partial_{x}-\frac{i}{2} \gamma^{j} h_{j}^{k} \partial_{k}+\frac{i}{8}(\not \partial h) & \rightarrow|g|^{\frac{1}{4}}\left(i \not \partial_{x}-\frac{i}{2} \gamma^{j} h_{j}^{k} \partial_{k}+\frac{i}{8}(\not \partial h)\right)|g|^{-\frac{1}{4}} \\
& =i \not \partial_{x}-\frac{i}{2} \gamma^{j} h_{j}^{k} \partial_{k}-\frac{i}{8}(\not \partial h) .
\end{aligned}
$$

The perturbation $\Delta P^{\left(d^{4} x\right)}$ of the transformed system is given by (2.1.70),

$$
\begin{aligned}
\Delta P^{\left(d^{4} x\right)}(x, y)=-\int d^{4} z & \left\{s(x, z)\left(-\frac{i}{2} \gamma^{j} h_{j}^{k} \frac{\partial}{\partial z^{k}}-\frac{i}{8}(\not \partial h)(z)\right) P(z, y)\right. \\
+ & \left.P(x, z)\left(-\frac{i}{2} \gamma^{j} h_{j}^{k} \frac{\partial}{\partial z^{k}}-\frac{i}{8}(\not \partial h)(z)\right) s(z, y)\right\} .
\end{aligned}
$$

The formula for the transformation of the Dirac sea to the original integration measure $\sqrt{|g|} d^{4} x$ is

$$
P(x, y)+\Delta P(x, y)=|g|^{-\frac{1}{4}}(x)|g|^{-\frac{1}{4}}(y)\left(P(x, y)+\Delta P^{\left(d^{4} x\right)}(x, y)\right) .
$$

Thus

$$
\Delta P(x, y)=\Delta P^{\left(d^{4} x\right)}(x, y)-\frac{1}{4}(h(x)+h(y)) P(x, y) .
$$

Since the factors $P(z, y)$ and $s(z, y)$ in 2.3.1) only depend on the difference vector $z-y$, we can rewrite the $z$-derivatives as $y$-derivatives,

$$
\frac{\partial}{\partial z^{k}} P(z, y)=-\frac{\partial}{\partial y^{k}} P(z, y), \quad \frac{\partial}{\partial z^{k}} s(z, y)=-\frac{\partial}{\partial y^{k}} s(z, y),
$$

which can be pulled out of the integral. Furthermore, the relations

$$
\begin{aligned}
\int d^{4} z P(x, z)\left(i \not \partial_{z} h(z)\right) s(z, y) & =\int d^{4} z P(x, z)\left[\left(i \not \partial_{z}-m\right), h(z)\right] s(z, y) \\
& =-P(x, y) h(y) \\
\int d^{4} z s(x, z)\left(i \not \partial_{z} h(z)\right) P(z, y) & =h(x) P(x, y)
\end{aligned}
$$

make it possible to simplify the factors $(\not h h)$ in the integral. In the resulting formula for $\Delta P(x, y)$, one recovers the perturbation by an electromagnetic potential. More precisely,

$$
\Delta P(x, y)=\left(-\frac{1}{8} h(x)-\frac{3}{8} h(y)\right) P(x, y)-\frac{i}{2} \frac{\partial}{\partial y^{k}} \Delta P\left[\gamma^{j} h_{j}^{k}\right](x, y),
$$

where $\Delta P\left[\gamma^{j} h_{j}^{k}\right](x, y)$ is the perturbation 2.1.70 of the Dirac sea corresponding to the electromagnetic potential $\mathcal{B}=\gamma^{j} h_{j}^{k}$. The light-cone expansion of $\Delta P(x, y)$ is obtained by substituting the light-cone expansion of $\Delta P\left[\gamma^{j} h_{j}^{k}\right](x, y)$ into 2.3.2 and computing the $y$-derivative.

\subsection{The Formalism of the Continuum Limit}

In Section 2.2 we developed a method for analyzing the unregularized kernel of the fermionic projector in position space (see the summary in $\$ 2.2 .8$ ). Our next goal is to extend these methods in order to include an ultraviolet regularization. Following the method of variable regularization (see Remark 1.2.1), the allowed class of regularizations should be as large as possible. Moreover, we need to analyze in detail how the causal 
action and the corresponding EL equations depend on the regularization. As we shall see, these issues can be treated conveniently in the so-called formalism of the continuum limit, which is also most suitable for explicit computations.

The formalism of the continuum limit was first introduced in [F7, Chapter 4], based on earlier considerations in the unpublished preprint [F1]. In particular, the analysis in [F7, Sections 4.3-4.5] puts the formalism on a rigorous basis. For better readability, we here follow the original ideas in [F1 and develop the formalism from a more computational perspective. This makes it possible to explain the main points of the formalism in a nontechnical way. Generalizing the concepts, we then obtain the formalism of the continuum limit. In order avoid repetitions, we only outline the general derivation and refer the reader interested in the details to [F7, Sections 4.3-4.5] and Appendix F.

2.4.1. Example: The $i \varepsilon$-Regularization. In Section 1.2 we introduced the UV regularization in Minkowski space using general regularization operators (see Definition 1.2.3 and the resulting regularized kernel in Proposition 1.2.7). In order to get a better idea of what the effect of the regularization is, we now consider an explicit example. To this end, we assume that the regularized kernel of the fermionic projector, denoted again by $P^{\varepsilon}(x, y)$, is homogeneous in the sense that it depends only on the difference vector $\xi:=y-x$. Then the kernel can be written as a Fourier integral

$$
P^{\varepsilon}(x, y)=\int \frac{d^{4} k}{(2 \pi)^{4}} \hat{P}^{\varepsilon}(k) e^{-i k(x-y)}
$$

with a distribution $\hat{P}^{\varepsilon}(k)$. From the computational point of view, the simplest possible regularization method is to modify the unregularized kernel $(1.2 .23)$ by inserting a convergence-generating exponential factor. This leads us to choosing

$$
\hat{P}^{\varepsilon}(k)=(\not k+m) \delta\left(k^{2}-m^{2}\right) \Theta\left(-k^{0}\right) \exp \left(\varepsilon k^{0}\right),
$$

where $\varepsilon>0$ is the regularization length. The convergence-generating factor ensures that the Fourier integral (2.4.1) converges pointwise for any vector $\xi \in \mathscr{M}$. Moreover, differentiating (2.4.1) with respect to $x$ or $y$ gives rise to powers of $k$. Since these polynomial factors are dominated by the convergence-generating exponential factor, the Fourier integral again converges pointwise. We thus conclude that $P^{\varepsilon}(x, y)$ is a smooth function,

$$
P^{\varepsilon}(., .) \in C^{\infty}(\mathscr{M} \times \mathscr{M}) .
$$

Therefore, all composite expressions in the kernel of the fermionic projector are welldefined (like the closed chain (1.1.14), its eigenvalues $\lambda_{1}^{x y}, \ldots \lambda_{2 n}^{x y}$, the Lagrangian (1.1.9), the integrands in (1.1.4) and (1.1.5) as well as the kernel $Q(x, y)$ in (1.4.16)). But clearly, the singularities on the light-cone reappear in the limit $\varepsilon \searrow 0$, and the composite expressions will diverge. In other words, the limit $\varepsilon \searrow 0$ is a singular limit. Our goal is to analyze this singular limit in detail.

The effect of the convergence-generating factor in 2.4 .2 can be described conveniently in position space. Namely, introducing the short notations

$$
\omega=k^{0} \quad \text { and } \quad \xi=(t, \vec{x}),
$$

one can combine the exponential with the phase factor of the Fourier transform,

$$
\exp \left(\varepsilon k^{0}\right) e^{i k \xi}=e^{i \omega(t-i \varepsilon)-i \vec{k} \vec{x}} .
$$

This shows that the regularization amounts to the replacement

$$
t \rightarrow t-i \varepsilon \text {. }
$$


This simple replacement rule motivates the name $i \varepsilon$-regularization.

In order to illustrate how to work with this regularization, we next derive explicit formulas for the fermionic projector of the vacuum with this regularization. Our starting point is the light-cone expansion of the unregularized fermionic projector (2.2.129). More specifically, pulling the Dirac matrices out of the Fourier integral $\sqrt{1.2 .25}$ and expanding in a Taylor series in the mass parameter using (2.2.118) and 2.2.117), we obtain

$$
P^{\mathrm{vac}}(x, y)=(i \not \partial+m) T_{m^{2}}=(i \not \partial+m)\left(\sum_{n=0}^{\infty} \frac{m^{2 n}}{n !} T^{(n)}+(\text { smooth contributions })\right) .
$$

Since we are again interested mainly in the behavior of the singularities, for simplicity we shall disregard the smooth contributions. Clearly, such smooth contributions are important, and they also affect the singularities of composite expressions on the light cone (for example if multiplied by a singular contribution when forming the closed chain). But of course, smooth contributions can be treated in composite expressions in a straightforward way. Therefore, we now focus on the singularities and do all computations modulo smooth contributions. Then the residual argument shows that the $T^{(n)}$ satisfy the same computation rules as the Green's functions in (2.2.17) and 2.2.19),

$$
\frac{\partial}{\partial x^{k}} T^{(l)}(x, y)=-\frac{\partial}{\partial y^{k}} T^{(l)}(x, y)=\frac{1}{2} \xi_{k} T^{(l-1)}(x, y)
$$

(again valid up to smooth contributions; for an explicit derivation see Exercise 2.21). We thus obtain the light-cone expansion

$$
P^{\mathrm{vac}}(x, y)=\frac{i \not}{2} \sum_{n=0}^{\infty} \frac{m^{2 n}}{n !} T^{(-1+n)}+\sum_{n=0}^{\infty} \frac{m^{2 n+1}}{n !} T^{(n)}
$$

(where in analogy to (2.2.24) we use (2.4.4) to define $\left.T^{(-1)}\right)$.

The next step is to apply the replacement rule $(2.4 .3)$. The factor $\sharp$ becomes

$$
\not \rightarrow \not \varepsilon:=(t-i \varepsilon) \gamma^{0}-\vec{\xi} \vec{\gamma} .
$$

In order to regularize the factors $T^{(l)}$, we first note that, applying the replacement rule (2.4.3) to the distribution $T_{a}$ computed in Lemma 1.2.9, one really obtains a smooth function. Moreover, using the series expansion $(2.2 .3)$, one can compute the factors $T^{(n)}$ as defined by (2.2.118) and (2.2.117). When doing so, it is most convenient to combine the principal part with the $\delta$-contribution as well as the logarithm with the Heaviside function by using the identities

$$
\begin{aligned}
\frac{\mathrm{PP}}{\xi^{2}}+i \pi \delta\left(\xi^{2}\right) \epsilon\left(\xi^{0}\right) & =\lim _{\nu \searrow 0} \frac{1}{\xi^{2}-i \nu \xi^{0}} \\
\log \left|(y-x)^{2}\right|+i \pi \Theta\left(\xi^{2}\right) \epsilon\left(\xi^{0}\right) & =\lim _{\nu \searrow 0}\left(\log \left(\xi^{2}-i \nu \xi^{0}\right)-i \pi\right),
\end{aligned}
$$

where the logarithm is understood in the complex plane which is cut along the positive real axis such that $\lim _{\nu \searrow 0} \log (x+i \nu)$ is real for $x>0$. This gives

$$
\begin{aligned}
& T^{(0)} \rightarrow-\frac{1}{8 \pi^{3}} \frac{1}{(t-i \varepsilon)^{2}-|\vec{\xi}|^{2}} \\
& T^{(1)} \rightarrow \frac{1}{32 \pi^{3}} \log \left((t-i \varepsilon)^{2}-|\vec{\xi}|^{2}\right),
\end{aligned}
$$


and similar for the other distributions $T^{(n)}$. These replacement rules are compatible with our earlier computation rules like (2.4.4). These rules can also be used to compute $T^{(-1)}$ via (2.2.24) to obtain

$$
T^{(-1)} \rightarrow-\frac{1}{2 \pi^{3}} \frac{1}{\left((t-i \varepsilon)^{2}-r^{2}\right)^{2}},
$$

where we set $r=|\vec{\xi}|$.

Next, in 2.4.5 we apply the replacement rule 2.4.6) and replace the factors $T^{(l)}$ according to rules like (2.4.7)-(2.4.9). We thus obtain the regularized fermionic projector of the vacuum $P^{\varepsilon}(x, y)$. The kernel $P^{\varepsilon}(y, x)$ is obtained by taking the conjugate with respect to the spin scalar product (see (1.1.15) or (2.1.68)). Then one can form the closed chain $A_{x y}$ by (1.1.14) and compute all other quantities of interest. In order to give a concrete example, let us consider the massless case. Then

$$
\begin{aligned}
P(x, y) & =\frac{i}{2} \notin T^{(-1)} \quad \text { and thus } \\
P^{\varepsilon}(x, y) & =-\frac{i}{4 \pi^{3}} \frac{(t-i \varepsilon) \gamma^{0}-\vec{\xi} \vec{\gamma}}{\left((t-i \varepsilon)^{2}-r^{2}\right)^{2}} \\
P^{\varepsilon}(y, x) & =P^{\varepsilon}(x, y)^{*}=\frac{i}{4 \pi^{3}} \frac{(t+i \varepsilon) \gamma^{0}-\vec{\xi} \vec{\gamma}}{\left((t+i \varepsilon)^{2}-r^{2}\right)^{2}} \\
A_{x y}^{\varepsilon} & =P^{\varepsilon}(x, y) P^{\varepsilon}(y, x) \\
& =\frac{1}{16 \pi^{6}} \frac{1}{\left|(t-i \varepsilon)^{2}-r^{2}\right|^{4}}\left((t-i \varepsilon) \gamma^{0}-\vec{\xi} \vec{\gamma}\right)\left((t+i \varepsilon) \gamma^{0}-\vec{\xi} \vec{\gamma}\right) .
\end{aligned}
$$

Simplifying the Dirac matrices according to

$$
\left.\left(\not{\$}-i \varepsilon \gamma^{0}\right)\left(\not{\$}+i \varepsilon \gamma^{0}\right)=\xi^{2}-i \varepsilon\left[\gamma^{0}, \not\right)\right]+\varepsilon^{2},
$$

we obtain

$$
A_{x y}^{\varepsilon}=\frac{1}{16 \pi^{6}} \frac{\left(t^{2}-r^{2}\right)-i \varepsilon\left[\gamma^{0}, \not\right]+\varepsilon^{2}}{\left|(t-i \varepsilon)^{2}-r^{2}\right|^{4}} .
$$

In order to compute the eigenvalues of this matrix, the task is to diagonalize the bilinear contribution $i \varepsilon\left[\gamma^{0}, \$\right]$. The calculation

$$
\left.\left(i \varepsilon\left[\gamma^{0}, \not\right)\right]\right)^{2}=-4 \varepsilon^{2} \gamma^{0}(\vec{\xi} \vec{\gamma}) \gamma^{0}(\vec{\xi} \vec{\gamma})=4 \varepsilon^{2} \gamma^{0} \gamma^{0}(\vec{\xi} \vec{\gamma})(\vec{\xi} \vec{\gamma})=-4 \varepsilon^{2}|\vec{\xi}|^{2}<0
$$

shows that this bilinear contribution has complex eigenvalues. Thus the regularization makes the spacelike region larger. As we shall see below, this happens in a much more general setting. It is a desirable effect because it decreases the causal action.

Clearly, the singular behavior of the resulting expressions in the limit $\varepsilon \searrow 0$ is rather complicated. However, one limiting case, which will be important later on, is relatively easy to handle. This limiting case is to consider the region close to the light cone and away from the origin. For simplicity, we restrict attention to the upper light cone $t \approx r$ (but clearly, the lower light cone can be treated similarly). Then "close to the light cone" means that $t-r$ is much smaller than $r$, whereas "away from the origin" means that $\varepsilon$ is much smaller that $r$. Under these assumptions, we have approximately

$$
(t-i \varepsilon)^{2}-r^{2}=(t+r-i \varepsilon)(t-r-i \varepsilon) \approx 2 r(t-r-i \varepsilon) .
$$


In order to make the approximation precise, we write the error term as

$$
(t-i \varepsilon)^{2}-r^{2}=2 r(t-r-i \varepsilon)\left(1+\mathcal{O}\left(\frac{t-r}{r}\right)+\mathcal{O}\left(\frac{\varepsilon}{r}\right)\right) .
$$

Computing up to error terms of this type, the above formulas 2.4.7 -2.4 .9 can be simplified to

$$
\begin{aligned}
T^{(0)} & \rightarrow-\frac{1}{8 \pi^{3}} \frac{1}{2 r(t-r-i \varepsilon)} \\
T^{(1)} & \rightarrow \frac{1}{32 \pi^{3}} \log (2 r(t-r-i \varepsilon)) \\
T^{(-1)} & \rightarrow-\frac{1}{8 \pi^{3} r^{2}} \frac{1}{(t-r-i \varepsilon)^{2}} .
\end{aligned}
$$

Using this approximation, the closed chain 2.4.11) simplifies to

$$
A_{x y}^{\varepsilon}=\frac{1}{256 \pi^{6} r^{4}} \frac{\left(t^{2}-r^{2}\right)-i \varepsilon\left[\gamma^{0}, \$\right]+\varepsilon^{2}}{|t-r-i \varepsilon|^{4}} .
$$

Moreover, the numerator can be further simplified. We first note that, since $\xi$ is close to the light cone, the factor $\xi^{2}$ can be arbitrarily small. Therefore, despite the factor $\varepsilon$, the summand $\varepsilon\left[\gamma^{0}, \not\right]$ cannot be left out. But the summand $\varepsilon^{2}$ is of higher order in $\varepsilon / r$ and can be omitted. We conclude that

$$
A_{x y}^{\varepsilon}=\frac{1}{256 \pi^{6} r^{4}} \frac{\left(t^{2}-r^{2}\right)-i \varepsilon\left[\gamma^{0}, \not\right)}{|t-r-i \varepsilon|^{4}}\left(1+\mathcal{O}\left(\frac{t-r}{r}\right)+\mathcal{O}\left(\frac{\varepsilon}{r}\right)\right) .
$$

Clearly, composite expressions diverge in the limit $\varepsilon \searrow 0$. In order to analyze this singular behavior, the proper method is to evaluate weakly in $t$ for fixed $r$. Thus one considers integrals of the form

$$
\int_{-\infty}^{\infty} \eta(t)(\cdots) d t
$$

for a smooth test function $\eta$, where "..." stands for a composite expression in the $T^{(n)}$ and $\overline{T^{(n)}}$. Then "..." is a meromorphic function in $t$ with poles at $t= \pm r \pm i \varepsilon$. This makes it possible to compute the integral with the help of residues. The reader interested in an explicit example is referred to Exercise 2.20. Here we proceed by compiling and explaining a few general conclusions which will be important later on.

(a) The integrand in (2.4.17) has poles at $t= \pm r \pm i \varepsilon$. Again restricting attention to the upper light cone, we only need to consider the poles at $t=r \pm i \varepsilon$. When computing the residues at these points, the variable $t-r$ is of the order $\varepsilon$. Therefore, the two error terms in 2.4 .12 become the same. For convenience, we usually write the error terms as

$$
\cdots+\text { (higher orders in } \varepsilon /|\vec{\xi}|) \text {. }
$$

Moreover, the theorem of residues gives rise to contributions where the test function $\eta$ is differentiated. Every such derivative gives rise to an additional factor of $\varepsilon$. In order to keep the dimensions of length, we write the resulting error terms in the form

$$
+\left(\text { higher orders in } \varepsilon / \ell_{\text {macro }}\right) \text {, }
$$

where $\ell_{\text {macro }}$ denotes the "macroscopic" length scale on which $\eta$ varies. 
(b) The scaling of the integral 2.4.17) in $\varepsilon$ and $r$ can be described by

$$
T^{(n)} \sim(\varepsilon|\vec{\xi}|)^{n-1} \quad \text { and } \quad d t \sim \varepsilon .
$$

The resulting scaling of a composite expression in powers of $1 /(\varepsilon|\vec{\xi}|)$ is referred to as the degree of the expression. One should carefully distinguish the powers of $1 /(\varepsilon|\vec{\xi}|)$ defining the degree from the factors $\varepsilon /|\vec{\xi}|$ appearing in the error terms in 2.4.18). To make this distinction, it is important that we have two independent variables $\varepsilon$ and $|\vec{\xi}|$, and that we consider the scaling behavior in both variables. In this way, when evaluating a sum of expressions of different degrees, our methods make it possible to evaluate each degree separately, each with error terms of the form 2.4.18) and 2.4.19).

(c) The scaling behavior of the factors $\xi^{\varepsilon}$ is more subtle, as we now explain. If a factor $\xi^{\varepsilon}$ is contracted to Dirac matrices or to a macroscopic function (like a gauge potential or the Dirac current), we may simply disregard the regularization (2.4.6), i.e.

$$
\begin{aligned}
\not^{\varepsilon} & =\not+(\text { higher orders in } \varepsilon /|\vec{\xi}|) \\
\xi_{j}^{\varepsilon} f^{j} & =\xi_{j} f^{j}+(\text { higher orders in } \varepsilon /|\vec{\xi}|)
\end{aligned}
$$

(where $f_{j}$ is a macroscopic vector field). We refer to such factors $\xi^{\varepsilon}$ as outer factors.

(d) Two factors $\xi^{\varepsilon}$ which are contracted to each other are called inner factors. Since the resulting function $\xi^{2}$ is very small on the light cone, the factor $\varepsilon$ in 2.4.6) must be taken into account, i.e.

$$
\left(\xi^{\varepsilon}\right)^{2}=(t-i \varepsilon)^{2}-|\vec{\xi}|^{2}=t^{2}-|\vec{\xi}|^{2}-2 i \varepsilon t-\varepsilon^{2} .
$$

But similar as in 2.4.12, the quadratic term in $\varepsilon$ may be dropped, i.e.

$$
\left(\xi^{\varepsilon}\right)^{2}=t^{2}-|\vec{\xi}|^{2}-2 i \varepsilon t+(\text { higher orders in } \varepsilon /|\vec{\xi}|) \text {. }
$$

The general rule is that in every contraction, the factors $i \varepsilon$ must be taken into account linearly. This means in particular that the regularized factors $\xi^{\varepsilon}$ are no longer real, but must be treated as complex-valued vectors. Taking their complex conjugate corresponds to flipping the sign of $\varepsilon$, i.e.

$$
\bar{\xi}^{\varepsilon}=(t+i \varepsilon, \vec{\xi}) .
$$

Taking the adjoint of $\not^{\varepsilon}$ (with respect to the spin scalar product), we need to take the complex conjugate of $\xi^{\varepsilon}$, i.e.

$$
\left(\bar{\xi}^{\varepsilon}\right)^{*}=\overline{\xi^{\varepsilon}} .
$$

One must carefully distinguish $\xi^{\varepsilon}$ and $\overline{\xi^{\varepsilon}}$ in all computations.

(e) Clearly, a factor $\xi^{\varepsilon}$ may also be contracted to a factor $\bar{\xi}^{\varepsilon}$, or two factors $\bar{\xi}^{\varepsilon}$ may be contracted to each other. In these cases, we again refer to the factors $\xi^{\varepsilon}$ and $\bar{\xi}^{\varepsilon}$ as inner factors. Since we only take into account $\varepsilon$ linearly, we get

$$
\begin{aligned}
\left(\bar{\xi}^{\varepsilon}\right)^{2} & =t^{2}-|\vec{\xi}|^{2}+2 i \varepsilon t+(\text { higher orders in } \varepsilon /|\vec{\xi}|) \\
\left(\xi^{\varepsilon}\right)_{j}\left(\bar{\xi}^{\varepsilon}\right)^{j} & =t^{2}-|\vec{\xi}|^{2}+(\text { higher orders in } \varepsilon /|\vec{\xi}|) .
\end{aligned}
$$

Comparing these formulas with 2.4.22, one sees that

$$
\left(\xi^{\varepsilon}\right)_{j}\left(\bar{\xi}^{\varepsilon}\right)^{j}=\frac{1}{2}\left(\left(\xi^{\varepsilon}\right)^{2}+\left(\bar{\xi}^{\varepsilon}\right)^{2}\right)+(\text { higher orders in } \varepsilon /|\vec{\xi}|) .
$$


This identity will appear later in a much more general context as the so-called contraction rule.

After applying this contraction rule, one gets products of the form $\left(\xi^{\varepsilon}\right)^{2} T^{(l)}$. We remark that such products can be further simplified. Namely, according to the residual argument, the rule (2.2.18) also holds for $S^{(l)}$ replaced by $T^{(l)}$, up to smooth contributions. In fact, this rule even holds with regularization, i.e. for all $l \geq 0$

$$
\left(\xi^{(p)}\right)^{2} T^{(l)}=-4 p T^{(l-1)}+(\text { smooth contributions) }
$$

(the smooth contributions are of course important, but they can be treated together with the other smooth contributions to the fermionic projector as outlined in \$2.2.8). The reader interested in the details of the derivation of the identity $(2.4 .24)$ is referred to Exercise 2.21,

(f) We mention one more structure which in the present example is easy to understand, and which will come up in a more general context later on. Namely, suppose that the composite expression "..." in 2.4.17) can be written as a time derivative. Then we can integrate by parts,

$$
\int_{-\infty}^{\infty} \eta(t) \frac{\partial F}{\partial t} d t=-\int_{-\infty}^{\infty}\left(\partial_{t} \eta(t)\right) F(t) d t
$$

Since derivatives of the test function scale like factors $1 / \ell_{\text {macro }}$, this contribution is much smaller than expected from the scalings 2.4 .20 . We write

$$
\int_{-\infty}^{\infty} \eta(t) \frac{\partial F}{\partial t} d t=0+\left(\text { higher orders in } \varepsilon / \ell_{\text {macro }}\right) \text {. }
$$

This relation shows that certain composite expressions in the factors $T^{(n)}$ and $\overline{T^{(n)}}$ vanish when evaluated weakly on the light cone. In other words, there are relations between composite expressions.

These relations are expressed most conveniently in terms of so-called integrationby-parts rules. The starting point for deriving these rules is the identity (2.4.4) which holds up to smooth contributions, i.e. for all $l \geq 0$

$$
\frac{\partial}{\partial x^{k}} T^{(l)}(x, y)=\frac{1}{2}(y-x)_{k} T^{(l-1)}(x, y)+(\text { smooth contributions })
$$

(recall that in the case $l=0$, this relation serves as the definition of $T^{(-1)}$ ). For an explicit derivation of the identity (2.4.26) we again refer to Exercise 2.21. Considering a derivative in time direction (and noting that $\partial_{t}=-\partial_{x^{0}}$ ), we obtain

$$
\frac{\partial}{\partial t} T^{(l)}(x, y)=-\frac{1}{2} t T^{(l-1)}(x, y)+(\text { smooth contributions }) .
$$

Near the upper light cone, we can write this identity as

$$
\begin{aligned}
\frac{1}{r} & \frac{\partial}{\partial t} T^{(l)}(x, y)=-\frac{1}{2} T^{(l-1)}(x, y) \\
& +(\text { smooth contributions) }+ \text { (higher orders in } \varepsilon /|\vec{\xi}|) .
\end{aligned}
$$

Introducing the abbreviation

we thus obtain the relations

$$
\nabla:=\frac{1}{t} \frac{\partial}{\partial t}
$$

$$
\nabla T^{(l)}=-\frac{1}{2} T^{(l-1)}
$$


Moreover, the identity 2.4 .25 can be written in the short symbolic form

$$
\nabla(\cdots)=0+(\text { smooth contributions })+(\text { higher orders in } \varepsilon /|\vec{\xi}|),
$$

where "..." again stands for a composite expression in the $T^{(n)}$ and $\overline{T^{(n)}}$.

We finally remark that, at this stage, neglecting all terms of the order (2.4.18) merely is a matter of convenience. In fact, one can also take into account the higher orders in $\varepsilon /|\vec{\xi}| \mid$ by performing an expansion in powers of $\varepsilon /|\vec{\xi}|$. Such an expansion is called regularization expansion. We will come back to the regularization expansion in 2.4 .5 . But before, we analyze the situation for more general regularizations.

2.4.2. Example: Linear Combinations of $i \varepsilon$-Regularizations. Clearly, the $i \varepsilon$ regularization is very special and ad-hoc. In order to get a first idea on what happens for more general regularizations, it is instructive to consider linear combinations of $i \varepsilon$ regularizations. To this end, we choose an integer $N$ and generalize 2.4.2 to

$$
\hat{P}^{\varepsilon}(k)=(\not k+m) \delta\left(k^{2}-m^{2}\right) \Theta\left(-k^{0}\right)\left(\sum_{a=1}^{N} c_{a} \exp \left(\varepsilon d_{a} k^{0}\right)\right)
$$

with positive parameters $d_{1}, \ldots, d_{N}$ and real numbers $c_{1}, \ldots, c_{N}$ which add up to one,

$$
c_{1}+\cdots+c_{N}=1 \text {. }
$$

In fact, by choosing $N$ sufficiently large, with this ansatz one can approximate any regularization of the form

$$
\hat{P}^{\varepsilon}(k)=(\not k+m) \delta\left(k^{2}-m^{2}\right) \Theta\left(-k^{0}\right) \hat{h}\left(k^{0}\right),
$$

corresponding to a regularization by convolution with a function $h(t)$ (being a special case of the regularizations in Example 1.2.4).

For regularizations of the form 2.4.30), we can again evaluate weakly on the light cone $(2.4 .17)$. It turns out that the scalings in $\varepsilon$ and $|\vec{\xi}|$ are exactly the same as for the $i \varepsilon$-regularization. In order to see this in a simple setting, one can consider a polynomial in $T^{(n)}$ and $\overline{T^{(n)}}$,

$$
T^{\left(l_{1}\right)} \cdots T^{\left(l_{\alpha}\right)} \overline{T^{\left(n_{1}\right)} \cdots T^{\left(n_{\beta}\right)}} .
$$

When evaluating weakly on the light cone, one can pull the sums of the linear combinations in 2.4.30 out of the integral, i.e.

$$
\begin{aligned}
& \int_{-\infty}^{\infty} \eta(t) T^{\left(l_{1}\right)} \cdots T^{\left(l_{\alpha}\right)} \overline{T^{\left(n_{1}\right)} \cdots T^{\left(n_{\beta}\right)}} d t \\
& =\sum_{a_{1}, \ldots, a_{\alpha}, b_{1}, \ldots, b_{\beta}=1}^{N} c_{a_{1}} \cdots c_{a_{\alpha}} c_{b_{1}} \cdots c_{b_{\beta}} \int_{-\infty}^{\infty} \eta(t) T_{d_{a_{1}}}^{\left(l_{1}\right)} \cdots T_{d_{a_{\alpha}}}^{\left(l_{\alpha}\right)} \overline{T_{d_{b_{1}}}^{\left(n_{1}\right)} \cdots T_{d_{b_{\beta}}}^{\left(n_{3}\right)}} d t
\end{aligned}
$$

where $T_{d}^{(n)}$ denotes the $i \varepsilon$-regularization with $\varepsilon$ replaced by $\varepsilon d$. Again computing up to the error terms 2.4.18) and 2.4.19), one can again use the explicit formulas for $T^{(n)}$ like (2.4.13)-2.4.15) and analyze the integral with residues. The only difference compared to the analysis of the $i \varepsilon$-regularization is that one has many poles at positions $t=r \pm i \varepsilon d_{a}$, and the residue theorem gives sums over these poles. But obviously, this has no effect on all scalings. 
The contraction of the inner factors must be handled with care, as we now explain. Using (2.4.6) and forming linear combinations, one sees that the factor $\$ T^{(n)}$ is to be regularized according to

$$
\Varangle T^{(n)} \rightarrow \sum_{a=1}^{N} c_{a}\left(\left(t-i \varepsilon d_{a}\right) \gamma^{0}-\vec{\xi} \vec{\gamma}\right) T_{d_{a}}^{(n)}
$$

(with $T_{d}^{(n)}$ again as in 2.4 .32$)$ ). When forming composite expressions, one must take into account that the regularized factors $\xi$ and $T^{(n)}$ both carry the same summation index. Therefore, one should regard the factors $T^{(n)}$ and $\sharp$ as belonging together. It is useful to make this connection explicit in the notation. Therefore, we discard (2.4.6) and introduce instead the more general rule

$$
\notin T^{(n)} \rightarrow \oiint^{(n)} T^{(n)}
$$

where the right side is a short notation for the sum in (2.4.33).

Contracting two inner factors $\xi$ in this formalism gives

$$
\begin{gathered}
\left(\xi^{(l)}\right)_{j} T^{(l)}\left(\xi^{(n)}\right)^{j} T^{(n)}=\sum_{a, b=1}^{N} c_{a} c_{b}\left(\left(t-i \varepsilon d_{a}\right), \vec{\xi}\right)_{j} T_{d_{a}}^{(l)}\left(\left(t-i \varepsilon d_{b}\right), \vec{\xi}\right)^{j} T_{d_{b}}^{(n)} \\
=\sum_{a, b=1}^{N} c_{a} c_{b} T_{d_{a}}^{(l)} T_{d_{b}}^{(n)}\left(t^{2}-i \varepsilon t d_{a}-i \varepsilon t d_{b}-\varepsilon^{2} d_{a} d_{b}-|\vec{\xi}|^{2}\right) .
\end{gathered}
$$

This is considerably more complicated than (2.4.21). However, if as in 2.4 .22 we drop the term quadratic in $\varepsilon$, the formula can be simplified to

$$
\begin{aligned}
& \left.{ }^{\left(\xi^{(p)}\right.}\right)_{j} T^{(l)}\left(\xi^{(q)}\right)^{j} T^{(n)} \\
& =\sum_{a, b=1}^{N} c_{a} c_{b} T_{d_{a}}^{(l)} T_{d_{b}}^{(n)}\left(t^{2}-i \varepsilon t d_{a}-i \varepsilon t d_{b}-|\vec{\xi}|^{2}\right)+(\text { higher orders in } \varepsilon /|\vec{\xi}|) \\
& =\frac{1}{2} \sum_{a, b=1}^{N} c_{a} c_{b} T_{d_{a}}^{(l)} T_{d_{b}}^{(n)}\left(\left(\left(t-i \varepsilon d_{a}\right), \vec{\xi}\right)^{2}+\left(\left(t-i \varepsilon d_{b}\right), \vec{\xi}\right)^{2}\right) \\
& \quad+(\text { higher orders in } \varepsilon /|\vec{\xi}|) \\
& =\frac{1}{2}\left(\left(\xi^{(l)}\right)^{2}+\left(\xi^{(n)}\right)^{2}\right) T^{(l)} T^{(n)}+(\text { higher orders in } \varepsilon /|\vec{\xi}|)
\end{aligned}
$$

where the squares in 2.4.36 denote the Minkowski inner product, and where in the last step we introduced the notation

$$
\left(\xi^{(l)}\right)^{2} T^{(l)}=\sum_{a=1}^{N} c_{a}\left(\left(t-i \varepsilon d_{a}\right)^{2}-|\vec{\xi}|^{2}\right) T_{d_{a}}^{(l)} .
$$

In this way, the contraction rules $(2.4 .22)$ can be generalized to

$$
\left(\xi^{(l)}\right)^{j}\left(\xi^{(n)}\right)_{j}=\frac{1}{2}\left(\left(\xi^{(l)}\right)^{2}+\left(\xi^{(n)}\right)^{2}\right)
$$

Similarly the contraction rule 2.4 .23$)$ becomes

$$
\left(\xi^{(l)}\right)^{j} \overline{\left(\xi^{(n)}\right)_{j}}=\frac{1}{2}\left(\left(\xi^{(l)}\right)^{2}+\overline{\left(\xi^{(n)}\right)^{2}}\right) .
$$


We remark that this product can again be simplified using (2.4.24), giving rise to the computation rule

$$
\left(\xi^{(p)}\right)^{2} T^{(l)}=-4 p T^{(l-1)}+(\text { smooth contributions }) .
$$

We also remark that the integration-by-parts rules $(2.4 .28)$ and 2.4 .29$)$ with $\nabla$ according to 2.4.27) remain valid, as one sees immediately by applying (2.4.28) to each summand in 2.4.30 and by noting that 2.4.25 holds for any regularization.

Working with linear combinations of $i \varepsilon$-regularization gives a first hint why one should disregard error terms of the form 2.4.18 and 2.4.19), as we now explain. Using the method of variable regularization (see Remark 1.2.1), we must show that the structure of the effective equations in the continuum limit does not depend on the details of the regularization. Evaluating weakly on the light cone and neglecting error terms of the form $(2.4 .18)$ and $(2.4 .19)$, one gets relatively simple computation rules (like 2.4 .39 ), (2.4.40) or (2.4.24) ), giving rise to a formalism which captures the structure of the EL equation independent of regularization details. However, for example the quadratic term in $\varepsilon$ in 2.4 .34

$$
-\varepsilon^{2} \sum_{a, b=1}^{N} c_{a} c_{b} d_{a} d_{b} T_{d_{a}}^{(l)} T_{d_{b}}^{(n)}
$$

has a different structure. Namely, even after prescribing linear moments as they appear in (2.4.33), there is a lot of freedom to give the quadratic term in 2.4.41) an arbitrary value. More generally, if we computed the terms 2.4.18) or 2.4.19), these contributions would depend on the regularization in a complicated way, so much so that without knowing the regularization in detail, it would be impossible to evaluate these contributions. This is the reason why we shall disregard these contributions. Clearly, at this stage, the above argument is not quite satisfying because notions like "complicated" and "knowing the regularization in detail" are somewhat vague. The argument will be made more precise in 2.4.5 using Fourier methods.

2.4.3. Further Regularization Effects. Working with linear combinations of $i \varepsilon$ regularizations, one is still in the restrictive class of regularizations of the form 2.4.31) where the unregularized distribution is multiplied in momentum space by a convergencegenerating function $\hat{h}\left(k^{0}\right)$. Considering more general regularizations gives rise to additional effects. We now list those regularization effects will be important later on:

- The support of the distribution in (2.4.31) can be slightly deformed from the hyperboloid to another hypersurface. It turns out that in this case, one can still perform a mass expansion of the form 2.4.5). But the regularization of the factors $T^{(n)}$ also depends on the power of the mass in the corresponding contribution to the fermionic projector. In order to implement this effect into our formalism, one adds a subscript [.] to the factors $T^{(n)}$ which counts the power in $m$. Thus we regularize the contributions to the light-cone expansion according to the rule

$$
m^{p} T^{(n)} \rightarrow m^{p} T_{[p]}^{(n)}
$$

For example, the regularization of the light-cone expansion of the vacuum 2.4.5 now takes the form

$$
P^{\varepsilon}(x, y)=\sum_{n=0}^{\infty} \frac{m^{2 n}}{n !} \frac{i \not^{(-1+n)}}{2} T_{[2 n]}^{(-1+n)}+\sum_{n=0}^{\infty} \frac{m^{2 n+1}}{n !} T_{[2 n+1]}^{(n)} .
$$


Regularizing the fermionic projector in the presence of an external potential, one gets contributions involving factors $T_{[p]}^{(n)}$ with the same $n$ but different values of $p$. These factors must be treated as being different (although they clearly coincide without regularization).

- The direction of the vector $k$ which appears in the factor $\not k$ in 2.4 .31 can be slightly changed by the regularization. This leads to the notion of the shear of surface states. This effect is of importance when inner factors are contracted. More precisely, one needs to modify the calculation rule (2.4.24) to

$$
\left(\xi^{(p)}\right)^{2} T_{[p]}^{(n)}=-4\left(n T_{[p]}^{(n+1)}+T_{\{p\}}^{(n+2)}\right)+(\text { smooth contributions }),
$$

where the factors $T_{\{p\}}^{(l)}$ with curly brackets have the same scaling behavior as the corresponding factors with square brackets but are regularized differently.

- There may be additional contributions to $\hat{P}(k)$ which lie outside the hyperboloid in 2.4.31) or the deformation thereof. It turns out that the resulting contributions can be absorbed into the error terms (2.4.18) and (2.4.19) (for details see 82.4 .5 ).

We also remark that the regularization of neutrinos is more involved because the regularization must break the chiral symmetry and because the corresponding Dirac sea can "mimic" a Dirac sea of a different mass. In order not to distract from the main points of our construction, these extensions of the formalism will be introduced later when we need them (see Section 4.2).

2.4.4. The Formalism of the Continuum Limit. After the above motivation and preparations, we now present the formalism of the continuum limit. In 2.4 .5 we shall outline the derivation of this formalism as first given in [F7, Chapter 4].

Before beginning, we point out that we work modulo smooth contributions throughout. The reason for this procedure is that the smooth contributions can be computed in a straightforward manner by first evaluating composite expressions away from the light cone (where they are smooth) and taking the limit when $y-x$ approaches the light cone. Clearly, computing the smooth contributions is important and not always easy (for details see Appendix $\mathrm{D}$. . But these computations are not related to the problem of the singularities on the light cone to be considered here.

Our starting point is the light-cone expansion of the unregularized fermionic projector $P(x, y)$ (as given in 2.2 .8 ). In order to regularize the light-cone expansion on the length scale $\varepsilon$, we proceed as follows. The smooth contributions are all left unchanged. For the regularization of the factors $T^{(n)}$, we employ the replacement rule

$$
m^{p} T^{(n)} \rightarrow m^{p} T_{[p]}^{(n)},
$$

where the factors $T_{[p]}^{(n)}$ are smooth functions of $\xi$. Fortunately, the rather complicated detailed form of the factors $T_{[p]}^{(n)}$ will not be needed here, because these functions can be treated symbolically using the following simple calculation rules. In computations one may treat the $T_{[p]}^{(n)}$ like complex functions. However, one must be careful when tensor indices of factors $\sharp$ are contracted with each other. Naively, this gives a factor $\xi^{2}$ which vanishes on the light cone and thus changes the singular behavior on the light cone. In order to describe this effect correctly, we first write every summand of the light cone expansion 2.2.129) such that it involves at most one factor $\$$ (this can always be arranged using the anti-commutation relations of the Dirac matrices). We now associate every 
factor $\&$ to the corresponding factor $T_{[p]}^{(n)}$. In short calculations, this can be indicated by putting brackets around the two factors, whereas in the general situation we add corresponding indices to the factor $\sharp$, giving rise to the replacement rule

$$
m^{p} \not T^{(n)} \rightarrow m^{p} \sharp_{[p]}^{(n)} T_{[p]}^{(n)} .
$$

For example, we write the regularized fermionic projector of the vacuum as

$$
P^{\varepsilon}=\frac{i}{2} \sum_{n=0}^{\infty} \frac{m^{2 n}}{n !} \sharp_{[2 n]}^{(-1+n)} T_{[2 n]}^{(-1+n)}+\sum_{n=0}^{\infty} \frac{m^{2 n+1}}{n !} T_{[2 n+1]}^{(n)} .
$$

The kernel $P(y, x)$ is obtained by taking the conjugate (see (2.1.68)). The conjugates of the factors $T_{[p]}^{(n)}$ and $\xi_{[p]}^{(n)}$ are the complex conjugates,

$$
\overline{T_{[p]}^{(n)}}:=\left(T_{[p]}^{(n)}\right)^{*} \quad \text { and } \quad \overline{\xi_{[p]}^{(n)}}:=\left(\xi_{[p]}^{(n)}\right)^{*} .
$$

One must carefully distinguish between these factors with and without complex conjugation. In particular, the factors $\sharp_{[p]}^{(n)}$ need not be symmetric,

$$
\left(\sharp_{[p]}^{(n)}\right)^{*} \neq \sharp_{[p]}^{(n)} \quad \text { in general . }
$$

When forming composite expressions, the tensor indices of the factors $\xi$ are contracted to other tensor indices. The factors $\xi$ which are contracted to other factors $\xi$ are called inner factors. The contractions of the inner factors are handled with the so-called contraction rules

$$
\begin{aligned}
\left(\xi_{[p]}^{(n)}\right)^{j}\left(\xi_{\left[p^{\prime}\right]}^{\left(n^{\prime}\right)}\right)_{j} & =\frac{1}{2}\left(z_{[p]}^{(n)}+z_{\left[p^{\prime}\right]}^{\left(n^{\prime}\right)}\right) \\
\left(\xi_{[p]}^{(n)}\right)^{j} \overline{\left(\xi_{\left[p^{\prime}\right]}^{\left(n^{\prime}\right)}\right)_{j}} & =\frac{1}{2}\left(z_{[p]}^{(n)}+\overline{z_{\left[p^{\prime}\right]}^{\left(n^{\prime}\right)}}\right) \\
z_{[p]}^{(n)} T_{[p]}^{(n)} & =-4\left(n T_{[p]}^{(n+1)}+T_{\{p\}}^{(n+2)}\right),
\end{aligned}
$$

which are to be complemented by the complex conjugates of these equations. Here the factors $z_{[p]}^{(n)}$ can be regarded simply as a book-keeping device to ensure the correct application of the rule 2.4.46). The factors $T_{\{p\}}^{(n)}$ have the same scaling behavior as the $T_{[p]}^{(n)}$, but their detailed form is somewhat different; we simply treat them as a new class of symbols. In cases where the lower index does not need to be specified we write $T_{\circ}^{(n)}$. After applying the contraction rules, all inner factors $\xi$ have disappeared. The remaining so-called outer factors $\xi$ need no special attention and are treated like smooth functions.

Next, to any factor $T_{0}^{(n)}$ we associate the degree $\operatorname{deg} T_{\circ}^{(n)}$ by

$$
\operatorname{deg} T_{\circ}^{(n)}=1-n .
$$

The degree is additive in products, whereas the degree of a quotient is defined as the difference of the degrees of numerator and denominator. The degree of an expression can be thought of as describing the order of its singularity on the light cone, in the sense that a larger degree corresponds to a stronger singularity (for example, the contraction rule (2.4.46) increments $n$ and thus decrements the degree, in agreement with the naive observation that the function $z=\xi^{2}$ vanishes on the light cone). Using formal Taylor 
series, we can expand in the degree. In all our applications, this will give rise to terms of the form

$$
\eta(x, y) \frac{T_{\circ}^{\left(a_{1}\right)} \cdots T_{\circ}^{\left(a_{\alpha}\right)} \overline{T_{\circ}^{\left(b_{1}\right)} \cdots T_{\circ}^{\left(b_{\beta}\right)}}}{T_{\circ}^{\left(c_{1}\right)} \cdots T_{\circ}^{\left(c_{\gamma}\right)} \overline{T_{\circ}^{\left(d_{1}\right)} \cdots T_{\circ}^{\left(d_{\delta}\right)}}} \quad \text { with } \eta(x, y) \text { smooth . }
$$

The quotient of the two monomials in this equation is referred to as a simple fraction.

A simple fraction can be given a quantitative meaning by considering one-dimensional integrals along curves which cross the light cone transversely away from the origin $\xi=0$. This procedure is called weak evaluation on the light cone. For our purpose, it suffices to integrate over the time coordinate $t=\xi^{0}$ for fixed $\vec{\xi} \neq 0$. Moreover, using the symmetry under reflections $\xi \rightarrow-\xi$, it suffices to consider the upper light cone $t \approx|\vec{\xi}|$. The resulting integrals diverge if the regularization is removed. The leading contribution for small $\varepsilon$ can be written as

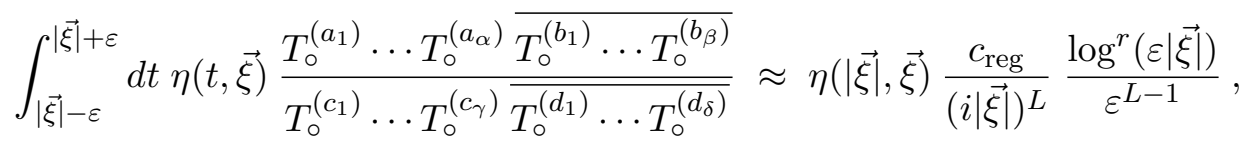

where $L$ is the degree of the simple fraction and $c_{\text {reg }}$, the so-called regularization parameter, is a real-valued function of the spatial direction $\vec{\xi} /|\vec{\xi}|$ which also depends on the simple fraction and on the regularization details (the error of the approximation will be specified below). The integer $r$ describes a possible logarithmic divergence. Apart from this logarithmic divergence, the scalings in both $\xi$ and $\varepsilon$ are described by the degree.

When analyzing a sum of expressions of the form (2.4.47), one must know if the corresponding regularization parameters are related to each other. In this respect, the integration-by-parts rules are important, which are described symbolically as follows. On the factors $T_{\circ}^{(n)}$ we introduce a derivation $\nabla$ by

$$
\nabla T_{\circ}^{(n)}=T_{\circ}^{(n-1)} .
$$

Extending this derivation with the Leibniz and quotient rules to simple fractions, the integration-by-parts rules state that

$$
\nabla\left(\frac{T_{\circ}^{\left(a_{1}\right)} \cdots T_{\circ}^{\left(a_{\alpha}\right)} \overline{T_{\circ}^{\left(b_{1}\right)} \cdots T_{\circ}^{\left(b_{\beta}\right)}}}{T_{\circ}^{\left(c_{1}\right)} \cdots T_{\circ}^{\left(c_{\gamma}\right)} \overline{T_{\circ}^{\left(d_{1}\right)} \cdots T_{\circ}^{\left(d_{\delta}\right)}}}\right)=0 .
$$

These rules give relations between simple fractions. The name is motivated by the integration-by-parts method as explained for the $i \varepsilon$-regularization in 2.4.25). Simple fractions which are not related to each other by the integration-by-parts rules are called basic fractions. As shown in [F7, Appendix E], there are no further relations between the basic fractions. Thus the corresponding basic regularization parameters are linearly independent.

The above symbolic computation rules give a convenient procedure to evaluate composite expressions in the fermionic projector, referred to as the analysis in the continuum limit: After applying the contraction rules and expanding in the degree, the EL equations can be rewritten as equations involving a finite number of terms of the form (2.4.47). By applying the integration-by-parts rules, we can arrange that all simple fractions are basic fractions. We evaluate weakly on the light cone 2.4.48 and collect the terms according to their scaling in $\xi$. Taking for every given scaling in $\xi$ only the leading pole in $\varepsilon$, we obtain equations which involve linear combinations of smooth functions and basic regularization parameters. We consider the basic regularization parameters as empirical 
parameters describing the unknown microscopic structure of space-time. We thus end up with equations involving smooth functions and a finite number of free parameters. We point out that these free parameters cannot be chosen arbitrarily because they might be constrained by inequalities (see the discussion after [F7, Theorem E.1]). Also, the values of the basic regularization parameters should ultimately be justified by an analysis of vacuum minimizers of the causal action principle.

We finally specify the error of the above expansions. By not regularizing the bosonic potentials and fermionic wave functions, we clearly disregard the

$$
\text { higher orders in } \varepsilon / \ell_{\text {macro }} \text {. }
$$

Furthermore, in 2.4.48 we must stay away from the origin, meaning that we neglect the

$$
\text { higher orders in } \varepsilon /|\vec{\xi}| \text {. }
$$

The higher oder corrections in $\varepsilon /|\vec{\xi}|$ depend on the fine structure of the regularization and thus seem unknown for principal reasons. Neglecting the terms in 2.4.50 and 2.4.51) also justifies the formal Taylor expansion in the degree. Clearly, leaving out the terms 2.4.51) is justified only if $|\vec{\xi}| \gg \varepsilon$. Therefore, whenever using the above formalism, we must always ensure that $|\vec{\xi}|$ is much larger than $\varepsilon$ (we will come back to this point in $\$ 2.6 .5$ 3.5.2 and Appendix A).

2.4.5. Outline of the Derivation. We now outline the derivation of the formalism of the continuum limit (for more details see [F7, Chapter 4]). The method relies on an asymptotic analysis of the Fourier integral 2.4.1,

$$
P^{\varepsilon}(x, y)=\int \frac{d^{4} k}{(2 \pi)^{4}} \hat{P}^{\varepsilon}(k) e^{i k \xi} .
$$

For simplicity, we begin the analysis for the scalar component, i.e. we consider the case

$$
\hat{P}^{\varepsilon}(p)=\phi(p) f(p)
$$

(the vector component will be treated after (2.4.82) below). We may assume that the spatial component of the vector $\xi$ points in the direction of the $x$-axis of our Cartesian coordinate system, i.e. $y-x=(t, r, 0,0)$ with $r>0$. Choosing cylindrical coordinates $\omega$, $k, \rho$ and $\varphi$ in momentum space, defined by $p=(\omega, \vec{p})$ and $\vec{p}=(k, \rho \cos \varphi, \rho \sin \varphi)$, the Fourier integral becomes

$$
P(x, y)=\frac{1}{(2 \pi)^{4}} \int_{-\infty}^{\infty} d \omega \int_{-\infty}^{\infty} d k \int_{0}^{\infty} \rho d \rho \int_{0}^{2 \pi} d \varphi \hat{P}^{\varepsilon}(\omega, k, \rho, \varphi) e^{i \omega t-i k r} .
$$

Since the exponential factor in this formula is independent of $\rho$ and $\varphi$, we can write the fermionic projector as the two-dimensional Fourier transform

$$
P(x, y)=2 \int_{-\infty}^{\infty} d \omega \int_{-\infty}^{\infty} d k h(\omega, k) e^{i \omega t-i k r}
$$

of a function $h$ defined by

$$
h(\omega, k)=\frac{1}{2(2 \pi)^{4}} \int_{0}^{\infty} \rho d \rho \int_{0}^{2 \pi} d \varphi(\phi f)(\omega, k, \rho, \varphi) .
$$

We want to analyze $P(x, y)$ close to the light cone $(y-x)^{2}=0$ away from the origin $y=x$. Without loss of generality, we may restrict attention to the upper light cone $t=r$. 
Thus we are interested in the region $t \approx r>0$. The "light-cone coordinates"

$$
s=\frac{1}{2}(t-r), \quad l=\frac{1}{2}(t+r)
$$

are well-suited to this region, because the "small" variable $s$ vanishes for $t=r$, whereas the "large" variable $l$ is positive and non-zero. Introducing also the associated momenta

$$
u=-k-\omega, \quad v=k-\omega,
$$

we can write the fermionic projector as

$$
P(s, l)=\int_{-\infty}^{\infty} d u \int_{-\infty}^{\infty} d v h(u, v) e^{-i(u s+v l)}
$$

Let us briefly discuss the qualitative form of the function $h,(2.4 .56$ ). Without regularization, the scalar component is given by the $\delta$-distribution on the lower mass shell $\hat{P}=m \delta\left(p^{2}-m^{2}\right) \Theta\left(-p^{0}\right)$. In this case, the integral 2.4.56) can be evaluated to be

$$
\begin{aligned}
h & =\frac{m}{2(2 \pi)^{4}} \int_{0}^{\infty} \rho d \rho \int_{0}^{2 \pi} d \varphi \delta\left(\omega^{2}-k^{2}-\rho^{2}-m^{2}\right) \Theta(-\omega) \\
& =\frac{m}{4(2 \pi)^{3}} \Theta\left(\omega^{2}-k^{2}-m^{2}\right) \Theta(-\omega)=\frac{m}{32 \pi^{3}} \Theta\left(u v-m^{2}\right) \Theta(u) .
\end{aligned}
$$

Thus integrating over $\rho$ and $\varphi$ yields a constant function in the interior of the two-dimensional "lower mass shell" $\omega^{2}-k^{2}=m^{2}, \omega<0$. From this we conclude that for small momenta, where the regularization should play no role, the function $h$ should have a discontinuity along the hyperbola $\left\{u v=m^{2}, u>0\right\}$, be zero below (i.e. for $u v<m^{2}$ ) and be nearly constant above. The precise form of $h$ for large energy or momentum can be arbitrary. We only know that $h$ decays at infinity.

It is instructive to discuss the energy scales. Clearly, one scale is given by the regularization length $\varepsilon$. In momentum space, this corresponds to the high energy scale $\varepsilon^{-1}$. We sometimes refer to the region $|\omega|+|k| \gtrsim \varepsilon^{-1}$ as the high energy region. The relevant low energy scale, on the other hand, is $\varepsilon m^{2}$ (it is zero for massless fermions). This is because the hyperbola $u v=m^{2}$ comes as close to the $v$-axis as as $v \sim \varepsilon m^{2}$ before entering the high energy region. Finally, the Compton scale $m$ lies between the low- and high energy scales,

$$
\varepsilon m^{2} \lesssim m \lesssim \varepsilon^{-1}
$$

Since we want to analyze the situation close to the light cone, we choose the "small" light-cone parameter $s$ on the regularization scale, i.e.

$$
s \lesssim \varepsilon .
$$

The "large" light-cone parameter $l$, on the other hand, is non-zero. We shall always choose this scale between the regularization scale and the Compton scale,

$$
\varepsilon \ll l \ll \frac{1}{m} .
$$

Since $\varepsilon m \ll 1$, the inequalities in $(2.4 .62)$ still leave us the freedom to vary $l$ on many orders of magnitude.

Our task is to evaluate the Fourier integral (2.4.59) using the scales (2.4.61) and (2.4.62). In preparation, we discuss and specify the function $h(u, v)$ for fixed $u$, also denoted by $h_{u}(v)$. Without regularization (2.4.60), the function $h_{u}$ has a discontinuous "jump" from zero to a finite value on the hyperbola. Therefore, we cannot expect that $h_{u}$ is continuous when a regularization is present. On the contrary, the decay for large $v$ 
suggests that $h_{u}$ might have another discontinuity for large $v$, where it might "jump" to zero. In order to keep the presentation reasonably simple, we assume that $h_{u}$ is always of this general form, i.e.

$$
h_{u}(v)=\left\{\begin{array}{cl}
0 & \text { for } v<\alpha_{u} \text { or } v>\beta_{u} \\
\text { smooth } & \text { for } \alpha_{u} \leq v \leq \beta_{u}
\end{array}\right.
$$

with parameters $\alpha_{u}<\beta_{u}$. The case of less than two discontinuities can be obtained from (2.4.63) by setting $h_{u}\left(\alpha_{u}\right)$ or $h_{u}\left(\beta_{u}\right)$ equal to zero, or alternatively by moving the position of the discontinuities $\alpha_{u}$ or $\beta_{u}$ to infinity. We remark that the discontinuity at $v=\beta_{u}$ will become irrelevant later; it is taken into account only in order to explain why the behavior of the fermionic projector on the light cone is independent of many regularization details.

Without regularization (2.4.60), the function $h_{u}(v)$ is constant for $v \geq \alpha_{u}$. Thus the $v$-dependence of $h_{u}(v)$ for $\alpha_{u} \leq v \leq \beta_{u}$ merely is a consequence of the regularization, and it is therefore reasonable to assume that the $v$-derivatives of $h_{u}(v)$ scale in powers of the regularization length $\varepsilon$. More precisely, we assume that there is a constant $c_{1} \ll l / \varepsilon$ such that

$$
\left|h_{u}^{(n)}(v)\right| \leq\left(c_{1} \varepsilon\right)^{n} \max \left|h_{u}\right| \quad \text { for } \alpha_{u} \leq v \leq \beta_{u},
$$

where the derivatives at $v=\alpha_{u}$ and $\beta_{u}$ are understood as the right- and left-sided limits, respectively. This regularity condition is typically satisfied for polynomial, exponential and trigonometric functions, but it excludes the case that the function $h_{u}$ has small-scale fluctuations. Clearly, we could also consider a more general ansatz for $h_{u}$ with more than two discontinuities or weaker regularity assumptions. But this does not seem to be the point because all interesting effects, namely the influence of discontinuities for small and large $v$ as well as of smooth regions, can already be studied in the setting (2.4.63), 2.4.64.

Let us analyze the $v$-integral of the Fourier transform 2.4.59,

$$
P_{u}(l):=\int_{-\infty}^{\infty} h_{u}(v) e^{-i v l} d v
$$

According to the left inequality in 2.4 .62 , the exponential factor in 2.4 .65 is highly oscillatory on the scale $v \sim 1 / \varepsilon$. Thus we can expect that the smooth component of $h_{u}$ only gives a small contribution to the integral 2.4.65), so that the discontinuities at $\alpha_{u}$ and $\beta_{u}$ should play the dominant role. In order to make this picture mathematically precise, in 2.4.65 we iteratively integrate $K$ times by parts,

$$
\begin{aligned}
P_{u}(l) & =\int_{\alpha_{u}}^{\beta_{u}} h_{u}(v) e^{-i v l} d v=-\frac{1}{i l} \int_{\alpha_{u}}^{\beta_{u}} d v h_{u}(v) \frac{d}{d v} e^{-i v l} \\
& =-\left.\frac{1}{i l} h_{u}(v) e^{-i v l}\right|_{\alpha_{u}} ^{\beta_{u}}+\frac{1}{i l} \int_{\alpha_{u}}^{\beta_{u}} h_{u}^{\prime}(v) e^{-i v l} d l=\cdots= \\
& =-\left.\frac{1}{i l} \sum_{n=0}^{K-1}\left(\frac{1}{i l}\right)^{n} h_{u}^{(n)}(v) e^{-i v l}\right|_{\alpha_{u}} ^{\beta_{u}}+\left(\frac{1}{i l}\right)^{K} \int_{\alpha_{u}}^{\beta_{u}} h_{u}^{(K)}(v) e^{-i v l} d l .
\end{aligned}
$$

If we bound all summands in 2.4 .66$)$ using the first inequality in 2.4 .62 and the regularity condition (2.4.64), each $v$-derivative appears in combination with a power of $l^{-1}$, and giving a factor $c_{1} \varepsilon / l \ll 1$. Thus in the limit $K \rightarrow \infty$, we may drop the integral in 2.4.66 to obtain

$$
P_{u}(l)=-\left.\frac{1}{i l} \sum_{n=0}^{\infty}\left(\frac{1}{i l}\right)^{n} h_{u}^{(n)}(v) e^{-i v l}\right|_{\alpha_{u}} ^{\beta_{u}} .
$$


This expansion converges, and its summands decay like $\left(c_{1} \varepsilon / l\right)^{n}$.

Using (2.4.65), we can write the Fourier transform (2.4.59) as

$$
P(s, l)=\int_{-\infty}^{\infty} P_{u}(l) e^{-i u s} d u .
$$

Notice that, apart from the constraints (2.4.62), the "large" variable $l$ can be freely chosen. We want to study the functional dependence of (2.4.68) on the parameter $l$. In preparation, we consider an integral of the general form

$$
\int_{a}^{b} f(u) e^{-i \gamma(u) l} d u
$$

where we assume that $(u, \gamma(u))$ is a curve in the high energy region in the sense that $\gamma \sim$ $1 / \varepsilon$. Furthermore, we assume that $\gamma$ is monotone with $\left|\gamma^{\prime}\right| \sim 1$ and that $(b-a) \sim 1 / \varepsilon$. By transforming the integration variable, we can then write 2.4 .69 as the Fourier integral

$$
\int_{\gamma(a)}^{\gamma(b)} f\left|\gamma^{\prime}\right|^{-1} e^{-i \gamma l} d \gamma
$$

If the function $f\left|\gamma^{\prime}\right|^{-1}$ is smooth, its Fourier transform 2.4.70 has rapid decay in the variable $l$. Under the stronger assumption that $f\left|\gamma^{\prime}\right|^{-1}$ varies on the scale $1 / \varepsilon$, we conclude that the length scale for this rapid decay is of the order $l \sim \varepsilon$. As a consequence, the rapid decay can be detected even under the constraint $l<l_{\max }$ imposed by 2.4 .62 , and we say that 2.4.70 has rapid decay in $l$. The reader who feels uncomfortable with this informal definition can immediately make this notion mathematically precise by an integration by parts argument similar to 2.4.66) imposing for $f\left|\gamma^{\prime}\right|^{-1}$ a condition of type 2.4.64). The precise mathematical meaning of rapid decay in $l$ for the integral (2.4.69) is that for every integer $k$ there should be constants $c \sim 1$ and parameters $l_{\min }, l_{\max }$ in the range $\varepsilon \ll l_{\min } \ll l_{\max } \ll 1 / m$ such that for all $l \in\left(l_{\min }, l_{\max }\right)$,

$$
\int_{a}^{b} f(u) e^{-i \gamma(u) l} d u \leq c\left(\frac{\varepsilon}{l}\right)^{k} \int_{a}^{b}|f(u)| d u .
$$

We return to the analysis of the integral (2.4.68). The boundary terms in 2.4.67) at $\beta_{u}$ yield contributions to $P(s, l)$ of the form

$$
-\left(\frac{1}{i l}\right)^{n+1} \int_{-\infty}^{\infty} h_{u}^{(n)}\left(\beta_{u}\right) e^{-i \beta_{u} l-i u s} d u .
$$

According to (2.4.61), the length scale for the oscillations of the factor $\exp (-i u s)$ is $u \sim 1 / \varepsilon$. Under the reasonable assumption that $\beta_{u}$ is monotone and that the functions $\left|\beta^{\prime}(u)\right|^{-1}$ and $h_{u}^{(n)}\left(\beta_{u}\right)$ vary on the scale $1 / \varepsilon$, the integral 2.4.71) is of the form 2.4.70), and the above consideration yields that 2.4.71) has rapid decay in $l$. We conclude that it suffices to consider the boundary terms in (2.4.67) at $\alpha_{u}$. Using this result in 2.4.68, we obtain

$$
P(s, l)=\sum_{n=0}^{\infty}\left(\frac{1}{i l}\right)^{n+1} \int_{-\infty}^{\infty} h_{u}^{(n)}\left(\alpha_{u}\right) e^{-i \alpha_{u} l-i u s} d u+(\text { rapid decay in } l) .
$$

The integral (2.4.72) cannot be estimated again using the "oscillation argument" after (2.4.69), because, according to (2.4.60), the function $\alpha_{u}$ tends asymptotically to 
zero for large $u$, so that the factor $\exp \left(-i \alpha_{u} l\right)$ is non-oscillating in this region. Instead, we expand this factor in a Taylor series,

$$
P(s, l)=\sum_{n, k=0}^{\infty} \frac{1}{k !}(i l)^{k-n-1} \int_{-\infty}^{\infty} h_{u}^{(n)}\left(\alpha_{u}\right)\left(-\alpha_{u}\right)^{k} e^{-i u s} d u .
$$

Let us discuss this expansion. Without regularization 2.4.60), the function $\alpha_{u}=m^{2} / u$ involves the mass. Therefore, expanding in powers of $\alpha_{u}$ corresponds precisely to the expansion in the mass expansion as considered earlier (see $(2.2 .8)$ and 2.2 .113$)$ and the explanations thereafter). With this in mind, we can regard (2.4.73) as a generalization of the mass expansion to the setting with regularization. This expansion is clearly justified if $\alpha_{u} l \ll 1$. However, as the function $m^{2} / u$ has a pole at $u=0$, the function $\alpha_{u}$ becomes large for small $u$, so that it is not clear whether the mass expansion is sensible. Indeed, this issue is closely related to the logarithmic mass problem which was mentioned in $2_{2.2 .6}$ and was resolved by working with the "regularized" distribution $T_{a}^{\mathrm{reg}},(2.2 .117$ ). In the present setting, this "regularization procedure" can be understood as follows: For small momenta $u \ll 1 / \varepsilon$, our oscillation argument after 2.4.69 again applies and shows that the resulting contribution to $P(s, l)$ decays rapidly in $l$. Therefore, disregarding contributions with rapid decay in $l$, we may restrict attention to the region $u \gtrsim \varepsilon$ where

$$
\alpha_{u}<\alpha_{\max } \ll l_{\max }^{-1} .
$$

Then $\alpha_{u} l \ll 1$, justifying the mass expansion (2.4.73).

For a fixed value of $k-n$, all summands in 2.4.73 have the same $l$-dependence. Let us compare the relative size of these terms. According to our regularity assumption (2.4.64), the derivatives of $h$ scale like $h_{u}^{(n)} \sim \varepsilon^{n}$. Using the bound (2.4.74), we conclude that, for a fixed power of $l$, the summands in (2.4.73) decrease like $\left(\varepsilon \alpha_{\max }\right)^{n}$. Thus it is a very good approximation to drop the summands for large $n$. At first sight, it might seem admissible to take into account only the first summand $n=0$. But the situation is not quite so simple. For example, it may happen that, when restricted to the curve $\left(u, \alpha_{u}\right)$, the function $h(u, v)$ is so small that the summands for $n=0$ in $(2.4 .73)$ are indeed not dominant. More generally, we need to know that for some $n_{0} \geq 0$, the function $h_{u}^{\left(n_{0}\right)}\left(\alpha_{u}\right)$ is really of the order given in 2.4.64, i.e.

$$
\left|h_{u}^{\left(n_{0}\right)}\left(\alpha_{u}\right)\right| \geq c\left(c_{1} \varepsilon\right)^{n_{0}} \max \left|h_{u}\right|
$$

with a positive constant $c$ which is of the order one. If this condition is satisfied, we may neglect all summands for $n>n_{0}$, and collecting the terms in powers of $l$, we conclude that

$$
\begin{aligned}
& P(s, l) \\
& =\frac{1}{(i l)^{n_{0}+1}} \sum_{k=0}^{\infty}(-i l)^{k} \sum_{n=\max \left(n_{0}-k, 0\right)}^{n_{0}} \frac{(-1)^{n_{0}-n}}{\left(k-n_{0}+n\right) !} \int_{-\infty}^{\infty} h_{u}^{(n)}\left(\alpha_{u}\right) \alpha_{u}^{k-n_{0}+n} e^{-i u s} d u \\
& \quad+\sum_{n=n_{0}+1}^{\infty} \frac{1}{(i l)^{n+1}} \int_{-\infty}^{\infty} h_{u}^{(n)}\left(\alpha_{u}\right) e^{-i u s} d u+(\text { rapid decay in } l) \\
& \left.\quad+\text { (higher orders in } \varepsilon \alpha_{\max }\right) .
\end{aligned}
$$

We point out that, according to (2.4.74),

$$
\varepsilon \alpha_{\max } \ll \varepsilon / l_{\max },
$$


and this explains why we disregard the higher orders in $\varepsilon \alpha_{\max }$. In our case, the function $h_{u}$ has in the low energy region according to 2.4 .60 the form $h_{u}\left(\alpha_{u}\right)=m /\left(32 \pi^{3}\right) \Theta(u)$. Hence it is natural to assume that 2.4 .75$)$ is satisfied for $n_{0}=0$. Introducing the shorter notation

$$
h(u):=h_{u}(\alpha(u)), \quad h^{[n]}(u):=h_{u}^{(n)}\left(\alpha_{u}\right), \quad \alpha(u):=\alpha_{u},
$$

we have thus derived the following result.

Expansion of the scalar component: Close to the light cone (2.4.61), (2.4.62), the scalar component 2.4.53) of the fermionic projector of the vacuum has the expansion

$$
\begin{aligned}
P(s, l)=\frac{1}{i l} & \sum_{k=0}^{\infty} \frac{(-i l)^{k}}{k !} \int_{-\infty}^{\infty} h \alpha^{k} e^{-i u s} d u \\
& +\sum_{n=1}^{\infty} \frac{1}{(i l)^{n+1}} \int_{-\infty}^{\infty} h^{[n]} e^{-i u s} d u \\
& \left.+(\text { rapid decay in } l)+\text { (higher orders in } \varepsilon \alpha_{\max }\right)
\end{aligned}
$$

with suitable regularization functions $h, h^{[n]}$ and $\alpha$. In the low energy region $u \ll 1 / \varepsilon$, the regularization functions are

$$
h(u)=\frac{m}{32 \pi^{3}} \Theta(u), \quad h^{[n]}(u)=0, \quad \alpha(u)=\alpha_{u}=\frac{m^{2}}{u} .
$$

In this expansion, the $l$-dependence is written out similar to a Laurent expansion. The main simplification compared to our earlier Fourier representation is that the dependence on the regularization is now described by functions of only one variable, denoted by $h$, $h^{[n]}$ and $\alpha$. In composite expressions in $P(s, l)$, we will typically get convolutions of these functions; such one-dimensional convolutions can be easily analyzed. The simplification to one-dimensional regularization functions became possible because many details of the regularization affect only the contribution with rapid decay in $l$, which we do not consider here. Notice that the summands in $(2.4 .78)$ and $(2.4 .79)$ decay like $\left(l \alpha_{\max }\right)^{k} / k ! \ll$ $\left(l / l_{\max }\right)^{k} / k$ ! and $(\varepsilon / l)^{n}$, respectively. In the low energy limit (2.4.81), the expansion 2.4.78 goes over to a power series in $m^{2}$, and we thus refer to (2.4.78) as the mass expansion. In the mass expansion, the regularization is described by only two functions $h$ and $\alpha$. The series $(2.4 .79)$, on the other hand, is a pure regularization effect and is thus called the regularization expansion. It involves an infinite number of regularization functions $h^{[n]}$. Accordingly, we will use the notions of mass and regularization expansions also for other expansions of type (2.4.76).

We now outline how to extend the previous analysis to the vector component. More precisely, we will analyze the Fourier integral 2.4.52) for

$$
\hat{P}^{\varepsilon}(p)=v_{j}(p) \gamma^{j} f(p)
$$

close to the light cone. We again choose light-cone coordinates $\left(s, l, x_{2}, x_{3}\right)$ with $y-x=$ $(s, l, 0,0)$ ( $s$ and $l$ are given by (2.4.57), while $x_{2}$ and $x_{3}$ are Cartesian coordinates in the orthogonal complement of the $s l$-plane). The associated momenta are denoted by $p=\left(u, v, p_{2}, p_{3}\right)$ with $u$ and $v$ according to 2.4.58). As in (2.4.55), we integrate out the coordinates perpendicular to $u$ and $v$,

$$
h_{j}(u, v):=\frac{1}{2(2 \pi)^{4}} \int_{-\infty}^{\infty} d p_{2} \int_{-\infty}^{\infty} d p_{3}\left(v_{j} f\right)\left(u, v, p_{2}, p_{3}\right) .
$$


We thus obtain a representation of the fermionic projector involving two-dimensional Fourier integrals

$$
P(s, l)=\gamma^{j} P_{j}(s, l)
$$

with

$$
P_{j}(s, l):=\int_{-\infty}^{\infty} d u \int_{-\infty}^{\infty} d v h_{j}(u, v) e^{-i(u s+v l)} .
$$

The tensor indices in (2.4.83) and 2.4.84) refer to the coordinate system $\left(s, l, x_{2}, x_{3}\right)$. For clarity, we denote the range of the indices by $j=s, l, 2,3$; thus

$$
\gamma^{s}=\frac{1}{2}\left(\gamma^{0}-\gamma^{1}\right), \quad \gamma^{l}=\frac{1}{2}\left(\gamma^{0}+\gamma^{1}\right),
$$

where $\gamma^{0}, \ldots, \gamma^{3}$ are the usual Dirac matrices of Minkowski space. Since without regularization, $\hat{P}=\not p \delta\left(p^{2}-m^{2}\right) \Theta\left(-p^{0}\right)$, the functions $h_{j}$ can be computed similar to 2.4.60 to be

$$
\gamma^{j} h_{j}(u, v)=\frac{1}{32 \pi^{3}}\left(-u \gamma^{s}-v \gamma^{l}\right) \Theta\left(u v-m^{2}\right) \Theta(u) .
$$

This limiting case specifies the regularized $h_{j}(u, v)$ for small energy-momentum $u, v \ll$ $1 / \varepsilon$. In order to keep the form of the functions $h_{j}$ in the high energy region sufficiently general, we merely assume in what follows that the functions $h_{j}$ have all the properties which se assumed for the function $h$ above. This gives the following result.

Expansion of the vector component: Close to the light cone (2.4.61), 2.4.62), the vector component 2.4.82 of the fermionic projector of the vacuum has the expansion $P=\gamma^{j} P_{j}$ with

$$
\begin{aligned}
P_{s}( & s, l)=\frac{1}{i l} \sum_{k=0}^{\infty} \frac{(-i l)^{k}}{k !} \int_{-\infty}^{\infty}-u g_{s} \alpha^{k} e^{-i u s} d u \\
& +\sum_{n=1}^{\infty} \frac{1}{(i l)^{n+1}} \int_{-\infty}^{\infty}-u g_{s}^{[n]} e^{-i u s} d u \\
& +(\text { rapid decay in } l)+\left(\text { higher orders in } \varepsilon \alpha_{\max }\right) \\
P_{l}(s, l) & =\frac{1}{(i l)^{2}} \sum_{k=0}^{\infty} \frac{(-i l)^{k}}{k !} \int_{-\infty}^{\infty}\left[(k-1) \alpha^{k}+k \frac{b}{u} \alpha^{k-1}\right] g_{l} e^{-i u s} d u \\
& +\sum_{n=1}^{\infty} \frac{1}{(i l)^{n+2}} \int_{-\infty}^{\infty}-(n+1) g_{l}^{[n]} e^{-i u s} d u \\
& +(\text { rapid decay in } l)+\left(\text { higher orders in } \varepsilon \alpha_{\max }\right) \\
P_{2 / 3} & (s, l)=\frac{1}{(i l)^{2}} \sum_{k=0}^{\infty} \frac{(-i l)^{k}}{k !} \int_{-\infty}^{\infty}\left[\alpha^{k}+k \frac{b_{2 / 3}}{u} \alpha^{k-1}\right] g_{2 / 3} e^{-i u s} d u \\
& +\sum_{n=1}^{\infty} \frac{1}{(i l)^{n+2}} \int_{-\infty}^{\infty} g_{2 / 3}^{[n]} e^{-i u s} d u \\
& +(\text { rapid decay in } l)+\left(\text { higher orders in } \varepsilon \alpha_{\max }\right)
\end{aligned}
$$

and suitable regularization functions $g_{j}, g_{j}^{[n]}, b, b_{2 / 3}$ and the mass regularization function $\alpha$ as in 2.4.78) and 2.4.81). In the low energy region $u \ll 1 / \varepsilon$, the regularization 
functions have the form

$$
\begin{aligned}
g_{s}(u) & =\frac{1}{32 \pi^{3}} \Theta(u), \quad g_{s}^{[n]}(u)=0 \\
g_{l}(u) & =\frac{1}{32 \pi^{3}} \Theta(u), \quad g_{l}^{[n]}(u)=b(u)=0 \\
g_{2 / 3}(u) & =g_{2 / 3}(u)=b_{2 / 3}(u)=0 .
\end{aligned}
$$

In order to explain these formulas, we consider the situation where, like in the case without regularization, the vector $v(p)$ in 2.4 .82 points into the direction $p$. In this case, we can write the vector component as

$$
\hat{P}^{\varepsilon}(p)=p_{j} \gamma^{j}(\phi f)(p),
$$

where $(\phi f)$ has the form of the scalar component as considered above. Since multiplication in momentum space corresponds to differentiation in position space, we obtain for (2.4.84)

$$
P(s, l)=-i\left(\gamma^{s} \frac{\partial}{\partial s}+\gamma^{l} \frac{\partial}{\partial l}+\gamma^{2} \frac{\partial}{\partial x^{2}}+\gamma^{3} \frac{\partial}{\partial x^{3}}\right) P_{\text {scalar }}(s, l),
$$

where $P_{\text {scalar }}$ is the scalar component 2.4 .59 with $h$ as in 2.4 .56$)$. We now substitute for $P_{\text {scalar }}$ the expansion on the light cone $(2.4 .78)-(2.4 .80)$ and carry out the partial derivatives. For the $s$ - and $l$-components, this gives exactly the expansions 2.4.87), 2.4.88 with

$$
g_{s}=g_{l}=h, \quad g_{s}^{[n]}=g_{l}^{[n]}=h^{[n]}, \quad b=0 .
$$

For the components $j=2,3$, the calculation of the partial derivatives is not quite so straightforward because the expansion of the scalar component (2.4.78)- 2.4.80) was carried out for fixed $x_{2}$ and $x_{3}$. Nevertheless, one can deduce also the expansion (2.4.89) from 2.4.78) -2.4 .80 if one considers $x_{2}$ and $x_{3}$ as parameters of the regularization functions $h, h^{[n]}$ and $\alpha$, and differentiates through, keeping in mind that differentiation yields a factor $1 / l$ (to get the scaling dimensions right). In this way, the simple example (2.4.93) explains the general structure of the expansions (2.4.87)-(2.4.89). We point out that the regularization function $b$ vanishes identically in (2.4.94). This means that $b$ is non-zero only when the direction of the vector field $v$ is modified by the regularization. Thinking in terms of the decomposition into the one-particle states, we refer to this regularization effect as the shear of the surface states.

The derivation of these formulas uses the same methods as for the scalar components. The analysis is a bit more subtle because one must carefully analyze the scaling of the different components. We refer the interested reader to [F7, Section 4.4].

Computing composite expressions using the above Fourier representations, one readily verifies the calculations rules stated in $\$ 2.4 .4$. The details can be found in $\mathbf{F 7}$, Section 4.5].

\subsection{Computation of the Local Trace}

When deriving the EL equations in $\$ 1.4 .1$, we showed in Proposition 1.4.1 that for every minimizer of the causal action principle, the local trace is constant in space-time. We also argued that this condition should be satisfied by the rescaling (1.4.11). In the Minkowski vacuum, the local trace is obviously constant because the kernel of the fermionic projector is translation invariant (see our ansatz (2.4.52)). But in the presence of an external potential, the local trace will in general no longer be constant, making it 
necessary to perform the rescaling 1.4.11). We now explain how to compute the local trace and discuss the effect of the rescaling (1.4.11).

We begin by noting that, using the abstract definition of the kernel of the fermionic projector 1.1.13), we know that the local trace can be computed by

$$
\operatorname{tr}(x)=\operatorname{Tr}_{S_{x}}\left(P^{\varepsilon}(x, x)\right) .
$$

In what follows, we usually omit the subscript $S_{x}$ and regard $\operatorname{Tr}$ as the trace of a $4 \times 4$ matrix. In the vacuum, one can compute this trace from 2.4.52 to conclude the scaling

$$
\operatorname{Tr}_{S_{x}}\left(P^{\varepsilon}(x, x)\right)=c \frac{m}{\varepsilon^{2}}(1+\mathcal{O}(m \varepsilon)),
$$

where the constant $c$ depends on the regularization method (for an explicit computation in the $i \varepsilon$-regularization see Exercise 2.22.

In the next proposition we specify how the local trace is affected by the external potential.

Proposition 2.5.1. In the presence of a smooth external chiral potential 2.2.25) with the properties as in Lemma 2.1.2, the contribution $\Delta P$ to the fermionic projector to order $n$ in perturbation theory influences the local trace only by an error term of the form

$$
\left|\operatorname{Tr}_{S_{x}}\left(\Delta P^{\varepsilon}(x, x)\right)\right| \leq \frac{C}{\varepsilon},
$$

where the constant $C$ depends on $m, n$ as well as on the potential $\mathcal{B}$ and its partial derivatives. Moreover, the function $\operatorname{Tr}_{S_{x}}\left(\Delta P^{\varepsilon}(x, x)\right)$ is smooth in $x$.

This result implies that, when rescaling the causal fermion system according to (1.4.11),

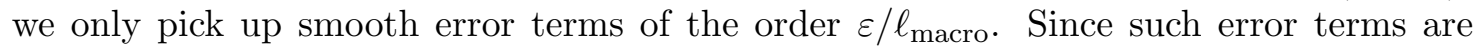
neglected in the continuum limit (see 2.4 .50$)$ ), we may disregard the rescaling (1.4.11). This is the reason why the rescaling 1 1.4.11) will not be considered further in this book.

Before coming to the proof of the above proposition, we note that for a gravitational field, the situation is more involved. Namely, for linear gravity as considered in Section 2.3. the change of the local trace is typically of the order

$$
\operatorname{Tr}_{S_{x}}\left(\Delta P^{\varepsilon}(x, x)\right) \sim \frac{m}{\varepsilon^{2}} \mathcal{O}(h) .
$$

Clearly, this is sufficient in order to treat a weak gravitational field. However, when constructing causal fermion systems non-perturbatively in curved space-time (as is done in [FR2, Section 4]), the macroscopic space-time dependence of the local trace must be taken into account, meaning that the rescaling procedure (1.4.11) will change the causal fermion system substantially. The same is true if a scalar potential is considered, because in this case the local trace takes the form

$$
\operatorname{Tr}_{S_{x}}\left(P^{\varepsilon}(x, x)\right)=\frac{c}{\varepsilon^{2}} \frac{\operatorname{Tr}_{S_{x}}(B(x))}{\operatorname{dim}\left(S_{x}\right)}+\mathcal{O}\left(\frac{1}{\varepsilon}\right),
$$

where the potential $B$ again includes the mass 2.2.10) (for the derivation see Exercise 2.23).

Proof of Proposition 2.5.1, As shown in Theorem 2.2.16, to every order in perturbation theory, the non-causal high energy contribution $\tilde{p}-\tilde{p}^{\text {res }}$ is a smooth function in $x$ and $y$. Therefore, it is even bounded for $x=y$, and we do not need to consider it here. Hence it suffices to consider the perturbation expansions of $\tilde{k}$ and $\tilde{p}^{\text {res }}$. These perturbation expansions must be regularized on the scale $\varepsilon$. The procedure for this is 
explained in the appendix (see Appendix F). In order to keep the presentation as simple as possible, here we shall not enter the regularized causal perturbation theory. Instead, we consider the unregularized perturbation expansion and make use of the fact that the regularization gives rise to a decay in momentum space on the scale $\varepsilon^{-1}$. This simplified procedure will be justified by a short remark at the end of the proof.

In view of (2.1.26) and (2.2.110), instead of $\tilde{k}$ and $\tilde{p}^{\text {res }}$ we can just as well consider the causal Green's functions $s^{\wedge}$ and $s^{\vee}$ (see 2.1.25) as well as the Green's functions $s^{+}$ and $s^{-}$(see (2.2.108)). For the causal Green's function, we can apply the structural results on the light-cone expansion stated in Theorem 2.2.4. Using the residual argument, this theorem holds just as well for the Green's functions $s^{ \pm}$. With this in mind, we may restrict attention to the causal Green's functions, which we again simply denote by $s$.

The formula 2.5.3 can also be expressed by saying that $S^{(0)} \sim \varepsilon^{-2}$. Since increasing the upper index gives a scaling factor $\xi^{2}$, which for $x=y$ is translated to a scaling factor $\varepsilon^{2}$, we have

$$
S^{(h)} \sim \varepsilon^{-2+2 h} .
$$

Moreover, every factor $\xi$ in the light-cone expansion gives rise to a scaling factor

$$
\xi \sim \varepsilon
$$

Applying these scalings to a contribution of the light-cone expansion in Theorem 2.2.4, we find that

$$
\text { (2.2.5) } \sim \varepsilon^{-2+2 h+|I|} .
$$

Therefore, our task is to show that all expressions of the form 2.2 .5 which contribute to the local trace satisfy the inequality

$$
2 h+|I|>0 .
$$

Using the identity (2.2.34), the inequality (2.5.7) is equivalent to

$$
k-1+\sum_{a=1}^{k}\left(\left|I_{a}\right|+2 p_{a}\right)>0 .
$$

Obviously, it suffices to consider the cases $k=0$ and $k=1$. If $k=0$, the fermionic projector is odd (i.e. it contains an odd number of Dirac matrices), so that the local trace vanishes. In the case $k=1$, on the other hand, the contribution involving the chiral potential is again odd and vanishes. The contribution involving the mass matrix $m Y$, on the other hand, is precisely the term $m Y S^{(0)}$ whose local trace was computed in 2.5.1). This concludes the proof, provided that the scalings $(2.5 .5)$ and $(2.5 .6)$ hold.

The scalings (2.5.5) and (2.5.6) are justified by the regularized causal perturbation theory developed in Appendix $\mathrm{F}$. We here explain the reason for the scalings: In the regularized causal perturbation calculation, the "causality" is built in by demanding that the resulting regularized light-cone expansion again only involves integrals along the line segment $\overline{x y}$ (and not integrals along the whole straight line through $x$ and $y$ ). In more technical terms, this is achieved by demanding that the contributions to the perturbation expansion remain bounded in the limit when the momentum of the external potential tends to zero (this method was first used in [F7, Appendix D]). This procedure ensures that a factor $\xi$ in the unregularized light-cone expansion really gives a scaling factor $\varepsilon,(2.5 .6)$. The scaling (2.5.5), on the other hand, follows immediately from the fact that the local trace is obtained by integrating over the momentum variables (similar as in Exercise 2.22), and that the regularization gives decay in momentum space on the scale $\varepsilon^{-1}$. 


\subsection{Spectral Analysis of the Closed Chain}

In this section we explain how to analyze the EL equations corresponding to the causal action in the continuum limit. Since the Lagrangian involves the eigenvalues of the closed chain, the main task is to compute the spectral decomposition of $A_{x y}^{\varepsilon}=$ $P^{\varepsilon}(x, y) P^{\varepsilon}(y, x)$. We first compute this spectral decomposition in the vacuum (2.6.1). This spectral decomposition has the special properties that the eigenvalues are non-real and form complex conjugate pairs, and that the corresponding eigenvectors are null (with respect to the spin scalar product). In order to simplify the subsequent computations, it is very convenient to choose a spinor basis which reflects these special properties of the closed chain of the vacuum. This so-called double null spinor frame is introduced in $\$ 2.6 .2$. In $\$ 2.6 .3$ we proceed by describing the interaction perturbatively using contour integral methods. In 2.6 .4 we derive a few general properties of the spectral representation of the closed chain. Finally, in 82.6 .5 we use the obtained spectral representation of the closed chain to rewrite the EL equations in a form suitable for an explicit analysis.

2.6.1. Spectral Decomposition of the Regularized Vacuum. In order to analyze the causal action principle, we clearly need to know the eigenvalues $\lambda_{i}^{x y}$ of the closed chain. Moreover, in order to bring the EL equations into a tractable form, we also need to know the corresponding eigenspaces. We now compute the spectral decomposition of the closed chain for the regularized fermionic projector of the vacuum. We first do the computation in general, and then rewrite it using the formalism of the continuum limit.

As in 1.2 .5 we assume that the regularized fermionic projector of the vacuum is homogeneous and has a vector-scalar structure (1.2.44). These assumptions are reasonable and sufficiently general for our purposes. Thus we assume that $P^{\varepsilon}(x, y)$ can again be written again as the Fourier integral $(2.4 .1)$, where $\hat{P}^{\varepsilon}$ now is a distribution of the form

$$
\hat{P}^{\varepsilon}(k)=\hat{g}_{j}(k) \gamma^{j}+\hat{h}(k)
$$

with real-valued distributions $\hat{g}_{j}$ and $\hat{h}$. Here the parameter $\varepsilon>0$ denotes the length scale of the regularization. Thus, expressed in momentum space, the distributions $\hat{g}_{j}$ and $\hat{h}$ should decay at infinity on the scale $k \sim \varepsilon^{-1}$. This means in position space that the kernel of the fermionic projector has the form

$$
P^{\varepsilon}(x, y)=g_{j}(x, y) \gamma^{j}+h(x, y)
$$

with smooth functions $g_{j}$ and $h$ whose derivatives scale at most in powers of $\varepsilon^{-1}$. As $\varepsilon$ tends to zero, the regularized fermionic projectors should go over to the unregularized fermionic projector,

$$
\lim _{\varepsilon \searrow 0} P^{\varepsilon}(x, y)=P(x, y) \quad \text { as a distribution. }
$$

According to (1.1.14), we introduce the corresponding closed chain by

$$
A_{x y}^{\varepsilon}=P^{\varepsilon}(x, y) P^{\varepsilon}(y, x) .
$$

In the next lemma we compute the roots of the characteristic polynomial of this matrix. For ease in notation we shall often omit the subscripts " $x y . "$

Lemma 2.6.1. The characteristic polynomial of the closed chain $A_{x y}^{\varepsilon}$ has two roots $\lambda_{ \pm}$. Either the $\lambda_{ \pm}$form a complex conjugate pair, $\overline{\lambda_{+}}=\lambda_{-}$, or else they are both real and have the same sign. The roots are given explicitly by

$$
\lambda_{ \pm}=g \bar{g}+h \bar{h} \pm \sqrt{(g \bar{g})^{2}-g^{2} \bar{g}^{2}+(g \bar{h}+h \bar{g})^{2}} .
$$


Proof. We write the fermionic projector in position space as

$$
P^{\varepsilon}(x, y)=g_{j}(x, y) \gamma^{j}+h(x, y), \quad P^{\varepsilon}(y, x)=\overline{g_{j}(x, y)} \gamma^{j}+\overline{h(x, y)} .
$$

Thus, omitting the arguments $x$ and $y$,

$$
A_{x y}^{\varepsilon}=(\not g+h)(\bar{g}+\bar{h}) .
$$

Omitting the superscript $\varepsilon$ and the subscript $x y$, we obtain

$$
A=\not g \bar{g}+h \overline{\not g}+\not g \bar{h}+h \bar{h} .
$$

It is useful to decompose $A$ in the form

$$
A=A_{1}+A_{2}+\mu
$$

with

$$
A_{1}=\frac{1}{2}[\phi, \bar{g}], \quad A_{2}=h \bar{g}+\not g \bar{h}, \quad \mu=g \bar{g}+h \bar{h}
$$

and $g \bar{g} \equiv g_{j} \overline{g^{j}}$. Then the matrices $A_{1}$ and $A_{2}$ anti-commute, and thus

$$
(A-\mu)^{2}=A_{1}^{2}+A_{2}^{2}=(g \bar{g})^{2}-g^{2} \bar{g}^{2}+(g \bar{h}+h \bar{g})^{2} .
$$

The right side of (2.6.7) is a multiple of the identity matrix, and so 2.6.7) is a quadratic equation for $A$. The roots $\lambda_{ \pm}$of this equation as given by (2.6.5) are the zeros of the characteristic polynomial of $A$. If the discriminant is negative, the $\lambda_{ \pm}$form a complex conjugate pair. If conversely the discriminant is positive, the $\lambda_{ \pm}$are both real. In order to show that they have the same sign, we compute their product,

$$
\begin{aligned}
\lambda_{+} \lambda_{-} & =(g \bar{g}+h \bar{h})^{2}-\left[(g \bar{g})^{2}-g^{2} \bar{g}^{2}+(g \bar{h}+h \bar{g})^{2}\right] \\
& =2(g \bar{g})|h|^{2}+|h|^{4}+g^{2} \bar{g}^{2}-(g \bar{h}+h \bar{g})^{2} \\
& =|h|^{4}+g^{2} \bar{g}^{2}-g^{2} \bar{h}^{2}-h^{2} \bar{g}^{2} \\
& =\left(g^{2}-h^{2}\right)\left(\bar{g}^{2}-\bar{h}^{2}\right) \geq 0 .
\end{aligned}
$$

This concludes the proof.

In the degenerate case that the two eigenvalues $\lambda_{+}$and $\lambda_{-}$coincide, the relation (2.6.7) shows that the matrix $A-\mu$ is nilpotent. However, in this case the matrix $A-\mu$ need not vanish (as one sees from (2.6.6)), giving examples where the matrix $A$ is not diagonalizable. Except for this degenerate case, the matrix $A$ is indeed diagonalizable and has two-dimensional eigenspaces:

Lemma 2.6.2. In the case $\lambda_{+} \neq \lambda_{-}$, the matrix $A_{x y}$ is diagonalizable and has twodimensional eigenspaces. It has the spectral representation

$$
A_{x y}=\sum_{s= \pm} \lambda_{s}^{x y} F_{s}^{x y}
$$

where the spectral projections are given by

$$
F_{ \pm}^{x y}=\frac{\mathbb{1}}{2} \pm \frac{\frac{1}{2}[\not, \bar{\phi}]+h \bar{\phi}+\not \bar{h}}{2 \sqrt{(g \bar{g})^{2}-g^{2} \bar{g}^{2}+(g \bar{h}+h \bar{g})^{2}}} .
$$


Proof. If we assume that $A$ is diagonalizable, then $\lambda_{ \pm}$are the two eigenvalues of $A$, and the corresponding spectral projectors $F_{ \pm}$are given by

$$
F_{ \pm}=\frac{\mathbb{1}}{2} \pm \frac{1}{\lambda_{+}-\lambda_{-}}\left(A-\frac{1}{2}\left(\lambda_{+}+\lambda_{-}\right) \mathbb{1}\right)
$$

Applying (2.6.5) gives (2.6.9). Taking their trace, one sees that the matrices $F_{+}$and $F_{-}$ both have rank two.

In order to prove that $A$ is diagonalizable, one takes formulas 2.6 .9 and shows by direct computation that (see Exercise 2.24)

$$
A F_{ \pm}=\lambda_{ \pm} F_{ \pm} \quad \text { and } \quad F_{+}+F_{-}=\mathbb{1} .
$$

This shows that the images of $F_{+}$and $F_{-}$are indeed eigenspaces of $A$ which span $\mathbb{C}^{4}$.

Our next step is to rewrite the spectral representation using the formalism of the continuum limit. Let us compute the leading singularity on the light cone. Then

$$
P(x, y)=\frac{i}{2} \not T_{[0]}^{(-1)}+(\operatorname{deg}<2),
$$

where for notational convenience we omitted the indices ${ }_{[0]}^{-1}$ of the factor $\xi$, and where the bracket $(\operatorname{deg}<2)$ stands for terms of degree at most one. Using this formula for the fermionic projector, the closed chain becomes

$$
A_{x y}=\frac{1}{4}\left(\Varangle T_{[0]}^{(-1)}\right)\left(\overline{\left(T_{[0]}^{(-1)}\right.}\right)+\Varangle(\operatorname{deg} \leq 3)+(\operatorname{deg}<3),
$$

where $\bar{\not}:=\overline{\xi_{j}} \gamma^{j}$. Its trace can be computed with the help of the contraction rules 2.4.45,

$$
\operatorname{Tr}\left(A_{x y}\right)=\left(\xi_{j} \overline{\xi^{j}}\right) T_{[0]}^{(-1)} \overline{T_{[0]}^{(-1)}}=\frac{1}{2}(z+\bar{z}) T_{[0]}^{(-1)} \overline{T_{[0]}^{(-1)}}+(\operatorname{deg}<3) .
$$

We next compute the square of the trace-free part of the closed chain,

$$
\begin{aligned}
& \left(A_{x y}-\frac{1}{4} \operatorname{Tr}\left(A_{x y}\right) \mathbb{1}\right)^{2}=\frac{1}{16}\left(\Varangle \bar{\ddagger}-\frac{z+\bar{z}}{2}\right)^{2}\left(T_{[0]}^{(-1)} \overline{T_{[0]}^{(-1)}}\right)^{2} \\
& =\frac{1}{16}\left(\Varangle \bar{\ddagger} \Varangle \bar{\ddagger}-(z+\bar{z}) \Varangle \bar{\ddagger}+\frac{1}{4}(z+\bar{z})^{2}\right)\left(T_{[0]}^{(-1)} \overline{T_{[0]}^{(-1)}}\right)^{2} \\
& =\frac{1}{64}(z-\bar{z})^{2}\left(T_{[0]}^{(-1)} \overline{T_{[0]}^{(-1)}}\right)^{2} .
\end{aligned}
$$

Combining these formulas, we see that to leading degree, the closed chain is a solution of the polynomial equation

$$
\left(A_{x y}-\frac{1}{8}(z+\bar{z}) T_{[0]}^{(-1)} \overline{T_{[0]}^{(-1)}}\right)^{2}=\left(\frac{1}{8}(z-\bar{z}) T_{[0]}^{(-1)} \overline{T_{[0]}^{(-1)}}\right)^{2} .
$$

We point out that the calculations so far are only formal, but they have a well-defined meaning in the formalism of the continuum, because to all our end formulas we will be able to apply the weak evaluation formula (2.4.48). Having this in mind, we can interpret the roots of the polynomial in 2.6.14

$$
\lambda_{+}=\frac{1}{4}\left(z T_{[0]}^{(-1)}\right) \overline{T_{[0]}^{(-1)}} \quad \text { and } \quad \lambda_{-}=\frac{1}{4} T_{[0]}^{(-1)} \overline{\left(z T_{[0]}^{(-1)}\right)}
$$


as the eigenvalues of the closed chain. Using the contraction rule 2.4 .46$)$, these eigenvalues simplify to (see also [F7, eq. (5.3.20)])

$$
\lambda_{+}=T_{[0]}^{(0)} \overline{T_{[0]}^{(-1)}}+(\operatorname{deg}<3), \quad \lambda_{-}=T_{[0]}^{(-1)} \overline{T_{[0]}^{(0)}}+(\operatorname{deg}<3) .
$$

The corresponding spectral projectors become (see also [F7, eq. (5.3.21)])

$$
F_{ \pm}=\frac{1}{2}\left(\mathbb{1} \pm \frac{[\not, \bar{\phi}]}{z-\bar{z}}\right)+\Varangle(\operatorname{deg} \leq 0)+(\operatorname{deg}<0) .
$$

Since in the formalism of the continuum limit, the factors $z$ and $\bar{z}$ are treated as two different functions, we do not need to worry about the possibility that the eigenvalues $\lambda_{+}$ and $\lambda_{-}$might coincide or that the denominator in 2.6.16) might vanish. Similarly, we can treat $\xi$ and $\bar{\xi}$ simply as two different vectors. Then the methods and results of Lemma 2.6.2 apply and show that the matrices $F_{+}$and $F_{-}$have rank two, so that the eigenvalues $\lambda_{+}$and $\lambda_{-}$are both two-fold degenerate. By direct computation, one finds that (see Exercise 2.25)

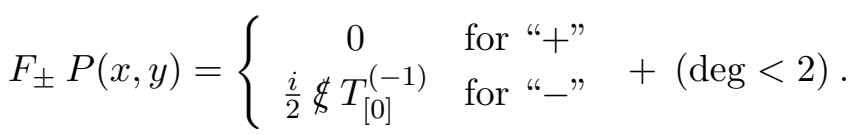

From 2.6.15) and 2.6.16) one sees that the eigenvalues of the closed chain form a complex conjugate pair and are both two-fold degenerate. Using this result in (1.1.9), one comes to the important conclusion that the Lagrangian vanishes identically, implying that, using the formalism of the continuum limit, the fermionic projector of the vacuum is a minimizer of the causal action. We will return to this point in a more general context in 2.6 .5 .

The lower degrees on the light cone can be computed in a straightforward way by expanding the formulas (2.6.9). To give an impression, we here list a few formulas:



$$
\begin{aligned}
& +T_{[1]}^{(0)} \overline{T_{[1]}^{(0)}} \mp \frac{T_{[1]}^{(0)} \overline{T_{[0]}^{(-1)}}-T_{[0]}^{(-1)} \overline{T_{[1]}^{(0)}}}{T_{[0]}^{(0)} \overline{T_{[0]}^{(-1)}}-T_{[0]}^{(-1)} \overline{T_{[0]}^{(0)}}}\left(T_{[1]}^{(0)} \overline{T_{[0]}^{(0)}}-T_{[0]}^{(0)} \overline{T_{[1]}^{(0)}}\right) \\
& +(\operatorname{deg}<2) \text {. } \\
& F_{ \pm} P(x, y)=\frac{i}{4}\left(\sharp T_{[0]}^{(-1)}\right)+(\operatorname{deg}<2) \\
& \pm \frac{i}{4} \frac{\left(\notin T_{[0]}^{(-1)}\right)\left(T_{[0]}^{(0)} \overline{T_{[0]}^{(-1)}}+T_{[0]}^{(-1)} \overline{T_{[0]}^{(0)}}\right)-2\left(\overline{\left(\$ T_{[0]}^{(-1)}\right.}\right) T_{[0]}^{(-1)} T_{[0]}^{(0)}}{T_{[0]}^{(0)} \overline{T_{[0]}^{(-1)}}-T_{[0]}^{(-1)} \overline{T_{[0]}^{(0)}}} .
\end{aligned}
$$

These formulas can be obtained more systematically with the perturbation expansion of the spectral decomposition which we now describe.

2.6.2. The Double Null Spinor Frame. Before entering the perturbation calculation, it is convenient to choose a specific eigenvector basis of the closed chain of the vacuum. This basis is referred to as the double null spinor frame and is denoted by $\left(\mathfrak{f}_{ \pm}^{L / R}\right)$. Performing computations in the double null spinor frame is an improvement of the method 
of "factorizing matrix traces" as introduced in [F7, Appendix G.2]. Following (2.6.13), we introduce the matrix

$$
\left.A_{x y}^{0}=\frac{1}{4}\left(\sharp T_{[0]}^{(-1)}\right) \overline{\left(\nsubseteq T_{[0]}^{(-1)}\right.}\right) .
$$

According to 2.6.15) and 2.6.16), in the formalism of the continuum limit the corresponding eigenvalues and spectral projectors are given by

$$
\begin{gathered}
\lambda_{+}=T_{[0]}^{(0)} \overline{T_{[0]}^{(-1)}}, \quad \lambda_{-}=T_{[0]}^{(-1)} \overline{T_{[0]}^{(0)}} \\
F_{ \pm}=\frac{1}{2}\left(\mathbb{1} \pm \frac{[\not,, \bar{\xi}]}{z-\bar{z}}\right)
\end{gathered}
$$

and they satisfy the relations

$$
F_{+} \not \bar{\sharp}=z F_{+}, \quad \text { and } \quad F_{-} \not \bar{\sharp}=\bar{z} F_{-} .
$$

Furthermore, the matrix $A_{x y}^{0}$ is invariant on the left- and right-handed components, and thus we may choose joint eigenvectors of the matrices $A_{0}$ and $\Gamma$. This leads us to introduce the four eigenvectors $\mathfrak{f}_{ \pm}^{L / R}$ by the relations

$$
\chi_{c} F_{s} \mathfrak{f}_{s}^{c}=\mathfrak{f}_{s}^{c}
$$

with $c \in\{L, R\}$ and $s \in\{+,-\}$, which define each of these vectors up to a complex factor. For clarity in notation, we again write the inner product on Dirac spinors $\bar{\psi} \phi \equiv \psi^{\dagger} \gamma^{0} \phi$ as $\prec \psi \mid \phi \succ$, and refer to it as the spin scalar product. Then the calculation

$$
\prec \mathfrak{f}_{+}^{L}\left|\mathfrak{f}_{+}^{L} \succ=\prec \chi_{L} \mathfrak{f}_{+}^{L}\right| \chi_{L} \mathfrak{f}_{+}^{L} \succ=\prec \mathfrak{f}_{+}^{L} \mid \chi_{R} \chi_{L} \mathfrak{f}_{+}^{L} \succ=0
$$

(and similarly for the other eigenvectors) shows that these vectors are indeed all null with respect to the spin scalar product. Moreover, taking the adjoint of (2.6.19) with respect to the spin scalar product, one sees that

$$
\left(F_{+}\right)^{*}=F_{-} .
$$

As a consequence, the inner products vanish unless the lower indices are different, for example

$$
\prec \mathfrak{f}_{+}^{L}\left|\mathfrak{f}_{+}^{R} \succ=\prec F_{+} \mathfrak{f}_{+}^{L}\right| F_{+} \mathfrak{f}_{+}^{R} \succ=\prec \mathfrak{f}_{+}^{L} \mid F_{-} F_{+} \mathfrak{f}_{+}^{R} \succ=0 .
$$

We conclude that all inner products between the basis vectors vanish except for the inner products $\prec \mathfrak{f}_{+}^{L}\left|\mathfrak{f}_{-}^{R} \succ, \prec \mathfrak{f}_{+}^{R}\right| \mathfrak{f}_{-}^{L} \succ$ as well as their complex conjugates $\prec \mathfrak{f}_{-}^{R} \mid \mathfrak{f}_{+}^{L} \succ$ and $\prec \mathfrak{f}_{-}^{L} \mid \mathfrak{f}_{+}^{R} \succ$. We assume that all the non-vanishing inner products are equal to one,

$$
\left|\prec \mathfrak{f}_{+}^{L}\right| \mathfrak{f}_{-}^{R} \succ|=1=| \prec \mathfrak{f}_{+}^{R}\left|\mathfrak{f}_{-}^{L} \succ\right|
$$

In order to specify the phases and relative scalings of the basis vectors, we introduce a space-like unit vector $u$ which is orthogonal to both $\xi$ and $\bar{\xi}$. Then the imaginary vector $v=i u$ satisfies the relations

$$
\langle v, \xi\rangle=0=\langle v, \bar{\xi}\rangle, \quad\langle v, v\rangle=1 \quad \text { and } \quad \bar{v}=-v .
$$

As a consequence, the operator $\psi$ commutes with $F_{+}$and $F_{-}$, and since it flips parity, we may set $\mathfrak{f}_{+}^{R}=\psi \mathfrak{f}_{+}^{L}$. Next, a straightforward computation using (2.6.19) gives the identities

$$
F_{-} \not=\not F_{+} \quad \text { and } \quad F_{-} \bar{\sharp}=\bar{\phi} F_{+} .
$$

These identities can be used as follows. The first identity implies that

$$
\left(\chi_{R} F_{-} \sharp\right) \mathfrak{f}_{+}^{L}=\sharp \chi_{L} F_{+} \mathfrak{f}_{+}^{L} \sim \notin \mathfrak{f}_{+}^{L},
$$


showing that the vectors $\oiint_{+}^{L}$ and $\mathfrak{f}_{-}^{R}$ are linearly dependent. The calculation

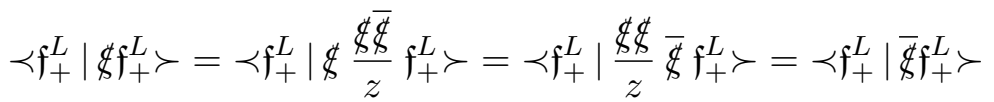

(where we used (2.6.20) and (2.6.19) ) shows that the vector $\oiint^{\prime} \mathfrak{f}_{+}^{L}$ is in fact a real multiple of $\mathfrak{f}_{-}^{R}$. Hence by normalizing $\mathfrak{f}_{+}^{L}$ appropriately, we can arrang $3^{3}$ that $\mathfrak{f}_{-}^{R}=\oiint_{\mathfrak{f}_{+}}^{L}$. Using the second identity in (2.6.24), we also find that $\mathfrak{f}_{-}^{R}=\bar{\sharp} \mathfrak{f}_{+}^{L}$. Similarly, we may also set $\mathfrak{f}_{-}^{L}=\not \mathfrak{f}_{+}^{R}=\bar{\sharp} \mathfrak{f}_{+}^{R}$. The resulting relations between our basis vectors are summarized in the following diagram:



With 2.6 .20$),(2.6 .22)$ and $(2.6 .25)$ we have introduced the double null spinor frame $\left(\mathfrak{f}_{ \pm}^{L / R}\right)$. The construction involves the freedom in choosing the operator $\psi$ according to (2.6.23); for given $\psi$, the basis vectors are unique up to an irrelevant common phase. The construction of the double null spinor frame is illustrated in Exercise 2.26.

We next explain how we can represent a given linear operator $B$ on the spinors in the double null frame $\left(\mathfrak{f}_{ \pm}^{L / R}\right)$. Following the notation in [F7, Appendix G], we denote the matrix element in the column $(c, s)$ and row $\left(c^{\prime}, s^{\prime}\right)$ by $\mathfrak{F}_{s s^{\prime}}^{c c^{\prime}}(B)$. These matrix entries are obtained by acting with $B$ on the vector $\mathfrak{f}_{s^{\prime}}^{c^{\prime}}$ and taking the inner product with the basis vector which is conjugate to $\mathfrak{f}_{s}^{c}$, i.e.

$$
\mathfrak{F}_{s s^{\prime}}^{c c^{\prime}}(B)=\prec \mathfrak{f}_{\bar{s}}^{\bar{c}} \mid B \mathfrak{f}_{s^{\prime}}^{c^{\prime}} \succ
$$

where the conjugation flips the indices according to $L \leftrightarrow R$ and $+\leftrightarrow-$. Similarly, we can also express the projectors $\chi_{c} F_{s}$ in terms of the basis vectors, for example

$$
\chi_{L} F_{+}=\left|\mathfrak{f}_{+}^{L} \succ \prec \mathfrak{f}_{-}^{R}\right| .
$$

For computing (2.6.26), we use the relations in 2.6.25) to express the vector $\mathfrak{f}_{s^{\prime}}^{c^{\prime}}$ in terms of $\mathfrak{f}_{+}^{L}$, choosing the relations which do not involve factors of $\bar{\xi}$. Similarly, we express the vector $\mathfrak{f}_{\bar{s}}^{\bar{c}}$ in terms of $\mathfrak{f}_{-}^{R}$, avoiding factors of $\not$. Applying (2.6.27), we can then rewrite the inner product as a trace involving the operator $F_{+}$. More precisely, a straightforward calculation yields

$$
\begin{aligned}
& \mathfrak{F}_{++}^{L L}(B)=\operatorname{Tr}\left(F_{+} \chi_{L} B\right) \quad, \quad \mathfrak{F}_{++}^{L R}(B)=\operatorname{Tr}\left(F_{+} \psi \chi_{L} B\right) \\
& \mathfrak{F}_{+-}^{L L}(B)=\operatorname{Tr}\left(\sharp F_{+} \psi \chi_{L} B\right) \quad, \quad \mathfrak{F}_{+-}^{L R}(B)=\operatorname{Tr}\left(\nsubseteq F_{+} \chi_{L} B\right) \\
& \mathfrak{F}_{-+}^{L L}(B)=\frac{1}{z} \operatorname{Tr}\left(F_{+} \psi \notin \chi_{L} B\right), \quad \mathfrak{F}_{-+}^{L R}(B)=\frac{1}{z} \operatorname{Tr}\left(F_{+} \sharp \chi_{L} B\right) \\
& \mathfrak{F}_{--}^{L L}(B)=\frac{1}{z} \operatorname{Tr}\left(\sharp F_{+} \sharp \chi_{L} B\right), \quad \mathfrak{F}_{--}^{L R}(B)=\frac{1}{z} \operatorname{Tr}\left(\sharp F_{+} \psi \notin \chi_{L} B\right)
\end{aligned}
$$

\footnotetext{
${ }^{3}$ Let us explain why we do not consider the opposite sign $\mathfrak{f}_{-}^{R}=-\not \mathfrak{f}_{+}^{L}$. To this end, we must show that $\prec \mathfrak{f}_{+}^{L} \mid \xi_{\mathfrak{f}_{+}}^{L} \succ>0$. Since for any given positive or definite spinor $\zeta$, the vector $\chi_{L} F_{+} \zeta$ is a multiple of $\mathfrak{f}_{+}^{L}$, it suffices to compute instead the sign of the combination $\prec \chi_{L} F_{+} \zeta \mid \not \chi_{L} F_{+} \zeta \succ$. Applying (2.6.21) and 2.6.24, this inner product simplifies to $\prec \zeta \mid \chi_{R} F_{-} \$ \zeta \succ$. With the help of 2.6.17) and (2.6.12), we can treat the factor $\$$ as an outer factor. Then our inner product simplifies to the expectation value $\prec \zeta \mid \chi_{R} \notin \zeta \succ$. This expectation value is positive if we follow the convention introduced before 2.4 .48 that $\xi^{0}>0$.
} 
(see also [F7, eq. (G.19)], where these relations are derived with a different method). Indeed, it suffices to compute the given eight matrix elements, because the other eight matrix elements are obtained by the replacements $L \leftrightarrow R$. Moreover, the matrix elements of the adjoint (with respect to the spin scalar product) are obtained by

$$
\mathfrak{F}_{s s^{\prime}}^{c c^{\prime}}\left(B^{*}\right)=\prec \mathfrak{f} \frac{\bar{c}}{s} \mid B^{*} \mathfrak{f}_{s^{\prime}}^{c^{\prime}} \succ=\overline{\prec \mathfrak{f}_{s^{\prime}}^{c^{\prime}} \mid B \mathfrak{f}_{\bar{s}}^{\bar{c}} \succ}=\overline{\mathfrak{F} \overline{\bar{c}^{\prime} c}(B)}
$$

A simple example for how to compute the matrix elements in the double null spinor frame is given in Exercise 2.27 .

2.6.3. Perturbing the Spectral Decomposition. Omitting the arguments $(x, y)$, we decompose the fermionic projector as

$$
P=P_{0}+\Delta P
$$

where $P_{0}$ is the vacuum fermionic projector (possibly modified by gauge phases). This gives rise to the decomposition of $A$

$$
A=A_{0}+\Delta A
$$

with

$$
\begin{aligned}
A_{0} & =P_{0}(x, y) P_{0}(y, x) \\
\Delta A & =\Delta P(x, y) P_{0}(y, x)+P_{0}(x, y) \Delta P(y, x)+\Delta P(x, y) \Delta P(y, x) .
\end{aligned}
$$

The eigenvalues and spectral projectors of $A_{0}$ were computed explicitly in 2.6.1. In view of later generalizations, we write the obtained spectral decomposition as

$$
A_{0}=\sum_{k=1}^{K} \lambda_{k} F_{k}
$$

with $K=2$, where $\lambda_{k}$ are distinct eigenvalues with corresponding spectral projections $F_{k}$. Since the perturbation $\Delta A$ will in general remove the degeneracies, we cannot expect that by perturbing $F_{k}$ we again obtain spectral projection operators. But we can form projectors $G_{k}$ on the space spanned by all eigenvectors of $A$ whose eigenvalues are sufficiently close to $\lambda_{k}$. The $G_{k}$ are most conveniently introduced using contour integrals. We choose $\varepsilon>0$ such that

$$
\left|\lambda_{i}-\lambda_{j}\right|<2 \varepsilon \quad \text { for all } i, j=1, \ldots, K \text { and } i \neq j \text {. }
$$

Then we set

$$
G_{k}=\frac{1}{2 \pi i} \oint_{\left|z-\lambda_{k}\right|=\varepsilon}(z-A)^{-1} d z
$$

Combining the resolvent identity with the Cauchy integral formula, one sees that $G_{k}$ is indeed an idempotent operator whose image is the invariant subspace corresponding to the eigenvalues near $\lambda_{k}$ (for details see Exercise 2.6). 
The integral formula 2.6 .32$)$ is very useful for a perturbation expansion. To this end, we substitute 2.6.29 into 22.6.32 and compute the inverse with the Neumann series,

$$
\begin{aligned}
G_{k} & =\frac{1}{2 \pi i} \oint_{\left|z-\lambda_{k}\right|=\varepsilon}\left(z-A_{0}-\Delta A\right)^{-1} d z \\
& =\frac{1}{2 \pi i} \oint_{\left|z-\lambda_{k}\right|=\varepsilon}\left(\mathbb{1}-\left(z-A_{0}\right)^{-1} \Delta A\right)^{-1}\left(z-A_{0}\right)^{-1} d z \\
& =\frac{1}{2 \pi i} \oint_{\left|z-\lambda_{k}\right|=\varepsilon} \sum_{n=0}^{\infty}\left(\left(z-A_{0}\right)^{-1} \Delta A\right)^{n}\left(z-A_{0}\right)^{-1} d z
\end{aligned}
$$

Interchanging the integral with the infinite sum gives the perturbation expansion,

$$
G_{k}=\sum_{n=0}^{\infty} \frac{1}{2 \pi i} \oint_{\left|z-\lambda_{k}\right|=\varepsilon}\left(\left(z-A_{0}\right)^{-1} \Delta A\right)^{n}\left(z-A_{0}\right)^{-1} d z,
$$

where $n$ is the order in perturbation theory. After substituting in the spectral representation for $\left(z-A_{0}\right)^{-1}$,

$$
\left(z-A_{0}\right)^{-1}=\sum_{l=1}^{K} \frac{F_{l}}{z-\lambda_{l}}
$$

the contour integral in 2.6.33 can be carried out with residues. For example, we obtain to second order,

$$
\begin{aligned}
G_{k}=F_{k} & +\sum_{l \neq k} \frac{1}{\lambda_{k}-\lambda_{l}}\left(F_{k} \Delta A F_{l}+F_{l} \Delta A F_{k}\right)+\mathcal{O}\left((\Delta A)^{3}\right) \\
& +\sum_{l, m \neq k} \frac{1}{\left(\lambda_{k}-\lambda_{l}\right)\left(\lambda_{k}-\lambda_{m}\right)} \\
& \quad \times\left(F_{k} \Delta A F_{l} \Delta A F_{m}+F_{l} \Delta A F_{k} \Delta A F_{m}+F_{l} \Delta A F_{m} \Delta A F_{k}\right) \\
- & \sum_{l \neq k} \frac{1}{\left(\lambda_{k}-\lambda_{l}\right)^{2}} \\
& \quad \times\left(F_{k} \Delta A F_{k} \Delta A F_{l}+F_{k} \Delta A F_{l} \Delta A F_{k}+F_{l} \Delta A F_{k} \Delta A F_{k}\right) .
\end{aligned}
$$

To order $n>2$, the corresponding formulas are clearly more complicated, but even then they involve matrix products which are all of the form

$$
F_{k_{1}} \Delta A F_{k_{2}} \Delta A \cdots F_{k_{n}} \Delta A F_{k_{n+1}} .
$$

An example of a first order perturbation computation is given in Exercise 2.28 .

2.6.4. General Properties of the Spectral Decomposition. We now derive a few general properties of the spectral decomposition of the closed chain.

Lemma 2.6.3. Assume that for a one-parameter family of fermionic projectors $P(\tau)$ and fixed $x, y \in M$, the matrices $A_{x y}$ and $A_{y x}$ are diagonalizable for all $\tau$ in a neighborhood of $\tau=0$, and that the eigenvalues of the matrix $\left.A_{x y}\right|_{\tau=0}$ are all non-real. Then the unperturbed closed chain $A_{x y}$ has a spectral representation

$$
\left.A_{x y}\right|_{\tau=0}=\sum_{k=1}^{4} \lambda_{k}^{x y} F_{k}^{x y}
$$


with the following properties. The last two eigenvalues and spectral projectors are related to the first two by

$$
\lambda_{3}^{x y}=\overline{\lambda_{1}^{x y}}, \quad F_{3}^{x y}=\left(F_{1}^{x y}\right)^{*} \quad \text { and } \quad \lambda_{4}^{x y}=\overline{\lambda_{2}^{x y}}, \quad F_{4}^{x y}=\left(F_{2}^{x y}\right)^{*} .
$$

The first order perturbation $\delta A_{x y}=\left.\partial_{\tau} A_{x y}\right|_{\tau=0}$ of the closed chain is diagonal in the bases of the non-trivial degenerate subspaces, i.e.

$$
F_{k}^{x y}\left(\delta A_{x y}\right) F_{l}^{x y}=0 \quad \text { if } k \neq l \text { and } \lambda_{k}^{x y}=\lambda_{l}^{x y} .
$$

The closed chain $A_{y x}$ has a corresponding spectral representation satisfying (2.6.37)2.6.39 with all indices ' $x y$ ' are replaced by ' $y x$ '. The spectral representations of $A_{x y}$ and $A_{y x}$ are related to each other by

$$
\lambda_{k}^{x y}=\lambda_{k}^{y x} \quad \text { and } \quad F_{k}^{x y} P(x, y)=P(x, y) F_{k}^{y x} .
$$

Proof. By continuity, the eigenvalues of the matrix $A_{x y}$ are non-real in a neighborhood of $\tau=0$. Moreover, by direct computation one sees that the matrix $A_{x y}$ is symmetric in the sense that $A_{x y}=A_{x y}^{*}=\gamma^{0} A_{x y}^{\dagger} \gamma^{0}$. Hence, using the idempotence of the matrix $\gamma^{0}$ together with the multiplicity of the determinant, we find that

$$
\operatorname{det}\left(A_{x y}-\lambda\right)=\operatorname{det}\left(\gamma^{0}\left(A_{x y}^{\dagger}-\lambda\right) \gamma^{0}\right)=\operatorname{det}\left(A_{x y}^{\dagger}-\lambda\right)=\overline{\operatorname{det}\left(A_{x y}-\bar{\lambda}\right)} .
$$

Hence if $\lambda$ is an eigenvalue of the matrix $A_{x y}$, so is $\bar{\lambda}$. Thus the eigenvalues must form complex conjugate pairs.

We first complete the proof in the case that there are no degeneracies. For any eigenvalue $\lambda$ of $A_{x y}$ we choose a polynomial $p_{\lambda}(z)$ with $p_{\lambda}(\lambda)=1$ and $p_{\lambda}(\mu)=0$ for all other spectral points $\mu$. Then the spectral projector on the eigenspace corresponding to $\lambda$, denoted by $F_{\lambda}^{x y}$, is given by

$$
F_{\lambda}^{x y}=p_{\lambda}\left(A_{x y}\right) .
$$

Taking the adjoint and possibly after reordering the indices $k$, we obtain the relations (2.6.37) and (2.6.38). The general matrix relation $\operatorname{det}(B C-\lambda)=\operatorname{det}(C B-\lambda)$ (see for example [F10, Section 3]) shows that the closed chains $A_{x y}$ and $A_{y x}$ have the same spectrum. Multiplying (2.6.41) by $P(x, y)$ and iteratively applying the relation

$$
A_{x y} P(x, y)=P(x, y) P(y, x) P(x, y)=P(x, y) A_{y x},
$$

we find that $F_{\lambda}^{x y} P(x, y)=P(x, y) F_{\lambda}^{y x}$. Thus we can label the eigenvalues of the matrix $A_{y x}$ such that (2.6.40) holds.

In the case with degeneracies, the assumption that $A_{x y}$ is diagonalizable in a neighborhood of $\tau=0$ allows us to diagonalize $\delta A_{x y}$ on the degenerate subspaces (see for example [Ba or the similar method for self-adjoint operators in [S2, Section 11.1.2]). This yields (2.6.39), whereas 2.6.38) can be arranged by a suitable ordering of the spectral projectors $F_{k}^{x y}$. In the degenerate subspaces of $A_{y x}$ we can choose the bases such that (2.6.37) and (2.6.38) hold (with ' $x y$ ' replaced by ' $y x$ ') and that (2.6.40) is satisfied. It remains to prove that (2.6.39) also holds for $A_{y x}$ : From (2.6.39) we know that for any pair $l, k$ with $\lambda_{l}^{x y}=\lambda_{k}^{x y}$,

$$
\begin{aligned}
0 & =F_{k}^{x y}\left(\delta A_{x y}\right) F_{l}^{x y}=F_{k}^{x y}(\delta P(x, y) P(y, x)+P(x, y) \delta P(y, x)) F_{l}^{x y} \\
& =F_{k}^{x y}(\delta P(x, y)) F_{l}^{y x} P(y, x)+P(x, y) F_{k}^{y x}(\delta P(y, x)) F_{l}^{x y},
\end{aligned}
$$


where in the last line we applied the second equation in 2.6.40). Multiplying by $P(y, x)$ from the left and by $P(x, y)$ on the right, we find

$$
0=P(y, x) F_{k}^{x y}(\delta P(x, y)) F_{l}^{y x} \lambda_{l}^{y x}+\lambda_{k}^{y x} F_{k}^{y x}(\delta P(y, x)) F_{l}^{x y} P(x, y) .
$$

After dividing by $\lambda_{l}^{y x}=\lambda_{k}^{y x}$ (note that the eigenvalues are non-zero because they are assumed to form complex conjugate pairs), we can again use the second equation in (2.6.40) to obtain

$$
\begin{aligned}
0 & =P(y, x) F_{k}^{x y}(\delta P(x, y)) F_{l}^{y x}+F_{k}^{y x}(\delta P(y, x)) F_{l}^{x y} P(x, y) \\
& =F_{k}^{y x}(P(y, x) \delta P(x, y)+\delta P(y, x) P(x, y)) F_{l}^{y x}=F_{k}^{y x}\left(\delta A_{y x}\right) F_{l}^{y x},
\end{aligned}
$$

concluding the proof.

2.6.5. Spectral Analysis of the Euler-Lagrange Equations. We now explain how the spectral decomposition of the closed chain can be used to analyze the causal action principle introduced in $\$ 1.1 .1$ as well as the corresponding EL equations as worked out in $\$ 1.4 .1$. For the regularized Dirac sea vacuum as considered in 2.6 .1 , the situation is quite simple. Namely, according to Lemma 2.6.1 (or more explicitly in (2.6.18)), the closed chain has two eigenvalues which form a complex conjugate pair. As a consequence, the eigenvalues all have the same absolute value. Writing the Lagrangian in the form (1.1.9), one sees that the Lagrangian vanishes identically. We come to the following conclusion:

In the formalism of the continuum limit, the regularized Dirac sea vacuum is a minimizer of the causal action.

If the fermionic projector of the vacuum is perturbed (for example by an external potential or by additional particle or antiparticle states), the degeneracy of the eigenvalues will in general disappear, so that the spectrum will consist of two complex conjugate pairs. As a consequence, the causal action will no longer vanish. In order to analyze whether we still have a critical point of the causal action, one needs to analyze the corresponding EL equations in Proposition 1.4.3. To this end, it is very convenient to rewrite these EL equations using the spectral decomposition of the closed chain, as we now explain.

For simplicity, we again restrict attention to Dirac spinors and spin dimension two. Moreover, we only consider the case that the Lagrange multipliers $\kappa$ and $\lambda$ in Proposition 1.4 .3 are both equal to zero. The generalization to higher spin dimension and to non-trivial $\kappa$ and $\lambda$ are straightforward and will be carried out later on (see Lemma 3.6.2, Lemma 3.7.1 and the similar results in 4.4.1). Writing the Lagrangian in the form 1.1.9), we have

$$
\mathcal{L}(x, y)=\frac{1}{8} \sum_{i, j=1}^{4}\left(\left|\lambda_{i}^{x y}\right|-\left|\lambda_{j}^{x y}\right|\right)^{2} .
$$

The relation 2.6.39) allows us to compute the variation of the eigenvalues by a standard first order perturbation calculation without degeneracies,

$$
\delta \lambda_{k}^{x y}=\operatorname{Tr}\left(F_{k}^{x y} \delta A_{x y}\right) .
$$

Using that that $\delta|\lambda|=\operatorname{Re}(\bar{\lambda} \delta \lambda /|\lambda|)$, we can compute the first variation of 2.6.43) by

$$
\delta \mathcal{L}(x, y)=\frac{1}{2} \operatorname{Re} \sum_{j, k=1}^{4}\left(\left|\lambda_{k}^{x y}\right|-\left|\lambda_{j}^{x y}\right|\right) \frac{\overline{\lambda_{k}^{x y}}}{\left|\lambda_{k}^{x y}\right|} \operatorname{Tr}\left(F_{k}^{x y} \delta A_{x y}\right) .
$$


We now insert the identity

$$
\delta A_{x y}=\delta P(x, y) P(y, x)+P(x, y) \delta P(y, x) .
$$

Cyclically commuting the arguments of the trace, we obtain

$$
\begin{aligned}
\delta \mathcal{L}(x, y)= & \frac{1}{2} \sum_{j, k=1}^{4}\left(\left|\lambda_{k}^{x y}\right|-\left|\lambda_{j}^{x y}\right|\right) \\
& \times \operatorname{Re} \operatorname{Tr}\left[\frac{\overline{\lambda_{k}^{x y}}}{\left|\lambda_{k}^{x y}\right|} P(y, x) F_{k}^{x y} \delta P(x, y)+\frac{\overline{\lambda_{k}^{x y}}}{\left|\lambda_{k}^{x y}\right|} F_{k}^{x y} P(x, y) \delta P(y, x)\right] .
\end{aligned}
$$

Using (2.6.38) and (2.6.40), one sees that the first summand in the square bracket is the adjoint of the second summand. Therefore, the trace of the square bracket is real-valued, so that it is unnecessary take the real part. Comparing with (1.4.16), we conclude that

$$
Q(x, y)=\frac{1}{2} \sum_{j, k=1}^{4}\left(\left|\lambda_{k}^{x y}\right|-\left|\lambda_{j}^{x y}\right|\right) \frac{\overline{\lambda_{k}^{x y}}}{\left|\lambda_{k}^{x y}\right|} F_{k}^{x y} P(x, y)
$$

(where we again used (2.6.40) $)$. In the vacuum, when the eigenvalues of the closed chain form a complex conjugate pair (2.6.18), the kernel $Q(x, y)$ vanishes identically in the formalism of the continuum limit. If the fermionic projector of the vacuum is perturbed, the first order perturbation of $Q(x, y)$ can be computed easily with the help of 2.6.44). The higher orders in perturbation theory can be treated systematically by using the contour method in $\$ 2.6 .3$ and by evaluating the resulting expressions in the formalism of the continuum limit.

The above methods give a mathematical meaning to $Q(x, y)$ in the formalism of the continuum limit. The remaining difficulty is that in the EL equations worked out in Proposition 1.4.3, the kernel $Q(x, y)$ appears inside an integral (1.4.18), and one must control the error terms 2.4.50) and 2.4.51) inside this integral. The method is to choose a vector $u \in \mathcal{H}$ such that its physical wave function $\psi^{u}$ is supported away from $x$, up to a small error. This method is referred to as testing on null lines. In a more physical picture, one chooses $\psi^{u}$ as an ultrarelativistic wave packet localized near a null curve which does not meet the space-time point $x$. Applying this method to (1.4.18), the left side is evaluated weakly on the light cone, whereas the right side vanishes. In this way, the EL equations in the continuum limit reduce to

$$
Q(x, y)=0 \quad \text { evaluated weakly on the light cone . }
$$

We refer for details to 33.5 .2 . The estimates of all the error terms are worked out in Appendix A.

\section{Exercises}

EXERCISE 2.1. (external field problem) In physics textbooks, the notions of a "particle" and "anti-particle" are often associated to the frequency (or equivalently the energy)

of the solutions: solutions of positive frequency are called particles, whereas the negativefrequency solutions are reinterpreted as describing anti-particle states. The aim of this exercise is to explain why these notions are ill-defined in the presence of a time-dependent potential. To this end, we consider the Dirac equation

$$
(i \not \partial+\mathcal{B}-m) \psi=0,
$$


where $\mathcal{B}$ is a "step potential in time" i.e.

$$
\mathcal{B}(t, \vec{x})=V \gamma^{0} \Theta(t) \Theta(1-t)
$$

with a real parameter $V$.

(a) Separate out the spatial dependence for any given $\vec{k} \in \mathbb{R}^{3}$ with the plane-wave ansatz

$$
\psi(t, \vec{x})=e^{i \vec{k} \vec{x}} \phi(t)
$$

(where $\phi$ is a spinor-valued function). Derive the resulting ordinary differential equation for $\phi(t)$.

(b) Clearly, the potential has discontinuities at $t=0$ and $t=1$. Show that there are two fundamental solutions $\phi_{1}, \phi_{2} \in C^{0}\left(\mathbb{R}, \mathbb{C}^{4}\right)$ which are smooth solutions of the ODE except at the points $t=0$ and $t=1$. Remark: This procedure is familiar to physics students from quantum mechanics textbooks where wave functions are "glued together" at discontinuities of step potentials. From the mathematical point of view, the "glueing" of the solutions can be justified by saying that $\phi_{1}$ and $\phi_{2}$ are a fundamental system of weak solutions of the ODE. To the reader who is not familiar with these concepts, it might be instructive to verify that the notion of "weak solution" really gives rise to a continuity condition for $\phi$. (Likewise, for a second order equation like the Schrödinger equation, the notion of "weak solution" gives rise to $C^{1}$-solutions whose second derivatives are discontinuous.)

(c) Consider a "scattering process" where for negative times the solution is of the form

$$
\phi(t)=e^{-i \omega t} \chi,
$$

where $\chi$ is a constant spinor and $\omega:=\sqrt{\vec{k}^{2}-m^{2}}$. Show that for time $t>1$, this solution can be written as

$$
\phi(t)=e^{-i \omega t} \chi_{+}+e^{+i \omega t} \chi_{-}
$$

with constant spinors $\chi_{+}$and $\chi_{-}$. Compute $\chi_{+}$and $\chi_{-}$explicitly as functions of $\chi$ and $V$. Verify in particular that $\chi_{-}$in general does not vanish.

(d) What does this mean for the interpretation of the solution in terms of "particles" and "anti-particles"? Why can the frequency of the solutions not be used for a global concept of particles and anti-particles? How can a pair creation/annihilation process be understood in our example? Remark: In order to avoid misunderstandings, we point out that the above arguments only show that the frequency cannot be used to obtain a global particle interpretation. They do not rule out the possibility that there may be a well-defined global particle interpretation using other properties of the solutions. In fact, such a global particle interpretation is provided by the causal perturbation expansion (or the corresponding functional analytic constructions in [FR2, FR3, FMR]). However, this global particle interpretation in general does not coincide with the "particles" and "anti-particles" as experienced by a local observer.

EXERCISE 2.2. This exercise is devoted to the advanced Green's function $s_{m}^{\vee}$ (for a more computational exercise on the advanced Green's function see Exercise 2.12 below).

(a) Assume that $m>0$. Show that the limit $\nu \searrow 0$ in 2.1 .9$)$ exist in the distributional sense.

(b) Show that the limit $\nu \searrow 0$ in $(2.1 .9$ also exists in the massless case $m=0$ and that

$$
\lim _{m \searrow 0} s_{m}^{\vee}(k)=s_{0}^{\vee}(k) \quad \text { as a distribution . }
$$


Hint: Proceed similar as in Exercise 1.12 .

(c) Consider the Fourier integral in the $q^{0}$-variable

$$
\int_{-\infty}^{\infty} \frac{1}{q^{2}-m^{2}-i \nu q^{0}} e^{i q^{0} t} d q^{0}
$$

Show with residues that this integral vanishes for sufficiently small $\nu$ if $t<0$.

(d) Argue with Lorentz invariance to prove the left side of 2.1.12.

ExERCISE 2.3. Modifying the location of the poles in 2.1 .9$)$ gives rise to the distribution

$$
s_{m}^{F}(k):=\lim _{\nu \searrow 0} \frac{\not k+m}{k^{2}-m^{2}+i \nu} .
$$

This is the well-known Feynman propagator, which is often described intuitively by saying that "positive frequencies move to the future and negative frequencies move to the past." Make this sentence precise by a computation similar to that in Exercise 2.2 (c).

EXERCise 2.4. (a) Assume that $m>0$. Give a detailed proof of the distributional relation 2.1.14). Hint: Argue similar as in Exercise 1.12.

(b) Prove that (2.1.14) also holds in the case $m=0$. Hint: The subtle point is to analyze the behavior at $q=0$. To this end, apply Lebesgue's dominated convergence theorem.

EXERCISE 2.5. (probability integral and current conservation) Let $\psi, \phi$ be two solutions of the Dirac equation 2.1.5 with a smooth potential $\mathcal{B}$ which is symmetric 2.1 .20 . Moreover, assume that $\psi$ and $\phi$ are smooth and have spatially compact support.

(a) Show that the integral $(2.1 .19)$ is independent of $t_{0}$.

(b) More generally, let $\mathcal{N}$ be a Cauchy surface in Minkowski space with future-directed normal $\nu$. Show that the integral

$$
\int_{\mathcal{N}} \bar{\psi}(\psi \phi) d \mu_{\mathcal{N}}
$$

is independent of the choice of the Cauchy surface (where $d \mu_{\mathcal{N}}$ is the volume measure corresponding to the induced Riemannian metric on $\mathcal{N})$. Hint: Show that the vector field $\bar{\psi} \gamma^{j} \phi$ is divergence-free and apply the Gauß divergence theorem.

EXERCISE 2.6. (resolvent and contour integrals) The aim of this exercise is to make the reader familiar with the notion of the resolvent and the contour integral representation of spectral projectors in the finite-dimensional setting. More details and generalizations to infinite dimensions can be found in the book by Kato $\mathbf{K a}$.

(a) Let $A \in \mathrm{L}\left(\mathbb{C}^{k}\right)$ be a $k \times k$-matrix. The resolvent set is the set of all $\lambda \in \mathbb{C}$ for which the matrix $(A-\lambda)$ is invertible. The spectrum is the complement of the resolvent set. For any $\lambda$ in the resolvent set, we define the resolvent $R_{\lambda}$ by

$$
R_{\lambda}=(A-\lambda \mathbb{1})^{-1}
$$

(we use this sign convention consistently, although some authors use the opposite sign convention). Prove the resolvent identity

$$
R_{\lambda} R_{\lambda^{\prime}}=\frac{1}{\lambda-\lambda^{\prime}}\left(R_{\lambda}-R_{\lambda^{\prime}}\right)
$$

valid for any $\lambda, \lambda^{\prime}$ in the resolvent set. Hint: Multiply the identity $\lambda^{\prime}-\lambda=(A-$ $\lambda)-\left(A-\lambda^{\prime}\right)$ from the left and right by a resolvent. 
(b) Assume that $A$ is a Hermitian matrix. Let $\Gamma$ be a contour which encloses only one eigenvalue $\lambda_{0}$ with winding number one. Show that the contour integral

$$
-\frac{1}{2 \pi i} \oint_{\Gamma} R_{\lambda} d \lambda
$$

is an orthogonal projection onto the corresponding eigenspace. Hint: Choose an eigenvector basis and apply the Cauchy integral formula.

(c) Now let $A$ be any matrix. Let $\Gamma$ be a contour which encloses a point $\lambda_{0}$ in the spectrum with winding number one. Show that the contour integral $(2.6 .48)$ is an idempotent operator whose image is the corresponding invariant subspace. Hint: Choose a Jordan representation of the matrix. Restrict attention to one Jordan block. Then the resolvent can be written as a Neumann series, which reduces to a finite sum. The resulting integral can be computed with residues.

(d) Derive the idempotence relation in (c) directly from the resolvent identity. Hint: A very similar computation is given in the proof of Theorem 2.1.6.

EXERCISE 2.7. In this exercise we explore an alternative and more computational proof of Lemma 2.1.8.

(a) Show by direct computation in momentum space that $\left.k_{m}\right|_{t_{0}} k_{m}=k_{m}$. Hint: Proceed similarly as in the derivation of (1.2.24) in the proof of Lemma 1.2.8.

(b) Show that due to current conservation (see Exercise 2.5 above), the operator $\left.\tilde{k}_{m}\right|_{t_{0}} \tilde{k}_{m}$ is independent of $t_{0}$. Therefore, it suffices to compute the limit $t_{0} \rightarrow-\infty$. In order to study this limit, assume for technical simplicity that $\mathcal{B}$ has compact support. Show with the help of (2.1.14) 2.1 .25 and 2.1.26 that for sufficiently small $t_{0}<0$,

$$
\begin{aligned}
\left.\tilde{k}_{m}\right|_{t_{0}} \tilde{k}_{m} & =\left.\frac{1}{4 \pi^{2}} \sum_{n, n^{\prime}=0}^{\infty}\left(-s_{m}^{\wedge} \mathcal{B}\right)^{n} s_{m}^{\wedge}\right|_{t_{0}} s_{m}^{\vee}\left(-\mathcal{B} s_{m}^{\vee}\right)^{n^{\prime}} \\
& =\left.\sum_{n, n^{\prime}=0}^{\infty}\left(-s_{m}^{\wedge} \mathcal{B}\right)^{n} k_{m}\right|_{t_{0}} k_{m}\left(-\mathcal{B} s_{m}^{\vee}\right)^{n^{\prime}} .
\end{aligned}
$$

(c) Apply the result of (a) together with 2.1.14 to conclude that $\left.\tilde{k}_{m}\right|_{t_{0}} \tilde{k}_{m}=\tilde{k}_{m}$.

EXERCISE 2.8. (causal perturbation expansion to second order)

(a) Compute $P^{\text {sea }}$ to second order in $\mathcal{B}$. Hint: Use (2.1.64) as well as the perturbation series for $\tilde{k}$. The resulting formulas are also listed in [FT2, Appendix A].

(b) The so-called residual fermionic projector is defined by modifying the integrand in 2.1.64 to

$$
P_{\text {res }}^{\text {sea }}=-\frac{1}{2 \pi i} \oint_{\Gamma_{-}} \tilde{R}_{\lambda} d \lambda
$$

Show that to first order in $\mathcal{B}$, the operators $P^{\text {sea }}$ and $P_{\text {res }}^{\text {sea }}$ coincide. However, there is a difference to second order in $\mathcal{B}$. Compute it. Hint: In order to simplify the computation, it is helpful to write the difference as

$$
P^{\text {sea }}-P_{\text {res }}^{\text {sea }}=\frac{1}{2 \pi i} \oint_{\Gamma_{-}}(\lambda+1) \tilde{R}_{\lambda} d \lambda,
$$

and to use that the factor $\lambda+1$ decreases the order of the pole at $\lambda=-1$.

EXERCISE 2.9. (the fundamental solution $\tilde{p}$ ) 
(a) Show that the operator $\tilde{k}$ has the contour integral representation

$$
\tilde{k}=-\frac{1}{2 \pi i} \oint_{\Gamma_{+} \cup \Gamma_{-}} \lambda \tilde{R}_{\lambda} d \lambda
$$

Hint: Use (2.1.54) or the functional calculus of Theorem 2.1.6.

(b) Conclude that the fermionic projector $P^{\text {sea }},(2.1 .64$ ), can be represented as

$$
P^{\text {sea }}=\frac{1}{2}(\tilde{p}-\tilde{k}),
$$

where $\tilde{p}$ is defined by

$$
\tilde{p}:=-\frac{1}{2 \pi i}\left(\oint_{\Gamma_{+}}-\oint_{\Gamma_{-}}\right) \lambda \tilde{R}_{\lambda} d \lambda .
$$

EXERCISE 2.10. (structural properties of $\tilde{p}$ : even number of factors $k$ ) The goal of this exercise is to show that every contribution to the perturbation expansion of $\tilde{p}$ contains an even number of factors $k$.

(a) Read off from $(2.1 .53)$ and 2.1 .55$)$ that every contribution to $\Delta k$ involves an odd number of factors $k$.

(b) Carry out the products $\cdot$ in the perturbation series for $\tilde{R}_{\lambda}$ in 2.1 .56 with the help of (2.1.57) and the multiplication rules 2.1 .52 and 2.1 .69 . Show that this gives rise to a sum of terms of the form

$$
\frac{c \lambda^{r}}{\left(1-\lambda^{2}\right)^{q}} C^{[p]} \mathcal{B} C^{[p-1]} \mathcal{B} \cdots \mathcal{B} C^{[0]}
$$

with operators $C^{[j]} \in\{k, p, s\}$, parameters $q \geq 0$ and $r \in \mathbb{Z}$ as well as a combinatorial factor $c$. Show that the total number of factors $p$ and $k$ in the above operator product is always odd. Show that the number of factors $p$ in the above operator product is even if and only if the parameter $r$ is even. Hint: Use the result of (a) and analyze how the number of factors $p$ and $k$ changes when the different multiplication rules are applied.

(c) Substitute (2.6.50) into 2.6 .49$)$ and analyze the symmetry of the resulting contour integral under the transformation $\lambda \rightarrow-\lambda$. Use the results of (b) to deduce that every contribution to the perturbation expansion of $\tilde{p}$ contains an even number of factors $k$.

EXERCISE 2.11. (structural properties of $\tilde{p}$ : replacing $k$ by $p$ ) In this exercise we compute what one gets if in the perturbation series for $\tilde{p}$ one replaces all factors $k$ by $p$.

(a) Show that replacing all factors $k$ by $p$, the formula 2.1.53 becomes

$$
\tilde{k}=\sum_{\beta=0}^{\infty}(-i \pi)^{2 \beta} b^{<} p(b p)^{2 \beta} b^{>} .
$$

Show that this gives rise to the simple multiplication rule

$$
\tilde{k} \cdot \tilde{k}=\tilde{k} .
$$

Hint: Use the multiplication rules 2.1.52) and 2.1.69). It might be helpful to insert the identity $\mathbb{1}=p+(\mathbb{1}-p)$.

(b) Use the result of (a) to deduce that $\tilde{p}=\tilde{p}^{\text {res }}=\tilde{k}$. Hint: Compute the contour integrals with residues. 
(c) Show that the perturbation expansion of $\tilde{p}^{\text {res }}$ involves no operators $k$. Hint: Use the explicit formulas 2.2.110, (2.2.108) and 2.2.109). Indeed, the perturbation expansion of $\tilde{p}^{\text {res }}$ coincides precisely with the series in (2.6.51).

(d) Combine the results of (b) and (c) to conclude that replacing all factors $k$ by $p$, the perturbation series of $\tilde{p}$ goes over to that of $\tilde{p}^{\text {res }}$.

EXERCISE 2.12. This exercise explains the notion of the light-cone expansion in simple examples.

(a) What is the light-cone expansion of a smooth function on $M \times M$ ? In which sense is it trivial? In which sense is it non-unique?

(b) Show that $A(x, y)=\log \left(|y-x|^{2}\right)$ is a well-defined distribution on $M \times M$. What is the order on the light cone? Write down a light-cone expansion.

(c) Now consider the distributional derivatives

$$
\left(\frac{\partial}{\partial x^{0}}\right)^{p} A(x, y) \quad \text { with } \quad p \in \mathbb{N}
$$

and $A(x, y)$ as in part (b). What is the order on the light cone? Write down a light-cone expansion.

(d) Consider the function

$$
E(x, y)=\sin \left((y-x)^{2}\right) \log \left(|y-x|^{2}\right) .
$$

Determine the order on the light cone and give a light cone expansion.

(e) Consider the function

$$
E(x, y)=\left\{\begin{array}{cl}
e^{-\frac{1}{(y-x)^{2}}} & \text { if }(y-x)^{2} \geq 0 \\
0 & \text { otherwise }
\end{array}\right.
$$

Determine the order on the light cone and give a light cone expansion.

(f) Show that the expression

$$
\lim _{\varepsilon \searrow 0} \frac{\log \left(|y-x|^{2}\right)}{(y-x)^{4}+i \varepsilon}
$$

is a well-defined distribution on $M \times M$. Derive its light-cone expansion.

ExERCISE 2.13. This exercise is devoted to computing the Fourier transform of the advanced Green's function (2.2.5) and deriving the series expansion (2.2.7).

(a) As in Lemma 1.2 .9 , we set $\xi=y-x$ and $\xi=(t, \vec{\xi})$ with $t>0$. Moreover, we choose polar coordinates $r=(|\vec{\xi}|, \vartheta, \varphi)$. Carry out the $\omega$-integration with residues and compute the angular integrals to obtain

$$
S_{m^{2}}^{\vee}(x, y)=\frac{i}{8 \pi r} \int_{0}^{\infty} \frac{p}{\omega(p)}\left(e^{-i p r}-e^{i p r}\right)\left(e^{i \omega(p) t}-e^{-i \omega(p) t}\right) d p,
$$

where $p=|\vec{p}|$ and $\omega(p):=\sqrt{\left|\vec{p}^{2}\right|+m^{2}}$. Justify this integral as the Fourier transform of a distribution and show that

$$
S_{m^{2}}^{\vee}(x, y)=\frac{i}{8 \pi r} \lim _{\varepsilon \searrow 0} \int_{0}^{\infty} e^{-\varepsilon p} \frac{p}{\omega(p)}\left(e^{-i p r}-e^{i p r}\right)\left(e^{i \omega(p) t}-e^{-i \omega(p) t}\right) d p
$$

with convergence as a distribution.

(b) Verify 2.2.6 in the case $m=0$ by setting $\omega(p)=p$ and using 1.2 .33 . 
(c) In order to analyze the behavior away from the light cone, it is most convenient to take the limit $r \searrow 0$ and use Lorentz invariance. Show that in this limit,

$$
\begin{aligned}
S_{m^{2}}^{\vee}(x, y) & =\frac{1}{4 \pi} \lim _{\varepsilon \searrow 0} \int_{0}^{\infty} e^{-\varepsilon p} \frac{p^{2}}{\omega(p)}\left(e^{i \omega(p) t}-e^{-i \omega(p) t}\right) d p \\
& =\frac{1}{4 \pi} \lim _{\varepsilon \searrow \omega} \int_{m}^{\infty} e^{-\varepsilon p} \sqrt{\omega^{2}-m^{2}}\left(e^{i \omega t}-e^{-i \omega t}\right) d \omega .
\end{aligned}
$$

Compute this integral using [GR, formula (3.961.1)] (similar as in the proof of Lemma 1.2.9. Use the relations between Bessel functions [OLBC, (10.27.6), (10.27.11)] to obtain 2.2.6) away from the light cone.

As an alternative method for computing the Fourier integral, one can begin from the integral representation for $J_{0}$ in OLBC, (10.9.12)], differentiate with respect to $x$ and use [OLBC, (10.6.3)].

(d) Combine the results of (b) and (c) to prove (2.2.6). Why is there no additional contribution at $\xi=0$ ?

(e) Use the series expansion OLBC, (10.2.2)] to derive (2.2.7).

(f) The series expansion $(2.2 .7)$ can also be derived without using Bessel functions. To this end, one expands (2.6.52) in powers of $m^{2}$ and computes the Fourier transform term by term. Verify explicitly that this procedure really gives 2.2 .7$)$.

ExERCISE 2.14. This exercise is devoted to the proof of Lemma 2.2 .3 as given in [F6. Lemma 2.2].

(a) Use 2.2.24 to derive the identity

$$
\int d^{4} z S^{(l)}(x, z) V(z)(y-z)_{k} S^{(-1)}(z, y)=-2 \frac{\partial}{\partial y^{k}}\left(S^{(l)} V S^{(0)}\right)(x, y) .
$$

(b) Apply Lemma 2.2.2 and carry out the $y$-derivative in 2.6.54 to obtain the formula in Lemma 2.2.3. Hint: Use the identity

$$
\partial_{k} \square^{n} V(z)=-\frac{1}{2(n+1)} \square_{z}^{n+1}\left(V(z)(y-z)_{k}\right)+\frac{1}{2(n+1)}\left(\square_{z}^{n+1} V(z)\right)(y-z)_{k}
$$

and shift the summation index.

ExERCISE 2.15. In this exercise we collect elementary properties of the ordered exponential.

(a) Assume that the matrix-valued function $F$ in Definition 2.2 .5 is commutative in the sense that

$$
[F(\alpha), F(\beta)]=0 \quad \text { for all } \alpha, \beta \in[a, b] .
$$

Show that the ordered exponential reduces to the ordinary exponential,

$$
\operatorname{Pexp}\left(\int_{a}^{b} F(\alpha) d \alpha\right)=\exp \left(\int_{a}^{b} F(\alpha) d \alpha\right) .
$$

Hint: Show inductively that

$$
\int_{a}^{b} d t_{0} F\left(t_{0}\right) \int_{t_{0}}^{b} d t_{1} F\left(t_{1}\right) \cdots \int_{t_{n-1}}^{b} d t_{n} F\left(t_{n}\right)=\frac{1}{(n+1) !}\left(\int_{a}^{b} F(t) d t\right)^{n+1} .
$$


(b) Assume that $F$ is continuous on $[a, b]$. Show that the Dyson series converges absolutely and that

$$
\left\|\operatorname{Pexp}\left(\int_{a}^{b} F(\alpha) d \alpha\right)\right\| \leq \exp \left(\int_{a}^{b}\|F(\alpha)\| d \alpha\right) .
$$

Hint: Estimate the integrals and apply (a).

(c) Show by direct computation that the ordered exponential satisfies the equations

$$
\begin{gathered}
\frac{d}{d a} \operatorname{Pexp}\left(\int_{a}^{b} F(\alpha) d \alpha\right)=-F(a) \operatorname{Pexp}\left(\int_{a}^{b} F(\alpha) d \alpha\right) \\
\operatorname{Pexp}\left(\int_{a}^{a} F(\alpha) d \alpha\right)=\mathbb{1} .
\end{gathered}
$$

Use the uniqueness theorem for solutions of ordinary differential equations to give an alternative definition in terms of the solution of an initial-value problem. Use this reformulation to show the group property

$$
\operatorname{Pexp}\left(\int_{a}^{b} F(\alpha) d \alpha\right) \operatorname{Pexp}\left(\int_{b}^{c} F(\alpha) d \alpha\right)=\operatorname{Pexp}\left(\int_{a}^{c} F(\alpha) d \alpha\right) .
$$

(d) Show that

$$
\frac{d}{d b} \operatorname{Pexp}\left(\int_{a}^{b} F(\alpha) d \alpha\right)=\operatorname{Pexp}\left(\int_{a}^{b} F(\alpha) d \alpha\right) F(b) .
$$

Hint: Differentiate the identity $(2.6 .57)$ in the case $c=a$ and use the group properties 2.6.56) and 2.6.57).

(e) Show that

$$
\operatorname{Pexp}\left(\int_{a}^{b} F(\alpha) d \alpha\right)^{*}=\operatorname{Pexp}\left(\int_{b}^{a}\left(-F(\alpha)^{*}\right) d \alpha\right) .
$$

Deduce that if $F(\alpha)$ is an anti-Hermitian matrix, then the ordered exponential is a unitary matrix. Hint: There are two alternative methods. One method is to argue using the differential equations 2.6 .55$)$ and 2.6 .58$)$ or with the group property. A more computational approach is to take the adjoint of the Dyson series and reparametrize the integrals.

EXERCISE 2.16. This exercise recalls the concept of local gauge transformations and gets the connection to the ordered exponential.

(a) An electromagnetic potential $A$ of the form $A_{j}=\partial_{j} \Lambda$ with a real-valued function $\Lambda$ is called a pure gauge potential. Show that $(i \not \partial+\not A-m)=U(i \not \partial-m) U^{-1}$, where $U$ is the phase factor $U=e^{i \Lambda}$. Conclude that every solution of the Dirac equation $(i \not \partial+$ $A-m) \tilde{\psi}=0$ can be written in the form $\tilde{\psi}=U \psi$, where $\psi$ is a solution of the vacuum Dirac equation. In other words, pure gauge potentials merely describe local phase transformations of the wave functions.

(b) Generalize the argument of (a) to the case of non-abelian gauge fields and an additional gauge potential using the relation

$$
U(i \not \partial+\not A-m \mathbb{1}) U^{-1}=i \not \partial+U A U^{-1}+i U\left(\not \partial U^{-1}\right)-m \mathbb{1},
$$

where now $U(x)$ is a unitary matrix (the mass matrix was left out for simplicity). How does the gauge potential transform under local unitary transformations of the spinors? 
(c) Prove that for a pure gauge potential $A=i U\left(\not \partial U^{-1}\right)$ the ordered exponential of Definition 2.2.5 simplifies to

$$
\operatorname{Pexp}\left(-i \int_{x}^{y} A^{j}(y-x)_{j}\right)=U(x) U(y)^{-1} .
$$

Hint: Apply the integration-by-parts method of Exercise 2.17 to the Dyson series. Alternatively, one can make use of the differential equation 2.6.55 with initial conditions (2.6.56).

EXERCISE 2.17. This exercise illustrates the handling of the tangential derivatives mentioned before Proposition 2.2.6. Let $z=\beta y+(1-\beta) x$ be a point on the line segment $\overline{x y}$. Show that

$$
\int_{z}^{y}[p, q \mid 0] f\left(z^{\prime}\right) d z^{\prime}=\int_{0}^{1} \alpha^{p}(1-\alpha)^{q} f(\alpha(1-\beta)(y-x)+z)
$$

Deduce the identity

$$
\begin{aligned}
& (y-x)^{j} \int_{z}^{y}[p, q \mid 0]\left(\partial_{j} f\right)\left(z^{\prime}\right) d z^{\prime} \\
& \quad=\frac{1}{1-\beta} \int_{0}^{1} \alpha^{p}(1-\alpha)^{q} \frac{d}{d \alpha} f(\alpha(1-\beta)(y-x)+z) d \alpha .
\end{aligned}
$$

In the case $p, q>0$, integrate by parts to derive the computation rule

$$
(y-x)^{j} \int_{z}^{y}[p, q \mid 0]\left(\partial_{j} f\right)\left(z^{\prime}\right) d z^{\prime}=-\frac{1}{1-\beta} \int_{z}^{y}(p[p-1, q \mid 0]-q[p, q-1 \mid 0]) f .
$$

What is the analogous computation rule in the cases $p=0$ and/or $q=0$ ?

ExERCiSE 2.18. This exercise explains how the Maxwell field tensor and the Maxwell current arise in the light cone expansion. To this end, we consider the first order perturbation of the massless Green's function by an electromagnetic potential $A$,

$$
\Delta s_{0}:=-s_{0} A s_{0} .
$$

(a) Show that the leading contributions to the light-cone expansion of $\Delta s_{m}$ have the form

$$
\begin{aligned}
\left(\Delta s_{0}\right)(x, y)=\frac{1}{2} & \int_{x}^{y} A_{i}(z) \xi^{i} \oiint S^{(-1)}(x, y) \\
& +\int_{x}^{y} d z[0,1 \mid 0]\left(\not \partial A_{i}\right)(z) \xi^{i} S^{(0)}(x, y) \\
& -\int_{x}^{y} d z[0,0 \mid 0] \not A(z) S^{(0)}(x, y) \\
& +\not A(x) S^{(0)}(x, y)+\not \mathcal{O}\left(\xi^{-2}\right)+\mathcal{O}\left(\xi^{0}\right),
\end{aligned}
$$

where $\xi:=y-x$. Hint: First compute $s_{0}$ using (2.2.20) and 2.2.24). Then perform the light-cone expansion of the first order perturbation by using Lemma 2.2.3 and then by differentiating similar as done in the displayed computation before (2.2.24). Finally, the resulting formulas can be simplified by using (2.2.18) and by integrating the tangential derivatives by part (see Exercise 2.17 or the proof of Proposition 2.2.6).

(b) Which of the above contributions are phase-free? Show that the contribution which is not phase-free can be understood as the first-order contribution to the gauge phase in (2.2.57). 
(c) Rewrite the phase-free contributions in an explicitly gauge-invariant way.

Hint: In (2.6.60) use the identity $\left.\not \partial A_{i}\right)(z) \xi^{i}=\gamma^{j} F_{j i} \xi^{i}-\xi^{j} \partial_{j} A$. Note that this generates a tangential derivative (see $(2.2 .58)$ ). Integrate it by parts as explained in Exercise 2.17 or in the proof of Proposition 2.2.6.

(d) Compute the contributions to the above light-cone expansion of the form $\sim \notin \cdots S^{(0)}$. There is a term involving $\square A$. Rewrite it in an explicitly gauge-invariant way using the Maxwell current $j_{i}:=\partial_{i k} A^{k}-\square A_{i}$.

(e) The reader who wants to get more computational practice may find it instructive to carry out the light-cone expansion up to the order $\mathcal{O}\left(\xi^{2}\right)$. In particular, there is a term $\sim(\square$ A $) S^{(1)}$. Rewriting the contributions again an explicitly gauge-invariant form, one thus obtains a contribution $\sim \gamma^{k} j_{k} S^{(1)}$. In fact, this contribution gives rise to the Maxwell current in the field equations in the continuum limit.

We note that all these computations are explained in more detail in [F6, Appendix A].

EXERCISE 2.19. (contour integral representation of the residual fermionic projector) In Exercise 2.9 (a) we derived a contour integral representation for the operator $\tilde{k}$ in 2.2.111). Thus it remains to derive a contour integral representation for the operator $\tilde{p}^{\text {res }}$ as defined by defined by (2.2.110). Verify to second order in perturbation theory (see Exercise 2.8) that $\tilde{p}^{\text {res }}$ has the contour integral representation

$$
\tilde{p}^{\mathrm{res}}=-\frac{1}{2 \pi i} \oint_{\Gamma_{+} \cup \Gamma_{-}} \tilde{R}_{\lambda} d \lambda .
$$

Remark: This equation indeed holds to every order in perturbation theory. This is a consequence of an underlying symmetry of the perturbation expansions with mass and spatial normalizations as explained in [FT2, Section 3.4].

EXERCISE 2.20. The goal of this exercise is to explore weak evaluation on the light cone in the example of the massless closed chain of the vacuum 2.4.11. Thus in view of (2.4.17), we want to analyze the integral

$$
\int_{-\infty}^{\infty} \eta(t) \frac{\left.\left(t^{2}-r^{2}\right)-i \varepsilon\left[\gamma^{0}, \not\right)\right]+\varepsilon^{2}}{\left|(t-i \varepsilon)^{2}-r^{2}\right|^{4}} d t
$$

for a test function $\eta \in C_{0}^{\infty}(\mathbb{R})$ asymptotically as $\varepsilon \searrow 0$.

(a) Choose $r>0$. Show that, changing the integral only by contributions which are bounded uniformly in $\varepsilon$, we may replace $\eta(t)$ by a test function supported in the interval $(r / 2,2 r)$ around the upper light cone.

(b) Use the identity

$$
\frac{1}{(t-i \varepsilon)^{2}-r^{2}}=\frac{1}{(t-i \varepsilon-r)(t-i \varepsilon+r)}=\frac{1}{2 r}\left(\frac{1}{t-i \varepsilon-r}-\frac{1}{t-i \varepsilon+r}\right)
$$

to rewrite the integrand in 2.6.63) in the form

$$
\sum_{p, q=0}^{2} \frac{\eta_{p, q}(t, r, \varepsilon)}{(t-i \varepsilon-r)^{p}(t+i \varepsilon-r)^{q}},
$$

with functions $\eta_{p, q}(t, r, \varepsilon)$ which in the limit $\varepsilon \searrow 0$ converge in $C^{\infty}$ to smooth functions $\eta_{p, q}(t, r)$, i.e.

$$
\lim _{\varepsilon \searrow 0} \partial_{t}^{\alpha} \partial_{r}^{\beta} \eta_{p, q}(t, r, \varepsilon)=\partial_{t}^{\alpha} \partial_{r}^{\beta} \eta_{p, q}(t, r) \quad \text { for all } \alpha, \beta \geq 0
$$


Compute the functions $\eta_{p, q}$. Verify that the contribution for $p=q=2$ agrees with the approximation (2.4.16).

(c) We now compute the leading contributions and specify what we mean by "leading." First compute the following integrals with residues:

$$
\begin{aligned}
& I_{0}(\varepsilon):=\int_{-\infty}^{\infty} \frac{1}{(t-i \varepsilon-r)^{2}(t+i \varepsilon-r)^{2}} d t \\
& I_{1}(\varepsilon):=\int_{-\infty}^{\infty} \frac{t-r}{(t-i \varepsilon-r)^{2}(t+i \varepsilon-r)^{2}} d t .
\end{aligned}
$$

Show that

$$
\begin{aligned}
& \int_{-\infty}^{\infty} \frac{\eta_{2,2}(t, r)}{(t-i \varepsilon-r)^{2}(t+i \varepsilon-r)^{2}} d t \\
& \quad=I_{0}(\varepsilon) \eta_{2,2}(r, r)+I_{1}(\varepsilon)\left(\partial_{t} \eta_{2,2}\right)(r, r)+\mathcal{O}(\varepsilon) .
\end{aligned}
$$

Hint: To estimate the error term, proceed similar as in Exercise 1.10 (a).

(d) We now analyze the dependence of the resulting terms on $r$. To this end, first compute $\eta_{2,2}(r, r)$ and $\left(\partial_{t} \eta_{2,2}\right)(r, r)$. Verify the rules (2.4.20). Verify the scaling of the error terms (2.4.18) and (2.4.19), where we use the convention that every derivative of $\eta$ gives rise to a factor $1 / \ell_{\text {macro }}$.

(e) Show that the integrals for $p<2$ or $q<2$ can be absorbed into the error terms. Also show that the term $\sim \varepsilon^{2}$ in 2.4.11) can be absorbed into the error terms.

(f) So far we analyzed the integrals with the simplified test functions $\eta_{p, q}(t, r)$. Show that replacing them by $\eta_{p, q}(t, r, \varepsilon)$ changes the integrals only by error terms of the form 2.4.18 and 2.4.19.

ExERCise 2.21. This exercise explains how the identities (2.4.24) and (2.4.26) can be derived by explicit computation.

(a) Use 2.2.118 together with 2.2.117) and the series expansion 2.2.97) to derive explicit formulas for $T^{(l)}$ for all $l \geq 0$. Use the relation $(2.4 .4)$ in the case $l=0$ to also compute $T^{(-1)}$.

(b) Show that for all $n \geq 0$,

$$
\xi^{2} T^{(l)}(x, y)=-4 T^{(l-1)}+(\text { smooth contributions }) .
$$

Why do the "smooth contributions" arise?

(c) Verify that the relation 2.6.64 remains valid for the $i \varepsilon$-regularization. Hint: One can argue without computations directly with a meromorphic extension using (2.4.3).

(d) Verify the identities 2.4.26 by explicit computation. What are the "smooth contributions"? Show that these identities remain valid for the $i \varepsilon$-regularization.

EXercise 2.22. (computation of the local trace) Compute $P^{\varepsilon}(x, x)$ in the Minkowski vacuum with $i \varepsilon$-regularization (see (2.4.1) and 2.4.2). How do the vector and scalar components scale in $m$ and $\varepsilon$ ? Verify the scaling of the local trace (2.5.1).

EXERCISE 2.23. (scalar potentials and the local trace) Consider a potential $\mathcal{B}$ composed of chiral potentials and a scalar potential, i.e. in generalization of 2.2.25),

$$
\mathcal{B}=\chi_{L} A_{R}+\chi_{R} A_{L}+\Phi(x) .
$$

(a) Show that the scalar potential can be combined with the mass terms to obtain a Dirac equation of the form 2.2.10 with $B$ as in 2.2.29), but now with $Y(x)$ depending on $x$. We remark that this so-called dynamical mass matrix was first 
introduced in [F6, Section 2] (also including a pseudoscalar potential); see also [F7, Section 2.5].

(b) Go through the proof of Theorem 2.2.4 and convince yourself that the statement of the theorem remains valid in the presence of a scalar potential if in 2.2 .33 the matrix $Y$ is replaced by $Y(x)$. Remark: This generalization of Theorem 2.2.4 is given in [F6, Theorem 2.3].

(c) Use this generalization of Theorem 2.2.4 together with the scaling argument in the proof of Proposition 2.5.1 to derive the formula for the local trace 2.5.4).

EXERCISE 2.24. (spectral representation of $A_{x y}$ ) Derive the formulas (2.6.11) by a straightforward computation using (2.6.6) and (2.6.9).

EXERCISE 2.25. (spectral representation in the continuum limit) Derive (2.6.17) by using 2.6.16), 2.6.12 as well as the contraction rules 2.4.44-2.4.46.

EXERCISE 2.26. In this exercise we consider the double null spinor frame in the example of the $i \varepsilon$-regularization.

(a) Consider a point $(t, \vec{\xi})$ on the upper light cone, i.e. $t=|\vec{\xi}|$ (more specifically one may choose $\vec{x}=(t, 0,0))$. Use (2.4.6) to and compute $z$ (up to errors of the form (2.4.18). Compute the spectral projectors (2.6.19). Verify the relations (2.6.24).

(b) Compute the solutions $\mathfrak{f}_{s}^{c}$ of the eigenvector equations 2.6.20). Normalize them according to 2.6 .22$)$. What is the remaining freedom to modify the eigenvectors.

(c) Choose a space-like unit vector $u$ which is orthogonal to $\xi$ and $\bar{\xi}$. What is the freedom in choosing this vector? Show that by suitably choosing the phases of the eigenvectors $\mathfrak{f}_{s}^{c}$ one can arrange that the relations in 2.6.25) hold. What is the remaining freedom in choosing the frame $\left(\mathfrak{f}_{s}^{c}\right)$ ?

(d) The diagram 2.6.25 implies in particular that $\oiint_{+}^{L}=\bar{\phi} \mathfrak{f}_{+}^{L}$. Explain how this identity can be understood in view of the error terms 2.4.18).

EXERCISE 2.27. (matrix elements in the double null spinor frame) Compute the matrix elements $\mathfrak{F}_{++}^{L L}(B), \mathfrak{F}_{+-}^{L L}(B), \mathfrak{F}_{+-}^{L R}(B)$ and $\mathfrak{F}_{+-}^{R R}(B)$ for $B$ given by

$$
B=\frac{i}{2} \chi_{L} \notin T_{[0]}^{(-1)} .
$$

Simplify the expression as far as possible. Hint: Use the cyclic property of the trace, the anti-commutation relations of the Dirac matrices and the contraction rules.

EXERCISE 2.28. (Perturbation of the eigenvalues of the closed chain) The light-cone expansion can be understood as giving corrections to the fermionic projector of lower order on the light cone. We now explore how these corrections affect the eigenvalues of the closed chain, and which of them are compatible with the EL equations. In order to work in a specific example, we assume that the unperturbed fermionic projector is

$$
P(x, y)=\frac{i}{2} \sharp T_{[0]}^{(-1)}
$$

(similar as considered in Exercise 2.27), whereas the perturbation has a left- and righthanded component,

$$
\Delta P(x, y)=\chi_{L} \psi_{L}+\chi_{R} \psi_{R},
$$

where $\nu_{L}$ and $\nu_{R}$ are given vectors in Minkowski space. 
(a) Compute the corresponding perturbation $\Delta \lambda_{k}^{x y}$ to leading order in the degree on the light cone. What is the leading degree? Which eigenvalues change, which remain the same? Hint: Use the usual formula for first order perturbations (see (2.6.44)) and rewrite it in the double null spinor frame.

(b) For which vectors $\nu_{L}$ and $\nu_{R}$ does the relation $\left|\lambda_{k}^{x y}\right|=\left|\lambda_{l}^{x y}\right|$ hold for all $k, l \in$ $\{1, \ldots, 4\}$ ? Show that these relations are a sufficient condition for the EL equations to be satisfied. What would one need to verify in order to conclude that these relations are necessary? Hint: Consider 2.6.44 and 2.6.46). Keep in mind that the EL equations are evaluated weakly on the light cone. 


\title{
CHAPTER 3
}

\section{An Action Principle for an Interacting Fermion System and its Analysis in the Continuum Limit}

\begin{abstract}
We introduce and analyze a system of relativistic fermions in a space-time continuum, which interact via an action principle as previously considered in a discrete space-time. The model is defined by specifying the vacuum as a sum of Dirac seas corresponding to several generations of elementary particles. The only free parameters entering the model are the fermion masses. We find dynamical field equations if and only if the number of generations is at least three. If the number of generations equals three, the dynamics is described by a massive axial potential coupled to the Dirac spinors. The coupling constant and the rest mass of the axial field depend on the regularization; for a given regularization method they can be computed as functions of the fermion masses. The bosonic mass term arises as a consequence of a symmetry breaking effect, giving an alternative to the Higgs mechanism. In addition to the standard loop corrections of quantum field theory, we find new types of correction terms to the field equations which violate causality. These non-causal corrections are too small for giving obvious contradictions to physical observations, but they might open the possibility to test the approach in future experiments.
\end{abstract}

\subsection{Introduction}

In [F7] it was proposed to formulate physics based on a new action principle in space-time. On the fundamental level, this action principle is defined in so-called discrete space-time for a finite collection of projectors in an indefinite inner product space (see also [F10]). An effect of spontaneous symmetry breaking [F9] leads to the emergence of a discrete causal structure (see [DFS] for an explanation in simple examples), which for many space-time points and many particles should go over to the usual causal structure of Minkowski space (for the connection between discrete and continuum space-times we also refer to $[\mathbf{F P}, \mathbf{F 9}, \mathbf{F 1 0}$ and the survey article [F12]). Furthermore, on a more phenomenological level, it is shown in [F7, Chapters 4-8] that the action can also be analyzed in Minkowski space in the so-called continuum limit, where the interaction is described effectively by classical gauge fields coupled to second-quantized Dirac fields. Finally, generalizing our approach has led to the mathematical framework of so-called causal fermion systems (cf. [FGS] and the references therein).

Apart from deriving the general formalism of the continuum limit, in [F7, Chapters 4$8]$ it is shown that for a suitable system involving 24 Dirac seas, the resulting effective gauge group as well as the coupling of the effective gauge fields to the Dirac fields have striking similarities to the standard model. However, the detailed form of the effective interaction so far has not been worked out.

This work is the first of a series of papers devoted to the detailed analysis of our action principle in the continuum limit and to the derivation of the resulting field equations. In order to make the presentation as clear and easily accessible as possible, our procedure 
is to begin with small systems, which are composed of only a few Dirac seas, and then to gradually build up larger and more complicated systems. In the present paper, we consider a system of several Dirac seas (corresponding to several "generations" of elementary particles) in the simplest possible configuration referred to as a single sector. The only free parameters entering the model are the masses of the Dirac particles of each generation. However, we do not specify the form of the interaction, which is completely determined by our action principle. Also, we do not put in coupling constants nor the masses of gauge bosons. The analysis of the model in the continuum limit reveals that we get dynamical field equations if and only if the number of generations is at least three. If the number of generations equals three, the dynamics can be described by a massive axial potential $A_{\mathrm{a}}$ coupled to the Dirac equation. The corresponding Dirac and field equations (stated for notational simplicity for one Dirac particle) become

$$
\left(i \not \partial+\Gamma A_{\mathrm{a}}-m\right) \psi=0, \quad C_{0} j_{\mathrm{a}}^{k}-C_{2} A_{\mathrm{a}}^{k}=12 \pi^{2} \bar{\psi} \Gamma \gamma^{k} \psi,
$$

where $j_{\mathrm{a}}^{k}=\partial^{k}{ }_{l} A_{\mathrm{a}}^{l}-\square A_{\mathrm{a}}^{k}$ is the corresponding axial current (here $\Gamma$ is the pseudoscalar matrix, which is often denoted by $\gamma^{5}$ ). The coupling constant and the rest mass of the axial gauge field are described by the constants $C_{0}$ and $C_{2}$, which for a given regularization method can be computed as functions of the fermion masses. The mass term of the gauge field arises as a consequence of a symmetry breaking effect, giving an alternative to the Higgs mechanism. The field equations involve surprising corrections which challenge the standard model of elementary particle physics: First, the field equations involve additional convolution terms of the form

$$
-f_{[0]} * j_{\mathrm{a}}^{k}+6 f_{[2]} * A_{\mathrm{a}}^{k}
$$

where $f_{[p]}$ are explicit Lorentz invariant distributions. These convolution terms give rise to small corrections which violate causality. Moreover, we get new types of higher order corrections to the field equations. We also find additional potentials which are non-dynamical in the sense that they vanish away from the sources.

In order to make the paper self-consistent, we introduce our fermion systems and the continuum limit from the basics. However, to avoid an excessive overlap with previous work, we present a somewhat different point of view, where instead of considering a discrete space-time or a space-time continuum of finite volume, we work exclusively in Minkowski space. Furthermore, we always restrict attention to a single sector. For clarity, we omit the more technical aspects of the regularization, relying instead on results from the corresponding chapters of the book [F7.

The paper is organized as follows. In Section 3.2 we introduce our action principle in a space-time continuum. In Sections 3.33 .5 we review and adapt the methods for analyzing this action principle in the continuum as developed in [F7. More precisely, in Section 3.3 we describe the vacuum by a system of regularized Dirac seas. We list all the assumptions on the vacuum state, either motivating them or explaining how they can be justified. In Section 3.4 we construct more general fermion configurations in Minkowski space by modifying and perturbing the vacuum state, also introducing particles and gauge fields. We also outline the mathematical methods for analyzing the unregularized fermionic projector with interaction. In Section 3.5, we explain how interacting systems are to be regularized, and how to treat the regularization in an effective way. This leads us to the formalism of the continuum limit, which allows us to analyze our action principle in the continuum, taking into account the unknown regularization details by a finite number of free parameters. In the following Sections 3.633 .10 the continuum 
limit of our action principle is worked out in detail; this is the main part of the paper where we present our new results. Section 3.6 is devoted to the leading singularities of the Euler-Lagrange equations on the light cone, where the vacuum contributions (\$3.6.1) are modified by phases coming from the chiral gauge potentials $(\$ 3.6 .2)$. The next lower orders of singularities are analyzed in Section 3.7. Then the currents of the gauge fields come into play, and we also get a mass term corresponding to the axial gauge field (3.7.1). Furthermore, we find a corresponding contribution of the Dirac current (\$3.7.2). A priori, the different current terms are not comparable, because the gauge currents have logarithmic poles on the light cone (3.7.3). But provided that the number of generations is at least three, these logarithmic poles can be compensated by a local axial transformation, as is developed in 3.7.4 3.7 .6 . After considering more general local transformations (3.7.7), in 3.7.8 we explain a basic shortcoming of local transformations. This motivates us to work instead with so-called microlocal transformations, which are developed in $\$ 3.7 .9$ 3.7.11.

Section 3.8 is devoted to the derivation and analysis of the field equations. In \$3.8.1 we show that the Euler-Lagrange equations corresponding to our action principle give rise to relations between the Dirac and gauge currents. If the number of generations equals three, we thus obtain field equations for the axial gauge potential (see Theorem 3.8.2). These field equations involve non-causal correction terms, which are analyzed and discussed in $\$ 3.8 .2$ and $\$ 3.8 .3$. In 33.8 .4 we explain schematically how the standard loop corrections of quantum field theory (QFT) appear in our framework, and how loop corrections of the non-causal terms could be obtained. In 33.8 .5 we get a connection to the Higgs mechanism and explain why our model involves no Higgs particle. We finally compute the coupling constant and the rest mass of the axial field for a few simple regularizations $(3.8 .6$.

In Section 3.9 we analyze and discuss further potentials and fields, including scalar and pseudoscalar potentials, bilinear potentials, as well as the gravitational field and a conformal axial field. In Section 3.10 we consider nonlocal potentials, which can be used to satisfy the Euler-Lagrange equations to higher order in an expansion near the origin.

In order not to interrupt the explanations in the main sections by longer calculations, the more technical parts are worked out in the appendices. Appendix A supplements the estimates needed for the derivation of the Euler-Lagrange equations in the continuum limit in 3.5.2. All the calculations in the formalism of the continuum limit as needed in Sections 3.6 3.9 are combined in Appendix B, which also reviews the general method as developed in [F7, Appendix G]. All the formulas given in this appendix have been obtained with the help of computer algebra. In Appendix C we give a general argument which explains why local transformation cannot be used to compensate the logarithmic poles of the current terms. In Appendix $\mathrm{D}$ we compute and analyze the smooth contributions to the fermionic projector as needed in 3.8.1 this is done by modifying a resummation technique first introduced in [F5. Finally, in Appendix E we outline how our constructions and results can be extended to the setting where the Dirac seas involve weight factors, as was proposed in [F11 and $[\mathbf{F H}]$.

\subsection{An Action Principle for Fermion Systems in Minkowski Space}

In relativistic quantum mechanics, a fermionic particle is described by a Dirac wave function $\psi$ in Minkowski space $(\mathscr{M},\langle.,\rangle$.$) . In order to describe a many-particle system,$ we consider an operator $P$ on the Dirac wave functions and interpret the vectors in the image of $P$ as the occupied fermionic states of the system (for a discussion of the Pauli 
exclusion principle and the connection to the fermionic Fock space formalism see [F7, Chapter 3 and Appendix A]). We assume that $P$ has an integral representation

$$
(P \psi)(x)=\int_{\mathscr{M}} P(x, y) \psi(y) d^{4} y
$$

with an integral kernel $P(x, y)$. Moreover, we assume for technical simplicity that $P(x, y)$ is continuous in both arguments $x$ and $y$; then the integral in (3.2.1) is clearly well-defined if for the domain of definition of $P$ we choose for example the space $C_{0}^{\infty}(\mathscr{M}, S \mathscr{M})$ of smooth wave functions with compact support. Moreover, we assume that $P$ is symmetric with respect to the Lorentz invariant inner product

$$
<\psi \mid \phi>=\int_{\mathscr{M}} \overline{\psi(x)} \phi(x) d^{4} x,
$$

where $\bar{\psi} \equiv \psi^{\dagger} \gamma^{0}$ is the usual adjoint spinor ( $\psi^{\dagger}$ is the complex conjugate spinor). In other words, we demand that

$$
<P \psi|\phi>=<\psi| P \phi>\quad \text { for all } \psi, \phi \in C_{0}^{\infty}(\mathcal{M}, S \mathcal{M}) .
$$

This condition can also be expressed in terms of the kernel by

$$
P(x, y)^{*} \equiv \gamma^{0} P(x, y)^{\dagger} \gamma^{0}=P(y, x) \quad \text { for all } x, y \in \mathscr{M},
$$

where the dagger denotes the transposed, complex conjugate matrix. We refer to $P$ as the fermionic projector. The vectors in the image of $P$ are referred to as the physical wave functions. We point out that for the moment, these wave functions do not need to be solutions of a Dirac equation.

For any space-time points $x$ and $y$, we next introduce the closed chain $A_{x y}$ by

$$
A_{x y}=P(x, y) P(y, x) .
$$

It is a $4 \times 4$-matrix which can be considered as a linear operator on the wave functions at $x$. For any such linear operator $A$ we define the spectral weight $|A|$ by

$$
|A|=\sum_{i=1}^{4}\left|\lambda_{i}\right|,
$$

where $\lambda_{1}, \ldots, \lambda_{4}$ are the eigenvalues of $A$ counted with algebraic multiplicities. For any $x, y \in \mathscr{M}$ we define the Lagrangian $\mathcal{L}$ by

$$
\mathcal{L}_{x y}[P]=\left|A_{x y}^{2}\right|-\frac{1}{4}\left|A_{x y}\right|^{2} .
$$

Integrating over space-time, we can furthermore introduce the functionals

$$
\begin{gathered}
\mathcal{S}[P] \stackrel{\text { formally }}{=} \iint_{\mathcal{M} \times \mathcal{M}} \mathcal{L}_{x y}[P] d^{4} x d^{4} y \\
\mathcal{T}[P] \stackrel{\text { formally }}{=} \iint_{\mathcal{M} \times \mathcal{M}}\left|A_{x y}\right|^{2} d^{4} x d^{4} y .
\end{gathered}
$$

These expressions are only formal because the integrands need not decay for large $x$ or $y$, and thus the integrals may be infinite (similar as in classical field theory, where the spacetime integral over the Lagrangian diverges without imposing suitable decay properties at infinity). The functional $\mathcal{S}$ is referred to as the causal action. Our variational principle is 
to minimize $\mathcal{S}$ under the constraint that $\mathcal{T}$ is kept fixed ${ }^{1}$. For this minimization, we vary the fermionic projector in the following sense. In order to prevent trivial minimizers, the variation should preserve the normalization of the wave functions. This normalization should be performed with respect to the Lorentz invariant inner product 3.2 .2 (in a more abstract language, we thus consider unitary variations in the Krein space as introduced in Remark 1.4.5). However, we do not want to assume that this inner product is finite for the wave functions $\psi$ in the image of $P$ (indeed, for physical wave functions, the inner product $\langle\psi \mid \psi\rangle$ is in general infinite because the time integral diverges). Our method for avoiding the divergences in $(3.2 .2)$ and $(3.2 .8)$ is to consider variations which outside a compact set are the identity.

Definition 3.2.1. An operator $U$ on the Dirac wave functions is called unitary in a compact region if

(i) $\langle U \psi \mid U \psi\rangle=\langle\psi \mid \psi\rangle$ for all compactly supported $\psi$.

(ii) The operator $V:=U-\mathbb{1}$ has the representation

$$
(V \psi)(x)=\int_{\mathscr{M}} v(x, y) \psi(y) d^{4} y
$$

with a smooth integral kernel $v(x, y)$ which has compact support, i.e. there is a compact set $K \subset \mathcal{M}$ such that

$$
v(x, y)=0 \quad \text { unless } x \in K \text { and } y \in K .
$$

Thus introducing a variation of the wave functions by the transformation $\psi \rightarrow U \psi$, all the wave functions are changed only in the compact region $K \subset \mathcal{M}$, in such a way that all inner products in this region, i.e. all the integrals

$$
\int_{K} \overline{\psi(x)} \phi(x) d^{4} x
$$

remain unchanged. Having introduced a well-defined notion of "varying the fermionic projector while respecting the inner product (3.2.2)," we can now specify what we mean by a minimizer.

Definition 3.2.2. A fermionic projector $P$ of the form (3.2.1) is a minimizer of the variational principle

$$
\text { minimize } \mathcal{S} \text { for fixed } \mathcal{T}
$$

if for any operator $U$ which is unitary in a compact region and satisfies the constraint

$$
\int_{\mathscr{M}} d^{4} x \int_{\mathscr{M}} d^{4} y\left(\left|A_{x y}[P]\right|^{2}-\left|A_{x y}\left[U P U^{-1}\right]\right|^{2}\right)=0,
$$

the functional $\mathcal{S}$ satisfies the inequality

$$
\int_{\mathscr{M}} d^{4} x \int_{\mathscr{M}} d^{4} y\left(\mathcal{L}_{x y}\left[U P U^{-1}\right]-\mathcal{L}_{x y}[P]\right) \geq 0 .
$$

We point out that, since $U$ changes the wave functions only inside a compact set $K$, the integrands in (3.2.10) and (3.2.11) clearly vanish if $x$ and $y$ are outside $K$. However, it is not obvious that the integrals over the region $x \in K$ and $y \in \mathcal{M} \backslash K$ (and similarly $x \in$

\footnotetext{
${ }^{1}$ Clearly, the constraint of keeping $\mathcal{T}$ fixed is stronger than the boundedness constraint 1.1 .5 which merely imposes that $\mathcal{T}$ must be bounded from above. Working with 1.1 .5 is preferable when working out the existence theory $\mathbf{F 1 3}$. However, for what follows here, it makes no difference if $\mathcal{T}$ is kept fixed or only stays bounded, because both variational principles give rise to the same Euler-Lagrange equations.
} 
$\mathcal{M} \backslash K$ and $y \in \mathscr{M})$ exist. By writing $(3.2 .10)$ and $(3.2 .11)$ we implicitly demand that the integrand in 3.2 .10 and the negative part of the integrand in (3.2.11) should be in $L^{1}(\mathscr{M} \times \mathscr{M}, \mathbb{R})$.

Before going on, we briefly discuss this action principle and bring it into the context of previous work. We first remark that, in contrast to $\mathbf{F 7}, \mathbf{F 1 0}$, we here ignore the condition that $P$ should be idempotent. This is done merely to simplify the presentation, anticipating that the idempotence condition will not be of relevance in this paper. The action principle (3.2.9) was first introduced in a discrete space-time in [F7, Section 3.5]. Apart from the obvious replacement of sums by integrals, the action here differs from that in [F7, Section 3.5] only by an irrelevant multiple of the constraint $\mathcal{T}$. This has the advantage that the Lagrangian (3.2.7) coincides with the so-called critical case of the auxiliary Lagrangian as introduced in [F10]; this is the case relevant in our setting of one sector. Note that this Lagrangian is symmetric (see [F10, eq. (13)]) and non-negative,

$$
\mathcal{L}_{x y}[P]=\mathcal{L}_{y x}[P] \quad \text { and } \quad \mathcal{L}_{x y}[P] \geq 0 .
$$

Moreover, the action principle 3.2 .9 can be regarded as an infinite volume limit of the variational principle in [F13, Section 3] (possibly also in the limit where the number of particles tends to infinity). In the special case of homogeneous systems, our variational principle is closely related to the variational principle in infinite volume as considered in [F13, Section 4]. Working with unitary transformations in a compact region, we can make sense of the action principle even in infinite space-time volume without assuming homogeneity; this procedure can be seen in analogy to considering variations of compact support in the Lagrangian formulation of classical field theory (like a variation $\delta A \in$ $C_{0}^{\infty}\left(\mathscr{M}, \mathbb{R}^{4}\right)$ of the electromagnetic potential in classical electrodynamics).

\subsection{Assuming a Vacuum Minimizer}

Apart from the general existence results in [F10, F13 and the simple examples in DFS, F13, almost nothing is known about the minimizers of our action principle. Therefore, before we can do physics, we need to assume the existence of a special minimizer which describes a physically meaningful vacuum. In this section, we compile our assumptions on this vacuum minimizer, and we outline in which sense and to what extent these assumptions have been justified in $[\mathbf{F 1 1}, \mathbf{F H}]$. At the end of this section, we will explain how to work with these assumptions in practice.

Taking Dirac's original concept seriously, we want to describe the vacuum by "completely filled Dirac seas" corresponding to the masses $m_{1}, \ldots, m_{g}$ of $g$ generations of elementary particles (later we will set $g=3$, but for the moment it is preferable not to specify the number of generations). Thus our first ansatz for the integral kernel of the

fermionic projector of the vacuum is the Fourier transform of the projectors $\frac{1}{2 m_{\beta}}\left(\not k+m_{\beta}\right)$ on the Dirac states on the lower mass shells,

$$
P(x, y)=\sum_{\beta=1}^{g} \int \frac{d^{4} k}{(2 \pi)^{4}}\left(\not k+m_{\beta}\right) \delta\left(k^{2}-m_{\beta}^{2}\right) \Theta\left(-k^{0}\right) e^{-i k(x-y)} .
$$

(Here $\Theta$ is the Heaviside function, and $k(x-y)$ is a short notation for the Minkowski inner product $\langle k, x-y\rangle$. The slash denotes contraction with the Dirac matrices, thus $\not k=k_{j} \gamma^{j}$. We always work in natural units $\hbar=c=1$, and for the signature of the Minkowski inner product we use the convention $(+---)$.) We always index the masses in increasing 
order,

$$
m_{1}<m_{2}<\ldots<m_{g}
$$

The Fourier integral (3.3.1) is well-defined as a distribution. If the vector $y-x$ is spacelike or timelike, the integral (3.3.1) exists even pointwise. However, if the vector $y-x$ is null, the distribution $P(x, y)$ is singular (for details see [F7, Section 2.5] or \$1.2.5). In physical terms, these singularities occur if $y$ lies on the light cone centered at $x$. Thus we refer to the singularities on the set where $(x-y)^{2}=0$ as the singularities on the light cone. As a consequence of these singularities, the pointwise product in (3.2.5) is ill-defined, and the Lagrangian (3.2.7) has no mathematical meaning. In order to resolve this problem, one needs to introduce an ultraviolet regularization. In position space, this regularization can be viewed as a "smoothing" on a microscopic length scale. It seems natural to identify this microscopic length scale with the Planck length $\ell_{P}$, although it may be even smaller. Thus we always assume that $\varepsilon \lesssim \ell_{P}$. Likewise, in momentum space the regularization corresponds to a cutoff or decay on the scale $\varepsilon^{-1}$, which is at least as large as the Planck energy $E_{P}=\ell_{P}^{-1}$. Clearly, the regularization scale is extremely small compared to the length scale $\ell_{\text {macro }}$ of macroscopic physics, and thus it seems reasonable to expand in powers of $\varepsilon / \ell_{\text {macro }}$. However, such an expansion would not be mathematically meaningful, because Taylor series can be performed only in continuous variables (but not in a constant, no matter how small). Therefore, it is preferable to denote the regularization length by the variable $\varepsilon$, which may vary in the range $0<\varepsilon \ll \ell_{\text {macro. }}$ We are thus led to a one-parameter family of regularizations. We assume that these regularized Dirac sea configurations are all minimizers. We also compile all assumptions on the regularization as introduced in [F7, Chapter 4].

Assumption 3.3.1. (regularized Dirac sea vacuum) There is a family $\left(P^{\varepsilon}\right)_{\varepsilon>0}$ of fermionic projectors whose kernels $P^{\varepsilon}(x, y)$ (as defined by (3.2.1) ) have the following properties:

(i) Every $P^{\varepsilon}(x, y)$ is a minimizer in the sense of Definition 3.2.2.

(ii) Every $P^{\varepsilon}(x, y)$ is homogeneous, i.e. it depends only on the variable $\xi:=y-x$.

(iii) Taking its Fourier transform,

$$
P^{\varepsilon}(x, y)=\int \frac{d^{4} k}{(2 \pi)^{4}} \hat{P}^{\varepsilon}(k) e^{-i k(x-y)},
$$

$\hat{P}^{\varepsilon}$ is a distribution with a vector-scalar structure, i.e.

$$
\hat{P}^{\varepsilon}(k)=\left(v_{j}^{\varepsilon}(k) \gamma^{j}+\phi^{\varepsilon}(k) \mathbb{1}\right) f^{\varepsilon}(p)
$$

with a vector field $v^{\varepsilon}$, a scalar field $\phi^{\varepsilon}$ and a distribution $f^{\varepsilon}$, which are all realvalued.

(iv) If the regularization is removed, $P^{\varepsilon}$ goes over to $P$ (as given by (3.3.1), i.e.

$$
\lim _{\varepsilon \searrow 0} \hat{P}^{\varepsilon}(k)=\hat{P}(k):=\sum_{\beta=1}^{g}\left(\not k+m_{\beta}\right) \delta\left(k^{2}-m_{\beta}^{2}\right) \Theta\left(-k^{0}\right)
$$

with convergence in the distributional sense.

The assumptions so far seem natural and are easy to state. In order to understand the following assumptions, one should notice that the singularities of $P(x, y)$ on the light cone arise because its Fourier transform $\hat{P}(k)$ is supported on the mass shells $k^{2}=m_{\beta}^{2}$, which are hypersurfaces being asymptotic to the mass cone $k^{2}=0$ (for details see $\mathbf{F 7}$, 
Section 4.2]). Thus in order to control the behavior of $P^{\varepsilon}$ near the light cone, we need to make suitable assumptions on $P^{\varepsilon}(\omega, \vec{k})$ for $\omega \approx-|\vec{k}| \sim \varepsilon^{-1}$.

(v) We assume that the distribution $\hat{P}^{\varepsilon}$ is supported on hypersurfaces described by graphs, i.e. the distribution $f^{\varepsilon}$ in (3.3.4) should be of the form

$$
f^{\varepsilon}(\omega, \vec{k})=\sum_{\beta=1}^{g} \delta\left(\omega+|\vec{k}|+\alpha_{\beta}(\vec{k})\right) .
$$

These hypersurfaces should be asymptotic to the mass cone in the sense that

$$
\alpha_{\beta}(\vec{k}) \sim \varepsilon \quad \text { if }|\vec{k}| \sim \varepsilon^{-1}
$$

Except for these singularities, $\hat{P}^{\varepsilon}(k)$ is so regular that the singularities as $\varepsilon \searrow 0$ of $P^{\varepsilon}(x, y)$ on the light cone are completely described by the behavior of $\hat{P}^{\varepsilon}(k)$ on the hypersurfaces (3.3.5), up to corrections of higher order in $\varepsilon$. We refer to this assumption as the restriction to surfaces states.

(vi) On the hypersurfaces (3.3.5) and for $|\vec{k}| \sim \varepsilon^{-1}$, the vector field $v^{\varepsilon}$ in $(3.3 .4)$ should be parallel to $k$, up to a small error term. More precisely, decomposing $v^{\varepsilon}$ as

$$
v^{\varepsilon}=s^{\varepsilon}(k) k+\vec{w}^{\varepsilon}(k)
$$

with a scalar function $s^{\varepsilon}$, the vector field $\vec{w}^{\varepsilon}$ should be bounded by

$$
\left|\vec{w}^{\varepsilon}(k)\right|<\varepsilon_{\text {shear }} \quad \text { where } \quad \varepsilon_{\text {shear }} \ll 1 .
$$

We refer to $\varepsilon_{\text {shear }}$ as the shear parameter. Considering the effect of this assumption in position space, we say that the vector component is null on the light cone.

(vii) The functions in (3.3.4) either vanish, $\phi^{\varepsilon}(k)=0=v^{\varepsilon}(k)$, or else $\phi^{\varepsilon}(k)>0$ and the vector field $v^{\varepsilon}$ is time-like and past-directed. Furthermore,

$$
v^{\varepsilon}(p)^{2}=\phi^{\varepsilon}(p)^{2} \text {. }
$$

For a discussion of the assumptions (v) and (vi) we refer to [F7, Chapter 4]. The condition (vii) requires a brief explanation. This assumption is clearly satisfied without regularization (3.3.1) (in which case we choose $v(p)=p /(2 \omega)$ and $\phi$ a positive function which on the mass shells takes the values $\left.m_{\alpha} /(2 \omega)\right)$. A closely related condition was first proposed in [F7, Chapter 4] as the assumption of half-occupied surface states. This condition was motivated by the wish to realize the Dirac sea configurations with as few occupied states as possible, noting that the condition (vii) implies that the matrix $\hat{P}^{\varepsilon}(k)$ has rank at most two. Furthermore, the condition (vii) implies that the image of the matrix $\hat{P}^{\varepsilon}(k)$ is negative definite with respect to the inner product $\bar{\psi} \phi$. From the mathematical point of view, this definiteness is crucial for our action principle to be mathematically welldefined (see the reformulation as a causal variational principle in [F13, FGS] as well as the general compactness result [F13, Theorem 4.2]). Thus the physical intuition and the mathematical requirements fit together. Moreover, in the case when $\hat{P}^{\varepsilon}(k)$ does not vanish, we can choose a suitably normalized orthogonal basis $\left(\psi_{p, 1}, \psi_{p, 2}\right)$ of the image of $\hat{P}^{\varepsilon}(k)$ such that $(2 \pi)^{4} \hat{P}^{\varepsilon}(k)=-\psi_{k, 1} \overline{\psi_{k, 1}}-\psi_{k, 2} \overline{\psi_{k, 2}}$. Substituting this representation into the Fourier integral (3.3.3) and using (3.3.5), we obtain

$$
P^{\varepsilon}(x, y)=-\sum_{\beta=1}^{g} \int_{\mathbb{R}^{3}} d \vec{k} \sum_{a=1,2} \psi_{\vec{k} \beta a}(x) \overline{\psi_{\vec{k} \beta a}(y)},
$$


where $\psi_{\vec{k} \beta a(x)}=\psi_{p, a} e^{-i p x}$ for $p=\left(-|\vec{k}|-\alpha_{\beta}(\vec{k}), \vec{k}\right)$. This representation is helpful because it shows that the regularized fermionic projector of the vacuum is composed of negative-energy wave functions; the index $a$ can be thought of as describing the two spin orientations.

We next outline the approach taken to justify the above assumptions. In [F11] a class of regularizations is constructed for which the action remains finite when the regularization is removed (more precisely, this is done by proving that the constructed regularizations satisfy the so-called assumption of a distributional $\mathcal{M} P$-product). These regularizations are spherically symmetric, but they break the Lorentz symmetry. However, after suitably removing the regularization, we obtain a well-defined Lorentz invariant action principle. This Lorentz invariant action principle is analyzed in $[\mathbf{F H}]$, and it is shown that for certain values of the masses and the so-called weight factors (which for simplicity we do not consider in the main text of this paper; see however Appendix E), the Dirac sea configuration (3.3.1) is indeed a minimizer, in a sense made precise using the notion of state stability. Following these results, "good candidates" for satisfying the above assumptions are obtained by regularizing the state stable Dirac sea configurations from $[\mathbf{F H}]$ according to the regularization scheme in [F11]. The remaining task for giving a rigorous justification of Assumption 3.3.1 is to use the freedom in choosing the regularization such as to obtain a minimizer in the sense of Definition 3.2.2. This task seems difficult and has not yet been accomplished. In [F13, Theorem 4.2] the existence of minimizers is proved within the class of homogeneous fermionic projectors; but this is considerably weaker than being a minimizer in the sense of Definition 3.2.2. In technical terms, the main difficulty is to quantify the influence of the spherically symmetric regularization on the action, even taking into account contributions which remain finite when the regularization is removed. Despite this difficult and technically challenging open problem, it is fair to say that the results of [F11, $[\mathbf{F H}]$ show that Dirac sea configurations tend to make our action small, thus explaining why Assumption 3.3.1 is a reasonable starting point for the continuum analysis.

We finally explain how to work with the above assumptions in practice. Ideally, the fields $v^{\varepsilon}, \phi^{\varepsilon}$ and the distribution $f^{\varepsilon}$ in $(3.3 .4$ could be determined by minimizing our action 3.2 .9 , thus giving detailed information on $P^{\varepsilon}$. Such a minimization process is indeed possible (see [F13, Theorem 4.2] for a general existence result and [FP] for a lattice formulation), but so far has not been analyzed in sufficient depth. Thus for the time being, there is a lot of freedom to choose the functions in (3.3.4). Our program is not to make a specific choice but to consider instead general functions $v^{\varepsilon}, \phi^{\varepsilon}$ and $f^{\varepsilon}$. Our subsequent analysis will clearly depend on the choice of these functions, and our task is to look for conclusions which are robust to regularization details. This so-called method of variable regularization (which is worked out in detail in [F7, Section 4.1]) leads to the formalism of the continuum limit which will be explained in Section 3.5 below.

\subsection{Introducing an Interaction}

Our next goal is to generalize the regularized fermionic projector $P^{\varepsilon}$ of the previous section such as to include an interaction. Postponing the treatment of the regularization to Section 3.5, we shall now extend the definition of the fermionic projector of the vacuum (3.3.1) to the case with interaction. We outline the methods developed in [F3, F5, F6]; see also [F7, Chapter 2] or Chapter 2 in this book. 
3.4.1. A Dirac Equation for the Fermionic Projector. First, it is useful to recover (3.3.1) as a solution of a Dirac equation: Replacing the ordinary sum in (3.3.1) by a direct sum, we introduce the so-called auxiliary fermionic projector $P^{\text {aux }}$ by

$$
P^{\operatorname{aux}}(x, y)=\bigoplus_{\beta=1}^{g} \int \frac{d^{4} k}{(2 \pi)^{4}}\left(\not k+m_{\beta}\right) \delta\left(k^{2}-m_{\beta}^{2}\right) \Theta\left(-k^{0}\right) e^{-i k(x-y)}
$$

(thus $P^{\text {aux }}(x, y)$ is represented by a $4 g \times 4 g$-matrix). It is a solution of the free Dirac equation

$$
\left(i \not \partial_{x}-m Y\right) P^{\operatorname{aux}}(x, y)=0
$$

where the mass matrix $Y$ is composed of the rest masses corresponding to the $g$ generations,

$$
m Y=\bigoplus_{\beta=1}^{g} m_{\beta}
$$

(here $m>0$ is an arbitrary mass parameter which makes $Y$ dimensionless and will be useful for expansions in the mass parameter; see also [F7, Section 2.3] or Section 2.2]. The fermionic projector of the vacuum is obtained from $P^{\text {aux }}$ by summing over the generation indices,

$$
P=\sum_{\alpha, \beta=1}^{g}\left(P^{\text {aux }}\right)_{\beta}^{\alpha}
$$

This summation removes the generation indices, leaving us with the configuration of one sector. In [F7 this operation is referred to as the partial trace. However, this notion might be confusing because it suggests that in (3.4.3) one should set $\alpha=\beta$ and sum over one index (for a more detailed discussion see [F7, paragraph after Lemma 2.6.1]). In order to avoid this potential source of confusion, in this book we always refer to the operator in (3.4.3) as the the sectorial projection.

The obvious idea for introducing an interaction is to replace the free Dirac equation $(3.4 .2)$ by a Dirac equation with interaction,

$$
(i \not \partial+\mathcal{B}-m Y) P^{\text {aux }}(x, y)=0,
$$

where $\mathcal{B}$ is a general perturbation operator, and to introduce the fermionic projector again forming the sectorial projection (3.4.3). In order to ensure that the resulting fermionic projector is again symmetric (3.2.3), we generalize the inner product (3.2.2) to the wave functions of the auxiliary Dirac equation by setting

$$
<\psi_{\text {aux }} \mid \phi_{\text {aux }}>=\sum_{\beta=1}^{g} \int_{\mathcal{M}} \overline{\psi_{\text {aux }}^{\beta}(x)} \phi_{\text {aux }}^{\beta}(x) d^{4} x,
$$

and demand that the auxiliary fermionic projector should be symmetric with respect to this new inner product,

$$
<P^{\text {aux }} \psi_{\text {aux }}\left|\phi_{\text {aux }}>=<\psi_{\text {aux }}\right| P^{\text {aux }} \phi_{\text {aux }}>\text { for all } \psi_{\text {aux }}, \phi_{\text {aux }} \in C_{0}^{\infty}(\mathscr{M}, S \mathscr{M})^{g} .
$$

In order to obtain a coherent framework, we shall always assume that the Dirac operator is symmetric with respect to this inner product,

$$
<(i \not \partial+\mathcal{B}-m Y) \psi_{\text {aux }}\left|\phi_{\text {aux }}>=<\psi_{\text {aux }}\right|(i \not \partial+\mathcal{B}-m Y) \phi_{\text {aux }}>.
$$

This equation gives a condition for the operator $\mathcal{B}$ describing the interaction. Apart from this condition and suitable regularity and decay assumptions, the operator $\mathcal{B}$ can 
be chosen arbitrarily; in particular, it can be time dependent. In typical applications, $\mathcal{B}$ is a multiplication or differential operator composed of bosonic potentials and fields. The choices of $\mathcal{B}$ relevant for this work will be discussed in $\$ 3.4 .5$ below.

3.4.2. The Interacting Dirac Sea. Clearly, the Dirac equation (3.4.4) has many different solutions, and thus in order to determine $P^{\text {aux }}$, we need to specify of which one-particle states $P^{\text {aux }}$ should be composed. In the vacuum (3.4.1), this can be done by taking all the negative-energy solutions, i.e. all states on the lower mass shells $\left\{k^{2}=\right.$ $\left.m_{\beta}^{2}, k^{0}<0\right\}$. Unfortunately, the concept of negative energy does not carry over to the situation of a time-dependent interaction (3.4.4), because in this case the energy of the Dirac wave functions is not conserved; this is the so-called external field problem (see [F7, Section 2.1] or Section 2.1). The clue for resolving this problem is the observation that the negative-energy states in (3.4.1) can be characterized alternatively using the causality of the Dirac Green's functions in a specific way. This causal approach generalizes to the situation (3.4.4) and makes it possible to extend the concept of the Dirac sea to the timedependent setting. It gives rise to a unique definition of the fermionic projector $P^{\text {sea }}$ in terms of a power series in $\mathcal{B}$. More precisely, the so-called causal perturbation expansion expresses $P^{\text {sea }}$ as sums of operator products

$$
P^{\text {sea }}=\sum_{k=0}^{\infty} \sum_{\alpha=0}^{\alpha_{\max }(k)} c_{\alpha} C_{1, \alpha} \mathcal{B} C_{2, \alpha} \mathcal{B} \cdots \mathcal{B} C_{k+1, \alpha},
$$

where the factors $C_{l, \alpha}$ are the Green's functions or fundamental solutions of the free Dirac equation (3.4.2), and the $c_{\alpha}$ are combinatorial factors (for details see [F3] and [F7, Sections 2.2-2.3]; for a more recent account on idempotence and unitarity questions see [FG1, FT2]). In the language of Feynman diagrams, each summand in (3.4.6) is a tree diagram. These tree diagrams are all finite, provided that $\mathcal{B}$ satisfies suitable regularity and decay assumptions at infinity (see [F7, Lemma 2.2.2.] or Lemma 2.1.2).

3.4.3. Introducing Particles and Anti-Particles. The fermionic projector $P^{\text {sea }}$ is interpreted as a generalization of completely filled Dirac seas to the interacting situation (3.4.4). In order to bring particles and anti-particles into the system, we add the projectors on states $\psi_{1}, \ldots, \psi_{n_{\mathrm{p}}}$ which are not contained in the image of the operator $P^{\text {sea }}$ (the particle states) and subtract the projectors on states $\phi_{1}, \ldots, \phi_{n_{\mathrm{a}}}$ which are in the image of $P^{\text {sea }}$ (the anti-particle states),

$$
P^{\text {aux }}(x, y)=P^{\text {sea }}(x, y)-\frac{1}{2 \pi} \sum_{k=1}^{n_{\mathrm{p}}} \psi_{k}(x) \overline{\psi_{k}(y)}+\frac{1}{2 \pi} \sum_{l=1}^{n_{\mathrm{a}}} \phi_{l}(x) \overline{\phi_{l}(y)} .
$$

Then the fermionic projector is again obtained by forming the sectorial projection 3.4.3). Here the wave functions in (3.4.7) are to be normalized such that they are orthonormal with respect to the usual integral over the probability density, i.e.

$$
\int_{\mathbb{R}^{3}}\left(\overline{\psi_{k}} \gamma^{0} \psi_{k^{\prime}}\right)(t, \vec{x}) d^{3} x=\delta_{k, k^{\prime}}, \quad \int_{\mathbb{R}^{3}}\left(\overline{\phi_{l}} \gamma^{0} \phi_{l^{\prime}}\right)(t, \vec{x}) d^{3} x=\delta_{l, l^{\prime}}
$$

The factors $\pm \frac{1}{2 \pi}$ in 3.4 .7 are needed for the proper normalization of the fermionic states (for details see [FT2] or $\$ 2.1 .7$ ). 
3.4.4. The Light-Cone Expansion and Resummation. We now outline the methods for analyzing the fermionic projector in position space (for details see [F5, F6 or Section 2.2). The following notion is very useful for describing the structure of the singularities on the light cone.

Definition 3.4.1. A distribution $A(x, y)$ on $\mathcal{M} \times \mathscr{M}$ is of the order $\mathcal{O}\left((y-x)^{2 p}\right)$, $p \in \mathbb{Z}$, if the product

$$
(y-x)^{-2 p} A(x, y)
$$

is a regular distribution (i.e. a locally integrable function). It has the light-cone expansion

$$
A(x, y)=\sum_{j=g_{0}}^{\infty} A^{[j]}(x, y)
$$

with $g_{0} \in \mathbb{Z}$ if the distributions $A^{[j]}(x, y)$ are of the order $\mathcal{O}\left((y-x)^{2 j}\right)$ and if $A$ is approximated by the partial sums in the sense that for all $p \geq g$,

$$
A(x, y)-\sum_{j=g_{0}}^{p} A^{[j]}(x, y) \quad \text { is of the order } \mathcal{O}\left((y-x)^{2 p+2}\right) .
$$

Thus the light-cone expansion is an expansion in the orders of the singularity on the light cone. As the main difference to a Taylor expansion, for any fixed $x$ the expansion parameter $(y-x)^{2}$ vanishes for all $y$ in an unbounded set, namely the whole light cone centered at $x$. In this sense, the light-cone expansion is a nonlocal expansion.

For a convenient formulation of the light-cone expansion of the fermionic projector, it is helpful to work with a generating function, i.e. a power series in a real parameter $a>0$ whose coefficients are functions in $(y-x)^{2}$ which are of increasing order on the light cone. The first ansatz for such a generating function is the Fourier transform $T_{a}(x, y)$ of the lower mass shell with $k^{2}=a$,

$$
T_{a}(x, y)=\int \frac{d^{4} k}{(2 \pi)^{4}} \delta\left(k^{2}-a\right) \Theta\left(-k^{0}\right) e^{-i k(x-y)} .
$$

Carrying out the Fourier integral and expanding the resulting Bessel functions, one obtains

$$
\begin{aligned}
T_{a}(x, y)= & -\frac{1}{8 \pi^{3}}\left(\frac{\mathrm{PP}}{\xi^{2}}+i \pi \delta\left(\xi^{2}\right) \epsilon\left(\xi^{0}\right)\right) \\
& +\frac{a}{32 \pi^{3}} \sum_{j=0}^{\infty}\left(\log \left|a \xi^{2}\right|+c_{j}+i \pi \Theta\left(\xi^{2}\right) \epsilon\left(\xi^{0}\right)\right) \frac{(-1)^{j}}{j !(j+1) !} \frac{\left(a \xi^{2}\right)^{j}}{4^{j}}
\end{aligned}
$$

where we again used the abbreviation $\xi=y-x$, and $\epsilon$ denotes the sign function (i.e. $\epsilon(x)=1$ if $x \geq 0$ and $\epsilon(x)=-1$ otherwise). The real coefficients $c_{j}$ are given explicitly in [F7, Section 2.5]. Unfortunately, due to the factor $\log \left|a \xi^{2}\right|$, the expression (3.4.10) is not a power series in $a$. In order to bypass this problem, we simply remove the logarithms in $a$ by subtracting suitable counter terms,

$$
T_{a}^{\mathrm{reg}}(x, y):=T_{a}(x, y)-\frac{a}{32 \pi^{3}} \log |a| \sum_{j=0}^{\infty} \frac{(-1)^{j}}{j !(j+1) !} \frac{\left(a \xi^{2}\right)^{j}}{4^{j}} .
$$


The resulting distribution $T_{a}^{\mathrm{reg}}$ is a power series in $a$, and it is indeed the right choice for our generating function. We denote its coefficients by

$$
T^{(n)}=\left.\left(\frac{d}{d a}\right)^{n} T_{a}^{\mathrm{reg}}\right|_{a=0} \quad(n=0,1,2, \ldots)
$$

and also introduce $T^{(-1)}$ via the distributional equation

$$
\frac{\partial}{\partial x^{k}} T^{(0)}(x, y)=\frac{1}{2}(y-x)_{k} T^{(-1)}(x, y) .
$$

We remark for clarity that removing the logarithmic poles in $a$ has similarity to an infrared regularization, because infrared problems also appear when the mass parameters tend to zero. This is the motivation for using the superscript "reg." But clearly, this "regularization" is not related to the ultraviolet regularization in Assumption 3.3.1.

Combining Fourier techniques with methods of hyperbolic partial differential equations, one can perform the light-cone expansion of each summand of the perturbation series (3.4.6). After suitably rearranging all the resulting contributions, one can partially carry out the infinite sums. This so-called resummation gives rise to an expansion of the interacting fermionic projector of the form

$$
\begin{aligned}
P^{\text {sea }}(x, y)= & \sum_{n=-1}^{\infty} \sum_{k} m^{p_{k}}(\text { phase-inserted nested line integrals }) \times T^{(n)}(x, y) \\
& +\tilde{P}^{\mathrm{le}}(x, y)+\tilde{P}^{\mathrm{he}}(x, y)
\end{aligned}
$$

Here the $n$-summands describe the different orders of the singularities on the light cone, whereas the $k$-sum describes all contributions to a given order on the light cone. The phase-inserted nested line integrals involve $\mathcal{B}$ and its partial derivatives, possibly sandwiched between time-ordered exponentials of chiral potentials. Since these nested line integrals are smooth functions in $x$ and $y$, the series in (3.4.14) is a light-cone expansion in the sense of Definition 3.4.1, provided that the $k$-sum is finite for every $n$. This is indeed the case if $\mathcal{B}$ is composed of scalar, pseudoscalar and chiral potentials $[\mathbf{F 6}$, whereas for a more general perturbation operator $\mathcal{B}$ this condition still needs to be verified. This expansion is causal in the sense that it depends on $\mathcal{B}$ and its partial derivatives only along the line segment $\overline{x y}$. The contributions $\tilde{P}^{\text {le }}$ and $\tilde{P}^{\text {he }}$, on the other hand, are not causal but depend instead on the global behavior of $\mathcal{B}$ in space-time. They can be written as a series of functions which are all smooth in $x$ and $y$. Their different internal structure gives rise to the names non-causal low energy contribution and non-causal high energy contribution, respectively.

For an introduction to the light-cone expansion and the required mathematical methods we refer to [F5] and [F6], the exposition in [F7, Section 2.5] or Chapter 2 in this book. The formulas of the light-cone expansion needed in this work are compiled in Appendix B.

3.4.5. Clarifying Remarks. The above constructions require a few explanations. We first point out that, although we are working with one-particle wave functions, the ansatz for the fermionic projector (3.4.7) describes a many-particle quantum state. In order to get a connection to the Fock space formalism, one can take the wedge product of the wave functions $\psi_{k}$ and $\phi_{l}$ to obtain a vector in the fermionic Fock space (for details see [F7, Appendix A]). We conclude that (3.4.7) describes second-quantized fermions. For the description of entangled states see [F14]. 
One should keep in mind that at this stage, the form of the potential $\mathcal{B}$ has not been specified; it can be an arbitrary operator. Indeed, we regard the operator $\mathcal{B}$ merely as a device for modifying or perturbing the fermionic projector. We do not want to preassume which of these perturbations are physically relevant; instead, we want to select the relevant perturbations purely on the basis of whether they are admissible for minimizers of our action principle (3.2.9). In other words, our action principle should decide how the physical interaction looks like, even quantitatively in the sense that our action principle should determine the corresponding field equations. Following this concept, we should choose $\mathcal{B}$ as general as possible, even allowing for potentials which are usually not considered in physics. We now give a brief overview over the potentials which will be of relevance in the present work. The most obvious choice is an electromagnetic potentia $2^{2}$,

$$
\mathcal{B}=A \text {. }
$$

More generally, one can choose chiral potentials, which may be non-diagonal in the generations,

$$
\mathcal{B}=\chi_{L} A_{R}+\chi_{R} A_{L},
$$

where $A_{L / R}=\left(A_{L / R}^{i}\right)_{\beta}^{\alpha}$ with generation indices $\alpha, \beta=1, \ldots, g$ and a vector index $i=$ $0, \ldots, 3$ (here $\chi_{L / R}=\frac{1}{2}(\mathbb{1} \mp \Gamma)$ are the chiral projectors, and $\Gamma=i \gamma^{0} \gamma^{1} \gamma^{2} \gamma^{3}$ is the usual pseudoscalar matrix). To describe a gravitational field, one needs to choose $\mathcal{B}$ as a differential operator of first order; more precisely,

$$
\mathcal{B}=\mathcal{D}-i \not \partial,
$$

where $\mathcal{D}$ is the Dirac operator in the presence of a gravitational field.

The above choices of $\mathcal{B}$ are of course motivated by physical fields observed in nature. However, we point out that we do not assume any field equations. Thus the electromagnetic potential in (3.4.15) does not need to satisfy Maxwell's equations, in (3.4.16) we do not assume Yang-Mills-type equations for the chiral gauge fields, and in (3.4.17) the Einstein equations are not imposed. This is because, as already pointed out above, our goal is to derive the classical field equations from our action principle (3.2.9).

Apart from the above choices of $\mathcal{B}$ motivated from physics, one can also choose other physically less obvious operators, like for example scalar or pseudoscalar potentials,

$$
\mathcal{B}=\Phi+i \Gamma \Xi
$$

with $\Phi=\Phi_{\beta}^{\alpha}, \Xi=\Xi_{\beta}^{\alpha}$ and $\alpha, \beta=1, \ldots, g$. Furthermore, one can consider a scalar differential operator,

$$
\mathcal{B}=i \Phi^{j} \partial_{j}
$$

or a higher order differential operator. More specifically, we will find a pseudoscalar differential potential useful,

$$
\mathcal{B}=\Gamma\left(v^{j} \partial_{j}+\partial_{j} v^{j}\right) .
$$

It is worth noting that one does not need to restrict attention to differential operators. Indeed, $\mathcal{B}$ can also be an integral operator, in which case we talk of nonlocal potentials. Clearly, one can also take linear combinations of all the above operators $\mathcal{B}$.

Next, it is worth noting that for the moment, we consider $\mathcal{B}$ as a-priori given, and thus at this stage, our system consists of Dirac particles moving in an external field.

\footnotetext{
${ }^{2}$ For convenience we shall always omit the coupling constant $e$ in the Dirac equation. Our convention is obtained from the usual choice $\mathcal{B}=e A$ by the transformation $A \rightarrow e^{-1} A$. The coupling constant clearly reappears in the Maxwell equations, which we write in natural units and with the Heaviside-Lorentz convention as $\partial_{j k} A^{k}-\square A_{k}=e^{2} \bar{\psi} \gamma_{k} \psi$. As usual, the fine structure constant is given by $\alpha=e^{2} /(4 \pi)$.
} 
However, our action principle $(3.2 .9)$ will give relations between the potentials contained in $\mathcal{B}$ and the Dirac wave functions in (3.4.7), and thus these potentials will be influenced by the Dirac wave functions. This leads to a mutual coupling of the potentials to the Dirac wave functions, giving rise to a fully interacting system. We also point out that the potentials and fields contained in $\mathcal{B}$ should be regarded as classical. Indeed, in this book we will always work with classical bosonic fields. However, as is worked out in [F14, F17, F20, the framework of the fermionic projector also allows for the description of second-quantized bosonic fields.

3.4.6. Relation to Other Approaches. Having outlined our approach, we can now give a short review of related works. In order to get a connection to our description of the Dirac sea in $\$ 3.4 .2$, we begin with the construction of quantum fields in an external field. Historically, this problem was first analyzed in the Fock space formalism. Klaus and Scharf [KS1, KS2] considered the Fock representation of the electron-positron field in the presence of a static external field. They noticed that the Hamiltonian needs to be regularized by subtracting suitable counter terms which depend on the external field. They also noticed that the electron-positron field operators in the external field form a Fock representation on the standard Fock space of free fields only if the external field satisfies a certain regularity condition. This regularity condition is quite restrictive and excludes many cases of physical interest (like a magnetic field [NS] and a Coulomb potential [K1]). In particular, these results show that different external fields in general give rise to nonequivalent Fock representations of the electron-positron field operators. More recently, in [HLS2, HLS1 the vacuum state was constructed for a system of Dirac particles with electrostatic interaction in the Bogoliubov-Dirac-Fock approximation. The conclusion of this analysis is that for mathematical consistency, one must take into account all the states forming the Dirac sea. Furthermore, the interaction mixes the states in such a way that it becomes impossible to distinguish between the particle states and the states of the Dirac sea.

In the time-dependent setting, Fierz and Scharf [FS] proposed that the Fock representation should be adapted to the external field as measured by a local observer. Then the Fock representation becomes time and observer dependent. This implies that the distinction between particles and anti-particles no longer has an invariant meaning, but it depends on the choice of an observer. In this formulation, the usual particle interpretation of quantum states only makes sense for the in- and outgoing scattering states, but it has no invariant meaning for intermediate times. For a related approach which allows for the construction of quantum fields in the presence of an external magnetic field see [DDMS]. In all the above approaches, the Dirac sea leads to divergences, which must be treated by an ultraviolet regularization and suitable counter terms.

As an alternative to working with Fock spaces, one can use the so-called point splitting renormalization method, which is particularly useful for renormalizing the expectation value of the energy-momentum tensor [Ch. The idea is to replace a function of one variable $T(x)$ by a two-point distribution $T(x, y)$, and to take the limit $y \rightarrow x$ after subtracting suitable singular distributions which take the role of counter terms. Analyzing the singular structure of the counter terms leads to the so-called Hadamard condition (see for example [FSW] $)$. Reformulating the Hadamard condition for the two-point function as a local spectral condition for the wave front set [Ra turns out to be very useful for the axiomatic formulation of free quantum field theory in curved space-time. As in the Fock space formalism, in the point splitting approach the particle interpretation depends on 
the observer. This is reflected mathematically by the fact that the Hadamard condition specifies the two-point distribution only up to smooth contributions, thus leaving the smooth particle wave functions undetermined. For a good introduction to free quantum fields in curved space-time we refer to the recent book $\mathbf{B F}$.

As mentioned at the beginning of $\$ 3.4 .5$, in our approach the connection to the Fock space formalism is obtained by choosing a basis of the image of the fermionic projector and taking the wedge product of the basis vectors (for details see [F7, Appendix A] or [F14]). If in this construction the states of the Dirac sea are taken into account, we get precisely the framework in [FS]. The connection to the Hadamard condition is even closer. Indeed, considering the light-cone expansion locally for $y$ near $x$, the summands in (3.4.14) coincide precisely with the singular distributions in the Hadamard construction. Since the non-causal contributions $\tilde{P}^{\text {he }}$ and $\tilde{P}^{\text {le }}$ are smooth functions, we conclude that the integral kernel of the fermionic projector satisfies the Hadamard condition, provided that the perturbation expansions of $\tilde{P}^{\text {he }}$ and $\tilde{P}^{\text {le }}$ converge (a subtle technical problem which we do not want to enter here). Thus in a given external field, $P^{\text {sea }}(x, y)$ can be interpreted as the two-point function, and using the methods of $[\mathbf{R a}, \mathbf{B F}]$ one could construct the corresponding free QFT. This construction has been carried out in FMR in the presence of an external potential.

A major difference of our approach is that our framework allows for the description of an interacting theory, where the coupling of the fermions to bosonic fields and the back-reaction of the bosonic fields to the fermions is taken into account. In this setting, the interaction is described by our action principle (3.2.8). The mathematical framework is no longer equivalent to standard QFT. In particular, $P(x, y)$ cannot be interpreted as the two-point function of a corresponding QFT, simply because the notions of QFT can no longer be used. But we still get a connection to the Feynman diagrams of QFT (as will be explained in $\$ 3.8 .4$ below).

Another major difference of our approach is that the distribution $P^{\text {sea }}$ as defined by the causal perturbation expansion (3.4.6) distinguishes a unique state which can be interpreted as the fermionic vacuum state where all Dirac seas are completely filled. Thus working relative to this distinguished state, there is a unique observer independent particle interpretation, even at intermediate times (see [F3, Section 5] for a discussion of this point). At first sight, this distinguished particle interpretation might seem of purely academic interest, because $P^{\text {sea }}$ is defined globally in space-time and is thus not accessible to a local observer. However, our action principle (3.2.8) does have access to quantities defined globally in space-time, and in this way the distinguished particle interpretation enters the physical equations. More precisely, $P^{\text {sea }}$ drops out of the Euler-Lagrange equations corresponding to our action principle, up to terms which are well-defined and explicitly computable, even including a uniquely determined smooth contribution. In this way, the arbitrariness of working modulo smooth contributions (in the Hadamard condition) or modulo regular counter terms (in the Fock space formalism) is removed. The corresponding smooth contributions to the physical equations will be analyzed in $\$ 3.8 .1$ and Appendix D. They are nonlocal and violate causality, as will be explained in $\$ 3.8 .2$.

A frequently asked question is how our approach relates to Connes' noncommutative geometry [2]. In particular, can our approach be thought of as a Lorentzian version of noncommutative geometry? Clearly, both approaches have in common that the Dirac operator plays a central role. Moreover, the light-cone expansion is the Lorentzian analog of local expansions of the resolvent or the heat kernel near the diagonal. A major difference is that instead of considering the whole spectrum of the Dirac operator, we 
only consider the eigenspaces corresponding to the masses $m_{\alpha}$ of the Dirac particles of our system. Furthermore, we only take "half the eigenspaces" by constructing Dirac seas, and we also build in additional particle and anti-particle states (3.4.7). Another major difference concerns the mathematical structure of our action principle (3.2.8). Namely, this action cannot be thought of as a spectral action, because it is impossible to express it in terms of spectral properties of the Dirac operator. This is obvious from the fact that in (3.2.7) and (3.2.8) we perform a nonlinear (and even non-analytic) transformation of the kernel $P(x, y)$ before integrating over $x$ and $y$. As a consequence, there is no connection to a regularized trace or Hilbert-Schmidt norm of $P$. The specific form of our action principle makes it possible to regard the structures of Minkowski space as emerging from a self-organization of the wave functions in discrete space time (see [F12]), an idea which has no correspondence in noncommutative geometry. On the other hand, noncommutative geometry has deep connections to Riemannian geometry, index theory and number theory. We conclude that despite superficial similarities, the aims, ideas and methods of our approach are quite different from those in noncommutative geometry.

\subsection{The Continuum Limit}

In Section 3.3 we described the vacuum by a family of regularized fermionic projectors $P^{\varepsilon}$. Our next goal is to use the information on the regularized vacuum to also regularize the fermionic projector with interaction. We cannot expect that this information will suffice to determine the interacting fermionic projector in all details, because it is unknown how the interaction affects the fermionic projector on the microscopic scale. But as shown in [F7, Chapter 4 and Appendix D], there is a canonical method to regularize the formulas of the light-cone expansion (3.4.14). This method also gives a meaning to composite expressions as needed for the analysis of the action principle introduced in Section 3.2. In particular, it allows us to analyze the corresponding Euler-Lagrange equations in the continuum, taking into account the unknown regularization details by a finite number of free parameters. We now outline this method, relying for all technical issues on the detailed analysis in [F7. The method in 33.5 .2 is a major improvement and simplification of the techniques in [F7, Appendix F]. An introduction to the methods is given in Section 2.4.

3.5.1. Weak Evaluation on the Light Cone. Our method relies on the physically reasonable assumption of macroscopic potentials and wave functions which states that both the bosonic potentials in (3.4.4) and the fermionic wave functions in (3.4.7) vary only on the macroscopic scale and are thus almost constant on the regularization scale $\varepsilon$. Then the idea is to regularize the perturbation expansion 3.4 .6 in such a way that the interaction modifies the fermionic projector also only on the macroscopic scale. As exemplified in [F7, Appendix D] in the perturbation expansion to first order, this idea can be realized by demanding that the perturbation expansion should be gauge invariant and should satisfy a causality condition. Performing the light-cone expansion of the thus regularized perturbation expansion and using the form of the regularized vacuum minimizers as specified in Assumption 3.3.1, one obtains a simple regularization scheme for the continuum fermionic projector (3.4.14), which we now describe.

The non-causal contributions $\tilde{P}^{\text {le }}$ and $\tilde{P}^{\text {he }}$, which are already smooth in $x$ and $y$, are not regularized. Likewise, the smooth nested line-integrals are not regularized. Thus we only regularize the singularities of the factors $T^{(n)}$ on the light cone, and this is done by 
the replacement rule

$$
m^{p} T^{(n)} \rightarrow m^{p} T_{[p]}^{(n)},
$$

where the factors $T_{[p]}^{(n)}(\xi)$ are smooth functions defined as Fourier integrals involving the functions $v^{\varepsilon}, \phi^{\varepsilon}$ and $f^{\varepsilon}$ in the ansatz (3.3.4). If the sectorial projection is formed, we clarify the handling of the generation index by accents, where' and ' denote the summation over an upper and lower generation index, respectively More precisely, we extend the replacement rule (3.5.1) to (see also [F7, Section 7.1])

$$
\sum_{\alpha, \beta, \gamma_{1}, \ldots, \gamma_{p-1}=1}^{g} m^{p} \underbrace{Y_{\gamma_{1}}^{\alpha} \cdots Y_{\gamma_{2}}^{\gamma_{1}} \cdots Y_{\beta}^{\gamma_{p-1}}}_{p \text { factors } Y} T^{(n)} \rightarrow m^{p} \dot{Y} Y \cdots \grave{Y} T_{[p]}^{(n)},
$$

and use the notation ${ }^{3}$

$$
\sum_{\alpha, \beta=1}^{g} m^{0} \delta_{\beta}^{\alpha} T^{(n)} \rightarrow m^{0} g T_{[0]}^{(n)} \quad \text { and } \quad \sum_{\alpha, \beta=1}^{g} m Y_{\beta}^{\alpha} T^{(n)} \rightarrow m \hat{Y} T_{[1]}^{(n)} .
$$

Fortunately, the rather complicated detailed form of the factors $T_{[p]}^{(n)}$ will not be needed here, because these functions can always be treated symbolically using the following simple calculation rules. In computations one may treat the $T_{[p]}^{(n)}$ like complex functions. However, one must be careful when tensor indices of factors $\dot{\&}$ are contracted with each other. Naively, this gives a factor $\xi^{2}$ which vanishes on the light cone and thus changes the singular behavior on the light cone. In order to describe this effect correctly, we first write every summand of the light cone expansion (3.4.14) such that it involves at most one factor $\sharp$ (this can always be arranged using the anti-commutation relations of the Dirac matrices). We now associate every factor $\$$ to the corresponding factor $T_{[p]}^{(n)}$. In simple calculations, this can be indicated by putting brackets around the two factors, whereas in the general situation we add an index to the factor $\$$, giving rise to the replacement rule

$$
m^{p} \not T^{(n)} \rightarrow m^{p} \ddot{q}_{[p]}^{(n)} T_{[p]}^{(n)} .
$$

The factors $\$$ which are contracted to other factors $\$$ are called inner factors. The contractions of inner factors can be handled with the so-called contraction rules

$$
\begin{aligned}
\left(\xi_{[p]}^{(n)}\right)^{j}\left(\xi_{\left[p^{\prime}\right]}^{\left(n^{\prime}\right)}\right)_{j} & =\frac{1}{2}\left(z_{[p]}^{(n)}+z_{\left[p^{\prime}\right]}^{\left(n^{\prime}\right)}\right) \\
\left(\xi_{[p]}^{(n)}\right)^{j} \overline{\left(\xi_{\left[p^{\prime}\right]}^{\left(n^{\prime}\right)}\right)_{j}} & =\frac{1}{2}\left(z_{[p]}^{(n)}+\overline{z_{\left[p^{\prime}\right]}^{\left(n^{\prime}\right)}}\right) \\
z_{[p]}^{(n)} T_{[p]}^{(n)} & =-4\left(n T_{[p]}^{(n+1)}+T_{\{p\}}^{(n+2)}\right),
\end{aligned}
$$

which are to be complemented by the complex conjugates of these equations. Here the factors $z_{[p]}^{(n)}$ can be regarded simply as a book-keeping device to ensure the correct application of the rule 3.5 .5 . The factors $T_{\{p\}}^{(n)}$ have the same scaling behavior as the $T_{[p]}^{(n)}$,

\footnotetext{
${ }^{3}$ In contrast to the convention in [F7, here we always write out the factors $g$ which count the number of generations (in [F7], the factor $g$ was absorbed into the factors $T_{[0]}^{(n)}$ and $\overline{T_{[0]}^{(n)}}$ ). The shorter notation in [F7. has the disadvantage that reinserting the factors of $g$ in the end is a potential source of confusion and may lead to computational errors. In the convention here, the factors $T_{\circ}^{(n)}$ without regularization always coincide with the distributions 3.4.12 and 3.4.13.
} 
but their detailed form is somewhat different; we simply treat them as a new class of symbols 4 . In cases where the lower index does not need to be specified we write $T_{0}^{(n)}$. After applying the contraction rules, all inner factors $\xi$ have disappeared. The remaining so-called outer factors $\xi$ need no special attention and are treated like smooth functions.

Next, to any factor $T_{0}^{(n)}$ we associate the degree $\operatorname{deg} T_{\circ}^{(n)}$ by

$$
\operatorname{deg} T_{\circ}^{(n)}=1-n .
$$

The degree is additive in products, whereas the degree of a quotient is defined as the difference of the degrees of numerator and denominator. The degree of an expression can be thought of as describing the order of its singularity on the light cone, in the sense that a larger degree corresponds to a stronger singularity (for example, the contraction rule (3.5.5) increments $n$ and thus decrements the degree, in agreement with the naive observation that the function $z=\xi^{2}$ vanishes on the light cone). Using formal Taylor expansions, we can expand in the degree. In all our applications, this will give rise to terms of the form

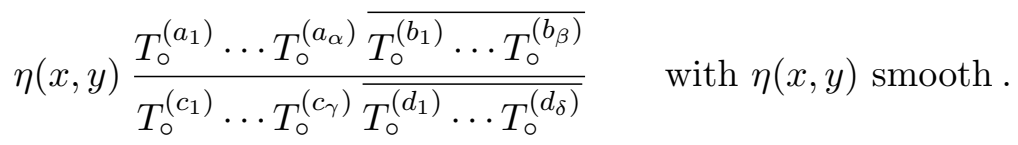

Here the quotient of the two monomials is referred to as a simple fraction.

A simple fraction can be given a quantitative meaning by considering one-dimensional integrals along curves which cross the light cone transversely away from the origin $\xi=0$. This procedure is called weak evaluation on the light cone. For our purpose, it suffices to integrate over the time coordinate $t=\xi^{0}$ for fixed $\vec{\xi} \neq 0$. Moreover, using the symmetry under reflections $\xi \rightarrow-\xi$, it suffices to consider the upper light cone $t \approx|\vec{\xi}|$. The resulting integrals will diverge if the regularization is removed. The leading contribution for small $\varepsilon$ can be written as

$$
\int_{|\vec{\xi}|-\varepsilon}^{|\vec{\xi}|+\varepsilon} d t \eta(t, \vec{\xi}) \frac{T_{\circ}^{\left(a_{1}\right)} \cdots T_{\circ}^{\left(a_{\alpha}\right)} \overline{T_{\circ}^{\left(b_{1}\right)} \cdots T_{\circ}^{\left(b_{\beta}\right)}}}{T_{\circ}^{\left(c_{1}\right)} \cdots T_{\circ}^{\left(c_{\gamma}\right)} \overline{T_{\circ}^{\left(d_{1}\right)} \cdots T_{\circ}^{\left(d_{\delta}\right)}}} \approx \eta(|\vec{\xi}|, \vec{\xi}) \frac{c_{\mathrm{reg}}}{(i|\vec{\xi}|)^{L}} \frac{\log ^{r}(\varepsilon|\vec{\xi}|)}{\varepsilon^{L-1}}
$$

where $L$ is the degree and $c_{\text {reg }}$, the so-called regularization parameter, is a real-valued function of the spatial direction $\vec{\xi} /|\vec{\xi}|$ which also depends on the simple fraction and on the regularization details (the error of the approximation will be specified below). The integer $r$ describes a possible logarithmic divergence; we postpone its discussion until when we need it (see \$3.7.3). Apart from this logarithmic divergence, the scalings in both $\xi$ and $\varepsilon$ are described by the degree.

When analyzing a sum of expressions of the form (3.5.6), one must know if the corresponding regularization parameters are related to each other. In this respect, the integration-by-parts rules are important, which are described symbolically as follows. On the factors $T_{\circ}^{(n)}$ we introduce a derivation $\nabla$ by

$$
\nabla T_{\circ}^{(n)}=T_{\circ}^{(n-1)} .
$$

\footnotetext{
${ }^{4}$ We remark that the functions $T_{\{p\}}^{(n)}$ will be of no relevance in this chapter, because they contribute to the EL equations only to degree three and lower; see 3.9 .4 .
} 
Extending this derivation with the Leibniz and quotient rules to simple fractions, the integration-by-parts rules states that

$$
\nabla\left(\frac{T_{\circ}^{\left(a_{1}\right)} \cdots T_{\circ}^{\left(a_{\alpha}\right)} \overline{\overline{T_{\circ}^{\left(b_{1}\right)} \cdots T_{\circ}^{\left(b_{\beta}\right)}}}}{T_{\circ}^{\left(c_{1}\right)} \cdots T_{\circ}^{\left(c_{\gamma}\right)} \overline{T_{\circ}^{\left(d_{1}\right)} \cdots T_{\circ}^{\left(d_{\delta}\right)}}}\right)=0 .
$$

These rules give relations between simple fractions (the name is motivated by the fact that when evaluating (3.5.8) weakly on the light cone (3.5.7), the rules state that the integral over a derivative vanishes). Simple fractions which are not related to each by the integration-by-parts rules are called basic fractions. As shown in [F7, Appendix E], there are no further relations between the basic fractions. Thus the corresponding basic regularization parameters are linearly independent.

We next specify the error of the above expansions. By not regularizing the bosonic potentials and fermionic wave functions, we clearly miss the

$$
\text { higher orders in } \varepsilon / \ell_{\text {macro }} \text {. }
$$

Furthermore, in (3.5.7) we must stay away from the origin, meaning that we neglect the

$$
\text { higher orders in } \varepsilon /|\vec{\xi}| \text {. }
$$

The higher oder corrections in $\varepsilon /|\vec{\xi}|$ depend on the fine structure of the regularization and thus seem unknown for principal reasons. Neglecting the terms in 3.5 .9 and $(3.5 .10)$ also justifies the formal Taylor expansion in the degree. Neglecting the terms 3.5.10) clearly makes it necessary to choose $|\vec{\xi}| \gg \varepsilon$. Finally, we disregard the higher order corrections in the parameter $\varepsilon_{\text {shear }}$ in $(3.3 .7)$.

The above symbolic computation rules give a convenient procedure to evaluate composite expressions in the fermionic projector, referred to as the analysis in the continuum limit: After applying the contraction rules and expanding in the degree, we obtain equations involving a finite number of terms of the form (3.5.6). By applying the integrationby-parts rules, we can arrange that all simple fractions are basic fractions. We evaluate weakly on the light cone (3.5.7) and collect the terms according to their scaling in $\xi$. Taking for every given scaling in $\xi$ only the leading pole in $\varepsilon$, we obtain equations which involve linear combinations of smooth functions and basic regularization parameters. We consider the basic regularization parameters as empirical parameters describing the unknown microscopic structure of space-time. We thus end up with equations involving smooth functions and a finite number of free parameters. We point out that these free parameters cannot be chosen arbitrarily because they might be constrained by inequalities (see the discussion after [F7, Theorem E.1]). Also, the values of the basic regularization parameters should ultimately be justified by an analysis of vacuum minimizers of our variational principle (as discussed at the end of Section 3.3).

In view of the later considerations in 3.8 .1 , we point out that the above calculation rules are valid only modulo smooth contributions to the fermionic projector. This can be understood from the fact that these rules only deal with the terms of the series in 3.4.14, but they do not take into account the smooth non-causal high and low energy contributions. But the above calculation rules affect these smooth contributions as well. To give a simple example, we consider the distribution $T^{(0)}$, which according to 3.4.10 3.4 .12 is given by

$$
T^{(0)}=-\frac{1}{8 \pi^{3}}\left(\frac{\mathrm{PP}}{\xi^{2}}+i \pi \delta\left(\xi^{2}\right) \varepsilon\left(\xi^{0}\right)\right) .
$$


Multiplying by $z=\xi^{2}$ in the distributional sense gives a constant

$$
z T^{(0)}=-\frac{1}{8 \pi^{3}} .
$$

On the other hand, the contraction rule 3.5 .5 yields

$$
z_{[0]}^{(0)} T_{[p]}^{(0)}=-4 T_{\{p\}}^{(2)} .
$$

The last relation gives much finer information than the distributional equation (3.5.11), which is essential when we want to evaluate composite expressions weakly on the light cone (3.5.7). However, the constant term in (3.5.11) does not appear in (3.5.12). The way to think about this shortcoming is that this constant term is smooth and can thus be taken into account by modifying the corresponding low energy contribution $\tilde{P}^{\mathrm{le}}(x, y)$ in (3.4.14). Indeed, this situation is not as complicated as it might seem at first sight. Namely, the smooth contributions to the fermionic projector need special attention anyway and must be computed using the resummation technique explained in Appendix $\mathrm{D}$. When performing this resummation, we can in one step also compute all the smooth contributions which were not taken into account by the formalism of the continuum limit. Thus altogether we have a convenient method where we first concentrate on the singularities on the light cone, whereas the neglected smooth contributions will be supplemented later when performing the resummation.

We note that the above procedure needs to be modified for the description of gravity, because in this case the gravitational constant makes it necessary to have relations between terms involving different powers of a fundamental length scale. These generalizations are worked out in Chapter 4.

3.5.2. The Euler-Lagrange Equations in the Continuum Limit. We now return to the action principle of Section 3.2. Our goal is to bring the conditions for a minimizer (3.2.10) and (3.2.11) into a form suitable for the analysis in the continuum limit. We begin by considering a smooth family $P(\tau)$ of fermionic projectors and compute the corresponding first variation of the action. We differentiate (3.2.11) with respect to $\tau$, treating the constraint (3.2.10) with a Lagrange multiplier (for the mathematical justification of this procedure see the related paper $[\mathbf{B F}]$ ). For convenience, we introduce the functional

$$
\mathcal{S}_{\mu}[P] \stackrel{\text { formally }}{=} \iint_{\mathcal{M} \times \mathcal{M}} \mathcal{L}_{\mu}\left[A_{x y}\right] d^{4} x d^{4} y \quad \text { with } \quad \mathcal{L}_{\mu}[A]=\left|A^{2}\right|-\mu|A|^{2} .
$$

Choosing $\mu=\frac{1}{4}$ gives precisely the action 3.2 .8 , whereas by allowing a general $\mu \in \mathbb{R}$ we take into account the Lagrange multiplier. We thus obtain the condition

$$
0=\delta \mathcal{S}_{\mu}[P]=\iint_{\mathscr{M} \times \mathcal{M}} \operatorname{Re} \operatorname{Tr}\left\{\nabla \mathcal{L}_{\mu}\left[A_{x y}\right] \delta P(x, y)\right\} d^{4} x d^{4} y,
$$

where $\delta P:=P^{\prime}(0)$. Here we consider $P(y, x)$ via

$$
P(y, x)=P(x, y)^{*} \equiv \gamma^{0} P(x, y)^{\dagger} \gamma^{0}
$$

as a function of $P(x, y)$, and $\nabla$ denotes the gradient where the real and imaginary parts of $P(x, y)$ are treated as independent variables, i.e.

$$
(\nabla f)_{\beta}^{\alpha}:=\frac{\partial f}{\partial \operatorname{Re} P(x, y)_{\alpha}^{\beta}}-i \frac{\partial f}{\partial \operatorname{Im} P(x, y)_{\alpha}^{\beta}},
$$


and $\alpha, \beta=1, \ldots, 4$ are spinor indices. Introducing the integral operator $R$ with kernel

$$
R(y, x):=\nabla \mathcal{L}_{\mu}\left[A_{x y}\right],
$$

we can write (3.5.14) as a trace of an operator product,

$$
\delta \mathcal{S}_{\mu}[P]=\operatorname{Re} \operatorname{tr}(R \delta P) .
$$

In order to get rid of the real part, it is convenient to replace $R$ by its symmetric part. More precisely, introducing the symmetric operator $Q$ with kernel

$$
Q(x, y)=\frac{1}{4}\left(R(x, y)+R(y, x)^{*}\right)
$$

we can write the variation as

$$
\delta \mathcal{S}_{\mu}[P]=2 \operatorname{tr}(Q \delta P) .
$$

As explained before Definition 3.2.1, we want to vary the fermionic projector by unitary transformations in a compact region. Thus the family of fermionic projectors $P(\tau)$ should be of the form

$$
P(\tau)=U^{-1}(\tau) P U(\tau)
$$

with a smooth family $U(\tau)$ of unitary transformations in a fixed compact region $K$ (see Definition 3.2.1) with $U(0)=\mathbb{1}$. Then the operator $B=-i U^{\prime}(0)$ has the integral representation

$$
(B \psi)(x)=\int_{\mathscr{M}} B(x, y) \psi(y) d^{4} y
$$

with a smooth compactly supported integral kernel $B \in C_{0}^{\infty}\left(K \times K, \mathbb{C}^{4 \times 4}\right)$. Differentiating (3.5.19) yields that $\delta P=i[P, B]$, and substituting this identity into (3.5.18) and cyclically commuting the operators inside the trace, we can rewrite the condition (3.5.14) as

$$
0=\operatorname{tr}([P, Q] B) \text {. }
$$

Since $B$ is arbitrary, we obtain the Euler-Lagrange (EL) equations

$$
[P, Q]=0,
$$

stating that two operators in space-time should commute. For more details on the derivation of the EL equations we refer to [F7, Section 3.5] and \$1.4.1.

When analyzing the commutator 3.5 .20 in the continuum limit, the kernel $Q(x, y)$ can be evaluated weakly using the formula (3.5.7). The subtle point is that, according to 3.5 .10$)$, this weak evaluation formula only applies if $x$ and $y$ stay apart. But writing the commutator in 3.5 .20 with integral kernels,

$$
[P, Q](x, y)=\int_{\mathcal{M}}(P(x, z) Q(z, y)-Q(x, z) P(z, y)) d^{4} z,
$$

we also integrate over the regions $z \approx y$ and $z \approx x$ where the kernels $Q(z, y)$ and $Q(x, z)$ are ill-defined. There are several methods to resolve this difficulty, which all give the same end result. The cleanest method is the method of testing on null lines. We now explain the ideas and results of this last method, referring for the rigorous derivation to Appendix A (for other methods of testing see [F7, Appendix F]). The idea is to take the expectation value of the commutator in (3.5.21) for two wave functions $\psi_{1}$ and $\psi_{2}$, one being in the kernel and one in the image of the operator $P$. Thus

$$
P \psi_{1}=0 \quad \text { and } \quad \psi_{2}=P \phi
$$


for a suitable wave function $\phi$. Then, using the symmetry of $P$ with respect to the indefinite inner product $(3.2 .2)$, we find

$$
<\psi_{1}\left|[P, Q] \phi>=<P \psi_{1}\right| Q \phi>-<\psi_{1}\left|Q P \phi>=-<\psi_{1}\right| Q \psi_{2}>\text {. }
$$

Now the commutator has disappeared, and the EL equations 3.5.20 give rise to the condition

$$
0=<\psi_{1} \mid Q \psi_{2}>=\iint_{\mathcal{M} \times \mathcal{M}} Q(x, y) \psi_{1}(x) \psi_{2}(y) d^{4} x d^{4} y .
$$

The hope is that by choosing suitable wave functions $\psi_{1}$ and $\psi_{2}$ of the form (3.5.22) having disjoint supports, we can evaluate the expectation value (3.5.24) weakly on the light cone (3.5.7), thus making sense of the EL equations in the continuum limit.

The key question is to what extent the constraints 3.5 .22 restrict the freedom in choosing the wave functions $\psi_{1}$ and $\psi_{2}$. For clarity, we here explain the situation in the simplified situation where $P$ is composed of one free Dirac sea of mass $m$,

$$
P(x, y)=\int \frac{d^{4} k}{(2 \pi)^{4}}(\not k+m) \delta\left(k^{2}-m^{2}\right) \Theta\left(-k^{0}\right) e^{-i k(x-y)}
$$

(but $Q$ can be a general operator for which the methods of Section 3.5 apply). The generalization to several generations and a $P$ with general interaction is worked out in Appendix A. In order to extract information from (3.5.24) and (3.5.7), it is desirable that the wave functions $\psi_{1}$ and $\psi_{2}$ are as much as possible localized in space-time. For the wave function $\psi_{1}$, this requirement is easy to fulfill by removing a strip of width $\Delta \omega$ around the lower mass shell in momentum space. For example, we can construct a wave function supported near the origin by choosing for a given parameter $\delta>0$ a smooth function $\eta$ supported in the ball of radius $\delta$ in Euclidean $\mathbb{R}^{4}$ and setting

$$
\psi_{1}(x)=\int \frac{d^{4} k}{(2 \pi)^{4}} \hat{\eta}(k) \chi_{\mathbb{R} \backslash[-\Delta \omega, \Delta \omega]}\left(k^{0}+\sqrt{|\vec{k}|^{2}+m^{2}}\right) e^{-i k x},
$$

where $\hat{\eta}$ is the Fourier transform of $\eta$, and $\chi_{I}$ is the characteristic function defined by $\chi_{I}(x)=1$ if $x \in I$ and $\chi_{I}(x)=0$ otherwise. In the limit $\Delta \omega \searrow 0$, the characteristic function in $(3.5 .26)$ becomes the identity, so that $\psi_{1}$ goes over to $\eta$. Moreover, for any $\Delta \omega>0$, the function $\psi_{1}$ is indeed in the kernel of the operator $P$, because it vanishes on the lower mass shell. Thus by choosing $\Delta \omega$ sufficiently small, we can arrange that $\psi_{1}$ is arbitrarily close to $\eta$ and satisfies the condition in 3.5 .22 (indeed, in finite space-time volume one cannot choose $\Delta \omega$ arbitrarily small, leading to small corrections which will be specified in Appendix A, see Remark A.0.4.

The construction of $\psi_{2}$ is a bit more difficult because $\psi_{2}$ must lie in the image of $P$, and thus it must be a negative-energy solution of the Dirac equation $(i \not \partial-m) \psi_{2}=0$. Due to current conservation, it is obviously not possible to choose $\psi_{2}$ to be localized in spacetime; the best we can do is to localize in space by considering a wave packet. According to the Heisenberg Uncertainty Principle, localization in a small spatial region requires large momenta, and thus we are led to considering an ultrarelativistic wave packet of negative energy moving along a null line $\mathfrak{L}$, which does not intersect the ball $B_{\delta}(0) \subset \mathbb{R}^{4}$ where $\psi_{1}$ is localized. By a suitable rotation and/or a Lorentz boost of our reference frame $(t, \vec{x})$, we can arrange that

$$
\mathfrak{L}=\{(\tau,-\tau+\ell, 0,0) \text { with } \tau \in \mathbb{R}\}
$$

with $\ell>0$. For $\psi_{2}$ we take the ansatz

$$
\psi_{2}=(i \not \partial+m)\left(e^{-i \Omega(t+x)} \phi(t+x-\ell, y, z)\right)+(\text { small corrections }),
$$




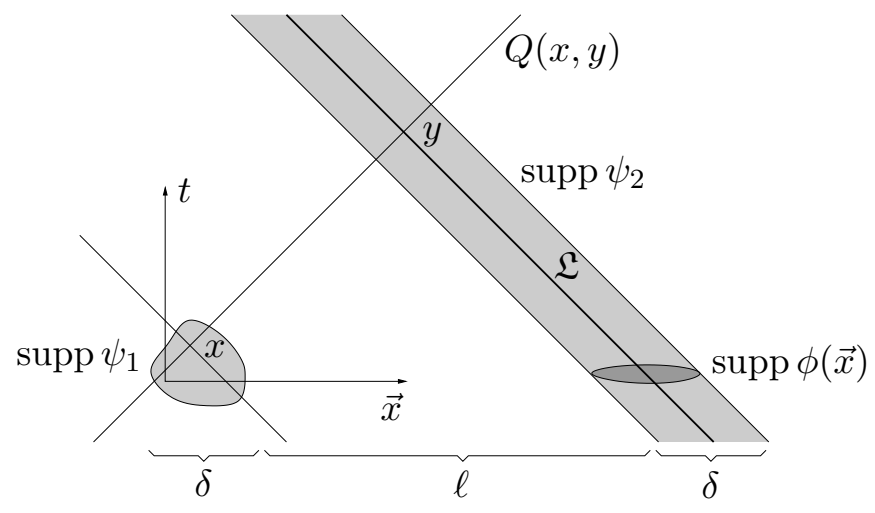

Figure 3.1. Intersection of the null line $\mathfrak{L}$ with the singular set of $Q(x, y)$

where the smooth function $\phi$ is supported in $B_{\delta}(\overrightarrow{0}) \subset \mathbb{R}^{3}$, and the frequency $\Omega<0$ as well as the length scales $\delta$ and $\ell$ are chosen in the range

$$
\varepsilon \ll|\Omega|^{-1} \ll \delta \ll \ell, \ell_{\text {macro }}, m^{-1} .
$$

The small corrections in 3.5.27) are due to the non-zero rest mass, the dispersion and the condition that $\psi_{2}$ must have no contribution of positive energy (for details see Appendix A].

Except for the small corrections to be specified in Appendix A, the support of the wave function $\psi_{1}$ in 3.5 .26 lies in $B_{\delta}(0)$, and thus it is disjoint from the support $B_{\delta}(\mathfrak{L})$ of the wave function $\psi_{2}$ in (3.5.27). Hence the integrals in (3.5.24) only involve the region $x \neq y$ where $Q(x, y)$ is well-defined in the continuum limit. Furthermore, the null line $\mathfrak{L}$ intersects the null cone around $x$ in precisely one point $y$ for which $\left|\xi^{0}\right|=|\vec{\xi}| \sim \ell$ (see Figure 3.1). Since this intersection is transverse, we can evaluate the expectation value (3.5.24) with the help of (3.5.7). In view of the freedom in choosing the parameter $\ell$ and the direction of $\mathfrak{L}$, we conclude that (3.5.7) itself must vanish,

$$
Q(x, y)=0 \quad \text { if evaluated weakly on the light cone . }
$$

The above consideration is made rigorous in Appendix A. More precisely, in Proposition A.0.2, the above arguments are extended to the setting involving several generations and a general interaction, and the scaling of the correction terms in (3.5.27) is specified to every order in perturbation theory. This proposition applies to our action principle 3.2 .9 and all interactions to be considered here, thus justifying (3.5.29) in all cases of interest in this book. Moreover, in Remark A.0.4 we consider the corrections to 3.5.26) which arise if the lifetime of the universe is finite. Using that this lifetime can be estimated by the time from the big bang as known from experiments, we show that the correction to 3.5 .26 ) can indeed be neglected for our universe.

To summarize, we saw that within the formalism of the continuum limit, the commutator in (3.5.20) vanishes only if $Q(x, y)$ itself is zero. This result is the strongest condition we could hope for, because in view of (3.5.18) it implies that arbitrary first variations of the action vanish, even if we disregard the constraint that $P$ must be a projector. We refer to 3.5 .29 ) as the Euler-Lagrange equations in the continuum limit. 
We finally remark that by replacing the null lines by null geodesics, the above method could immediately be generalized to situations involving a gravitational field. However, the estimates of Appendix $\mathrm{A}$ would become more demanding.

\subsection{The Euler-Lagrange Equations to Degree Five}

We proceed with the analysis of the EL equations in the continuum limit 3.5 .29 using the methods outlined in Sections 3.4 and 3.5. For clarity, we begin in the vacuum and then introduce more and more interaction terms. Furthermore, we consider the contributions to the EL equations to decreasing degree on the light cone. In this section, we consider the most singular contributions of degree five. The contributions of degree four will be analyzed in Section 3.7, whereas the contributions to even lower degree are discussed in Section 3.9 .

We point out that many results of this section were already obtained in [F7, Chapters 5 and 6] for more general systems, which however involve only one generation. In order to lay consistent foundations for the new calculations of Sections 3.7 3.9, we here present all calculations in a self-contained way.

3.6.1. The Vacuum. In order to perform the light-cone expansion of the fermionic projector of the vacuum, we first pull the Dirac matrices out of the Fourier integral (3.4.1) and use (3.4.9) to obtain

$$
P^{\operatorname{aux}}(x, y)=\left.\bigoplus_{\beta=1}^{g}\left(i \not \partial_{x}+m_{\beta}\right) T_{a}(x, y)\right|_{a=m_{\beta}^{2}}
$$

After removing the logarithmic mass terms by the replacement $T_{a} \rightarrow T_{a}^{\mathrm{reg}}$, the light-cone expansion reduces to a Taylor expansion in the mass parameter $a$. Restricting attention to the leading degree on the light cone, it suffices to consider the first term of this expansion. Using (3.4.13) and forming the sectorial projection (3.4.3), we obtain for the regularized fermionic projector (for the factors of $g$ see Footnote 3 on page 184 )

$$
P(x, y)=\frac{i g}{2} \notin T_{[0]}^{(-1)}+(\operatorname{deg}<2),
$$

where for notational convenience we omitted the indices ${ }_{[0]}^{-1}$ of the factor $\xi$, and where the bracket $(\operatorname{deg}<2)$ stands for terms of degree at most one.

Using this formula for the fermionic projector, the closed chain 3.2 .5 becomes

$$
A_{x y}=\frac{g^{2}}{4}\left(\not T_{[0]}^{(-1)}\right)\left(\overline{\sharp T_{[0]}^{(-1)}}\right)+\sharp(\operatorname{deg} \leq 3)+(\operatorname{deg}<3),
$$

where $\bar{\phi}:=\overline{\xi_{j}} \gamma^{j}$. Its trace can be computed with the help of the contraction rules (3.5.4),

$$
\operatorname{Tr}\left(A_{x y}\right)=g^{2}\left(\xi_{j} \overline{\xi^{j}}\right) T_{[0]}^{(-1)} \overline{T_{[0]}^{(-1)}}=\frac{g^{2}}{2}(z+\bar{z}) T_{[0]}^{(-1)} \overline{T_{[0]}^{(-1)}}+(\operatorname{deg}<3) .
$$


Next we compute the square of the trace-free part of the closed chain,

$$
\begin{aligned}
& \left(A_{x y}-\frac{1}{4} \operatorname{Tr}\left(A_{x y}\right) \mathbb{1}\right)^{2}=\frac{g^{4}}{16}\left(\Varangle \bar{\ddagger}-\frac{z+\bar{z}}{2}\right)^{2}\left(T_{[0]}^{(-1)} \overline{T_{[0]}^{(-1)}}\right)^{2} \\
& =\frac{g^{4}}{16}\left(\Varangle \bar{\ddagger} \Varangle \bar{\ddagger}-(z+\bar{z}) \Varangle \bar{\ddagger}+\frac{1}{4}(z+\bar{z})^{2}\right)\left(T_{[0]}^{(-1)} \overline{T_{[0]}^{(-1)}}\right)^{2} \\
& =\frac{g^{4}}{64}(z-\bar{z})^{2}\left(T_{[0]}^{(-1)} \overline{T_{[0]}^{(-1)}}\right)^{2} .
\end{aligned}
$$

Combining these formulas, we see that to leading degree, the closed chain is a solution of the polynomial equation

$$
\left(A_{x y}-\frac{g^{2}}{8}(z+\bar{z}) T_{[0]}^{(-1)} \overline{T_{[0]}^{(-1)}}\right)^{2}=\left(\frac{g^{2}}{8}(z-\bar{z}) T_{[0]}^{(-1)} \overline{T_{[0]}^{(-1)}}\right)^{2} .
$$

We point out that the calculations so far are only formal, but they have a well-defined meaning in the formalism of the continuum, because to our end formulas we will be able to apply the weak evaluation formula (3.5.7). Having this in mind, we can interpret the roots of the polynomial in 3.6.4

$$
\lambda_{+}=\frac{g^{2}}{4}\left(z T_{[0]}^{(-1)}\right) \overline{T_{[0]}^{(-1)}} \quad \text { and } \quad \lambda_{-}=\frac{g^{2}}{4} T_{[0]}^{(-1)} \overline{\left(z T_{[0]}^{(-1)}\right)}
$$

as the eigenvalues of the closed chain. Using the contraction rule (3.5.5), these eigenvalues simplify to (see also [F7, eq. (5.3.20)] or 2.6.15)

$$
\lambda_{+}=g^{2} T_{[0]}^{(0)} \overline{T_{[0]}^{(-1)}}+(\operatorname{deg}<3), \quad \lambda_{-}=g^{2} T_{[0]}^{(-1)} \overline{T_{[0]}^{(0)}}+(\operatorname{deg}<3) .
$$

The corresponding spectral projectors, denoted by $F_{ \pm}$, are given by

$$
F_{+}=\frac{A_{x y}-\lambda_{-}}{\lambda_{+}-\lambda_{-}}, \quad F_{+}=\frac{A_{x y}-\lambda_{+}}{\lambda_{-}-\lambda_{+}} ;
$$

a short calculation yields (see also [F7, eq. (5.3.21)] or $(2.6 .16)$ )

$$
F_{ \pm}=\frac{1}{2}\left(\mathbb{1} \pm \frac{[\not,, \bar{\phi}]}{z-\bar{z}}\right)+\Varangle(\operatorname{deg} \leq 0)+(\operatorname{deg}<0) .
$$

Since in the formalism of the continuum limit, the factors $z$ and $\bar{z}$ are treated as two different functions, we do not need to worry about the possibility that the denominator in (3.6.6) might vanish. Similarly, we can treat $\xi$ and $\bar{\xi}$ simply as two different vectors. Then the matrices $F_{+}$and $F_{-}$have rank two, so that the eigenvalues $\lambda_{+}$and $\lambda_{-}$are both two-fold degenerate. A straightforward calculation yields

$$
A_{x y}=\lambda_{+} F_{+}+\lambda_{-} F_{-}+\$(\operatorname{deg} \leq 3)+(\operatorname{deg}<3),
$$

showing that our spectral decomposition is indeed complete. An important general conclusion from (3.6.5) and (3.6.6) is that in the vacuum, the eigenvalues of the closed chain form a complex conjugate pair, and are both two-fold degenerate.

We now give the corresponding operator $Q$ which appears in the EL equations of the continuum limit (3.5.29). 
Proposition 3.6.1. For the fermionic projector of the vacuum (3.6.2), the operator $Q$ as defined by (3.5.17) and (3.5.16) takes the form

$$
Q(x, y)=i \nsubseteq g^{3}(1-4 \mu) T_{[0]}^{(0)} T_{[0]}^{(-1)} \overline{T_{[0]}^{(-1)}}+(\operatorname{deg}<5) .
$$

In order not to distract from the main points, we first discuss the consequences of this result and derive it afterwards. According to the EL equations in the continuum limit (3.5.29), the expression (3.6.8) must vanish. This determines the value of the Lagrange multiplier $\mu=\frac{1}{4}$. Thus the action 3.5.13) reduces to the action in (3.2.8), and we conclude that

$$
P \text { is a critical point of } \mathcal{S},
$$

disregarding the constraint $\mathcal{T}=$ const. This result can be understood immediately from the form of the Lagrangian (3.2.7) and the fact that the eigenvalues of $A_{x y}$ form a complex conjugate pair. Namely, writing the spectral weights in (3.2.7) via (3.2.6) as sums over the eigenvalues $\lambda_{ \pm}^{x y}$ (both of multiplicity two), we obtain

$$
\mathcal{L}_{x y}[P]=\left(\left|\lambda_{+}\right|-\left|\lambda_{-}\right|\right)^{2} .
$$

The expression $\left|\lambda_{+}\right|-\left|\lambda_{-}\right|$clearly vanishes for a complex conjugate pair, and the fact that it appears quadratically is the reason why even first variations of $\mathcal{L}_{x y}[P]$ vanish, explaining (3.6.9).

In the last argument we only used that the eigenvalues of $A_{x y}$ form a complex conjugate pair. Therefore, we can use this argument to show that $Q$ vanishes in a more general sense: First, a straightforward calculation yields that the eigenvalues of the closed chain $A_{x y}$ form a complex conjugate pair to every degree on the light cone (for details see [F7, Section 5.3] or Section 2.6), and thus $Q$ vanishes identically in the formalism of the continuum limit. Moreover, going beyond the formalism of the continuum limit, in F11] it is shown that there are regularizations of the vacuum for which the operator $Q$ vanishes up to contributions which stay finite in the limit $\varepsilon \searrow 0$. Furthermore, in [F11] it is shown that restricting attention to such regularizations does not give any constraints for the regularization parameters $c_{\mathrm{reg}}$ in (3.5.7). Since we are here interested in the singularities of $Q(x, y)$ in the limit $\varepsilon \searrow 0$ as described by the weak evaluation formula (3.5.7), we can in what follows assume that in the vacuum, the operator $Q$ vanishes identically.

The remainder of this section is devoted to deriving the result of Proposition 3.6.1. For the derivation it is preferable to bypass the computation of the gradient $(3.5 .16)$ by determining $Q$ directly from (3.5.18). For later use, we assume a more general spectral decomposition of $A_{x y}$ with eigenvectors $\lambda_{1}^{x y}, \ldots, \lambda_{4}^{x y}$ and corresponding one-dimensional spectral projectors $F_{1}^{x y}, \ldots, F_{4}^{x y}$. This setting can be obtained from (3.6.7) by choosing pseudo-orthonormal bases in the degenerate eigenspaces and letting $F_{k}^{x \bar{y}}$ be the projectors onto the span of these basis vectors. It is convenient to choose these bases according to Lemma 2.6.3.

For later use, we next compute the operator $Q$ in the general setting of the previous lemma. Noting that the function $\mathcal{L}_{\mu}$ in 3.5 .13 depends only on the absolute values of the eigenvalues, we can write

$$
\mathcal{L}_{\mu}\left[A_{x y}\right]=\mathcal{L}_{\mu}\left(\left|\lambda_{1}^{x y}\right|, \ldots,\left|\lambda_{4}^{x y}\right|\right) .
$$

The partial derivatives of the function $\mathcal{L}_{\mu}\left(\left|\lambda_{1}^{x y}\right|, \ldots,\left|\lambda_{4}^{x y}\right|\right)$ will be denoted by $D_{k}$. 
Lemma 3.6.2. Under the assumptions of Lemma 2.6.3, the operator $Q$ in (3.5.18) is given by

$$
Q(x, y)=\sum_{k=1}^{4} D_{k} \mathcal{L}_{\mu}\left(\left|\lambda_{1}^{x y}\right|, \ldots,\left|\lambda_{4}^{x y}\right|\right) \frac{\overline{\lambda_{k}^{x y}}}{\left|\lambda_{k}^{x y}\right|} F_{k}^{x y} P(x, y)
$$

Proof. The relation 2.6.39 allows us to compute the variation of the eigenvalues by a standard first order perturbation calculation without degeneracies,

$$
\delta \lambda_{k}^{x y}=\operatorname{Tr}\left(F_{k}^{x y} \delta A_{x y}\right) .
$$

Using that that $\delta|\lambda|=\operatorname{Re}(\bar{\lambda} \delta \lambda /|\lambda|)$, we can compute the first variation of this function with the help of (3.6.11),

$$
\delta \mathcal{L}_{\mu}\left[A_{x y}\right]=\operatorname{Re} \sum_{k=1}^{4} D_{k} \mathcal{L}_{\mu}\left(\left|\lambda_{1}^{x y}\right|, \ldots,\left|\lambda_{4}^{x y}\right|\right) \frac{\overline{\lambda_{k}^{x y}}}{\left|\lambda_{k}^{x y}\right|} \operatorname{Tr}\left(F_{k}^{x y} \delta A_{x y}\right) .
$$

In the last trace we substitute the identity

$$
\delta A_{x y}=\delta P(x, y) P(y, x)+P(x, y) \delta P(y, x)
$$

and cyclically commute the arguments to obtain

$$
\begin{aligned}
\operatorname{Tr}\left(F_{k}^{x y} \delta A_{x y}\right) & =\operatorname{Tr}\left(F_{k}^{x y} P(x, y) \delta P(y, x)+P(y, x) F_{k}^{x y} \delta P(x, y)\right) \\
& =\operatorname{Tr}\left(F_{k}^{x y} P(x, y) \delta P(y, x)+F_{k}^{y x} P(y, x) \delta P(x, y)\right),
\end{aligned}
$$

where in the last step we applied (2.6.40). Substituting this formula into (3.6.12) and integrating over $x$ and $y$, we can exchange the names of $x$ and $y$ such that only $\delta P(y, x)$ appears. We thus obtain

$$
\delta \mathcal{S}_{\mu}[P]=2 \operatorname{Re} \iint_{M} d^{4} x d^{4} y \operatorname{Tr}(Q(x, y) \delta P(y, x))
$$

with the integral kernel $Q(x, y)$ given by $(3.6 .10)$. Using Lemma 2.6 .3 , one sees that the operator corresponding to this integral kernel is symmetric (i.e. $Q(x, y)^{*}=Q(y, x)$ ). As a consequence, the integral in $(3.6 .13)$ is real, so that it is unnecessary to take the real part. Comparing with (3.5.18), we conclude that the operator with kernel (3.6.10) indeed coincides with the operator $Q$ in (3.5.18). We note that due to the sum in (3.6.10), it is irrelevant how the bases were chosen on the degenerate subspaces of $A_{x y}$.

Proof of Proposition 3.6.1. Let us specialize the general formula 3.6 .10 to our spectral representation with eigenvalues (3.6.5) and spectral projectors (3.6.6). First, from $(3.5 .13)$ we readily obtain that

$$
D_{k} \mathcal{L}_{\mu}\left(\left|\lambda_{1}^{x y}\right|, \ldots,\left|\lambda_{4}^{x y}\right|\right)=2\left|\lambda_{k}\right|-2 \mu \sum_{l=1}^{4}\left|\lambda_{l}\right|=2(1-4 \mu)\left|\lambda_{-}\right| .
$$

The product $F_{k}^{x y} P(x, y)$ can be computed with the help of 3.6 .2 and 3.6 .6 as well as the relations

$$
[\not, \bar{\phi}] \not=2\langle\bar{\xi}, \xi\rangle \notin-2 \xi^{2} \bar{\xi}=-(z-\bar{z}) \notin,
$$

where in the last step we treated the factors $\notin$ and $\bar{\phi}$ as outer factors and applied the contraction rules (3.5.3) and (3.5.4). We thus obtain (see also [F7, eq. (5.3.23)] or (2.6.17))

$$
F_{+}^{x y} P(x, y)=(\operatorname{deg}<2), \quad F_{-}^{x y} P(x, y)=\frac{i g}{2} \sharp T_{[0]}^{(-1)}+(\operatorname{deg}<2) .
$$

Substituting these formulas into (3.6.10) and using (3.6.5), the result follows. 
3.6.2. Chiral Gauge Potentials. We now begin the study of interacting systems by introducing chiral potentials. Thus we choose the operator $\mathcal{B}$ in the auxiliary Dirac equation with interaction (3.4.4) according to (3.4.16) with two real vector fields $A_{L}$ and $A_{R}$. Sometimes it is convenient to write $\mathcal{B}$ in the form

$$
\mathcal{B}=A_{\mathrm{v}}+\Gamma A_{\mathrm{a}}
$$

with a vector potential $A_{\mathrm{v}}$ and an axial potential $A_{\mathrm{a}}$ defined by

$$
A_{\mathrm{v}}=\left(A_{L}+A_{R}\right) / 2 \quad \text { and } \quad A_{\mathrm{a}}=\left(A_{L}-A_{R}\right) / 2 .
$$

To the considered highest degree on the light cone, the chiral gauge potentials merely describe phase transformations of the left- and right-handed components of the fermionic projector (for details see [F6], [F7, Section 2.5] or Section 2.2). More precisely, the fermionic projector is obtained from 3.6 .2 by inserting the phase factors

$$
P(x, y)=\frac{i g}{2}\left(\chi_{L} e^{-i \Lambda_{L}^{x y}}+\chi_{R} e^{-i \Lambda_{R}^{x y}}\right) \notin T_{[0]}^{(-1)}+(\operatorname{deg}<2),
$$

where the functions $\Lambda_{L / R}^{x y}$ are integrals of the chiral potentials along the line segment $\overline{x y}$,

$$
\Lambda_{L / R}^{x y}=\int_{x}^{y} A_{L / R}^{j} \xi_{j}:=\left.\int_{0}^{1} A_{L / R}^{j}\right|_{\tau y+(1-\tau) x} \xi_{j} d \tau .
$$

Consequently, the closed chain is obtained from (3.6.3) by inserting phase factors,

$$
\left.A_{x y}=\frac{g^{2}}{4}\left(\chi_{L} \nu_{L}+\chi_{R} \nu_{R}\right)\left(\sharp T_{[0]}^{(-1)}\right) \overline{\left(\$ T_{[0]}^{(-1)}\right.}\right)+\notin(\operatorname{deg} \leq 3)+(\operatorname{deg}<3),
$$

where

$$
\nu_{L}=\overline{\nu_{R}}=e^{-i\left(\Lambda_{L}^{x y}-\Lambda_{R}^{x y}\right)}=\exp \left(-2 i \int_{x}^{y} A_{\mathrm{a}}^{j} \xi_{j}\right) .
$$

From 3.6.19) one sees that the matrix $A_{x y}$ is invariant on the left- and right-handed subspaces (i.e. on the image of the operators $\chi_{L}$ and $\chi_{R}$ ). On each of these invariant subspaces, it coincides up to a phase with the closed chain of the vacuum (3.6.3). Using these facts, the eigenvalues $\left(\lambda_{s}^{c}\right)_{c \in\{L, R\}, s \in\{+,-\}}$ and corresponding spectral projectors $F_{s}^{c}$ are immediately computed by

$$
\lambda_{ \pm}^{L / R}=\nu_{L / R} \lambda_{ \pm} \quad \text { and } \quad F_{ \pm}^{L / R}=\chi_{L / R} F_{ \pm}
$$

with $\lambda_{s}$ and $F_{s}$ as in 3.6 .5 and $(3.6 .6)$. We conclude that the eigenvalues of the closed chain are again complex, but in general they now form two complex conjugate pairs. Since the eigenvalues $\lambda_{c}^{L}$ and $\lambda_{c}^{R}$ differ only by a phase, we see that all eigenvalues have the same absolute value,

$$
\left|\lambda_{+}^{L}\right|=\left|\lambda_{+}^{R}\right|=\left|\lambda_{-}^{L}\right|=\left|\lambda_{-}^{R}\right| .
$$

Writing the Lagrangian (3.2.7) as

$$
\mathcal{L}_{x y}[P]=\sum_{c, s}\left|\lambda_{s}^{c}\right|^{2}-\frac{1}{4}\left(\sum_{c, s}\left|\lambda_{s}^{c}\right|\right)^{2}=\frac{1}{8} \sum_{c, c^{\prime} \in\{L, R\}} \sum_{s, s^{\prime} \in\{ \pm\}}\left(\left|\lambda_{s}^{c}\right|-\left|\lambda_{s^{\prime}}^{c^{\prime}}\right|\right)^{2}
$$

(where we sum over $c \in\{L, R\}$ and $s \in\{ \pm\}$ ), we find that $\mathcal{L}$ vanishes identically. Since the Lagrangian is quadratic in $\left|\lambda_{s}^{c}\right|-\left|\lambda_{s^{\prime}}^{c^{\prime}}\right|$, also first variations of $\mathcal{L}$ vanish, suggesting that the operator $Q(x, y)$ should again vanish identically. This is indeed the case, as is verified immediately by applying Lemmas 2.6.3 and 3.6.2. We conclude that for chiral potentials, the EL equations in the continuum limit (3.5.29) are satisfied to degree five on the light cone. 
We end this section by explaining how the line integrals in $(3.6 .18)$ and the phase factors in (3.6.17) and (3.6.19) can be understood from an underlying local gauge symmetry (for more details in the general context of non-abelian gauge fields see [F7, Section 6.1]). The local phase transformation $\psi(x) \rightarrow e^{i \Lambda(x)} \psi(x)$ with a real function $\Lambda$ describes a unitary transformation of the wave functions (with respect to the inner product (3.2.2)). Transforming all objects unitarily, we obtain the transformation laws

$$
\begin{aligned}
i \not \partial+\mathcal{B}-m Y & \rightarrow e^{i \Lambda(x)}(i \not \partial+\mathcal{B}-m Y) e^{-i \Lambda(x)}=i \not \partial+\mathcal{B}-m Y+(\not \partial \Lambda) \\
P(x, y) & \rightarrow e^{i \Lambda(x)} P(x, y) e^{-i \Lambda(y)} \\
A_{x y} & \rightarrow e^{i \Lambda(x)} P(x, y) e^{-i \Lambda(y)} e^{i \Lambda(y)} P(y, x) e^{-i \Lambda(x)}=A_{x y} .
\end{aligned}
$$

The transformation of the Dirac operator corresponds to a transformation of the vector and axial potentials by

$$
A_{\mathrm{v}} \rightarrow A_{\mathrm{v}}+\partial \Lambda \quad \text { and } \quad A_{\mathrm{a}} \rightarrow A_{\mathrm{a}} .
$$

These are the familiar gauge transformations of electrodynamics. Using the formula

$$
\Lambda(y)-\Lambda(x)=\left.\int_{0}^{1} \frac{d}{d \tau} \Lambda\right|_{\tau y+(1-\tau) x} d \tau=\int_{x}^{y}\left(\partial_{j} \Lambda\right) \xi^{j} d \tau,
$$

the phases in 3.6 .25 can be described similar to (3.6.18) in terms of line integrals. This explains why the phase factors in (3.6.17) describe the correct behavior under gauge transformations. According to $(3.6 .26)$, the closed chain $A_{x y}$ is gauge invariant. This is consistent with the fact that in (3.6.19) and (3.6.20) only the axial potential enters, which according to (3.6.27) is also gauge invariant.

In order to transform the axial potential, one can consider the local transformation $\psi(x) \rightarrow e^{-i \Gamma \Lambda(x)} \psi(x)$. In contrast to the above gauge transformation, this transformation is not unitary (with respect to the inner product (3.2.2)), and the requirement that the Dirac operator and the fermionic projector must be symmetric operators leads us to the transformations

$$
\begin{aligned}
i \not \partial+\mathcal{B}-m Y & \rightarrow e^{i \Gamma \Lambda(x)}(i \not \partial+\mathcal{B}-m Y) e^{i \Gamma \Lambda(x)} \\
& =i \not \partial+e^{i \Gamma \Lambda(x)}(\mathcal{B}-m Y) e^{i \Gamma \Lambda(x)}+\Gamma(\not \partial \Lambda) \\
P(x, y) & \rightarrow e^{-i \Gamma \Lambda(x)} P(x, y) e^{-i \Gamma \Lambda(x)}
\end{aligned}
$$

Thus the vector and axial potentials transform as desired by

$$
A_{\mathrm{v}} \rightarrow A_{\mathrm{v}} \quad \text { and } \quad A_{\mathrm{a}} \rightarrow A_{\mathrm{a}}+\partial \Lambda
$$

(and also the term $m Y$ is modified, but this is of no relevance for the argument here). The point is that when we now consider the transformation of the closed chain,

$$
A_{x y} \rightarrow e^{i \Gamma \Lambda(x)} P(x, y) e^{i \Gamma \Lambda(y)} e^{i \Gamma \Lambda(y)} P(y, x) e^{i \Gamma \Lambda(x)},
$$

the local transformations do not drop out. This explains why in 3.6 .19$)$ phases involving the axial potentials appear.

For clarity, we point out that the field tensors and the currents of the chiral gauge potentials also affect the fermionic projector, in a way which cannot be understood from the simple gauge transformation laws considered above. The corresponding contributions to the operator $Q$ will be of degree four, and we shall consider them in the next section. 


\subsection{The Euler-Lagrange Equations to Degree Four}

We come to the analysis of the EL equations to the next lower degree four on the light cone. In preparation, we bring the EL equations into a convenient form.

Lemma 3.7.1. To degree four, the EL equations in the continuum limit 3.5.29) are equivalent to the equation

$$
\mathcal{R}:=\frac{\Delta\left(\left|\lambda_{-}^{L}\right|-\left|\lambda_{-}^{R}\right|\right)}{\left|\lambda_{-}\right|} g^{3} T_{[0]}^{(0)} T_{[0]}^{(-1)} \overline{T_{[0]}^{(-1)}}=0+(\operatorname{deg}<4),
$$

where $\Delta$ denotes the perturbation of the eigenvalues (3.6.21) to degree two.

Proof. According to (3.6.21), the eigenvalues to degree three are all non-real. Since this property is stable under perturbations of lower degree, we can again apply Lemmas 2.6.3 and 3.6.2. Noting that before (3.6.9), we fixed the Lagrange multiplier to $\mu=\frac{1}{4}$, we consider the Lagrangian (3.2.7), which we now write in analogy to (3.6.23) as

$$
\mathcal{L}_{x y}[P]=\frac{1}{8} \sum_{k, l=1}^{4}\left(\left|\lambda_{k}^{x y}\right|-\left|\lambda_{l}^{x y}\right|\right)^{2} .
$$

Then the relation 3.6 .10 can be written as

$$
Q(x, y)=\frac{1}{2} \sum_{k, l=1}^{4}\left\{\left|\lambda_{k}^{x y}\right|-\left|\lambda_{l}^{x y}\right|\right\} \frac{\overline{\lambda_{k}^{x y}}}{\left|\lambda_{k}^{x y}\right|} F_{k}^{x y} P(x, y) .
$$

According to (3.6.22), the curly brackets vanish for the unperturbed eigenvalues. This has the convenient consequence that to degree four, it suffices to take into account the perturbation of the curly brackets, whereas everywhere else we may work with the unperturbed spectral decomposition (3.6.21),

$$
Q(x, y)=\frac{1}{2} \sum_{k, l=1}^{4} \Delta\left(\left|\lambda_{k}^{x y}\right|-\left|\lambda_{l}^{x y}\right|\right) \frac{\overline{\lambda_{k}^{x y}}}{\left|\lambda_{k}^{x y}\right|} F_{k}^{x y} P(x, y)+(\operatorname{deg}<4) .
$$

Using (3.6.14), we see that we only get a contribution if $\lambda_{k}$ equals $\lambda_{-}^{L}$ or $\lambda_{-}^{R}$. Furthermore, we can apply (2.6.38), numbering the eigenvalues such that $\overline{\lambda_{L}^{ \pm}}=\lambda_{R}^{\mp}$. We thus obtain

$$
Q(x, y)=\sum_{c \in\{L, R\}} \Delta\left(\left|\lambda_{-}^{c}\right|-\left|\lambda_{+}^{c}\right|\right) \frac{\overline{\lambda_{-}^{c}}}{\left|\lambda_{-}^{c}\right|} \chi_{c} \frac{i \not}{2} g T_{[0]}^{(-1)}+(\operatorname{deg}<4) .
$$

The EL equations 3.5 .29 imply that the left- and right-handed components of this expression must vanish separately. Thus, again applying (2.6.38), we obtain the sufficient and necessary condition

$$
\Delta\left(\left|\lambda_{-}^{L}\right|-\left|\lambda_{-}^{R}\right|\right) \frac{\overline{\lambda_{-}}}{\left|\lambda_{-}\right|} g T_{[0]}^{(-1)}+(\operatorname{deg}<4)=0 .
$$

The explicit formulas $(3.6 .21)$ and $(3.6 .5$ yield the result.

It is important to observe that the EL equations only involve the difference of the absolute values of the left- and right-handed eigenvalues. This can immediately be understood as follows. To the leading degree three, the eigenvalues of $A_{x y}$ form two complex conjugate pairs (see (3.6.21)). Since this property is preserved under perturbations, we can again write the Lagrangian in the form (3.6.23). Hence the Lagrangian vanishes 
identically unless the absolute values of the eigenvalues are different for the two pairs. This explains the term $\Delta\left(\left|\lambda_{-}^{L}\right|-\left|\lambda_{-}^{R}\right|\right)$ in (3.7.1).

As explained on page 193 , the expression $\Delta\left(\left|\lambda_{-}^{L}\right|-\left|\lambda_{-}^{R}\right|\right)$ vanishes in the vacuum. Furthermore, the phase factors in (3.6.21) drop out of this expression. But new types of contributions to the interacting fermionic projector come into play, as we now explain.

3.7.1. The Axial Current Terms and the Mass Terms. An interaction by chiral potentials $(3.4 .16)$ as introduced in $\$ 3.6 .2$ affects the fermionic projector in a rather complicated way. For clarity, we treat the different terms in succession, beginning with the contributions near the origin $\xi=0$ (the contributions away from the origin will be considered in Section 3.10). For the Taylor expansion around $\xi=0$ we note that when evaluated weakly on the light cone (3.5.7), a simple fraction of degree $L$ has a pole $|\vec{\xi}|^{-L}$. This leads us to say that a term of the form (3.5.6) is of the order $k$ at the origin if the smooth function $\eta$ vanishes at the origin to the order $k+L$.

DEFinition 3.7.2. An expression of the form (3.5.6) is said to be of order $o\left(|\vec{\xi}|^{k}\right)$ at the origin if the function $\eta$ is in the class $o\left(\left(\left|\xi^{0}\right|+|\vec{\xi}|\right)^{k+L}\right)$.

In the next lemma we specify the contributions to the EL equations to degree four on the light cone, to leading order at the origin.

Lemma 3.7.3. For an interaction described by vector and axial potentials (3.4.16), the expression $\mathcal{R}$ as defined by (3.7.1) takes the form

$$
\mathcal{R}=-i \xi_{k}\left(j_{\mathrm{a}}^{k} N_{1}-m^{2} A_{\mathrm{a}}^{k} N_{2}\right)+(\operatorname{deg}<4)+o\left(|\vec{\xi}|^{-3}\right),
$$

where $j_{\mathrm{a}}$ is the axial current

$$
j_{\mathrm{a}}^{k}=\partial_{j}^{k} A_{\mathrm{a}}^{j}-\square A_{\mathrm{a}}^{k},
$$

and $N_{1}, N_{2}$ are the simple fractions

$$
\begin{aligned}
& N_{1}=\frac{g^{3}}{6 \overline{T_{[0]}^{(0)}}}\left[\left(T_{[0]}^{(0)} T_{[0]}^{(0)}-2 T_{[0]}^{(1)} T_{[0]}^{(-1)}\right) \overline{T_{[0]}^{(0)} T_{[0]}^{(-1)}}-c . c .\right] \\
& N_{2}=-\frac{2}{\overline{T_{[0]}^{(0)}}}\left[\left(g \hat{Y}^{2} T_{[0]}^{(-1)} T_{[0]}^{(0)} \overline{T_{[1]}^{(0)} T_{[1]}^{(0)}}+g^{2} \dot{Y} \grave{Y} T_{[0]}^{(-1)} T_{[2]}^{(1)} \overline{T_{[0]}^{(-1)} T_{[0]}^{(0)}}\right)-c . c .\right] .
\end{aligned}
$$

Here "c.c." denotes the complex conjugate of the preceding simple fraction; the accents were defined in (3.5.2).

In order not to distract from the main ideas, we postpone the proof of this lemma to Appendix $B$ and proceed right away with the physical discussion. From the mathematical point of view, the appearance of the axial current $j_{\mathrm{a}}$ is not surprising, because the lightcone expansion of the fermionic projector involves derivatives of the potentials. In physical terms, this shows that the axial potential affects the fermionic projector not only via the phases in (3.6.17), but also via the axial current. The term $-i \xi_{k} j_{a}^{k} N_{1}$ is referred to as the current term. The other term $-i \xi_{k} m^{2} A_{a}^{k} N_{2}$ could not appear in ordinary Yang-Mills theories because it would not be gauge invariant. However, as pointed out after (3.6.28), the axial $U(1)$-transformations do not correspond to a local gauge symmetry, because they are not unitary. Instead, they describe relative phase transformations of the leftand right-handed components of the fermionic projector, thereby changing the physics of the system. Only the phase transformations (3.6.26) correspond to a local gauge 
symmetry, and in view of (3.6.27), the term $-i \xi_{k} m^{2} A_{a}^{k} N_{2}$ is indeed consistent with this local $U(1)$-symmetry.

Since the direction $\xi$ can be chosen arbitrarily on the light cone, the condition (3.7.1) implies that the bracket in (3.7.3) must vanish,

$$
j_{\mathrm{a}}^{k} N_{1}-m^{2} A_{\mathrm{a}}^{k} N_{2}=0 .
$$

If $N_{1}$ and $N_{2}$ could be treated as constants, this equation would go over to field equations for the axial potential $A_{\mathrm{a}}$ with rest mass $m^{2} N_{2} / N_{1}$. For this reason, we refer to the term $-i \xi_{k} m^{2} A_{a}^{k} N_{2}$ in (3.7.3) as the mass term. It is remarkable that in our framework, the bosonic mass term appears naturally, without the need for the Higgs mechanism of spontaneous symmetry breaking (for a detailed discussion of this point see s3.8.5). We also point out that the simple fraction $N_{2}$ involves the mass matrix $Y$, and thus the mass term in (3.7.7) depends on the masses of the fermions of the system.

In order to make the argument after 3.7.7 precise, we need to analyze the simple fractions $N_{1}$ and $N_{2}$ weakly on the light cone. Before this will be carried out in 3.7 .3 , we specify how the Dirac current enters the EL equations.

3.7.2. The Dirac Current Terms. As explained in $\$ 3.4 .3$, the particles and antiparticles of the system enter the auxiliary fermionic projector via (3.4.7), where we orthonormalize the wave functions according to (3.4.8). Introducing the left- and righthanded component of the Dirac current by

$$
J_{L / R}^{i}=\sum_{k=1}^{n_{\mathrm{p}}} \overline{\psi_{k}} \chi_{R / L} \gamma^{i} \psi_{k}-\sum_{l=1}^{n_{\mathrm{a}}} \overline{\phi_{l}} \chi_{R / L} \gamma^{i} \phi_{l},
$$

a decomposition similar to (3.6.16) leads us to define the axial Dirac current by

$$
J_{\mathrm{a}}^{i}=\sum_{k=1}^{n_{\mathrm{p}}} \overline{\psi_{k}} \Gamma \gamma^{i} \psi_{k}-\sum_{l=1}^{n_{\mathrm{a}}} \overline{\phi_{l}} \Gamma \gamma^{i} \phi_{l} .
$$

The next lemma gives the corresponding contribution to the EL equations, to leading order at the origin.

Lemma 3.7.4. Introducing the axial Dirac current by the particle and anti-particle wave functions in (3.4.7) leads to a contribution to $\mathcal{R}$ of the form

$$
\mathcal{R} \asymp i \xi_{k} J_{\mathrm{a}}^{k} N_{3}+(\operatorname{deg}<4)+o\left(|\vec{\xi}|^{-3}\right),
$$

where

$$
N_{3}=\frac{g^{2}}{8 \pi} \frac{1}{\overline{T_{[0]}^{(0)}}}\left[T_{[0]}^{(-1)} \overline{T_{[0]}^{(0)} T_{[0]}^{(-1)}}-\text { c.c. }\right] .
$$

Here the symbol " $\asymp$ " means that we merely give the contribution to $\mathcal{R}$ by the Dirac current, but do not repeat the earlier contributions given in Lemma 3.7.3. The proof of this lemma is again postponed to Appendix B.

3.7.3. The Logarithmic Poles on the Light Cone. Combining the results of Lemmas 3.7.1, 3.7.3 and 3.7.4, the Euler-Lagrange equations give rise to the equation

$$
\xi_{k}\left(j_{\mathrm{a}}^{k} N_{1}-m^{2} A_{\mathrm{a}}^{k} N_{2}-J_{a}^{k} N_{3}\right)=0,
$$

which involves the axial potential $A_{\mathrm{a}}$ (see $(3.6 .15)$ ), the corresponding axial bosonic current (3.7.4) and the axial Dirac current (3.7.8). At first sight, this equation resembles a 
bosonic field equation, which describes the coupling of the Dirac spinors to the bosonic field and involves a bosonic mass term. However, the situation is not quite so simple, because the factors $N_{1}, N_{2}$ and $N_{3}$ (see (3.7.5), (3.7.6) and (3.7.9p) have a mathematical meaning only when evaluated weakly on the light cone (3.5.7). Let us analyze the weak evaluation in more detail. The simple fraction $N_{3}$ is composed of the functions $T_{[0]}^{(0)}$, $T_{[0]}^{(-1)}$ and their complex conjugates, which according to 3.4.10 - 3.4.13 all have poles of the order $\xi^{-2}$ or $\xi^{-4}$. In particular, no logarithmic poles appear, and thus we may apply (3.5.7) with $r=0$ to obtain

$$
\int_{|\vec{\xi}|-\varepsilon}^{|\vec{\xi}|+\varepsilon} d t \eta \xi_{k} J_{a}^{k} N_{3}=\frac{c_{3}^{\text {reg }}}{\varepsilon^{3}|\vec{\xi}|^{4}} \eta(x) \xi_{k} J_{a}^{k}(x)+(\operatorname{deg}<4)+o\left(|\vec{\xi}|^{-3}\right)
$$

with a regularization parameter $c_{3}^{\text {reg }}$, where we omitted error terms of the form 3.5 .9 ) and 3.5.10. The simple fractions $N_{1}$ and $N_{2}$, on the other hand, involve in addition the functions $T_{0}^{(1)}$ and $\overline{T_{0}^{(1)}}$, which according to $3.4 .10-(3.4 .12)$ involve a factor $\log \left|\xi^{2}\right|$ and thus have a logarithmic pole on the light cone. As a consequence, in (3.5.7) we also obtain contributions with $r=1$,

$$
\begin{aligned}
& \int_{|\vec{\xi}|-\varepsilon}^{|\vec{\xi}|+\varepsilon} d t \eta \xi_{k}\left(j_{\mathrm{a}}^{k} N_{1}-m^{2} A^{k} N_{2}\right)=(\operatorname{deg}<4)+o\left(|\vec{\xi}|^{-3}\right) \\
& +\frac{1}{\varepsilon^{3}|\vec{\xi}|^{4}} \eta(x) \xi_{k}\left[j_{\mathrm{a}}^{k}\left(c_{1}^{\mathrm{reg}}+d_{1}^{\mathrm{reg}} \log (\varepsilon|\vec{\xi}|)\right)-m^{2} A_{\mathrm{a}}^{k}\left(c_{2}^{\mathrm{reg}}+d_{2}^{\mathrm{reg}} \log (\varepsilon|\vec{\xi}|)\right)\right],
\end{aligned}
$$

involving four regularization parameters $c_{1 / 2}^{\mathrm{reg}}$ and $d_{1 / 2}^{\mathrm{reg}}$. Combining the above weak evaluation formulas, the freedom in choosing the radius $|\vec{\xi}|$ and the spatial direction $\vec{\xi} /|\vec{\xi}|$ implies that the logarithmic and non-logarithmic terms must vanish separately,

$$
\begin{aligned}
& j_{\mathrm{a}}^{k} d_{1}^{\mathrm{reg}}-m^{2} A_{\mathrm{a}}^{k} d_{2}^{\mathrm{reg}}=0 \\
& j_{\mathrm{a}}^{k} c_{1}^{\text {reg }}-m^{2} A_{\mathrm{a}}^{k} c_{2}^{\text {reg }}=J_{a}^{k} c_{3}^{\text {reg }},
\end{aligned}
$$

where $c_{1 / 2}^{\text {reg }}$ and $d_{1 / 2}^{\text {reg }}$ are constants depending on the particular regularization.

Unfortunately, the system of equations (3.7.10) into (3.7.11) is overdetermined. Thus turns out to be too restrictive for physical applications, as we now explain. We begin with the case of a generic regularization for which the constants $c_{1}^{\text {reg }}, c_{3}^{\text {reg }}$ and $d_{1}^{\text {reg }}$ are nonzero. Thus solving (3.7.10) for $j_{\mathrm{a}}$ and substituting into (3.7.11), one obtains an algebraic equation involving $J_{\mathrm{a}}$ and $A_{\mathrm{a}}$. This means that either $J_{\mathrm{a}}$ must vanish identically, or else the gauge potential $A_{\mathrm{a}}$ is fixed to a constant times $J_{\mathrm{a}}$ and thus cannot be dynamical. Both cases are not interesting from a physical point of view. The basic reason for this shortcoming is that the bosonic current and mass terms have logarithmic poles on the light cone, whereas the Dirac current terms involve no such logarithms. Our method for overcoming this problem is to insert additional potentials into the Dirac equation, with the aim of compensating the logarithmic poles of the bosonic current and mass terms. Before entering these constructions in $\$ 3.7 .4$, we now briefly discuss alternative methods for treating the logarithmic poles.

An obvious idea for reducing the system 3.7 .10 and 3.7 .11 to a single equation is to restrict attention to non-generic regularizations where the constants $c_{i}^{\text {reg }}$ and/or $d_{i}^{\text {reg }}$ take special values. In particular, it seems tempting to demand that $d_{1}^{\text {reg }}=d_{2}^{\text {reg }}=0$, so that (3.7.10) is trivially satisfied, leaving us with the field equations (3.7.11). This 
method does not work, as the following consideration shows. Differentiating (3.4.11) and using (3.4.10), one sees that

$$
32 \pi^{3} T_{\circ}^{(1)}=\log \left|\xi^{2}\right|+c_{0}+i \pi \Theta\left(\xi^{2}\right) \epsilon\left(\xi^{0}\right) .
$$

Evaluating near the upper light cone $\xi^{0} \approx|\vec{\xi}|$, we can apply the relation $\log \left|\xi^{2}\right|=$ $\log \left|\xi^{0}+\right| \vec{\xi}||+\log \left|\xi^{0}-\right| \vec{\xi}||$ to obtain

$$
32 \pi^{3} T_{\circ}^{(1)}=\log |2 \vec{\xi}|+\log \left|\xi^{0}-\right| \vec{\xi}||+i \pi \Theta\left(\xi^{0}-|\vec{\xi}|\right)+c_{0}+\mathcal{O}\left(\xi^{0}-|\vec{\xi}|\right) .
$$

When evaluating the corresponding simple fraction weakly (3.5.7), the first term in 3.7.13) gives rise to the $\log |\vec{\xi}|$-dependence, the second term gives the $\log \varepsilon$-dependence, whereas all the other terms do not involve logarithms or are of higher order in $\varepsilon$. Obviously, the same is true for the complex conjugate $\overline{T_{0}^{(1)}}$. Since in $(3.5 .7)$ the vector $\vec{\xi}$ is fixed, the vanishing of the $\log |\vec{\xi}|$-dependent contribution to the integral (3.5.7) implies that the simple fraction still vanishes if the factors $T_{\circ}^{(1)}$ and $\overline{T_{0}^{(1)}}$ are replaced by constants. Inspecting the $T^{(1)}$-dependence of (3.7.5) and (3.7.6) and comparing with (3.7.9), we find that

$$
d_{1}^{\text {reg }}=0 \Longleftrightarrow d_{2}^{\text {reg }}=0 \quad \text { and } \quad d_{1}^{\text {reg }}=0 \Longrightarrow c_{3}^{\text {reg }}=0 \text {. }
$$

Thus if the constants $d_{1}^{\text {reg }}$ and $d_{2}^{\text {reg }}$ in $(3.7 .10)$ vanish, then 3.7 .10$)$ becomes trivial as desired. But then the constant $c_{3}^{\text {reg }}$ in (3.7.11) is also zero, so that the Dirac current drops out of the field equation. Again, we do not end up with physically reasonable equations.

Sticking to the idea of considering regularizations where the regularization constants have special values, the remaining method is to assume that all regularization constants in (3.7.10) and (3.7.11) vanish. Then the EL equations would be trivially satisfied to degree four on the light cone, and one would have to proceed to the analysis to degree three on the light cone. This method does not seem to be promising for the following reasons. First, it is not clear whether there exist regularizations for which all the regularization constants in 3.7.10 and 3.7.11 vanish. In any case, it seems difficult to satisfy all these conditions, and the resulting regularizations would have to be of a very special form. This would not be fully convincing, because one might prefer not to restrict the class of admissible regularizations at this point. Secondly, there is no reason to believe that the situation to degree three would be better, at least not without imposing additional relations between regularization constants, giving rise to even more constraints for the admissible regularizations.

We conclude that assuming special values for the regularization constants in (3.7.10) and (3.7.11) does not seem to be a promising strategy. Thus in what follows we shall not impose any constraints on the regularization constants, which also has the advantage that our constructions will apply to any regularization. Then the only possible strategy is to try to compensate the logarithmic poles by a suitable transformation of the fermionic projector.

3.7.4. A Pseudoscalar Differential Potential. Compensating the logarithmic poles of the bosonic current and mass terms by a suitable transformation of the fermionic projector is not an easy task, because it is not at all obvious how such a transformation should look like. We approach the problem in several steps, following the original path which eventually led us to the microlocal transformation to be introduced in $\$ 3.7 .10$. The most obvious method is to inserting additional potentials into the auxiliary Dirac equation (3.4.4) and to analyze the effect on the fermionic projector. In order to get 
contributions of comparable structure, these potentials should involve a vector field $v$, which should be equal either to the axial potential $A_{\mathrm{a}}$ or to the corresponding axial current $j_{\mathrm{a}}$ (see Lemma 3.7.3). Since contracting the vector index of $v$ with the Dirac matrices would again give rise to chiral potentials (3.4.16), we now prefer to contract $v$ with partial derivatives. Moreover, since we want to compensate contributions which are odd under parity transformations (i.e. which change signs if we flip the left- and righthanded components), the resulting operator must involve the pseudoscalar matrix $\Gamma$. The requirement that the Dirac operator should be symmetric with respect to the inner product 3.2.2 leads us to the ansatz involving an anti-commutator

$$
\mathcal{B}=\Gamma\left\{v^{j}, \partial_{j}\right\}=2 \Gamma v^{j} \partial_{j}+\Gamma\left(\partial_{j} v^{j}\right) .
$$

We refer to this ansatz as a pseudoscalar differential potential. Our ansatz seems unusual because such differential potentials do not occur in the standard model nor in general relativity. However, as explained in 3.4 .5 , we are free to modify the Dirac equation arbitrarily.

The corresponding leading contribution to the fermionic projector is of the form (for details see equation (B.2.22) in Appendix B

$$
\begin{aligned}
P(x, y) \asymp & \frac{g}{2} \Gamma \xi_{i}\left(v^{i}(y)+v^{i}(x)\right) T^{(-1)} \\
& +\frac{g}{2} \Gamma \xi_{i} \int_{x}^{y}\left[\not \not,\left(\not \partial v^{i}\right)\right] T^{(-1)}+(\operatorname{deg}<2) .
\end{aligned}
$$

This contribution has a pole of order $\xi^{-4}$ on the light cone and is therefore much more singular than the desired logarithmic pole. A straightforward calculation shows that (3.7.15) does contribute to the expression $\mathcal{R}$ in Lemma 3.7.1, and thus we conclude that 3.7 .15 is not suitable for compensating the logarithmic pole.

The key for making use of the pseudoscalar differential potential (3.7.14) is to observe that the required logarithmic poles do appear to higher order in a mass expansion. More precisely, to leading order at the origin, the cubic contribution to the fermionic projector is

$$
P(x, y) \asymp \frac{m^{3}}{4} \Gamma\left[v_{j}^{(3)}(x)+\mathcal{O}\left(\left|\xi^{0}\right|+|\vec{\xi}|\right)\right]\left(\not \xi^{j} T^{(0)}-2 \gamma^{j} T^{(1)}\right)+(\operatorname{deg}<-1),
$$

where $v^{(3)}$ is a Hermitian matrix composed of $v$ and $Y$,

$$
v^{(3)}=i(v Y Y Y-Y v Y Y+Y Y v Y-Y Y Y v)
$$

(for details see equation $(\mathrm{B} .2 .23)$ in Appendix B . Thus there is hope that the logarithmic poles can be compensated, provided that we can arrange that the contributions by (3.7.14) to $\mathcal{R}$ of order $m^{0}, m$ and $m^{2}$ in a mass expansion vanish. The last requirement cannot be met if we consider one Dirac sea, because the term (3.7.15) does contribute to $\mathcal{R}$. But if we consider several Dirac seas, we have more freedom, as the pseudoscalar differential potential (3.7.14) can be chosen differently for each Dirac sea. For example, we can multiply the potentials acting on the different Dirac seas by real constants $\mathfrak{g}_{\alpha}$,

$$
(\mathcal{B})_{\beta}^{\alpha}=\mathfrak{g}_{\alpha} \delta_{\alpha \beta} \Gamma\left\{v^{j}, \partial_{j}\right\} \quad \text { with } \quad \alpha, \beta=1, \ldots, g .
$$

Using this additional freedom, it is indeed possible to arrange that the contribution (3.7.15) drops out of $\mathcal{R}$. This consideration explains why we must consider several generations of elementary particles.

The critical reader might object that there might be other choices of the operator $\mathcal{B}$ which could make it possible to compensate the logarithmic poles without the need for 
several generations. However, the following consideration shows that (3.7.14) is indeed the only useful ansatz, provided that we work with local operators (for nonlocal operators see 3.7 .10 and Section 3.10). The only zero order operator are the chiral potentials $(3.4 .16)$, which were already considered in \$3.6.2. Apart from (3.7.14), the only first order differential operator involving the vector field $v$ and the pseudoscalar matrix $\Gamma$ is the operator

$$
\Gamma\left\{v_{j} \sigma^{j k}, \partial_{k}\right\},
$$

where $\sigma^{j k}=\frac{i}{2}\left[\gamma^{j}, \gamma^{k}\right]$ are the bilinear covariants. This ansatz can be shown to be useless, basically because the calculations in the continuum limit give rise to contractions with the vector $\xi$, which vanish (see also \$3.9.2). Differential operators of higher order must involve the wave operator $\square$, which applied to the Dirac wave functions gives rise to lower order operators. This shows that it is not useful to consider differential operators of order higher than one. We conclude that (3.7.14) and its generalizations to several generations (like (3.7.18) ) are indeed the only possible ansätze for compensating the logarithmic poles.

We end the discussion by having a closer look at the matrix $v^{(3)},(3.7 .17)$. Note that the ansatz (3.7.18) is diagonal in the generation index and thus commutes with the mass matrix $Y$. As a consequence, the matrix $v^{(3)}$ vanishes. This means that for compensating the logarithmic poles, the ansatz (3.7.18) is not sufficient, but we must allow for non-zero off-diagonal elements in the generation index. Thus we replace the factors $b_{\alpha}$ in (3.7.18) by a Hermitian matrix $\mathfrak{g}=\left(\mathfrak{g}_{\beta}^{\alpha}\right)_{\alpha, \beta=1, \ldots, g}$, the so-called generation mixing matrix. Later on, the generation mixing matrix will depend on the space-time point $x$. This leads us to generalize 3.7 .18 by the ansatz

$$
(\mathcal{B})_{\beta}^{\alpha}=\Gamma\left\{\mathfrak{g}_{\beta}^{\alpha}(x) v^{j}(x), \partial_{j}\right\},
$$

thus allowing that the pseudoscalar differential potential mixes the generations.

3.7.5. A Vector Differential Potential. Modifying the auxiliary Dirac equation (3.4.4) by a first oder operator 3.7.14) or 3.7.19) changes the behavior of its solutions drastically. In particular, it is not clear whether the operator $\mathcal{B}$ can be treated perturbatively (3.4.6). In order to analyze and resolve this problem, we begin by discussing the case when the potential $v$ in (3.7.14) is a constant vector field, for simplicity for one Dirac sea of mass $m$. Then taking the Fourier transform, the Dirac equation reduces to the algebraic equation

$$
\left(\not k-2 i \Gamma v^{j} k_{j}-m\right) \hat{\psi}(k)=0 .
$$

Multiplying from the left by the matrix $\left(\not k-2 i \Gamma v^{j} k_{j}+m\right)$, we find that the momentum of a plane-wave solution must satisfy the dispersion relation

$$
k^{2}-4\left(v^{j} k_{j}\right)^{2}-m^{2}=0 .
$$

Rewriting this equation as

$$
g^{i j} k_{i} k_{j}-m^{2}=0 \quad \text { with } \quad g^{i j}:=\eta^{i j}-4 v^{i} v^{j},
$$

where $\eta^{i j}=\operatorname{diag}(1,-1,-1,-1)$ is again the Minkowski metric, we see that the new dispersion relation is the same as that for the Klein-Gordon equation in a space-time with Lorentzian metric $g^{i j}$. In particular, the characteristics of the Dirac equation become the null directions of the metric $g^{i j}$. In other words, the light cone is "deformed" to that of the new metric $g^{i j}$.

This deformation of the light cone leads to a serious problem when we want to compensate the logarithmic poles, as we now discuss. Suppose that we introduce a pseudoscalar 
differential potential which according to 3.7 .18$)$ or 3.7 .19$)$ depends on the generation index. In the case (3.7.18), the Dirac seas feel different dispersion relations. In particular, the singularities of the fermionic projector $P(x, y)$ will no longer be supported on one light cone, but will be distributed on the union of the light cones corresponding to the Lorentzian metrics $g_{\alpha}^{i j}=\eta^{i j}-4 v_{\alpha}^{i} v_{\alpha}^{j}$. The ansatz (3.7.19) leads to a similar effect of a "dissociation of the light cone." In the EL equations, this would lead to large additional contributions, which are highly singular on the light cone and can certainly not compensate the logarithmic poles.

Our method for bypassing this problem is to introduce another differential potential which transforms the dispersion relation back to that of the Klein-Gordon equation in Minkowski space. In the case of a constant vector field $v$ and one generation, this can be achieved by choosing matrices $G^{j}$ which satisfy the anti-commutation relations

$$
\left\{G^{i}, G^{j}\right\}=2 \eta^{i j}+8 v^{i} v^{j} \quad \text { and } \quad\left\{\Gamma, G^{i}(x)\right\}=0,
$$

and by modifying 3.7 .20 to

$$
\left(G^{j} k_{j}-2 i \Gamma v^{j} k_{j}-m\right) \hat{\psi}(k)=0 .
$$

This modification of the Dirac matrices can be interpreted as introducing a constant gravitational potential corresponding to the metric $\eta^{i j}+4 v_{\alpha}^{i} v_{\alpha}^{j}$. This construction is extended to the general case (3.7.19) as follows. We choose $(4 g \times 4 g)$-matrices $G^{j}(x)$ which are symmetric with respect to the inner product $\bar{\psi} \phi$ on the Dirac spinors and satisfy the anti-commutation relations

$$
\left\{G^{i}(x), G^{j}(x)\right\}=2 \eta^{i j}+8 \mathfrak{g}(x)^{2} v^{i}(x) v^{j}(x) \quad \text { and } \quad\left\{\Gamma, G^{i}(x)\right\}=0 .
$$

In the auxiliary Dirac equation (3.4.4) we insert the additional operator

$$
\mathcal{B}=i\left(G^{j}(x)-\gamma^{j}\right) \partial_{j}+G^{j}(x) E_{j}(x),
$$

where the matrices $E_{j}$ involve the spin connection coefficients and are not of importance here (for details see for example [F7, Section 1.5]). We refer to $(3.7 .22$ as a vector differential potential. In the case (3.7.18), this construction can be understood as introducing for each Dirac sea a gravitational potential corresponding to the metric $\eta^{i j}+4 \mathfrak{g}_{\alpha}^{2} v_{\alpha}^{i} v_{\alpha}^{j}$, whereas in case (3.7.19), the interpretation is bit more complicated due to the off-diagonal terms.

3.7.6. Recovering the Differential Potentials by a Local Axial Transformation. By introducing the differential potentials $(3.7 .19)$ and $(3.7 .22)$ with $G^{j}$ according to 3.7.21), we inserted differential operators into the auxiliary Dirac equation (3.4.4). We will now show that the effect of these operators on the solutions of the auxiliary Dirac equation can be described by a local transformation

$$
\psi_{\text {aux }}(x) \rightarrow U(x) \psi_{\text {aux }}(x),
$$

which is unitary with respect to the inner product (3.4.5).

Recall that we introduced the vector differential potential 3.7 .22 with the goal of transforming the dispersion relation back to the form in the vacuum. Thus if $v$ is a constant vector field, the combination $(3.7 .19)+(3.7 .22)$ leaves the momenta of planewave solutions unchanged. This suggests that the sum $(3.7 .19)+(3.7 .22)$ might merely describe a unitary transformation of the Dirac wave functions. Thus we hope that there might be a unitary matrix $U(x)$ such that

$$
U(i \not \partial-m Y) U^{-1}=i \not \partial+3.7 .14+3.7 .22 \text {. }
$$


Let us verify that there really is such a unitary transformation. The natural ansatz for $U$ is an exponential of an axial matrix involving the vector field $v$ and the generation mixing matrix,

$$
U(x)=\exp \left(-i \mathfrak{g}(x) \Gamma \gamma^{j} v_{j}(x)\right) .
$$

Writing out the exponential series and using that $\left(\Gamma \gamma^{j} v_{j}\right)^{2}=-v^{2}$, we obtain

$$
U(x)=\cos (\mathfrak{g} \varphi) \mathbb{1}-i \frac{\sin (\mathfrak{g} \varphi)}{\varphi} \Gamma \psi, \quad U(x)^{-1}=\cos (\mathfrak{g} \varphi) \mathbb{1}+i \frac{\sin (\mathfrak{g} \varphi)}{\varphi} \Gamma \psi,
$$

where the angle $\varphi:=\sqrt{-v^{2}}$ is real or imaginary (note that $(3.7 .25)$ is well-defined even in the limit $\varphi \rightarrow 0)$. A short calculation yields

$$
U \gamma^{j}-\gamma^{j} U=-i \frac{\sin (\mathfrak{g} \varphi)}{\varphi}\left[\Gamma \psi, \gamma^{j}\right]=-2 i \Gamma v^{j} \frac{\sin (\mathfrak{g} \varphi)}{\varphi}
$$

and thus

$$
\begin{aligned}
& U(i \not \partial-m Y) U^{-1}=i U \gamma^{j} U^{-1} \partial_{j}+U \gamma^{j}\left(i \partial_{j} U^{-1}\right)-m U Y U^{-1} \\
& \quad=i \not \partial+2 \Gamma v^{j} \frac{\sin (\mathfrak{g} \varphi)}{\varphi} U^{-1} \partial_{j}+U \gamma^{j}\left(i \partial_{j} U^{-1}\right)-m U Y U^{-1} \\
& \quad=i \not \partial+\Gamma \frac{\sin (2 \mathfrak{g} \varphi)}{\varphi} v^{j} \partial_{j}+2 i \frac{\sin ^{2}(\mathfrak{g} \varphi)}{\varphi^{2}} \psi v^{j} \partial_{j}+U \gamma^{j}\left(i \partial_{j} U^{-1}\right)-m U Y U^{-1} .
\end{aligned}
$$

In order to verify that the resulting Dirac operator allows us to recover both 3.7 .19 and 3.7 .22 , we assume that $v^{2}$ is so small that $\sin (2 \mathfrak{g} \varphi) \approx 2 \mathfrak{g} \varphi$ and $\sin ^{2}(\mathfrak{g} \varphi) \approx \mathfrak{g}^{2} \varphi^{2}$. Then the second summand in 3.7 .26 reduces precisely to the differential operator in the relation (3.7.19). The third summand in (3.7.26) gives precisely the differential operator in (3.7.22), noting that (3.7.21) has the solution $G^{j}=\gamma^{j}+2 \mathfrak{g}^{2} \psi v^{j}+\mathcal{O}\left(v^{4}\right)$. Likewise, a direct calculation shows that the multiplication operators in $(3.7 .19)$ and $(3.7 .22)$ are contained in the fourth summand in (3.7.26). Writing out the fourth and fifth summands in (3.7.26), one finds a rather complicated combination of additional chiral, scalar, pseudoscalar and even bilinear potentials. These additional potentials do not cause any problems; on the contrary, they guarantee that the total transformation of the Dirac wave functions simply is the local transformation (3.7.23). We conclude that with (3.7.26) we have found a Dirac operator which includes the differential potentials in (3.7.19) and $(3.7 .22$. It has the nice property that it can easily be treated non-perturbatively by the simple local transformation (3.7.23). We refer to the transformation $(3.7 .23)$ with $U$ according to (3.7.24) as the local axial transformation.

The local axial transformation was analyzed in detail in a previous version of the present work (see arXiv:0908.1542v3 [math-ph]). There are two reasons why the local axial transformation will no longer be used here. First, the unitarity of (3.7.23) turns out not to be essential, as it can be dropped in the more general construction given in \$3.7.7 below. Second and more importantly, compensating the logarithmic poles of the current and and mass terms by a local axial transformation leads to additional contributions to the fermionic projector of higher order in the local transformation (for details see Appendix C in arXiv:0908.1542v3 [math-ph]). It turns out that the gauge phases which appear in these additional contributions (which were not considered in arXiv:0908.1542v3 [math-ph]) enter the EL equations in a way which makes it impossible to satisfy these equations. This problem was first observed when working out the follow-up paper on systems involving neutrinos (see Chapter 4). The method for overcoming this problem 
also led to major revisions of the present paper. The basic problem will be explained in $\$ 3.7 .8$ and resolved in 3.7 .9 .

Before entering the generalizations and discussing the shortcomings of the local axial transformation, we now briefly review how the local axial transformation can be used to compensate the logarithmic poles of the current and mass terms. For simplicity, we only consider a perturbation expansion to first order in $v$. Then the transformation (3.7.24) simplifies to

$$
U(x)=\mathbb{1}-i \mathfrak{g} \Gamma \psi(x)+\mathcal{O}\left(v^{2}\right) .
$$

Transforming the auxiliary fermionic projector (3.4.1) by $U$ and forming the sectorial projection (3.4.3), we obtain for the perturbation of the fermionic projector the expression

$$
P \asymp-i \Gamma \psi \dot{\mathfrak{g}} \grave{P}+i \dot{P} \grave{\mathfrak{g}} \Gamma \psi+\mathcal{O}\left(v^{2}\right),
$$

where we denoted the sectorial projection similar to 3.5 .2 by accents. Here we always sum over one index of the generation mixing matrix. Thus it is convenient to introduce real functions $c_{\alpha}$ and $d_{\alpha}$ by

$$
\sum_{\alpha=1}^{g} \mathfrak{g}_{\beta}^{\alpha}=c_{\beta}+i d_{\beta} \quad \text { and } \quad \sum_{\beta=1}^{g} \mathfrak{g}_{\beta}^{\alpha}=c_{\alpha}-i d_{\alpha},
$$

where the last equation is verified by taking the adjoint of the first and using that $\mathfrak{g}$ is Hermitian. Combining these equations with the fact that the auxiliary fermionic projector of the vacuum is diagonal on the generations, we can write (3.7.28) as

$$
P \asymp \sum_{\beta=1}^{g}\left(-i\left[c_{\beta} \Gamma \psi, P_{\beta}\right]+\left\{d_{\beta} \Gamma \psi, P_{\beta}\right\}\right)+\mathcal{O}\left(v^{2}\right),
$$

where the $P_{\beta}$ stand for the direct summands in (3.4.1). The next lemma shows that the functions $c_{\beta}$ drop out of the EL equations; the proof is again given in Appendix $B$.

LEMMA 3.7.5. The perturbation of the fermionic projector by the functions $c_{\beta}$ in (3.7.30) does not contribute linearly to the EL equations.

This leaves us with the $g$ real coefficients $d_{\beta}$. In order to study their effect, we expand $P_{\beta}$ in 3.7 .30 in powers of the mass. The zeroth order in the mass expansion vanishes in view of the identity

$$
\sum_{\beta=1}^{g} d_{\beta}=0
$$

(which follows immediately from (3.7.29) by summing over the free generation index). The first order in the mass expansion yields instead of a logarithmic pole a stronger singularity $\sim \xi^{-2}$ and contributes to the EL equations even to degree five on the light cone. In order for these contributions to vanish, we need to impose that

$$
\sum_{\beta=1}^{g} m_{\beta} d_{\beta}=0 \text {. }
$$

The remaining contributions to EL equations are indeed of degree four. They involve logarithmic poles, making it possible to compensate the corresponding poles of the current and mass terms.

It is worth noting that this procedure only works if the number of generations equals three. Namely, in the case $g<3$, the equations (3.7.31) and 3.7.32 only have the trivial 
solution $d_{\beta} \equiv 0$. In the case $g=3$, the conditions 3.7 .31$)$ and 3.7 .32 leave one free constant, which is uniquely fixed by compensating the logarithmic pole. In the case $g>3$, however, there are free constants even after compensating the logarithmic pole, so that the resulting field equations are underdetermined.

In order to avoid confusion, we finally point out that it is crucial that the local axial transformation is performed before taking the sectorial projection. Namely, if we performed a similar transformation after taking the sectorial projection,

$$
P(x, y) \rightarrow V(x) P(x, y) V(y)^{*} \quad \text { with } \quad V(x)=e^{-i \Gamma \not B(x)}
$$

with a real vector field $V$, then $V(x)$ would be a unitary transformation on the spinors. Such a transformation can be regarded as a local gauge transformation (see [F7, Sections 1.5 and 3.1]). Since our action is gauge invariant (see [F7, Section 3.5] or [F10]), it would have no effect on our physical system. By performing a gauge transformation (3.7.33) with $B=-\hat{g} v$, we can always arrange that the matrix $\mathfrak{g}$ in the local axial transformation $(3.7 .24)$ has the property

$$
\hat{g}(x)=0 \quad \text { for all } x .
$$

3.7.7. General Local Transformations. We now generalize the local axial transformation (3.7.23) and bring it in connection to the causal perturbation expansion of \$3.4.2 Thus suppose that $U(x)$ is a linear transformation of the spinors at every space-time point. For simplicity, we assume that $U(x)$ is invertible and depends smoothly on $x$, but it need not be a symmetric operator. We then transform the Dirac operator with interaction locally by $U(x)^{-1}$,

$$
i \not \partial+\mathcal{B}-m Y \longrightarrow\left(U(x)^{-1}\right)^{*}\left(i \not \partial_{x}+\mathcal{B}-m Y\right) U^{-1}(x) .
$$

Writing the transformed Dirac operator in the form $i \not \partial+\mathcal{B}-m Y$ with a new perturbation operator $\mathcal{B}$, we can again introduce the corresponding fermionic projector as outlined in Section 3.4 with the causal perturbation expansion followed by the light-cone expansion and a resummation of the perturbation expansion.

Introducing the perturbation operator by the local transformation 3.7 .35 has the advantage that the effect on the fermionic projector can be described explicitly. In preparation, we consider the advanced and retarded Green's functions $s^{\vee}$ and $s^{\wedge}$, which are characterized by the equations

$$
(i \not \partial+\mathcal{B}-m Y) s^{\wedge}(x, y)=\delta^{4}(x-y)=(i \not \partial+\mathcal{B}-m Y) s^{\vee}(x, y)
$$

and the fact that they are supported in the upper and lower light cone, respectively (for details see [F7, Section 2.2], [F6] or Section 2.1]. A direct calculation shows that under the transformation (3.7.35), the Green's functions simply transform locally by

$$
s^{\vee / \wedge}(x, y) \longrightarrow U(x) s^{\vee / \wedge}(x, y) U(y)^{-1} .
$$

The transformation of the auxiliary fermionic projector is more involved, because one has nonlocal contributions and must satisfy normalization conditions (for details see [FG1, FT2 and [F6]). However, the residual argument (see [F6, Section 3.1], [FG1, Section 6] or Section 2.2 shows that the poles of the auxiliary fermionic projector have the same structure as those of the causal Green's functions, i.e.

$$
\tilde{P}^{\text {aux }}(x, y)=U(x) \tilde{P}_{\mathcal{B}}^{\text {aux }}(x, y) U(y)^{*}+(\text { smooth contributions }),
$$

where $\tilde{P}_{\mathcal{B}}^{\text {aux }}$ denotes the auxiliary fermionic projector in the presence of the external field $\mathcal{B}$. Thus as long as we are concerned with the poles of the fermionic projector, we can 
work with the simple local transformation 3.7.36) of the auxiliary fermionic projector. The fermionic projector is again obtained by forming the sectorial projection (3.4.3). Again using the notation (3.5.2), we thus obtain

$$
\tilde{P}(x, y)=\dot{U}^{\prime}(x)(x, y) \tilde{P}_{\mathcal{B}}^{\text {aux }}(x, y) \grave{U}(y)^{*}+(\text { smooth contributions }) .
$$

This construction shows that the unitarity condition for $U(x)$ used in (3.7.23) is not needed. Moreover, since the construction is based on the causal perturbation expansion for the new perturbation $\mathcal{B}$ (which also incorporates the local transformation), we do not need to worry about the normalization of the fermionic states. In other words, the auxiliary fermionic projector defined by (3.7.36) is idempotent (as is made precise in [FT2 ). The prize we pay is that the additional smooth contributions in (3.7.37) are not known explicitly. But they could be computed with the resummation technique developed in Appendix D.

3.7.8. The Shear Contributions by the Local Axial Transformation. In order to explain the problem of the local axial transformation (3.7.27), we need to analyze its effect on the fermionic projector to higher order in the vector field $v$. The nonlinear dependence has two reasons: First, $U$ depends nonlinearly on $v$ (see (3.7.24) and second, the transformation (3.7.37) involves two factors of $U$. For clarity, we want to begin with the second effect. To this end, we simplify $U$ to a transformation which is linear in $v$,

$$
U(x)=\mathbb{1}+i E(x) \quad \text { with } \quad E(x)=-\mathfrak{g} \Gamma \psi(x) .
$$

Note that, in contrast to (3.7.27), we no longer have an error term. As a consequence, the transformation $U(x)$ is no longer unitary. Nevertheless, the general construction in $\$ 3.7 .7$ applies (see (3.7.35)-(3.7.37)). Clearly, the linear ansatz (3.7.38) leaves us with the freedom to perform additional local transformations which are of higher order in $v$; these will be considered in Appendix C.

Using (3.7.34), the effect of the local axial transformation (3.7.37), (3.7.38) on the vacuum fermionic projector (3.4.1) is described by the transformation

$$
P(x, y) \rightarrow P(x, y)+\Delta P(x, y)
$$

where $\Delta P$ is computed by

$$
\begin{aligned}
\Delta P(x, y)= & i m\left(\dot{E}(x) \grave{Y}-\dot{Y} \grave{E}(y)^{*}\right) T_{[1]}^{(0)} \\
& +\frac{i}{2} \dot{E}(x) \not ̈ \grave{E}(y)^{*} T_{[0]}^{(-1)} \\
& +m \dot{E}(x) Y \grave{E}(y)^{*} T_{[1]}^{(0)}+\notin(\operatorname{deg}<2)+(\operatorname{deg}<1) .
\end{aligned}
$$

The contribution 3.7 .39 was already considered in $\$ 3.7 .6$, it vanishes in view of 3.7 .32 . The logarithmic poles on the light cone are contained in the error terms in (3.7.41). To higher order in $v$, we get the additional contributions (3.7.40) and (3.7.41), which need to be taken into account. 
Let us analyze the effect of the most singular contribution 3.7 .40 in more detail. Using (3.7.38), we obtain

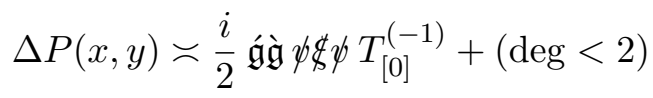

$$
\begin{aligned}
& \Delta A_{x y} \asymp \Delta P(x, y) P(y, x)+P(x, y) \Delta P(y, x)+\mathcal{O}\left(v^{4}\right) \\
& \stackrel{3.6 .2}{-} \frac{g}{4} \mathfrak{g} \mathfrak{\mathfrak { g }}(\psi \notin \psi \psi \bar{\ddagger}+\sharp \psi \bar{\ddagger} \psi) T_{[0]}^{(-1)} \overline{T_{[0]}^{(-1)}}+\mathcal{O}\left(v^{4}\right) \\
& =g \mathfrak{g} \mathfrak{\mathfrak { g }}\langle v, \xi\rangle^{2} T_{[0]}^{(-1)} \overline{T_{[0]}^{(-1)}}+\mathcal{O}\left(v^{4}\right)+(\operatorname{deg}<4) \\
& \Delta \lambda_{ \pm} \asymp g \mathfrak{g} \mathfrak{g}\langle v, \xi\rangle^{2} T_{[0]}^{(-1)} \overline{T_{[0]}^{(-1)}}+\mathcal{O}\left(v^{4}\right)+(\operatorname{deg}<4) .
\end{aligned}
$$

Thus we get a contribution to the closed chain which is of degree four on the light cone and is thus more singular than the closed chain of the vacuum (see (3.6.3)). We call this contribution the shear contribution of the local axial transformation (the connection to the parameter $\varepsilon_{\text {shear }}$ in (3.3.7) will become clear in $\$ 3.7 .9$ below). The resulting contribution to the eigenvalues is also of degree four on the light cone. This by itself is not a problem because the contribution to $A_{x y},(3.7 .43$, is a real multiple of the identity matrix. Thus it changes both eigenvalues by the same real amount (see (3.7.44)). As a consequence, the eigenvalues $\lambda_{ \pm}$remain complex conjugate pairs $(3.6 .22)$, so that the Lagrangian is still zero.

However, the situation becomes more involved when the gauge phases are taken into account. Namely, according to (3.6.17) and (3.6.18), we need to multiply the fermionic projector of the vacuum by the phase factor $\left(\chi_{L} e^{-i \Lambda_{L}^{x y}}+\chi_{R} e^{-i \Lambda_{R}^{x y}}\right)$. As the local axial transformation is odd and thus flips the chirality, 3.7.42 becomes

$$
\Delta P(x, y) \asymp \frac{i}{2} \mathfrak{g} \mathfrak{g}\left(\chi_{L} e^{-i \Lambda_{R}^{x y}}+\chi_{R} e^{-i \Lambda_{L}^{x y}}\right)(\psi \Varangle \psi) T_{[0]}^{(-1)} .
$$

In view of (3.6.17), the phases cancel in the closed chain (3.7.43). We conclude that the contribution to the eigenvalues (3.7.44) does not involve any gauge phases, i.e.

$$
\Delta \lambda_{ \pm}^{L / R} \asymp \kappa \quad \text { with } \quad \kappa:=g \mathfrak{g} \mathfrak{g}\langle v, \xi\rangle^{2} T_{[0]}^{(-1)} \overline{T_{[0]}^{(-1)}}+\mathcal{O}\left(v^{4}\right)+(\operatorname{deg}<4) .
$$

Combining this result with (3.6.21), we obtain

$$
\lambda_{ \pm}^{L / R}=\nu_{L / R} \lambda_{ \pm}+\kappa
$$

As a consequence, the eigenvalues $\lambda_{ \pm}^{L / R}$ will in general no longer all have the same absolute value (see Figure 3.2). Thus the Lagrangian (3.6.23) does not vanish, and the EL equations are no longer satisfied to degree five on the light cone.

As a possible method to bypass this problem, one could hope to compensate the contribution (3.7.40) by other local transformations of the form (3.7.36). For example, one could work with a local transformation involving a bilinear potential $B_{i j}$ with

$$
v_{i} v_{j}=B_{i k} B_{j}^{k} \text {. }
$$

Another potential method is to work with the contributions to 3.7 .25 of higher order in $v$ (which were disregarded in (3.7.38)). It turns out that all these methods necessarily fail. We now merely explain the underlying reason and refer for the detailed analysis to Appendix C. The basic problem of the local axial transformation is that it generates a shear contribution to the closed chain (see (3.7.43)). The appearance of such contributions can be understood as follows. The vector $\xi=y-x$ is null on the light cone. As a 


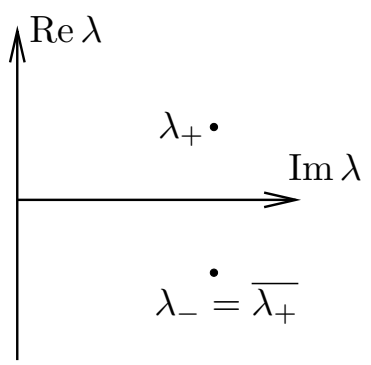

(a)



(b)

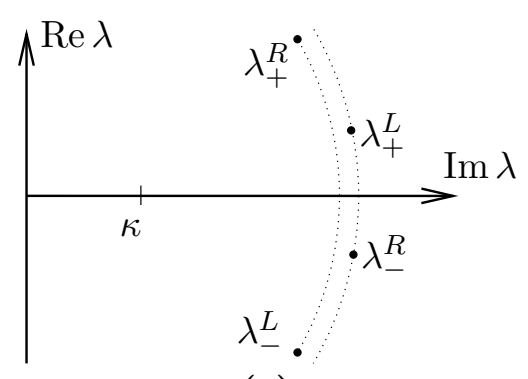

(c)

Figure 3.2. The eigenvalues of $A_{x y}$ to degree five on the light cone in the vacuum (a), in the presence of chiral gauge fields (b), and after a local axial transformation $(\mathbf{c})$

consequence, contracting the vectors $\xi$ and $\bar{\xi}$ gives factors $z$ and $\bar{z}$, which vanish on the light cone (see the calculation (3.6.3)-(3.6.5)). If, as a consequence of the local axial transformation, the vector $\xi$ is no longer null on the light cone, then the inner product $\langle\xi, \bar{\xi}\rangle$ no longer vanishes on the light cone. This gives rise to contributions to the closed chain which are more singular on the light cone. These are precisely the shear contributions. Thus in order to prevent shear contributions, one must make sure that the factors $\xi$ remain lightlike on the light-cone.

A lightlike vector $\xi$ has the special property that the bilinear form $\bar{\psi} \not \phi$ is positive semi-definite, but degenerate (i.e. it is not positive definite). Indeed, these two properties encode the fact that $\xi^{2}=0$. A local transformation $\sharp \rightarrow V^{*} \notin V$ preserves the definiteness of the corresponding bilinear form, because $\bar{\psi}\left(V^{*} \& V\right) \phi=\overline{V \psi} \notin(V \phi) \geq 0$. If $V$ is invertible, the inner product also remains degenerate. However, the transformation $\grave{U}^{*}: \mathbb{C}^{4} \rightarrow \mathbb{C}^{4 g}$ in 3.7 .37 is certainly not invertible. As a consequence, the bilinear form $\bar{\psi} \dot{U}^{\prime} \grave{U}^{*} \phi$ will in general be positive definite, giving rise to the undesirable shear contributions. The condition that the bilinear form $\bar{\psi} \dot{U} \dot{s}^{*} \phi$ be degenerate gives rise to strong constraints for the local transformation. As worked out in Appendix C, these constraints are not compatible with local axial transformations.

3.7.9. Homogeneous Transformations in the High-Frequency Limit. In order to resolve the problems caused by the shear contributions, we now reconsider the local axial transformation in the special case of a constant transformation. We again write $U$ in the form (3.7.38). Transforming the vacuum fermionic projector (3.4.1) and disregarding the smooth contributions in (3.7.37), we obtain

$$
\tilde{P}=\dot{U} P^{\text {aux }} \grave{U}^{*}=P-i \Gamma \psi\left(\dot{\mathfrak{g}} \grave{P}^{\mathrm{aux}}\right)+i\left(\dot{P}^{\mathrm{aux}} \grave{\mathfrak{g}}\right) \Gamma \psi+\Gamma \psi\left(\dot{\mathfrak{g}} P^{\text {aux }} \grave{\mathfrak{g}}\right) \Gamma \psi .
$$

As outlined in 33.7 .6 , the contributions linear in $v$ give rise to the desired axial terms with logarithmic poles on the light cone. The problematic shear contribution, however, is quadratic in $v$ (see (3.7.42) and the discussion thereafter). For the unregularized fermionic projector (3.3.1), this shear contribution can be written in momentum space as

$$
\tilde{P} \asymp \sum_{\beta=1}^{g} \dot{\mathfrak{g}}_{\alpha} \psi k\left\langle\psi \grave{\mathfrak{g}}^{\alpha} \delta\left(k^{2}-m_{\alpha}^{2}\right) \Theta\left(-k^{0}\right)\right.
$$




$$
=\sum_{\beta=1}^{g} \mathfrak{g}_{\alpha}\left(2\langle v, k\rangle \psi-v^{2} \not k\right) \grave{\mathfrak{g}}^{\alpha} \delta\left(k^{2}-m_{\alpha}^{2}\right) \Theta\left(-k^{0}\right) .
$$

(With regularization, one transforms similarly the distribution (3.3.4). This has no effect on the following consideration if one keeps in mind that the vector field $v^{\varepsilon}$ is time-like and past-directed according to our assumption (vii).) Since the momentum $k$ is timelike and past-directed, a short calculation shows that the vector $2\langle v, k\rangle v-v^{2} k$ is non-spacelike and past-directed (no matter how $v$ is chosen). As a consequence, adding up the contributions by different Dirac seas, we necessarily obtain a timelike and past-directed vector, even if by the replacement $v \rightarrow v_{\beta}$ we allowed the vector field in $(3.7 .49)$ to depend on the generation index $\beta$. This means that the contributions by different Dirac seas cannot compensate each other. Transforming to position space, we necessarily get a contribution to $\tilde{P}$ which is highly singular on the light cone, leading to the problems discussed in \$3.7.8. Using the decomposition (3.3.6), one sees that $\tilde{P}$ violates the condition (3.3.7). We can thus say that the local axial transformation introduces a shear of the surface states (see also [F7, Section 4.4]). This also explains why we referred to (3.7.43) as the shear contribution.

In order to bypass the problems caused by the shear contributions, we need to introduce an axial contribution to $\tilde{P}$ without generating a vectorial contribution of the form (3.7.49). To explain the method, we consider in generalization of (3.7.47) the transformation

$$
\tilde{P}(k)=\dot{U}(k) P^{\operatorname{aux}}(k) \grave{U}(k)^{*}=\sum_{\beta=1}^{g} U_{\beta}(k) P_{\beta}(k) U_{\beta}(k)^{*},
$$

where $U(k)$ is a multiplication operator in momentum space. Before going on, we briefly discuss this ansatz. We first point out that the operator $U(k)$ can be an arbitrary multiplication operator in momentum space. Representing it in position space gives rise to a convolution operator with an integral kernel $U(x-y)$ which will in general be non-zero if $x \neq y$. Thus $(3.7 .50$ ) describes a nonlocal homogeneous transformation. We shall see that the freedom to choose $U$ as a function of $k$ will be essential for our construction to work. Clearly, 3.7.50 gives a lot of freedom to modify the fermionic projector. On the other hand, we will see in the subsequent calculations that 3.7.50 is quite restrictive is the sense that it imposes inequalities on the vector and axial components of $\tilde{P}(k)$. The basic reason for these restrictions can be understood from the fact that $-\tilde{P}(k)$ is necessarily a positive operator, meaning that

$$
-\bar{\psi} \tilde{P}(k) \psi \geq 0 \quad \text { for every spinor } \psi \text {. }
$$

Namely, taking the expectation value of 3.7 .50 , we obtain

$$
\bar{\psi} \tilde{P}(k) \psi=\sum_{\beta=1}^{g} \overline{\left(U_{\beta}^{*} \psi\right)} P_{\beta}(k)\left(U_{\beta}^{*} \psi\right) \leq 0,
$$

where in the last step we used the fact that the operators $-\left(\not k+m_{\beta}\right)$ are obviously positive for $k$ on the lower mass shell. We remark that this positivity of $(-\tilde{P})$ in momentum space gives rise to corresponding positivity properties of the fermionic projector in position space; these are worked out in Appendix C. For general properties of positive operators in indefinite inner product space we refer to [F10, Section 4].

We first analyze the effect of the above transformations in the high-frequency limit. Thus we consider a momentum $k=(\omega, \vec{k})$ on the mass cone and consider the asymptotics 
as $\Omega:=|\omega| \rightarrow \infty$. Then the projector onto the states of momentum $k$ of rest mass $m$ converges after suitable rescaling,

$$
\frac{1}{\Omega}(\not k+m) \stackrel{\Omega \rightarrow \infty}{\longrightarrow} \hat{\not k} \quad \text { with } \quad \hat{k}:=(-|\vec{k}|, \vec{k}) .
$$

Note that the operator $\hat{k}$ is nilpotent and negative semi-definite (in the sense that $\prec \psi \mid \hat{\not k} \psi \succ \leq 0$ for every spinor $\psi$ ). We want to generate an axial contribution of the form $\Gamma \psi$ (independent of $k$ ). Thus in view of the scaling (3.7.51), our goal is to perturb the operator $\hat{k}$ such as to generate an axial contribution of the form

$$
\frac{1}{\Omega} \Gamma \psi \quad \text { with a timelike vector } v \text {. }
$$

If this perturbation is described by a unitary transformation, the resulting operator will again be nilpotent and negative semi-definite. According to the methods in \$3.7.7, however, the perturbation can also be described by a non-unitary transformation. But even then the semi-definiteness is preserved, and the operator will also remain close to a nilpotent operator provided that we want to vary its eigenvalues only slightly. Therefore, in order to to get an idea for how to perturb the states, it is helpful to characterize the general form of nilpotent and negative semi-definite operators.

Lemma 3.7.6. Suppose that the linear operator $A$ is nilpotent and negative semidefinite. Then A has the representation

$$
A=(\not 1+a i \Gamma)(\mathbb{1}+i(\not+b i \Gamma))
$$

with a non-spacelike past-directed vector $q$ and a non-spacelike vector $u$ as well as real parameters $a$ and $b$ such that

$$
\langle q, u\rangle=a b
$$

and moreover one of the following two alternative conditions holds:

$$
\left\{\begin{array}{lll}
q^{2}=a^{2} & \text { and } & -1 \leq u^{2}-b^{2} \leq 0 \\
q^{2}>a^{2} & \text { and } & -1=u^{2}-b^{2} .
\end{array}\right.
$$

Proof. It is convenient to interpret the component proportional to $i \Gamma$ as an additional spatial coordinate. To this end, we introduce the matrices $\Gamma^{0}, \ldots, \Gamma^{4}$ by $\Gamma^{i}=\gamma^{i}$ if $i=0, \ldots, 3$ and $\Gamma^{4}=i \Gamma$. These matrices generate a 5 -dimensional Clifford algebra of signature $(1,4)$. Introducing the vectors $\underline{q}=(q, a), \underline{u}=(u, b) \in \mathbb{R}^{1,4}$, the lemma can be restated that there is a non-spacelike past-directed vector $\underline{q} \in \mathbb{R}^{1,4}$ and a vector $\underline{u} \in \mathbb{R}^{1,4}$ with $-1 \leq \underline{u}^{2} \leq 0$ such that

$$
A=\Gamma^{a} q_{a}\left(\mathbb{1}+i \Gamma^{b} u_{b}\right) \quad \text { with } \quad\langle\underline{q}, \underline{u}\rangle=0 \quad \text { and } \quad \underline{q}^{2}=0 \text { or } \underline{u}^{2}=-1 .
$$

The calculation

$$
\begin{aligned}
A^{2} & =\Gamma^{a} q_{a}\left(\mathbb{1}+i \Gamma^{b} u_{b}\right) \Gamma^{c} q_{c}\left(\mathbb{1}+i \Gamma^{d} u_{d}\right) \\
& =\Gamma^{a} q_{a} \Gamma^{c} q_{c}\left(\mathbb{1}-i \Gamma^{b} u_{b}\right)\left(\mathbb{1}+i \Gamma^{d} u_{d}\right)=\underline{q}^{2}\left(1+\underline{u}^{2}\right)=0
\end{aligned}
$$

shows that every matrix of the form 3.7 .55 is indeed nilpotent. In order to show conversely that any nilpotent matrix $A$ can be written in the form $(3.7 .55)$, we introduce the bilinear covariants by $\Sigma^{a b}=\frac{i}{2}\left[\Gamma^{a}, \Gamma^{b}\right]$. Then the matrices $\mathbb{1}, \Gamma^{a}$ and $\Sigma^{a b}$ form a basis of 
the symmetric linear operators. Since a nilpotent matrix is trace-free, we know that $A$ has the basis representation

$$
A=\Gamma^{a} q_{a}+\Sigma^{a b} B_{a b}
$$

with a vector $q \in \mathbb{R}^{1,4}$ and an anti-symmetric tensor $B$. Using the anti-commutation relations, it follows that

$$
\begin{aligned}
0=A^{2} & =\underline{q}^{2}+\left(\Sigma^{a b} B_{a b}\right)^{2}+\left\{\Gamma^{a} q_{a}, \Sigma^{a b} B_{a b}\right\} \\
& =\underline{q}^{2}+B_{a b} B^{a b}+\frac{1}{4} \epsilon^{a b c d e} B_{a b} B_{c d} \Gamma_{e}+\epsilon^{a b c d e} B_{a b} q_{c} \Sigma_{d e},
\end{aligned}
$$

where $\epsilon^{a b c d e}$ is the totally antisymmetric symbol in $\mathbb{R}^{1,4}$. It follows that the expression $\epsilon^{a b c d e} B_{a b} q_{c}=0$ vanishes, which implies that $B$ can be written as the anti-symmetrized tensor product of $\underline{q}$ with another vector $\underline{u}, B_{a b}=q_{[a} u_{b]}$. Then the vectorial component of $A^{2}$ also vanishes, i.e.

$$
0=A^{2}=\underline{q}^{2}+B_{a b} B^{a b}=\underline{q}^{2}+\langle\underline{q}, \underline{u}\rangle^{2}-\underline{u}^{2} \underline{q}^{2} .
$$

In the case $\underline{q}^{2}=0$, it follows that $\langle\underline{q}, \underline{u}\rangle=0$. On the other hand if $\underline{q}^{2} \neq 0$, by adding a multiple of $q$ to $\underline{u}$ we can arrange that again $\langle q, \underline{u}\rangle=0$. We conclude that $\Sigma^{a b} B_{a b}=$ $i \Gamma^{a} q_{a} \Gamma^{b} u_{b}$, giving the desired representation in 3.7 .55 . Finally, the relations $\underline{q}^{2}=0$ or $\underline{u}^{2}=-1$ again follow from (3.7.56).

It remains to show that $q$ is non-spacelike and past-directed and that $-1 \leq \underline{u}^{2} \leq 0$. To verify these inequalities, it is most convenient to arrange by a Lorentz transformation in $R^{1,4}$ that the subspace spanned by $q$ and $u$ lies in the $(0,1)$ or $(1,2)$ plane or is a null surface. A straightforward calculation in each of these cases using that $A$ is negative semi-definite gives the result.

Let us discuss the result of this lemma. Multiplying out, the representation 3.7.53 becomes

$$
A=\underbrace{\not q}_{\text {vectorial }}+\underbrace{a i \Gamma}_{\text {pseudoscalar }}+\underbrace{\frac{i}{2}[\not, \mu]}_{\text {bilinear }}-\underbrace{a \Gamma \not+b \Gamma \not}_{\text {axial }} .
$$

The operator $\hat{k}$ can be realized by choosing $q=\hat{k}, u=0$ and $a=b=0$. Then we are clearly in the first case in (3.7.54). The simplest operator in the second case is obtained by choosing $u=0, a=0$ and $b=\mp 1$, giving $A=\chi_{L / R} q$, where $q$ is any non-spacelike past-directed vector. In view of this example, we refer to the second case in (3.7.54) as the chiral limit. Because of the equation $u^{2}-b^{2}=-1$, the chiral limit cannot be obtained by continuously deforming the operator $\hat{k}$. Since we have a perturbation of $\hat{k}$ in mind, in what follows we always restrict attention to the first case in (3.7.54). If we choose $a=0$, the vector $q$ must be light-like. Moreover, the axial contribution in 3.7 .57 is proportional to $\Gamma q$, making it impossible to generate a contribution of the form $(3.7 .52)$. We conclude that we must choose $a \neq 0$. This implies that we necessarily get a pseudoscalar contribution and a timelike vectorial contribution. Furthermore, in order for the axial contribution to point into a general direction $v$, we also need to choose $u \neq 0$. This implies that we also get a bilinear contribution. We conclude that when generating the desired axial contribution 3.7.52, we necessarily generate error terms having a vectorial, a pseudoscalar and a bilinear contribution. The appearance of such error terms can be understood similar to the shear contribution in (3.7.49). The 
good news is that now the error terms can be arranged to be much smaller than the shear contributions, as we now make precise by specifying the scalings.

Choosing a unitary matrix $U$ with an axial and a vector component,

$$
U=\exp \left(\frac{i}{\sqrt{\Omega}} Z\right) \quad \text { with } \quad Z=\nu \Gamma \psi+\lambda \psi, \quad \lambda, \nu \in \mathbb{R}
$$

we obtain

$$
\begin{aligned}
Z^{2}= & \left(-\nu^{2}+\lambda^{2}\right) v^{2} \\
Z \not k Z= & (\nu \Gamma+\lambda) \psi \not k \psi(-\nu \Gamma+\lambda)=(\nu \Gamma+\lambda)^{2} \not \not k \psi \\
= & \left(\nu^{2}+\lambda^{2}+2 \lambda \nu \Gamma\right)\left(2\langle v, k\rangle \psi-v^{2} \not k\right) \\
U \not k U^{-1}= & \not k+\frac{i}{\sqrt{\Omega}}[Z, \not k]-\frac{1}{2 \Omega}\left\{Z^{2}, \not k\right\}+\frac{1}{\Omega} Z \not k Z+\mathcal{O}\left(\Omega^{-\frac{3}{2}}\right) \\
= & (\not k+m)+\frac{1}{\sqrt{\Omega}}(\nu 2\langle k, v\rangle i \Gamma+\lambda i[\psi, \not k])+\frac{1}{\Omega}\left(\nu^{2}-\lambda^{2}\right) v^{2} \not k \\
& +\frac{1}{\Omega}\left(\nu^{2}+\lambda^{2}+2 \lambda \nu \Gamma\right)\left(2\langle v, k\rangle \psi-v^{2} \not k\right)+\mathcal{O}\left(\Omega^{-\frac{3}{2}}\right)
\end{aligned}
$$

and thus

$$
\begin{aligned}
U(\not k+m) U^{-1}= & (\not k+m)+\frac{1}{\sqrt{\Omega}}(\nu 2\langle v, k\rangle i \Gamma+\lambda i[\psi, \not k]) \\
& +\frac{2}{\Omega}\left(\nu^{2}+\lambda^{2}+2 \lambda \nu \Gamma\right)\langle v, k\rangle \psi \\
& -\frac{2}{\Omega}\left(\lambda^{2}+\lambda \nu \Gamma\right) v^{2} \not k+\mathcal{O}\left(\Omega^{-\frac{3}{2}}\right) .
\end{aligned}
$$

The last contribution 3.7 .62 points into the direction $\not k$. It can be compensated by the subsequent transformation

$$
U(\not k+m) U^{-1} \longrightarrow V U(\not k+m) U^{-1} V^{*}
$$

with

$$
V=\mathbb{1}+\frac{1}{\Omega}\left(\lambda^{2} v^{2}+\lambda \nu v^{2} \Gamma\right)
$$

(this transformation describes a unitary pseudoscalar transformation combined with a scaling by the factor $\left.\left(1+\lambda^{2} v^{2} / \Omega\right)^{2}\right)$. In view of this transformation, we can drop (3.7.62). The remaining contributions (3.7.60) and (3.7.61) can be understood in analogy to our discussion of (3.7.57). Namely, the desired axial contribution is contained in (3.7.61), which also involves a vectorial error term. The second summand in 3.7 .60$)$ is the error term composed of the pseudoscalar and the bilinear components. By inspecting (3.7.57), one verifies that the above scaling is optimal in the sense that the error terms must be at least of the order $\Omega^{-\frac{1}{2}}$. By repeating the above calculation for a more general matrix $Z$, one can verify that the form of the contributions in (3.7.60) and (3.7.61) is uniquely determined, so that the only arbitrariness is to choose the two free parameters $\lambda$ and $\nu^{5}$, For what follows, it is important that the error terms in 3.7.60 do not have a fixed sign. Thus when considering several generations, it will be possible to arrange that the contributions 3.7.60) cancel each other. The vectorial error term in (3.7.61), on the other

\footnotetext{
${ }^{5}$ We remark that the ansatz 3.7 .58 for $Z$ can be can be generalized to $Z=\Gamma \not g+\not h$ involving two vectors $g$ and $h$. This gives additional freedom to modify the vector component, but without any influence on the axial component to be considered here. For details we refer to 4.4 .3
} 
hand, has a definite sign, leading to a timelike and past-directed contribution. But it is by a factor $1 / \Omega$ smaller than the corresponding shear terms in $(3.7 .49$.

Let us extend the last computation to a system of $g$ Dirac seas and generate the desired logarithmic pole on the light cone. Our goal is to generate an axial contribution of the form

$$
\tilde{P}(x, y) \asymp \Gamma \psi \log \left(\xi^{2}\right)
$$

with a timelike vector $v$. Taking the Fourier transform, one sees that the contribution should be proportional to the derivative of the $\delta$ distribution,

$$
\Gamma \not \delta^{\prime}\left(k^{2}\right) \Theta\left(-k^{0}\right) \text {. }
$$

Similar to 3.7.49, we can transform each sea independently. Thus

$$
\tilde{P}=\sum_{\beta=1}^{g} U_{\beta}(k)\left(\not k+m_{\beta}\right) U_{\beta}(k)^{-1} \delta\left(k^{2}-m_{\beta}^{2}\right),
$$

where the operators $U_{\beta}$ are again of the form 3.7.58). Using (3.7.60) and (3.7.61), we obtain

$$
\begin{aligned}
& \tilde{P}(k)=\sum_{\beta=1}^{g}\left[\left(\not k+m_{\beta}\right)+\frac{1}{\sqrt{\Omega}}\left(\nu_{\beta} 2\langle v, k\rangle i \Gamma+\lambda_{\beta} i[\psi, \not k]\right)\right. \\
&\left.+\frac{4\langle v, k\rangle}{\Omega}\left(\lambda_{\beta} \nu_{\beta} \Gamma \psi+(\text { vectorial })\right)\right] \delta\left(k^{2}-m_{\beta}^{2}\right) \Theta\left(-k^{0}\right) .
\end{aligned}
$$

Expanding the $\delta$-distributions according to $\delta\left(k^{2}-m_{\beta}^{2}\right)=\delta\left(k^{2}\right)-m_{\beta}^{2} \delta^{\prime}\left(k^{2}\right)+(\operatorname{deg}<0)$, we obtain the conditions

$$
\begin{aligned}
\sum_{\beta=1}^{g} \lambda_{\beta} & =0, & \sum_{\beta=1}^{g} \nu_{\beta} & =0 \\
\sum_{\beta=1}^{g} \lambda_{\beta} \nu_{\beta} & =0, & \sum_{\beta=1}^{g} m_{\beta}^{2} \lambda_{\beta} \nu_{\beta} & =-\frac{\Omega}{4\langle v, k\rangle} \neq 0 .
\end{aligned}
$$

Since $k$ scales like $\Omega$, we see that these equations have the correct scaling if we assume that the parameters $\nu_{\beta}$ and $\lambda_{\beta}$ all scale $\sim \Omega^{0}$.

Let us verify that the equations (3.7.67) and (3.7.68) do not admit solutions if the number of generations $g<3$. In the case $g=1$, the equations (3.7.67) only have the trivial solution, in contradiction to the right equation in 3.7 .68 . In the case $g=2$, the equations (3.7.67) imply that $\nu_{2}=-\nu_{1}$ and $\lambda_{2}=-\lambda_{1}$. Using these relations on the left of (3.7.68), it follows that $\lambda_{1}=\nu_{1}=0$, again in contradiction to the right equation in $(3.7 .68)$.

In order to analyze the case of three generations, it is convenient to arrange with the transformation $\nu_{\beta} \rightarrow-\nu_{\beta} \Omega /(4\langle w, k\rangle)$ that the inhomogeneity in (3.7.68) equals one. Moreover, introducing the parameters

$$
a_{\beta}=\frac{1}{2}\left(\lambda_{\beta}+\nu_{\beta}\right), \quad b_{\beta}=\frac{1}{2}\left(\lambda_{\beta}-\nu_{\beta}\right),
$$


the equations 3.7 .67$)$ and $(3.7 .68)$ become

$$
\begin{aligned}
\sum_{\beta=1}^{3} a_{\beta} & =0, & \sum_{\beta=1}^{g} b_{\beta} & =0 \\
\sum_{\beta=1}^{3}\left(a_{\beta}^{2}-b_{\beta}^{2}\right) & =0, & \sum_{\beta=1}^{3} m_{\beta}^{2}\left(a_{\beta}^{2}-b_{\beta}^{2}\right) & =1 .
\end{aligned}
$$

We solve the linear equations $(3.7 .69)$ for $a_{3}=a_{3}\left(a_{1}, a_{2}\right)$ and $b_{3}=b_{3}\left(b_{1}, b_{2}\right)$. In order to analyze the remaining quadratic equations 3.7 .70 for $a_{1}, a_{2}$ and $b_{1}, b_{2}$, it is most convenient to introduce the scalar products $\langle., .\rangle_{0}$ and $\langle., .\rangle_{2}$ by

$$
\sum_{\beta=1}^{3} a_{\beta}^{2}=\left\langle\left(\begin{array}{l}
a_{1} \\
a_{2}
\end{array}\right),\left(\begin{array}{l}
a_{1} \\
a_{2}
\end{array}\right)\right\rangle_{0}, \quad \sum_{\beta=1}^{3} m_{\beta}^{2} a_{\beta}^{2}=\left\langle\left(\begin{array}{l}
a_{1} \\
a_{2}
\end{array}\right),\left(\begin{array}{l}
a_{1} \\
a_{2}
\end{array}\right)\right\rangle_{2},
$$

making it possible to write 3.7 .70 as

$$
\langle a, a\rangle_{0}=\langle b, b\rangle_{0} \quad \text { and } \quad\langle a, a\rangle_{2}-\langle b, b\rangle_{2}=1
$$

(where $a=\left(a_{1}, a_{2}\right)$ and $\left.a=\left(b_{1}, b_{2}\right)\right)$. Representing the scalar products with signature matrices $S_{0}$ and $S_{2}$,

$$
\langle a, a\rangle_{0}=\left\langle a, S_{0} a\right\rangle_{\mathbb{R}^{2}}, \quad\langle a, a\rangle_{2}=\left\langle a, S_{2} a\right\rangle_{\mathbb{R}^{2}},
$$

we can rewrite (3.7.71) with the scalar product $\langle., .\rangle_{0}$,

$$
\langle a, a\rangle_{0}=\langle b, b\rangle_{0} \quad \text { and } \quad\left\langle a,\left(S_{0}^{-1} S_{2}\right) a\right\rangle_{0}-\left\langle b,\left(S_{0}^{-1} S_{2}\right) b\right\rangle_{0}=1 .
$$

Thus we seek for vectors $a$ and $b$ having the same norm, such that the difference of their expectation values of the operator $\left(S_{0}^{-1} S_{2}\right)$ equals one. Having two equations for four unknowns, we can clearly not expect a unique solution. But we get a unique solution by imposing that the error terms should be as small as possible, which means that we want to the norms on the left to be minimal. For the computation, it is useful that the operator $\left(S_{0}^{-1} S_{2}\right)$ is symmetric with respect to $\langle.,\rangle_{0}$. Thus we can choose an eigenvector basis $e_{1}, e_{2}$ of $\left(S_{0}^{-1} S_{2}\right)$ which is orthonormal with respect to $\langle., .\rangle_{0}$. Representing $a=\alpha_{k} e_{k}$ and $b=\beta_{k} e_{k}$ in this basis, we obtain the equations

$$
\alpha_{1}^{2}+\alpha_{2}^{2}=\beta_{1}^{2}+\beta_{2}^{2} \quad \text { and } \quad \mu_{1}\left(\alpha_{1}^{2}-\beta_{1}^{2}\right)+\mu_{2}\left(\alpha_{2}^{2}-\beta_{2}^{2}\right)=1
$$

where the eigenvalues $\mu_{1 / 2}$ are computed by

$$
\mu_{1 / 2}=\frac{1}{3}\left(m_{1}^{2}+m_{2}^{2}+m_{3}^{2} \pm \sqrt{m_{1}^{4}+m_{2}^{4}+m_{3}^{4}-m_{1}^{2} m_{2}^{2}-m_{2}^{2} m_{3}^{2}-m_{1}^{2} m_{3}^{2}}\right) .
$$

Now our minimization problem leads us to choose $\alpha_{1}^{2}$ as large as possible and $\beta_{1}^{2}$ as small as possible, giving the unique solution

$$
\alpha_{1}=\beta_{2}=\frac{1}{\sqrt{\mu_{1}-\mu_{2}}}, \quad \alpha_{2}=\beta_{1}=0 .
$$

We thus obtain the following result.

Proposition 3.7.7. Considering homogeneous transformations of the vacuum fermionic projector, it is impossible to generate a logarithmic pole (3.7.63) if the number of generations is smaller than three. If the number of generations equals three, by minimizing the error terms we get a unique solution of the resulting equations (3.7.67) and (3.7.68). 
Thus for sufficiently large $\Omega$, there are transformations $U_{\beta}(k)$ such that the transformed fermionic projector (3.7.65) is of the form

$$
\begin{aligned}
\tilde{P}(k)= & P(k)+\Gamma \psi \sum_{\beta=1}^{3} C_{\beta} \delta\left(k^{2}-m_{\beta}^{2}\right)+(\text { vectorial }) \delta\left(k^{2}\right)\left(1+\mathcal{O}\left(\Omega^{-1}\right)\right) \\
& +(\text { pseudoscalar or bilinear }) \sqrt{\Omega} \delta^{\prime}\left(k^{2}\right)\left(1+\mathcal{O}\left(\Omega^{-1}\right)\right) .
\end{aligned}
$$

The coefficients $C_{\beta}$ satisfy the relations

$$
\begin{aligned}
\sum_{\beta=1}^{3} C_{\beta} & =0, \quad \sum_{\beta=1}^{3} m_{\beta}^{2} C_{\beta}=-1, \\
\sum_{\beta=1}^{3} m_{\beta}^{2} \log \left(m_{\beta}^{2}\right) C_{\beta} & =-\frac{\sum_{\beta=1}^{3} m_{\beta}^{2} \log \left(m_{\beta}^{2}\right)\left(2 m_{\beta}^{2}-\sum_{\alpha \neq \beta} m_{\alpha}^{2}\right)}{\sum_{\beta=1}^{3} m_{\beta}^{2}\left(2 m_{\beta}^{2}-\sum_{\alpha \neq \beta} m_{\alpha}^{2}\right)} .
\end{aligned}
$$

Proof. Introducing the abbreviation $C_{\beta}=\lambda_{\beta} \nu_{\beta} 4\langle v, k\rangle / \Omega$, the relations (3.7.74) follow immediately from (3.7.68). The identity (3.7.75) is obtained by a straightforward calculation using the explicit form of the parameters $\lambda_{\beta}$ and $\nu_{\beta}$.

We remark that our analysis could be carried out similarly in the case of more than three generations. We expect that, similar as explained after 3.7 .32 for the local axial transformation, the equation should be underdetermined for than three generations. But we will not enter the details here. Instead, we shall always choose $g=3$, noting that this is the smallest number for which one can compensate the logarithmic poles on the light-cone.

3.7.10. The Microlocal Chiral Transformation. The setting in the previous section was rather special because we only considered homogeneous transformations in the high-frequency limit. But the methods and results can be generalized in a straightforward way, leading to the so-called microlocal chiral transformation. We now explain these generalizations.

We first recall that with the ansatz (3.7.58) we built in a specific scaling which was suitable to describe the asymptotic behavior in the high-frequency limit $\Omega \rightarrow \infty$. More precisely, we showed that there are transformations $U_{\beta}(k)$ such that the transformed fermionic projector (3.7.73) involves the desired axial contribution (3.7.64) as well as vectorial, pseudoscalar and bilinear error terms. By adding lower order terms in $\Omega$ to (3.7.58), one could extend the analysis to also include correction terms which decay faster for large $\Omega$. More generally, one can consider transformations of the form $U(k)=e^{i Z(k)}$ with $Z(k)$ as in (3.7.58) without taking the high-energy limit (for ease in notation, we now combined the factor $1 / \sqrt{\Omega}$ in $(3.7 .58)$ with the operator $Z$ ). Then using $(3.7 .50)$, a state of the fermionic projector transforms to

$$
\begin{aligned}
& U(k)(\not k+m) U(k)^{*}=e^{i Z}(\not k+m) e^{-i Z} \\
& \quad=\left(\cos (\alpha)+\frac{i Z}{\alpha} \sin (\alpha)\right) \not k\left(\cos (\alpha)-\frac{i Z}{\alpha} \sin (\alpha)\right)+m,
\end{aligned}
$$

where we set $\alpha=\sqrt{Z^{2}}$ and used that, according to (3.7.59), $Z^{2}$ is a multiple of the identity matrix. A straightforward computation shows that by choosing $Z$ appropriately, one can generate an arbitrary axial contribution, at the cost of generating vectorial, bilinear and 
pseudoscalar error terms. Such homogeneous transformations in the low-frequency region will be analyzed systematically in $\$ 3.10 .1$ below. Here we simply choose $Z(k)$ in the lowfrequency region such that the coefficients $C_{\beta}$ in $(3.7 .73)$ are constants. Taking the Fourier transform, we thus obtain the following result.

LEMMA 3.7.8. There is a homogeneous transformation $U(k)$ such that the transformed fermionic projector 3.7.50 is of the form

$$
\begin{aligned}
\tilde{P}(x, y)= & P(x, y)+\Gamma \psi T_{[3]}^{(1)}+\Gamma \psi(\operatorname{deg}<0)+(\text { smooth contributions }) \\
& +\psi(\operatorname{deg}<2)+(\text { pseudoscalar or bilinear })(\operatorname{deg}<1) .
\end{aligned}
$$

We note that the smooth axial contributions can be computed explicitly from 3.7.73 and (3.7.75). The smooth vectorial, pseudoscalar and bilinear contributions, however, are undetermined.

Our next step is to extend our methods to the non-homogeneous setting where the vector $v$ in 3.7 .63 is a smooth vector field. In order to determine the relevant length scales, we first read off from 3.7.58) (for constant $\lambda$ and $\nu$ ) that $U(k)$ varies on the scale $m$ of the rest masses of the Dirac seas. Thus in position space, the distribution $U(x, y)$ decays for $x-y$ on the Compton scale. Moreover, the vector field $v$ varies on the scale $\ell_{\text {macro }}$ of macroscopic physics. We want to treat $v$ as a slowly varying function in space-time. To this end, we need to assume that the difference vector $x-y$ of the fermionic projector is much smaller than the macroscopic length scale. This leads us to evaluate weakly (3.5.7) for $\vec{\xi}$ on the scale

$$
\varepsilon \ll|\vec{\xi}| \ll \ell_{\text {macro }} .
$$

Under this assumption, we can write (3.7.63) as

$$
\left.\tilde{P}(x, y) \asymp \Gamma \psi\left(\frac{x+y}{2}\right) \log \left(\xi^{2}\right)+\text { (higher orders in }|\vec{\xi}| / \ell_{\text {macro }}\right) .
$$

In order to clarify the dependence on the vector $v$, we now denote the homogeneous nonlocal transformation of Lemma 3.7 .8 by $U(k, v)$. We introduce the distribution $U(x, y)$ by

$$
U(x, y)=\int \frac{d^{4} k}{(2 \pi)^{4}} U\left(k, v\left(\frac{x+y}{2}\right)\right) e^{-i k(x-y)}
$$

and consider it as the integral kernel of a corresponding operator $U$. We generalize the transformation 3.7 .50 by

$$
\tilde{P}:=\dot{U} P^{\text {aux }} \grave{U}^{*} \text {. }
$$

The Fourier integral in (3.7.78) resembles the so-called Weyl map which is used to transform the Wigner distribution to an integral kernel in position space. More generally, the so-called quasi-homogeneous ansatz $(3.7 .78)$ is frequently used in microlocal analysis in order to approximately localize functions simultaneously in position and in momentum space. For this reason, we refer to $U$ as the microlocal chiral transformation.

Let us specify the error of the quasi-homogeneous approximation. As the kernel $U(x, y)$ decays on the Compton scale, at first sight one might expect a relative error of the or$\operatorname{der}\left(m \ell_{\text {macro }}\right)^{-1}$. In fact, the situation is improved by using (3.7.77), as the following argument shows. The kernel $U(x, y)$ will in general be singular at $x=y$, but it can be chosen to be smooth otherwise. Hence we can decompose $U(x, y)$ as

$$
U(x, y)=U_{|\vec{\xi}|}(x, y)+U_{\text {smooth }}(x, y)
$$


where $U_{\text {smooth }}$ is smooth and $U_{|\vec{\xi}|}$ is supported for $|\vec{x}-\vec{y}| \leq|\vec{\xi}| / 2$. Using this decomposition in 3.7 .79$)$, one sees that the error is of the order $|\vec{\xi}| / \ell_{\text {macro }}$, up to smooth contributions. We thus obtain the following result.

Proposition 3.7.9. If the number of generations equals three, then for any macroscopic vector field $v$ there is a microlocal chiral transformation (3.7.78) such that the transformed fermionic projector (3.7.79) is of the form

$$
\begin{aligned}
\tilde{P}(x, y)= & P(x, y)+\Gamma \psi T_{[3]}^{(1)}\left(1+\mathcal{O}\left(|\vec{\xi}| / \ell_{\text {macro }}\right)\right)+\Gamma \psi(\operatorname{deg}<0)+\psi(\operatorname{deg}<2) \\
& +(\text { pseudoscalar or bilinear })(\operatorname{deg}<1)+(\text { smooth contributions }) .
\end{aligned}
$$

We now give the corresponding contribution to $\mathcal{R}$; the derivation is postponed to Appen$\operatorname{dix}$ B.

Lemma 3.7.10. The perturbation of the fermionic projector by the microlocal chiral transformation of Proposition 3.7.9 leads to a contribution to $\mathcal{R}$ of the form

$$
\mathcal{R} \asymp i \xi_{k} v^{k} N_{4}\left(1+\mathcal{O}\left(|\vec{\xi}| / \ell_{\text {macro }}\right)\right)+(\operatorname{deg}<4)+o\left(|\vec{\xi}|^{-3}\right),
$$

where

$$
N_{4}=-\frac{g}{\overline{T_{[0]}^{(0)}}}\left[T_{[3]}^{(1)} T_{[0]}^{(-1)} \overline{T_{[0]}^{(-1)} T_{[0]}^{(0)}}-\text { c.c. }\right] .
$$

We note that the smooth contributions in (3.7.81) also affect $\mathcal{R}$; this will be analyzed in detail in 3.8 .1 .

It is a shortcoming of the transformation 3.7 .79 that it does not necessarily preserve the normalization of the fermionic projector (note that the operator defined by the quasihomogeneous ansatz (3.7.78) is in general not unitary). We now explain how this problem can be fixed by adapting the methods in 3.7.7. Similar to 3.7.35, we transform the Dirac operator by $U^{-1}$, and rewrite the transformed Dirac operator as

$$
\left(U^{-1}\right)^{*}\left(i \not \partial_{x}+\mathcal{B}-m Y\right) U^{-1}=i \not \not_{x}+\mathfrak{n}-m Y \text {. }
$$

As $U$ is non-local, the operator $\mathfrak{n}$ will in general be a nonlocal potential (i.e. it can be written as an integral operator with a nonlocal integral kernel $\mathfrak{n}(x, y))$. Now we can again perform the causal perturbation expansion. The resulting fermionic projector is properly normalized (for details see [FT2]). As explained in (3.7.36) and (3.7.37), the residual argument yields that the fermionic projector defined by the causal perturbation expansion coincides with 3.7 .79 up to smooth contributions.

We conclude by pointing out that the previous constructions involve several correction terms which will not be analyzed further in this book: First, there are the corrections of the order $|\vec{\xi}| / \ell_{\text {macro }}$ in (3.7.81). Moreover, 3.7.81) involves corrections of the order $\left(m \ell_{\text {macro }}\right)^{-1}$ to the smooth contributions, generated by the operator $U_{\text {smooth }}$ in 3.7 .80 . Finally, the causal perturbation expansion corresponding to the nonlocal Dirac operator (3.7.83) involve smooth corrections to 3.7 .79$)$. Since these corrections vanish for homogeneous perturbations (because in this case, the transformation $U(k)$ is unitary), they should again be of the order $\left(m \ell_{\text {macro }}\right)^{-1}$. We thus expect that all these correction terms are smaller than the quantum corrections (3.1.1) to be considered in $\$ 3.8 .1$, which are of the order $k / m$ (where $k$ is the momentum of the bosonic field). This is the reason why in this paper, we shall not enter the analysis of these correction terms. 
3.7.11. The Shear Contributions by the Microlocal Chiral Transformation. With the constructions in $\$ 3.7 .9$ and $\$ 3.7 .10$, we could avoid the shear contribution 3.7 .40 caused by the local axial transformation. Instead, the microlocal chiral transformation gives rise to the vectorial contribution in (3.7.61). We now analyze the effect of this contribution in detail and compare the situation to that of the shear terms in 3.7 .8 . The vectorial contribution in (3.7.61) can be generated by the transformation of the Dirac states

$$
(\not k+m) \rightarrow \not k+\frac{2}{\Omega}\left(\nu^{2}+\lambda^{2}\right)\langle v, k\rangle \psi+m .
$$

Writing the fermionic projector in the form (3.3.4), this transformation describes a change of the direction of the vector field $v^{\varepsilon}$. Using the notion in $(3.3 .6)$ and (3.3.7), this transformation again introduces a shear of the surface states. We thus refer to the resulting contributions to the fermionic projector as the shear contributions by the microlocal chiral transformation. In Proposition 3.7.7, Lemma 3.7.8 and Proposition 3.7.9, the shear contributions were included in the error term $\psi(\operatorname{deg}<2)$. A short computation shows that they can be written as

$$
\Delta P(x, y)=\frac{c}{m^{2}} \psi T_{[1]}^{(0)}\left(1+\mathcal{O}\left(|\vec{\xi}| / \ell_{\text {macro }}\right)\right),
$$

where $c$ is a real-valued, dimensionless constant depending on the ratios of the fermionic masses (the reason for the subscript [1] is that, according to (3.7.61), this contribution can be obtained form the contribution $\sim m$ to $\not k+m$ by multiplication with a function which is constant as $\Omega \rightarrow \infty$ ). Perturbing the fermionic projector of the vacuum, we obtain similar to 3.7 .43

$$
\Delta A_{x y}=\frac{i g c}{2 m^{2}}\left(\not \psi T_{[0]}^{(-1)} \overline{T_{[1]}^{(0)}}-\psi \notin T_{[1]}^{(0)} \overline{T_{[0]}^{(-1)}}\right)+(\operatorname{deg}<3) .
$$

As this contribution is invariant under the replacements $L \leftrightarrow R$, it clearly drops out of the EL equations.

The situation becomes more subtle when the gauge phases are taken into account. Similar to (3.7.45), the microlocal chiral transformation flips the chirality. Thus (3.7.84) becomes

$$
\Delta P(x, y)=\frac{c}{m^{2}}\left(\chi_{L} e^{-i \Lambda_{R}^{x y}}+\chi_{R} e^{-i \Lambda_{L}^{x y}}\right) \psi T_{[1]}^{(0)}\left(1+\mathcal{O}\left(|\vec{\xi}| / \ell_{\text {macro }}\right)\right) .
$$

Also using (3.6.17) and (3.6.19), we obtain

$$
\begin{aligned}
A_{x y}= & \frac{g^{2}}{4}\left(\chi_{L} \nu_{L}+\chi_{R} \nu_{R}\right)\left(\sharp T_{[0]}^{(-1)}\right)\left(\overline{\left(T_{[0]}^{(-1)}\right.}\right) \\
& +\frac{i g c}{2 m^{2}}\left(\sharp \psi T_{[0]}^{(-1)} \overline{T_{[1]}^{(0)}}-\psi \notin T_{[1]}^{(0)} \overline{T_{[0]}^{(-1)}}\right)+\Varangle(\operatorname{deg} \leq 3)+(\operatorname{deg}<3) .
\end{aligned}
$$

We now give the corresponding eigenvalues of the closed chain; the proof is again given in Appendix B.

Lemma 3.7.11. The closed chain 3.7.86 has the eigenvalues

$$
\lambda_{+}^{L / R}=\nu_{L / R} g^{2} T_{[0]}^{(0)} \overline{T_{[0]}^{(-1)}}-\frac{i g c}{m^{2}} v_{k} \xi^{k} T_{[1]}^{(0)} \overline{T_{[0]}^{(-1)}}+(\operatorname{deg}<3),
$$

and the eigenvalues $\lambda_{-}^{L / R}$ are obtained by complex conjugation (see (2.6.38)).

This lemma shows that, similar to (3.7.46), the eigenvalues of the closed chain are perturbed by a contribution which does not involve the chiral phases. This means that, just as shown in Figure 3.2 , in general the eigenvalues will no longer have the same absolute 
value. In other words, the fermionic projector again involves shear contributions which violate the EL equations to degree five on the light cone.

However, now the situation is much better, because the perturbation (3.7.85) is of degree one on the light cone (and not of degree two as (3.7.45)). This makes it possible to "modify the phase" of (3.7.85) by the following construction. The auxiliary fermionic projector $\tilde{P}$ in the presence of chiral gauge fields and including the microlocal chiral transformation is defined by (cf. (3.7.79) ) The auxiliary fermionic projector $\tilde{P}$ in the presence of chiral gauge fields and including the microlocal chiral transformation is a solution of the Dirac equation

$$
\mathcal{D} \tilde{P}^{\text {aux }}=0,
$$

where the operator $\mathcal{D}$ is obtained from the Dirac operator with chiral gauge fields (as given in (3.4.4) and (3.4.16) ) by performing the nonlocal transformation (3.7.83),

$$
\mathcal{D}:=\left(U^{-1}\right)^{*}\left(i \not_{x}+\chi_{L} A_{R}+\chi_{R} A_{L}-m Y\right) U^{-1} \text {. }
$$

As explained after (3.7.83), one can take this Dirac equation as the starting point and introduce the fermionic projector by the causal perturbation expansion as outlined in Section 3.4. The resulting fermionic projector coincides agrees with (3.7.83), up to smooth contributions which guarantee the desired normalization. We now modify the operator $U$, which implicitly changes the potentials in the Dirac operator 3.7.88), and, via the causal perturbation expansion, also the fermionic projector. More precisely, we introduce the new Dirac operator

$$
\mathcal{D}_{\text {flip }}:=\left(U_{\text {flip }}^{-1}\right)^{*}\left(i \not \partial_{x}+\chi_{L} A_{R}+\chi_{R} A_{L}-m Y\right) U_{\text {flip }}^{-1},
$$

where for $U_{\text {flip }}$ we make the ansatz

$$
U_{\text {flip }}=\mathbb{1}+(U-\mathbb{1}) V,
$$

and $V$ is defined similar to $(3.4 .6)$ by a perturbation series

$$
V=\mathbb{1}+\sum_{k=1}^{\infty} \sum_{\alpha=0}^{\alpha_{\max }(k)} c_{\alpha} \mathcal{B}_{1, \alpha} C_{1, \alpha} \mathcal{B}_{2, \alpha} \cdots \mathcal{B}_{k, \alpha} C_{k, \alpha}
$$

with combinatorial factors $c_{\alpha}$ and Green's functions or fundamental solutions $C_{\ell, \alpha}$. Here the factors $\mathcal{B}_{\ell, \alpha}$ are chiral potentials which can be freely chosen, giving a lot of freedom to modify the gauge phases in the fermionic projector. However, our ansatz (3.7.90) ensures that these modified phases only enter the contributions generated by the microlocal chiral transformation (using the notation (3.7.58), $V$ only affects the orders $1 / \sqrt{\Omega}$ or higher of $P$, whereas the leading order $\sim \Omega^{0}$ is not altered by $V$ ). Taking the Dirac equation

$$
\mathcal{D}_{\text {flip }} \tilde{P}^{\text {aux }}=0
$$

as the starting point, one can again introduce the fermionic projector by the causal perturbation expansion as outlined in Section 3.4. The next proposition shows that by a suitable choice of the operator $V$, one can arrange that the gauge phases in (3.7.85) are flipped. The proof will again be given in Appendix B.

Proposition 3.7.12. By a suitable choice of the perturbation series (3.7.91), one can arrange that the auxiliary fermionic projector $\tilde{P}^{\text {aux }}$ defined by 3.7 .92 differs from that defined by (3.7.87) in that the contribution (3.7.85) is modified to

$$
\Delta P(x, y)=\frac{c}{m^{2}}\left(\chi_{L} e^{-i \Lambda_{L}^{x y}}+\chi_{R} e^{-i \Lambda_{R}^{x y}}\right) \psi T_{[1]}^{(0)}\left(1+\mathcal{O}\left(|\vec{\xi}| / \ell_{\text {macro }}\right)\right) .
$$


All the other contributions to the fermionic projector remain unchanged, up to error terms of the order $o(|\vec{\xi}|)(\operatorname{deg}<2)$.

Following this result, the closed chain (3.7.86) and the eigenvalue $\lambda_{+}$become

$$
\begin{aligned}
A_{x y}= & \left.\left(\chi_{L} \nu_{L}+\chi_{R} \nu_{R}\right)\left[\frac{g^{2}}{4}\left(\sharp T_{[0]}^{(-1)}\right) \overline{\left(\sharp T_{[0]}^{(-1)}\right.}\right)+\frac{i g c}{2 m^{2}}\left(\sharp \psi T_{[0]}^{(-1)} \overline{T_{[1]}^{(0)}}-\psi \notin T_{[1]}^{(0)} \overline{T_{[0]}^{(-1)}}\right)\right] \\
& +\Varangle(\operatorname{deg} \leq 3)+(\operatorname{deg}<3) \\
\lambda_{+}^{L / R}= & \nu_{L / R}\left[g^{2} T_{[0]}^{(0)} \overline{T_{[0]}^{(-1)}}-\frac{i g c}{m^{2}} v_{k} \xi^{k} T_{[1]}^{(0)} \overline{T_{[0]}^{(-1)}}\right]+(\operatorname{deg}<3) .
\end{aligned}
$$

In particular, one sees that the eigenvalues all have the same absolute value, so that the EL equations are satisfied to degree five. To degree four, the error terms $\tilde{P}(x, y) \sim$ $\mathcal{O}\left(|\vec{\xi}|^{2}\right)(\operatorname{deg} \leq 1)$ appear. But as they involve at least two factors $\xi$, they are of one order higher in $|\vec{\xi}|$ than the current and mass terms. For this reason, we shall not consider them here. We conclude that the shear contributions drop out of the EL equations.

\subsection{The Field Equations}

Having developed a method for compensating the logarithmic poles on the light cone, we are now in the position to derive and analyze the field equations. We again point out that our method only works if the number of generations is at least three (see Proposition 3.7.7). In what follows, we assume that the number of generations equals three.

3.8.1. The Smooth Contributions to the Fermionic Projector at the Origin. We add the contributions from Lemmas 3.7.3, 3.7.4 and 3.7.10 and collect all the terms which involve factors of $T_{0}^{(1)}$ or $\overline{T_{0}^{(1)}}$. Using $(3.7 .12)$, we find that the contribution to $\mathcal{R}$ involving factors of $\log \left|\xi^{2}\right|$ has the form

$$
\begin{aligned}
\mathcal{R} \asymp & -\frac{i \xi_{k}}{16 \pi^{3}}\left\{\frac{j_{\mathrm{a}}^{k}}{6}-m^{2} \dot{Y} \grave{Y} A_{\mathrm{a}}^{k}-\frac{v^{k}}{2}\right\} \frac{\log \left|\xi^{2}\right|}{\overline{T_{[0]}^{(0)}}} g^{2} T_{[0]}^{(-1)} \overline{T_{[0]}^{(-1)}}\left(T_{[0]}^{(0)}-\overline{T_{[0]}^{(0)}}\right) \\
& +(\operatorname{deg}<4)+o\left(|\vec{\xi}|^{-3}\right) .
\end{aligned}
$$

As explained after (3.7.13), this term must vanish. This leads us to choose $v$ as

$$
v=\frac{j_{\mathrm{a}}}{3}-2 m^{2} \grave{Y} \grave{Y} A_{\mathrm{a}}
$$

Then the logarithmic poles of $\mathcal{R}$ have disappeared.

Before analyzing the remaining contributions to $\mathcal{R}$, we must have a closer look at the non-causal low- and high energy contributions $\tilde{P}^{\text {le }}$ and $\tilde{P}^{\text {he }}$ in the light cone expansion (3.4.14). These smooth contributions to the fermionic projector were disregarded in the formalism of the continuum limit as outlined in Section 3.5. This is justified as long as singular contributions to the fermionic projector are considered. In particular, contributions to $P(x, y)$ involving the functions $T_{0}^{(-1)}, T_{0}^{(0)}$ or their complex conjugates have poles on the light cone, and therefore smooth corrections would be of lower degree on the light cone, meaning that the corresponding contributions to the EL equations would be negligible corrections of the form $(3.5 .9)$. However, the factors $T_{0}^{(1)}$ and $\overline{T_{0}^{(1)}}$ only have a logarithmic pole, and after the above cancellations of the logarithmic poles, the remaining leading contributions are indeed bounded functions. Thus smooth corrections become relevant. We conclude that it is necessary to determine the smooth contributions 
to the fermionic projector $P(x, y)$ at the origin $x=y$. This analysis is carried out in Appendix $\mathrm{D}$ to first order in the bosonic potentials using a resummation technique. In what follows, we use these results and explain them.

In order to introduce a convenient notation, we write the factors $T_{[p]}^{(1)}$ in generalization of $(3.7 .12)$ as

$$
T_{[p]}^{(1)}=\frac{1}{32 \pi^{3}}\left(\log \left|\xi^{2}\right|+i \pi \Theta\left(\xi^{2}\right) \epsilon\left(\xi^{0}\right)\right)+s_{[p]},
$$

where the real-valued functions $s_{[p]}$, which may depend on the masses and the bosonic potentials, will be specified below. Taking the complex conjugate of $(3.7 .12)$, we get a similar representation for $\overline{T_{[p]}^{(1)}}$. Substituting these formulas into $\mathcal{R}$, the factors $\log \left|\xi^{2}\right|$ cancel each other as a consequence of (3.8.1) and (3.8.2). Moreover, a short calculation shows that the factors $i \pi \Theta\left(\xi^{2}\right) \epsilon\left(\xi^{0}\right)$ in (3.8.3) also drop out. Applying Lemma 3.7.1, the EL equations to degree four yield the vector equation

$$
j_{a} N_{5}-m^{2} A_{a} N_{6}=J_{a} N_{3}
$$

with $N_{3}$ as in $(3.7 .9$ and

$$
\begin{aligned}
N_{5}= & \frac{g^{3}}{6 \overline{T_{[0]}^{(0)}}}\left[T_{[0]}^{(0)} T_{[0]}^{(0)} \overline{T_{[0]}^{(0)} T_{[0]}^{(-1)}}-c . c .\right] \\
& +g\left(s_{[0]}-s_{[3]}\right) \frac{g^{2}}{3 \overline{T_{[0]}^{(0)}}} T_{[0]}^{(-1)} \overline{T_{[0]}^{(-1)}}\left(T_{[0]}^{(0)}-\overline{T_{[0]}^{(0)}}\right) \\
N_{6}= & -\frac{2 g \hat{Y}^{2}}{\overline{T_{[0]}^{(0)}}}\left[T_{[0]}^{(-1)} T_{[0]}^{(0)} \overline{T_{[1]}^{(0)} T_{[1]}^{(0)}}-c . c .\right] \\
& +\left(s_{[2]}-s_{[3]}\right) \frac{2 g^{2} \hat{Y} \grave{Y}}{\overline{T_{[0]}^{(0)}}} T_{[0]}^{(-1)} \overline{T_{[0]}^{(-1)}}\left(T_{[0]}^{(0)}-\overline{T_{[0]}^{(0)}}\right) .
\end{aligned}
$$

By direct inspection one verifies that the integration-by-parts rules 3.5.8 do not yield relations between the simple fractions. In other words, the appearing simple fractions are all basic fractions. When evaluating weakly on the light cone (3.5.7), all basic fractions are of degree four, thus producing the same factor $\varepsilon^{-3}(i|\vec{\xi}|)^{-4}$. Using furthermore that no logarithmic divergences appear, we conclude that (3.8.4) must hold if the basic fractions are replaced by the corresponding regularization parameters. We can thus rewrite (3.8.4) as

$$
\left(c_{0}-c_{1}\left(s_{[0]}-s_{[3]}\right)\right) j_{a}-m^{2}\left(c_{2} \hat{Y}^{2}+c_{3} \dot{Y} \grave{Y}-2 c_{1}\left(s_{[2]}-s_{[3]}\right) \dot{Y} \grave{Y}\right) A_{a}=\frac{c_{1}}{8 \pi} J_{a}
$$

where the constants $c_{0}, \ldots, c_{3}$ are the four regularization parameters corresponding to the basic fractions appearing in 3.7 .9 and $3.8 .5-3.8 .8$. Since we are free to multiply (3.8.9) by a non-zero constant, our field equations (3.8.9) involve three regularization parameters. For a given regularization scheme, these parameters can be computed to obtain numerical constants, as will be explored further in 33.8.6. Alternatively, these parameters can be regarded as empirical constants which take into account the unknown microscopic structure of space-time. Apart from these three constants, all the quantities in (3.8.9) are objects of macroscopic physics, defined independent of the regularization.

It remains to determine $s_{[0]}, s_{[2]}$ and $s_{[3]}$. As in (3.8.1), we again consider the leading order at the origin, and thus it suffices to compute the functions $s_{[p]}(x, y)$ at $x=y$. Let us 
begin with the calculation of $s_{[3]}$. Since the factors $T_{[3]}^{(1)}$ and $\overline{T_{[3]}^{(1)}}$ only appear in 3.7 .82 , the function $s_{[3]}$ is obtained by computing the smooth contributions to the fermionic projector generated by the microlocal chiral transformation. The vector contribution clearly drops out of (3.7.1). The pseudoscalar and bilinear contributions are even. As a consequence, the leading contribution to $\mathcal{R}$ involves a factor of $m$ and is thus of lower degree on the light cone (for details see $\$ 3.9 .1$ and $\$ 3.9 .2$ and Lemma B.3.1). Thus it remains to consider the axial contribution to the fermionic projector. According to (3.7.65), we obtain contributions from the different Dirac seas. Using the formulas 3.4.10)- 3.4.13) for the Fourier transform of the lower mass shell, we find that the relevant contribution of the $\beta^{\text {th }}$ Dirac sea involving the logarithmic pole and the constant term is given by

$$
P_{\beta}(x, y) \asymp \frac{m_{\beta}^{3}}{32 \pi^{3}} \log \left(m_{\beta}^{2}\left|\xi^{2}\right|\right)+c,
$$

where $c$ equals the constant $c_{0}$ in 3.4 .10$)$. Since in 3.8 .9 ) only the differences of the functions $s_{[p]}$ appear, we may always disregard this constant. Then the smooth contribution at the origin is given by (3.7.75). More precisely, collecting all prefactors and comparing with (3.8.3), we obtain

$$
s_{[3]}=\frac{1}{32 \pi^{3}} \frac{\sum_{\beta=1}^{3} m_{\beta}^{2} \log \left(m_{\beta}^{2}\right)\left(2 m_{\beta}^{2}-\sum_{\alpha \neq \beta} m_{\alpha}^{2}\right)}{\sum_{\beta=1}^{3} m_{\beta}^{2}\left(2 m_{\beta}^{2}-\sum_{\alpha \neq \beta} m_{\alpha}^{2}\right)} .
$$

The functions $s_{[0]}$ and $s_{[2]}$ are more difficult to compute. Therefore, we first state the result and discuss it afterwards.

Lemma 3.8.1. The operators $s_{[0]}$ and $s_{[2]}$ appearing in 3.8 .3 and $(3.8 .9$ ) have the form

$$
\begin{aligned}
s_{[0]} j_{\mathrm{a}} & =\frac{1}{3 \cdot 32 \pi^{3}} \sum_{\beta=1}^{3}\left(\log \left(m_{\beta}^{2}\right) j_{a}+f_{[0]}^{\beta} * j_{\mathrm{a}}\right)+\mathcal{O}\left(A_{a}^{2}\right) \\
s_{[2]} A_{\mathrm{a}} & =\frac{1}{32 \pi^{3} m^{2} \dot{Y} \grave{Y}} \sum_{\beta=1}^{3} m_{\beta}^{2}\left(\log \left(m_{\beta}^{2}\right) A_{\mathrm{a}}+f_{[2]}^{\beta} * A_{\mathrm{a}}\right)+\mathcal{O}\left(A_{a}^{2}\right),
\end{aligned}
$$

where the star denotes convolution, i.e.

$$
\left(f_{[p]}^{\beta} * h\right)(x)=\int f_{[p]}^{\beta}(x-y) h(y) d^{4} y .
$$

The convolution kernels are the Fourier transforms of the distributions

$$
\begin{aligned}
& \hat{f}_{[0]}^{\beta}(q)=6 \int_{0}^{1}\left(\alpha-\alpha^{2}\right) \log \left|1-\left(\alpha-\alpha^{2}\right) \frac{q^{2}}{m_{\beta}^{2}}\right| d \alpha \\
& \hat{f}_{[2]}^{\beta}(q)=\int_{0}^{1} \log \left|1-\left(\alpha-\alpha^{2}\right) \frac{q^{2}}{m_{\beta}^{2}}\right| d \alpha .
\end{aligned}
$$

Postponing the proof of this lemma to Appendix D, we here merely discuss the result. For clarity, we first remark that the convolution operators in (3.8.11) and (3.8.12) can also be regarded as multiplication operators in momentum space, defined by

$$
f_{[p]}^{\beta} * e^{-i q x}=\hat{f}_{[p]}^{\beta}(q) e^{-i q x}
$$


with the functions $\hat{f}_{[p]}^{\beta}$ as in 3.8.13 and 3.8.14. Next, we note that the integrands in 3.8 .13 and 3 3.8.14 only have logarithmic poles, so that the integrals are finite. In Appendix D, these integrals are even computed in closed form (see Lemma D.0.2 and Figure D.1). Next, we point out that these integrals vanish if $q^{2}=0$, because then the logarithm in the integrand is zero. Therefore, the convolutions by $f_{[p]}^{\beta}$ can be regarded as higher order corrections in $q^{2}$ to the field equations. Thus we can say that $s_{[0]}$ and $s_{[2]}$ are composed of constant terms involving logarithms of the Dirac masses, correction terms $f_{[p]}^{\beta}$ taking into account the dependence on the momentum $q^{2}$ of the bosonic potential, and finally correction terms of higher order in the bosonic potential.

The constant term in $s_{[0]}$ can be understood from the following simple consideration (the argument for $s_{[2]}$ is similar). The naive approach to determine the constant contribution to the $\beta^{\text {th }}$ Dirac sea is to differentiate 3.4 .10 at $a=m_{\beta}^{2}$ to obtain

$$
T_{[0]}^{(1)} \asymp \frac{1}{32 \pi^{3}}\left(\log \left(m_{\beta}^{2}\right)+1\right),
$$

where we again omitted the irrelevant constant $c_{0}$. Forming the sectorial projection and comparing with (3.8.3), we obtain the contribution

$$
s_{[0]} \asymp \frac{1}{3 \cdot 32 \pi^{3}} \sum_{\beta=1}^{3}\left(\log \left(m_{\beta}^{2}\right)+1\right) .
$$

This naive guess is wrong because there is also a contribution to the fermionic projector of the form $\sim \notin \xi_{k} j_{\mathrm{a}}^{k} T_{[0]}^{(0)}$, which when contracted with $\Varangle$ yields another constant term which is not taken into account by the formalism of Section 3.5 (see the term $\sqrt{\mathrm{B} .2 .3}$ in Appendix B). This additional contribution cancels the summand +1 in (3.8.15), giving the desired constant term in (3.8.11).

Next, it is instructive to consider the scaling behavior of the functions $s_{[p]}$ in the fermion masses. To this end, we consider a joint scaling $m_{\beta} \rightarrow L m_{\beta}$ of all masses. Sine the expressions (3.8.10), 3.8.11) and (3.8.12) have the same powers of the masses in the numerator and denominator, our scaling amounts to the replacement $\log \left(m_{\beta}^{2}\right) \rightarrow$ $\log \left(m_{\beta}^{2}\right)+2 \log L$. Using the specific form of the operators $s_{[p]}$, one easily verifies that the transformation of the constant terms can be described by the replacement $s_{[p]} \rightarrow$ $s_{[p]}+2 /\left(32 \pi^{3}\right) \log L$. We conclude that for differences of these operators as appearing in (3.8.9), the constant terms are indeed scaling invariant. In other words, the constant terms in the expressions $s_{[0]}-s_{[3]}$ and $s_{[2]}-s_{[3]}$ depend only on quotients of the masses $m_{1}$, $m_{2}$ and $m_{3}$.

Before discussing the different correction terms in (3.8.11) and (3.8.12), it is convenient to combine all the constant terms in (3.8.11), (3.8.12) and (3.8.9). More precisely, multiplying (3.8.9) by $96 \pi^{3} / c_{0}$ gives the following result.

THEOREM 3.8.2. The EL equations to degree four on the light cone give rise to the condition

$$
\left(C_{0}-f_{[0]} *\right) j_{\mathrm{a}}-\left(C_{2}-6 f_{[2]} *\right) A_{\mathrm{a}}=12 \pi^{2} J_{\mathrm{a}}+\mathcal{O}\left(A_{\mathrm{a}}^{2}\right)
$$

involving the axial bosonic potential $A_{\mathrm{a}}$, the corresponding axial current $j_{\mathrm{a}}$ and the axial Dirac current $J_{\mathrm{a}}$ (see (3.6.16), (3.7.4) and (3.7.8)). Here the convolution kernels are the 
Fourier transforms of the distributions

$$
\begin{aligned}
& \hat{f}_{[0]}(q)=\sum_{\beta=1}^{3} \hat{f}_{[0]}^{\beta}(q)=\sum_{\beta=1}^{3} 6 \int_{0}^{1}\left(\alpha-\alpha^{2}\right) \log \left|1-\left(\alpha-\alpha^{2}\right) \frac{q^{2}}{m_{\beta}^{2}}\right| d \alpha \\
& \hat{f}_{[2]}(q)=\sum_{\beta=1}^{3} m_{\beta}^{2} \hat{f}_{[0]}^{\beta}(q)=\sum_{\beta=1}^{3} m_{\beta}^{2} \int_{0}^{1} \log \left|1-\left(\alpha-\alpha^{2}\right) \frac{q^{2}}{m_{\beta}^{2}}\right| d \alpha
\end{aligned}
$$

(with the functions $\hat{f}_{[p]}^{\beta}$ as defined by (3.8.13) and (3.8.14)). The constants $C_{0}$ and $m_{1}^{2} C_{2}$ depend only on the regularization and on the ratios of the masses of the fermions.

With this theorem, we have derived the desired field equations for the axial potential $A_{\mathrm{a}}$. They form a linear hyperbolic system of equations involving a mass term, with corrections in the momentum squared and of higher order in the potential. It is remarkable that the corrections in the momentum squared are described by explicit convolutions, which do not involve any free constants. In order to make the effect of the convolution terms smaller, one must choose the constants $C_{0}$ and $C_{2}$ larger, also leading to a smaller coupling of the Dirac current. Thus the effect of the convolution terms decreases for a smaller coupling constant, but it cannot be arranged to vanish completely.

We proceed by explaining and analyzing the above theorem, beginning with the convolution operators $f_{[p]}(\$ 3.8 .2$ and the higher orders in the potential (\$3.8.3). In $\$ 3.8 .4$ we explain how the standard loop corrections of QFT appear in our model. In 3.8 .5 we explain why the Higgs boson does not appear in our framework. Finally, in $\$ 3.8 .6$ we compute the coupling constants and the bosonic rest mass for a few simple regularizations.

3.8.2. Violation of Causality and the Vacuum Polarization. In this section we want to clarify the significance of the convolution operators in the field equations (3.8.16). Our first step is to bring the convolution kernels into a more suitable form. For any $a>0$, we denote by $S_{a}$ the following Green's function of the Klein-Gordon equation,

$$
\begin{aligned}
S_{a}(x, y) & =\int \frac{d^{4} k}{(2 \pi)^{4}} \frac{\mathrm{PP}}{k^{2}-a} e^{-i k(x-y)} \\
& =-\frac{1}{2 \pi} \delta\left(\xi^{2}\right)+\frac{a}{4 \pi} \frac{J_{1}\left(\sqrt{a \xi^{2}}\right)}{\sqrt{a \xi^{2}}} \Theta\left(\xi^{2}\right),
\end{aligned}
$$

where in the last step we again set $\xi=y-x$ and computed the Fourier integral using the Bessel function $J_{1}$. This Green's function is obviously causal in the sense that it vanishes for spacelike $\xi$. Due to the principal part, it is the mean of the advanced and retarded Green's function; this choice has the advantage that $S_{a}$ is symmetric, meaning that $\overline{S_{a}(x, y)}=S_{a}(y, x)$. Expanding the Bessel function in a power series, the square roots drop out, showing that $S_{a}$ is a power series in $a$. In view of the explicit and quite convenient formula (3.8.18), it seems useful to express the convolution kernels in terms of $S_{a}$. This is done in the next lemma. 
Lemma 3.8.3. The distributions $f_{[p]}^{\beta}$ as defined by (3.8.13) and (3.8.14 can be written as

$$
\begin{aligned}
& f_{[0]}^{\beta}(x-y)=\int_{4 m_{\beta}^{2}}^{\infty}\left(S_{a}(x, y)+\frac{\delta^{4}(x-y)}{a}\right) \sqrt{a-4 m_{\beta}^{2}}\left(a+2 m_{\beta}^{2}\right) \frac{d a}{a^{\frac{3}{2}}} \\
& f_{[2]}^{\beta}(x-y)=\int_{4 m_{\beta}^{2}}^{\infty}\left(S_{a}(x, y)+\frac{\delta^{4}(x-y)}{a}\right) \sqrt{a-4 m_{\beta}^{2}} \frac{d a}{\sqrt{a}} .
\end{aligned}
$$

Proof. We first compute the Fourier transform of the distribution $\log \left|1-q^{2} / b\right|$ for given $b>0$. Using that $\lim _{a \rightarrow \infty} \log \left|1-q^{2} / a\right|=0$ with convergence as a distribution, we have

$$
\log \left|1-\frac{q^{2}}{b}\right|=-\int_{b}^{\infty} \frac{d}{d a} \log \left|1-\frac{q^{2}}{a}\right| d a=\int_{b}^{\infty}\left(\frac{\mathrm{PP}}{q^{2}-a}+\frac{1}{a}\right) d a .
$$

Now we can compute the Fourier transform with the help of 3.8 .17$)$. Setting $b=m_{\beta}^{2} /(\alpha-$ $\alpha^{2}$ ), we obtain

$$
\int \frac{d^{4} q}{(2 \pi)^{4}} \log \left|1-\left(\alpha-\alpha^{2}\right) \frac{q^{2}}{m_{\beta}^{2}}\right| e^{-i q(x-y)}=\int_{\frac{m_{\beta}^{2}}{\alpha-\alpha^{2}}}^{\infty}\left(S_{a}(x, y)+\frac{\delta^{4}(x-y)}{a}\right) d a .
$$

We finally integrate over $\alpha$, interchange the orders of integration,

$$
\int_{0}^{1} d \alpha\left(\alpha-\alpha^{2}\right)^{r} \int_{\frac{m_{\beta}^{2}}{\alpha-\alpha^{2}}}^{\infty} d a(\cdots)=\int_{4 m_{\beta}^{2}}^{\infty} d a(\cdots) \int_{0}^{1} d \alpha\left(\alpha-\alpha^{2}\right)^{r} \Theta\left(a-\frac{m_{\beta}^{2}}{\alpha-\alpha^{2}}\right),
$$

and compute the last integral.

Qualitatively speaking, this lemma shows that the distributions $f_{[p]}(x, y)$ can be obtained by integrating the Green's function $S_{a}$ over the mass parameter $a$ and by subtracting a suitable counter term localized at $\xi=0$. The interesting conclusion is that the convolution kernels $f_{[p]}(x, y)$ in the field equations 3.8.16) are weakly causal in the sense that they vanish for spacelike $\xi$. But they are not strictly causal in the sense that the past influences the future and also the future influences the past.

Before discussing whether and how such a violation of causality could be observed in experiments, we give a simple consideration which conveys an intuitive understanding for how the non-causal contributions to the field equations come about. For simplicity, we consider the linear perturbation $\Delta P$ of a Dirac sea of mass $m$ by a potential $\mathcal{B}$,

$$
\Delta P(x, y)=-\int d^{4} z\left(s_{m}(x, z) V(z) t_{m}(z, y)+t_{m}(x, z) V(z) s_{m}(z, y)\right),
$$

where $s_{m}$ is the Dirac Green's function and $t_{m}$ denotes the Dirac sea of the vacuum, i.e.

$$
t_{m}=(i \not \partial+m) T_{m^{2}} \quad \text { and } \quad s_{m}=(i \not \partial+m) S_{m^{2}}
$$

and $T_{m^{2}}$ and $S_{m^{2}}$ as defined by (3.4.9) and 3.8.17) (for details see [F5, eqs (2.4) and (2.5)] or Section 2.1). Let us consider the support of the integrand in (3.8.21). The Green's function $s_{m}$ vanishes outside the light cone (see (3.8.18)), whereas the distribution $t_{m}$ is non-causal (see (3.4.10) $)$. Thus in (3.8.21) we integrate over the union of the double light cone (meaning the interior of the light cones and their boundaries) centered at the points $x$ and $y$; see the left of Figure 3.3. In the limit $x \rightarrow y$, the integral in (3.8.21) will diverge, as becomes apparent in the poles of light cone expansion. But after subtracting these divergent contributions, we can take the limit $x \rightarrow y$ to obtain a well-defined integral 


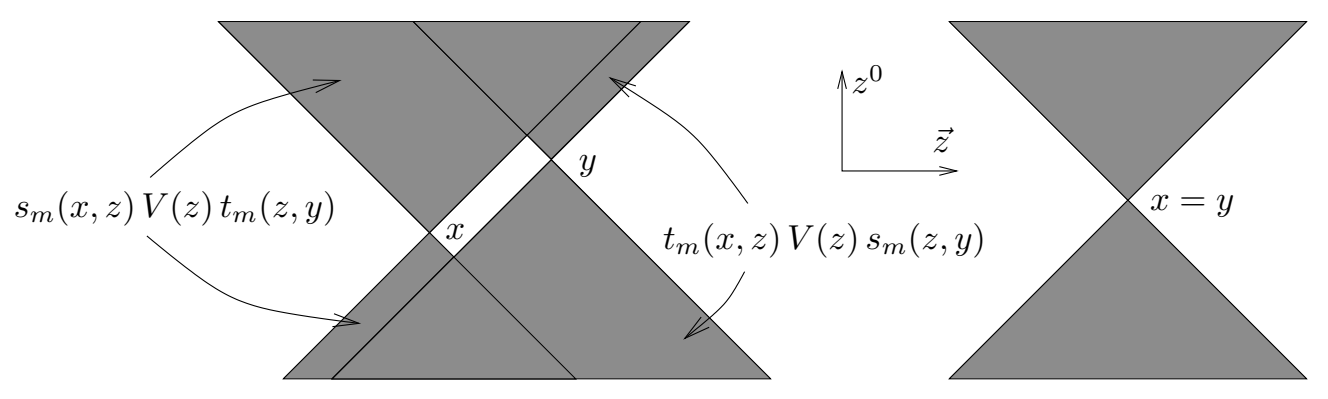

Figure 3.3. The support of the integrand in (3.8.21)

over the double light cone centered at the point $x=y$; see the right of Figure 3.3. Indeed, the finite contribution at the origin described by this integral corresponds precisely to the smooth contribution to the fermionic projector as considered in $\$ 3.8 .1$. This consideration explains why the distributions $f_{[p]}^{\beta}(x-y)$ vanish for spacelike $\xi$. Moreover, one sees that the distributions $f_{[p]}^{\beta}(x, y)$ are closely related to the pointwise product in position space of the Bessel functions appearing in the distributions $T_{m^{2}}(x, y)$ and $S_{m^{2}}(x, y)$ for timelike $\xi$. Going into more details, this argument could even be elaborated to an alternative method for computing the convolution kernels. However, for actual computations this alternative method would be less convenient than the resummation technique of Appendix D.

One might object that the above violation of causality occurs simply because in (3.8.21) we are working with the wrong Green's functions. Indeed, if in (3.8.21) the first and second factors $s_{m}$ were replaced by the regarded and advanced Green's function, respectively, the support of the integral would become strictly causal in the sense that $z$ must lie in the causal past of $x$ or $y$. However, modifying the Green's functions in this way is not admissible, as it would destroy the property that the Dirac sea is composed only of half of the solutions of the Dirac equation. More generally, the uniqueness of the perturbation expansion of the fermionic projector follows from a causality argument (see [F7, Section 2.2] or Section 2.1). Thus there is no freedom in modifying the perturbation expansion, and thus the above violation of causality cannot be avoided.

The violation of causality in the field equations breaks with one of the most fundamental physical principles. The immediate question is whether and how this effect could be verified in experiments. We conclude this section by discussing this question. Before beginning, we point out that the present paper is concerned with a simple fermion system, and one should be careful to draw physical conclusions from this oversimplified physical model. Also, the author has no expertise to address experimental issues. Nevertheless, it seems worth exploring the potential consequences of the causality violation in a few "Gedanken experiments," just to make sure that we do not get immediate contradictions to physical observations. In order to be closer to everyday physics, let us consider what happened if we inserted the nonlocal convolution term into Maxwell's equations. For simplicity, we consider one Dirac wave function $\psi$ of mass $m$. Thus dropping the mass term in (3.8.16) and choosing for convenience the Lorentz gauge, the modified Dirac-Maxwell equations become

$$
(i \not \partial+A-m) \psi=0, \quad-\left[1-\frac{e^{2}}{12 \pi^{2}} f_{[0]} *\right] \square A_{k}=e^{2} \bar{\psi} \gamma_{k} \psi,
$$


where we chose the constant $C_{0}$ such that without the convolution terms, the Maxwell equations take the familiar form $-\square A_{k}=e^{2} \bar{\psi} \gamma_{k} \psi$ (note that we again use the convention where the Dirac equation involves no coupling constants; see also Footnote 2 on page 180 . In view of Lemma 3.8.3, the square bracket is an integral operator which vanishes for spacelike distances. Furthermore, we see from (3.8.13) and 3.8.14) (for more details see Lemma D.0.2 that the functions $\hat{f}_{[0]}^{\beta}(q)$ diverge for large $q^{2}$ only logarithmically. Thus in view of the smallness of the fine structure constant $e^{2} / 4 \pi \approx 1 / 137$, for the energy range accessible by experiments the square bracket in $(3.8 .23)$ is an invertible operator. Thus we may write our modified Dirac-Maxwell equations as

$$
(i \not \partial+\not A-m) \psi=0, \quad-\square A_{k}=\left[1-\frac{e^{2}}{12 \pi^{2}} f_{[0]^{*}}\right]^{-1} e^{2} \bar{\psi} \gamma_{k} \psi,
$$

showing that the convolution term can be regarded as a modification of the source term. Alternatively, one may write the Maxwell equation in the standard form

$$
-\square \tilde{A}_{k}=e^{2} \bar{\psi} \gamma_{k} \psi
$$

with a so-called auxiliary potential $\tilde{A}$ and take the point of view that the convolution term only affects the coupling of the electromagnetic potential to the Dirac equation,

$$
(i \not \partial+\not A-m) \psi=0 \quad \text { with } \quad A:=\left[1-\frac{e^{2}}{12 \pi^{2}} f_{[0]^{*}}\right]^{-1} \tilde{A}
$$

(note that the wave and convolution operators commute, as they are both multiplication operators in momentum space). Both the "source form" (3.8.24) and the "coupling form" 3.8.25) and 3.8.26) are useful; they give different points of view on the same system of equations. We point out that, as the inverse of a causal operator, the operator on the right of (3.8.24) and (3.8.26) is again causal in the sense that its integral kernel vanishes for spacelike distances. Moreover, for large timelike distances, the kernel $f_{[0]}$ is oscillatory and decays. More specifically, writing the Green's function $S_{a}$ in (3.8.19) with Bessel functions and using their asymptotic expansion for large $\xi^{2}$, one finds that

$$
f_{[0]}^{\beta}(x-y) \sim m_{\beta}\left(\xi^{2}\right)^{-\frac{3}{2}} \cos \left(\sqrt{4 m_{\beta}^{2} \xi^{2}}+\varphi\right) \quad \text { if } \xi^{2} \gg m_{\beta}^{2}
$$

(where $\varphi$ is an irrelevant phase).

The formulation 3.8.25 and 3.8.26) reveals that our modified Dirac-Maxwell equations are of variational form. More precisely, they can be recovered as the EL equations corresponding to the modified Dirac-Maxwell action

$$
\mathcal{S}_{\mathrm{DM}}=\int_{\mathcal{M}}\left\{\bar{\psi}\left(i \not \partial+\left[1-\frac{e^{2}}{12 \pi^{2}} f_{[0]^{*}}\right]^{-1} \tilde{A}-m\right) \psi-\frac{1}{4 e^{2}} \tilde{F}_{i j} \tilde{F}^{i j}\right\} d^{4} x,
$$

where $\tilde{F}$ is the field tensor corresponding to the auxiliary potential. Hence by applying Noether's theorem, we obtain corresponding conserved quantities, in particular the total electric charge and the total energy of the system. Thus all conservation laws of the classical Dirac-Maxwell system still hold, but clearly the form of the conserved quantities must be modified by suitable convolution terms.

The simplest idea for detecting the convolution term is to expose an electron to a laser pulse. Then the convolution term in the Dirac equation (3.8.26) might seem to imply that the electron should "feel" the electromagnetic wave at a distance, or could even be influenced by a laser beam flying by in the future, at a time when the electron may already have moved away. However, such obvious violations of causality are impossible for the 
following reason: An electromagnetic wave satisfies the vacuum Maxwell equations $\square \tilde{A}=$ 0 (see (3.8.25)). Thus the momentum squared of the electromagnetic wave vanishes, implying that $f_{[0]} * \tilde{A}=0$, so that the convolution term in 3.8 .26 drops out. In more general terms, the convolution terms are constant if the bosonic field is on-shell. We conclude that the convolution terms can be detected only by off-shell bosonic fields, which according to 3.8 .25$)$ occur only at the electromagnetic sources.

Another idea for observing the convolution term is that, according to (3.8.24), it modifies the way the Dirac current generates an electromagnetic field. Due to the prefactor $e^{2} /\left(12 \pi^{2}\right)$ and in view of the fact that the kernel $f_{[0]}$ decays and has an oscillatory behavior (3.8.27), this effect will not be large, but it could nevertheless be observable. In particular, one may ask whether the positive and negative charges of protons and electrons still compensate each other in such a way that macroscopic objects appear neutral. If this were not the case, this would have drastic consequences, because then the electromagnetic forces would dominate gravity on the large scale. To analyze this question we consider for example a crystal containing exactly as many positive and negative charges. Then the corresponding auxiliary potential $\tilde{A}$ vanishes outside the crystal (except for dipole effects, which fall off rapidly with increasing distance). As a consequence, the potential $A$ defined by $(3.8 .26)$ also vanishes outside the crystal, and thus there are no observable electrostatic forces outside the crystal, in agreement with physical observations.

More generally, the above considerations show that the convolution term can lead to observable effects only if the sources of the electromagnetic field and the Dirac particles on which it acts are very close to each other, meaning that the whole interaction must take place on the scale of the Compton length of the electron. One conceivable way of measuring this effect is by considering electron-electron scattering. In order to concentrate on the violation of causality, it seems preferable to avoid the noise of the usual electromagnetic interactions by considering two wave packets which stay causally separated, but nevertheless come as close as the Compton length. In this case, an electron in the future could even affect the motion of an electron in the past. However, due to the Heisenberg uncertainty principle, localizing a wave packet on the Compton scale implies that the energy uncertainty is of the order of the rest mass, so that pair creation becomes a relevant effect. Therefore, arranging such wave packets seems a very difficult task.

Another potential method for observing the convolution term is to get a connection to the high-precision measurements of atomic spectra. Thus we conclude the discussion by considering the static situation. Integrating the Green's function (3.8.17) over time, we can compute the remaining spatial Fourier integral with residues to obtain the familiar Yukawa potential,

$$
\begin{aligned}
V_{a}(\vec{\xi}) & :=\int_{-\infty}^{\infty} S_{a}(x, y) d \xi^{0}=-\int_{\mathbb{R}^{3}} \frac{d \vec{k}}{(2 \pi)^{3}} \frac{e^{-i \vec{k} \vec{\xi}}}{|\vec{k}|^{2}+a} \\
& =-\frac{1}{(2 \pi)^{2}} \int_{0}^{\infty} \frac{k^{2} d k}{k^{2}+a} \int_{-1}^{1} e^{-i k r \cos \vartheta} d \cos \vartheta \\
& =\frac{1}{(2 \pi)^{2} i r} \int_{-\infty}^{\infty} \frac{k}{k^{2}+a} e^{-i k r} d k=-\frac{1}{4 \pi} \frac{e^{-\sqrt{a} r}}{r}
\end{aligned}
$$


where we set $r=|\vec{\xi}|$. Hence in the static case, the convolution operator reduces to the three-dimensional integral

$$
\left(f_{[0]} * h\right)(\vec{x})=\int_{\mathbb{R}^{3}} f_{[0]}(\vec{x}-\vec{y}) h(\vec{y}) d \vec{y}
$$

involving the kernel

$$
f_{[0]}(\vec{\xi})=\frac{1}{3} \sum_{\beta=1}^{3} \int_{4 m_{\beta}^{2}}^{\infty}\left[V_{a}(\vec{\xi})+\frac{\delta^{3}(\vec{\xi})}{a}\right] \sqrt{a-4 m_{\beta}^{2}}\left(a+2 m_{\beta}^{2}\right) \frac{d a}{a^{\frac{3}{2}}} .
$$

We now consider a classical point charge $Z e$ located at the origin. In order to compute the corresponding electric field $A_{0}$, we consider the corresponding Maxwell equation (3.8.24),

$$
\Delta A_{0}(\vec{x})=\left[1-\frac{e^{2}}{12 \pi^{2}} f_{[0]^{*}}\right]^{-1} Z e^{2} \delta^{3}(\vec{x})=Z e^{2} \delta^{3}(\vec{x})+\frac{Z e^{4}}{12 \pi^{2}} f_{[0]}(\vec{x})+\mathcal{O}\left(e^{6}\right) .
$$

In order to solve for $A_{0}$, we convolute both sides with the Newtonian potential $V_{0}(\xi)=$ $-1 /(4 \pi r)$. To compute the resulting convolution of the Newtonian potential with $f_{[0]}$, we first observe that $(3.8 .28)$ involves the Yukawa potential $V_{a}$. Since convolution corresponds to multiplication in momentum space, we can use the simple transformation

$$
\frac{1}{|\vec{k}|^{2}} \frac{1}{|\vec{k}|^{2}+a}=\frac{1}{a}\left(-\frac{1}{|\vec{k}|^{2}+a}+\frac{1}{|\vec{k}|^{2}}\right)
$$

to conclude that

$$
\left(V_{0} * V_{a}\right)(\vec{x})=\frac{1}{a}\left(V_{a}(\vec{x})-V_{0}(\vec{x})\right) .
$$

We thus obtain

$$
A_{0}(\vec{x})=-\frac{Z e^{2}}{4 \pi r}+\frac{Z e^{4}}{12 \pi^{2}} \sum_{\beta=1}^{3} \int_{4 m_{\beta}^{2}}^{\infty}\left[-\frac{e^{-\sqrt{a}|\vec{\xi}|}}{4 \pi r} \frac{1}{a}\right] \sqrt{a-4 m_{\beta}^{2}}\left(a+2 m_{\beta}^{2}\right) \frac{d a}{a^{\frac{3}{2}}} .
$$

Here the first summand is the Coulomb potential, whereas the second summand is an additional short-range potential. This is very similar to the situation for the relativistic correction described by the Darwin term (a relativistic correction to the Schrödinger equation; see for example [S. Section 3.3]). Concentrating the short range potential at the origin by the replacement

$$
\frac{e^{-\sqrt{a} r}}{4 \pi r} \rightarrow \frac{1}{a} \delta^{3}(\vec{x})
$$

we can carry out the $a$-integral to obtain

$$
A_{0}(\vec{x})=-\frac{Z e^{2}}{4 \pi r}-\frac{Z e^{4}}{60 \pi^{2}} \sum_{\beta=1}^{3} \frac{1}{m_{\beta}^{2}} \delta^{3}(\vec{x}) .
$$

We thus end up with a correction to the Dirac Hamiltonian of the form

$$
H_{\text {noncausal }}=\frac{Z e^{4}}{60 \pi^{2}} \sum_{\beta=1}^{3} \frac{1}{m_{\beta}^{2}} \delta^{3}(\vec{x}) .
$$

This coincides precisely with the Uehling potential which describes the one-loop vacuum polarization in the static situation (see for example [PS, eq. (7.94) and (7.95)]). At first sight, it might be surprising that we get the same result as in perturbative QFT, although we did not consider a fermionic loop diagram and did not encounter the usual 
ultraviolet divergences. In order to see the connection, it is preferable to reconsider the original derivation by Uehling and Serber $[\mathbf{U}$, Se], which was based on earlier papers by Dirac D2] and Heisenberg [He. Similar to (3.3.8), Dirac considers the sum over all sea states,

$$
R\left(t, \vec{x} ; t^{\prime}, \vec{x}^{\prime}\right)=\sum_{l \text { occupied }} \psi_{l}(t, \vec{x}) \overline{\psi_{l}\left(t^{\prime}, \vec{x}^{\prime}\right)}
$$

where the wave functions $\psi_{l}$ are solutions of the Dirac equation

$$
(i \not \partial+e A(t, \vec{x})-m) \psi_{l}(t, \vec{x})=0 .
$$

Thus up to an irrelevant overall constant, $R$ coincides precisely with the kernel of the fermionic projector $P(x, y)$. Dirac realizes that $R$ has singularities on the light cone and discusses their form. Heisenberg pushes the calculation a bit further and, using physical conservation laws and the requirement of gauge invariance, he brings the singular contribution to $R$ into a canonical form. The he argues that this singular contribution to $R$ should simply be dropped. Uehling and Serber took the remaining regular contribution to $R$ as the starting point to derive the corresponding correction to the Maxwell equations. Since the regular contribution to $R$ coincides precisely with the non-causal contributions in (3.4.14) (albeit in a less explicit form where the underlying causal structure is not apparent), it is clear that 3.8 .29 coincides with the usual Uehling potential.

We conclude that the non-causal correction reproduces the usual vacuum polarization as described by the Uehling potential. The main difference in our approach is that the singular contributions to the fermionic projector are not disregarded or removed, but they are carried along in our analysis. These singular contributions then drop out of the Euler-Lagrange equations corresponding to our action principle $(3.2 .9)$. In this way, all divergences disappear. The remaining finite contributions to the fermionic projector give rise to the bosonic current and mass terms in the resulting field equations (3.8.16), and also yield the convolution terms which describe the vacuum polarization. The main advantage of the fermionic projector approach is that no counter terms are needed. The back-reaction of the Dirac sea on the electromagnetic field is finite, no divergences occur. Moreover, as we do not need counter terms computed from the Minkowski vacuum, the setting immediately becomes background independent. It is to be expected (although it has not yet been worked out in detail) that the singularities of the fermionic projector will also drop out of the Euler-Lagrange equations if one sets up the theory in curved space-time.

We finally remark that the connection to Feynman diagrams will be explained in more detail in 3.8 .4 .

3.8.3. Higher Order Non-Causal Corrections to the Field Equations. The non-causal convolution terms in the previous section were obtained by computing the non-causal contributions in (3.4.14) at the origin, considering the first order of the perturbation expansion (3.4.6). Likewise, the higher orders of this expansion also contribute to $\tilde{P}^{l e}$ and $\tilde{P}^{\text {he }}$, giving rise to higher order non-causal corrections to the field equations. In this section we briefly discuss the structure of these correction terms (computing them in detail goes beyond the scope of this book).

It is natural to distinguish between the low and high energy contributions. The noncausal low energy contribution $\tilde{P}^{\text {le }}$ in 3.4 .14 can be computed at the origin to every order in $\mathcal{B}$ by extending the resummation technique of Appendix $D$ to higher order (more precisely, according to the residual argument, we again get sums of the form D.0.12, 
but with nested line integrals and multiple series, which are to be carried out iteratively). Similar as explained to first order after Lemma 3.8.3, the resulting corrections to the field equation are weakly causal in the sense that they can be described by convolutions with integral kernels which vanish for spacelike distances. Thus they have the same mathematical structure, but are clearly much smaller than the convolution terms in $\$ 3.8 .2$.

The non-causal high energy contribution $\tilde{P}^{\text {he }}$ in (3.4.14) is more interesting, because it gives rise to corrections of different type. For simplicity, we explain their mathematical structure only in the case of one generation and only for the leading contribution to $\tilde{P}^{\text {he }}$ (see [FG1] for details)

$$
\tilde{P}^{\text {he }}=-\frac{\pi^{2}}{4}\left(t_{m} \mathcal{B} \overline{t_{m}} \mathcal{B} t_{m}-\overline{t_{m}} \mathcal{B} t_{m} \mathcal{B} \overline{t_{m}}\right)+\mathcal{O}\left(\mathcal{B}^{3}\right),
$$

where we set

$$
t_{m}=(i \not \partial+m) T_{m^{2}} \quad \text { and } \quad \overline{t_{m}}=(i \not \partial+m) \overline{T_{m^{2}}},
$$

and $\overline{T_{a}}$ is the complex conjugate of the distribution $T_{a},(3.4 .9)$. Thus the distributions $t_{m}$ and $\overline{t_{m}}$ are supported on the lower and upper mass shell, respectively. Evaluating this expression at the origin gives

$$
\begin{array}{r}
\tilde{P}^{\text {he }}(x, x)=-\frac{\pi^{2}}{4} \int_{\mathscr{M}} d^{4} z_{1} \int_{\mathscr{M}} d^{4} z_{2}\left(t_{m}\left(x, z_{1}\right) \mathcal{B}\left(z_{1}\right) \overline{t_{m}}\left(z_{1}, z_{2}\right) \mathcal{B}\left(z_{2}\right) t_{m}\left(z_{2}, x\right)\right. \\
\left.-\overline{t_{m}}\left(x, z_{1}\right) \mathcal{B}\left(z_{1}\right) t_{m}\left(z_{1}, z_{2}\right) \mathcal{B}\left(z_{2}\right) \overline{t_{m}}\left(z_{2}, x\right)\right)+\mathcal{O}\left(\mathcal{B}^{3}\right) .
\end{array}
$$

This is similar to a second order tree diagram, but instead of Green's functions it involves the projectors onto the lower and upper mass shells, which appear in alternating order. The expression is well-defined and finite (see [F7, Lemma 2.2.2] or Lemma 2.1.2). Similar to the correction terms in Theorem 3.8.2, our expression is a convolution, but now it involves two integrals, each of which contains one factor of $\mathcal{B}$. Consequently, the integral kernel depends on two arguments $z_{1}$ and $z_{2}$. The interesting point is that this integral kernel does not vanish even if the vectors $z_{1}-x$ or $z_{2}-x$ are space-like. Thus the corresponding corrections to the field equations violate causality even in the strong sense that in addition to an influence of the future on the past, there are even interactions for spacelike distances. This surprising result is in sharp contrast to conventional physical theories. However, since for space-like separation the kernels $t_{m}$ decay exponentially fast on the Compton scale, the effect is extremely small. In particular, describing this exponential decay by the Yukawa potential, this effect could be described similar to the correction (3.8.28) and (3.8.29). But compared to the latter first order correction, the second order correction by $P^{\text {he }}$ would be smaller by a factor $e^{2}$. In view of the discussion in 3.8 .2 , measuring this correction is at present out of reach. Thus it seems that the only promising approach for detecting an effect of the high energy contribution is to look for an experiment which is sensitive to interactions between regions of space-time with spacelike separation, without being affected by any causal interactions.

3.8.4. The Standard Quantum Corrections to the Field Equations. We now explain how the quantum corrections due to the Feynman loop diagrams arise in our model. We will recover all the standard quantum corrections. Moreover, we will obtain quantum corrections of the previously described non-causal terms (see \$3.8.2 and $\$ 3.8 .3$ ). For clarity, we proceed in several steps and begin by leaving out the non-causal convolution terms in the field equations (3.8.16). Furthermore, we consider only one Dirac 
particle of mass $m$. Under this simplifying assumption, the interaction is described by the coupled Dirac-Yang/Mills equations

$$
(i \not \partial+\Gamma \not A-m) \psi=0, \quad \partial_{k l} A^{l}-\square A_{k}-M^{2} A_{k}=e^{2} \bar{\psi} \Gamma \gamma_{k} \psi,
$$

where $A$ is the axial potential, and the bosonic rest mass $M$ and the coupling constant $e$ are determined from (3.8.16) by setting $M^{2}=C_{2} / C_{0}$ and $e^{2}=12 \pi^{2} / C_{0}$. We point out that the wave function $\psi$ and the bosonic field $A$ in (3.8.31) are classical in the sense that no second quantization has been performed.

The equations (3.8.31) form a coupled system of nonlinear hyperbolic partial differential equations. For such a system, standard methods give local existence and uniqueness results (see for example $[\mathbf{J}$, Section 5.3] or [T, Chapter 16]), but constructing global solutions is a very difficult task. Therefore, we must rely on a perturbative treatment, giving us a connection to Feynman diagrams. Although this connection is quite elementary, it does not seem to be well-known to mathematicians working on partial differential equations. In physics, on the other hand, Feynman diagrams are usually derived from second quantized fields, where the connection to nonlinear partial differential equations is no longer apparent. Therefore, we now explain the procedure schematically from the basics, hopefully bridging a gap between the mathematics and physics communities. In order to be in a simpler and more familiar setting, we consider instead of (3.8.31) the Dirac-Maxwell equations in the Lorentz gauge, as considered in quantum electrodynamics (see for example [BD] $)^{6}$

$$
(i \not \partial+e \not A-m) \psi=0, \quad-\square A_{k}=e \bar{\psi} \gamma_{k} \psi .
$$

The natural question in the theory of hyperbolic partial differential equations is the Cauchy problem, where we seek for solutions of (3.8.32) for given initial values

$$
\left.\psi(t, \vec{x})\right|_{t=0}=\psi_{0}(\vec{x}),\left.\quad A(t, \vec{x})\right|_{t=0}=A_{0}(\vec{x}),\left.\quad \partial_{t} A(t, \vec{x})\right|_{t=0}=A_{1}(\vec{x}) .
$$

In preparation, we formulate the equations as a system which is of first order in time. To this end, we introduce the field $\Phi$ with components

$$
\Phi=\left(\begin{array}{c}
\psi \\
A \\
i \partial_{t} A
\end{array}\right),
$$

and write the system in the Hamiltonian form

$$
i \partial_{t} \Phi(t, \vec{x})=H(\Phi(t, \vec{x})):=H_{0} \Phi+e B(\Phi),
$$

6 In order to bring the system (3.8.31) into a comparable form, one first takes the divergence of the Yang/Mills equation to obtain

$$
-M^{2} \partial_{k} A^{k}=e^{2} \overline{\partial_{k} \psi} \Gamma \gamma^{k} \psi+e^{2} \bar{\psi} \Gamma \gamma^{k} \partial_{k} \psi=-2 i e^{2} m \bar{\psi} \Gamma \psi,
$$

where in the last step we used the Dirac equation. In particular, the divergence of $A$ in general does not vanish. It seems convenient to subtract from $A$ the gradient of a scalar field $\Phi$,

$$
B_{k}:=A_{k}-\partial_{k} \Phi
$$

in such a way that the new potential $B$ becomes divergence-free. This leads to the system of equations

$$
(i \not \partial-m+\Gamma \not B+\Gamma(\not \partial \Phi)) \psi=0, \quad-\square \Phi=-\frac{2 i e^{2} m}{M^{2}} \bar{\psi} \Gamma \psi, \quad\left(-\square-M^{2}\right) B_{k}=e^{2} \bar{\psi} \Gamma \gamma_{k} \psi+M^{2} \partial_{k} \Phi .
$$

This system has the same structure as 3.8.32, and it can be analyzed with exactly the same methods. For the handling of the factors $e$ see Footnote 2 on page 180 
where in the last step we decomposed the Hamiltonian into its linear and non-linear parts given by

$$
H_{0}=\left(\begin{array}{ccc}
-i \gamma^{0} \vec{\gamma} \vec{\nabla}+\gamma^{0} m & 0 & 0 \\
0 & 0 & 1 \\
0 & -\Delta & 0
\end{array}\right), \quad B(\Phi)=\left(\begin{array}{c}
-\gamma^{0} A \psi \\
0 \\
\bar{\psi} \gamma \psi
\end{array}\right) .
$$

In the case $e=0$, we have a linear equation, which is immediately solved formally by exponentiation,

$$
\Phi(t)=e^{-i t H_{0}} \Phi_{0}
$$

where we set $\Phi_{0}=\left.\Phi\right|_{t=0}$. This equation is given a rigorous meaning by writing the so-called time evolution operator $e^{-i t H_{0}}$ as an integral operator in the spatial variables.

Lemma 3.8.4. For any $t \geq 0$, the operator $e^{-i t H_{0}}$ can be written as

$$
\left(e^{-i t H_{0}} \Phi\right)(\vec{x})=\int_{\mathbb{R}^{3}} R_{t}(\vec{x}-\vec{y}) \Phi(\vec{y}) d \vec{y},
$$

where the integral kernel is the distribution

$$
R_{t}(\vec{x})=\left(\begin{array}{ccc}
s_{m}^{\wedge}(t, \vec{x})\left(i \gamma^{0}\right) & 0 & 0 \\
0 & -\partial_{t} S_{0}^{\wedge}(t, \vec{x}) & i S_{0}^{\wedge}(t, \vec{x}) \\
0 & -i \Delta S_{0}^{\wedge}(t, \vec{x}) & -\partial_{t} S_{0}^{\wedge}(t, \vec{x})
\end{array}\right),
$$

which involves the retarded Green's functions defined by

$$
\begin{aligned}
S_{a}^{\wedge}(x) & =\lim _{\varepsilon \searrow 0} \int \frac{d^{4} k}{(2 \pi)^{4}} \frac{e^{-i k x}}{k^{2}-m^{2}+i \varepsilon k^{0}} \\
s_{m}^{\wedge}(t, \vec{x}) & =\left(i \not \partial_{x}+m\right) S_{m^{2}}^{\wedge}(x) .
\end{aligned}
$$

Proof. Using that for any $t>0$, the Green's function $S_{a}^{\wedge}$ is a solution of the KleinGordon equation $(-\square-a) S_{a}(x)=0$, a short calculation using (3.8.40) shows that (3.8.37) is a solution of the equation $\left(i \partial_{t}-H_{0}\right)\left(e^{-i t H_{0}} \Phi\right)=0$. In order to verify the correct initial conditions, we differentiate $S_{a}^{\wedge}$ with respect to time and carry out the $t$-integration with residues to obtain

$$
\begin{array}{r}
\lim _{t \searrow 0} \partial_{t}^{n} S_{a}^{\wedge}(t, \vec{x})=\frac{1}{(2 \pi)^{4}} \int_{\mathbb{R}^{3}} d \vec{k} e^{i \vec{k} \vec{x}} \lim _{\varepsilon, t \searrow 0} \int_{-\infty}^{\infty} \frac{(-i \omega)^{n}}{\omega^{2}-|\vec{k}|^{2}-m^{2}+i \varepsilon \omega} e^{-i \omega t} d \omega \\
=\left.\frac{1}{(2 \pi)^{4}} \int_{\mathbb{R}^{3}} d \vec{k} e^{i \vec{k} \vec{x}}(-2 \pi i) \frac{(-i \omega)^{n}}{2 \omega}\right|_{\omega= \pm \sqrt{|\vec{k}|^{2}+m^{2}}}=\left\{\begin{array}{cc}
0 & \text { if } n=0 \\
-\delta^{3}(\vec{x}) & \text { if } n=1 .
\end{array}\right.
\end{array}
$$

Using this result in 3.8 .40 and $(3.8 .38)$ shows that indeed $\lim _{t \searrow 0} R_{t}(\vec{x})=\delta^{3}(\vec{x})$.

In the nonlinear situation $e \neq 0$, it is useful to work in the so-called "interaction picture" (see for example [S2, Section 8.5]). We thus employ the ansatz

$$
\Phi(t)=e^{-i t H_{0}} \Phi_{\text {int }}(t),
$$

giving rise to the nonlinear equation

$$
i \partial_{t} \Phi_{\text {int }}=e B_{\text {int }}\left(\Phi_{\text {int }}(t)\right)
$$

where

$$
B_{\text {int }}\left(\Phi_{\text {int }}(t)\right)=e^{i t H_{0}} B\left(e^{-i t H_{0}} \Phi_{\text {int }}(t)\right) .
$$


We regard (3.8.42) as an ordinary differential equation in time, which in view of (3.8.37) is nonlocal in space. From 3.8 .41 one sees that $\Phi_{\text {int }}$ comes with the initial data $\left.\Phi_{\text {int }}\right|_{t=0}=$ $\Phi_{0}$. Taking a power ansatz in $e$,

$$
\Phi_{\text {int }}(t)=\Phi_{\text {int }}^{(0)}(t)+e \Phi_{\text {int }}^{(1)}(t)+e^{2} \Phi_{\text {int }}^{(2)}(t)+\cdots,
$$

a formal solution of the Cauchy problem for $\Phi_{\text {int }}$ is obtained by integrating 3.8 .42 inductively order by order,

$$
\begin{aligned}
\Phi_{\text {int }}^{(0)}(t) & =\Phi_{0}, \quad \Phi_{\text {int }}^{(1)}(t)=-i \int_{0}^{t} B_{\text {int }}\left(\Phi_{\text {int }}^{(0)}(\tau)\right) d \tau \\
\Phi_{\text {int }}^{(2)}(t) & =-i \int_{0}^{t} \nabla B_{\text {int }}\left(\Phi_{\text {int }}^{(0)}(\tau)\right) \cdot \Phi_{\text {int }}^{(1)}(\tau) d \tau \\
& =(-i)^{2} \int_{0}^{t} d \tau \nabla B_{\text {int }}\left(\Phi_{\text {int }}^{(0)}(\tau)\right) \int_{0}^{\tau} d \sigma B_{\text {int }}\left(\Phi_{\text {int }}^{(0)}(\sigma)\right), \quad \ldots
\end{aligned}
$$

(here $\nabla B$ denotes the Jacobi matrix of $B$, where as in 3.5 .15 we consider the real and imaginary parts of the arguments as independent variables). Substituting these formulas into (3.8.41), we obtain the desired solution $\Phi$ of the original Cauchy problem expressed as a sum of iterated time integrals, involving intermediate factors of the free time evolution operator $e^{-i \tau H_{0}}$. In particular, we obtain to second order

$$
\begin{aligned}
\Phi(t)= & e^{-i t H_{0}} \Phi_{0}-i e \int_{0}^{t} e^{-i(t-\tau) H_{0}} B\left(e^{-i \tau H_{0}} \Phi_{0}\right) d \tau \\
& -e^{2} \int_{0}^{t} d \tau e^{-i(t-\tau) H_{0}} \nabla B\left(e^{-i \tau H_{0}} \Phi_{0}\right) \int_{0}^{\tau} d \sigma e^{-i(\tau-\sigma) H_{0}} B\left(e^{-i \sigma H_{0}}\right)+\mathcal{O}\left(e^{3}\right) .
\end{aligned}
$$

We remark that in the case when $B(\Phi)$ is linear in $\Phi$, this expansion simplifies to the wellknown Dyson series (also referred to as the time-ordered exponential). In view of (3.8.34), we have derived a unique formal solution of the Cauchy problem $(3.8 .32)$ and 33.8 .33$)$.

Combining the above expansion of $\Phi(t)$ with the formula for the time evolution operator in Lemma 3.8.4, one can write the above perturbation expansion in a manifestly covariant form. Namely, when multiplying the operators $R_{t}$ with $B$ (or similarly $\nabla B$ or higher derivatives), the factors $\gamma^{0}$ in the first component of (3.8.38) and in the formula for $B$ in (3.8.36) cancel each other, giving the Lorentz invariant expression $s^{\wedge} A$. Likewise, the Dirac current in 3.8 .36 multiplies the retarded Green's function $S_{0}^{\wedge}$. Moreover, we can combine the spatial and time time integrals to integrals over Minkowski space. In this way, we can identify the contributions to the perturbation expansion with the familiar Feynman diagrams. More precisely, every integration variable corresponds to a vertex of the diagram, whereas the bosonic and fermionic Green's functions $S_{0}^{\wedge}$ and $s_{m}^{\wedge}$ are written as wiggled and straight lines, respectively. Denoting the argument of the solution $\Phi(t, \vec{y})$ by $y$, whereas $x=(0, \vec{x})$ stands for the argument of the initial values, we obtain all tree diagrams as exemplified in Figure 3.4 (left). We come to the following conclusion:

- All tree diagrams are obtained from the nonlinear system of partial differential equations 3.8.32, working purely with classical fields.

We point out that the bosonic loop diagrams are missing as a consequence of the strict time ordering in the solution of the Cauchy problem. However, if one introduces a microscopic background field or takes into account the mechanism of microscopic mixing, then one also gets bosonic loop diagrams (as for example in Figure 3.4 (right)). We shall not enter these constructions here but refer the interested reader to [FT1, F17. 

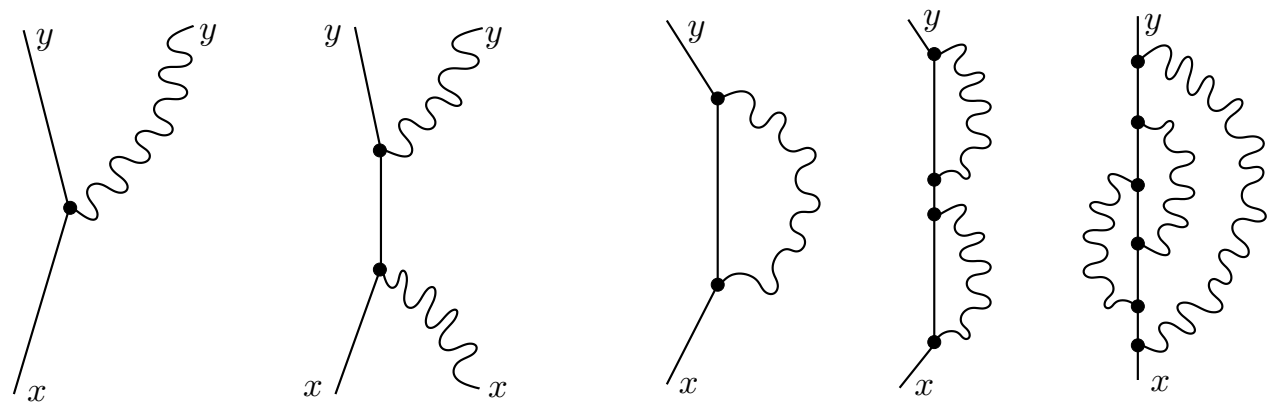

FiguRE 3.4. Feynman tree diagrams (left) and bosonic loop diagrams (right)

In order to make the connection to QFT clearer, we point out that in quantum physics one usually does not consider the initial value problem (3.8.33). Instead, one is interested in the $n$-point functions, which give information about the correlation of the fields at different space-time points. The two-point function is obtained by choosing initial values involving $\delta^{3}$-distributions. Similarly, all the $n$-point functions can be recovered once the solution of the Cauchy problem is known. Thus from a conceptual point of view, the only difference between our expansion and the Feynman diagrams in QFT is that, since in quantum physics the Feynman diagrams do not come from an initial value problem, there is a freedom in choosing the Green's function. Note that in the setting of the Cauchy problem, one necessarily gets the retarded Green's function (see (3.8.38)). In contrast, in QFT one is free to work instead with any other Green's function. Indeed, different choices lead to different approaches for handling the perturbation series. The most common choice is the so-called Feynman propagator (see for example [BD]), which is motivated from the physical picture that the positive frequencies (describing particles) move to the future, whereas the negative frequencies (corresponding to anti-particles) move to the past. In this standard approach, the loop diagrams diverge. This problem is bypassed in the renormalization program by first regularizing the diagrams, and then removing the regularization while simultaneously adjusting the masses and coupling constants (see for example [C1]). A QFT is called renormalizable if this renormalization procedure works to all orders in perturbation theory, involving only a finite number of effective constants. There are different equivalent renormalization procedures, the most common being dimensional renormalization (see for example [PS]). But the Feynman propagator is not a canonical choice, and indeed this choice suffers from the problem of not being invariant under general coordinate transformations (for more details see [F7, Section 2.1]). An alternative method, which seems natural but has not yet been worked out, would be to extend the choice of Green's functions in the causal perturbation expansion 3.4.6 (see also [FG1, FT2 ) to the loop diagrams. Yet another method is the so-called causal approach based on ideas of Epstein and Glaser [EG], which uses the freedom in choosing the Green's function to avoid the divergences of QFT (see also [S1]). We also mention that our above derivation of Feynman diagrams is certainly not the most sophisticated or most elegant method. Maybe the cleanest method for the formal perturbation expansion is obtained in the framework of path integrals (see for example $[\mathbf{K 2}, \mathbf{P}]$ ).

Recall that one simplification of the system (3.8.31) was that we considered only one Dirac particle and disregarded the interaction of this particle with the states of the Dirac sea. In particular, we did not allow for the creation of a particle/anti-particle pair. This 

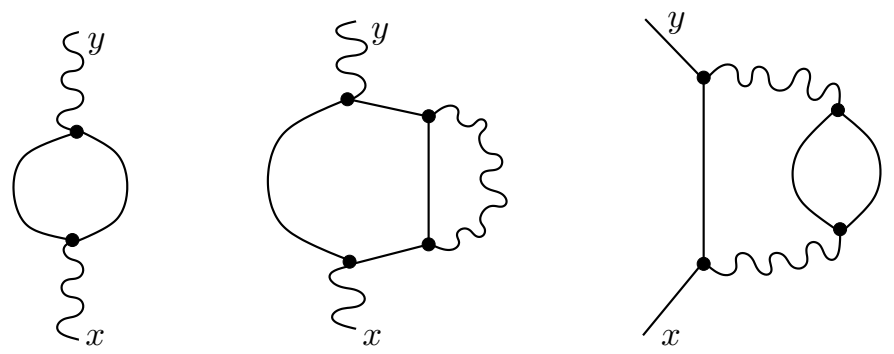

Figure 3.5. Typical Feynman diagrams involving fermion loops

shortcoming is reflected in our perturbation expansion in that the fermionic loop diagrams are missing (see Figure 3.5 for a few examples). However, we already encountered the loop diagram in Figure 3.5 (left) when discussing the vacuum polarization in $\$ 3.8 .2$. This shows that the fermion loops appear once we take into account the convolution terms in 3.8.16) and the other non-causal corrections mentioned in 3.8.3. In order to include these corrections in the above expansion, we simply add them to the perturbation operator $e B$ in 3.8.35). We conclude that

- The framework of the fermionic projector in the continuum limit yields all tree diagrams and all fermionic loop diagrams.

We point out that this statement does not imply that the framework of the fermionic projector is equivalent to perturbative QFT. As a major difference, the perturbation expansion of the fermionic projector involves a non-trivial combinatorics of operator products involving different types of Green's functions and fundamental solutions (see [FG1] and Section 2.1 for details or 3.8.30 for an example of a contribution which is absent in standard QFT). This difference has no influence on the singularities of the resulting Feynman diagrams, and thus we expect that the renormalizability of the theory is not affected. But the higher-loop radiative corrections should depend on the detailed combinatorics, giving the hope to obtain small deviations from standard QFT which might be tested experimentally.

We close this section with two remarks. First, we again point out that in the above derivation of the Feynman diagrams, we worked with classical bosonic fields. This raises the question why a quantization of the bosonic fields is at all needed, and what this "quantization" actually means. Here we shall not enter a discussion of this point, but refer the reader to [F8, Section 4] and to the constructions in [F14, [F17]. The second remark is that the described method of first taking the continuum limit, then expanding the resulting equations in terms of Feynman diagrams and renormalizing these diagrams should be considered as preliminary. This method has the great advantage that it gives a simple connection to Feynman diagrams and to the renormalization program, making it easier to compare our approach to standard QFT. But ultimately, a fully convincing theory should work exclusively with the regularized fermionic projector, thereby completely avoiding the ultraviolet divergences of QFT. Before one can attack this program, one needs to have a better understanding of our variational principle in discrete space-time.

3.8.5. The Absence of the Higgs Boson. In this section we compare the mechanism leading to the mass term in the field equations (see (3.7.7) and (3.8.16) ) with the Higgs mechanism of the standard model. Clearly, our framework is considerably different from that of the standard model, so that no simple comparison is possible. But in order 
to make the connection between the formalisms as close as possible, we can consider the effective action of the continuum limit and compare it to the action of a corresponding model involving a Higgs field. For simplicity leaving out the non-causal convolution terms and considering only one particle of mass $m$, the field equations (3.8.16) coupled to the Dirac equation are recovered as the EL equations corresponding to the action

$$
\mathcal{S}_{\mathrm{DYM}}=\int_{\mathscr{M}}\left\{\bar{\psi}(i \not \partial+\Gamma \not A-m) \psi-\frac{1}{4 e^{2}} F_{i j} F^{i j}+\frac{M^{2}}{2 e^{2}} A_{j} A^{j}\right\} d^{4} x,
$$

where $A$ denotes the axial potential and $F_{i j}=\partial_{i} A_{j}-\partial_{j} A_{i}$ is the corresponding field tensor. The coupling constant $e$ and the bosonic mass $M$ are related to the constants in (3.8.16) by $e^{2}=12 \pi^{2} / C_{0}$ and $M^{2}=C_{2} / C_{0}$. We point out that this action is not invariant under the axial gauge transformation

$$
\psi(x) \rightarrow e^{-i \Gamma \Lambda(x)} \psi(x), \quad A \rightarrow A+\partial \Lambda,
$$

because both the fermionic mass term $m \bar{\psi} \psi$ and the bosonic mass term $M^{2} A_{j} A^{j} /\left(2 e^{2}\right)$ have no axial symmetry. As explained in $\$ 3.6 .2$, the absence of an axial symmetry can be understood from the fact that the transformation of the wave function in (3.8.44) is not unitary, and thus it does not correspond to a local symmetry of our functionals in (3.2.8) (see also (3.6.28) and (3.6.29)).

The axial gauge transformation (3.8.44 can be realized as a local symmetry by adding a Higgs field $\phi$, in complete analogy to the procedure in the standard model. More precisely, we introduce $\phi$ as a complex scalar field which behaves under axial gauge transformations as

$$
\phi(x) \rightarrow e^{-2 i \Lambda(x)} \phi(x) .
$$

The fermionic mass term can be made gauge invariant by inserting suitable factors of $\phi$. Moreover, in view of (3.8.44), we can introduce a corresponding gauge-covariant derivative $D$ by

$$
D_{j}=\partial_{j}+2 i A_{j}
$$

Thus the Dirac-Yang/Mills-Higgs action defined by

$$
\begin{aligned}
\mathcal{S}_{\mathrm{DYMH}}=\int_{\mathcal{M}}\left\{\bar{\psi}(i \not \partial+\Gamma \not A) \psi-m \bar{\psi}\left(\phi \chi_{L}+\bar{\phi} \chi_{R}\right) \psi\right. \\
\left.\quad-\frac{1}{4 e^{2}} F_{i j} F^{i j}+\frac{M^{2}}{8 e^{2}}\left(\overline{D_{j} \phi}\right)\left(D^{j} \phi\right)-V\left(|\phi|^{2}\right)\right\} d^{4} x
\end{aligned}
$$

is invariant under the axial gauge transformation (3.8.44) and (3.8.45). We now follow the construction of spontaneous symmetry breaking in the standard model. For $V$ we choose a double well potential having its minimum at $|\phi|^{2}=1$. Then the Higgs field $\phi$ has a non-trivial vacuum with $|\phi|=1$. Thus choosing an axial gauge where $\phi$ is real and positive, we can write $\phi$ as

$$
\phi(x)=1+h(x)
$$

with a real-valued field $h$. Since $h$ vanishes in the vacuum, we may expand the action in powers of $h$. Taking the leading orders in $h$, we obtain the action after spontaneous symmetry breaking

with

$$
\mathcal{S}_{\mathrm{DYMH}}=\mathcal{S}_{\mathrm{DYM}}+\mathcal{S}_{\mathrm{Higgs}}
$$

$$
\mathcal{S}_{\text {Higgs }}=\int_{\mathcal{M}}\left\{-m h \bar{\psi} \psi+\frac{M^{2} h}{e^{2}} A_{j} A^{j}+\frac{M^{2}}{8 e^{2}}\left(\partial_{j} h\right)\left(\partial^{j} h\right)-2 V^{\prime \prime}(1) h^{2}\right\} d^{4} x .
$$


We conclude that for the action (3.8.46), the Higgs mechanism yields an action which reproduces the effective action of the continuum limit (3.8.43), but gives rise to additional terms involving a real Higgs boson $h$. The Higgs boson has a rest mass as determined by the free parameter $V^{\prime \prime}(1)$. It couples to both the wave function $\psi$ and the axial potential $A$.

The Higgs field $h$ can also be described in the setting of the fermionic projector, as we now explain. Note that the coupling terms of the Higgs field to $\psi$ and $A$ can be obtained from 3.8 .43 by varying the masses according to

$$
m \rightarrow(1+h(x)) m, \quad M \rightarrow(1+h(x)) M .
$$

Taking into account that in our framework, the bosonic masses are given in terms of the fermion masses (see (3.8.9) ), this variation is described simply by inserting a scalar potential into the Dirac equation. Likewise, for a system involving several generations, we must scale all fermion masses by a factor $1+h$. This is implemented in the auxiliary Dirac equation (3.4.4) by choosing

$$
\mathcal{B}=-m h(x) Y
$$

The remaining question is whether scalar perturbations of the form (3.8.48) occur in the setting of the fermionic projector, and whether our action principle (3.2.9) reproduces the dynamics of the Higgs field as described by (3.8.47). A-priori, any symmetric perturbation of the Dirac equation is admissible, and thus we can certainly consider the scalar perturbation (3.8.48). Since (3.8.48) is even under parity transformations, the corresponding leading perturbations of the eigenvalues $\lambda_{s}^{L}$ and $\lambda_{s}^{R}$ will be the same. In view of (3.7.1) and the formulas for the unperturbed eigenvalues (3.6.21), we find that the leading contributions by 3.8 .48 drop out of the EL equations. We thus conclude that, although a Higgs field can be described in our framework, the action principle 3.2 .9 ) does not describe a dynamics of this field, but instead predicts that the Higgs field must vanish identically (for more details on scalar perturbations see Lemma B.3.1).

We point out that for systems involving the direct sum of several sectors as studied in Chapters 4 and 5, it is conceivable that scalar/pseudoscalar potentials indeed give rise to dynamical degrees of freedom which can be identified with the Higgs field. The corresponding field equations could be obtained by analyzing the EL equations to degree three on the light cone (see also Section 5.5).

3.8.6. The Coupling Constant and the Bosonic Mass in Examples. The regularization parameters $c_{0}, \ldots, c_{3}$ in the field equations (3.8.9) are given in terms of the simple fractions in (3.7.9) and (3.8.5) - 3.8.8). For a given regularization method, we can evaluate these simple fractions and compute the coupling constant and the bosonic rest mass. We now exemplify the procedure by considering the two simplest methods of regularization:

(A) The $i \varepsilon$-regularization: Exactly as in 2.4 .1 , we define the distribution $\hat{P}^{\varepsilon}$ in 3.3 .3 by inserting an exponential convergence generating factor into the integrand of (3.3.1),

$$
\hat{P}^{\varepsilon}(k)=\sum_{\beta=1}^{g}\left(\not k+m_{\beta}\right) \delta\left(k^{2}-m^{2}\right) \Theta\left(-k^{0}\right) \exp \left(\varepsilon k^{0}\right) .
$$



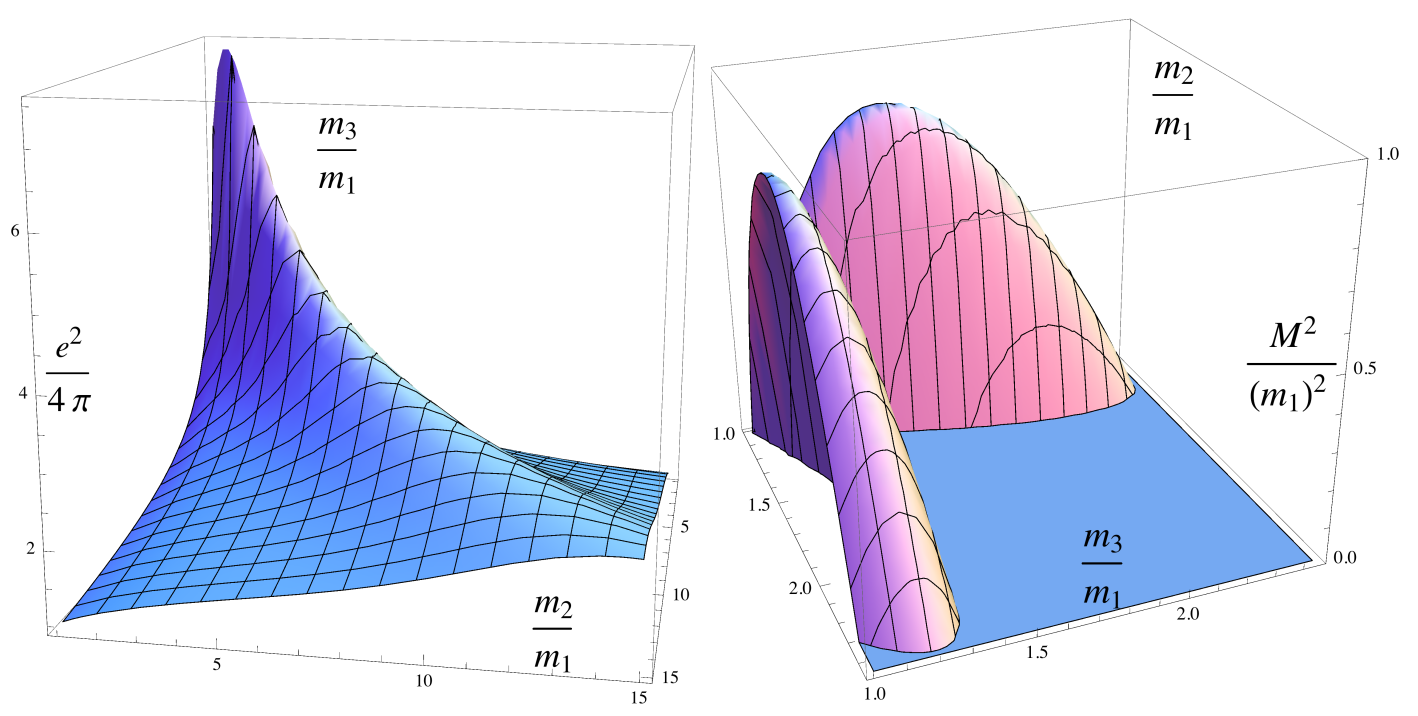

Figure 3.6. The coupling constant and the bosonic mass for the regularization 3.8.49

To the considered leading degree on the light cone, this regularization corresponds to the simple replacements (cf. 2.4.7) and (2.4.9) )

$$
T_{[p]}^{(0)} \rightarrow-\frac{1}{8 \pi^{3}} \frac{1}{2 r(t-r-i \varepsilon)}, \quad T_{[p]}^{(-1)} \rightarrow-\frac{2}{r} \frac{\partial}{\partial t} T_{[p]}^{(0)}=-\frac{1}{8 \pi^{3} r^{2}} \frac{1}{(t-r-i \varepsilon)^{2}},
$$

and similarly for the complex conjugates. Using these formulas in (3.8.9), the basic fractions all coincide up to constants, giving the equation

$$
\left(-\frac{3}{2}-96 \pi^{3}\left(s_{[0]}-s_{[3]}\right)\right) j_{a}+2 m^{2}\left(\hat{Y}^{2}+96 \pi^{3}\left(s_{[2]}-s_{[3]}\right) \dot{Y} \grave{Y}\right) A_{a}=12 \pi^{2} J_{a} .
$$

According to Lemma 3.8.1 and 3.8.10, the functions $s_{[p]}$ involve the masses of the fermions and also the convolution terms $f_{[p]}^{\beta}$. For clarity, we here leave out the convolution terms (which are analyzed in detail in 33.8 .2 and Appendix D). Then we can write the field equation in the usual form

$$
j_{a}-M^{2} A_{a}=e^{2} J_{a}
$$

where the coupling constant $e$ and the mass $M$ are given functions of the ratios $m_{2} / m_{1}$ and $m_{3} / m_{1}$ of the fermion masses. In Figure 3.6, these constants are plotted as functions of the mass ratios. The coupling constant is of the order one; it is largest if the fermion masses are close to each other. The term $M^{2}$ is always positive, so that the mass term has the correct sign. The bosonic mass has the same order of magnitude as the fermion masses.

(B) A cutoff in momentum space: In (A) we considered a regularization which was "soft" in the sense that it was smooth in momentum space. To give a complementary example, we now consider the "hard" regularization obtained by inserting a Heaviside function into the integrand of (3.3.1). Thus we define the distribution $\hat{P}^{\varepsilon}$ in $(3.3 .3$ by

$$
\hat{P}^{\varepsilon}(k)=\sum_{\beta=1}^{g}\left(\not k+m_{\beta}\right) \delta\left(k^{2}-m^{2}\right) \Theta\left(-k^{0}\right) \Theta\left(1+\epsilon k^{0}\right) .
$$


This regularization is described in analogy to 3.8 .49 by

$$
T_{[p]}^{(0)} \rightarrow-\frac{1}{16 \pi^{3}} \frac{1-e^{-\frac{i(t-r)}{\varepsilon}}}{2 r(t-r)}, \quad T_{[p]}^{(-1)} \rightarrow-\frac{2}{r} \frac{\partial}{\partial t} T_{[p]}^{(0)} .
$$

Thus as expected, the cutoff in momentum space gives rise to rapid oscillations in position space. Using these formulas in (3.8.9), the resulting basic fractions are no longer multiples of each other. But the weak evaluation integrals (3.5.7) can still be computed in closed form. We thus obtain the field equation

$$
\left(-\frac{9}{4}-96 \pi^{3}\left(s_{[0]}-s_{[3]}\right)\right) j_{a}+3 m^{2}\left(\hat{Y}^{2}+64 \pi^{3}\left(s_{[2]}-s_{[3]}\right) \dot{Y} \grave{Y}\right) A_{a}=12 \pi^{2} J_{a} .
$$

This equation can be analyzed exactly as in example (A), giving the same qualitative results. It is remarkable that the constants in the field equations in example (A) and (B) differ at most by a factor $3 / 2$, indicating that our results do not depend sensitively on the method of regularization.

\subsection{The Euler-Lagrange Equations to Degree Three and Lower}

The wave functions in (3.4.7) do not only have a vector and axial component as considered in 3.7 .2 , but they also have scalar, pseudoscalar and bilinear components. We will now analyze the effect of these contributions on the EL equations (\$3.9.1 and 3.9.2). Moreover, we will insert further potentials into the Dirac equation and analyze the consequences. More precisely, in 3.9 .2 we consider bilinear potentials, whereas in 33.9 .3 we consider scalar and pseudoscalar potentials and discuss the remaining possibilities in choosing other potentials and fields. We conclude this section with a discussion of the structure of the EL equations to degree three and lower (\$3.9.4).

3.9.1. Scalar and Pseudoscalar Currents. In analogy to the Dirac currents in $\$ 3.7 .2$, we introduce the scalar Dirac current $J_{\mathrm{s}}$ and the pseudoscalar Dirac current $J_{\mathrm{p}}$ by

$$
J_{\mathrm{s}}=\sum_{k=1}^{n_{\mathrm{p}}} \overline{\psi_{k}} \psi_{k}-\sum_{l=1}^{n_{\mathrm{a}}} \overline{\phi_{l}} \phi_{l} \quad \text { and } \quad J_{\mathrm{p}}=\sum_{k=1}^{n_{\mathrm{p}}} \overline{\psi_{k}} i \Gamma \psi_{k}-\sum_{l=1}^{n_{\mathrm{a}}} \overline{\phi_{l}} i \Gamma \phi_{l} .
$$

According to (3.4.7), these currents lead to a perturbation of the fermionic projector. In view of the fact that the scalar and pseudoscalar currents involve no Dirac matrix which could be contracted with a factor of $\xi$, one expects that the resulting contribution to the EL equations should be one degree lower than that of the axial current (see Lemma 3.7.4. Thus to leading order at the origin, one might expect contributions of the form

$$
\left.Q(x, y) \asymp J_{\mathrm{p}} \text { (monomial of degree three }\right)+(\operatorname{deg}<3) .
$$

However, perturbing the fermionic projector of the vacuum by (3.9.1), one sees that the corresponding contribution to $Q(x, y)$ of degree three on the light cone vanishes (see Lemma B.3.1, where it also explained how the cancellations come about). Taking into account the axial potentials, we do get contributions to $Q(x, y)$ of degree three on the light cone, which are of the form

$$
\left.Q(x, y) \asymp A_{a}^{j} \xi_{j} J_{\mathrm{s}} \text { (monomial of degree three }\right)+(\operatorname{deg}<3) .
$$

However, these contribution have the same tensor structure as the contributions of the axial potentials and currents of degree three, which will be discussed in 3.9 .4 below. 
3.9.2. Bilinear Currents and Potentials. The particles and anti-particle currents in (3.4.7) also have a bilinear component, leading us to introduce the bilinear Dirac current $J_{\mathrm{b}}$ by

$$
J_{\mathrm{b}}^{i j}=\sum_{k=1}^{n_{\mathrm{p}}} \overline{\psi_{k}} \sigma^{i j} \psi_{k}-\sum_{l=1}^{n_{\mathrm{a}}} \overline{\phi_{l}} \sigma^{i j} \phi_{l}
$$

(here $\sigma^{j k}=\frac{i}{2}\left[\gamma^{j}, \gamma^{k}\right]$ are again the bilinear covariants). Likewise, one may want to insert a bilinear potential

$$
\mathcal{B}=H_{i j}(x) \sigma^{i j}
$$

into the auxiliary Dirac equation (3.4.4) (where $H$ is an anti-symmetric tensor field).

Let us briefly discuss the effect of a bilinear current and a bilinear potential on the EL equations. The bilinear current corresponds to a perturbation of the fermionic projector of the form

$$
\Delta P(x, y)=-\frac{1}{8 \pi} J_{\mathrm{b}}^{i j} \sigma_{i j}+o\left(|\vec{\xi}|^{0}\right) .
$$

The bilinear potential, on the other hand, gives rise to different types of contributions to the light-cone expansion which involve $H$ and its partial derivatives (for details see F5. Appendix A.5]). When computing the perturbations of the eigenvalues $\lambda_{ \pm}^{L / R}$ of the closed chain (cf. [F7, Appendix G] or Appendix B], all Dirac matrices in $\Delta P$ are contracted with outer factors $\xi$. As a consequence, the contribution of the bilinear current drops out. Moreover, due to the anti-symmetry of $H$, all the bilinear contributions of the bilinear potential to the EL equations vanish. What remains are terms involving the divergence $\partial_{j} H^{i j}$ of the bilinear potential or derivatives of the divergence. All these terms can be interpreted as effective vector or axial potentials or derivatives thereof. Furthermore, the resulting contributions to the EL equations are of degree three on the light cone. Such contributions will again be discussed in $\$ 3.9 .4$ below.

3.9.3. Further Potentials and Fields. Having considered many different perturbations of the Dirac operator, we are now in the position to draw a few general conclusions, and to discuss a few potentials and fields which are not covered by the previous analysis. First of all, we point out that in Lemmas 3.7 .3 and 3.7 .4 the vector components dropped out, so that we got no contribution by an electromagnetic field. This cancellation can be understood from the general structure of our action principle, namely from the fact that the Lagrangian (3.6.23) involves the differences of the absolute values of the left- and right-handed eigenvalues. As a consequence, any perturbation which affects the eigenvalues $\lambda_{s}^{L}$ and $\lambda_{s}^{R}$ in the same way necessarily drops out of the EL equations. In other words, the EL equations are only affected by perturbations of the fermionic projector which have odd parity.

The fact that the electromagnetic field does not enter the EL equations does not necessarily imply that the electromagnetic field must vanish. But it means that no electromagnetic fields are generated in the system, so that the only possible electromagnetic field must be radiation coming from infinity. Having isolated systems without incoming radiation in mind, we conclude that our system involves no electromagnetic field.

The above consideration for the electromagnetic field also applies to the gravitational field, as we now explain. Introducing gravitational fields (see for example [F7, Section 1.5]) gives rise to contributions to the light-cone expansion which involve the metric, the curvature and the derivatives of curvature (see [F5, AppendixB]). The main effect 
of the gravitational field can be understood as a "deformation" of the light cone corresponding to the fact that the light cone is now generated by the null geodesics. The corresponding contributions to the light-cone expansion drop out of the EL equations if we make the action principle diffeomorphism invariant simply by replacing the measure $d^{4} x$ in $(3.2 .8)$ by $\sqrt{\left|\operatorname{det} g_{i j}\right|} d^{4} x$. In addition, there are terms involving the curvature of space-time, whose singularities on the light cone are of so small degree that the corresponding closed chain can again be treated perturbatively. Since these curvature terms are even under parity transformations, they drop out of the EL equations. We conclude that our model involves no gravitational field.

From the physical point of view, it might seem disappointing that our model involves no electromagnetic and gravitational fields. However, the simple explanation is that our system of one sector is too small to involve these fields. If one considers systems of several sectors, the equation analogous to 3.6.23 will involve the differences of the eigenvalues $\lambda_{s}^{c}$ and $\lambda_{s^{\prime}}^{c^{\prime}}$ in different sectors. Then potentials no longer drop out even if they have even parity, provided that they are not the same in all sectors. Only a detailed analysis of systems involving several sectors will show whether the electromagnetic and gravitational fields will appear in the physically correct way (see the Chapters 4 and 5).

Having understood why gravitational fields drop out of the EL equations, one might want to consider instead an axial gravitational field as described by a perturbation of the form

$$
\mathcal{B}=i \Gamma \gamma_{i} h^{i j} \partial_{j}+\text { (lower order terms) }
$$

Such a field cannot occur for the following reason. As just mentioned, a gravitational field describes a deformation of the light cone, which due to the diffeomorphism invariance of our action principle does not enter the EL equations. Similarly, an axial gravitational field (3.9.4) describes a deformation of the light cone, but now differently for the leftand right-handed components of the Dirac sea. Thus considering a perturbation of the form (3.9.4), the light cone would "split up" into two separate light cones being the singular sets of the left- and right-handed components of the fermionic projector, respectively. As a consequence, the leading singularities of the closed chain could no longer compensate each other in the EL equations, so that the EL equations would be violated to degree five on the light cone. Thus for the EL to be satisfied, the axial gravitational field must vanish.

In order to avoid the problem of the axial deformations of the light cone, one may want to consider a so-called axial conformal field

$$
\mathcal{B}=i \Xi(x) \Gamma \gamma^{j} \partial_{j}+\text { (lower order terms) . }
$$

But this field is of no use, as the following consideration shows. Let us consider for a given real function $\Lambda$ the so-called axial scaling transformation $U=e^{\Gamma \Lambda(x)}$. This transformation is unitary, and thus it has no effect on the EL equations. Transforming the Dirac operator according to

$$
\begin{aligned}
(i \not \partial-m) \rightarrow & U(i \not \partial-m) U^{-1}=i U^{2} \not \partial-m+i \Gamma(\not \partial \Lambda) \\
& =\cosh (2 \Lambda(x)) i \not \partial+\sinh (2 \Lambda(x)) i \Gamma \not \partial-m+i \Gamma(\not \partial \Lambda),
\end{aligned}
$$

the summand $\sinh (2 \Lambda(x)) i \Gamma \not \partial$ can be identified with the first-order term in (3.9.5). The summand $\cosh (2 \Lambda(x)) i \not \partial$, on the other hand, is a conformal gravitational field, and we already saw that gravitational fields do not enter the EL equations. Thus in total, we are left with a perturbation of the Dirac operator by chiral potentials, as considered earlier in this chapter. 
Next, we briefly consider a scalar or pseudoscalar potential (3.4.18). The leading contributions to the fermionic projector involve the potentials $\Xi$ and $\Phi$, whereas to lower degree on the light cone also derivatives of these potentials appear. Since $\Phi$ has even parity, its leading contribution to the EL equations vanishes. For the potential $\Xi$, the leading contribution cancels in analogy to (3.9.2) (see also Lemma B.3.1). But to degree four on the light cone, one gets cross terms similar to 3.9 .3 which also involve the axial potential. In order for these additional terms to vanish, we are led to setting the scalar and pseudoscalar potentials equal to zero. Thus there seems no point in considering scalar or pseudoscalar potentials. Nevertheless, scalar or pseudoscalar perturbations might enter the EL equations to degree three and lower, as will be discussed in \$3.9.4 below.

In the analysis of the axial potential we made one assumption which requires a brief explanation. Namely, when introducing the axial potential $A_{\mathrm{a}}$ in $\$ 3.6 .2$, we assumed that it couples to all generations in the same way (see (3.6.15)). In view of the constructions in $3.7 .4-33.7 .6$, where it was essential that the potentials were different for each generation, the ansatz (3.6.15) seems rather special, and one might wonder what would happen if we replaced the potential $A_{\mathrm{a}}$ in 3.6 .15 by a matrix potential acting non-trivially on the generations. Indeed, this scenario was already discussed in [F7, Remark 6.2.3], and thus here we briefly repeat the main argument. Suppose that the potential $A_{\mathrm{a}}$ in $(3.6 .15)$ were a matrix. Then the exponentials in (3.6.17) would have to be replaced by ordered exponentials of the form

$$
\operatorname{Texp}\left(-i \int_{x}^{y} A_{\mathrm{a}}^{j} \xi_{j}\right) .
$$

This is a unitary matrix whose eigenvalues can be regarded as different phase factors. Thus when forming the sectorial projection, we do not get a single phase factor, but instead a linear combination of different phases. As a consequence, the relations 3.6.22 will in general be violated, so that we get a contribution to the EL equations to degree five on the light cone. Reverting this argument, we can say that the EL equations to degree five imply that the eigenvalues (3.6.21) should involve only one phase, meaning that the axial potential must in fact be of the form 3.6 .15 with a vector field $A_{\mathrm{a}}$.

\subsubsection{The Non-Dynamical Character of the EL Equations to Lower De-} gree. In our analysis of the EL equations we began with the leading degree five on the light cone. The analysis to degree four revealed the field equations and thus described the dynamics. Generally speaking, to degree three and lower on the light cone, we get many more conditions, but on the other hand, we also get much more freedom to modify the fermionic projector. Namely, to degree three the Dirac currents as well as vector and axial potentials give rise to many terms, which in general do not cancel each other, even if the field equations of Section 3.8 are satisfied. For example, the factors $T_{\{p\}}^{(n)}$ involving curly brackets come into play (see (3.5.5)), and we also get contributions involving higher derivatives of the potentials. To degree two and one on the light cone, we get even more terms, involving the cross terms of different potentials and the terms generated by the mass expansion of the fermionic projector. In order to satisfy the EL equations to lower degree, all these terms must cancel each other. The good news is that we also get more and more free parameters. For example, we can consider scalar and pseudoscalar potentials (3.4.18), or bilinear potentials, or the other potentials discussed in 3.9.3. All these potentials can be chosen independently for each generation. Taking into account that to lower degree on the light cone, more and more cross terms between different perturbations 
come into play, we obtain a very complicated structure involving a large number of free parameters to modify the EL equations to degree three and lower.

In view of this complexity, it is not clear whether the EL equations can be satisfied to every degree on the light cone or not. The analysis becomes so complicated that it seems impossible to answer this question even with more computational effort. A possible philosophy to deal with this situation would be to take the pragmatic point of view that one should simply satisfy the EL equations as far as possible, but stop once the equations can no longer be handled. Since we do not find this point of view convincing, we now go one step further and explain why the analysis of the EL to lower degree (no matter what the results of this analysis would or will be) will have no influence on the dynamics of the system.

The field equations of Section 3.8 were dynamical in the sense that they involved partial differential equations of the potentials, and by solving these equations one finds that the potential is non-trivial even away from the sources. This property of the field equations is a consequence of the fact that the leading contributions to the fermionic projector (namely the phase factors in (3.6.17)) dropped out of the EL equations. This made is possible that the derivative terms became relevant (although they were of lower degree on the light cone), leading to dynamical field equations. The set of perturbations which can lead to dynamical field equations is very limited, because this requires that the potential itself must drop out of the EL equations, meaning that the potential must correspond to a local symmetry of the system. All in this sense dynamical perturbations have been considered in this chapter. This implies that all further potentials and fields will be non-dynamical in the sense that the potentials themselves (and not their derivatives) would enter the EL equations. This would give rise to algebraic relations between these potentials. In particular, these potentials would vanish away from the sources. Thus they do not describe a dynamical interaction, also making it difficult to observe them experimentally.

A possible idea for avoiding non-dynamical potentials is to choose the potentials differently for each generation, in such a way that the most singular contribution vanishes when forming the sectorial projection. This would open the possibility that the leading contribution to the EL equations might involve derivatives, thereby giving rise to dynamical field equations. We now give an argument which explains why this idea does not seem to work: Suppose that the potential is chosen as a matrix on the generations. Then for the potential to drop out of the EL equations, we must impose that a certain sectorial projection involving the potential must vanish at every space-time point. But this implies that this sectorial projection also vanishes if the potential is replaced by its derivatives. In particular, these derivative terms again drop out of the EL equations, making it impossible to get dynamical field equations. We remark that the last argument fails if the derivative terms also involve factors of the mass matrix, in which case the sectorial projection might be non-zero. But this situation seems rather artificial, and we shall not enter its analysis here.

This concludes our analysis of local potentials. Our treatment was exhaustive in the sense that we considered all multiplication operators and considered all relevant first order operators. Our results gave a good qualitative picture of how the fermionic projector is affected by different kinds of perturbations of the Dirac operator, and what the resulting contributions to the EL equations are. But clearly, the present paper cannot cover all possible perturbations, and some details still remain unsettled. Nevertheless, as explained 
above, our analysis covers all perturbations which should be of relevance for the dynamics of the fermions in our model.

\subsection{Nonlocal Potentials}

So far, we only analyzed the EL equations at the origin, i.e. for the leading contribution in an expansion in the parameter $\xi=y-x$. But clearly, the EL equations should also be satisfied away from the origin. In this section, we will explore whether this can be accomplished by introducing nonlocal potentials into the Dirac equation. We shall see that this method indeed makes it possible to satisfy the EL equations to every order in an expansion around $\xi=0$ (see Theorem 3.10.5). However, it will not become possible to satisfy the EL equations globally for all $x$ and $y$. This will lead us to the conclusion that all nonlocal potentials should vanish in the continuum limit.

In order to introduce the problem, we consider the perturbation of the fermionic projector by a particle wave function $\psi$, i.e. in view of (3.4.7)

$$
P(x, y) \asymp-\frac{1}{2 \pi} \psi(x) \overline{\psi(y)} .
$$

In the variable $y-x$, this contribution oscillates on the scale of the Compton wave length, whereas in the variable $y+x$, it varies typically on the larger atomic or macroscopic scale. For this reason, it is appropriate to begin by analyzing homogeneous perturbations which depend only on the variable $y-x$ (see $3.10 .1-33.10 .3$ ). In $\$ 3.10 .4$ we shall extend our constructions to build in an additional dependence on the variable $y+x$. The results and physical significance of our analysis will be discussed in 3.10 .5 .

3.10.1. Homogeneous Transformations in the Low-Frequency Regime. We now return to the homogeneous transformation of the fermionic projector (3.7.76). In $\$ 3.7 .10$ we analyzed this transformation, choosing $Z(k)$ such that the coefficients $C_{\beta}$ in (3.7.73) were constants. This had the advantage that the corresponding smooth contribution to the fermionic projector was easily computable (see $(3.8 .10)$ ). However, $Z(k)$ can be chosen more generally as an arbitrary function of $k$. In order to complement the analysis of 3.7.9, where the emphasis was on the singularities on the light cone, we now concentrate on smooth contributions to the fermionic projector. We thus assume that $Z(k)$ has rapid decay. For technical simplicity, we assume that $Z$ is a Schwartz function in momentum space, $Z \in \mathcal{S}(\hat{\mathscr{M}})$. As a consequence, the function $\delta P(x, y)$ is smooth. Clearly, more general functions could be realized by approximation.

In order to explain the basic idea in the homogeneous setting, we again consider the system 3.5.25) of one Dirac sea of mass $m$. Transforming each state according to 3.7.76 gives rise to a perturbation of the fermionic projector of the form

$$
\begin{aligned}
\delta P(x, y)=\int & \frac{d^{4} k}{(2 \pi)^{4}} \Gamma \psi(k) \delta\left(k^{2}-m^{2}\right) \Theta\left(-k^{0}\right) e^{-i k(x-y)} \\
& +(\text { smooth vectorial, pseudoscalar or bilinear contributions })
\end{aligned}
$$

where $v$ is any vector field of rapid decay. Exactly as explained in the derivation of (3.8.10), the vectorial contribution drops out of (3.7.1), whereas the pseudoscalar and bilinear contributions drop out of the EL equations to degree four. Hence it suffices to consider the axial contribution. Moreover, in the EL equations, $\delta P(x, y)$ is always 
contracted with a factor $\xi$. Thus it suffices to consider the expression

$$
\frac{1}{4} \operatorname{Tr}(\Gamma \not \delta P(x, y))=-\int \frac{d^{4} k}{(2 \pi)^{4}}\langle\xi, v(k)\rangle T_{m^{2}}(k) e^{i k \xi},
$$

where the distribution $T_{m^{2}}(k):=\delta\left(k^{2}-m^{2}\right) \Theta\left(-k^{0}\right)$ is supported on the lower mass shell. For what follows, it will be useful to write the inner product $\langle\xi, v(k)\rangle=\xi^{i} v_{i}$ in components and to analyze the components separately. Dividing by $\xi^{i}$, we can reduce (3.10.3) to four scalar Fourier integrals of the form

$$
f(\xi)=\int \frac{d^{4} k}{(2 \pi)^{4}} \hat{f}(k) T_{m^{2}}(k) e^{i k \xi} .
$$

3.10.2. Homogeneous Perturbations by Varying the Momenta. Another homogeneous perturbation which was not considered so far is to vary the momenta of the states, dropping the mass shell condition. For simplicity, we begin with the system (3.5.25) of one Dirac sea for a given rest mass $m>0$. This system is composed of states $\psi_{k}(x)=\chi(k) e^{-i k x}$ which are plane waves of momentum $k$ on the lower mass shell. Considering a variation $\delta k$ of the momentum leads us to replace $\psi_{k}(x)$ by the plane wave

$$
\chi(k) e^{-i(k+\delta k) x} .
$$

Then to first order in $\delta k$, the individual states are varied by

$$
\delta \psi_{k}(x)=-i\langle x, \delta k\rangle \psi_{k}(x)+\mathcal{O}\left((\delta k)^{2}\right),
$$

and this yields the following perturbation of the fermionic projector,

$$
\delta P(x, y)=i \int \frac{d^{4} k}{(2 \pi)^{4}}\langle\xi, \delta k\rangle(\not k+m) \delta\left(k^{2}-m^{2}\right) \Theta\left(-k^{0}\right) e^{-i k(x-y)} .
$$

The vector field $\delta k(k)$ can be chosen arbitrarily on the lower mass shell, giving us a lot of freedom to vary $P(x, y)$. However, due to the factor $\xi$ inside the inner product, the variation necessarily vanishes at the origin $x=y$. For technical simplicity we again assume that $\delta k$ has rapid decay.

We want to explore whether such a variation of the momenta allows us to modify the EL equations. First of all, we remind the reader that only the axial component of the currents enters the EL equations to degree four (whereas the scalar, pseudoscalar and bilinear components drop out; see 33.9 .1 and $\$ 3.9 .2)$. But the perturbation (3.10.6) does not have an axial component, and thus we must generalize $(3.10 .6)$ such as to include a perturbation which is odd under parity transformations. To this end, we choose a vector field $q$ in momentum space with the properties

$$
\langle k, q(k)\rangle=0 \quad \text { and } \quad q(k)^{2}=-1 .
$$

Then the operators

$$
\Pi_{ \pm}(k):=\frac{1}{2}(1 \mp \Gamma q(k))
$$

are projectors which commute with the fermionic projector of the vacuum; they project onto the two spin orientations in the direction of $q$ and $-q$, respectively. Thus multiplying the fermionic projector by $\Pi_{ \pm}$,

$$
P_{ \pm}(k)=\Pi_{ \pm}(k)(\not k+m) \delta\left(k^{2}-m^{2}\right) \Theta\left(-k^{0}\right),
$$

we decompose the Dirac sea into two "subseas" $P_{ \pm}$, which are still composed of solutions of the free Dirac equation. We remark that this decomposition was already used in $[\mathbf{F 7}$, 
Appendix C.1], where it was shown that in a suitable limit $m \searrow 0$, the projectors $P_{ \pm}(k)$ go over to chiral Dirac seas composed of left- or right-handed states. Here the above decomposition gives us the freedom to vary the momenta of each subsea independently. Thus we generalize $(3.10 .6)$ by

$$
\delta P(x, y)=i \sum_{s= \pm} \int \frac{d^{4} k}{(2 \pi)^{4}}\left\langle\xi, \delta k_{s}\right\rangle P_{s}(k) e^{-i k(x-y)},
$$

where $\delta k_{+}$and $\delta k_{-}$are two vector fields on the lower mass shell. For our purposes, it will be sufficient to always assume that these vector fields are smooth and have rapid decay. Thus we can extend them to Schwartz functions in momentum space. As a consequence, the function $\delta P(x, y)$ is smooth (but due to the restriction to the mass shell in (3.10.10), it will in general not have rapid decay).

In the EL equations, $\delta P(x, y)$ is contracted with a factor $\$$. The corresponding vector and axial components are computed by

$$
\begin{aligned}
\frac{1}{4} \operatorname{Tr}(\sharp \delta P(x, y)) & =\frac{i}{2} \int \frac{d^{4} k}{(2 \pi)^{4}}\left\langle\xi, \delta k_{+}+\delta k_{-}\right\rangle\langle\xi, k\rangle T_{m^{2}}(k) e^{i k \xi} \\
\frac{1}{4} \operatorname{Tr}(\Gamma \& \delta P(x, y)) & =-\frac{i}{2} \int \frac{d^{4} k}{(2 \pi)^{4}}\left\langle\xi, \delta k_{+}-\delta k_{-}\right\rangle\langle\xi, m q\rangle T_{m^{2}}(k) e^{i k \xi}
\end{aligned}
$$

(where again $T_{m^{2}}(k)=\delta\left(k^{2}-m^{2}\right) \Theta\left(-k^{0}\right)$ ). Collecting the factors of $\xi$, these expressions can be written in the form $\xi_{j} \xi_{l} A^{j l}$ with a symmetric tensor field $A^{j l}(\xi)$. We first want to eliminate the tensor indices, leaving us with scalar Fourier transforms. Thus suppose that for a given smooth tensor field $A^{j l}(\xi)$ we want to find the corresponding vector fields $\delta k_{ \pm}$ and $q$ in (3.10.11) or 3.10.12). In the vector component 3.10.11), we can rewrite the factor $\langle\xi, k\rangle$ as a $\xi$-derivative, leading to the equation

$$
\xi^{j} \frac{\partial}{\partial \xi^{j}} f_{l}(\xi)=\xi^{j} A_{j l}(\xi)
$$

where $f_{l}$ is the Fourier integral

$$
f_{l}=\frac{1}{2} \int \frac{d^{4} k}{(2 \pi)^{4}}\left(\delta k_{+}+\delta k_{-}\right)_{l} T_{m^{2}}(k) e^{i k \xi} .
$$

Integrating the ordinary differential equation 3.10 .13 gives the solution

$$
f_{l}(\xi)=\xi^{j} \int_{0}^{1} A_{j l}(\tau \xi) d \tau
$$

being a smooth vector field. Thus for every choice of the vector index $l$, we must again solve the equation (3.10.4), if we set $f=f_{l}$ and $\hat{f}=\left(\delta k_{+}+\delta k_{-}\right)_{l} / 2$. In this way, we have reduced (3.10.11) to scalar Fourier integrals of the form 3.10.4). The same can be accomplished for the axial component 3.10 .12 with the following construction. We write the factor $\xi$ in 3.10 .12 , which is contracted with $q$, as a $k$-derivative of the factor $e^{i k \xi}$ and integrate by parts. Using the relation

$$
q^{j} \frac{\partial}{\partial k^{j}} T_{m^{2}}(k)=q^{j} \frac{\partial}{\partial k^{j}}\left(\delta\left(k^{2}-m^{2}\right) \Theta\left(-k^{0}\right)\right)=2 q^{j} k_{j} \delta^{\prime}\left(k^{2}-m^{2}\right) \Theta\left(-k^{0}\right)=0,
$$

where in the last step we applied the orthogonality relation in (3.10.7), we obtain the equation

$$
\xi^{j} A_{j l}(\xi)=\frac{1}{2} \int \frac{d^{4} k}{(2 \pi)^{4}}\left[\frac{\partial}{\partial k^{j}}\left(\left(\delta k_{+}-\delta k_{-}\right)_{l} m q^{j}\right)\right] T_{m^{2}}(k) e^{i k \xi}
$$


We again fix the index $l$, but now set $f(\xi)=\xi^{j} A_{j l}(\xi)$. Furthermore, we introduce the vector field $v^{j}=\left(\delta k_{+}-\delta k_{-}\right)_{l} m q^{j}$. Suppose that the smooth scalar function $f$ can be represented in the form $(3.10 .4)$ with a suitable Schwartz function $\hat{f}$. Then the remaining task is to satisfy on the lower mass shell the equation

$$
\frac{\partial}{\partial k^{j}} v^{j}(k)=\hat{f}
$$

In order to verify that this equation always has a solution, it is useful to rewrite it as a geometric PDE defined intrinsically on the hyperbola

$$
\mathcal{H}:=\left\{k \in \hat{M} \mid k^{2}=m^{2}, k^{0}<0\right\} .
$$

Namely, the orthogonality condition in (3.10.7) implies that the vector $v$ is tangential to $\mathcal{H}$, and the derivatives in 3.10.14 can be rewritten as the covariant divergence on $\mathcal{H}$,

$$
\nabla_{j} v^{j}=\hat{f} \in \mathcal{S}(\mathcal{H})
$$

(where $\nabla$ is the Levi-Civita connection on $\mathcal{H}$ ). Conversely, for a given vector field $v$ on $\mathcal{H}$ which satisfies 3.10 .15 , extending $v$ to a vector field on $\hat{\mathcal{M}}$ which is everywhere orthogonal to $k$ gives the desired solution of (3.10.14). A simple solution of (3.10.15) is obtained by first solving the Poisson equation $\Delta_{\mathcal{H}} \phi=\hat{f}$ (for example using the explicit form of the Green's function on the hyperbola) and setting $v=\nabla \phi$. However, this solution has the disadvantage that $v$ has no rapid decay at infinity. In order to do better, we must exploit that our function $f(\xi)=\xi^{j} A_{j l}(\xi)$ vanishes at the origin, and thus the integral of $\hat{f}$ vanishes,

$$
\int_{\mathcal{H}} \hat{f} d \mu_{\mathcal{H}}=0
$$

Combining this fact with the freedom to add to $v$ an arbitrary divergence-free vector field, one can indeed construct a solution $v$ of (3.10.15) within the Schwartz class, as is shown in the following lemmet

Lemma 3.10.1. For every function $\hat{f} \in \mathcal{S}(\mathcal{H})$ whose integral vanishes 3.10 .16 , there is a vector field $v \in \mathcal{S}(\mathcal{H})$ which satisfies 3.10.15.

Proof. We parametrize the hyperbola $\mathcal{H}$ by

$$
\left(-\sqrt{m^{2}+\rho^{2}}, \rho \cos \vartheta, \rho \sin \vartheta \cos \varphi, \rho \sin \vartheta \sin \varphi\right) \in \hat{\mathscr{M}},
$$

where $\rho:=|\vec{k}|$, and $(\vartheta, \varphi)$ are the standard polar coordinates on the 2 -sphere. Then the metric on $\mathcal{H}$ is diagonal,

$$
g_{i j}=\operatorname{diag}\left(\frac{m^{2}}{\rho^{2}+m^{2}}, \rho^{2}, \rho^{2} \sin ^{2} \vartheta\right) .
$$

For the vector field $v$ we take the ansatz as the sum of a radial part $u^{j}=\left(u^{\rho}, 0,0\right)$ and an angular part $w^{j}=\left(0, w^{\vartheta}, w^{\varphi}\right)$. We also regard $w$ as a vector field on the sphere. Then the equation 3.10.15 can be written as

$$
\operatorname{div}_{\mathcal{H}}(u)+\operatorname{div}_{S^{2}}(w)=\hat{f} .
$$

\footnotetext{
${ }^{7}$ I thank Bernd Ammann for the idea of solving the equation on the leaves of a foliation, after subtracting the mean value of $f$.
} 
Taking the average of $\hat{f}$ over spheres defines a spherically symmetric Schwartz function $\bar{f}$,

$$
\bar{f}(\rho):=\frac{1}{4 \pi} \int_{S^{2}} \hat{f}(\rho, \vartheta, \varphi) d \varphi d \cos \vartheta \in \mathcal{S}(\mathcal{H})
$$

Writing the integration measure as $d \mu_{\mathcal{H}}=\sqrt{\operatorname{det} g} d \rho d \vartheta d \varphi$ and using Fubini, the condition 3.10.16 implies that

$$
\int_{0}^{\infty} \bar{f}(\rho) \frac{\rho^{2} d \rho}{\sqrt{\rho^{2}+m^{2}}}=0 .
$$

Solving the Poisson equation $\Delta_{\mathcal{H}} \phi=\bar{f}$, the resulting function $\phi$ is smooth and again spherically symmetric. Setting $u=\nabla \phi$, we obtain a smooth radial vector field, being a solution of the equation

$$
\operatorname{div}_{\mathcal{H}}(u)=\bar{f} .
$$

Writing the covariant divergence as

$$
\operatorname{div} \mathcal{H}(u)=\frac{1}{\sqrt{\operatorname{det} g}} \frac{\partial}{\partial x^{j}}\left(\sqrt{\operatorname{det} g} u^{j}\right)=\frac{\sqrt{\rho^{2}+m^{2}}}{\rho^{2}} \frac{\partial}{\partial \rho}\left(\frac{\rho^{2} u^{\rho}}{\sqrt{\rho^{2}+m^{2}}}\right),
$$

the divergence condition 3.10 .19 becomes an ordinary differential equation, having the explicit solution

$$
u^{\rho}(\rho)=\frac{\sqrt{\rho^{2}+m^{2}}}{\rho^{2}} \int_{0}^{\rho} \bar{f}(\tau) \frac{\tau^{2} d \tau}{\sqrt{\tau^{2}+m^{2}}} .
$$

Using (3.10.18), one immediately verifies that the vector field $u$ and all its derivatives have rapid decay at infinity. Hence $u$ is in the desired Schwartz class.

Using (3.10.19) in (3.10.17), it remains to consider the differential equation

$$
\operatorname{div}_{S^{2}}(w)=\hat{f}-\bar{f} .
$$

Since the function $\hat{f}-\bar{f}$ has mean zero on every sphere, it can be expanded in terms of spherical harmonics starting at $l=1$,

$$
(\hat{f}-\bar{f})(\rho, \vartheta, \varphi)=\sum_{l=1}^{\infty} \sum_{k=-l}^{l} c_{l k}(\rho) Y_{l k}(\vartheta, \varphi) .
$$

Since $\hat{f}-\bar{f}$ is a Schwartz function, the coefficients $c_{l k}$ are all smooth in $\rho$, and these coefficients together with all their $\rho$-derivatives have rapid decay in both $\rho$ and $l$, uniformly in $k$. Hence the Poisson equation $\Delta_{S^{2}} \phi=\hat{f}-\bar{f}$ can be solved explicitly by

$$
\phi(\rho, \vartheta, \varphi)=-\sum_{l=1}^{\infty} \sum_{k=-l}^{l} \frac{c_{l k}(\rho)}{l(l+1)} Y_{l k}(\vartheta, \varphi)
$$

defining again a Schwartz function on $\mathcal{H}$. Introducing the vector field $w$ by $w^{j}=\nabla_{S^{2}}^{j} \phi=$ $\left(0, \partial_{\vartheta} \phi, \cos ^{-2} \vartheta \partial_{\varphi} \phi\right)$ gives the desired solution of 3.10 .20$)$ in the Schwartz class. 
3.10.3. The Analysis of Homogeneous Perturbations on the Light Cone. With the above constructions, we have reduced the analysis of the homogeneous transformation $(3.10 .12)$ as well as the perturbation by varying of the momenta $(3.10 .10)$ to the scalar Fourier transform (3.10.4). Having a weak evaluation on the light cone (3.5.7) in mind, we may restrict attention to the light cone $L=\left\{\xi \mid \xi^{2}=0\right\}$. Thus our task is to analyze the Fourier integral

$$
f(\xi)=\int \frac{d^{4} k}{(2 \pi)^{4}} \hat{f}(k) \delta\left(k^{2}-m^{2}\right) \Theta\left(-k^{0}\right) e^{i k \xi} \quad \text { for } \xi \in L .
$$

More precisely, for a given smooth function $f \in C^{\infty}(\mathscr{M})$ we want to find a Schwartz function $\hat{f} \in \mathcal{S}(\hat{M})$ such that the Fourier integral 3.10.21) coincides on the light cone with $f$. The question is for which $f$ such a function $\hat{f}$ exists. We begin the analysis in the simple case that the function $\hat{f}$ when restricted to the mass shell depends only on the variable $\omega=k^{0}$ (by linearity, we can later realize more general functions $\hat{f}$ by superposition). Then the resulting Fourier integral is spherically symmetric, so that the function $f(\xi)$ will only depend on the time and radial variables $t=\xi^{0}$ and $r=|\vec{\xi}|$. Restricting attention to the light cone $t= \pm r$, we end up with a one-dimensional problem. More precisely, setting $p=|\vec{k}|$ and denoting the angle between $\vec{\xi}$ and $\vec{k}$ by $\vartheta$, the Fourier integral (3.10.21) becomes

$$
\begin{aligned}
f(t, r) & =\frac{1}{8 \pi^{3}} \int_{-\infty}^{0} d \omega \hat{f}(\omega) e^{i \omega t} \int_{0}^{\infty} p^{2} d p \delta\left(\omega^{2}-p^{2}-m^{2}\right) \int_{-1}^{1} d \cos \vartheta e^{-i p r \cos \vartheta} \\
& =\frac{i}{8 \pi^{3} r} \int_{-\infty}^{0} d \omega \hat{f}(\omega) e^{i \omega t} \int_{0}^{\infty} p d p \delta\left(\omega^{2}-p^{2}-m^{2}\right)\left(e^{-i p r}-e^{i p r}\right) \\
& =\frac{i}{16 \pi^{3} r} \int_{-\infty}^{-m} d \omega \hat{f}(\omega) e^{i \omega t}\left(e^{-i \sqrt{\omega^{2}-m^{2}} r}-e^{i \sqrt{\omega^{2}-m^{2}} r}\right) .
\end{aligned}
$$

Hence on the light cone $t= \pm r$ we obtain the representation

$$
\text { it } f(t)=\frac{1}{16 \pi^{3}} \int_{-\infty}^{-m} d \omega \hat{f}(\omega)\left(e^{i \omega_{+} t}-e^{i \omega_{-} t}\right),
$$

where we set

$$
\omega_{ \pm}:=\omega \pm \sqrt{\omega^{2}-m^{2}}
$$

The right side of $(3.10 .22)$ differs from an ordinary Fourier integral in two ways: First, the integrand does not involve one plane wave, but the difference of the two plane waves $e^{i \omega_{ \pm} t}$, whose frequencies are related to each other by $\omega_{+} \omega_{-}=m^{2}$. Second, in (3.10.22) only negative frequencies appear. Let us discuss these two differences after each other. The appearance of the combination $\left(e^{i \omega_{+} t}-e^{i \omega_{-} t}\right)$ in $(3.10 .22)$ means that the coefficients of the plane waves cannot be chosen arbitrarily, but a contribution for a frequency $\omega_{-}<-m$ always comes with a corresponding contribution of frequency $\omega_{+}>-m$. This frequency constraint makes it impossible to represent a general negative-frequency function $f$; for example, it is impossible to represent a function $t f(t)$ whose frequencies are supported in the interval $[-\infty,-m)$. However, this frequency constraint can be regarded as a shortcoming of working with a single Dirac sea. If we considered instead a realistic system of several Dirac seas (3.3.1), the Fourier integral 3.10 .22 ) would involve a sum over the generations,

$$
\text { it } f(t)=\frac{1}{16 \pi^{3}} \sum_{\beta=1}^{g} \int_{-\infty}^{-m_{\beta}} d \omega \hat{f}^{\beta}(\omega)\left(e^{i \omega_{+}^{\beta} t}-e^{i \omega_{-}^{\beta} t}\right)
$$


with $\omega_{ \pm}^{\beta}(\omega):=\omega \pm\left(\omega^{2}-m_{\beta}^{2}\right)^{-\frac{1}{2}}$ and $g>1$. Then the freedom in choosing $g$ independent functions $\hat{f}^{\beta}$ would indeed make it possible to approximate any negative-frequency function, as the following lemma shows.

Lemma 3.10.2. Assume that the number of generations $g \geq 2$. Assume furthermore that the one-dimensional Fourier transform $\hat{f}$ of a given Schwartz function $f \in \mathcal{S}(\mathbb{R})$ is supported in the interval $(-\infty, 0)$. Then there is a sequence of Schwartz functions $\hat{f}_{n}^{\beta} \in$ $\mathcal{S}(\mathbb{R})$ such that the corresponding functions $f_{n}(t)$ defined by the Fourier integrals 3.10 .23 . as well as all their derivatives converge uniformly to $f(t)$,

$$
\sup _{t \in \mathbb{R}}\left|\partial_{t}^{K}\left(f_{n}(t)-f(t)\right)\right| \stackrel{n \rightarrow \infty}{\longrightarrow} 0 \quad \text { for all } K \geq 0 .
$$

Proof. We want to find functions $\hat{f}^{\beta}$ in 3.10 .23 such that the right side of 3.10 .23$)$ gives the plane wave $e^{i \Omega t}$ with $\Omega<0$. Again ordering the masses according to 3.3 .2$)$, we choose $\omega_{1}$ such that $\omega_{+}^{g}\left(\omega_{1}\right)=\Omega$ or $\omega_{-}^{g}\left(\omega_{1}\right)=\Omega$, i.e.

$$
\omega_{1}=\frac{\Omega^{2}+m_{g}^{2}}{2 \Omega} .
$$

Then choosing $\hat{f}^{g}(\omega)= \pm \delta\left(\omega-\omega_{1}\right)$, we obtain the desired plane wave $e^{i \Omega t}$, but as an error term we get the plane wave $-e^{i \Omega_{1} t}$ with $\Omega_{1}=m_{g}^{2} / \Omega$. In order to compensate the error, we next choose $\omega_{2}$ such that $\omega_{+}^{1}\left(\omega_{2}\right)=\Omega_{1}$ or $\omega_{-}^{1}\left(\omega_{2}\right)=\Omega_{1}$. Choosing $\hat{f}^{1}(\omega)=\delta\left(\omega-\omega_{2}\right)$, the plane wave $-e^{i \Omega_{1} t}$ drops out, but we obtain instead the plane wave $e^{i \Omega_{2} t}$ with $\Omega_{2}=$ $m_{1}^{2} / \Omega_{1}=m_{1}^{2} \Omega / m_{g}^{2}$. We proceed by compensating the plane waves in turns by the last Dirac sea and the first Dirac sea. After $n$ iteration steps, the functions $\hat{f}^{1}$ and $\hat{f}^{g}$ take the form

$$
\hat{f}_{n}^{1}(\omega)=-\sum_{l=1}^{n} \delta\left(\omega-\frac{\Omega_{2 n+1}^{2}+m_{1}^{2}}{2 \Omega_{2 n+1}}\right), \quad \hat{f}_{n}^{g}(\omega)=\sum_{l=0}^{n-1} \delta\left(\omega-\frac{\Omega_{2 n}^{2}+m_{g}^{2}}{2 \Omega_{2 n}}\right),
$$

where

$$
\Omega_{2 n}=\frac{m_{1}^{2 n}}{m_{g}^{2 n}} \Omega \quad \text { and } \quad \Omega_{2 n+1}=\frac{m_{g}^{2 n+2}}{m_{1}^{2 n} \Omega} .
$$

The Fourier integral 3.10 .23 gives rise to the plane waves

$$
e^{i \Omega t}-e^{i \lambda^{n} \Omega t} \quad \text { where } \quad \lambda:=\frac{m_{1}^{2}}{m_{g}^{2}}<1 .
$$

In order to form superpositions of these plane waves, we next multiply by a Schwartz function $\hat{h}(\Omega)$ and integrate over $\Omega$. Then the Fourier integral 3.10 .23 becomes

$$
i t f_{n}(t)=\int_{-\infty}^{0} \hat{h}(\Omega)\left(e^{i \Omega t}-e^{i \lambda^{n} \Omega t}\right) d \Omega .
$$

Choosing $\hat{h}(\omega)=-\partial_{\omega} \hat{f}(\omega) /(2 \pi)$, we can extend the integration to the whole real line. After integrating by parts, we can carry out the Fourier integral to obtain

$$
f_{n}(t)=\frac{1}{2 \pi} \int_{-\infty}^{\infty} \hat{f}(\Omega)\left(e^{i \Omega t}-\lambda^{n} e^{i \Omega\left(\lambda^{n} t\right)}\right) d \Omega=f(t)-\lambda^{n} f\left(\lambda^{n} t\right) .
$$

From this explicit formula it is obvious that the functions $f_{n}$ converge in the limit $n \rightarrow \infty$ in the sense (3.10.24). 
As is immediately verified, the functions $f_{n}$ as well as all their derivatives also converge in $L^{2}(\mathbb{R})$. However, we point out that for the functions $\hat{f}_{n}^{1}$ and $\hat{f}_{n}^{g}$, the convergence is a bit more subtle. Namely, from (3.10.25) one sees that for large $n$, these functions involve more and more contributions for large $\omega$. A direct calculation shows that in the limit $n \rightarrow \infty$, these functions converge to smooth functions which decay at infinity only $\sim 1 / \omega$.

Let us now discuss the consequences of the fact that $(3.10 .22)$ only involves negative frequencies. This restriction is already obvious in the Fourier integral (3.10.10), before the reduction to scalar Fourier integrals (3.10.21) or 3.10.22). Since contractions with factors $\xi$ merely correspond to differentiations in momentum space which preserve the sign of the frequencies, the following considerations apply in the same way before or after the contractions with $\xi$ have been performed. We point out that the contribution by a Dirac wave function (3.10.1) can be composed of positive frequencies (=particles) or negative frequencies (=anti-particles), and thus in (3.10.1) we cannot restrict attention to negative frequencies. This raises the question whether a contribution to 3.10.1 of positive frequency can be compensated by a contribution to 3.10.21 of negative frequency. The answer to this question is not quite obvious, because the EL equations involve both $P(x, y)$ and its adjoint $P(y, x)=P(x, y)^{*}$. Since taking the adjoint reverses the sign of the frequencies, a negative-frequency contribution to $P(x, y)$ affects the EL equations by contributions of both positive and negative frequency. Thus one might hope that perturbations of $P(x, y)$ of positive and negative frequency could compensate each other in the EL equations. However, such a compensation is impossible, as the following lemma shows.

Lemma 3.10.3. Assume that $\hat{f}$ and $\hat{g}$ are the Fourier transforms of chiral perturbations of the fermionic projector, such that $\hat{f}$ has a non-vanishing contribution inside the upper mass cone, whereas $\hat{g}$ is supported inside the lower mass cone. Then the linear contributions of $f$ and $g$ to the EL equations to degree four cannot compensate each other for all $\xi$.

Proof. By linearity, we may restrict attention to the spherically symmetric situation, so that $f$ and $g$ restricted to the light cone are functions of one variable $t$. Since the negative-frequency component of $f$ can clearly be compensated by $g$, we can assume that $f$ and $g$ are composed purely of positive and negative frequencies, respectively. The perturbation $g$ affects the EL equations to degree four by (see Lemma (3.7.4) and its proof in Appendix B

$$
\mathcal{R} \asymp c\left(M_{1} g(\xi)+M_{2} \overline{g(\xi)}\right)+(\operatorname{deg}<4),
$$

where

$$
M_{1}=T_{[0]}^{(-1)} \overline{T_{[0]}^{(-1)}} \quad \text { and } \quad M_{2}=-T_{[0]}^{(-1)} \overline{T_{[0]}^{(-1)}} \frac{T_{[0]}^{(0)}}{\overline{T_{[0]}^{(0)}}},
$$

and $c>0$ is an irrelevant constant. Evaluating weakly on the light cone (3.5.7), we obtain the contribution

$$
c_{1} g(t)+c_{2} \overline{g(t)},
$$

where $c_{1}$ and $c_{2}$ are real regularization parameters (real because the degree is even). Similarly, the perturbation $f$ yields the contribution

$$
c_{1} f(t)+c_{2} \overline{f(t)} \text {. }
$$


In order for (3.10.28) to compensate (3.10.27), both the negative and positive frequencies must cancel each other, leading to the conditions

$$
c_{1} g(t)=c_{2} \overline{f(t)} \quad \text { and } \quad c_{2} \overline{g(t)}=c_{1} f(t) .
$$

Taking the complex conjugate of the first equation, multiplying it by $c_{2}$ and subtracting $c_{1}$ times the second equation, we get

$$
\left(c_{1}^{2}-c_{2}^{2}\right) f(t)=0 .
$$

We thus obtain the condition

$$
c_{1}= \pm c_{2} \text {. }
$$

If this condition holds, we can indeed satisfy $(3.10 .29)$ by setting $g(t)= \pm \overline{f(t)}$.

We conclude that $f$ and $g$ can compensate each other if and only if we impose the relation 3.10 .30$)$ between the regularization parameters corresponding to the basic fractions $M_{1}$ and $M_{2}$. Imposing relations between the regularization parameters was not used previously in this paper, and one could simply reject 3.10 .30 by saying that we do not want to restrict the class of admissible regularization by introducing such relations. However, this argumentation would not be fully convincing, as it would not allow for the possibility that the microscopic structure of space-time on the regularization scale $\varepsilon$ corresponds to a regularization which does have the special property (3.10.30). This possibility is ruled out by the following argument which shows that there are in fact no regularizations which satisfy (3.10.30): As only the real parts of basic fractions enter (3.5.7), it suffices to consider the real parts of $M_{1}$ and $M_{2}$. Using the specific form of these monomials in (3.10.26), we obtain

$$
\frac{\operatorname{Re}\left(M_{1}+M_{2}\right)}{2}=\left|\frac{T_{[0]}^{(-1)}}{T_{[0]}^{(0)}}\right|^{2}\left(\operatorname{Im} T_{[0]}^{(0)}\right)^{2}, \quad \frac{\operatorname{Re}\left(M_{1}-M_{2}\right)}{2}=\left|\frac{T_{[0]}^{(-1)}}{T_{[0]}^{(0)}}\right|^{2}\left(\operatorname{Re} T_{[0]}^{(0)}\right)^{2} .
$$

Both these expressions are non-negative, and thus there cannot be cancellations between positive and negative contributions, no matter how we regularize. Without a regularization, we know from $(3.4 .10)-3.4 .12$ that

$$
\operatorname{Re} T^{(0)}=-\frac{1}{8 \pi^{3}} \frac{\mathrm{PP}}{\xi^{2}} \quad \text { and } \quad \operatorname{Im} T_{[0]}^{(0)}=-\frac{i}{8 \pi^{2}} \delta\left(\xi^{2}\right) \epsilon\left(\xi^{0}\right) .
$$

Regularizing these terms, we find that for any regularization, both $M_{1}+M_{2}$ and $M_{1}-M_{2}$ are non-zero to degree four on the light cone. Hence 3.10 .30 is violated.

We come to the definitive conclusion that using perturbations of the form (3.10.10), it is in general impossible to satisfy the EL equations to degree four globally for all $\xi$. But, as we will now show, it is possible to satisfy the EL equations locally near $\xi=0$, in the sense that we can compensate all contributions in a Taylor expansion in $\xi$ to an arbitrarily high order. We consider the obvious generalization of (3.10.10 to several generations

$$
\delta P(x, y)=i \sum_{\beta=1}^{g} \sum_{s= \pm} \int \frac{d^{4} k}{(2 \pi)^{4}}\left\langle\xi, \delta k_{s}^{\beta}\right\rangle P_{ \pm}^{\beta}(k) e^{-i k(x-y)}
$$

where $P_{\beta}^{ \pm}$is obtained from 3.10 .9 if one replaces the vector field $q$ in 3.10 .8 by a vector field $q^{\beta}$ for the corresponding generation. 
Proposition 3.10.4. Suppose that the number of generations $g \geq 2$. Then for any given smooth functions $h_{\mathrm{v}}, h_{\mathrm{a}} \in C^{\infty}(\mathcal{M})$ and every parameter $L>2$, there are vector fields $\delta k_{ \pm}^{\beta}$ and $q^{\beta}$ in the Schwartz class such that for all multi-indices $\kappa$ with $1 \leq|\kappa| \leq L$,

$$
\left.\partial_{\xi}^{\kappa}\left[\operatorname{Tr}(\sharp \delta P(x, y))-h_{\mathrm{v}}(\xi)\right]\right|_{\xi=0}=0=\left.\partial_{\xi}^{\kappa}\left[\operatorname{Tr}(\Gamma \notin \delta P(x, y))-h_{\mathrm{a}}(\xi)\right]\right|_{\xi=0} .
$$

Proof. Following the arguments after $(3.10 .3)$ as well as after $(3.10 .10)$ and applying Lemma 3.10.1, it again suffices to analyze scalar Fourier integrals. Furthermore, using the polarization formula for the multi-index $\kappa$, we may restrict attention to the spherically symmetric situation (3.10.23 with Schwartz functions $\hat{f}^{\beta} \in \mathcal{S}(\mathbb{R})$. Keeping track of the factors $\xi$ in the arguments after 3.10 .3 and 3.10 .10 , it remains to show that for every smooth function $h(t)$ there are functions $\hat{f}_{\beta} \in \mathcal{S}(\hat{\mathcal{M}})$ such that the corresponding function $f(t)$ defined by (3.10.23) satisfies the conditions

$$
\left.\frac{d^{l}}{d t^{l}}(f(t)-h(t))\right|_{t=0}=0 \quad \text { for all } l=0, \ldots, L .
$$

We choose a test function $\hat{\eta} \in C_{0}^{\infty}((-1,1))$ and denote its Fourier transform by $\eta$. Furthermore, we choose a parameter $\Omega_{0}<-4 m_{g}$ and introduce the function $\hat{\eta}_{\Omega_{0}} \in$ $C_{0}^{\infty}\left(\left(-5 m_{g},-3 m_{g}\right)\right)$ by

$$
\hat{\eta}_{\Omega_{0}}(\Omega)=\frac{1}{\left|4 \Omega_{0}\right|} \hat{\eta}\left(\frac{\Omega-\Omega_{0}}{4 \Omega_{0}}\right) .
$$

Thus $\hat{\eta}_{\Omega_{0}}$ is supported for large negative frequencies. For any such frequency $\Omega \in$ $\operatorname{supp} \hat{\eta}_{\Omega_{0}}$, we want to construct the plane wave $e^{i \Omega t}$, with an error term which is again of large negative frequency. To this end, we proceed similar as in the proof of Lemma 3.10 .2 by iteratively perturbing the first and last Dirac seas, but now beginning with the first sea. Thus we first construct the plane wave $e^{i \Omega t}$ by perturbing the first sea, and compensate the error term by perturbing the last Dirac sea. This gives in analogy to 3.10.25

$$
\hat{f}^{1}(\omega)=\delta\left(\omega-\frac{\Omega^{2}+m_{1}^{2}}{2 \Omega}\right), \quad \hat{f}^{g}(\omega)=-\delta\left(\omega-\frac{\Omega m_{g}^{2}}{2 m_{1}^{2}}-\frac{m_{1}^{2}}{2 \Omega}\right),
$$

giving rise to the plane wave

$$
e^{i \Omega t}-e^{i \Omega t m_{g}^{2} / m_{1}^{2}} \text {. }
$$

Multiplying by $\hat{\eta}_{\Omega_{0}}$ and integrating over $\Omega$, we find that the function

$$
f(t):=\frac{1}{i t}\left(e^{i \Omega_{0} t} \eta\left(4 \Omega_{0} t\right)-e^{i \Omega_{1} t} \eta\left(4 \Omega_{1} t\right)\right) \quad \text { with } \quad \Omega_{1}=\frac{m_{g}^{2}}{m_{1}^{2}} \Omega_{0}
$$

has the desired Fourier representation $(3.10 .23)$. Since differentiating 3.10 .23 with respect to $t$ merely generates factors of $\omega_{ \pm}^{\beta}$, the functions $f^{(l)}(t):=t^{-1} \partial_{t}^{l}(t f(t)), l=$ $1, \ldots, L$, can again be represented in the form (3.10.23). By a suitable choice of the function $\hat{\eta}$, we can clearly arrange that the parameters $f(0), f^{(1)}(0), \ldots, f^{(L)}(0)$ are linearly independent. Thus by adding to $f$ a suitable linear combination of the functions $f^{(l)}$, we can arrange (3.10.31).

For clarity, we point out that the function $f$ in (3.10.31) will in general not be a good global approximation to $h$. In particular, it is impossible to pass to the limit $L \rightarrow \infty$. We also remark that this proposition also holds in the case $g=1$ of only Dirac sea. However, in this case it would not be possible to compensate the error term, so that instead of 3.10 .32 we would have to work with the combination $e^{i \Omega t}-e^{i t m^{2} / \Omega}$. This has 
the disadvantage that in the limit $\Omega \rightarrow-\infty$, we would get contributions of low frequency, making it impossible to generalize the result to the non-homogeneous situation (see the proof of Theorem 3.10.5 below). This is why Proposition 3.10.4 was formulated only in the case $g \geq 2$.

3.10.4. Nonlocal Potentials, the Quasi-Homogeneous Ansatz. We now want to extend the previous results to the non-homogeneous situation. For notational simplicity, we will write all formulas only for one generation. But as we only consider perturbations which are diagonal on the generations, all constructions immediately carry over to several generations by taking sums. Our method is to first describe our previous perturbations of the fermionic projector $(3.10 .10)$ by homogeneous perturbations of the Dirac operator. Replacing this perturbation operator by a nonlocal operator will then make it possible to describe the desired non-homogeneous perturbations of the fermionic projector.

We first note that the perturbation of the fermionic projector $(3.10 .2)$ came about by the unitary transformation (3.7.76). We can describe it alternatively by perturbing the Dirac operator in momentum space to

$$
\not k+\mathcal{B}(k)-m \quad \text { with } \quad \mathcal{B}(k)=U(k) \not k U(k)^{*}-\not k .
$$

Similarly, the plane wave $(3.10 .5)$ is a solution of the Dirac equation $(i \not \partial-\delta \not k-m) \psi=$ $\mathcal{O}\left((\delta k)^{2}\right)$. Thus the linear perturbation 3.10 .6 can be described equivalently by working with the perturbed Dirac operator in momentum space

$$
\not k-\delta \not k(k)-m \text {. }
$$

Likewise, for the perturbation (3.10.10), we must find a perturbation $\mathfrak{n}$ of the Dirac operator which is symmetric and, when restricted to the image of the operators $P_{ \pm}(k)$, reduces to the operators $-\delta \not k_{ \pm}$. In order to determine $\mathfrak{n}$, it is convenient to decompose the vector fields $\delta k_{ \pm}$as

$$
\delta k_{ \pm}=V \pm(\phi q+A) \quad \text { where } \quad\langle A, q\rangle=0
$$

(thus $V$ is the vector part, whereas $\phi$ and $A$ describe the axial components parallel and orthogonal to $q$, respectively). Then using (3.10.7) together with the relations

$$
\Gamma \not q \Pi_{ \pm}=\mp \Pi_{ \pm},
$$

we obtain

$$
\begin{aligned}
-\delta \not k_{ \pm} P_{ \pm} & =(-V \mp(\phi \not q+A)) P_{ \pm}=-V P_{ \pm}+(\phi \not q+A)(\Gamma \not) P_{ \pm} \\
& =(-V+\phi \Gamma+\Gamma \not \not A) P_{ \pm}=\left[-V+\frac{\phi}{m} \Gamma \not k+\Gamma \not q A\right] P_{ \pm},
\end{aligned}
$$

where in the last step we used that $(\not k-m) P_{ \pm}=0$. The square bracket has the desired properties of $\mathfrak{n}$. Thus the perturbation 3.10 .10 is equivalently described by the Dirac operator in momentum space

$$
\not k+\mathfrak{n}+m \quad \text { with } \quad \mathfrak{n}(k)=-V(k)+\frac{\phi(k)}{m} \Gamma \not k+\Gamma \not k(k) A(k),
$$

and the fields $V, A$ and $\phi$ as defined by (3.10.33). Note that $\mathfrak{n}$ is composed of three terms, which can be regarded as a vector, an axial and a bilinear perturbation of the Dirac operator. The perturbations in (3.10.34) are all homogeneous. The reader interested in the perturbation expansion for the corresponding Dirac solutions is referred to [F7, Appendix C.1]. 
In order to generalize to non-homogeneous perturbations, we write the operator $\mathfrak{n}$ as the convolution operator in position space

$$
(\mathfrak{n} \psi)(x)=\int_{\mathscr{M}} \mathfrak{n}(x, y) \psi(y) d^{4} y
$$

with the integral kernel

$$
\mathfrak{n}(x, y)=\int \frac{d^{4} k}{(2 \pi)^{4}} \mathfrak{n}(k) e^{i k \xi} .
$$

Now we can replace $\mathfrak{n}(x, y)$ by a general nonlocal kernel. We refer to the operator $\mathfrak{n}$ as a nonlocal potential in the Dirac equation. For technical convenience, it seems appropriate to make suitable decay assumptions at infinity, for example by demanding that

$$
\mathfrak{n}(x, y) \in \mathcal{S}(\mathscr{M} \times \mathscr{M}) .
$$

Then the corresponding fermionic projector can be introduced perturbatively exactly as outlined in $\$ 3.4 .4$. Before we can make use of the general ansatz (3.10.35) and 3.10.36), we must specify $\mathfrak{n}(x, y)$. To this end, we fix the variable $\zeta:=y+x$ and consider the fermionic projector as a function of the variable $\xi$ only. Then we are again in the homogeneous setting, and we can choose the operator $\mathfrak{n}$ as in (3.10.34). In order to clarify the dependence on the parameter $\zeta$, we denote this operator by $\mathfrak{n}(k, \zeta)$. As explained at the beginning of this section, we consider $y+x$ as a macroscopic variable, whereas $k$ is the momentum of the quantum mechanical oscillations. This motivates us to introduce the kernel $\mathfrak{n}(x, y)$ similar to 3.7 .78 by the quasi-homogeneous ansatz

$$
\mathfrak{n}(x, y)=\int \frac{d^{4} k}{(2 \pi)^{4}} \mathfrak{n}(k, y+x) e^{i k(y-x)} .
$$

We remark for clarity that this procedure generalizes our method $(3.7 .83)$ of rewriting the microlocal chiral transformation in terms of a perturbation of the Dirac operator. Now $\mathfrak{n}$ may involve additional nonlocal potentials which describe variations of the momenta of the plain-wave solutions.

The quasi-homogeneous ansatz makes it possible to satisfy the EL equations to degree four locally around every space-time point, as is made precise in the following theorem.

THEOREM 3.10.5. Suppose that the number of generations $g \geq 2$, and that we are given an integer $L \geq 2$ and a parameter $\delta>0$. Then for any given smooth functions $h_{\mathrm{v}}, h_{\mathrm{a}} \in$ $\mathcal{S}(\mathscr{M} \times \mathscr{M})$, there is a nonlocal potential $\mathfrak{n}$ of the form (3.10.35) with a kernel $\mathfrak{n}(x, y) \in$ $\mathcal{S}(\mathscr{M} \times \mathscr{M})$ of the form (3.10.37) such that for all multi-indices $\kappa$ with $1 \leq|\kappa| \leq L$,

$$
\left.\left|\partial_{\xi}^{\kappa}\left[\operatorname{Tr}(\notin \Delta P(x, y))-h_{\mathrm{v}}(x, y)\right]\right|_{x=y}|+| \partial_{\xi}^{\kappa}\left[\operatorname{Tr}(\Gamma \notin \Delta P(x, y))-h_{\mathrm{a}}(x, y)\right]\right|_{x=y} \mid<\delta .
$$

Here $\Delta P$ denotes the perturbation of the fermionic projector to first order in the nonlocal potential $\mathfrak{n}$.

Proof. For a fixed choice of the parameter $\Omega_{0}$ and for any given $\zeta$, we choose the homogeneous perturbation $\delta P$ as in the proof of Proposition 3.10.4 and rewrite it according to (3.10.34) as a homogeneous perturbation $\mathfrak{n}(k, \zeta)$ of the Dirac equation. Introducing the nonlocal potential by (3.10.35) and (3.10.37), the rapid decay of the functions $h_{\mathrm{v}}$ and $h_{\mathrm{a}}$ implies that the kernel $\mathfrak{n}(x, y)$ has rapid decay also in $\zeta$ (the rapid decay in $\xi$ is obvious because $\mathfrak{n}(., \zeta) \in \mathcal{S}(\hat{\mathscr{M}}))$. Since the same is true for all derivatives, we conclude that $\mathfrak{n}(x, y) \in \mathcal{S}(\mathscr{M} \times \mathscr{M})$. 
The corresponding perturbation of the fermionic projector $\Delta P$ can be analyzed with the methods introduced in [F5] (see also Section 2.2). We first pull out the Dirac matrices to obtain

$$
\Delta P(x, y)=\left(i \not \partial_{x}+m\right)\left(-i \frac{\partial}{\partial y^{k}}+m\right) \Delta T_{m^{2}}[\mathfrak{n}](x, y) \gamma^{k},
$$

where $\Delta T_{m^{2}}$ is the perturbation of the corresponding solution of the inhomogeneous Klein-Gordon equation (see [F5, eqs (2.4) and (2.5)])

$$
\begin{aligned}
& \Delta T_{m^{2}} {[\mathfrak{n}](x, y)=-\int_{\mathscr{M}} d^{4} z_{1} \int_{\mathcal{M}} d^{4} z_{2} } \\
& \quad \times\left(S_{m^{2}}\left(x, z_{1}\right) \mathfrak{n}\left(z_{1}, z_{2}\right) T_{m^{2}}\left(z_{2}, y\right)+T_{m^{2}}\left(x, z_{1}\right) \mathfrak{n}\left(z_{1}, z_{2}\right) S_{m^{2}}\left(z_{2}, y\right)\right)
\end{aligned}
$$

(where $T_{a}$ and $S_{a}$ are again given by $(3.4 .9)$ and $(3.8 .17)$ ). We next transform to momentum space. Setting

$$
\mathfrak{n}(p, q)=\int_{\mathscr{M}} \mathfrak{n}(p, \zeta) e^{\frac{i q \zeta}{2}} d^{4} \zeta
$$

with the "macroscopic" momentum vector $q$, the above formula for $\Delta T_{m^{2}}$ becomes (see [F5, eqs (3.8) and (3.9)])

$$
\begin{aligned}
& \Delta T_{m^{2}}[\mathfrak{n}]\left(p+\frac{q}{2}, p-\frac{q}{2}\right) \\
& \quad=-S_{m^{2}}\left(p+\frac{q}{2}\right) \mathfrak{n}(p, q) T_{m^{2}}\left(p-\frac{q}{2}\right)-T_{m^{2}}\left(p+\frac{q}{2}\right) \mathfrak{n}(p, q) S_{m^{2}}\left(p-\frac{q}{2}\right) .
\end{aligned}
$$

Now we can perform the light-cone expansion exactly as in [F5, Section 3]. This gives (cf. [F5, eq. (3.21)])

$$
\begin{aligned}
& \Delta T_{m^{2}}[\mathfrak{n}]\left(p+\frac{q}{2}, p-\frac{q}{2}\right)=-\mathfrak{n}(p, q) \\
& \quad \times \sum_{n=0}^{\infty} \frac{(-1)^{n}}{n !}\left(\frac{q^{2}}{4}\right)^{n} \sum_{k=0}^{\infty} \frac{1}{(2 k+1) !} \sum_{l=0}^{k}\left[\begin{array}{c}
2 k \\
l
\end{array}\right]\left(\frac{q^{2}}{2}\right)^{l}\left(\frac{q^{j}}{2} \frac{\partial}{\partial p^{j}}\right)^{2 k-2 l} T_{m^{2}}^{(n+1+l)}(p),
\end{aligned}
$$

where the curly brackets are combinatorial factors whose detailed form is not needed here (see [F5, eq. (3.13)]).

Let us discuss how the Fourier transform of this expansion behaves in the limit $\Omega_{0} \rightarrow$ $-\infty$. According to the construction of the functions $\hat{f}_{\beta}$ in the proof of Proposition 3.10.4. the function $\mathfrak{n}(p, q)$ is supported in the region $p^{2} \sim \Omega_{0}^{2}$. Moreover, the $p$-derivatives of $\mathfrak{n}$ scale in powers of $1 / \Omega_{0}$. This implies that every derivative of the factor $T_{m^{2}}$ gives a scaling factor of $\Omega_{0}^{-2}$. Since every such derivative comes with factor $q^{2}$, we obtain a scaling factor $\left(q^{2} / \Omega_{0}^{2}\right)^{n+l}$. Thus in the limit $\Omega_{0} \rightarrow-\infty$, it suffices to consider the lowest summand in $n+l$,

$$
\Delta T_{m^{2}}[\mathfrak{n}]=-\mathfrak{n}(p, q) \sum_{k=0}^{\infty} \frac{1}{(2 k+1) !}\left(\frac{q^{j}}{2} \frac{\partial}{\partial p^{j}}\right)^{2 k} T_{m^{2}}^{(1)}(p)\left[1+\mathcal{O}\left(\frac{q^{2}}{\Omega_{0}^{2}}\right)\right] .
$$

When transforming to position space, the $p$-derivatives can be integrated by parts. If they act on the function $\mathfrak{n}(p, q)$, this generates scale factors of the order $\mathcal{O}\left(|q| /\left|\Omega_{0}\right|\right)$ which again tend to zero as $\Omega_{0} \rightarrow-\infty$. Thus it remains to consider the case when these derivatives 
act on the plane wave $e^{i p \xi}$. We thus obtain

$$
\Delta T_{m^{2}}[\mathfrak{n}](x, y)=-\int \frac{d^{4} p}{(2 \pi)^{4}} \int \frac{d^{4} q}{(2 \pi)^{4}} \mathfrak{n}(p, q) \sum_{k=0}^{\infty} \frac{(-q \xi)^{2 k}}{2^{k}(2 k+1) !} T_{m^{2}}^{(1)}(p) e^{i p \xi-\frac{i q \zeta}{2}}+\mathcal{O}\left(\frac{1}{\Omega_{0}}\right) .
$$

We now consider a Taylor expansion in $\xi$ around $\xi=0$ up to the given order $L$. This amounts to replacing the factor $e^{e^{i p \xi}}$ by its power series and collecting the powers of $\xi$. The remaining task is to compare the factors $p \xi$ with $q \xi$. This is a subtle point, because the fact that $p^{2} \sim \Omega_{0}^{2}$ does not imply that the inner product $p \xi$ is large. Indeed, this effect was responsible for the appearance of low frequencies in the Fourier integral (3.10.22). However, in the proof of Proposition 3.10 .4 we arranged by a suitable choice of $\hat{f}_{1}$ and $\hat{f}_{g}$ that these low-frequency contributions cancel, so that we were working only with the high-frequency terms (3.10.32). Restating this fact in the present context, we can say that for the leading contribution to $\Delta T_{m^{2}}$, the factor $p \xi$ is larger than $q \xi$ by a factor of the order $\mathcal{O}\left(|q| /\left|\Omega_{0}\right|\right)$. Thus it suffices the consider the summand $k=0$,

$$
\Delta T_{m^{2}}[\mathfrak{n}](x, y)=-\int \frac{d^{4} p}{(2 \pi)^{4}} \int \frac{d^{4} q}{(2 \pi)^{4}} \mathfrak{n}(p, q) T_{m^{2}}^{(1)}(p) e^{i p \xi-\frac{i q \zeta}{2}}+\mathcal{O}\left(\frac{1}{\Omega_{0}}\right) .
$$

Now we can carry out the $q$-integration to obtain

$$
\Delta T_{m^{2}}[\mathfrak{n}](x, y)=-16 \int \frac{d^{4} q}{(2 \pi)^{4}} \mathfrak{n}(p, y+x) T_{m^{2}}^{(1)}(p) e^{i p \xi}+\mathcal{O}\left(\frac{1}{\Omega_{0}}\right) .
$$

Using this result in (3.10.38), we can carry out the derivatives to recover precisely the homogeneous perturbation (3.10.10) for fixed $\zeta$.

The scaling argument used in the last proof can be understood non-technically as follows. It clearly suffices to consider the region where $y$ lies in a small neighborhood of $x$. Thus we may perform the rescaling $\xi \rightarrow \xi / \lambda$ with a scale factor $\lambda \gg 1$, leaving $\zeta$ unchanged. This corresponds to changing the momentum scale by $\Omega_{0} \rightarrow \lambda \Omega_{0}$. In the limiting case $\lambda \rightarrow \infty$, the fermionic projector depends on $\xi$ on a smaller and smaller scale. On this scale, the dependence on the variable $x+y$ drops out, so that the quasi-homogeneous ansatz (3.10.37) becomes exact. For this argument to work, one must ensure that in the homogeneous setting all frequencies scale like $\Omega_{0}$, as was arranged in 3.10 .32 .

3.10.5. Discussion and Concluding Remarks. The previous analysis puts us into the position to discuss the scope and significance of nonlocal potentials. It is remarkable that by choosing suitable nonlocal potentials, one can satisfy the EL equations to any order in an expansion in powers of $\xi$ (see Theorem 3.10.5). However, this is not sufficient because, as shown in 3.5.2 the EL equations $(3.5 .29)$ must be satisfied globally (i.e. for any $x, y$ with $|\vec{\xi}| \gg \varepsilon$; see (3.5.10). Such global solutions of the EL equations, however, cannot be constructed with the help of nonlocal potentials (see Lemma 3.10 .3 and the paragraph before this lemma). More specifically, as one sees from 3.10.32, every plane wave $e^{i \Omega t}$ comes with an error term of the same magnitude. This raises the question whether nonlocal potentials are at all useful for fulfilling the EL equations.

In order to answer this question, it is helpful to first consider the EL equations on the more restrictive scale (3.7.77). On this scale, we could satisfy the EL equations by working with local potentials and the microlocal chiral transformation. Introducing additional nonlocal potentials does not seem helpful because in view of $(3.10 .32)$, it becomes impossible to satisfy the EL equation for all $x, y$ in the range (3.7.77). More specifically, by 
arranging that the constants $C_{\beta}$ in 3.7 .73 were constant, we achieved that the contribution by the microlocal chiral transformation was the desired logarithmic pole plus smooth contributions which varied on the scale $\ell_{\text {macro }}$. According to (3.10.32), additional nonlocal potentials would introduce contributions which oscillate on the macroscopic scale, thus violating the EL equations on the scale (3.7.77). We conclude that in order to satisfy the EL equations in a weak evaluation for any $|\vec{\xi}|$ in the range $(3.7 .77)$, the nonlocal potentials considered in this section must necessarily vanish.

We point out that with our methods, it seems impossible to satisfy the EL equations globally (i.e. to fulfill $(3.5 .29)$ for $x, y$ with $|\vec{\xi}| \gtrsim \ell_{\text {macro }}$ ). At first sight, this might seem to imply that the EL equations are overdetermined and cannot be solved. On the other hand, the general compactness results in $[\mathbf{F 1 3}$ indicate that our action principle does have non-trivial minimizers, so that the EL equations are expected to admit solutions. Thus there should be a way to compensate the above nonlocal error terms. A possible method is to modify the wave functions globally in space-time. Whether and how in detail this is supposed to work is a difficult question which we cannot answer here. Instead, we explain what this situation means physically: Suppose that a physical system is described by a minimizer of our action principle. Then the corresponding EL equations to degree four do not only yield the field equations, but they give rise to additional conditions which are nonlocal and can therefore not be specified by a local observer. In [F8, Section 7] such so-called nonlocal quantum conditions were proposed to explain phenomena which in ordinary quantum mechanics are probabilistic.

More specifically, the fact that the EL equations cannot in general be satisfied globally might explain the tendency for quantum mechanical wave functions to be localized, as we now outline. Suppose that the fermionic projector is perturbed by a fermionic wave function (3.10.1). At the origin $\xi=0$, this perturbation leads to the field equations as worked out in Section 3.8. The higher orders in $\xi$ can be compensated by nonlocal potentials. But the contribution for large $\xi$ cannot be compensated, thereby increasing our action. Thus seeking for minimizers, our action principle should try to arrange that the contribution (3.10.1) vanishes for large $\xi$. This might explain why quantum mechanical wave functions are usually not spread out over large distances, but are as much as possible localized, even behaving as point particles. This idea is explained further in $\mathbf{F 1 5}$. 



\title{
CHAPTER 4
}

\section{The Continuum Limit of a Fermion System Involving Neutrinos: Weak and Gravitational Interactions}

\begin{abstract}
We analyze the causal action principle for a system of relativistic fermions composed of massive Dirac particles and neutrinos. In the continuum limit, we obtain an effective interaction described by a left-handed, massive $\mathrm{SU}(2)$ gauge field and a gravitational field. The off-diagonal gauge potentials involve a unitary mixing matrix, which is similar to the Maki-Nakagawa-Sakata matrix in the standard model.
\end{abstract}

\subsection{Introduction}

In F7 it was proposed to formulate physics based on a new action principle in space-time. In Chapter 3, this action principle was worked out in detail in the so-called continuum limit for a simple model involving several generations of massive Dirac particles. We now extend this analysis to a model which includes neutrinos. In the continuum limit, we shall obtain an effective interaction described by a left-handed massive $\mathrm{SU}(2)$ gauge field and a gravitational field.

More specifically, we again consider the causal action principle introduced in [F7. Thus we define the causal Lagrangian by

$$
\mathcal{L}\left[A_{x y}\right]=\left|A_{x y}^{2}\right|-\frac{1}{8}\left|A_{x y}\right|^{2},
$$

where $A_{x y}=P(x, y) P(y, x)$ denotes the closed chain corresponding to the fermionic projector $P(x, y)$, and $|A|=\sum_{i=1}^{8}\left|\lambda_{i}\right|$ is the spectral weight (where $\lambda_{i}$ are the eigenvalues of $A$ counted with algebraic multiplicities). We introduce the action $\mathcal{S}$ and the constraint $\mathcal{T}$ by

$$
\mathcal{S}[P]=\iint_{\mathcal{M} \times \mathcal{M}} \mathcal{L}\left[A_{x y}\right] d^{4} x d^{4} y, \quad \mathcal{T}[P]=\iint_{\mathcal{M} \times \mathcal{M}}\left|A_{x y}\right|^{2} d^{4} x d^{4} y,
$$

where $(\mathscr{M},\langle.,\rangle$.$) denotes Minkowski space. The causal action principle is to$

$$
\text { minimize } \mathcal{S} \text { for fixed } \mathcal{T} \text {. }
$$

This action principle is given a rigorous meaning in Section 3.2. Every minimizer is a critical point of the so-called auxiliary action

$$
\mathcal{S}_{\mu}[P]=\iint_{\mathcal{M} \times \mathcal{M}} \mathcal{L}_{\mu}\left[A_{x y}\right] d^{4} x d^{4} y, \quad \mathcal{L}_{\mu}\left[A_{x y}\right]=\left|A_{x y}^{2}\right|-\mu\left|A_{x y}\right|^{2},
$$

which involves a Lagrange multiplier $\mu \in \mathbb{R}$.

We model the configuration of the fermions by a system consisting of a doublet of two sectors, each composed of three generations. Thus we describe the vacuum by the fermionic projector

$$
P(x, y)=P^{N}(x, y) \oplus P^{C}(x, y),
$$


where the charged sector $P^{C}$ is formed exactly as the fermionic projector in Chapter 3 as a sum of Dirac seas, i.e.

$$
P^{C}(x, y)=\sum_{\beta=1}^{3} P_{m_{\beta}}^{\mathrm{vac}}(x, y),
$$

where $m_{\beta}$ are the masses of the fermions and $P_{m}^{\mathrm{vac}}$ is the distribution

$$
P_{m}^{\mathrm{vac}}(x, y)=\int \frac{d^{4} k}{(2 \pi)^{4}}(\not k+m) \delta\left(k^{2}-m^{2}\right) \Theta\left(-k^{0}\right) e^{-i k(x-y)} .
$$

For the neutrino sector $P^{N}$ we consider two different ansätze. The first ansatz of chiral neutrinos is to take a sum of left-handed, massless Dirac seas,

$$
P^{N}(x, y)=\sum_{\beta=1}^{3} \chi_{L} P_{0}^{\mathrm{vac}}(x, y) .
$$

The configuration of Dirac seas (4.1.4), 4.1.5) and (4.1.7) models precisely the leptons in the standard model. It was considered earlier in [F7, Section 5.1]. The chiral ansatz 4.1.7) has the shortcoming that the neutrinos are necessarily massless, in contradiction to experimental observations. In order to describe massive neutrinos, we proceed as follows. As the mass mixes the left- and right-handed spinor components in the Dirac equation, for massive Dirac particles it is impossible to to restrict attention to one chirality. This leads us to the ansatz of massive neutrinos

$$
P^{N}(x, y)=\sum_{\beta=1}^{3} P_{\tilde{m}_{\beta}}^{\mathrm{vac}}(x, y) .
$$

Here the neutrino masses $\tilde{m}_{\beta} \geq 0$ will in general be different from the masses $m_{\beta}$ in the charged sector. Except for the different masses, the ansätze (4.1.5) and (4.1.8) are exactly the same. In particular, it might seem surprising that 4.1.8 does not distinguish the left- or right-handed component, in contrast to the observation that neutrinos are always left-handed. In order to obtain consistency with experiments, if working with (4.1.8) we need to make sure that the interaction distinguishes one chirality. For example, if we described massive neutrinos by (4.1.8) and found that the neutrinos only couple to lefthanded gauge fields, then the right-handed neutrinos, although being present in (4.1.8), would not be observable. With this in mind, working with 4.1.8 seems a possible approach, provided that we find a way to break the chiral symmetry in the interaction. It is a major goal of this paper to work out how this can be accomplished.

Working out the continuum limit for the above systems gives the following results. First, we rule out the chiral ansatz 4.1.7 by showing that it does not admit a global minimizer of the causal action principle. Thus in the fermionic projector approach, we must necessarily work with the massive ansatz 4.1.8). We find that at least one of the neutrino masses $\tilde{m}_{\beta}$ must be strictly positive. In order to break the chiral symmetry, we introduce additional right-handed states into the neutrino sector. It is a delicate question how this should be done. We discuss different approaches, in particular the so-called shear states and general surface states. The conclusion is that if the right-handed states and the regularization are introduced suitably, then the continuum limit is well-defined. Moreover, the structure of the effective interaction in the continuum limit is described as follows. The fermions satisfy the Dirac equation coupled to a left-handed SU(2)-gauge 
potential $A_{L}$,

$$
\left[i \not \partial+\chi_{R}\left(\begin{array}{cc}
A_{L}^{11} & A_{L}^{12} U_{\mathrm{MNS}}^{*} \\
\mathcal{A}_{L}^{21} U_{\mathrm{MNS}} & -\mathcal{A}_{L}^{11}
\end{array}\right)-m Y\right] \psi=0,
$$

where we used a block matrix notation (where the matrix entries are $3 \times 3$-matrices). Here $m Y$ is a diagonal matrix composed of the fermion masses,

$$
m Y=\operatorname{diag}\left(\tilde{m}_{1}, \tilde{m}_{2}, \tilde{m}_{3}, m_{1}, m_{2}, m_{3}\right),
$$

and $U_{\mathrm{MNS}}$ is a unitary $3 \times 3$-matrix. In analogy to the standard model, we refer to $U_{\mathrm{MNS}}$ as the Maki-Nakagawa-Sakata (MNS) matrix. The gauge potentials $A_{L}$ satisfy a classical Yang-Mills-type equation, coupled to the fermions. More precisely, writing the isospin dependence of the gauge potentials according to $A_{L}=\sum_{\alpha=1}^{3} A_{L}^{\alpha} \sigma^{\alpha}$ in terms of Pauli matrices, we obtain the field equations

$$
\partial_{l}^{k}\left(A_{L}^{\alpha}\right)^{l}-\square\left(A_{L}^{\alpha}\right)^{k}-M_{\alpha}^{2}\left(A_{L}^{\alpha}\right)^{k}=c_{\alpha} \bar{\psi}\left(\chi_{L} \gamma^{k} \sigma^{\alpha}\right) \psi,
$$

valid for $\alpha=1,2,3$. Here $M_{\alpha}$ are the bosonic masses and $c_{\alpha}$ the corresponding coupling constants. The masses and coupling constants of the two off-diagonal components are equal, i.e. $M_{1}=M_{2}$ and $c_{1}=c_{2}$, but they may be different from the mass and coupling constant of the diagonal component $\alpha=3$.

Moreover, our model involves a gravitational field described by the Einstein equations

$$
R_{j k}-\frac{1}{2} R g_{j k}+\Lambda g_{j k}=\kappa T_{j k},
$$

where $R_{j k}$ denotes the Ricci tensor, $R$ is scalar curvature, and $T_{j k}$ is the energy-momentum tensor of the Dirac field. Moreover, $\kappa$ and $\Lambda$ denote the gravitational and the cosmological constants, respectively. We find that the gravitational constant scales like $\kappa \sim \delta^{2}$, where $\delta$ is the length scale on which the shear and general surface states become relevant. The dynamics in the continuum limit is described by the coupled Dirac-Yang/Mills-Einstein equations 4.1.9), 4.1.10 and 4.1.11). These equations are of variational form, meaning that they can be recovered as Euler-Lagrange equations corresponding to an "effective action." The effective continuum theory is manifestly covariant under general coordinate transformations.

For ease in notation, the field equations 4.1.10 (and similarly the Einstein equations (4.1.11)) were written only for one fermionic wave function $\psi$. But clearly, the equations hold similarly for many-fermion systems (see Theorem 4.8.1). In this context, it is worth noting that, although the states of the Dirac sea are explicitly taken into account in our analysis, they do not enter the Einstein equations. Thus the naive "infinite negative energy density" of the sea drops out of the field equations, making it unnecessary to subtract any counter terms.

Similar as explained in Chapter 3 for an axial field, we again obtain corrections to the field equations which are nonlocal and violate causality in the sense that the future may influence the past. Moreover, for a given regularization one can compute the coupling constant, the bosonic mass, and the gravitational constant.

We remark that in this book, we always assume that the regularization and the corresponding scales $\varepsilon$ and $\delta$ are constant in space-time. Although this seems a good approximation locally, it is conceivable that the regularization does change on the astrophysical or cosmological scale. In this case, the gravitational constant would no longer be constant in space-time, but would become dynamical. The resulting effect, referred 
to as dynamical gravitational coupling, will not be covered in this book, but we refer the interested reader to $[\mathbf{F R}$.

We note that in this paper, we restrict attention to explaining our computations and results; for all conceptual issues and more references we refer to Chapter 3 and the survey article [F16].

\subsection{Regularizing the Neutrino Sector}

In this section, we explain how the neutrino sector is to be regularized. We begin in $\$ 4.2 .1$ by reviewing the regularization method used in [F7] (see also Section 2.4). Then we give an argument why this method is not sufficient for our purposes (see 4.2.2). This leads us to extending our methods (see 4.2 .3 ), and we will explain why these methods only work for the ansatz of massive neutrinos (see 4.2 .4 ). In 4.2 .5 we introduce the resulting general regularization scheme for the vacuum neutrino sector. In 4.2 .6 we explain how to introduce an interaction, relying for the more technical aspects on Appendix $\mathrm{F}$. Finally, in 4.2 .7 we introduce a modification of the formalism of the continuum limit which makes some computations more transparent.

4.2.1. A Naive Regularization of the Neutrino Sector. As in Section 3.3 we denote the regularized fermionic projector of the vacuum by $P^{\varepsilon}$, where the parameter $\varepsilon$ is the length scale of the regularization. This regularization length can be thought of as

the Planck length, but it could be even smaller. Here we shall always assume that $P^{\varepsilon}$ is homogeneous, meaning that it depends only on the difference vector $\xi:=y-x$. This is a natural physical assumption as the vacuum state should not distinguish a specific point in space-time. The simplest regularization method for the vacuum neutrino sector is to replace the above distribution $P^{N}(x, y)$ (see 4.1.7) ) by a function $P_{\varepsilon}^{N}$ which is again left-handed,

$$
P_{\varepsilon}^{N}(x, y)=\chi_{L} g_{j}(\xi) \gamma^{j}
$$

Such a regularization, in what follows referred to as a naive regularization, was used in [F7] (see [F7, eq. (5.3.1)]). It has the effect that the corresponding closed chain vanishes due to so-called chiral cancellations (see [F7, eq. 5.3.2]),

$$
A_{x y}^{N}:=P_{\varepsilon}^{N}(x, y) P_{\varepsilon}^{N}(y, x)=\chi_{L} \not g(x, y) \chi_{L} \not g(y, x)=\chi_{L} \chi_{R} \not g(x, y) \not g(y, x)=0 .
$$

Regularizing the charged sector as explained in [F7, Chapter 4] or Chapter 3, the closed chain of the regularized fermionic projector $P^{\varepsilon}$ of the whole system is of the form

$$
A_{x y}=P^{\varepsilon}(x, y) P^{\varepsilon}(y, x)=0 \oplus A_{x y}^{C} .
$$

Hence the closed chain has the eigenvalue zero with multiplicity four as well as the nontrivial eigenvalues $\lambda_{+}$and $\lambda_{-}$, both with multiplicity two (see [F7, Section 5.3]). Let us recall from [F7, Chapter 5] how by a specific choice of the Lagrange multiplier $\mu$ we can arrange that the EL equations are satisfied: The operator $Q$ corresponding to the action 4.1.3) is computed by (see [F7, Section 3.5] or Section 3.6)

$$
Q(x, y)=(-4 \mu) \oplus[(1-4 \mu)] \sum_{s= \pm} \overline{\lambda_{s}} F_{s} P(x, y) .
$$

In order for the operator $Q$ to vanish on the charged sector, we must choose

$$
\mu=\frac{1}{4} \text {. }
$$


Then

$$
Q(x, y)=-\sum_{s= \pm} \overline{\lambda_{s}} F_{s} P_{\varepsilon}^{N}(x, y) \oplus 0
$$

and multiplying by $P(y, z)$, we again get chiral cancellations to obtain

$$
Q(x, y) P(y, z)=-\sum_{s= \pm} \overline{\lambda_{s}} F_{s} \chi_{L} \not g(x, z) \chi_{L} \not g(z, y) \oplus 0=0
$$

Similarly, the pointwise product $P(x, y) Q(y, z)$ also vanishes, showing that the EL equations $[P, Q]$ are indeed satisfied in the vacuum.

Before going on, we note for clarity that in [F7], the chiral regularization ansatz (4.2.1) was overridden on the large scale in order to arrange a suitable normalization of the chiral fermionic states (see [F7, Appendix C]). More precisely, $P_{\varepsilon}^{N}$ was constructed by projecting out half of the states of a Dirac sea of mass $m$. The formula (4.2.1) was recovered in the limit $m \searrow 0$. In this so-called singular mass limit, the normalization integrals did not converge, making it possible to arrange a proper normalization, although for the limit (4.2.1) the normalization integral would vanish due to chiral cancellations. However, in [F7, Appendix C.1] it was explained that the formalism of the continuum limit is well-behaved in the singular mass limit, thus justifying why we were allowed to describe the regularized chiral Dirac seas by (4.2.1).

4.2.2. Instability of the Naively Regularized Neutrino Sector. We now give an argument which shows that if the neutrino sector is regularized in the neutrino sector according to (4.2.1), the system 4.1.4) cannot be an absolute minimum of the causal action principle (4.1.2). Suppose conversely that a fermionic projector $P^{\varepsilon}$, which in the neutrino sector is regularized according to (4.2.1), is an absolute minimum of the action principle (4.1.2). Then any variation of the fermionic projector can only increase the action. Evaluating this condition for specific variations leads to the notion of state stability, which we now recall (for details see [F7, Section 5.6] or [FH]). This notion makes it necessary to assume that our regularization is macroscopic away from the light cone, meaning that the difference $P^{\varepsilon}(x, y)-P(x, y)$ should be small pointwise except if the vector $y-x$ is close to the light cone (see [F7, Section 5.6]). This condition seems to be fulfilled for any reasonable regularization, and thus we shall always assume it from now on. Suppose that the state $\psi$ is occupied by a particle (i.e. that $\psi$ lies in the image of the operator $P^{\varepsilon}$ ), whereas the state $\phi$ is not occupied. We assume that $\psi$ and $\phi$ are suitably normalized and negative definite with respect to the indefinite inner product

$$
<\psi \mid \phi>=\int_{\mathscr{M}} \overline{\psi(x)} \phi(x) d^{4} x .
$$

Then the ansatz

$$
\delta P^{\varepsilon}(x, y)=\psi(x) \overline{\psi(y)}-\phi(x) \overline{\phi(y)}
$$

describes an admissible perturbation of $P^{\varepsilon}$. Since the number of occupied states is very large, $\delta P^{\varepsilon}$ is a very small perturbation (which even becomes infinitesimally small in the infinite volume limit). Thus we may consider $\delta P$ as a first order variation and treat the constraint in 4.1 .2 with a Lagrange multiplier. We point out that the set of possible variations $\delta P^{\varepsilon}$ does not form a vector space, because it is restricted by additional conditions. This is seen most easily from the fact that $-\delta P^{\varepsilon}$ is not an admissible variation, as it does not preserve the rank of $P^{\varepsilon}$. The fact that possible variations $\delta P^{\varepsilon}$ are restricted has the consequence that we merely get the variational inequality

$$
\mathcal{S}_{\mu}\left[P^{\varepsilon}+\delta P^{\varepsilon}\right] \geq \mathcal{S}_{\mu}\left[P^{\varepsilon}\right],
$$


valid for all admissible variations of the form (4.2.4).

Next, we consider variations which are homogeneous, meaning that $\psi$ and $\phi$ are plane waves of momenta $k$ respectively $q$,

$$
\psi(x)=\hat{\psi} e^{-i k x}, \quad \phi(x)=\hat{\phi} e^{-i q x} .
$$

Then both $P^{\varepsilon}$ and the variation $\delta P$ depend only on the difference vector $\xi=y-x$. Thus after carrying out one integral in 4.1.3), we obtain a constant, so that the second integral diverges. Thinking of the infinite volume limit of a system in finite 4-volume, we can remove this divergence simply by omitting the second integral. Then 4.2.5 simplifies to the state stability condition

$$
\int_{\mathscr{M}} \delta \mathcal{L}_{\mu}[A(\xi)] d^{4} \xi \geq 0
$$

In order to analyze state stability for our system (4.1.4), we first choose the Lagrange multiplier according to 4.2.2). Moreover, we assume that $\psi$ is a state of the charged sector, whereas $\phi$ is in the neutrino sector,

$$
\hat{\psi}=0 \oplus \hat{\psi}^{C}, \quad \hat{\phi}=\hat{\phi}^{N} \oplus 0 .
$$

Since $\psi$ should be an occupied state, it must clearly be a solution of one of the Dirac equations $\left(i \not \partial-m_{\alpha}\right) \psi=0$ with $\alpha \in\{1,2,3\}$. The state $\phi$, on the other hand, should be unoccupied; we assume for simplicity that its momentum $q$ is outside the support of $P_{\varepsilon}^{N}$,

$$
q \notin \operatorname{supp} \hat{g}
$$

(where $\hat{g}$ is the Fourier transform of the vector field $g$ in 4.2.1)). Thus our variation removes a state from a Dirac sea in the charged sector and occupies instead an unoccupied state in the neutrino sector with arbitrary momentum $q$ (in particular, $\phi$ does not need to satisfy any Dirac equation). Let us compute the corresponding variation of the Lagrangian. First, using that the spectral weight is additive on direct sums, we find that

$$
\begin{aligned}
\delta \mathcal{L}_{\frac{1}{4}} & =\delta\left(\left|A^{2}\right|-\frac{1}{4}|A|^{2}\right)=\delta\left|A^{2}\right|-\frac{1}{2}|A| \delta|A| \\
& =\delta\left|\left(A^{C}\right)^{2}\right|+\delta\left|\left(A^{N}\right)^{2}\right|-\frac{1}{2}\left(\left|A^{C}\right|+\left|A^{N}\right|\right)\left(\delta\left|A^{C}\right|+\delta\left|A^{N}\right|\right) .
\end{aligned}
$$

This formula simplifies if we use that $A^{N}$ vanishes due to chiral cancellations. Moreover, the first order variation of $\left(A^{N}\right)^{2}$ vanishes because

$$
\delta\left(\left(A^{N}\right)^{2}\right)=\left(\delta A^{N}\right) A^{N}+A^{N}\left(\delta A^{N}\right)=0 .
$$

Finally, $\delta\left|A^{N}\right|=\left|\left(A^{N}+\delta A^{N}\right)\right|-\left|A^{N}\right|=\left|\delta A^{N}\right|$. This gives

$$
\delta \mathcal{L}_{\frac{1}{4}}=\delta\left(\left|\left(A^{C}\right)^{2}\right|-\frac{1}{4}\left|A^{C}\right|^{2}\right)-\frac{1}{2}\left|A^{C}\right|\left|\delta A^{N}\right| .
$$

Note that $\psi$ only affects the first term, whereas $\phi$ influences only the second term. In the first term the neutrino sector does not appear, and thus the state stability analysis for one sector as carried out in [F7, Section 5.6] and [FH] applies. From this analysis, we know that the charged sector should be regularized in compliance with the condition of a distributional $\mathcal{M} P$-product (see also [F11]). Then the first term in 4.2.10 leads to a finite variation of our action. The point is that the second term in (4.2.10) is negative. In the next lemma we show that it is even unbounded below, proving that our system indeed violates the state stability condition (4.2.7). 
Lemma 4.2.1. Suppose that $P^{\varepsilon}$ is a regularization of the distribution (4.1.4) which is macroscopic away from the light cone and which in the neutrino sector is of the form 4.2.1. Then for any constant $C>0$ there is a properly normalized, negative definite wave function $\phi$ satisfying (4.2.6), (4.2.8) and (4.2.9) such that the corresponding variation of the fermionic projector

$$
\delta P^{\varepsilon}(x, y)=-\phi(x) \overline{\phi(y)}
$$

satisfies the inequality

$$
\int_{\mathscr{M}}\left|A^{C}\right|\left|\delta A^{N}\right| d^{4} \xi>C
$$

Proof. For convenience, we occupy two fermionic states of the same momentum $q$ such that

$$
\delta P_{\varepsilon}^{N}(x, y)=(\not p+m) e^{-i q(y-x)},
$$

where $p$ is a vector on the lower hyperboloid $\mathcal{H}_{m}:=\left\{p \mid p^{2}=m^{2}\right.$ and $\left.p^{0}<0\right\}$, and $m$ is a positive parameter which involves the normalization constant. For this simple ansatz one easily verifies that the image of $\delta P^{N}$ is indeed two-dimensional and negative definite. By occupying the two states in two separate steps, one can decompose 4.2 .12 into two variations of the required form (4.2.11). Therefore, it suffices to prove the lemma for the variation 4.2.12.

Using (4.2.1) and 4.2.12, the variation of $A^{N}$ is computed to be

$$
\delta A^{N}=\chi_{L} \not p(x, y)(\not p+m) e^{i q \xi}+\chi_{R}(\not p+m) \not g(y, x) e^{-i q \xi} .
$$

To simplify the notation, we omit the arguments $x$ and $y$ and write $g(\xi)=g(x, y)$. Then $g$ is a complex vector field with $\overline{g(\xi)}=g(y, x)$. Using that our regularization is macroscopic away from the light cone, there clearly is a set $\Omega \subset \mathscr{M}$ of positive Lebesgue measure such that both the vector field $g$ and the function $\left|A^{C}\right|$ are non-zero for all $\xi \in \Omega$. Then we can choose a past directed null vector $\mathfrak{n}$ such that $\langle\mathfrak{n}, g\rangle$ is non-zero on a set $\Omega^{\prime} \subset \Omega$ again of positive measure. We now consider a sequence of vectors $p_{l} \in \mathcal{H}_{m}$ which converge to the ray $\mathbb{R}^{+} \mathfrak{n}$ in the sense that there are coefficients $c_{l}$ with

$$
p_{l}-c_{l} \mathfrak{n} \rightarrow 0 \quad \text { and } \quad c_{l} \rightarrow \infty
$$

Then on $\Omega^{\prime}$, the inner product $\left\langle p_{l}, g\right\rangle$ diverges as $l \rightarrow \infty$. A short computation shows that in this limit, the eigenvalues of the matrix $\delta A_{l}^{N}$ also diverge. Computing these eigenvalues asymptotically, one finds that

$$
\left|\delta A_{l}^{N}\right| \geq 4\left|\left\langle p_{l}, g\right\rangle\right|+\mathcal{O}\left(l^{0}\right)
$$

Hence for large $l$,

$$
\int_{\mathscr{M}}\left|A^{C}\right|\left|\delta A_{l}^{N}\right| \geq \int_{\Omega^{\prime}}\left|A^{C}\right|\left|\left\langle p_{l}, g\right\rangle\right| \stackrel{l \rightarrow \infty}{\longrightarrow} \infty
$$

completing the proof.

It is remarkable that the above argument applies independent of any regularization details. We learn that regularizing the neutrino sector by a left-handed function 4.2.1) necessarily leads to an instability of the vacuum. The only way to avoid this instability is to consider more general regularizations where $P_{\varepsilon}^{N}$ also involves a right-handed component. 


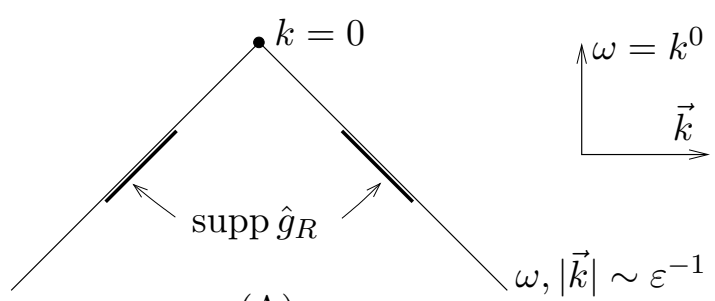

(A)

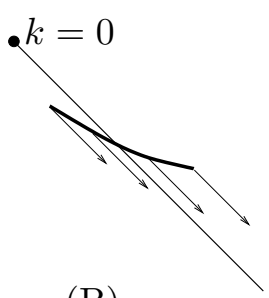

(B)

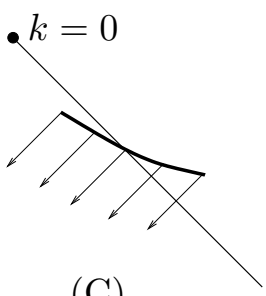

(C)

FiguRE 4.1. Plots of $\hat{g}_{R}$ exemplifying different regularization mechanisms in the neutrino sector

\subsubsection{Regularizing the Vacuum Neutrino Sector - Introductory Discus-}

sion. We begin by explaining our regularization method for one massless left-handed Dirac sea,

$$
P(x, y)=\chi_{L} P_{0}^{\mathrm{vac}}(x, y)
$$

(several seas and massive neutrinos will be considered later in this section). Working with a left-handed Dirac sea is motivated by the fact that right-handed neutrinos have never been observed in nature. To be precise, this physical observation only tells us that there should be no right-handed neutrinos in the low-energy regime. However, on the regularization scale $\varepsilon^{-1}$, which is at least as large as the Planck energy $E_{P}$ and therefore clearly inaccessible to experiments, there might well be right-handed neutrinos. Thus it seems physically admissible to regularize $P$ by

$$
P^{\varepsilon}(x, y)=\chi_{L} \not \phi_{L}(x, y)+\chi_{R} \not_{R}(x, y),
$$

provided that the Fourier transform $\hat{g}_{R}(k)$ vanishes if $\left|k^{0}\right|+|\vec{k}| \ll \varepsilon^{-1}$.

In order to explain the effect of such a right-handed high-energy component, we begin with the simplest example where $\hat{g}_{R}$ is supported on the lower mass cone,

$$
\hat{g}_{R}(k)=8 \pi^{2} \not k \hat{h}(\omega) \delta\left(k^{2}\right),
$$

where $\omega \equiv k^{0}$, and the non-negative function $\hat{h}$ is supported in the high-energy region $\omega \sim$ $\varepsilon^{-1}$ (see Figure $4.1(\mathrm{~A})$ ). We compute the Fourier integrals by

$$
\begin{aligned}
\not_{R}(\xi) & =8 \pi^{2} \int \frac{d^{4} k}{(2 \pi)^{4}} \not k \hat{h}(\omega) \delta\left(k^{2}\right) e^{i k \xi}=-8 i \pi^{2} \not \not_{\xi} \int \frac{d^{4} k}{(2 \pi)^{4}} \hat{h}(\omega) \delta\left(k^{2}\right) e^{i k \xi} \\
& =-2 i \not \partial_{\xi} \int_{-\infty}^{0} \frac{d \omega}{2 \pi} \hat{h}(\omega) e^{i \omega t} \int_{0}^{\infty} p^{2} d p \delta\left(\omega^{2}-p^{2}\right) \int_{-1}^{1} d \cos \vartheta e^{-i p r \cos \vartheta} \\
& =2 \not \partial_{\xi}\left[\frac{1}{r} \int_{-\infty}^{0} \frac{d \omega}{2 \pi} \hat{h}(\omega) e^{i \omega t} \int_{0}^{\infty} p d p \delta\left(\omega^{2}-p^{2}\right)\left(e^{-i p r}-e^{i p r}\right)\right] \\
& =-\not \partial_{\xi}\left[\frac{1}{r} \int_{-\infty}^{0} \frac{d \omega}{2 \pi} \hat{h}(\omega) e^{i \omega t}\left(e^{-i \omega r}-e^{i \omega r}\right)\right],
\end{aligned}
$$

where we set $t=\xi^{0}, r=|\vec{\xi}|$ and chose polar coordinates $(p=|\vec{k}|, \vartheta, \varphi)$. This gives the simple formula

$$
\not_{R}(\xi)=-\not \partial_{\xi} \frac{h(t-r)-h(t+r)}{r},
$$

where $h$ is the one-dimensional Fourier transform of $\hat{h}$. Under the natural assumption that the derivatives of $\hat{h}$ scale in powers of $\varepsilon$, the function $h$ decays rapidly on the regularization scale. Then $g_{R}$ vanishes except if $\xi$ is close to the light cone, so that the regularization 
is again macroscopic away from the light cone. But the contribution (4.2.14) does affect the singularities on the light cone, and it is thus of importance in the continuum limit. More specifically, on the upper light cone away from the origin $t \approx r \gg \varepsilon$, we obtain the contribution

$$
\begin{aligned}
g_{R}(\xi)= & -\not \partial_{\xi} \frac{h(t-r)}{r}=-\left(\gamma^{0}-\gamma^{r}\right) \frac{h^{\prime}(t-r)}{r}+\gamma^{r} \frac{h(t-r)}{r^{2}} \\
& +(\text { rapid decay in } r),
\end{aligned}
$$

where we set $\gamma^{r}=(\vec{\xi} \vec{\gamma}) / r$. This contribution is compatible with the formalism of the continuum limit, because it has a similar structure and the same scaling as corresponding contributions by a regularized Dirac sea (see [F11], where the same notation and sign conventions are used).

Regularizing the neutrino sector of our fermionic projector 4.1.4 using a righthanded high-energy component has the consequence that no chiral cancellations occur. Hence the EL equations become

$$
\sum_{i}\left(\left|\lambda_{i}\right|-\mu \sum_{l}\left|\lambda_{l}\right|\right) \frac{\overline{\lambda_{i}}}{\left|\lambda_{i}\right|} F_{i} P(x, y)=0,
$$

where $i$ labels the eigenvalues of $A_{x y}$. For these equations to be satisfied, we must choose

$$
\mu=\frac{1}{8}
$$

and furthermore we must impose that the eigenvalues of $A_{x y}$ all have the same absolute values in the sense that

$$
\left(\left|\lambda_{i}\right|-\left|\lambda_{j}\right|\right) \frac{\overline{\lambda_{i}}}{\left|\lambda_{i}\right|} F_{i} P(x, y)=0 \quad \text { for all } i, j
$$

In simple terms, the matrix $A^{N}$ must have the same spectral properties as $A^{C}$.

This consideration points to a shortcoming of the regularization (4.2.14). Namely, the expression 4.2.15 does not involve a mass parameter, and thus the corresponding contribution to the closed chain $A^{N}$ cannot have the same spectral properties as $A^{C}$, which has a non-trivial mass expansion. A possible solution to this problem is to consider states on a more general hypersurface, as we now explain again in the example of a spherically symmetric regularization. We choose

$$
\hat{g}_{R}(k)=-4 \pi^{2}\left(\gamma^{0}+\gamma^{k}\right) \hat{h}(\omega) \delta(|\vec{k}|-K(\omega)),
$$

where $\gamma^{k}=\vec{k} \vec{\gamma} / k$, and $h$ is chosen as in 4.2.14. We again assume that $\hat{\not g}$ is supported in the high-energy region, meaning that

$$
\hat{h}(\omega)=0 \quad \text { if }|\omega| \ll \varepsilon^{-1} .
$$


Setting $K=-\omega$, we get back to (4.2.14); but now the function $K$ gives a more general dispersion relation (see Figure 4.1(B)). Carrying out the Fourier integrals, we obtain

$$
\begin{aligned}
g_{R}^{0}(\xi)= & -4 \pi^{2} \int \frac{d^{4} k}{(2 \pi)^{4}} \hat{h}(\omega) \delta(|\vec{k}|-K(\omega)) e^{i k \xi} \\
= & -\int_{-\infty}^{0} \frac{d \omega}{2 \pi} \hat{h}(\omega) e^{i \omega t} \int_{0}^{\infty} p^{2} d p \delta(p-K(\omega)) \int_{-1}^{1} d \cos \vartheta e^{-i p r \cos \vartheta} \\
= & -\frac{i}{r} \int_{-\infty}^{0} \frac{d \omega}{2 \pi} \hat{h}(\omega) e^{i \omega t} \int_{0}^{\infty} p d p \delta(p-K(\omega))\left(e^{-i p r}-e^{i p r}\right) \\
= & \frac{i}{r} \int_{-\infty}^{0} \frac{d \omega}{2 \pi} \hat{h}(\omega) K(\omega) e^{i \omega t}\left(e^{i K r}-e^{-i K r}\right) \\
\left(\vec{\gamma} \vec{g}_{R}\right)(\xi)= & -4 \pi^{2}(i \vec{\gamma} \vec{\nabla}) \int \frac{d^{4} k}{(2 \pi)^{4}} \hat{h}(\omega) \delta(k-K(\omega)) \frac{1}{|\vec{k}|} e^{i k \xi} \\
= & -\vec{\gamma} \vec{\nabla}\left[\frac{1}{r} \int_{-\infty}^{0} \frac{d \omega}{2 \pi} \hat{h}(\omega) e^{i \omega t}\left(e^{i K r}-e^{-i K r}\right)\right] \\
= & -\frac{i \gamma^{r}}{r} \int_{-\infty}^{0} \frac{d \omega}{2 \pi} \hat{h}(\omega) K(\omega) e^{i \omega t}\left(e^{i K r}+e^{-i K r}\right) \\
& +\frac{\gamma^{r}}{r^{2}} \int_{-\infty}^{0} \frac{d \omega}{2 \pi} \hat{h}(\omega) e^{i \omega t}\left(e^{i K r}-e^{-i K r}\right)
\end{aligned}
$$

Evaluating as in 4.2.15 on the upper light cone away from the origin, we conclude that

$$
\begin{aligned}
g_{R}(\xi)= & \int_{-\infty}^{0} \frac{d \omega}{2 \pi} \hat{h}(\omega)\left(i \frac{\gamma^{0}-\gamma^{r}}{r} K(\omega)+\frac{\gamma^{r}}{r^{2}}\right) e^{i(\omega t+K r)} \\
& +(\text { rapid decay in } r) .
\end{aligned}
$$

For ease in notation, from now on we will omit the rapidly decaying error term. Rearranging the exponentials, we obtain

$$
g_{R}(\xi)=\int_{-\infty}^{0} \frac{d \omega}{2 \pi} e^{i(\omega+K) r} \hat{h}(\omega)\left(i \frac{\gamma^{0}-\gamma^{r}}{r} K(\omega)+\frac{\gamma^{r}}{r^{2}}\right) e^{i \omega(t-r)} .
$$

Now the mass expansion can be performed by expanding the factor $\exp (i(\omega+K) r)$,

$$
\begin{aligned}
g_{R}(\xi) & =\sum_{n=0}^{\infty} \frac{(i r)^{n}}{n !} \int_{-\infty}^{0} \frac{d \omega}{2 \pi} \hat{h}(\omega)(\omega+K)^{n}\left(i \frac{\gamma^{0}-\gamma^{r}}{r} K(\omega)+\frac{\gamma^{r}}{r^{2}}\right) e^{i \omega(t-r)} \\
& =\int_{-\infty}^{0} \frac{d \omega}{2 \pi} \hat{h}(\omega)\left(\frac{\gamma^{r}}{r^{2}}+i \frac{K \gamma^{0}+\omega \gamma^{r}}{r}+\cdots\right) e^{i \omega(t-r)} .
\end{aligned}
$$

We conclude that the general ansatz 4.2 .18 gives rise to a mass expansion which is similar to that for a massive Dirac sea (see [F7, Chaper 4] or Section 2.2). By modifying the geometry of the hypersurface $\{|\vec{k}|=K(\omega)\}$, we have a lot of freedom to modify the contributions to the mass expansion. We point out that, in contrast to the mass expansion for a massive Dirac sea, the mass expansion in 4.2.20 involves no logarithmic poles. This is because here we only consider high-energy states 4.2.19), whereas the logarithmic poles are a consequence of the low-frequency behavior of the massive Dirac seas (for details see the discussion of the logarithmic mass problem in [F7, Sections 2.5 and 4.3]). 
We now come to another regularization effect. The regularizations 4.2 .14 and 4.2 .18 considered so far have the property that $\not_{R}$ is a multiple of the matrix $\chi_{L}\left(\gamma^{0}+\gamma^{k}\right)$, as is indicated in Figure (4.1) (B) by the arrows (to avoid confusion with the signs, we note that on the lower mass shell, $\left.\not k=\omega \gamma^{0}-\vec{k} \vec{\gamma}=\omega\left(\gamma^{0}+\gamma^{k}\right)\right)$. Clearly, we could also have flipped the sign of $\gamma^{k}$, i.e. instead of 4.2.18,

$$
\hat{g}_{R}(k)=-4 \pi^{2}\left(\gamma^{0}-\gamma^{k}\right) \hat{h}(\omega) \delta(|\vec{k}|-K(\omega))
$$

(see Figure 4.1 (C)). In order to explain the consequence of this sign change in the simplest possible case, we consider the two functions

$$
\hat{\phi}_{ \pm}(k)=8 \pi^{2} \omega\left(\gamma^{0} \pm \gamma^{k}\right) \hat{h}(\omega) \delta\left(k^{2}\right),
$$

whose Fourier transforms are given in analogy to 4.2.15 on the upper light cone by

$$
\phi_{ \pm}(\xi)=-\left(\gamma^{0} \mp \gamma^{r}\right) \frac{h^{\prime}(t-r)}{r} \mp \gamma^{r} \frac{h(t-r)}{r^{2}} .
$$

When multiplying $\phi_{+}$by itself, the identity $\left(\gamma^{0}+\gamma^{r}\right)^{2}=0$ gives rise to a cancellation. For example, in the expression

$$
\frac{1}{4} \operatorname{Tr}\left(g_{+}(\xi) \not \phi_{+}(\xi)^{*}\right)=\frac{2 \operatorname{Re}\left(h^{\prime}(t-r) \overline{h(t-r)}\right)}{r^{3}}-\frac{|h(t-r)|^{2}}{r^{4}}
$$

the term $\sim r^{-2}$ has dropped out. The situation is different if we multiply $\phi_{+}$by $\not_{-}$. For example, in

$$
\frac{1}{4} \operatorname{Tr}\left(\not_{+}(\xi) \not q_{-}(\xi)^{*}\right)=\frac{2\left|h^{\prime}(t-r)\right|^{2}}{r^{2}}-\frac{2 i \operatorname{Im}\left(h^{\prime}(t-r) \overline{h(t-r)}\right)}{r^{3}}+\frac{|h(t-r)|^{2}}{r^{4}}
$$

no cancellation occurs, so that the term $\sim r^{-2}$ is present. From this consideration we learn that by flipping the sign of $\gamma^{r}$ as in (4.2.21), we can generate terms in the closed chain which have a different scaling behavior in the radius.

In order to clarify the last construction, it is helpful to describe the situation in terms of the general notions introduced in [F7, Section 4.4]. The fact that the leading term in 4.2.15) is proportional to $\left(\gamma^{0}-\gamma^{r}\right)$ can be expressed by saying that the vector component is null on the light cone. When forming the closed chain, the term quadratic in the leading terms drops out, implying that $A_{x y} \sim r^{-3}$. In momentum space, this situation corresponds to the fact that the vector $\hat{g}(k)$ points almost in the same direction as $k$. In other words, the shear of the surface states is small. Thus in (4.2.14) and (4.2.18) as well as in $g_{+}$, the shear is small, implying that the vector component is null on the light cone, explaining the cancellation of the term $\sim r^{-2}$ in 4.2.23). The states in 4.2.21) and $g_{-}$, however, have a large shear. Thus the corresponding vector component is not null on the light cone, explaining the term $\sim r^{-2}$ in 4.2.24. We point out that states of large shear have never been considered before, as in [F7] we always assumed the shear to be small. For simplicity, we refer to the states in (4.2.21) and $g_{-}$as shear states.

We next outline how the above considerations can be adapted to the general ansätze (4.1.7) and (4.1.8). In order to describe several chiral Dirac seas, one simply adds regularized Dirac seas, each of which might involve a right-handed high-energy component and/or shear states. In other words, in the chiral ansatz 4.1.7) one replaces each summand by a Dirac sea regularized as described above. In the massive ansatz 4.1.8, we regularize every massive Dirac sea exactly as described in [F7, Chapter 4]. Moreover, in order to distinguish the neutrino sector from a massive sector, we add one or several 
right-handed high-energy contributions. In this way, the regularization breaks the chiral symmetry.

We finally make a few remarks which clarify our considerations and bring them into the context of previous work.

REMARK 4.2.2. (1) We point out that the above assumption of spherical symmetry was merely a technical simplification. But this assumption is not crucial for the arguments, and indeed it will be relaxed in 4.2.5. We also point out that in all previous regularizations, the occupied states formed a hypersurface in momentum space. In this paper, we will always restrict attention to such surface states (see [F7, Section 4.3]). The underlying guiding principle is that one should try to build up the regularized fermionic projector with as few occupied states as possible. This can be understood from the general framework of causal variational principles as introduced in $[\mathbf{F 1 0}, \mathbf{F 1 3}$. Namely, in this framework the minimum of the action decreases if the number of particles gets larger ${ }^{1}$. Thus to construct minimizers, one should always keep the number of particles fixed. Conversely, one could also construct minimizers by keeping the action fixed and decreasing the number of particles. With this in mind, a regularization involving fewer particles corresponds to a smaller action and is thus preferable.

(2) It is worth mentioning that in all the above regularizations we worked with null states, meaning that for every $k$, the image of the operator $\hat{P}(k)$ is null with respect to the spin scalar product. Such null states can be obtained from properly normalized negative definite states by taking a singular mass limit, similar as worked out in [F7, Appendix C].

(3) At first sight, our procedure for regularizing might seem very special and adhoc. However, it catches all essential effects of more general regularizations, as we now outline. First, states of large shear could be used just as well for the regularization of massive Dirac seas, also in the charged sector. However, our analysis in Section 4.7 will reveal that the EL equations will only involve the difference in the regularization used in the charged sector compared to that in the neutrino sector. Thus it is no loss of generality to regularize the charged sector simply according to [F7, Chapter 4], and to account for shear states only in the neutrino sector. Next, in the high-energy region one could also work with massive states. In order to break the chiral symmetry, one could project out one spin state with the ansatz

$$
\not g(p)=\frac{1}{2}(\mathbb{1}-\rho \not)(\not k+m) \hat{h}(k)
$$

with $p^{2}=m^{2}, q^{2}=-1$ and $\langle q, k\rangle=0$ (see [F7, eq. (C.1.5)], where a corresponding Dirac sea is considered before taking the singular mass limit). However, this procedure would have two disadvantages. First, massive states would yield additional contributions to the fermionic projector, whereas 4.2.25 even gives rise to bilinear and pseudoscalar contributions, which would all cause technical complications. Secondly, massive states involve both left- and right-handed

\footnotetext{
${ }^{1}$ To be precise, this results holds for operators in the class $\mathcal{P}^{f}$ (see [F10, Definition 2.7]) if the fermionic operator is rescaled such that its trace is independent of $f$. In the formulation with local correlation matrices (see [F13, Section 3.2]) and under the trace constraint, the canonical embedding $\mathbb{C}^{f} \hookrightarrow \mathbb{C}^{f+1}$ allows one to regard a system of $f$ particles as a special system of $f+1$ particles. Since varying within the set of $f+1$-particle systems gives more freedom, it is obvious that the action decreases if $f$ gets larger.
} 
components, which are coupled together in such a way that it would be more difficult to introduce a general interaction. Apart from these disadvantages, working with massive states does not seem to lead to any interesting effects. This is why we decided not to consider them in this paper.

(4) We mention that for a fully convincing justification of the vacuum fermionic projector 4.1.4 and of our regularization method, one should extend the state stability analysis from [FH to a system of a charged sector and a neutrino sector. Since this analysis only takes into account the behavior of the fermionic projector away from the light cone, the high-energy behavior of $P^{\varepsilon}$ plays no role, so that one could simply work with the explicit formula for the unregularized fermionic projector (4.1.4). Then the methods of [FH] apply to each of the sectors. However, the two sectors are coupled by the term $|A|^{2}$ in the Lagrangian. The results of this analysis will depend on the value of the Lagrange multiplier (4.2.17) as well as on the choice of all lepton masses (including the neutrino masses). Clearly, the details of this analysis are too involved for predicting results. For the moment, all one can say is that there is no general counter argument (in the spirit of $\$ 4.2 .2$ which might prevent state stability.

4.2.4. Ruling out the Chiral Neutrino Ansatz. In this section, we give an argument which shows that for chiral neutrinos there is no regularization which gives rise to a stable minimum of the causal action principle. More precisely, we will show that even taking into account the regularization effects discussed in the previous section, it is impossible to arrange that the vacuum satisfies the EL equations in the continuum limit 4.2.16 and 4.2.17). Our argument applies in such generality (i.e. without any specific assumptions on the regularization) that it will lead us to drop the ansatz of chiral neutrinos (4.1.7), leaving us with the ansatz of massive neutrinos (4.1.8).

Considering massive neutrinos is clearly consistent with the experimental observation of neutrino oscillations. Based on these experimental findings, we could also have restricted attention to the ansatz (4.1.8) right away. On the other hand, considering also chiral neutrinos (4.1.7) has the advantage that we can conclude that massive neutrinos are needed even for mathematical consistency. This conclusion is of particular interest because in the neutrino experiments, the mass of the neutrinos is observed indirectly from the fact that different generations of neutrinos are converted into each other. This leaves the possibility that neutrinos might be massless, and that the neutrino oscillations can be explained instead by modifying the weak interaction. The following argument rules out this possibility by giving an independent reason why there must be massive neutrinos.

Recall that the Dirac seas in the charged sector $P^{C}, 4.1 .5$, can be written as

$$
P_{m}^{\mathrm{vac}}(x, y)=\left(i \not \partial_{x}+m\right) T_{m^{2}}(x, y),
$$

where $T_{m^{2}}$ is the Fourier transform of the lower mass shell,

$$
T_{m^{2}}(x, y)=\int \frac{d^{4} k}{(2 \pi)^{4}} \delta\left(k^{2}-m^{2}\right) \Theta\left(-k^{0}\right) e^{-i k(x-y)} .
$$

Computing this Fourier integral and expanding the resulting Bessel functions gives the expansion in position space

$$
\begin{aligned}
T_{m^{2}}(x, y)=- & \frac{1}{8 \pi^{3}}\left(\frac{\mathrm{PP}}{\xi^{2}}+i \pi \delta\left(\xi^{2}\right) \varepsilon\left(\xi^{0}\right)\right) \\
& +\frac{m^{2}}{32 \pi^{3}}\left(\log \left|m^{2} \xi^{2}\right|+c+i \pi \Theta\left(\xi^{2}\right) \epsilon\left(\xi^{0}\right)\right)+\mathcal{O}\left(\xi^{2} \log \left(\xi^{2}\right)\right) .
\end{aligned}
$$


(see [F7, Section 2.5] or 93.4 .4 ). The point for what follows is that the light-cone expansion of $P_{m}^{\mathrm{vac}}(x, y)$ involves a logarithmic pole $\sim \log \left(\xi^{2}\right)$. As a consequence, in the EL equations (4.2.16) we get contributions to 4.2.16) which involve the logarithm of the radius $|\vec{x}-\vec{y}|$ (for details see 33.5 .1 or the weak evaluation formula 4.2.31) below). In order to satisfy the EL equations, these logarithmic contributions in the charged sector must be compensated by corresponding logarithmic contributions in the neutrino sector.

Now assume that we consider the chiral neutrino ansatz (4.1.7). Then the light-cone expansion of $T_{N}$ does not involve logarithmic poles (indeed, the distribution $P_{0}^{\mathrm{vac}}$ can be given explicitly in position space by taking the limit $m \searrow 0$ in 4.2.26 and 4.2.27). Thus the logarithmic contributions in the radius must come from the high-energy component to the fermionic projector. However, as one sees explicitly from the formulas 4.2.20 and 4.2.22), the high-energy component is a Laurent series in the radius and does not involve any logarithms. This explains why with chiral neutrinos alone it is impossible to satisfy the EL equations.

This problem can also be understood in more general terms as follows. The logarithmic poles of $P_{m}^{\mathrm{vac}}(x, y)$ are an infrared effect related to the fact that the square root is not an analytic function (see the discussion of the so-called logarithmic mass problem in [F7, Sections 2.5 and 4.5]). Thus in order to arrange logarithmic contributions in the high-energy region, one would have to work with states on a surface with a singularity. Then the logarithm in the radius would show up in the next-to leading order on the light cone. Thus in order to compensate the logarithms in (4.2.27), the contribution by the high-energy states would be just as singular on the light cone as the contribution by the highest pole in 4.2.27). Apart from the fact that it seems difficult to construct such highenergy contributions, such constructions could no longer be regarded as regularizations of Dirac sea structures. Instead, one would have to put in specific additional structures ad hoc, in contrast to the concept behind the method of variable regularization (see [F7, Section 4.1] or Remark 1.2.1).

The above arguments show that at least one generation of neutrinos must be massive. In particular, we must give up the ansatz (4.1.7) of chiral neutrinos. Instead, we shall always work with massive neutrinos (4.1.8), and we need to assume that at least one of the masses $\tilde{m}_{\beta}$ is non-zero.

For clarity, we finally remark that our arguments also leave the possibility to choose another ansatz which involves a combination of both chiral and massive neutrinos, i.e.

$$
P^{N}(x, y)=\sum_{\beta=1}^{\beta_{0}} \chi_{L} P_{0}^{\mathrm{vac}}(x, y)+\sum_{\beta=\beta_{0}+1}^{3} P_{m_{\beta}}^{\mathrm{vac}}(x, y) \quad \text { with } \quad \beta_{0} \in\{1,2\} .
$$

The only reason why we do not consider this ansatz here is that it seems more natural to describe all neutrino generations in the same way. All our methods could be extended in a straightforward way to the ansatz 4.2.28.

4.2.5. A Formalism for the Regularized Vacuum Fermionic Projector. In the following sections 4.2 .5 and 4.2 .6 , we incorporate the regularization effects discussed in $\$ 4.2 .3$ to the formalism of the continuum limit. Beginning with the vacuum, we recall that in [F7, Section 4.5] we described the regularization by complex factors $T_{[p]}^{(n)}$ and $T_{\{p\}}^{(n)}$ (see also 3.5 .1 ). The upper index $n$ tells about the order of the singularity on the light cone, whereas the lower index keeps track of the orders in a mass expansion. In 4.2 .3 , we considered a chiral decomposition (4.2.13) and chose the left- and right-handed components independently. This can be indicated in our formalism by a chiral index $c \in\{L, R\}$, 
which we insert into the subscript. Thus we write the regularization (4.2.13) and 4.2.14) symbolically as

$$
P^{\varepsilon}(x, y)=\frac{i}{2}\left(\chi_{L} \not T_{[L, 0]}^{(-1)}+\chi_{R} \sharp T_{[R, 0]}^{(-1)}\right) .
$$

If the regularization effects of the previous section are not used in the left- or right-handed component, we simply omit the chiral index. Thus if we work with general surface states or shear states only in the right-handed component, we leave out the left-handed chiral index,

$$
P^{\varepsilon}(x, y)=\frac{i}{2}\left(\chi_{L} \sharp T_{[0]}^{(-1)}+\chi_{R} \sharp T_{[R, 0]}^{(-1)}\right) .
$$

When using the same notation as in the charged sector, we always indicate that we assume the corresponding regularizations to be compatible. Thus for factors $T_{0}^{(n)}$ without a chiral index, we shall use the same calculation rules in the neutrino and in the charged sector. This will also make it possible to introduce an interaction between these sectors (for details see 4.2 .6 and Appendix F). If we consider a sector of massive neutrinos 4.1.8, we first perform the mass expansion of every Dirac sea

$$
P_{m}^{\varepsilon}=\frac{i \not}{2} \sum_{n=0}^{\infty} \frac{m^{2 n}}{n !} T_{[2 n]}^{(-1+n)}+\sum_{n=0}^{\infty} \frac{m^{2 n+1}}{n !} T_{[2 n+1]}^{(n)}
$$

and then add the chiral index to the massless component,

$$
\begin{aligned}
P_{m}^{\varepsilon}(x, y)= & \frac{i}{2}\left(\chi_{L} \oiint T_{[0]}^{(-1)}+\chi_{R} \sharp T_{[R, 0]}^{(-1)}\right) \\
& +\frac{i \notin}{2} \sum_{n=1}^{\infty} \frac{m^{2 n}}{n !} T_{[2 n]}^{(-1+n)}+\sum_{n=0}^{\infty} \frac{m^{2 n+1}}{n !} T_{[2 n+1]}^{(n)} .
\end{aligned}
$$

Now the regularization effects of the previous section can be incorporated by introducing more general factors $T_{[c, p]}^{(n)}$ and $T_{\{c, p\}}^{(n)}$ and by imposing suitable computation rules. Before beginning, we point out that the more general factors should all comply with our weak evaluation rule

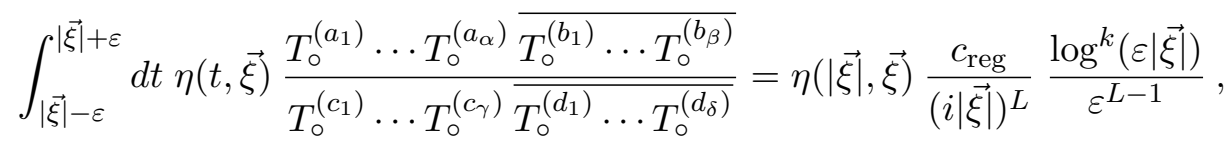

which holds up to

$$
\text { (higher orders in } \left.\varepsilon / \ell_{\text {macro }} \text { and } \varepsilon /|\vec{\xi}|\right) \text {. }
$$

Here $L$ is the degree defined by $\operatorname{deg} T_{\circ}^{(n)}=1-n$, and $c_{\text {reg }}$ is a so-called regularization parameter (for details see again [F7, Section 4.5] or \$3.5.1). The quotient of products of factors $T_{\circ}^{(n)}$ and $\overline{T_{\circ}^{(n)}}$ in 4.2 .31 is referred to as a simple fraction. In order to take into account the mass expansion 4.2.20), we replace every factor $T_{[c, 0]}^{(-1)}$ by the formal series

$$
\sum_{n=0}^{\infty} \frac{1}{n !} \frac{1}{\delta^{2 n}} T_{[c, 2 n]}^{(-1+n)} .
$$

This notation has the advantage that it resembles the even part of the standard mass expansion 4.2.29). In order to get the scaling dimensions right, we inserted a factor $\delta^{-2 n}$, where the parameter $\delta$ has the dimension of a length. The scaling of $\delta$ will be specified 
later (see 4.4.19), Section 4.5 and Section 4.9). For the moment, in order to make sense of the mass expansion, we only need to assume that the

$$
\text { length scale } \delta \gg \varepsilon \text {. }
$$

But $\delta$ could be much smaller than the Compton wave length of the fermions of the system. It could even be on the same scale as the regularization length $\varepsilon$. We thus replace (4.2.30) by

$$
\begin{aligned}
P_{m}^{\varepsilon}(x, y)= & \chi_{L} \frac{i \not}{2} T_{[0]}^{(-1)}+\chi_{R} \frac{i \not}{2} \sum_{n=0}^{\infty} \frac{1}{n !} \frac{1}{\delta^{2 n}} T_{[R, 2 n]}^{(-1+n)} \\
& +\frac{i \nsubseteq}{2} \sum_{n=1}^{\infty} \frac{m^{2 n}}{n !} T_{[2 n]}^{(-1+n)}+\sum_{n=0}^{\infty} \frac{m^{2 n+1}}{n !} T_{[2 n+1]}^{(n)} .
\end{aligned}
$$

The effect of large shear can be incorporated in our contraction rules, as we now explain. Recall that our usual contraction rules read

$$
\begin{aligned}
\left(\sharp_{[p]}^{(n)}\right)^{j}\left(\sharp_{\left[p^{\prime}\right]}^{\left(n^{\prime}\right)}\right)_{j} & =\frac{1}{2}\left(z_{[p]}^{(n)}+z_{\left[p^{\prime}\right]}^{\left(n^{\prime}\right)}\right)+(\text { higher orders in } \varepsilon /|\vec{\xi}|) \\
z_{[p]}^{(n)} T_{[p]}^{(n)} & =-4\left(n T_{[p]}^{(n+1)}+T_{\{p\}}^{(n+2)}\right)
\end{aligned}
$$

(and similarly for the complex conjugates, cf. [F7, Section 4.5] or 3.5.1). We extend the first rule in the obvious way by inserting lower chiral indices. In the second rule we insert a factor $\delta^{-2}$,

$$
z_{[c, p]}^{(n)} T_{[c, p]}^{(n)}=-4\left(n T_{[c, p]}^{(n+1)}+\frac{1}{\delta^{2}} T_{\{c, p\}}^{(n+2)}\right) .
$$

The factor $\delta^{-2}$ has the advantage that it ensures that the factors with square and curly brackets have the same scaling dimension (as one sees by comparing 4.2.38) with 4.2.33) or 4.2.29; we remark that this point was not taken care of in [F7] and Chapter 3 . simply because the factors with curly brackets played no role). The term $\delta^{-2} T_{\{c, p\}}^{(n+2)}$ can be associated precisely to the shear states. For example, in the expression

$$
\frac{1}{8} \operatorname{Tr}\left(\left(\Varangle T_{[0]}^{(-1)}\right)\left(\Varangle T_{[R, 0]}^{(-1)}\right)\right)=T_{[0]}^{(0)} T_{[R, 0]}^{(-1)}+T_{[0]}^{(-1)} T_{[R, 0]}^{(0)}-T_{\{0\}}^{(1)} T_{[R, 0]}^{(-1)}-\frac{1}{\delta^{2}} T_{[0]}^{(-1)} T_{\{R, 0\}}^{(1)},
$$

the last summand involves an additional scaling factor of $r$ and can thus be used to describe the effect observed in 4.2.24. Using again 4.2.34, we can reproduce the scaling of the first summand in 4.2.24).

In the weak evaluation formula (4.2.31), one can integrate by parts. This gives rise to the following integration-by-parts rules. On the factors $T_{\circ}^{(n)}$ we introduce a derivation $\nabla$ by

$$
\nabla T_{\circ}^{(n)}=T_{\circ}^{(n-1)}
$$

Extending this derivation with the Leibniz and quotient rules, the integration-by-parts rules states that

$$
\nabla\left(\frac{T_{\circ}^{\left(a_{1}\right)} \cdots T_{\circ}^{\left(a_{\alpha}\right)} \overline{T_{\circ}^{\left(b_{1}\right)} \cdots T_{\circ}^{\left(b_{\beta}\right)}}}{T_{\circ}^{\left(c_{1}\right)} \cdots T_{\circ}^{\left(c_{\gamma}\right)} \overline{T_{\circ}^{\left(d_{1}\right)} \cdots T_{\circ}^{\left(d_{\delta}\right)}}}\right)=0 .
$$

As shown in [F7, Appendix E], there are no further relations between the factors $T_{\circ}^{(a)}$.

We finally point out that the chiral factors $T_{[c, p]}^{(n)}$ and $T_{\{c, p\}}^{(n)}$ were introduced in such a way that the weak evaluation formula 4.2.31 remains valid. However, one should keep 
in mind that these chiral factors do not have logarithmic singularities on the light cone, which implies that they have no influence on the power $k$ in 4.2.31). This follows from the fact that the chiral factors only describe high-energy effects, whereas the logarithmic poles are a consequence of the low-frequency behavior of the massive Dirac seas (see also the explicit example 4.2 .20 and the explanation thereafter).

4.2.6. Interacting Systems, Regularization of the Light-Cone Expansion. We now extend the previous formalism such as to include a general interaction; for the derivation see Appendix F. For simplicity, we restrict attention to the system 4.1.4 with massive neutrinos (4.1.8) and a non-trivial regularization of the neutrino sector by right-handed high-energy states. But our methods apply to more general systems as well (see Remark 4.2.3 below). In preparation, as in [F7, Section 2.3] and \$3.4.1 it is helpful to introduce the auxiliary fermionic projector as the direct sum of all Dirac seas. In order to allow the interaction to be as general as possible, it is preferable to describe the right-handed high-energy states by a separate component of the auxiliary fermionic projector. Thus we set

$$
P^{\mathrm{aux}}=P_{\mathrm{aux}}^{N} \oplus P_{\mathrm{aux}}^{C},
$$

where

$$
P_{\mathrm{aux}}^{N}=\left(\bigoplus_{\beta=1}^{3} P_{\tilde{m}_{\beta}}^{\mathrm{vac}}\right) \oplus 0 \quad \text { and } \quad P_{\mathrm{aux}}^{C}=\bigoplus_{\beta=1}^{3} P_{m_{\beta}}^{\mathrm{vac}} .
$$

Note that $P^{\text {aux }}$ is composed of seven direct summands, four in the neutrino and three in the charged sector. As the fourth component of the neutrino sector is reserved for right-handed high-energy neutrinos (possibly occupying shear or general surface states), the corresponding component vanishes without regularization 4.2.41).

In order to recover $P^{\text {aux }}$ from a solution of the Dirac equation, we introduce the chiral asymmetry matrix $X$ by

$$
X=\left(\mathbb{1}_{\mathbb{C}^{3}} \oplus \tau_{\text {reg }} \chi_{R}\right) \oplus \mathbb{1}_{\mathbb{C}^{3}} .
$$

Here $\tau_{\text {reg }}$ is a dimensionless parameter, which we always assume to take values in the range

$$
0<\tau_{\text {reg }} \leq 1
$$

It has two purposes. First, it indicates that the corresponding direct summand involves a non-trivial regularization. This will be useful below when we derive constraints for the interaction. Second, it can be used to modify the amplitude of the regularization effects. In the limit $\tau_{\text {reg }} \searrow 0$, the general surface states and shear states are absent, whereas in the case $\tau_{\text {reg }}=1$, they have the same order of magnitude as the regular states.

Next, we introduce the mass matrix $Y$ by

$$
Y=\frac{1}{m} \operatorname{diag}\left(\tilde{m}_{1}, \tilde{m}_{2}, \tilde{m}_{3}, 0, m_{1}, m_{2}, m_{3}\right)
$$

(here $m$ is an arbitrary mass parameter which makes $Y$ dimensionless and is useful for the mass expansion; see also [F7, Section 2.3] or $\$ 3.4 .1$. In the limiting case $\tau_{\text {reg }} \searrow 0$, we can then write $P^{\text {aux }}$ as

$$
P^{\text {aux }}=X t=t X^{*} \quad \text { with } \quad t:=\bigoplus_{\beta=1}^{7} P_{m Y_{\beta}^{\beta}}^{\mathrm{vac}} .
$$

In the case $\tau_{\text {reg }}>0$, the fourth direct summand will contain additional states. We here model these states by a massless Dirac sea (the shear, and general surface states will be obtained later from these massless Dirac states by building in a non-trivial regularization). 
Thus we also use the ansatz 4.2.44 in the case $\tau_{\text {reg }}>0$. Since $t$ is composed of Dirac seas, it is a solution of the Dirac equation

$$
(i \not \partial-m Y) t=0 \text {. }
$$

In order to introduce the interaction, we insert an operator $\mathcal{B}$ into the Dirac equation,

$$
(i \not \partial+\mathcal{B}-m Y) \tilde{t}=0 \text {. }
$$

Just as explained in [F7, Section 2.2] and [FG1], the causal perturbation theory defines $\tilde{t}$ in terms of a unique perturbation series (see also Section 2.1). The light-cone expansion (see [F7, Section 2.5] and the references therein or Section 2.2) is a method for analyzing the singularities of $\tilde{t}$ near the light cone. This gives a representation of $\tilde{t}$ of the form

$$
\begin{aligned}
\tilde{t}(x, y)= & \left.\sum_{n=-1}^{\infty} \sum_{k} m^{p_{k}} \text { (nested bounded line integrals }\right) \times T^{(n)}(x, y) \\
& +\tilde{P}^{\mathrm{le}}(x, y)+\tilde{P}^{\mathrm{he}}(x, y),
\end{aligned}
$$

where $\tilde{P}^{\mathrm{le}}(x, y)$ and $\tilde{P}^{\text {he }}(x, y)$ are smooth to every order in perturbation theory. The remaining problem is to insert the chiral asymmetry matrix $X$ into the perturbation series to obtain the auxiliary fermionic projector with interaction $\tilde{P}^{\text {aux }}$. As is shown in Appendix $\mathrm{F}$, the operator $\tilde{P}^{\text {aux }}$ can be uniquely defined in full generality, without any assumptions on $\mathcal{B}$. However, for the resulting light-cone expansion to involve only bounded line integrals, we need to assume the causality compatibility condition

$$
(i \not \partial+\mathcal{B}-m Y) X=X^{*}(i \not \partial+\mathcal{B}-m Y) \quad \text { for all } \tau_{\text {reg }} \in(0,1] .
$$

A similar condition is considered in [F7, Definition 2.3.2]. Here the additional parameter $\tau_{\text {reg }}$ entails the further constraint that the right-handed neutrino states must not interact with the regular sea states. This constraint can be understood from the fact that gauge fields or gravitational fields should change space-time only on the macroscopic scale, but they should leave the microscopic space-time structure unchanged. This gives rise to conditions for the admissible interactions of the high-energy states. As is worked out in Appendix F, the gauge fields and the gravitational field must not lead to a "mixing" of the right-handed high-energy states with other states.

Assuming that the causality compatibility condition holds, the auxiliary fermionic projector of the sea states $P^{\text {sea }}$ is obtained similar to 4.2 .44 by multiplication with the chiral asymmetry matrix. Incorporating the mass expansion similar to 4.2 .33 leads to the following formalism. We multiply the formulas of the light-cone expansion by $X$ from the left or by $X^{*}$ from the right (which as a consequence of (4.2.48) gives the same result). The regularization is built in by the formal replacements

$$
\begin{aligned}
m^{p} T^{(n)} & \rightarrow m^{p} T_{[p]}^{(n)}, \\
\tau_{\text {reg }} T^{(n)} & \rightarrow \tau_{\text {reg }} \sum_{k=0}^{\infty} \frac{1}{k !} \frac{1}{\delta^{2 k}} T_{[R, 2 n]}^{(k+n)} .
\end{aligned}
$$

Next, we introduce particles and anti-particles by occupying additional states or removing states from the sea, i.e.

$$
P^{\mathrm{aux}}(x, y)=P^{\mathrm{sea}}(x, y)-\frac{1}{2 \pi} \sum_{k=1}^{n_{\mathrm{p}}} \psi_{k}(x) \overline{\psi_{k}(y)}+\frac{1}{2 \pi} \sum_{l=1}^{n_{\mathrm{a}}} \phi_{l}(x) \overline{\phi_{l}(y)} .
$$


For the normalization of the particle and anti-particle states we refer to [F7, Section 2.8] and 3.4 .3 . Finally, we introduce the regularized fermionic projector $P$ by forming the sectorial projection (see also [F7, Section 2.3] or (3.4.3)),

$$
(P)_{j}^{i}=\sum_{\alpha, \beta}\left(\tilde{P}^{\text {aux }}\right)_{(j, \beta)}^{(i, \alpha)},
$$

where $i, j \in\{1,2\}$ is the sector index, whereas the indices $\alpha$ and $\beta$ run over the corresponding generations (i.e., $\alpha \in\{1, \ldots 4\}$ if $i=1$ and $\alpha \in\{1,2,3\}$ if $i=2$ ). We again indicate the sectorial projection of the mass matrices by accents (see [F7, Section 7.1] or $(3.5 .2)$ ),

$$
\hat{Y}=\sum_{\alpha} Y_{\alpha}^{\alpha}, \quad \grave{Y} Y \cdots \grave{Y}=\sum_{\alpha, \beta, \gamma_{1}, \ldots, \gamma_{p-1}} Y_{\gamma_{1}}^{\alpha} \cdots Y_{\gamma_{2}}^{\gamma_{1}} \cdots Y_{\beta}^{\gamma_{p-1}} .
$$

REMARK 4.2.3. (Regularizing general systems with interaction) We now outline how the above construction fits into a general framework for describing interacting fermion system with chiral asymmetry. Suppose we consider a system which in the vacuum is composed of a direct sum of sums of Dirac seas, some of which involve non-trivial regularizations composed of right- or left-handed high-energy shear or general surface states. Then the interaction can be introduced as follows: To obtain the auxiliary fermionic projector, we replace the sums by direct sums. For each Dirac sea which should involve a non-trivial regularization, we add a direct summand involving a left- or right-handed massless Dirac sea. After reordering the direct summands, we thus obtain

$$
P^{\mathrm{aux}}=\left(\bigoplus_{\ell=1}^{\ell_{1}} P_{m_{j}}^{\mathrm{vac}}\right) \oplus\left(\bigoplus_{\ell=\ell_{1}+1}^{\ell_{2}} \chi_{L} P_{0}^{\mathrm{vac}}\right) \oplus\left(\bigoplus_{\ell=\ell_{2}+1}^{\ell_{\max }} \chi_{R} P_{0}^{\mathrm{vac}}\right)
$$

with parameters $1 \leq \ell_{1} \leq \ell_{2} \leq \ell_{\max }$. In order to keep track of which direct summand belongs to which sector, we form a partition $L_{1}, \ldots, L_{N}$ of $\left\{1, \ldots, \ell_{\max }\right\}$ such that $L_{i}$ contains all the seas in the $i^{\text {th }}$ sector. Then the fermionic projector of the vacuum is obtained by forming the sectorial projection as follows,

$$
P_{j}^{i}=\sum_{\alpha \in L_{i}} \sum_{\beta \in L_{j}}\left(P^{\text {aux }}\right)_{\beta}^{\alpha}, \quad i, j=1, \ldots, N .
$$

The next step is to specify the intended form of the regularization by parameters $\tau_{1}^{\text {reg }}, \ldots, \tau_{p}^{\text {reg }}$ with $p \in \mathbb{N}_{0}$. The rule is that to every left- or right-handed massless Dirac sea which corresponds to a non-trivial regularization we associate a parameter $\tau_{k}^{\text {reg }}$. Regularizations which we consider to be identical are associated the same parameter; for different regularizations we take different parameters. Introducing the chiral asymmetry matrix $X$, the mass matrix $Y$, and the distribution $t$ by

$$
\begin{aligned}
m Y & =\left(m_{1}, \ldots, m_{\ell_{1}}\right) \oplus(0, \ldots, 0) \oplus(0, \ldots, 0) \\
X & =(1, \ldots, 1) \oplus \chi_{L}\left(1, \ldots, 1, \tau_{k_{1}}^{\mathrm{reg}}, \ldots \tau_{k_{a}}^{\mathrm{reg}}\right) \oplus \chi_{R}\left(1, \ldots, 1, \tau_{k_{a+1}}^{\mathrm{reg}}, \ldots \tau_{k_{b}}^{\mathrm{reg}}\right) \\
t & =\left(\bigoplus_{\ell=1}^{\ell_{1}} P_{m_{\ell}}^{\mathrm{vac}}\right) \oplus\left(\bigoplus_{\ell=\ell_{1}+1}^{\ell_{2}} P_{0}^{\mathrm{vac}}\right) \oplus\left(\bigoplus_{\ell=\ell_{2}+1}^{\ell_{\max }} P_{0}^{\mathrm{vac}}\right),
\end{aligned}
$$

the interaction can again be described by inserting an operator $\mathcal{B}$ into the Dirac equation 4.2.46). Now the causality compatibility condition 4.2.48 must hold for all values 
of the regularization parameters $\tau_{1}^{\mathrm{reg}}, \ldots, \tau_{k}^{\mathrm{reg}}$, thus allowing for an interaction only between seas with identical regularization. Using the causal perturbation expansion and the light-cone expansion, we can again represent $\tilde{t}$ in the form (4.2.47). The regularization is again introduced by setting $P^{\text {sea }}=t X^{*}$ and applying the replacement rules 4.2 .49 as well as

$$
\chi_{L / R} \tau_{j}^{\mathrm{reg}} T^{(n)} \rightarrow \chi_{L / R} \tau_{j}^{\mathrm{reg}} \sum_{k=0}^{\infty} \frac{1}{k !} \frac{1}{\delta^{2 k}} T_{[R / L, 2 n, j]}^{(k+n)},
$$

where the additional index $j$ in the subscript $[R / L, 2 n, j]$ indicates that the factors $T_{0}^{(n)}$ corresponding to different parameters $\tau_{j}$ must be treated as different functions. This means that the basic fractions formed of these functions are all linearly independent in the sense made precise in [F7, Appendix E]. Finally, we introduce particles and antiparticles again by 4.2 .51 and obtain the fermionic projector by forming the sectorial projection 4.2.55).

4.2.7. The $\iota$-Formalism. In the formalism of the continuum limit reviewed in $\$ 4.2 .5$, the regularization is described in terms of contraction rules. While this formulation is most convenient for most computations, it has the disadvantage that the effect of the regularization on the inner factors $\not_{0}^{(n)}$ is not explicit. The $\iota$-formalism remedies this shortcoming by providing more detailed formulas for the regularized fermionic projector in position space. The formalism will be used in 4.3 .2 , 4.5 and $\$ 4.6 .2$. It will also be important for the derivation of the Einstein equations in Section 4.9 . Here we introduce the formalism and illustrate its usefulness in simple examples.

We begin for clarity with one Dirac sea in the charged sector. Then the mass expansion gives (cf. 4.2.29); see also [F7, Section 4.5])

$$
P_{m}^{\varepsilon}=\frac{i}{2} \sum_{n=0}^{\infty} \frac{m^{2 n}}{n !} \sharp T_{[2 n]}^{(-1+n)}+\sum_{n=0}^{\infty} \frac{m^{2 n+1}}{n !} T_{[2 n+1]}^{(n)} .
$$

We choose a vector $\check{\xi}$ which is real-valued, lightlike and approximates $\xi$, i.e.

$$
\check{\xi}^{2}=0, \quad \bar{\xi}=\check{\xi} \quad \text { and } \quad \check{\xi}=\xi+(\text { higher orders in } \varepsilon /|\vec{\xi}|) .
$$

Replacing all factors $\xi$ in $P_{m}^{\varepsilon}$ by $\check{\xi}$, we obtain the function $\check{P}_{m}^{\varepsilon}$,

$$
\check{P}_{m}^{\varepsilon}:=\frac{i}{2} \sum_{n=0}^{\infty} \frac{m^{2 n}}{n !} \check{\ddagger} T_{[2 n]}^{(-1+n)}+\sum_{n=0}^{\infty} \frac{m^{2 n+1}}{n !} T_{[2 n+1]}^{(n)} .
$$

Clearly, this function differs from $P_{m}^{\varepsilon}$ by vectorial contributions. We now want to determine these additional contributions by using that the contraction rules 4.2.36 and 4.2.37) hold. It is most convenient to denote the involved vectors by $\iota_{[p]}^{(n)}$, which we always normalize such that

$$
\left\langle\check{\xi}, \iota_{\circ}^{(n)}\right\rangle=1 .
$$

Then the contraction rules 4.2.36 and (4.2.37) are satisfied by the ansatz

$$
P_{m}^{\varepsilon}=\check{P}_{m}^{\varepsilon}-i \sum_{n=0}^{\infty} \frac{m^{2 n}}{n !} \psi_{[2 n]}^{(-1+n)}\left((n-1) T_{[2 n]}^{(n)}+T_{\{2 n\}}^{(n+1)}\right),
$$

as is verified by a straightforward calculation. To explain the essence of this computation, let us consider only the leading contribution in the mass expansion,

$$
P^{\varepsilon}=\frac{i}{2} \check{\not} T_{[0]}^{(-1)}+i \psi_{[0]}^{(-1)} T_{[0]}^{(0)}+(\operatorname{deg}<-1)+\mathcal{O}(m) .
$$


Taking the square, we obtain

$$
\begin{aligned}
\left(P^{\varepsilon}\right)^{2} & =-\left\langle\check{\xi}, \iota_{[0]}^{(-1)}\right\rangle T_{[0]}^{(-1)} T_{[0]}^{(0)}-\left\langle\iota_{[0]}^{(-1)}, \iota_{[0]}^{(-1)}\right\rangle T_{[0]}^{(0)} T_{[0]}^{(0)}+(\operatorname{deg}<-2)+\mathcal{O}(m) \\
& =-T_{[0]}^{(-1)} T_{[0]}^{(0)}-\left\langle\iota_{[0]}^{(-1)}, \iota_{[0]}^{(-1)}\right\rangle T_{[0]}^{(0)} T_{[0]}^{(0)}+(\operatorname{deg}<-2)+\mathcal{O}(m) .
\end{aligned}
$$

The first summand reproduces the contraction rules (4.2.36) and (4.2.37). Compared to this first summand, the second summand is of higher order in $\varepsilon /|\vec{\xi}|$. It is thus omitted in the formalism of the continuum limit, where only the leading contribution in $\varepsilon /|\vec{\xi}|$ is taken into account (for details see [F7, Chapter 4]). More generally, when forming composite expressions of 4.2.61 in the formalism of the continuum limit, only the mixed products $\left\langle\xi_{\circ}^{(n)}, \iota_{\circ}^{\left(n^{\prime}\right)}\right\rangle$ need to be taken into account, whereas the products $\left\langle\iota_{\circ}^{(n)}, \iota_{\circ}^{\left(n^{\prime}\right)}\right\rangle$ involving two factors $\iota_{0}^{(\cdot)}$ may be disregarded. With this in mind, one easily sees that the ansatz 4.2.61) indeed incorporates the contraction rules 4.2.36) and (4.2.37). Concerning the uniqueness of the representation (4.2.61), there is clearly the freedom to change the vectors $\iota_{0}^{(n)}$, as long as the relations 4.2.60 are respected. Apart from this obvious arbitrariness, the representation 4.2.61) is unique up to contributions of higher order in $\varepsilon /|\vec{\xi}|$, which can be neglected in a weak evaluation on the light cone.

In order to extend the above formalism to include the regularization effects in the neutrino sector, we define $\check{P}_{m}^{\varepsilon}$ by replacing all factors $\xi$ in 4.2 .35 by $\check{\xi}$. Writing

$$
\begin{aligned}
P_{m}^{\varepsilon}(x, y)=\check{P}_{m}^{\varepsilon} & -i \chi_{L} \psi_{[0]}^{(-1)}\left(-T_{[0]}^{(0)}+T_{\{0\}}^{(1)}\right) \\
& -i \chi_{R} \sum_{n=0}^{\infty} \frac{1}{n !} \frac{1}{\delta^{2 n}} \psi_{[2 n]}^{(-1+n)}\left((n-1) T_{[R, 2 n]}^{(n)}+\frac{1}{\delta^{2}} T_{\{R, 2 n\}}^{(n+1)}\right) \\
& -i \sum_{n=1}^{\infty} \frac{m^{2 n}}{n !} \psi_{[2 n]}^{(-1+n)}\left((n-1) T_{[2 n]}^{(n)}+T_{\{2 n\}}^{(n+1)}\right)
\end{aligned}
$$

a direct calculation shows that the contraction rules 4.2.36, 4.2.37) and 4.2.38 are indeed respected.

Clearly, the $\iota$-formalism is equivalent to the standard formalism of $\$ 4.2 .5$. However, it makes some computations more transparent, as we now explain. For simplicity, we again consider the leading order in the mass expansion 4.2.62 and omit all correction terms, i.e.

$$
\begin{aligned}
& P^{\varepsilon}(x, y)=\frac{i}{2} \check{\not} T_{[0]}^{(-1)}+i \psi_{[0]}^{(-1)} T_{[0]}^{(0)} \\
& P^{\varepsilon}(y, x)=P^{\varepsilon}(x, y)^{*}=-\frac{i}{2} \check{\Varangle} \overline{T_{[0]}^{(-1)}}-\overline{i \psi_{[0]}^{(-1)} T_{[0]}^{(0)}} .
\end{aligned}
$$

Suppose we want to compute the eigenvalues of the closed chain. As we already saw in the example 4.2.62, contractions between two factors $\iota_{\circ}^{(n)}$ are of higher order in $\varepsilon /|\vec{\xi}|$. Thus, in view of the relations 4.2.59), it suffices to take into account the mixed terms, i.e.

$$
A_{x y}=\frac{1}{2} \psi_{[0]}^{(-1)} \check{\dot{\xi}} T_{[0]}^{(0)} \overline{T_{[0]}^{(-1)}}+\frac{1}{2} \check{\dot{\xi}} \overline{\psi_{[0]}^{(-1)}} T_{[0]}^{(-1)} \overline{T_{[0]}^{(0)}}+(\text { higher orders in } \varepsilon /|\vec{\xi}|) .
$$

When taking powers of $A_{x y}$, any product of the first summand in 4.2 .67 with the second summand in (4.2.67) vanishes, because we get two adjacent factors $\sharp$. Similarly, we also 
get zero when the second summand is multiplied by the first summand, because in this case we get two adjacent factors $\psi$. We thus obtain

$$
\left(A_{x y}\right)^{p}=\left(\frac{1}{2} \psi_{[0]}^{(-1)} \check{\dot{\xi}} T_{[0]}^{(0)} \overline{T_{[0]}^{(-1)}}\right)^{p}+\left(\frac{1}{2} \check{\not}_{\bar{\psi}}^{(-1)} T_{[0]}^{(-1)} \overline{T_{[0]}^{(0)}}\right)^{p},
$$

where we again omitted the higher orders in $\varepsilon /|\vec{\xi}|$. Moreover, powers of products of $\sharp$ and $\psi$ can be simplified using the anti-commutation relations; for example,

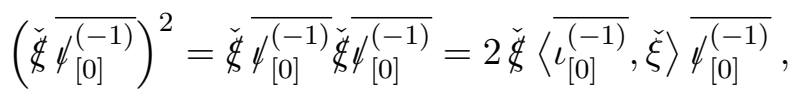

and applying (4.2.60) together with the fact that $\check{\xi}$ is real, we obtain

$$
\left(\check{\check{\ddagger}} \overline{\psi_{[0]}^{(-1)}}\right)^{2}=2 \check{\ddagger} \overline{\psi_{[0]}^{(-1)}} \text {. }
$$

This shows that the Dirac matrices in 4.2.68) in the first and second summand in 4.2.68 both have the eigenvalues two and zero. From this fact we can immediately read off the eigenvalues of (4.2.67) to be

$$
\lambda_{+}=T_{[0]}^{(0)} \overline{T_{[0]}^{(-1)}} \quad \text { and } \quad \lambda_{-}=T_{[0]}^{(-1)} \overline{T_{[0]}^{(0)}} .
$$

Clearly, these formulas were obtained earlier in the usual formalism (for details see [F7, Sections 5.3 and 6.1] or \$3.6.1). But the above consideration gives a more direct understanding for how these formulas come about.

Another advantage is that it becomes clearer how different contributions to the fermionic projector influence the eigenvalues. We explain this in the example of a lefthanded contribution of the form

$$
P(x, y) \asymp \chi_{L} \psi .
$$

The corresponding contribution to the left-handed component of the closed chain is given by

$$
\chi_{L} A_{x y} \asymp \chi_{L} \psi P^{\varepsilon}(y, x) .
$$

If we substitute $P^{\varepsilon}(y, x)$ according to 4.2.66), the factor $\iota$ will be contracted in any composite expression either with $u$ or with another factor $\iota$. In both cases, we get contributions of higher order in $\varepsilon /|\vec{\xi}|$. Hence we can disregard the factor $\iota$,

$$
\chi_{L} A_{x y} \asymp-\frac{i}{2} \chi_{L} \psi \check{\check{q}} \overline{T_{[0]}^{(-1)}} .
$$

When multiplying with 4.2.67), the product with the second summand vanishes. Even more, using the anti-commutation relations, one finds that

$$
\left(A_{x y}\right)^{p} \psi \check{\psi} \check{\xi}\left(A_{x y}\right)^{q}=\langle u, \check{\xi}\rangle\left(\frac{1}{2} \psi_{[0]}^{(-1)} \check{\xi} T_{[0]}^{(0)} \overline{T_{[0]}^{(-1)}}\right)^{p+q} .
$$

This implies that only the eigenvalue $\lambda_{L+}$ is influenced; more precisely,

$$
\lambda_{L+} \asymp-\frac{i}{2} u_{j} \xi^{j} \overline{T_{[0]}^{(-1)}} \quad \text { and } \quad \lambda_{L-} \asymp 0 .
$$

Of course, this result is consistent with earlier computations (see for example the proof of Lemma 3.7.4 in Appendix B. 


\subsection{The Euler-Lagrange Equations to Degree Five}

Before entering the analysis of the EL equations, we briefly recall the basics. Counting with algebraic multiplicities, the closed chain $A_{x y}$ has eight eigenvalues, which we denote by $\lambda_{n c s}^{x y}$, where $n \in\{1,2\}, c \in\{L, R\}$ and $s \in\{+,-\}$. The corresponding spectral projectors are denoted by $F_{n c s}^{x y}$. In case of degeneracies, we usually omit the lower indices on which the eigenvalues do not depend. For example, in the case of the four-fold degeneracy $\lambda_{1 L+}=\lambda_{2 L+}=\lambda_{1 R+}=\lambda_{2 R+}$, we simply denote the corresponding eigenvalue by $\lambda_{+}$and the spectral projector onto the four-dimensional eigenspace by $F_{+}$.

The considerations in the previous section led us to choosing the Lagrange multiplier $\mu=\frac{1}{8}$ (see 4.2.17) ), and thus a minimizer $P$ is a critical point of the auxiliary action

$$
\mathcal{S}[P]=\iint_{\mathcal{M} \times \mathcal{M}} \mathcal{L}\left[A_{x y}\right] d^{4} x d^{4} y
$$

with $\mathcal{L}$ according to (4.1.1),

$$
\mathcal{L}\left[A_{x y}\right]=\sum_{n, c, s}\left|\lambda_{n c s}^{x y}\right|^{2}-\frac{1}{8}\left(\sum_{n, c, s}\left|\lambda_{n c s}^{x y}\right|\right)^{2}=\frac{1}{16} \sum_{n, c, s} \sum_{n^{\prime}, c^{\prime}, s^{\prime}}\left(\left|\lambda_{n c s}^{x y}\right|-\left|\lambda_{n^{\prime} c^{\prime} s^{\prime}}^{x y}\right|\right)^{2} .
$$

Considering first order variations of $P$, one gets the EL equations (see [F7, Section 3.5] or for more details 3.5 .20 )

$$
[P, Q]=0,
$$

where the operator $Q$ has the integral kernel (see [F7, Sections 3.5 and 5.4])

$$
\begin{aligned}
Q(x, y) & =\frac{1}{2} \sum_{n c s} \frac{\partial \mathcal{L}}{\partial \lambda_{n c s}^{x y}} F_{n c s}^{x y} P(x, y) \\
& =\sum_{n, c, s}\left[\left|\lambda_{n c s}^{x y}\right|-\frac{1}{8} \sum_{n^{\prime}, c^{\prime}, s^{\prime}}\left|\lambda_{n^{\prime} c^{\prime} s^{\prime}}^{x y}\right|\right] \frac{\overline{\lambda_{n c s}^{x y}}}{\left|\lambda_{n c s}^{x y}\right|} F_{n c s}^{x y} P(x, y) .
\end{aligned}
$$

By testing on null lines (see $\$ 3.5 .2$ and Appendix A , one sees that the commutator (4.3.1) vanishes if and only if $Q$ itself is zero. We thus obtain the EL equations in the continuum limit

$$
Q(x, y)=0 \quad \text { if evaluated weakly on the light cone . }
$$

By relating the spectral decomposition of $A_{x y}$ to that of $A_{y x}$ (see [F7, Lemma 3.5.1]), one sees that the operator $Q$ is symmetric, meaning that

$$
Q(x, y)^{*}=Q(y, x) .
$$

As in Chapter 3 we shall analyze the EL equations 4.3 .3 degree by degree on the light cone. In this section, we consider the leading degree five, both in the vacuum and in the presence of gauge potentials. In Section 4.4 we then consider the next degree four.

4.3.1. The Vacuum. Applying the formalism of $\$ 4.2 .5$ and $\$ 4.2 .6$ to the ansatz 4.1.4 , 4.1.5) and 4.1.8) and forming the sectorial projection, we obtain according to 4.2.40 and (4.2.41) for the vacuum fermionic projector the expression

$$
P(x, y)=\frac{i}{2}\left(\begin{array}{cc}
3 \nsubseteq T_{[0]}^{(-1)}+\chi_{R} \tau_{\text {reg }} \sharp T_{[R, 0]}^{(-1)} & 0 \\
0 & 3 \notin T_{[0]}^{(-1)}
\end{array}\right)+(\operatorname{deg}<2),
$$


where we used a matrix notation in the isospin index. Thus

$$
\begin{aligned}
\chi_{L} A_{x y}= & \frac{3}{4} \chi_{L}\left(\begin{array}{cc}
3 \sharp T_{[0]}^{(-1)} \overline{\sharp T_{[0]}^{(-1)}}+\tau_{\text {reg }} \sharp T_{[0]}^{(-1)} \bar{\sharp} T_{[R, 0]}^{(-1)} & 0 \\
0 & 3 \sharp T_{[0]}^{(-1)} \overline{\sharp T_{[0]}^{(-1)}}
\end{array}\right) \\
& +\notin(\operatorname{deg}<3)+(\operatorname{deg}<2),
\end{aligned}
$$

and the right-handed component is obtained by taking the adjoint. The eigenvalues can be computed in the charged and neutrino sectors exactly as in 3.6.1 to obtain

$$
\lambda_{2+L}=\lambda_{2+R}=\overline{\lambda_{2-R}}=\overline{\lambda_{2-L}}=9 T_{[0]}^{(0)} \overline{T_{[0]}^{(-1)}}+(\operatorname{deg}<3)
$$

and

$$
\begin{aligned}
& \lambda_{1+L}=\overline{\lambda_{1-R}}=3 T_{[0]}^{(0)}\left(3 \overline{T_{[0]}^{(-1)}}+\tau_{\text {reg }} \overline{T_{[R, 0]}^{(-1)}}\right)+(\operatorname{deg}<3) \\
& \lambda_{1+R}=\overline{\lambda_{1-L}}=\left(3 T_{[0]}^{(0)}+\tau_{\text {reg }} T_{[R, 0]}^{(0)}\right) 3 \overline{T_{[0]}^{(-1)}}+(\operatorname{deg}<3) .
\end{aligned}
$$

The corresponding spectral projectors can be computed exactly as in [F7, Sections 5.3 and 6.1] or Section 3.6 to

$$
F_{1 c s}=\left(\begin{array}{cc}
\chi_{c} F_{s} & 0 \\
0 & 0
\end{array}\right), \quad F_{2 c s}=\left(\begin{array}{cc}
0 & 0 \\
0 & \chi_{c} F_{s}
\end{array}\right)
$$

where $F_{ \pm}$are given by

$$
F_{ \pm}:=\frac{1}{2}\left(\mathbb{1} \pm \frac{[\not, \bar{\phi}]}{z-\bar{z}}\right)+\$(\operatorname{deg} \leq 0)+(\operatorname{deg}<0) .
$$

Here the omitted indices of the factors $\xi, z$ and their complex conjugates are to be chosen in accordance with the corresponding factors $T_{\circ}^{(-1)}$ and $\overline{T_{\circ}^{(-1)}}$, respectively. In the charged sector, this simply amounts to adding indices ${ }_{[0]}^{(-1)}$ to all such factors. In the neutrino sector, however, one must keep in mind the contributions involving $\tau_{\text {reg }}$, making it necessary to keep track of the factors $T_{[R, \circ]}^{(n)}$. More precisely, setting

$$
L_{[p]}^{(n)}=T_{[p]}^{(n)}+\frac{1}{3} \tau_{\mathrm{reg}} T_{[R, p]}^{(n)},
$$

we obtain

$$
\begin{aligned}
2 \chi_{R} F_{ \pm} & =\mathbb{1} \pm \frac{1}{4 L_{[0]}^{(0)}-L_{[0]}^{(-1)} \overline{z_{[0]}^{(-1)}}}\left[\oiint_{[0]}^{(-1)} T_{[0]}^{(-1)}+\frac{1}{3} \tau_{\mathrm{reg}} \oiint_{[R, 0]}^{(-1)} T_{[R, 0]}^{(-1)}, \overline{\sharp_{[0]}^{(-1)}}\right] \\
2 \chi_{L} F_{ \pm} & =\mathbb{1} \pm \frac{1}{z_{[0]}^{(-1)} \overline{L_{[0]}^{(-1)}}-4 \overline{L_{[0]}^{(0)}}}\left[\oiint_{[0]}^{(-1)}, \overline{3 \oiint_{[0]}^{(-1)} T_{[0]}^{(-1)}+\tau_{\mathrm{reg}} \sharp_{[R, 0]}^{(-1)} T_{[R, 0]}^{(-1)}}\right]
\end{aligned}
$$

with the error terms as in 4.3.7). Moreover, a direct computation shows that (cf. [F7, eq. (5.3.23)])

$$
\begin{aligned}
& F_{n c+} P(x, y)=(\operatorname{deg}<0) \\
& F_{1 c-} P(x, y)=\chi_{c}\left(\begin{array}{ll}
1 & 0 \\
0 & 0
\end{array}\right) P(x, y)+(\operatorname{deg}<0) \\
& F_{2 c-} P(x, y)=\chi_{c}\left(\begin{array}{ll}
0 & 0 \\
0 & 1
\end{array}\right) P(x, y)+(\operatorname{deg}<0) .
\end{aligned}
$$


Evaluating the EL equations (4.3.3) by substituting the above formulas into 4.3.2), we obtain the three conditions

$$
\begin{gathered}
\left(2\left|\overline{T_{[0]}^{(-1)}} T_{[0]}^{(0)}\right|-\left|\overline{L_{[0]}^{(-1)}} T_{[0]}^{(0)}\right|-\left|\overline{T_{[0]}^{(-1)}} L_{[0]}^{(0)}\right|\right) \frac{\overline{T_{[0]}^{(-1)}} T_{[0]}^{(0)}}{\left|\overline{T_{[0]}^{(-1)}} T_{[0]}^{(0)}\right|} T_{[0]}^{(-1)}=0 \\
\left(3\left|\overline{L_{[0]}^{(-1)}} T_{[0]}^{(0)}\right|-2\left|\overline{T_{[0]}^{(-1)}} T_{[0]}^{(0)}\right|-\left|\overline{T_{[0]}^{(-1)}} L_{[0]}^{(0)}\right|\right) \frac{\bar{L}_{[0]}^{(-1)} T_{[0]}^{(0)}}{\left|\overline{L_{[0]}^{(-1)}} T_{[0]}^{(0)}\right|} L_{[0]}^{(-1)}=0 \\
\left(3\left|\overline{T_{[0]}^{(-1)}} L_{[0]}^{(0)}\right|-2\left|\overline{T_{[0]}^{(-1)}} T_{[0]}^{(0)}\right|-\left|\overline{L_{[0]}^{(-1)}} T_{[0]}^{(0)}\right|\right) \frac{\frac{T_{[0]}^{(-1)}}{T_{[0]}^{(0)}}}{\left|\overline{T_{[0]}^{(-1)}} L_{[0]}^{(0)}\right|} T_{[0]}^{(-1)}=0 .
\end{gathered}
$$

These three equations must be satisfied in a weak evaluation on the light cone.

To summarize, evaluating the EL equations for the fermionic projector of the vacuum (4.3.5), we obtain a finite hierarchy of equations to be satisfied in a weak evaluation on the light cone. As the detailed form of these equations is quite lengthy and will not be needed later on, we omit the explicit formulas.

4.3.2. The Gauge Phases. Let us introduce chiral gauge potentials. As the auxiliary fermionic projector 4.2.40 has seven components, the most general ansatz for chiral potentials would correspond to the gauge group $\mathrm{U}(7)_{L} \times \mathrm{U}(7)_{R}$. However, the causality compatibility conditions (4.2.48) reduce the gauge group to

$$
\mathrm{U}(6)_{L} \times \mathrm{U}(6)_{R} \times \mathrm{U}(1)_{R}
$$

where the groups $\mathrm{U}(6)_{L}$ and $\mathrm{U}(6)_{R}$ act on the first and last three components, whereas the group $\mathrm{U}(1)_{R}$ acts on the fourth component. Similar as in $\$ 3.6 .2$, to degree five the gauge potentials describe phase transformations of the left- and right-handed components of the fermionic projector,

$$
P^{\operatorname{aux}}(x, y) \rightarrow\left(\chi_{L} U_{L}(x, y)+\chi_{R} U_{R}(x, y)\right) P^{\operatorname{aux}}(x, y)+(\operatorname{deg}<2) .
$$

However, as the gauge group (4.3.16) is non-abelian, the unitary operators $U_{L / R}$ now involve the ordered exponential (for details see [F7, Section 2.5] or [F6, Section 2.2])

$$
U_{L / R}=\operatorname{Pexp}\left(-i \int_{x}^{y} A_{L / R}^{j} \xi_{j}\right) .
$$

Substituting the gauge potentials corresponding to the gauge group (4.3.16) and forming the sectorial projection, we obtain

$$
\begin{aligned}
& \chi_{L} P(x, y)=\chi_{L} \frac{i \nsubseteq}{2} T_{[0]}^{(-1)}\left(\begin{array}{cc}
\hat{U}_{L}^{11} & \hat{U}_{L}^{12} \\
\hat{U}_{L}^{21} & \hat{U}_{L}^{22}
\end{array}\right)+(\operatorname{deg}<2) \\
& \chi_{R} P(x, y)=\chi_{R} \frac{i \notin}{2}\left[T_{[0]}^{(-1)}\left(\begin{array}{cc}
\hat{U}_{R}^{11} & \hat{U}_{R}^{12} \\
\hat{U}_{R}^{21} & \hat{U}_{R}^{22}
\end{array}\right)+\left(\begin{array}{ccc}
V T_{[R, 0]}^{(-1)} & 0 \\
0 & 0
\end{array}\right)\right]+(\operatorname{deg}<2),
\end{aligned}
$$

where

$$
U_{L / R}=\left(\begin{array}{cc}
U_{L / R}^{11} & U_{L / R}^{12} \\
U_{L / R}^{21} & U_{L / R}^{22}
\end{array}\right) \in \mathrm{U}(6), \quad V \in \mathrm{U}(1)
$$


and the hat denotes the sectorial projection,

$$
\hat{U}_{L}^{i j}=\sum_{\alpha, \beta=1}^{3}\left(U_{L}^{i j}\right)_{\beta}^{\alpha}, \quad \hat{U}_{R}=\sum_{\alpha, \beta=1}^{3}\left(U_{R}\right)_{\beta}^{\alpha} .
$$

At this point it is important to observe that our notation in 4.3 .19 is oversimplified because it does not make manifest that the four matrices $U_{L / R}^{11}$ and $U_{L / R}^{22}$ on the block diagonal describe a mixing of three regularized Dirac seas. Thus when the sectorial projection is formed, one gets new linear combinations of the regularized Dirac seas, which are then described effectively by the factor $T_{[0]}^{(-1)}$. The analysis in [F11] gives a strong indication that an admissible regularization can be obtained only by taking a sum of several Dirac seas and by delicately adjusting their regularizations (more precisely, the property of a distributional $\mathcal{M}$-product can be arranged only for a sum of at least three Dirac seas). This means that if we take a different linear combination of our three regularized Dirac seas, we cannot expect that the resulting regularization is still admissible. In order to avoid this subtle but important problem, we must impose that each of the four matrices $U_{L}^{11}, U_{L}^{22}, U_{R}^{11}$ and $U_{R}^{22}$ is a multiple of the identity matrix, because only in this case we get up to a constant the same linear combination of regularized Dirac seas as in the vacuum (for more details and similar considerations see [F7, Remark 6.2.3] and 3.9 .3 . This argument shows that the matrices $U_{L}^{11}, U_{L}^{22}, U_{R}^{11}$ and $U_{R}^{22}$ must be multiples of the identity matrix. The following lemma tells us what these conditions mean for $U_{L}$ and $U_{R}$.

Lemma 4.3.1. Suppose that $\mathcal{G} \subset \mathrm{U}(6)$ is a Lie subgroup such that in the standard representation on $\mathbb{C}^{6}$, every $g \in \mathcal{G}$ is of the form

$$
g=\left(\begin{array}{cc}
a \mathbb{1}_{\mathbb{C}^{3}} & * \\
* & c \mathbb{1}_{\mathbb{C}^{3}}
\end{array}\right) \quad \text { with } a, c \in \mathbb{R}
$$

where we used $a(3 \times 3)$ block matrix notation, and the stars stand for arbitrary $3 \times 3$ matrices. Then there is a matrix $U \in \mathrm{U}(3)$ such that every $g \in \mathcal{G}$ has the representation

$$
g=\left(\begin{array}{cc}
a \mathbb{1}_{\mathbb{C}^{3}} & \bar{b} U^{*} \\
b U & c \mathbb{1}_{\mathbb{C}^{3}}
\end{array}\right) \quad \text { with } \quad\left(\begin{array}{ll}
a & \bar{b} \\
b & c
\end{array}\right) \in \mathrm{U}(2) .
$$

In particular, $\mathcal{G}$ is isomorphic to a Lie subgroup of $\mathrm{U}(2)$.

Proof. For any $A \in T_{e} \mathcal{G}$ we consider the one-parameter subgroup $V(\tau)=e^{i \tau A}$ $(\tau \in \mathbb{R})$. Evaluating 4.3.21 to first order in $\tau$, we find that

$$
A=\left(\begin{array}{cc}
a \mathbb{1}_{\mathbb{C}^{3}} & Z^{*} \\
Z & c \mathbb{1}_{\mathbb{C}^{3}}
\end{array}\right)
$$

with a $3 \times 3$-matrix $Z$. Considering (4.3.21) for the quadratic terms in $\tau$, we find that the matrices $Z Z^{*}$ and $Z^{*} Z$ are multiples of the identity matrix. Taking the polar decomposition of $Z$, we find that there is a unitary matrix $U$ such that

$$
A=\left(\begin{array}{cc}
a \mathbb{1}_{\mathbb{C}^{3}} & \bar{b} U^{*} \\
b U & c \mathbb{1}_{\mathbb{C}^{3}}
\end{array}\right) \quad \text { with } a, c \in \mathbb{R} \text { and } b \in \mathbb{C} .
$$

Exponentiating, one finds that $V(\tau)$ is of the required form (4.3.22), but with $U$ depending on $A$. 
We next choose two matrices $A, \tilde{A} \in T_{e} \mathcal{G}$ and represent them in the form 4.3.23) (where tildes always refer to $\tilde{A}$ ). It remains to show that $U$ and $\tilde{U}$ coincide up to a phase,

$$
\tilde{U}=e^{i \varphi} U \quad \text { with } \varphi \in \mathbb{R} \text {. }
$$

To this end, we consider the one-parameter subgroup $V(\tau)=e^{i \tau(A+\tilde{A})}$. Evaluating 4.3.21 to second order in $\tau$, we obtain the condition

$$
\{A, \tilde{A}\}=\left(\begin{array}{cc}
d \mathbb{1}_{\mathbb{C}^{3}} & * \\
* & e \mathbb{1}_{\mathbb{C}^{3}}
\end{array}\right) \quad \text { with } d, e \in \mathbb{R} .
$$

Writing out this condition using 4.3.23, we find that

$$
a \tilde{a}+\bar{b} U^{*} \tilde{b} \tilde{U}=d \mathbb{1}_{\mathbb{C}^{3}} .
$$

Let us show that there is a parameter $\varphi \in \mathbb{R}$ such that (4.3.24 holds. If $b$ or $\tilde{b}$ vanish, there is nothing to prove. Otherwise, we know from (4.3.25) that the matrix $U^{*} \tilde{U}$ is a multiple of the identity matrix. Since this matrix is unitary, it follows that $U^{*} \tilde{U}=e^{i \varphi} \mathbb{1}_{\mathbb{C}^{3}}$, proving 4.3.24.

We point out that the matrix $U \in \mathrm{U}(3)$ is the same for all $g \in \mathcal{G}$; this means that $U$ will be a constant matrix in space-time.

Using the representation 4.3.22 in 4.3.19), the left-handed component of the fermionic projector becomes

$$
\chi_{L} P(x, y)=\chi_{L} \frac{i \not}{2} T_{[0]}^{(-1)}\left(\begin{array}{cc}
U_{L}^{11} & U_{L}^{12} U_{\mathrm{MNS}}^{*} \\
U_{L}^{21} U_{\mathrm{MNS}} & U_{L}^{22}
\end{array}\right)+(\operatorname{deg}<2),
$$

where $U_{L} \in U(2)$, and $U_{\mathrm{MNS}} \in \mathrm{U}(3)$ is a constant matrix. The matrix $U_{\mathrm{MNS}}$ can be identified with the MNS matrix in the electroweak theory. In 4.3.26), we still need to make sense of the expressions

$$
\hat{U}_{\mathrm{MNS}} T_{[0]}^{(-1)} \quad \text { and } \quad \hat{U}_{\mathrm{MNS}}^{*} T_{[0]}^{(-1)} .
$$

Again, the matrix $U_{\text {MNS }}$ describes a mixing of regularized Dirac seas, now even combining the seas with different isospin. Since $U_{\text {MNS }}$ is constant, one can take the point of view that we should adjust the regularizations of all six Dirac seas in such a way that the expressions in (4.3.27) are admissible (in the sense that the fermionic projector has the property of a distributional $\mathcal{M} P$-product; see [F11]).

For the right-handed component, the high-energy component $T_{[R, 0]}^{(-1)}$ makes the argument a bit more involved. Applying Lemma 4.3.1 to the right-handed component, we obtain a representation of the form

$$
\chi_{R} P(x, y)=\chi_{R} \frac{i \notin}{2}\left[T_{[0]}^{(-1)}\left(\begin{array}{cc}
U_{R}^{11} & U_{R}^{12} U^{*} \\
U_{R}^{21} U & U_{R}^{22}
\end{array}\right)+\tau_{\text {reg }} T_{[R, 0]}^{(-1)}\left(\begin{array}{ll}
V & 0 \\
0 & 0
\end{array}\right)\right]+(\operatorname{deg}<2)
$$

with $\left(U_{R}, V\right) \in U(2) \times U(1)$ and a fixed matrix $U \in U(3)$. As explained after (4.3.20), our notation is again a bit too simple in that it does not make manifest that the three Dirac seas and the right-handed high-energy states will in general all be regularized differently, and that only their linear combination is described effectively by the factors $T_{\circ}^{(-1)}$. With this in mind, we can repeat the argument after 4.3.20 to conclude that the relative prefactor of the regularization functions in the upper left matrix entry should not be affected by the gauge potentials, i.e.

$$
U_{R}^{11} T_{[0]}^{(-1)}+\tau_{\text {reg }} V T_{[R, 0]}^{(-1)}=\kappa\left(T_{[0]}^{(-1)}+\tau_{\text {reg }} T_{[R, 0]}^{(-1)}\right) \quad \text { with } \kappa \in \mathbb{C} .
$$


In particular, one sees that $U_{R}^{11}$ must be a phase factor, and this implies that $U_{R}$ must be a diagonal matrix. Moreover, we find that $V=U_{R}^{11}$.

Putting our results together, we conclude that the admissible gauge group is

$$
\mathcal{G}=\mathrm{U}(2)_{L} \times \mathrm{U}(1)_{R} \times \mathrm{U}(1)_{R}
$$

Choosing a corresponding potential $\left(A_{L}, A_{R}^{C}, A_{R}^{N}\right) \in \mathfrak{u}(2) \times \mathfrak{u}(1) \times \mathfrak{u}(1)$, the interaction is described by the operator

$$
\mathcal{B}=\chi_{R}\left(\begin{array}{cc}
A_{L}^{11} & A_{L}^{12} U_{\mathrm{MNS}}^{*} \\
A_{L}^{21} U_{\mathrm{MNS}} & A_{L}^{22}
\end{array}\right)+\chi_{L}\left(\begin{array}{cc}
A_{R}^{N} & 0 \\
0 & A_{R}^{C}
\end{array}\right) .
$$

Thus the $\mathrm{U}(1)$-potentials $A_{R}^{N}$ and $A_{R}^{C}$ couple to the right-handed component of the two isospin components. The $\mathrm{U}(2)$-potential $A_{L}$, on the other hand, acts on the left-handed components, mixing the two isospin components. The $U_{\mathrm{MNS}}-$ matrix describes a mixing of the generations in the off-diagonal isospin components of $A_{L}$.

In order to analyze the EL equations to degree five in the presence of the above gauge potentials, we need to compute the eigenvalues of the closed chain (see 4.3.3 and (4.3.2) $)$. Combining 4.3.19) with the form of the gauge potentials as specified in 4.3.28 and 4.3.29), we obtain

$$
\begin{aligned}
\chi_{L} P(x, y) & =\frac{3}{2} \chi_{L} i \not T_{[0]}^{(-1)}\left(\begin{array}{cc}
U_{L}^{11} & \bar{c} U_{L}^{12} \\
c U_{L}^{21} & U_{L}^{22}
\end{array}\right)+(\operatorname{deg}<2) \\
\chi_{R} P(x, y) & =\frac{3}{2} \chi_{R} i \notin\left(\begin{array}{cc}
V_{R}^{N} L_{[0]}^{(-1)} & 0 \\
0 & V_{R}^{C} T_{[0]}^{(-1)}
\end{array}\right)+(\operatorname{deg}<2)
\end{aligned}
$$

with $U_{L} \in U(2)$ and $V_{R}^{N}, V_{R}^{C} \in U(1)$, where we again used the notation 4.3.9 and introduced the complex number

$$
c=\frac{1}{3} \hat{U}_{\mathrm{MNS}}
$$

It follows for the closed chain that

$$
\begin{aligned}
\chi_{L} A_{x y}= & \frac{9}{4} \chi_{L}\left(\begin{array}{cc}
U_{L}^{11} & \bar{c} U_{L}^{12} \\
c U_{L}^{21} & U_{L}^{22}
\end{array}\right)\left(\begin{array}{cc}
V_{R}^{N} & 0 \\
0 & V_{R}^{C}
\end{array}\right)\left(\begin{array}{cc}
\sharp T_{[0]}^{(-1)} \overline{\sharp L_{[0]}^{(-1)}} & 0 \\
0 & \sharp T_{[0]}^{(-1)} \overline{\sharp T_{[0]}^{(-1)}}
\end{array}\right) \\
& +\notin(\operatorname{deg}<3)+(\operatorname{deg}<2) .
\end{aligned}
$$

When diagonalizing the matrix 4.3.33, the factor $\overline{L_{[0]}^{(-1)}}$ causes major difficulties because it leads to microscopic oscillations of the eigenvectors. Let us explain this problem in detail. First, it is convenient to use the $\iota$-formalism, because then, similar as explained after 4.2.67), the contributions $\sim \psi(\check{\xi}$ and $\sim \check{\dot{\phi}} \psi$ act on complementary subspaces. Thus it remains to diagonalize the $2 \times 2$-matrices

$$
\left(\begin{array}{cc}
U_{L}^{11} & \bar{c} U_{L}^{12} \\
c U_{L}^{21} & U_{L}^{22}
\end{array}\right)\left(\begin{array}{cc}
V_{R}^{N} T_{[0]}^{(0)} \overline{L_{[0]}^{(-1)}} & 0 \\
0 & V_{R}^{C} T_{[0]}^{(0)} \overline{T_{[0]}^{(-1)}}
\end{array}\right)
$$

and

$$
\left(\begin{array}{cc}
U_{L}^{11} & \bar{c} U_{L}^{12} \\
c U_{L}^{21} & U_{L}^{22}
\end{array}\right)\left(\begin{array}{cc}
V_{R}^{N} T_{[0]}^{(-1)} \overline{L_{[0]}^{(0)}} & 0 \\
0 & V_{R}^{C} T_{[0]}^{(-1)} \overline{T_{[0]}^{(0)}}
\end{array}\right) .
$$


The characteristic polynomial involves square roots of linear combinations of the inner matrix elements, describing non-trivial fluctuations of the eigenvalues on the regularization scale $\varepsilon$. Such expressions are ill-defined in the formalism of the continuum limit. A first idea for overcoming this problem would be to extend the formalism such as to include square roots of linear combinations of simple fractions. However, even if one succeeded in extending the continuum limit in this way, it would be unclear how the resulting square root expressions after weak evaluation would depend on the smooth parameters $U_{L}^{i j}$ and $V_{R}^{N / C}$. The basic difficulty is that integrating over the microscopic oscillations will in general not preserve the square root structure (as a simple example, an integral of the form $\int_{0}^{\infty} \sqrt{a+x} f(x) d x$ cannot in general be written again as a square root of say the form $\sqrt{a b+c}$ ). This is the reason why the complications related to the factor $L_{[0]}^{(-1)}$ in 4.3 .33 seem to arise as a matter of principle.

In order to bypass this difficulty, we must restrict attention to a parameter range where the eigenvalues of the above matrices can be computed perturbatively. In order to make the scaling precise, we write $\tau_{\text {reg }}$ as

$$
\tau_{\text {reg }}=(m \varepsilon)^{p_{\text {reg }}} \quad \text { with } \quad 0<p_{\text {reg }}<2 .
$$

Under this assumption, we know that that the relation

$$
T_{[p]}^{(n)}=L_{[p]}^{(n)}\left(1+\mathcal{O}\left((m \varepsilon)^{p_{\text {reg }}}\right)\right) \quad \text { holds pointwise }
$$

(by "holds pointwise" we mean that if we multiply $T_{[p]}^{(n)}-L_{[p]}^{(n)}$ by any simple fraction and evaluate weakly according to (4.2.31), we get zero up to an error of the specified order). Making $\tau_{\text {reg }}$ small in this sense does not necessarily imply that the above matrices can be diagonalized perturbatively, because we need to compare $\tau_{\text {reg }}$ to the size of the offdiagonal matrix elements $U_{R}^{12}$ and $U_{R}^{21}$. As they are given as line integrals over the chiral potentials (cf. (4.3.18), their size is described by

$$
\left\|A_{L}^{12}\right\| \cdot|\vec{\xi}| \quad \text { and } \quad\left\|A_{L}^{21}\right\| \cdot|\vec{\xi}|
$$

(where $\|$.$\| is a Euclidean norm defined in the same reference frame as \vec{\xi}$ ). This leads us to the following two cases:

$$
\text { (i) } \quad|\vec{\xi}| \gg \frac{(m \varepsilon)^{p_{\text {reg }}}}{\left\|A_{L}^{12}\right\|+\left\|A_{L}^{21}\right\|}, \quad \text { (ii) } \quad|\vec{\xi}| \ll \frac{(m \varepsilon)^{p_{\text {reg }}}}{\left\|A_{L}^{12}\right\|+\left\|A_{L}^{21}\right\|} \text {. }
$$

In fact, the computations are tractable in both cases, as we now explain.

Case (i). We expand in powers of $\tau_{\text {reg. }}$. We begin with the case $\tau_{\text {reg }}=0$. Then in the vacuum, 4.3.35 implies that the relations 4.3.13 4.3 .15 are trivially satisfied. If gauge potentials are present, in the above matrices we can factor out the scalar functions $T_{[0]}^{(0)} \overline{T_{[0]}^{(-1)}}$ and $T_{[0]}^{(-1)} \overline{T_{[0]}^{(0)}}$, respectively. Thus it remains to compute the eigenvalues and spectral projectors of the $2 \times 2$-matrix

$$
\left(\begin{array}{cc}
U_{L}^{11} & \bar{c} U_{L}^{12} \\
c U_{L}^{21} & U_{L}^{22}
\end{array}\right)\left(\begin{array}{cc}
V_{R}^{N} & 0 \\
0 & V_{R}^{C}
\end{array}\right)
$$

LEMma 4.3.2. The matrix in (4.3.37) is normal (i.e. it commutes with its adjoint). Moreover, its eigenvalues have the same absolute value. 
Proof. We denote the matrix in (4.3.37) by $B$ and write the two factors in 4.3.37) in terms of Pauli matrices as

$$
B=(a \mathbb{1}+i \vec{v} \vec{\sigma}) e^{i \varphi}(b \mathbb{1}+i \vec{w} \vec{\sigma})
$$

with $a, b, \varphi \in \mathbb{R}$ and $\vec{v}, \vec{w} \in \mathbb{R}^{3}$. Using the multiplication rules of Pauli matrices, one finds that

$$
e^{-i \varphi} B=(a b-\vec{v} \vec{w}) \mathbb{1}+i(a \vec{w}+b \vec{v}+\vec{v} \wedge \vec{w}) \vec{\sigma} .
$$

A short calculation shows that this matrix is normal. Moreover, the eigenvalues of $B$ are computed by

$$
e^{i \varphi}((a b-\vec{v} \vec{w}) \pm i|a \vec{w}+b \vec{v}+\vec{v} \wedge \vec{w}|) .
$$

Obviously, these eigenvalues have the same absolute value.

We denote the eigenvalues and corresponding spectral projection operators of the matrix in 4.3.37) by $\nu_{n L}$ and $I_{n}$. Then, according to the above lemma,

$$
\left|\nu_{1 L}\right|=\left|\nu_{2 L}\right| \quad \text { and } \quad I_{n}^{*}=I_{n} .
$$

For the left-handed component of the closed chain 4.3 .33 we thus obtain the eigenvalues $\lambda_{n L s}$ and spectral projectors $F_{n L s}$ given by

$$
\lambda_{n L s}=\nu_{n L} \lambda_{s}, \quad F_{n L s}=\chi_{L} I_{n} F_{s},
$$

where $\lambda_{ \pm}$and $F_{s}$ are given by (cf. (4.3.6) and (4.3.8),

$$
\begin{gathered}
\lambda_{+}=9 T_{[0]}^{(0)} \overline{T_{[0]}^{(-1)}}+(\operatorname{deg}<3), \quad \lambda_{-}=9 T_{[0]}^{(-1)} \overline{T_{[0]}^{(0)}}+(\operatorname{deg}<3) \\
F_{ \pm}=\frac{1}{2}\left(\mathbb{1} \pm \frac{[\not, \bar{\phi}]}{z-\bar{z}}\right)+\Varangle(\operatorname{deg} \leq 0)+(\operatorname{deg}<0) .
\end{gathered}
$$

The spectral decomposition of $\chi_{R} A_{x y}$ is obtained by complex conjugation,

$$
\lambda_{n R \pm}=\nu_{n R} \lambda_{ \pm}=\overline{\lambda_{n L \mp}}=\overline{\nu_{n L}} \lambda_{ \pm}, \quad F_{n L \pm}=F_{n R \mp}^{*} .
$$

Combining these relations with (4.3.39) and (4.3.41), we conclude that all the eigenvalues of the closed chain have the same absolute value. Thus in view of $(4.3 .2)$, the EL equations are indeed satisfied for $\tau_{\text {reg }}=0$. In order to treat the higher orders in $\tau_{\text {reg }}$, one performs a power expansion up to the required order in the Planck length. The EL equations can be satisfied to every order in $\tau_{\text {reg }}$ by imposing suitable conditions on the regularization functions. Thus one gets a finite hierarchy of equations to be satisfied in a weak evaluation on the light cone.

Case (ii). We perform a perturbation expansion in the off-diagonal elements $U_{L}^{21}$ and $U_{L}^{12}$. If we set these matrix elements to zero, we again get a spectral representation of the form 4.3.40-4.3.43, but now with

$$
I_{1}=\left(\begin{array}{ll}
1 & 0 \\
0 & 0
\end{array}\right), \quad I_{2}=\left(\begin{array}{ll}
0 & 0 \\
0 & 1
\end{array}\right)
$$

and

$$
\nu_{1}=U_{L}^{11} V_{R}^{N}, \quad \nu_{2}=U_{R}^{22} V_{R}^{C} .
$$

Since the diagonal elements of any $U(2)$-matrix have the same absolute value, it follows that 4.3.39 again holds. Hence the EL equations are again satisfied in the case $U_{L}^{21}=$ $0=U_{L}^{12}$. Expanding in powers of $U_{L}^{21}$ and $U_{L}^{12}$ again gives a finite hierarchy of equations to be evaluated weakly on the light cone, which can again be satisfied by imposing suitable conditions on the regularization functions. 
We conclude that to degree five on the light cone, the EL equations can be satisfied by a suitable choice of the regularization functions, whenever the EL equations have a welldefined continuum limit. Clearly, the detailed computation of admissible regularizations is rather involved. Fortunately, we do not need to work out the details, because they will not be needed later on.

\subsection{The Euler-Lagrange Equations to Degree Four}

We now come to the analysis of the EL equations to degree four on the light cone. Before beginning, we clarify our scalings. Recall that the mass expansion increases the upper index of the factors $T_{\circ}^{(n)}$ and thus decreases the degree on the light cone. In view of the weak evaluation formula (4.2.31), the mass expansion gives scaling factors $m^{2} \varepsilon|\vec{\xi}|$.

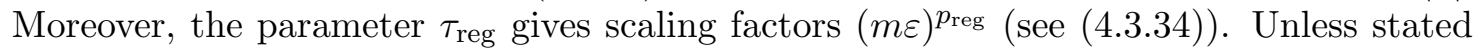
otherwise, we shall only consider the leading order in $(m \varepsilon)^{p_{\text {reg }}}$, meaning that we allow for an error term of the form

$$
\left(1+\mathcal{O}\left((m \varepsilon)^{p_{\text {reg }}}\right)\right) .
$$

Finally, the weak evaluation formulas involve error terms of the form 4.2.32). Since the contributions to the EL equations to degree four on the light cone involve at least one scaling factor $m^{2} \varepsilon|\vec{\xi}|$ (from the mass expansion) or a factor with the similar scaling $\varepsilon|\vec{\xi}| / \ell_{\text {macro }}^{2}$ (from the light-cone expansion), the factors $\varepsilon /|\vec{\xi}|$ (which arise from the regularization expansion) give rise to at least one factor $m^{2} \varepsilon^{2}$, which can be absorbed into the error term (4.4.1). Hence, unless stated otherwise, in all the subsequent calculations we neglect the

$$
\text { (higher orders in } \left.\varepsilon / \ell_{\text {macro }} \text { and }(m \varepsilon)^{p_{\text {reg }}}\right) \text {. }
$$

For ease in notation, in most computations we omit to write out the corresponding error term $\left(1+\mathcal{O}\left(\varepsilon / \ell_{\text {macro }}\right)+\mathcal{O}\left((m \varepsilon)^{p_{\text {reg }}}\right)\right)$.

4.4.1. General Structural Results. We again denote the eigenvalues of the closed chain $A_{x y}$ by $\lambda_{n c s}^{x y}$. These eigenvalues will be obtained by perturbing the eigenvalues with gauge phases as given in 4.3 .40 and 4.3 .43 . As a consequence, they will again form complex conjugate pairs, i.e.

$$
\lambda_{n R \pm}^{x y}=\overline{\lambda_{n L \mp}^{x y}} .
$$

As the unperturbed eigenvalues all have the same absolute value (see (4.3.40), 4.3.39) and (4.3.41)), to degree four we only need to take into account the perturbation of the square bracket in (4.3.2). Thus the EL equations reduce to the condition

$$
0=\Delta Q(x, y):=\sum_{n, c, s}\left[\Delta\left|\lambda_{n c s}^{x y}\right|-\frac{1}{8} \sum_{n^{\prime}, c^{\prime}, s^{\prime}} \Delta\left|\lambda_{n^{\prime} c^{\prime} s^{\prime}}^{x y}\right|\right] \frac{\overline{\lambda_{n c s}^{x y}}}{\left|\lambda_{n c s}^{x y}\right|} F_{n c s}^{x y} P(x, y),
$$

where we again evaluate weakly on the light cone and consider the perturbation of the eigenvalues to degree two (also, the superscript $x y$ clarifies the dependence of the eigenvalues on the space-time points).

Here the unperturbed spectral projectors $F_{n c s}$ were computed explicitly in (4.3.40) and 4.3.42). Moreover, the relations 4.3.10 4.3 .12 can be written in the shorter form

$$
F_{+}^{x y} \sharp=(\operatorname{deg}<0), \quad F_{-}^{x y} \not=\sharp+(\operatorname{deg}<0) .
$$


Combining these relations with the explicit formulas for the corresponding unperturbed eigenvalues (see 4.3.40) and 4.3.41) as well as using 4.4.3), we can write $\Delta Q(x, y)$ as

$$
\Delta Q(x, y)=\frac{i}{2} \sum_{n, s}\left[\mathcal{K}_{n c}(x, y)-\frac{1}{4} \sum_{n^{\prime}, c^{\prime}} \mathcal{K}_{n^{\prime} c^{\prime}}(x, y)\right] I_{n} \chi_{c} \nLeftarrow+(\operatorname{deg}<4),
$$

where

$$
\mathcal{K}_{n c}(x, y):=\frac{\Delta\left|\lambda_{n c-}^{x y}\right|}{\left|\lambda_{-}\right|} 3^{3} T_{[0]}^{(0)} T_{[0]}^{(-1)} \overline{T_{[0]}^{(-1)}}
$$

(for more details see the proof of Lemma 3.7.1). Since the smooth factors in 4.4.5) are irrelevant, the EL equations (4.4.4) reduce to the conditions

$$
\mathcal{K}_{1 L}=\mathcal{K}_{2 L}=\mathcal{K}_{1 R}=\mathcal{K}_{2 R} \quad \bmod (\operatorname{deg}<4) .
$$

For all the contributions to the fermionic projector of interest in this paper, it will suffice to compute $\Delta\left|\lambda_{n c+}^{x y}\right|$ in a perturbation calculation of first or second order. Then the complex numbers $\mathcal{K}_{n c}$ can be recovered as traces of $I_{n}$ with suitable $2 \times 2$-matrices, as the following lemma shows.

LEMMA 4.4.1. In a perturbation calculation to first order, there are $2 \times 2$-matrices $\mathcal{K}_{L}$ and $\mathcal{K}_{R}$ such that

$$
\mathcal{K}_{n c}=\operatorname{Tr}_{\mathbb{C}^{2}}\left(I_{n} \mathcal{K}_{c}\right)+(\operatorname{deg}<4) .
$$

In a second order perturbation calculation, one can again arrange (4.4.8), provided that the gauge phases $\nu_{n c}$ in the unperturbed eigenvalues 4.3.40 and 4.3.43 must not to be taken into account and that the perturbation vanishes on the degenerate subspaces in the sense that

$$
F_{+}(\Delta A) F_{+}=0 .
$$

Proof. In view of (4.4.6), it clearly suffices to show that $\Delta\left|\lambda_{n c+}\right|$ can be written as such a trace. Writing

$$
\Delta\left|\lambda_{n c+}\right|=\frac{1}{2\left|\lambda_{+}\right|}\left(\left(\Delta \lambda_{n c+}\right) \overline{\lambda_{+}}+\lambda_{+} \overline{\left(\Delta \lambda_{n c+}\right)}\right)
$$

and using 4.4.3), one concludes that it suffices to show that

$$
\Delta \lambda_{n c s}=\operatorname{Tr}_{\mathbb{C}^{2}}\left(I_{n} B\right)
$$

for a suitable $2 \times 2$-matrix $B=B(c, s)$.

The linear perturbation is given by

$$
\Delta \lambda_{n c s}=\operatorname{Tr}\left(F_{n c s} \Delta A\right) .
$$

As the unperturbed spectral projectors involve a factor $I_{n}$ (see 4.3.40) and 4.3.43), this is obviously of the form 4.4.10).

Using 4.4.9, we have to second order

$$
\Delta \lambda_{n c s}=\sum_{n^{\prime}, c^{\prime}} \frac{1}{\lambda_{n c s}-\lambda_{n^{\prime} c^{\prime}(-s)}} \operatorname{Tr}\left(F_{n c s} \Delta A F_{n^{\prime} c^{\prime}(-s)} \Delta A\right) .
$$


Disregarding the gauge phases $\nu_{c s}$ in 4.3 .40 ) and 4.3 .43 , we get

$$
\begin{aligned}
\Delta \lambda_{n c s} & =\sum_{n^{\prime}, c^{\prime}} \frac{1}{\lambda_{s}-\lambda_{-s}} \operatorname{Tr}\left(F_{n c s} \Delta A F_{n^{\prime} c^{\prime}(-s)} \Delta A\right) \\
& =\frac{1}{\lambda_{s}-\lambda_{-s}} \operatorname{Tr}\left(\chi_{c} I_{n} F_{s} \Delta A F_{-s} \Delta A\right),
\end{aligned}
$$

where in the last line we used the form of the spectral projectors in 4.3 .40 and 4.3 .43 ) and carried out the sums over $n^{\prime}$ and $c^{\prime}$. This is again of the form 4.4.10).

Instead of analyzing the conditions (4.4.7), we shall always analyze the stronger conditions

$$
\mathcal{K}_{L}(x, y)=\mathcal{K}_{R}(x, y)=c(\xi) \mathbb{1}_{\mathbb{C}^{2}}
$$

This requires a detailed explanation, depending on the two cases in 4.3.36). In Case (i), when the projectors $I_{n}$ are determined by the chiral gauge potentials, the condition 4.4.12 can be understood in two different ways. The first, more physical argument is to note that the spectral projectors $I_{n}$ of the matrix product (4.3.37) depend on the local gauge potentials $A_{L}$ and $A_{R}$. In order for these potentials to be dynamical, the EL equations should not give algebraic constraints for these potentials (i.e. constraints which involve the potentials but not their derivatives). This can be achieved by demanding that the conditions (4.4.7) should be satisfied for any choice of the potentials. In view of 4.4.8), this implies that (4.4.12) must hold.

To give the alternative, more mathematical argument, let us assume conversely that one of the matrices $\mathcal{K}_{L}$ or $\mathcal{K}_{R}$ is not a multiple of the identity matrix. Then the perturbation calculation would involve terms mixing the free eigenspaces corresponding to $\lambda_{1 c s}$ and $\lambda_{2 c s}$. More precisely, to first order one would have to diagonalize the perturbation on the corresponding degenerate subspace. To second order, the resulting contribution to the perturbation calculation would look similar to (4.4.11), but it would also involve factors of $\left(\lambda_{1 c s}-\lambda_{2 c s}\right)^{-1}$. In both cases, the perturbed eigenvalues would no longer be a power series in the bosonic potentials. Analyzing these non-analytic contributions in the EL equations (4.4.7), one finds that they must all vanish identically. Working out this argument in more detail, one could even derive (4.4.12) from the EL equations.

In Case (ii) in 4.3.36), the projectors $I_{n}$ are isospin-diagonal 4.3.44), so that (4.4.7) only tests the diagonal elements of $\mathcal{K}_{c}$. Thus at first sight, 4.4 .12 seems a too strong condition. However, even in this case the condition $(4.4 .12$ can be justified as follows. The left-handed gauge potentials modify the left-handed component of the fermionic projector by generalized phase transformations. If the involved gauge potential is off-diagonal, it makes an off-diagonal components of $P(x, y)$ diagonal and vice versa. As a consequence, satisfying (4.4.7) in the presence of off-diagonal gauge potentials is equivalent to satisfying (4.4.7). We will come back to this argument in more detail in Section 4.8.

We finally use 4.4.8 in 4.4.5 to obtain a useful representation of $\Delta Q$ :

Corollary 4.4.2. Under the assumptions of Lemma 4.4.1, the kernel $\Delta Q(x, y)$ in (4.4.4) has the representation

$$
\Delta Q(x, y)=\frac{i}{2} \sum_{n, c} \operatorname{Tr}_{\mathbb{C}^{2}}\left(I_{n} \mathcal{Q}_{c}\right) I_{n} \chi_{c} \sharp,
$$

where

$$
\mathcal{Q}_{L}:=\mathcal{K}_{L}-\frac{1}{4} \operatorname{Tr}_{\mathbb{C}^{2}}\left(\mathcal{K}_{L}+\mathcal{K}_{R}\right) \mathbb{1}_{\mathbb{C}^{2}}
$$


(and $\mathcal{Q}_{R}$ is obtained by the obvious replacements $L \leftrightarrow R$ ).

The stronger condition 4.4.12 is then equivalent to demanding that the relations

$$
\mathcal{Q}_{L}(x, y)=0=\mathcal{Q}_{R}(x, y)
$$

hold in a weak evaluation on the light cone.

4.4.2. The Vacuum. We begin by analyzing the eigenvalues of the closed chain in the vacuum. As the fermionic projector is diagonal in the isospin index, we can consider the charged sector and the neutrino sector after each other. In the charged sector, the eigenvalues can be computed exactly as in [F7, Section 5.3] or \$3.6.1. Using the notation and conventions in Chapter 3, we obtain

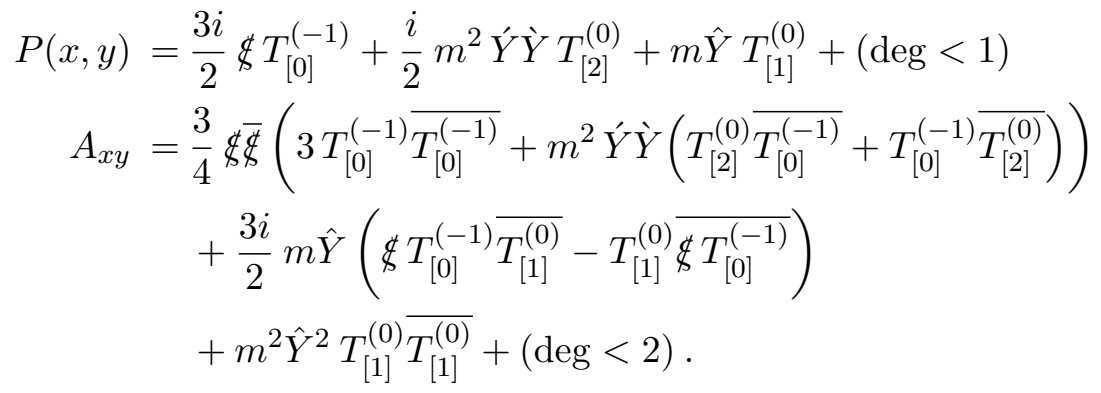

A straightforward calculation shows that the closed chain has two eigenvalues $\lambda_{ \pm}$, both with multiplicity two. They have the form

$$
\begin{aligned}
& \lambda_{+}=9 T_{[0]}^{(0)} \overline{T_{[0]}^{(-1)}}+m^{2}(\cdots)+(\operatorname{deg}<2) \\
& \lambda_{-}=9 T_{[0]}^{(-1)} \overline{T_{[0]}^{(0)}}+m^{2}(\cdots)+(\operatorname{deg}<2),
\end{aligned}
$$

where $(\cdots)$ stands for additional terms, whose explicit form will not be needed here (for details see [F7, eq. (5.3.24)]).

In the neutrino sector, by using 4.2.35) in the ansatz (4.1.8) and 4.2.41), after forming the sectorial projection we obtain

$$
\begin{aligned}
& P(x, y)=\frac{3 i \not}{2} T_{[0]}^{(-1)}+\chi_{R} \tau_{\text {reg }} \frac{i \not}{2}\left(T_{[R, 0]}^{(-1)}+\delta^{-2} T_{[R, 2]}^{(0)}\right) \\
& +\frac{i}{2} \notin m^{2} \hat{Y} \grave{Y} T_{[2]}^{(0)}+m \hat{Y} T_{[1]}^{(0)}+(\operatorname{deg}<1) \\
& \chi_{L} A_{x y}=\frac{3}{4} \chi_{L} \notin \bar{\sharp} T_{[0]}^{(-1)} \overline{\left(3 T_{[0]}^{(-1)}+\tau_{\text {reg }} T_{[R, 0]}^{(-1)}+\tau_{\text {reg }} \delta^{-2} T_{[R, 2]}^{(0)}\right)} \\
& +\frac{3}{4} \Varangle \bar{\Varangle} m^{2} \dot{Y} \grave{Y}\left(T_{[2]}^{(0)} \overline{T_{[0]}^{(-1)}}+T_{[0]}^{(-1)} \overline{T_{[2]}^{(0)}}\right)+m^{2} \hat{Y}^{2} T_{[1]}^{(0)} \overline{T_{[1]}^{(0)}} \\
& +\frac{3 i}{2} m \hat{Y}\left(\sharp T_{[0]}^{(-1)} \overline{T_{[1]}^{(0)}}-T_{[1]}^{(0)} \overline{\& T_{[0]}^{(-1)}}\right)+(\operatorname{deg}<2) \text {. }
\end{aligned}
$$

The contraction rules 4.2.36) and (4.2.38) yield $(\Varangle \bar{\phi})^{2}=(z+\bar{z}) \notin \bar{\phi}+z \bar{z}$ and thus

$$
(\not \bar{\ddagger}-z)(\not \bar{\phi}-\bar{z})=0 \text {. }
$$


This shows that the matrix $\Varangle \bar{\phi}$ has the eigenvalues $z$ and $\bar{z}$. Also applying (4.3.35), the eigenvalues of the closed chain are computed by

$$
\begin{aligned}
\lambda_{L+} & =\frac{3}{4} z T_{[0]}^{(-1)} \overline{\left(3 L_{[0]}^{(-1)}+\tau_{\text {reg }} \delta^{-2} T_{[R, 2]}^{(0)}\right)}+m^{2}(\cdots) \\
& =9 T_{[0]}^{(0)} \overline{L_{[0]}^{(-1)}}+3 \tau_{\text {reg }} \delta^{-2} T_{[0]}^{(0)} \overline{T_{[R, 2]}^{(0)}}+m^{2}(\cdots)+(\operatorname{deg}<2) \\
\lambda_{L-} & =\frac{3}{4} T_{[0]}^{(-1)} \overline{z\left(3 T_{[0]}^{(-1)}+\tau_{\text {reg }} T_{[R, 0]}^{(-1)}+\tau_{\text {reg }} \delta^{-2} T_{[R, 2]}^{(0)}\right)}+m^{2}(\cdots) \\
& =9 T_{[0]}^{(-1)} \overline{L_{[0]}^{(0)}}-3 \tau_{\text {reg }} \delta^{-2} T_{[0]}^{(-1)} \overline{T_{\{R, 0\}}^{(1)}}+m^{2}(\cdots)+(\operatorname{deg}<2),
\end{aligned}
$$

where $L_{\circ}^{(n)}$ is again given by 4.3 .9$)$, and $m^{2}(\cdots)$ denotes the same contributions as in 4.4.16) with the masses $m_{\beta}$ replaced by the corresponding neutrino masses $\tilde{m}_{\beta}$. The two other eigenvalues are again obtained by complex conjugation (4.4.3).

The first summands in (4.4.17) and (4.4.18) are of degree three on the light cone and were already analyzed in Section 4.3. Thus the point of interest here are the summands involving $\delta$. Before analyzing them in detail, we point out that they arise for two different reasons: The term in (4.4.17) is a consequence of the mass expansion of general surface states. The term in (4.4.18), on the other hand, corresponds to the last term in the contraction rule 4.2.38, which takes into account the shear of the surface states.

Let us specify the scaling of the terms involving $\delta$. Recall that the parameter $\tau_{\text {reg scales }}$ according to (4.3.34), whereas $\delta$ is only specified by (4.2.34). We want that the general surface and shear states make up for the fact that the masses $m_{\beta}$ of the charged fermions are different from the neutrino masses $\tilde{m}_{\beta}$. Therefore, it would be natural to impose that the summands involving $\delta$ should have the same scaling as the contributions $m^{2}(\cdots)$ arising in the standard mass expansion. This gives rise to the scaling

$$
\frac{\tau_{\text {reg }}}{\delta^{2}} \approx m^{2}
$$

and thus $\delta \approx m(m \varepsilon)^{\frac{p_{\text {reg }}}{2}}$. But $\delta$ can also be chosen smaller. In this case, the terms involving $\delta$ in 4.4.17) and 4.4.18 could dominate the contributions by the standard mass expansion. But they do not need to, because their leading contributions may cancel when evaluated weakly on the light cone. With this in mind, we allow for the scaling

$$
\varepsilon \ll \delta \lesssim \frac{1}{m}(m \varepsilon)^{\frac{p_{\text {reg }}}{2}} .
$$

Assuming this scaling, by choosing the regularization parameters corresponding to the factors $T_{[R, 2]}^{(0)}$ and $T_{\{R, 0\}}^{(1)}$ appropriately, we can arrange that 4.4.4 holds. This procedure works independent of the masses $m_{\beta}$ and $\tilde{m}_{\beta}$.

4.4.3. The Current and Mass Terms. We now come to the analysis of the interaction. More precisely, we want to study the effect of the fermionic wave functions in 4.2.51) and of the chiral potentials 4.3.29) in the Dirac operator 4.2.46) on the EL equations to degree four. As in Section 3.7 we consider the contribution near the origin in a Taylor expansion around $\xi=0$.

Definition 4.4.3. The integrand in 4.2.31) is said to be of order $o\left(|\vec{\xi}|^{k}\right)$ at the origin if the function $\eta$ is in the class o $\left(\left(\left|\xi^{0}\right|+|\vec{\xi}|\right)^{k+L}\right)$. Likewise, a contribution to the fermionic projector of the form $P(x, y) \asymp \eta(x, y) T^{(n)}$ is of the order o( $\left.|\vec{\xi}|^{k}\right)$ if $\eta \in$ $o\left(\left(\left|\xi^{0}\right|+|\vec{\xi}|\right)^{k+1-n}\right)$. 
Before stating the main result, we define the bosonic current $j_{L / R}$ and the Dirac current $J_{L / R}$ by

$$
\begin{aligned}
j_{L / R}^{k} & =\partial_{j}^{k} A_{L / R}^{j}-\square A_{L / R} \\
\left(J_{L / R}^{k}\right)_{(j, \beta)}^{(i, \alpha)} & =\sum_{l=1}^{n_{\mathrm{p}}} \overline{\psi_{l}^{(j, \beta)}} \chi_{R / L} \gamma^{k} \psi_{l}^{(i, \alpha)}-\sum_{l=1}^{n_{\mathrm{a}}} \overline{\phi_{l}^{(j, \beta)}} \chi_{R / L} \gamma^{k} \phi_{l}^{(i, \alpha)} .
\end{aligned}
$$

Note that, due to the dependence on the isospin and generation indices, these currents are $6 \times 6$-matrices. We also point out that for the sake of brevity, in 4.4 .20 we omitted the terms quadratic in the potentials which arise for a non-abelian gauge group. But as the form of these quadratic terms is uniquely determined from the well-known behavior under gauge transformations, they could be inserted into all our equations in an obvious way. Similar to the notation (4.2.53), we denote the sectorial projection by $\hat{\jmath}$ and $\hat{J}$. Moreover, we introduce the $2 \times 2$-matrix-valued vector field $\mathfrak{J}_{L}$ by

$$
\begin{aligned}
\mathfrak{J}_{L}^{k}= & \hat{J}_{R}^{k} K_{1}+\hat{\jmath}_{L}^{k} K_{2}+\hat{\jmath}_{R}^{k} K_{3} \\
& -3 m^{2}\left(\hat{A}_{L}^{k} Y \grave{Y}+\hat{Y} Y \grave{A}_{L}^{k}\right) K_{4} \\
& +m^{2}\left(\hat{A}_{L}^{k} \dot{Y} \grave{Y}+\dot{Y} \grave{Y} \hat{A}_{L}^{k}\right) K_{4} \\
& -3 m^{2}\left(\hat{A}_{R}^{k} Y \grave{Y}-2 \dot{Y} A_{L}^{k} \grave{Y}+\hat{Y} Y \grave{A}_{R}^{k}\right) K_{5} \\
& -6 m^{2}\left(\hat{A}_{L}^{k} \grave{Y} \hat{Y}+\hat{Y} \dot{Y} \grave{A}_{L}^{k}\right) K_{6} \\
& +6 m^{2}\left(\hat{Y} \hat{A}_{L}^{k} \grave{Y}+\dot{Y} \grave{A}_{L}^{k} \hat{Y}\right) K_{7} \\
& +m^{2}\left(\hat{A}_{L}^{k} \hat{Y} \hat{Y}+2 \hat{Y} \hat{A}_{R}^{k} \hat{Y}+\hat{Y} \hat{Y} \hat{A}_{L}^{k}\right) K_{6} \\
& -m^{2}\left(\hat{A}_{R}^{k} \hat{Y} \hat{Y}+2 \hat{Y} \hat{A}_{L}^{k} \hat{Y}+\hat{Y} \hat{Y} \hat{A}_{R}^{k}\right) K_{7},
\end{aligned}
$$

where $K_{1}, \ldots, K_{7}$ are the expressions

$$
\begin{aligned}
& K_{1}=-\frac{3}{16 \pi} \frac{1}{\overline{T_{[0]}^{(0)}}}\left[T_{[0]}^{(-1)} T_{[0]}^{(0)} \overline{T_{[0]}^{(-1)}}-c . c .\right] \\
& K_{2}=\frac{3}{4} \frac{1}{\overline{T_{[0]}^{(0)}}}\left[T_{[0]}^{(0)} T_{[0]}^{(0)} \overline{T_{[0]}^{(-1)} T_{[0]}^{(0)}}-c . c .\right] \\
& K_{3}=\frac{3}{2} \frac{1}{\overline{T_{[0]}^{(0)}}}\left[T_{[0]}^{(-1)} T_{[0]}^{(1)} \overline{T_{[0]}^{(-1)} T_{[0]}^{(0)}}-c . c .\right] \\
& K_{4}=\frac{1}{4} \frac{1}{\overline{T_{[0]}^{(0)}}}\left[T_{[0]}^{(0)} T_{[2]}^{(0)} \overline{T_{[0]}^{(-1)} T_{[0]}^{(0)}}-c . c .\right] \\
& K_{5}=\frac{1}{4} \frac{1}{\overline{T_{[0]}^{(0)}}}\left[T_{[0]}^{(-1)} T_{[2]}^{(1)} \overline{T_{[0]}^{(-1)} T_{[0]}^{(0)}}-c . c .\right] \\
& K_{6}=\frac{1}{12} \frac{T_{[0]}^{(0)} \overline{T_{[0]}^{(0)}}}{\overline{T_{[0]}^{(0)}}} \frac{\left(T_{[1]}^{(0)} \overline{T_{[0]}^{(-1)}}-T_{[0]}^{(-1)} \overline{T_{[1]}^{(0)}}\right)^{2}}{T_{[0]}^{(0)} \overline{T_{[0]}^{(-1)}}-T_{[0]}^{(-1)} \overline{T_{[0]}^{(0)}}}
\end{aligned}
$$




$$
K_{7}=\frac{1}{12} \frac{T_{[0]}^{(-1)} \overline{T_{[0]}^{(-1)}}}{\overline{T_{[0]}^{(0)}}} \frac{\left(T_{[1]}^{(0)} \overline{T_{[0]}^{(0)}}-T_{[0]}^{(0)} \overline{T_{[1]}^{(0)}}\right)^{2}}{T_{[0]}^{(0)} \overline{T_{[0]}^{(-1)}}-T_{[0]}^{(-1)} \overline{T_{[0]}^{(0)}}}
$$

evaluated weakly on the light cone 4.2.31) (and c.c. denotes the complex conjugate). Similarly, the matrix $\mathfrak{J}_{R}$ is defined by the replacements $L \leftrightarrow R$.

Lemma 4.4.4. The contribution of the bosonic current 4.4.20) and of the Dirac current 4.4.21) to the order $(\operatorname{deg}<4)+o\left(|\vec{\xi}|^{-3}\right)$ are of the form 4.4.13 and 4.4.14) with

$$
\mathcal{K}_{L / R}=i \xi_{k} \mathfrak{J}_{L / R}^{k}+(\operatorname{deg}<4)+o\left(|\vec{\xi}|^{-3}\right)
$$

Proof. The perturbation of the eigenvalues is obtained by a perturbation calculation to first and second order (see [F7, Appendix G] and Appendix B). The resulting matrix traces are computed most conveniently in the double null spinor frame $\left(\mathfrak{f}_{ \pm}^{L / R}\right)$ with the methods described in Appendix $\mathrm{B}$. One finds that $\Delta A$ is diagonal on the degenerate subspaces, so that the second order contribution is given by 4.4.11). Moreover, the gauge phases $\nu_{n c}$ in the unperturbed eigenvalues 4.3.40 and (4.3.43) only affect the error term $o\left(|\vec{\xi}|^{-3}\right)$. We conclude that Lemma 4.4.1 applies, and thus $\mathcal{K}_{L}$ and $\mathcal{K}_{R}$ are well-defined.

In order to compute $\mathcal{K}_{L / R}$, we need to take into account the following contributions to the light-cone expansion of the fermionic projector:

$$
\begin{aligned}
\chi_{L} P(x, y) \asymp & -\frac{1}{2} \chi_{L} \sharp \xi_{i} \int_{x}^{y}[0,0 \mid 1] j_{L}^{i} T^{(0)} \\
& -\chi_{L} \int_{x}^{y}[0,2 \mid 0] j_{L}^{i} \gamma_{i} T^{(1)} \\
& -i m \chi_{L} \xi_{i} \int_{x}^{y} Y A_{R}^{i} T^{(0)} \\
& +\frac{i m}{2} \chi_{L} \sharp \int_{x}^{y}\left(Y A_{R}-A_{L} Y\right) T^{(0)} \\
& +i m \chi_{L} \int_{x}^{y}[0,1 \mid 0]\left(Y\left(\partial_{j} A_{R}^{j}\right)-\left(\partial_{j} A_{L}^{j}\right) Y\right) T^{(1)} \\
& +\frac{m^{2}}{2} \chi_{L} \sharp \xi_{i} \int_{x}^{y}[1,0 \mid 0] Y Y A_{L}^{i} T^{(0)} \\
& +\frac{m^{2}}{2} \chi_{L} \sharp \xi_{i} \int_{x}^{y}[0,1 \mid 0] A_{L}^{i} Y Y T^{(0)} \\
& +m^{2} \chi_{L} \int_{x}^{y}[1,0 \mid 0] Y Y A_{L} T^{(1)} \\
& -m^{2} \chi_{L} \int_{x}^{y}[0,0 \mid 0] Y A_{R} Y T^{(1)} \\
& +m^{2} \chi_{L} \int_{x}^{y}[0,1 \mid 0] A_{L} Y Y T^{(1)}
\end{aligned}
$$


(for the derivation see [F7, Appendix B] and [F6, Appendix A]; cf. also Appendix B]. A long but straightforward calculation (which we carried out with the help of the $\mathrm{C}++$ program class_commute and an algorithm implemented in Mathematica) gives the result ${ }^{2}$.

We finally mention a rather subtle point in the calculation: According to 4.3.40) and 4.3.43), our unperturbed eigenvalues involve gauge phases and can thus be expanded in powers of $A_{c}^{k} \xi_{k}$. As a consequence, we must take into account contributions of the form 4.4.11) where the factors $\Delta A$ involve no gauge potentials, but the unperturbed eigenvalues $\lambda_{n c s}$ are expanded linearly in $A_{c}^{k} \xi_{k}$. In this case, the corresponding contributions involving no factors of $A_{c}^{k} \xi_{k}$ can be identified with contributions to the eigenvalues in the vacuum in 4.4.16) and 4.4.17), 4.4.18). Using that the vacuum eigenvalues all have the same absolute value, the contributions linear in $A_{c}^{k} \xi_{k}$ can be simplified to obtain the formulas for $\mathfrak{J}_{c}^{k}$ listed above. Another, somewhat simpler method to get the same result is to use that the operator $Q$ is symmetric (4.3.4) (see [F7, Lemma 3.5.1]). Thus it suffices to compute the symmetric part $\left(\Delta Q(x, y)+\Delta Q(y, x)^{*}\right) / 2$ of the operator $\Delta Q$ as defined by (4.4.4). This again gives the above formulas for $\mathfrak{J}_{c}^{k}$, without using any relations between the vacuum eigenvalues.

Let us briefly discuss the obtained formula for $\mathfrak{J}_{R}$. The summands in 4.4 .22 involve the chiral gauge currents and Dirac currents; they can be understood in analogy to the current terms in $\$ 3.7 .1$ and $\$ 3.7 .2$. The contributions 4.4.23)-4.4.29) are the mass terms. They are considerably more complicated than in 3.7.1. These complications are caused by the fact that we here consider left- and right-handed gauge potentials acting on two sectors, involving a mixing of the generations. In order to clarify the structure of the mass terms, it is instructive to look at the special case of a $U(1)$ vector potential, i.e. $A_{L}=A_{R}=A \cdot \mathbb{1}_{\mathbb{C}^{2}}$ (with a real vector field $A$ ). In this case, the terms 4.4.23) and 4.4.24 cancel each other (note that $\hat{A} Y \cdots=3 \hat{A} Y \cdots$ ), and (4.4.25) vanishes. Similarly, the summand 4.4.26 cancels 4.4.28), and 4.4.27) cancels 4.4.29). Thus the mass terms are zero, in agreement with local gauge invariance.

4.4.4. The Microlocal Chiral Transformation. The simple fractions $K_{3}$ and $K_{5}$ involve factors $T_{\circ}^{(1)}$ which have a logarithmic pole on the light cone. Before working out the field equations, we must compensate these logarithmic poles by a suitable transformation of the fermionic projector. We again work with a microlocal chiral transformation as developed in 3.7.8 3.7.11. As the generalizations to a system of two sectors is not straightforward, we give the necessary constructions step by step. Before beginning, we mention for clarity that in the following sections 4.4 .4 and 4.4 .5 we will construct contributions to $P(x, y)$ which enter the EL equations to degree four only linearly. Therefore, it is obvious that Lemma 4.4.1 again applies.

As in $\$ 3.7 .9$ we begin in the homogeneous setting and work in momentum space. Then the logarithmic poles on the light cone correspond to a contribution to the fermionic projector of the form

$$
\tilde{P}(k) \asymp\left(\chi_{L} \psi_{L}+\chi_{R} \psi_{R}\right) \delta^{\prime}\left(k^{2}\right) \Theta\left(-k^{0}\right),
$$

where the vector components $v_{L}^{j}$ are Hermitian $2 \times 2$-matrices acting on the sector index. In order to generate the desired contribution 4.4.30, we consider a homogeneous

\footnotetext{
${ }^{2}$ The $\mathrm{C}++$ program class_commute and its computational output as well as the Mathematica worksheets were included as ancillary files to the arXiv submission arXiv:1211.3351 [math-ph].
} 
transformation of the fermionic projector of the vacuum of the form

$$
\tilde{P}(k)=\dot{U}(k) P^{\operatorname{aux}}(k) \grave{U}(k)^{*}
$$

with a multiplication operator in momentum space $U(k)$. With the operator $U(k)$ we want to modify the states of vacuum Dirac sea with the aim of generating a contribution which can compensate the logarithmic poles. We denote the absolute value of the energy of the states by $\Omega=\left|k^{0}\right|$. We are mainly interested in the regime $m \ll \Omega \ll \varepsilon^{-1}$ where regularization effects play no role. Therefore, we may disregard the right-handed highenergy states and write the vacuum fermionic projector according to 4.1.4, 4.1.6) and 4.1.8. Expanding in the mass, we obtain

$$
P^{\text {aux }}=(\not k+m Y) \delta\left(k^{2}\right) \Theta\left(-k^{0}\right)-(\not k+m Y) m^{2} Y^{2} \delta^{\prime}\left(k^{2}\right) \Theta\left(-k^{0}\right)+(\operatorname{deg}<0) .
$$

For the transformation $U(k)$ in 4.4 .31 we take the ansatz

$$
U(k)=\mathbb{1}+\frac{i}{\sqrt{\Omega}} Z(k) \quad \text { with } \quad Z=\chi_{L} L^{j} \gamma_{j}+\chi_{R} R^{j} \gamma_{j},
$$

where $L^{j}$ and $R^{j}$ are $6 \times 6$-matrices (not necessarily Hermitian) which act on the generation and sector indices. For simplicity, we assume that the dependence on the vector index can be written as

$$
L^{j}=L v_{L}^{j} \quad \text { and } \quad R^{j}=R v_{R}^{j}
$$

with real vector fields $v_{L}$ and $v_{R}$ (and $6 \times 6$-matrices $L$ and $R$ ). The ansatz 4.4.33 can be regarded as the linear Taylor expansion of the exponential $U=\exp (i Z / \sqrt{\Omega})$, giving agreement to 3.7 .9 (in view of the fact that the quadratic and higher orders of this Taylor expansion dropped out in $\$ 3.7 .9$, for simplicity we leave them out here). Note that the operator $U(k)$ is in general not unitary (for details see Remark 4.4 .8 below).

Applying the transformation (4.4.31) and 4.4.33) to (4.4.32), only the isospin matrices are influenced. A short calculation gives

$$
\begin{aligned}
\chi_{L} \dot{U} & (\not k+m Y) \grave{U}^{*}=\chi_{L}(3 \not k+m \hat{Y}) \\
& +\frac{i}{\sqrt{\Omega}} \chi_{L}\left(\hat{L} \not k-\not k \hat{\mathbb{R}}^{*}\right)+\frac{i m}{\sqrt{\Omega}} \chi_{L}\left(\dot{L} \grave{Y}-\dot{Y} \grave{L}^{*}\right) \\
& +\frac{1}{\Omega} \chi_{L} \dot{L} \not k \grave{L}^{*}+\frac{m}{\Omega} \chi_{L} \dot{L} Y \grave{R}^{*} \\
\chi_{L} \dot{U} & (\not k+m Y) m^{2} Y^{2} \grave{U}^{*}=\chi_{L}\left(\not k m^{2} \dot{Y} \grave{Y}+m^{3} \dot{Y} Y \grave{Y}\right) \\
& +\frac{i m^{2}}{\sqrt{\Omega}} \chi_{L}\left(\dot{L} Y \grave{Y} \not k-\not k \dot{Y} Y \grave{R}^{*}\right)+\frac{i m^{3}}{\sqrt{\Omega}} \chi_{L}\left(\dot{L} Y Y \grave{Y}-\dot{Y} Y Y \grave{L}^{*}\right) \\
& +\frac{m^{2}}{\Omega} \chi_{L} \dot{L} \not k Y^{2} \grave{L}^{*}+\frac{m^{3}}{\Omega} \chi_{L} \dot{L} Y^{3} \grave{R}^{*}
\end{aligned}
$$

(and similarly for the right-handed component). Let us discuss the obtained contributions. Clearly, the terms 4.4 .35 and 4.4 .38$)$ are the unperturbed contributions. Generally speaking, due to the factor $\delta\left(k^{2}\right)$ in (4.4.32), the contributions (4.4.36) and (4.4.39) are singular on the light cone and should vanish, whereas the desired logarithmic contribution 4.4.30 must be contained in 4.4.37) or 4.4.40). The terms 4.4.36) of order $\Omega^{-\frac{1}{2}}$ contribute to the EL equations to degree five on the light cone. Thus in order for them 
to vanish, we need to impose that

$$
\begin{aligned}
\hat{L} & =0=\hat{R} \\
\dot{L} \grave{Y}-\dot{Y} \grave{L}^{*} & =0=\hat{R} \grave{Y}-\grave{Y} \grave{R}^{*} .
\end{aligned}
$$

The last summand in (4.4.37) does not involve a factor $\not k$ and is even. As a consequence, it only enters the EL equations in combination with another factor of $m$, giving rise to a contribution of degree three on the light cone (for details see Lemma B.3.1). With this in mind, we may disregard the last summand in (4.4.37). Similarly, the last summand in 4.4.40 and the first summand in 4.4 .39 are even and can again be omitted. In order for the second summand in 4.4 .39 to vanish, we demand that

$$
\dot{L} Y Y \grave{Y}-\dot{Y} Y Y \grave{L}^{*}=0=\dot{R} Y Y \grave{Y}-\dot{Y} Y Y \grave{R}^{*} .
$$

Then it remains to consider the first summand in 4.4.37) and the first summand in 4.4.40. We thus end up with a left-handed (and similarly right-handed) contribution to the fermionic projector of the form

$$
\chi_{L} \tilde{P}(k) \asymp \frac{1}{\Omega} \chi_{L} \dot{L} \not \grave{L}^{*} \delta\left(k^{2}\right) \Theta\left(-k^{0}\right)-\frac{m^{2}}{\Omega} \chi_{L} \dot{L} \not k Y^{2} \grave{L}^{*} \delta^{\prime}\left(k^{2}\right) \Theta\left(-k^{0}\right) .
$$

Note that the conditions (4.4.41)- 4.4.43) are linear in $L$ and $R$, whereas the contribution 4.4.44 is quadratic.

Before going on, we remark that at first sight, one might want to replace the conditions 4.4.42 and 4.4.43 by the weaker conditions

$$
\begin{gathered}
\dot{L} \grave{Y}-\dot{Y} \grave{L}^{*}=\dot{R} \grave{Y}-\dot{Y} \grave{R}^{*}=i v_{1}(k) \mathbb{1}_{\mathbb{C}^{2}} \\
\dot{L} Y Y \grave{Y}-\dot{Y} Y Y \grave{L}^{*}=\dot{R} Y Y \grave{Y}-\dot{Y} Y Y \grave{R}^{*}=i v_{3}(k) \mathbb{1}_{\mathbb{C}^{2}}
\end{gathered}
$$

involving two real-valued vector fields $v_{1}$ and $v_{3}$. Namely, as the resulting contribution to the fermionic projector acts trivially on the isospin index and is symmetric under the replacement $L \leftrightarrow R$, it perturbs the eigenvalues of the closed chain in a way that the absolute values of all eigenvalues remain equal, so that the EL equations are still satisfied. However, this argument is too simple because the gauge phases must be taken into account. For the contributions in 4.4.44, the methods in 3.7 .11 make it possible to arrange that the gauge phases enter in a way which is compatible with the EL equations. For the contributions corresponding to 4.4.45, however, it is impossible to arrange that the gauge phases drop out of the EL equations. Hence $v_{1}$ and $v_{3}$ would necessarily enter the EL equations. As the scaling factors $1 / \sqrt{\Omega}$ in $(4.4 .36$ and 4.4 .39 give rise to a different $|\vec{\xi}|$-dependence, these contributions to the EL equations would have a different scaling behavior in the radius. As a consequence, the EL equations would only be satisfied if $v_{1} \equiv v_{3} \equiv 0$.

For clarity, we want to focus our attention to the component of 4.4.44 which will give the dominant contribution to the EL equations. For the moment, we only motivate in words how this component is chosen; the detailed justification that the other components can really be neglected will be given in the proof of Proposition 4.4 .6 below. In the EL equations, the chiral component (4.4.44) is contracted with a factor $\xi$. This means in momentum space that the main contribution of (4.4.44) to the EL equations is obtained by contracting with a factor $k$ (this will be justified in detailed in the proof of Proposition 4.4 .6 below). Therefore, we use the anti-commutation relations to rewrite (4.4.44) as

$$
\tilde{P}(k)=\chi_{L} P_{L}^{j}(k) \gamma_{j}+\chi_{R} P_{R}^{j}(k) \gamma_{j}
$$


(here we use the specific form 4.4.34 of our ansatz). We now contract with $k$ to obtain

$$
\begin{aligned}
P_{L}[k]:=P_{L}^{j}(k) k_{j}= & \frac{1}{\Omega}\left(2 \dot{L}_{i} \grave{L}_{j}^{*} k^{i} k^{j}-k^{2} \dot{L}^{j} \grave{L}_{j}^{*}\right) \delta\left(k^{2}\right) \Theta\left(-k^{0}\right) \\
& -\frac{m^{2}}{\Omega}\left(2 \dot{L}_{i} Y^{2} \grave{L}_{j}^{*} k^{i} k^{j}-k^{2} \dot{L}^{j} Y^{2} \grave{L}_{j}^{*}\right) \delta^{\prime}\left(k^{2}\right) \Theta\left(-k^{0}\right) .
\end{aligned}
$$

As the factor $k^{2}$ vanishes on the mass shell, we may omit the resulting terms (for details see again the proof of Proposition 4.4.6 below). We thus obtain

$$
P_{L}[k]=\frac{2}{\Omega} L[k] L[k]^{*} \delta\left(k^{2}\right) \Theta\left(-k^{0}\right)-\frac{2}{\Omega} L[k] m^{2} Y^{2} L[k]^{*} \delta^{\prime}\left(k^{2}\right) \Theta\left(-k^{0}\right),
$$

where we set $L[k]=L_{j}(k) k^{j}$ (note that $L[k]$ is a $2 \times 6$-matrix, and the star simply denotes the adjoint of this matrix). The right-handed component is obtained by the obvious replacements $L \rightarrow R$.

Let us work out the conditions needed for generating a contribution of the desired form 4.4.30. Similar as explained in 3.7.9, the first summand in 4.4.47 necessarily gives a contribution to the fermionic projector. For this contribution to drop out of the EL equations, we need to impose that it is vectorial and proportional to the identity matrix, i.e.

$$
L[k] L[k]^{*}=R[k] R[k]^{*}=\mathfrak{c}_{0}(k) \mathbb{1}_{\mathbb{C}^{2}}
$$

with some constant $\mathfrak{c}_{0}(k)$. In order to better justify that (4.4.48) is a necessary condition, we remark that the contribution to the fermionic projector corresponding to the first summand in 4.4.47) is of the form $P \simeq \chi_{L} \psi T_{[1]}^{[0]}$. The resulting contribution to the eigenvalues of the closed chain is $\Delta \lambda_{L+} \simeq i v_{j} \xi^{j} T_{[1]}^{(0)} \overline{T_{[0]}^{(-1)}}$, and a direct computation shows that this gives rise to a non-trivial contribution to the EL equations unless 4.4.48 holds.

The second summand in 4.4.47) is of the desired form 4.4.30). Keeping in mind that we may again allow for a vector contribution proportional to the identity, we get the conditions

$$
\begin{aligned}
L[k] m^{2} Y^{2} L[k]^{*} & =\frac{\Omega}{2} v_{L}[k]+\mathfrak{c}_{2}(k) \mathbb{1}_{\mathbb{C}^{2}} \\
R[k] m^{2} Y^{2} R[k]^{*} & =\frac{\Omega}{2} v_{R}[k]+\mathfrak{c}_{2}(k) \mathbb{1}_{\mathbb{C}^{2}},
\end{aligned}
$$

where we set $v_{L / R}[k]=v_{L / R}^{j}(k) k_{j}$ (and $\mathfrak{c}_{2}$ is another free constant). Our task is to solve the quadratic equations 4.4.48) and 4.4.49) under the linear constraints 4.4.41 4.4.43. Moreover, in order to compute the smooth contribution to the fermionic projector, we need to determine the expectation values involving the logarithms of the masses

$$
L[k]\left(m^{2} Y^{2} \log (m Y)\right) L[k]^{*} \quad \text { and } \quad R[k]\left(m^{2} Y^{2} \log (m Y)\right) R[k]^{*} .
$$

We next describe a method for treating the quadratic equations $(4.4 .48)$ and 4.4 .49 under the linear constraints 4.4.41) (the linear constraints 4.4.42 and 4.4.43) will be treated afterwards). We first restrict attention to the left-handed component and consider the corresponding equations in 4.4.41), 4.4.48) and 4.4.49) (the right-handed component can be treated similarly). We write the matrix $L[k]$ in components,

$$
L[k]=\left(\begin{array}{llllll}
l_{11} & l_{12} & l_{13} & l_{14} & l_{15} & l_{16} \\
l_{21} & l_{22} & l_{23} & l_{24} & l_{25} & l_{26}
\end{array}\right)
$$


where the matrix entries $l_{a b}$ are complex numbers. We use the linear relations (4.4.41) to express the third and sixth columns of the matrices by

$$
l_{a 3}=-l_{a 1}-l_{a 2}, \quad l_{a 6}=-l_{a 4}-l_{a 5} \quad(a=1,2) .
$$

This reduces the number of free parameters to 8 complex parameters, which we combine to the matrix

$$
\psi=\left(\begin{array}{l}
\psi_{1} \\
\psi_{2}
\end{array}\right) \quad \text { with } \quad \psi_{a}=\left(l_{a 1}, l_{a 2}, l_{a 4}, l_{a 5}\right) .
$$

We introduce on $\mathbb{C}^{4}$ the scalar product $\langle., .\rangle_{0}$ as well as the positive semi-definite inner product $\langle., .\rangle_{2}$ by

$$
\left\langle\psi_{a}, \psi_{b}\right\rangle_{0}=\left(L[k] L[k]^{*}\right)_{b}^{a} \quad \text { and } \quad\left\langle\psi_{a}, \psi_{b}\right\rangle_{2}=\left(L[k] m^{2} Y^{2} L[k]^{*}\right)_{b}^{a}
$$

(where we implicitly use 4.4.52 to determine the third and sixth columns of $L[k]$ ). We represent these scalar products with signature matrices,

$$
\langle\psi, \phi\rangle_{0}=\left\langle\psi, S_{0} \phi\right\rangle_{\mathbb{C}^{4}}, \quad\langle\psi, \phi\rangle_{2}=\left\langle\psi, S_{2} \phi\right\rangle_{\mathbb{C}^{4}} .
$$

Expressing $\langle., .\rangle_{2}$ in terms of $\langle., .\rangle_{0}$,

$$
\langle\psi, \phi\rangle_{2}=\left\langle\psi, S_{0}^{-1} S_{2} \phi\right\rangle_{0},
$$

the resulting linear operator $S_{0}^{-1} S_{2}$ is symmetric with respect to $\langle., .\rangle_{0}$. Thus by diagonalizing the matrix $S_{0}^{-1} S_{2}$, one can construct an eigenvector basis $e_{1}, \ldots, e_{4}$ which is orthonormal with respect to $\langle., .\rangle_{0}$, i.e.

$$
\left\langle e_{a}, e_{b}\right\rangle_{0}=\delta_{a b}, \quad\left\langle e_{a}, e_{b}\right\rangle_{2}=\mu_{a} \delta_{a b} .
$$

As the matrices have real-valued entries, we can choose the eigenvectors $e_{a}$ such that all their components are real. Moreover, as the matrices $S_{0}$ and $S_{2}$ are block-diagonal in the isospin index, we may choose the eigenvectors such that $e_{1}$ and $e_{2}$ have isospin up, whereas $e_{3}$ and $e_{4}$ have isospin down, i.e.

$$
e_{1}, e_{2}=(*, *, 0,0), \quad e_{3}, e_{4}=(0,0, *, *)
$$

(where the star stands for an arbitrary real-valued entry). Finally, we always order the eigenvalues and eigenvectors such that

$$
0 \leq \mu_{1} \leq \mu_{2} \quad \text { and } \quad \mu_{3} \leq \mu_{4} .
$$

Writing the vectors $\psi_{a}$ in 4.4 .53 in this eigenvector basis,

$$
\psi_{a}=\sum_{d=1}^{4} \psi_{a}^{d} e_{d}
$$

we can express (4.4.54) in the simpler form

$$
\left(L[k] L[k]^{*}\right)_{b}^{a}=\sum_{d=1}^{4} \overline{\psi_{a}^{d}} \psi_{b}^{d}, \quad\left(L[k] m^{2} Y^{2} L[k]^{*}\right)_{b}^{a}=\sum_{d=1}^{4} \mu_{d} \overline{\psi_{a}^{d}} \psi_{b}^{d} .
$$

Moreover, the linear condition 4.4.41) is satisfied.

In order to treat the remaining linear constraints 4.4 .42 and 4.4 .43 , we decompose the coefficients in (4.4.57) into their real and imaginary parts,

$$
\psi_{1 / 2}^{d}=a_{1 / 2}^{d}+i b_{1 / 2}^{d}, \quad(d=1, \ldots, 4) .
$$

Considering the diagonal entries of 4.4 .42 and 4.4 .43 shows that

$$
b_{1}^{1}=b_{1}^{2}=b_{2}^{3}=b_{2}^{4}=0 .
$$


The off-diagonal entries make it possible to express $a_{1}^{3}, a_{1}^{4}$ in terms of $a_{2}^{1}, a_{2}^{2}$ and $b_{1}^{3}, b_{1}^{4}$ in terms of $b_{2}^{1}, b_{2}^{2}$, leaving us with the eight real parameters $a_{1}^{1}, a_{1}^{2}, a_{2}^{3}, a_{2}^{4}$ and $a_{2}^{1}, a_{2}^{2}, b_{2}^{1}, b_{2}^{2}$.

In order to simplify the setting, it is useful to observe that all our constraints are invariant if we multiply the rows of the matrix $\psi$ in 4.4 .53 by phase factors according to

$$
\psi_{1} \rightarrow e^{i \varphi} \psi_{1}, \quad \psi_{2} \rightarrow e^{-i \varphi} \psi_{2} \quad \text { with } \quad \varphi \in \mathbb{R} .
$$

These transformations only affect the off-diagonal isospin components of the left-handed matrix in (4.4.49). With this in mind, we can assume that this matrix has real components and can thus be decomposed in terms of Pauli matrices as

$$
L[k] m^{2} Y^{2} L[k]^{*}=t \mathbb{1}+x \sigma^{1}+z \sigma^{3} .
$$

Using this equation in 4.4.58 and evaluating the real part of the off-diagonal elements of 4.4.48), one finds that $b_{2}^{1}=0=b_{2}^{2}$, leaving us with the six real parameters $a_{1}^{1}, a_{1}^{2}, a_{2}^{1}, a_{2}^{2}, a_{2}^{3}, a_{2}^{4}$. With these six parameters, we need to satisfy three quadratic relations in (4.4.60) and three quadratic relations in 4.4.48). This suggests that for given parameters $\mathfrak{c}_{0}$ and $\mathfrak{c}_{2}$ as well as $t, x, z$, there should be a discrete (possibly empty) set of solutions.

In preparation, we analyze the case when all potentials are diagonal in the isospin index.

EXAMPLE 4.4.5. (isospin-diagonal potentials) Assume that the parameter $x$ in 4.4.60 vanishes. Evaluating the real part of the off-diagonal components of 4.4.48 and (4.4.60), one finds that $a_{2}^{1}=0=a_{2}^{2}$. The diagonal components of (4.4.48) and 4.4.60) give the quadratic equations

$$
\begin{aligned}
\left(a_{1}^{1}\right)^{2} & =\frac{-t-z+\mathfrak{c}_{0} \mu_{2}}{\mu_{2}-\mu_{1}}, & \left(a_{2}^{1}\right)^{2} & =\frac{t+z-\mathfrak{c}_{0} \mu_{1}}{\mu_{2}-\mu_{1}} \\
\left(a_{3}^{2}\right)^{2} & =\frac{-t+z+\mathfrak{c}_{0} \mu_{4}}{\mu_{4}-\mu_{3}}, & \left(a_{4}^{2}\right)^{2} & =\frac{t-z-\mathfrak{c}_{0} \mu_{3}}{\mu_{4}-\mu_{3}} .
\end{aligned}
$$

For these equations to admit solutions, we need to assume the non-degeneracies

$$
\mu_{2} \neq \mu_{1} \quad \text { and } \quad \mu_{3} \neq \mu_{4} .
$$

Then there are solutions if and only if all the squares are non-negative. In view of our sign conventions 4.4.56), we obtain the conditions

$$
\mathfrak{c}_{0} \mu_{1} \leq t+z \leq \mathfrak{c}_{0} \mu_{2} \quad \text { and } \quad \mathfrak{c}_{0} \mu_{3} \leq t-z \leq \mathfrak{c}_{0} \mu_{4} .
$$

Provided that these inequalities hold, the matrix entries $a_{1}^{1}, a_{2}^{1}, a_{3}^{2}$ and $a_{4}^{2}$ are uniquely determined up to signs. For any solution obtained in this way, one can compute the logarithmic expectation value 4.4.50.

In order to analyze the conditions (4.4.63), we first note that changing the constant $\mathfrak{c}_{2}$ corresponds to adding a constant to the parameter $t$ (see 4.4.60 and 4.4.49). Hence we can always satisfy 4.4 .63 by choosing $\mathfrak{c}_{0}$ and $\mathfrak{c}_{2}$ sufficiently large, provided that

$$
\mu_{1} \leq \mu_{4} \quad \text { and } \quad \mu_{3} \leq \mu_{2} .
$$

If conversely these conditions are violated, it is impossible to satisfy 4.4.63 in the case $z=0$. The physical meaning of the inequalities 4.4.64 will be discussed in Remark 4.4 .9 below.

In the next proposition, we use a perturbation argument to show that the inequalities 4.4.64 guarantee the existence of the desired homogeneous transformations even if off-diagonal isospin components are present. 
Proposition 4.4.6. Assume that the parameters $\mu_{1}, \ldots, \mu_{4}$ as defined by 4.4.55) and 4.4.56) satisfy the inequalities 4.4.64). Then for any choice of the chiral potentials $v_{L}$ and $v_{R}$ in (4.4.30), there is a homogeneous chiral transformation of the form 4.4.33 such that the transformed fermionic projector 4.4.31) is of the form

$$
\begin{aligned}
\tilde{P}(k)= & P(k)+\left(\chi_{L} \psi_{L}+\chi_{R} \psi_{R}\right) T_{[3, \mathfrak{c}]}^{(1)} \\
& + \text { (vectorial) } \mathbb{1}_{\mathbb{C}^{2}} \delta\left(k^{2}\right)\left(1+\mathcal{O}\left(\Omega^{-1}\right)\right) \\
& + \text { (vectorial) } \mathbb{1}_{\mathbb{C}^{2}} \delta^{\prime}\left(k^{2}\right)\left(1+\mathcal{O}\left(\Omega^{-\frac{1}{2}}\right)\right) \\
& +(\text { pseudoscalar or bilinear }) \sqrt{\Omega} \delta^{\prime}\left(k^{2}\right)\left(1+\mathcal{O}\left(\Omega^{-1}\right)\right) \\
& +(\text { higher orders in } \varepsilon /|\vec{\xi}|) .
\end{aligned}
$$

Before coming to the proof, we point out that the values of the parameters $\mathfrak{c}_{0}$ and $\mathfrak{c}_{2}$ are not determined by this proposition. They can be specified similar as in $\$ 3.7 .9$ by choosing the homogeneous transformation such that $\mathfrak{c}_{0}$ is minimal (see also Section 4.8). In order

to clarify the dependence on $\mathfrak{c}_{0}$ and $\mathfrak{c}_{2}$, we simply added a subscript $\mathfrak{c}$ to the factor $T_{[3]}^{(1)}$. Similar to (3.8.3), this factor can be written in position space as

$$
T_{[3, \mathfrak{c}]}^{(1)}=\frac{1}{32 \pi^{3}}\left(\log \left|\xi^{2}\right|+i \pi \Theta\left(\xi^{2}\right) \epsilon\left(\xi^{0}\right)\right)+s_{[3, \mathfrak{c}]},
$$

where $s_{[3, \mathfrak{c}]}$ is a real-valued smooth function which depends on the choice of $\mathfrak{c}_{0}$ and $\mathfrak{c}_{2}$. In fact, $s_{[p, \mathfrak{c}]}$ may even depend on the isospin components of $v_{L}$ and $v_{R}$; but for ease in notation we shall not make this possible dependence explicit.

Proof of Proposition 4.4.6. We first show that for sufficiently large $\mathfrak{c}_{0}$ and $\mathfrak{c}_{2}$, there are solutions of (4.4.60) and of the left equation in 4.4.48). Evaluating the real part of the off-diagonal components of 4.4.48) and 4.4.60), we get linear equations in $a_{2}^{1}$ and $a_{2}^{2}$, making it possible to express $a_{2}^{1}$ and $a_{2}^{2}$ in terms of $a_{1}^{1}, a_{1}^{2}, a_{2}^{3}, a_{2}^{4}$. These relations do not involve $\mathfrak{c}_{0}$ nor $\mathfrak{c}_{2}$. As a consequence, the diagonal components of (4.4.48) and 4.4.60) give a system of equations, which for large parameters $\mathfrak{c}_{0}$ and $\mathfrak{c}_{2}$ are a perturbation of the system 4.4.61) and 4.4.62). Hence for sufficiently large $\mathfrak{c}_{0}$ and $\mathfrak{c}_{2}$, there are solutions by the implicit function theorem.

Repeating the above arguments for the right-handed potentials, we obtain matrices $L[k]$ and $R[k]$ such that $(4.4 .48)$ and $(4.4 .49)$ hold. Moreover, it is clear from our constructions that (4.4.41), 4.4.42) and 4.4.43) are satisfied. It remains to go through all the contributions (4.4.35)- 4.4.40) and to verify that they are of the form 4.4.65)-4.4.69). Clearly, 4.4.35) and (4.4.38) combine to the summand $P(k)$ in 4.4.65). The contributions in (4.4.36) vanish due to 4.4.41 and 4.4.42). The second summand in 4.4.37) as well as the first summand in (4.4.39) are of the form (4.4.68). The second summand in 4.4.39 vanishes in view of (4.4.43). Hence it really suffices to consider the first summand in 4.4.37) and the first summand in 4.4.40, which were combined earlier in (4.4.44).

It remains to justify the contraction with the momentum $k$, which led us to analyze (4.4.47). To this end, we need to consider the derivation of the weak evaluation formulas on the light cone in [F7, Chapter 4]. More precisely, the expansion of the vector component in [F7, eq. (4.4.6)-(4.4.8)] shows that $k$ and $\xi$ are collinear, up to errors of the order $\varepsilon /|\vec{\xi}|$. Moreover, the terms in 4.4.46) which involve a factor $k^{2}$ are again of the 
order $\varepsilon /|\vec{\xi}|$ smaller than the terms where the factors $k$ are both contracted to $\dot{L}$ or $\grave{L}^{*}$. This explains the error term 4.4.69).

We remark that the error term 4.4 .69 could probably be improved by analyzing those components of $\dot{L}^{j}(k)$ which vanish in the contraction $\dot{L}^{j}(k) k^{j}$. Here we shall not enter this analysis because errors of the order $\varepsilon /|\vec{\xi}|$ appear anyway when evaluating weakly on the light cone (4.2.31).

Exactly as in $\$ 3.7 .10$, one can use a quasi-homogeneous ansatz to extend the above methods to a microlocal chiral transformation of the form

$$
U(x, y)=\int \frac{d^{4} k}{(2 \pi)^{4}} U\left(k, v_{L / R}\left(\frac{x+y}{2}\right)\right) e^{-i k(x-y)},
$$

and one introduced the auxiliary fermionic projector is defined via the Dirac equation

$$
\left(U^{-1}\right)^{*}(i \not \partial-m Y) U^{-1} \tilde{P}^{\text {aux }}=0 .
$$

This gives the following result.

Proposition 4.4.7. Assume that the parameters $\mu_{1}, \ldots, \mu_{4}$ as defined by 4.4.55) and (4.4.56) satisfy the inequalities 4.4.64). Then for any choice of the chiral potentials $v_{L}$ and $v_{R}$ in (4.4.30), there is a microlocal chiral transformation of the form 4.4.70) such that the transformed fermionic projector $\tilde{P}:=U^{\prime} P^{a u x} \grave{U}^{*}$ is of the form

$$
\begin{aligned}
\tilde{P}(x, y)= & P(x, y)+\left(\chi_{L} \psi_{L}+\chi_{R} \psi_{R}\right) T_{[3, \mathfrak{c}]}^{(1)}\left(1+\mathcal{O}\left(|\vec{\xi}| / \ell_{\text {macro }}\right)\right) \\
& +(\text { vectorial }) \mathbb{1}_{\mathbb{C}^{2}}(\operatorname{deg}<2)+(\text { pseudoscalar or bilinear })(\operatorname{deg}<1) \\
& +(\text { smooth contributions })+(\text { higher orders in } \varepsilon /|\vec{\xi}|) .
\end{aligned}
$$

We conclude this section with two remarks.

REMARK 4.4.8. (Unitarity of $U$ ) We now explain why it would be preferable that the operator $U$ in the microlocal transformation were unitary, and how and to which extent this can be arranged. We begin with the homogeneous setting 4.4.31) and 4.4.33. As pointed out after (4.4.33), the operator $U$ as given by (4.4.33) is in general not unitary. However, the following construction makes it possible to replace $U$ by a unitary operator without effecting out results: We first consider the left-handed matrices $L^{j}(k)$. Note that our analysis only involved the sectorial projection $\dot{L}[k]$ of these matrices contracted with $k$. Moreover, by multiplying the columns by a phase 4.4 .59 we could arrange that all the components in 4.4.50 were real. In this situation, a straightforward analysis shows that there is indeed a Hermitian $6 \times 6$-matrix whose sectorial projection coincides with 4.4.50). By choosing the other components of $L^{j}(k)$ appropriately, one can arrange that the matrices $L^{j}(k)$ are all Hermitian, and 4.4.50 still holds. Similarly, one can also arrange that the matrices $R^{j}(k)$ are Hermitian. Replacing the ansatz 4.4.33 by $U=\exp (i Z / \sqrt{\Omega})$, we get a unitary operator. A straightforward calculation shows that expanding the exponential in a Taylor series, the second and higher orders of this expansion only effect the error terms in Proposition 4.4.6 (for a similar calculation see \$3.7.9).

Having arranged that $U$ is unitary has the advantage that the auxiliary fermionic projector defined via the Dirac equation 4.4 .71 ) is simply given by $\tilde{P}^{\text {aux }}=U P U^{*}$ (whereas if $U$ were not unitary, the auxiliary fermionic projector would involve unknown smooth correction terms; see the similar discussion for local transformations in 33.7.7).

In the microlocal setting (4.4.70), the transformation $U$ will no longer be unitary, even if the used homogeneous transformations $U\left(., v_{L / R}\right)$ are unitary for every $v_{L / R}$. Thus 
it seems unavoidable that the fermionic projector defined via the Dirac equation 4.4.71) will differ from the operator $U P U^{*}$ by smooth contributions on the light cone (see also the discussion after (3.7.82)). But even then it is of advantage to choose the homogeneous transformations $U\left(., v_{L / R}\right)$ to be unitary, because then the correction terms obviously vanish in the limit $\ell_{\text {macro }} \rightarrow \infty$. More precisely, a straightforward analysis shows that these correction terms are of the order $|\vec{\xi}| / \ell_{\text {macro }}$.

REMARK 4.4.9. (Lower bound on the largest neutrino mass) The inequalities (4.4.63) give constraints for the masses of the fermions, as we now explain. Thinking of the interactions of the standard model, we want to be able to treat the case when a left-handed gauge field but no right-handed gauge fields are present. In this case, $\mathfrak{c}_{0}$ is non-zero, but the parameter $z$ vanishes for the right-handed component. In view of our sign conventions (4.4.56), the first inequality in (4.4.63) implies that $\mathfrak{c}_{0}>0$. Then the inequalities (4.4.63) yield the necessary conditions (4.4.64). More precisely, the eigenvalues $\mu_{1}, \ldots, \mu_{4}$ are given in terms of the lepton masses by (see also $(3.7 .72)$

$$
\begin{aligned}
& \mu_{1 / 2}=\frac{1}{3}\left(\tilde{m}_{1}^{2}+\tilde{m}_{2}^{2}+\tilde{m}_{3}^{2} \mp \sqrt{\tilde{m}_{1}^{4}+\tilde{m}_{2}^{4}+\tilde{m}_{3}^{4}-\tilde{m}_{1}^{2} \tilde{m}_{2}^{2}-\tilde{m}_{2}^{2} \tilde{m}_{3}^{2}-\tilde{m}_{1}^{2} \tilde{m}_{3}^{2}}\right) \\
& \mu_{3 / 4}=\frac{1}{3}\left(m_{1}^{2}+m_{2}^{2}+m_{3}^{2} \mp \sqrt{m_{1}^{4}+m_{2}^{4}+m_{3}^{4}-m_{1}^{2} m_{2}^{2}-m_{2}^{2} m_{3}^{2}-m_{1}^{2} m_{3}^{2}}\right) .
\end{aligned}
$$

The first inequality in 4.4.64 is satisfied once the mass $m_{3}$ of the $\tau$-lepton is much larger than the neutrino masses, as is the case for present experimental data. However, the second inequality in (4.4.64) demands that the largest neutrino mass $\tilde{m}_{3}$ must be at least of the same order of magnitude as $m_{2}$. In particular, our model does not allow for a description of the interactions in the standard model if all neutrino masses are too small.

Before comparing this prediction with experiments, one should clearly take into account that we are working here with the naked masses, which differ from the physical masses by the contributions due to the self-interaction (with a natural ultraviolet cutoff given by the regularization length $\varepsilon$ ). Moreover, one should consider the possibility of heavy and yet unobserved so-called sterile neutrinos.

We finally point out that here our method was to compensate all the logarithmic poles by a microlocal chiral transformation. Following the method of treating the algebraic constraints which will be introduced in $\$ 4.7 .1$ below, one can take the alternative point of view that it suffices to compensate the logarithmic poles in the direction of the dynamical gauge potentials. This alternative method is preferable because it gives a bit more freedom in choosing the microlocal chiral transformation. For conceptual clarity, we postpone this improved method to Chapter 5, where a system involving quarks is analyzed (see $\$ 5.4 .2$ ).

4.4.5. The Shear Contributions. We proceed by analyzing the higher orders in an expansion in the chiral gauge potentials. Qualitatively speaking, these higher order contributions describe generalized phase transformations of the fermionic projector. Our task is to analyze how precisely the gauge phases come up and how they enter the EL equations. The most singular contributions to discuss are the error terms

$$
\text { (vectorial) } \mathbb{1}_{\mathbb{C}^{2}}(\operatorname{deg}=1)
$$

in Proposition 4.4.7. If modified by gauge phases, these error terms give rise to the socalled shear contributions by the microlocal chiral transformation. In the setting of one sector, these shear contributions were analyzed in detail in 3.7.11. As the adaptation 
to the present setting of two sectors is not straightforward, we give the construction in detail.

Recall that the gauge phases enter the fermionic projector to degree two according to 4.3.30) and 4.3.31). In order to ensure that the error term 4.4.75) drops out of the EL equations, it must depend on the gauge phases exactly as 4.3.30), i.e. it must be modified by the gauge phases to

$$
\left[\chi_{L}\left(\begin{array}{cc}
U_{L}^{11} & \bar{c} U_{L}^{12} \\
c U_{L}^{21} & U_{L}^{22}
\end{array}\right)+\chi_{R}\left(\begin{array}{cc}
V_{R}^{N} & 0 \\
0 & V_{R}^{C}
\end{array}\right)\right] \times(\text { vectorial }) \mathbb{1}_{\mathbb{C}^{2}}(\operatorname{deg}=1) .
$$

Namely, if (4.4.76) holds, then the corresponding contributions to the closed chain involve the gauge phases exactly as in 4.3.33), and a straightforward calculation using 4.3.38 (as well as (4.3.9) and (4.3.35) ) shows that the eigenvalues of the closed chain all have the same absolute value. If conversely $(4.4 .76)$ is violated, then the eigenvalues of the closed chain are not the same, and the EL equations will be violated (at least without imposing conditions on the regularization functions). We conclude that the transformation law (4.4.76) is necessary and sufficient for the EL equations to be satisfied to degree five on the light cone.

In order to arrange 4.4.76), we follow the procedure in $\$ 3.7 .11$ and write down the Dirac equation for the auxiliary fermionic projector

$$
\mathcal{D}_{\text {flip }} \tilde{P}^{\text {aux }}=0,
$$

where $\mathcal{D}_{\text {flip }}$ is obtained from the Dirac operator with chiral gauge fields by

$$
\mathcal{D}_{\text {flip }}:=\left(U_{\text {flip }}^{-1}\right)^{*}\left(i \not \partial_{x}+\chi_{L} A_{R}+\chi_{R} A_{L}-m Y\right) U_{\text {flip }}^{-1},
$$

where $U_{\text {flip }}$ is obtained from the operator $U$ in (4.4.71) by

$$
U_{\text {flip }}=\mathbb{1}+(\mathrm{U}-\mathbb{1}) V \text {, }
$$

and $V$ is the unitary perturbation flow which changes the gauge potentials from $A_{L / R}$ to $A_{L / R}^{\mathrm{even}}$,

$$
V=U_{\text {flow }}\left[\chi_{L} A_{R}^{\text {even }}+\chi_{R} A_{L}^{\text {even }}\right] U_{\text {flow }}\left[\chi_{L} A_{R}+\chi_{R} A_{L}\right]^{-1}
$$

(see (3.7.88), 3.7.90) and (B.4.1)). Exactly as in the proof of Proposition 3.7.12, one sees that the component $\sim \Omega^{-1}$ of $P$ satisfies the Dirac equation involving the chiral gauge potentials $A_{L / R}^{\text {even }}$. In view of 4.4.44 and 4.4.48, we find that the left-handed contribution of 4.4 .75 is modified by the chiral gauge potentials to

$$
L[k] \operatorname{Pexp}\left(-i \int_{x}^{y}\left(A_{R}^{\mathrm{even}}\right)_{j} \xi^{j}\right) L[k]^{*} .
$$

Thus similar as in (4.3.17), gauge phases appear. The difference is that the chirality is flipped, and moreover here the new potentials $A_{L / R}^{\text {even }}$ enter. A-priori, these potentials can be chosen arbitrarily according to the gauge group 4.3.16).

For the right-handed component of the fermionic projector, we can use that $A_{R}$ is sector-diagonal (see 4.3 .29 ) . Thus we obtain agreement between the phase transformations corresponding to $A_{R}$ and the transformation law 4.4.80) (with $L$ exchanged by $R$ ) simply by choosing

$$
A_{L}^{\text {even }}=\left(\begin{array}{cc}
A_{R}^{N} & 0 \\
0 & A_{R}^{C}
\end{array}\right) .
$$

For the left-handed component of the fermionic projector, the basic difficulty is that the matrix $L[k]$ is non-trivial in the generation index (see 4.4.51)-4.4.53). Moreover, the 
gauge potential $A_{L}$ involves the MNS matrix $U_{\mathrm{MNS}}$ (see (4.3.26)). Therefore, it is not obvious how 4.4.80) can be related to 4.3.26). But the following construction shows that for a specific choice of $A_{R}^{\text {even }}$ the connection can be made: We denote the two column vectors of $L[k]^{*}$ by $\ell_{1}, \ell_{2} \in \mathbb{C}^{6}$. In view of (4.4.48), these vectors are orthogonal. We set $\mathfrak{e}_{1}=\ell_{1} /\left\|\ell_{1}\right\|$ and $\mathfrak{e}_{4}=\ell_{2} /\left\|\ell_{2}\right\|$ and extend these two vectors to an orthonormal basis $\mathfrak{e}_{1}, \ldots, \mathfrak{e}_{6}$ of $\mathbb{C}^{6}$. We choose $A_{R}^{\text {even }}$ such that in this basis it has the form

$$
A_{R}^{\text {even }}(k, x)=\left(\begin{array}{cc}
A_{L}^{11}(x) & A_{L}^{12}(x) V(x)^{*} \\
A_{L}^{21}(x) V(x) & A_{L}^{22}(x)
\end{array}\right),
$$

where we used a block matrix representation in the two $\operatorname{subspaces} \operatorname{span}\left(\mathfrak{e}_{1}, \mathfrak{e}_{2}, \mathfrak{e}_{3}\right)$ and $\operatorname{span}\left(\mathfrak{e}_{4}, \mathfrak{e}_{5}, \mathfrak{e}_{6}\right)$. Here the potentials $A_{L}^{i j}$ are chosen as in 4.3 .29 , and $V(x) \in \mathrm{U}(3)$ is an arbitrary unitary matrix. We point out that the whole construction depends on the momentum $k$ of the homogeneous transformation in 4.4 .80 , as is made clear by the notation $A_{R}^{\text {even }}(k, x)$. Substituting the ansatz (4.4.82) in (4.4.80) and using that the columns of $L[k]^{*}$ are multiples of $\mathfrak{e}_{1}$ and $\mathfrak{e}_{4}$, we obtain

$$
\begin{aligned}
L[k] & \operatorname{Pexp}\left(-i \int_{x}^{y}\left(A_{R}^{\mathrm{even}}\right)_{j} \xi^{j}\right) L[k]^{*} \\
& =\left(\begin{array}{cc}
U_{L}^{11} & \bar{d} U_{L}^{12} \\
d U_{L}^{21} & U_{L}^{22}
\end{array}\right) L[k] L[k]^{*} \stackrel{4.4 .48}{=}\left(\begin{array}{cc}
U_{L}^{11} & \bar{d} U_{L}^{12} \\
d U_{L}^{21} & U_{L}^{22}
\end{array}\right) \mathfrak{c}_{0}(k) \mathbb{1}_{\mathbb{C}^{2}},
\end{aligned}
$$

where $U_{L}^{i j}$ as in 4.3 .30 and $d=V_{1}^{1}$. Choosing $V$ such that $d$ coincides with the parameter $c$ in (4.3.30), we recover the transformation law of the left-handed component in (4.4.76). Repeating the above construction for the right-handed component (by flipping the chirality and replacing $L[k]$ by $R[k]$ ), we obtain precisely the transformation law 4.4.76.

In order to get into the microlocal setting, it is useful to observe that the $k$-dependence of $A_{R}^{\text {even }}$ can be described by a unitary transformation,

$$
A_{R}^{\text {even }}(k, x)=W(k) A_{L}(x) W(k)^{*} \quad \text { with } \quad W(k) \in \mathrm{U}(6) .
$$

Interpreting $W$ as a multiplication operator in momentum space and $A_{L}$ as a multiplication operator in position space, we can introduce $A_{R}^{\text {even }}$ as the operator product

$$
A_{R}^{\text {even }}=W A_{L} W^{*} .
$$

We point out that the so-defined potential $A_{R}^{\text {even }}$ is non-local. As the microlocal chiral transformation is non-local on the Compton scale, one might expect naively that the same should be true for $A_{R}^{\text {even }}$. However, $A_{R}^{\text {even }}$ can be arranged to be localized on the much smaller regularization scale $\varepsilon$, as the following argument shows: The $k$-dependence of $W$ is determined by the matrix entries of $L[k]$. The analysis in 4.4 .4 shows that the matrix entries of $L[k]$ vary in $k$ on the scale of the energy $\varepsilon^{-1}$ (in contrast to the matrix $Z$, which in view of the factor $1 / \sqrt{\Omega}$ in $(4.4 .33$ ) varies on the scale $m$ ). Taking the Fourier transform, the operator $W$ decays in position space on the regularization scale.

This improved scaling has the positive effect that the error term caused by the quasilocal ansatz 4.4.85) is of the order $\varepsilon / \ell_{\text {macro }}$. Hence the gauge phases enter the left-handed component of the error term 4.4.75 as

$$
\chi_{L}\left(\begin{array}{cc}
U_{L}^{11} & \bar{c} U_{L}^{12} \\
c U_{L}^{21} & U_{L}^{22}
\end{array}\right)\left(1+\mathcal{O}\left(\varepsilon / \ell_{\text {macro }}\right)\right)(\text { vectorial }) \mathbb{1}_{\mathbb{C}^{2}}(\operatorname{deg}=1) .
$$


Carrying out a similar construction for the right-handed component, we obtain the following result.

Proposition 4.4.10. Introducing the potentials $A_{L / R}^{\text {even }}$ in 4.4.79 according to 4.4.81 and 4.4.85, the error term 4.4.75) in Proposition 4.4.7 transforms to

$$
\left[\chi_{L}\left(\begin{array}{cc}
U_{L}^{11} & \bar{c} U_{L}^{12} \\
c U_{L}^{21} & U_{L}^{22}
\end{array}\right)+\chi_{R}\left(\begin{array}{cc}
V_{R}^{N} & 0 \\
0 & V_{R}^{C}
\end{array}\right)\right]\left(1+\mathcal{O}\left(\varepsilon / \ell_{\text {macro }}\right)\right)(\text { vectorial }) \mathbb{1}_{\mathbb{C}^{2}}(\operatorname{deg}=1) .
$$

In this way, we have arranged that the EL equations are satisfied to degree five on the light cone. Note that the above construction involves the freedom in choosing the basis vectors $\mathfrak{e}_{2}, \mathfrak{e}_{3}, \mathfrak{e}_{5}, \mathfrak{e}_{6}$ as well as the unitary matrix $V$ in 4.4 .82 . This will be analyzed in more detail in 4.6 .1 .

We finally point out that in general, the Dirac operator 4.4 .78 violates the causality compatibility condition 4.2.48). This implies that the light-cone expansion of the auxiliary fermionic projector may involve unbounded line integrals. However, this causes no problems because these unbounded line integrals drop out when taking the sectorial projection. Thus the fermionic projector is again causal in the sense that its light-cone expansion only involves bounded line integrals.

\subsection{The Energy-Momentum Tensor and the Curvature Terms}

In this section we compute other relevant contributions to the EL equations to degree four: the contributions by the energy-momentum tensor and the curvature of space-time.

4.5.1. The Energy-Momentum Tensor of the Dirac Field. Considering the contribution of the particle and anti-particle wave functions in (4.2.51) at the origin $x=y$ gives rise to the Dirac current terms as considered in $\$ 4.4 .3$ (for details see also 33.7 .2 ). We now go one order higher in an expansion around the origin $\xi=0$. Setting $z=(x+y) / 2$ and expanding in powers of $\xi$ according to

$$
\begin{aligned}
\psi(x) & =\psi(z-\xi / 2)=\psi(z)-\frac{1}{2} \xi^{j} \partial_{j} \psi(z)+o(|\vec{\xi}|) \\
\psi(y) & =\psi(z+\xi / 2)=\psi(z)+\frac{1}{2} \xi^{j} \partial_{j} \psi(z)+o(|\vec{\xi}|) \\
\psi(x) \overline{\psi(y)} & =\psi(z) \overline{\psi(z)}-\frac{1}{2} \xi^{j}\left(\left(\partial_{j} \psi(z)\right) \overline{\psi(z)}-\psi(z)\left(\partial_{j} \bar{\psi}(z)\right)\right)+o(|\vec{\xi}|),
\end{aligned}
$$

we can write the contribution by the particles and anti-particles as

$$
P(x, y) \asymp-\frac{1}{8 \pi} \sum_{c=L / R} \chi_{c} \gamma_{k}\left(\hat{J}_{c}^{k}-i \xi_{l} \hat{T}_{c}^{k l}\right)+o(|\vec{\xi}|)+(\text { even contributions }),
$$

where

$$
\left(T_{L / R}^{k l}\right)_{(j, \beta)}^{(i, \alpha)}=-\operatorname{Im} \sum_{a=1}^{n_{\mathrm{p}}} \overline{\psi_{a}^{(j, \beta)}} \chi_{R / L} \gamma^{k} \partial^{l} \psi_{a}^{(i, \alpha)}+\operatorname{Im} \sum_{b=1}^{n_{\mathrm{a}}} \overline{\phi_{b}^{(j, \beta)}} \chi_{R / L} \gamma^{k} \partial^{l} \phi_{b}^{(i, \alpha)},
$$

and similar to 4.2.53), the hat denotes the sectorial projection. We denote the vectorial component by

$$
T^{k l}:=T_{L}^{k l}+T_{R}^{k l}
$$

Taking the trace over the generation and isospin indices, we obtain the energy-momentum tensor of the particles and anti-particles. We now compute the resulting contribution to 
the matrices $\mathcal{K}_{L / R}$ as introduced in Lemma 4.4.1. The proof of this lemma is obtained similar to that of Lemma 4.4.4 by a straightforward computation.

LEMMA 4.5.1. The tensors $T_{L / R}^{k l}$, 4.5.1, give the following contribution to the matrices $\mathcal{K}_{L / R}$ in 4.4.13 and 4.4.14,

$$
\mathcal{K}_{L / R} \asymp \hat{T}_{R / L}^{k l} \xi_{k} \xi_{l} K_{8}+(\operatorname{deg}<4)+o\left(|\vec{\xi}|^{-2}\right),
$$

where $K_{8}$ is the simple fraction

$$
K_{8}=\frac{3}{16 \pi} \frac{1}{\overline{T_{[0]}^{(0)}}}\left[T_{[0]}^{(-1)} T_{[0]}^{(0)} \overline{T_{[0]}^{(-1)}}+c . c .\right]
$$

(note that $K_{8}$ differs from $K_{1}$ on page 298 in that the term -c.c. has been replaced by + c.c.).

The energy-momentum tensor of the gauge field will be computed in 4.5 .3 below.

4.5.2. The Curvature Terms. The obvious idea for compensating the above contributions to the EL equations is to modify the Lorentzian metric. At first sight, one might want to introduce a metric which depends on the isospin index. However, such a dependence cannot occur, as the following argument shows: The singular set of the fermionic projector $P(x, y)$ is given by the pair of points $(x, y)$ with light-like separation. If the metric depended on the isospin components, the singular set would be different in different isospin components. Thus the light cone would "split up" into two separate light cones. As a consequence, the leading singularities of the closed chain could no longer compensate each other in the EL equations, so that the EL equations would be violated to degree five on the light cone.

Strictly speaking, this argument leaves the possibility to introduce a conformal factor which depends on the isospin (because a conformal transformation does not affect the causal structure). However, as the conformal weight enters the closed chain to degree five on the light cone, the EL equations will be satisfied only if the conformal factor is independent of isospin.

The above arguments readily extend to a chiral dependence of the metric: If the leftand right-handed component of the fermionic projector would feel a different metric, then the singular sets of the left- and right-handed components of the closed chain would again be different, thereby violating the EL equations to degree five (for a similar argument for an axial gravitational field see the discussion in \$3.9.3).

Following these considerations, we are led to introducing a Lorentzian metric $g_{i j}$. Linear perturbations of the metric were studied in [F5, Appendix B] (see also Section 2.3). The contributions to the fermionic projector involving the curvature tensor were computed by

$$
\begin{aligned}
P(x, y) \asymp & \frac{i}{48} R_{j k} \xi^{j} \xi^{k} \sharp T^{(-1)} \\
& +\frac{i}{24} R_{j k} \xi^{j} \gamma^{k} T^{(0)}+\Varangle(\operatorname{deg} \leq 1)+(\operatorname{deg}<1),
\end{aligned}
$$

where $R_{j k}$ denotes the Ricci tensor (we only consider the leading contribution in an expansion in powers of $\left.|\vec{\xi}| / \ell_{\text {macro }}\right)$. We refer to 4.5.3) and (4.5.4) as the curvature terms. More generally, in [FG2, Appendix A] the singularity structure of the fermionic projector was analyzed on a globally hyperbolic Lorentzian manifold (for details see also [G]). Transforming the formulas in $[\mathbf{F G 2}, \mathbf{G}]$ to the the coordinate system and gauge used 
in [F5], one sees that (4.5.3) and (4.5.4) also hold non-perturbatively. In particular, the results in FG2 show that, to the considered degree on the light cone, no quadratic or even higher order curvature expressions occur. In what follows, we consider 4.5.3) and (4.5.4) as a perturbation of the fermionic projector in Minkowski space. This is necessary because at present, the formalism of the continuum limit has only been worked out in Minkowski space. Therefore, strictly speaking, the following results are perturbative. But after extending the formalism of the continuum limit to curved space-time (which seems quite straightforward because the framework of the fermionic projector approach is diffeomorphism invariant), our results would immediately carry over to a globally hyperbolic Lorentzian manifold.

Let us analyze how the curvature terms enter the eigenvalues of the closed chain. We first consider the case when we strengthen (4.4.19) by assuming that

$$
\varepsilon \ll \delta \ll \frac{1}{m}(m \varepsilon)^{\frac{p_{\text {reg }}}{2}}
$$

(the case $\delta \simeq m(m \varepsilon)^{\frac{p_{\text {reg }}}{2}}$ will be discussed in Section 4.9). The assumption 4.5.5) makes it possible to omit the terms $\sim m^{2} R_{i j}$.

Lemma 4.5.2. The curvature of the Lorentzian metric gives the following contribution to the matrices $\mathcal{K}_{L / R}$ in 4.4.8,

$$
\begin{aligned}
\mathcal{K}_{c} \asymp & \frac{5}{24} \frac{1}{48} R_{k l} \xi^{k} \xi^{l} A_{x y}^{(0)} P^{(0)}(x, y) \\
& +\delta_{c L} \frac{\tau_{\text {reg }}}{\delta^{2}} R_{k l} \xi^{k} \xi^{l}\left(\begin{array}{ll}
1 & 0 \\
0 & 0
\end{array}\right) K_{16} \\
& +m^{2} R_{k l} \xi^{k} \xi^{l}(\operatorname{deg}=4)+(\operatorname{deg}<4)+o\left(|\vec{\xi}|^{-2}\right),
\end{aligned}
$$

where $K_{16}$ is the following simple fraction of degree four,

$$
K_{16}=\frac{27}{32}\left|T_{[0]}^{(-1)}\right|^{2}{\overline{T_{[0]}^{(0)}}}^{-1}\left(T_{[R, 2]}^{(1)} \overline{L_{[0]}^{(0)}}+L_{[0]}^{(0)} \overline{T_{[R, 2]}^{(1)}}\right)
$$

(and $P^{(0)}(x, y)$ and $A_{x y}^{(0)}$ denote the vacuum fermionic projector and the closed chain of the vacuum, respectively).

PROOF. The contribution 4.5.3 multiplies the fermionic projector of the vacuum by a scalar factor. Thus it can be combined with the vacuum fermionic projector $P^{(0)}$ to the expression

$$
c_{x y} P^{(0)}(x, y) \quad \text { with } \quad c_{x y}:=1+\frac{1}{24} R_{j k} \xi^{j} \xi^{k} .
$$

Hence the closed chain and the eigenvalues are simply multiplied by a common prefactor,

$$
A_{x y}=c_{x y}^{2} A_{x y}^{(0)}, \quad \lambda_{n c s}=c_{x y}^{2} \lambda_{n c s}^{(0)} .
$$

As a consequence, the contribution 4.5.3 can be written in the form 4.5.6).

The summand (4.5.4) is a bit more involved, and we treat it in the $\iota$-formalism. The closed chain is computed by

$$
\begin{aligned}
A_{x y} \asymp & \frac{3}{16} R_{j k} \check{\xi}^{j} \gamma^{k} \check{\dot{\phi}} T_{[0]}^{(0)} \overline{T_{[0]}^{(-1)}} \\
& +\frac{3}{16} R_{j k} \check{\not}^{\check{\xi}} \check{\xi}^{j} \gamma^{k} T_{[0]}^{(-1)} \overline{T_{[0]}^{(0)}} .
\end{aligned}
$$


Similar as explained for the chiral contribution after 4.2 .69 , the eigenvalues $\lambda_{n c+}$ are only perturbed by 4.5.10. More precisely,

$$
\lambda_{n c+} \asymp \frac{3}{16} R_{j k} \xi^{j} \xi^{k} T_{[0]}^{(0)} \overline{T_{[0]}^{(-1)}},
$$

and the other eigenvalues are obtained by complex conjugation (4.4.3). In particular, one sees that the eigenvalues are perturbed only by a common prefactor. Combining the perturbation with the eigenvalues of the vacuum, we obtain

$$
\lambda_{n c+}=\left(1+\frac{1}{48} R_{j k} \xi^{j} \xi^{k}\right) \lambda_{n c+}^{(0)} .
$$

In view of (4.4.3), this relation also holds for the eigenvalues $\lambda_{n c-}$. We conclude that (4.5.4) can again be absorbed into 4.5.6. A short calculation using 4.4.6) shows that the contributions so far combine precisely to 4.5.6.

It remains to consider the effects of shear and of the general surface terms. The shear contribution is described by a homogeneous transformation of the spinors which is localized on the scale $\varepsilon$ (for details see Appendix F). Since this transformation does not effect the macroscopic prefactor $c_{x y}$ in 4.5 .9 , the eigenvalues are again changed only by a common prefactor. Hence (4.5.3) drops out of the EL equations for the shear states. The contributions (4.5.10) and (4.5.11), on the other hand, do not involve $\iota$, and are thus absent for the shear states. We conclude that also 4.5.4) drops out of the EL equations for the shear states.

We finally consider the general surface states. As 4.5 .10 is a smooth factor times the vacuum fermionic projector, the Ricci tensor again drops out of the EL equations. For the remaining term (4.5.11), the replacement rule 4.2.50) yields the contribution of the general mass expansion

$$
\chi_{R} P^{\varepsilon}(x, y) \asymp \frac{i}{24} R_{j k} \xi^{j} \gamma^{k} \frac{\tau_{\mathrm{reg}}}{\delta^{2}}\left(\begin{array}{cc}
T_{[R, 2]}^{(1)} & 0 \\
0 & 0
\end{array}\right) .
$$

As a consequence,

$$
\begin{aligned}
\chi_{R} A_{x y} & \asymp \frac{3}{48} R_{j k} \xi^{j} \gamma^{k} \bar{\xi} \frac{\tau_{\text {reg }}}{\delta^{2}}\left(\begin{array}{cc}
T_{[R, 2]}^{(1)} \overline{T_{[0]}^{(-1)}} & 0 \\
0 & 0
\end{array}\right) \\
\lambda_{n R+} & \asymp \frac{3}{48} R_{j k} \xi^{j} \xi^{k} \frac{\tau_{\text {reg }}}{\delta^{2}} T_{[R, 2]}^{(1)} \overline{T_{[0]}^{(-1)}} \operatorname{Tr}_{\mathbb{C}^{2}}\left(I_{n}\left(\begin{array}{ll}
1 & 0 \\
0 & 0
\end{array}\right)\right) \\
\mathcal{K}_{L} & \asymp \frac{27}{32} R_{j k} \xi^{j} \xi^{k} \frac{\tau_{\text {reg }}}{\delta^{2}}\left(T_{[R, 2]}^{(1)} \overline{L_{[0]}^{(0)}}+L_{[0]}^{(0)} \overline{T_{[R, 2]}^{(1)}}\right) \frac{1}{\overline{T_{[0]}^{(0)}}}\left|T_{[0]}^{(-1)}\right|^{2}\left(\begin{array}{ll}
1 & 0 \\
0 & 0
\end{array}\right) .
\end{aligned}
$$

Similarly,

$$
\chi_{L} A_{x y} \asymp \frac{3}{48} \sharp R_{j k} \xi^{j} \gamma^{k} \frac{\tau_{\mathrm{reg}}}{\delta^{2}}\left(\begin{array}{cc}
T_{[0]}^{(-1)} \overline{T_{[R, 2]}^{(1)}} & 0 \\
0 & 0
\end{array}\right),
$$

and a straightforward computation yields $\lambda_{n L+} \asymp 0$ and $\mathcal{K}_{R} \asymp 0$. This concludes the proof. 
4.5.3. The Energy-Momentum Tensor of the Gauge Field. We now compute the effect of the energy-momentum tensor of the chiral gauge field. We denote the chiral field tensor by $F_{c}^{j k}=\partial^{j} A_{c}^{k}-\partial^{k} A_{c}^{j}$.

Lemma 4.5.3. The field tensor of the gauge fields gives the following contribution to the matrix $\mathcal{K}_{L}$ in (4.4.13) and 4.4.14),

$$
\begin{aligned}
& \mathcal{K}_{L} \asymp-\frac{g^{2}}{3} \dot{F}_{k i}^{L} \grave{F}_{L}^{k j} \xi^{i} \xi_{j} \frac{\left|T_{[0]}^{(-1)}\right|^{2}}{\overline{T_{[0]}^{(0)}}}\left(T_{[0]}^{(1)} \overline{T_{[0]}^{(0)}}+T_{[0]}^{(0)} \overline{T_{[0]}^{(1)}}\right) \\
& -\frac{g^{2}}{24} \dot{F}_{k i}^{R} \grave{F}_{R}^{k j} \xi^{i} \xi_{j} T_{[0]}^{(0)}\left(T_{[0]}^{(0)} \overline{T_{[0]}^{(-1)}}+T_{[0]}^{(-1)} \overline{T_{[0]}^{(0)}}\right) \\
& +\frac{g}{8} \hat{F}_{k i}^{L} \hat{F}_{R}^{k j} \xi^{i} \xi_{j} T_{[0]}^{(0)} \frac{\left(T_{[0]}^{(0)} T_{[0]}^{(0)} \overline{T_{[0]}^{(-1)} T_{[0]}^{(-1)}}-c . c .\right)}{T_{[0]}^{(0)} \overline{T_{[0]}^{(-1)}}-T_{[0]}^{(-1)} \overline{T_{[0]}^{(0)}}} \\
& -\frac{g}{8} \epsilon_{i j k l} \xi^{i} \xi_{a} \hat{F}_{R}^{a j} \hat{F}_{L}^{k l} \frac{T_{[0]}^{(0)}\left|T_{[0]}^{(-1)} T_{[0]}^{(0)}\right|^{2}}{T_{[0]}^{(0)} \overline{T_{[0]}^{(-1)}}-T_{[0]}^{(-1)} \overline{T_{[0]}^{(0)}}} \\
& -\frac{g}{8} \epsilon_{i j k l} \xi^{i} \xi_{a} \hat{F}_{L}^{a j} \hat{F}_{R}^{k l} \frac{T_{[0]}^{(-1)} T_{[0]}^{(-1)} T_{[0]}^{(0)} \overline{T_{[0]}^{(0)} T_{[0]}^{(0)}}}{T_{[0]}^{(0)} \overline{T_{[0]}^{(-1)}}-T_{[0]}^{(-1)} \overline{T_{[0]}^{(0)}}} \\
& -\frac{g}{8} \epsilon_{i j k l} \hat{F}_{R}^{i j} \xi^{k} \xi_{a} \hat{F}_{L}^{a l} \frac{T_{[0]}^{(0)} T_{[0]}^{(0)} T_{[0]}^{(0)} \overline{T_{[0]}^{(-1)} T_{[0]}^{(-1)}}}{T_{[0]}^{(0)} \overline{T_{[0]}^{(-1)}}-T_{[0]}^{(-1)} \overline{T_{[0]}^{(0)}}} \\
& -\frac{g}{8} \epsilon_{i j k l} \hat{F}_{L}^{i j} \xi^{k} \xi_{a} \hat{F}_{R}^{a l} \frac{T_{[0]}^{(-1)} T_{[0]}^{(0)} T_{[0]}^{(0)} \overline{T_{[0]}^{(-1)} T_{[0]}^{(0)}}}{T_{[0]}^{(0)} \overline{T_{[0]}^{(-1)}}-T_{[0]}^{(-1)} \overline{T_{[0]}^{(0)}}} \\
& +(\operatorname{deg}<4)+o\left(|\vec{\xi}|^{-2}\right) .
\end{aligned}
$$

The contribution to $\mathcal{K}_{R}$ is obtained by the replacements $L \leftrightarrow R$.

The proof of this lemma is given in Appendix B.5. The main reason why the formulas are rather complicated is that the eigenvalues of the closed chain must be computed to second order in perturbation theory.

The last lemma reveals the general problem that 4.5.12 involves logarithmic poles on the light cone. This comes about because $P(x, y)$ involves a term quadratic in the field tensor with a logarithmic pole on the light cone, which when contracted with a factor $\$$ has the form

$$
\frac{1}{4} \operatorname{Tr}\left(\sharp \chi_{L / R} P(x, y)\right) \asymp-\frac{i}{3} F_{k i}^{L / R} F_{L / R}^{k j} \xi^{i} \xi_{j} T_{[0]}^{(1)}+o\left(|\vec{\xi}|^{2}\right)
$$

(for the detailed computations see again Appendix B.5). Similar as explained in $\$ 3.7 .2$ for the logarithmic poles of the current terms, the logarithmic pole must again be compensated by a suitable microlocal transformation. This can indeed be accomplished, as we now explain. For clarity, we outline and discuss the method before entering the details. First, it suffices to consider the homogeneous situation in the high-frequency limit, because the macroscopic space-time dependence of the energy-momentum tensor can be 
taken into account just as in $\$ 3.7 .10$ by a corresponding quasi-homogeneous ansatz of the form (3.7.78). Generally speaking, our method is to generate a contribution of the form as in Proposition 4.4.6, but with the logarithmic contributions in 4.4.65) and 4.4.67) of the more specific form that they are vectorial and act trivially on the sector index, i.e.

$$
\begin{aligned}
\tilde{P}(k)= & P(k)+(\text { vectorial }) \mathbb{1}_{\mathbb{C}^{2}} T_{[3, \mathrm{c}]}^{(1)}\left(1+\mathcal{O}\left(\Omega^{-\frac{1}{2}}\right)\right) \\
& +(\text { vectorial }) \mathbb{1}_{\mathbb{C}^{2}} \delta\left(k^{2}\right)\left(1+\mathcal{O}\left(\Omega^{-1}\right)\right) \\
& \left.+(\text { pseudoscalar or bilinear }) \sqrt{\Omega} \delta^{\prime}\left(k^{2}\right)\left(1+\mathcal{O}\left(\Omega^{-1}\right)\right)+\text { (higher orders in } \varepsilon /|\vec{\xi}|\right) .
\end{aligned}
$$

These contributions to the fermionic projector change the matrices $\mathcal{K}_{L}$ and $\mathcal{K}_{R}$ in 4.4.14 only by a multiple of the identity matrix and thus drop out of the EL equations (4.4.4). However, the above contributions 4.5.20 and (4.5.21) are modified by gauge phases, and our strategy is to arrange these gauge phases in such a way that the modification linear in the gauge potential will give the desired bilinear logarithmic contribution. In order to understand the scaling in $\xi$, one should keep in mind that the term 4.5.20 gives a contribution to $\mathcal{K}_{L / R}$ of the form $v_{j} \xi^{j}\left(K_{2}+K_{3}\right)$ (cf. 4.4.22)). Therefore, the first order modification by a gauge potential $A$ will be of the form

$$
\sim v_{j} \xi^{j} A_{k} \xi^{k}(\operatorname{deg}=4)
$$

involving as desired two factors of $\xi$. Next, we need to explain how the potential $A$ in 4.5.22 is to be chosen. It is essential for our construction that the gauge phases of the contributions by the microlocal chiral transformation are determined by the potentials $A_{L / R}^{\text {even }}$ in 4.4.79 (see 4.4.80). It is very helpful that the potentials $A_{L / R}^{\text {even }}$ can be chosen independent of the chiral potentials $A_{L / R}$ in 4.3.29. This makes it possible to arrange that the contribution (4.5.22) involves the desired bilinear logarithmic term needed to compensate the contribution 4.5.19), without affecting the gauge phases as analyzed in $\$ 4.3 .2$.

The detailed construction is carried out in the following lemma.

Lemma 4.5.4. Assume that the chiral potentials are of the form 4.3 .29 with a lefthanded $\mathrm{SU}(2)$-potential (i.e. $A_{R}^{N}=A_{R}^{C}=0$ and $A_{L}^{11}=A_{L}^{22}$ ). Then the logarithmic pole of the contribution to the fermionic projector 4.5.19 can be compensated by the shear contributions corresponding to a microlocal chiral transformation for a suitable choice of the potentials $A_{L / R}^{\text {even }}$ in 4.4.79.

Proof. The first step is to arrange the contributions 4.5.20 and 4.5.21) by specializing the transformation used in 4.4.4. Thus we again choose the ansatz (4.4.33), but now with a pure vector component, i.e.

$$
U(k)=\mathbb{1}+\frac{i}{\sqrt{\Omega}} L^{j} \gamma_{j}
$$

with $6 \times 6$-matrices $L^{j}$. Next, we choose the matrices $L^{j}$ as diagonal matrices involving one vector field $v$,

$$
L^{j} \gamma_{j}=\psi \operatorname{diag}\left(\lambda_{1}, \ldots, \lambda_{6}\right) .
$$

This ansatz has the advantage that the conditions 4.4.41) -4.4.43 give rise to independent conditions in the two sectors. In the charged sector, we satisfy these conditions by 
arranging that

$$
\begin{gathered}
\left\langle\left(\begin{array}{l}
\lambda_{4} \\
\lambda_{5} \\
\lambda_{6}
\end{array}\right),\left(\begin{array}{l}
1 \\
1 \\
1
\end{array}\right)\right\rangle_{\mathbb{C}^{3}}=0 \\
\operatorname{Im}\left\langle\left(\begin{array}{l}
\lambda_{4} \\
\lambda_{5} \\
\lambda_{6}
\end{array}\right),\left(\begin{array}{l}
m_{1} \\
m_{2} \\
m_{3}
\end{array}\right)\right\rangle_{\mathbb{C}^{3}}=0=\operatorname{Im}\left\langle\left(\begin{array}{l}
\lambda_{4} \\
\lambda_{5} \\
\lambda_{6}
\end{array}\right),\left(\begin{array}{l}
m_{1}^{3} \\
m_{2}^{3} \\
m_{3}^{3}
\end{array}\right)\right\rangle_{\mathbb{C}^{3}} .
\end{gathered}
$$

In the neutrino sector, we obtain impose similar relations for the vector $\left(\lambda_{1}, \lambda_{2}, \lambda_{3}\right)$. Moreover, the formulas 4.4.48) and 4.4.49) give rise to the conditions

$$
\begin{gathered}
\left|\lambda_{1}\right|^{2}+\left|\lambda_{2}\right|^{2}+\left|\lambda_{3}\right|^{2}=\left|\lambda_{4}\right|^{2}+\left|\lambda_{5}\right|^{2}+\left|\lambda_{6}\right|^{2} \\
\tilde{m}_{1}^{2}\left|\lambda_{1}\right|^{2}+\tilde{m}_{2}^{2}\left|\lambda_{2}\right|^{2}+\tilde{m}_{3}^{2}\left|\lambda_{3}\right|^{2}=m_{1}^{2}\left|\lambda_{4}\right|^{2}+m_{2}^{2}\left|\lambda_{5}\right|^{2}+m_{3}^{2}\left|\lambda_{6}\right|^{2} .
\end{gathered}
$$

By choosing the parameters $\lambda_{1}, \ldots \lambda_{6}$ according to the above conditions, we can arrange a contribution to the fermionic projector of the form 4.5.20 and 4.5.21).

Next, we need to specify the gauge potentials $A_{L / R}^{\text {even }}$. Since the bilinear logarithmic contribution to be compensated 4.5 .19 is sector diagonal, we also choose the potentials sector diagonal, i.e.

$$
A_{L / R}^{\text {even }}=\left(\begin{array}{cc}
A_{L / R}^{N} & 0 \\
0 & A_{L / R}^{C}
\end{array}\right),
$$

where we used the same block matrix notation as in (4.3.29). This makes it possible to again analyze the two sectors separately. We only consider the charged sector, because the neutrino sector can be treated in exactly the same way. It is convenient to choose the vectors in $\mathbb{C}^{3}$

$$
f_{1}=\left(\begin{array}{l}
1 \\
1 \\
1
\end{array}\right), \quad f_{2}=\left(\begin{array}{c}
\lambda_{4} \\
\lambda_{5} \\
\lambda_{6}
\end{array}\right), \quad f_{3}=\left(\begin{array}{c}
m_{1} \\
m_{2} \\
m_{3}
\end{array}\right)
$$

(which to avoid trivialities we may assume to be all non-zero). We need to ensure that the gauge phases do not give rise to contributions which are more singular than the logarithmic poles. This is a rather subtle point, which we explain in detail. First, the construction of 4.4 .5 yields that the gauge phases of the gauge potentials $A_{L / R}^{\text {even }}$ enter exactly as those of the chiral potentials $A_{L / R}$, except that the chirality is flipped (see 4.4.80). Next, we must keep in mind that the chirality of the gauge phase flips at every factor of the mass matrix $Y$ (see the relations $(\mathrm{B} .2 .8)$ and $(\overline{B .2 .9})$ ). We need to make sure that the gauge potentials drop out of the expressions in (4.4.41) and (4.4.42). More specifically, it is no problem if a vectorial gauge phase comes up, because the corresponding phase factor is the same for the left- and right-handed components. Arranging that it is also the same in both sectors, such a phase factor drops out of the EL equations. Therefore, our task is to ensure that the expressions in 4.4.41) and (4.4.42) only involve a vectorial phase factor. This leads to the conditions

$$
\begin{gathered}
A_{L}^{C} f_{1}=0=A_{R}^{C} f_{1} \\
\left\langle f_{2},\left(A_{L}^{C}\right)^{p} f_{3}\right\rangle_{\mathbb{C}^{3}}=\left\langle f_{3},\left(A_{R}^{C}\right)^{p} f_{2}\right\rangle_{\mathbb{C}^{3}} \quad \text { for all } p \in \mathbb{N} .
\end{gathered}
$$

Next, we need to make sure that also the contribution (4.5.21) involves only a vectorial phase factor. Here it suffices to consider the linear contribution in the potential, because 
higher orders in the potential may be disregarded in the EL equations. Thus we need to arrange that

$$
\left\langle f_{2}, A_{L}^{C} f_{2}\right\rangle_{\mathbb{C}^{3}}=\left\langle f_{2}, A_{R}^{C} f_{2}\right\rangle_{\mathbb{C}^{3}}
$$

Finally, we need to arrange that the axial potential does modify the contribution 4.5.20, because this will give rise to the desired term used to compensate the bilinear logarithmic term 4.5.19. By scaling we may impose that

$$
\left\langle f_{2},\left\{\left(A_{L}^{C}-A_{R}^{C}\right), Y^{2}\right\} f_{2}\right\rangle_{\mathbb{C}^{3}}=1,
$$

were $Y^{2}$ is the mass matrix in the charged sector, i.e. $m Y=\operatorname{diag}\left(m_{1}, m_{2}, m_{3}\right)$ (here the anti-commutator comes about in view of (B.2.14) and (B.2.15)).

In order to construct potentials satisfying the conditions (4.5.27)-(4.5.30), we make the ansatz

$$
A_{L / R}^{C}=\left|\psi_{L / R}\right\rangle\left\langle\psi_{L / R}\right| \quad \text { with } \psi_{L / R} \in \mathbb{C}^{3} .
$$

Then the conditions 4.5.27) and 4.5.29 become

$$
\begin{gathered}
\left\langle\psi_{L}, f_{1}\right\rangle_{\mathbb{C}^{3}}=0=\left\langle\psi_{R}, f_{1}\right\rangle_{\mathbb{C}^{3}} \\
\left|\left\langle\psi_{L}, f_{2}\right\rangle_{\mathbb{C}^{3}}\right|^{2}=\left|\left\langle\psi_{R}, f_{2}\right\rangle_{\mathbb{C}^{3}}\right|^{2} .
\end{gathered}
$$

Next, the condition 4.5.28 for $p=1$ yields

$$
\left\langle f_{2}, \psi_{L}\right\rangle_{\mathbb{C}^{3}}\left\langle\psi_{L}, f_{3}\right\rangle_{\mathbb{C}^{3}}=\left\langle f_{3}, \psi_{R}\right\rangle_{\mathbb{C}^{3}}\left\langle\psi_{R}, f_{2}\right\rangle_{\mathbb{C}^{3}}
$$

Since the matrices $A_{L / R}^{C}$ have rank one, their powers in 4.5.28 are scalar multiples times the matrices themselves. Therefore, the conditions 4.5.28) for general $p$ are satisfied if and only if the vectors $\psi_{L}$ and $\psi_{R}$ have the same norm,

$$
\left\|\psi_{L}\right\|_{\mathbb{C}^{3}}=\left\|\psi_{R}\right\|_{\mathbb{C}^{3}}
$$

In order to verify whether the above system of equations admits non-trivial solutions, we first count the number of degrees of freedom. Since the phases of $\psi_{L}$ and $\psi_{R}$ do not influence the corresponding potentials (4.5.31), we begin with ten real degrees of freedom (five for $\psi_{L}$ and five for $\psi_{R}$ ). The equations 4.5.32 4.5.35 give $4+1+2+1=8$ conditions, leaving us with two free parameters. This suggest that there should indeed be non-trivial solutions. In order to construct these solutions, it is useful to choose an orthonormal basis $\left(e_{1}, e_{2}\right)$ of the orthogonal complement of the vector $f_{1} \in \mathbb{C}^{3}$. Since $f_{2}$ is orthogonal to $f_{1}$ (see 4.5.24) ), we can choose the first basis vector $e_{1}$ in the direction of $f_{2}$, so that $f_{2}=\left\|f_{2}\right\| e_{1}$. The conditions 4.5 .32 mean that the vectors $\psi_{L}$ and $\psi_{R}$ are in the span of $e_{1}$ and $e_{2}$. Combining the conditions (4.5.33) and 4.5.35) with the freedom in changing the phase of both $\psi_{L}$ and $\psi_{R}$, we can arrange that in the basis $\left(e_{1}, e_{2}\right)$, the vectors $\psi_{L}$ and $\psi_{R}$ have the form

$$
\psi_{L}=\left(\begin{array}{c}
a \\
e^{i \varphi_{L} b}
\end{array}\right) \quad \text { and } \quad \psi_{R}=\left(\begin{array}{c}
a \\
e^{i \varphi_{R} b}
\end{array}\right)
$$

with $a, b \geq 0$ and suitable phases $\varphi_{L / R}$. Then $\left\langle f_{2}, \psi_{L}\right\rangle_{\mathbb{C}^{3}}=\left\langle f_{2}, \psi_{R}\right\rangle_{\mathbb{C}^{3}}=a\left\|f_{2}\right\|$, so that the condition (4.5.34) simplifies to

$$
\left\langle\psi_{L}, f_{3}\right\rangle_{\mathbb{C}^{3}}=\overline{\left\langle\psi_{R}, f_{3}\right\rangle_{\mathbb{C}^{3}}} .
$$

The relation 4.5.30 becomes

$$
\operatorname{Re}\left(\left\langle f_{2}, \psi_{L}\right\rangle_{\mathbb{C}^{3}}\left\langle\psi_{L}, Y^{2} f_{2}\right\rangle_{\mathbb{C}^{3}}-\left\langle f_{2}, \psi_{R}\right\rangle_{\mathbb{C}^{3}}\left\langle\psi_{R}, Y^{2} f_{2}\right\rangle_{\mathbb{C}^{3}}\right)=1,
$$


which in our basis representation simplifies to

$$
a\left\|f_{2}\right\| \operatorname{Re}\left\langle\psi_{L}-\psi_{R}, Y^{2} f_{2}\right\rangle_{\mathbb{C}^{3}}=1 .
$$

The previous construction make it possible to arrange a contribution to $\mathcal{K}_{L / R}$ of the form 4.5.22 for an arbitrary choice of the vector fields $v$ and $A$. This is not quite sufficient for compensating a contribution by a symmetric tensor field of the form $T_{j k} \xi^{j} \xi^{k}$ because the tensor field does not need to be decomposable into a product of two vector fields. The tensor field can only be written as a linear combination of such products, i.e.

$$
T_{j k}=\sum_{p=1}^{p_{\max }}\left(v_{j}^{(p)} A_{k}^{(p)}+v_{k}^{(p)} A_{j}^{(p)}\right)
$$

for a suitable parameter $p_{\max } \in \mathbb{N}$ and vector fields $v^{(p)}$ and $A^{(p)}$. This leads us to generalize the ansätze 4.5.23 and 4.5.26) to

$$
L^{j} \gamma_{j}=\sum_{b=1}^{B} \psi^{(b)} \operatorname{diag}\left(\lambda_{1}^{(b)}, \ldots, \lambda_{6}^{(b)}\right) \quad \text { and } \quad A_{L / R}^{\text {even }}=\sum_{b=1}^{B}\left(\begin{array}{cc}
A_{L / R}^{N,(b)} & 0 \\
0 & A_{L / R}^{C,(b)}
\end{array}\right)
$$

for a suitable parameter $B$. Then the linear conditions 4.5.24 and 4.5.25 can be satisfied for each $b$ separately. The quadratic conditions and relations, however, connect the vector fields for $b$ and $b^{\prime}$ with $b \neq b^{\prime}$. A detailed analysis of the resulting equations shows that that it is indeed possible to compensate the logarithmic terms for a general symmetric tensor $T_{j k}$.

\subsection{Structural Contributions to the Euler-Lagrange Equations}

In this section, we analyze additional contributions to the EL equations to degree four on the light cone. These contributions will not enter the field equations, but they are nevertheless important because they give constraints for the form of the admissible gauge fields and thus determine the structure of the interaction. For this reason, we call them structural contributions.

4.6.1. The Bilinear Logarithmic Terms. We now return to the logarithmic singularities on the light cone. In $\$ 4.4 .3$ we computed the corresponding contributions to the EL equations to the order $o\left(|\vec{\xi}|^{-3}\right)$ at the origin. In $\$ 4.4 .4$, we succeeded in compensating the logarithmic singularities by a microlocal chiral transformation. The remaining question is how the logarithmic singularities behave in the next order in a Taylor expansion around $\xi=0$. It turns out that the analysis of this question yields constraints for the form of the admissible gauge fields, as is made precise by the following proposition.

Proposition 4.6.1. Assume that the parameter $\mathfrak{c}_{2}$ in 4.4 .49 is sufficiently large and that the chiral potentials in (4.3.29) satisfy the conditions

$$
\begin{array}{ll}
A_{L}^{11}-A_{R}^{N}= \pm\left(A_{L}^{22}-A_{R}^{C}\right) & \text { at all space-time points, and } \\
A_{L}^{11}-A_{R}^{N}=-\left(A_{L}^{22}-A_{R}^{C}\right) & \text { at all space-time points with } A_{L}^{12} \neq 0 .
\end{array}
$$

Moreover, in Case (i) in 4.3.36 we assume that the MNS matrix and the mass matrix satisfy the relation

$$
\left(\begin{array}{cc}
0 & \dot{U}_{M N S}^{*} \\
\dot{U}_{M N S} & 0
\end{array}\right) Y \grave{Y}=\dot{Y} Y\left(\begin{array}{cc}
0 & \grave{U}_{M N S}^{*} \\
\grave{U}_{M N S} & 0
\end{array}\right)
$$


Then one can arrange by a suitable choice of the basis $\mathfrak{e}_{1}, \ldots, \mathfrak{e}_{6}$ and the unitary matrix $V$ in 4.4 .82 that the contributions to the EL equations $\sim|\vec{\xi}|^{-3} \log |\vec{\xi}|$ vanish.

If conversely (4.6.1) does not hold and if we do not assume any relations between the regularization parameters, then the EL equations of order $|\vec{\xi}|^{-3} \log |\vec{\xi}|$ are necessarily violated at some space-time point.

The importance of this proposition is that it poses a further constraint on the form of the chiral gauge potentials.

The remainder of this section is devoted to the proof of Proposition 4.6.1. Generally speaking, our task is to analyze how the gauge phases enter the logarithmic singularities of the fermionic projector. We begin with the logarithmic current term

$$
\chi_{L} P^{\text {aux }}(x, y) \asymp-2 \chi_{L} \int_{x}^{y}[0,0 \mid 1] j_{L}^{i} \gamma_{i} T^{(1)},
$$

which gives rise to the last summand in 4.4.22 (and similarly for the right-handed component; for details see (B.2.6) and (B.2.7) or [F7, Appendix B]). According to the general rules for inserting ordered exponentials (see [F6, Definition 2.9], [F7, Definition 2.5.5] or Definition 2.2.7), the gauge potentials enter the logarithmic current term according to

$$
-2 \chi_{L} \int_{x}^{y}[0,0 \mid 1] \mathrm{Pe}^{-i \int_{x}^{z} A_{L}^{k}(z-x)_{k}} j_{L}^{i}(z) \gamma_{i} \mathrm{Pe}^{-i \int_{z}^{y} A_{L}^{l}(y-z)_{l}} T^{(1)}
$$

(where Pe $\equiv$ Pexp again denotes the ordered exponential 4.3.18)). Performing a Taylor expansion around $\xi=0$ gives

$$
\begin{aligned}
\chi_{L} P^{\operatorname{aux}}(x, y) \asymp & -\frac{1}{3} \chi_{L} j_{L}^{i}\left(\frac{x+y}{2}\right) \gamma_{i} T^{(1)} \\
& +\frac{i}{6} \chi_{L}\left(A_{L}^{j} \xi_{j} j_{L}^{i} \gamma_{i}+j_{L}^{i} \gamma_{i} A_{L}^{j} \xi_{j}\right) T^{(1)}+o(|\vec{\xi}|)
\end{aligned}
$$

(note that in 4.6.5) it plays no role if the functions are evaluated at $x$ or $y$ because the difference can be combined with the error term).

We arranged by the microlocal chiral transformation that the logarithmic singularity of 4.6.4 is compensated by the second summand in 4.4.72). Since both 4.6.4 and (4.4.70) involve the argument $(x+y) / 2$, the logarithmic singularities compensate each other even if $x$ and $y$ are far apart (up to the error terms as specified in (4.6.5) and Proposition 4.4.7). Thus it remains to analyze how the gauge phases enter 4.4 .72$)$. To this end, we adapt the method introduced after 4.4.80) to the matrix products in 4.4.49). Beginning with the left-handed component, the square of the mass matrix is modified by the gauge phases similar to (4.6.3) and (4.6.4), 4.6.5) by

$$
\begin{aligned}
\chi_{L} m^{2} Y^{2} \rightarrow & \chi_{L} \int_{x}^{y} \mathrm{Pe}^{-i \int_{x}^{z} A_{L}^{k}(z-x)_{k}} m^{2} Y^{2} \mathrm{Pe}^{-i \int_{z}^{y} A_{L}^{l}(y-z)_{l}} d z \\
& =\chi_{L} m^{2} Y^{2}-\frac{i}{2} \chi_{L} m^{2}\left(A_{L}^{j} \xi_{j} Y^{2}+Y^{2} A_{L}^{j} \xi_{j}\right)+o(|\vec{\xi}|)
\end{aligned}
$$

(for details see [F6, Section 2 and Appendix A]). When using this transformation law in 4.4.49, we need to take into account that, similar to 4.4.80), the chiral gauge potentials $A_{L / R}$ must be replaced by $A_{R / L}^{\text {even }}$. Thus we need to compute the expectation values

$$
\chi_{L} P(x, y) \asymp-\frac{i}{2} m^{2} L[k]\left(A_{R}^{\text {even }}[\xi] Y^{2}+Y^{2} A_{R}^{\text {even }}[\xi]\right) L[k]^{*},
$$

where the square bracket again denotes a contraction, $A_{R}^{\text {even }}[\xi] \equiv\left(A_{R}^{\text {even }}\right)_{k} \xi^{k}$. 
Again choosing the basis $\mathfrak{e}_{1}, \ldots, \mathfrak{e}_{6}$, the potential $A_{R}^{\text {even }}$ is of the form 4.4.82. Now we must treat the diagonal and the off-diagonal elements of $A_{L}$ separately. Obviously, the diagonal entries in 4.4.82 map the eigenvectors $\mathfrak{e}_{1}$ and $\mathfrak{e}_{4}$ to each other. Hence 4.6.6) gives rise to the anti-commutator

$$
\begin{aligned}
\chi_{L} P(x, y) \asymp-\frac{i}{2}\left\{\left(\begin{array}{cc}
A_{L}^{11}[\xi] & 0 \\
0 & A_{L}^{22}[\xi]
\end{array}\right), L[k] m^{2} Y^{2} L[k]^{*}\right\} \\
\stackrel{4.4 .49}{=}-\frac{i}{2}\left\{\left(\begin{array}{cc}
A_{L}^{11}[\xi] & 0 \\
0 & A_{L}^{22}[\xi]
\end{array}\right), \frac{\Omega}{2} v_{L}[k]+\mathfrak{c}_{2}(k) \mathbb{1}_{\mathbb{C}^{2}}\right\} .
\end{aligned}
$$

For the off-diagonal elements of $A_{L}$, the matrices $V$ and $V^{*}$ in 4.4 .82 make the situation more complicated. For example, the lower left matrix entry in 4.4.82 maps $\mathfrak{e}_{1}$ to a nontrivial linear combination of $\mathfrak{e}_{4}, \mathfrak{e}_{5}, \mathfrak{e}_{6}$, i.e. for any $6 \times 6$-matrix $\bar{B}$,

$$
L[k] B\left(\begin{array}{cc}
0 & 0 \\
V & 0
\end{array}\right) L[k]^{*}=\left(\begin{array}{cc}
\left\|\ell_{1}\right\|^{2}\left(B_{4}^{1} V_{1}^{1}+B_{5}^{1} V_{1}^{2}+B_{6}^{1} V_{1}^{3}\right) & 0 \\
\left\|\ell_{2}\right\|\left\|\ell_{1}\right\|\left(B_{4}^{4} V_{1}^{1}+B_{5}^{4} V_{1}^{2}+B_{6}^{4} V_{1}^{3}\right) & 0
\end{array}\right) .
$$

Similarly, the upper right matrix entry in 4.4.82 maps $\mathfrak{e}_{4}$ to a non-trivial linear combination of $\mathfrak{e}_{1}, \mathfrak{e}_{2}, \mathfrak{e}_{3}$. As a consequence, the off-diagonal elements of $A_{L}$ yield a contribution to 4.6.6 of the general form

$$
\chi_{L} P(x, y) \asymp A_{L}^{12}[\xi] G(k)+A_{L}^{21}[\xi] G(k)^{*} \quad \text { with } \quad G=\left(\begin{array}{cc}
G^{11} & 0 \\
G^{12} & G^{22}
\end{array}\right) .
$$

Here the $2 \times 2$-matrix $G(k)$ depends on $\mathfrak{c}_{2}$ and $v_{L}[k]$ as well as on the choice of the basis vectors $\mathfrak{e}_{2}, \mathfrak{e}_{3}, \mathfrak{e}_{5}, \mathfrak{e}_{6}$ and the matrix $V$ in 4.4 .82 . Counting the number of free parameters, one sees that $G(k)$ can be chosen arbitrarily, up to inequality constraints which come from the fact that $V$ must be unitary and that the entries of the matrix $m^{2} Y^{2}$ in the basis $\left(\mathfrak{e}_{1}, \ldots, \mathfrak{e}_{6}\right)$ cannot be too large due to the Schwarz inequality. These inequality constraints can always be satisfied by suitably increasing the parameter $\mathfrak{c}_{2}$. For this reason, we can treat $G(k)$ as an arbitrary matrix involving three free real parameters.

The right-handed component of the microlocal chiral transformation can be treated similarly. The only difference is that the right-handed gauge potentials are already diagonal in view of 4.3.29. Thus we obtain in analogy to 4.6.7

$$
\chi_{R} P(x, y) \asymp-\frac{i}{2}\left\{\left(\begin{array}{cc}
A_{R}^{N}[\xi] & 0 \\
0 & A_{R}^{C}[\xi]
\end{array}\right), \frac{\Omega}{2} v_{R}[k]+\mathfrak{c}_{2}(k) \mathbb{1}_{\mathbb{C}^{2}}\right\},
$$

whereas 4.6.9 has no correspondence in the right-handed component.

Comparing 4.6.3 with 4.6.7), 4.6.9) and 4.6.10), one sees that the transformation laws are the same for the diagonal elements of $A_{L}$ and $A_{R}$. For the off-diagonal elements of $A_{L}$, we can always choose $G(k)$ such as to get agreement with 44.6 .3$)$. We conclude that by a suitable choice of $G(k)$ we can arrange that the transformation law 4.6.3 agrees with (4.6.7), 4.6.9) and (4.6.10). As a consequence, the logarithmic poles of the current terms are compensated by the microlocal chiral transformation, even taking into account the gauge phases to the order $o(|\xi|)$. 
We next consider the logarithmic mass terms

$$
\begin{aligned}
\chi_{L} P^{\operatorname{aux}}(x, y) \asymp & m^{2} \chi_{L} \int_{x}^{y}[1,0 \mid 0] Y Y A_{L} T^{(1)} \\
& -m^{2} \chi_{L} \int_{x}^{y}[0,0 \mid 0] Y A_{R} Y T^{(1)} \\
& +m^{2} \chi_{L} \int_{x}^{y}[0,1 \mid 0] A_{L} Y Y T^{(1)},
\end{aligned}
$$

which give rise to the summand (4.4.25) (for details see $(\mathrm{B} .2 .18)-(\sqrt{\mathrm{B} .2 .20})$ or $[\mathbf{F 7}$, Appen$\operatorname{dix} \mathrm{B}]$ ). Here the gauge phases enter somewhat differently, as we now describe.

Lemma 4.6.2. Contracting the logarithmic mass terms 4.6.11-4.6.13 with a factor $\xi$ and including the gauge phases, we obtain

$$
\begin{aligned}
\frac{1}{2} \operatorname{Tr} & \left(i \not \chi_{L} P^{\operatorname{aux}}(x, y)\right) \\
\asymp & \frac{i m^{2}}{2}\left(A_{L}^{j}\left(z_{1}\right) Y Y-2 Y A_{R}^{j}\left(z_{2}\right) Y+Y Y A_{L}^{j}\left(z_{3}\right)\right) \xi_{j} T^{(1)} \\
& +\frac{m^{2}}{8}\left(\left(A_{L}^{j} \xi_{j}\right)\left(A_{L}^{k} \xi_{k}\right) Y Y+2\left(A_{L}^{j} \xi_{j}\right) Y Y\left(A_{L}^{k} \xi_{k}\right)+Y Y\left(A_{L}^{j} \xi_{j}\right)\left(A_{L}^{k} \xi_{k}\right)\right) T^{(1)} \\
& -\frac{m^{2}}{2} Y\left(A_{R}^{j} \xi_{j}\right)\left(A_{R}^{k} \xi_{k}\right) Y T^{(1)}+o\left(|\vec{\xi}|^{2}\right),
\end{aligned}
$$

where

$$
z_{1}=\frac{3 x+y}{4}, \quad z_{2}=\frac{x+y}{2}, \quad z_{3}=\frac{x+3 y}{4} .
$$

The right-handed component is obtained by the obvious replacements $L \leftrightarrow R$.

Proof. Following the method of [F6, proof of Theorem 2.10] (see the proof of Theorem 2.2.8), we first choose a special gauge and then use the behavior of the fermionic projector under chiral gauge transformations. More precisely, with a chiral gauge transformation we can arrange that $A_{L}$ and $A_{R}$ vanish identically along the line segment $\overline{x y}$. In the new gauge, the mass matrix $Y$ is no longer constant, but it is to be replaced by dynamical mass matrices $Y_{L / R}(x)$ (see [F6, eq. (2.8)], [F7, eq. (2.5.9)] or (2.2.28)). Performing the light-cone expansion in this gauge, a straightforward calculation yields

$$
\frac{1}{2} \operatorname{Tr}\left(i \notin \chi_{L} P^{\text {aux }}(x, y)\right) \asymp m^{2}\left(Y_{L}(x) Y_{R}(y)-\int_{x}^{y}\left(Y_{L} Y_{R}\right)(z) d z\right) T^{(1)}
$$

Transforming back to the original gauge amounts to inserting ordered exponentials according to the rules in [F6, Definition 2.9] (see also [F7, Definition 2.5.5] or Definition 2.2.7). We thus obtain

$$
\begin{aligned}
& \frac{1}{2} \operatorname{Tr}\left(i \notin \chi_{L} P^{\mathrm{aux}}(x, y)\right) \asymp m^{2} Y \mathrm{Pe}^{-i \int_{x}^{y} A_{R}^{j} \xi_{j}} Y T^{(1)} \\
& \quad-m^{2} \int_{x}^{y} \mathrm{Pe}^{-i \int_{x}^{z} A_{L}^{j}(z-x)_{j}} Y Y \mathrm{Pe}^{-i \int_{z}^{y} A_{L}^{j}(y-z)_{j}} d z T^{(1)} .
\end{aligned}
$$

Expanding in powers of $\xi$ gives the result.

The contribution (4.6.14) is the mass term which we already encountered in 4.4.25). In contrast to 4.6.4) and (4.4.70), the term (4.6.14) does not only depend on the variable $(x+y) / 2$. However, forming the sectorial projection, for a diagonal potential $A_{L}$ we 
obtain in view of 4.3 .29 that

$$
\begin{aligned}
\left(\hat{A}_{L}^{j}\left(z_{1}\right) Y \grave{Y}+\grave{Y} Y \grave{A}_{L}^{j}\left(z_{3}\right)\right) \xi_{j} & =\left(A_{L}^{j}\left(z_{1}\right)+A_{L}^{j}\left(z_{3}\right)\right) \xi_{j} \grave{Y} \grave{Y} \\
& =2 A_{L}^{j}\left(\frac{x+y}{2}\right) \xi_{j} \grave{Y} \grave{Y}+o(|\vec{\xi}|) .
\end{aligned}
$$

This makes it possible to compensate the logarithmic singularity of (4.6.14) by the second term in 4.4.72, up to the specified error terms. For the off-diagonal potentials, the situation is more complicated and depends on the two cases in (4.3.36). If we are in Case (i) and (4.6.2) is satisfied, then (4.6.18) also holds for the off-diagonal potentials. As a consequence, the logarithmic singularity of (4.6.14) can again be compensated by the second term in 4.4.72). However, if we do not impose (4.6.2), then it seems impossible to compensate the off-diagonal logarithmic terms by a microlocal chiral transformation 4.4.72). If we assume instead that we are in Case (ii) in (4.3.36), then the spectral projectors $I_{n}$ are diagonal (4.3.44), so that off-diagonal potentials are irrelevant as they do not enter the EL equations (4.4.7). We conclude that we can compensate the logarithmic singularities of (4.6.14) in Case (i) under the additional assumptions 4.6.2), and in Case (ii) without any additional assumptions.

The terms 4.6.15) and 4.6.16), on the other hand, are quadratic in the chiral gauge potentials. Analyzing whether these terms are compatible with the transformation law 4.6.7), 4.6.9) and 4.6.10) of the microlocal chiral transformation gives the following result.

Lemma 4.6.3. Consider the component of the fermionic projector which involves a bilinear tensor field and has a logarithmic pole on the light cone,

$$
P(x, y) \asymp\left(\chi_{L} h_{L}^{i j}(x, y) \gamma_{i} \xi_{j}+\chi_{R} h_{R}^{i j}(x, y) \gamma_{i} \xi_{j}\right) T^{(1)}
$$

(where $h_{L / R}^{i j}$ is a smooth tensor field acting as a $2 \times 2$-matrix on the isospin index). If (4.6.1) holds and $\mathfrak{c}_{2}$ is sufficiently large, then one can arrange by a suitable choice of the basis $\mathfrak{e}_{1}, \ldots, \mathfrak{e}_{6}$ and of the unitary matrix $V$ in 4.4 .82 that

$$
\chi_{L} h_{L}^{i j}+\chi_{R} h_{R}^{i j}=h^{i j} \grave{Y} \grave{Y}
$$

(where $h^{i j}$ is a suitable tensor field which acts trivially on the isospin index). If conversely (4.6.1) does not hold, then (4.6.20 is necessarily violated at some space-time point.

Proof. We first analyze the right-handed component. If 4.6.14 is transformed according to (4.6.10), we could argue just as for the logarithmic current terms to conclude that the contribution of the form (4.6.19) vanishes. Therefore, it suffices to consider the terms obtained by subtracting from (4.6.14 - 4.6.16) the term 4.6.14 transformed according to 4.6.6), giving rise to the expression

$$
\begin{aligned}
B_{L}:= & -\frac{m^{2}}{4}\left\{A_{R}^{\text {even }}[\xi],\left(A_{L}[\xi]\left(z_{1}\right) Y Y-2 Y A_{R}[\xi] Y+Y Y A_{L}[\xi]\right)\right\} T^{(1)} \\
& \left.+\frac{m^{2}}{8}\left(A_{L}[\xi]^{2} Y Y+2 A_{L}[\xi] Y Y A_{L}[\xi]+Y Y A_{L}[\xi]^{2} \xi_{k}\right)\right) T^{(1)} \\
& -\frac{m^{2}}{2} Y A_{R}[\xi]^{2} Y T^{(1)}
\end{aligned}
$$


and similarly for the right-handed component. We must arrange that $\hat{B}_{L}$ and $\hat{B}_{R}$ are multiples of the identity matrix and coincide. We first analyze $B_{R}$. Then according to 4.4.82, the potential $A_{L}^{\text {even }}$ coincides with $A_{R}$ and is sector diagonal. We thus obtain

$$
\begin{aligned}
B_{R}= & -\frac{m^{2}}{8}\left(A_{R}[\xi]^{2} Y Y+2 A_{R}[\xi] Y Y A_{R}[\xi]+Y Y A_{R}[\xi]^{2}\right) T^{(1)} \\
& +\frac{m^{2}}{2}\left(A_{R}[\xi] Y A_{L}[\xi] Y-Y A_{L}[\xi]^{2} Y+Y A_{L}[\xi] Y A_{R}[\xi]\right) T^{(1)} .
\end{aligned}
$$

We decompose $A_{L}$ into its diagonal and off-diagonal elements, denoted by

$$
A_{L}[\xi]=A_{L}^{d}[\xi]+A_{L}^{o}[\xi] .
$$

A straightforward calculation using the identity

$$
A_{L}[\xi]^{2}=A_{L}^{d}[\xi]^{2}+A_{L}^{o}[\xi]^{2}+\left\{A_{L}^{d}[\xi], A_{L}^{o}[\xi]\right\}
$$

gives

$$
\begin{aligned}
B_{R}= & -\frac{m^{2}}{2} Y\left(\left(A_{R}[\xi]-A_{L}^{d}[\xi]\right)^{2}+A_{L}^{o}[\xi]^{2}\right) Y T^{(1)} \\
& +\frac{m^{2}}{2} Y\left\{A_{R}[\xi]-A_{L}^{d}[\xi], A_{L}^{o}[\xi]\right\} Y T^{(1)} .
\end{aligned}
$$

Clearly, the matrix $A_{L}^{o}[\xi]^{2}$ is a multiple of the identity matrix. The matrix $\left(A_{R}[\xi]-A_{L}^{d}\right)^{2}$, on the other hand, is a multiple of the identity matrix if and only if 4.6.1 holds. The anti-commutator in 4.6.23 is zero on the diagonal. It vanishes provided that 4.6.1 holds. We conclude that the contributions 4.6.22 and 4.6.23 act trivially on the isospin index if and only if 4.6.1 holds. In this case,

$$
B_{R}=-\frac{m^{2}}{2} Y\left(\left(A_{R}[\xi]-A_{L}^{d}[\xi]\right)^{2}+A_{L}^{o}[\xi]^{2}\right) Y T^{(1)} .
$$

It remains to show that under the assumption (4.6.1), we can arrange that the corresponding left-handed contribution $\hat{B}_{L}$ is also a multiple of the identity matrix, and that it coincides with (4.6.24). Now $A_{R}^{\text {even }}$ is given by (4.4.82). The diagonal entries of $A_{R}^{\text {even }}$ coincide with those of $A_{L}$, giving rise to the contribution

$$
\begin{aligned}
B_{L} \asymp & -\frac{m^{2}}{4}\left\{A_{L}^{d}[\xi],\left(A_{L}[\xi] Y Y-2 Y A_{R}[\xi] Y+Y Y A_{L}[\xi]\right)\right\} T^{(1)} \\
& +\frac{m^{2}}{8}\left(A_{L}[\xi]^{2} Y Y+2 A_{L}[\xi] Y Y A_{L}[\xi]+Y Y A_{L}[\xi]^{2}\right) T^{(1)}-\frac{m^{2}}{2} Y A_{R}[\xi]^{2} Y T^{(1)} .
\end{aligned}
$$

Similar as in 4.6.9), we can add contributions which involve $A_{L}^{12}$ or $A_{L}^{21}$. A short calculation shows that in this way, we can indeed arrange that $\hat{B}_{L}$ coincides with $\hat{B}_{R}$ as given by 4.6 .24 .

REMARK 4.6.4. (necessity of a mixing matrix) The proof of Lemma 4.6.3 even gives an explanation why the mixing matrix $U_{\text {MNS }}$ must occur. Namely, the following consideration shows that the method of proof fails if $U_{\mathrm{MNS}}$ is trivial: Suppose that $U_{\mathrm{MNS}}=$ $\mathbb{1}$. Then the parameter $c$ in 4.3.32 is equal to one. As a consequence, the parameter $d$ in (4.4.83 also equals one, implying that $V_{1}^{1}=1$. Since $V$ is unitary, it follows that $V_{1}^{2}=$ $V_{1}^{3}=0$. As a consequence, the freedom in choosing $V(x)$ does not make it possible to modify (4.6.8). Thus the matrix $G(k)$ in 4.6 .9$)$ can no longer be chosen arbitrarily, in general making it impossible to arrange 4.6.20). 
We note that in $\$ 5.3 .5$, a different argument is given which also yields that the mixing matrix must be non-trivial (see Lemma 5.3.19).

The next lemma gives the connection to the EL equations.

LEMMA 4.6.5. The contributions to the EL equations $\sim|\vec{\xi}|^{-3} \log |\vec{\xi}|$ vanish if and only if the condition 4.6.20 holds.

Proof. A direct computation shows that the terms of the form 4.6.19 contribute to the EL equations of the order $|\vec{\xi}|^{-3} \log |\vec{\xi}|$ unless 4.6 .20 holds. Therefore, our task is to show that it is impossible to compensate a term of the form 4.6 .19 by a generalized microlocal chiral transformation. It clearly suffices to consider the homogeneous setting in the high-frequency limit as introduced in $\$ 3.7 .9$. Transforming to momentum space, the contribution 4.6 .19 corresponds to the distribution

$$
\gamma^{i} h_{i j} k^{j} \delta^{\prime \prime}\left(k^{2}\right) \Theta\left(-k^{0}\right) .
$$

Having only three generations to our disposal, such a contribution would necessarily give rise to error terms of the form

$$
\frac{1}{m^{2}} \gamma^{i} h_{i j} k^{j} \delta^{\prime}\left(k^{2}\right) \Theta\left(-k^{0}\right) \quad \text { or } \quad \frac{1}{m^{4}} \gamma^{i} h_{i j} k^{j} \delta\left(k^{2}\right) \Theta\left(-k^{0}\right) .
$$

These error terms are as large as the shear contributions by local axial transformation as analyzed in $\$ 3.7 .8$, causing problems in the EL equations (for details see 3.7 .8 and Appendix C). Instead of going through these arguments again, we here rule out (4.6.25) with the following alternative consideration: In order to generate the contribution (4.6.25), at least one of the Dirac seas would have to be perturbed by a contribution with the scaling

$$
\frac{1}{m^{4}} \gamma^{i} h_{i j} k^{j} \delta\left(k^{2}-m_{\alpha}^{2}\right) \Theta\left(-k^{0}\right) \text {. }
$$

Due to the factor $k^{j}$, this perturbation is by a scaling factor $\Omega$ larger than the perturbations considered in $\$ 3.7 .9$. Thus one would have to consider a transformation of the form (cf. 3.7 .58$)$ )

$$
U=\exp (i Z) \quad \text { with } \quad Z=\mathcal{O}\left(\Omega^{0}\right) .
$$

This transformation does not decay in $\Omega$ and thus cannot be treated perturbatively. Treating it non-perturbatively, the resulting shear contributions violate the EL equations.

Combining Lemmas 4.6 .3 and 4.6 .5 gives Proposition 4.6.1.

4.6.2. The Field Tensor Terms. We now come to the analysis of the contributions to the fermionic projector

$$
\begin{aligned}
\chi_{L} P(x, y) & \asymp \frac{1}{4} \chi_{L} \oiint \int_{x}^{y} F_{L}^{i j} \gamma_{i} \gamma_{j} T^{(0)}-\chi_{L} \xi_{i} \int_{x}^{y}[0,1 \mid 0] F_{L}^{i j} \gamma_{j} T^{(0)} \\
& =\frac{1}{2} \chi_{L} \int_{x}^{y}(2 \alpha-1) \xi_{i} F_{L}^{i j} \gamma_{j} T^{(0)}+\frac{i}{4} \chi_{L} \int_{x}^{y} \epsilon_{i j k l} F_{L}^{i j} \xi^{k} \Gamma \gamma^{l} T^{(0)},
\end{aligned}
$$

which we refer to as the field tensor terms (see [F5, Appendix A], [F6, Appendix A] and Appendix B; note that here we only consider the phase-free contributions, to which gauge phases can be inserted according to the rules in [F6] or Definition 2.2.7). In Chapter 3 , the field tensor terms were disregarded because they vanish when the Dirac matrices are contracted with outer factors $\xi$. Now we will analyze the field tensor terms in the 
$\iota$-formalism introduced in 4.2 .7 . This will give additional constraints for the form of the admissible gauge fields (see relation 4.6 .39 below).

In this section, the corrections in $\tau_{\text {reg }}$ are essential. It is most convenient to keep the terms involving $\tau_{\text {reg }}$ in all computations. We assume that we evaluate weakly for such a small vector $\xi$ that we are in Case (ii) in 4.3.36) (this will be discussed in Section 4.8). It then suffices to consider the sector-diagonal elements of the closed chain. Moreover, by restricting attention to the first or second isospin component, we can compute the spectral decomposition of the closed chain in the neutrino sector $(n=1)$ and the chiral sector $(n=2)$ separately. For a uniform notation, we introduce the notation

$$
M_{n}^{(l)}=\left\{\begin{array}{rr}
L_{[0]}^{(l)} & \text { if } n=1 \\
T_{[0]}^{(l)} & \text { if } n=2
\end{array}\right.
$$

with $L_{\circ}^{(l)}$ as given by 4.3 .9 . Then the unperturbed eigenvalues are given by

$$
\lambda_{n L-}=9 T_{[0]}^{(-1)} \overline{M_{n}^{(0)}}, \quad \lambda_{n R-}=9 M_{n}^{(-1)} \overline{T_{[0]}^{(0)}} .
$$

Moreover, using the calculations

$$
\begin{gathered}
\frac{\overline{\lambda_{n L-}}}{\left|\lambda_{n L-}\right|} \chi_{L} P(x, y)=3 i \chi_{L} \nLeftarrow \frac{M_{n}^{(0)} \overline{T_{[0]}^{(-1)}}}{\left|T_{[0]}^{(-1)} M_{n}^{(0)}\right|} T_{[0]}^{(-1)}=3 i \chi_{L} \sharp \frac{\left|T_{[0]}^{(-1)}\right|}{\left|M_{n}^{(0)}\right|} M_{n}^{(0)} \\
\frac{\overline{\lambda_{n R-}}}{\left|\lambda_{n R-}\right|} \chi_{R} P(x, y)=3 i \chi_{R} \nLeftarrow \frac{\left|M_{n}^{(-1)}\right|}{\left|T_{[0]}^{(0)}\right|} T_{[0]}^{(0)}
\end{gathered}
$$

in (4.4.4), we can write the EL equations as

$$
\begin{aligned}
& \left(\Delta\left|\lambda_{n L-}\right|-\frac{1}{4} \sum_{n^{\prime}, c^{\prime}} \Delta\left|\lambda_{n^{\prime} c^{\prime}-}\right|\right) \frac{\left|T_{[0]}^{(-1)}\right|}{\left|M_{n}^{(0)}\right|} M_{n}^{(0)}=0 \\
& \left(\Delta\left|\lambda_{n R-}\right|-\frac{1}{4} \sum_{n^{\prime}, c^{\prime}} \Delta\left|\lambda_{n^{\prime} c^{\prime}-}\right|\right) \frac{\left|M_{n}^{(-1)}\right|}{\left|T_{[0]}^{(0)}\right|} T_{[0]}^{(0)}=0 .
\end{aligned}
$$

Note that in the case $\tau_{\text {reg }}=0$, these equations reduce to our earlier conditions (4.4.7) and 4.4.6).

Our task is to analyze how 4.6.26) influences the eigenvalues $\lambda_{n c-}$ of the closed chain.

Lemma 4.6.6. The field tensor terms 4.6.26 contribute to the eigenvalues $\lambda_{n c-}$ by

$$
\begin{aligned}
\lambda_{n L-} \asymp & \frac{3 i}{2} \int_{x}^{y}(2 \alpha-1) \operatorname{Tr}_{\mathbb{C}^{2}}\left(I_{n} \hat{F}_{L}^{i j} \check{\xi}_{i}\left(\iota_{[0]}^{(-1)}\right)_{j}\right) T_{[0]}^{(0)} \overline{M_{n}^{(0)}} \\
& +\frac{3}{4} \int_{x}^{y} \operatorname{Tr}_{\mathbb{C}^{2}}\left(I_{n} \epsilon_{i j k l} \hat{F}_{L}^{i j} \check{\xi}^{k}\left(\iota_{[0]}^{(-1)}\right)^{l}\right) T_{[0]}^{(0)} \overline{M_{n}^{(0)}}+(\operatorname{deg}<2) \\
\lambda_{n R-} \asymp & \frac{3 i}{2} \int_{x}^{y}(2 \alpha-1) \operatorname{Tr}_{\mathbb{C}^{2}}\left(I_{n} \hat{F}_{R}^{i j} \check{\xi}_{i}\left(\iota_{[0]}^{(-1)}\right)_{j}\right) M_{n}^{(0)} \overline{T_{[0]}^{(0)}} \\
& -\frac{3}{4} \int_{x}^{y} \operatorname{Tr}_{\mathbb{C}^{2}}\left(I_{n} \epsilon_{i j k l} \hat{F}_{R}^{i j} \check{\xi}^{k}\left(\iota_{[0]}^{(-1)}\right)^{l}\right) M_{n}^{(0)} \overline{T_{[0]}^{(0)}}+(\operatorname{deg}<2) .
\end{aligned}
$$


Proof. We first consider the effect of a left-handed field on the left-handed eigenvalues. Every summand in 4.6.26) involves a factor $\xi T^{(0)}$. As the factor $\iota^{(0)}$ gives no contribution (see 4.2.65), we regularize 4.6.26) in the $\iota$-formalism by

$$
\chi_{L} P(x, y) \asymp \frac{1}{2} \chi_{L} \int_{x}^{y}(2 \alpha-1) \check{\xi}_{i} \hat{F}_{L}^{i j} \gamma_{j} T_{[0]}^{(0)}+\frac{i}{4} \chi_{L} \int_{x}^{y} \epsilon_{i j k l} \hat{F}_{L}^{i j} \check{\xi}^{k} \Gamma \gamma^{l} T_{[0]}^{(0)}
$$

(where the hat again denotes the sectorial projection). For computing the effect on the eigenvalues, we first multiply by the vacuum fermionic projector $P^{(0)}(y, x)$ to form the closed chain. Then we multiply by powers of the vacuum chain (4.2.68) and take the trace. Since the number of factors $\iota$ in 4.2 .68 always equals the number of factors $\hat{\xi}$, and taking into account that 4.6 .33 vanishes when contracted with a factor $\hat{\xi}$, we conclude that the factor $P^{(0)}(y, x)$ must contain a factor $\iota$. In view of 4.2 .65$)$, this means that we only need to take into account the contribution $P^{(0)}(y, x) \asymp-3 i \psi_{[0]}^{(-1)} L_{[0]}^{(0)}$. We thus obtain

$$
\begin{aligned}
\chi_{L} A_{x y} \asymp & \frac{3 i}{2} \chi_{L} \int_{x}^{y}(2 \alpha-1) \check{\xi}_{i} \hat{F}_{L}^{i j} \gamma_{j} T_{[0]}^{(0)} \overline{\psi_{[0]}^{(-1)} M_{n}^{(0)}} \\
& -\frac{3}{4} \chi_{L} \int_{x}^{y} \epsilon_{i j k l} \hat{F}_{L}^{i j} \check{\xi}^{k} \Gamma \gamma^{l} T_{[0]}^{(0)} \overline{\psi_{[0]}^{(-1)} M_{n}^{(0)}} .
\end{aligned}
$$

Since the last Dirac factor involves $\iota$, this contribution vanishes when multiplied by the first summand in 4.2.68). Thus our field tensor term only influences the eigenvalue $\lambda_{n L-}$. A short calculation gives 4.6.29) and 4.6.30). Similarly, a right-handed field only influences the corresponding right-handed eigenvalues by (4.6.31) and 4.6.32). The result follows by linearity.

Before going on, we remark that the above contributions do not appear in the standard formalism of the continuum limit, where all factors $\xi$ which are contracted to macroscopic functions are treated as outer factors. In order to get back to the standard formalism, one can simply impose that $F_{i j} \check{\xi}^{i} \iota^{j}=0$. However, this procedure, which was implicitly used in Chapter 3 , is not quite convincing because it only works if the regularization is adapted locally to the field tensor. If we want to construct a regularization which is admissible for any field tensor (which should of course satisfy the field equations), then the contributions by Lemma 4.6.6 must be taken into account.

COROLlaRY 4.6.7. Introducing the macroscopic functions

$$
\begin{aligned}
a_{n L / R}= & \frac{3 i}{4} \int_{x}^{y}(2 \alpha-1) \operatorname{Tr}_{\mathbb{C}^{2}}\left(I_{n} \hat{F}_{L / R}^{i j} \check{\xi}_{i}\left(\iota_{[0]}^{(-1)}\right)_{j}\right) \\
& \pm \frac{3}{8} \int_{x}^{y} \operatorname{Tr}_{\mathbb{C}^{2}}\left(I_{n} \epsilon_{i j k l} \hat{F}_{L / R}^{i j} \check{\xi}^{k}\left(\iota_{[0]}^{(-1)}\right)^{l}\right),
\end{aligned}
$$

the absolute values of the eigenvalues are perturbed by the field tensor terms (4.6.26) according to

$$
\begin{aligned}
& \Delta\left|\lambda_{n L-}\right|=\frac{\left|M_{n}^{(0)}\right|}{\left|T_{[0]}^{(-1)}\right|}\left(a_{n L} T_{[0]}^{(0)} \overline{T_{[0]}^{(-1)}}+\overline{a_{n L}} T_{[0]}^{(-1)} \overline{T_{[0]}^{(0)}}\right) \\
& \Delta\left|\lambda_{n R-}\right|=\frac{\left|T_{[0]}^{(0)}\right|}{\left|M_{n}^{(-1)}\right|}\left(a_{n R} M_{n}^{(0)} \overline{M_{n}^{(-1)}}+\overline{a_{n R}} M_{n}^{(-1)} \overline{M_{n}^{(0)}}\right) .
\end{aligned}
$$


Proof. Writing the result of Lemma 4.6.6 as

$$
\Delta \lambda_{n L-}=2 a_{n L} T_{[0]}^{(0)} \overline{M_{n}^{(0)}}, \quad \Delta \lambda_{n R-}=2 a_{n R} M_{n}^{(0)} \overline{T_{[0]}^{(0)}},
$$

we obtain

$$
\Delta\left|\lambda_{n L-}\right|=\frac{1}{\left|T_{[0]}^{(-1)} \overline{M_{n}^{(0)}}\right|} \operatorname{Re}\left(a_{n L} T_{[0]}^{(0)} \overline{M_{n}^{(0)}} M_{n}^{(0)} \overline{T_{[0]}^{(-1)}}\right)=\frac{\left|M_{n}^{(0)}\right|}{\left|T_{[0]}^{(-1)}\right|} \operatorname{Re}\left(a_{n L} T_{[0]}^{(0)} \overline{T_{[0]}^{(-1)}}\right) .
$$

The calculation for $\Delta\left|\lambda_{n R-}\right|$ is analogous.

After these preparations, we are ready to analyze the EL equations (4.6.27), (4.6.28). We begin with the case $\tau_{\text {reg }}=0$. Then we can set $M_{n}^{(l)}=T_{[0]}^{(l)}$, giving the conditions 4.4.7), where now

$$
\mathcal{K}_{n c}=\Delta\left|\lambda_{n L-}\right| \frac{\left|T_{[0]}^{(-1)}\right|}{\left|T_{n}^{(0)}\right|} M_{n}^{(0)}=a_{n c} T_{[0]}^{(0)} T_{[0]}^{(0)} \overline{T_{[0]}^{(-1)}}+\overline{a_{n c}} T_{[0]}^{(0)} T_{[0]}^{(-1)} \overline{T_{[0]}^{(0)}} .
$$

This formula can be simplified further with the integration-by-parts rules. Namely, applying (4.2.39), we obtain

$$
0=\nabla\left(T_{[0]}^{(0)} T_{[0]}^{(0)} \overline{T_{[0]}^{(0)}}\right)=2 T_{[0]}^{(0)} T_{[0]}^{(-1)} \overline{T_{[0]}^{(0)}}+T_{[0]}^{(0)} T_{[0]}^{(0)} \overline{T_{[0]}^{(-1)}} .
$$

Using this relation, we conclude that

$$
\mathcal{K}_{n c}=-\left(2 a_{n c}-\overline{a_{n c}}\right) T_{[0]}^{(0)} T_{[0]}^{(-1)} \overline{T_{[0]}^{(0)}}=-\left(\operatorname{Re}\left(a_{n c}\right)+3 \operatorname{Im}\left(a_{n c}\right)\right) T_{[0]}^{(0)} T_{[0]}^{(-1)} \overline{T_{[0]}^{(0)}} .
$$

If any non-trivial gauge field is present, the four macroscopic functions $\operatorname{Re}\left(a_{n c}\right)+3 \operatorname{Im}\left(a_{n c}\right)$ will not all be the same (note that even for a vectorial field which acts trivially on the isospin index, the contribution 4.6.35 has opposite signs for $a_{n L}$ and $a_{n R}$ ). This implies that 4.4.7) can be satisfied only if we impose the regularization condition

$$
T_{[0]}^{(0)} T_{[0]}^{(-1)} \overline{T_{[0]}^{(0)}}=0 \quad \text { in a weak evaluation on the light cone . }
$$

In order to compute the effect of $\tau_{\text {reg }}$, we first note that the perturbations $\Delta\left|\lambda_{2 c-}\right|$ do not involve $\tau_{\text {reg }}$ (as is obvious from Corollary 4.6.7). Moreover, the contribution of these

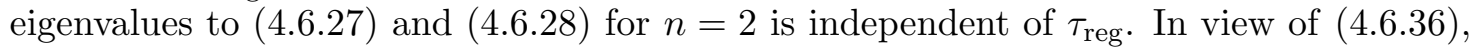
these contributions drop out of the EL equations. Next, the eigenvalue $\lambda_{1 L-}$ contributes to (4.6.27) and (4.6.28) for $n=2$ by

$$
-\frac{1}{4} \Delta\left|\lambda_{1 L-}\right| \frac{\left|T_{[0]}^{(-1)}\right|}{\left|T_{[0]}^{(0)}\right|} T_{[0]}^{(0)}=\frac{\left|L_{[0]}^{(0)}\right|}{\left|T_{[0]}^{(0)}\right|} T_{[0]}^{(0)}\left(a_{n L} T_{[0]}^{(0)} \overline{T_{[0]}^{(-1)}}+\overline{a_{n L}} T_{[0]}^{(-1)} \overline{T_{[0]}^{(0)}}\right) .
$$

This is in general non-zero. Thus in order to allow for left-handed gauge fields in the neutrino sector, we need to impose additional conditions on the regularization functions. The simplest method is to impose that

$$
\left|L_{[0]}^{(0)}\right|=\left|T_{[0]}^{(0)}\right|\left(1+\mathcal{O}\left((m \varepsilon)^{2 p_{\text {reg }}}\right)\right) \quad \text { pointwise . }
$$

Then (4.6.37) again vanishes as a consequence of (4.6.36), up to terms quadratic in $\tau_{\text {reg. }}$. We note that this is compatible with 4.3.35) and poses an additional condition on the regularization in the case $n=0$ and $p=0$. We also remark that 4.6 .38 could be replaced by a finite number of equations to be satisfied in a weak evaluation on the light 
cone. But as these equations are rather involved, we here prefer the stronger pointwise condition 4.6.38). We also note that, in contrast to the condition 4.6.36 for the regularization of ordinary Dirac seas, the relation 4.6.38 imposes a constraint only on the right-handed high-energy states.

It remains to consider the terms involving $T_{[R, 0]}^{(-1)}$. These are $\Delta\left|\lambda_{1 R-}\right|$ as well as the factor $\left|M_{n}^{(-1)}\right|$ in 4.6 .28 in case $n=1$. Collecting all the corresponding contributions to the EL equations, we get a finite number of equations to be satisfied in a weak evaluation on the light cone. Again, we could satisfy all these equations by imposing suitable conditions on the regularization. However, these additional conditions would basically imply that $T_{[R, 0]}^{(-1)}=0$ vanishes, meaning that there are no non-trivial regularization effects. For this reason, our strategy is not to impose any more regularization conditions. Then the EL equations 4.6.27) and 4.6.28 are satisfied if and only if there is no right-handed gauge field in the neutrino sector and if the vectorial component is trace-free, because only under these conditions all the equations involving $T_{[R, 0]}^{(-1)}$ or $\overline{T_{[R, 0]}^{(-1)}}$ are satisfied. If the field tensor vanishes everywhere, we can arrange by a global gauge transformation that also the corresponding potential vanishes globally. We have thus derived the following result.

Proposition 4.6.8. Taking into account the contributions by the field tensor terms in Lemma 4.6.6, the EL equations to degree four can be satisfied only if the regularization satisfies the conditions 4.6.36) and 4.6.38) (or a weaker version of (4.6.38) involving weak evaluations on the light cone). If no further regularization conditions are imposed, then the chiral potentials must satisfy at all space-time points the conditions

$$
\operatorname{Tr}\left(\Im_{1} A_{R}\right)=0 \quad \text { and } \quad \operatorname{Tr}\left(A_{L}+A_{R}\right)=0,
$$

where $\mathfrak{I}_{1}$ is the projection on the neutrino sector. If conversely the conditions 4.6.36), (4.6.38) and 4.6.39) are satisfied, then the field tensor terms do not contribute to the EL equations of degree four.

We note for clarity that in the vacuum, the operator $\mathfrak{I}_{1}$ coincides with the projection operator $I_{1}$ in 4.3 .39 ). However, the operator $I_{1}$ in general depends on the gauge potentials. The operator $\mathfrak{I}_{1}$, however, is a fixed matrix projecting on the neutrino sector. The reason why $\mathfrak{I}_{1}$ comes up is that the conditions 4.6 .39 are derived in Case (ii) in 4.3.36 where the matrix $I_{1}$ is given by (4.3.44).

\subsection{The Effective Action in the Continuum Limit}

4.7.1. Treating the Algebraic Constraints. Let us briefly review our general strategy for deriving the field equations in the continuum limit. The starting point is the fermionic projector of the vacuum, being composed of solutions of the free Dirac equation (cf. 4.2.45)

$$
(i \not \partial-m Y) \psi=0 \text {. }
$$

As explained in $\$ 4.4 .3$, a wave function $\psi$ gives rise to a contribution to the fermionic projector of the form (cf. 4.2.51)

$$
-\frac{1}{2 \pi} \bar{\psi}(x) \psi(x)
$$


which enters the EL equations. Our method for satisfying the EL equations is to introduce a suitable potential into the Dirac equation (see 4.2.46)

$$
(i \not \partial+\mathcal{B}-m Y) \psi=0 .
$$

After compensating the resulting logarithmic singularities by a microlocal chiral transformation (see 4.4.4), we can hope that the remaining contributions to the fermionic projector by the bosonic current can compensate the contribution by the Dirac current 4.7.1. This general procedure was worked out for an axial potential in Chapter 3 .

In the present setting of two sectors, the situation is more complicated because the potential $\mathcal{B}$ in 4.7 .2 must satisfy additional equations which involve the chiral potentials without derivatives (see 4.3 .29 and the conditions arising from the structural contributions in Section 4.6). We refer to these equations as the algebraic constraints. These constraints imply that the corresponding bosonic currents must be of a specific form. As a consequence, we cannot expect to compensate an arbitrary contribution of the form 4.7.1 in the EL-equations 4.3.3. Graphically speaking, we can only hope to compensate those contributions to (4.7.1) which are "parallel" to the bosonic degrees of freedom. The problem is that it is not obvious how to decompose the Dirac current (4.7.1) into contributions "parallel" and "orthogonal" to the degrees of freedom of the bosonic current, simply because there is no obvious scalar product on the contributions to $Q$. The goal of this section is to give a systematic procedure for deriving the field equations in the continuum limit, taking into account the algebraic constraints. These field equations will be recovered as the critical points of a corresponding effective action.

In order to understand the concept behind the derivation of the effective action, one should keep in mind that we regard only the fermionic states (including the states of the Dirac sea) as the basic physical objects. The bosonic fields, however, are merely auxiliary objects used for describing the collective behavior of the fermionic states. With this in mind, in contrast to the usual Lagrangian formalism, we do not need to derive the Dirac equation from an action principle. On the contrary, the Dirac equation 4.7.2 serves as the definition of $\mathcal{B}$ introduced for describing the behavior of the fermionic states. Then the appearance of algebraic constraints like 4.3 .29 can be understood as constraints for the admissible variations of the fermionic projector. Such constraints are typically handled by demanding that the action should be critical only under the admissible variations. Thus in our setting, the natural idea is to demand that the first variation of the action (see (3.5.18)

$$
\delta \mathcal{S}_{\mu}[P]=2 \operatorname{tr}(Q \delta P)
$$

should vanish for all variations $\delta P$ which are admissible in the sense that they are described by variations of $\mathcal{B}$ which satisfy the algebraic constraints. Unfortunately, this method cannot be implemented directly because, in order to obtain information independent of regularization details, the operator $Q$ must be evaluated weakly on the light-cone (see 3.5 .1 and $\$ 3.5 .2$. This means that the kernel $\delta P(x, y)$ of the operator $\delta P$ must satisfy the two conditions that it be smooth and that it vanishes in a neighborhood of the diagonal $x=y$,

$$
\delta P(x, y) \in C^{\infty}(\mathscr{M} \times \mathscr{M}) \quad \text { and } \quad \delta P(x, y)=0 \text { unless }|\vec{\xi}| \gg \varepsilon .
$$

However, the perturbation $\delta P$ corresponding to a perturbation of the bosonic potentials does not satisfy these two conditions, because in this case $\delta P(x, y)$ is singular on the light cone and non-zero at $x=y$. 
Our method to overcome this shortcoming is to take $\delta P$ as obtained from a variation of $\mathcal{B}$, and to arrange the additional requirements (4.7.4) by smoothing $\delta P(x, y)$ and by setting it to zero in a neighborhood of $x=y$. This procedure can be understood as follows: The bosonic potentials satisfying the algebraic constraints tell us about the admissible directions for varying $P$. But these variations need not necessarily be performed for all the Dirac states simultaneously. Instead, it seems reasonable that only the low-energy states (i.e. the states with frequencies $\ll \varepsilon^{-1}$ ) are varied. Then $\delta P$ is smooth. Moreover, by combining different such variations, one can arrange that $\delta P$ vanishes at the origin. The resulting variations satisfy (4.7.4), and we use them for testing in (4.7.3).

The goal of this section is to use the just-described method to derive EL equations in the continuum limit. For clarity, we first treat the chiral gauge field, whereas the gravitational field will be considered afterwards in a similar manner. In preparation, we note that quantities like currents and fields take values in the Hermitian $6 \times 6$-matrices and have a left- and right-handed component. Thus, taking the direct sum of the two chiral components, it is useful to introduce the real vector space

$$
\mathfrak{S}_{6}:=\operatorname{Symm}\left(\mathbb{C}^{6}\right) \oplus \operatorname{Symm}\left(\mathbb{C}^{6}\right),
$$

where $\operatorname{Symm}\left(\mathbb{C}^{6}\right)$ denotes the Hermitian $6 \times 6$-matrices. For example, the Dirac current 4.4.21 can be regarded as an element of $\mathfrak{S}_{6}$,

$$
\mathcal{J}:=\left(J_{L}, J_{R}\right) \in \mathfrak{S}_{6}
$$

(here we disregard the tensor indices, which will be included later in a straightforward way). In the EL equations, the Dirac current enters only after forming the sectorial projection. In what follows, it is convenient to consider the sectorial projection as an operation

$$
\therefore: \mathfrak{S}_{6} \rightarrow \mathfrak{S}_{2} \subset \mathfrak{S}_{6},
$$

where in the last inclusion we regard a symmetric $2 \times 2$-matrix as a $6 \times 6$-matrix which acts trivially on the generation index (and $\mathfrak{S}_{2}$ denotes similar to 4.7 .5 the chiral Hermitian $2 \times 2$-matrices). The chiral gauge potential and current 4.4.20) can also be regarded as elements of $\mathfrak{S}_{6}$. However, they can take values only in a subspace of $\mathfrak{S}_{6}$, as we now make precise. We denote the gauge group corresponding to the admissible gauge potentials by $\mathcal{G} \subset \mathrm{U}(6)_{L} \times \mathrm{U}(6)_{R}$ and refer to it as the dynamical gauge group (recall that in $\$ 4.3 .2$ we found the group 4.3 .28 , and taking into account the additional constraints encountered in Section 4.6, the dynamical gauge group is a proper subgroup of (4.3.28)). The dynamical gauge potentials are elements of the corresponding Lie algebra $\mathfrak{g}=T_{e} \mathcal{G}$, the so-called dynamical gauge algebra. It can be identified with a subspace of $\mathfrak{S}_{6}$. The dynamical potentials and corresponding bosonic currents take values in the dynamical subspace,

$$
\mathcal{A}:=\left(A_{L}, A_{R}\right) \in \mathfrak{g} \quad \text { and } \quad\left(j_{L}, j_{R}\right) \in \mathfrak{g} .
$$

We now evaluate 4.7 .3 for $\delta P$ being a variation in direction of the dynamical subspace. In order to evaluate this equation, we need to analyze how the potentials and currents enter the EL equations (4.4.7). We consider the contributions to degree four on the light cone after compensating the logarithmic poles and evaluate weakly on the light cone. The corresponding contribution $\Delta Q$ is given in Corollary 4.4.2. In order to determine the variation $\delta P$, one should keep in mind that it is vectorial, and that the the left- and right-handed gauge potentials affect the left- and right-handed components of $\delta P$, respectively. Moreover, $\delta P$ involves a sectorial projection. We thus obtain

$$
\operatorname{Tr}_{\mathbb{C}^{8}}\left(\Delta Q\left(\chi_{L} \hat{A}_{L}+\chi_{R} \hat{A}_{R}\right) \psi\right)=0 \quad \text { for all } \mathcal{A}=\left(A_{L}, A_{R}\right) \in \mathfrak{g} .
$$


Here $u$ is an arbitrary vector field, whose only purpose is to get a contraction with the factor $\$$ in 4.4.13. As explained before 4.4.12), we want to consider the stronger conditions which are independent of the projectors $I_{n}$. Using the form of $\Delta Q$ in Corollary 4.4.2, we thus obtain the conditions

$$
\operatorname{Tr}_{\mathbb{C}^{2}}\left(\mathcal{Q}_{L} \hat{A}_{R}+\mathcal{Q}_{R} \hat{A}_{L}\right)=0 \quad \text { for all } \mathcal{A}=\left(A_{L}, A_{R}\right) \in \mathfrak{g}
$$

(see also 4.4.15) and note that the chirality flips at the factor $\$$ in 4.4 .13 ).

In order to recover (4.7.9) from an effective variational principle, our goal is to choose a Dirac Lagrangian $\mathcal{L}_{\text {Dirac }}$ and a Yang-Mills Lagrangian $\mathcal{L}_{\text {YM }}$ such that varying the gauge potentials in the effective gauge algebra gives the left side of 4.7 .9 with $\mathcal{A}$ replaced by the variation $\delta \mathcal{A}$ of the potentials. In order to keep track of the contractions of the tensor indices, it is useful to again use the matrix-valued vector field $\mathfrak{J}_{L / R}^{k}$ in Lemma 4.4.4. Similar to 4.4.14 we set

$$
\mathcal{Q}_{c}^{k}:=\mathfrak{J}_{c}^{k}-\frac{1}{4} \operatorname{Tr}_{\mathbb{C}^{2}}\left(\mathfrak{J}_{L}^{k}+\mathfrak{J}_{R}^{k}\right) \mathbb{1}_{\mathbb{C}^{2}}
$$

Then we would like to choose $\mathcal{L}_{\text {Dirac }}$ and a Yang-Mills Lagrangian $\mathcal{L}_{\text {YM }}$ such that

$$
K(\varepsilon, \xi) \frac{\delta}{\delta \mathcal{A}}\left(\mathcal{L}_{\text {Dirac }}+\mathcal{L}_{\mathrm{YM}}\right)=\operatorname{Tr}_{\mathbb{C}^{2}}\left(\mathcal{Q}_{L}^{k}[\hat{\mathcal{J}}, \mathcal{A}]\left(\delta \hat{A}_{R}\right)_{k}+\mathcal{Q}_{R}^{k}[\hat{\mathcal{J}}, \mathcal{A}]\left(\delta \hat{A}_{L}\right)_{k}\right)
$$

for any $\delta \mathcal{A}=\left(\delta A_{L}, \delta A_{R}\right) \in \mathfrak{g}$. The square brackets $[\hat{\mathcal{J}}, \mathcal{A}]$ clarify the dependence on the chiral potentials and on the sectorial projection of the Dirac current. Our notation also points out that for example the left-handed component $\mathcal{Q}_{L}^{k}$ may depend on both the left- and right-handed components of the currents (as becomes explicit in $(4.4 .22)$ (4.4.29). The way the equation (4.7.11) is to be understood is that the right side is to be evaluated weakly according to (4.2.31). We demand that the dependence on the regularization length $\varepsilon$ and on the direction $\xi$ can be absorbed in the prefactor $K$. If this has been accomplished, the continuum limit of the EL equations corresponding to the causal action principle can be recovered by seeking for critical points of the effective action

$$
\mathcal{S}_{\text {eff }}=\int_{\mathbb{R}^{4}}\left(\mathcal{L}_{\text {Dirac }}+\mathcal{L}_{\mathrm{YM}}\right) d^{4} x
$$

The above construction can be adapted in a straightforward way to the gravitational field. To this end, we consider the contribution to $\Delta Q$ by the energy-momentum and the Ricci tensor as computed in Section 4.5. Since the gravitational field couples to the right- and left-handed components of all fermions in the same way, it corresponds to a variation in the direction $(\mathbb{1}, \mathbb{1}) \in \mathfrak{S}_{2}$. We thus obtain in analogy to 4.7.8) the condition

$$
\operatorname{Tr}_{\mathbb{C}^{8}}(\Delta Q \psi)=0 \text {. }
$$

Similar to 4.7.11 we want to recover this condition as the critical point of an effective Lagrangian. In order to recover the Einstein equations, we want to add the EinsteinHilbert action. Moreover, in curved space-time one clearly replaces the integration measure in 4.7 .12 by $\sqrt{-\operatorname{det} g} d^{4} x$, where $g$ again denotes the Lorentzian metric. Moreover, the Dirac action should clearly involve the Dirac operator in curved space-time. In order to treat the tensor indices properly, we introduce a matrix-valued symmetric 2 -tensor $\mathcal{Q}^{k l}$ by

$$
\operatorname{Tr}_{\mathbb{C}^{8}}(\Delta Q \psi)=i \xi_{j} u^{j} \mathcal{Q}^{k l}[\hat{T}, g] \xi_{k} \xi_{l} .
$$

(where the factors $\xi_{i} \xi_{j}$ are precisely those in 4.5.2 and similarly in Lemma 4.5.2). The square bracket $[\hat{T}, g]$ clarifies the dependence on the energy-momentum tensor (which 
involves a sectorial projection) and the metric. Our goal is to find an effective action such that, in analogy to 4.7.11,

$$
i K(\varepsilon, \xi) \frac{\delta}{\delta g}\left(\left(\mathcal{L}_{\text {Dirac }}+\mathcal{L}_{\mathrm{YM}}+\mathcal{L}_{\mathrm{EH}}\right) \sqrt{-\operatorname{deg} g}\right)=\mathcal{Q}^{k l}[\hat{T}, g] \delta g_{k l}
$$

with the Einstein-Hilbert action

$$
\mathcal{L}_{\mathrm{EH}}=\frac{1}{\kappa(\varepsilon, \delta)}(R+2 \Lambda)
$$

(where $R$ denotes scalar curvature and $\Lambda \in \mathbb{R}$ is the cosmological constant). We point out that the gravitational coupling constant $\kappa$ may depend on the length scales $\varepsilon$ and $\delta$ (recall that the parameter $\delta$ gives the length scale for the shear contributions; see (4.2.33) and (4.2.34)). The dependence on $\varepsilon$ or $\delta$ is needed in order to take into account that the gravitational constant is not dimensionless. This procedure will also make it possible to link the Planck length to the regularization lengths $\varepsilon$ or $\delta$.

We thus obtain the effective action

$$
\mathcal{S}_{\text {eff }}=\int_{\mathcal{M}}\left(\mathcal{L}_{\text {Dirac }}+\mathcal{L}_{\mathrm{YM}}+\mathcal{L}_{\mathrm{EH}}\right) \sqrt{-\operatorname{deg} g} d^{4} x
$$

Varying the chiral potentials in $\mathfrak{g}$ gives the bosonic field equations, whereas varying the metric gives the equations for gravity. We again point out that the variation of the effective action must always be performed under the constraint that the Dirac equation 4.7.2 holds. Thus we do not need to derive the Dirac equation from the effective action. Instead, the Dirac equation holds a-priori and must be respected by the variation. The resulting procedure for computing variations will be explained in 4.7 .3 .

4.7.2. The Effective Dirac Action. Our goal is to find Lagrangians such that the equations (4.7.11) and 4.7.15) hold. The main task is to choose the Dirac Lagrangian such that the coupling of the Dirac wave functions to the chiral potentials and the gravitational fields as described by $\Delta Q$ is compatible with the variations of the Dirac Lagrangian in (4.7.11) and 4.7.15). Usually, the coupling of the Dirac wave functions to the bosonic fields is described by the Dirac Lagrangian, which in our context takes the form

$$
\mathcal{L}_{\text {Dirac }}=\operatorname{Re} \bar{\psi}(i \not \partial+\mathcal{B}-m Y) \psi
$$

(note that using the symmetry of the Dirac operator, the real part can be omitted if one integrates over space-time). The corresponding Dirac action has the nice feature that varying the Dirac wave functions gives the Dirac equation (4.2.46). The standard method would be to add to 4.7.18 a Yang-Mills Lagrangian, in such a way that varying the bosonic potentials gives the effective EL equations (4.7.9). However, in our situation this standard method does not work, because according to (4.7.11), the effective EL equations involve the sectorial projection of the Dirac current, whereas varying $\mathcal{B}$ in $(4.7 .18)$ yields the Dirac current without a sectorial projection. A similar problem occurs when we try to recover the equations for the gravitational field (4.7.15) from a variational principle. The standard procedure is to add the Einstein-Hilbert action. But then varying the metric would give the energy-momentum tensor of the Dirac wave functions without a sectorial projection, in contrast to the sectorial projection $\hat{T}_{j k}$ in 4.7.15).

In order to resolve this problem, we need to modify the Dirac Lagrangian in such a way that the sectorial projection is built in correctly. It is now convenient to describe the 
sectorial projection by a projection operator $\check{\pi}$,

$$
\check{\pi}=\frac{1}{3}\left(\begin{array}{lll}
1 & 1 & 1 \\
1 & 1 & 1 \\
1 & 1 & 1
\end{array}\right): \mathbb{C}^{3} \rightarrow \mathbb{C}^{3},
$$

acting on the generations. In agreement with our earlier notation, $\check{\pi}$ acts on $\mathbb{C}^{6}$ as the block-diagonal matrix

$$
\left(\begin{array}{cc}
\check{\pi} & 0 \\
0 & \check{\pi}
\end{array}\right): \mathbb{C}^{6} \rightarrow \mathbb{C}^{6}
$$

Likewise, $\check{\pi}$ may act on the left- and right-handed components. Then the operation in (4.7.6) can be realized by acting with $\check{\pi}$ from the left and from the right; for example

$$
\hat{\jmath}=9 \check{\pi} \mathscr{J} \check{\pi} .
$$

The most obvious idea is to insert a sectorial projection into 4.7.18,

$$
\operatorname{Re}(\bar{\psi} 3 \check{\pi}(i \not \partial+\mathcal{B}-m Y) \psi) .
$$

Then varying the metric gives the desired energy-momentum term $\hat{T}_{i j}$. However, when varying the chiral potential, the mixing matrix $U_{\text {MNS }}$ comes up in the wrong way. This leads us to also take the sectorial projection of $\mathcal{B}$. We thus choose

$$
\mathcal{L}_{\text {Dirac }}=\operatorname{Re}(\bar{\psi} 3 \check{\pi}(i \not \partial+\check{\pi} \mathcal{B} \check{\pi}-m Y) \psi) .
$$

Then varying the bosonic potentials also gives agreement with the sectorial projections of the factors $\hat{g}_{L}$ and $\hat{g}_{R}$ in 4.7 .9 . We point out that varying the Dirac wave functions in the Dirac action corresponding to 4.7.18) does not give the Dirac equation (4.7.18). This is not a general problem because, as explained in 4.7 .1 , in our approach the Dirac equation holds trivially as the defining equation for the bosonic potentials. Nevertheless, at first sight it might seem that the Dirac Lagrangian 4.7.18) should be inconsistent with the Dirac equation. In 4.7 .3 we will see that there are indeed no inconsistencies if the variations are handled properly.

There is one more modification which we want to implement in the Dirac Lagrangian 4.7.19). Namely, in order to have more freedom to modify the coupling of the right-handed neutrinos to the gravitational field, we insert a parameter $\tau$ into $\check{\pi}$ which modifies the left-handed component of the upper isospin component,

$$
\check{\pi}_{\tau}:=\left(\begin{array}{cc}
1+\tau \chi_{L} & 0 \\
0 & 1
\end{array}\right) \check{\pi} \quad \text { with } \tau \in \mathbb{R} .
$$

We define our final Dirac Lagrangian by

$$
\mathcal{L}_{\text {Dirac }}=\operatorname{Re}\left(\bar{\psi} 3 \check{\pi}_{\tau}(i \not \partial+\check{\pi} \mathcal{B} \check{\pi}-m Y) \psi\right) .
$$

If a left-handed gauge field $\mathcal{B}$ is varied, then the parameter $\tau$ drops out because the righthanded neutrinos do not couple to the chiral gauge fields. However, the parameter $\tau$ will make a difference when considering variations of the metric. We will come back to this point in Section 4.9 below.

The effective action is obtained as usual by adding to 4.7.20 suitable Lagrangians involving the chiral gauge field and scalar curvature. They will be worked out in detail in Sections 4.8 and 4.9 . 
4.7.3. Varying the Effective Dirac Action. We now explain how the effective action 4.7.12 with the Dirac Lagrangian 4.7.20 is to be combined with the Dirac equation (4.7.2) (or similarly the action 4.7.17) with the corresponding Dirac equation in the gravitational field).

We again point out that in our approach, the Dirac equation 4.7 .2 is trivially satisfied, because it serves as the definition of the bosonic potentials in $\mathcal{B}$. The bosonic potentials in $\mathcal{B}$ are merely a device for describing the behavior of the wave functions $\psi$ in the fermionic projector. With this concept in mind, the method of varying the bosonic potentials for fixed wave functions (as used after (4.7.12)) is not the proper procedure. The procedure is not completely wrong, because in many situations the wave functions do not change much when varying the bosonic potentials, and in these cases it is admissible to consider them as being fixed. But in general, it is not a consistent procedure to vary $\mathcal{B}$ for fixed $\psi$, because then the Dirac equation 4.7.2 will be violated. Taking the Dirac equation as the definition of $\mathcal{B}$, the only way to vary the bosonic potentials is to also vary the wave functions according to 4.7 .2 , and to consider the the effective Lagrangian under the resulting joint variations of $\mathcal{B}$ and $\psi$.

Let us compute such variations, for simplicity for a variation of the bosonic potential in Minkowski space (the method works similarly in the presence of a gravitational field and for variations of the metric).

Proposition 4.7.1. Varying the potential $\mathcal{B}$ in the Dirac action corresponding to the Dirac Lagrangian 4.7.20 under the constraint that the Dirac equation 4.7.2 holds, we obtain the first variation

$$
\begin{aligned}
& \delta \mathcal{S}_{\text {Dirac }}=\operatorname{Re} \int_{\mathscr{M}} \prec \psi \mid X_{\tau}(\delta \mathcal{B}) \psi \succ d^{4} x \\
& -\operatorname{Re} \int_{\mathscr{M}} \prec \psi \mid 3 \check{\pi}_{\tau}(\delta \mathcal{B})(\mathbb{1}-\check{\pi}) \psi \succ d^{4} x \\
& -\operatorname{Re} \int_{\mathscr{M}}\left(\prec \delta \psi\left|3 \check{\pi}_{\tau} \mathcal{B}(\mathbb{1}-\check{\pi}) \psi \succ+\prec \psi\right| 3 \check{\pi}_{\tau}(\mathbb{1}-\check{\pi}) \mathcal{B} \delta \psi \succ\right) d^{4} x \\
& \left.-\operatorname{Re} \int_{\mathscr{M}} \prec \psi \mid\left((\mathcal{B}-m Y) X_{\tau}^{*}-X_{\tau}(\mathcal{B}-m Y)\right) \delta \psi \succ\right) d^{4} x
\end{aligned}
$$

(where $\prec \psi \mid \phi \succ \equiv \bar{\psi} \phi$ denotes the spin scalar product). Here $X_{\tau}$ is the matrix

$$
X_{\tau}=\left(\begin{array}{cc}
1+\tau \chi_{L} & 0 \\
0 & 1
\end{array}\right) \otimes \mathbb{1}_{\mathbb{C}^{3}}
$$

and the variation of the wave function $\delta \psi$ is given by

$$
\delta \psi=-\tilde{s}(\delta \mathcal{B}) \psi,
$$

where $\tilde{s}$ is a Green's function of the Dirac equation 4.7.2,

$$
(i \not \partial+\mathcal{B}-m Y) \tilde{s}=\mathbb{1} .
$$

Proof. Let $\delta B$ be the variation of $\mathcal{B}$. In order to satisfy the Dirac equation, we must vary the wave function according to 4.7.26. The variation of the Dirac wave function does not have compact support, making it necessary to take into account boundary terms when integrating by parts. In order to treat these boundary terms properly, we multiply the variation of the wave function by a test function $\eta \in C_{0}^{\infty}(\mathscr{M})$. Thus instead of 4.7.26 we consider the variation

$$
\widetilde{\delta \psi}=-\eta \tilde{s}(\delta \mathcal{B}) \psi \text {. }
$$


At the end, we will remove the test function by taking the limit $\eta \rightarrow 1$ in which $\eta$ goes over to the function constant one.

The resulting variation of the Dirac action is computed by

$$
\begin{aligned}
\delta \mathcal{S}_{\text {Dirac }}= & \int_{\mathscr{M}} \delta \mathcal{L}_{\text {Dirac }} d^{4} x=\operatorname{Re} \int_{\mathcal{M}}\left(\prec \widetilde{\delta \psi} \mid 3 \check{\pi}_{\tau}(i \not \partial+\check{\pi} \mathcal{B} \check{\pi}-m Y) \psi \succ\right. \\
& \left.+\prec \psi\left|3 \check{\pi}_{\tau}(\delta(\check{\pi} \mathcal{B} \check{\pi})) \psi \succ+\prec \psi\right| 3 \check{\pi}_{\tau}(i \not \partial+\check{\pi} \mathcal{B} \check{\pi}-m Y) \widetilde{\delta \psi} \succ\right) d^{4} x .
\end{aligned}
$$

Using that $\psi$ satisfies the Dirac equation, and that $\widetilde{\delta \psi}$ satisfies the inhomogeneous Dirac equation

$$
(i \not \partial+\mathcal{B}-m Y) \widetilde{\delta \psi}=-i(\not \partial \eta) \tilde{s}(\delta \mathcal{B}) \psi-\eta(\delta \mathcal{B}) \psi
$$

we obtain

$$
\begin{aligned}
\delta \mathcal{S}_{\text {Dirac }}= & \operatorname{Re} \int_{\mathscr{M}} \eta\left(\prec \delta \psi\left|3 \check{\pi}_{\tau}(\check{\pi} \mathcal{B} \check{\pi}-\mathcal{B}) \psi \succ+\prec \psi\right| 3 \check{\pi}_{\tau}(\check{\pi} \mathcal{B} \check{\pi}-\mathcal{B}) \delta \psi \succ\right) d^{4} x \\
& +\operatorname{Re} \int_{\mathscr{M}}\left(\prec \psi\left|3 \check{\pi}_{\tau}(\delta(\check{\pi} \mathcal{B} \check{\pi})-\eta(\delta \mathcal{B})) \psi \succ-i \prec \psi\right| 3 \check{\pi}_{\tau}(\not \partial \eta)(\tilde{s}(\delta \mathcal{B}) \psi) \succ\right) d^{4} x .
\end{aligned}
$$

In the last term we decompose the matrix $3 \check{\pi}_{\tau}$ into its diagonal and off-diagonal parts,

$$
3 \check{\pi}_{\tau}=X_{\tau}+Z \quad \text { with } \quad Z=3 \check{\pi}-\mathbb{1}_{\mathbb{C}^{3}}
$$

and $X_{\tau}$ according to 4.7.25. Thus, using 4.7.26,

$$
\begin{aligned}
& -i \int_{\mathcal{M}} \prec \psi \mid 3 \check{\pi}_{\tau}(\not \partial \eta)(\tilde{s}(\delta \mathcal{B}) \psi) \succ d^{4} x \\
& \quad=i \int_{\mathcal{M}}\left(\partial_{j} \eta\right) \prec \psi\left|X_{\tau} \gamma^{j} \delta \psi \succ d^{4} x+i \int_{\mathscr{M}}\left(\partial_{j} \eta\right) \prec \psi\right| Z \gamma^{j} \delta \psi \succ d^{4} x .
\end{aligned}
$$

In the first integral we integrate by parts,

$$
\begin{aligned}
& i \int_{\mathscr{M}}\left(\partial_{j} \eta\right) \prec \psi \mid X_{\tau} \gamma^{j} \delta \psi \succ d^{4} x \\
& \quad=\int_{\mathscr{M}} \eta\left(\prec i \partial_{j} \psi\left|X_{\tau} \gamma^{j} \delta \psi \succ-\prec \psi\right| X_{\tau} \gamma^{j} i \partial_{j}(\delta \psi) \succ\right) d^{4} x .
\end{aligned}
$$

The pseudoscalar matrix in $X_{\tau}$ anti-commutes with the Dirac matrix $\gamma^{j}$. Since the pseudoscalar matrix is anti-symmetric with respect to the spin scalar product, we can express this anti-commutation by

$$
X_{\tau} \gamma^{j}=\gamma^{j} X_{\tau}^{*}
$$

Then we can rewrite the partial derivatives $i \partial_{j}$ with the Dirac equation 4.7.2) and the inhomogeneous Dirac equation equation for $\delta \psi$,

$$
(i \not \partial+\mathcal{B}-m Y) \delta \psi=-(\delta \mathcal{B}) \psi .
$$


This gives

$$
\begin{aligned}
& i \int_{\mathcal{M}}\left(\partial_{j} \eta\right) \prec \psi \mid X_{\tau} \gamma^{j} \delta \psi \succ d^{4} x \\
& =\int_{\mathscr{M}} \eta\left(\prec i \not \partial \psi\left|X_{\tau}^{*} \gamma^{j} \delta \psi \succ-\prec \psi\right| X_{\tau} i \not \partial(\delta \psi) \succ\right) d^{4} x \\
& =-\int_{\mathcal{M}} \eta \prec \psi \mid\left((\mathcal{B}-m Y) X_{\tau}^{*}-X_{\tau}(\mathcal{B}-m Y)\right)(\delta \psi) \succ d^{4} x \\
& \quad+\int_{\mathscr{M}} \eta \prec \psi \mid X_{\tau}(\delta \mathcal{B}) \psi \succ d^{4} x .
\end{aligned}
$$

Combining all the terms, we obtain

$$
\begin{aligned}
& \delta \mathcal{S}_{\text {Dirac }}=\operatorname{Re} \int_{\mathcal{M}} \eta\left(\prec \delta \psi\left|3 \check{\pi}_{\tau}(\check{\pi} \mathcal{B} \check{\pi}-\mathcal{B}) \psi \succ+\prec \psi\right| 3 \check{\pi}_{\tau}(\check{\pi} \mathcal{B} \check{\pi}-\mathcal{B}) \delta \psi \succ\right) d^{4} x \\
& +\operatorname{Re} \int_{\mathcal{M}} \prec \psi \mid 3 \check{\pi}_{\tau}(\delta(\check{\pi} \mathcal{B} \check{\pi})-\eta(\delta \mathcal{B})) \psi \succ d^{4} x \\
& +\operatorname{Re} \int_{\mathscr{M}} i\left(\partial_{j} \eta\right) \prec \psi \mid Z \gamma^{j} \delta \psi \succ d^{4} x \\
& -\operatorname{Re} \int_{\mathcal{M}} \eta \prec \psi \mid\left((\mathcal{B}-m Y) X_{\tau}^{*}-X_{\tau}(\mathcal{B}-m Y)\right)(\delta \psi) \succ d^{4} x \\
& +\operatorname{Re} \int_{\mathscr{M}} \eta \prec \psi \mid X_{\tau}(\delta \mathcal{B}) \psi \succ d^{4} x .
\end{aligned}
$$

Now we may take the limit $\eta \rightarrow 1$. In this limit, the integral $(4.7 .29)$ goes to zero, as will be justified in Lemma 4.7 .2 below. Rearranging the terms using the relation $\check{\pi}_{\tau} \check{\pi}=\check{\pi}_{\tau}$ gives the result.

We now explain why the integral (4.7.29) tends to zero if $\eta \rightarrow 1$. Since this is a rather subtle point, we give the details. However, for technical simplicity we assume that the bosonic potential has compact support. The result could be extended in a straightforward manner to the case that the potential has suitable decay properties at infinity by estimating the Lippmann-Schwinger equation (we refer the interested reader to the exposition in [FKT] and to similar methods in [FMR]).

Lemma 4.7.2. Assume that the fermion masses are different in the generations, i.e.

$$
m_{\alpha} \neq m_{\beta} \quad \text { and } \quad \tilde{m}_{\alpha} \neq \tilde{m}_{\beta} \quad \text { for all } \alpha, \beta \in\{1,2,3\} \text { and } \alpha \neq \beta .
$$

Moreover, assume that the potential $\mathcal{B}$ and its variation $\delta \mathcal{B}$ are smooth and have compact support, and that $\psi$ is smooth. Then for any test function $\eta \in C_{0}^{\infty}\left(\mathbb{R}^{4}\right)$ which is constant in a neighborhood of the origin,

$$
\lim _{L \rightarrow \infty} \int_{\mathcal{M}} \prec \psi \mid Z \gamma^{j}(\tilde{s}(\delta \mathcal{B}) \psi) \succ \frac{\partial}{\partial x^{j}} \eta\left(\frac{x}{L}\right) d^{4} x=0 .
$$

Proof. By choosing $L$ sufficiently large, we can arrange that $\eta$ is constant on the support of $\mathcal{B}$ and $\delta B$. Then we may replace $\psi$ and $\phi:=\tilde{s}(\delta \mathcal{B}) \psi$ by smooth solutions of the vacuum Dirac equation $(\not \not \partial-m Y) \psi=0=(i \not \partial-m Y) \phi$. Since the matrix $\mathbb{1}-3 \check{\pi}_{\tau}$ vanishes on the diagonal and only mixes the wave functions within each sector, the integral 4.7.32 can be rewritten as a finite sum of integrals of the form

$$
\int_{\mathscr{M}} \prec \psi_{\alpha} \mid \gamma^{j} \phi_{\beta} \succ \partial_{j} \eta_{L}(x) d^{4} x
$$


where $\eta_{L}(x):=\eta(x / L)$, and where $\psi_{\alpha}$ and $\phi_{\beta}$ are solutions of the Dirac equation for different masses,

$$
\left(i \not \partial-m_{\alpha}\right) \psi_{\alpha}=0=\left(i \not \partial-m_{\beta}\right) \phi_{\beta} \quad \text { and } m_{\alpha} \neq m_{\beta} .
$$

Writing the solutions as distributions in momentum space,

$$
\hat{\psi}_{\alpha}(k)=f(k) \delta\left(k^{2}-m_{\alpha}^{2}\right), \quad \hat{\phi}_{\beta}(k)=g(k) \delta\left(k^{2}-m_{\beta}^{2}\right),
$$

the smoothness of $\psi_{\alpha}$ and $\phi_{\beta}$ implies that the functions $f$ and $g$ can be chosen to have rapid decay. Then the integral in 4.7 .33 can be rewritten in momentum space as

$$
-i \int_{\hat{\mathscr{M}}} \prec \hat{\psi}_{\alpha} \mid \not k\left(\hat{\eta}_{L} * \hat{\phi}_{\beta}\right) \succ \frac{d^{4} k}{(2 \pi)^{4}}
$$

where the star denotes the convolution of the distribution $\hat{\phi}_{\beta}$ with the test function $\hat{\eta}_{L}$ giving a Schwartz function (note that the smoothness of $\eta_{L}$ implies that $\hat{\eta}_{L}$ has rapid decay), and the integral is to be understood that the distribution $\hat{\psi}_{\alpha}$ is applied to this Schwartz function. Since the functions $f$ and $g$ have rapid decay, for any $\varepsilon>0$ there is a compact set $K \subset \hat{M}$ such that

$$
\left|\int_{\hat{M} \backslash K} \prec \hat{\psi}_{\alpha}\right| \not k\left(\hat{\eta}_{L} * \hat{\phi}_{\beta}\right) \succ d^{4} k \mid<\varepsilon,
$$

uniformly in $L$. For any fixed $K$, the supports of the distributions $\delta\left(k^{2}-m_{\alpha}^{2}\right)$ and $\delta\left(k^{2}-\right.$ $m_{\beta}^{2}$ ) have a finite separation (measured in the Euclidean norm on $\mathbb{R}^{4}$ in a chosen reference frame). Since $\hat{\eta}_{L}(k)=L^{4} \hat{\eta}(L k)$, by increasing $L$ we can arrange that the function $\hat{\eta}_{L}$ decays on a smaller and smaller scale. Since $\hat{\eta}$ has rapid decay, this implies that the integral over $K$ tends to zero,

$$
\lim _{L \rightarrow \infty} \int_{K} \prec \hat{\psi}_{\alpha} \mid \not k\left(\hat{\eta}_{L} * \hat{\phi}_{\beta}\right) \succ d^{4} k=0 .
$$

Since $\varepsilon$ is arbitrary, the result follows.

Combining the result of Proposition 4.7.1 with the variation of the Yang-Mills Lagrangian in 4.7.12) (which can be computed in the standard way, see Section 4.8), one obtains field equations describing the dynamics of the chiral gauge field and its coupling to the Dirac particles and anti-particles. Together with the Dirac equation (4.7.2), one obtains a consistent set of equations which we regard as the effective EL-equations in the continuum limit.

Let us discuss the structure of the resulting field equations: The first term 4.7.21 differs from the standard contribution obtained by varying the bosonic potential in the Dirac Lagrangian 4.7 .20 by the fact that the sectorial projection has disappeared. This is desirable because the resulting contribution looks very much like the variation of the standard Lagrangian (4.7.18). The only difference is the additional factor $X_{\tau}$. However, this factor comes into play only if one considers gauge fields which couple to the righthanded neutrinos. Such gauge fields will be ruled out in the present paper. They also do not appear in the standard model. Therefore, the factor $X_{\tau}$ in 4.7.21 seems consistent with observations.

The terms 4.7.22)-4.7.24) are additional contributions which are absent in the standard Lagrangian formulation. They can be understood as corrections which are needed in order to get consistency with the Dirac equation 4.7.2). We refer to the terms 4.7.22 4.7.24 as the sectorial corrections to the field equations. The term 4.7.22 modifies the 
coupling of those chiral gauge potentials which involve a non-trivial mixing matrix. The correction (4.7.23) can be understood similarly. As a difference, it involves the Green's function $\tilde{s}$ and is therefore nonlocal (we note that the choice of the Green's function $\tilde{s}$ in (4.7.26) is uniquely determined by the causal perturbation expansion FT2; see also Section 2.1). The correction term 4.7.24 is also nonlocal and comes into play when the neutrinos are massive. The appearance of these nonlocal correction terms are a prediction of the fermionic projector approach. It is conceivable that these corrections are testable in experiments. More specifically, the corrections vanish if the mixing matrices do not come into play and if the Dirac wave functions are eigenstates of the mass matrix. Thinking of the analogous situation for the standard model, the corrections vanish for example for electrons with an electromagnetic interaction. However, they come play in an interaction via $W$-bosons if the wave function $\psi$ is a non-trivial superposition of for example an electron and a muon. The detailed mechanism triggered by the nonlocal effects is unclear and still needs to investigated. All we can say for the moment is that the corrections 4.7.23 and (4.7.24) cease to play any role as soon as the Dirac Lagrangian no longer involves cross terms of electrons and muons.

We finally point out that the effective action cannot be regarded as some kind of "continuum limit" of the causal action principle. It is merely a method for recovering the EL equations corresponding to the causal action principle in the continuum limit from an effective variational principle. The basic difference of the causal action principle and the effective action can be understood already from the fact that the causal action is minimized, whereas for the effective action one only seeks for critical points. Thus the effective action should be regarded merely as a convenient method for getting the connection to the standard Lagrangian formalism. In particular, by applying Noether's theorem to the effective action, one can immediately deduce conservation laws for the effective EL equations.

\subsection{The Field Equations for Chiral Gauge Fields}

We now use the methods of Section 4.7 to compute the effective action for the coupling of the Dirac field to the gauge fields. In order to determine the dynamical gauge algebra $\mathfrak{g} \subset \mathfrak{S}_{6}$ (defined before (4.7.7)), we first recall that in $\$ 4.3 .2$ we derived the admissible gauge group (4.3.28) together with the representation of the gauge potentials 4.3 .29 ). In Section 4.6, we obtained further restrictions for the gauge potentials. Namely, the analysis of the bilinear logarithmic terms in 4.6 .1 revealed that the diagonal elements must satisfy the constraint 4.6.1). The field tensor terms in $\$ 4.6 .2$, on the other hand, gave us the two linear constraints 4.6 .39 for the field tensor, which due to gauge symmetry we can also regard as constraints for the potentials. Putting these conditions together, we conclude that the dynamical gauge potentials must be of one of the two alternative forms

$$
\begin{aligned}
& \mathcal{B}=\chi_{R}\left(\begin{array}{cc}
A_{L}^{11} & 0 \\
0 & 0
\end{array}\right)+\chi_{L}\left(\begin{array}{cc}
0 & 0 \\
0 & -A_{L}^{11}
\end{array}\right) \text { or } \\
& \mathcal{B}=\chi_{R}\left(\begin{array}{cc}
A_{L}^{11} & A_{L}^{12} U_{\mathrm{MNS}}^{*} \\
A_{L}^{21} U_{\mathrm{MNS}} & -\mathscr{A}_{L}^{11}
\end{array}\right)+\chi_{L}\left(\begin{array}{ll}
0 & 0 \\
0 & 0
\end{array}\right) .
\end{aligned}
$$

The potentials of the form 4.8.1) do not form a Lie algebra (because taking a commutator, the resulting potential has the same sign on the two isospin components, in contradiction to (4.8.1)). This means that the structure of 4.8.1) is not preserved under local gauge 
transformations corresponding to the potentials of the form 4.8.1). As this seems to be inconsistent, we disregard this case. We thus restrict attention to the remaining case 4.8.2), where $\mathfrak{g}$ is the Lie algebra $\mathrm{su}(2)$, which acts on the left-handed component of the spinors and involves the MNS mixing matrix.

Using the results of Section 4.4, it is straightforward to compute the right side of 4.7.11). Namely, applying Lemma 4.4.4 together with 4.4.22 and 4.7.10 (and using that $\delta A_{c}$ is traceless), for the right side of (4.7.11) we obtain the contribution

$$
\operatorname{Tr}_{\mathbb{C}^{2}}\left(\mathcal{Q}_{R}^{k}[\hat{\jmath}, \mathcal{A}]\left(\delta \hat{A}_{L}\right)_{k}\right) \asymp K_{1} \operatorname{Tr}_{\mathbb{C}^{2}}\left(J_{L}^{k}\left(\delta \hat{A}_{L}\right)_{k}\right) .
$$

This is compatible with the variation of the Dirac Lagrangian 4.7.20) (for fixed wave functions) if we choose

$$
K(\varepsilon, \xi)=3 K_{1}
$$

It is worth pointing out that this compatibility involves both the fact that the left-handed gauge potentials couple only to the left-handed component of the Dirac current and also that only the sectorial projection of the potentials and currents appears. In particular, our method would fail if 4.4 .22 involved the left-handed component of the Dirac current.

The contributions by the bosonic current and mass terms as listed in 4.4.22 - 4.4.29) are a bit more difficult to handle because the logarithmic poles must be removed with the help of the microlocal chiral transformation (see Proposition 4.4.7). If this is done, the resulting contributions have a rather complicated form. However, the general structure is easy to understand: First, writing the $\mathrm{SU}(2)_{L}$-gauge potentials in 4.3 .29 in components

$$
A_{L}^{\alpha}=\frac{1}{2} \operatorname{Tr}\left(\sigma^{\alpha} A_{L}\right)
$$

it is obvious from the symmetries that all contributions involving $A_{L}^{\alpha} \cdot \delta A_{L}^{\beta}$ and $j_{L}^{\alpha} \cdot \delta A_{L}^{\beta}$ with $\alpha \neq \beta$ vanish. Second, in view of the symmetry under relative phase transformations of the isospin components

$$
\psi \rightarrow\left(\begin{array}{cc}
e^{i \varphi} & 0 \\
0 & e^{-i \varphi}
\end{array}\right) \psi
$$

the contributions involving $A_{L}^{\alpha} \cdot \delta A_{L}^{\alpha}$ and $j_{L}^{\alpha} \cdot \delta A_{L}^{\alpha}$ coincide for $\alpha=1$ and $\alpha=2$. Hence the equation 4.7.11 can be satisfied for a bosonic Lagrangian of the form

$$
\begin{aligned}
\mathcal{L}_{\mathrm{YM}}=a_{1} & \left(\left(\partial_{j} A_{L}^{1}\right)\left(\partial^{j} A_{L}^{1}\right)+\left(\partial_{j} A_{L}^{2}\right)\left(\partial^{j} A_{L}^{2}\right)\right)+a_{3}\left(\partial_{j} A_{L}^{3}\right)\left(\partial^{j} A_{L}^{3}\right) \\
+ & \left.b_{1}\left(\left(A_{L}^{1}\right)^{2}+\left(A_{L}^{2}\right)\right)+b_{3}\left(A_{L}^{3}\right)^{2}\right)
\end{aligned}
$$

and suitable constants $a_{1}, a_{2}$ and $b_{1}, b_{3}$. We thus obtain the following result.

THEOREM 4.8.1. Expressing the $\mathrm{SU}(2)_{L}$-gauge potentials in Pauli matrices acting on the isospin (4.8.3) (and similarly for the currents), the field equations read

$$
j_{L}^{\alpha}-M_{\alpha}^{2} A_{L}^{\alpha}=c_{\alpha} J_{L}^{\alpha}+\left(f_{[0]} * j_{L}\right)^{\alpha}+\left(f_{[2]} * A_{L}\right)^{\alpha},
$$

where $j_{L}$ and $J_{R}$ are the currents 4.4.20 and 4.4.21, respectively. The mass parameters $M_{\alpha}$ and the coupling constants $c_{\alpha}$ satisfy the relations

$$
M_{1}=M_{2} \quad \text { and } \quad c_{1}=c_{2} .
$$

Finally, the distributions $f_{[0]}$ and $f_{[2]}$ are convolution kernels.

The convolution kernels take into account the following corrections: 
- The corrections due to the smooth, noncausal contributions to the fermionic projector. These corrections include the vacuum polarization due to the fermion loops. These corrections are discussed further in 3.8.1-33.8.3.

- The corrections due to the microlocal chiral transformation (see the last paragraph in $\$ 3.7 .10$ ).

- The sectorial corrections (see Proposition 4.7.1 and the explanation after the proof of Lemma 4.7.2.

Qualitatively speaking, this theorem can be understood similar to the results in Chapter 3. Also, the calculations use exactly the same methods. The appearance of bosonic masses and the connection to a spontaneous breaking of the gauge symmetry is explained in 33.8 .5 . In particular, the convolution kernels $f_{[0]}$ and $f_{[2]}$ are computed and interpreted just as in $\$ 3.8 .1$ and $\$ 3.8 .2$. In view of these similarities, we here omit the detailed computations and only point to two steps in the computations which are not quite straightforward. First, as mentioned after Proposition 4.4.6, the constants $\mathfrak{c}_{0}$ and $\mathfrak{c}_{2}$ are not determined by this proposition. Following the strategy used in $\$ 3.7 .9$, we can fix these constants by minimizing $\mathfrak{c}_{0}$. Thus we choose the microlocal chiral transformation in such a way that the vectorial contribution 4.4.48 to the fermionic projector is as small as possible. Using this method, for a given regularization one can also compute the coupling constants and the masses similar as in 3.8 .6 .

The second step which requires an explanation concerns the computation of the coupling constants and bosonic masses for a given regularization in the spirit of \$3.8.6. Here one must distinguish the two Cases (i) and (ii) in (4.3.36). Which of these cases applies depends crucially on the choice of the parameter $p_{\text {reg }}$ in (4.3.34). In particular, by choosing $p_{\text {reg }}$ sufficiently small (and thus the parameter $\tau_{\text {reg }}$ in (4.2.42) sufficiently large), we can arrange that we are in Case (ii). In order to keep the setting as general as possible, we deliberately left open which of the cases should be physically relevant. We found that all our computations up to and including Section 4.4 apply in the same way in both cases. In the analysis of the bilinear logarithmic terms in 4.6.1, however, our constructions apply in Case (i) only under the additional assumption (4.6.2). The analysis of the field tensor terms in 4.6.2 was carried out only in Case (ii) (and at present it is unclear how the results could be extended to Case (i)). This gives a strong indication that the physically relevant scaling should indeed be described by Case (ii). This scaling can be realized by choosing the parameter $p_{\text {reg }}$ in 4.3 .34 sufficiently small. Thus in a physical model, the the parameter $\tau_{\text {reg }}$ in 4.2 .42 should be chosen sufficiently large.

Arranging in this way that we are in Case (ii), it remains to justify the transition from the EL equations (4.4.7) to the stronger conditions 4.4.12). We already indicated an argument before Corollary 4.4.2. We are now in the position to make this argument precise: Recall that in Case (ii), the spectral projectors $I_{n}$ are isospin-diagonal (4.3.44). The perturbation of these spectral projectors by the gauge phases leads to a finite hierarchy of equations to be satisfied in a weak evaluation on the light cone. With this in mind, it suffices to satisfy 4.4.7) with $I_{n}$ according to 4.3.44). But clearly, we must take into account that the gauge phases enter the matrices $\mathcal{K}_{n c}$, as we now explain. We begin with the Dirac current terms. As the left-handed component of a wave function is modified by the gauge phases in the obvious way by

$$
\chi_{L} \psi(y) \rightarrow \chi_{L} \exp \left(-i \int_{x}^{y} A_{L}^{j} \xi_{j}\right) \psi(y)
$$


the gauge potential enters the Dirac current term as described by the replacement

$$
J_{L} \rightarrow\left(1-i A_{L}^{j}\left(\frac{x+y}{2}\right) \xi_{j}\right) J_{L}
$$

In this way, the off-diagonal components of the Dirac current enter the diagonal matrix entries of $\mathcal{K}_{n}$ and thus the EL equations (4.4.7). Since the gauge currents have the same behavior under gauge transformations (see (4.6.3)), their off-diagonal elements enter the EL equations in the same way. For the mass terms, there is the complication that they have a different behavior under gauge transformations (for the logarithmic terms, this was studied in (4.6.17), whereas for the contributions of the second order perturbation calculation, the dependence on the gauge phases can be read off from the formulas given in [F7, Definition 7.2.1]). This different behavior under gauge transformation does not cause problems for the logarithmic poles, because we saw in 4.6 .1 that the logarithmic poles on the light cone can be arranged to vanish. Thus the only effect of the different gauge behavior of the mass terms is that it modifies the values of the bosonic mass corresponding to the off-diagonal gauge potentials. The easiest method to describe this effect quantitatively is to again work with the EL equations (4.4.12), but to modify the off-diagonal matrix elements of $\mathcal{K}_{L}$ and $\mathcal{K}_{R}$ by multiplying the contributions (4.4.23)4.4.29 with numerical factors which take into account the linear behavior under offdiagonal left-handed gauge transformations. It is planned to work out the masses and coupling constants for a specific example of an admissible regularization in a separate publication.

One might ask whether all coupling constants and masses in Theorem 4.8.1 should be the same, i.e. if also

$$
M_{1}=M_{3} \quad \text { and } \quad c_{1}=c_{3} .
$$

Indeed, the contribution by the bosonic current to $\mathcal{Q}_{L}$ in 4.4 .22 suggests that the derivative terms in the bosonic Lagrangian can be written as

$$
\operatorname{Tr}_{\mathbb{C}^{2}}\left(\left(\partial_{j} \hat{A}_{L}\right)\left(\partial^{j} \hat{A}_{L}\right)\right) \text {. }
$$

However, since the microlocal chiral transformation involves the masses of the Dirac particles, which may be different in the two isospin components, there is no reason why the more elegant form 4.8.4 should be preserved when the microlocal chiral transformation is taken into account. For the mass terms, on the other hand, it is obvious from 4.4.23 4.4.29 that the masses of the Dirac particles are involved. Thus again, there is no reason why there should be a simple relation between the masses $M_{1}$ and $M_{3}$.

\subsection{The Einstein Equations}

Our first task is to compute the symmetric tensor $\mathcal{Q}^{k l}$ as defined by 4.7.14). If we used the form of $\Delta Q$ in Corollary 4.4.2, we would get zero, because

$$
\sum_{n, c} \operatorname{Tr}_{\mathbb{C}^{2}}\left(I_{n} \mathcal{Q}_{c}\right) \operatorname{Tr}_{\mathbb{C}^{2}} I_{n}=\sum_{n, c} \operatorname{Tr}_{\mathbb{C}^{2}}\left(I_{n} \mathcal{Q}_{c}\right)=\sum_{c} \operatorname{Tr}_{\mathbb{C}^{2}}\left(\mathcal{Q}_{c}\right)=0
$$

where in the last step we used 4.4.14). This means that in order to compute 4.7.13), we need to evaluate (4.4.4) to higher order in $(m \varepsilon)^{p_{\text {reg }}}$ (note that these contributions were neglected in 4.4 .1 according to 4.4 .2 ).

Expanding (4.4.4) to higher oder in powers of $(m \varepsilon)^{p_{\text {reg }}}$ is a bit subtle because there might be contributions to $\Delta\left|\lambda_{n c s}^{x y}\right|$ which are linear in $(m \varepsilon)^{p_{\text {reg }}}$ but do not involve curvature. In this case, we would have to take into account the effect of curvature on the 
factors

$$
\frac{\overline{\lambda_{n c s}^{x y}}}{\left|\lambda_{n c s}^{x y}\right|} F_{n c s}^{x y} P(x, y)
$$

in (4.4.4). The resulting contributions to $Q(x, y)$ would not be proportional to $\$$, giving rise to additional equations to be satisfied in the continuum limit. Moreover, we would have to take into account the effect of the Dirac and bosonic currents to (4.9.1), giving rise to even more equations to be satisfied in the continuum limit. For this reason, we must assume that our regularization is such that in the vacuum, the quantities $\left|\lambda_{n c s}^{x y}\right|$ coincide pointwise up to the order $(m \varepsilon)^{2 p_{\text {reg }}}$. Such a regularization condition was already imposed in 4.6.38). Now we need to complement it by a similar condition for the upper index minus one,

$$
\left|L_{[0]}^{(-1)}\right|=\left|T_{[0]}^{(-1)}\right|\left(1+\mathcal{O}\left((m \varepsilon)^{2 p_{\text {reg }}}\right)\right) \quad \text { pointwise } .
$$

We note that this is compatible with 4.3.35) and poses an additional condition on the regularization in the case $n=-1$ and $p=0$. Similar as explained after (4.6.38), the pointwise condition 4.9.2 could be replaced by a number of conditions to be satisfied in a weak evaluation on the light cone, but we do not enter this analysis here. Generally speaking, the conditions (4.6.38) and 4.9.2 seem to indicate that the right-handed neutrino states should affect the factors $L_{[0]}^{(-1)}$ and $L_{[0]}^{(0)}$ only by phase factors, up to errors of the order $\mathcal{O}\left((m \varepsilon)^{2 p_{\text {reg }}}\right)$.

Lemma 4.9.1. Assume that the regularization has the properties 4.9.2) and 4.6.38). Then the energy-momentum tensor gives the following contribution to $\mathcal{Q}^{k l}$,

$$
\begin{aligned}
\mathcal{Q}^{k l} \asymp & \frac{1}{2} K_{8}\left\{\left(\left(\hat{T}_{L}^{k l}\right)_{1}^{1}-3\left(\hat{T}_{R}^{k l}\right)_{1}^{1}+\left(\hat{T}_{L}^{k l}\right)_{2}^{2}+\left(\hat{T}_{R}^{k l}\right)_{2}^{2}\right)\right. \\
& \left.+\frac{L_{[0]}^{(0)}}{T_{[0]}^{(0)}}\left(-\left(\hat{T}_{L}^{k l}\right)_{1}^{1}+3\left(\hat{T}_{R}^{k l}\right)_{1}^{1}-\left(\hat{T}_{L}^{k l}\right)_{2}^{2}-\left(\hat{T}_{R}^{k l}\right)_{2}^{2}\right)\right\} \\
& +\mathcal{O}\left((m \varepsilon)^{2 p_{\mathrm{reg}}}\right)(\operatorname{deg}=4)+(\operatorname{deg}<4) .
\end{aligned}
$$

Proof. The matrices $\mathcal{K}_{L}$ and $\mathcal{K}_{R}$ were computed in Lemma 4.5.1. Substituting these formulas into the representation of Corollary 4.4.2 and computing the trace in 4.7.13 gives zero. More generally, one sees from 4.4.4 that the trace in 4.7.13 vanishes no matter what the perturbations of the eigenvalues $\Delta \lambda_{n c s}^{x y}$ are, provided that we approximate the last term in (4.4.4) by its leading asymptotics on the light cone

$$
\begin{aligned}
\frac{\overline{\lambda_{n c s}^{x y}}}{\left|\lambda_{n c s}^{x y}\right|} F_{n c s}^{x y} P(x, y)=\delta_{s-} & \frac{i}{2} \frac{T_{[0]}^{(0)} \overline{T_{[0]}^{(-1)}}}{\left|T_{[0]}^{(0)} T_{[0]}^{(-1)}\right|} g T_{[0]}^{(-1)} I_{n} \chi_{c} \sharp+(\operatorname{deg}<4) \\
& +\left(\text { higher orders in } \varepsilon / \ell_{\text {macro }} \text { and }(m \varepsilon)^{p_{\text {reg }}}\right),
\end{aligned}
$$

where for clarity we wrote out the error terms in 4.4 .2 . Therefore, it suffices to compute the correction to 4.9 .3 to next order in $(m \varepsilon)^{p_{\text {reg }}}$. To this end, we must carefully distinguish between factors $T_{[p]}^{(n)}$ and $L_{[p]}^{(n)}$, similar as done in 4.3.13-4.3.15. A straightforward calculation using (4.9.2) and 4.6.38) gives the result. 
Lemma 4.9.2. Curvature gives the following contribution to $\mathcal{Q}^{k l}$,

$$
\begin{aligned}
\mathcal{Q}^{k l} \asymp & \frac{\tau_{\text {reg }}}{2 \delta^{2}} R^{k l} K_{16}\left(1-\frac{L_{[0]}^{(0)}}{T_{[0]}^{(0)}}\right) \\
& +\mathcal{O}\left((m \varepsilon)^{2 p_{\text {reg }}}\right)(\operatorname{deg}=4)+o\left(|\vec{\xi}|^{-4}\right)+(\operatorname{deg}<4) .
\end{aligned}
$$

Proof. The matrices $\mathcal{K}_{L}$ and $\mathcal{K}_{R}$ were computed in Lemma 4.5.2. Again, substituting these formulas into the representation of Corollary 4.4 .2 and computing the trace in 4.7.13 gives zero. Therefore, just as in the proof of Lemma 4.9.1, we need to take into

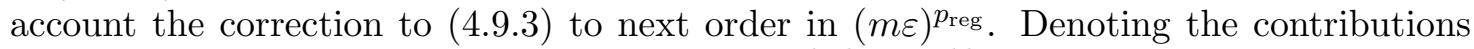
to $\mathcal{K}_{L / R}$ in Lemma 4.5.2 leaving out the factors $\xi^{k} \xi^{l}$ by $\mathcal{K}_{L / R}^{k l}$, we thus obtain (cf. 4.4.13), (4.4.14) and (4.7.14))

$$
\begin{aligned}
\mathcal{Q}^{k l} \asymp & \frac{1}{2}\left\{\left(\left(\mathcal{K}_{L}^{k l}\right)_{1}^{1}-3\left(\mathcal{K}_{R}^{k l}\right)_{1}^{1}+\left(\mathcal{K}_{L}^{k l}\right)_{2}^{2}+\left(\mathcal{K}_{R}^{k l}\right)_{2}^{2}\right)\right. \\
& \left.+\frac{L_{[0]}^{(0)}}{T_{[0]}^{(0)}}\left(-\left(\mathcal{K}_{L}^{k l}\right)_{1}^{1}+3\left(\mathcal{K}_{R}^{k l}\right)_{1}^{1}-\left(\mathcal{K}_{L}^{k l}\right)_{2}^{2}-\left(\mathcal{K}_{R}^{k l}\right)_{2}^{2}\right)\right\} \\
& +\mathcal{O}\left((m \varepsilon)^{2 p_{\text {reg }}}\right)(\operatorname{deg}=4)+(\operatorname{deg}<4) .
\end{aligned}
$$

The term 4.5.6) drops out everywhere. Computing the contribution by (4.5.7) gives the result.

The next step is to satisfy 4.7.15). In fact, the results of the previous lemmas are compatible with 4.7.15 if we choose the parameter $\tau$ in the Dirac Lagrangian 4.7.20 as

$$
\tau=-4
$$

and the Lagrangian $\mathcal{L}_{\mathrm{EH}}$ according to 4.7.16) with

$$
\kappa=\frac{\delta^{2}}{\tau_{\text {reg }}} \frac{K_{17}}{K_{18}}
$$

where $K_{17}$ and $K_{18}$ are the composite expressions

$$
K_{17}=\frac{1}{2} K_{16}\left(1-\frac{L_{[0]}^{(0)}}{T_{[0]}^{(0)}}\right) \quad \text { and } \quad K_{18}=\frac{1}{2} K_{8}\left(1-\frac{L_{[0]}^{(0)}}{T_{[0]}^{(0)}}\right)
$$

(which are both to be evaluated weakly on the light cone 4.2.31)). These findings are summarized as follows.

ThEOREM 4.9.3. Assume that the parameters $\delta$ and $p_{\text {reg }}$ satisfy the scaling (4.5.5), and that the regularization satisfies the conditions (4.6.38) and (4.9.2). Then the EL equations in the continuum limit 4.3.2 can be expressed in terms of the effective action (4.7.17). The parameter $\tau$ in the Dirac Lagrangian (4.7.20) is determined to have the value $\tau=-4$. The gravitational constant $\kappa$ is given by 4.9.4).

Combined with the equations for the chiral gauge fields in Theorem 4.8.1, this theorem shows that the structure of the interaction is described completely by the underlying EL equations 4.3.3 corresponding to the causal action principle 4.1.2. 
We point out that our results imply that the right-handed component of the neutrinos must couple to the Einstein equations with a relative factor of -3 . In particular, the righthanded component of the neutrinos has a negative energy density, thus violating the usual energy conditions. This might give a possible explanation for the anomalous acceleration of the universe.

One should keep in mind that the effective Lagrangian is determined only up to terms which contribute to the EL equations 4 4.3.2 to degree three or lower. In particular, if the Ricci tensor is a multiple of the metric, the term $R_{j k} \xi^{j} \xi^{k}$ in Lemma 4.5.2 is of degree one on the light cone, giving rise to a contribution which can be absorbed in the error term. In other words, to the considered degree four on the light cone, the Ricci tensor is determined only up to multiples of the metric. This gives precisely the freedom to add the cosmological Lagrangian

$$
\int_{\mathscr{M}} \frac{2 \Lambda}{\kappa} \sqrt{-\operatorname{det} g} d^{4} x
$$

for an arbitrary value of the cosmological constant $\Lambda$. In principle, the cosmological constant could be determined in our approach by evaluating the EL equations to degree three on the light cone. But this analysis goes beyond the scope of the present work.

We point out that our results exclude corrections to the Einstein-Hilbert action of higher order in the curvature tensor. Note that the simple fractions $K_{17}$ and $K_{18}$ are both of degree four, and thus their quotient is of the order one. Hence

$$
\kappa \sim \delta^{2} .
$$

This means that the Planck length is to be identified with the length scale $\delta$ describing the shear and general surface states (see (4.2.33) and (4.2.38)).

We next explain how this theorem could be extended to the case

$$
\delta \simeq \frac{1}{m}(m \varepsilon)^{\frac{p_{\mathrm{reg}}}{2}} .
$$

In this case, the terms $\sim m^{2} R_{j k} \xi^{j} \xi^{k}$ in Lemma 4.5.2 are of the same order as those $\sim$ $\tau_{\text {reg }} / \delta^{2} R_{j k} \xi^{j} \xi^{k}$ and must be taken into account. They can be obtained by a straightforward computation. The statement of Theorem 4.9 .3 will remain the same, except that the form of $K_{16}$ will of course be modified. The only structural difference is that (4.5.8) will then involve factors $T_{[0]}^{(1)}$, which have logarithmic poles on the light cone. It does not seem possible to compensate these logarithmic poles by a microlocal transformation. Therefore, in order for the logarithmic poles to drop out of the EL equations, one must impose that

$$
\sum_{\alpha=1}^{3} m_{\alpha}^{2}=\sum_{\alpha=1}^{3} \tilde{m}_{\alpha}^{2} .
$$

This constraint for the neutrino masses can be understood similar as in Remark 4.4.9. Working out the detailed computations seems an interesting project for the future.

We next point out that our method of perturbing the regularized Minkowski vacuum by a gravitational field implies in particular that the unperturbed regularization is homogeneous, so that the parameters $\varepsilon$ and $\delta$ are constant in space-time. Although this seems a good approximation locally, it is conceivable that the regularization does change on the astrophysical or cosmological scale. In this case, the gravitational constant would no longer be constant in space-time, but would become dynamical. We refer the reader interested in this effect of "dynamical gravitational coupling" to the paper [FR]. 
We finally note how the energy-momentum tensor of the gauge field enters the Einstein equations. The contribution of this energy-momentum tensor to the EL-equations to degree four on the light cone was computed in Lemma 4.5.3. Since our gauge field is left-handed, only the term 4.5.12 contributes. Due to the factor $T_{[0]}^{(1)}$, this contribution has a logarithmic pole on the light cone. As shown in Lemma 4.5.4, this logarithmic pole on the light cone can be compensated by a suitable microlocal transformation. As a result, the energy-momentum tensor enters the EL equations. After this has been done, the energy-momentum tensor of the gauge field enters the EL equations similar to the energy-momentum tensor of the Dirac field in 4.5.2). The only difference is that, since the simple fraction in 4.5.12 has a different form than the simple fraction $K_{8}$ in Lemma 4.5.1, the energy-momentum tensor of the gauge field comes with an additional regularization parameter. A-priori, this regularization parameter is to be treated as a free empirical parameter of the effective continuum theory. However, it can be fixed uniquely by the following mathematical consistency condition: The Einstein equations imply that the total energy-momentum tensor must be divergence-free. On the other hand, the energy-momentum tensor obtained by varying the metric in the effective action 4.7.17) gives rise to a divergence-free vector field being a specific linear combination of the energymomentum tensor of the Dirac field and the energy-momentum tensor of the gauge field. In general, these two energy-momentum tensors are not divergence-free separately, only the specific linear combination is divergence-free as a consequence of the Dirac equation and the field equations for the gauge fields. Therefore, in order to allow for non-trivial solutions, we are forced by mathematical consistency of the equations to choose the regularization parameter in such a way that the energy-momentum tensors enter the EL equations to degree four in the same linear combination as obtained by varying the effective action (4.7.17).

These considerations complete the analysis of the EL equations to degree four on the light cone up to errors of the order

$$
Q(x, y)=(\operatorname{deg}=4) \cdot o\left(|\vec{\xi}|^{-2}\right)+(\operatorname{deg}<4) .
$$




\title{
CHAPTER 5
}

\section{The Continuum Limit of a Fermion System Involving Leptons and Quarks: Strong, Electroweak and Gravitational Interactions}

\begin{abstract}
The causal action principle is analyzed for a system of relativistic fermions composed of massive Dirac particles and neutrinos. In the continuum limit, we obtain an effective interaction described by classical gravity as well as the strong and electroweak gauge fields of the standard model.
\end{abstract}

\subsection{Introduction}

In this chapter, we consider a system which is composed of seven massive sectors and one neutrino sector, each containing three generations of fermions. Analyzing the Euler-Lagrange equations of the causal action principle in the continuum limit, we obtain a unification of gravity with the strong and electroweak forces of the standard model.

More precisely, we obtain three main results. The first main result is the so-called spontaneous block formation (see Theorem 5.3.2), stating that the eight sectors form pairs, so-called blocks. The block involving the neutrinos can be regarded as the leptons, whereas the three other blocks correspond to the quarks (in three colors). The index distinguishing the two sectors within each block can be identified with the isospin. The interaction can be described effectively by $\mathrm{U}(1) \times \mathrm{SU}(2) \times \mathrm{SU}(3)$-gauge potentials acting on the blocks and on the isospin index. In this way, one recovers precisely the gauge potentials of the standard model together with their correct coupling to the fermions.

Our second main result is to derive the field equations for the gauge fields. Theorem 5.4.1 gives the general structure of the electroweak theory of the standard model after spontaneous symmetry breaking, but the masses and coupling constants involve more free parameters than in the standard model. In Theorem 5.4.2 it is shown that one gets precise agreement with the electroweak theory if one imposes three additional relations between the free parameters. Finally, in Proposition 5.4.3 it is shown that these three additional relations hold in the limit when the mass of the top quark is much larger than the lepton masses. We thus obtain agreement with the strong and electroweak theory up to small corrections. These corrections are discussed, and some of them are specified quantitatively.

Our third main result is to derive the gravitational interaction and the Einstein equations (see Theorem 5.4.4).

We point out that the continuum limit gives the correspondence to the standard model and to general relativity on the level of second-quantized fermionic fields coupled to classical bosonic fields. For the connection to second-quantized bosonic fields we refer to [F17, F20. We also point out that we do not consider a Higgs field. This is why we get the correspondence to the standard model after spontaneous symmetry breaking 
without the Higgs field (i.e. for a constant Higgs potential). But in Section 5.5 it is explained that the Higgs potential can possibly be identified with scalar potentials in the Dirac equation.

\subsection{Preliminaries}

In this section we repeat constructions used in Chapters 3 and 4 and adapt them to the system of Dirac seas to be considered here.

5.2.1. The Fermionic Projector and its Perturbation Expansion. We want to extend the analysis in Chapters 3 and 4 to a system involving quarks. Exactly as explained in [F7, Section 5.1], the quarks are described by additional sectors of the fermionic projector. More precisely, we describe the vacuum similar to (4.1.4) by the fermionic projector

$$
P(x, y)=P^{N}(x, y) \oplus P^{C}(x, y),
$$

where the charged component $P^{C}$ is formed as the direct sum of seven identical sectors, each consisting of a sum of three Dirac seas,

$$
P^{C}(x, y)=\bigoplus_{a=1}^{7} \sum_{\beta=1}^{3} P_{m_{\beta}}^{\mathrm{vac}}(x, y)
$$

where $m_{\beta}$ are the masses of the fermions and $P_{m}^{\mathrm{vac}}$ is the distribution

$$
P_{m}^{\mathrm{vac}}(x, y)=\int \frac{d^{4} k}{(2 \pi)^{4}}(\not k+m) \delta\left(k^{2}-m^{2}\right) \Theta\left(-k^{0}\right) e^{-i k(x-y)} .
$$

Thus every massive sector has the form as considered in (3.3.1). For the neutrino sector $P^{N}$ we choose the ansatz of massive neutrinos (cf. 4.1.8)

$$
P^{N}(x, y)=\sum_{\beta=1}^{3} P_{\tilde{m}_{\beta}}^{\mathrm{vac}}(x, y) .
$$

The neutrino masses $\tilde{m}_{\beta} \geq 0$ will in general be different from the masses $m_{\beta}$ in the charged sector. For a discussion of this ansatz we also refer to 4.2 .4 , where the alternative ansatz of chiral neutrinos is ruled out.

We introduce an ultraviolet regularization on the length scale $\varepsilon$. The regularized vacuum fermionic projector is denoted by $P^{\varepsilon}$. We again use the formalism of the continuum limit as developed in [F7, Chapter 4] and described in Section 3.5. In the neutrino sector, we work exactly as in $\$ 4.2 .5$ with a non-trivial regularization by right-handed high-energy states.

In order to describe an interacting system, we proceed exactly as described in [F7, Section 2.3], Section 3.4 and 4.2 .6 . We first introduce the auxiliary fermionic projector by

where

$$
P^{\mathrm{aux}}=P_{\mathrm{aux}}^{N} \oplus P_{\mathrm{aux}}^{C},
$$

$$
P_{\mathrm{aux}}^{N}=\left(\bigoplus_{\beta=1}^{3} P_{\tilde{m}_{\beta}}^{\mathrm{vac}}\right) \oplus 0 \quad \text { and } \quad P_{\mathrm{aux}}^{C}=\bigoplus_{a=1}^{7} \bigoplus_{\beta=1}^{3} P_{m_{\beta}}^{\mathrm{vac}} .
$$

Note that $P^{\text {aux }}$ is composed of 25 direct summands, four in the neutrino and 21 in the charged sector. The fourth direct summand of $P_{\text {aux }}^{N}$ has the purpose of describing the 
right-handed high-energy states. Moreover, we introduce the chiral asymmetry matrix $X$ and the mass matrix $Y$ by (cf. 4.2.42) and 4.2.43)

$$
\begin{aligned}
X & =\left(\mathbb{1}_{\mathbb{C}^{3}} \oplus \tau_{\text {reg }} \chi_{R}\right) \oplus \bigoplus_{a=1}^{7} \mathbb{1}_{\mathbb{C}^{3}} \\
m Y & =\operatorname{diag}\left(\tilde{m}_{1}, \tilde{m}_{2}, \tilde{m}_{3}, 0\right) \oplus \bigoplus_{a=1}^{7} \operatorname{diag}\left(m_{1}, m_{2}, m_{3}\right),
\end{aligned}
$$

where $m$ is an arbitrary mass parameter. Here $\tau_{\text {reg }} \in(0,1]$ is a dimensionless parameter for which we always assume the scaling

$$
\tau_{\text {reg }}=(m \varepsilon)^{p_{\text {reg }}} \quad \text { with } \quad 0<p_{\text {reg }}<2 .
$$

This allows us to rewrite the vacuum fermionic projector as

$$
P^{\text {aux }}=X t=t X^{*} \quad \text { with } \quad t:=\bigoplus_{\beta=1}^{25} P_{m Y_{\beta}^{\beta}}^{\mathrm{vac}} .
$$

Now $t$ is a solution of the Dirac equation

$$
(i \not \partial-m Y) t=0 .
$$

In order to introduce the interaction, we insert an operator $\mathcal{B}$ into the Dirac equation,

$$
(i \not \partial+\mathcal{B}-m Y) \tilde{t}=0 .
$$

The causal perturbation theory (see [F7, Section 2.2], [FT2 or Section 2.1) defines $\tilde{t}$ in terms of a unique perturbation series. The light-cone expansion (see [F7, Section 2.5] and the references therein or Section 2.2 is a method for analyzing the singularities of $\tilde{t}$ near the light cone. This gives a representation of $\tilde{t}$ of the form

$$
\begin{aligned}
\tilde{t}(x, y)= & \sum_{n=-1}^{\infty} \sum_{k} m^{p_{k}}(\text { nested bounded line integrals }) \times T^{(n)}(x, y) \\
& +\tilde{P}^{\mathrm{le}}(x, y)+\tilde{P}^{\mathrm{he}}(x, y)
\end{aligned}
$$

where $\tilde{P}^{\text {le }}(x, y)$ and $\tilde{P}^{\text {he }}(x, y)$ are smooth to every order in perturbation theory. For the resulting light-cone expansion to involve only bounded line integrals, we need to assume the causality compatibility condition

$$
(i \not \partial+\mathcal{B}-m Y) X=X^{*}(i \not \partial+\mathcal{B}-m Y) \quad \text { for all } \tau_{\text {reg }} \in(0,1] .
$$

Then the auxiliary fermionic projector of the sea states $P^{\text {sea }}$ is obtained similar to 5.2 .5 by multiplication with the chiral asymmetry matrix.

As in 4.2 .6 we built the regularization into the formulas of the light-cone expansion by the formal replacements

$$
\begin{aligned}
m^{p} T^{(n)} & \rightarrow m^{p} T_{[p]}^{(n)}, \\
\tau_{\text {reg }} T^{(n)} & \rightarrow \tau_{\text {reg }} \sum_{k=0}^{\infty} \frac{1}{k !} \frac{1}{\delta^{2 k}} T_{[R, 2 n]}^{(k+n)} .
\end{aligned}
$$


Moreover, we introduce particles and anti-particles by occupying additional states or by removing states from the sea, i.e.

$$
P^{\text {aux }}(x, y)=P^{\mathrm{sea}}(x, y)-\frac{1}{2 \pi} \sum_{k=1}^{n_{\mathrm{p}}} \psi_{k}(x) \overline{\psi_{k}(y)}+\frac{1}{2 \pi} \sum_{l=1}^{n_{\mathrm{a}}} \phi_{l}(x) \overline{\phi_{l}(y)}
$$

(for the normalization of the particle and anti-particle states we refer to [F7, Section 2.8] or $\$ 3.4 .3$ and [FT2]). Finally, we introduce the regularized fermionic projector $P$ by forming the sectorial projection (see also [F7, Section 2.3], (3.4.3) or 4.2.52),

$$
(P)_{j}^{i}=\sum_{\alpha, \beta}\left(\tilde{P}^{\text {aux }}\right)_{(j, \beta)}^{(i, \alpha)}
$$

where $i, j \in\{1, \ldots, 8\}$ is the sector index, and the indices $\alpha$ and $\beta$ run over the corresponding generations (i.e., $\alpha \in\{1, \ldots 4\}$ if $i=1$ and $\alpha \in\{1,2,3\}$ if $i=2, \ldots, 8$ ). We again indicate the sectorial projection of the mass matrices by accents (see [F7, Section 7.1], (3.5.2) or 4.2 .53$)$ ),

$$
\hat{Y}=\sum_{\alpha} Y_{\alpha}^{\alpha}, \quad \grave{Y} Y \cdots \grave{Y}=\sum_{\alpha, \beta, \gamma_{1}, \ldots, \gamma_{p-1}} Y_{\gamma_{1}}^{\alpha} \cdots Y_{\gamma_{2}}^{\gamma_{1}} \cdots Y_{\beta}^{\gamma_{p-1}}
$$

As in Chapter 4, we need assumptions on the regularization. Namely, again setting

$$
L_{[p]}^{(n)}=T_{[p]}^{(n)}+\frac{1}{3} \tau_{\mathrm{reg}} T_{[R, p]}^{(n)},
$$

we impose the following regularization conditions (see 44.6.36), 4.6.38) and (4.9.2)

$$
\begin{array}{r}
T_{[0]}^{(0)} T_{[0]}^{(-1)} \overline{T_{[0]}^{(0)}}=0 \quad \text { in a weak evaluation on the light cone } \\
\left|L_{[0]}^{(n)}\right|=\left|T_{[0]}^{(n)}\right|\left(1+\mathcal{O}\left((m \varepsilon)^{\left.2 p_{\text {reg }}\right)}\right) \quad \text { for } n=0,-1\right. \text { pointwise }
\end{array}
$$

Here by weak evaluation we mean that we multiply by a test function $\eta$ and integrate, staying away from the origin. More precisely, we use the weak evaluation formula (for details see 3.5 .1 (3.

$$
\int_{|\vec{\xi}|-\varepsilon}^{|\vec{\xi}|+\varepsilon} d t \eta(t, \vec{\xi}) \frac{T_{\circ}^{\left(a_{1}\right)} \cdots T_{\circ}^{\left(a_{\alpha}\right)} \overline{T_{\circ}^{\left(b_{1}\right)} \cdots T_{\circ}^{\left(b_{\beta}\right)}}}{T_{\circ}^{\left(c_{1}\right)} \cdots T_{\circ}^{\left(c_{\gamma}\right)} \overline{T_{\circ}^{\left(d_{1}\right)} \cdots T_{\circ}^{\left(d_{\delta}\right)}}}=\eta(|\vec{\xi}|, \vec{\xi}) \frac{c_{\mathrm{reg}}}{(i|\vec{\xi}|)^{L}} \frac{\log ^{k}(\varepsilon|\vec{\xi}|)}{\varepsilon^{L-1}}
$$

which holds up to

$$
\text { (higher orders in } \varepsilon / \ell_{\text {macro }} \text { and } \varepsilon /|\vec{\xi}| \text { ). }
$$

Here $L$ is the degree defined by $\operatorname{deg} T_{\circ}^{(n)}=1-n$, and $c_{\text {reg }}$ is a so-called regularization parameter (for details see again [F7, Section 4.5] or \$3.5.1). In (5.2.10 by pointwise we mean that if we multiply $T_{[p]}^{(n)}-L_{[p]}^{(n)}$ by any simple fraction and evaluate weakly on the light cone, we get zero up to an error of the specified order. We remark that 5.2.10 could be replaced by a finite number of equations to be satisfied in a weak evaluation on the light cone. But in order to keep our analysis reasonably simple, we always work with the easier pointwise conditions 5.2 .10 . 
5.2.2. Chiral Gauge Potentials and Gauge Phases. Similar as in $\$ 3.6 .2$ and 4.3 .2 we consider chiral gauge potentials. Thus the operator $\mathcal{B}$ in the Dirac equation (5.2.6) is chosen as

$$
\mathcal{B}=\chi_{L} A_{R}+\chi_{R} A_{L}
$$

where $A_{L}^{j}$ and $A_{R}^{j}$ are Hermitian $25 \times 25$-matrices acting on the sectors. A-priori, the chiral gauge potentials can be chosen according to the gauge group

$$
\mathrm{U}(25)_{L} \times \mathrm{U}(25)_{R}
$$

This gauge group is too large for mathematical and physical reasons. First, exactly as in 4.3 .2 , the causality compatibility condition (5.2.7) inhibits that non-trivial high-energy contributions are mixed with the Dirac seas, giving rise to the smaller gauge group

$$
\mathrm{U}(24)_{L} \times \mathrm{U}(24)_{R} \times \mathrm{U}(1)_{R},
$$

(where the $\mathrm{U}(24)$ acts on the first three direct summands of $P_{\mathrm{aux}}^{N}$ and on the 21 direct summands in $P_{\text {aux }}^{M}$ in (5.2.4). Similar as described in $\$ 3.6 .2$ and $\$ 4.3 .2$, to degree three on the light cone the gauge potentials describe generalized phase transformations of the left- and right-handed components of the fermionic projector,

$$
P^{\operatorname{aux}}(x, y) \rightarrow\left(\chi_{L} U_{L}(x, y)+\chi_{R} U_{R}(x, y)\right) P^{\operatorname{aux}}(x, y)+(\operatorname{deg}<2),
$$

where the unitary operators $U_{c}$ are ordered exponentials (for details see [F7, Section 2.5], [F6, Section 2.2] or (4.3.17),

$$
U_{c}=\operatorname{Pexp}\left(-i \int_{x}^{y} A_{c}^{j} \xi_{j}\right)
$$

The fermionic projector is obtained from (5.2.14) by forming the sectorial projection (5.2.8). Summing over the generation indices has the effect that wave functions are superimposed which may involve different gauge phases. In other words, the sectorial projection in general involves relative gauge phases. In order to simplify the form of the gauge potentials, we now argue that such relative gauge phases should be absent. In fact, if such relative phases occurred, the different Dirac seas forming the fermionic projector would get out of phase, implying that all relations for the fermionic projector would have to be satisfied for each Dirac sea separately. This would give rise to many additional constraints for the regularization, which seem impossible to satisfy. We remark that a similar argument is given in 4.3 .2 . Moreover, the physical picture is similar for the gravitational field, where it was argued in Section 4.5 that the metric tensor must be independent of the isospin index.

The simplest method to avoid such relative phases would be to choose gauge potentials which do not depend on the generation index, i.e.

$$
\left(A_{L}\right)_{(j, \beta)}^{(i, \alpha)}=\left(A_{L}^{\mathrm{sec}}\right)_{j}^{i} \delta_{\beta}^{\alpha}
$$

(where the superscript "sec" clarifies that the potential carries only sector indices). In order to be compatible with the $U(1)_{R}$-subgroup in (5.2.13) acting on the right-handed high-energy states in the neutrino sector, we need to choose the potentials in 5.2.15 corresponding to the gauge group

$$
\mathrm{U}(8)_{L} \times \mathrm{U}(1)_{R} \times \mathrm{U}(7)_{R}
$$

where the $\mathrm{U}(7)$ acts on the seven direct summands in 5.2 .2 but is trivial on the neutrinos (5.2.3). The ansatz (5.2.15) can be slightly generalized by allowing for unitary 
transformations in each sector. This leads to the ansatz

$$
\mathcal{B}=\chi_{R} U_{L}^{\mathrm{mix}} \mathscr{A}_{L}^{\mathrm{sec}}\left(U_{L}^{\mathrm{mix}}\right)^{*}+\chi_{L} U_{R}^{\mathrm{mix}} \mathcal{A}_{R}^{\mathrm{sec}}\left(U_{R}^{\mathrm{mix}}\right)^{*},
$$

where the potentials $A_{c}^{\mathrm{sec}}$ are again of the form (5.2.15), and the matrices $U_{c}^{\text {mix }}$ are constant unitary matrices which are diagonal in the sector index,

$$
\left(U_{c}^{\operatorname{mix}}\right)_{(j, \beta)}^{(i, \alpha)}=\delta_{j}^{i}\left(U_{c}^{i}\right)_{\beta}^{\alpha} \quad \text { with } \quad U_{c}^{i} \in \mathrm{U}(3) .
$$

Thus we allow for a different mixing matrix for every sector. Also, the mixing matrices may be different for the left- and right-handed components of the spinors. The fact that the mixing matrices are constant could be justified by using arguments similar to those worked out for two sectors in Lemma 4.3.1. Here we do not enter such arguments again but simply take (5.2.17) as our ansatz for the chiral gauge potentials. It seems the most general ansatz which avoids relative phases when forming the sectorial projection. Specializing the chiral gauge fields to the ansatz (5.2.17), the matrices $U_{c}$ in (5.2.14) become

$$
U_{c}=U_{c}^{\mathrm{mix}} \operatorname{Pexp}\left(-i \int_{x}^{y} A_{c}^{j} \xi_{j}\right)\left(U_{c}^{\mathrm{mix}}\right)^{*} .
$$

5.2.3. The Microlocal Chiral Transformation. Exactly as in 3.7 .10 and 3.4 .4 , our method is to compensate the logarithmic singularities of the current and mass terms by a microlocal chiral transformation. To this end, one considers a Dirac equation of the form

$$
\left(U^{-1}\right)^{*}(i \not \partial+\mathcal{B}-m Y) U^{-1} \tilde{P}^{\text {aux }}=0,
$$

where $U$ is an integral operator with an integral kernel $U(x, y)$, which we write in the microlocal form

$$
U(x, y)=\int \frac{d^{4} k}{(2 \pi)^{4}} U\left(k, \frac{x+y}{2}\right) e^{-i k(x-y)},
$$

where $U(k, z)$ is a chiral transformation

$$
U(k, z)=\mathbb{1}+\frac{i}{\sqrt{\Omega}} Z(k, z) \quad \text { with } \quad Z(z)=\chi_{L} L^{j}(k, z) \gamma_{j}+\chi_{R} R^{j}(k, z) \gamma_{j} .
$$

Writing the Dirac equation $(5.2 .20)$ in the form 5.2 .6 with a nonlocal operator $\mathcal{B}$, the perturbative methods of $\$ 5.2 .1$ again apply.

More specifically, the matrices $L$ and $R$ in (5.2.21) are chosen such that the matrices $L[k, x]:=\dot{L}_{j}(k, x) k^{j}$ and $R[k, x]:=\dot{L}_{j}(k, x) k^{j}$ satisfy the conditions

$$
\begin{aligned}
L[k, x] L[k, x]^{*} & =R[k, x] R[k, x]^{*}=\mathfrak{c}_{0}(k, x) \mathbb{1}_{\mathbb{C}^{2}} \\
L[k, x] m^{2} Y^{2} L[k, x]^{*} & =\frac{\Omega}{2} v_{L}(x)+\mathfrak{c}_{2}(k, x) \mathbb{1}_{\mathbb{C}^{2}} \\
R[k, x] m^{2} Y^{2} R[k, x]^{*} & =\frac{\Omega}{2} v_{R}(x)+\mathfrak{c}_{2}(k, x) \mathbb{1}_{\mathbb{C}^{2}},
\end{aligned}
$$

where $\mathfrak{c}_{0}$ and $\mathfrak{c}_{2}$ are real parameters, and $\Omega=\left|k^{0}\right|$ denotes the frequency of the fourmomentum $k$. The vector fields $v_{L}$ and $v_{R}$ are the currents or potentials which multiply the logarithmic singularities to be compensated.

Writing the Dirac equation (5.2.20) raises the question how the potential $\mathcal{B}$ is to be chosen. The most obvious procedure would be to choose $\mathcal{B}$ equal to the chiral potentials in (5.2.12). However, as shown in \$3.7.11 and $\$ 4.4 .5$, this is not the correct choice, intuitively speaking because the microlocal chiral transformation in 5.2 .20 has contributions which flip the chirality, making it necessary to also modify the potentials in the 
Dirac operator. To this end, we write down the Dirac equation for the auxiliary fermionic projector as

$$
\mathcal{D}_{\text {flip }} \tilde{P}^{\text {aux }}=0 \text {, }
$$

where $\mathcal{D}_{\text {flip }}$ is obtained from the Dirac operator with chiral gauge fields by

$$
\mathcal{D}_{\text {flip }}:=\left(U_{\text {flip }}^{-1}\right)^{*}\left(i \not \partial_{x}+\chi_{L} A_{R}+\chi_{R} A_{L}-m Y\right) U_{\text {flip }}^{-1} \text {. }
$$

Here $U_{\text {flip }}$ is obtained from the operator $U$ in 5.2 .20 by

$$
U_{\text {flip }}=\mathbb{1}+(\mathrm{U}-\mathbb{1}) V,
$$

and $V$ is the unitary perturbation flow which changes the gauge potentials from $A_{L / R}$ to $A_{L / R}^{\text {even }}$,

$$
V=U_{\text {flow }}\left[\chi_{L} \mathscr{A}_{R}^{\text {even }}+\chi_{R} \mathscr{A}_{L}^{\text {even }}\right] U_{\text {flow }}\left[\chi_{L} \mathscr{A}_{R}+\chi_{R} \mathscr{A}_{L}\right]^{-1} .
$$

For details we refer to (3.7.88), (3.7.90) and (B.4.1).

5.2.4. The Causal Action Principle. We again consider the causal action principle introduced in [F7]. The action is

$$
\mathcal{S}[P]=\iint_{\mathcal{M} \times \mathcal{M}} \mathcal{L}\left[A_{x y}\right] d^{4} x d^{4} y
$$

with the Lagrangian

$$
\mathcal{L}\left[A_{x y}\right]=\left|A_{x y}^{2}\right|-\frac{1}{32}\left|A_{x y}\right|^{2},
$$

where $A_{x y}=P(x, y) P(y, x)$ denotes the closed chain and $|A|=\sum_{i=1}^{8}\left|\lambda_{i}\right|$ is the spectral weight. As shown in $\$ 3.5 .2$, the Euler-Lagrange equations in the continuum limit can be written as

$$
Q(x, y)=0 \text { if evaluated weakly on the light cone }
$$

where $Q(x, y)$ is defined as follows. Similar as explained in 4.3 .1 , we count the eigenvalues of the the closed chain $A_{x y}$ with algebraic multiplicities and denote them by $\lambda_{n c s}^{x y}$, where $n \in\{1, \ldots, 8\}, c \in\{L, R\}$ and $s \in\{+,-\}$. The corresponding spectral projectors are denoted by $F_{n c s}^{x y}$. Then $Q(x, y)$ is given by

$$
\begin{aligned}
Q(x, y) & =\frac{1}{2} \sum_{n c s} \frac{\partial \mathcal{L}}{\partial \lambda_{n c s}^{x y}} F_{n c s}^{x y} P(x, y) \\
& =\sum_{n, c, s}\left[\left|\lambda_{n c s}^{x y}\right|-\frac{1}{8} \sum_{n^{\prime}, c^{\prime}, s^{\prime}}\left|\lambda_{n^{\prime} c^{\prime} s^{\prime}}^{x y}\right|\right] \frac{\overline{\lambda_{n c s}^{x y}}}{\left|\lambda_{n c s}^{x y}\right|} F_{n c s}^{x y} P(x, y) .
\end{aligned}
$$

The equation (5.2.26) is satisfied in the vacuum (see 3.6 .1 and 4.3 .1 ). When evaluating the EL equations in the interacting situation, in most cases it will be sufficient to consider (5.2.26) for perturbations of the eigenvalues,

$$
0=\Delta Q(x, y):=\sum_{n, c, s}\left[\Delta\left|\lambda_{n c s}^{x y}\right|-\frac{1}{8} \sum_{n^{\prime}, c^{\prime}, s^{\prime}} \Delta\left|\lambda_{n^{\prime} c^{\prime} s^{\prime}}^{x y}\right|\right] \frac{\overline{\lambda_{n c s}^{x y}}}{\left|\lambda_{n c s}^{x y}\right|} F_{n c s}^{x y} P(x, y) \text {. }
$$




\subsection{Spontaneous Block Formation}

The goal of this section is to derive constraints for the form of the admissible gauge fields. The arguments are similar in style to those in [F7, Chapter 7]. However, as a main difference, we here consider the effect of the sectorial projection and the mixing of the generations, whereas in [F7, Chapter 7] the contributions of higher order in a mass expansion (which are of lower degree on the light cone) were analyzed. The analysis given here supersedes the arguments in [F7, Chapter 7], which with the present knowledge must be regarded as being preliminary.

5.3.1. The Statement of Spontaneous Block Formation. Analyzing the EL equations to degree five and degree four on the light cone gives rise to a number of equations which involve the chiral potentials without derivatives. These equations do not describe a dynamics of the potentials and fields, but merely pose constraints for the structure of the possible interactions. We refer to these equations as the algebraic constraints for the gauge potentials. The algebraic constraints trigger a mechanism where the eight sectors form pairs, the so-called blocks. Describing the interaction within and among the four blocks by chiral gauge fields gives rise to precisely the gauge groups and couplings in the standard model.

In order to introduce a convenient notation, we denote those chiral potentials of the form (5.2.17) which satisfy all the algebraic constraints as admissible. Since ordered exponentials of the chiral potentials appear (see for example (5.2.14) and (5.2.19) ), it seems necessary for mathematical consistency to consider a set of admissible chiral gauge potentials which forms a Lie algebra, the so-called dynamical gauge algebra $\mathfrak{g}$. More precisely, the commutator of two elements $\mathcal{A}=\left(A_{L}, A_{R}\right)$ and $\tilde{A}=\left(\tilde{A}_{L}, \tilde{A}_{R}\right)$ in $\mathfrak{g}$ is defined by

$$
[\mathcal{A}, \tilde{\mathcal{A}}]=\left(\left[A_{L}, \tilde{A}_{L}\right],\left[A_{R}, \tilde{A}_{R}\right]\right)
$$

(where the brackets [.,.] is the commutator of symmetric $8 \times 8$-matrices; note that the mixing matrices in (5.2.17) drop out of all commutators). The assumption that $\mathfrak{g}$ is a Lie algebra is the implication $\mathcal{A}, \tilde{\mathcal{A}} \in \mathfrak{g} \Rightarrow i[\mathcal{A}, \tilde{\mathcal{A}}] \in \mathfrak{g}$. The corresponding Lie group will be a Lie subgroup of the gauge group in (5.2.16). We denote this Lie group by $\mathcal{G} \subset$ $\mathrm{U}(8)_{L} \times \mathrm{U}(1)_{R} \times \mathrm{U}(7)_{R}$ and refer to it as the dynamical gauge group .

The potentials in the dynamical gauge algebra should be regarded as describing the physical interactions of the system. In order to understand the algebraic constraints, we clearly want to find all the potentials which satisfy the algebraic constraints. Therefore, we always choose $\mathcal{G}$ maximal in the sense that $\mathcal{G}$ has no Lie group extension extension $\tilde{\mathcal{G}}$ with $\mathcal{G} \varsubsetneqq \tilde{\mathcal{G}} \subset \mathrm{U}(8)_{L} \times \mathrm{U}(1)_{R} \times \mathrm{U}(7)_{R}$ which is also generated by admissible chiral potentials.

We begin with the following definition.

Definition 5.3.1. An admissible chiral potential $\mathcal{A}=\left(A_{L}, A_{R}\right) \in \mathfrak{g}$ is a free gauge potential if it has the following properties:

(a) The potential is vectorial: $A_{L}=A_{R}=: A$.

(b) The potential does not depend on the generation index: $A_{(j, \beta)}^{(i, \alpha)}=\delta_{\beta}^{\alpha}\left(A^{\mathrm{sec}}\right)_{j}^{i}$.

(c) The potential commutes with the mass matrix: $[A, m Y]=0$.

The Lie group generated by all free gauge potentials is referred to as the free gauge group $\mathcal{G}_{\text {free }} \subset \mathcal{G}$. 
Since the conditions (a)-(c) are linear and invariant under forming the Lie bracket, $\mathcal{G}_{\text {free }}$ is indeed a Lie subgroup of $\mathcal{G}$.

A free gauge potential has the desirable property that it corresponds to a gauge symmetry of the system (because it describes isometries of the spin spaces; see 33.6 .2 ). As a consequence, the mass terms vanish, implying that the corresponding bosonic fields are necessarily massless (see 33.8 .5 ). Moreover, chiral potentials with the above properties (a)-(c) satisfy all algebraic constraints (see \$5.3.2 $\$ 5.3 .4$ below) and are thus admissible.

Here is the main result of this section:

THEOREM 5.3.2. (spontaneous block formation) Consider the setting introduced in \$5.2.1 and assume that the following conditions hold:

(i) The admissible gauge potentials involve non-abelian left- or right-handed gauge potentials.

(ii) The mixing matrices $U_{c}^{\text {mix }}$ in (5.2.17) are chosen such that the dimension of the free gauge group is maximal.

Then the effective gauge group is given by

$$
\mathcal{G}=\mathrm{U}(1) \times \mathrm{SU}(2) \times \mathrm{SU}(3) .
$$

By relabeling the massive sectors and performing constant phase transformations of the wave functions, we can arrange that the corresponding gauge potentials $A^{\mathrm{em}} \in \mathfrak{u}(1), W \in$ $\mathfrak{s u}(2)$ and $G \in \mathfrak{s u}(3)$ enter the operator $\mathcal{B}$ in the Dirac equation (5.2.6) as follows,

$$
\begin{aligned}
\mathcal{B}\left[A^{\mathrm{em}}\right] & =A^{\mathrm{em}} \operatorname{diag}\left(0,-1, \frac{2}{3},-\frac{1}{3}, \frac{2}{3},-\frac{1}{3}, \frac{2}{3},-\frac{1}{3}\right) \\
\mathcal{B}[W] & =\chi_{R}\left(W_{\mathrm{MNS}} \oplus W_{\mathrm{CKM}} \oplus W_{\mathrm{CKM}} \oplus W_{\mathrm{CKM}}\right) \\
\mathcal{B}[G] & =\left(\mathbb{1} \oplus \mathcal{G}_{\mathrm{r}}\right) \otimes \mathbb{1}_{\mathbb{C}^{2}},
\end{aligned}
$$

where

$$
W_{\mathrm{MNS}}=\left(\begin{array}{cc}
(W)^{11} & (W)^{12} U_{\mathrm{MNS}}^{*} \\
(W)^{21} U_{\mathrm{MNS}} & (W)^{22}
\end{array}\right), \quad W_{\mathrm{CKM}}=\left(\begin{array}{cc}
(W)^{11} & (W)^{12} U_{\mathrm{CKM}}^{*} \\
(W)^{21} U_{\mathrm{CKM}} & (W)^{22}
\end{array}\right),
$$

and $U_{\mathrm{MNS}}, U_{\mathrm{CKM}} \in \mathrm{U}(3)$ are fixed unitary matrices. If one of these matrices is non-trivial, the other is also non-trivial and

$$
\hat{U}_{\mathrm{MNS}}=\hat{U}_{\mathrm{CKM}} \text {. }
$$

If the masses of the charged leptons and neutrinos (5.2.2) and (5.2.3) are different in the sense that

$$
\sum_{\beta=1}^{3} m_{\beta}^{2} \neq \sum_{\beta=1}^{3} \tilde{m}_{\beta}^{2},
$$

then the mixing matrices are necessarily non-trivial,

$$
U_{\mathrm{MNS}}, U_{\mathrm{CKM}} \neq \mathbb{1}_{\mathbb{C}^{3}} .
$$

To clarify the notation, we first note that we always identify $\mathfrak{u}(n)$ with the Hermitian $n \times n$-matrices, and $\mathfrak{s u}(n)$ are the corresponding traceless matrices. Next, the diagonal matrix in (5.3.2) acts on the eight sectors. The potential in 5.3.3 only couples to the left-handed component of the spinors. Each of the four direct summands acts on two sectors (i.e. $W_{\mathrm{MNS}}$ on the first and second sector, the next summand $W_{\mathrm{CKM}}$ on the third and fourth sector, etc.). In (5.3.4) the direct sum $\mathbb{1}+\phi_{t}$ is a $4 \times 4$ matrix acting on pairs 
of sectors as indicated by the factor $\mathbb{1}_{\mathbb{C}^{2}}$ (i.e. the first column acts on the first and second sector, the second column on the third and fourth sector, etc.).

The specific form of the potentials in the above theorem can be understood as a mechanism where the sectors form pairs, referred to as blocks. Thus the first two sectors form the first block (referred to as the lepton block), the third and fourth sectors form the second block (referred to as the first quark block), and so on. The potentials in (5.3.3) are the same in each of the four blocks, except for the mixing matrices $U_{\mathrm{MNS}}$ and $U_{\mathrm{CKM}}$, which may be different in the lepton and in the quark blocks. The potentials in (5.3.4) describe an interaction of the three quark blocks. Clearly, the potentials $A^{\mathrm{em}}$ and $G$ correspond to the electromagnetic and the strong potentials in the standard model. The potential $W$ corresponds to the gauge potentials of the weak isospin. The reduction from the large gauge group (5.2.16) to its subgroup (5.3.1) and to gauge potentials of the specific form (5.3.2)-(5.3.4) can be regarded as a spontaneous breaking of the gauge symmetry. We refer to this effect as spontaneous block formation.

We point out that without any additional assumptions (like (i) and (ii) above), the dynamical gauge group will not be uniquely determined. This is due to the fact that the algebraic constraints are nonlinear, and therefore these constraints will in general be satisfied by different Lie algebras. Thus in general, there will be a finite (typically small) number of possible dynamical gauge groups, leaving the freedom to choose one of them as being the "physical" one. The above assumptions (i) and (ii) give a way to single out a unique dynamical gauge group, corresponding to the choice which we consider to be physically relevant. Clearly, this procedure can be criticized as not deriving the structure of the physical interactions purely from the causal action principle and the form of the vacuum. But at least, the choice of the dynamical gauge group is global in space-time, i.e. it is to be made once and forever. Moreover, our procedure clarifies the following points:

- The gauge groups and couplings of the gauge fields to the fermion as used in the standard model follow uniquely from general assumptions on the interaction, which do not involve any specific characteristics of the groups or of the couplings.

- The gauge groups of the standard model are maximal in the sense that no additional chiral potentials are admissible. Thus we get an explanation why there are not more physical gauge fields than those in the standard model.

As an example of a dynamical gauge group which we do not consider as being physically relevant, one could choose $\mathcal{G}_{\text {free }}$ as the Lie group $\mathrm{U}(7)$ acting on the 7 massive sectors. Forming $\mathcal{G}$ as a maximal extension gives a dynamical gauge group where the corresponding left- and right-handed gauge potentials are all abelian. This explains why an assumption like (i) above is needed.

We remark that the specific form of assumption (i) is a major simplification of our analysis, because it makes it possible to disregard the situation that there are non-abelian admissible potentials, but that every such potential is a mixture of a left- and right-handed component. We expect that assumption (i) could be weakened by refining our methods, but we leave this as a problem for future research.

The remainder of this section is devoted to the proof of Theorem 5.3.2. We first work out all the constraints for the gauge potentials $(\$ 5.3 .2-\$ 5.3 .4)$ and then combine our findings to infer the theorem $(\$ 5.3 .5)$.

5.3.2. The Sectorial Projection of the Chiral Gauge Phases. Similar as explained in Section 4.3, we shall now analyze the effect of the gauge phases in the EL equations to degree five on the light cone. Combining (5.2.14), (5.2.19) and (5.2.8), the 
closed chain is computed by (see also $\$ 4.3 .2$

$$
\chi_{L} A_{x y}=\chi_{L} \hat{U}_{L} \hat{U}_{R}^{*} A_{x y}^{\mathrm{vac}}+(\operatorname{deg}<3) .
$$

Here $A_{x y}^{\mathrm{vac}}$ is the closed chain in the vacuum. It is diagonal in the sector index and has the form (cf. 4.3 .1 )

$$
\chi_{L} A_{x y}^{\mathrm{vac}}=\left\{\begin{array}{cl}
\frac{3}{4} \chi_{L}\left(3 \notin T_{[0]}^{(-1)} \bar{\sharp} T_{[0]}^{(-1)}+\tau_{\mathrm{reg}} \sharp T_{[0]}^{(-1)} \overline{\sharp T_{[R, 0]}^{(-1)}}\right) & \text { on the neutrino sector } \\
\frac{3}{4} \chi_{L} 3 \sharp T_{[0]}^{(-1)} \bar{\sharp} T_{[0]}^{(-1)} & \text { on the massive sectors },
\end{array}\right.
$$

up to contributions of the form $\sharp(\operatorname{deg}<3)+(\operatorname{deg}<2)$. In $\$ 4.3 .2$ the size of $\tau$ is discussed, leading to the two cases (i) and (ii) (see 4.3.36)). For brevity, we here only consider Case (i), noting that Case (ii) can be treated exactly as in 4.3 .2 . Thus we assume that $\tau$ is so small that the factor $T_{[R, 0]}^{(-1)}$ may be disregarded, so that the closed chain of the vacuum simplifies to

$$
\chi_{L} A_{x y}^{\mathrm{vac}}=\frac{9}{4} \chi_{L} \nLeftarrow T_{[0]}^{(-1)} \overline{\sharp T_{[0]}^{(-1)}} .
$$

In order to satisfy the EL equations to degree five, the non-trivial eigenvalues of the matrix (5.3.8) must all have the same absolute value. Since the matrix (5.3.9) commutes with the matrices $\hat{U}_{L}$ and $\hat{U}_{R}^{*}$, the eigenvalues of the closed chain are simply the products of the eigenvalues of $\chi_{L} A_{x y}^{\mathrm{vac}}$ and the eigenvalues of $\hat{U}_{L} \hat{U}_{R}^{*}$. Since the nontrivial eigenvalues of $\chi_{L} A_{x y}^{\mathrm{vac}}$ form a complex conjugate pair, the EL equations to degree five are satisfied if and only if

$$
\text { the eigenvalues } \hat{U}_{L} \hat{U}_{R}^{*} \text { all have the same absolute value . }
$$

This leads to constraints for the gauge potentials, which we now work out.

In preparation, we introduce a convenient notation. Our goal is to determine the dynamical gauge group $\mathcal{G}$. At the moment, we only know that it should be a Lie subgroup of the group in (5.2.16). The admissible chiral gauge potentials are vectors in the corresponding Lie algebra $\mathfrak{g}=T_{e} \mathcal{G}$. More precisely, in view of (5.2.17), these chiral potentials have the form

$$
\mathfrak{g} \ni \mathcal{A}=\left(A_{L}, A_{R}\right) \quad \text { and } \quad A_{c}=U_{c}^{\mathrm{mix}} A_{c}^{\mathrm{sec}}\left(U_{c}^{\mathrm{mix}}\right)^{*},
$$

where $A_{c}^{\text {sec }}$ are Hermitian $8 \times 8$-matrices acting on the sectors. Moreover, the matrix $A_{R}$ does not mix the first with the other 7 sectors, i.e.

$$
A_{R}=\left(\begin{array}{cccc}
\left(A_{R}\right)_{1}^{1} & 0 & \cdots & 0 \\
0 & \left(A_{R}\right)_{2}^{2} & \cdots & \left(A_{R}\right)_{8}^{2} \\
\vdots & \vdots & \ddots & \vdots \\
0 & \left(A_{R}\right)_{2}^{8} & \cdots & \left(A_{R}\right)_{8}^{8}
\end{array}\right) .
$$

LEMmA 5.3.3. Assume that for any $\left(U_{L}, U_{R}\right) \in \mathcal{G}$, the eigenvalues of the matrix $\hat{U}_{L} \hat{U}_{R}^{*}$ all have the same absolute value. Then for any $\mathcal{A}=\left(A_{L}, A_{R}\right) \in \mathfrak{g}$ there is a real num$\operatorname{ber} c(\mathcal{A})$ such that the matrix

$$
\hat{A}_{L} \grave{A}_{L}+\hat{A}_{R} \grave{A}_{R}-\hat{A}_{L}^{2}-\hat{A}_{R}^{2}-c(\mathcal{A}) \mathbb{1}_{\mathbb{C}^{8}}
$$

vanishes on all the eigenspaces of the matrix $\hat{A}_{L}-\hat{A}_{R}$. 
Proof. For simplicity, we only consider the situation that the group element $\left(U_{L}, U_{R}\right)$ is in a neighborhood of the identity $e \in \mathcal{G}$. Then, since $\mathcal{G}$ is assumed to be a Lie group, we can represent the group element with the exponential map,

$$
U_{c}=\exp \left(-i A_{c}\right)=\mathbb{1}-i A_{c}-\frac{1}{2} A_{c}^{2}+\mathcal{O}\left(\mathcal{A}^{3}\right) .
$$

Forming the sectorial projection, we obtain

$$
\hat{U}_{c}=\exp \left(-i A_{c}\right)=\mathbb{1}-i \hat{A}_{c}-\frac{1}{2} \hat{A}_{c} \grave{A}_{c}+\mathcal{O}\left(\mathcal{A}^{3}\right) .
$$

The effect of the sectorial projection becomes clearer when comparing with the unitary matrix obtained by exponentiating the sectorial projection of $A_{c}$,

$$
\exp \left(-i \hat{A}_{c}\right)=\mathbb{1}-i \hat{A}_{c}-\frac{1}{2} \hat{A}_{c}^{2}+\mathcal{O}\left(\mathcal{A}^{3}\right) .
$$

This gives

$$
\begin{aligned}
\hat{U}_{c} & =\exp \left(-i \hat{A}_{c}\right)+\frac{1}{2}\left(\hat{A}_{c}^{2}-\dot{A}_{c} \grave{A}_{c}\right)+\mathcal{O}\left(\mathcal{A}^{3}\right) \\
& =\exp \left(-i \hat{A}_{c}\right)\left(\mathbb{1}+\frac{1}{2}\left(\hat{A}_{c}^{2}-\grave{A}_{c} \grave{A}_{c}\right)\right)+\mathcal{O}\left(\mathcal{A}^{3}\right),
\end{aligned}
$$

showing that $\hat{U}_{c}$ is unitary up to a contribution to second order which is Hermitian. As a consequence,

$$
\hat{U}_{L} \hat{U}_{R}^{*}=\exp \left(-i \hat{A}_{L}\right)\left\{\mathbb{1}+\frac{1}{2}\left(\hat{A}_{L}^{2}-\hat{A}_{L} \grave{A}_{L}+\hat{A}_{R}^{2}-\hat{A}_{R} \grave{A}_{R}\right)\right\} \exp \left(i \hat{A}_{R}\right)+\mathcal{O}\left(\mathcal{A}^{3}\right) .
$$

The curly brackets enclose a Hermitian matrix. Moreover, to the considered second order in $\mathcal{A}$, the curly brackets can be commuted to the left or right. This shows that the matrix $\hat{U}_{L} \hat{U}_{R}^{*}$ is normal (i.e. it commutes with its adjoint). Therefore, the eigenvalues can be computed with a standard perturbation calculation with degeneracies. To first order in $\mathcal{A}$, we need to diagonalize the matrix $\hat{A}_{L}-\hat{A}_{R}$. The exponentials in (5.3.12) are unitary and thus only change the eigenvalues by a phase. Therefore, the change of the absolute values of the eigenvalues is described by a first order perturbation calculation for the matrix in the curly brackets. This gives the result.

The condition (5.3.11) arising from this lemma is difficult to analyze because the eigenspaces of the matrix $A_{L}-\hat{A}_{R}$ are unknown and depend on the potential in a complicated non-linear way. A good strategy for satisfying the conditions for all $\mathcal{A} \in \mathfrak{g}$ is to demand that the matrix in (5.3.11) vanishes identically, i.e.

$$
\hat{A}_{L} \grave{A}_{L}+\hat{A}_{R} \grave{A}_{R}-\hat{A}_{L}^{2}-\hat{A}_{R}^{2}=c(\mathcal{A}) \mathbb{1}_{\mathbb{C}^{8}} .
$$

Clearly, this is a stronger condition than (5.3.11). But by perturbing the potentials in $\mathfrak{g}$, one could also get information on the matrix elements of $(5.3 .13)$ which mix different eigenspaces of $\hat{A}_{L}-\hat{A}_{R}$, suggesting that the assumptions of Lemma 5.3 .3 even imply that (5.3.13) holds. Making this argument precise would make it necessary to study third order perturbations. In order to keep our analysis reasonably simple, we shall not enter higher oder perturbation theory. Instead, in what follows we take 5.3 .13 as a necessary condition which all admissible potentials $\mathcal{A}=\left(A_{L}, A_{R}\right) \in \mathfrak{g}$ must satisfy. 
Let us reformulate $(5.3 .13)$ in a convenient notation. First, we let $\check{\pi}: \mathbb{C}^{3} \rightarrow \mathbb{C}^{3}$ be the orthogonal projection onto the subspace spanned by the vector $(1,1,1)$. We introduce the vector space

$$
T:=\mathbb{C}^{8} \times \mathbb{C}^{3}
$$

of vectors carrying a sector and a generation index. We also consider $\check{\pi}$ as an operator on $T$ which acts on the second factor (i.e. on the generation index). Then the sectorial projections in (5.3.13) can be written as

$$
\sum_{c=L, R} \check{\pi} A_{c}(\mathbb{1}-\check{\pi}) A_{c} \check{\pi}=c(\mathcal{A}) \mathbb{1}_{T} .
$$

We introduce the subspaces $I:=\check{\pi}(T)$ and $J:=(\mathbb{1}-\check{\pi})(T)$; they are 8- respectively 16-dimensional. Moreover, we introduce the operators

$$
B_{c}=(\mathbb{1}-\check{\pi}) A_{c} \check{\pi}: I \rightarrow J .
$$

Combining the left- and right-handed matrices,

$$
B:=\left(\begin{array}{c}
B_{L} \\
B_{R}
\end{array}\right): I \rightarrow K:=J \oplus J,
$$

we can write the condition $(5.3 .14)$ as

$$
\langle B u \mid B u\rangle=c(\mathcal{A})\|u\|^{2} \quad \text { for all } u \in I
$$

(where the scalar product and the norm refer to the canonical scalar products on $K$ and $I$, respectively). In other words, the matrix $B$ must be a multiple of an isometry. We denote the possible values of $B$ by $\mathcal{B}$,

$$
\mathcal{B}:=\left\{\left(\begin{array}{l}
(1-\check{\pi}) A_{L} \check{\pi} \\
(1-\check{\pi}) A_{R} \check{\pi}
\end{array}\right): I \rightarrow K \quad \text { with } \quad \mathcal{A} \in \mathfrak{g}\right\}
$$

Then $\mathcal{B}$ is a real vector space of matrices. The condition (5.3.17) must hold on the whole vector space,

$$
\langle B u \mid B u\rangle=c(B)\|u\|^{2} \quad \text { for all } B \in \mathcal{B} \text { and } u \in I .
$$

The analysis of (5.3.19) bears some similarity to the "uniform splitting lemma" used in [F7, Lemma 7.1.3]. In fact, if $\mathcal{B}$ were a complex vector space, we could polarize (5.3.19) to conclude that

$$
\left\langle B u \mid B^{\prime} u\right\rangle=c\left(B, B^{\prime}\right)\|u\|^{2} \quad \text { for all } B, B^{\prime} \in \mathcal{B} \text { and } u \in I,
$$

making it possible to apply [F7, Lemma 7.1.3]. However, there is the subtle complication that $\mathcal{B}$ is only a real vector space, implying that the above polarization is in general wrong. This makes it necessary to modify the method such that we work purely with real vector spaces. To this end, we consider $I$ and $K$ as real vector spaces, for clarity denoted by a subscript $\mathbb{R}$. These vector spaces have the real dimensions 16 respectively 64. On $I_{\mathbb{R}}$ and $K_{\mathbb{R}}$ we introduce the scalar product

$$
\langle. \mid .\rangle_{\mathbb{R}}:=\operatorname{Re}\langle. \mid .\rangle .
$$

We encode the complex structure in a real linear operator $\mathbb{I}$ acting on $I_{\mathbb{R}}$ and $K_{\mathbb{R}}$ with the properties

$$
\mathbb{I}^{*}=-\mathbb{I} \quad \text { and } \quad \mathbb{I}^{2}=-\mathbb{1} .
$$

Next, we let Re $I$ be the subspace of $I$ formed of all vectors with real components. We also consider $\operatorname{Re} I$ as an 8-dimensional subspace of $I_{\mathbb{R}}$. Moreover, we let $\operatorname{Re}: I_{\mathbb{R}} \rightarrow \operatorname{Re} I$ be 
the orthogonal projection to the real part. By restricting to $\operatorname{Re} I$, every operator $B \in \mathcal{B}$ gives rise to a mapping

$$
B_{\mathbb{R}}:=\left.B\right|_{\operatorname{Re} I}: \operatorname{Re} I \rightarrow K_{\mathbb{R}} .
$$

Note that the operator $B_{\mathbb{R}}$ is represented by a $64 \times 8$-matrix. Knowing $B_{\mathbb{R}}$, we can uniquely reconstruct the corresponding $B$ by "complexifying" according to

$$
B u=B \operatorname{Re} u-\mathbb{I} B \operatorname{Re}(\mathbb{I} u) .
$$

Lemma 5.3.4. There is an isometry $V: K_{\mathbb{R}} \rightarrow K_{\mathbb{R}}$ and a basis $B_{1}, \ldots B_{L}$ of $\mathcal{B}$ (with $L \geq 0)$ such that the matrices $\left(B_{\ell}\right)_{\mathbb{R}}$ have the representation

$$
\left(B_{\ell}\right)_{\mathbb{R}}=V M_{\ell}
$$

with operators $M_{\ell}: I_{\mathbb{R}} \rightarrow K_{\mathbb{R}}$ which in the canonical bases have the block matrix representation

$$
M_{1}=\left(\begin{array}{c}
\mathbb{1} \\
0 \\
\vdots \\
0 \\
0
\end{array}\right), \quad M_{2}=\left(\begin{array}{c}
0 \\
\mathbb{1} \\
\vdots \\
0 \\
0
\end{array}\right), \ldots, \quad M_{L}=\left(\begin{array}{c}
0 \\
0 \\
\vdots \\
\mathbb{1} \\
0
\end{array}\right) .
$$

Here the upper $L$ matrix entries are $8 \times 8$-matrices, whereas the lowest matrix entry is a $(64-8 L) \times 8$-matrix.

Proof. We rewrite (5.3.19) in real vector spaces as

$$
\left\langle B_{\mathbb{R}} u \mid B_{\mathbb{R}} u\right\rangle_{\mathbb{R}}=c(B)\|u\|_{R}^{2} \quad \text { for all } B \in \mathcal{B} \text { and } u \in \operatorname{Re} I \text {. }
$$

Using the symmetry of the real scalar product, polarization gives

$$
\left\langle B_{\mathbb{R}} u \mid B_{\mathbb{R}}^{\prime} u\right\rangle_{\mathbb{R}}=c\left(B, B^{\prime}\right)\langle u \mid v\rangle_{\mathbb{R}}^{2} \quad \text { for all } B, B^{\prime} \in \mathcal{B} \text { and } u \in \operatorname{Re} I \text {. }
$$

Now we can proceed as in the proof of [F7, Lemma 7.1.3]: Let $\left(e_{1}, \ldots, e_{8}\right)$ be the canonical basis of $\operatorname{Re} I$. We introduce the subspaces

$$
E_{i}=\operatorname{span}\left\{B_{\mathbb{R}} e_{i} \text { with } B \in \mathcal{B}\right\} \subset K_{\mathbb{R}}
$$

as well as the mappings

$$
\kappa_{i}: \mathcal{B} \rightarrow E_{i}, \quad B \mapsto B_{\mathbb{R}} e_{i} .
$$

The property $(5.3 .20)$ implies that for all $B, B^{\prime} \in \mathcal{B}$,

$$
\left\langle B_{\mathbb{R}} e_{i} \mid B_{\mathbb{R}}^{\prime} e_{j}\right\rangle_{\mathbb{R}}=c\left(B, B^{\prime}\right) \delta_{i j} .
$$

If $i \neq j$, this relation shows that the subspaces $\left(E_{i}\right)_{i=1, \ldots, p_{1}}$ are orthogonal. Moreover, in the case $i=j$, the relation (5.3.21) yields that the scalar products $\left\langle\kappa_{i}\left(B^{\prime}\right) \mid \kappa_{i}\left(B^{\prime}\right)\right\rangle_{\mathbb{R}}$ are independent of $i$. Thus the mappings $\kappa_{i}$ are isometrically equivalent, and so we can arrange by an isometry $V$ that the $\kappa_{i}$ have the matrix representations

$$
\kappa_{1}=\left(\begin{array}{c}
\kappa \\
\vdots \\
0 \\
0
\end{array}\right), \ldots, \quad \kappa_{L}=\left(\begin{array}{c}
0 \\
\vdots \\
\kappa \\
0
\end{array}\right)
$$

where $\kappa: \mathcal{B} \rightarrow \mathbb{R}^{8}$.

Finally, we choose a basis $B_{1}, \ldots, B_{L}$ of $\mathcal{B}$ such that $\kappa\left(B_{1}\right)=(1, \ldots, 0), \kappa\left(B_{2}\right)=$ $(0,1, \ldots, 0)$, etc. This gives the result. 
Counting dimensions, this lemma shows in particular that the dimension of $\mathcal{B}$ is at most 8 . In our applications we need the following refined counting of dimensions.

Corollary 5.3.5. Assume that the images of the matrices $B_{1}, \ldots, B_{L}: I \rightarrow K$ span an $N$-dimensional subspace of $K$. Then the dimension of $\mathcal{B}$ is bounded from above by

$$
L \leq \frac{N}{4}
$$

Proof. Note that the real dimension of the image of $\left.\left(B_{\ell}\right)\right|_{\mathbb{R}}: I_{\mathbb{R}} \rightarrow K_{\mathbb{R}}$ is twice the complex dimension of $B_{\ell}: I \rightarrow K$.

5.3.3. The Bilinear Logarithmic Terms. In 4.6 .1 the left-handed component of the bilinear logarithmic terms quadratic in the mass matrices were computed by (see 4.6.21)

$$
\begin{aligned}
B_{L}:= & -\frac{m^{2}}{4}\left\{A_{R}^{\text {even }}[\xi],\left(A_{L}[\xi] Y Y-2 Y A_{R}[\xi] Y+Y Y A_{L}[\xi]\right)\right\} T^{(1)} \\
& \left.+\frac{m^{2}}{8}\left(A_{L}[\xi]^{2} Y Y+2 A_{L}[\xi] Y Y A_{L}[\xi]+Y Y A_{L}[\xi]^{2} \xi_{k}\right)\right) T^{(1)} \\
& -\frac{m^{2}}{2} Y A_{R}[\xi]^{2} Y T^{(1)} .
\end{aligned}
$$

The right-handed component is obtained similarly by the replacements $L \leftrightarrow R$. Exactly as shown in Lemma 4.6.5, the EL equations in the continuum limit are satisfied only if the matrices $\hat{B}_{L}$ and $\hat{B}_{R}$ coincide and are multiples of the matrix $\dot{Y} \grave{Y}$.

Let us specify the potentials $A_{c}^{\text {even }}$ in 5.2 .25 . Exactly as shown in $\$ 4.4 .5$, the shear contributions vanish only if, in a suitable basis, the matrix $A_{L}^{\text {even }}$ coincides with $A_{R}$ and $A_{R}^{\text {even }}$ coincides with $A_{L}$, up to the choice of the mixing matrices. More precisely, in order to introduce $A_{L}^{\text {even }}$, we let $\mathfrak{e}_{i \alpha}(k, x)$ with $i \in\{1, \ldots, 8\}$ and $\alpha \in\{1,2,3\}$ be an orthonormal basis of $\mathbb{C}^{8 \times 3}$ such that the vectors $\mathfrak{e}_{i 1}$ are multiples of the eight columns of the matrix $L[k, x]^{*}$ (note that these column vectors are orthogonal according to 5.2 .22$)$ ). In this basis, the potential $A_{L}^{\text {even }}$ is defined by

$$
A_{L}^{\mathrm{even}}=V_{R} A_{R}^{\mathrm{sec}} V_{R}^{*}
$$

where $A_{R}^{\mathrm{sec}}$ is the potential in (5.2.17) (in the standard basis), and $V_{R}$ are unitary matrices which are diagonal in the sector index,

$$
\left(V_{R}\right)_{(j, \beta)}^{(i, \alpha)}(x)=\delta_{j}^{i}\left(V_{R}^{i}\right)_{\beta}^{\alpha}(x) \quad \text { with } \quad V_{c}^{i}(x) \in \mathrm{U}(3) .
$$

This is analogous to (5.2.17) and 5.2.18), with the only difference that different mixing matrices $V_{c}^{i}$ appear, which may even depend on the space-time point $x$. In order to introduce $A_{R}^{\text {even }}$, one chooses similarly a basis $\mathfrak{e}_{i \alpha}(k, x)$ such that the vectors $\mathfrak{e}_{i 1}$ are multiples of the eight columns of the matrix $R[k, x]^{*}$, and in this basis one sets

$$
A_{R}^{\text {even }}=V_{L} A_{L}^{\mathrm{sec}} V_{L}^{*}
$$

with a sector-diagonal unitary matrix $V_{L}(x)$. We point out that the construction of the potentials $A_{L / R}^{\text {even }}$ depends on the momentum $k$ of the microlocal chiral transformation. As a consequence, these potentials are non-local operators (for details see the discussion in 4.4 .5 .

When using (5.3.24) and (5.3.25) in (5.3.23), the freedom in choosing the matrices $V_{c}^{i}$ gives many free parameters to modify $B_{L}$ and $B_{R}$, making the situation rather complicated. In order to derive necessary conditions, it suffices to consider particular choices 
for the potentials for which the matrices $V_{c}^{i}$ do not come into play. One possibility is to assume that $\mathfrak{g}$ contains a right-handed potential $\mathcal{A}=\left(0, A_{R}\right) \in \mathfrak{g}$. Then $A_{R}^{\text {even }}$ and $A_{L}$ vanish, so that

$$
B_{L}=-\frac{m^{2}}{2} \grave{Y} A_{R}[\xi]^{2} \grave{Y} T^{(1)}
$$

This must be a multiple of the matrix $Y \dot{Y}$. Proceeding similarly for left-handed potentials gives the following result.

Lemma 5.3.6. Suppose that $\mathcal{A}=\left(A_{L}, 0\right) \in \mathfrak{g}$ (or $\left.\mathcal{A}=\left(0, A_{R}\right) \in \mathfrak{g}\right)$ is a left-handed (respectively right-handed) admissible gauge potential. Then the matrix $A_{L}[\xi]^{2}$ (respectively $A_{R}^{2}[\xi]$ ) is a multiple of the identity matrix at every space-time point and for all directions $\xi$.

The next lemma gives additional information on left-handed or right-handed admissible gauge potentials. For notational simplicity, we only state the result for the left-handed potentials.

Lemma 5.3.7. Suppose that $\mathcal{A}=\left(A_{L}, 0\right) \in \mathfrak{g}$ does not depend on the generation index, i.e.

$$
\left(A_{L}\right)_{(j, \beta)}^{(i, \alpha)}=\delta_{\beta}^{\alpha}\left(A^{\mathrm{sec}}\right)_{j}^{i}
$$

Then

$$
\dot{Y} A_{L}[\xi]^{2} \grave{Y}=\hat{A}_{L}[\xi] Y^{2} \grave{A}_{L}[\xi] .
$$

Proof. According to (5.3.27), we may compute $B_{L}$ according to 5.3.23 with $A_{R}^{\text {even }}=$ $A_{L}$. Then

$$
B_{L}=-\frac{m^{2}}{8}\left(A_{L}[\xi]^{2} Y^{2}+2 A_{L}[\xi] Y^{2} A_{L}[\xi]+Y^{2} A_{L}[\xi]^{2}\right) T^{(1)} .
$$

This matrix must coincide with $B_{R}$, which is computed similar to (5.3.26) by

$$
B_{R}=-\frac{m^{2}}{2} Y A_{L}[\xi]^{2} Y T^{(1)} .
$$

Applying Lemma 5.3.6, the matrix $A_{L}[\xi]^{2}$ is a multiple of the identity and thus commutes with $Y$. This gives the result.

5.3.4. The Field Tensor Terms. The methods in 4.6 .2 also apply to the present situation of eight sectors. In particular, Proposition 4.6 .8 can be restated as follows:

Proposition 5.3.8. Taking into account the contributions by the field tensor terms, the EL equations to degree four can be satisfied only if the regularization satisfies the conditions (5.2.10) and (5.2.9). If no further regularization conditions are imposed, then the chiral potentials must satisfy at all space-time points the conditions

$$
\operatorname{Tr}\left(\Im_{1} A_{R}\right)=0 \quad \text { and } \quad \operatorname{Tr}\left(A_{L}+A_{R}\right)=0,
$$

where $\mathfrak{I}_{1}$ is the projection on the neutrino sector. If conversely the conditions (5.2.10), (5.2.9) and (5.3.28) are satisfied, then the field tensor terms do not contribute to the EL equations of degree four. 
5.3.5. Proof of Spontaneous Block Formation. Instead of working with gauge groups, it will usually be more convenient to consider the corresponding Lie algebras. This is no restriction because the corresponding Lie groups can then be recovered by exponentiation. When forming the Lie algebra of a product of groups, this gives rise to the direct sum of the algebras, like for example

$$
T_{e}\left(\mathrm{U}(8)_{L} \times \mathrm{U}(1)_{R} \times \mathrm{U}(7)_{R}\right)=\mathfrak{u}(8)_{L} \oplus \mathfrak{u}(1)_{R} \oplus \mathfrak{u}(7)_{R} .
$$

The proof of spontaneous block formation will be given in several steps, which are organized in separate paragraphs.

Left-handed $\mathfrak{s u}(2)$-potentials. We now evaluate our assumption (i) that $\mathfrak{g}$ should contain left- or right-handed non-abelian potentials.

We first note that $\mathfrak{g}$ cannot contain right-handed potentials:

LEMma 5.3.9. The dynamical gauge algebra $\mathfrak{g}$ does not contain potentials of the form $\left(0, A_{R}\right)$ with $A_{R} \neq 0$.

Proof. Assume conversely that $\mathcal{A}=\left(0, A_{R}\right) \in \mathfrak{g}$ is a non-trivial admissible righthanded potential. It follows from Lemma 5.3.6 that $A^{2}$ is a multiple of the identity. On the other hand, combining (5.3.10) with the fact that the right-handed potential vanishes on the neutrino sector (see the first equation in (5.3.28), we find that $A_{R}$ must be of the form

$$
A_{R}=\left(\begin{array}{cccc}
0 & 0 & \cdots & 0 \\
0 & \left(A_{R}\right)_{2}^{2} & \cdots & \left(A_{R}\right)_{8}^{2} \\
\vdots & \vdots & \ddots & \vdots \\
0 & \left(A_{R}\right)_{2}^{8} & \cdots & \left(A_{R}\right)_{8}^{8}
\end{array}\right)
$$

As a consequence, $A_{R}^{2}$ cannot be a multiple of the identity, a contradiction.

Thus it remains to consider the case that $\mathfrak{g}$ contains non-abelian left-handed potentials. The left-handed potentials form a Lie subalgebra of $\mathfrak{g}$,

$$
\mathfrak{g}_{L}:=\left\{\mathcal{A}=\left(A_{L}, 0\right) \in \mathfrak{g}\right\} \subset \mathfrak{g} .
$$

Again applying Lemma 5.3.6, we know that every $\mathcal{A}=\left(A_{L}, 0\right) \in \mathfrak{g}$ has the property that $A^{2}$ is a multiple of the identity. The following general lemma gives an upper bound for the dimension of $\mathfrak{g}_{L}$.

Lemma 5.3.10. Let $\mathfrak{h} \subset \mathfrak{s u}(N)$ be a Lie algebra with the additional property that

$$
A^{2} \sim \mathbb{1}_{\mathbb{C}^{N}} \quad \text { for all } A \in \mathfrak{h} .
$$

Then $\mathfrak{h}$ is isomorphic to a subalgebra of $\mathfrak{s u}(2)$.

Proof. Polarizing (5.3.31), we find that for all $A, A^{\prime} \in \mathfrak{h}$,

$$
\left\{A, A^{\prime}\right\}=k\left(A, A^{\prime}\right) \mathbb{1}_{\mathbb{C}^{N}}
$$

with a bilinear form $k: \mathfrak{h} \times \mathfrak{h} \rightarrow \mathbb{R}$. Since the square of a non-zero Hermitian matrix is positive semi-definite and non-zero, we conclude that $k$ is positive definite and thus defines a scalar product on $\mathfrak{h}$. Hence $\mathfrak{h}$ generates a Clifford algebra $\mathfrak{C} \ell(\mathfrak{h}, \mathbb{R})$. Since $\mathfrak{h}$ is also a Lie algebra, the commutator of two elements in $\mathfrak{h}$ is again an element of $\mathfrak{h}$. This means for the Clifford algebra that the bilinear covariants $[u, v]$ with $u, v \in \mathfrak{h}$ are all multiples of the generators of the Clifford algebra. This in turn implies that the dimension of the Clifford algebra is at most three (for details see the classification of Clifford algebras in [LM]). Moreover, $\mathfrak{h}$ is a Lie algebra isomorphic to a subalgebra of $\mathfrak{s u}(2)$. 
Since every proper Lie subalgebra of $\mathfrak{s u}(2)$ is abelian we immediately obtain the following result.

COROLlary 5.3.11. The left-handed dynamical gauge group $\mathfrak{g}_{L}$, 5.3.30, is Lie algebra isomorphic to $\mathfrak{s u}(2)$.

We now write $\mathfrak{g}_{L}$ more explicitly as matrices.

Lemma 5.3.12. There is a unitary matrix $V \in \mathrm{U}(8)$ (acting on the generations) and a basis $\left(A_{L, \alpha}\right)_{\alpha=1,2,3}$ of $\mathfrak{g}_{L}$ such that

$$
A_{L, \alpha}=U_{L}^{\text {mix }} V\left(\begin{array}{cccc}
\sigma_{\alpha} & 0 & 0 & 0 \\
0 & \sigma_{\alpha} & 0 & 0 \\
0 & 0 & \sigma_{\alpha} & 0 \\
0 & 0 & 0 & \sigma_{\alpha}
\end{array}\right) V^{*}\left(U_{L}^{\text {mix }}\right)^{*}
$$

where $\sigma^{\alpha}$ are the Pauli matrices, and $U_{L}^{\mathrm{mix}}$ is the matrix in 5.2.18.

Proof. Using (5.2.17), we can pull out the mixing matrices and work with $8 \times 8$ matrices. Since $\mathfrak{g}_{L}$ is Lie algebra isomorphic to $\mathfrak{s u}(2)$, it can be regarded as a representation of $\mathfrak{s u}(2)$ on $\mathbb{C}^{8}$. We decompose this representation into irreducible components. The fact that the matrix $\left(A_{L, \alpha}\right)^{2}$ is a multiple of the identity implies that every irreducible component is the fundamental representation (because all the other irreducible representations are not generators of a Clifford algebra). This gives the result.

Arranging the free gauge group of maximal dimension. We denote the commutant of $\mathfrak{g}_{L}$ by $\mathfrak{g}_{L}^{\prime}$,

$$
g_{L}^{\prime}=\left\{\mathcal{A}^{\prime} \in \mathfrak{u}(8)_{L} \oplus \mathfrak{u}(1)_{R} \oplus \mathfrak{u}(7)_{R} \text { with }\left[\mathcal{A}, \mathcal{A}^{\prime}\right]=0 \quad \forall \mathcal{A} \in \mathfrak{g}_{L}\right\} .
$$

Lemma 5.3.13. The dynamical gauge algebra is contained in the direct sum

$$
\mathfrak{g} \subset \mathfrak{g}_{L} \oplus \mathfrak{g}_{L}^{\prime} .
$$

Proof. For any $\mathcal{A}=\left(A_{L}, 0\right) \in \mathfrak{g}_{L}$ and $\tilde{\mathcal{A}}=\left(\tilde{A}_{L}, \tilde{A}_{R}\right) \in \mathfrak{g}$, the commutator is lefthanded

$$
[\mathcal{A}, \tilde{\mathcal{A}}]=\left(\left[A_{L}, \tilde{A}_{L}\right], 0\right) .
$$

Therefore, this commutator must be an element of $\mathfrak{g}_{L}$. In this way, every $\tilde{\mathcal{A}} \in \mathfrak{g}$ gives rise to a linear endomorphism of $\mathfrak{g}_{L}$. In view of the structure equations for $\mathfrak{s u}(2)$ (which in the usual notation with Pauli matrices can be written as $\left[\sigma_{\alpha}, \sigma_{\beta}\right]=i \varepsilon_{\alpha \beta \gamma} \sigma_{\gamma}$ with $\varepsilon$ the totally anti-symmetric Levi-Civita symbol), this endomorphism of $\mathfrak{g}_{L}$ can be realized uniquely as an inner endomorphism, i.e. there is a unique $\hat{\mathcal{A}}=\left(\hat{A}_{L}, 0\right) \in \mathfrak{g}_{L}$ with

$$
[\mathcal{A}, \tilde{\mathcal{A}}]=[\mathcal{A}, \hat{\mathcal{A}}] \quad \text { for all } \mathcal{A} \in \mathfrak{g}_{L} .
$$

As a consequence, the potential $\mathcal{A}^{c}:=\tilde{\mathcal{A}}-\hat{\mathcal{A}}$ lies in the commutant $\mathfrak{g}_{L}^{\prime}$. We thus obtain a unique decomposition

$$
\tilde{\mathcal{A}}=\hat{\mathcal{A}}+\mathcal{A}^{c} \quad \text { with } \quad \hat{\mathcal{A}} \in \mathfrak{g}_{L} \text { and } \mathcal{A}^{c} \in \mathfrak{g}_{L}^{\prime} .
$$

This concludes the proof. 
In particular, this lemma gives information on the left-handed component of $\mathfrak{g}$, denoted by

$$
\operatorname{pr}_{L} \mathfrak{g}=\left\{A_{L} \text { with }\left(A_{L}, A_{R}\right) \in \mathfrak{g}\right\} \subset \mathfrak{u}(8)
$$

Note that the projection $\operatorname{pr}_{L} \mathfrak{g}$ is a Lie algebra which contains $\mathfrak{g}_{L}$.

LEMma 5.3.14. The left-handed component of the dynamical gauge algebra satisfies the inclusion

$$
\operatorname{pr}_{L} \mathfrak{g} \subset \mathfrak{g}_{L} \oplus \mathfrak{u}(4)
$$

Here the elements of $\mathfrak{u}(4)$ come with the matrix representation

$$
U_{L}^{\text {mix }} V\left(A \otimes \mathbb{1}_{\mathbb{C}^{2}}\right) V^{*}\left(U_{L}^{\text {mix }}\right)^{*}, \quad A \in \mathfrak{u}(4),
$$

where the factor $\mathbb{1}_{\mathbb{C}^{2}}$ acts on the block matrix entries in (5.3.32).

Proof. The commutant of the matrices in (5.3.32) is computed to be all matrices whose block matrix entries are the identity. Taking a unitary transformation gives the result.

Let us consider what this lemma tells us about the possible form of the free gauge algebra. Since $\mathfrak{g}$ does not contain right-handed gauge potentials (see Lemma 5.3.9), the left-handed component of the free gauge potentials is disjoint from $\mathfrak{g}_{L}$. Hence, using (5.3.33),

$$
\operatorname{pr}_{L} \mathfrak{g}_{\text {free }} \subset \mathfrak{u}(4)
$$

where the potentials in $\mathfrak{u}(4)$ are again of the form $(5.3 .34)$, plus possibly vectors of $\mathfrak{g}_{L}$. The right-handed component of $\mathfrak{g}_{\text {freee }}$, on the other hand, must be of the form 5.3 .29 . Combining these findings gives the following result.

LEMma 5.3.15. Choosing the mixing matrices such that the free gauge group has the maximal dimension, we obtain

$$
\mathfrak{g}_{\text {free }}=\mathfrak{u}(1) \times \mathfrak{s u}(3) .
$$

In a suitable global gauge, the gauge potentials in $\mathfrak{g}_{L}$ (see (5.3.30) and Lemma 5.3.12) have a basis $\left(A_{L, \alpha}\right)_{\alpha=1,2,3}$ with

$$
A_{L, \alpha}=U_{L}^{\operatorname{mix}}\left(\begin{array}{cccc}
\sigma_{\alpha} & 0 & 0 & 0 \\
0 & \sigma_{\alpha} & 0 & 0 \\
0 & 0 & \sigma_{\alpha} & 0 \\
0 & 0 & 0 & \sigma_{\alpha}
\end{array}\right)\left(U_{L}^{\mathrm{mix}}\right)^{*}
$$

where the mixing matrix $U_{L}$ is a diagonal matrix on the sectors of the form

$$
U_{L}^{\text {mix }}=\operatorname{diag}\left(U_{1}, U_{2}, \mathbb{1}, U_{\mathrm{CKM}}, \mathbb{1}, U_{\mathrm{CKM}}, \mathbb{1}, U_{\mathrm{CKM}}\right)
$$

with unitary matrices $U_{1}, U_{2}, U_{\mathrm{CKM}} \in \mathrm{U}(3)$. The free $\mathfrak{u}(1)$ - and $\mathfrak{s u}(3)$-potentials, on the other hand, have the respective matrix representations

$$
\begin{array}{ll}
A_{L}=A_{R}=B \operatorname{diag}\left(0,-1, \frac{2}{3},-\frac{1}{3}, \frac{2}{3},-\frac{1}{3}, \frac{2}{3},-\frac{1}{3}\right) & \text { with } B \in \mathfrak{u}(1)=\mathbb{R} \\
A_{L}=A_{R}=\left(\begin{array}{cc}
0 & 0 \\
0 & C
\end{array}\right) \otimes \mathbb{1}_{\mathbb{C}^{2}} & \text { with } C \in \mathfrak{s u}(3) .
\end{array}
$$

Proof. The free gauge potentials are those vectorial potentials which are compatible with both 5.3.35 and 5.3.29). The zero matrix entries in 5.3.29 imply that at least one of the rows and columns of $\mathfrak{u}(4)$ must be zero. But it is possible to realize the subgroup $\mathfrak{u}(3)$ by considering the potentials of the form 5.3 .39 (but with $C \in \mathfrak{u}(3)$ ). In 
order to get consistency, we must make sure that all mixing matrices drop out of (5.3.34). This forces us to choose the mixing matrix of the form

$$
U_{L}^{\text {mix }}=\operatorname{diag}\left(U_{1}, U_{2}, U_{3}, U_{4}, U_{3}, U_{4}, U_{3}, U_{4}\right)
$$

with four unitary matrices $U_{j} \in \mathrm{U}(3)$. Since a joint unitary transformations of all sectors has no influence on the potentials in (5.2.17), it is no loss of generality to choose $U_{3}=\mathbb{1}$. This gives (5.3.37). In order to satisfy the second relation in $(5.3 .28)$, the matrix $C$ must be trace-free. This gives (5.3.39).

In order to find all the remaining vectorial potentials which are compatible with both (5.3.35) and (5.3.29), one must keep in mind that the left-handed component may be formed of linear combinations of (5.3.34) and the potentials in $\mathfrak{g}_{L}$ of the form (5.3.36). In order for the first row and column to vanish, the only possibility is to form the linear combinations of $A_{L, 3}$ and $\mathbb{1} \in \mathfrak{u}(4)$

$$
B \operatorname{diag}(0,-1,0,-1,0,-1,0,-1) \text {. }
$$

In view of the second relation in (5.3.28), we must remove the trace by adding a multiple of the potential (5.3.39) with $C=\mathbb{1}_{\mathbb{C}^{3}}$. This gives (5.3.38) and concludes the proof.

Proof that $\mathfrak{g}$ is maximal. So far, we constructed the dynamical gauge algebra

$$
\mathfrak{g}_{L} \oplus \mathfrak{g}_{\text {free }} \simeq \mathfrak{s u}(2) \oplus \mathfrak{u}(1) \oplus \mathfrak{s u}(3) .
$$

Let us show that this dynamical gauge algebra is maximal. To this end, assume that there is a chiral potential $\mathcal{A}^{\text {new }}=\left(A_{L}^{\text {new }}, A_{R}^{\text {new }}\right) \in \mathfrak{u}(8) \oplus \mathfrak{u}(7)$ which is an element of $\mathfrak{g}$ but not of $\mathfrak{g}_{L} \oplus \mathfrak{g}_{\text {free }}$,

$$
\mathcal{A}^{\text {new }} \in \mathfrak{g} \backslash\left(\mathfrak{g}_{L} \oplus \mathfrak{g}_{\text {free }}\right) .
$$

We want to deduce a contradiction. Since right-handed potentials were excluded in Lemma 5.3.9, we can assume that $A_{L}^{\text {new }} \neq 0$. According to Lemma 5.3.14, it suffices to consider the potentials in $\mathfrak{g}_{L} \oplus \mathfrak{u}(4)$ (where the $\mathfrak{u}(4)$ )-potentials are represented similar to 5.3 .39 as $C \times \mathbb{1}_{\mathbb{C}^{2}}$ with $\left.C \in \mathfrak{u}(4)\right)$. Moreover, by adding suitable potentials in $\mathfrak{g}_{L} \oplus \mathfrak{g}_{\text {free }}$ and using the freedom to conjugate with exponentials of potentials in $\mathfrak{g}_{\text {free }}$, we can arrange that $A_{L}^{\text {new }}$ is of the form

$$
A_{L}^{\text {new }}=\alpha\left(\begin{array}{cccc}
\mathbb{1}_{\mathbb{C}^{2}} & 0 & 0 & 0 \\
0 & 0 & 0 & 0 \\
0 & 0 & 0 & 0 \\
0 & 0 & 0 & 0
\end{array}\right)+\beta U_{L}^{\text {mix }}\left(\begin{array}{cccc}
0 & \mathbb{1}_{\mathbb{C}^{2}} & 0 & 0 \\
\mathbb{1}_{\mathbb{C}^{2}} & 0 & 0 & 0 \\
0 & 0 & 0 & 0 \\
0 & 0 & 0 & 0
\end{array}\right)\left(U_{L}^{\text {mix }}\right)^{*}
$$

with real parameters $\alpha$ and $\beta$. By subtracting multiples of the potentials $A_{L, 3}$ and the $\mathfrak{u}(1)$-potential, we can even arrange that $A_{L}^{\text {new }}$ is of the form

$$
A_{L}^{\text {new }}=\alpha\left(\begin{array}{cccc}
0 & 0 & 0 & 0 \\
0 & \mathbb{1}_{\mathbb{C}^{2}} & 0 & 0 \\
0 & 0 & \mathbb{1}_{\mathbb{C}^{2}} & 0 \\
0 & 0 & 0 & \mathbb{1}_{\mathbb{C}^{2}}
\end{array}\right)+\beta U_{L}^{\text {mix }}\left(\begin{array}{cccc}
0 & \mathbb{1}_{\mathbb{C}^{2}} & 0 & 0 \\
\mathbb{1}_{\mathbb{C}^{2}} & 0 & 0 & 0 \\
0 & 0 & 0 & 0 \\
0 & 0 & 0 & 0
\end{array}\right)\left(U_{L}^{\text {mix }}\right)^{*} .
$$

The corresponding right-handed component $A_{R}^{\text {new }}$ is unknown, except that it must be of the form (5.3.29). In particular, $A_{R}^{\text {new }}$ might involve a non-trivial mixing matrix.

Our strategy is to evaluate off-diagonal matrix elements of $\hat{B}_{R}$ (see $(5.3 .23)$ with $L$ and $R$ exchanged) for specific choices of the potential. The vanishing of these matrix elements gives us constraints for $\mathcal{A}^{\text {new }}$ which in turn imply that the parameters $\alpha$ and $\beta$ in 5.3.40 must vanish. We begin with the parameter $\beta$. 
Lemma 5.3.16. If $\mathfrak{g}$ contains a potential $\mathcal{A}=\left(A_{L}, A_{R}\right)$ with $A_{L}$ of the form 5.3.40) with $\beta \neq 0$, then necessarily

$$
U_{1}=\mathbb{1}_{\mathbb{C}^{3}} \quad \text { and } \quad U_{2} U_{\mathrm{CKM}}^{*}=\mathbb{1}_{\mathbb{C}^{3}} .
$$

Proof. By conjugating with $\mathrm{SU}(3)$-transformations in the free gauge group, we can transform the potential $A_{L}^{\text {new }}$ from 5.3 .40 to the matrix

$$
A_{L}=\alpha\left(\begin{array}{cccc}
0 & 0 & 0 & 0 \\
0 & \mathbb{1}_{\mathbb{C}^{2}} & 0 & 0 \\
0 & 0 & \mathbb{1}_{\mathbb{C}^{2}} & 0 \\
0 & 0 & 0 & \mathbb{1}_{\mathbb{C}^{2}}
\end{array}\right)+\beta U_{L}^{\text {mix }} M\left(U_{L}^{\text {mix }}\right)^{*}
$$

where $M$ can be any of the six matrices

$$
\begin{gathered}
\left(\begin{array}{llll}
0 & \mathbb{1} & 0 & 0 \\
\mathbb{1} & 0 & 0 & 0 \\
0 & 0 & 0 & 0 \\
0 & 0 & 0 & 0
\end{array}\right), \quad\left(\begin{array}{llll}
0 & 0 & \mathbb{1} & 0 \\
0 & 0 & 0 & 0 \\
\mathbb{1} & 0 & 0 & 0 \\
0 & 0 & 0 & 0
\end{array}\right), \\
\left(\begin{array}{cccc}
0 & -i \mathbb{1} & 0 & 0 \\
i \mathbb{1} & 0 & 0 & 0 \\
0 & 0 & 0 & 0 \\
0 & 0 & 0 & 0
\end{array}\right), \quad\left(\begin{array}{cccc}
0 & 0 & -i \mathbb{1} & 0 \\
0 & 0 & 0 & 0 \\
i \mathbb{1} & 0 & 0 & 0 \\
0 & 0 & 0 & 0
\end{array}\right), \quad\left(\begin{array}{cccc}
0 & 0 & 0 & \mathbb{1} \\
0 & 0 & 0 & 0 \\
0 & 0 & 0 & 0 \\
\mathbb{1} & 0 & 0 & 0
\end{array}\right),
\end{gathered}
$$

If (5.3.41) is violated, then the corresponding matrices $(1-\check{\pi}) A_{L} \check{\pi}$ are obviously nontrivial and linearly independent. As a consequence, the vector space $\mathcal{B}$ (see (5.3.18) has dimension at least six. If the mixing matrix $U_{\mathrm{CKM}}$ is non-trivial, the operators $B$ corresponding to the left-handed potentials $A_{L, 1}$ and $A_{L, 2}$ in 5.3.36 are also non-zero, increasing the dimension of $\mathcal{B}$ to at least eight. We now show that these dimensions contradict the upper bound of Corollary 5.3.5. We treat the cases separately when $U_{\mathrm{CKM}}$ is trivial and non-trivial.

In the case that the matrix $U_{\mathrm{CKM}}$ is non-trivial, the form of the right-handed component of the potentials $(5.3 .29)$ implies that the first row cannot contribute to the operators $B$. As a consequence, the dimension $N$ in Corollary 5.3.5 is at most $2 \times(8+7)=30$. Hence 5.3.22 yields that the dimension of $\mathcal{B}$ is at most 7 , a contradiction.

In the case that the matrix $U_{\mathrm{CKM}}$ is trivial, the mixing of the generations comes about only as a consequence of the matrices $U_{1}$ and $U_{2}$ in (5.3.37). Hence the matrices $B_{L}$ (see (5.3.15) ) are of the general form

$$
(1-\check{\pi})\left(\begin{array}{cccccccc}
0 & \star U_{1} U_{2}^{*} & \star U_{1} & \star U_{1} & \star U_{1} & \star U_{1} & \star U_{1} & \star U_{1} \\
\star U_{2} U_{1}^{*} & 0 & \star U_{2} & \star U_{2} & \star U_{2} & \star U_{2} & \star U_{2} & \star U_{2} \\
\star U_{1}^{*} & \star U_{2}^{*} & 0 & 0 & 0 & 0 & 0 & 0 \\
\star U_{1}^{*} & \star U_{2}^{*} & 0 & 0 & 0 & 0 & 0 & 0 \\
\star U_{1}^{*} & \star U_{2}^{*} & 0 & 0 & 0 & 0 & 0 & 0 \\
\star U_{1}^{*} & \star U_{2}^{*} & 0 & 0 & 0 & 0 & 0 & 0 \\
\star U_{1}^{*} & \star U_{2}^{*} & 0 & 0 & 0 & 0 & 0 & 0 \\
\star U_{1}^{*} & \star U_{2}^{*} & 0 & 0 & 0 & 0 & 0 & 0
\end{array}\right) \check{\pi}
$$

where the stars $\star$ denote complex factors. Evaluating more specifically the six possible choices for the matrix $M$ in $(5.3 .42)$, one immediately verifies that the span of the images of the corresponding matrices $B_{L}$ has dimension 8 (note that the terms involving $\alpha$ drop out, and that the first two rows have a six-dimensional image, whereas the other six rows have a two-dimensional image). Hence the dimension $N$ in Corollary 5.3.5 is at 
most $8+2 \times 7=22$. The inequality $(5.3 .22)$ implies that the dimension of $\mathcal{B}$ is at most 5 . This is again a contradiction.

Lemma 5.3.17. Assume that the dynamical gauge algebra $\mathfrak{g}$ contains a potential $\mathcal{A}^{\text {new }}=$ $\left(A_{L}^{\text {new }}, A_{R}^{\text {new }}\right) \in \mathfrak{u}(8) \oplus \mathfrak{u}(7)$ with $A_{L}^{\text {new }}$ of the form 5.3.40). Then $\beta$ vanishes.

Proof. For a parameter $\varepsilon>0$, we consider the family of potentials

$$
\mathcal{A}=\mathcal{A}^{\text {old }}+\varepsilon \mathcal{A}^{\text {new }} \quad \text { with } \quad \mathcal{A}^{\text {old }}=\left(A_{L}^{\text {old }}=A_{L, 3}, 0\right)
$$

and $A_{L, 3}$ as in (5.3.36). We compute the terms linear in $\varepsilon$. Moreover, we consider the matrix entry of $B_{R}$ in the third row and first column (where we again consider $B_{R}$ as an $8 \times 8$-matrix on the sectors). Using that $A_{L}^{\text {old }}$ is sector-diagonal and the matrix component $\left(A_{R}\right)_{3}^{1}$ vanishes, the matrix $A_{R}$ drops out. Similarly, the matrix $A_{L}^{\text {even }}$ drops out (see (5.3.24) $)$, which also has the desirable effect that the corresponding mixing matrix $V_{R}$ does not enter. We thus obtain

$$
\begin{aligned}
\left(B_{R}\right)_{1}^{3} & =-\frac{m^{2}}{2} \varepsilon\left(Y\left(A_{L}^{\text {old }} A_{L}^{\text {new }}+A_{L}^{\text {new }} A_{L}^{\text {old }}\right) Y\right)_{1}^{3} T^{(1)}+\mathcal{O}\left(\varepsilon^{2}\right) \\
& =-\frac{m^{2}}{2} \varepsilon Y_{3}^{3}\left(\left(A_{L}^{\text {old }}\right)_{3}^{3}\left(A_{L}^{\text {new }}\right)_{1}^{3}+\left(A_{L}^{\text {new }}\right)_{1}^{3}\left(A_{L}^{\text {old }}\right)_{1}^{1}\right) Y_{1}^{1} T^{(1)}+\mathcal{O}\left(\varepsilon^{2}\right) \\
& =m^{2} \varepsilon Y_{3}^{3}\left(A_{L}^{\text {new }}\right)_{1}^{3} Y_{1}^{1} T^{(1)}+\mathcal{O}\left(\varepsilon^{2}\right) \\
& =m^{2} \beta \varepsilon Y_{3}^{3} U_{1}^{*} Y_{1}^{1} T^{(1)}+\mathcal{O}\left(\varepsilon^{2}\right),
\end{aligned}
$$

where in the last step we used (5.3.40) together with the form of the mixing matrix (5.3.37). Taking the sectorial projection and applying Lemma 5.3 .16 gives the result.

The argument which shows that $\alpha$ vanishes is somewhat different:

Lemma 5.3.18. Assume that the dynamical gauge algebra $\mathfrak{g}$ contains a potential $\mathcal{A}^{\text {new }}=$ $\left(A_{L}^{\text {new }}, A_{R}^{\text {new }}\right) \in \mathfrak{u}(8) \oplus \mathfrak{u}(7)$ with $A_{L}^{\text {new }}$ of the form 5.3.40 and $\beta=0$. Then $\alpha$ vanishes.

Proof. Assume conversely that there is an admissible potential $\mathcal{A}=\left(A_{L}, A_{R}\right) \in \mathfrak{g}$ with

$$
A_{L}=\left(\begin{array}{cc}
0 & 0 \\
0 & \mathbb{1}_{\mathbb{C}^{3}}
\end{array}\right) \otimes \mathbb{1}_{\mathbb{C}^{2}}
$$

This potential involves no mixing matrices. Thus we may compute $B_{L}$ according to 5.3 .23 with $A_{R}^{\text {even }}=A_{L}$. Since the left-handed component is sector-diagonal, we obtain

$$
B_{L}=-\frac{m^{2}}{2} Y\left(A_{L}-A_{R}\right)^{2} Y
$$

The sectorial projection of this matrix must be a multiple of $Y \dot{Y}$. In view of $(5.3 .29$, this implies that $\left(A_{L}-A_{R}\right)^{2}=0$, and thus $A_{L}=A_{R}$. But the resulting potential violates the second equation in (5.3.28) and is thus not admissible. This is a contradiction.

The previous lemmas show that the parameters $\alpha$ and $\beta$ in 5.3 .40 are both zero. But then $A_{L}^{\text {new }}$ vanishes, a contradiction. We conclude that $\mathfrak{g}=\mathfrak{g}_{L} \oplus \mathfrak{g}_{\text {free }}$ is maximal. 
Non-triviality of the mixing matrices. We now analyze the properties of the mixing matrices $U_{\mathrm{MNS}}$ and $U_{\mathrm{CKM}}$. Suppose that one of these matrices is non-trivial. Then the matrix $B$ (see (5.3.16) ) corresponding to the left-handed potentials $A_{L, 1}$ and $A_{L, 2}$ in $(5.3 .36)$ is non-zero. The representation of Lemma 5.3.4 yields in particular that the columns of the matrix $B$ all have the same norm. This implies that

$$
\left\|(\mathbb{1}-\check{\pi}) U_{\mathrm{MNS}} \check{\pi}\right\|=\left\|(\mathbb{1}-\check{\pi}) U_{\mathrm{CKM}} \check{\pi}\right\| \text {. }
$$

This shows that if one of the matrices $U_{\mathrm{MNS}}$ and $U_{\mathrm{CKM}}$ is non-trivial, the other is also non-trivial. Moreover, by a global phase transformation, we can arrange that the relation 5.3.5 holds.

In the next lemma we show that 5.3.6 implies (5.3.7).

Lemma 5.3.19. Suppose that the masses of the charged leptons and neutrinos are different in the sense (5.3.6). Then the mixing matrices $U_{\mathrm{MNS}}$ and $U_{\mathrm{CKM}}$ are non-trivial.

Proof. Assume conversely that the potentials in $\mathfrak{g}_{L}$ do not involve mixing matrices. Then for any $\mathcal{A} \in \mathfrak{g}_{L}$, we can compute $B_{L}$ according to $(5.3 .23)$ with $A_{R}^{\text {even }}=A_{L}$. Considering the first sector and taking the sectorial projection, we obtain

$$
\Im_{1} \hat{B}_{L} \Im_{1}=-\frac{m^{2}}{4} \Im_{1}\left(\dot{Y} \grave{Y} A_{L}[\xi]^{2}+A_{L}[\xi] \grave{Y} \grave{Y} A_{L}[\xi]\right) \mathfrak{I}_{1} T^{(1)}
$$

where $\mathfrak{I}_{1}$ denotes the projection on the neutrino sector. Since the right-handed component of $\mathcal{A}$ vanishes, we can also compute $B_{R}$ with $A_{L}^{\text {even }}=A_{R}$. This gives

$$
\mathfrak{I}_{1} \hat{B}_{R} \mathfrak{I}_{1}=-\frac{m^{2}}{2} \mathfrak{I}_{1} \dot{Y} \grave{Y} A_{L}[\xi]^{2} \mathfrak{I}_{1} T^{(1)} .
$$

We conclude that

$$
\begin{aligned}
\mathfrak{I}_{1}\left(\hat{B}_{L}-\hat{B}_{R}\right) \mathfrak{I}_{1} & =\frac{m^{2}}{4} \mathfrak{I}_{1}\left(\dot{Y} \grave{Y} A_{L}[\xi]^{2}-A_{L}[\xi] \dot{Y} \grave{Y} A_{L}[\xi]\right) \mathfrak{I}_{1} T^{(1)} \\
& =\frac{m^{2}}{4} \mathfrak{I}_{1} A_{L}[\xi]^{2} \mathfrak{I}_{1} T^{(1)} \sum_{\alpha=1}^{3}\left(\tilde{m}_{\alpha}^{2}-m_{\alpha}^{2}\right) .
\end{aligned}
$$

This is non-zero for the potentials $A_{L, 1}$ and $A_{L, 2}$ in (5.3.36), a contradiction.

Proof that $\mathfrak{g}$ is admissible. We have shown that, under the assumptions of Theorem 5.3.2, the dynamical gauge potentials are necessarily of the form (5.3.2)-(5.3.4). It remains to show that these potentials are indeed admissible in the sense that they satisfy all algebraic constraints. This can be done explicitly as follows: In order to study the sectorial projection of the gauge phases, one can use the fact that the phases of the free gauge potentials 5.3.2 and 5.3.4 drop out of the closed chain and thus do not enter the EL equations. Therefore, it suffices to consider the weak potentials (5.3.3). Since the weak potentials are block-diagonal, one may consider the lepton block and the charged blocks separately. For the lepton block, the computations were carried out in 4.3 .2 . In the charged blocks, the computation is even easier because there are no shear contribution; it reduces to applying Lemma 4.3.2. For the bilinear logarithmic terms one can again use that the free gauge potentials drop out. Therefore, one can analyze the neutrino block and the charged blocks exactly as in 84.6 .1 . For the field tensor terms, the relevant contributions are linear in the field. Therefore, one may choose a basis on the sectors where the field tensor is diagonal and use the computations and results of 4.6 .2 . 
This completes the proof of Theorem 5.3.2.

\subsection{The Effective Action}

In this section we rewrite the EL equations in the continuum limit $(5.2 .28)$ as the Euler-Lagrange equations of an effective action. We adapt the methods introduced in Chapter 4. This adaptation is straightforward because the dynamical gauge potentials as obtained in Theorem 5.3.2 either act separately within each block (the weak and electromagnetic gauge potentials), or else they mix identical blocks (the strong gauge potentials). This makes it possible to analyze the blocks separately. For each block, we can proceed just as in Sections 4.7 and 4.8 .

5.4.1. The General Strategy. Our goal is to rewrite (5.2.28) as the Euler-Lagrange equations of an effective action of the form

$$
\mathcal{S}_{\text {eff }}=\int_{\mathscr{M}}\left(\mathcal{L}_{\text {Dirac }}+\mathcal{L}_{\mathrm{YM}}+\mathcal{L}_{\mathrm{EH}}\right) \sqrt{-\operatorname{deg} g} d^{4} x
$$

involving a Dirac Lagrangian (which describes the coupling of the Dirac wave functions to the gauge fields and gravity), a Yang-Mills-type Lagrangian for the gauge fields and the Einstein-Hilbert action. Moreover, the Dirac wave functions should satisfy the Dirac equation (see (5.2.6)

$$
(i \not \partial+\mathcal{B}-m Y) \psi=0 .
$$

Exactly as explained in $\$ 4.7 .2$, one must take into account that the Dirac equation 5.4 .2 holds for the auxiliary fermionic projector (without taking the sectorial projection), whereas the current and the energy-momentum tensor, which are to be obtained by varying the Dirac Lagrangian in (5.4.1), involve a sectorial projection. This leads us to choose the effective Dirac Lagrangian as

$$
\mathcal{L}_{\text {Dirac }}=\operatorname{Re}\left(\bar{\psi} 3 \check{\pi}_{\tau}(i \not \partial+\check{\pi} \mathcal{B} \check{\pi}-m Y) \psi\right),
$$

where the operator $\check{\pi}_{\tau}$ has the form

$$
\check{\pi}_{\tau}:=\left(1+\tau \chi_{L} \mathfrak{I}_{1}\right) \check{\pi} \quad \text { with } \tau \in \mathbb{R},
$$

and $\mathfrak{I}_{1}$ is again the projection on the neutrino sector. The sectorial projections in (5.4.3) are needed in order to get the correct coupling of the Dirac wave functions to the bosonic fields. The parameter $\tau$ in (5.4.4) gives us the freedom to modify the coupling of the right-handed component of the neutrinos to the gravitational field.

Our goal is to choose the Lagrangians $\mathcal{L}_{\mathrm{YM}}$ and $\mathcal{L}_{\mathrm{EH}}$ such that their first variation is compatible with 5.2.28. In order to treat the gauge fields, one first rewrites $\Delta Q$ as

$$
\Delta Q(x, y)=\frac{i}{2} \sum_{n, c} \operatorname{Tr}_{\mathbb{C}^{2}}\left(I_{n} \mathcal{Q}_{c}\right) I_{n} \chi_{c} \notin,
$$

and represents the factors $\mathcal{Q}_{c}$ by

$$
\mathcal{Q}_{L}:=\mathcal{K}_{L}-\frac{1}{4} \operatorname{Tr}_{\mathbb{C}^{2}}\left(\mathcal{K}_{L}+\mathcal{K}_{R}\right) \mathbb{1}_{\mathbb{C}^{2}}
$$

(and similarly for $\mathcal{Q}_{R}$ ), where the matrices $\mathcal{K}_{c}$ are defined by

$$
\operatorname{Tr}_{\mathbb{C}^{2}}\left(I_{n} \mathcal{K}_{c}\right)=\frac{\Delta\left|\lambda_{n c-}^{x y}\right|}{\left|\lambda_{-}\right|} 3^{3} T_{[0]}^{(0)} T_{[0]}^{(-1)} \overline{T_{[0]}^{(-1)}}+(\operatorname{deg}<4) .
$$


In Chapter 4 , the matrices $\mathcal{K}_{c}$ are computed in the neutrino block, and these computations apply just as well to the quark blocks. Next, in order to treat the tensor indices properly, one writes $\mathcal{K}_{c}$ as

$$
\mathcal{K}_{c}=i \xi_{k} \mathfrak{J}_{c}^{k}+(\operatorname{deg}<4)+o\left(|\vec{\xi}|^{-3}\right)
$$

and sets

$$
\mathcal{Q}_{c}^{k}:=\mathcal{K}_{c}^{k}-\frac{1}{4} \operatorname{Tr}_{\mathbb{C}^{2}}\left(\mathcal{K}_{L}^{k}+\mathcal{K}_{R}^{k}\right) \mathbb{1}_{\mathbb{C}^{2}} .
$$

The Lagrangian $\mathcal{L}_{\text {YM }}$ must be chosen such as to satisfy the conditions

$$
K(\varepsilon, \xi) \frac{\delta}{\delta \mathcal{A}}\left(\mathcal{L}_{\text {Dirac }}+\mathcal{L}_{\mathrm{YM}}\right)=\operatorname{Tr}_{\mathbb{C}^{8}}\left(\mathcal{Q}_{L}^{k}[\hat{\mathcal{J}}, \mathcal{A}]\left(\delta \hat{A}_{R}\right)_{k}+\mathcal{Q}_{R}^{k}[\hat{\mathcal{J}}, \mathcal{A}]\left(\delta \hat{A}_{L}\right)_{k}\right)
$$

where $K$ is a constant and $\delta \mathcal{A}=\left(\delta A_{L}, \delta A_{R}\right) \in \mathfrak{g}$ is a dynamical gauge potential. Here the square brackets $[\hat{\mathcal{J}}, \mathcal{A}]$ clarify the dependence on the chiral potentials and on the sectorial projection of the Dirac current.

In order to treat the gravitational field, we rewrite the trace component of $\Delta Q$ as

$$
\operatorname{Tr}_{\mathbb{C}^{8 \times 4}}(\Delta Q \not u)=i \xi_{j} u^{j} \mathcal{Q}^{k l}[\hat{T}, g] \xi_{k} \xi_{l} .
$$

Our task is to satisfy the relation

$$
i K(\varepsilon, \xi) \frac{\delta}{\delta g}\left(\left(\mathcal{L}_{\text {Dirac }}+\mathcal{L}_{\mathrm{YM}}+\mathcal{L}_{\text {curv }}\right) \sqrt{-\operatorname{deg} g}\right)=\mathcal{Q}^{k l}[\hat{T}, g] \delta g_{k l} .
$$

Once we have arranged (5.4.6) and (5.4.7), we may consider (5.4.1) as the effective action in the continuum limit. Varying the chiral potentials in $\mathfrak{g}$ gives the bosonic field equations, whereas varying the metric gives the equations for gravity. We point out that the variation of the effective action must always be performed under the constraint that the Dirac equation $(5.4 .2$ holds. As explained in 4.7 .3 , this gives rise to the so-called sectorial corrections to the field equations. Since these corrections are computed exactly as in Chapter 4, we do not enter the calculations again. Instead, we restrict attention to deriving the effective action and to discussing our findings.

\subsubsection{The Effective Lagrangian for Chiral Gauge Fields.}

General structure of the effective Lagrangian. We begin with a general result on the structure of the effective Lagrangian for the gauge fields. The connection to the Lagrangian of the standard model will be explained afterwards.

Theorem 5.4.1. Denoting the dynamical gauge potentials as in Theorem 5.3.2 and decomposing the weak potentials as $W=\sum_{\alpha=1}^{3} W^{\alpha} \sigma^{\alpha} \in \mathfrak{s u}(2)$, the EL equations in the continuum limit are of variational form (5.4.6), where the effective Lagrangians is of the form (5.4.3) and

$$
\begin{aligned}
\mathcal{L}_{\mathrm{YM}}= & c_{1}\left(\operatorname{Tr}_{\mathbb{C}^{3}}\left(\left(\partial_{j} G\right)\left(\partial^{j} G\right)\right)+\frac{4}{3}\left(\partial_{j} A^{\mathrm{em}}\right)\left(\partial^{j} A^{\mathrm{em}}\right)\right) \\
& +c_{2}\left(\left(\partial_{j} W^{1}\right)\left(\partial^{j} W^{1}\right)+\left(\partial_{j} W^{2}\right)\left(\partial^{j} W^{2}\right)\right)+c_{3}\left(\partial_{j} W^{3}\right)\left(\partial^{j} W^{3}\right) \\
& +c_{4}\left(\partial_{j} A^{\mathrm{em}}\right)\left(\partial^{j} W^{3}\right)+M_{1}^{2}\left(W^{1} W^{1}+W^{2} W^{2}\right)+M_{3}^{2} W^{3} W^{3} .
\end{aligned}
$$

Here $c_{1}, c_{2}, c_{3}, c_{4}$ and $M_{1}, M_{3}$ are parameters which depend on the regularization. 
Proof. The matrix-valued vector fields $\mathfrak{J}_{c}$ in 5.4 .5 were computed in $\$ 4.4 .3$. Combining (5.2.9) with the integration-by-parts rule

$$
0=\nabla\left(T_{[0]}^{(0)} T_{[0]}^{(0)} \overline{T_{[0]}^{(0)}}\right)=2 T_{[0]}^{(0)} T_{[0]}^{(-1)} \overline{T_{[0]}^{(0)}}+T_{[0]}^{(0)} T_{[0]}^{(0)} \overline{T_{[0]}^{(-1)}},
$$

one sees that the following simple fraction vanishes,

$$
K_{2}:=\frac{3}{4} \frac{1}{\overline{T_{[0]}^{(0)}}}\left[T_{[0]}^{(0)} T_{[0]}^{(0)} \overline{T_{[0]}^{(-1)} T_{[0]}^{(0)}}-\text { c.c. }\right]=0 .
$$

As a consequence, one obtains

$$
\begin{aligned}
\mathfrak{J}_{L}^{k}= & \hat{J}_{R}^{k} K_{1}+\hat{\jmath}_{R}^{k} K_{3} \\
& -3 m^{2}\left(\hat{A}_{L}^{k} Y \grave{Y}+\dot{Y} Y \grave{A}_{L}^{k}\right) K_{4} \\
& +m^{2}\left(\hat{A}_{L}^{k} \dot{Y} \grave{Y}+\dot{Y} \grave{Y} \hat{A}_{L}^{k}\right) K_{4} \\
& -3 m^{2}\left(\hat{A}_{R}^{k} Y \grave{Y}-2 \dot{Y} A_{L}^{k} \grave{Y}+\hat{Y} Y \grave{A}_{R}^{k}\right) K_{5} \\
& -6 m^{2}\left(\hat{A}_{L}^{k} \grave{Y} \hat{Y}+\hat{Y} \grave{Y}_{L}^{k}\right) K_{6} \\
& +6 m^{2}\left(\hat{Y} \hat{A}_{L}^{k} \grave{Y}+\hat{Y} \grave{A}_{L}^{k} \hat{Y}\right) K_{7} \\
& +m^{2}\left(\hat{A}_{L}^{k} \hat{Y} \hat{Y}+2 \hat{Y} \hat{A}_{R}^{k} \hat{Y}+\hat{Y} \hat{Y} \hat{A}_{L}^{k}\right) K_{6} \\
& -m^{2}\left(\hat{A}_{R}^{k} \hat{Y} \hat{Y}+2 \hat{Y} \hat{A}_{L}^{k} \hat{Y}+\hat{Y} \hat{Y} \hat{A}_{R}^{k}\right) K_{7}
\end{aligned}
$$

with the simple fractions $K_{1}, \ldots, K_{7}$ as given in 4.4.22 -4.4 .29 (and $\mathfrak{J}_{R}^{k}$ is obtained by the obvious replacements $L \leftrightarrow R$ ). For the Dirac current, we thus obtain

$$
\operatorname{Tr}_{\mathbb{C}^{8}}\left(\mathcal{Q}_{L}^{k}[\hat{\mathcal{J}}, \mathcal{A}]\left(\delta \hat{A}_{R}\right)_{k}+\mathcal{Q}_{R}^{k}[\hat{\mathcal{J}}, \mathcal{A}]\left(\delta \hat{A}_{L}\right)_{k}\right) \asymp K_{1} \operatorname{Tr}_{\mathbb{C}^{8}}\left(J_{R}^{k}\left(\delta \hat{A}_{R}\right)_{k}+J_{L}^{k}\left(\delta \hat{A}_{L}\right)_{k}\right) .
$$

This is compatible with (5.4.6) and the variation of the Dirac Lagrangian (5.4.3) (for fixed wave functions) if we choose

$$
K(\varepsilon, \xi)=3 K_{1}
$$

For the bosonic current and mass terms, one must compensate the logarithmic poles on the light cone by a microlocal chiral transformation, just as described in $\$ 4.4 .4$. For the free gauge potentials $\left(A^{\mathrm{em}}, G\right) \in \mathfrak{u}(1) \oplus \mathfrak{s u}(3)$, the mass terms vanish. A direct computation gives

$$
\begin{gathered}
\operatorname{Tr}_{\mathbb{C}^{8}}\left(\mathcal{Q}_{L}^{k}[\hat{\mathcal{J}}, \mathcal{A}]\left(\delta \hat{A}_{R}\right)_{k}+\mathcal{Q}_{R}^{k}[\hat{\mathcal{J}}, \mathcal{A}]\left(\delta \hat{A}_{L}\right)_{k}\right) \\
\asymp K_{19} \operatorname{Tr}_{\mathbb{C}^{8}}\left(j^{k}\left[A^{\mathrm{em}}\right] \delta A^{\mathrm{em}}+j^{k}[G] \delta G\right)
\end{gathered}
$$

for a suitable simple fraction $K_{19}$, where $j[A]^{k}=\partial^{k} A^{j}-\square A^{k}$ is the bosonic current. If only the potential $W$ is considered, we can compute the right side of 5.4 .6 exactly as in Section 4.8 to obtain

$$
\begin{aligned}
\operatorname{Tr}_{\mathbb{C}^{8}}\left(\mathcal{Q}_{L}^{k}[\hat{\mathcal{J}}, \mathcal{A}]\left(\delta \hat{A}_{R}\right)_{k}+\mathcal{Q}_{R}^{k}[\hat{\mathcal{J}}, \mathcal{A}]\left(\delta \hat{A}_{L}\right)_{k}\right) \\
\asymp K_{20}\left(j^{k}\left[W^{1}\right]\left(\delta W^{1}\right)_{k}+j^{k}\left[W^{2}\right]\left(\delta W^{2}\right)_{k}\right)+K_{21} j^{k}\left[W^{3}\right]\left(\delta W^{3}\right)_{k} \\
\quad+m^{2} K_{22}\left(\left(W^{1}\right)^{k}\left(\delta W^{1}\right)_{k}+\left(W^{2}\right)^{k}\left(\delta W^{2}\right)_{k}\right)+m^{2} K_{23}\left(W^{3}\right)^{k}\left(\delta W^{3}\right)_{k}
\end{aligned}
$$


for suitable simple fractions $K_{\ell}$. Finally, we must take into account cross terms of $A^{\mathrm{em}}$ and $W^{3}$. These have the form

$$
\begin{aligned}
\operatorname{Tr}_{\mathbb{C}^{8}}\left(\mathcal{Q}_{L}^{k}[\hat{\mathcal{J}}, \mathcal{A}]\left(\delta \hat{A}_{R}\right)_{k}+\mathcal{Q}_{R}^{k}[\hat{\mathcal{J}}, \mathcal{A}]\left(\delta \hat{A}_{L}\right)_{k}\right) \\
\asymp K_{24} j^{k}\left[W^{3}\right]\left(\delta A^{\mathrm{em}}\right)_{k}+K_{25} j^{k}\left[A^{\mathrm{em}}\right]\left(\delta W^{3}\right)_{k} \\
\quad+m^{2} K_{26}\left(W^{3}\right)^{k}\left(\delta A^{\mathrm{em}}\right)_{k}+m^{2} K_{27}\left(A^{\mathrm{em}}\right)^{k}\left(W^{3}\right)_{k} .
\end{aligned}
$$

Let us consider the terms (5.4.19). In order to be compatible with the variational ansatz (5.4.6), we must impose that

$$
K_{24}=K_{25}
$$

This relation is automatically satisfied if we use the form of the current terms in 5.4.11). However, one should keep in mind that $K_{3}$ has a logarithmic pole which must be compensated by a microlocal chiral transformation. We thus obtain the condition for the microlocal chiral transformation that it should preserve (5.4.21).

Moreover, the factors $K_{26}$ and $K_{27}$ vanish, as we now explain. First, the potential $A^{\mathrm{em}}$ does not contribute to the mass terms, implying that $K_{27}$ is zero. Moreover, direct inspection of the contributions (5.4.12)-(5.4.18) shows that for a sector-diagonal potential which does not involve a mixing matrix, the mass terms depends only on the combination $A_{L}-A_{R}$. This also implies that

$$
\mathcal{Q}_{L}^{k}=-\mathcal{Q}_{R}^{k}
$$

On the other hand, for a variation by an electromagnetic potential, $\delta A_{L}=\delta A_{R}$. Therefore, the right side of (5.4.6) vanishes by symmetry, implying that $K_{26}$ is zero. Similar as explained above for (5.4.21), the microlocal chiral transformation must be performed in such a way that $(5.4 .22)$ is respected.

Combining all the terms gives the result.

Correspondence to electroweak theory. Let us discuss the form of the effective Lagrangian obtained in Theorem 5.4.1. The first summand in (5.4.8) is precisely the Lagrangian of the strong interaction in the standard model. The second summand (5.4.8) is the Lagrangian of the electromagnetic field. One difference to the standard model is that the coupling constants of the strong and electromagnetic fields are not independent, but are related to each other by an algebraic relation. In order to understand this relation, one should keep in mind that the masses and coupling constants appearing in Theorem 5.4.1 should be regarded as the "naked" constants, which coincide with the physical constants only at certain energy scale which can be thought of as being very large (like for example the Planck energy). Thus a relation for the "naked" constants does not mean that this relation should be valid also for the physical constants. This situation is indeed very similar to that in grand unified theories (GUTs); we refer the reader for example to the textbook [Ro. The terms in $(5.4 .9)$ and $(5.4 .10)$ have a similarity to the Lagrangian of the weak potential after spontaneous symmetry breaking. Indeed, one obtains complete agreement for specific values of the constants:

TheOREm 5.4.2. Assume that the parameters in the effective Lagrangian of Theorem 5.4.1 satisfy the conditions

$$
c_{2}=c_{3}=c_{4} \quad \text { and } \quad M_{1}=M_{3} .
$$


Then the effective Lagrangian coincides with the Lagrangian of the standard model after spontaneous symmetry breaking excluding the Higgs field. The coupling constants of the strong and weak gauge potentials as well of weak hypercharge are given by

$$
g_{\text {strong }}=\frac{1}{2 \sqrt{c_{1}}}, \quad g_{\text {weak }}=\frac{2}{\sqrt{c_{2}}}, \quad g_{\text {hyp }}=\frac{1}{2}\left(\frac{16}{3} c_{1}-c_{2}\right)^{-\frac{1}{2}} .
$$

Under the assumptions of this theorem, one can introduce the $Z$ and $W^{ \pm}$-potentials by forming the usual linear combinations of the weak potential and the potential of weak hypercharge. The masses $m_{Z}$ and $m_{W}$ of the corresponding gauge bosons are related to each other by

$$
m_{Z}=\frac{m_{W}}{\cos \Theta_{W}},
$$

where the Weinberg angle $\Theta_{W}$ is given as usual by

$$
\cos \Theta_{W}=\frac{g_{\text {weak }}}{\sqrt{g_{\text {weak }}^{2}+g_{\text {hyp }}^{2}}} .
$$

Proof. So far, we parametrized the isospin diagonal electroweak potentials by the electromagnetic potential $A^{\mathrm{em}}$ and the weak potential $W^{3}$. The standard model, however, is usually formulated instead in terms of the potential of weak hypercharge $A^{\text {hyp }}$ and the weak potential. Since the transformation from one parametrization to the other also changes the weak potential, we denote the weak potential in the parametrization of the standard model by an additional tilde. Then the potentials are related by

$$
A^{\mathrm{em}}=2 A^{\text {hyp }}, \quad W^{3}=\tilde{W}^{3}-A^{\text {hyp }} .
$$

Using these relations in $(5.4 .8)-(5.4 .10)$, the relevant part of the Lagrangian transforms to

$$
\begin{aligned}
\mathcal{L}_{\mathrm{YM}} \asymp & \frac{16}{3} c_{1}\left(\partial_{j} A^{\mathrm{hyp}}\right)\left(\partial^{j} A^{\mathrm{hyp}}\right) \\
& +c_{3}\left(\partial_{j} A^{\mathrm{hyp}}\right)\left(\partial^{j} A^{\mathrm{hyp}}\right)-2 c_{3}\left(\partial_{j} A^{\mathrm{hyp}}\right)\left(\partial^{j} \tilde{W}^{3}\right)+c_{3}\left(\partial_{j} \tilde{W}^{3}\right)\left(\partial^{j} \tilde{W}^{3}\right) \\
& +2 c_{4}\left(\partial_{j} A^{\mathrm{hyp}}\right)\left(\partial^{j} \tilde{W}^{3}\right)-2 c_{4}\left(\partial_{j} A^{\mathrm{hyp}}\right)\left(\partial^{j} A^{\mathrm{hyp}}\right) \\
& +M_{3}^{2}\left(\tilde{W}^{3}-A^{\mathrm{hyp}}\right)\left(\tilde{W}^{3}-A^{\mathrm{hyp}}\right)
\end{aligned}
$$

and thus

$$
\begin{aligned}
\mathcal{L}_{\mathrm{YM}} \asymp & \left(\frac{16}{3} c_{1}+c_{3}-2 c_{4}\right)\left(\partial_{j} A^{\mathrm{hyp}}\right)\left(\partial^{j} A^{\mathrm{hyp}}\right)+c_{3}\left(\partial_{j} \tilde{W}^{3}\right)\left(\partial^{j} \tilde{W}^{3}\right) \\
& -2\left(c_{3}-c_{4}\right)\left(\partial_{j} A^{\mathrm{hyp}}\right)\left(\partial^{j} \tilde{W}^{3}\right)+M_{3}^{2}\left(\tilde{W}^{3}-A^{\mathrm{hyp}}\right)\left(\tilde{W}^{3}-A^{\mathrm{hyp}}\right) .
\end{aligned}
$$

This differs from the Lagrangian of the standard model in that the kinetic term of the standard model does not involve the cross terms $\sim\left(\partial_{j} A^{\text {hyp }}\right)\left(\partial^{j} \tilde{W}^{3}\right)$. But using the equation $c_{3}=c_{4}$ in $(5.4 .23)$, this cross term vanishes. We thus obtain for the full Lagrangian

$$
\begin{aligned}
\mathcal{L}_{\mathrm{YM}}= & c_{1} \operatorname{Tr}_{\mathbb{C}^{3}}\left(\left(\partial_{j} G\right)\left(\partial^{j} G\right)\right)+\left(\frac{16}{3} c_{1}-c_{3}\right)\left(\partial_{j} A^{\mathrm{hyp}}\right)\left(\partial^{j} A^{\mathrm{hyp}}\right) \\
& +c_{2}\left(\left(\partial_{j} W^{1}\right)\left(\partial^{j} W^{1}\right)+\left(\partial_{j} W^{2}\right)\left(\partial^{j} W^{2}\right)\right)+c_{3}\left(\partial_{j} \tilde{W}^{3}\right)\left(\partial^{j} \tilde{W}^{3}\right) \\
& +M_{1}^{2}\left(W^{1} W^{1}+W^{2} W^{2}\right)+M_{3}^{2}\left(\tilde{W}^{3}-A^{\mathrm{hyp}}\right)\left(\tilde{W}^{3}-A^{\mathrm{hyp}}\right) .
\end{aligned}
$$


The constants in front of the quadratic derivative terms can be absorbed into the coupling constants by rescaling the potentials. To this end, we introduce the coupling constants

$$
g_{\text {strong }}=\frac{1}{2 \sqrt{c_{1}}}, \quad g_{\text {hyp }}=\frac{1}{2}\left(\frac{16}{3} c_{1}-c_{3}\right)^{-\frac{1}{2}}, \quad g_{2}=\frac{1}{2 \sqrt{c_{2}}}, \quad g_{3}=\frac{1}{2 \sqrt{c_{3}}} .
$$

Rescaling the potentials according to

$$
G \rightarrow g_{\text {strong }} G, \quad A^{\text {hyp }} \rightarrow g_{\text {hyp }} A^{\text {hyp }}, \quad W^{1 / 2} \rightarrow g_{2} W^{1 / 2}, \quad W^{3} \rightarrow g_{3} W^{3},
$$

the Lagrangian becomes

$$
\begin{aligned}
\mathcal{L}_{\mathrm{YM}}= & \frac{1}{4} \operatorname{Tr}_{\mathbb{C}^{3}}\left(\left(\partial_{j} G\right)\left(\partial^{j} G\right)\right)+\frac{1}{4}\left(\partial_{j} A^{\mathrm{hyp}}\right)\left(\partial^{j} A^{\mathrm{hyp}}\right) \\
& +\frac{1}{4}\left(\left(\partial_{j} W^{1}\right)\left(\partial^{j} W^{1}\right)+\left(\partial_{j} W^{2}\right)\left(\partial^{j} W^{2}\right)+\left(\partial_{j} \tilde{W}^{3}\right)\left(\partial^{j} \tilde{W}^{3}\right)\right) \\
& +M_{1}^{2} g_{2}^{2}\left(W^{1} W^{1}+W^{2} W^{2}\right)+M_{3}^{2}\left(g_{3} \tilde{W}^{3}-g_{\mathrm{hyp}} A^{\mathrm{hyp}}\right)\left(g_{3} \tilde{W}^{3}-g_{\mathrm{hyp}} A^{\mathrm{hyp}}\right) .
\end{aligned}
$$

Now the kinetic term of the Lagrangian looks just as in the standard model. Clearly, the rescaling of the potentials (5.4.28) must also be performed in the Dirac Lagrangian 5.4.3. This amounts to inserting coupling constants into the gauge covariant derivative, which thus becomes

$$
D_{j}=\partial_{j}-i g_{\text {strong }} G_{j}-i g_{\text {hyp }} A_{j}^{\text {hyp }} y-i g_{2} \chi_{L}\left(W_{j}^{1} \sigma_{\text {iso }}^{1}+W_{j}^{2} \sigma_{\text {iso }}^{2}\right)-i g_{3} \chi_{L} W_{j}^{3} \sigma_{\text {iso }}^{3},
$$

where $\sigma_{\text {iso }}^{\alpha}$ are the Pauli matrices acting on isospin, and $y$ is the generator of the weak hypercharge,

$$
y=\chi_{L} \operatorname{diag}\left(-1,-1, \frac{1}{3}, \frac{1}{3}, \frac{1}{3}, \frac{1}{3}, \frac{1}{3}, \frac{1}{3}\right)+\chi_{R} \operatorname{diag}\left(0,-2, \frac{4}{3},-\frac{2}{3}, \frac{4}{3},-\frac{2}{3}, \frac{4}{3},-\frac{2}{3}\right) .
$$

In the standard model, there is only one coupling constant for the $\mathfrak{s u}(2)$-potentials. This leads us to impose the equation $c_{2}=c_{3}$ in (5.4.23). Then $g_{2}=g_{3}=: g_{\text {weak }}$. The last relation in (5.4.23) is needed in order for the mass matrix after spontaneous symmetry breaking to be compatible with (5.4.27) (see for example [PS, Section 20.2]). This concludes the proof.

Additional relations between the regularization parameters. The remaining important question is whether the relations $(5.4 .23)$ hold for suitable regularizations of the fermionic projector. Do they always hold? Or are there in general deviations?

The general answer is that the relations $(5.4 .23)$ do not need to hold in general. But as will be specified in Proposition 5.4.3 below, the relations 5.4.23 do hold in the limiting cases when the masses of the leptons are much larger than the masses of the neutrinos, and the mass of the top quark is much larger than the mass of the leptons. Therefore, using the hierarchy of the fermion masses of the standard model, we obtain agreement with the standard model. In view of the experimental observations

$$
\frac{m_{\nu_{\tau}}^{2}}{m_{\tau}^{2}} \lesssim 8 \times 10^{-5} \quad \text { and } \quad \frac{m_{\tau}^{2}}{m_{\text {top }}^{2}} \approx 10^{-4}
$$

it seems that our limiting case should be an excellent approximation. But for general regularizations, we expect deviations for the masses and coupling constants of electroweak theory of the order (5.4.29). Unfortunately, since at the moment we do not have detailed information on how the microscopic structure of the physical regularization is, we cannot make a prediction for the deviations. 
Proposition 5.4.3. Assume that all the mass parameters in (5.2.2) and (5.2.3) are dominated by the mass of the heaviest charged fermion, i.e.

$$
m_{3} \gg m_{1}, m_{2}
$$

and

$$
m_{3} \gg \tilde{m}_{1}, \tilde{m}_{2}, \tilde{m}_{3} .
$$

Moreover, assume that the physical (=renormalized) mass of the top quark is much larger than that of the leptons,

$$
m_{\mathrm{top}} \gg m_{e}, m_{\nu}, m_{\tau} \text {. }
$$

Then the parameters in the effective Lagrangian of Theorem 5.4.1 satisfy the relations in (5.4.23) up to relative errors of the order

$$
\frac{m_{1}^{2}+m_{2}^{2}}{m_{3}^{2}}, \quad \frac{\tilde{m}_{1}^{2}+\tilde{m}_{2}^{2}+\tilde{m}_{3}}{m_{3}^{2}} \quad \text { and } \quad \frac{m_{e}^{2}+m_{\nu}^{2}+m_{\tau}^{2}}{m_{\mathrm{top}}^{2}} .
$$

The remainder of this section is devoted to the derivation of this proposition. Our derivation will not be a mathematical proof. Instead, we are content with explaining the involved approximations in the non-rigorous style common in theoretical physics. We begin by noting that the term involving the bosonic currents in (5.4.11) contributes to the right side of $(5.4 .6)$ by

$$
\begin{aligned}
\operatorname{Tr}_{\mathbb{C}^{8}}\left(\mathcal{Q}_{L}^{k}[\hat{\jmath}, \mathcal{A}]\left(\delta \hat{A}_{R}\right)_{k}+\mathcal{Q}_{R}^{k}[\hat{\jmath}, \mathcal{A}]\left(\delta \hat{A}_{L}\right)_{k}\right) \asymp K_{3} \operatorname{Tr}_{\mathbb{C}^{8}}\left(\hat{\jmath}_{R}^{k}\left(\delta \hat{A}_{R}\right)_{k}+\hat{\jmath}_{L}^{k}\left(\delta \hat{A}_{L}\right)_{k}\right) \\
=\frac{16}{3} K_{3} 9 j\left[A_{\mathrm{em}}\right]^{k}\left(\delta A_{\mathrm{em}}\right)_{k}+4 K_{3} 9\left(j\left[A_{\mathrm{em}}\right]^{k}\left(\delta W^{3}\right)_{k}+j\left[W^{3}\right]^{k}\left(\delta A_{\mathrm{em}}\right)_{k}\right) \\
+8 K_{3}\left(9 j\left[W^{3}\right]^{k}\left(\delta W_{3}\right)_{k}+j\left[\hat{W}^{1}\right]^{k}\left(\delta \hat{W}_{1}\right)_{k}+j\left[\hat{W}^{2}\right]^{k}\left(\delta \hat{W}_{2}\right)_{k}\right)
\end{aligned}
$$

(the factors of 9 come up whenever we leave out the sectorial projection). This is of variational form, leading us to choose

$$
\begin{aligned}
\mathcal{L}_{\mathrm{YM}} \asymp & \left.\frac{8}{3} K_{3} 9\left(\partial_{j} A_{\mathrm{em}}\right)\left(\partial^{j} A_{\mathrm{em}}\right)+4 K_{3} 9\left(\partial_{j} A_{\mathrm{em}}\right)\left(\partial^{j} W^{3}\right)\right) \\
& +4 K_{3}\left(9\left(\partial_{j} W^{3}\right)\left(\partial^{j} W_{3}\right)+\left(\partial_{j} \hat{W}^{1}\right)\left(\partial_{j} \hat{W}^{1}\right)+\left(\partial_{j} \hat{W}^{2}\right)\left(\partial_{j} \hat{W}^{2}\right)\right) .
\end{aligned}
$$

This is of the general form of Theorem 5.4.1, but with $c_{3}=c_{4}$. Thus one of the relations in 5.4.23 is automatically satisfied. Moreover, the coupling constants $c_{1}$ and $c_{3}$ are related by

$$
c_{1}=\frac{c_{3}}{2} \text {. }
$$

The relation $c_{2}=c_{3}$ is violated because of the sectorial projection of the mixing matrix. However, keeping in mind that the Dirac Lagrangian (5.4.3) as well as the mass terms also involve sectorial projections, these sectorial projections indeed play no role. This will be explained at the end of this section. If we disregard the sectorial projection for the moment, the relation $c_{2}=c_{3}$ is also satisfied. We conclude that the structure of how the bosonic currents enter the EL equations in the continuum limit is consistent with the relations on the left of (5.4.23). Moreover, one has the additional relation (5.4.34).

The subtle point is that $K_{3}$ has a logarithmic pole which must be compensated by a microlocal chiral transformation. Thus in order to decide if the relations on the left of (5.4.23) or the relation (5.4.34) remain valid, we need to analyze whether the microlocal chiral transformation respects these relations. This is not easy to tell because the analysis in 4.4 .4 depends in a complicated way on the ratios of the fermion masses. Moreover, 
the parameters $\mathfrak{c}_{0}$ and $\mathfrak{c}_{2}$ were not determined explicitly. But at least, we can analyze the behavior of the microlocal chiral transformation if we make use of the mass hierarchies, as we now explain.

Before beginning, we need to adapt our method of compensating the logarithmic poles to the construction of the effective Lagrangian in (5.4.6). Recall that when introducing the microlocal chiral transformation in $\$ 3.7 .9$, \$3.7.10 and $\$ 4.4 .4$, we always compensated all the logarithmic poles. However, in the construction of the effective Lagrangian as introduced in $\$ 4.7 .1$, we argued that the EL equations in the continuum limit (5.2.28) should be satisfied only in the "directions parallel to the bosonic degrees of freedom." This is implemented mathematically by the fact that (5.4.6) involves testing with a dynamical gauge potential $\delta \mathcal{A} \in \mathfrak{g}$. As a consequence, it is no longer necessary to compensate the logarithmic poles completely. It suffices to arrange that the logarithmic poles drop out of (5.4.6). More precisely, the contributions $\left(j_{L}, j_{R}\right)$ involving logarithmic poles which remain after the microlocal chiral transformation must satisfy the condition

$$
\operatorname{Tr}_{\mathbb{C}^{8}}\left(j_{L} \delta A_{L}+j_{R} \delta A_{R}\right)=0 \quad \forall \delta \mathcal{A} \in \mathfrak{g} .
$$

In order to express this condition in a convenient way, we introduce the real vector space

$$
\mathfrak{S}_{8}:=\operatorname{Symm}\left(\mathbb{C}^{8}\right) \oplus \operatorname{Symm}\left(\mathbb{C}^{8}\right),
$$

where $\operatorname{Symm}\left(\mathbb{C}^{8}\right)$ denotes the Hermitian $8 \times 8$-matrices. Moreover, we introduce the bilinear form

$$
\langle., .\rangle_{\mathfrak{S}_{8}}: \mathfrak{S}_{8} \times \mathfrak{g} \rightarrow \mathbb{C}, \quad\left\langle\left(j_{L}, j_{R}\right), \mathcal{A}\right\rangle_{\mathfrak{S}_{8}}=\operatorname{Tr}_{\mathbb{C}^{8}}\left(j_{L} A_{L}+j_{R} A_{R}\right) .
$$

Then (5.4.35) can be expressed by saying that the logarithmic contribution must be orthogonal to $\mathfrak{g}$ with respect to the bilinear form (5.4.36).

We begin by considering sector-diagonal transformations. The microlocal chiral transformation is worked out explicitly in Example 4.4.5. The transformation involves the eigenvalues $\mu_{1}, \ldots, \mu_{4}$ of the matrix $S_{0}^{-1} S_{2}$. In the lepton block, these eigenvalues are given by (see also 3.7.72)

$$
\begin{aligned}
& \mu_{1 / 2}=\frac{1}{3}\left(\tilde{m}_{1}^{2}+\tilde{m}_{2}^{2}+\tilde{m}_{3}^{2} \mp \sqrt{\tilde{m}_{1}^{4}+\tilde{m}_{2}^{4}+\tilde{m}_{3}^{4}-\tilde{m}_{1}^{2} \tilde{m}_{2}^{2}-\tilde{m}_{2}^{2} \tilde{m}_{3}^{2}-\tilde{m}_{1}^{2} \tilde{m}_{3}^{2}}\right) \\
& \mu_{3 / 4}=\frac{1}{3}\left(m_{1}^{2}+m_{2}^{2}+m_{3}^{2} \mp \sqrt{m_{1}^{4}+m_{2}^{4}+m_{3}^{4}-m_{1}^{2} m_{2}^{2}-m_{2}^{2} m_{3}^{2}-m_{1}^{2} m_{3}^{2}}\right) .
\end{aligned}
$$

In the quark blocks, one has similarly the eigenvalues $\mu_{3 / 4}$, both with multiplicity two. As explained in Example 4.4.5, the amplitude $\kappa$ of the microlocal chiral transformation in each sector can vary in the range (see 4.4.63)

$$
\mathfrak{c}_{0} \mu_{1} \leq \kappa \leq \mathfrak{c}_{0} \mu_{2} \quad \text { and } \quad \mathfrak{c}_{0} \mu_{3} \leq \kappa \leq \mathfrak{c}_{0} \mu_{4} .
$$

The general strategy is to compensate the logarithmic poles choosing $\mathfrak{c}_{0}$ as small as possible. The eigenvalues $\mu_{1}, \ldots, \mu_{4}$ scale like the masses squared. Therefore, if the neutrino masses are much smaller than the masses of the lepton and quarks (5.4.31), then the microlocal chiral transformation has no effect in the neutrino sector. Let us assume in addition that one of the masses of the charged leptons dominates (5.4.30). Then

$$
\mu_{3}=\mathcal{O}\left(\frac{m_{1}^{2}+m_{2}^{2}}{m_{3}^{2}}\right), \quad \mu_{4}=\frac{2}{3} m_{3}^{2}+\mathcal{O}\left(\frac{m_{1}^{2}+m_{2}^{2}}{m_{3}^{2}}\right)
$$


As a consequence, the inequalities in 5.4.37 reduce to the interval

$$
0 \leq \kappa \leq \frac{2}{3} \mathfrak{c}_{0} m_{3}^{2}
$$

We conclude that for a sector-diagonal transformation, our freedom in choosing the microlocal chiral transformation reduces to selecting for the left- and right-handed component of every charged sector a parameter $\kappa$ in the range (5.4.39). We denote these parameters by $\kappa_{a c}$ with $a \in\{2, \ldots, 8\}$ and $c \in\{L, R\}$. In order to minimize $\mathfrak{c}_{0}$, the best strategy is to choose every parameter $\kappa_{a c}$ at one of the boundary points of the interval, i.e.

$$
\kappa_{a c}=0 \quad \text { or } \quad \kappa_{a c}=\frac{2}{3} \mathfrak{c}_{0} m_{3}^{2}
$$

(with errors as specified in (5.4.38)). Let us try this strategy for the current corresponding to $A^{\text {hyp }}$. As this current is sector-diagonal, testing in (5.4.6) gives zero if $\delta \mathcal{A}$ is the potential $\mathcal{A}\left[\delta W^{1}\right]$ or $\mathcal{A}\left[\delta W^{2}\right]$. Moreover, this current is invariant under the action of the strong $\mathrm{SU}(3)$, implying that (5.4.6) also vanishes if $\delta \mathcal{A}$ is a strong potential. Therefore, it suffices to consider the cases that $\delta \mathcal{A}$ is $\mathcal{A}\left[\delta A^{\text {hyp }}\right]$ or $\mathcal{A}\left[\delta \tilde{W}^{3}\right]$ (the tilde again clarifies that we parametrize the potentials by $\left.\left(A^{\text {hyp }}, \tilde{W}^{3}\right)\right)$. The terms with logarithmic poles generated by the current of weak hypercharge are collinear to $\mathcal{A}\left[\delta A^{\text {hyp }}\right]$ and orthogonal to $\mathcal{A}\left[\delta \tilde{W}^{3}\right]$ (with respect to the bilinear form (5.4.36)). Thus we need to make sure that the logarithmic pole is compensated when testing with $\mathcal{A}\left[\delta A^{\text {hyp }}\right]$, but that we get no contribution when testing with $\mathcal{A}\left[\delta \tilde{W}^{3}\right]$. This can be arranged by the two choices

$$
\left(\kappa_{a L}\right)=\frac{2}{3} \mathfrak{c}_{0} m_{3}^{2}(0,0,0,0,0,0,0,0) \quad \text { and } \quad\left(\kappa_{a R}\right)=\frac{2}{3} \mathfrak{c}_{0} m_{3}^{2}(0,0,1,0,1,0,1,0)
$$

or alternatively

$$
\left(\kappa_{a L}\right)=\frac{2}{3} \mathfrak{c}_{0} m_{3}^{2}(0,0,0,0,0,0,0,0) \quad \text { and } \quad\left(\kappa_{a R}\right)=\frac{2}{3} \mathfrak{c}_{0} m_{3}^{2}(0,0,0,1,0,1,0,1) .
$$

Indeed, since the contributions generated by the microlocal chiral transformation have a definite sign (see (4.4.49), we need both cases, depending on whether the bosonic current is future or past directed. By adjusting $\mathfrak{c}_{0}$, we can arrange that the contributions involving logarithmic poles satisfy the condition (5.4.35) and thus drop out of (5.4.6).

The next step is to compute the corresponding smooth contributions generated by the microlocal chiral transformation. Again using that the largest mass dominates 5.4.30, the contribution by the microlocal chiral transformation is simply given by the corresponding Dirac sea, i.e.

$$
P(x, y) \sim \log \left|m^{2} \xi^{2}\right|+c+i \pi \Theta\left(\xi^{2}\right) \epsilon\left(\xi^{0}\right)
$$

with a numerical constant $c$ (see [F7, Section 2.5] or 33.4.4). Therefore, the smooth contribution is explicit. It is proportional to the original contribution involving the logarithmic pole. This is very useful because we conclude that the current term after compensating the logarithmic pole is again orthogonal to $\mathcal{A}\left(\delta \tilde{W}^{3}\right)$ (with respect to the bilinear form (5.4.36) ). This means that in the kinetic term of the resulting Lagrangian, there is no cross term of $A^{\text {hyp }}$ and $\tilde{W}^{3}$. Comparing with (5.4.24), this gives precisely the relation $c_{1}=c_{3}$. We conclude that the logarithmic pole of weak of the bosonic current corresponding to weak hypercharge is compensated such that the relation $c_{1}=c_{3}$ is preserved (up to error terms as mentioned above).

We now proceed similarly for the current corresponding to $\tilde{W}^{3}$. Thus we want to choose parameters $\kappa_{a c}$ of the form (5.4.40) which respect the strong SU(3)-symmetry, 
such that the logarithmic poles of the current are removed, but the resulting contribution is orthogonal to $\mathcal{A}\left[\delta A^{\text {hyp }}\right]$. A short computation shows that the only two solutions are

$$
\begin{array}{rlrl}
\left(\kappa_{a L}\right) & =\frac{2}{3} \mathfrak{c}_{0} m_{3}^{2}(0,1,1,0,1,0,1,0), & \left(\kappa_{a R}\right) & =\frac{2}{3} \mathfrak{c}_{0} m_{3}^{2}(0,0,0,0,0,0,0,0) \\
\left(\kappa_{a L}\right) & =\frac{2}{3} \mathfrak{c}_{0} m_{3}^{2}(0,1,0,1,0,1,0,1), \quad\left(\kappa_{a R}\right)=\frac{2}{3} \mathfrak{c}_{0} m_{3}^{2}(0,0,0,0,0,0,0,0) .
\end{array}
$$

Note that these ansätze have a contribution in the charged lepton sector. As will be explained below, this leads to difficulties. The only method for avoiding these difficulties is to give up (5.4.40) and to allow for the parameters $\kappa_{a c}$ to take values in the interior of the interval (5.4.39). This makes it possible to choose the parameters $\kappa_{a c}$ such that they vanish in the lepton block. Namely, a direct computation gives the solutions

$$
\begin{array}{rlrl}
\left(\kappa_{a L}\right) & =\frac{2}{3} \mathfrak{c}_{0} m_{3}^{2}(0,0, a, 0, a, 0, a, 0), & \left(\kappa_{a R}\right) & =\frac{2}{3} \mathfrak{c}_{0} m_{3}^{2}(0,0, b, c, b, c, b, c) \\
\left(\kappa_{a L}\right) & =\frac{2}{3} \mathfrak{c}_{0} m_{3}^{2}(0,0,0, a, 0, a, 0, a), & \left(\kappa_{a R}\right)=\frac{2}{3} \mathfrak{c}_{0} m_{3}^{2}(0,0, b, c, b, c, b, c),
\end{array}
$$

where the parameters $a, b, c$ are to be chosen such that

$$
a+4 b-2 c=0, \quad 0 \leq a, b, c \leq 1 \quad \text { and } \quad \max (a, b, c)=1 .
$$

Let us explain the consequence of these different solutions. In the case $(5.4 .42)$, the relation 5.4.41) again holds. This implies that the relation 5.4.34 will hold after removing the logarithmic poles. In the case (5.4.43), however, the relation (5.4.41) no longer holds, because all three Dirac seas contribute substantially to the microlocal chiral transformation. This makes the situation much more complicated, and we do want to not enter the details here. For our purposes, it suffices to make the following remarks. First, the parameters $\kappa_{a c}$ must necessarily be chosen in accordance to the relation $c_{1}=c_{3}$, because otherwise (5.4.6) could not be satisfied, and the EL equations in the continuum limit would no longer be of variational form. Moreover, since (5.4.41) is violated, the relation (5.4.34) will no longer hold after the logarithmic poles have been removed. This makes it necessary to treat $c_{1}$ and $c_{3}$ as independent effective parameters, giving rise to independent effective coupling constants $g_{\text {hyp }}$ and $g_{\text {weak }}$.

We next consider non-sectordiagonal transformations. Since the ansatz (5.4.43) only affects the quark blocks, it can immediately be generalized to non-sectordiagonal transformations. Namely, since the microlocal transformation can be performed independently for the two chiral components, it suffices to consider for example the left-handed component. Then one can use an U(2)-transformation to diagonalize the logarithmic contribution. Using the degeneracy of the masses in each block, this $\mathrm{U}(2)$-transformation can also be performed for the local chiral transformation by

$$
L[k] \rightarrow U L[k] U^{*} \quad \text { with } U \in \mathrm{U}(2) .
$$

In this way, the constructions and results of Example 4.4.5 can also be used for the nonsectordiagonal transformations in the quark blocks. This implies in particular that the constant $c_{2}$ in the dynamical term of the gauge fields $W^{1}$ and $W^{2}$ in $(5.4 .9)$ coincides with the corresponding constant $c_{3}$ for the gauge field $W^{3}$. We point out that this $\mathrm{U}(2)-$ transformation cannot be used in the lepton block because the masses of the neutrinos are different from those of the charged leptons. In particular, it is not clear if and how the ansatz 5.4 .42 can be generalized to non-sectordiagonal transformations.

Next, we need to analyze the mass terms. This is considerably more complicated because we must analyze the contributions (5.4.12)-(5.4.18). The only contribution with 
a logarithmic pole is the term (5.4.14). For the $W^{3}$-potential, we can compensate the logarithm as explained above, choosing for example

$$
\begin{aligned}
\left(\kappa_{a L}\right) & =\frac{2}{3} \mathfrak{c}_{0} m_{3}^{2}\left(0,0, \frac{1}{2}, 0, \frac{1}{2}, 0, \frac{1}{2}, 0\right), & \left(\kappa_{a R}\right) & =\frac{2}{3} \mathfrak{c}_{0} m_{3}^{2}(0,0,0,1,0,1,0,1) \\
\left(\kappa_{a L}\right) & =\frac{2}{3} \mathfrak{c}_{0} m_{3}^{2}(0,0,0,1,0,1,0,1), & \left(\kappa_{a R}\right) & =\frac{2}{3} \mathfrak{c}_{0} m_{3}^{2}\left(0,0, \frac{1}{2}, 0, \frac{1}{2}, 0, \frac{1}{2}, 0\right) .
\end{aligned}
$$

The resulting contribution is orthogonal to the electromagnetic component, implying that the parameter $K_{27}$ in (5.4.20) again vanishes. Hence we only need to take into account the contributions where the mass terms are tested by the left-handed weak potentials. In view of (5.4.6), it thus suffices to consider $\mathcal{J}_{R}$. Moreover, as the mass terms vanish identically for free gauge fields, it suffices to consider 5.4.12 - 5.4.18 for a left-handed weak potential. Hence the relevant contribution by the mass terms reduces to

$$
\begin{aligned}
\mathfrak{J}_{R}^{k} \asymp & -3 m^{2}\left(\hat{A}_{L}^{k} Y \grave{Y}+\dot{Y} Y \grave{A}_{L}^{k}\right) K_{5} \\
& +2 m^{2} \hat{Y} \hat{A}_{L}^{k} \hat{Y} K_{6}-m^{2}\left(\hat{A}_{L}^{k} \hat{Y} \hat{Y}+\hat{Y} \hat{Y} \hat{A}_{L}^{k}\right) K_{7} .
\end{aligned}
$$

The following argument shows that the contribution (5.4.44) drops out of the effective EL equations: In view of the hierarchy (5.4.30), the logarithmic pole of the mass term is of the form (5.4.41). Since the contribution by the microlocal chiral transformation is of the same form, it cancels the contribution by (5.4.44) including the smooth contributions. As a result, 5.4.44 drops out of the effective EL equations.

The remaining contribution (5.4.45) has the following structure. In the three quark blocks, the factors $\hat{Y}$ are constants, so that the mass term can be written as $c \hat{W}$. In the lepton block, however, the fact that the neutrino masses are different from the masses of the charged leptons implies that the mass terms for $W^{3}$ have a different structure than those for $W^{1}$ and $W^{2}$. This implies that the constants $M_{1}$ and $M_{3}$ in (5.4.10) will in general be different.

We now give an argument which shows that $M_{1}$ and $M_{3}$ coincide in the limiting case (5.4.32) when the quark masses are much larger than the lepton masses. This argument will also explain why the solution 5.4 .42 must be dismissed, leaving us with the ansatz (5.4.43) for the microlocal chiral transformation. Our argument makes use of the concept that the masses $m_{\beta}$ in (5.2.1) are the "naked" masses, and that these masses are modified by the self-interaction to the physical masses. Having this concept in mind, it is a natural idea that the physical mass of the gauge bosons should again be described by (5.4.45) if only the masses of in the mass matrix $m Y$ are replaced by the physical fermion masses. This idea is motivated by the renormalization program which states that for a renormalizable theory the self-interaction describes a shift of the masses and coupling constants but leaves the structure of the interaction unchanged. However, it must be said that the renormalization of the fermionic projector is work in progress. If we take the results of the normalization program for granted and combine them with the mass hierarchy (5.4.32), then we conclude that all the contributions involving the fermion masses are much smaller in the lepton block than in the quark blocks. In particular, in the ansatz $(5.4 .42$ we must replace the sequences $0,1, \ldots$ by $0, \delta, \ldots$ with $\delta \ll 1$. But then the resulting contribution is no longer orthogonal to $\mathcal{A}\left[\delta A^{\mathrm{hyp}}\right]$. Therefore, the ansätze $(5.4 .42)$ must be dismissed. For the mass terms in (5.4.45), we conclude that the main contribution comes from the quark sectors, giving rise to an effective mass Lagrangian of the form

$$
M^{2}\left(\hat{W}^{1} \hat{W}^{1}+\hat{W}^{2} \hat{W}^{2}+9 W^{3} W^{3}\right)
$$


which involves only one mass parameter.

It remains to analyze the effect of the sectorial projection of the potentials $W^{1}$ and $W^{2}$. For notational simplicity, we only consider the potential $W^{1}$. By inspecting (5.4.3), (5.4.33) and (5.4.46), one sees that only the sectorial projection of the potential $W^{1}$ enters. Thus varying $\hat{W}^{1}$, one sees that the rest mass of the bosonic field remained unchanged if all the sectorial projections were left out. Moreover, varying the Dirac Lagrangian as explained in $\$ 4.7 .3$, one sees that the coupling to the Dirac particles has the same form as without the sectorial projection, except for the sectorial corrections mentioned after (5.4.7). This explains the last equation in (5.4.23) and thus establishes Proposition 5.4.3.

\subsubsection{The Effective Lagrangian for Gravity.}

Theorem 5.4.4. Assume that the parameters $\delta$ and $p_{\text {reg }}$ satisfy the scaling

$$
\varepsilon \ll \delta \ll \frac{1}{m}(m \varepsilon)^{\frac{p_{\mathrm{reg}}}{2}},
$$

and that the regularization satisfies the conditions (5.2.10). Then the EL equations in the continuum limit (5.2.27) can be expressed in terms of the effective action (5.4.1) with the Einstein-Hilbert action

$$
\mathcal{L}_{\mathrm{EH}}=\frac{1}{\kappa(\varepsilon, \delta)}(R+2 \Lambda)
$$

(where $R$ denotes scalar curvature and $\Lambda \in \mathbb{R}$ is the cosmological constant). Here the gravitational constant $\kappa$ is given by

$$
\kappa=\frac{\delta^{2}}{\tau_{\text {reg }}} \frac{K_{17}}{K_{18}}
$$

where $K_{17}$ and $K_{18}$ are the simple fractions

$$
K_{17}=\frac{1}{2} K_{16}\left(1-\frac{L_{[0]}^{(0)}}{T_{[0]}^{(0)}}\right) \quad \text { and } \quad K_{18}=\frac{1}{2} K_{8}\left(1-\frac{L_{[0]}^{(0)}}{T_{[0]}^{(0)}}\right)
$$

with $K_{16}$ and $K_{8}$ as in 4.5.7) and 4.5.2 (here all expressions are to be evaluated weakly on the light cone (5.2.11)). The parameter $\tau$ in the Dirac Lagrangian (5.4.3) is determined to have the value $\tau=-16$.

Proof. One proceeds exactly as in Section 4.9. The variation of the matrices $\mathcal{Q}^{k l}$ is computed as in Lemma 4.9.1 and 4.9.2. In order to satisfy (5.4.7) one must choose $\tau=$ -16 . Then the result follows immediately.

We finally explain how the energy-momentum tensor of the gauge fields comes into play. The effect of the field tensor of the gauge fields was computed in Lemma 4.5.3. In Lemma 4.5.4 we saw how the resulting logarithmic poles can be compensated for an axial potential. We now need to generalize these results to the electroweak and strong potentials as they appear in Theorem 5.3.2. If only a left-handed weak potential is present, we can apply Lemma 4.5 .3 directly, because after compensating the axial component of the weak potentials, the remaining contribution is vectorial and is the same in all sectors. Hence it drops out of the EL equations. If more general gauge potentials are present, the situation is more involved because the contribution to be compensated is different in every sector. In the following lemma we explain how to treat this situation in the simple setting that only an electromagnetic potential is present. 
Lemma 5.4.5. Assume that the chiral potentials have the form $\mathcal{B}\left[A^{\mathrm{em}}\right]$ given in $(5.3 .2)$. Then the logarithmic poles of the contribution to the fermionic projector 4.5.19) can be compensated by the shear contributions corresponding to a microlocal chiral transformation for a suitable choice of the potentials $A_{L / R}^{\mathrm{even}}$ in 4.4.79.

Proof. As in the proof of Lemma 4.5.4, the first step is to specify the microlocal transformation such that, similar to 4.5.20 and 4.5.21), the contribution to the fermionic projector is of the form

$$
\begin{aligned}
\tilde{P}(k)= & P(k)+(\text { vectorial }) \mathbb{1}_{\mathbb{C}^{8}} T_{[3, \mathrm{c}]}^{(1)}\left(1+\mathcal{O}\left(\Omega^{-\frac{1}{2}}\right)\right) \\
& +(\text { vectorial }) \mathbb{1}_{\mathbb{C}^{8}} \delta\left(k^{2}\right)\left(1+\mathcal{O}\left(\Omega^{-1}\right)\right) \\
& \left.+(\text { pseudoscalar or bilinear }) \sqrt{\Omega} \delta^{\prime}\left(k^{2}\right)\left(1+\mathcal{O}\left(\Omega^{-1}\right)\right)+\text { (higher orders in } \varepsilon /|\vec{\xi}|\right) .
\end{aligned}
$$

Since all the following transformations will be diagonal on the sectors, we may consider the sectors separately. Thus, omitting the sector index, our transformations only act on the generation index. We denote the masses in the considered sector by $m_{1}, m_{2}$ and $m_{3}$.

We again employ the ansatz 4.4.33), but now with a pure vector component, i.e.

$$
U(k)=\mathbb{1}+\frac{i}{\sqrt{\Omega}} L^{j} \gamma_{j}
$$

with $3 \times 3$-matrices $L^{j}$. Next, we choose the matrices $L^{j}$ as diagonal matrices involving one vector field $v$,

$$
L^{j} \gamma_{j}=\psi \operatorname{diag}\left(\lambda_{1}, \lambda_{2}, \lambda_{3}\right)
$$

for simplicity with real parameters $\lambda_{\alpha}$. Then the conditions 4.4.41-4.4.43 can be arranged by imposing that

$$
\lambda_{1}+\lambda_{2}+\lambda_{3}=0 .
$$

Moreover, the formulas 4.4.48 and 4.4.49) give rise to the conditions

$$
\begin{aligned}
\left|\lambda_{1}\right|^{2}+\left|\lambda_{2}\right|^{2}+\left|\lambda_{3}\right|^{2} & =\mathfrak{c}_{0} \\
m_{1}^{2}\left|\lambda_{1}\right|^{2}+m_{2}^{2}\left|\lambda_{2}\right|^{2}+m_{3}^{2}\left|\lambda_{3}\right|^{2} & =\mathfrak{c}_{2},
\end{aligned}
$$

where the parameters $\mathfrak{c}_{0}$ and $\mathfrak{c}_{2}$ should be the same in every sector. By choosing the parameters $\lambda_{\alpha}$ according to the above conditions, we can arrange a contribution to the fermionic projector of the form (5.4.47) and (5.4.48).

Next, we need to specify the gauge potentials $A_{L / R}^{\text {even }}$ in the considered sector. We choose this gauge potential to be vectorial and make similar to 4.5.31 the ansatz

$$
A_{L}^{\text {even }}=A_{R}^{\text {even }}=|\psi\rangle\langle\psi|,
$$

but now with a real vector $\psi \in \mathbb{R}^{3}$. It is convenient to choose the vectors in $\mathbb{C}^{3}$

$$
f_{1}=\left(\begin{array}{c}
1 \\
1 \\
1
\end{array}\right), \quad f_{2}=\left(\begin{array}{c}
\lambda_{1} \\
\lambda_{2} \\
\lambda_{3}
\end{array}\right)
$$

Then we impose the conditions

$$
\begin{aligned}
& \left\langle\psi, f_{1}\right\rangle_{\mathbb{C}^{3}}=0 \\
& \left\langle\psi, f_{2}\right\rangle_{\mathbb{C}^{3}}=\mathfrak{c}_{3}
\end{aligned}
$$


(where the parameter $\mathfrak{c}_{3}$ is again the same in all sectors). These two linear equations reduce the degrees of freedom in $\psi$ to one free parameter. This free parameter may be used to prescribe the expectation value

$$
\left\langle f_{2}, Y^{2} A_{L / R}^{\text {even }} f_{2}\right\rangle_{\mathbb{C}^{3}}=\left\langle f_{2}, Y^{2} \psi\right\rangle_{\mathbb{C}^{3}}\left\langle\psi, f_{2}\right\rangle_{\mathbb{C}^{3}}
$$

With (5.4.50) and 5.4.51) (and keeping in mind that the parameters $\lambda_{\alpha}$ and $\psi_{\alpha}$ are all real) we have arranged that the gauge phases drop out of 4.4.41) and 4.4.42) (note that the relations similar to (4.5.27), 4.5.28) and (4.5.29) are satisfied). The contribution 5.4 .52 can be used to compensate the logarithmic pole of an energy-momentum tensor of the form $T_{j k} \sim v_{j} A_{k}^{\text {even }}+v_{k} A_{j}^{\text {even }}$.

Exactly as explained in the proof of Lemma 4.5.4, the above construction generalizes to energy-momentum tensors of the form 4.5.38.

If combinations of electroweak and strong gauge potentials are present, the resulting contributions with logarithmic poles are no longer diagonal on the sectors. As a consequence, the microlocal chiral transformation must involve off-diagonal contributions in the sector index. Since the resulting computations are rather tedious and not very instructive, we do not give them here.

After the logarithmic poles have been compensated, we are in the same situation as explained at the end of Section 4.9. The energy-momentum tensor of the gauge fields enters the EL equations similar to the energy-momentum tensor of the Dirac field, albeit potentially with a different coupling as determined by the corresponding regularization parameters. In order to get mathematically consistent equations, these regularization parameters must be adjusted such as to give the same couplings as obtained by varying the effective action with respect to the metric. In this way, the effective field equations in

the continuum limit include the Einstein equations with the energy-momentum tensors of both the Dirac field and the gauge fields.

\subsection{The Higgs Field}

As explained in $\$ 3.8 .5$, the Higgs potential of the standard model can be identified with suitable scalar/pseudoscalar potentials in the Dirac equation. As shown in Lemma B.3.1, the contributions by the pseudoscalar potentials to the fermionic projector drop out of the EL equations. The scalar potentials, on the other hand, contribute to the EL equations to degree three on the light cone. As the detailed computations are rather involved, we postpone the analysis of these contributions to a future publication. 



\section{APPENDIX A}

\section{Testing on Null Lines}

In this appendix we justify the EL equations in the continuum limit $(3.5 .29)$ by specifying the wave functions $\psi_{1}$ and $\psi_{2}$ used for testing the EL equations (3.5.24) in the setting with a general interaction and for systems involving several generations. Our method is to adapt the causal perturbation expansion 3.4.6 to obtain corresponding expansions of $\psi_{1}$ and $\psi_{2}$. We then consider the scaling of these terms to every order in perturbation theory. We rely on results from [F7] and [FG1, also using the same notation.

We begin with the Dirac equation for the auxiliary fermionic projector of the general form (3.4.4), where we assume that $\mathcal{B}$ is a multiplication operator which is smooth and decays so fast at infinity that

$$
\int_{\mathcal{M}}\left|x^{I} \partial_{x}^{J} \mathcal{B}(x)\right| d^{4} x<\infty \quad \text { for all multi-indices } I \text { and } J \text { with }|I| \leq 2 .
$$

Under these assumptions, every Feynman diagram of the causal perturbation expansion (3.4.6) is well-defined and finite (see [F7, Lemma 2.2.2] or Lemma 2.1.2). As in [F7, Section 2.6], we denote the spectral projectors of the operator $(i \not \partial+\mathcal{B}-m Y-\mu \mathbb{1})$ by $\tilde{p}_{+\mu}$. In contrast to (3.5.26), where we cut out an $\omega$-strip around the mass shell, it is here more convenient to remove a neighborhood in the mass parameter by setting

$$
\psi_{1}=\eta-\int_{-\Delta m}^{\Delta m} \widehat{\tilde{p}}_{+\mu} \eta d \mu
$$

where the tilde again denotes the sectorial projection. When taking the product $P \psi_{1}$, we get cross terms involving different generations. However, as in the proof of [F7, Theorem 2.6.1] one sees that these cross terms vanish in the infinite volume limit. Thus $\psi_{1}$ indeed lies in the kernel of the Dirac operator. Moreover, by choosing $\Delta m$ sufficiently small, we can make the difference $\psi_{1}-\eta$ as small as we like.

The construction of $\psi_{2}$ is a bit more involved. In order to get into the framework involving several generations, we first extend the wave packet in (3.5.27) to an object with $4 g$ components,

$$
\psi:=(i \not \partial+m Y) \theta \quad \text { with } \quad \theta=\left(e^{-i \Omega(t+x)} \phi(t+x-\ell, y, z)\right) \oplus \underbrace{0 \oplus \cdots \oplus 0}_{g-1 \text { summands }} .
$$

For $\psi_{2}$ we make an ansatz involving a sectorial projection,

$$
\psi_{2}=\sum_{\beta=1}^{g}\left(\psi_{\beta}+\Delta \psi_{\beta}^{\mathrm{D}}+\Delta \psi_{\beta}^{\mathrm{E}}\right)
$$

where the corrections $\psi_{\beta}^{\mathrm{D}}$ and $\Delta \psi_{\beta}^{\mathrm{E}}$ should take into account that the auxiliary Dirac equation must hold and that the generalized energy must be negative, respectively. In 
order to specify $\Delta \psi_{\beta}^{\mathrm{D}}$, we first apply the free Dirac operator to $\psi$,

$$
\begin{aligned}
& (i \not \partial-m Y) \psi=(i \not \partial-m Y)(i \not \partial+m Y)\left(e^{-i \Omega(t+x)} \phi(t+x-\ell, y, z) \oplus 0 \oplus \cdots \oplus 0\right) \\
& \quad=-\left(\square+m^{2}\right)\left(e^{-i \Omega(t+x)} \phi(t+x-\ell, y, z)\right) \oplus 0 \oplus \cdots \oplus 0=\left(\partial_{y}^{2}+\partial_{z}^{2}-m^{2}\right) \theta
\end{aligned}
$$

where $m \equiv m_{1}$ is the mass of the first generation. Note that the $x$ - and $y$-parameters dropped out. This implies that the obtained expression depends on the "large" parameter $\Omega$ only via a phase; in this sense it is a small error term. In order for $\psi+\Delta \psi_{\beta}^{\mathrm{D}}$ to satisfy the auxiliary Dirac equation (3.4.4), the wave function $\Delta \psi_{\beta}^{\text {Dirac }}$ must be a solution of the inhomogeneous Dirac equation

$$
(i \not \partial+\mathcal{B}-m Y) \Delta \psi^{\mathrm{D}}=-\left(\partial_{y}^{2}+\partial_{z}^{2}-m^{2}\right) \theta-\mathcal{B} \psi .
$$

Solutions of this equation could be constructed rigorously with energy estimates (see for example $[\mathbf{J}]$ ). But here we are content with a perturbative treatment. Denoting the Green's function of the zero mass free Dirac operator by $s$, i.e.

$$
i \not \supset s=1,
$$

we can solve for $\Delta \psi^{\mathrm{D}}$ in terms of the perturbation series

$$
\Delta \psi^{\mathrm{D}}=-\sum_{k=0}^{\infty}(-s(\mathcal{B}-m Y))^{k} s\left[\left(\partial_{y}^{2}+\partial_{z}^{2}-m^{2}\right) f+\mathcal{B} \psi\right] .
$$

We point out that $\Delta \psi^{\mathrm{D}}$ is not uniquely determined, and this non-uniqueness is reflected in the fact that there is the freedom in choosing different Green's functions, like the advanced or retarded Green's functions or the Feynman propagator (for details on the above operator expansions and the different Green's functions see [F7] or [FG1]). For our purpose, it is preferable to work with the retarded Green's function $s^{\wedge}$, whose kernel $s^{\wedge}(x, y)$ is given explicitly by (see [F7, Section 2.5] or (2.1.9) and (2.2.4), (2.2.7)

$$
s^{\wedge}(x, y)=-\frac{1}{2 \pi} i \not \not_{x} \delta\left(\xi^{2}\right) \Theta\left(-\xi^{0}\right) .
$$

This has the advantage that the support of $\Delta \psi^{D}$ lies in the future of $\psi$, and thus it is disjoint from the support of $\psi_{1}$ (see Figure 3.1 on page 190).

The function $\psi+\Delta \psi^{\mathrm{D}}$ solves the auxiliary Dirac equation, but in general it will have a component of generalized positive energy. This positive-energy contribution must be subtracted in order to obtain a vector in the image of $P$. Formally, this can be achieved by setting

$$
\Delta \psi^{\mathrm{E}}=-(1-P)\left(\psi+\Delta \psi^{\mathrm{D}}\right)
$$

In order to give this equation a meaning, one must keep in mind that the normalization of the fermionic projector involves a $\delta$-distribution in the mass parameters, i.e. $P_{+\mu} P_{+\mu^{\prime}}=$ $\delta\left(\mu-\mu^{\prime}\right) P_{+\mu}$. Thus using the formalism introduced in [FG1, Section 2], we can make sense of A.0.7) as an operator product simply by omitting all resulting $\delta$-distributions (see also Section 2.1. With A.0.4) as well as A.0.3, A.0.5 and A.0.7), we have introduced $\psi_{2}$ in terms of a well-defined perturbation series.

We now estimate $\Delta \psi^{\mathrm{E}}$ for large $|\Omega|$, with a similar method as previously used in $[\mathbf{F 6}$, Theorem 3.4] for the estimate of the non-causal high energy contribution. 
Lemma A.0.1. To very order $n$ in perturbation theory and for every $\nu \in \mathbb{N}$, there is a constant $C(n, \nu)$ such that the wave function $\Delta \psi^{E}$ as defined by (A.0.7) satisfies the inequality

$$
\sup _{x \in \mathscr{M}}\left|\left(\Delta \psi^{E}\right)^{(n)}(x)\right| \leq \frac{C(n, \nu)}{|\Omega|^{\nu}}
$$

Proof. The $n^{\text {th }}$ order contribution $\left(\Delta \psi^{\mathrm{E}}\right)^{(n)}$ can be written as a finite number of terms of the form

$$
g:=C_{n} \mathcal{B} C_{n-1} \mathcal{B} \cdots \mathcal{B} C_{0} \phi,
$$

where every factor $C_{l}$ is a linear combination of the operators $p, k$, and $s$. Here $\phi$ stands either for the wave function $\psi$ in A.0.3 or for the square bracket in A.0.5. In either case, $\psi$ is given explicitly and involves the free parameter $\Omega$. It is preferable to proceed in momentum space. The regularity and decay assumption A.0.1 implies that

$$
\sup _{k \in \hat{M}}\left|k^{J} \partial^{I} \hat{\mathcal{B}}(k)\right|<\infty \quad \text { for all multi-indices } I \text { and } J \text { with }|I| \leq 2 .
$$

Setting $F_{0}=\hat{\phi}$ and

$$
F_{l}(k)=\int \frac{d^{4} q}{(2 \pi)^{4}} \hat{\mathcal{B}}(k-q) C_{l}(q) F_{l-1}(q) \quad(\text { where } 1 \leq l \leq n),
$$

we can write the Fourier transform of $g$ as

$$
\hat{g}(k)=C_{n}(k) F_{n}(k) .
$$

It clearly suffices to prove the lemma for $\nu$ an even number. Let us show inductively that the functions $F_{l}$ satisfy the bounds

$$
\sup _{(\omega, \vec{k}) \in \hat{M}}(\omega-\Omega)^{\nu}\left(\left|F_{l}(\omega, \vec{k})\right|+\sum_{i=0}^{3}\left|\partial_{i} F_{l}(\omega, \vec{k})\right|\right)<C(l, \nu) \quad \text { uniformly in } \Omega .
$$

In the case $l=0$, the claim follows immediately from the explicit form of $\phi$. To prove the induction step, we use the inequality

$$
(\omega-\Omega)^{\nu} \leq c(\nu)\left(\left(\omega-\omega^{\prime}\right)^{\nu}+\left(\omega^{\prime}-\Omega\right)^{\nu}\right)
$$

to obtain the estimate

$$
\begin{aligned}
& \left|(\omega-\Omega)^{\nu} F_{l}(\omega, \vec{k})\right| \\
& \leq c(\nu)\left|\int_{-\infty}^{\infty} \frac{d \omega^{\prime}}{2 \pi} \int_{\mathbb{R}^{3}} \frac{d \vec{k}^{\prime}}{(2 \pi)^{3}}\left[\left(\omega-\omega^{\prime}\right)^{\nu} \hat{\mathcal{B}}\left(\omega-\omega^{\prime}, \vec{k}-\vec{k}^{\prime}\right)\right] C_{l}\left(\omega^{\prime}, \vec{k}^{\prime}\right) F_{l-1}\left(\omega^{\prime}, \vec{k}^{\prime}\right)\right| \\
& \quad+c(\nu)\left|\int_{-\infty}^{\infty} \frac{d \omega^{\prime}}{2 \pi} \int_{\mathbb{R}^{3}} \frac{d \vec{k}^{\prime}}{(2 \pi)^{3}} \hat{\mathcal{B}}\left(\omega-\omega^{\prime}, \vec{k}-\vec{k}^{\prime}\right) C_{l}\left(\omega^{\prime}, \vec{k}^{\prime}\right)\left[\left(\omega^{\prime}-\Omega\right)^{\nu} F_{l-1}\left(\omega^{\prime}, \vec{k}^{\prime}\right)\right]\right| .
\end{aligned}
$$

Furthermore, the factors $C_{l}$ involve at most first derivatives; more precisely, they are bounded in terms of Schwartz norms by (see for example [F7, Proof of Lemma 2.2.2])

$$
\left|C_{l}(f)\right| \leq \text { const }\|f\|_{4,1} \quad \text { for all } f \in \mathcal{S} .
$$

Combining these inequalities, we can use the induction hypothesis together with A.0.10 to bound the expression $\left|(\omega-\Omega)^{\nu} F_{l}(\omega, \vec{k})\right|$ uniformly in $\Omega$ and $(\omega, \vec{k})$. The expression $\mid(\omega-$ $\Omega)^{\nu} \partial_{i} F_{l}(\omega, \vec{k}) \mid$ can be estimated in exactly the same way if one keeps in mind that if we differentiate (A.0.11) with respect to $k$, the derivative acts only on the potential $\hat{\mathcal{B}}$, but not on the factor $F_{l-1}$. This proves A.0.13. 
We next consider the operators in $($ A.0.9 $)$ in more detail. The factor $(1-P)$ in (A.0.7) can be regarded as a projection operator onto the generalized positive-energy solutions of the Dirac equation. The perturbation expansion of the fermionic projector can be arranged in such a way that each operator product involves at least one factor $p-k$ which projects onto the negative-energy solutions (for details see [FG1]). Similarly, the factor $(1-P)$ in (A.0.7) implies that we can arrange the operator products such that every contribution (A.0.9) involves at least one factor $p+k$, being supported on the upper mass cone. Thus in the corresponding induction step, we may replace an arbitrary even number of factors $(\omega-\Omega)$ by factors of $|\Omega|$. In the following induction steps we proceed as in A.0.13. At the end, we apply A.0.12 to obtain the result.

We can now prove the main result of this appendix.

Proposition A.0.2. Consider a fermion system in Minkowski space with an interaction $\mathcal{B}$ which is a multiplication operator satisfying the regularity and decay assumptions A.0.1). Assume furthermore that the pole of $Q$ is of order $o\left(|\vec{\xi}|^{-4}\right)$ at the origin (see Definition 3.7.2). Then the EL equations (3.5.20) imply that the operator $Q$ vanishes identically in the continuum limit 3.5.29).

Proof. We introduce the wave functions $\psi_{1}$ and $\psi_{2}$ perturbatively via $\mathrm{A} .0 .2$ and A.0.4 with $\psi, \Delta \psi^{\mathrm{D}}$ and $\Delta \psi^{\mathrm{E}}$ according to A.0.3, A.0.5 and A.0.7). Evaluating the commutator $[P, Q]$ as in (3.5.23) gives the condition (3.5.24). Following the arguments in 3.5 .2 , the leading terms give (3.5.29), and thus it remains to consider all correction terms. The corrections of $\psi_{1}$ can be made arbitrarily small by choosing the parameter $\Delta m$ in A.0.2 sufficiently small. Working in A.0.5 with the retarded Green's function A.0.6, the support of $\Delta \psi^{\mathrm{D}}$ does not intersect the support of $\eta$, so that the corresponding contribution to 3.5 .24 ) is well-defined in the continuum limit.

The wave function $\Delta \psi^{\mathrm{E}}$ is more problematic, because it will in general not vanish on the support of $\eta$. But according to Lemma A.0.1. we can make $\Delta \psi^{\mathrm{E}}$ arbitrarily small by choosing $|\Omega|$ sufficiently large. This is not quite good enough for two reasons: First, the integrand in $(3.5 .24)$ becomes more and more oscillatory as $|\Omega|$ is increased, so that the leading contribution to (3.5.24) will also become small as $|\Omega|$ gets large. And secondly, even if $\Delta \psi^{\mathrm{E}}$ is small, it gives rise to a contribution at $x=y$ where $Q(x, y)$ is ill-defined. The first problem can be treated by noting that the oscillations in the integrand of (3.5.24) will give rise to a polynomial decay in $\Omega$ (typically a $1 / \Omega$ behavior), whereas according to A.0.8), the wave function $\Delta \psi^{\mathrm{E}}$ decays in $\Omega$ even rapidly. Thus we can indeed arrange that (A.0.8) is arbitrarily small compared to the leading contribution in 3.5.24). For the second problem we need to use that the pole of $Q$ is of order $o\left(|\vec{\xi}|^{-4}\right)$ at the origin: Due to this assumption, the integrand in (3.5.24) will be at most logarithmically divergent at $x=y$. By modifying $\psi_{2}$ by a suitable negative-energy solution of the Dirac equation (for example a wave packet of negative energy, whose amplitude is fine-tuned), one can arrange that this logarithmic divergence drops out. Then the integrals in $(3.5 .24)$ become finite, and by choosing $|\Omega|$ sufficiently large, we can arrange that the contribution of $\Delta \psi^{\mathrm{E}}$ to 3.5 .24 is much smaller than the leading contribution which yields 3.5.29).

Before discussing the result of this proposition, we estimate an operator product which is similar to A.0.9 but involves a nonlocal potential as considered in $\$ 3.10 .4$.

LEMMA A.0.3. We consider the expression

$$
g=C_{n} B_{n} C_{n-1} B_{n-1} \cdots B_{1} C_{0} \phi,
$$


where every factor $C_{l}$ stands for one of the operators $p, k$, or $s$. As in the proof of Lemma A.0.1. the function $\phi$ is either the wave function $\psi$ in A.0.3 or the square bracket in (A.0.5). Each factor $B_{l}$ either stands for the multiplication operator $\mathcal{B}$ satisfying (A.0.1), or else it is a nonlocal operator $\mathfrak{n}$ in the Schwartz class (3.10.36). We assume that at least one factor $B_{l}$ is a nonlocal operator. Then for every integer $\nu$ there is a constant $C(\nu)$ such that

$$
\sup _{x \in \mathcal{M}}|g(x)| \leq \frac{C(\nu)}{|\Omega|^{\nu}}
$$

Proof. As in the proof of Lemma A.0.1, we proceed inductively in momentum space. Suppose that $p$ is the smallest index such that $B_{p}=\mathfrak{n}$. Then for all $l<p$, only the potential $\mathcal{B}$ is involved, and the functions $F_{l}$ defined by A.0.11 again satisfy the inequalities A.0.13). In the $p^{\text {th }}$ induction step, we must replace (A.0.11) by

$$
F_{p}(k)=\int \frac{d^{4} q}{(2 \pi)^{4}} \hat{n}(k, q) C_{l}(q) F_{l-1}(q),
$$

where $\hat{n} \in \mathcal{S}(\hat{M} \times \hat{M})$ denotes the Fourier transform of $n(x, y)$. Using the induction hypothesis A.0.13 together with the rapid decay of $\hat{n}(p, q)$ in the variable $q$, we obtain a factor $|\Omega|^{-\nu}$. In the remaining induction steps, we can use the simpler method of Lemma 2.1.2 to obtain the result.

Clearly, the setting of Proposition A.0.2 is too special for our applications. But the methods and results can readily be extended in the following ways:

- Taking into account the wave functions of the particles and anti-particles: We first note that, being solutions of the Dirac equation, the wave functions of the particles and anti-particles in (3.4.7) are orthogonal to the wave function $\psi_{1}$ (as is obvious from $($ A.0.2 $)$ ). Furthermore, by choosing $|\Omega|$ much larger than the energies of all particle and anti-particle wave functions, we can arrange that these wave functions are also orthogonal to $\psi_{2}$. Then all the wave functions drop out of (3.5.24), so that we are back in the setting of Proposition A.0.2.

- Handling the microlocal chiral transformation: Following the constructions in $\$ 3.7 .10$, we must apply the microlocal chiral transformation (3.7.78) to the fermionic projector before forming the sectorial projection. Likewise, we here apply this transformation to the wave functions $\psi_{1}$ and $\psi_{2}$ before forming the sectorial projection. If this is done, all our arguments still go through.

- Arranging the right order of the pole of $Q$ at the origin: As we saw in Section 3.6. the operator $Q$ vanishes identically to degree five on the light cone. Thus the leading contribution to $Q$ is of degree four on the light cone. Since $Q$ always involves a factor $\sharp$ (see $(3.7 .2)$ ), the pole of $Q$ at the origin is indeed of the required order $o\left(|\vec{\xi}|^{-4}\right)$.

One might object that near the origin $x=y$, where the continuum limit of $Q(x, y)$ is not well-defined, the arguments of Section 3.6 do not apply, and thus there might be a non-zero contribution to $Q$ which scales like $(3.6 .8)$, thus having a pole $\sim|\vec{\xi}|^{-4}$. However, as explained after 3.6 .9 , we may assume that in the vacuum, the operator $Q$ vanishes identically, even at the origin where the formalism of the continuum limit does not apply. Since to degree five, an interaction only leads to phase transformations (see (3.6.21) ), the operator $Q$ will then again vanish identically. As a consequence, the pole of $Q$ will indeed scale like $|\vec{\xi}|^{-3}$, even without relying on the formalism of the continuum limit. 
- Handling nonlocal potentials: Proposition A.0.2 does not apply to nonlocal potentials as introduced in $\$ 3.10 .4$. Another difficulty is that the support argument used for $\Delta \psi^{\mathrm{D}}$ no longer applies. But as shown in Lemma A.0.3, any contribution to $\Delta \psi^{\mathrm{D}}$ or $\Delta \psi^{\mathrm{E}}$ which involves a nonlocal potential satisfies the inequality A.0.14 and can thus be made arbitrarily small by choosing $|\Omega|$ sufficiently large. Following the arguments in the proof of Proposition A.0.2, this gives us control of all error terms due to the nonlocal potentials, to every order in perturbation theory.

We conclude that with the help of Proposition A.0.2, we can justify the EL equations of the continuum limit (3.5.29) for all fermion systems considered in this book.

We finally analyze the scalings in a universe of finite life time.

REMARK A.0.4. (A universe of finite life-time) Suppose that instead of Minkowski space, we consider a more realistic universe of finite life time $t_{\max }$, like a cosmology with a "big bang" and a "big crunch." In this case, the Fourier integral (3.5.25) still gives a good local description of a Dirac sea (this is made precise in the example of a closed FRW geometry in [FR1, Theorem 5.1]). However, one can no longer expect a continuum of states, and therefore the condition $P \psi_{1}=0$ can no longer be satisfied by removing an arbitrarily thin strip around the mass shell. More precisely, the width $\Delta \omega$ of the strip in (3.5.26) should be at least as large as the "coarseness" of the states in momentum space. This gives rise to the scaling (for details in the example of the closed FRW geometry see [FR1, Section 5])

$$
\Delta \omega \sim \frac{1}{t_{\max }}
$$

The corresponding contribution to the Fourier integral 3.5.26 scales as follows,

$$
\Delta \psi_{1}:=\psi_{1}(x)-\eta(x) \sim \sup |\hat{\eta}| \frac{\Delta \omega}{\delta^{3}} \sim \Delta \omega \delta|\eta(0)| \sim \frac{\delta}{t_{\max }}\left|\psi_{1}(0)\right| .
$$

As a consequence, the wave function $\psi_{1}$ no longer vanishes on $\mathfrak{L}$, but

$$
\left.\psi_{1}\right|_{\mathfrak{L}} \sim \frac{\delta}{t_{\max }} \sup \left|\psi_{1}\right| .
$$

Furthermore, since $\Delta \psi_{1}$ is supported near the lower mass shell in momentum space, it decays in position space at infinity like the fundamental solution $\left(p_{m}-k_{m}\right)(0, y)$, smeared out on the scale $\delta$. Combining these statements, we find that the corresponding contribution to the expectation value (3.5.24) scales like

$$
<\Delta \psi_{1}\left|Q \psi_{2}>\sim \sup \right| \psi_{1}|\sup | \psi_{2} \mid \delta^{4} \frac{\delta}{t_{\max }} \frac{1}{\varepsilon^{L-1}} \varepsilon^{3-p} .
$$

where $p$ denotes the order of the pole at the origin, being defined as the smallest integer $p$ such that

$$
\limsup _{x \rightarrow y}\left(\left|\xi^{0}\right|+|\vec{\xi}|\right)^{L-p}|\eta(x, y)|<\infty .
$$

In comparison, the main contribution on the light cone around the origin scales like

$$
<\psi_{1}\left|Q \psi_{2}>\sim \sup \right| \psi_{1}|\sup | \psi_{2} \mid \delta^{4} \frac{1}{\varepsilon^{L-1}} \ell^{-p} \frac{\delta^{2}}{|\Omega|},
$$

and thus

$$
\frac{<\Delta \psi_{1} \mid Q \psi_{2}>}{<\psi_{1} \mid Q \psi_{2}>} \sim \frac{\varepsilon^{3-p} \ell^{p}|\Omega|}{t_{\max } \delta}=\left(\frac{\varepsilon}{\ell}\right)^{3-p} \varepsilon|\Omega| \frac{\ell^{3}}{\varepsilon l_{\max } \delta} .
$$


This equation involves the fundamental length scale $\sqrt{\varepsilon l_{\max }}$. The time since the big bang is estimated to about 13 billion years, which is the same order of magnitude as the size of the visible universe, estimated to 28 billion parsec. Thus it seems reasonable to assume that

$$
t_{\text {max }}>10^{10} \text { years } \sim 10^{26} \text { meters } .
$$

Taking for $\varepsilon$ the Planck length $\varepsilon \sim 10^{-35}$ meters, we obtain

$$
\sqrt{\varepsilon l_{\max }} \sim 10^{-4} \text { meters . }
$$

It is remarkable that this is about the length scale of macroscopic physics. Thus by choosing $\varepsilon|\Omega|$ sufficiently small, we can make the quotient A.0.15) arbitrarily small without violating the scalings $(3.5 .28)$. We conclude that even if the life time of our universe were finite, this would have no effect on the statement of Proposition A.0.2. 



\section{APPENDIX B}

\section{Spectral Analysis of the Closed Chain}

In this appendix we analyze how different contributions to the fermionic projector influence the EL equations. In particular, we shall give the proofs of Lemmas 3.7.3. 3.7.4 3.7.5 and 3.7.10. Furthermore, we will analyze a pseudoscalar differential potential (see (B.2.22) and (B.2.23) ) and a scalar/pseudoscalar potential (Lemma B.3.1). Finally, we prove Proposition 3.7 .12 on the shear contributions caused by the microlocal chiral transformation.

\section{B.1. The General Procedure}

We first review the methods and the general procedure. The behavior of the fermionic projector near the light cone is described by the light-cone expansion (3.4.14). We concentrate on the singular behavior on the light cone as described by the series in (3.4.14), disregarding the smooth non-causal contributions $\tilde{P}^{\text {le }}$ and $\tilde{P}^{\text {he }}$ (for the smooth contributions see Appendix $\mathrm{D}$ and $\$ 3.8 .3$ also cf. the end of $\$ 3.5 .1)$. The terms of the light-cone expansion can be computed as described in [F7, Section 2.5] and Section 2.2 (for more details see [F5 and [F6]). The main task is to calculate the corresponding perturbation of the eigenvalues $\lambda_{ \pm}^{L / R}$, because then the effect on the EL equations is given by Lemma 3.7.1. In principle, the perturbation of the eigenvalues can be determined in a straightforward manner by substituting the summands of the light-cone expansion into the closed chain $A_{x y}(3.2 .5)$, and by performing a standard perturbation calculation for the eigenvalues of the $(4 \times 4)$-matrix $A_{x y}$. However, the combinatorics of the tensor contractions inside the closed chain makes this direct approach so complicated that it is preferable to use a more efficient method developed in [F7, Appendix G]. We now outline this method, giving at the same time a somewhat different viewpoint.

The first step is to perform the light-cone expansion of the fermionic projector (as introduced in Section 2.2p. For the spectral analysis of the closed chain we use the methods introduced in Section 2.6. More precisely, we first compute the matrix elements of the fermionic projector in the double null spinor frame $\mathfrak{f}_{s}^{c}$ (see $(2.6 .28)$ in $\left.\$ 2.6 .3\right)$. Transforming to the double null spinor frame at such an early stage has the advantage that the contractions of the tensor indices (which arise by taking traces of products of Dirac matrices) are relatively easy to compute. After forming the closed chain $A_{x y}$ in our double-null spinor basis, we can compute the eigenvalues of $A_{x y}$ with a standard perturbation calculation as explained in 2.6 .3 . As the unperturbed operator we choose the closed chain (3.6.19) which involves the axial phases. This is particularly convenient because the unperturbed operator is diagonal in our double null spinor basis, and moreover the unperturbed eigenvalues are non-degenerate according to (3.6.21). Thus it suffices to use simple perturbation theory without degeneracies. Next, it is useful that the unperturbed eigenvalues (3.6.21) form two complex conjugate pairs. This will remain true if perturbations of lower degree are taken into account, so that $\overline{\lambda_{s}^{c}}=\lambda \frac{\bar{c}}{s}$. Therefore, it 
suffices to consider the eigenvalue $\lambda_{+}^{L}$. The eigenvalue $\lambda_{+}^{R}$ is then obtained by the replacement $L \leftrightarrow R$, whereas the eigenvalues $\lambda_{-}^{L / R}$ are obtained by complex conjugation. Expressing the perturbation calculation for $\lambda_{+}^{L}$ in terms of the traces (2.6.28), one finds that to the considered degree on the light cone, the vector $v$ drops out.

In order to avoid computational errors, the light-cone expansion was carried out with the help of the $\mathrm{C}++$ program class_commute, which was originally developed for the calculations in [F5] and [F6]. The traces in (2.6.28), which involve the contractions of tensor indices, are also computed with the help of class_commute. The resulting matrix elements of the fermionic projector are exported to the computer algebra program Mathematica (from this moment on, the tensor indices are simply treated as fixed text strings). The perturbation calculation as well as the expansions around the origin are then carried out by an algorithm implemented in Mathematica. This also has the advantage that the standard simplification algorithms of Mathematica and the comfortable front end are available 1 .

\section{B.2. Vector and Axial Contributions}

We now list the results of these calculations, also giving some intermediate steps. We note that some of these results were already obtained in [F7, Appendix G.3], however without using the algorithm implemented in Mathematica.

Proof of Lemma 3.7.3. Using for line integrals as in [F6 the short notation 3.6.18) and (see also (2.2.31))

$$
\int_{x}^{y}[p, q \mid r] f:=\int_{0}^{1} \alpha^{p}(1-\alpha)^{q}\left(\alpha-\alpha^{2}\right)^{r} f(\alpha y+(1-\alpha) x) d \alpha
$$

the relevant contributions to the light-cone expansion can be written as (cf. [F7, Appen$\operatorname{dix} \mathrm{B}]$ and [F6. Appendix A])

$$
\begin{aligned}
\chi_{L} P(x, y)= & \frac{i}{2} \chi_{L} e^{-i \Lambda_{L}^{x y}} \sharp T^{(-1)} \\
& -\frac{1}{2} \chi_{L} \notin \xi_{i} \int_{x}^{y}[0,0 \mid 1] j_{L}^{i} T^{(0)} \\
& +\frac{1}{4} \chi_{L} \notin \int_{x}^{y} F_{L}^{i j} \gamma_{i} \gamma_{j} T^{(0)} \\
& -\chi_{L} \xi_{i} \int_{x}^{y}[0,1 \mid 0] F_{L}^{i j} \gamma_{j} T^{(0)} \\
& -\chi_{L} \xi_{i} \int_{x}^{y}[0,1 \mid 1] \not j_{L}^{i} T^{(1)} \\
& -\chi_{L} \int_{x}^{y}[0,2 \mid 0] j_{L}^{i} \gamma_{i} T^{(1)} \\
& -i m \chi_{L} \xi_{i} \int_{x}^{y} Y A_{R}^{i} T^{(0)} \\
& +\frac{i m}{2} \chi_{L} \oiint \int_{x}^{y}\left(Y A_{R}-A_{L} Y\right) T^{(0)}
\end{aligned}
$$

\footnotetext{
${ }^{1}$ The $\mathrm{C}++$ program class_commute and its computational output as well as the Mathematica worksheets were included as ancillary files to the arXiv submission arXiv:1211.3351 [math-ph].
} 


$$
\begin{aligned}
& +i m \chi_{L} \xi_{i} \int_{x}^{y}[0,0 \mid 1] Y j_{R}^{i} T^{(1)} \\
& -\frac{i m}{2} \chi_{L} \int_{x}^{y}[1,0 \mid 0] Y F_{R}^{i j} \gamma_{i} \gamma_{j} T^{(1)} \\
& -\frac{i m}{2} \chi_{L} \int_{x}^{y}[0,1 \mid 0] F_{L}^{i j} \gamma_{i} \gamma_{j} Y T^{(1)} \\
& +i m \chi_{L} \int_{x}^{y}[0,1 \mid 0]\left(Y\left(\partial_{j} A_{R}^{j}\right)-\left(\partial_{j} A_{L}^{j}\right) Y\right) T^{(1)} \\
& +\frac{m^{2}}{2} \chi_{L} \xi_{i} \int_{x}^{y}[1,0 \mid 0] Y Y A_{L}^{i} T^{(0)} \\
& +\frac{m^{2}}{2} \chi_{L} \xi_{i} \xi_{x}^{y}[0,1 \mid 0] A_{L}^{i} Y Y T^{(0)} \\
& -m^{2} \chi_{L} \xi_{i} \int_{x}^{y}[0,0 \mid 1] Y Y F_{L}^{i j} \gamma_{j} T^{(1)} \\
& -m^{2} \chi_{L} \xi_{i} \int_{x}^{y}[0,2 \mid 0] F_{L}^{i j} \gamma_{j} Y Y T^{(1)} \\
& +m^{2} \chi_{L} \int_{x}^{y}[1,0 \mid 0] Y Y A_{L} T^{(1)} \\
& -m^{2} \chi_{L} \int_{x}^{y}[0,0 \mid 0] Y A_{R} Y T^{(1)} \\
& +m^{2} \chi_{L} \int_{x}^{y}[0,1 \mid 0] A_{L} Y Y T^{(1)} \\
& +\notin(\operatorname{deg}<1)+(\operatorname{deg}<0)+\mathcal{O}\left(A_{L / R}^{2}\right),
\end{aligned}
$$

where $F_{c}^{j k}=\partial^{j} A_{c}^{k}-\partial^{k} A_{c}^{j}$ is the chiral field tensor and $j_{c}^{k}=\partial_{j}^{k} A_{c}^{j}-\square A_{c}^{k}$ is the corresponding chiral current. The term (B.2.2) is our unperturbed fermionic projector (3.6.17); all other summands form our perturbation. We form the sectorial projection, expand in powers of $\xi$ and compute the matrix elements (2.6.28). Contracting the field tensor in (B.2.4 and $(\mathrm{B} .2 .5)$ with $\xi$, we get the term $F_{j k} \xi^{j} \xi^{k}$ which vanishes because we treat both factors $\xi$ as outer factors (for a more careful analysis of these field tensor terms see 4.6 .2 ). Moreover, the error terms of the form $\$(\operatorname{deg}<1)$ are contracted in the computations with another factor $\sharp$ or $\bar{\xi}$, giving rise to a term of lower degree. Likewise, the higher orders in $A_{L / R}$ give rise to terms either of lower degree on the light cone or of higher order in the expansion around the origin. Computing $\Delta \lambda_{+}^{L}$ by a first order perturbation calculation gives

$$
\begin{aligned}
\Delta \lambda_{+}^{L}= & \frac{i g^{2}}{3} j_{L}^{i} \xi_{i} T_{[0]}^{(1)} \overline{T_{[0]}^{(-1)}}-\frac{i g^{2}}{6} j_{R}^{i} \xi_{i} T_{[0]}^{(0)} \overline{T_{[0]}^{(0)}} \\
& -2 i g m^{2} A_{\mathrm{a}}^{i} \xi_{i} \hat{Y} \grave{Y}\left(T_{[2]}^{(1)} \overline{T_{[0]}^{(-1)}}+T_{[0]}^{(0)} \overline{T_{[2]}^{(0)}}\right) \\
& -2 i m^{2} A_{\mathrm{a}}^{i} \xi_{i} \hat{Y}^{2} \frac{T_{[1]}^{(0)} \overline{T_{[0]}^{(-1)}}\left(T_{[1]}^{(0)} \overline{T_{[0]}^{(0)}}+\text { c.c. }\right)-c . c .}{T_{[0]}^{(0)} \overline{T_{[0]}^{(-1)}}-T_{[0]}^{(-1)} \overline{T_{[0]}^{(0)}}}+(\operatorname{deg}<2)+o\left(|\vec{\xi}|^{-1}\right) .
\end{aligned}
$$


The other eigenvalues are obtained by the replacement $L \leftrightarrow R$ and by complex conjugation. Substituting the resulting formulas into (3.7.1) gives the result for the current terms.

For the computation of the mass term, we must consider another contribution where the mass expansion of the fermionic projector of the vacuum

$$
P(x, y) \asymp m Y T^{(0)}+\frac{i}{2} m^{2} Y^{2} \sharp T^{(0)}
$$

is taken into account in a perturbation calculation to second order,

$$
\begin{aligned}
\Delta \lambda_{+}^{L} \asymp & m^{2} \hat{Y}^{2} T_{[1]}^{(0)} \overline{T_{[1]}^{(0)}}+g m^{2} \dot{Y} \grave{Y} T_{[0]}^{(0)} \overline{T_{[2]}^{(0)}} \\
& +\frac{g^{2} m^{2} \hat{Y}^{2}}{\lambda_{+}^{L}-\lambda_{-}^{R}}\left(T_{[1]}^{(0)} \overline{T_{[0]}^{(-1)}}-T_{[0]}^{(-1)} \overline{T_{[1]}^{(0)}}\right)\left(T_{[0]}^{(0)} \overline{T_{[1]}^{(0)}}-T_{[1]}^{(0)} \overline{T_{[0]}^{(0)}}\right) .
\end{aligned}
$$

The analogous formulas for the other eigenvalues are obtained by the replacements $L \leftrightarrow R$ and $\nu \leftrightarrow \bar{\nu}$ as well as by complex conjugation. In order to compute $\left|\Delta \lambda_{+}^{L}\right|$, we use the identity

$$
\Delta\left|\lambda_{+}^{L}\right| \asymp \frac{1}{2\left|\lambda_{+}^{L}\right|}\left(\lambda_{-}^{R} \Delta \lambda_{+}^{L}+\lambda_{+}^{L} \Delta \lambda_{-}^{R}\right)
$$

and express the unperturbed eigenvalues in terms of (3.6.21). Expanding the phase factors $\nu$ and $\bar{\nu}$ in powers of the axial potential (see (3.6.20) ) and keeping the linear term in $A_{\mathrm{a}}$, we obtain

$$
\begin{aligned}
\Delta\left|\lambda_{+}^{L}\right| \asymp & \frac{g^{2} m^{2} \hat{Y}^{2}}{\left|\lambda_{+}\right|} A_{\mathrm{a}}^{i} \xi_{i} T_{[1]}^{(0)} \overline{T_{[1]}^{(0)}}\left(T_{[0]}^{(-1)} \overline{T_{[0]}^{(0)}}-T_{[0]}^{(0)} \overline{T_{[0]}^{(-1)}}\right) \\
& +\frac{g^{3} m^{2} \hat{Y} \grave{Y}}{\left|\lambda_{+}\right|} A_{\mathrm{a}}^{i} \xi_{i} T_{[0]}^{(0)} \overline{T_{[0]}^{(0)}}\left(T_{[0]}^{(-1)} \overline{T_{[2]}^{(0)}}-T_{[2]}^{(0)} \overline{T_{[0]}^{(-1)}}\right),
\end{aligned}
$$

and similarly for the other eigenvalues. Substituting these formulas into (3.7.1) completes the proof.

Proof of Lemma 3.7.4. According to (3.7.8) and (3.4.7), the perturbation $\Delta P(x, y)$ by the Dirac current satisfies the relations

$$
\operatorname{Tr}\left(\gamma^{j} \Delta P(x, y)\right)=-\frac{1}{2 \pi} J_{\mathrm{v}}^{j}, \quad \operatorname{Tr}\left(\Gamma \gamma^{j} \Delta P(x, y)\right)=-\frac{1}{2 \pi} J_{\mathrm{a}}^{j}
$$

and thus

$$
\Delta P(x, x)=-\frac{1}{8 \pi} \gamma_{j} J_{\mathrm{v}}^{j}+\frac{1}{8 \pi} \Gamma \gamma_{j} J_{\mathrm{a}}^{j} .
$$

The corresponding perturbation of the eigenvalue $\Delta \lambda_{+}^{L}$ is computed to be

$$
\Delta \lambda_{+}^{L}=\frac{i g}{8 \pi} J_{L}^{i} \xi_{i} \overline{T_{[0]}^{(-1)}} .
$$

The formula for $\mathcal{R}$ follows by a direct calculation.

Proof of LEMma 3.7.11. The result follows exactly as in the proof of Lemma 3.7.4. noting that (3.7.84) is obtained from (B.2.21) by setting $J_{L}=J_{R}=v$ and multiplying by $-8 \pi c / m^{2}$. Moreover, (3.7.86) tells us how to insert the gauge phases. 
We now come to the pseudoscalar differential potential (3.7.14). The contribution to the fermionic projector linear in $v$ has the form

$$
P(x, y) \asymp \frac{1}{2} \Gamma \xi \xi_{i} \int_{x}^{y} \not \partial v^{i} T^{(-1)}+\Gamma \xi_{i} v^{i}(x) T^{(-1)}+(\operatorname{deg}<2) .
$$

After applying the relation $2 \notin \not \partial v^{i}=2 \xi^{j} \partial_{j} v^{i}+\left[\not, \not \partial v^{i}\right]$, in the first term we can integrate by parts to obtain (3.7.15). The light-cone expansion to lower degree involves many terms, which we shall not give here. To higher order in the mass, the contributions become less singular on the light cone. In particular, the leading term cubic in the mass takes the form

$$
\begin{aligned}
P(x, y) \asymp & -\frac{i m^{3}}{2} \Gamma \notin \xi_{i} \int_{x}^{y}[0,1 \mid 0]\left(Y v^{i} Y Y-v^{i} Y Y Y\right) T^{(0)} \\
& -\frac{i m^{3}}{2} \Gamma \not \xi_{i} \int_{x}^{y}[1,0 \mid 0]\left(Y Y Y v^{i}-Y Y v^{i} Y\right) T^{(0)} \\
& +i m^{3} \Gamma \int_{x}^{y}[0,1 \mid 0](Y \psi Y Y-\psi Y Y Y) T^{(1)} \\
& +i m^{3} \Gamma \int_{x}^{y}[1,0 \mid 0](Y Y Y \psi-Y Y \psi Y) T^{(1)} \\
& +\&(\operatorname{deg}<1)+(\operatorname{deg}<0) .
\end{aligned}
$$

Expanding in powers of $\xi$, we obtain (3.7.16) and (3.7.17).

We point out that for the scalar differential potential, the higher orders in perturbation theory are difficult to handle because they are not of lower degree on the light cone. Moreover, a resummation procedure similar to that for chiral potential does not seem to work. For a constant potential, this problem corresponds to the effect of the "deformation of the light cone" as discussed after (3.7.20). In the more general setting here, this problem means that the scalar differential potential cannot be treated perturbatively in a convincing way. This serious difficulty was our original motivation for introducing the vector differential potential (3.7.22), and to rewrite the combination of these potentials by the local axial transformation (3.7.24).

For the local axial transformation (3.7.24), the fermionic projector can easily be computed non-perturbatively. To first order in $v$, we obtain the contribution 3.7.30). We now give a general symmetry argument which shows that this contribution drops out of the EL equations.

Proof of Lemma 3.7.5. In order to avoid a case-by-case analysis of the different orders in a mass expansion, as the unperturbed fermionic projector $P_{0}(x, y)$ we choose the fermionic projector of the vacuum (3.6.1). After regularization, we then obtain a fermionic projector with a vector-scalar structure which we write in the form

$$
P(x, y)=\not g+h, \quad P(y, x)=\bar{\not}+\bar{h}
$$

with a complex vector field $g(x, y)$ and a complex scalar $h(x, y)$. The corresponding closed chain $A_{x y}=P(x, y) P(y, x)$ has two eigenvalues $\lambda_{ \pm}$, both with multiplicity two, and the corresponding spectral projectors can be written as

$$
F_{ \pm}=\frac{1}{\lambda_{ \pm}-\lambda_{\mp}}\left(A_{x y}-\lambda_{\mp}\right)
$$


(for details see [F7, Section 5.3]). Consequently, a linear mapping on the eigenspace corresponding to $\lambda_{s}$ (with $s \in\{+,-\}$ ) can be written as

$$
X F_{s} \quad \text { with } \quad X=\alpha \mathbb{1}+\beta_{1} \Gamma \psi_{1}+\beta_{2} \Gamma \psi_{2}+i \beta_{3} \psi_{1} \psi_{2},
$$

where $\alpha, \beta_{1}, \beta_{2}, \beta_{3}$ are complex parameters and $u_{1}, u_{2}$ are two vector fields which are orthogonal to $g$ and $\bar{g}$. Our task is to show that the linear perturbation of the fermionic projector by the functions $c_{\beta}$ in (3.7.30) has no effect on the eigenvalues of the closed chain. To this end, we must show that $\Delta A_{x y}$ vanishes on the degenerate subspaces, i.e.

$$
\operatorname{Tr}\left(X F_{s} \Delta A_{x y}\right)=0 .
$$

Writing $P(y, x)$ as $P(x, y)^{*}$ and omitting the arguments $(x, y)$, we have

$$
\Delta A_{x y}=(\Delta P) P^{*}+P(\Delta P)^{*} \quad \text { and } \quad \Delta P=-i \sum_{\beta=1}^{g}\left[c_{\beta} \Gamma \psi, P_{\beta}\right] .
$$

Combining these formulas, one sees that it suffices to show that

$$
\operatorname{Tr}\left(X F_{s}\left[\Gamma \psi, P_{\beta}\right] P^{*}\right)=0,
$$

because all other contributions are then obtained by conjugation and by taking linear combinations.

By cyclically commuting the operators and using that $X$ commutes with $P^{*}$, we obtain

$$
\operatorname{Tr}\left(X F_{s}\left[\Gamma \psi, P_{\beta}\right] P^{*}\right)=\operatorname{Tr}\left(\Gamma \psi\left[P_{\beta}, X P^{*} F_{s}\right]\right) .
$$

It suffices to consider the vector component of $P_{\beta}$ (otherwise the commutator vanishes). Moreover, since the trace of an odd number of Dirac matrices vanishes, we may restrict attention to the even contribution to $X P^{*} F_{s}$. The bilinear contribution to $X P^{*} F_{s}$ gives rise to a vector contribution to the commutator, so that the trace in (B.2.28) vanishes. Moreover, the scalar contribution to $X P^{*} F_{s}$ drops out of the commutator. Hence it remains to consider the pseudoscalar contribution to $X P^{*} F_{s}$.

In view of (B.2.26), (B.2.25) and (B.2.24), the only way to obtain a pseudoscalar contribution is to combine four linearly independent Dirac matrices,

$$
X P^{*} F_{s} \asymp i \beta_{3} \psi_{1} \psi_{2}\left(P^{*} F_{s}\right)_{\text {bilinear }},
$$

where the subscript means that we only take into account the bilinear contribution. Again using (B.2.25) and (B.2.24), this bilinear contribution can be written as

$$
\left(P^{*} F_{ \pm}\right)_{\text {bilinear }}=\frac{1}{\lambda_{ \pm}-\lambda_{\mp}}\left(P^{*} P P^{*}\right)_{\text {bilinear }}=\frac{1}{\lambda_{ \pm}-\lambda_{\mp}}(\bar{h} \not g \bar{\phi}+\bar{\phi} h \bar{\phi}+\bar{\phi} \not \phi \bar{h})_{\text {bilinear }} .
$$

Using the anti-commutation relations, one finds that the first and third summands combine to a scalar, and that the second summand is also a scalar. Thus the bilinear component of $P^{*} F_{s}$ vanishes.

Proof of Lemma 3.7.10. Let us consider the different contributions in (3.7.81). The vector component clearly drops out of (3.7.1). The pseudoscalar and bilinear contributions are even. Thus in the perturbation calculation for $\lambda_{s}^{c}$, they only effect the odd powers in the mass expansion. As a consequence, the leading contribution is of the form $\Delta \lambda_{s}^{c}=m(\operatorname{deg}<2)$ and can thus be omitted. It remains to consider the axial contribution,

$$
P(x, y) \asymp \Gamma \psi T_{[3]}^{(1)}+(\operatorname{deg}<0)
$$


The corresponding perturbation of the eigenvalue $\Delta \lambda_{+}^{L}$ is given by

$$
\Delta \lambda_{+}^{L} \asymp-i g v_{j} \xi^{j} T_{[3]}^{(1)} \overline{T_{[0]}^{(-1)}}+(\operatorname{deg}<2) .
$$

The other eigenvalues are again obtained by the replacement $L \leftrightarrow R$ and by complex conjugation. Substituting the resulting formulas into (3.7.1) gives the result.

\section{B.3. Scalar and Pseudoscalar Contributions}

We next consider the perturbation of the fermionic projector by the scalar and pseudoscalar Dirac current (3.9.1). According to (3.4.7), the corresponding perturbation of the fermionic projector is given by

$$
\Delta P(x, y)=-\frac{1}{8 \pi}\left(J_{\mathrm{s}}+i \Gamma J_{\mathrm{a}}\right)+o\left(|\vec{\xi}|^{0}\right) .
$$

Lemma B.3.1. The first order contribution of the perturbation (B.3.1) to the operator $Q(x, y)$ is of degree two on the light cone.

Proof. A first order perturbation calculation yields

$$
\Delta \lambda_{+}^{L}=\frac{J_{\mathrm{s}}}{4 \pi} m \hat{Y} \frac{T_{[0]}^{(0)}\left(T_{[0]}^{(-1)} \overline{T_{[1]}^{(0)}}-\text { c.c. }\right)+\overline{T_{[0]}^{(-1)}}\left(T_{[1]}^{(0)} \overline{T_{[0]}^{(0)}}-\text { c.c. }\right)}{T_{[0]}^{(0)} \overline{T_{[0]}^{(-1)}}-T_{[0]}^{(-1)} \overline{T_{[0]}^{(0)}}}+(\operatorname{deg}<1) .
$$

Note that the pseudoscalar current dropped out. This cancellation can also be understood from the following consideration. The pseudoscalar contribution in (B.3.1) can be written symbolically as

$$
\Delta P(x, y)=i[\Gamma \psi, P(x, y)],
$$

where $\psi(x, y)$ is a suitable function. This perturbation has the same form as that in (B.2.27). Proceeding as in the proof of Lemma 3.7.5, we conclude that the pseudoscalar contribution drops out of the EL equations.

The resulting first order contribution to the EL equations is obtained by considering first variations of the Lagrangian of the form (3.6.23) and by substituting the formulas for $\Delta \lambda_{s}^{c}$. Counting degrees, this contribution is expected to be of degree three on the light cone. However, since (B.3.2) has even parity, whereas the first variation of the Lagrangian involves factors $\left(\left|\lambda_{s}^{L}\right|-\left|\lambda_{s}^{R}\right|\right)$ of odd parity, this expected contribution of degree three vanishes.

\section{B.4. Shear Contributions}

We now come to the analysis of the Dirac equation (3.7.89), where we interchanged the chirality of the potentials in the even component of the Dirac operator.

Proof of Proposition 3.7.12. It suffices to consider the homogeneous transformation (3.7.58) because then the result immediately carries over to the microlocal transformation by considering just as in 3.7 .10 the corresponding quasi-homogeneous ansatz (3.7.78). Moreover, it suffices to show that the auxiliary fermionic projector defined in analogy to 3.7 .79 by

$$
\tilde{P}^{\text {aux }}=\dot{U}_{\text {flip }} P^{\text {aux }} \grave{U}_{\text {flip }}^{*}
$$


has the desired properties (where $P^{\text {aux }}$ satisfies the Dirac equation $\left(i \not \partial_{x}+\chi_{L} A_{R}+\chi_{R} A_{L}-\right.$ $\left.m Y) P^{\text {aux }}=0\right)$. Namely, this fermionic operator obviously satisfies the Dirac equation (3.7.92). This implies that it differs from the fermionic projector defined from 3.7 .92$)$ via the causal perturbation expansion only by smooth contributions, giving the result.

Expanding (3.7.58) in powers of $1 / \sqrt{\Omega}$, we obtain

$$
\begin{aligned}
U_{\text {flip }}=\mathbb{1} & +\left(\frac{i Z}{\sqrt{\Omega}}-\frac{Z^{2}}{2 \Omega}\right) V+\mathcal{O}\left(\Omega^{-\frac{3}{2}}\right) \\
\tilde{P}^{\text {aux }}= & P^{\text {aux }}+\frac{i}{\Omega}\left(Z V P^{\text {aux }}-P^{\text {aux }} V^{*} Z\right) \\
& +\frac{1}{\Omega} Z V P^{\text {aux }} V^{*} Z-\frac{1}{2 \Omega}\left(Z^{2} V P^{\text {aux }}+P^{\text {aux }} V Z^{2}\right)+\mathcal{O}\left(\Omega^{-\frac{3}{2}}\right) .
\end{aligned}
$$

Performing the light-cone expansion order by order in perturbation theory, one finds that the factors $V$ and $V^{*}$ modify the fermionic projector by phases and give rise to additional contributions of lower degree on the light cone. In particular, the contributions of or$\operatorname{der} \mathcal{O}\left(\Omega^{-\frac{3}{2}}\right)$ are of order $o(|\vec{\xi}|)(\operatorname{deg}<2)$ on the light cone. Moreover, the relations (3.7.67) imply that the linear terms in $Z$ again drop out. Moreover, the contributions involving $Z^{2}$ can be compensated just as explained for the contribution (3.7.62). We conclude that

$$
\tilde{P}=P+\frac{1}{\Omega} \dot{Z} V P^{\text {aux }} V^{*} \grave{Z}+o(|\vec{\xi}|)(\operatorname{deg}<2) .
$$

Hence, as desired, the transformation $V$ only modifies the contribution generated by the microlocal chiral transformation.

In order to specify $V$, it is most convenient to work with the unitary perturbation flow, which makes it possible to obtain the fermionic projector in the external potential $P^{\text {aux }}$ from the vacuum fermionic projector $P^{\mathrm{vac}}$ by conjugation with an operator $U_{\text {flow }}$,

$$
P^{\text {aux }}=U_{\text {flow }} P^{\text {vac }} U_{\text {flow }}^{*}
$$

(see [FG1, Section 5] or the more explicit and systematic treatment with spatial or mass normalization in [FT2, Section 4]). For clarity, we denote the dependence of $U_{\text {flow }}$ on the external potential in square brackets, i.e. $U_{\text {flow }}=U_{\text {flow }}\left[\chi_{L} A_{R}+\chi_{R} A_{L}\right]$. In order for $V$ to flip the chirality of the potentials, we simply choose

$$
V=U_{\text {flow }}\left[\chi_{L} A_{L}+\chi_{R} A_{R}\right] U_{\text {flow }}\left[\chi_{L} A_{R}+\chi_{R} A_{L}\right]^{-1}
$$

(note that $U_{\text {flow }}$ can be inverted with a Neumann series as a formal power series in the external potential). Then the operator $V P^{\text {aux }} V^{*}$ coincides with the fermionic projector in the presence of the chiral potential $\chi_{L} A_{L}+\chi_{R} A_{R}$. This concludes the proof.

\section{B.5. The Energy-Momentum Tensor of Chiral Gauge Fields}

We proceed by computing the energy-momentum tensor of chiral gauge fields. The relevant contributions to the kernel of the fermionic projector are given by

$$
\begin{aligned}
\chi_{L} P(x, y) & \asymp-i \chi_{L} \sharp \int_{x}^{y}[0,1 \mid 1] d z_{1} \int_{z_{1}}^{y}[0,1 \mid 0] d z_{2} F_{k i}^{L}\left(z_{1}\right) F_{L}^{k j}\left(z_{2}\right) \xi^{i} \xi_{j} T_{[0]}^{(0)}+(\operatorname{deg}<1) \\
& =-\frac{i}{24} \chi_{L} \sharp F_{k i}^{L} F_{L}^{k j} \xi^{i} \xi_{j} T_{[0]}^{(0)}+(\operatorname{deg}<1)+o\left(|\vec{\xi}|^{2}\right) .
\end{aligned}
$$


From the contributions involving $T_{\circ}^{(1)}$, it suffices to compute the contraction with $\Varangle$ :

$$
\begin{aligned}
\frac{1}{4} \operatorname{Tr} & \left(\$ \chi_{L / R} P(x, y)\right) \\
\asymp & \pm \frac{1}{2} \int_{x}^{y}[0,2 \mid 0] d z_{1} \int_{z_{1}}^{y}[0,0 \mid 0] d z_{2} \epsilon_{i j k l} \xi^{i} F_{L / R}^{j a}\left(z_{1}\right) \xi_{a} F_{L / R}^{k l}\left(z_{2}\right) T_{[0]}^{(1)} \\
& \pm \frac{1}{2} \int_{x}^{y}[0,2 \mid 0] d z_{1} \int_{z_{1}}^{y}[0,1 \mid 0] d z_{2} \epsilon_{i j k l} \xi^{i} F_{L / R}^{j k}\left(z_{1}\right) F_{L / R}^{l a}\left(z_{2}\right) \xi_{a} T_{[0]}^{(1)} \\
& -i \int_{x}^{y}[0,2 \mid 0] d z_{1} \int_{z_{1}}^{y}[1,0 \mid 0] d z_{2} F_{k i}^{L / R}\left(z_{1}\right) F_{L / R}^{k j}\left(z_{2}\right) \xi^{i} \xi_{j} T_{[0]}^{(1)} \\
& -4 i \int_{x}^{y}[0,1 \mid 1] d z_{1} \int_{z_{1}}^{y}[0,1 \mid 0] d z_{2} F_{k i}^{L / R}\left(z_{1}\right) F_{L / R}^{k j}\left(z_{2}\right) \xi^{i} \xi_{j} T_{[0]}^{(1)} \\
= & -\frac{i}{3} F_{k i}^{L / R} F_{L / R}^{k j} \xi^{i} \xi_{j} T_{[0]}^{(1)}+o\left(|\vec{\xi}|^{2}\right)
\end{aligned}
$$

where $\epsilon^{i j k l}$ is the totally anti-symmetric Levi-Civita symbol. Note that the terms involving $\epsilon^{i j k l}$ vanish to leading order at the origin. This is due to the following lemma.

Lemma B.5.1. For any anti-symmetric tensor $F$ and any $\xi$ on the light cone,

$$
\epsilon_{i j k l} \xi^{i} F^{j a} \xi_{a} F^{k l}=0 \text {. }
$$

Proof. We extend $\xi$ to a basis $\left(\xi=e_{1}, e_{2}, e_{3}, e_{4}\right)$ being a null frame in the sense that

$$
\left\langle e_{1}, e_{2}\right\rangle=\left\langle e_{2}, e_{1}\right\rangle=1, \quad\left\langle e_{3}, e_{3}\right\rangle=\left\langle e_{4}, e_{4}\right\rangle=-1,
$$

and all other Minkowski inner products vanish. Then $F$ can be represented as

$$
F=\sum_{i, j=1}^{4} F^{i j} e_{i} \wedge e_{j}
$$

(where for ease in notation we assume that $F^{i j}=-F^{j i}$ ). Since $\xi$ is null, the index $a$ in (B.5.3) must not be equal to one. Moreover, due to the total anti-symmetrization, none of the indices $j, k, l$ must be equal to one. Therefore, we may assume that all indices of $F$ are not equal to one, i.e.

$$
F=\sum_{i, j=2}^{4} F^{i j} e_{i} \wedge e_{j} .
$$

Next, by performing a rotation of the basis vectors $e_{3}$ and $e_{4}$, we can arrange that $F^{23}$ vanishes, i.e.

$$
F=2 F^{24} e_{2} \wedge e_{4}+2 F^{34} e_{3} \wedge e_{4} .
$$

Now, in view of the left side of (B.5.4), the index $a$ in (B.5.3) must be equal to two. Consequently, the index $j$ is equal to four. On the other hand, we see from (B.5.5) that one of the indices $k$ or $l$ in B.5.3 must also be equal to four. Hence anti-symmetrizing in the indices $j, k$ and $l$ gives zero.

In order to compute the perturbation of the eigenvalues of the closed chain, one needs to take into account the contributions $B$ B.5.1) and (B.5.2) to first order, and the 
contributions $\mathrm{B} .2 .4$ and $\mathrm{B} .2 .5$ to second order in perturbation theory. This gives

$$
\begin{aligned}
\Delta \lambda_{+}^{L}= & -\frac{2}{3} g \hat{F}_{k i}^{L} \grave{F}_{L}^{k j} \xi^{i} \xi_{j} T_{[0]}^{(1)} \overline{T_{[0]}^{(-1)}} \\
& -\frac{g}{12} \hat{F}_{k i}^{R} \grave{F}_{R}^{k j} \xi^{i} \xi_{j} T_{[0]}^{(0)} \overline{T_{[0]}^{(0)}} \\
& -\frac{1}{4}\left(\hat{F}_{k i}^{L} \hat{F}_{R}^{k j} \xi^{i} \xi_{j}+\epsilon_{i j k l} \xi^{i} \xi_{a} \hat{F}_{L}^{a j} \hat{F}_{R}^{k l}\right) T_{[0]}^{(0)} \overline{T_{[0]}^{(0)}} \\
& -\frac{1}{4}\left(\hat{F}_{k i}^{L} \hat{F}_{L}^{k j}+\hat{F}_{k i}^{R} \hat{F}_{L}^{k j}\right) \xi^{i} \xi_{j} \frac{T_{[0]}^{(0)} T_{[0]}^{(0)} \overline{T_{[0]}^{(-1)} T_{[0]}^{(0)}}}{T_{[0]}^{(0)} \overline{T_{[0]}^{(-1)}}-T_{[0]}^{(-1)} \overline{T_{[0]}^{(0)}}} \\
& -\frac{1}{2} \hat{F}_{k i}^{L} \hat{F}_{R}^{k j} \xi^{i} \xi_{j} \frac{T_{[0]}^{(-1)} T_{[0]}^{(0)} \overline{T_{[0]}^{(0)}} T_{[0]}^{(0)}}{T_{[0]}^{(0)}} \overline{T_{[0]}^{(-1)}}-T_{[0]}^{(-1)} \overline{T_{[0]}^{(0)}} \\
& -\frac{1}{4} \epsilon_{i j k l} \xi^{i} \xi_{a} \hat{F}_{R}^{a j} \hat{F}_{L}^{k l} \frac{T_{[0]}^{(0)} T_{[0]}^{(0)} \overline{T_{[0]}^{(-1)} T_{[0]}^{(0)}}}{T_{[0]}^{(0)} \overline{T_{[0]}^{(-1)}}-T_{[0]}^{(-1)} \overline{T_{[0]}^{(0)}}} \\
& -\frac{1}{4} \epsilon_{i j k l} \xi^{i} \xi_{a} \hat{F}_{L}^{a j} \hat{F}_{R}^{k l} \frac{T_{[0]}^{(-1)} \frac{T_{[0]}^{(0)} \overline{T_{[0]}^{(0)} T_{[0]}^{(0)}}}{T_{[0]}^{(0)} \overline{T_{[0]}^{(-1)}}-T_{[0]}^{(-1)} \overline{T_{[0]}^{(0)}}}}{}
\end{aligned}
$$

More precisely, the contributions $(\mathrm{B} .5 .6)$ and $(\mathrm{B}$.5.7) are obtained by a first order perturbation calculation for $\Delta A=\Delta P(x, y) P(y, x)+P(x, y) \Delta P(y, x)$ with $\Delta P$ being quadratic in the field strength. The terms (B.5.8) arises in a first order perturbation calculation for $\Delta A=\Delta P(x, y) \Delta P(y, x)$ with $\Delta P$ being linear in the field strength. The remaining contributions (B.5.9) - B.5.12) are the result of a second order perturbation calculation for $\Delta A=\Delta P(x, y) P(y, x)+P(x, y) \Delta P(y, x)$ with $\Delta P$ being linear in the field strength.

Now Lemma 4.5 .3 follows by a straightforward calculation using 4.4.6). It is worth noting that the contribution $($ B.5.9 can be written as

$$
i c \lambda_{+} \quad \text { with } \quad c=\frac{i}{36}\left(\hat{F}_{k i}^{L} \hat{F}_{L}^{k j}+\hat{F}_{k i}^{R} \hat{F}_{L}^{k j}\right) \xi^{i} \xi_{j} \frac{T_{[0]}^{(0)} \overline{T_{[0]}^{(0)}}}{T_{[0]}^{(0)} \overline{T_{[0]}^{(-1)}}-T_{[0]}^{(-1)} \overline{T_{[0]}^{(0)}}} \in \mathbb{R}
$$

and $\lambda_{+}$as in (3.6.5). Hence this contribution changes the eigenvalue only by a phase. As a consequence, it drops out of (4.4.6). This explains why the formula in Lemma 4.5.3 does not involve contributions $\sim \hat{F}_{L} \hat{F}_{L}$ or $\sim \hat{F}_{R} \hat{F}_{R}$ in which both field tensors have the same chirality. 


\section{APPENDIX C}

\section{Ruling out the Local Axial Transformation}

We saw in $\$ 3.7 .8$ that the local axial transformation yields a shear contribution to the closed chain which is of degree four on the light cone (see $(3.7 .42)$ and $(3.7 .43)$ ). The resulting contribution $\kappa$ to the eigenvalues $\lambda^{L / R}$ violates the EL equations (see (3.7.46) and Figure 3.2). We now analyze the same mechanism for the general local transformation (3.7.37). We will find that only a very restrictive class of local transformations respects the EL equations. In particular, this class involves does not include local axial transformations.

We first observe that the shear contributions appear only if the two factors $\xi$ and $\bar{\xi}$ contained in $P(x, y)$ and $P(y, x)$ are not contracted to each other (because otherwise we get a factor $\langle\xi, \bar{\xi}\rangle$ which decreases the degree on the light cone; see the calculation (3.6.3)(3.6.5)). Therefore, the factors $\xi$ and $\bar{\xi}$ are contracted to the local transformation. Thus our task is to analyze how the operator $\not$ (and similarly $\bar{\not}$ ) is affected by a local transformation. Moreover, to the leading degree on the light cone, it suffices to fix a space-time point $x$ and to consider the transformation (3.7.37) in the limiting case $U(y)=U(x) \equiv U$. Finally, as we are interested in the singularities on the light cone, we may clearly disregard the smooth corrections in (3.7.37). This leads us to consider the transformation

$$
\sharp \rightarrow \dot{U}^{\prime} \grave{U}^{*} \text {. }
$$

As $U$ enters this formula only via $U^{\prime}$ and its adjoint, it is convenient to write the generations in components,

$$
\dot{U}=\left(U_{1}, \ldots, U_{g}\right) .
$$

Then the transformation C.0.1 can be written as

$$
\sharp \rightarrow \sum_{a=1}^{g} U_{a} \sharp U_{a}^{*} .
$$

In the formalism of the continuum limit, the vector $\xi$ is null on the light cone, i.e.

$$
\operatorname{Tr}\left(\dot{\phi}^{2}\right)=4\langle\xi, \xi\rangle=(\operatorname{deg}<0) .
$$

Likewise, a general lightlike vector $v$ satisfies the relation $\operatorname{Tr} \psi^{2}=0$. The next proposition specifies under which assumptions on $U$ this relation is preserved by the transformation C.0.1.

Proposition C.0.1. Assume that for every light-like vector v,

$$
\operatorname{Tr}\left(\left(\dot{U} \psi \grave{U}^{*}\right)^{2}\right)=0 \text {. }
$$

Then for all $a, b \in\{1, \ldots, g\}$, there are real parameters $\alpha$ and $\beta$ such that

$$
U_{a}^{*} U_{b}=\alpha \mathbb{1}+\beta \Gamma .
$$


Under the additional assumption that one of the operators $U_{a}$ invertible, there are complex parameters $\alpha_{1}, \ldots, \alpha_{g}$ and $\beta_{1}, \ldots, \beta_{g}$ as well as an invertible linear mapping $A$ on the spinors at $x$ such that

$$
U_{a}=A\left(\alpha_{a}+\beta_{a} \Gamma\right)
$$

For the proof we need a preparatory lemma. We denote the space of four-component spinors at a given space-time point $x$ by $(V, \prec . \mid . \succ$ ) (where $\prec . \mid . \succ$ again denotes the spin scalar product $\left.\prec \phi \mid \psi \succ=\phi^{\dagger} \gamma^{0} \psi\right)$. We say that a linear operator $B$ on $V$ is positive if

$$
\prec \psi \mid B \psi \succ \geq 0 \quad \text { for all } \psi \in V .
$$

Lemma C.0.2. For every positive linear operator $B$ on $V$ the following implication holds:

$$
\operatorname{Tr}\left(B^{2}\right)=0 \quad \Longrightarrow \quad B^{2}=0 .
$$

Proof. According to [F10, Lemma 4.2], the zeros of the characteristic polynomial of $B$ are all real. Hence the assumption $\operatorname{Tr}\left(B^{2}\right)=0$ implies that $B$ is nilpotent. Thus it remains to show that the Jordan chains of $B$ have length at most two.

Let us assume conversely that $B$ has a Jordan chain of length three. Then there is a spinor basis $\left(\mathfrak{f}_{\alpha}\right)_{\alpha=1, \ldots, 4}$ in which $B$ has the matrix representation

$$
B=\left(\begin{array}{llll}
0 & 1 & 0 & 0 \\
0 & 0 & 1 & 0 \\
0 & 0 & 0 & 0 \\
0 & 0 & 0 & 0
\end{array}\right)
$$

The positivity of $B$ clearly implies that $B$ is symmetric with respect to the spin scalar product. An elementary consideration shows that by a suitable change of basis which respects (C.0.6), one can arrange that the signature matrix $S$ defined by $S_{\alpha \beta}=\prec f_{\alpha} \mid f_{\beta} \succ$ has the form

$$
S= \pm\left(\begin{array}{cccc}
0 & 0 & 1 & 0 \\
0 & 1 & 0 & 0 \\
1 & 0 & 0 & 0 \\
0 & 0 & 0 & -1
\end{array}\right)
$$

(for details see the proof of Lemma 4.4 in $[\mathbf{F 1 3}$ ). A direct computation shows that the matrix $S B$ has a negative eigenvalue, in contradiction to the positivity of $B$.

In the case that $B$ has a Jordan chain of length four, we repeat the last argument for the matrices

$$
B=\left(\begin{array}{llll}
0 & 1 & 0 & 0 \\
0 & 0 & 1 & 0 \\
0 & 0 & 0 & 1 \\
0 & 0 & 0 & 0
\end{array}\right) \quad \text { and } \quad S= \pm\left(\begin{array}{cccc}
0 & 0 & 0 & 1 \\
0 & 0 & 1 & 0 \\
0 & 1 & 0 & 0 \\
1 & 0 & 0 & 0
\end{array}\right)
$$

This concludes the proof.

Proof of Proposition C.0.1. Choosing a future-directed lightlike vector $v$, the bilinear form $\prec . \mid \psi \cdot \succ$ is positive semi-definite. As a consequence, the product $\dot{U} \psi \grave{U}^{*}$ is a positive operator on $(V, \prec . \mid . \succ)$. In view of the assumption (C.0.3), we can apply Lemma C.0.2 to conclude that

$$
\left(\dot{U} \not \grave{U}^{*}\right)^{2}=0
$$


Assume that $\prec \psi \mid \psi \psi \succ$ vanishes for a given spinor $\psi$. Then $\psi$ lies in the null space of the inner product $\prec . \mid \psi \cdot \succ$. Since the spin scalar product is non-degenerate, it follows that $\psi \psi=0$. We conclude that the following implication holds:

$$
\prec \psi \mid \psi \psi \succ=0 \quad \Longrightarrow \quad \psi \psi=0 \text {. }
$$

We now apply this implication to (C.0.7). Choosing $\psi=\grave{U}^{*} \dot{U} \psi \grave{U}^{*} \chi$ with an arbitrary spinor $\chi$, the equation (C.0.7) implies that $\prec \psi \mid \psi \psi \succ=0$. Applying (C.0.8) and using that $\chi$ is arbitrary, we obtain the equation

$$
\psi \grave{U}^{*} \grave{U}_{\psi} \grave{U}^{*}=0 \text {. }
$$

Taking the adjoint of this equation and choosing $\psi=\psi \grave{U}^{*} \grave{U}^{\prime} \chi$, we can again apply (C.0.8) to obtain

$$
\psi \grave{U}^{*} \grave{U}^{\prime} \psi=0 \text {. }
$$

Using the notation C.0.2, we obtain for all $a, b \in\{1, \ldots, g\}$ the equations

$$
\psi U_{a}^{*} U_{b} \psi=0 \text {. }
$$

Multiplying from the left and right by chiral projectors, one sees that the even and odd components of $U_{a} U_{b}^{*}$ can be treated separately. A short calculation using the anticommutation relations shows that the operators $U_{a}^{*} U_{b}$ can have only scalar and pseudoscalar components. This proves (C.0.4).

To prove (C.0.5), we assume that $U_{a}$ is invertible. We set $A=\left(U_{a}^{*}\right)^{-1}$. Then for every $b \in\{1, \ldots, g\}$, we can apply C.0.4 to obtain

$$
U_{b}=\left(A U_{a}^{*}\right) U_{b}=A(\alpha \mathbb{1}+\beta \Gamma) .
$$

Setting $\alpha_{b}=\alpha$ and $\beta_{b}=\beta$ gives the claim.

We now work out the consequences of Proposition C.0.1. We first note that in the vacuum, $U(x)$ is the identity, and thus $U_{a}=\mathbb{1}$ for all $a$. Therefore, we can assume that, at least for a weakly interacting system, one of the matrices $U_{a}$ is invertible. Thus Proposition C.0.1 implies that if the condition C.0.3 holds, then $U(x)$ must have the representation (C.0.2) and C.0.5).

The representation C.0.5 means in words that the local transformation consists of the transformation $A$ which acts trivially on the generation index, and of additional scalar and pseudoscalar transformations acting on the components of the generation index. If we start with a local axial transformation (3.7.27) and add other potentials (like scalar, pseudoscalar, vector or bilinear components) plus possibly higher order contributions, we will never get a transformation of the form C.0.5. Namely, it was essential for compensating the logarithmic poles that the local axial transformation acts non-trivially on the generation index, contrary to C.0.5). We conclude that local transformations of the form C.0.2 and C.0.5 do not include local axial transformations.

This consideration also shows that if a local axial transformation is present, then the condition (C.0.3) is necessarily violated at some space-time point $x$ for some lightlike vector $v$. The leading contribution to the closed chain (3.6.3) transforms to

$$
A_{x y} \asymp \frac{1}{4} \dot{U}(x) \dot{\sharp} \grave{U}(y) \dot{U}(y) \bar{\xi} \grave{U}(x)\left|T_{[0]}^{(-1)}\right|^{2} .
$$

Choosing a point $y$ in a small neighborhood of $x$ such that the difference vector $\xi=y-x$ is a multiple of $v$, we obtain a shear contribution to the closed chain. This shows that the formalism of the continuum limit is no longer valid. In particular, we get contributions 
to the eigenvalues of the closed chain which are more singular on the light cone than in the vacuum.

The above argument does not necessarily imply that the EL equations are violated. Namely, it leaves the possibility that the gauge phases enter the shear contributions in agreement with (3.6.17), in which case the argument of Figure 3.2 would not apply and the eigenvalues $\lambda_{ \pm}^{L / R}$ would still have the same absolute value. In order to rule out this case, we now take into account the gauge phases and restrict attention to those contributions to the closed chain for which these phases drop out.

We first point out that the gauge phases enter the factors $\xi$ in $($ C.0.9 only via the transformation (3.6.17). The local transformation, however, cannot involve any gauge phases, because the gauge phases are obtained as integrals along a straight line joining two space-time points $x$ and $y$ (see (3.6.18) ) and thus cannot be encoded in a function $U(x)$ of one variable. Therefore, to select the contributions to the closed chain which do not involve gauge phases, we simply take those contributions to C.0.9 for which both factors $\xi$ have the same chirality,

$$
A_{x y} \asymp \frac{1}{4} \sum_{c=L, R} \dot{U}(x) \chi_{c} \nLeftarrow \grave{\dot{U}}(y) \dot{U}(y) \chi_{c} \bar{\phi} \grave{U}(x)\left|T_{[0]}^{(-1)}\right|^{2} .
$$

Again considering the limiting case $y=x$, we are led to the condition that the equation

$$
\sum_{c=L, R} \operatorname{Tr}\left(\left(\dot{U} \chi_{c} \psi \grave{U}^{*}\right)^{2}\right)=0
$$

should hold for every lightlike vector $v$. As the operator $U^{\prime} \chi_{c} \psi \grave{U^{*}}$ is positive, the trace of its square is necessarily positive. Hence both summands must vanish separately,

$$
\operatorname{Tr}\left(\left(\dot{U} \chi_{c} \psi \grave{U}^{*}\right)^{2}\right) \quad \text { for } c=L \text { or } R \text {. }
$$

We now combine the chiral projectors with the local transformation to obtain (for example in the case $c=L)$,

$$
0=\operatorname{Tr}\left(\left(\dot{U} \chi_{L} \psi \grave{U}^{*}\right)^{2}\right)=\operatorname{Tr}\left(\left(\left(\dot{U}^{\prime} \chi_{L}\right) \psi\left(\dot{U} \chi_{L}\right)^{*}\right)^{2}\right) .
$$

For the new local transformation $U^{\prime} \chi_{L}$, the components $U_{a} \chi_{L}$ are clearly not invertible, so that C.0.5 no longer applies. But we can still apply the first part of Proposition C.0.1 to obtain

$$
\chi_{R} U_{a}^{*} U_{b} \chi_{L}=\alpha \mathbb{1}+\beta \Gamma .
$$

This equation is satisfied if and only if the product $U_{a}^{*} U_{b}$ is even, i.e.

$$
\left[\Gamma, U_{a}^{*} U_{b}\right]=0 \text {. }
$$

This condition is considerably weaker than the representation C.0.5). In particular, it is indeed fulfilled for special choices of $U_{a}$ which do involve axial fields. But we can nevertheless rule out local axial transformations in a perturbation expansion around the vacuum. Namely, inserting the perturbation ansatz

$$
U_{a}=\mathbb{1}+i E_{a}+\mathcal{O}\left(E_{a}^{2}\right),
$$

we obtain the condition

$$
\left[\Gamma, E_{a}^{*}-E_{b}\right]=0 .
$$

Thus the odd contribution to $E_{a}$ must be of the form

$$
E_{a}=i(\psi+\Gamma \psi)+(\text { even }) \quad \text { for all } a=1, \ldots, g
$$


with two vector fields $u$ and $v$. In particular, the axial transformation acts trivially on the generation index, making it impossible to compensate the logarithmic poles on the light cone. 



\section{APPENDIX D}

\section{Resummation of the Current and Mass Terms at the Origin}

As pointed out in $\$ 3.4 .4$, the distribution $T_{a}$ is not a power series in $a$, and thus it cannot be expanded in a Taylor series around $a=0$ (see 3.4 .10 and the explanation thereafter). The method of subtracting suitable counter terms (3.4.11) has the shortcoming that the subsequent calculations are valid only modulo smooth contributions on the light cone. This method is suitable for analyzing the singularities on the light cone, but it is not sufficient when smooth contributions to the fermionic projector become important (cf. the discussion after (3.5.11) and the beginning of 33.8.1). We now present a convenient method for computing the smooth contributions to the fermionic projector. Our method is based on the resummation technique developed in [F5, Section 4] and is outlined as follows. We first perform the mass expansion not around zero mass, but around a given mass parameter $a>0$. Then, according to 3.4 .10 , the distribution $T_{a}$ is smooth in $a$, and we may set

$$
T_{a}^{(n)}=\left(\frac{d}{d a}\right)^{n} T_{a}
$$

Adapting the method of the light-cone expansion, we can express any Feynman tree diagram as a sum of terms of the form

$$
P^{\text {sea }}(x, y)=\sum_{n=-1}^{\infty} \sum_{k} m^{p_{k}} \text { (phase-inserted nested line integrals) } \times T_{a}^{(n)}(x, y),
$$

where for each $n$, the $k$-sum is finite, whereas the $n$-sum is to be understood as a formal power series. Note that, in contrast to the series in (3.4.14), the infinite sum in (D.0.2) is not a light-cone expansion in the sense of Definition 3.4.1 because the distributions $T_{a}^{(n)}$ all involve smooth contributions and are thus only of the order $\mathcal{O}\left((y-x)^{0}\right)$. We proceed by partially carrying out the series in (D.0.2) to obtain explicit smooth contributions on the light cone. After this resummation has been performed, we recover (3.4.14), but now with an explicit formula for $\tilde{P}^{\text {le }}(x, y)$.

For simplicity, we develop the method only for the contributions to the fermionic projector needed in this book: the vector and axial components of the fermionic projector perturbed by chiral potentials to first order. But the method generalizes in a straightforward way to arbitrary Feynman tree diagrams. Furthermore, we begin by considering a single Dirac sea (the generalization to several generalizations will then be straightforward; see the proof of Lemma 3.8.1 below). We thus consider the contribution to the fermionic projector

$$
\Delta P=-s_{m}\left(\chi_{L} A_{R}+\chi_{R} A_{L}\right) t_{m}-t_{m}\left(\chi_{L} A_{R}+\chi_{R} A_{L}\right) s_{m}
$$

with the spectral projector $t_{m}$ and the Green's function $s_{m}$ as in (3.8.22). In order to concentrate on the vector and axial components, we want to consider the expression $\operatorname{Tr}\left(\not \chi_{L} \Delta P(x, y)\right)$, being a well-defined distribution. The singular part of this distribution on the light cone can be computed by inserting the formulas of the light-cone 
expansion (B.2.2) - B.2.20) and using the contraction rule

$$
\xi^{2} T^{(n)}(x, y)=-4 n T^{(n+1)}(x, y)+(\text { smooth contribution }), \quad n \in\{-1,0\}
$$

(which is immediately verified from the explicit formulas 3.4.10 (3.4.13)). We thus obtain

$$
\begin{aligned}
& \frac{1}{2} \operatorname{Tr}\left(\not \chi_{L} \Delta P(x, y)\right)=2 \int_{x}^{y} \xi_{k} A_{L}^{k} T_{[0]}^{(0)}(x, y) \\
& \quad-2 \int_{x}^{y}\left(\alpha-\alpha^{2}\right) \xi_{k} j_{L}^{k} T_{[0]}^{(1)}(x, y)+m^{2} \int_{x}^{y} \xi_{k}\left(A_{L}^{k}-A_{R}^{k}\right) T_{[2]}^{(1)}(x, y) \\
& \quad+\xi^{k} f_{k}(x, y)+(\operatorname{deg}<0),
\end{aligned}
$$

where we added subscripts [.] in order to indicate how these factors are to be regularized (although we do not need a regularization at this point), and $f_{k}(x, y)$ are yet undetermined smooth functions (clearly, the summand in (D.0.5) is the gauge term as discussed in \$3.6.2. whereas the summands in (D.0.6) correspond to the current and mass terms considered in 3.7 .1$)$. Our goal is to compute the functions $f_{k}(x, y)$ at the origin $x=y$.

Our first step is to perform a mass expansion of the Feynman diagram (D.0.3) around a given $a \neq 0$. To this end, we need suitable calculation rules which are derived in the next lemma.

Lemma D.0.1. The distributions $T_{a}^{(n)}$, D.0.1), satisfy for all $n \in \mathbb{N}_{0}$ the calculation rules

$$
\begin{aligned}
\left(-\square_{x}-a\right) T_{a}^{(n)}(x, y) & =n T_{a}^{(n-1)}(x, y) \\
\frac{\partial}{\partial x^{k}} T_{a}^{(n+1)}(x, y) & =\frac{1}{2} \xi_{k} T_{a}^{(n)}(x, y) \\
\xi^{2} T_{a}^{(n)}(x, y) & =-4 n T_{a}^{(n+1)}(x, y)-4 a T_{a}^{(n+2)}(x, y) .
\end{aligned}
$$

In the case $n=-1$, the rule (D.0.9) can be used to define the distribution $\xi_{k} T_{a}^{(-1)}$. Using this definition, the rule (D.0.10) also holds in the case $n=-1$.

Proof. The relations (D.0.8) and (D.0.9) were already derived in [F6] (see [F6, eqs (3.5) and (3.6)]). For self-consistency we here repeat the proof. Clearly, $T_{a}$ is a distributional solution of the Klein-Gordon equation,

$$
\left(-\square_{x}-a\right) T_{a}(x, y)=0 \text {. }
$$

Differentiating $n$ times with respect to $a$ gives (D.0.8). Next, we differentiate the identity in momentum space

$$
T_{a}(p)=\delta\left(p^{2}-a\right) \Theta\left(-p^{0}\right)
$$

with respect to $p_{k}$ to obtain

$$
\frac{\partial}{\partial p^{k}} T_{a}(p)=2 p_{k} T_{a}^{(1)}(p)
$$

Using that differentiation in momentum space corresponds to multiplication in position space and vice versa, we find

$$
\xi_{k} T_{a}(x, y)=2 \frac{\partial}{\partial x^{k}} T_{a}^{(1)}(x, y) .
$$

Differentiating $n$ times with respect to $a$ gives D.0.9). 
To derive (D.0.10), we first combine D.0.9 with the product rule to obtain

$$
\square_{x} T_{a}^{(1)}=\partial_{x}^{k}\left(\frac{1}{2} \xi_{k} T_{a}^{(0)}\right)=-2 T_{a}^{(0)}+\frac{1}{2} \xi_{k} \partial_{x}^{k} T_{a}^{(0)}=-2 T_{a}^{(0)}+\frac{1}{4} \xi^{2} T_{a}^{(-1)} .
$$

On the other hand, we know from (D.0.8) that

$$
\square_{x} T_{a}^{(1)}=-T_{a}^{(0)}-a T_{a}^{(1)} .
$$

Solving for $\xi^{2} T_{a}^{(-1)}$, we obtain

$$
\xi^{2} T_{a}^{(-1)}=4 T_{a}^{(0)}-4 a T_{a}^{(1)} .
$$

We finally differentiate this relation $n+1$ times with respect to $a$, giving (D.0.10).

Alternatively, this lemma could be proved working with the series representation 3.4.10). We also remark that in the case $n=-1$, the rule (D.0.9) is consistent with our earlier definition (3.4.13).

Using the relations (D.0.8) and (D.0.9), the mass expansion of the first order Feynman diagram (D.0.3) was first performed in [F5] (see [F5, Theorem 3.3], where the mass expansion is referred to as the "formal light-cone expansion"). More generally, for the advanced and retarded Green's function, we have the expansion

$$
\begin{aligned}
& \left(S_{a}^{(l)} V S_{a}^{(r)}\right)(x, y) \\
& \quad=\sum_{n=0}^{\infty} \frac{1}{n !} \int_{0}^{1} \alpha^{l}(1-\alpha)^{r}\left(\alpha-\alpha^{2}\right)^{n}\left(\square^{n} V\right)_{\mid \alpha y+(1-\alpha) x} d \alpha S_{a}^{(n+l+r+1)}(x, y),
\end{aligned}
$$

which is proved exactly as in the case $a=0$ (see [F6, Lemma 2.1], [F7, Lemma 2.5.2] or Lemma 2.2.2). The residual argument (cf. [F6, Section 3.1]) also generalizes immediately to the case $a>0$, making it possible to extend (D.0.11) to the so-called residual fermionic projector (the non-residual part of the fermionic projector is precisely the non-causal high energy contribution, which can be analyzed as indicated in $\$ 3.8 .3$ for details see $\mathbf{F 6}$, Section 3.2]). Applied to our problem, we obtain the expansion

$$
\left(S_{a} V T_{a}+T_{a} V S_{a}\right)(x, y)=\sum_{n=0}^{\infty} \frac{1}{n !} \int_{x}^{y}\left(\alpha-\alpha^{2}\right)^{n}\left(\square^{n} V\right) d \alpha T_{a}^{(n+1)}(x, y),
$$

where for the line integrals we again used the short notation (B.2.1), and $S_{a}$ is the symmetric Green's function (3.8.17). The mass expansion of (D.0.3) is now readily obtained by applying the differential operators $(i \not \partial+m)$ and simplifying the Dirac matrices using the rules (D.0.9) and (D.0.10). Multiplying by $\sharp \chi_{L}$ and taking the trace, a straightforward calculation using again (D.0.10) yields

$$
\begin{aligned}
\frac{1}{2} \operatorname{Tr}\left(\$ \chi_{L} P(x, y)\right) \asymp 2 \sum_{n=0}^{\infty} \frac{1}{n !} \int_{x}^{y}\left(\alpha-\alpha^{2}\right)^{n} \xi_{k}\left(\square^{n} A_{L}^{k}\right) T_{m^{2}}^{(n)}(x, y) \\
\quad-2 \sum_{n=0}^{\infty} \frac{1}{n !} \int_{x}^{y}(2 \alpha-1)\left(\alpha-\alpha^{2}\right)^{n}\left(\square^{n} \partial_{i} A_{L}^{i}\right) T_{m^{2}}^{(n+1)}(x, y) \\
\quad-m^{2} \sum_{n=0}^{\infty} \frac{1}{n !} \int_{x}^{y}\left(\alpha-\alpha^{2}\right)^{n} \xi_{k}\left(\square^{n} A_{L}^{k}+\square^{n} A_{R}^{k}\right) T_{m^{2}}^{(n+1)}(x, y) \\
\quad+2 m^{2} \sum_{n=0}^{\infty} \frac{1}{n !} \int_{x}^{y}(2 \alpha-1)\left(\alpha-\alpha^{2}\right)^{n}\left(\square^{n} \partial_{i} A_{L}^{i}\right) T_{m^{2}}^{(n+2)}(x, y)
\end{aligned}
$$


(this result was again obtained with the help of class_commute; see page 394). Integrating the line integrals by parts,

$$
\int_{x}^{y}(2 \alpha-1)\left(\alpha-\alpha^{2}\right)^{n}\left(\square^{n} \partial_{i} A_{L}^{i}\right)=\frac{1}{n+1} \int_{x}^{y}\left(\alpha-\alpha^{2}\right)^{n+1} \xi^{k}\left(\square^{n} \partial_{i k} A_{L}^{i}\right),
$$

the divergence terms can be rewritten to recover the chiral currents. In particular, in the case $A_{L}=A_{R}$ of a vector potential, one immediately verifies that D.0.13)-D.0.16 has the correct behavior under gauge transformations. Furthermore, one readily sees that the expansion (D.0.13) $-(\overline{D .0 .16})$ is compatible with $(\overline{D .0 .5})-(\overline{D .0 .7})$ in the sense that the singularities on the light cone coincide. We now subtract (D.0.13)-(D.0.16) from (D.0.5)(D.0.7) and solve for $\xi^{k} f_{k}(x, y)$.

In order to compute $f_{k}(x, x)$, it suffices to take into account the constant counter term in (3.4.11), as can be done by the replacement

$$
T_{a}(x, y) \longrightarrow T_{a}^{\mathrm{reg}}+N(a) \quad \text { with } \quad N(a):=\frac{1}{32 \pi^{3}} a \log |a|,
$$

and similarly for the $a$-derivatives. Moreover, in the line integrals we may set $x=y$. In order to keep the formulas simple, we also specialize to the situation where only the axial potential in (3.6.16) is present. We thus obtain

$$
\begin{aligned}
& f^{k}(x, x)=2 \sum_{n=0}^{\infty} \frac{1}{n !} \int_{0}^{1}\left(\alpha-\alpha^{2}\right)^{n}\left(\square^{n} A_{\mathrm{a}}^{k}(x)\right) N^{(n)}\left(m^{2}\right) d \alpha \\
& \quad-2 \sum_{n=0}^{\infty} \frac{1}{(n+1) !} \int_{0}^{1}\left(\alpha-\alpha^{2}\right)^{n+1}\left(\square^{n} \partial_{i}^{k} A_{\mathrm{a}}^{i}(x)\right)\left(N^{(n+1)}\left(m^{2}\right)-m^{2} N^{(n+2)}\left(m^{2}\right)\right) d \alpha .
\end{aligned}
$$

By linearity, it suffices to consider the case that $A_{\mathrm{a}}$ is a plane wave of momentum $q$,

$$
A_{\mathrm{a}}^{k}(z)=\hat{A}_{\mathrm{a}}^{k} e^{-i q(z-x)} .
$$

Then the above sums are recognized as Taylor series,

$$
\begin{aligned}
\sum_{n=0}^{\infty} \frac{\lambda^{n}}{n !} N^{(n)}\left(m^{2}\right) & =N^{(0)}\left(m^{2}+\lambda\right) \\
\sum_{n=0}^{\infty} \frac{\lambda^{n}}{(n+1) !} N^{(n+\ell)}\left(m^{2}\right) & =\frac{N^{(\ell-1)}\left(m^{2}+\lambda\right)-N^{(\ell-1)}\left(m^{2}\right)}{\lambda}
\end{aligned}
$$

where we introduced the abbreviation $\lambda=-\left(\alpha-\alpha^{2}\right) q^{2}$ (the second equation in D.0.18) can be derived from the first by integration over $\lambda)$. We thus obtain

$$
\begin{aligned}
f^{k}(x, x)= & 2 \int_{0}^{1} A_{\mathrm{a}}^{k}(x) N\left(m^{2}+\lambda\right) d \alpha \\
& -\left.\frac{2}{\lambda} \int_{0}^{1}\left(\alpha-\alpha^{2}\right) \partial_{i}^{k} A_{\mathrm{a}}^{i}(x)\left(N^{(0)}-m^{2} N^{(1)}\right)\left(m^{2}+\nu\right)\right|_{\nu=0} ^{\nu=\lambda} d \alpha \\
= & \frac{1}{16 \pi^{3}} \int_{0}^{1} A_{\mathrm{a}}^{k}(x)\left(m^{2}+\lambda\right) \log \left|m^{2}+\lambda\right| d \alpha \\
& -\frac{1}{16 \pi^{3}} \int_{0}^{1}\left(\alpha-\alpha^{2}\right) \partial_{i}^{k} A_{\mathrm{a}}^{i}(x) \log \left|m^{2}+\lambda\right| d \alpha
\end{aligned}
$$


where in the last step we substituted the explicit formula for $N(a)$ in (D.0.17). In order to rewrite the last result in terms of the axial current, we use the identity $\lambda A_{\mathrm{a}}^{k}=\left(\alpha-\alpha^{2}\right) \square A_{\mathrm{a}}^{k}$ to conclude

$$
\begin{aligned}
f^{k}(x, x)= & \frac{m^{2}}{16 \pi^{3}} A_{\mathrm{a}}^{k}(x) \int_{0}^{1} \log \left|m^{2}-\left(\alpha-\alpha^{2}\right) q^{2}\right| d \alpha \\
& -\frac{1}{16 \pi^{3}} j_{\mathrm{a}}^{k}(x) \int_{0}^{1}\left(\alpha-\alpha^{2}\right) \log \left|m^{2}-\left(\alpha-\alpha^{2}\right) q^{2}\right| d \alpha .
\end{aligned}
$$

Substituting this result into the light-cone expansion (D.0.5)-(D.0.7) evaluated at the origin and using that $\int_{0}^{1}\left(\alpha-\alpha^{2}\right)=1 / 6$, one sees that the term $\xi_{k} f^{k}(x, x)$ can be incorporated into the formulas of the light-cone expansion by the replacements

$$
\left.\begin{array}{l}
T_{[0]}^{(1)} \rightarrow T_{[0]}^{(1)}+\frac{\log \left(m^{2}\right)}{32 \pi^{3}}+\frac{6}{32 \pi^{3}} \int_{0}^{1}\left(\alpha-\alpha^{2}\right) \log \left|1-\left(\alpha-\alpha^{2}\right) \frac{q^{2}}{m^{2}}\right| d \alpha \\
T_{[2]}^{(1)} \rightarrow T_{[2]}^{(1)}+\frac{\log \left(m^{2}\right)}{32 \pi^{3}}+\frac{1}{32 \pi^{3}} \int_{0}^{1} \log \left|1-\left(\alpha-\alpha^{2}\right) \frac{q^{2}}{m^{2}}\right| d \alpha .
\end{array}\right\}
$$

To clarify the above construction, we point out that the radius of convergence of the Taylor series in (D.0.18) is $|\lambda|=m^{2}$. Thus in the case $|\lambda|>m^{2}$, these series do not converge absolutely, so that (D.0.18) can be understood only on the level of formal Taylor series. For the reader who feels uncomfortable with formal power series, we remark that all formal expansions could be avoided by regularizing the distribution $T_{a}$ according to (3.4.11) before performing the light-cone expansion, making a later resummation unnecessary. However, this method seems technically complicated and has not yet been carried out (see also the discussions in [F6, Section 3.3] and after (3.8.21)). In this book, we will be content with the formal character of (D.0.18).

We are now ready to prove the main result of this appendix.

Proof of Lemma 3.8.1. We return to the situation with three generations and a general axial potential $A_{\mathrm{a}}(z)$. As the axial potential is diagonal on the generation index, the auxiliary fermionic projector splits into the direct sum of three fermionic projectors, corresponding to the Dirac seas of masses $m_{1}, m_{2}$, and $m_{3}$. Thus the sectorial projection (3.4.3) reduces to a sum over the generation index. Decomposing $A_{\mathrm{a}}$ into Fourier modes,

$$
A_{\mathrm{a}}(z)=\int_{\mathscr{M}} \frac{d^{4} z}{(2 \pi)^{4}} \hat{A}_{\mathrm{a}}(q) e^{-i q(z-x)}
$$

for every $\hat{A}_{\mathrm{a}}(q)$ and for every generation we may apply the replacement rules (D.0.19). Rewriting the multiplication in momentum space by a convolution in position space gives the formulas (3.8.11)-(3.8.14).

In order to check the prefactors, it is convenient to verify whether the arguments of the logarithms can be combined to give dimensionless quantities. This is indeed the case with the expressions

$$
\begin{gathered}
\log \left|\xi^{2}\right|+\frac{1}{3} \sum_{\beta=1}^{3} \log \left(m_{\beta}^{2}\right)=\frac{1}{3} \sum_{\beta=1}^{3} \log \left|m_{\beta}^{2} \xi^{2}\right| \\
\log \left|\xi^{2}\right|+\frac{1}{m^{2} \dot{Y} \grave{Y}} \sum_{\beta=1}^{3} m_{\beta}^{2} \log \left(m_{\beta}^{2}\right)=\frac{1}{m^{2} \dot{Y} \grave{Y}} \sum_{\beta=1}^{3} m_{\beta}^{2} \log \left|m_{\beta}^{2} \xi^{2}\right|
\end{gathered}
$$


explaining the prefactors in 3.8 .11$)$ and $(3.8 .12)$ relative to those in 3.8 .3$)$.

We finally need to verify that the smooth contributions which were disregarded in the formalism of \$3.5.1 really enter the EL equations according to the simple replacement rules (D.0.19). The subtle point is that the contraction rule in the continuum limit (3.5.5) is not the same as the corresponding distributional identity (D.0.4), and this might give rise to additional terms which are not captured by (D.0.19). Fortunately, such additional terms do not appear, as the following consideration shows: To degree four on the light cone, the smooth contributions to $P(x, y)$ enter the EL equations only if the smooth term is contracted with a factor $\sharp$ without generating a factor $\xi^{2}$ (the contributions involving $\xi^{2}$ are of degree three on the light cone). Thus for the smooth contributions, the contraction rule 3.5 .5 is not applied, and therefore it could here be replaced by the simpler distributional identities (D.0.4) and (D.0.10).

We finally carry out the $\alpha$-integrals in $(\mathrm{D} .0 .19)$ in closed form and discuss the result. This result will not be used in this book. But it is nevertheless worth stating, because it gives more explicit information on the structure of the non-causal correction terms.

Lemma D.0.2. The functions $\hat{f}_{[p]}^{\beta}$ defined by 3.8.13 and 3.8.14 can be written as

$$
\hat{f}_{[p]}^{\beta}(q)=\lim _{\varepsilon \searrow 0} g_{[p]}\left(\frac{q^{2}+i \varepsilon}{4 m_{\beta}^{2}}\right)
$$

where the functions $g_{[p]}(z)$ are defined in the upper half plane by

$$
\begin{aligned}
& g_{[0]}(z)=-\frac{3+5 z}{3 z}+\frac{1+z-2 z^{2}}{2 z \sqrt{z(z-1)}}[\log (1-2 z+\sqrt{z(z-1)})-i \pi \Theta(z-1)] \\
& g_{[2]}(z)=-2-\frac{\sqrt{z(z-1)}}{z}[\log (1-2 z+\sqrt{z(z-1)})-i \pi \Theta(z-1)],
\end{aligned}
$$

where the logarithm in the complex plane is cut along the ray $-i \mathbb{R}^{+}$(and $\Theta$ is the Heaviside function, extended continuously to the upper half plane).

Proof. Writing the logarithm of the absolute value for any $x \in \mathbb{R}$ as

$$
\log |1-x|=\lim _{\delta \searrow 0}(\log (1-(x+i \delta))+i \pi \Theta((x+i \delta)-1)),
$$

we obtain the representation D.0.20 with

$$
\begin{aligned}
& g_{[0]}(z)=6 \int_{0}^{1}\left(\alpha-\alpha^{2}\right)\left(\log \left(1-4\left(\alpha-\alpha^{2}\right) z\right)+i \pi \Theta\left(4\left(\alpha-\alpha^{2}\right) z-1\right)\right) d \alpha \\
& g_{[2]}(z)=\int_{0}^{1}\left(\log \left(1-4\left(\alpha-\alpha^{2}\right) z\right)+i \pi \Theta\left(4\left(\alpha-\alpha^{2}\right) z-1\right)\right) d \alpha .
\end{aligned}
$$

It remains to calculate these integrals for $z$ in the upper half plane, thus avoiding the singularities on the real line. The term involving the Heaviside function is readily computed in closed form. Thus it remains to consider for $\ell=0,1$ the integrals

$$
\int_{0}^{1}\left(\alpha-\alpha^{2}\right)^{\ell} \log \left(1-4\left(\alpha-\alpha^{2}\right) z\right) d \alpha=\frac{1}{2} \int_{0}^{1} \log (1-x z)\left\{\left(\frac{x}{4}\right)^{\ell} \frac{1}{\sqrt{1-x}}\right\} d x
$$

where in the last step we transformed to the integration variable $x:=4\left(\alpha-\alpha^{2}\right)$. After computing the indefinite integral of the expression inside the curly brackets, we can integrate by parts. Then the logarithm in the integrand disappears, and the calculation of the integral becomes elementary. 


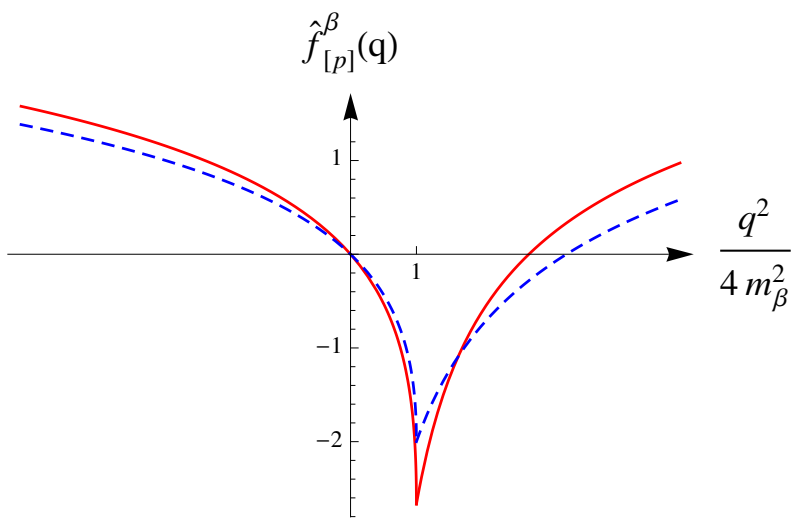

Figure D.1. The functions $\hat{f}_{[0]}^{\beta}$ (red, solid) and $\hat{f}_{[2]}^{\beta}$ (blue, dashed)

In Figure D.1 the functions $\hat{f}_{[0]}^{\beta}$ and $\hat{f}_{[2]}^{\beta}$ are plotted. One sees that these functions attain their minimum if $q^{2}=4 m_{\beta}^{2}$, and for this value of $q^{2}$ the function has a cusp. The asymptotics for large $\left|q^{2}\right|$ is obtained by dropping the summand one in the argument of the logarithm in (3.8.13) and (3.8.14),

$$
\begin{aligned}
& \hat{f}_{[0]}^{\beta}(q) \sim 6 \int_{0}^{1}\left(\alpha-\alpha^{2}\right) \log \left|\left(\alpha-\alpha^{2}\right) \frac{q^{2}}{m_{\beta}^{2}}\right| d \alpha=-\frac{5}{3}+\log \left(\frac{q^{2}}{m_{\beta}^{2}}\right) \\
& \hat{f}_{[2]}^{\beta}(q) \sim \int_{0}^{1} \log \left|\left(\alpha-\alpha^{2}\right) \frac{q^{2}}{m_{\beta}^{2}}\right| d \alpha=-2+\log \left(\frac{q^{2}}{m_{\beta}^{2}}\right),
\end{aligned}
$$

revealing a logarithmic divergence as $q^{2} \rightarrow \pm \infty$. For small momenta, the functions have the asymptotics

$$
\hat{f}_{[0]}^{\beta}(q)=-\frac{q^{2}}{5 m_{\beta}^{2}}+\mathcal{O}\left(q^{4}\right), \quad \hat{f}_{[2]}^{\beta}(q)=-\frac{q^{2}}{6 m_{\beta}^{2}}+\mathcal{O}\left(q^{4}\right),
$$

describing a non-trivial low energy effect. 



\section{APPENDIX E}

\section{The Weight Factors $\rho_{\beta}$}

In [F11] the ansatz for the vacuum (3.3.1) was generalized by introducing so-called weight factors $\rho_{\beta}$ for the Dirac seas,

$$
P(x, y)=\sum_{\beta=1}^{g} \rho_{\beta} \int \frac{d^{4} k}{(2 \pi)^{4}}\left(\not k+m_{\beta}\right) \delta\left(k^{2}-m_{\beta}^{2}\right) \Theta\left(-k^{0}\right) e^{-i k(x-y)} .
$$

This generalization turns out to be useful when considering an action principle for the masses of Dirac particles $[\mathbf{F H}]$; for a physical discussion see [F11, Appendix A]. All the constructions in this book could immediately be extended to the setting with weight factors, as we now explain.

The weight factors are introduced into the auxiliary fermionic projector of the vacuum (3.4.1) by the replacement

$$
\bigoplus_{\beta=1}^{g} \rightarrow \bigoplus_{\beta=1}^{g} \rho_{\beta}
$$

For our systems, the causality compatibility condition (see [F11, eq. (A.1)] or (4.2.48)) does not cause problems, because all our potentials are either diagonal in the generation index, or else they can be described by a microlocal chiral transformation (see $\$ 3.7 .10$, in which case the causality compatibility condition is irrelevant. We conclude that the causal perturbation series as well as the light-cone expansion remain well-defined. The weight factors are taken into account simply by inserting them into the resulting formulas. More precisely, the number of generations is to be replaced by the sum of the weights,

$$
g \rightarrow \sum_{\beta=1}^{g} \rho_{\beta} .
$$

Moreover, the weights must be introduced into the sectorial projections by the replacements

$$
\hat{Y} \rightarrow \sum_{\alpha, \beta=1}^{g} \rho_{\alpha} Y_{\beta}^{\alpha}, \quad \underbrace{\dot{Y} Y \cdots \grave{Y}}_{p \text { factors } Y} \rightarrow \sum_{\alpha, \beta, \gamma_{1}, \ldots, \gamma_{p-1}=1}^{g} \rho_{\alpha} Y_{\gamma_{1}}^{\alpha} \cdots Y_{\gamma_{2}}^{\gamma_{1}} \cdots Y_{\beta}^{\gamma_{p-1}},
$$

or more generally using the rule

$$
\dot{B} \rightarrow \sum_{\beta=1}^{g} \rho_{\beta} B^{\beta}
$$

(to avoid confusion, we note that, due to the causality compatibility condition, the weight factors could just as well be inserted at the last instead of the first summation index). When performing the microlocal chiral transformation, the weight factors must be inserted in the obvious way into (3.7.66). After these straightforward modifications, all our formulas and results remain valid. It seems a promising strategy for the construction of 
realistic physical models to choose the fermion masses and the weight factors according to state stable vacuum configurations as exemplified in $[\mathbf{F H}]$. 
APPENDIX F

\section{The Regularized Causal Perturbation Theory with Neutrinos}

\section{F.1. The General Setting}

For clarity, we begin with a single Dirac sea (i.e. with one direct summand of (4.2.41) or (4.2.54)). Thus without regularization, the vacuum is described as the product of the Fourier integral 4.1.6 with a chiral asymmetry matrix,

$$
P=X t \quad \text { with } t=P_{m} \text { and } X=\mathbb{1}, \chi_{L} \text { or } \chi_{R},
$$

under the constraint that $X=\mathbb{1}$ if $m>0$. We again denote the regularization by an index $\varepsilon$. We always assume that the regularization is homogeneous, so that $P^{\varepsilon}$ is a multiplication operator in momentum space, which sometimes we denote for clarity by $\hat{P}^{\varepsilon}$. If $m>0$, we assume that the regularization satisfies all the conditions in [F7, Chapter 4]; see also the compilation in Section 3.3. In the case $m=0$, we relax the conditions on the shear and allow for general surface states, as explained in 4.2 .3 . In the low-energy regime, $P^{\varepsilon}$ should still be of the form (F.1.1), i.e.

$$
\hat{P}^{\varepsilon}(k)=\left\{\begin{array}{ll}
(\not k+m) \delta\left(k^{2}-m^{2}\right) & \text { if } m>0 \\
X \not k \delta\left(k^{2}\right) & \text { if } m=0
\end{array} \quad\left(\left|k^{0}\right|+|\vec{k}| \ll \varepsilon^{-1}\right) .\right.
$$

However, in the high-energy regime, $P^{\varepsilon}$ will no longer satisfy the Dirac equation. But in preparation of the perturbation expansion, we need to associate the states $P^{\varepsilon}$ to eigenstates of the Dirac operator (not necessarily to the eigenvalue $m$ ). To this end, we introduce two operators $V_{\text {shift }}$ and $V_{\text {shear }}$ with the following properties. The operator $V_{\text {shift }}$ has the purpose of changing the momentum of states such that general surface states (as in Figure $4.1(\mathrm{~B}))$ are mapped onto the mass cone, i.e.

$$
\left(V_{\text {shift }} \psi\right)(k):=\psi\left(v_{\text {shift }}(k)\right),
$$

where $v_{\text {shift }}: \hat{M} \rightarrow \hat{M}$ is a diffeomorphism. The operator $V_{\text {shear }}$, on the other hand, is a unitary multiplication operator in momentum space, which has the purpose of introducing the shear of the surface states (i.e. it should map the states in Figure 4.1 (B) to those in Figure $4.1(\mathrm{C}))$,

$$
\left(V_{\text {shear }} \psi\right)(k)=\hat{V}_{\text {shear }}(k) \psi(k) \quad \text { with } \quad \hat{V}_{\text {shear }}(k) \text { unitary . }
$$

These operators are to be chosen such that the operator $\check{P}^{\varepsilon}$ defined by

$$
P^{\varepsilon}=V_{\text {shear }} V_{\text {shift }} \check{P}^{\varepsilon} V_{\text {shift }}^{-1} V_{\text {shear }}^{-1}
$$

is of the following form,

$$
\check{P}^{\varepsilon}(k)= \begin{cases}d(k)(\not k+m(k) \mathbb{1}) \delta\left(k^{2}-m(k)^{2}\right) & \text { if } m>0 \\ d(k) X \not k \delta\left(k^{2}\right) & \text { if } m=0\end{cases}
$$


with $X$ as in (F.1.1). Thus in the massive case, $\check{P}^{\varepsilon}$ should be composed of Dirac eigenstates corresponding to an energy-dependent mass $m(k)>0$, and it should have vectorscalar structure. In the massless case, we demand that $k^{2}=0$, so that the states of $\check{P}^{\varepsilon}$ are all neutral. The ansatz $(\mathrm{F} .1 .5)$ is partly a matter of convenience, and partly a requirement needed for the perturbation expansion (see Proposition F.3.1 below). Moreover, we assume for convenience that $\check{P}^{\varepsilon}$ is composed only of states of negative energy,

$$
\check{P}^{\varepsilon}(k)=0 \quad \text { if } k^{2}<0 \text { or } k^{0}>0 .
$$

In view of (F.1.2), it is easiest to assume that $V_{\text {shift }}$ and $V_{\text {shear }}$ are the identity in the low-energy regime, i.e.

$$
\hat{V}_{\text {shear }}(k)=\mathbb{1} \quad \text { and } \quad v_{\text {shift }}(k)=k \quad \text { if }\left|k^{0}\right|+|\vec{k}| \ll \varepsilon^{-1} .
$$

Then, by comparing (F.1.2) with (F.1.5), one finds that

$$
d(k)=\mathbb{1} \quad \text { and } \quad m(k)=m \quad \text { if }\left|k^{0}\right|+|\vec{k}| \ll \varepsilon^{-1} .
$$

The required regularization of $P^{\varepsilon}(x, y)$ on the scale $\varepsilon$ is implemented by demanding that

$$
d(k) \text { decays on the scale }\left|k^{0}\right|+|\vec{k}| \sim \varepsilon^{-1} .
$$

In view of their behavior in the low-energy regime, it is natural to assume that the functions in (F.1.7) and (F.1.8) should be smooth in momentum space and that their derivatives scale in powers of the regularization length, i.e.

$$
\begin{aligned}
\left|\nabla_{k}^{\gamma} d(k)\right| & \sim \varepsilon^{|\gamma|}|d(k)|, & \left|\nabla_{k}^{\gamma} m(k)\right| & \sim \varepsilon^{|\gamma|}|m(k)| \\
\left|\nabla_{k}^{\gamma} \hat{V}_{\text {shear }}(k)\right| & \sim \varepsilon^{|\gamma|}\left|\hat{V}_{\text {shear }}(k)\right|, & \left|\nabla_{k}^{\gamma} v_{\text {shift }}(k)\right| & \sim \varepsilon^{|\gamma|}\left|v_{\text {shift }}(k)\right| .
\end{aligned}
$$

Clearly, the above conditions do not uniquely determine the function $d$ and the operators $V_{\text {shift }}$ and $V_{\text {shear }}$. But we shall see that the results of our analysis will be independent of the choice of these operators. We remark that the transformation $V_{\text {shear }}$ is analogous to the transformations $U_{l}$ considered in [F7, Appendix D] (see [F7, eq. (D.22)]), except that here we consider only one unitary transformation.

The last construction immediately generalizes to a system of Dirac seas. Namely, suppose that without regularization, the auxiliary fermionic projector of the vacuum is a direct sum of Dirac seas (see for example 4.2.41) or 4.2.54),

$$
P^{\text {aux }}=\bigoplus_{\ell=1}^{\ell_{\max }} X_{\ell} t_{\ell}
$$

Then we introduce $P^{\varepsilon}$ simply by taking the direct sum of the corresponding regularized seas

$$
P^{\text {aux }}:=\bigoplus_{\ell=1}^{\ell_{\max }} P_{\ell}^{\varepsilon}, \quad V_{\text {shift }}:=\bigoplus_{\ell=1}^{\ell_{\max }} V_{\text {shift }}^{\ell}, \quad V_{\text {shear }}:=\bigoplus_{\ell=1}^{\ell_{\max }} V_{\text {shear }}^{\ell} .
$$

Setting

$$
P^{\text {aux }}=V_{\text {shear }} V_{\text {shift }} \check{P}^{\varepsilon} V_{\text {shift }}^{-1} V_{\text {shear }}^{-1},
$$

the operator $\check{P}^{\varepsilon}$ satisfies the Dirac equation in momentum space

$$
(\not k-m Y(k)) \widehat{\widetilde{P}^{\varepsilon}}(k)=0,
$$


where the mass matrix is given by (cf. 4.2.43 or 4.2.56)

$$
m Y(k)=\bigoplus_{\ell=1}^{\ell_{\max }} m_{\ell}
$$

In the low-energy regime, we know furthermore that

$$
\left.\begin{array}{c}
\hat{V}_{\text {shear }}(k)=\mathbb{1} \quad \text { and } \quad v_{\text {shift }}(k)=k \\
\hat{P}^{\text {aux }}(k)=X t
\end{array}\right\} \quad \text { if }\left|k^{0}\right|+|\vec{k}| \ll \varepsilon^{-1},
$$

where $X$ and $t$ are given as in (4.2.44). Clearly, the regularity assumptions (F.1.10) are imposed similarly for $\hat{P}^{\text {aux }}$. Finally, we need to specify what we mean by saying that two Dirac seas are regularized in the same way. The difficulty is that, as mentioned above, different choices of $d, \hat{V}_{\text {shear }}$ and $\hat{V}_{\text {shift }}$ may give rise to the same regularization effects. In order to keep the situation reasonably simple, we use the convention that if we want two Dirac seas to show the same regularization effects, we choose the corresponding functions $d$ as well as $\hat{V}_{\text {shear }}$ and $\hat{V}_{\text {shift }}$ to be exactly the same. If conversely two Dirac seas should show different regularization effects, we already choose the corresponding functions $d$ to be different. Then we can say that two Dirac seas labeled by $a$ and $b$ are regularized in the same way if $d_{a} \equiv d_{b}$. In this case, our convention is that also $\left(\hat{V}_{\text {shear }}\right)_{a}=\left(\hat{V}_{\text {shear }}\right)_{b}$ and $\left(\hat{V}_{\text {shift }}\right)_{a}=\left(\hat{V}_{\text {shift }}\right)_{b}$. This notion gives rise to an equivalence relation on the Dirac seas. In the formalism of $\$ 4.2 .6$, the equivalence classes will be labeled by the parameters $\tau_{i}^{\text {reg }}$ (see 4.2.42 and 4.2.57)).

\section{F.2. Formal Introduction of the Interaction}

Now the interaction can be introduced most conveniently by using the unitary perturbation flow [FG1, Section 5] (see also [FT2, Section 4]). In order not to get confused with the mass matrix, we introduce an additional spectral parameter $\mu$ into the free Dirac equation, which in momentum space reads

$$
(\not k-m Y(k)-\mu \mathbb{1}) \hat{\psi}(k)=0 .
$$

For this Dirac equation, we can introduce the spectral projectors $p$, the causal fundamental solutions $k$ and the symmetric Green's functions $s$ can be introduced just as in $[\mathbf{F 7}$, Section 2.2], if only in the formulas in momentum space we replace $m$ by $m Y(k)$. For clarity, we denote the dependence on $\mu$ by an subscript $+\mu$ (this notation was used similarly in [F7, Section 2.6]; see also [F7, Appendix C.3] for an additional "modified mass scaling", which we will for simplicity not consider here). We describe the interaction by inserting an operator $\mathcal{B}$ into the Dirac operator,

$$
\mathcal{D}=i \not \partial+\mathcal{B}-m Y(k) \text {. }
$$

After adding the subscript $+\mu$ to all factors $p, k$ or $s$ in the operator products in FG1, Section 5], we obtain an operator $U$ which associates to every solution $\psi$ of the free Dirac equation $(i \not \partial-m Y-\mu \mathbb{1}) \psi=0$ a corresponding solution $\tilde{\psi}$ of the interacting Dirac equation $(i \not \partial+\mathcal{B}-m Y-\mu \mathbb{1}) \psi=0$,

$$
U(\mathcal{B}): \psi \mapsto \tilde{\psi} .
$$

The operator $U$ is uniquely defined in terms of a formal power series in $\mathcal{B}$. Taking $\mu$ as a free parameter, in [FG1, Section 5] the operator $U$ is shown to be unitary with respect 
to the indefinite inner product (4.2.3). We now use $U$ to unitarily transform all the Dirac states contained in the operator $\dot{P}^{\varepsilon}$ and set

$$
\tilde{P}^{\text {aux }}=V_{\text {shear }} V_{\text {shift }} U(\mathcal{B}) \check{P}^{\varepsilon} U(\mathcal{B})^{-1} V_{\text {shift }}^{-1} V_{\text {shear }}^{-1} .
$$

This construction uniquely defines the regularized auxiliary fermionic projector with interaction $\tilde{P}^{\text {aux }}$ in terms of a formal power expansion in $\mathcal{B}$. The fermionic projector is then obtained by forming the sectorial projection (see 4.2 .52 ) or 4.2 .55$)$ ).

\section{F.3. Compatibility Conditions for the Interaction}

In order to derive the structure of the admissible $\mathcal{B}$, we first consider a perturbation calculation to first order and assume that $\mathcal{B}$ is a multiplication operator in position space having the form of a plane wave of momentum $q$,

$$
\mathcal{B}(x)=\mathcal{B}_{q} e^{-i q x} .
$$

In this case (cf. [F7, eq. (D.14)]),

$$
\Delta \check{P}^{\text {aux }}=-\int_{-\infty}^{\infty} d \mu\left(s_{+\mu} \mathcal{B} p_{+\mu} \check{P}^{\varepsilon}+\check{P}^{\varepsilon} p_{+\mu} \mathcal{B} s_{+\mu}\right)
$$

Using a matrix notation in the direct sums with indices $a, b \in\left\{1, \ldots, \ell_{\max }\right\}$, we obtain in momentum space (for the notation see [F7, Chapter 2] or [FG1])

$$
\begin{aligned}
&\left(\Delta \check{P}^{\varepsilon}\right)_{b}^{a}(k+q, k)=-\int_{-\infty}^{\infty} d\left\{s_{m_{a}+\mu}(k+q)\left(\mathcal{B}_{q}\right)_{b}^{a} p_{m_{b}+\mu}(k)\left(\check{P}^{\varepsilon}\right)_{b}^{b}(k)\right. \\
&\left.\left.+\left(\check{P}^{\varepsilon}\right)_{a}^{a}(k+q) p_{m_{a}+\mu}(k+q)\left(\mathcal{B}_{q}\right)_{b}^{a} s_{m_{b}+\mu}(k)\right)\right\} .
\end{aligned}
$$

This equation was already considered in [F5, Section 3] and [F7, Appendix D]. However, here we analyze the situation more systematically and in a more general context, pointing out the partial results which were obtained previously.

For clarity, we analyze (F.3.2) step by step, beginning with the diagonal elements. For ease in notation, we assume that $\mathcal{B}_{q}$ has only one non-trivial component, which is on the diagonal,

$$
\left(\mathcal{B}_{q}\right)_{b}^{a}=\delta^{a \ell} \delta_{b \ell} \mathcal{B}
$$

with $\ell \in\left\{1, \ldots, \ell_{\max }\right\}$ and $\mathcal{B}$ a matrix acting on Dirac spinors. Shifting the integration variable according to $m_{\ell}+\mu \rightarrow \mu$, we obtain (cf. [F7, eq. (D.15)])

$$
\begin{aligned}
&\left(\Delta \check{P}^{\varepsilon}\right)_{\ell}^{\ell}(k+q, k) \\
&=-\int_{-\infty}^{\infty} d \mu\left\{s_{\mu}(k+q) \mathcal{B} p_{\mu}(k)\left(\check{P}^{\varepsilon}\right)_{\ell}^{\ell}(k)+\left(\check{P}^{\varepsilon}\right)_{\ell}^{\ell}(k+q) p_{\mu}(k+q) \mathcal{B} s_{\mu}(k)\right\} \\
&=-\int_{-\infty}^{\infty} d \mu \epsilon(\mu)\left\{\frac{\mathrm{PP}}{(k+q)^{2}-\mu^{2}}(\not k+\not k+\mu) \mathcal{B}(\not k+\mu) \delta\left(k^{2}-\mu^{2}\right)\left(\check{P}^{\varepsilon}\right)_{\ell}^{\ell}(k)\right. \\
&\left.+\left(\check{P}^{\varepsilon}\right)_{\ell}^{\ell}(k+q) \delta\left((k+q)^{2}-\mu^{2}\right)(\not k+\not q+\mu) \mathcal{B}(\not k+\mu) \frac{\mathrm{PP}}{k^{2}-\mu^{2}}\right\} \\
&=-\int_{-\infty}^{\infty} d \mu \epsilon(\mu) \frac{\mathrm{PP}}{2 k q+q^{2}}\left\{(\not k+\not k+\mu) \mathcal{B}(\not k+\mu) \delta\left(k^{2}-\mu^{2}\right)\left(\check{P}^{\varepsilon}\right)_{\ell}^{\ell}(k)\right. \\
&\left.-\left(\check{P}^{\varepsilon}\right)_{\ell}^{\ell}(k+q) \delta\left((k+q)^{2}-\mu^{2}\right)(\not k+\not k+\mu) \mathcal{B}(\not k+\mu)\right\},
\end{aligned}
$$


where in the last step we used that the argument of the $\delta$-distribution vanishes. Carrying out the $\mu$-integration gives (cf. [F7, eq. (D.15)])

$$
\begin{aligned}
\left(\Delta \check{P}^{\varepsilon}\right)_{\ell}^{\ell}(k+q, k)=-\frac{\mathrm{PP}}{4 k q+2 q^{2}}\{ & ((\not k+\not q) \mathcal{B}+\mathcal{B} \not k)\left(\check{P}^{\varepsilon}\right)_{\ell}^{\ell}(k) \\
& \left.-\left(\check{P}^{\varepsilon}\right)_{\ell}^{\ell}(k+q)((k+q) \mathcal{B}+\mathcal{B} \not k)\right\} .
\end{aligned}
$$

Here the principal part has poles if $2 k q+q^{2}=0$, leading to a potential divergence of $\Delta \check{P}^{\varepsilon}$. In order to explain the nature of this divergence, we first point out that if $\mathcal{B}$ had been chosen to be a smooth function with rapid decay, then $\Delta \check{P}$ would have been finite (see the proof of [F7, Lemma 2.2.2]). Thus the potential divergence is related to the fact that the plane wave in (F.3.1) does not decay at infinity. A more detailed picture is obtained by performing the light-cone expansion (see [F5 and [F7, Appendix F]). Then one can introduce the notion that (F.3.4) is causal if its light-cone expansion only involves integrals along a line segment $\overline{x y}$. Since such integrals are uniformly bounded, it follows immediately that all contributions to the light-cone expansion are finite for all $q$. If conversely (F.3.4) diverges, then the analysis in [F7, Appendix F] reveals that individual contributions to the light-cone expansion do diverge, so that unbounded line integrals must appear (see also the explicit light-cone expansions in [F2]). In this way, one gets a connection between the boundedness of (F.3.4) and the causality of the light-cone expansion.

Unbounded line integrals lead to contributions to the EL equations whose scaling behavior in the radius is different from all other contributions. Therefore, the EL equations are satisfied only if all unbounded line integrals drop out. The easiest way to arrange this is to demand that the fermionic projector itself should not involve any unbounded line integrals. This is our motivation for imposing that

$$
(\Delta \hat{P})^{\varepsilon}(k+q, k) \text { should be bounded locally uniformly in } q .
$$

Let us analyze this boundedness condition for (F.3.4). Since the denominator in (F.3.4) vanishes as $q \rightarrow 0$, we clearly get the necessary condition that the curly brackets must vanish at $q=0$,

$$
\left[\{\not k, \mathcal{B}\}, \check{P}^{\varepsilon}(k)\right]=0 .
$$

Using (F.1.5) together with the identity

$$
[\{\not k, \mathcal{B}\}, \not k]=\left[k^{2}, \mathcal{B}\right]+\not k \mathcal{B} \not k-\not k \mathcal{B} \not k=0,
$$

we find that $($ F.3.6 $)$ is automatically satisfied in the case $X=\mathbb{1}$. The situation is more interesting if a chiral asymmetry is present. If for example $X=\chi_{L}$, we get the condition

$$
\left[\{\not k, \mathcal{B}\}, \chi_{L} \not k\right]=0 \text {. }
$$

This condition is again trivial if $\mathcal{B}$ is odd (meaning that $\{\mathcal{B}, \Gamma\}=0$ ). However, if $\mathcal{B}$ is even, we conclude that

$$
0=\frac{\Gamma}{2}\{\{\not k, \mathcal{B}\}, \not k\}=\Gamma \not k \mathcal{B} \not k
$$

(where in the last step we used that $k^{2}=0$ in view of $($ F.1.5 ). As $k$ is any state on the lower mass shell, this rules out that $\mathcal{B}$ is a bilinear potential, leaving us with a scalar or a pseudoscalar potential. In order to rule out these potentials, we next choose a vector $\hat{q}$ 
with $\hat{q} k=0$, set $q=\varepsilon \hat{q}$ and consider $($ F.3.4 $)$ in the limit $\varepsilon \rightarrow 0$. Then the denominator in $\left(\right.$ F.3.4 diverges like $\varepsilon^{-2}$, so that the curly brackets must tend to zero even $\sim \varepsilon^{2}$.

$$
((\not k+\varepsilon \hat{q}) \mathcal{B}+\mathcal{B} \not k) \check{P}^{\varepsilon}(k)-\check{P}^{\varepsilon}(k+\varepsilon \hat{q})((\not k+\varepsilon \hat{q}) \mathcal{B}+\mathcal{B} \not k)=\mathcal{O}\left(\varepsilon^{2}\right) .
$$

Using that $\check{P}^{\varepsilon}(k)$ is left-handed and that $\mathcal{B}$ is even, we find that the first summand in (F.3.7) is right-handed, whereas the second summand is left-handed. Hence both summand must vanish separately, and thus

$$
0=((\not k+\hat{q}) \mathcal{B}+\mathcal{B} \not k) \check{P}^{\varepsilon}(k)=\hat{q} \mathcal{B} \not k d(k) \delta\left(k^{2}\right) .
$$

This condition implies that $\mathcal{B}$ must vanish. We conclude that if $X=\chi_{L}$, only odd potentials may occur. We can write this result more generally as

$$
\mathcal{B} X=X^{*} \mathcal{B} \text {. }
$$

We have thus derived the causality compatibility condition (4.2.48) from our boundedness condition (F.3.5). This derivation is an alternative to the method in [F7, Section 2.3], where the same condition was introduced by the requirement that it should be possible to commute the chiral asymmetry matrix through the perturbation expansion.

So far, we considered $(\mathrm{F} .3 .5)$ in the limit $q \rightarrow 0$. We now analyze this condition for general q. Using (F.1.5) and (F.3.8), a short calculation gives

$$
\left(\Delta \check{P}^{\varepsilon}\right)_{\ell}^{\ell}(k+q, k)=-X \frac{(\not k+\not q) \mathcal{B} \not k}{4 k q+2 q^{2}}\left(d(k) \delta\left(k^{2}-m^{2}\right)-d(k+q) \delta\left((k+q)^{2}-m^{2}\right)\right),
$$

where we set $m=m_{\ell}$. If $d(k)=d(k+q)$, the transformations

$$
\begin{aligned}
\int_{0}^{1} \delta^{\prime}\left(k^{2}-m^{2}+\tau\left(2 k q+q^{2}\right)\right) d \tau & =\frac{1}{2 k q+q^{2}} \int_{0}^{1} \frac{d}{d \tau} \delta\left(k^{2}-m^{2}+\tau\left(2 k q+q^{2}\right)\right) d \tau \\
& =\frac{1}{2 k q+q^{2}}\left(\delta\left((k+q)^{2}-m^{2}\right)-\delta\left(k^{2}-m^{2}\right)\right)
\end{aligned}
$$

show that $\Delta \check{P}^{\varepsilon}$ is indeed a bounded distribution for any $q$. Thus it remains to be concerned about the contribution if $\delta(k) \neq \delta(k+q)$,

$$
X \frac{(\not k+q) \mathcal{B} \not k}{4 k q+2 q^{2}} \delta\left(k^{2}-m^{2}\right)(d(k+q)-d(k)) .
$$

Unless in the trivial case $\mathcal{B}=0$, this contribution is infinite at the poles of the denominator. We conclude that in order to comply with the condition $($ F.3.5 $)$, we must impose that the weight function $d(k)$ in $(\mathrm{F} .1 .5)$ is constant on the mass shell $k^{2}=m(k)^{2}$. This is indeed the case in the low-energy regime (F.1.8). However, in the high-energy region, the function $d(k)$ is in general not a constant (and indeed, assuming that $d(k)$ is constant would be in contradiction to (F.1.9p). Our way out of this problem is to observe that (F.3.9) implies that the light-cone expansion of (F.3.4 $)$ is in general not causal, in the sense that it involves unbounded line integrals. However, using that $d(k+q)-d(k) \sim q|\nabla d|$, the scalings $q \sim \ell_{\text {macro }}^{-1}$ and F.1.10 show that these non-causal contributions to the light-cone expansion are of

$$
\text { higher order in } \varepsilon / \ell_{\text {macro }} \text {. }
$$

This consideration shows that the perturbation expansion will give rise to error terms of higher order in $\varepsilon / \ell_{\text {macro }}$. In what follows, we will always neglect such error terms. If this is done, the above assumptions are consistent and in agreement with (F.3.5), provided that the causality compatibility condition (F.3.8) holds. 
Before moving on to potentials which mix different Dirac seas, we remark that the above arguments can also be used to derive constraints for the possible form of $\hat{P}^{\varepsilon}(k)$, thus partly justifying our ansatz F.1.5.

Proposition F.3.1 (Possible form of $\left.\check{P}^{\varepsilon}\right)$. Suppose that $\Delta \check{P}^{\varepsilon}$ as given by (F.3.4) satisfies the condition (F.3.5). We renounce the assumptions on $\check{P}^{\varepsilon}$ (see (F.1.5), (F.1.6), (F.1.8) and (F.1.10) $)$, but we assume instead that the admissible interaction includes chiral or axial potentials. Then for every $k$, there are complex coefficients $a, b, c, d \in \mathbb{C}$ such that

$$
\check{P}^{\varepsilon}(k)=a \mathbb{1}+i b \Gamma+c \not k+d \Gamma \not k .
$$

Proof. We again analyze the necessary condition (F.3.6). This condition is clearly satisfied if $\mathcal{B}$ is a vector potential. Thus by linearity, we may assume that $\mathcal{B}=\Gamma \mathcal{A}$ is a vector potential. It follows that

$$
0=\left[\{\not k, \Gamma \not A\}, \check{P}^{\varepsilon}(k)\right]=\left[\Gamma[\not A, \not k], \check{P}^{\varepsilon}(k)\right] .
$$

Decomposing $\check{P}^{\varepsilon}(k)$ into its even and odd components, by linearity we can again consider these components after each other. If $\check{P}^{\varepsilon}(k)$ is odd, i.e.

$$
\check{P}^{\varepsilon}(k)=\psi+\Gamma \psi,
$$

we obtain

$$
0=\Gamma\left\{[A, \not k], \check{P}^{\varepsilon}(k)\right\}=4 \Gamma((k u) \not A-(A u) \not k)+4((k v) \not A-(A v) \not k) .
$$

Since $A$ is arbitrary, it follows that $u$ and $v$ must be multiples of $k$.

If $\check{P}^{\varepsilon}(k)$ is even, we obtain the condition

$$
\left[[A, \not k], \check{P}^{\varepsilon}(k)\right]=0 \text {. }
$$

This condition is obviously satisfied if $\check{P}^{\varepsilon}(k)$ is a scalar or a pseudoscalar. Writing the bilinear component of $\check{P}^{\varepsilon}(k)$ in the form $F_{i j} \gamma^{i} \gamma^{j}$ with an anti-symmetric tensor field $F$, we get the condition

$$
0=\left[[\mathcal{A}, \not k], F_{i j} \gamma^{i} \gamma^{j}\right]=2 F_{i j} k^{i}\left[\not A, \gamma^{j}\right]-2 F_{i j} A^{i}\left[\not k, \gamma^{j}\right] .
$$

Since $A$ is arbitrary, it follows that $F=0$, concluding the proof.

The step from (F.3.11) to our the stronger assumption (F.1.2 could be justified by the assumption that the image of $P^{\varepsilon}$ should be negative definite or neutral, and furthermore by assuming that without chiral asymmetry the matrix $\Gamma$ is absent, whereas the chiral asymmetry is then introduced simply by multiplying with $\chi_{L}$ or $\chi_{R}$. We finally remark that in [F7, Appendix D], the condition (F.3.6) is analyzed for $\mathcal{B}$ a scalar potential to conclude that $\check{P}^{\varepsilon}(k)$ should commute with the Dirac operator (see [F7, eq. (D.16) and eq. (D.17)]). This is consistent with our ansatz (F.1.5), which is even a solution of the Dirac equation (F.1.11). However, we here preferred to avoid working with scalar potentials, which do not seem crucial for physically realistic models.

Next, instead of (F.3.3) we consider a general potential $\mathcal{B}_{q}$ which may have offdiagonal terms in the direct summands. Then F.3.2 can be evaluated similar as in the computation after (F.3.3), but the calculation is a bit more complicated. Therefore, we 
first compute the integral of the first summand in (F.3.2),

$$
\begin{aligned}
& \int_{-\infty}^{\infty} d \mu s_{m_{a}+\mu}(k+q)\left(\mathcal{B}_{q}\right)_{b}^{a} p_{m_{b}+\mu}(k)\left(\check{P}^{\varepsilon}\right)_{b}^{b}(k) \\
&= \int_{-\infty}^{\infty} d \mu \epsilon\left(m_{b}+\mu\right) \frac{\mathrm{PP}}{(k+q)^{2}-\left(m_{a}+\mu\right)^{2}} \delta\left(k^{2}-\left(m_{b}+\mu\right)^{2}\right) \\
&=\sum_{\mu= \pm|k|-m_{b}} \frac{1}{m_{b}+\mu} \mathfrak{B}_{b}^{a}\left(\check{P}^{\varepsilon}\right)_{b}^{b}(k),
\end{aligned}
$$

where we set

$$
\mathfrak{B}_{b}^{a}=\frac{1}{2} \operatorname{PP}\left(\frac{\left(\not k+\not l+m_{a}+\mu\right)\left(\mathcal{B}_{q}\right)_{b}^{a}\left(\not k+m_{b}+\mu\right)}{2 k q+q^{2}-\left(m_{a}^{2}-m_{b}^{2}\right)-2 \mu\left(m_{a}-m_{b}\right)}\right)
$$

and $|k|=\sqrt{k^{2}}$ (note that, in view of our assumption $(\overline{\mathrm{F} .1 .6})$, the factor $\left(\check{P}^{\varepsilon}\right)_{b}^{b}(k)$ guarantees that the above expression vanishes if $\left.k^{2}<0\right)$. Treating the second summand in $($ F.3.2 similarly, we obtain

$$
\left(\Delta \check{P}^{\varepsilon}\right)_{b}^{a}(k+q, k)=\sum_{\mu= \pm|k+q|-m_{a}} \frac{\left(\check{P}^{\varepsilon}\right)_{a}^{a}(k+q)}{m_{a}+\mu} \mathfrak{B}_{b}^{a}-\sum_{\mu= \pm|k|-m_{b}} \mathfrak{B}_{b}^{a} \frac{\left(\check{P}^{\varepsilon}\right)_{b}^{b}(k)}{m_{b}+\mu} .
$$

This formula is rather involved, but fortunately we do not need to enter a detailed analysis. It suffices to observe that $($ F.3.13 $)$ has poles in $q$, which lead to singularities of (F.3.12). Thus the only way to satisfy the condition $(\overline{F .3 .5})$ is to arrange that contributions of the first and second expression on the right of (F.3.14) cancel each other. In view of (F.1.5), the first expression involves $d_{a}(k+q)$, whereas in the second expression the term $d_{b}(k)$ appears. This shows that in order to get the required cancellations, the functions $d_{a}$ and $d_{b}$ must coincide. Using the notion introduced on page 421, we conclude that $\mathcal{B}$ may describe an interaction of Dirac seas only if they are regularized in the same way. An interaction of Dirac seas with different regularization, however, is prohibited by the causality condition for the light-cone expansion. For brevity, we also say that the interaction must be regularity compatible.

\section{F.4. The Causal Perturbation Expansion with Regularization}

We are now ready to perform the causal perturbation expansion. In FG1, Section 5], the unitary perturbation flow is introduced in terms of an operator product expansion. Replacing the Green's functions and fundamental solutions in this expansion by the corresponding operators of the free Dirac equation (F.1.11), we can write the operator $U(\mathcal{B}) \check{P}^{\varepsilon} U(\mathcal{B})^{-1}$ as a series of operator products of the form

$$
z:=C_{1} \mathcal{B} \cdots \mathcal{B} C_{p} \mathcal{B} \check{P}^{\varepsilon} \mathcal{B} C_{p+1} \mathcal{B} \cdots \mathcal{B} C_{k},
$$

where the factors $C_{l}$ are the Green's functions or fundamental solutions of the free Dirac equation (F.1.11). The operators $C_{l}$ are diagonal in momentum space, whereas the potential $\mathcal{B}$ varies on the macroscopic scale and thus changes the momentum only on the scale $\ell_{\text {macro }}^{-1}$. Thus all the factors $C_{l}$ will be evaluated at the same momentum $p$, up to errors of the order $\ell_{\text {macro }}^{-1}$. We refer to this momentum $p$, determined only up to summands of the order $\ell_{\text {macro }}^{-1}$, as the considered momentum scale. In view of the regularity assumptions on the functions $d$ and $m$ in $($ F.1.10), we may replace them by the constants $d(p)$ and $m(p)$, making an error of the order (F.3.10). This evaluation of the regularization 
functions is referred to as the fixing of the momentum scale, and we indicate it symbolically by $\left.\right|_{\text {scale } p}$. Since $\mathcal{B}$ is regularity compatible, we may then commute the constant matrix $d$ to the left. Moreover, we can apply the causality compatibility condition (F.3.8) together with the form of $X$ in $(\mathrm{F} .1 .5)$ and $(\mathrm{F} .1 .2)$ to also commute the chiral asymmetry matrix $X$ to the left. We thus obtain the expansion

$$
\begin{aligned}
\left.U(\mathcal{B}) \check{P}^{\varepsilon} U(\mathcal{B})^{-1}\right|_{\text {scale } p}=\sum_{k=0}^{\infty} \sum_{\alpha=0}^{\alpha_{\max }(k)} c_{\alpha} & \left.X d C_{1, \alpha} \mathcal{B} C_{2, \alpha} \mathcal{B} \cdots \mathcal{B} C_{k+1, \alpha}\right|_{\text {scale } p} \\
& +\left(\text { higher orders in } \varepsilon / \ell_{\text {macro }}\right)
\end{aligned}
$$

where we set

$$
X=\bigoplus_{\ell=1}^{\ell_{\max }} X_{\ell} \quad \text { and } \quad d=\bigoplus_{\ell=1}^{\ell_{\max }} d_{\ell}(p),
$$

and $c_{\alpha}$ are combinatorial factors. Here the combinatorics of the operator products coincides precisely with that of the causal perturbation expansion for the fermionic projector as worked out in detail in [FG1, FT2].

\section{F.5. The Behavior under Gauge Transformations}

In order to analyze the behavior of the above expansion under $U(1)$-gauge transformations, we consider the case of a pure gauge potential, i.e. $\mathcal{B}=\not \partial \Lambda$ with a real-valued function $\Lambda$. Then the gauge invariance of the causal perturbation expansion yields

$$
\left.\left.U(\mathcal{B}) \check{P}^{\varepsilon} U(\mathcal{B})^{-1}\right|_{\text {scale } p}=\left.\left(e^{i \Lambda} \check{P}^{\varepsilon} e^{-i \Lambda}\right)\right|_{\text {scale } p}+\text { (higher orders in } \varepsilon / \ell_{\text {macro }}\right) \text {. }
$$

According to (F.2.1), we obtain $\tilde{P}^{\text {aux }}$ by applying with $V_{\text {shift }}$ and $V_{\text {shear }}$. The transformation $V_{\text {shift }}$ is a subtle point which requires a detailed explanation. We first consider its action on a multiplication operator in momentum space $M(k)$. Then, according to the definition (F.1.3),

$$
\begin{aligned}
\left(V_{\text {shift }} M V_{\text {shift }}^{-1} \psi\right)(k) & =\left(M V_{\text {shift }}^{-1} \psi\right)\left(v_{\text {shift }}(k)\right) \\
& =M\left(v_{\text {shift }}(k)\right)\left(V_{\text {shift }}^{-1} \psi\right)\left(v_{\text {shift }}(k)\right)=M\left(v_{\text {shift }}(k)\right) \psi(k),
\end{aligned}
$$

showing that the transformation again yields a multiplication operator, but with a transformed argument. In order to derive the transformation law for multiplication operators in position space, we first let $f=e^{-i q x}$ be the operator of multiplication by a plane wave. Then

$$
\begin{aligned}
\left(V_{\text {shift }} f V_{\text {shift }}^{-1} \psi\right)(k) & =\left(f V_{\text {shift }}^{-1} \psi\right)\left(v_{\text {shift }}(k)\right) \\
& =\left(V_{\text {shift }}^{-1} \psi\right)\left(v_{\text {shift }}(k)-q\right)=\psi\left(v_{\text {shift }}^{-1}\left(v_{\text {shift }}(k)-q\right)\right) .
\end{aligned}
$$

This can be simplified further if we assume that the momentum $q \sim \ell_{\text {macro }}^{-1}$ is macroscopic. Namely, the scaling of the function $v_{\text {shift }}$ in (F.1.10) allows us to expand in a Taylor series in $q$,

$$
\left(V_{\text {shift }} f V_{\text {shift }}^{-1} \psi\right)(k)=\psi\left(k-\left.D v_{\text {shift }}^{-1}\right|_{v_{\text {shift }}(k)} q\right)+\left(\text { higher orders in } \varepsilon / \ell_{\text {macro }}\right) .
$$

Thus $V_{\text {shift }} f V_{\text {shift }}^{-1}$ is again a multiplication operator in position space, but now corresponding to the new momentum

$$
L(k) q \quad \text { with } \quad L(k):=\left.D v_{\text {shift }}^{-1}\right|_{v_{\text {shift }}(k)} .
$$


Again in view of the regularity assumptions (F.1.10), when fixing the momentum scale we may replace the argument $k$ by $p$, i.e.

$$
\left.V_{\text {shift }} e^{-i q x} V_{\text {shift }}^{-1}\right|_{\text {scale } p}=e^{-i(L(p) q) x}+\left(\text { higher orders in } \varepsilon / \ell_{\text {macro }}\right) .
$$

Using the relation $(L(p) q) x=q L(p)^{*} x$, we can rewrite this transformation law simply as a linear transformation of the space-time coordinates. Then the transformation law generalizes by linearity to a general multiplication operator by a function $f$ which varies on the macroscopic scale, i.e.

$$
\left.\left.V_{\text {shift }} f(x) V_{\text {shift }}^{-1}\right|_{\text {scale } p}=f\left(L(p)^{*} x\right)+\text { (higher orders in } \varepsilon / \ell_{\text {macro }}\right) .
$$

With (F.5.2) and (F.5.3), we can transform (F.5.1) to the required form (F.2.1). Using that $V_{\text {shear }}$ commutes with scalar and macroscopic multiplication operators (again up to higher orders in $\left.\varepsilon / \ell_{\text {macro }}\right)$, we obtain in view of (F.1.4)

$$
\left.P^{\varepsilon}(x, y)\right|_{\text {scale } \mathrm{p}}=e^{i \Lambda\left(L(p)^{*} x\right)} P^{\varepsilon}(x, y) e^{-i \Lambda\left(L(p)^{*} y\right)}+\left(\text { higher orders in } \varepsilon / \ell_{\text {macro }}\right) .
$$

Except for the factors $L(p)^{*}$, this formula describes the usual behavior of the fermionic projector under gauge transformations. In particular, if we do not consider general surface states and $V_{\text {shift }}=\mathbb{1}$, then our perturbation expansion is gauge invariant. However, if we consider general surface states described by a non-trivial operator $V_{\text {shift }}$, then the matrix $L$ will in in general not be the identity, and the transformation law (F.5.4 violates gauge invariance.

Our method to recover gauge invariance is to replace the gauge potential $A$ by a more general operator $\mathcal{A}$, which in momentum space has the form

$$
\mathcal{A}\left(p+\frac{q}{2}, p-\frac{q}{2}\right):=\hat{A}\left(L(p)^{-1} q\right),
$$

where $\hat{A}$ is the Fourier transform of the classical potential to be used when no regularization is present. In the case $A=\not \partial \Lambda$ of a pure gauge field and fixing the momentum scale, we then find that $\mathcal{A}$ coincides with the multiplication operator $A\left(\left(\left(L(p)^{*}\right)^{-1} x\right)\right)$, just compensating the factors $L(p)^{*}$ in (F.5.4). In view of the regularity assumptions (F.1.10), the matrix $L$ scales in powers of the regularization length. Thus $A$ is a nonlocal operator, but only on the microscopic scale $\varepsilon$. On the macroscopic scale, however, it coincides with the classical local potential. We also point out that the compatibility conditions worked out in Section F.3 under the assumption that $\mathcal{B}$ is a multiplication operator are valid just as well for the nonlocal potential (F.5.5), because after fixing the momentum scale, $\mathcal{A}$ reduces to a multiplication operator, so that our previous considerations again apply.

\section{F.6. The Regularized Light-Cone Expansion}

We are now in the position to perform the light-cone expansion. Our starting point is the operator product expansion (F.4.1). We choose $\hat{\mathcal{B}}$ to be the Fourier transform of a general multiplication or differential operator and introduce $\mathcal{B}$ in analogy to (F.5.5) as a non-local operator. After fixing the momentum scale, this operator reduces again to a multiplication or differential operator. Then the light-cone expansion can be performed exactly as described in [F5 and [F7, Section 2.5] (see also Section 2.2). Finally, one can transform the obtained formulas with $V_{\text {shift }}$ and $V_{\text {shear }}$ (again using the rules (F.5.2) and (F.5.3)). Since the resulting line integrals do not depend on the momentum scale, the regularization only affects the factors $T^{(n)}$. The condition that $\mathcal{B}$ should be regularity 
compatible can be described by the parameters $\tau_{i}^{\text {reg }}$. We thus obtain the formalism described in 4.2 .5 and 4.2 .6 .

We finally compare our constructions with those in [F7, Appendix D]. Clearly, the constructions here are much more general because they apply to any order in perturbation theory and may involve a chiral asymmetry. Moreover, the momentum shift operator $V_{\text {shift }}$ makes it possible to describe general surface states, and also we allow for a large shear of the surface states (whereas in [F7, Appendix D] we always assumed the shear to be small). Nevertheless, the basic idea that in order to preserve the gauge invariance in the presence of a regularization, one should replace the classical potentials by operators which are nonlocal on the microscopic scale already appeared in [F7, Appendix D] (see the explanation after $[\mathbf{F 7}$, eq. (D.26)]). Thus [F7, Appendix D] can be regarded as a technical and conceptual preparation which is superseded by the constructions given here. 



\section{Bibliography}

[BF] C. Bär and K. Fredenhagen (eds), Quantum Field Theory on Curved Spacetimes, Lecture Notes in Physics, vol. 786, Springer Verlag, Berlin, 2009.

[Ba] H. Baumgärtel, Analytic Perturbation Theory for Matrices and Operators, Operator Theory: Advances and Applications, vol. 15, Birkhäuser Verlag, Basel, 1985.

$[\mathrm{BF}] \mathrm{Y}$. Bernard and F. Finster, On the structure of minimizers of causal variational principles in the non-compact and equivariant settings, arXiv:1205.0403 [math-ph], Adv. Calc. Var. 7 (2014), no. 1, $27-57$.

[BD] J.D. Bjorken and S.D. Drell, Relativistic Quantum Mechanics, McGraw-Hill Book Co., New York, 1964.

[B1] V.I. Bogachev, Measure theory. Vol. I, Springer-Verlag, Berlin, 2007.

[B2] J. Bognár, Indefinite Inner Product Spaces, Springer-Verlag, New York, 1974, Ergebnisse der Mathematik und ihrer Grenzgebiete, Band 78.

[BLMS] L. Bombelli, J. Lee, D. Meyer, and R.D. Sorkin, Space-time as a causal set, Phys. Rev. Lett. 59 (1987), no. 5, 521-524.

[BDF] R. Brunetti, M. Dütsch, and K. Fredenhagen, Perturbative algebraic quantum field theory and the renormalization groups, arXiv:0901.2038 [math-ph], Adv. Theor. Math. Phys. 13 (2009), no. 5, 1541-1599.

[Ch] S.M. Christensen, Vacuum expectation value of the stress tensor in an arbitrary curved background: the covariant point-separation method, Phys. Rev. D (3) 14 (1976), no. 10, 2490-2501.

[CL] E.A. Coddington and N. Levinson, Theory of Ordinary Differential Equations, McGraw-Hill Book Company, Inc., New York-Toronto-London, 1955.

[C1] J.C. Collins, Renormalization, Cambridge Monographs on Mathematical Physics, Cambridge University Press, Cambridge, 1984.

[C2] A. Connes, Noncommutative Geometry, Academic Press Inc., San Diego, CA, 1994.

[DDMS] D.-A. Deckert, D. Dürr, F. Merkl, and M. Schottenloher, Time-evolution of the external field problem in quantum electrodynamics, arXiv:0906.0046v2 [math-ph], J. Math. Phys. 51 (2010), no. 12, $122301,28$.

[DFS] A. Diethert, F. Finster, and D. Schiefeneder, Fermion systems in discrete space-time exemplifying the spontaneous generation of a causal structure, arXiv:0710.4420 [math-ph], Int. J. Mod. Phys. A 23 (2008), no. 27/28, 4579-4620.

[D1] J. Dieudonné, Foundations of Modern Analysis, Academic Press, New York-London, 1969, Enlarged and corrected printing, Pure and Applied Mathematics, Vol. 10-I.

[D2] P.A.M. Dirac, Discussion of the infinite distribution of electrons in the theory of the positron, Proc. Camb. Philos. Soc. 30 (1934), 150-163.

[EG] H. Epstein and V. Glaser, The role of locality in perturbation theory, Ann. Inst. H. Poincaré Sect. A (N.S.) 19 (1973), 211-295.

[FS] H. Fierz and G. Scharf, Particle interpretation for external field problems in QED, Helv. Phys. Acta 52 (1979), no. 4, 437-453.

[F1] F. Finster, Derivation of field equations from the principle of the fermionic projector, arXiv:grqc/9606040 (unpublished preprint in German) (1996).

[F2] , Light-cone expansion of the Dirac sea with light cone integrals, arXiv:funct-an/9707003 unpublished preprint (1997).

[F3] _ _ Definition of the Dirac sea in the presence of external fields, arXiv:hep-th/9705006, Adv. Theor. Math. Phys. 2 (1998), no. 5, 963-985.

[F4] _ Local U(2,2) symmetry in relativistic quantum mechanics, arXiv:hep-th/9703083, J. Math. Phys. 39 (1998), no. 12, 6276-6290. 
[F5] _ Light-cone expansion of the Dirac sea to first order in the external potential, arXiv:hepth/9707128, Michigan Math. J. 46 (1999), no. 2, 377-408.

[F6] , Light-cone expansion of the Dirac sea in the presence of chiral and scalar potentials, arXiv:hep-th/9809019, J. Math. Phys. 41 (2000), no. 10, 6689-6746.

[F7] , The Principle of the Fermionic Projector, hep-th/0001048, hep-th/0202059, hepth/0210121, AMS/IP Studies in Advanced Mathematics, vol. 35, American Mathematical Society, Providence, RI, 2006.

[F8] - The principle of the fermionic projector: An approach for quantum gravity?, arXiv:grqc/0601128, Quantum Gravity (B. Fauser, J. Tolksdorf, and E. Zeidler, eds.), Birkhäuser Verlag, Basel, 2006, pp. 263-281.

[F9] _ Fermion systems in discrete space-time - outer symmetries and spontaneous symmetry breaking, arXiv:math-ph/0601039, Adv. Theor. Math. Phys. 11 (2007), no. 1, 91-146.

[F10] - A variational principle in discrete space-time: Existence of minimizers, arXiv:mathph/0503069, Calc. Var. Partial Differential Equations 29 (2007), no. 4, 431-453.

[F11] _ On the regularized fermionic projector of the vacuum, arXiv:math-ph/0612003, J. Math. Phys. 49 (2008), no. 3, 032304, 60.

[F12] _ From discrete space-time to Minkowski space: Basic mechanisms, methods and perspectives, arXiv:0712.0685 [math-ph], Quantum Field Theory (B. Fauser, J. Tolksdorf, and E. Zeidler, eds.), Birkhäuser Verlag, Basel, 2009, pp. 235-259.

[F13] Causal variational principles on measure spaces, arXiv:0811.2666 [math-ph], J. Reine Angew. Math. 646 (2010), 141-194.

[F14] - Entanglement and second quantization in the framework of the fermionic projector, arXiv:0911.0076 [math-ph], J. Phys. A: Math. Theor. 43 (2010), 395302.

[F15] The fermionic projector, entanglement, and the collapse of the wave function, arXiv:1011.2162 [quant-ph], J. Phys.: Conf. Ser. 306 (2011), 012024.

[F16] - A formulation of quantum field theory realizing a sea of interacting Dirac particles, arXiv:0911.2102 [hep-th], Lett. Math. Phys. 97 (2011), no. 2, 165-183.

[F17] , Perturbative quantum field theory in the framework of the fermionic projector, arXiv:1310.4121 [math-ph], J. Math. Phys. 55 (2014), no. 4, 042301.

[F18] _ Causal fermion systems - an overview, arXiv:1505.05075 [math-ph], Quantum Mathematical Physics: A Bridge between Mathematics and Physics (F. Finster, J. Kleiner, C. Rken, and J. Tolksdorf, eds.), Birkhäuser Verlag, Basel, 2016, pp. 313-380.

[F19] _ The chiral index of the fermionic signature operator, arXiv:1404.6625 [math-ph], to appear in Math. Res. Lett. (2016).

[F20] F. Finster et al, The quantum field theory limit of causal fermion systems, in preparation.

[FG1] F. Finster and A. Grotz, The causal perturbation expansion revisited: Rescaling the interacting Dirac sea, arXiv:0901.0334 [math-ph], J. Math. Phys. 51 (2010), 072301.

[FG2] _ A Lorentzian quantum geometry, arXiv:1107.2026 [math-ph], Adv. Theor. Math. Phys. 16 (2012), no. 4, 1197-1290.

[FG3] _ On the initial value problem for causal variational principles, arXiv:1303.2964 [math-ph], to appear in J. Reine Angew. Math. (2016).

[FGS] F. Finster, A. Grotz, and D. Schiefeneder, Causal fermion systems: A quantum space-time emerging from an action principle, arXiv:1102.2585 [math-ph], Quantum Field Theory and Gravity (F. Finster, O. Müller, M. Nardmann, J. Tolksdorf, and E. Zeidler, eds.), Birkhäuser Verlag, Basel, 2012, pp. 157182.

[FH] F. Finster and S. Hoch, An action principle for the masses of Dirac particles, arXiv:0712.0678 [math-ph], Adv. Theor. Math. Phys. 13 (2009), no. 6, 1653-1711.

[FK] F. Finster and N. Kamran, Spinors on singular spaces and the topology of causal fermion systems, arXiv:1403.7885 [math-ph] (2014).

[FK1] F. Finster and J. Kleiner, Causal fermion systems as a candidate for a unified physical theory, arXiv:1502.03587 [math-ph], J. Phys.: Conf. Ser. 626 (2015), 012020.

[FK2] - Noether-like theorems for causal variational principles, arXiv:1506.09076 [math-ph], Calc. Var. Partial Differential Equations 55:35 (2016), no. 2, 41.

[FK3] - A Hamiltonian formulation of causal variational principles, in preparation.

[FKT] F. Finster, J. Kleiner, and J.-H. Treude, An Introduction to the Fermionic Projector and Causal Fermion Systems, in preparation. 
[FM] F. Finster and O. Müller, Lorentzian spectral geometry for globally hyperbolic surfaces, arXiv:1411.3578 [math-ph], to appear in Adv. Theor. Math. Phys. (2017).

[FMR] F. Finster, S. Murro, and C. Röken, The fermionic projector in a time-dependent external potential: Mass oscillation property and Hadamard states, arXiv:1501.05522 [math-ph], J. Math. Phys. 57 (2016), 072303.

[FP] F. Finster and W. Plaum, A lattice model for the fermionic projector in a static and isotropic space-time, arXiv:0712.067 [math-ph], Math. Nachr. 281 (2008), no. 6, 803-816.

[FR1] F. Finster and M. Reintjes, The Dirac equation and the normalization of its solutions in a closed Friedmann-Robertson-Walker universe, arXiv:0901.0602 [math-ph], Classical Quantum Gravity 26 (2009), no. 10, 105021.

[FR2] _ A non-perturbative construction of the fermionic projector on globally hyperbolic manifolds I - Space-times of finite lifetime, arXiv:1301.5420 [math-ph], Adv. Theor. Math. Phys. 19 (2015), no. $4,761-803$.

[FR3] , A non-perturbative construction of the fermionic projector on globally hyperbolic manifolds II - Space-times of infinite lifetime, arXiv:1312.7209 [math-ph], to appear in Adv. Theor. Math. Phys. (2016).

[FR] F. Finster and C. Röken, Dynamical gravitational coupling as a modified theory of general relativity, arXiv:1604.03872 [gr-qc] (2016).

[FS] F. Finster and D. Schiefeneder, On the support of minimizers of causal variational principles, arXiv:1012.1589 [math-ph], Arch. Ration. Mech. Anal. 210 (2013), no. 2, 321-364.

[FT1] F. Finster and J. Tolksdorf, Bosonic loop diagrams as perturbative solutions of the classical field equations in $\phi^{4}$-theory, arXiv:1201.5497 [math-ph], J. Math. Phys. 53 (2012), no. 5, 052305.

[FT2] _ Perturbative description of the fermionic projector: Normalization, causality and Furry's theorem, arXiv:1401.4353 [math-ph], J. Math. Phys. 55 (2014), no. 5, 052301.

[Fr] F.G. Friedlander, Introduction to the Theory of Distributions, second ed., Cambridge University Press, Cambridge, 1998, With additional material by M. Joshi.

[FSW] S.A. Fulling, M. Sweeny, and R.M. Wald, Singularity structure of the two-point function quantum field theory in curved spacetime, Comm. Math. Phys. 63 (1978), no. 3, 257-264.

[GLR] I. Gohberg, P. Lancaster, and L. Rodman, Indefinite Linear Algebra and Applications, Birkhäuser Verlag, Basel, 2005.

[GR] I.S. Gradshteyn and I.M. Ryzhik, Table of Integrals, Series, and Products, Fourth edition prepared by Ju. V. Geronimus and M. Ju. Ceitlin, Academic Press, New York, 1965.

[G] A. Grotz, A Lorentzian quantum geometry, Dissertation Universität Regensburg, urn:nbn:de:bvb:355epub-231289, 2011.

[HLS1] C. Hainzl, M. Lewin, and E. Séré, Existence of a stable polarized vacuum in the Bogoliubov-DiracFock approximation, arXiv:math-ph/0403005, Comm. Math. Phys. 257 (2005), no. 3, 515-562.

[HLS2] , Self-consistent solution for the polarized vacuum in a no-photon QED model, arXiv:physics/0404047, J. Phys. A: Math. Theor. 38 (2005), no. 20, 4483-4499.

[Ha] P.R. Halmos, Measure Theory, Springer, New York, 1974.

[He] W. Heisenberg, Bemerkungen zur Diracschen Theorie des Positrons, Z. Phys. 90 (1934), $209-231$.

[J] F. John, Partial Differential Equations, fourth ed., Applied Mathematical Sciences, vol. 1, SpringerVerlag, New York, 1991.

[JZKGKS] E. Joos, H.D. Zeh, C. Kiefer, D. Giulini, J. Kupsch, and I.-O. Stamatescu, Decoherence and the Appearance of a Classical World in Quantum Theory, second ed., Springer-Verlag, Berlin, 2003.

[Ka] T. Kato, Perturbation Theory for Linear Operators, Classics in Mathematics, Springer-Verlag, Berlin, 1995.

[K1] M. Klaus, Nonregularity of the Coulomb potential in quantum electrodynamics, Helv. Phys. Acta 53 (1980), no. 1, 36-39.

[KS1] M. Klaus and G. Scharf, The regular external field problem in quantum electrodynamics, Helv. Phys. Acta 50 (1977), no. 6, 779-802.

[KS2] _ _ Vacuum polarization in Fock space, Helv. Phys. Acta 50 (1977), no. 6, 803-814.

[K2] H. Kleinert, Path Integrals in Quantum Mechanics, Statistics, Polymer Physics, and Financial Markets, fourth ed., World Scientific Publishing Co. Pte. Ltd., Hackensack, NJ, 2006.

[LL] L.D. Landau and E.M. Lifshitz, The Classical Theory of Fields, Revised second edition. Course of Theoretical Physics, Vol. 2. Translated from the Russian by Morton Hamermesh, Pergamon Press, Oxford, 1962. 
[L] H. Langer, Spectral functions of definitizable operators in Krein spaces, Functional Analysis (Dubrovnik, 1981), Lecture Notes in Math., vol. 948, Springer, Berlin, 1982, pp. 1-46.

[LM] H.B. Lawson, Jr. and M.-L. Michelsohn, Spin Geometry, Princeton Mathematical Series, vol. 38, Princeton University Press, Princeton, NJ, 1989.

[Lax] P.D. Lax, Functional Analysis, Pure and Applied Mathematics (New York), Wiley-Interscience [John Wiley \& Sons], New York, 2002.

[NS] G. Nenciu and G. Scharf, On regular external fields in quantum electrodynamics, Helv. Phys. Acta 51 (1978), no. 3, 412-424.

[OLBC] F.W.J. Olver, D.W. Lozier, R.F. Boisvert, and C.W. Clark (eds.), Digital Library of Mathematical Functions, National Institute of Standards and Technology from http://dlmf.nist.gov/ (release date 2011-07-01), Washington, DC, 2010.

[PS] M.E. Peskin and D.V. Schroeder, An Introduction to Quantum Field Theory, Addison-Wesley Publishing Company Advanced Book Program, Reading, MA, 1995.

[P] S. Pokorski, Gauge Field Theories, second ed., Cambridge Monographs on Mathematical Physics, Cambridge University Press, Cambridge, 2000.

[Ra] M.J. Radzikowski, Micro-local approach to the Hadamard condition in quantum field theory on curved space-time, Comm. Math. Phys. 179 (1996), no. 3, 529-553.

[RS1] M. Reed and B. Simon, Methods of Modern Mathematical Physics. I, Functional analysis, second ed., Academic Press Inc., New York, 1980.

[RS2] - Methods of modern mathematical physics. II. Fourier analysis, self-adjointness, Academic Press, New York-London, 1975.

[Ro] G.G. Ross, Grand Unified Theories, Frontiers in Physics, vol. 60, Benjamin/Cummings Publishing Co., Inc., Advanced Book Program, Reading, MA, 1984.

[Ru] W. Rudin, Principles of Mathematical Analysis, third ed., McGraw-Hill Book Co., New YorkAuckland-Düsseldorf, 1976, International Series in Pure and Applied Mathematics.

[S] J.J. Sakurai, Advanced Quantum Mechanics, Addison-Wesley Publishing Company, 1967.

[S1] G. Scharf, Finite Quantum Electrodynamics, Texts and Monographs in Physics, Springer-Verlag, Berlin, 1989.

[S2] F. Schwabl, Quantum Mechanics, third ed., Springer-Verlag, Berlin, 2002.

[Se] R. Serber, Linear modifications of the Maxwell field equations, Phys. Rev. 48 (1935), 49-54.

[T] M.E. Taylor, Partial Differential Equations. III, Applied Mathematical Sciences, vol. 117, SpringerVerlag, New York, 1997.

[U] E.A. Uehling, Polarization effects in the positron theory, Phys. Rev. 48 (1935), 55-63. 


\section{Notation Index}

$\left(\mathcal{H},\langle. \mid .\rangle_{\mathcal{H}}\right)$ - complex Hilbert space, 1,14

$\mathrm{L}(\mathcal{H})$ - bounded linear operators on $\mathcal{H}, 1$

$\mathcal{F} \subset \mathrm{L}(\mathcal{H})$ - set of linear operators on $\mathcal{H}, 1$

$\rho$ - universal measure, measure on $\mathcal{F}, 1$

$\lambda_{1}^{x y}, \ldots, \lambda_{2 n}^{x y}-$ non-trivial eigenvalues of $x y, 1$. 3, 5

| . | - spectral weight, 2,170

$\mathcal{L}(x, y)$ - Lagrangian, $2,170,263,353$

$\mathcal{S}(\rho)$ - causal action, $20170,263.353$

$\operatorname{tr}-\operatorname{trace}$ on $\mathcal{H}, 2$

$\mathcal{T}(\rho)$ - functional in boundedness constraint, 2

170, 263

\|. . - sup-norm on L $(\mathcal{H}), 2$

supp - support of measure, 3

$M:=\operatorname{supp} \rho-$ space-time, 3

$\mathcal{C}(x, y)$ - distinguishes time direction, 4, 32, 33

$S_{x}:=x(\mathcal{H})-$ spin space, 5

$\left(S_{x} M, \prec . \mid \cdot \succ_{x}\right)$ - spin space, $5,6,15,22$

$\pi_{x}$ - orthogonal projection on spin space, 5

$P(x, y)$ - kernel of fermionic projector, $5,7,25$

$\operatorname{Tr}_{S_{x}}$ - trace on spin space, 5

$A_{x y}$ - closed chain, $5,170,263,353$

$\prec . \mid . \succ_{x}-$ spin scalar product, $5,23,148$

|. | - absolute value of symmetric operator on $\mathcal{H}, 6,8$

$C^{0}(M, S M)$ - continuous wave functions, 6

$\psi^{u}$ - physical wave function, 6

$\Psi$ - wave evaluation operator, 7

$\operatorname{Symm}\left(S_{x}\right)$ - symmetric linear operators on $S_{x}$, 7

$\langle.,$.$\rangle - inner product on Clifford subspace, 7,10$

$<. \mid$. $>$ - inner product on Krein space, 8170

$\langle\langle. \mid\rangle$.$\rangle - scalar product on Krein space, 8$

$(\mathcal{K},<. \mid .>)-$ Krein space, 8

$P$ - fermionic projector, 9169

$D_{x, y}$ - spin connection, 9,11

$s_{x}$ - Euclidean sign operator, 10

$T_{x}$ - tangent space, 10

$\nabla_{x, y}-$ metric connection, 12

$\mathfrak{R}$ - curvature of causal fermion system, 12

C $\ell-$ Clifford section, 13

$(\mathscr{M},\langle.,\rangle$.$) - Minkowski space, 13$

$d \mu=d^{4} x$ - volume measure in Minkowski space, 13

$\left(S_{x} \mathscr{M}, \prec . \mid . \succ_{x}\right)$ - spinor space, $13,15,22,23$ $\bar{\psi} \phi=\prec \psi \mid \phi \succ-$ inner product on spinors, 13 . $23,148,170$

$\gamma^{j}-$ Dirac matrices, 13

(.|.) - scalar product on Dirac wave functions, 1470

$F(x)$ - local correlation operator, 14

$F_{*} \mu$ - push-forward measure, 14

$F(x)$ - local correlation operator, 14

$\varepsilon$ - regularization length, $15,173,266,348$

$\ell_{P}-$ Planck length, 15, 31, 49,173

$C^{0}(K, S \mathcal{M})$ - continuous Dirac wave functions on $K \subset \mathcal{M}, 18$

$C_{0}^{\infty}(K, S \mathcal{M})$ - smooth and compactly supported Dirac wave functions on $K \subset \mathcal{M}, 18$

$|\cdot|_{C^{k}(K)}-C^{k}$-norm on Dirac wave functions, 18 $\mathfrak{R}_{\varepsilon}$ - regularization operator, 18

$F^{\varepsilon}(x)$ - local correlation operator with UV regularization, 20

$\rho^{\varepsilon}$ - universal measure with UV regularization, 20 ]

$M^{\varepsilon}$ - regularized space-time, 20

$e_{x}^{\varepsilon}: \mathcal{H} \rightarrow S_{x} \mathscr{M}$ - evaluation map, 22

$\iota_{x}^{\varepsilon}:=\left(e_{x}^{\varepsilon}\right)^{*}$ - adjoint of evaluation map, 22

$P^{\varepsilon}(x, y)$ - regularized kernel of fermionic projector, 23, 25, 122, $132,134,173,266$. 348

$\delta$ - Dirac's delta distribution, 25

$\Theta$ - Heaviside function, 25

$\not k$ - Feynman dagger, 25

$T_{a}(x, y)$ - Fourier transform of lower mass shell, 26, 27, 85,178

$\mathrm{PP}$ - principal value, 27, 74

$\xi$ - short notation for Minkowski vector $y-x$, 27

$J_{1}, Y_{1}$ and $K_{1}-$ Bessel functions, 27

$\epsilon$ - sign function, 27

$\mathcal{S}_{\kappa, \lambda, \nu}$ - causal action including Lagrange parameters, 36

$Q(x, y)$ - first variation of the Lagrangian, 39 188, $194,285,353$

$Q$ - integral operator on Krein space $\mathcal{K}, 40$

$\mathfrak{I} \subset \mathcal{F}$ - inner region, 46

$\mathcal{S}_{\mathfrak{I}}[\rho, \phi]$ - inner variational principle, 46

$\mathcal{B}$ - external potential, 47, 50, 67, 70, 176,280 339349 
$\left(\mathcal{H}_{m},(. \mid .)_{m}\right)$ - Hilbert space of Dirac solutions of mass $m, 47$

$<. \mid$. $>$ - inner product on Dirac solutions, 48

$\mathcal{S}$ - fermionic signature operator, 48

$\hat{\Psi}(x), \hat{\Psi}(x)^{*}$ - fermionic field operators, 48

$Q^{\varepsilon}(x, y)$ - first variation of the Lagrangian with regularization, 49

$n_{\mathrm{p}}, n_{\mathrm{a}}-$ number of particles and anti-particle states, $50,66,177$

$\psi_{k}, \phi_{l}-$ particle and anti-particle states, 50,66 . 83, 177

$P_{m}^{\mathrm{vac}}(x, y)$ - fermionic projector corresponding to a vacuum Dirac sea of mass $m, 66,68$ 71, 264, 348

$p_{m}, k_{m}$ - fundamental solutions of the vacuum Dirac equation, 68

$s_{m}^{\vee}, s_{m}^{\wedge}$ - causal Green's functions of the vacuum Dirac equation, 68, 85,86

supp - support of distribution, 68

$J_{x}^{\vee}, J_{x}^{\wedge}-$ causal future and past, 68

$(. \mid .)_{t_{0}}$ - scalar product on Dirac wave functions, computed at time $t_{0}, 70$

$\tilde{s}_{m}^{\vee}, \tilde{s}_{m}^{\wedge}$ - causal Green's functions of the Dirac equation in an external potential, 71,86

$\tilde{k}_{m}$ - causal fundamental solution of the Dirac equation in an external potential, 72,78

$\mathcal{S}\left(\mathbb{R}^{4}, \mathbb{C}^{4}\right)$ - Schwartz space, 72

$\|\cdot\|_{p, q}-$ Schwartz norm, 72

$s_{m}$ - symmetric Green's functions of the vacuum Dirac equation, 74227

$b_{m}^{<}, b_{m}, b_{m}^{>}-$series of operator products, 76

$P^{\text {sea }}$ - fermionic projector describing Dirac seas, 78, 80, 119, 177, 179

$\tilde{R}_{\lambda}$ - resolvent in presence of external potential, 79

$R_{\lambda}$ - resolvent in Minkowski vacuum, 79

$\left.\right|_{t}$ - spatial normalization integral, 80

$\mathcal{N}_{t}$ - Cauchy surface at time $t, 83$

$\Pi^{\text {sea }}-$ projection operator on Dirac sea states, 83

$\mathcal{O}\left((y-x)^{2 p}\right)$ - order on the light cone, 84,178

$S_{m^{2}}$ - symmetric Green's functions of the

Klein-Gordon equation, 226

$S_{m^{2}}^{\vee}, S_{m^{2}}^{\wedge}$ - causal Green's functions of the Klein-Gordon equation, 85,235

$B=\mathcal{B}-m-$ external potential combined with the mass, 86

$S^{(l)}$ - mass expansion of $S_{a}, 87,91$

$\chi_{L / R}$ - chiral projectors, 91,180

$\Gamma$ - pseudoscalar matrix, $91,168,180$

$A_{L}, A_{R}-$ chiral potentials, 91, 180, 195, 287. 351

$m$ - parameter used for mass expansion, 92 . 176, 265, 279, 349

$Y$ - mass matrix, 92, 176, 265, 279, 349 $\int_{x}^{y}[l, r \mid n] \cdots-$ short notation for line integrals, 93394

$\partial_{z}^{J},(y-x)^{J}-$ multi-index notation, 93

Pexp, Pe - ordered exponential, 99

$s^{ \pm}$- Feynman propagator of the vacuum Dirac equation, 111

$S_{a}^{ \pm}-$Feynman propagator of the vacuum Klein Gordon equation, 111

$\tilde{s}^{ \pm}$- Feynman propagator of the Dirac equation in an external potential, 111

$\tilde{P}^{\text {res }}(x, y)$ - residual fermionic projector, 112

$\tilde{p}^{\text {res }}(x, y)$ - residual fundamental solution, 112

$T_{a}^{\text {reg }}-T_{a}$ with log-terms in $a$ removed, 114,179

$T^{(l)}$ - mass expansion of $T_{a}^{\mathrm{reg}}, 114,179$

$\tilde{P}^{\text {causal }}$ - causal contribution to fermionic projector, 114

$\tilde{P}^{\text {le }}$ - non-causal low energy contribution to fermionic projector, 114, $179,222,233$

$\tilde{P}^{\text {he }}-$ non-causal high energy contribution to fermionic projector, $115,179,222,233$

$g_{j k}$ - Lorentzian metric, 120

$\eta_{j k}-$ Minkowski metric, 120

$h_{j k}$ - linear perturbation of metric, 120

$\xi^{\varepsilon}-$ Minkowski vector $y-x$ with $i \varepsilon$-regularization, 123

$\ell_{\text {macro }}$ - macroscopic length scale, 125,173

$\nabla$ - derivation on the light cone, $127,133,186$. 278

$T_{[p]}^{(n)}-$ ultraviolet regularized $T^{(n)}, 130132$ 184278

$T_{\{p\}}^{(n)}$ - factor in continuum limit describing the shear of surface states, 131, 132, 184, 278

\begin{tabular}{lllll}
$\xi_{[p]}^{(n)}-$ ultraviolet regularized factor $\xi$, & 132 & 184 \\
\hline
\end{tabular} 278

$z_{[p]}^{(n)}-$ abbreviation for $\left(\xi_{[p]}^{(n)}\right)^{2}, 132,184,278$

$T_{\circ}^{(n)}$ - stands for $T_{\{p\}}^{(n)}$ or $T_{[p]}^{(n)}, 132185$

deg - degree on light cone, 132 ,185

$L$ - degree of simple fraction, 133, 185, 277

$c_{\text {reg }}$ - regularization parameter, $133,[185,277$

$s, l$ - light-cone coordinates, 135

$u, v$ - momenta associated to light-cone coordinates, 135

$\left(\mathfrak{f}_{ \pm}^{L / R}\right)$ - double null spinor frame, 147

$A_{x y}^{0}$ - unperturbed closed chain, 148

$\lambda_{ \pm}, F_{ \pm}$- eigenvalues and spectral projections of unperturbed closed chain, 148, 192

$\psi$ - operator commuting with $F_{ \pm}, 148$

$\mathfrak{F}_{s s^{\prime}}^{c c^{\prime}}$ - matrix elements in double null spinor frame, 149

$g$ - number of generations, 172,184

$m_{\beta}-$ masses of charged fermions, $172,264,348$

$E_{P}-$ Planck energy, 173

$\varepsilon_{\text {shear }}$ - shear parameter, $174,186,211$

$P^{\text {aux }}$ - auxiliary fermionic projector, 176,191

279348 
$e$ - coupling constant, 180,241

$\Phi$ - scalar potential, 180

$\Xi$ - pseudoscalar potential, 180

, ' ... ' - short notation for sectorial projection, $184,281,350$

$\mathcal{S}_{\mu}$ - causal action involving one Lagrange multiplier, 187,263

$\mathcal{L}_{\mu}$ - Lagrangian involving one Lagrange multiplier, 187,263

$D$ - partial derivative of $\mathcal{L}_{\mu}, 193$

$A_{\mathrm{v}}$ - vector potential, 195

$A_{\mathrm{a}}-$ axial potential, 195

$\Lambda_{L / R}^{x y}$ - integrated chiral potentials, 195

$\nu_{L / R}$ - chiral phases, 195

$\lambda_{ \pm}^{L / R}, F_{ \pm}^{L / R}$ - eigenvalues and corresponding spectral projectors of closed chain, 195

$\mathcal{R}$ - appears in EL equations to degree four, 197

$o\left(|\vec{\xi}|^{k}\right)$ - order at the origin, 198,298

$j_{\mathrm{a}}-$ axial current, 198

c.c. - complex conjugate simple fraction, 198

$J_{L / R}$ - chiral Dirac current, 199,298

$J_{\mathrm{a}}-$ axial Dirac current, 199

$\asymp-$ denotes a contribution, 199

$\mathfrak{g}$ - generation mixing matrix, 203

$U(x)$ - local axial transformation, 204

$U(x)$ - local axial transformation, 205

$c_{\alpha}, d_{\alpha}-$ sectorial projection of $\mathfrak{g}, 206$

$U(x)$ - general local transformation, 207

$U(k)$ - nonlocal homogeneous transformation, 211

$\epsilon_{i j k l}-$ totally antisymmetric tensor in Minkowski space, 213

$U(x, y)$ - microlocal chiral transformation, 218 307,352

$s_{[p]}-$ smooth contribution to $T_{[p]}^{(1)}, 223$

\begin{tabular}{ll|l|l|l|l|}
$f_{[p]}^{\beta}-$ contribution to $s_{[p]}, 224$ & 225 & 227 \\
\hline
\end{tabular}

* - convolution, 224

$\hat{f}_{[p]}^{\beta}$ - Fourier transform of $f_{[p]}^{\beta}, 224,414$

$C_{0}, C_{2}$ - regularization parameters, 225

$t_{m}-$ vacuum Dirac sea, 227

$\tilde{A}-$ auxiliary potential, 229

$r$ - radius $|\vec{\xi}|, 231$

$\overline{t_{m}}$ - upper Dirac mass shell, 233

$H$ - full Hamiltonian, 235

$H_{0}$ - free Hamiltonian, 235

$B$ - perturbation of Hamiltonian, 235

$B_{\text {int }}$ - perturbation in interaction picture, 235

$F_{i j}$ - field tensor, 239

$M$ - bosonic mass, 239,241

$J_{\mathrm{s}}-$ scalar Dirac current, 242

$J_{\mathrm{p}}-$ pseudoscalar Dirac current, 242

$J_{\mathrm{b}}$ - bilinear Dirac current, 243

$\mathcal{S}(\hat{\mathcal{M}})$ - Schwartz functions in momentum space, 247

$P_{ \pm}-$half filled Dirac sea, 249

$\mathfrak{n}(x, y)$ - nonlocal kernel, 258
$\mathcal{S}(\mathscr{M} \times \mathscr{M})$ - Schwartz kernel in Minkowski space, 258

$P^{N}(x, y)$ - neutrino sector of the vacuum fermionic projector, 264,348

$P^{C}(x, y)$ - charged component of the vacuum fermionic projector, 264, 348

$\tilde{m}_{\beta}-$ neutrino masses, 264348

$U_{\text {MNS }}$ - Maki-Nakagawa-Sakata (MNS) matrix, $265,289,355$

$M_{\alpha}$ - bosonic masses, 265

$c_{\alpha}$ - coupling constant, 265

$R_{j k}-$ Ricci tensor, 265, 312

$R$ - scalar curvature, 265

$T_{j k}$ - energy-momentum tensor, 265312

$\Lambda$ - cosmological constant, 265, 345 381

$\kappa$ - gravitational constant, 265 344 381

$\delta$ - length scale of shear and general surface states, 277

$P_{\text {aux }}^{N}$ - neutrino sector of auxiliary fermionic projector, 279 , 348

$P_{\text {aux }}^{C}$ - charged component of auxiliary fermionic projector, 279,348

$X$ - chiral asymmetry matrix, 279,349

$\tau_{\text {reg }}$ - dimensionless parameter for high-energy states, 279, 291,349

$t$ - distribution composed of vacuum Dirac seas, 279349

$\tilde{t}$ - distribution composed of Dirac seas in the presence of an external potential, 280

$\check{\xi}$ - real lightlike vector in $\iota$-formalism, 282

$\check{P}_{m}^{\varepsilon}-$ lightlike component of vacuum fermionic projector in $\iota$-formalism, 282

$\iota_{\circ}^{(n)}$ - vector describing regularization in $\iota$-formalism, 282

$\lambda_{n c s}^{x y}, F_{n c s}^{x y}-$ eigenvalues and corresponding spectral projectors of closed chain, 285

$L_{[p]}^{(n)}=T_{[p]}^{(n)}+\tau_{\mathrm{reg}} T_{[R, p]}^{(n)} / 3,286,350$

$U_{c}$ - unitary matrix involving gauge phases, 287

$A_{L}$ - left-handed gauge potential, 290, 321

$A_{R}^{N}$ - right-handed potential in neutrino sector, $290,319,321$

$A_{R}^{C}$ - right-handed potential in charged sector, $290,319,321$

$p_{\text {reg }}-$ determines scaling $\tau_{\text {reg }}=(m \varepsilon)^{p_{\text {reg }}}, 291$

$\nu_{n c}, I_{n c}$ - eigenvalues and corresponding spectral projectors of matrix involving phases, 292

$\Delta Q(x, y)$ - first order perturbation of $Q(x, y)$, 293353

$\mathcal{K}_{n c}-$ matrices entering the EL equations to degree four, 294

$\mathcal{K}_{c}$ - matrices entering the EL equations to degree four, 294

$\mathcal{Q}_{c}$ - matrices entering the EL equations to degree four, 296

$j_{L / R}-$ chiral bosonic current, 298,395 
$\mathfrak{J}_{c}$ - matrix composed of current and mass terms, 298

$U(k)$ - homogeneous transformation, 301

$\Omega$ - absolute value of energy, 301, 352

$Z(k)$ - generator of homogeneous transformation, 301

$L(k), R(k)$ - chiral components of $Z(k), 301$

$\mathfrak{c}_{0}(k), \mathfrak{c}_{2}(k)$ - parameters in microlocal chiral transformation, 303,352

$S_{0}, S_{2}$ - signature matrices, 304

$\mathcal{D}_{\text {flip }}$ - Dirac operator including microlocal chiral transformation, 309,353

$\mathfrak{e}_{1}, \ldots, \mathfrak{e}_{6}-$ orthonormal basis, 310

$P^{(0)}(x, y), A_{x y}^{(0)}, \lambda_{n c s}^{(0)}-$ objects of the vacuum, 313

$F_{L / R}^{j k}-$ chiral field tensor, 315,395

$M_{n}^{(l)}$ - short notation for factors $T_{[0]}^{(l)}$ or $L_{[0]}^{(l)}$, 326

$\operatorname{Symm}\left(\mathbb{C}^{n}\right)-$ Hermitian $n \times n$-matrices, 331

$\mathfrak{S}_{n}=\operatorname{Symm}\left(\mathbb{C}^{6}\right) \oplus \operatorname{Symm}\left(\mathbb{C}^{6}\right)-$ left- and right-handed matrices, 331

$\mathcal{G}$ - dynamical gauge group, 331,354

$\mathfrak{g}$ - dynamical gauge algebra, 331

$\mathcal{S}_{\text {eff }}$ - effective action, $332,333,370$

$\check{\pi}$ - sectorial projection operator, 334

$\check{\pi}_{\tau}$ - sectorial projection with modified right-handed neutrino coupling, 334

$A_{L}^{\mathrm{sec}}, A_{R}^{\mathrm{sec}}-$ chiral potentials carrying only sector indices, 351

$U_{L}^{\operatorname{mix}}, U_{R}^{\operatorname{mix}}$ - general mixing matrices, 352

$Z(k, z)$ - generator of microlocal chiral transformation, 352

$L(k, z), R(k, z)$ - chiral components of $Z(k, z)$, 352

$\mathfrak{g}$ - dynamical gauge algebra, 354

$\mathcal{A}=\left(A_{L}, A_{R}\right)$ - element of dynamical gauge algebra, 354

$\mathcal{S}_{\text {free }} \subset \mathcal{G}$ - free gauge group, 355

$A^{\mathrm{em}}$ - free $\mathfrak{u}(1)$-potential, 355

$W$ - left-handed $\mathfrak{s u}(2)$-potential, 355

$G$ - free $\mathfrak{s u}(3)$-potential, 355

$W_{\mathrm{MNS}}-W$-potential in neutrino block, 355

$W_{\mathrm{CKM}}-W$-potential in quark blocks, 355

$U_{\mathrm{CKM}}$ - Cabibbo-Kobayashi-Maskawa (CKM) matrix, 355

$A_{x y}^{\mathrm{vac}}$ - closed chain of the vacuum, 357

$\check{\pi}: \mathbb{C}^{3} \rightarrow \mathbb{C}^{3}-$ orthogonal projection to $\operatorname{span}(1,1,1), 359$

$\mathfrak{I}_{1}$ - projection on neutrino sector, 362

$\mathfrak{g}_{L} \subset \mathfrak{g}$ - left-handed dynamical gauge potentials, 363

$\mathfrak{g}_{L}^{\prime}-$ commutant of $\mathfrak{g}_{L}, 364$

$\mathrm{pr}_{L}$ - projection on left-handed component, 365

$m_{W}, m_{Z}-$ masses of $W$ and $Z$ bosons, 374

$\Theta_{W}-$ Weinberg angle, 374
$T_{a}^{(n)}$ - term of mass expansion for $a>0,409$

$f(x, y)$ - smooth contribution to $\chi_{L} P(x, y)$, 410,412

$N(a)$ - smooth contribution to $T_{a}, 412$

$\rho_{\beta}$ - weight factor, 417

$V_{\text {shift }}, V_{\text {shear }}$ - operators in the regularized causal perturbation theory, 419 


\section{Subject Index}

$\iota$-formalism, 282

action

causal, 2, see also causal action

effective, 239 see also effective action

adjoint spinor, $13,23,148,170$

anti-particles, 50, 83,177

block, 354,356

lepton, 356

quark, 356

bosonic field

classical, vii, 41, 51, 177, 181

quantized, viii, $41,53,55,181$

Cauchy problem, 45,234

causal action, 2, 170, 263, 353

Euler-Lagrange equations of, 39, 41, 188,190

minimizer, 153

causal action principle, 2

finite-dimensional setting, 2

infinite-dimensional setting, 2

causal diamond, 73

causal fermion system, 1

causal fundamental solution, 69, 72,78

causal perturbation expansion, 51 $58,177,280$

regularized, 51

regularized with neutrinos, 419

causal structure, 3,46

causal variational principle, 2, see also causal action principle

in the compact setting, 45

causality, $3,34,46$

of the light-cone expansion, 119

of the perturbation expansion, 68, 84, 119

causality compatibility condition, 280 349,424

causality violation, 226, 228

for spacelike distances, 233

in experiments, 228

chiral asymmetry matrix, 279, 349

chiral cancellation, 266, 271

classical field

bosonic, vii $41,51,177,181$

Clifford extension, 10

Clifford section, 13

Clifford subspace, 7,13 closed chain, 5, 170, 263,353

spectral analysis of, $145,152,393$

Compton scale, 43

computer algebra, $101,300,394$

conservation law

for charge, 43

for energy and momentum, 44

constraint

boundedness, 2

trace, 2

volume, 2

continuum limit, 49, 131,183

analysis in the, 133, 186

formalism of the, 122

contraction rule, $127,132,184,278$

correspondence to Minkowski space, 13,34

$M^{\varepsilon} \leftrightarrow \mathscr{M}, 20$

$S_{x} M \leftrightarrow S_{x} \mathscr{M}, 22$

causal structure, 30

kernel of the fermionic projector, 23

physical wave function, 23

time direction, 34

cosmological constant, 265 345, 381

coupling constant

electroweak, 374

gravitational, 265, 344, 381

naked, 373

of axial field, 168,240

strong, 374

current

axial, 198

axial Dirac, 199

bilinear Dirac, 243

chiral Dirac, 199,396

pseudoscalar Dirac, 242, 399

scalar Dirac, 242, 399

curvature

of causal fermion system, 12

decoherent replica of the universal measure, 55

degree on the light cone, 126, 132, 185, 277, 279

Dirac equation

vacuum, 13

Dirac sea, 14, 25, 52,66

Dirac sea configuration, $14,25,66$

Dirac wave function, 13 
Dirac-Maxwell equations, 234

modified by convolution terms, 228

Dirac-Yang/Mills equations, 234

Dirac-Yang/Mills-Higgs action, 239

distribution

of Hadamard form, 48

regular, 27

tempered, 72

double null spinor frame, 147

dynamical gravitational coupling, $52,266,345$

Dyson series, 236

effective action, $239,330,332,370$

effective Lagrangian

Dirac, $333,334,370$

Einstein-Hilbert, 333, 371, 381

Yang-Mills, 340, 371

Einstein equations, 52, 265, 345

electromagnetic field, 243

energy-momentum tensor

of Dirac field, 312

of gauge fields, $315,381,400$

equivalence principle, 35

error term

higher order in $\varepsilon /|\vec{\xi}|, 125,134,186$

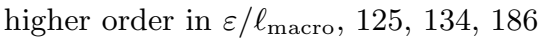

smooth contribution, 123 131 186

Euler-Lagrange equations, 39, 41, 188, 190

in the continuum limit, 190,388

structural contributions, 319

evaluation map, 22,23

evaluation on the light cone

pointwise, 291

weak, $133,185,277,350$

external field problem, 47, 67, 177, 181

external potential, 46, 47, 181

fermionic field

quantized, vii 51,179

fermionic operator

kernel of, 5,6

fermionic projector, 9170

auxiliary, 176, 279 348

axial current term, 198

bilinear logarithmic term, 323, 361

causal contribution, 114

chiral bosonic current term, 298

curvature term, 313

Dirac current term, 199,298

energy-momentum term, 311

field tensor term, 325,362

homogeneous, 122,173

in the presence of an external potential, 80

119,179

in the presence of linearized gravity, 121

kernel of, 5, 6, 23, 170

light-cone expansion of, 119,179 non-causal high energy contribution, 115 .

222233

non-causal low energy contribution, 114,222 233

of the vacuum, 66, 172,264

regularized kernel of, $23,25,122,132,134$

173

residual, 112

smooth contributions to, 115,222

unregularized in position space, 119,179

vector-scalar structure, 33173

fermionic signature operator, 47

Feynman dagger, 25

Feynman diagram, 234

bosonic loop, 236

fermionic loop, 238

tree diagram, 73, 177,236

Feynman propagator, 111

field equations in the continuum limit, 52,168 347

corrections, 340

due to microlocal chiral transformation, 219341

due to smooth, noncausal contributions to fermionic projector, 223, 227, 232, 341

sectorial corrections, 338, 341

for gauge fields, 340

Fock space, 179,181

frequency

constraint of negative, 252,254

functional calculus

in causal perturbation expansion, 79

gauge algebra

dynamical, 331,354

gauge field, 91

classical, vii, $41,51,177$

non-abelian, 92

quantized, viii $41,53,55$

gauge group

dynamical, 331, 354

free, 355

gauge potential, 51,91

algebraic constraint for, 354

dynamical, 331

free, 354

gauge symmetry, 196

gauge transformation, 196

axial, 239

behavior of regularized fermionic projector, 427

generation mixing matrix, 203

generations

why several, 202

grand unified theory (GUT), 373

gravitational field, 47, 180, 187, 243

effect on local trace, 142 
linearized, 120

Green's function

advanced, 68,86

retarded, 68, $85,86,235$

symmetric, 74,226

Hadamard condition, 181

Hadamard state, 48

Higgs field, 238, 239,383

Higgs mechanism, 199, 238, 383

Hilbert space

effective, 17

separable, 1,14

homogeneous perturbation analysis on the light cone, 252

by varying the momenta, 248

homogeneous transformation

in the high-frequency limit, 211

in the low-frequency region, 218, 247

identity of indiscernibles, principle of, 21

index theory, 48

inherent structures, 3

initial value problem, 45

inner factor $\xi$, 126, 132,184

integration-by-parts rule, $127,133,185,278$

interaction picture, 235

jet bundle, 47

Krein space, 8

Lagrangian, 2170

causal, 263,353

effective, see also effective Lagrangian, 333

life-time of universe, 390

light-cone coordinates, 135

light-cone expansion, $84,178,280$

explicit formulas, 394 397 400

for the residual fermionic projector, 113

in momentum space, 110,111

of fermionic projector, 119,179

of the Green's function, 93

to first order, 88

phase-free contribution, 100

phase-inserted contribution, 100

regularization, 131, 184, 428

resummation of, 98,179

lightlike, 3

local correlation operator, 14,20

rescaling of, 37

local gauge freedom, 35

local gauge principle, 35

local gauge transformation, 35

local trace, 36,142

arrange to be constant, 37

effect of chiral potentials on, 142

logarithmic mass problem, 114 logarithmic pole on the light cone, 200

Lorentzian manifold

globally hyperbolic, 47

Maki-Nakagawa-Sakata (MNS) matrix, 289

mass cone, 173

mass expansion, 86, 139, 272, 277, 282

mass matrix, 92 , 176 265, 279, 349

dynamical, 92

mass oscillation property, 48

mass shell, 25,173

mass term, 199

measure

convex combination of, 53

locally finite, 36

push-forward, 14

regular Borel, 2

support of, 3

universal, 1] see also universal measure

microscopic mixing of physical wave functions, 55

Minkowski space, 13

mixing matrix

Cabibbo-Kobayashi-Maskawa (CKM) matrix, 355

general, 352

Maki-Nakagawa-Sakata (MNS) matrix, 265. 289355

necessity of, 324369

modified Dirac-Maxwell equations

in variational form, 229

multi-index, 93

neutrino

chiral, 264, 275

massive, 264

non-causal correction

by convolution terms, 226

of higher order, 232

noncommutative geometry, 182

normalization

mass, 70

spatial, 70,71

order

at the origin, 198,298

ordered exponential, 99

outer factor $\xi, 126,132,185$

particles and anti-particles, 50, 83,177

Pauli exclusion principle, 34

perturbation expansion

causal, 51

regularized causal, 51

Planck energy, 173

Planck length, $15,31,49,173$

potential

admissible, 354 
algebraic constraint for, 330

auxiliary, 229

axial, 195, 394

axial conformal, 244

axial gravitational, 244

bosonic, 51, 67, 176, $177,280,349$

chiral, 91, 180 195, 287, 351

external, 47, 67, 70, 176, 177, 181, 280, 349

gauge, 51 see also gauge potential

non-dynamical, 246

nonlocal, 219, 247, 258, 390

\begin{tabular}{llllll} 
pseudoscalar, & 92 & 180 & 245 & 399 \\
\hline
\end{tabular}

pseudoscalar differential, $180,202,397$

scalar, 92, 180 240, 245,399

effect on local trace, 142

vector, 195,394

vector differential, 204

principal value, 27,74

quantum corrections, 233

quantum field

bosonic, viii, 41 , $53,55,181$

fermionic, vii, 51,179

quasi-homogeneous ansatz, 218, 258

regularization, 181

$i \varepsilon-, 122,240$

by cutoff, 241

by mollification, 19

linear combination of $i \varepsilon-, 128$

macroscopic away from light cone, 267, 271

method of variable, 16, 175

naive, 266,267

non-generic, 200, 255

of the light-cone expansion, 131, 184,282

spherically symmetric, 270,271

ultraviolet (UV), $14,18,121,173,348$

regularization expansion, 128, 139

regularization length, 15,173

regularization operator, 18

regularization parameter, 50, 133, 185,277

basic, $133,134,186$

renormalization, 237

by counter terms, 181

point splitting method, 181

residual argument, 112

resolvent

in causal perturbation expansion, 79 resummation

of current and mass terms, 409

of light-cone expansion, 98,179

of smooth contributions, 115,409

Schwartz norm, 72

Schwartz space, 72

sector, 263

charged, 264 neutrino, 264

sectorial projection, 176, $184,281,350$

sheaf, 12

shear contribution

analysis of, 399

by the local axial transformation, 209, 210

by the microlocal chiral transformation, 220. 308

shear of surface states, 131, 141

shear state, 273

sign operator

directional, 11 ,

Euclidean, 10

simple fraction, $133,185,277$

singular mass limit, 267

space-time, 3

space-time point

regular, 10

singular, 10

spacelike, 3, 46.

spectral geometry, 48

spectral weight, 2,170

spin connection, 9,11

spin dimension, 1

spin scalar product, $5,23,148$

spin space, 5

spin structure, 13

spin-connectable, 11

spinor space, 13

splice map, 12

spontaneous block formation, 347, 355, 356

spontaneous symmetry breaking, 199,239

state

massive, 274

null, 274

state stability, 267, 275

surface layer integral, 42

surface state

half-occupied, 174

restriction to, 174

symmetry

approximate, 44

of the Lagrangian, 43

tangent cone measure, 13

tangent space, 10

tangential derivative, 99

testing on null lines, $154,188,385$

time direction, 4, 32,33

time evolution, 45

timelike, 3,46

properly, 10

topological fermion system, 13

topological measure space, 3

topological spinor bundle, 13

topological vector bundle, 12

transformation of the fermionic projector 
general local, 207

local axial, 205, 397

ruling out this transformation, 403

microlocal chiral, 218, 300, 352, 389

ultrarelativistic wave packet, 154, 189, 385

unitary in a compact region, 171

unitary variation in Krein space, 41, 171

units, 180

universal measure, 1,20

variation of, 2 see also variation of universal measure

variation of physical wave functions, 38

smooth and compact, 38

variation of universal measure, 2

by multiplication, 36

by push-forward, 36

by unitary variation in Krein space, 41,171

by variation of physical wave functions, see also variation of physical wave functions

variational principle

causal, 2,45

inner, 46

vector component

is null on the light cone, 174

wave evaluation operator, 7

wave function, 6

continuous, 6

Dirac, 13

physical, 6, 170

weight factor, 417 



\title{
Back Cover
}

\begin{abstract}
About this book
This monograph introduces the basic concepts of the theory of causal fermion systems, a recent approach to the description of fundamental physics. The theory yields quantum mechanics, general relativity and quantum field theory as limiting cases and is therefore a candidate for a unified physical theory. From the mathematical perspective, causal fermion systems provide a general framework for describing and analyzing non-smooth geometries and "quantum geometries." The dynamics is described by a novel variational principle, called the causal action principle.

In addition to the basics, the book provides all the necessary mathematical background and explains how the causal action principle gives rise to the interactions of the standard model plus gravity on the level of second-quantized fermionic fields coupled to classical bosonic fields. The focus is on getting a mathematically sound connection between causal fermion systems and physical systems in Minkowski space.

The book is intended for graduate students entering the field, and is furthermore a valuable reference work for researchers in quantum field theory and quantum gravity.
\end{abstract}

\section{About the author}

Felix Finster studied physics and mathematics at the University of Heidelberg, where he graduated in 1992 with Claus Gerhardt and Franz Wegner. In 1992-1995 he wrote his PhD thesis at ETH Zürich with Konrad Osterwalder. In 1996-1998 he was a postdoc with Shing-Tung Yau at Harvard University. From 1998-2002 he was member of the Max Planck Institute for Mathematics in the Sciences in Leipzig in the group of Eberhard Zeidler. Since 2002 he has been full professor of mathematics at the University of Regensburg. He works on problems in general relativity and quantum field theory. 